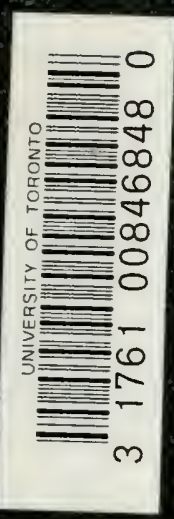



Digitized by the Internet Archive in 2007 with funding from Microsoft Corporation 


Thes $x$ X 


\section{A TEX'T BOOK}

OF

\section{P.H Y S I O L O.G Y}

BY

M. FOSTER, M.A., M.D., LL.D., F.R.S.

PROFESSOR OF PHYSIOLOGY IN THE UNIVERSITY OF CAMBRIDGE AND FELLOW OF TRINITY COLLEGE, CAMBRIDGE

REVISED AND ABRIDGED

FROM TIE AUTIIOR'S TEXT BOOK OF PIIYSIOLOGY

IN FIVE VOLUMES

WITH AN APPENDIX ON

The Chemeal Basis of the Animal Body

By A. SIERIDAN LEA, M.A., D.Sc, F.R.S.

UNIVERSITY LeCTIRER IN PHYSIOLOG IN THE UNIVERSTTY OF CAMBRIDGE

FELLOW OF CAIUS TOLLEGE, CAMBRIDGE

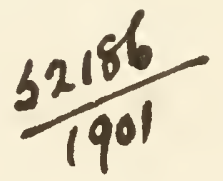

Nom Mork

THE MACMILLAN COMPANY

LONDON: MACMILLAN \& CO., LTD.

1900 
Copyright, 1894,

Br MACMILLAN AND CO.

New Edition, with Additions.

Copyrigh, 1596,

By THE MACMILLAN COMPANY.

New edition printed August, 1896. Reprinted August, 1897: September, 1898 ; August, Igoo.

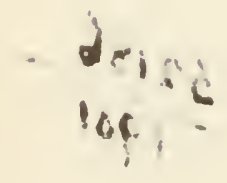

Norbood 3 Bress

J. S. Cunhing \& $\mathrm{C} 0$, Berwick \& Smith Nurwood Mess. U.S.A. 


\section{PREFACE.}

Is accordance with the wishes of some influential friends, I have prepared this abridged edition of my text book. The abridgment has been effected by omitting all the histological matter, and all discussions of a too theoretical nature. Otherwise, beyond such changes as the advance of science seems to call for, the text which is left is the same as in the full edition.

II. FOSTER.

SEPTEMBer, 1894. 



\section{CONTENTS.}

\section{INIRODUCTION.}

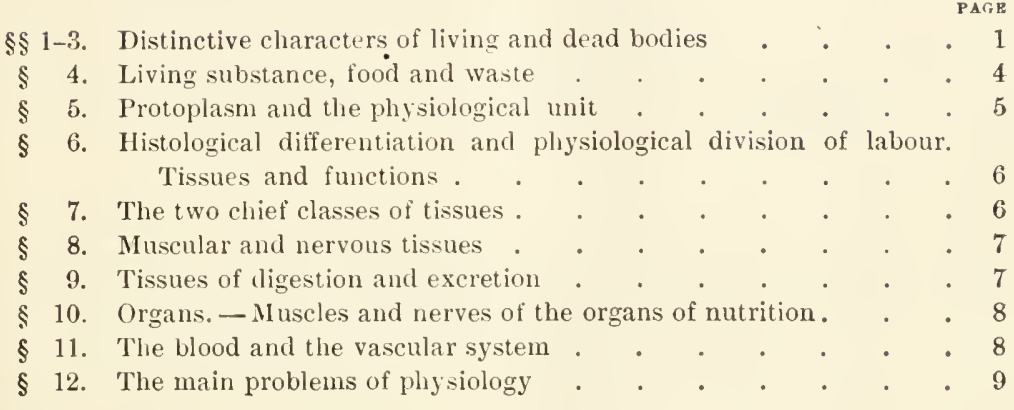

\section{BOOK I.}

BLOOD. THE TISSUES OF MOVEMENT.

THE VASCULAR MECHANISM.

\section{CHAPTER I.}

BLOOD.

$\S$ 13. The general work of the blood.

\section{SECTION I.}

The Clottixg of Blood.

$\S 14$. The phenomena of clotting . . . . . . . . 15

$\S 15$. The characters of fibrin . . . . . . . . . 17

$\S 16$. The features of serum. Paraglobulin: its characters . . . 18

$\S$ 17. Serum-albumin: its characters . . . . . . . . 19

$\S$ 18. The circumstances which affect the rapidity of clotting . . . 20 
\$ 19. The preparation of piasmine and fibrinogen . . . . . 22

$\$ 20$. Fibrin-ferment: its action. Nature of the process of clotting . . 23

$\$ 21$. Why blood clots when shed . . . . . . . . . . 26

$\S 22$. The influence on clotting exerted by the living blood vessels . . 27

$\S 23$. The nature of this influence; the action of the white corpuscles . . 29

\section{SECTION II.}

\section{The Corpuscles of tile Blood.}

\section{The Red Corpuscles.}

$\S 24$. The structure of the red corpuscles; laky blood; stroma, and hæmoglobin

$\$ 25$. The number of red corpuscles in human blood; method of enumeration . . . . . . . . . . 33

$\$ 26$. The destruction of red corpuscles . . . . . . . . . . 34

$\$ 27$. The formation of red corpuscles in the embryo and in the adult; hæmatoblasts . . . . . . . . . . . 35

\section{The White Corpuscles.}

$\$ 28$. The structure of the white corpuscles; characters of the cell-substance

$\S 29$. The chemical bodies present in white corpuscles . . . . .

$\$ 30$. The white corpuscles as a type of living matter; metabolism, katabolic and anabolic changes. The nature and relations of the 'granules '; living substance, food and waste. . . . . . 39

\$31. The origin of the white corpuscles. Leucocytes . . . . . . 42

$\S 32$. The disappearance of the white corpuscles. Their influence on the plasma. Different kinds of white corpuscles . . . . . 13

\section{Blood Platelets.}

§33. 'The characters of blood platelets . . . . . . . . 44

\section{SECTION III.}

The Chemical Composition of Blood.

\$34. General chemical characters . . . . . . . . . 46

\$3. Chemical composition of serum . . . . . . . . . . 46

\$36. Chemical composition of red corpuscles _ . . . . . . . 47

\$37. Chemical composition of white corpuseles . . . . . . 48

\section{SECTION IV.}

The Quantity of Bloon and its Distribution in tile Bony.

$\S 38$. The determination of the quantity of blood in the body, and the main

facts of its distribution . . . . . . . . . . . . 49 


\section{CHAPTER II.}

\section{The Contractile Tissues.}

§39. The movements of the body carried out by means of various kinds of contractile tissues . . . . . . . . . 51

\section{SECTION I.}

The Phenonena of Muscle and Nerve.

Muscular and Nervous Irritability.

§40. Irritability ; contractility; stimuli . . . . . . . 53

$\S 41$. Independent muscular irritability ; action of urari . . . . . 54

$\S 42$. Simple and tetanic contractions . . . . . . . . . . 55

$\S 43$. The muscle-nerve preparation . . . . . . . . . 55

$\S 44$. Various forms of stimuli. Induction Coil. Key. Magnetic Interruptor. Electrodes. Method of graphic record . . . . 56

The Phenomena of a Simple Muscular Contraction.

$\S 45$. The muscle-curve. Myographs, Time measurements. Signals • 65 $\S 46$. Analysis of a simple muscle-curve . . . . . . . . . 71

$\S 47$. Variations of the muscle-curve. The shortening accompanied by thickening. . . . . . . . . . . 74

$\S 48$. Simple muscular contractions rare in the living body . . . . $\quad$. 75

Tetanic Contractions.

$\S 49$. Tetanic contractions. Analysis of the curve of tetanus _ . $\quad$ - 75

$\S 50$. Various degrees of tetanic contractions _ . . . . . . . 79

$\S 51$. Diminution and disappearance of irritability after death . . . 80

\section{SECTION II.}

\section{ON the Changes which take place in a Muscle during a Contraction.}

\section{The Change in Form.}

§52. Gross structure of muscle, arrangement of muscular fibres, blood vessels and nerves . . . . . . . . . . . 82

$\S 53$. The wave of contraction; its length, velocity, and other characters . 83

§54. The visible changes which take place in a muscular fibre during a contraction

The Chemistry of Muscle.

§ 55. Contrast of living and dead muscle ; rigor mortis _ . . . . 86

$\S 56$. Chemical bodies present in dead muscle; myosin, syntonin . . 87 
§ 57. Chemistry of living muscle ; inuscle-plasina, muscle-clot, and muscleserum, myoglobulin, histo-hæmatin

PAGE

cid reaction of rigid nuscle; development of carbonic acid in rigor mortis

§59. Other constituents of muscl

$\S 60$. Chemical changes during contraction ; development of carbonic acid and acid reaction

$\S 61$. Summary of the chemistry of muscle .

\section{Thermal Changes.}

$\S 62$. Heat given out during a contraction. Comparison of muscle with a steam-engine

\section{Electrical Changes.}

$\S 63$. Non-polarizable electrodes. Muscle currents ; their distribution and

§64. Negative variation of the muscle current; currents of action. The rheoscopic frog

The Changes in a Nerve during the passage of a Nervous Impulse.

$\S 65$. The changes constituting what is called a nervous impulse propagated along the nerve . . . . . . . . . 10

$\S 66$. The chemistry of a nerve; cholesterin, lecithin, cerebrin, protagon

$\S 67$. The nervous impulse; the electrical changes accompanying it. These changes travel in both directions along the nerve. . . . 106

$\S 68$. Summary of the changes occurring in a muscle and nerve as the result of stimulation

\section{SECTION III.}

The Nature of the Changes through which an Electric Curlient is able to generate a Nervous Impulse.

\section{Action of the Constant Current.}

§69. Action of the constant current ; making and breaking contractions .

$\S 70$. Electrotonus. Effect of the constant current on the irritability of the nerve. Katelectrotonus. Anelectrotonus . . . . 112

$\S 71$. Electrotonic currents . . . . . . . . . . 114

$\$ 72$. Relation of electrotonus to nervous impulses, and to the effects of the constant current . . . . . . . . . . 116

$\S 73$. Action of the constant current on muscle . . . . . . 118 


\section{SECTION IV.}

The Mugcle-Nerve preparation as a Machine.

$\$ 74$. The influence of the nature and mode of application of the stimulus on the magnitude of the contraction. Maximal and minimal stimuli. Influence of abruptuess and duration of stimulus. Some parts of a nerve more irritable than others . . . . .

$\S 75$. Frequency of repetition necessary to produce tetanus; pale and red muscles. The muscular sound . . . . . . . 121

$\S 76$. The influence of the load; effect of resistance. The work done . 123

$\S 77$. The influence of the size and form of the muscle . . . . 124

\section{SECTION $V$.}

Tire Circumstances which determine the Degree of Irritability of Muscles and Nerves.

$\S 78$. Diminution and disappearance of irritability after severance from the body. Effect of division of nerves; degeneration of nerve fibres

§ 79. The influence of temperature . . . . . . . . 127

$\S 80$. The influence of blood supply . . . . . . . . . . . . 128

$\S 81$. The influence of functional activity. Exercise. Fatigue. The causes of exhaustion . . . . . . . . . . 128

\section{SECTION VI.}

On some other Forms of Contractile Tissue.

Plain, Smooth, or Unstriated Muscular Tissue.

§82. Muscular tissue . . . . . . . . . . . . . 131

$\S 83$. The chemistry of unstriated muscle . $\quad$. $\quad . \quad$. . . 131

$\S 84$. The characters of the contraction of unstriated muscle. Peristaltic contractions. 'Spontaneous' contractions. Touic contractions . 131

\section{Ciliary Movement.}

$\S 85$. The action of cilia . . . . . . . . . .

$\S 86$. Nature of ciliary movement. Circumstances affecting ciliary movements

Amoboid Movements.

$\S 87$. Nature of an amœboid movement; its relation to a muscular contraction 


\section{CHAPTER III.}

On the more General Features of Nervous Tissues.

$\S 88$. The general arrangement of the nervous system. Cerebro-spinal and splanchnic or sympathetic system; somatic and splanchnic nerves . . . . . . . . . . .

$\S 89$. Grey matter and white matter of the central nervous system. Structure of a nerve cell of the spinal cord; axis-cylinder process. Functions of nerve cells

$\S 90$. Reflex actions; the machinery required. The circumstances determining the nature of a reflex action. Reflex actions often purposeful . . . . . . . . . . . . 144

$\S$ 91. Automatic actions . . . . . . . . . . . 146

$\S 92$. Inhibitory nerves $. \quad . \quad$. . . . . . . . 148

\section{CHAPTER IV.}

\section{The Vascular Mechanism.}

\section{SECTION I.}

The Structure and Main Features of the Vascular Apparatus.

$\S 93$. The chief work of the blood carried on in the capillaries and other minute vessels . . . . . . . . . 149

$\S 94$. The main features of the vascular apparatus . . . . 150

\section{SECTION II.}

\section{The Maln Facts of the Circulation.}

$\S 95$. Behaviour of arteries contrasted with that of veins $\quad . \quad$. 153

$\S 96$. Blood pressure in an artery and in a vein _ . . . . 154

$\S 97$. Methods of registering blood pressure; mercurial manometer. Kymograph. The blood pressure curve . . . . . 156

$\S 98$. Characters of the blood pressure in various arteries and veins. Blood pressure in the capillaries. Fall of blood pressure in the minute vessels . . . . . . . . .

$\S 99$. The circulation through the capillaries, and small vessels. Peripheral resistance . . . . . . . . . . 161

\section{Hydraulic Principles of the Circulation.}

$\S 100$. The three main physical facts of the circulation ; the central pump, the peripheral resistance, and the elastic tubing 
$\S 101$. The conversion of the intermittent into a continuous flow by means of the elastic reaction of the arteries . . . . . . 164

\$102. Artificial Model. Arterial and venous pressure with great and with little peripheral resistance . . . . . . . . . . 166

$\S 103$. Additional aids to the circulation in the living body . . . . 171

\section{Circumstances determining the Rate of the Flow.}

$\S 104$. Methods of determining the rate of the flow. Hæmadromometer, Rheometer, Hænatachometer. The plethysmographic method.

The rate of flow in arteries, veins, and capillaries . . . 172

$\S 105$. The rate of flow dependent on the width of the bed _ . . 176

$\$ 106$. The time of the entire circuit . . . . . . . . . . 178

$\S 107$. Summary of the main physical facts of the circulation . . . 179

\section{SECTION III.}

The Heart.

\section{The Phenomena of the Normal Beat.}

$\S 108$. The visible movements . . . . . . . . . . 181

$\S 109$. The cardiac cycle ; the series of events constituting a beat . . 182

$\$ 110$. The change of form . . . . . . . . . . 185

$\S 111$. The cardiac impulse . . . . . . . . . . . . 187

$\$ 112$. The sounds of the heart . . . . . . . . . . . . 188

$\$ 113$. Endocardiac pressure. Methods of determining this. Cardiac sound and tambour. Piston and membrane nanometers. General features of the curve of endocardiac pressure . . . . 191

$\S 114$. The output of the heart; the methods of determining this . . 197

\section{The Mechanism of the Beat.}

$\S 115$. The curves obtained by means of cardiograph and the myocardiograph. The curve of ventricular pressure compared with these

$\S 116$. The pressure in the ventricle compared with that in the aorta. The differential manometer or manometer balance. 'The teachings of this comparison . . . . . . . . .

$\S 117$. Minimum and maximum manometers. The negative pressure in the cardiac cavities . . . . . . . . . 210

$\S 118$. The duration of the several phases of the cardiac cycle . . . 212

$\S 119$. Summary of the events constituting a beat . . . . . 215

$\S 120$. The work done . . . . . . . . . . . . 217

\section{SECTION IV.}

The Pulse.

$\S 121$. Methods of recording the pulse. The sphygmograph, sphygmoscope, and other instruments. The pulse curve.

$\S 122$. Pulse tracing from an artificial model; the nature of the pulse wave 
$\S 123$. The characters of the pulsc curve; influence of pressure exerted by lever

$\$ 124$. The changes undergone by the pulse wave along the arterial tract 227

$\$ 125$. The velocity of the pulse wave . . . . . . . . 228

$\$ 126$. The length of the pulse wave . . . . . . . . . 229

$\$ 127$. Secondary waves. Katacrotic and anacrotic tracings . . . 230

$\$ 128$. The dicrotic wave $:$ its causes . . . . . . . . 2:32

$\$ 129$. Circumstances letermining the prominence of the dicrotic wave . $2: 34$

$\S 130$. The predicrotic wave. Anacrotic waves . . . . . . 2:35

$\S 131$. Venous pulse . . . . . . . . . . . 236

\section{SECTION V.}

Tie Regulation and Adaptation of the Vascular Mechanism.

The Regulation of the Beat of the Heart.

$\S 132$. The two great regulators; changes in the heart beat and changes in the peripheral mechanism

The Development of the Normal Beat.

$\S 133$. Graphic record of the heart beat. The beat of the frog's heart. The sequence of events, and the descending scale of rbythmic power . . . . . . . . . . .

$\S 134$. Some features of the heart beat in the mammal . . . . 244

The Government of the Heart Beat by the Nervous System.

$\S 135$. Inhibition in the frog by stimulation of vagus nerves. Features of inhibition . . . . . . . . .

$\S 136$. Augmentation of the heart beat in the frog. Antagonism of augmentation and inhibition. Course of augmentor fibres in the frog . . . . . . . . . . 246

§ 137. Reflex inhibition. Cardio-inhibitory centre . . . . . 249

$\S 138$. Inhibition in the mammal ; effect on blood pressure. Reflcx inhibition. Course of augmentor fibres in the dog . . . . 250

$\S 139$. Action of atropin and muscarin . . . . . . . . 257

Other Infuences regulating or modifying the Beat of the Heart.

$\$ 140$. Influences of blood, and substances contained in the blood. Influence of the distension of the cavities. Relation of heart beat to blood pressure

\section{SECTION VI.}

Changes in the Calibre of the Minute Arteries. Vaso-motor Actions.

$\S 141$. Changes of calivrc in artcries as seen in the web of a frog's foot and elsewhere. Vaso-motor nerves . . . . . . 262

§142. 'The vascular phenomena in a rabbit's ear . . . . . . 263 
$\$ 143$. The effects on the vessels of the ear of dividing and stimulating the cervical sympathetic nerve . . . . . . . 264

$\S 144$. Course of vaso-motor fibres of the ear . . . . . . . 265

$\$ 145$. The effects on the vessels of the submaxillary gland of stimulating the chorda tympani nerve; vaso-constrictor and vaso-dilator fibres. . . . . . . . . .

$\S 146$. Vaso-motor nerves of other parts of the body. Constrictor and dilator fibres in the sciatic and brachial nerves . . . .

\section{The Course of Vaso-motor Fibres.}

$\S 147$. The course of vaso-constrictor fibres . . . . . . 273

$\S 148$. The course of vaso-dilator fibres . . . . . . . 275

\section{The Effects of Vaso-motor Actions.}

$\$ 149$. Local and general effects of the constriction and dilation of an artery or set of arteries

Vaso-motor Functions of the Central Nervous System.

$\$ 150$. Vaso-dilator fibres usually employed as part of a reflex action

$\$ 151$. Loss of tone resulting from the division of the spinal chord at various levels. Vaso-motor centre in the spinal bulb . . . 278

$\$ 152$. The Depressor nerve . . . . . . . . . . 280

$\$ 153$. Rise of blood pressure from stimulation of afferent nerves; pressor effects . . . . . . . . . . . . 281

$\S 154$. The limits of the bulbar vaso-motor centre _ . . . . . . 282

$\$ 155$. The relation of the bulbar vaso-motor centre to other spinal vasomotor centres. Dilation, tone, and constriction of blood vessels

$\S 156$. Summary of vaso-motor actions . . . . . . .

$\$ 157$. Instances of vaso-motor actions. Blushing. Effect of warmth on skin. Vascular clranges in kidney and alimentary canal . . 286

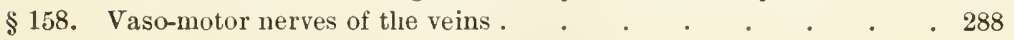

\section{SECTION VII.}

\section{The Capillary Circulation.}

$\$ 159$. The normal capillary circulation. The axial core and the plasmatic layer . . . . . . . . . . 289

$\S 160$. Changes in the capillary circulation induced by irritants. The phenomena of inflammation . . . . . . . . 290

$\S 161$. The migration of white corpuscles. Stasis . . . . . 292

$\S 162$. Nature of the inflammatory changes . . . . . . 243

$\S 163$. Changes in the peripheral resistance due to changes in the blood . 294

\section{SECTION VIII.}

\section{Changes in the Quantity of Blood.}

$\S 164$. Effects of increasing and of diminishing the total quantity of blood 


\section{SECTION IX.}

A Review of some of tile Features of the Circelation.

$\$ 165$. The constant and variable factors

$\S 166$. The influence of the venous inflow and of the distension of the cavities of the heart . . . . . . . . . 300

$\$ 167$. The heart beat influenced by the quantity and quality of the blood flowing through the heart . . . . . . . . 300

$\S 168$. The causes of an irregular heart beat . . . . . . 301

$\$ 169$. The causes of the sudden cessation of the heart beat and of sudden death. . . . . 302

$\S 170$. Instances of the working of the vaso-constrictor mechanism . . 303

$\S 171$. The influence of bodily exercise on the vascular mechanism . . 304

$\S 172$. The influence of food on the vascular mechanism . . . . . 306

$\$ 173$. The vascular mechanism and the nervous system . . . . 307

\section{BOOK II.}

THE TISSUES OF CHEMICAL ACTION WITH THEIR RESPECTIVE MECHANISMS. NUTRITION.

\section{CHAPTER I.}

The Tissues and Mechanisms of Digestion.

$\S 174$. Food-stuffs. The several stages of digestion . . . . . . 311

\section{SECTION I.}

The Characters and Properties of Saliva and Gastric Juice.

\section{Saliva.}

$\S 175$. The chemical characters of saliva. Mucir 313

$\S 176$. The properties of saliva. The action of saliva on starch. Characters of starch, dextrins, dextrose, maltose . . . . . . . 314

$\S 177$. The nature of the anylolytic action of saliva. The amylolytic ferment . . . . . . . . . . • .

$\S 178$. The characters of parotid, submaxillary, sublingual, and mixed saliva

\section{Gastric Juice.}

$\S 179$. The chemical characters of gastric juice .

$\S 180$. The action of gastric juice on proteids. Artificial gastric juice.

The characters of the more important proteid bodies. Formation of acid-albumin 
$\S 181$. Peptone and albumose. Parapeptone. Classification of proteids . 325

$\S 182$. Circumstances affecting gastric digestion; acidity, temperature . 327

$\S 183$. The nature of the action of gastric juice. Pepsin . . . . . 328

$\S 184$. The action of gastric juice on gelatin, chondrin, \&c. . 329

$\S 185$. The action of gastric juice on milk. Curdling of milk. Casein. Rennin .

\section{SECTION II.}

The Act of Secretion of Saliva and Gastric Juice and the Nervods Mechanisus which regulate it.

$\S 186$. The evidences of the existence of a nervous mechanism . . . . 332

$\$ 187$. The nerves of the submaxillary gland.$\quad$. . . . . . . 383

$\S 188$. The reflex secretion of saliva by means of the chorda tympani nerve . . . . . . . . . . .

$\S 189$. The nature of the action of the chorda tympani nerve. Influence of atropin . . . . . . . . . . . . . . 335

$\S 190$. The effects on the submaxillary gland of stimulating the cervical syinpathetic nerve. . . . . . . . . . . 337

$\S 191$. The nervous mechanism of the parotid gland . . . . . 338

$\$ 192$. The general features of the secretion of gastric juice . 338

$\S 193$. The nervous supply of the stomach. The action of the nerves obscure . . . . . . . . . . . . . . . 339

$\S 194$. The influence of the absorption of food in promoting secretion $\quad$. 339

\section{The Changes in a Gland constituting the Act of Secretion.}

$\S 195$. The appearances presented by the pancreas during secretion; the histological changes . . . . . . . . 340

$\S 196$. The changes in an albuminous gland during secretion . . . 342

$\S 197$. The changes in a mucous gland during secretioll . . . . . . 344

$\S 198$. The changes in the central cells of the stomach during secretion . 346

$\S 199$. The general nature of secretion. Loading and discharge $\quad . \quad 347$

$\S 200$. The formation of the ferment; zymogen, trypsill, and trypsinogen, pepsin and pepsinogen. .0 .0 .0 .0 .348

$\S 201$. The nature of the act of secretion itself. The flow of fluid. "Secretory' and 'trophic' fibres . . . . . . . . . 350

$\$ 202$. The functions of the epithelium of the ducts . . . . . 351.

$\S 203$. The formation of the free acid of gastric juice . . . . . . 352

$\S 204$. Why the stomach does not digest itself . . . . . . . 352

\section{SECTION III.}

The Properties and Characters of Bile, Pancreatic Juice, and Succus Entericus.

Bile.

$\S 205$. The characters of bile

$\$ 206$. The pigments of bile. Bilirubin 
$\$ 207$. The bile salts; glycochulic and taurocholic acids . . . . 356

$\$ 208$. The action of bile on foud . . . . . . . . . . 357

\section{Pancreatic Juice.}

$\$ 209$. The characters of pancreatic juice . . . . . . . 358

$\S 210$. The action of pancreatic juice on proteids; leucin, tyrosin. Its action on filts and on sturch. . . . . . . . . 359

Succus Entericus.

\$211. Nature and action of succus entericus . . . . . . 363

\$ 212. Gallstones . . . . . . . . . . . . 364

\section{SECTION IV.}

The Secretiox of Paxcreatic Júce axd of Bile.

$\S 213$. The secretion of pancreatic juice . . . . . . . 365

$\S 214$. The discharge of and the secretion of bile . . . . . . 366

$\$ 215$. The vascular conditions of the liver and their relations to the secretion of bile. The influence of absorbed products of digestion . . . . . . . . . 367

\$216. The pressure at wlich bile is secreted . . . . . . \$69

$\$ 217$. The resorption of bile. . . . . . . . . . 370

\section{SECTION V.}

The Mescular Meciasisms of Digestion.

\$218. Peristaltie movements. Mastication . . . . . . 371

$\$ 219$. Deglutition ; its phases, nature, and nervous mechanism . . . 372

$\$ 220$. The movements of the esophagus . . . . . . . 375

$\$ 221$. The movements of the stomach. . . . . . . . 377

\$22. Vomiting . . . . . . . . . . . 378

223. The movements of the small intestine . . . . . . . 380

$\$ 224$. The movements of the large intestine $\quad . \quad+\quad . \quad+\quad . \quad$. $\quad . \quad 381$

$\$ 225$. Defiecation . . . . . . . . . . . 381

$\S 226$. The nervous mechanisms of gastric and intestinal movements. The

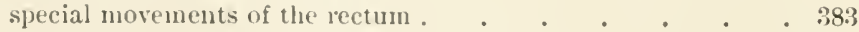

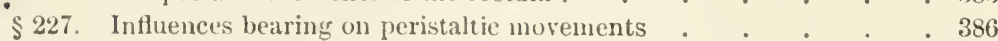

\section{SECTION VI.}

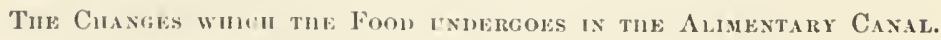

§228. The changes in the mouth . . . . . . . . . 387

The Changes in the Stomach.

$\$ 229$. The changes in the stomach. Chyme. Absorption from the stomach. Flatulence . . . . . . . . 388 
In the Small Intestine.

$\$ 230$. The changes in the sinall intestine. Action of bile and pancreatic PAGE juice . . . . . . . . . . 390

$\$ 231$. Influence of bile on peptic digestion . . . . . . . . . 392

$\$ 232$. The action of micro-organisms in the alinentary canal. Indol, \&c. 393

\section{In the Large Intestine.}

$\$ 233$. The changes in the large intestine. Digestion of cellulose . . 395

\section{The Faces.}

$\$ 234$. The nature and constituents of the fæces 396

\section{SECTION VII.}

The Lacteals and the Lxiphatic Srstem.

$\$ 235$. The general arrangement of the lymphatics . . . . . . 398

\section{SEC'TION VIII.}

The Nature axp Movements of Lymph (fncluding Cirle).

$\$ 236$. Movements of lymph continually going on in the living body . 399

\section{The Characters of Lymph.}

$\$ 237$. The microscopical characters of lymph . . . . . . . 400

$\$ 2: 38$. The chemical composition of lymph . . . . . . . . . . 401

$\$ 239$. The changes taking place in lymph during its passage . . . . 401

$\$ 240$. The chemical characters of serous fluids . . . . . . 402

$\$ 241$. The characters of chyle . . . . . . . . 402

\section{The Hovements of Lymph.}

$\$ 242$. The causes which maintain the flow of lymph . . . . . 40?

$\$ 243$. The influence of the nervous system on the flow of lymph . . 405

$\$ 244$. The phenomena of transudation. The relation of transudation to filtration and diffusion . . . . . . . . . 406

$\$ 245$. Cdema or dropsy ; its causes . . . . . . . . . . . 409

\section{SECTION IX.}

\section{Absorption from tile Alimentary Canal.}

$\$ 246$. The main products of digestion, and the two paths of absorption

open for them . . . . . . . . . . . 412

The Course taken by the Several Products of Digestion.

\$247. The course taken by the fats . . . . . . . . 413

$\S 248$. The course taken by water and salts . $\quad . \quad . \quad . \quad . \quad$. 414 
$\$ 249$. The course taken by sugar.

The Mechanism of Absorption.

$\S 251$. The mechanism of the absorption of the fats .

$\S 252$. The pumping action of the villi .

$\S 253$. The mechanism of the absorption of diffusible substances and of water. Relations of the process to diffusion. Action of the cells. The two stages of the act of absorption; their nature.

\section{CHAPTER II.}

$\S 254$. Respiration . 424

\section{SECTION I.}

Tue Mechanics of Puluonary Respiration.

$\S 255$. The entrance and exit of air into and from the lungs; tidal and stationary air

$\S 256$. Complemental, reserve or supplemental, and residual air. Results of an opening into the pleural chamber . . . . . . 426

$\S 257$. The lungs before birth and the changes at birth . . . . 4 4.7

$\S 258$. The pressure exerted in breathing and the quantity of air moved . 427

$\S 259$. The graphic records of the respiratory movements; pneumatograph 428

$\S 260$. The curve of respiratory movements . . . . . . . 432

\section{The Respiratory Movements.}

$\S 261$. The visible movements . . . . . . . . . 439?

\$262. The movements of inspiration. The movements of the diaphragm 4:;:3

$\S 263$. The elevation of the ribs . . . . . . . . . 4:34

$\S 264$. The muscles which move the ribs . . . . . . . 43:5

$\S 265$. The muscles of laboured inspiration . . . . . . . . . . 436

$\S 266$. Expiration. The expiratory muscles . . . . . . . 4397

$\S 267$. Facial and laryngeal respiration . . . . . . . 438

\section{SECTION II.}

\section{The Chasges of the Air in Respiration.}

$\S 268$. The changes in temperature . . . . . . . . 440

$\$ 269$. The aqueous vapour in expiration . . . . . . . 440

$\S 270$. The gaseous changes . . . . . . . . . . . 440

$\$ 271$. The diminution in volume . . . . . . . . . . 441

$\S 272$. The organic inpurities in expired air . . . . . . . . 442

\section{SECTION III.}

The Resphatoli Cinanges in the Blood.

$\S 273$. The gases of arterial and venous blood. The mereurial gas pump . 
The Relations of Orygen in the Blood.

$\$ 274$. The absorption of oxygen by blood is not according to the law of PAGE pressures '

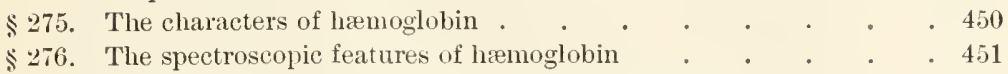

$\$ 277$. The spectroscopic features of reduced hæmoglobin . . . . 45:3

$\$ 278$. The oxygenation and reduction of hemoglobin . . . . . 455

$\$ 279$. The colour of venous and arterial blood . . . . . . . 455

\$280. Carbonic-oxide-hæmoglobin . . . . . . . . 457

Products of the Decomposition of Hamoglobin.

\$281. Hæmoglobin splits up into hæmatin and a proteid . . . . 458

$\S 282$. The features of hæmatin. Hæmin. Methæmoglobin . • . 460

The Relations of the Carbonic Acid in the Blood.

$\S 283$. The carbonic acid of the blood not simply absorbed _ . . 461

The Relations of the Nitrogen in the Blood.

\$284. The nitrogen simply absorbed . . . . . . . . 461

\section{SECTION IV.}

The Respiratory Changes in tile Lungs.

$\$ 285$. The relations of the oxygen of the blood to pressure. Association of oxygen with, and dissociation from hæmoglobin. The problem stated . . . . . . . . . 462

$\S 286$. The experimental evidence . . . . . . . . . 464

$\$ 287$. The relations of the oxygen in laboured breathing and asphyxia . 465

\section{The Exit of Carbonic Acid.}

$\S 288$. The exit of carbonic acid from the blood into the pulmonary alveolus the result of ordinary diffusion . . . . . . . 466

\section{SECTION $\mathrm{V}$.}

The Respiratory Changes in the Tissues.

$\$ 289$. The oxidations of the body take place mainly in the tissues and not in the circulating blond. The respiration of muscle . . .

$\$ 290$. The respiration of other tissues. The taking in of oxygen separate from the giving out of carbonic acid . . . . . . . . 469

$\S 291$. A summary of respiration in its chemical aspects . . . . 470

\section{SECTION VI.}

Tile Nervols Mechasisu of Respiration.

$\S 292$. Respiration an involuntary act. The efferent nerves, the respiratory centre 
$\$ 298$. The complex nature of the medullary respiratory centre; the subsidiary spinal mechanisms . . . . . . . . . 47:

$\$ 294$. The action of the centre automatic . . . . . . . 474

$\$ 295$. The centre influenced by afferent impulses. The respiratory action of the vagus nerve. Inhibitory and augmentor effects . . 475

$\$ 296$. The double action of the centre, inspiratory and expiratory. Antagonism between the two. . . . . . . . .

$\$ 297$. The effects of inflation and suction. Double action of the vagus fibres. Self-regulating mechanism . . . . . . 480

$\$ 298$. The influence on the respiratory centre of various afferent impulses 483

$\$ 299$. 'The respiratory centre composed of two lateral halves . . . . 484

$\$ 300$. The respiratory centre influenced by the condition of the blood brought to it. Eupncea, hyperpncea, and dyspnoea . . . 484

$\$ 301$. 'The direct character of these influences. Effects of heat on the respiratory centre. . . . . . . . .

$\S 302$. The relative shares taken by deficiency of oxygen and excess of carbonic acid in the above influences . . . . . .

$\S 303$. Changes in the blood other than differences in the gases influence the respiratory effect. The effects of muscular exercise . . 488

$\$ 304$. The phenomena and causes of apnœa . . . . . . . . 489

$\S 305$. Secondary respiratory rhythn. The Cheyne-Stokes respiration . 490

\section{SECTION VII.}

The Effects of Cunges in tile Composition and Pregsure OF TIL AIR BREATIED.

$\$ 306$. The phenomena of asphyxia . . . . . . . . . . . 491

$\$ 307$. The effects on respiration of breathing various foreign gases . . 494

$\$ 308$. The effects of a diminution of pressure in the air breathed . . 494

$\$ 309$. The effects of an increase of atmospheric pressure . . . . . 496

\section{SECTION VIII.}

The Relations of the Rispiratory Sistem to the Vascular AND OTHER SYSTEMS.

$\$ 310$. The respiratory movements influence the circulation. The respiratory undulations of the blood-pressure curve . . . .

$\$ 311$. The effects of the expansion of the thorax and its return on the flow of blood to and from the heart and so on the bloodpressure . . . . . . . . . .

\$312. The effects of the expansion and return of the thorax on the flow of blood through the lungs . . . . . . . . . . 502

\$313. The effects on the circulation of artificial respiration . . . . 503

$\$ 314$. An action of the cardio-inhibitory centre synchronous with that of the respiratory centre . . . . . . . . . 504

\$315. The effects of impeded respiration on the circulation in general.

Traube-Hering curves. The circulation in asphyxia . 
$\$ 316$. The effects on respiration of changes in the circulation through the PAGE respiratory centre and through the lungs . . . . . . 508

$\S 317$. The effects of exercise on respiration _ . . . . . . 509

\section{SEC'TION IX.}

Modfiei, Respiratole Movements.

$\$ 318$. Sighing, yawning, hiccough, sobbing, coughing, sneezing, laughing, and crying . . . . . . . . . . 511

\section{CHAP'TER III.}

\section{The Flimation of IVAste l'roducts.}

$\S 319$. The chief waste products and their channels of exit 513

\section{SECTION I.}

Tie Conposition ani Cilaracters of Urine.

$\S 320$. The general characters of urine . . . . . . . 515

$\S 321$. The normal organic constituents of urine . . . . . . 516

$\$ 322$. The inorganic salts . . . . . . . . . . 517

$\$ 323$. The non-nitrogenous constituents of the urine . . . . . 518

$\$ 324$. The pigments of the urine . . . . . . . . . . . 519

$\$ 325$. Ferments and other bodies present in urine . . . . . . . . 519

$\S 326$. The relative quantities of the more important constituents of urine 520

$\$ 327$. The acidity of urine . . . . . . . . . . 521

$\$ 328$. The abnormal constituents of urine . . . . . . . . 522

\section{SECTION II.}

\section{The Secretion of Utine.}

$\$ 329$. The double nature of urinary secretion . . . . . . . 524

$\$ 330$. The vaso-motor mechanisms of the kidney. The renal oncometer and oncograph . . . . . . . . . . .

$\$ 331$. The relation of the flow of blood through the kidney to variations in blood-pressure and to vaso-motor changes in general . . 528

$\$ 332$. 'The vaso-constrictor nerves of the kidney . . . . . . . 530

$\$ 333$. The vaso-dilator nerves of the kidney . . . . . . . . 531

$\S 334$. The influence on the renal circulation of chemical substances in the blood . . . . . . . . . . 531

$\$ 335$. The connection between changes in the renal circulation and the secretioll of urine. . . . . . . . . . 532 


\section{Secretion by the Renal Epithelium.}

$\S 336$. The evidence of the secretory activity of the epithelium. Experiments on amphibia. The results of injecting sulphindigotate of sodium .

$\S 337$. The nature of glomerular secretion; its relation to filtration and

diffusion. Albuminous urine . . . . . . . 535

$\S 338$. The nature of the work of the epithelium as regards the secretion of urea . . . . . . . . . . . . . 538

$\S 339$. The formation of hippuric acid . $\quad . \quad$. $\quad . \quad$. $\quad . \quad$. 539

$\$ 340$. The relations of the secretory activity of the kidney to the secretory activity of the skin . . . . . . . 540

$\S 341$. The relations of the secretion of urine to food and drink . . 541

$\$ 342$. Diuretics . . . . . . . . . . . . 542

$\S 343$. Direct action of the nervous system on the kidney . . . . 543

\section{SECTION III.}

The Discharge of Urine.

$\S 344$. The movements of the ureter . . . . . . . . 544

\section{Micturition.}

$\S 345$. The muscles of the bladder, their action, the nerves governing them; the sphincter vesicæ . . . . . . . . 545

$\S 346$. The varying tone of the bladder . . . . . . . . 546

$\$ 347$. The general nervous mechanism of micturition . . . . 547

$\$ 348$. Involuntary and voluntary micturition . . . . . . . 548

$\S 349$. Changes of the urine during its stay in the bladder . . . . 549

\section{SECTION IV.}

The Nature and Amount of Perspiration.

$\S 350$. Sensible and insensible perspiration. The characters and constituents of sweat

Cutaneous Respiration.

$\S 351$. The nature and amount of cutaneous respiration. The effects of varnishing the skin . . . . . . . . . . 552

§352. Absorption by the skin . . . . . . . . . . . 553

\section{SECTION V.}

The Mecrunisu of the Secretion of Sweat.

$\S 353$. The relation of sweating to vascular changes. The nervous mechanism of the sweat-glands . . . . . . . . . 555

$\S 354$. The sweat-nerves, their origin and course . . . . . . 557

$\S 355$. Inhibitory sweat-nerves . . . . . . . . . 558 


\section{CHAPTER IV.}

The Metabolic Processes of the Body.

\section{SECTION I.}

The History of Glycogex.

\$357. The characters of glycogen . . . . . . . . . 561

$\$ 358$. The conversion of glycogen into sugar by the liver . _ . 562

$\$ 359$. The influence of various foods in storing up glycogen. The storage of glycogen in the winter frog . . . . . . . . 563

$\$ 360$. The detailed characters of the hepatic cells in the frog . . . 566

$\$ 361$. The histological changes induced by food and circumstances in the hepatic cells of the frog . . . . . . . . . . 567

$\$ 362$. The corresponding changes in the mammal . . . . . 568

363. The nature and meaning of these changes _ . . . . . 568

$\$ 364$. Views as to the manner in which glycogen is stored in the hepatic cells. By simple dehydration of sugar _ . . . . . 569

$\$ 365$. The glycogen formed by a product of the metabolisin of the hepatic cells. Comparison of the two views . . . . . . 570

$\S 366$. The uses of glycogen. The formation of fat as a store of carbonholding naterial . . . . . . . . . 571

$\$ 367$. Glycogen in muscle . . . . . . . . . . . 573

$\S 368$. Glycogen in the placenta and in various tissues . . . . 574

\section{Diabetes.}

\$369. Artificial diabetes . . . . . . . . . . 575

$\$ 370$. The nervous mechanism of the diabetic puncture . . . . 576

$\$ 371$. Temporary diabetes from the use of drugs. Natural diabetes. The 576

diminution of hepatic glycogen by arsenic and other agents . 576

\section{SECTION II.}

The Spleen.

$\$ 372$. The movements of the spleen. The spleen curve . . . . 579

$\S 373$. The spleen pulp; the white and red corpuscles. Changes undergone by the latter . . . . . . . . . . 581

$\S 374$. The chemical constituents of the spleen . . . . . . 582

\section{SECTION III.}

The Formation of the Constituents of Bile.

\$375. The formation of bilirubin from hæenoglobin . . . . . 584

$\$ 376$. The nature of and preparations towards this formation . . . 585

$\S 377$. The formation of bile acids . . . . . . . . 586 
$\$ 378$. The relations of the secretion of bilirubin to the secretion of bile acids

$\$ 379$. The relation of the secretion of bile to the formation of glycogen . 587

SEC'TION IV.

Ox Urea axd on Nitrogenous Metabolism in gexeral.

$\$ 380$. Urea the end-product of the metabolisn of proteid matter . . . 589

$\$ 381$. Urea probably the result of a series of changes _ . . . . 590

$\$ 382$. The kreatin of muscle in its relations to urea . . . . . . 590

$\$ 383$. Difficulties presented by the normal presence of kreatin in urine . 591

$\$ 384$. The nitrogenous metabolism of the nervous tissues . . . . . 592

$\$ 385$. The nitrogenous metabolism of the glandular structures. The

increase of urea due directly to food . . . . . . . 593

$\$ 386$. The formation of urea in the liver . . . . . . . . . 594

$\$ 387$. The synthesis of urea . . . . . . . . . . . . 595

$\$ 388$. Uric acid . . . . . . . . . . . . 596

$\$ 389$. Other nitrogenous crystalline bodies, such as xanthin, \&c. . . 597

$\$ 390$. Relations of urea to cyanogen compounds . . . . . . 598

$\$ 391$. A summary of nitrogenous metabolism . . . . . . . 599

\section{SECTION $\mathrm{V}$.}

On some Structires any Processes of an obscere Nature.

$\$ 392$. The structure and functions of the thyroid body . . . . 600

$\$ 393$. The pituitary body . . . . . . . . . . 601

$\$ 394$. The structure, chemical constituents, and functions of the supra-

renal bodies. . . . . . . . . 601

$\$ 395$. The structure, nature, and functions of the thymus _ . . 602

\section{SECTION VI.}

The History of Fat. Adipose 'Tissue.

$\$ 396$. Adipose tissue; its clianges . . . . . . . . 604

$\$ 397$. The structure of adipose tissue . . . . . . . . . 604

$\$ 398$. 'Ihe disappearanee of fat from adipose tissue . . . . . . 605

$\S 399$. The nature of fat in adipose tissue . . . . . . . . 606

500 . Fat is formed in the body. . . . . . . . . 606

$\$ 401$. Formation of fat from carbohydrates and from proteids . . . 608

$\$ 402$. Limits to the construction of fat . . . . . . . 609

\section{SECTION VII.}

\section{'The Manuary Gland.}

$\S 403$. The general structure of the mammary gland . . . . . . 610

$\$ 404$. The structure of the alveoli ; the varying appearances of the epithelial cells 
$\$ 405$. The characters of the dormant mammary gland . . . . 611

$\$ 406$. The connective-tissue of the mammary gland . . . . . 612

$\$ 407$. The nature of milk; its constituents; their relative quantities in different animals . . . . . . . . . 612

$\$$ 408. Colostrun, its chemical and microscopical characters _. . . 614

$\$ 409$. The manmary gland at birth . . . . . . . . . 615

$\$ 410$. The nature of the act of secretion of milk . . . . . . . 615

$\$ 411$. The secretion of milk typical of metabolism in general . . . 616

$\$ 412$. The relations of the gland to the nervous system . . . . 618

CHAP'TER V.

Nutiltiox.

SECTION I.

Tine Statistics of Nutrition.

$\$ 413$. The composition of the animal body . . . . . . . 619

$\$ 414$. 'The composition of the starving body and the general phenomena of starvation . . . . . . . . . . . . . . 620

Comparison of Income and Output of Material.

$\$$ 415. The inethod of determining the income and output. The respiration chamber. The calculation of changes in the body based on a comparison of the income and output . · · ·

$\$ 416$. Nitrogenous metabolism. 'Tissue' proteids and 'floating' proteids. Luxus consumption

$\$ 417$. The effects of fatty and carbohydrate food . . . . . . 627

$\$ 418$. The effects of gelatin as food . . . . . . . . . . 629

$\$$ 419. Peptone as food . . . . . . . . . . . . . . 629

$\$ 420$. The effects of salts as food. . . . . . . . . . 630

\section{SECTION II.}

Tine Exergy of the Body.

The Income of Energy.

$\$ 421$. The available potential energy of the several food-stuffs and of an ordinary diet.

\section{The Expenditure of Energy.}

$\$ 422$. The daily expenditure as heat and as work done. Calorimeters . $\quad 634$ $\$ 423$. The energy of mechanical work does not arise exclusively from proteid metabolism ; urea and muscular exercise . . .

\section{Animal Heat.}

$\$ 424$. The sources and distribution of heat; the muscles the chief source $\$ 425$. The temperature of the body; cold-blooded and warm-blooded animals . 
$\$ 426$. The regulation of the temperature of the body by variation in the loss of heat. The skin the great regulator.

PAGE

$\$ 427$. The production of lieat, the circumstances determining it; the
effects of meals and of labour.

$\$ 427$. The production of leat, the circumstances determining it the
effects of meals and of labour . . . . . . 641 643

$\S 428$. The influence of the nervous mechanism in determining the production of heat; the effects of heat and cold on the metabolisin of the body are produced through the nervous system . .

$\S 429$. The portions of the central nervous system concerned in this regulation of heat.

$\$ 430$. The narrow limits within which the bodily temperature is maintained

\$431. Abnormally high temperatures, pyrexia . . . . . . 648

$\$ 432$. The effects of great heat . . . . . . . . . . 649

$\$ 433$. The effects of great cold . . . . . . . . . . 650

\section{SECTION III.}

\section{On Nutrition in general.}

$\S 434$. The general features of metabolism .

651

$\S 435$. The metabolism of muscle as typical of the metabolism of tissues; the nature of the food of muscle . . . . . . . 652

$\S 436$. The fate of the lactic acid produced by muscle . . . . . 653

$\$ 437$. A comparison of the metabolisin of muscular tissue with that of other tissues . . . . . . . . . . 653

$\S 438$. Proteid substance the pivot of metabolism . . . . . 654

$\S 439$. The influence of nerves on inetabolism; katabolic and anabolic nerves. The influence of the nervous system on the general nutrition of the tissues. The phenomena of disease, \&c.; trophic nerves

\section{SECTION IV.}

\section{ON Diet.}

$\S 440$. The normal diet, statistical and experimental .

$\S 441$. The necessity for all three food-stuffs, proteids, fats, and carbohydrates. The necessity and importance of salts including extractives; alcohol, \&c.

$\$$ 442. The chemical value of articles of food to be corrected by their

$\$$ 443. The physiological value of a purely vegetable diet . . . .

$\$ 444$. The modifications of a normal diet needed to meet variations in size

$\S 445$. The modifications of a normal diet needed to meet changes of climate

$\$ 446$. The modifications of a normal diet needed to promote or prevent fattening

$\S 447$. The modifications of a normal diet needed to meet muscular and mental labour 


\title{
BOOK III.
}

\section{THE CENTRAL NERVOUS SYSTEM AND ITS INSTRUMENTS.}

\author{
CHAPTER I.
}

The Spinal Cord.

\section{SECTION I.}

On some Features of the Spinal Nerves.

\$ 448. The spinal nerves

$\S 449$. On efferent and afferent impulses . . . . . . . . . 676

$\S 450$. Efferent fibres run in the anterior root and afferent fibres in the posterior root . . . . . . . . . . 677

$\$ 451$. The "trophic" influence of the ganglion of the posterior root; the degeneration of nerve fibres . . . . . . . . . . 678

\section{SECTION II.}

The Structure of the Spinal Cord.

$\S 452$. The general features of the cord; grey and white matter. The grouping of the nerve cells. The cells of the anterior and posterior horn, the lateral group, Clarke's column, and the lateral horn. The tracts of white matter. Median posterior column, external posterior column. The evidence of the differentiation of the white matter into tracts. Ascending and descending degeneration. Descending tracts : crossed and direct pyrainidal tracts, antero-lateral descending tract. Ascending tracts : cerebellar tract, antero-lateral ascending tract, median posterior tract

$\S 453$. The special features of the several regions of the spinal cord. The conus medullaris, the lumbar, and cervical swellings. Variations in the sectional area of the white matter . . . . . 689

$\S 454$. Variations in the sectional area of the grey matter . . . . 691

$\$ 455$. The relative size, form, and features of transverse sections of the cord at different levels . . . . . . . . . 692

$\S 456$. Variations in the several columns of white matter at different levels 693

\section{SECTION III.}

The Reflex Actions of the Cord.

$\$ 457$. The difficulties attending the experimental investigation of the central nervous system ; 'shock' and other effects of an operation

$\S 458$. The differences, as regards reflex movements, between different kinds of animals. The features of a reflex act dependent on the character of the afferent impulses . 
$\$ 459$. The characters of a reflex movement dependent on the strength of the stimulus .

PAG

$\$ 460$. The characters of a reflex movement dependent on the part of the body to which the stimulus is applied .

$\$ 461$. The complexity of many reflex movements; their relation to intel-

$\$ 462$. Reflex movements coordinated by afferent impulses other than the exciting impulses . . . . . . . . . . 702

$\$ 463$. The character's of a reflex movement determined by the intrinsic condition of the cord . . . . . . . . 703

$\$ 464$. The reflex movements carried out by the spinal cord in man . . 704

$\$ 465$. Reflex actions resulting in changes other than movements . . 706

$\$ 466$. The inhibition of reflex actions . . . . . . . . 707

$\$ 467$. The time required for reflex actions . . . . . . . 709

\section{SECTION IV.}

\section{The Automatic Actions of the Spinal Cord.}

$\$ 468$. Automatic actions of the spinal cord in the frog and in the dog . 711

$\$ 469$. Automatic activity dependent on afferent impulses . . . . 712

$\$$ 470. Tone of skeletal muscles . . . . . . . . . 713

$\$ 471$. Tendon phenomena, knee jerk . . . . . . . . 717

$\S 472$. Rigidity of muscles through spinal action . . . . . . 717

\section{CHAPTER II.}

The Brain.

\section{SECTION 1}

\section{On the Phenomena exilbited by an Aximal deprived of its} Cerebral Hemisplleres.

$\S 473$. The absence of distinct signs of volition and intelligence . . 719

$\$ 474$. The characters of the movements of a brainless frog . . . 720

$\$ 475$. The phenomena exhibited by birds after removal of their cerebral hemispheres . . . . . . . . . . 723

$\S 476$. The effects of removing the cerebral hemispheres in mammals . 725 $\$ 477$. The effects of removing the cerebral hemispheres in the dog . . 727

\section{SECTION II}

The Macminery of Cookbinated Movements.

\$478. The effects of injury to the semicircular canals. Our appreciation of the position of our body, the sense of equilibrium. $\quad$ fferent impulses and sensations as factors of the coordination of movenents 
§479. The phenomena and causation of vertigo .

$\S 481$. The parts of the middle brain concerned in the coordination of movements

\section{SEC'TION III.}

On Voluntary Movements.

$\$ 482$. The real distinction between voluntary and involuntary movements

$\$ 483$. The cortical motor areas of the $\mathrm{dog}$; the characters of the movements resulting from cortical stimulation . . . . . 740

$\$ 484$. The cortical motor areas in the monkey . . . . . . 744

$\$ 485$. The cortical motor areas in the anthropoid ape . . . . 748

$\$ 486$. The movements of cortical origin carried out by means of the pyramidal tract; the nature of the movements so carried out .

$\$ 48$. The results of the removal of a cortical area in dog and in the monkey . . . . . . . . . . . . 762

$\$ 488$. The cortical motor areas in man; the area for speech . . . 764

$\$ 489$. The nature of the action of a motor area in carrying out a voluntary movement; the characters of aphasia. 'The same as illustrated by the area for a limb in the dog; the influence of sensory impulses. . . . . . . . .

$\$ 490$. The relations of the motor area to other parts of the central nervous system ; the motor area employed in movements usually called involuntary . . . . . . . . . . .

$\S 491$. The passage of volitional impulses along the spinal cord in animals

$\$ 492$. Their passage in man . . . . . . . . .

$\S 493$. A summary of the chief facts concerning the carrying out of voluntary movements . . . . . . . .

\section{SECTION IV.}

On tue Derelopmext withix the Cextrat, Nervols Srstem of Visual aND OF SOME other SENSations,

$\$$ 494. Visual impulses and sensations; visual fields, and binocular vision

$\$ 495$. The decussation of the optic nerves in the optic chiasma. . . 781

$\$ 496$. The course of the optic tract. The endings of the optic tract in the lateral corpus geniculatum, the pulvinar and the anterior corpus quadrigeminum; the results of degeneration and atrophy experiments. . . . . . . . .

$\$ 497$. The connection of the three above bodies with the cerebral cortex ; the meaning of the terms, blindness total and complete or partial, hemianopsia, amblyopia. The difficulties of interpretation attending experiments on the vision of animals .

$\S 498$. The nature of the movements of the eyes caused by stimulation of the occipital cortex . . . . . . . . .

$\S 493$. The effects on vision of removing parts of the occipital cortex in monkeys and in dogs ; the teachings of clinical histories 
$\$ 500$. 'The probable progressive development of visual sensations; lower and higher visual centres . . . . . . . . . 793

$\$ 501$. Sensations of smell. The cortical area for smell . . . . . . 794

$\S 502$. Sensations of taste . . . . . . . . . . . . . . 795

$\$ 503$. Sensations of hearing . . . . . . . . . . . . . . 790

\section{SECTION $\mathrm{V}$.}

On the Develophent of Cutaneous and Some Orher Sensatons.

$\$ 504$. Sensations of touch, heat, cold, and pain .

$\$ 50$. Theoretical difticulties touching the cortical localisation of cutaneous sensations. The effects on cutaneous sensations of removing regions of the cortex

$\$ 506$. The afferent tracts from the spinal cord, their endings in the brain .

$\$ 507$. The effect of sections of the spinal cord on the transmission of afferent impulses infiuencing the vasomotor centre. Other experiments on animals as to the effects of sections of the spinal cord on the transmission of sensory impulses . . .

$\$ 508$. The teachings of clinical histories; different paths for different sensory impulses . . . . . . . . . . . .

$\$ 509$. General considerations on the development of sensations along the spinal cord. 'The cerebellar tract, the median posterior tract, the grey matter and internuncial tracts

$\$ 510$. The terms 'sensory' and 'motor' not an adequate description of the processes in the central nervous systen

$\S 511$. The transmission of sensations within the brain. The relations of the cerebellum

\section{SECTION VI.}

On Some Other Aspects of the Functions of the Brain.

\$512. Considerations touching the cerebellum . . . . . . 81:

$\$ 513$. Considerations touching the corpora quadrigemina . . . . 811

$\S 514$. The splanchnic functions of the brain . . . . . . 816

\section{SECTION VII.}

\section{On the Time takex up by Cerebral Operatioxs.}

$\S 515$. The reaction period or reaction time .

$\$ 516$. Elementary analysis of psychical processes, the time taken up by each. The time required for discrimination, for the development of perception, and of the will; the circumstances influencing them 


\section{SECTIUN VIII}

The Lymphatic Arrangeuents of the Brain and Spinal Cord.

§517. The characters of the cerebro-spinal fluid . . . . . . . 824

$\$ 518$. The renewal of the cerebro-spinal fluid. The purposes served by the fluid. . . . . . . . . 825

\section{SECIION IX.}

The Vascular Arrangemexts of the Brain and Spinal Cord.

$\S 519$. The distribution and characters of the arteries of the brain . . 827

$\S 520$. The venous arrangements of the brain . . . . . . . 828

$\S 521$. The supply of blood to the brain relatively small. The methods of investigating the circulation of the brain . . . . . 829

$\S 522$. The supply of blood to the brain modified by the respiration and by changes in the general arterial pressure. 'The want of clear proof of special vasomotor nerves for the cerebral arteries . .

$\S 523$. The flow of blood through the brain nevertheless influenced by changes taking place in the brain itself . . . . . 833

\section{CHAP'TER III.}

Sight.

\section{SECTION I.}

On tile General Structure of the Eye, axd on the Formation of the Retisal Image.

$\S 521$. Dioptic mechanisms and visual impulses .

$\S 525$. The general structure of the eye. The formation of the retinal image

\$526. A simple optic system; its cardinal points. The refractive surfaces and media of the eye . . . . . . . . . . 839

$\S 527$. The optic constants of the eye. The diagrammatic eye . . . 841

$\S 528$. The paths of the rays of light through the eye . . . . . 843

$\S 529$. The retinal image in relation to the sensations excited by it . . 845

\section{SECTION II.}

\section{Tue Facts of Accommonation.}

$\S 530$. The eye can accommodate for far and near objects; far and near

limits of acconmodation . . . . . . . . 846

$\S 531$. Scheiner's experiment . . . . . . . . . 847 
S 532. Emmetropic, myopic, hypermetropic, and preslyopic eyes

$\$ 533$. The changes observed in the eye during accommodation . . . 850

\section{SECTIOY III.}

The Mechanisy of Acconnodation asd the Motemexts of THE PUPIL.

$\$ 534$. The mechanism for changing the anterior cursature of the lens . 854

$\$ 535$. The eridence that such a mechanism does effect the result . . 856

\section{The Changes in the Pupit.}

$\$ 536$. Circumstances leading to constriction and to dilation of the pupil . 85\%

$\S 53 \%$. Constriction and dilation . . . . . . . . . 858

\$53. The nerves supplying the pupil . . . . . . . . $\$ 59$

$\$ 539$. Constriction a reflex act by means of the optic and oculo-motor nerves. . . . . . . . . 860

$\$ 540$. Changes in the pupil through the action of the cervical sympathetic nerre . . . . . . . . 86i.

\$41. The nature of the dilating mechanism . . . . . . 86:;

$\$ 54$. . Direct action of drugs and other agencies on the pupil . . . 86ti

$\$$ 543. The nervous mechanism of accommodation . . . . . 868

$\$$ it4. The association of the morements of accommodation and the morements of the pupil. . . . . . . . 868

\section{SECTIOS IY.}

IMPERFECTIONS IN THE Dioptric Apparatus.

\$5.5. Imperfections of accommodation . . . . . . . 870

\$ 546 . Spherical aberration . . . . . . . . . 871

\$54. Astigmatism . . . . . . . . . 871

\$5. Chromatic aberration . . . . . . . . . . $87 \%$

549. Entoptic phenomena. . . . . . . . . . sit

\section{SECTION V.}

Ox Some Gexeral Featcres of Viscal sexsatioss.

$\$ 550$. The relation of the sensation to the intensity of the stimulus;

Weber's law . . . . . . . . . . . 878

$\$ 551$. The relation of the sensation to the duration of the stimulus . . 881

$\$ 552$. Flickering and continuous sensations . . . . . . 883

55.3. Sensations produced by variuts changes in the retina referred to some external source of light . . . . . . . . 888

$\S 554$. Localisation of risual sensations . . . . . . . . 885

$\$ 555$. The conditions of discrete risual sensations . . . . . 886

$\$ 556$. The region of distinct rision. The limits of distinct vision . . 887

$\$ 557$. Nature of the discreteness of visual sensations; retinal visual units 889 


\section{SECTIUN VI.}

\section{Ox Colour Sexsations.}

$\$ 558$. The existence of many kinds of colour sensations . . . . 891

$\$ 559$. The mixing of colour sensations . . . . . . . . 892

$\$ 560$. The several usual colour sensations result from the mixture of simpler, primary sensations . . . . . . . . . . 893

$\$ 561$. The conditions which determine the characters of colour sensations 895

$\$ 562$. Complementary colours . . . . . . . . . . 896

$\$ 563$. Any colour sensation produced by the suitable mixture of three colour sensations . . . . . . . . . . 897

$\$ 564$. The Young-Hehnholtz theory of colour sensations . . . . 898

$\$ 565$. Hering's theory of colour sensations. A comparison of the two theories

$\$ 566$. Variations in colour vision. Colour blindness. The different kinds of colour blindness; red blind and green blind; the Young-Helmholtz explanation of them . . . . . 906

$\$ 567$. The explanation of colour blindness on Hering's theory . . . . 909

$\$ 568$. The probable subjective condition of the colour blind . . . . 911

$\S 569$. Blue or violet blindness; absolute colour blindness . . . . . . 912

$\$ 570$. Colour blindness in the periphery of the retina . . . . 913

$\$ 571$. 'The influence of the yellow spot . . . . . . . . . 913

$\$ 572$. Colour sensations in relation to the intensity of the stimulus . . . 913

\section{SECTION VII.}

\section{Ox the Developient of Visual Impulses.}

\$78. The blind spot . . . . . . . . . . . 916

\$574. Purkinjè's figures; their import . . . . . . . . . 917

$\$ 575$. Possible theories as to the mode of origin of visual sensations. . 920

$\$ 576$. Photochemistry of the retina; visual purple; the pigment epithelium . . . . . . . . . . . . 922

$\$ 577$. The functions of the layer of rods and cones. The ophthalmoscope 926

$\$ 578$. Possible differences of function of rods and cones . . . . 927

$\$ 579$. Electric currents in the retina . . . . . . . . 928

\section{SEC'TION VIII.}

Ox Some Features of Visual Sexsations especially ix Relation to Visual Perceptioxs.

$\$ 580$. Simultaneous visual sensations; the visual field $\quad$ • . . . 929

$\$ 581$. The psychological and physiological methods; sensations and perceptions ; their want of agreement . . • . . . 929

§582. Irradiation . . . . . . . . . . . . 932

$\$ 583$. Simultaneous contrast . . . . . . . . . . . . 932

\$584. After-images. Successive contrast . . . . . . . . . 933 
$\S 585$. The phenomena of 'contrast' in their bearing on the theories of colour vision .

PAGE

§586. Recurrent sensations. Ocular phantoms or hallucinations . . 936

\section{SECTION IX.}

\section{Binoculak Vision.}

$\$ 587$. The movements of the eye-ball ; their limitations. Centre of rotation, visual axis, visual plane . . . . . . . . 939

$\S 588$. The visual field and field of sight of one eye and of both eyes . . 940

$\$ 589$. Corresponding or identical points . . . . . . . . . 942

$\S 590$. The movements of the eye-ball ; the primary position and secondary positions ; the kind of movements which are possible $\quad$. 944

$\$ 591$. Listing's Law ; the experimental proof . . . . . . . 946

$\$ 592$. The muscles of the eye-ball . . . . . . . . . 948

$\$ 593$. The action of the ocular muscles $\quad . \quad$ c c c c c c c c c c 949

$\$ 594$. The nervous mechanism of the movements of the eye-balls; the coordination of the movements . . . . . . . 952

$\S 595$. The nervous centres for the movements of the eye-balls . . . 956

$\S 596$. The Horopter . . . . . . . . . . 957

\section{SECTION $\mathrm{x}$.}

On Some Features of Visual Perceptions and on Visual Judguents.

$\S 597$. On the differences between the objective field of sight and the sub-

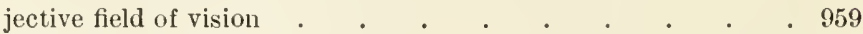

$\S 598$. The psychical processes belonging to visual perceptions; illusions and visual judgments . . . . . . . . . . . 961

$\S 599$. A ppreciation of apparent size . . . . . . . . . . . 962

$\S 600$. Judgment of distance and of actual size . . . . . . . . . 964

$\S 601$. The judgment of solidity . . . . . . . . . . 966

$\S 602$. The struggle of the two fields of vision . . . . . . 967

\section{SECTION XI.}

\section{The Nutrition of the Ere.}

$\S 603$. The arrangement of the blood vessels _ . . . . . 969

$\S 604$. The vaso-motor changes in the eye . . . . . . . 970

The Lymphatics of the Eye.

$\$ 605$. The lymphatic vessels and lymph spaces of the eye . . . . 970

$\S 606$. The aqueous humour; the changes taking place in it; how effected 972

$\$ 607$. The vitreous humour; the changes taking place in it . . . 974 


\title{
SECTION XII
}

The Protective Mechanisus of the Eye.

PAGE

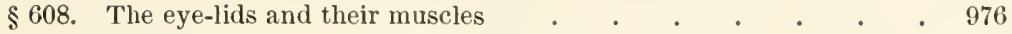

$\S 609$. The conjunctiva and its glands. Tears. The secretion of tears . 977

\section{CHAPTER IV.}

\author{
Hearisg.
}

\section{SECTION I.}

On the General Structure of the Ear and on the Structure and Functions of the Subsibiary Auditory Apparatus.

$\S 610$. The embryonic history of the ear. The otic vesicle . . .

$\$ 611$. The general relations of the parts of the ear; vestibule and cochlea, membranous and bony labyrinth, tympanum, auditory ossicles, membrana tympani and external meatus . . . . 981

$\$ 612$. The general use of the several parts _ . . . . . . . 986

\section{The Conduction of Sound through the Tympanum.}

$\$ 613$. The chain of ossicles as a lever . . . . . . . 988

$\S 614$. Longitudinal and transversal sonorous vibrations. The vibrations

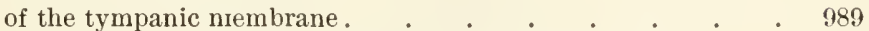

$\S 615$. The conduction of vibrations through the chain of ossicles . . 991

$\S 616$. The conduction of vibrations through the bones of the skull . . 992

$\$ 617$. The action of the tensor tympani and stapedius muscles _ . 993

$\S 618$. The Eustachian tube. . . . . . . . . . . 995

\section{SECTION II.}

\section{On Auditory Sexsations.}

\$619. Noises and musical sounds _ . . . . . . . 998

$\$ 620$. The characters of musical sounds; loudness, pitch and quality;

fundamental and partial tones . . . . . . . 998

$\$ 621$. The limits of auditory sensations . . . . . . . 1000

$\$ 622$. Appreciation of differences of pitch . . . . . . . . . 1001

$\$ 629$. The number of vibrations needed to excite a sensation . . . . 1001

$\S 624$. The characters of noises . . . . . . . . . 1002

$\$ 625$. The effects of exhaustion . . . . . . . . . . 1002

$\$ 626$. The fusion of auditory sensations . . . . . . . . . 1003

$\$ 627$. The interference of vibrations. Beats . . . . . . 1004 


\section{SECTION III}

ON THE DevelopMeNT of Aritory IMpelses.

$\$ 628$. The transmission of impulses through the labyrinth; the functions of the hair cells of the cochlea . . . . . . . 1007

$\$ 629$. The analysis of complex waves of sound; theories as to the mode of action of the organ of Corti . . . . . . . 1013

$\$ 630$. The appreciation of the nature of sounds ultimately a psychical process. . . . . . . . . . . . 1015

$\S 631$. Auditory functions of the vestibular labyrinth . . . . 1016

\section{SEC'TION IV.}

On Aumtory Perceptions and Judguexts.

$\S 632$. Auditory phantoms . . . . . . . . . . 1020

$\$ 633$. The appreciation of outwardness in sounds is connected with the tympanum . . . . . . . . . . . 1021

$\$ 634$. Hearing binaural. The judgment of the direction of sounds . . 1022

$\$ 6: 35$. Judgment of the distance of sounds. . . . . . . . 1023

\section{CHAPTER V. \\ TASTE AND SMELL.}

\section{SECTION I.}

Olfactory Sensations.

$\$ 6: 36$. The sensation due to contact of particles with the membrane $\quad 1025$

$\$ 637$. The chief characters of olfactory sensations . . . . . 1026

$\$ 638$. Olfactory judgments. The olfactory nerve the nerve of smell $\quad 1027$

\section{SECTION II.}

\section{Gustatory Sensations.}

$\S 639$. Sensations of taste usually or frequently accompanied by other sensations . . . . . . . . . . . 1029

$\$ 640$. The different kinds of taste. Sensations of taste provoked by mechanical and electrical stimulation . . . . . 1029

$\$ 641$. The conditions under which taste sensations are excited . . 1031

$\S 642$. The distribution of the several kinds of tastes. Theories as to the mode of origin of taste sensations . . . . . . 1032

$\$ 643$. The nerres of tastc; the chorda tympani . . . . . 1035 


\section{CHAPTER VI.}

\section{On Cutaneous and Some Other Sensations.}

\section{SECTION I.}

The General Features of Cutaneols sensations.

$\$ 644$. Three kinds of cutaneous sensations, of pressure, of heat and cold, and of pain . . . . . . . . . . . 1037

Tactile Sensations or Sensations of Pressure.

$\S 645$. The general characters of tactile sensations . . . . . 1037

$\$ 646$. The localisation of tactile sensations _ . . . . . 1039

\section{Sensations of Heat and Cold.}

$\$ 647$. Sensations of heat and cold due to sudden changes in the temperature of the skin . . . . . . . . 1041

$\S 648$. The general characters of temperature sensations . . . . 1042

$\$ 649$. Tactile and temperature sensations in parts other than the external skin . . . . . . . . . . 1043

\section{SECTION II.}

On Painful and Some Other Kinds of Sensation.

$\S 650$. Sensations of pain distinct from other sensations . . . . . 1044

$\$ 651$. Sensations of pain are extreme degrees of common sensibility $\quad .1044$

$\S 652$. Special nerve endings not necessary for sensations of pain . . 1047

$\S 653$. Hunger and thirst . . . . . . . . . . 1048

\section{SECTION III.}

On the Mode of Development of Cutaneous Sensations.

$\S 654$. The specific energy of nerves. Special terminal organs necessary for the sensations of touch and temperature as distinguished from sensations of pain . $\cdot \cdot \cdot \cdot \cdot \cdot \cdot \cdot \cdot$ for sensations of temperature . . . . . . . 1054

$\$ 656$. The terminal organs for sensations of heat different from those for sensations of cold . . . . . . . . . 1055

$\$ 657$. The importance of contrast in cutaneous sensations _ . . 1056 $\S 658$. The nature of the terminal organs . . . . . . . 1057 


\section{SEC'TION IV}

The Muscular Sense.

$\S 659$. We possess a sense of 'movement,' of 'position,' and of 'effort' 1059

$\$ 660$. 'The muscular sense distinguished from the sense of effort . . 1060

$\$ 601$. The afferent impulses forming the basis of the muscular sense are

distinct from cutaneous impulses . . . . • . 1061

$\S 662$. They are derived from the muscles, ligaments, and tendons . . 1063

\section{SECTION $V$.}

\section{On Tactile Perceptions and Judgments.}

$\$ 663$. The ties between touch and the muscular sense . . . . 1066

$\S 664$. The ties between touch and sight . . . . . . . . 1067

$\$ 665$. Cutaneous sensations may arise otherwise than from cutaneous events.

$\S 666$. Tactile Illusions

\section{CHAP'TER VII.}

\section{On Some Special Muscular Mechanisus.}

\section{SECTION I.}

TIne Volce.

$\S 667$. The laryngoscopic view of the larynx

$\S 668$. The fundamental features of the voice; loudness, pitch, and quality. The main conditions of the utterance of voice; adluction and tightening of the vocal cords . . . . 1074

$\$ 669$. The muscles of the larynx . . . . . . . . 1076

$\$ 670$. The action of the muscles in reference to narrowing and widening the glottis and to tightening and slackening the vocal cords . 1080

$\$ 671$. The nervous mechanisms of the larynx. The respiratory movements of the larynx . . . . . . . . . 1081

$\$ 672$. The nervous mechanism of phonation . . . . . . 1084

$\$ 673$. The cortical area for movements of the larynx _ . . . . 1084

$\S 674$. The different kinds of voice. Changes in the glottis other than those of mere adduction and general tension . . . . . 1085

$\S 675$. Chest-voice and head-voice. The registers of the voice. The complexity of the laryngeal movements . . . . . . 1087

$\$ 676$. The uses of the ventricles and other parts of the larynx . . 1091

$\$$ 6i7. 'The 'break' in the voice at puberty . . . . . . 1091 


\title{
SEC'TION II.
}

\section{SPEECH.}

\begin{abstract}
$\$ 678$. Speech, a mixture of musical sounds and of noises _ . . 1092
§679. Vowels and consonants . . . . . . . . . 1092

$\$ 680$. The manner of formation of the several vowels . . . . 1093

$\$ 681$. The manner of formation of the several groups of consonants . 1096

$\$ 682$. The manner of formation of the more important individual con-
\end{abstract}

sonants . . . . . . . . . . 1097

\section{SECTION III.}

ON SOME LOCOMOTOR MECHANisus.

$\S 683$. The general characters of the actions of skeletal muscles . . 1101

$\S 684$. The erect posture . . . . . . . . . . 1102

$\S 685$. Walking . . . . . . . . . . . . 1102

\section{BOOK IV.}

THE TISSUES AND MECHANISMS OF REPRODUCTION.

$\S 686$. The general features of reproduction $\quad$. $\quad$. $\quad$. $\quad$. 1109

CHAPTER I.

IMPREGNATION.

\section{SECTION I}

Menstruation.

$\$ 687$. The transference of the ovum from the ovary to the uterus. The changes in the uterine mucous membrane . . . . . 1111

\section{SECTION II.}

The Male Organs. 
$\S 690$. Erectile tissue . . . . . . . . . . 1115

$\$ 6$. The nature of erection . . . . . . . . . . . 1116

$\S 692$. The emission of semen . . . . . . . . . 1117

\section{CHAPTER II.}

Pregnaxcy and Birth.

\section{SECTION I.}

The Placenta.

$\S 693$. The spermatozoon enters and unites with the ovum _ . . 1119

$\$ 694$. The formation of the decidua . . . . . . . . . 1119

$\S 695$. The decidua serotina is transformed into the placenta. The shedding of the placenta . . . . . . . . . 1120

\section{SECTION II.}

\section{The Nutrition of the Embryo.}

$\S 696$. The embryo breathes by and feeds upon the maternal blood of the placenta . . . . . . . . . . . .

$\$ 697$. The blood and blood-flow in the unbilical arteries and umbilical vein . . . . . . . . . 1124

$\S 698$. The amniotic fluid, its nature and origin, its relations to the nutri-

tion of the foetus . . . . . . . . . 1126

$\$ 699$. 'The transmission of food material from the mother to the foetus . 1128

$\$ 700$. Glycogen in the fœtus . . . . . . . . . 1129

$\S 701$. 'Tlie movements of the foetus . • . . . . . . . . 1130

$\$ 702$. The digestive functions of the foetus . . . . . . 1130

$\S 703$. The foetal circulation towards the close of uterine life . . . 1132

$\S 704$. 'The cause of the first breath . . . . . . . . 1134

$\S 705$. 'The changes in the circulation taking place at birth . . . 1134

\section{SECTION III.}

\section{Parturition.}

$\S 706$. The period of gestation . . . . . . . . . 1136

$\S 707$. The events of "labour" . . . . . . . . . . 1137

$\S 708$. The reflex nature of parturition . . . . . . . 1140

$\S 709$. The nerves concerned in the act . . . . . . . $11+1$

$\$ 710$. The causes determining the onset of labour . . . . . 1142

$\S 711$. 'The inhibition of parturition . . . . . . . . 114\% 


\section{CHAPTER IIT.}

\section{The Phases of Life.}

$\$ 712$. The composition of the babe as compared with the adult . . 1144

$\S 713$. The curve of growth from birth onwards . . . . . 1145

$\$ 714$. The characters of the nutrition of the babe and infant . . . 1146

$\$ 715$. The nervous system of the babe . . . . . . . 1148

$\$ 716$. Dentition . . . . . . . . . . . . 1149

\$717. Puberty. Differences of sex . . . . . . . . 1150

$\S 718$. Old age . . . . . . . . . . . . 1151

$\$ 719$. Periodical events . . . . . . . . . . . 115:3

$\$ 720$. Sleep . . . . . . . . . . . . . 1153

$\$ 721$. Other diurnal changes in the functions . . . . . . 1156

\section{CHAPTER IV.}

DеАтн.

$\S 722$. The general causation of death

APPENDIX
INDEX




\section{LIST OF FIGURES.}

FIG.

1. A muscle-nerve preparation . . . . . . . . . 56

2. Diagram of du Bois-Reymond key . . . . . . . . . . . 58

3. Diagram illustrating apparatus arranged for experiments with muscle and nerve . . . . . . . . . 60

4. Diagram of an induction coil . . . . . . . . . . . $\quad$. 62

5. 'The magnetic interruptor . . . . . . . . . . . . . 63

6. 'The magnetic interruptor with Helmholtz's arrangement for equalizing the nilke and break shocks . . . . . . . . . . . 64

7. A muscle-curve from the gastrocnemius of a frog . . . . . . 66

8. 'The same, with the recording surface moving slowly . . . . . 66

9. The same, with the recolding surface travelling very rapidly . . 67

10. The penduluin myograph . . . . . . . . . . . 68

11. Diagram of an arrangement of a vibrating tuning-fork with a Desprez signal . . . . . . . . . . . . . . . 70

12. Curves illustrating the measurement of the velocity of a nervous impulse 73

13. Tracing of a double muscle-curve . . . . . . . . . . 76

14. Muscle-curve. Single induction-shocks repeated slowly . . . . 76

15. The same, repeated more rapidly . . . . . . . . . 77

16. The same, repeated still more rapidly . . . . . . . $\quad$. 77

17. Tetanus produced with the ordinary magnetic interruptor . . . 78

18. Non-polarizable electrodes . . . . . . . . . . . . 98

19. Diagram illustrating the electric currents of nerve and muscle . $\quad 99$

20. Diagram illustrative of the progression of electric changes . . . . 10:

21. Diagram of ascending and descending constant current . . 112

22. Diagram of the electrotonic changes in irritability . . . . . 114

23. Diagram illustrating electrotonic currents . . . . . . 115

24. Sclieine of the nerves of a segment of the spinal cord . . . . 140

25. Apparatus for investigating blood pressure . . . . . . . 157

26. Tracing of arterial pressure in $\mathbf{d o g} . \quad$. . . . . . . . . 158

27. 'Tracing of arterial pressure in rabbit . . . . . . . . . . 159

28. Ludwig's kymograpl . . . . . . . . . . . 160

29. Diagram of fall of blood pressure in arteries, capillaries and veins . 161

30. Arterial scheine . . . . . . . . . . . 167

81. Tracing from arterial nodel with little peripheral resistance . . 168

22. 'The same with increased peripheral resistance . . . . . 169

33. Kudwig's stromuhr . : . . . . . . . . . . . 173

34. Chauveau and Lortet's hrematachometer . . . . . . . . 174

35. Diagram illustrating causes determining the velocity of the flow . . 176

36. 'Tracing from heart of cat . . . . . . . . . . . . 186 
37. Marey's tambour, with cardiac sound .

38. Tracings from right auricle and ventricle of horse (Chauveau and Marey) 193

39. Curves of endocardiac pressure by means of piston manometer . . 194

40. The membrane manometer of Hürthle . . . . . . . . 194

41. Diagram of the same . . . . . . . . . . . 195

42. Curve of ventricular pressure : membrane manometer . . . . 196

43. Stolnikow's apparatus for measuring the output of the heart . . 198

44. Cardiometer of Roy and Adami . . . . . . . . . . 199

45. 'Tracing from the heart of a cat, by means of a light lever . . . 200

46. Cardiograms . . . . . . . . . . 201

47. Myocardiogram . . . . . . . . . . . . 202

48. Diagram of application of aortic and ventricular catheters . . . 203

49. Simultaneous tracings of ventricular and aortic pressure . . . 204

50. Diagram of the differential manometer of Hürthle _ . . . . 204

51. Simultaneous curves of aortic and ventricular pressure, and of the differential manometer; descending systolic plateau . . . 205

52. The same, with the recording surface travelling rapidly . . . 205

53. Simultaneous curves of aortic and ventricular pressure and of the differential manometer; ascending systolic plateau . . . . 208

54. Diagram of ventricular and aortic pressure and of the cardiac impulse 209

55. Maximum and minimum manometer . . . . . . . . 210

56. Fick's spring manometer . . . . . . . . . 220

57. Diagram of a sphygmograph . . . . . . . . . . 221

58. Pulse tracing from radial artery . . . . . . . . . . 22:3

59. Diagram of artificial pulse tracings . . . . . . . . 224

60. Diagram of progression of pulse wave . . . . . . . . 225

61. Pulse tracing with different pressures . . . . . . . 226

62. Pulse tracing from dorsalis pedis artery . . . . . . 227

63. Pulse tracing from carotid artery . . . . . . . . 230

64. Anacrotic pulse tracing . . . . . . . . . . . . 231

65. Dicrotic pulse tracing . . . . . . . . . . . . 231

66. A perfusion cannula . . . . . . . . . . 240

67. Diagram of apparatus for registering the beat of a frog's heart . . 241

68. Inhibition of heart beat in the frog . . . . . . . . 245

69. Diagram of the course of cardiac augmentor fibres in the frog . . 247

70. Cardiac inhibition in the mammal. . . . . . . . 250

71. The course of cardial inhibitory and augmentor fibres in the dog. . 254

72. Diagram of the course of vaso-constrictor fibres . . . . . 266

73. Diagram of the nerves of the submaxillary gland . . . . . . 267

74. The depressor nerve . . . . . . . . . . . . 281

75. Rise of pressure due to stimulation of the sciatic nerve . . . 282

76. Diagrammatic representation of the submaxillary gland of the dog with its nerves and blood vessels . . . . . . . 334

77. Alveoli of the pancreas of a rabbit at rest and in activity . . . 341

78. Changes in the parotid gland during secretion . . . . . . 343

79. Sections of the parotid gland of the rabbit at rest and after stimulation of the cervical sympathetic nerve . . . . . . 344

80. Mucous cells from the fresh submaxillary gland of the dog . . . 344

81. Alveoli of dog's submaxillary gland in loaded and in discharged phases 345

82. Gastric gland of mammal (bat) during activity . . . . . . 346 
FIG.

83. Diagram illustrating the influence of food on the secretion of the pancreatic juice

84. Diagram to illustrate the nerves of the alimentary canal in the dog . 384

85. Apparatus for taking tracings of the movements of the column of air in respiration.

86. Tracing of thoracic respiratory movements .

87. Diagram of Ludwig's mercurial gas-pump . .

88. Diagran of Alvergniat's pump

89. The spectra of oxy-hæmoglobin in different grades of concentration, of (reduced) hæmoglobin, and of carbonic-oxide-hæmoglobin . 45:

90. Spectra of some derivatives of hæmoglobin . . . . . . . . . 459

91. Curve of the effect on respiration of section of one vagus . . . 476

92. Curve of the effect on respiration of section of both vagus nerves . 477

9.). Curve of the quickening of respiration by gentle stimulation of the central end of the vagus trunk . . . . . . . . 477

94. Curve of respiratory increase due to stimulation of vagus nerve . 478

95. Curve of the inhibitory effects of stimulation of the superior laryngeal nerve.

96. Curves illustrating the effects of distension and collapse of the lung .

97. Curve shewing the effects of repeated inflations of the lungs . .

98. Curve shewing the effects of repeated suctions of the lungs _. . 482

99. Curves of blood-pressure and intra-thoracic pressure taken together. 498

100. Curves of blood-pressure during a suspension of breathing . . 505

101. Curve shewing Traube-Hering undulations . . . . . . 508

102. Renal onconter . . . . . . . . . . . 526

103. Oncograph . . . . . . . . . . . . . 527

104. Tracing from renal oncometer . . . . . . . . . 528

105. Section of the liver of frog . . . . . . . . . . . 564

106. Three pliases of the hepatic cells of the frog . . . . . . 565

107. Section of mammalian liver rich in glycogen . . . . . . 568

108. Section of manmalian liver containing little or no glycogen _ . 568

109. Normal spleen curve from dog . . . . . . . . 580

110. A transverse dorso-ventral section of the spinal cord (human) at the level of the sixtlı thoracic nerve . . . . . . .

111. Transverse dorso-ventral section of the spinal cord (human) at the level of the sixth cervical nerve . . . . . . .

112. Transverse dorso-ventral section of the spinal cord (human) at the level of the third lumbar nerve . . . . . . .

113. Diagram to illustrate the general arrangement of the several tracts of white matter in the spinal cord . . . . . . 6 686-687

114. Diagram illustrating some of the features of the spinal cord at different levels .

115. Diagram shewing the united sectional areas of the spinal nerves proceeding from below upwards. . . . . . . .

116. Diagram shewing the variations in the sectional area of the grey matter of the spinal cord, along its length . . . . .

117. Diagram shewing the relative sectional areas of the spinal nerves as they join the spinal cord .

118. Diagram shewing the variations in the sectional area of the lateral columis of the spinal cord, along its length. 
119. Diagram shewing the variations in the sectional area of the anterior columns of the spinal cord, along its length .

120. Diagram shewing the variations in the sectional area of the posterior columns of the spinal cord, along its length

121. The areas of the cerebral convolutions of the dog . . . . 741

122. Outline of brain of monkey to slew the principal sulei and gyri . 744

123. Left hemisplere of the brain of monkey viewed from the left side and from above . . . . . . . . . . . . . . . 745

124. Mesial aspect of the left half of the brain of inonkey . . . . 747

125. Outline of horizontal section of brain, to shew the internal capsule . 750

126. Outline of a sagittal section through the hemisphere . . . . 751

127. Outline of a transverse dorso-ventral section of the right half of the brain

128. Transverse dorso-ventral section through the crus and anterior corpora quadrigemina .

129. Transverse dorso-ventral section through the fore part of the pons . 754

130. Transverse dorso-ventral section through the pons at the exit of the fiftlı nerve

131. Transverse dorsal section through the bulb at the widest part of the fourtl ventricle

132. Transverse dorso-ventral section through the bulb just belind the pons

133. Diagram to illustrate the relative size of the pyramidal tract in man, monkey and $\operatorname{dog}$. . . . . . . . .

134. Diagran of the convolutions and fissures on the lateral surface of the

right cerebral hemisphere of man . . . . . .

135. The same on the mesial surface . . . . . . .

136. The right lateral aspect of the cerebrum of man in outline, to illustrate the cortical areas . . . . . . . . .

137. Mesial surface of the right cerebral hemisphere of man in outline, to illustrate the cortical areas . . . . . . . . . 768

138. Diagram to illustrate the nervous apparatus of vision in man . $\quad 783$

139. Diagrammatic outline of a horizontal section of the eye, to illustrate the relations of the various parts . . . . . . . . . . 8836

140. Diagram of simple optical system . . . . . . . . . $\quad$. 840

141. Diagram of the schematic or diagrammatic eye . . . . 843

142. Diagram of the formation of a retinal inage . . . . . 844

143. Diagram of Scheiner's experiment . . . . . . . . 848

144. Diagram of images reflected from the eye . . . . . 852

145. Diagram of the ciliary nuuscle as seen in a vertical radial section of the ciliary region . . . . . . . . . . 855

146. Diagram to illustrate accommodation . . . . . . . 856

147. Diagrammatic representation of the nerves governing the pupil . 859

148. Diagram illustrating chromatic aberration . . . . . 873

149. Diagram to illustrate entoptical images . . . . . . . . $\quad$. 876

150. Diagram of three primary colour sensations _ . . . . . 899

151. Diagram to illustrate Hering's theory of colour vision _ . . 902

152. Diagram illustrating the formation of Purkinjé's figures when the illumination is directed through the sclerotic . . . . . 918

153. Diagram illustrating the formation of Purkinje's figures when the illumination is directed through the cornea. . . . . 
FIS.

154. Diagram to illustrate the principles of a simple form of opthalmoscope

155. Figure to illustrate irradiation .

156. The visual field of the right eye

157. 'The visual fields (fields of sight) of the two eyes when the eyes converge to the same fixed point .

158. Diagram illustrating corresponding points . . . . . . 94?

159. Figure to illustrate the insertions of the ocular muscles . . . 949

160. I)iagram to illustrate the actions of the ocular muscles . . . 950

161. Diagram illustrating a simple horopter . . . . . . . . 95̃

162. Figure to illustrate the appreciation of apparent size $\quad$. . . 962

163. The same . . . . . . . . . . . . 96\%

164. Figure to illustrate an optical effect produced by parallel slanting lines

165. Figure to illustrate binocular vision .

166. Diagram to illustrate the general structure of the ear

167. The bony labyrinth .

168. The membrana tympani .

169. Diagram to illustrate the relations of auditory passage, tympanum and Eustachian tube

170. Frontal section through the tympanum

171. Diagram of the median wall of the tympanum

172. 'The auditory ossicles

173. The ossicles in position

174. The ligaments of the ossicles

175. The malleus and incus in position

176. Diagram of the outer wall of the tympanum as seen from the mesial side

177. The stapes in position

178. The membranous labyrinth as seen from above . . . . 1008

179. The membranous labyrinth and the endings of the auditory nerve . 1009

180. Diagram of a transverse section of a whorl of the cochlea $\quad$. 1010

181. Diagram of the organ of Corti _ . . . . . . . . 1011

18:. Diagrain of the constituents of the organ of Corti . . . . 1012

18.. Diagram of a laryngoscopic view of the larynx _ . . . . 1071

184. Diagram of the superior aperture of the larynx _ . . . . 1072

185. Diagram of the larynx in vertical section . . . . . . . 1072

186. Diagran of the larynx in vertical transverse section . . . . 1073

187. The larynx as seen by means of the laryngoscope in different conditions of the glottis . . . . . . . . . . 1075

188. Diagram of the transverse and oblique arytenoid and of the posterior crico-arytenoid muscles . . . . . . . . . . . . . 1076

189. Diagram to illustrate the thyro-arytenoid muscles . . . . . 107

190. The internal thyro-arytenoid muscle . . . . . . 1078

191. The lateral crico-arytenoid muscle . . . . . . . 1079

192. The crico-thyroid muscle . . . . . . . . . . . . . 1079

19:. Diagram to illustrate the contact of the feet with the ground in walking

194. Diagram to illustrate running

195. Diagram to illustrate the fotal circulation 
FIG.

196. Zinc sarcolactate. (After Kïhne) . . . . . . . . 12:4

197. Calcium sarcolactate. (After Kïuhne) . . . . . . . 12:34

198. Calcium oxalate. (After Funke) . . . . . . . 1285

199. Cholesterin crystals. (After Funke) . . . . . . 12:37

200. Chareot's crystals. (Krukenberg) . . . . . . . . 1241

201. Glycine crystals. (After Funke) . . . . . . . 1243

202. Leucine crystals. (Krukenberg) . . . . . . . 1241

20:3. 'Taurine erystals. (After Kiilne) . . . . . . . 1246

204. Creatine erystals. (Krukenberg after Kïhne) . . . . 1247

205. Creatinine crystals. (Krukeuberg after Kühne) . . . . 124!)

206. Creatinine-zinc-chloride erystals. (Kirukeuberg after Kïhne) 1249

207. Cystine crystals. (After Funke) . . . . . . . 1251

208. Urea erystals separated by slow evaporation from aqueous solution. (After Funke) . . . . . . . . . . . . . 1254

209. Crystals of nitrate of urea. (Krukenberg after Kühne). . . 1255

210. Crystals of oxalate of urea. (Krukenberg after Kiihne) . . 1256

211. Crystals of uric aeid. (Krukenberg after Kïhne) . . . . 1258

212. Crystals of uric acid. (After Funke). . . . . . . . 1259

213. (Krukenberg after Kühne) . . . . . . . . . . 1259

214. (Krukenberg after Kïühne) . . . . . . . . . . 1260

215. Crystals from concentrated urine of calf. (After Kühne) • 1262

216. Crystals of allantoin prepared by the oxidation of uric acid. (After Kühne) . . . . . . . . . . . . . . . 1263

217. Xanthine hydrochloride, $\mathrm{C}_{5} \mathrm{II}_{4} \mathrm{~N}_{4} \mathrm{O}_{2} \cdot \mathrm{HCl}$. (Kühne) . . . 1265

218. Xanthine nitrate, $\mathrm{C}_{5} \mathrm{H}_{4} \mathrm{~N}_{4} \mathrm{O}_{2} \cdot \mathrm{HNO}_{3}$. (Kühne) . . . . 1265

219. Crystals of xanthine silver-nitrate, $\mathrm{C}_{5} \mathrm{H}_{4} \mathrm{~N}_{4} \mathrm{O}_{2} \cdot \mathrm{AgNO}$. (Krukenberg after Kï̈hne) . . . . . . . . . . . . . . . . . .

220. Hypoxanthine silver-nitrate, $\mathrm{C}_{5} \mathrm{H}_{4} \mathrm{~N}_{4} \mathrm{O}, \mathrm{AgNO}_{3}$. (Krukenberg after Kühne) . . . . . . . . . . . . . . . 1268

221. Hypoxanthine-nitrate, $\mathrm{C}_{5} \mathrm{H}_{4} \mathrm{~N}_{4} \mathrm{O}$ ) $\mathrm{HNO}_{3}$. (Kühne) . . $\quad 1268$

222. Hypoxanthine-hydrochloride, $\mathrm{C}_{5} \mathrm{H}_{4} \mathrm{~N}_{4}($ ). $\mathrm{HCl}$. (Kühne) . 1269

223. Guanine hydrochloride, $\mathrm{C}_{5} \mathrm{H}_{5} \mathrm{~N}_{5} \mathrm{O}$. IICl $+\mathrm{H}_{2} \mathrm{O}$. (After Kïihne) 1270

224. Guanine nitrate, $\mathrm{C}_{5} \mathrm{H}_{5} \mathrm{~N}_{5} \mathrm{O}$. IINO $\mathrm{H}_{3}+1 \frac{1}{2} \mathrm{II}_{2} \mathrm{O}$. (After Kühne) . 1270

2.25. Hippuric acid clystals. (After Funke) . . . . . . 127:3

226. Tyrosine crystals. (Krukenberg) . . . . . . . 1275

227. Crystals of kynurenic acid. (After Kithne) . . . . . . 1276

228. Crystals of barium kynurenate. (After Kühne) . . . . 1276

229. Inosite crystals. (After Kühne) . . . . . . . . . 1277

230. Crystals of oxy-hæmoglobin. (After Funke) . . . . . . 1292

2:31. Iremin crystals from a drop of blood. (Kühne) • . . . 1300

232. Hæmin erystals. (After Preyer) . . . . . . 1300

23:3. Hæmatoidin crystals. (Frey after Funke) . . . . . $\quad 1302$

234. Bilirubin erystallized from carbon-disulphide. (Krukenberg) • 1304 



\section{INTRODUCTION.}

§1. Dissection, aided by microscopical examination, teaches us that the body of man is made up of certain kinds of material, so differing from each other in optical and other physical characters and so built up together as to give the body.certain structural features. Chemical examination further teaches us that these kinds of material are composed of various chemical substances, a large number of which have this characteristic that they possess a considerable amount of potential energy capable of being set free, rendered actual, by oxidation or some other chemical change. Thus the body as a whole may, from a chemical point of view, be considered as a mass of various chemical substances, representing altogether a considerable capital of potential energy.

§2. This body may exist either as a living body or (for a certain time at least) as a dead body, and the living body may at any time become a dead body. At what is generally called the moment of death (but artificially so, for as we shall see the processes of death are numerous and gradual) the dead body so far as structure and chemical composition are concerned is exceedingly like the living body; indeed the differences between the two are such as can be determined only by very careful examination, and are still to a large extent estimated by drawing inferences rather than actually observed. At any rate the dead body at the moment of death resembles the living body in so far as it represents a capital of potential energy. From that moment onwards however the capital is expended; by processes which are largely those of oxidation, the energy is gradually dissipated, leaving the body chiefly in the form of heat. While these chemical processes are going on the structural features disappear, and the body, with the loss of nearly all its energy, is at last resolved into "dust and ashes." 
The characteristic of the dead body then is that, being a mass of substances of considerable potential energy, it is always more or less slowly losing energy never gaining energy; the capital of energy present at the moment of death is more or less slowly diminished, is never increased or replaced.

$\$ 3$. When on the other hand we study a living body we are struck with the following salient facts.

1. The living body moves of itself, either moving one part of the body on another or moving the whole body from place to place. These movements are active; the body is not simply pulled or pushed by external forees, but the motive power is in the body itself, the energy of each movement is supplied by the body itself.

2. These movements are determined and influenced, indeed often seem to be started, by changes in the surroundings of the body. Sudden contact between the surface of the body and some foreign object will often call forth a movement. The body is sensitive to changes in its surroundings, and this sensitiveness is manifested not only by movements but by other changes in the body.

3. It is continually generating heat and giving out heat to surrounding things, the production and loss of heat, in the case of man and certain other animals, being so adjusted that the whole body is warm, - that is, of a temperature higher than that of surrounding things.

4. From time to time it eats, - that is to say, takes in to itself supplies of certain substances known as food, these substances being in the main similar to those which compose the body and being like them chemical bodies of considerable potential energy, capable through oxidation or other chemical changes of setting free a considerable quantity of energy.

5. It is continually breathing, - that is, taking in from the surrounding air supplies of oxygen.

6. It is continually, or from time to time, discharging from itself into its surroundings so-called waste matters, which waste matters may be broadly described as products of oxidation of the substances taken in as food, or of the substances composing the body.

Hence the living body may be said to be distinguished from the dead body by three main features.

The living lody like the dead is continually losing energy (and losing it more rapidly than the dead body, the special breathing arrangements permitting a more rapid oxidation of its substance), but unlike the dead body is hy means of food continually restoring its substance and replenishing its store of energy.

The energy set free in the dead body by the oxidation and other chemical changes of its substance leaves the body almost exclusively in the form of heat, whereas a great deal of energy leaves the living borly as mechanical work, the result of various movements of the body; and as we shall see a great deal of the 
energy which ultimately leaves the body as heat exists for a while within the living body in other forms than heat, though eventually transformed into heat.

The changes in the surroundings affect the. dead body at a slow rate and in a general way only, simply lessening or increasing the amount or rate of chemical change and the quantity of heat thereby set free, but never diverting the energy into some other form, such as that of movement; whereas changes in the surroundings may in the case of the living body rapidly, profoundly, and in special ways affect not only the amount but also the kind of energy set free. The dead body left to itself slowly falls to pieces, slowly dissipates its store of energy, and slowly gives out heat. A higher or lower temperature, more or less moisture, a free or scanty supply of oxygen, the advent of many or few putrefactive organisms, - these may quicken or slacken the rate at which energy is being dissipated but do not divert that energy from heat into motion; whereas in the living body so slight a change of surroundings as the mere touch by a hair of some particular surface, may so affect the setting free of energy as to lead to such a discharge of energy in the form of movement that the previously apparently quiescent body nay be suddenly thrown into the most violent convulsions.

The differences therefore between living substance and dead substance though recondite are very great, and the ultimate object of Physiology is to ascertain how it is that living substance can do what dead substance cannot, - can renew its substance and replenish the energy which it is continually losing, and can according to the nature of its surroundings vary not only the amount but also the kind of energy which it sets free. Thus there are two great divisions of Physiology: one having to do with the renewal of substance and the replenishment of energy, the other having to do with the setting free of energy.

$\$ 4$. Now, the body of man (or one of the higher animals) is a very complicated structure consisting of different kinds of material which we call tissues, such as nuscular, nervous, connective, and the like, variously arranged in organs, such as heart, lungs, muscles, skin, etc., all built up to form the body according to certain morphological laws. But all this complication, though advantageous and indeed necessary for the fuller life of man, is not essential to the existence of life. The amœba is a living being ; it renews its substance, replenishes its store of energy, and sets free energy now in one form, now in another; and yet the amœba may be said to have no tissues and no organs; at all events this is true of closely allied but not so well-known simple beings. Using the more familiar amoba as a type, and therefore leaving on one side the nucleus, and any distinction between endosarc and ectosare, we may say that its body is homogeneous in the sense that if we divided it into small pieces, each piece would be like all 
the others. In another sense it is not homogeneous; for we know that the amoba receives into its substance material as food, and that this food or part of it remains lodged in the body until it is made use of and built up into the living substance of the body; and each piece of the living substance of the body must have in or near it some of the material which it is about to build up into itself. Further, we know that the anœba gives out waste matters, such as carbonic aeid and other substances; and each piece of the amœeba must contain some of these waste matters about to be, but not yet, discharged from the piece. Each piece of the ancha will therefore contain these three things: the actual living substance, the food about to become living substance, and the waste matters which have ceased to be living substance.

Moreover, we have reasons to think that the living substance does not break down into the waste matters which leave the body at a single bound, but that there are stages in the downward progress between the one and the other. Similarly, though our knowledge on this point is less sure, we have reason to think that the food is not incorporated into the living substance at a single step, but that there are stages in the upward progress from the dead food to the living substance. Each piece of the body of the amoba will therefore contain substances representing various stages of becoming living, and of ceasing to be living, as well as the living substance itself. And we may safely make this statement though we are quite unable to draw the line where the dead food on its way up becomes living, or the living substance on its way down becomes dead.

$\$ 5$. Nor is it necessary for our present purpose to be able to point out under the microscope, or to describe from a histological point of view, the parts which are living and the parts which are dead food or dead waste. The body of the amoba is frequently spoken of as consisting of 'protoplasm.' The name was originally given to the matter forming the primordial utricle of the vegetable cell as distinguished from the cell wall on the one hand, and from the fluid contents of the cell or cell sap on the other, and also we may add from the nucleus. It has since been applied very generally to such parts of animal bodies as resemble, in their general features, the primordial utricle. Thus the body of a white blood corpuscle, or of a gland cell, or of a nerve cell, is said to consist of protoplasin. Such parts of animal bodies as do not in their general features resemble the matter of the primordial utricle are not called protoplasin, or, if they at some earlier stage did bear such resemblance, but no longer do so, are sometimes, as in the case of the substance of a muscular fibre, called 'differentiated protoplasin.' Protoplasm in this sense sometimes appears, as in the outer part of most amœbæ, as a mass of glassy-looking material, either continuous or interrupted by more or less spherical spaces or vacuoles filled with fluid, sometimes as in a gland cell as a more 
refractive, cloudy-looking, or finely granular material ar'ranged in a more or less irregular network, or spongework, the interstices of which are occupied either by fluid or by some material different from itself. We shall return however to the features of this "protoplasm' when we come to treat of white blood corpuseles and other 'protoplasmic' structures. 'Meanwhile it is sufficient for our present purpose to note that lodged in the protoplasm, discontinuous with it, and forming no part of it, are in the first place collections of fluid, of watery solutions of various substances, occupying the more regular vacuoles or the more irregular spaces of the network, and in the second place discrete granules of one kind or another, also forming no part of the protoplasm itself, but lodged either in the bars or substance of the protoplasm or in the vacuoles or meshes.

Now, there can be little doubt that the fluids and the discrete gramules are dead food or dead waste, but the present state of our knowledge will not permit us to make any very definite statement about the protoplasm itself. We may probably conclude, indeed we may be almost sure, that protoplasm in the above sense is not all living substance; that it is made up partly of the real living substance, and partly of material which is becoming living or has ceased to be living; and in the case where protoplasm is described as forming a network, it is possible that some of the material occupving the meshes of the network may be, like part of the network itself, really alive. 'Protoplasm' in fact, as in the sense in which we are now using it, and shall continue to use it, is a morphological term; but it must be borne in mind that the same word 'protoplasm' is also frequently used to denote what we have just now called 'the real living substance.' The word then embodies a physiological idea; so used it may be applied to the living substance of all living structures, whatever the microscopical features of those structures; in this sense it cannot at present, and possibly never will be recognised by the microscope, and our knowledge of its nature must be based on inferences.

Keeping then to the phrase 'living substance' we may say that each piece of the body of the amoba consists of living substance in which are lodged, or with which are built up in some way or other, food and waste in various stages.

Now, an amcba may divide itself into two, each half exhibiting all the phenomena of the whole; and we can easily imagine the process to be repeated until the amœba was divided into a multitude of exceedingly minute amœbæ, each having all the properties of the original. But it is obvious, as in the like division of a mass of a chemical substance, that the division could not be repeated indefinitely. Just as in division of the chemical mass we come to the chemical molecule, further division of which changes the properties of the substance, so in the continued division of the amœba we should come to a stage in which further division interfered with the physiological actions; we should come 
to a physiological unit, corresponding to but greatly more complex than the chemical molecule. This unit to remain a physiological unit and to continue to live must contain not only a portion of the living substance but also the food for that living substance, in several at least of the stages, from the initial raw food up to the final 'living' stages, and must similarly contain various stages of waste.

§6. Now the great characteristic of the typically simple amoba (leaving out the nucleus) is that, so far as we can ascertain, all the physiological units are alike; they all do the same things. Each and every part of the body receives food more or less raw and builds it up into its own living substance; each and every part of the body may be at one time quiescent and at another in motion; each and every part is sensitive and responds by movement or otherwise to various changes in its surroundings.

The body of man, in its first stage, while it is as yet an ovum, if we leave aside the nucleus and neglect differences caused by the unequal distribution of food material or yolk, may also be said to be composed of like parts or like physiological units.

By the act of segmentation however the ovum is divided into parts or cells which early shew differences from each other; and these differences rapidly increase as development proceeds. Some cells put on certain characters and others other characters; that is to say, the cells undergo histological differentiation. And this takes place in such a way that a number of cells lying together in a group become eventually converted into a tissue; and the whole body becomes a collection of such tissues arranged together according to inorphological laws, each tissue having a definite structure, its cellular nature being sometimes preserved, sometines obscured or even lost.

This histological differentiation is accompanied by a physiological division of labour. Each tissue may be supposed to be composed of physiological units, the units of the same tissue being alike but differing from the units of other tissues; and corresponding to this difference of structure, the units of different tissues behave or act differently. Instead of all the units as in the amoba doing the same things equally well, the units of one tissue are told off as it were to do one thing especially well, or especially fully, and thus the whole labour of the body is divided among the several tissues.

s. The several tissues may thus be classified according to the work which they have to do; and the first great distinction is into (1) the tissues which are concerned in the setting free of energy in special ways, and (2) the tissues which are concerned in replenishing the substance and so renewing the energy of the body.

Each physiological unit of the amœba while it is engaged in 
setting free energy so as to move itself, and by reason of its sensitiveness so directing that energy as to produce a movement suitable to the conditions of its surroundings, has at the same time to bear the labour of taking in raw food, of selecting that part of the raw food which is useful and rejecting that which is useless, and of working up the accepted part through a variety of stages into its own living substance; that is to say, it has at the same time that it is feeling and moving to carry on the work of digesting and assimilating. It has moreover at the same time to throw out the waste matters arising from the changes taking place in its own substance, having first brought these waste matters into a condition suitable for being thrown out.

$\S 8$. In the body of man, movements, as we shall see, are broadly speaking carried out by means of muscular tissue, and the changes in muscular tissue which lead to the setting free of energy in the form of movement are directed, governed, and adapted to the surroundings of man, by means of nervous tissue. Rays of light fall on the nervous substance of the eye called the retina, and set up in the retina changes which induce in the optic nerve other changes, which in turn are propagated to the brain as nervous impulses, both the excitation and the propagation involving an expenditure of energy. These nervous impulses reaching the brain may induce other nervous impulses which travelling down certain nerves to certain muscles may lead to changes in those muscles by which they suddenly grow short and pull upon the bones or other structures to which they are attached, in which case we say the man starts; or the nervous impulses reaching the brain may produce some other effects. Similarly, sound falling on the ear, or contact between the skin and some foreign body, or some change in the air or other surroundings of the body, or some change within the body itself may so affect the nervous tissue of the body that nervous impulses are started and travel to this point or to that, to the brain or elsewhere, and eventually may either reach some muscular tissue and so give rise to movements, or may reach other tissues and produce some other effect.

The muscular tissue then may be considered as given up to the production of movement, and the nervous tissue as given up to the generation, transformation, and propagation of nervous impulses. In each case there is an expenditure of energy, which in the case of the muscle, as we shall see, leaves the body partly as heat, and partly as work done, but in the case of nervous tissue is wholly or almost wholly transformed into heat before it leaves the body; and this expenditure necessitates a replenishment of energy and a renewal of substance.

\$9. In order that these inaster tissues - the nervous and muscular tissues - may carry on their important works to the best advantage, they are relieved of much of the labour which falls upon each physiological unit of the amoba. They are not presented 
with raw food; they are not required to carry out the necessary transformations of their immediate waste matters. The whole of the rest of the body is engaged (1) in so preparing the raw food, and so bringing it to the nervous and muscular tissues that these may build it up into their own substance with the least trouble; and (2) in receiving the waste matters which arise in muscular and nervous tissues, and preparing them for rapid and easy ejection from the body.

Thus to certain tissues, which we may speak of broadly as 'tissues of digestion,' is allotted the duty of acting on the food and preparing it for the use of the muscular and nervous tissues; and to other tissues, which we may speak of as 'tissues of excretion,' is allotted the duty of clearing the body from the waste matters generated by the inuscular and nervous tissues.

$\$ 10$. These tissues are for the most part arranged in machines or mechanisms called organs, and the working of these organs involves movement. The movements of these organs are carried out, like the other movements of the body, chiefly by means of muscular tissue governed by nervous tissue. Hence we may make a distinction between the muscles which are concerned in producing an effect on the world outside man's body - the muscles by which man does his work in the world - and the muscles which are concerned in carrying ont the movements of the internal organs; and we may similarly make a distinction between the nervous tissue concerned in carrying out the external work of the body and that concerned in regulating the movements and, as we shall see, the general conduct of the internal organs. But these two classes of muscular and nervous tissue though distinct in work and, as we shall see, often different in structure, are not separated or isolated. On the contrary, while it is the main duty of the nervous tissue as a whole (the nervous system, as we may call it) to carry out, by means of nervous impulses passing hither and thither, what may be spoken of as the work of man, and in this sense is the master tissue, it also serves as a bond of union between itself and the muscles doing external work on the one hand, and the organs of digestion or excretion on the other, so that the activity and conduct of the latter may be adequately adapted to the needs of the former.

$\$ 11$. Lastly, the food prepared and elaborated by the digestive organs is carried and presented to the muscular and nervous tissues in the form of a complex fluid known as blood, which driven by means of a complicated mechanism known as the vascular system, circulates all over the body, visiting in turn all the tissues of the body, and by a special arrangement known as the respiratory mechanism, carrying in itself to the several tissues a supply of oxygen as well as of food more properly so called.

The motive power of this vascular system is supplied as in the case of the digestive system by means of muscular tissue, the 
activity of which is similarly governed by the nervous system, and hence the flow of blood to this part or that part is regulated according to the needs of the part.

$\$ 12$. The above slight sketch will perhaps suffice to shew not only how numerous but how varied are the problems with which Physiology has to deal.

In the first place there are what may be called general problems, such as, How the food after its preparation and elaboration into blood is built up into the living substance of the several tissues? How the living substance breaks down into the dead waste? How the building up and breaking down differ in the different tissues in such a way that energy is set free in different modes, - the inuscular tissue contracting, the nervous tissue thrilling with a nervous impulse, the secreting tissue doing chemical work, and the like? To these general questions the answers which we can at present give can hardly be called answers at all.

In the second place there are what may be called special problems, such as, What are the various steps by which the blood is kept replenished with food and oxygen, and kept free from an accumulation of waste, and how is the activity of the digestive, respiratory, and excretory organs, which effect this, regulated and adapted to the stress of circumstances? What are the details of the working of the vascular mechanism by which each and every tissue is forever bathed with fresh blood, and how is that working delicately adapted to all the varied changes of the body? And, compared with which all other special problems are insignificant and preparatory only, How do nervous impulses so flit to and fro within the nervous system as to issue in the movements which make up what we sometimes call the life of man? It is to these special problems that we must chiefly confine our attention, and we may fitly begin with a study of the blood. 



\section{B00K I.}

BLOOD. THE TISSUES OF MOVEMENT. THE VASCULAR MECHANISM. 



\title{
CHAPTER I.
}

\author{
BLOOD.
}

$\$ 13$. The several tissues are traversed by minute tubes, - the capillary blood vessels, - to which blood is brought by the arteries, and from which blood is carried away by the veins. These capillaries form networks the meshes of which, differing in form and size in the different tissues, are occupied by the elements of the tissue which consequently lie outside the capillaries.

The blood flowing along the capillaries consists, under normal conditions, of an almost colourless fluid, the plasma, in which are carried a number of bodies, the red and the white corpuscles. Outside the capillary walls, filling up such spaces as exist between the capillary walls and the cells or fibres of the tissue, or between the elements of the tissue themselves, is found a colourless fluid, resembling in many respects the plasma of blood and called lymph. Thus all the elements of the tissue and the outsides of all the capillaries are bathed with lymph, which, as we shall see hereafter, is continually flowing away from the tissue along special channels to pass into lymphatic vessels and thence into the blood.

As the blood flows along the capillaries certain constituents of the plasma (together with, at times, white corpuseles, and under exceptional circumstances red corpuscles) pass through the capillary wall into the lymph, and certain constituents of the lymph pass throngh the capillary wall into the blood within the capillary. There is thus an interchange of material between the blood within the capillary and the lymph outside. A similar interchange of material is at the same time going on between the lymph and the tissue itself. Hence, by means of the lymph acting as middleman, a double interchange of material takes place between the blood within the capillary and the tissue outside the capillary. In every tissue, so long as life lasts and the blood flows through the blood vessels, a double stream, now rapid now slow, is passing from the blood to the tissue and from the tissue to the blood. The stream from the blood to the tissue carries to the tissue the material which the tissue needs for building itself up and for doing its work, including the all-important oxygen. The 
stream from the tissue to the blood carries into the blood certain of the products of the chemical changes which have been taking place in the tissue, - products which may be simple waste, to be cast out of the body as soon as possible, or which may be bodies capable of being made use of by some other tissue.

A third stream, that from the lymph lying in the chinks and crannies of the tissue along the lymph channels to the larger lymph vessels, carries away from the tissue such parts of the material coming from the blood as are not taken up by the tissue itself and such parts of the material coming from the tissue as do not find their way into the blood vessel.

In most tissues, as in muscle for instance, the capillary network is so close set and the muscular fibre lies so near to the blood vessel that the lymph between the two exists only as a very thin sheet; but in some tissues, as in cartilage, the blood vessels lie on the outside of a large mass of tissue, the interchange between the central parts of which and the nearest capillary blood ressel is carried on through a long stretch of lymph. But in each case the principle is the same; the tissue, by the help of lymph, lives on the blood; and when in succeeding pages we speak of changes between the blood and the tissues, it will be understood, whether expressly stated so or no, that the changes are effected by means of the lymph. The blood may thus be regarded as an internal medium bearing the same relations to the constituent tissues that the external medium, the world, does to the whole individual. Just as the whole organism lives on the things around it, its air and its food, so the several tissues live on the complex fluid by which they are all bathed and which is to them their immediate air and food.

All the tissues take up oxygen from the blood and give up carbonic acid to the blood, but not always at the same rate or at the same time. Moreover the several tissues take up from the blood and give up to the blood either different things or the same things at different rates or at different times.

From this it follows, on the one hand, that the composition and characters of the blood must be for ever varying in different parts of the body and at different times; and on the other hand, that the united action of all the tissues must tend to establish and maintain an average uniform composition of the whole mass of blood. The special changes which blood is known to undergo while it passes through the several tissues will best be dealt with when the individual tissues and organs come under our consideration. At present it will be sufficient to study the main features which are presented by blood, brought, so to speak, into a state of equilibrium by the common action of all the tissues.

of all these main features of blood, the most striking if not the most important is the property it possesses of clotting when shed. 


\section{SEC. 1. THE CLOTTING OF BLOOD.}

$\$ 14$. Blood, when shed from the blood vessels of a living body, is perfectly fluid. In a short time it becomes viscid : it flows less readily from vessel to vessel. The viscidity increases rapidly until the whole mass of blood under observation becomes a complete jelly. The vessel into which it has been shed can at this stage be inverted without a drop of the blood being spilt. The jelly is of the same bulk as the previously fluid blood, and if carefully shaken out will present a complete mould of the interior of the vessel. If the blood in this jelly stage be left untouched in a glass vessel, a few drops of an almost colourless fluid soon make their appearance on the surface of the jelly. Increasing in number, and running together, the drops after a while form a superficial layer of pale straw-coloured fluid. Later on, similar layers of the same fluid are seen at the sides and finally at the bottom of the jelly, which, shrunk to a smaller size and of firmer consistency, now forms a clot or crassamentum, floating in a perfectly fluid serum. The shrinking and condensation of the clot, and the corresponding increase of the serum, continue for some time. The upper surface of the clot is generally slightly concave. A portion of the clot examined under the microscope is seen to consist of a feltwork of fine granular fibrils, in the meshes of which are entangled the red and white corpuscles of the blood. In the serum nothing can be seen but a few stray corpuscles, chiefly white. The fibrils are composed of a substance called fibrin. Hence we may speak of the clot as consisting of fibrin and corpuscles; and the act of clotting is obviously a substitution for the plasma of fibrin and serum, followed by a separation of the fibrin and corpuscles from the serum.

In man, blood when shed becomes viscid in about two or three minutes, and enters the jelly stage in about five or ten minutes. After the lapse of another few minutes the first drops of serum are seen, and clotting is generally complete in from one 
to several hours. The times however will be found to vary according to circumstances. Among animals the rapidity of clotting varies exceedingly in different species. The blood of the horse clots with remarkable slowness; so slowly indeed that many of the red and also some of the white corpuscles (both these being specifically heavier than the plasma) have time to sink before viscidity sets in. In consequence there appears on the surface of the blood an upper layer of colourless plasma, containing in its deeper portions many colourless corpuscles (which are lighter than the red). This layer clots like the other parts of the blood, forming the socalled 'buffy coat.' A similar buffy coat is sometimes seen in the blood of man, in certain abnormal conditions of the body.

If a portion of horse's blood be surrounded by a cooling mixture of ice and salt, and thus kept at about $0^{\circ} \mathrm{C}$., clotting may be almost indefinitely postponed. Under these circumstances a more complete descent of the corpuscles takes place, and a considerable quantity of colourless transparent plasma free from blood-corpuscles may be obtained. A portion of this plasma removed from the freezing mixture clots in the same manner as does the entire blood. It first becomes viscid and then forms a jelly, which subsequently separates into a colourless slirunken clot and serum. This shews that the corpuscles are not an essential part of the clot.

If a few cubic centimetres of this colourless plasma, or of a similar plasma which may be obtained from almost any blood by means which we will presently describe, be diluted with many times its bulk of a 0.6 p.c. solution of sodium chloride ${ }^{1}$ clotting is much retarded, and the various stages may be more easily watched. As the fluid is becoming viscid, fine fibrils of fibrin will be seen to be developed in it, especially at the sides of the containing vessel. As these fibrils multiply in number, the fluid becomes more and more of the consistence of a jelly and at the same time somewhat opaque. Stirred or pulled about with a needle, the fibrils shrink up into a small, opaque, stringy mass; and a very considerable bulk of the jelly may by agitation be resolved into a minute fragment of shrunken fibrin floating in a quantity of what is really diluted serum. If a specimen of such diluted plasma be stirred from time to time, as soon as clotting begins, with a needle or glass rod, the fibrin may be removed piecemeal as it forms, and the jelly stage may be altogether done away with. When fresh blood which has not yet had time to clot is stirred or whipped with a bundle of rods (or anything presenting a large amount of rough surface), no jelly-like clotting takes place, but the rods become covered with a mass of shrunken fibrin. Blood thus whipped until fibrin ceases to be deposited, is found to have entirely lost its power of clotting.

1 A solution of sodium chloride of this strength will hereafter be spoken of as 'normal saline solution.' 
Putting these facts together, it is very clear that the phenomena of the clotting of blood are caused by the appearance in the plasma of tine fibrils of fibrin. So long as these are scanty, the blood is simply viscid. When they become sufficiently numerous, they give the blood the firmmess of a jelly. Soon after their formation they begin to shrink, and while shrinking enclose in their meshes the corpuscles but squeeze out the fluid parts of the blood. Hence the appearance of the shrunken coloured clot and the colourless serum.

$\$ 15$. Fibrin, whether obtained by whipping freshly-shed blood, or by washing either a normal clot, or a clot obtained from colourless plasma, exhibits the same general characters. It belongs to that class of complex unstable nitrogenous bodies called proteids which form a large portion of all living bodies and an essential part of all living structures.

Our knowledge of proteids is at present too imperfect, and probably none of them have yet been prepared in adequate purity, to justify us in attempting to assign to them any definite formula; but it is important to remember their general composition. 100 parts of a proteid contain rather more than 50 parts of carbon, rather more than 15 of nitrogen, about 7 of hydrogen, and rather more than 20 of oxygen; that is to say, they contain about half their weight of carbon, and only about $\frac{1}{6}$ th their weight of nitrogen ; and yet as we shall see they are eminently the nitrogenous substances of the body. They usually contain a small quantity ( 1 or 2 p.c.) of sulphur, and many also have some phosphorus attached to them in some way or other. When burnt they leave a variable quantity of ash, consisting of inorganic salts of which the bases are chiefly sodium and potassium and the acids chiefly hydrochloric, sulphuric, phosphoric, and carbonic.

They all give certain reactions, by which their presence may be recognised; of these the most characteristic are the following: Boiled with nitric acid they give a yellow colour, which deepens into orange upon the addition of ammonia. This is called the xanthoproteie test; the colour is due to a product of decomposition. Boiled with the mixture of mercuric and mercurous nitrates known as Millon's reagent they give a pink colour. Mixed with a strong solution of sodic hydrate they give on the addition of a drop or two of a very weak solution of cupric sulphate a violet colour which deepens on heating. These are artificial reactions, not throwing much if any light on the constitution of proteids; but they are useful as practical tests enabling us to detect the presence of proteids.

The several members of the proteid group are at present distinguished from each other chiefly by their respective solubilities, especially in various saline solutions. Fibrin is one of the least soluble; it is insoluble in water, almost insoluble in dilute neutral saline solutions, very sparingly soluble in more concentrated 
neutral saline solutions and in dilute acids and alkalis, but is easily dissolved in strong acids and alkalis. In the process of solution it becomes changed into something which is no longer fibrin. In dilute acids it swells up and becomes transparent, but when the acid is neutralized returns to its previous condition. When suspended in water and heated to $100^{\circ} \mathrm{C}$. or even to $75^{\circ} \mathrm{C}$., it becomes changed; it is still less soluble than before. It is said in this case to be eoagulated by the heat; and as we shall see, nearly all proteids have the property of being changed in nature, of undergoing coagulation and so becoming less soluble than before, by being exposed to a certain high temperature.

Fibrin then is a proteid distinguished from other proteids by its smaller solubility; it is further distinguished by its peculiar filamentous structure, the other proteids when obtained in a solid form appearing either in amorphous granules or at most in viscid inasses.

$\$ 16$. We may now return to the serum.

This is perfectly fluid, and remains fluid until it decomposes. It is of a faint straw colour, due to the presence of a special pigment substance, differing fron the red matter which gives redness to the red corpuscles. Tested by the xanthoproteic and other tests it obviously contains a large quantity of proteid matter, and upon examination we find that at least two distinct proteid substances are present in it.

If crystals of magnesium sulphate be added to serum and gently stirred until they dissolve, it will be seen that the serum as it approaches saturation with the salt becomes turbid instead of remaining clear, and eventually a white amorphous granular or flocculent precipitate makes its appearance. This precipitate may be separated by decantation or filtration, washed with saturated solutions of magnesium sulphate, in which it is insoluble, until it is freed from all other constituents of the sermm, and thus obtained fairly pure. It is then found to be a proteid body, distinguished by the following characters among others:-

1. It is (when freed from any adherent magnesium sulphate) insoluble in distilled water; it is insoluble in concentrated solutions of neutral saline bodies, such as magnesium sulphate, sodinm chloride, \&c., but readily soluble in dilute (e.g. 1 p.c) solutions of the same neutral saline bodies. Hence from its solutions in the latter it may be precipitated either by adding more neutral saline substance or by removing by dialysis the small quantity of saline substance present. When obtained in a precipitated form, and suspended in distilled water, it readily dissolves into a clear solution upon the addition of a small quantity of some neutral saline body. By these various solutions and precipitations it is not really changed in nature.

2. It readily dissolves in very dilute acids (e.g. in hydro- 
chloric acid even when diluted to far less than 1 p.c.), and it is sinnlarly soluble in dilute alkalis; but in being thus dissolved it is changed in nature, and the solutions of it in dilute acid and dilute alkalis give reactions quite different from those of the solution of the substance in dilute neutral saline solutions. By the acid it is converted into what is called acid-albumin, by the alkali into alkali-albumin, both of which bodies we shall have to study later on.

3. When it is suspended in water and heated it becomes altered in character, coagulated, and all its reactions are changed. It is no longer soluble in dilute neutral saline solutions, not even in dilute acids and alkalis; it has become coagulated proteid, and is now even less soluble than fresh fibrin. When a solution of it in dilute neutral saline solution is similarly heated, a similar change takes place: a precipitate falls down which on examination is found to be coagulated proteid. The temperature at which this change takes place is somewhere about $75^{\circ} \mathrm{C}$., though shifting slightly according to the quantity of saline substance present in the solution.

The above three reactions are given by a number of proteid bodies forming a group called globulins, and the particular globulin present in blood-serum, is called paraglobulin.

One of the proteids present in blood-serum is then paraglobulin, characterised by its solubility in dilute neutral saline solutions; its insolubility in distilled water and concentrated saline solutions; its ready solubility, and at the same time conversion into other bodies, in dilute acids and alkalis; and in its becoming converted into coagulated proteid, and so being precipitated from its solutions at $75^{\circ} \mathrm{C}$.

The amount of it present in blood-serum varies in various animals, and apparently in the same animal at different times. In 100 parts by weight of serum there are generally present about 8 or 9 parts of proteids altogether; and of these some 3 or 4 , more or less, may be taken as paraglobulin.

$\$ 17$. If the serum from which the paraglobulin has been precipitated by the addition of neutral salt, and removed by filtration, be subjected to dialysis, the salt added may be removed, and a clear, somewhat diluted serum free from paraglobulin may be obtained. This still gives abundant proteid reactions, so that the serum still contains a proteid, or some proteids still more soluble than the globulins, since they will remain in solution, and are not precipitated, even when dialysis is continued until the serum is practically freed from both the neutral salt added to it and the diffusible salts previously present in the natural serum. When this serum is heated to $75^{\circ} \mathrm{C}$. a precipitate makes its appearance; the proteids still present are eoagulated at this temperature. 
We have some reasons for thinking that more than one proteid is present; but they are all closely allied to each other, and we may for the present speak of them as if they were one, and call the proteid left in serum, after removal of the paraglobulin, by the name of albumin, or, to distingnish it from other albumins found elsewhere, serum-albumin. Serum-albumin is distinguished by being more soluble than the globulins, since it is soluble in distilled water, even in the absence of all neutral salts. Like the globulins, though with much less ease, it is converted by dilute acids and dilute alkalis into acid- or into alkali-albumin.

The percentage amount of serum-albumin in serum may be put down as 4 or 5 , more or less; but it varies, and sometimes is less abundant than paraglobulin. In some animals (snakes) it is said to disappear during starvation.

The more important characters of the three proteids which we have just studied may be stated as follows:-

Soluble in water and in saline solutions of all strengths . . . . . . . . . . serum-albumin.

Insoluble in water, readily soluble in dilute saline solutions, insoluble in concentrated saline solutions .............. paraglobulin.

Insoluble in water, hardly soluble at all in dilute saline solutions, and very little soluble in more concentrated saline solutions . fibrin.

Besides paraglobulin and serum-albumin, serum contains a very large number of substances, generally in small quantity, which, since they have to be extracted by special methods, are called extractives; of these some are nitrogenous, some nonnitrogenous. Serum contains in addition important inorganic saline substances; but to these we shall return.

$\$ 18$. With the knowledge which we have gained of the proteids of clotted blood we may go back to the question: Clotting being due to the appearance in blood plasma of a proteid substance, fibrin, which previously did not exist in it as such, what are the causes which lead to the appearance of fibrin?

We learn something by studying the most important external circumstances which affect the rapidity with which the blood of the same individual clots when shed. These are as follows:-

A temperature of about $40^{\circ} \mathrm{C}$., which is about or slightly above the temperature of the blood of warm-blooded animals, is perhaps the most favourable to clotting. A further rise of a few degrees is apparently also beneficial, or at least not injurious ; but upon a still further rise the effect changes, and when blood is rapidly heated to $56^{\circ} \mathrm{C}$. no clotting at all may take place. At this temperature certain proteids of the blood are coagulated and precipitated before clotting can take place, and with this change the power of the blood to elot is wholly lost. If however the heating be not 
very rapid, the blood may clot before this change has time to come on. When the temperature instead of being raised is lowered below $40^{\circ} \mathrm{C}$. the clotting becomes delayed and prolonged; and at the temperature of $0^{\circ}$ or $1^{\circ} \mathrm{C}$. the blood will remain fluid, and yet capable of clotting when withdrawn from the adverse circumstances, for a very long, it might almost be said for an indefinite, time.

A small quantity of blood shed into a small vessel clots sooner than a large quantity shed into a larger one; and in general the greater the amount of foreign surface with which the blood comes in contact the more rapid the clotting. When shed blood is stirred or "whipped" the fibrin makes its appearance sooner than when the blood is left to clot in the ordinary way; so that here too the accelerating influence of contact with foreign bodies makes itself felt. Similarly, movement of shed blood hastens clotting, since it increases the amount of contact with foreign bodies. So also the addition of spongy platinum or of powdered charcoal, or of other inert powders, to tardily clotting blood, will by influence of surface, hasten clotting. Conversely, blood brought into contact with pure oil does not clot so rapidly as when in contact with glass or metal; and blood will continue to How for a longer time without clotting through a tube smeared inside with oil than through a tube not so smeared. The influence of the oil in such cases is a physical not a chemical one; any pure, neutral, inert oil will do. As far as we know, these inflnences affect only the rapidity with which the clotting takes place; that is, the rapidity with which the fibrin makes its appearance, not the amount of clot, not the quantity of fibrin formed, though when clotting is very much retarded by cold changes may ensue whereby the amount of clotting which eventually takes place is indirectly affected.

Mere exposure to air exerts apparently little influence on the process of clotting. Blood collected direct from a blood-vessel over mercury so as wholly to exclude the air, clots, in a general way, as readily as blood freely exposed to the air. It is only when blood is much laden with carbonic acid, the presence of which is antagonistic to clotting, that exclusion of air, by hindering the escape of the excess of carbonic acid, delays clotting.

These facts teach us that fibrin does not as was once thought make its appearance in shed blood because the blood when shed ceases to share in the movement of the circulation, or because the blood is cooled on leaving the warm body, or because the blood is then more freely exposed to the air; they further suggest the view that the fibrin is the result of some chemical change, the conversion into fibrin of something which is not fibrin, the change like other chemical changes being most active at an optimum temperature, and like so many other chemical changes being assisted by the influences exerted by the presence of inert bodies.

And we have direct experimental evidence that plasma does contain an antecedent of fibrin which by chemical change is converted into fibrin. 
$\$ 19$. If blood be received direct from the blood-vessels into one-third its bulk of a saturated solution of some neutral salt such as magnesium sulphate, and the two gently but thoroughly mixed, clotting, especially at a moderately low temperature, will be deferred for a very long time. If the mixture be allowed to stand, the corpuscles will sink, and a colourless plasma will be obtained similar to the plasma gained from horse's blood by cold, except that it contains an excess of the neutral salt. The presence of the neutral salt has acted in the same direction as cold: it has prevented the occurrence of clotting. It has not destroyed the fibrin; for if some of the plasma be diluted with from five to ten times its bulk of water, it will clot speedily in quite a normal fashion, with the production of quite normal fibrin.

The separation of the fluid plasma from the corpuscles and from other bodies heavier than the plasma is much facilitated by the use of the centrifugal machine. This consists essentially of a tireless wheel with several spokes, placed in a horizontal position and made to revolve with great velocity (1000 revolutions per minute for instance) round its axis. Tubes of metal or very strong glass are suspended at the ends of the spokes by carefully adjusted joints. As the wheel rotates with increasing velocity, each tube gradually assumes a horizontal position, bottom outwards, without spilling any of its contents. As the rapid rotation continues the corpuscles and heavier particles are driven to the bottom of the tube, and if a very rapid movement be continued for a long time will form a compact cake at the bottom of the tube. When the rotation is stopped the tubes gradually return to their upright position again without anything being spilt, and the clear plasma in each tube can then be decanted off.

If some of the colourless, transparent plasma, obtained either by the action of neutral salts from any blood, or by the help of cold from horse's blood, be treated with some solid neutral salt, such as sodium chloride, to saturation, a white, flaky, somewhat sticky precipitate will make its appearance. If this precipitate be removed, the fluid no longer possesses the power of clotting (or very slightly so), even though the nentral salt present be removed by dialysis, or its influence lessened by dilution. With the removal of the substance precipitated, the plasma has lost its power of clotting.

If the precipitate itself, after being washed with a saturated solution of the neutral salt (in which it is insoluble) so as to get rid of all serum and other constituents of the plasma, be treated with a small quantity of water, it readily dissolves, ${ }^{1}$ and the solution rapidly filtered gives a clear, colourless filtrate, which is at first perfectly fluid. Soon, however, the fluidity gives way to

1 The substance itself is not soluble in distilled water, but a quantity of the neutral salts al ways elings to the precipitate, and thus the addition of water virtually gives rise to a dilute saline solution, in which the substance is readily soluble. 
viscidity, and this in turn to a jelly condition, and finally the jelly shrinks into a clot floating in a clear fluid; in other words, the filtrate clots like plasma. Thus there is present in cooled plasma, and in plasma kept from clotting by the presence of neutral salts, a something, precipitable by saturation with nentral salts; a something which, since it is solnble in very dilute saline solutions, cannot be fibrin itself, but which in solution speedily gives rise to the appearance of fibrin. To this substance its discoverer, Denis, gave the name of plasmine.

The substance thus precipitated is not however a single body but a nixtnre of at least two bodies. If sodium chloride be carefully added to plasma to an extent of about 13 per cent, a white, flaky, viscid precipitate is thrown down very much like plasmine. If after the removal of the first precipitate more sodium chloride and especially if magnesium sulphate be added, a second precipitate is thrown down, less viscid and more granular than the first.

The second precipitate when examined is found to be identical with the paraglobulin, coagulating at $75^{\circ} \mathrm{C}$., which we have already seen to be a constituent of serum.

The first precipitate is also a proteid belonging to the globulin group, but differs from paraglobulin not only in being more readily precipitated by sodium chloride, and in being when precipitated more viscid, but also in other respects, and especially in being coagnlated at a far lower temperature than paraglobulin, viz. at $56^{\circ} \mathrm{C}$. Now, while isolated paraglobulin cannot by any means known to us be converted into fibrin, and its presence in the so-called plasmine does not seem to be essential to the formation of fibrin out of plasmine, the presence in plasmine of the body coagnlating at $56^{\circ} \mathrm{C}$. does seem essential to the conversion of plasnime into tibrin; and we have reason for thinking that it is itself converted, in part at least, into fibrin. Hence it has received the name of fibrinogen.

$\S 20$. The reasons for this view are as follows.

Besides blood, which clots naturally when shed, there are certain Huids in the body which do not clot naturally, either in the body or when shed, but which by certain artificial means may be made to clot, and in clotting to yield quite normal fibrin. Thus the so-called serous fluid taken some hours after death 1 from the pericardial, pleural, or peritoneal cavities, the fluid found in the enlarged serous sac of the testis, known as hydrocele fluid, and other similar fluids, will in the majority of cases, when obtained free from blood or other admixtmres, remain fluid ahmost indefinitely, shewing no disposition whatever to clot. ${ }^{2}$ Yet in most cases at

1 If it he removed immediately after death it generally clots readily and firmly, giving a colourless clot consisting of fibrin and white corpuscles.

2 In some specimens, however, a spontaneous coagulation, generally slight, but in exceptional cases massive, may be observed. 
all events, these fluitis, when a little blood, or a piece of blood clot, or a little serum is added to them, will clot rapidly and firmly, giving rise to an unmistakeable clot of normal fibrin, differing only from the clot of blood in that, when serum is used, it is colourlcss, being free from red corpuscles.

Now, blood (or blood clot, or serum) contains many things, to any one of which the clotting power thus seen might be attributed. But it is found that in many cases clotting may be induced in the Huids of which we are speaking by the mere addition and that even in exceedingly small quantity, of a substance which can be extracted from blood, or from serum, or from blood clot, or even from washed fibrin, or indeed from other sources, - a substance whose cxact nature is uncertain, it being doubtful whether it is a proteid at all, and whose action is peculiar.

If serum, or whipped blood, or a broken-up clot be mixed with a large quantity of alcohol and allowed to stand some days, the proteids present are in time so changed by the alcohol as to become insoluble in water. Hence if the copious precipitate caused by the alcohol, after long standing, be separated by filtration from the alcohol, dried at a low temperature, not exceeding $40^{\circ} \mathrm{C}$., and extracted with distilled water, the aqueous extract contains very little proteid mattcr, - indeed vcry little organic matter at all. Nevertheless even a small quantity of this aqueous extract added alone to certain specimens of hydrocele fluid or other of the fluids spoken of above, will bring about a speedy clotting. The same aqueous extract has also a remarkable effect in hastening the clotting of fluids which, though they will eventually clot, do so very slowly. Thus, plasma may, by the careful addition of a certain quantity of neutral salt and water, be reduced to such a condition that it clots very slowly indeed, taking perhaps days to complete the process. The addition of a small quantity of the aqueous extract wc are describing will, however, bring about a clotting which is at once rapid and complete.

The active substance, whatever it be, in this aqueous extract exists in small quantity only, and its clotting virtues are at once and for ever lost when the solution is boiled. Further, there is no reason to think that the active substance actually cnters into the formation of the fibrin to which it gives rise. It appears to belong to a class of bodies playing an important part in physiological processes and called ferments, of which we shall have more to say hereafter. We may thcrefore speak of it as the fibrin ferment, the name given to it by its discovercr Alcxander Schmidt.

This fibrin ferment is present in and may be extracted from clotted or whipped blood, and from both the clot ${ }^{2}$ and the serum of clotted blood; and since in most if not all cases where blood or

1 In a fow cases no coagulation can thus be induced.

2 A powerful solution of fibrin ferment may be readily prepared by simply extracting a washed blood clot with a 10 p.c. solution of solium chloride. 
blood clot or serum produces clotting in hydrocele or pericardial Huid, an exactly similar clotting may be induced by the mere addition of tibrin ferment, we seem justified in concluding that the clotting virtues of the former are due to the ferment which they contain.

Now, when fibrinogen is precipitated from plasma as above described by sodium chloride, re-dissolved, and reprecipitated, more than once, it may be obtained in solution, by help of a dilute neutral saline solution, in an approximately pure condition, at all events free from other proteids. Such a solution will not clot spontaneously; it may remain tluid indefinitely; and yet on the addition of a little fibrin ferment it will clot readily and firmly, yielding quite normal fibrin.

This body fibrinogen is also present and may be separated out from the specimens of hydrocele, pericardial, and other tluids which clot on the addition of fibrin ferment; and when the fibrinogen has been wholly removed from these fluids they refuse to clot on the adilition of fibrin ferment.

Paraglobulin, on the other hand, whether prepared from plasmine by separation of the fibringgen, or from serum, or from other fluids in which it is found, camnot be converted by fibrin ferment or indeed by any other means into fibrin. And fibrinogen isolated as described above, or serous fluids which contain fibrinogen, can be made, by means of fibrin ferment, to yield quite normal fibrin in the complete absence of paraglobulin. A solution of paraglobulin obtained from serum or blood clot will, it is true, clot pericardial or hydrocele fluids containing fibrinogen, or indeed a solution of fibrinogen; but this is apparently due to the fact that the paraglobulin has in these cases some fibrin ferment mixed with it; it is also possible that under certain conditions the presence of paraglobulin may be favourable to the action of the ferment.

When the so-called plasmine is precipitated as directed in $\$ 19$, fibrin ferment is carried down with the fibrinogen and paraglobulin; and when the plasmine is re-dissolved the ferment is present in the solution and ready to act on the fibrinogen. Hence the re-dissolved plasmine clots spontaneously. When fibrinogen is isolated from plasma by repeated precipitation and solution, the ferment is washed away from it, and the pure ferment-free fibrinogen, ultimately obtained, does not clot spontaneously.

So far it seems clear that there does exist a proteid body, fibrinogen, which may by the action of fibrin ferment be directly, without the intervention of other proteids, converted into the less soluble fibrin. Our knowledge of the constitution of proteid bodies is too imperfect to enable us to make any very definite statement as to the exact nature of the change thus effected; but we may say this much: Fibrinogen and fibrin have about the same elementary composition, fibrin containing a tritle more 
nitrogen. When fibrinogen is converted into fibrin by means of fibrin ferment, the weight of the fibrin produced is always less than that of the fibrinogen which is consumed, and there is always produced at the same time a certain quantity of another proteid, belonging to the globulin family. There are reasons however why we cannot speak of the ferment as splitting up fibrinogen into fibrin and a globulin. It seems more probable that the ferment converts the fibrinogen first into a body which we might call soluble fibrin, and then turns this body into veritable fibrin; but further inquiries on the subject are needed.

The action of the fibrin ferment on fibrinogen is dependent on other conditions besides temperature; for instance, the presence of a calcium salt seems to be necessary. If blood be shed into a dilute solution of potassium oxalate, the mixture, which need not contain more than 1 p.c. of the oxalate, remains fluid indefinitely, but clots readily on the addition of a small quantity of a calcium salt. Apparently the oxalate, by precipitating the calcium salts present in the blood, prevents the conversion of the fibrinogen into fibrin. So also a solution of fibrinogen which has been deprived of its calcium salts, by diffusion for instance, will not clot on the addition of fibrin ferment similarly deprived of its calcium salts; but the mixture clots readily on the addition of a minute quantity of calcium sulphate. We shall have to speak later on of a somewhat analogous part played by calcium salts in the curdling of milk. It may be added that the presence of other neutrai salts, such as sodium chloride, appears to influence clotting.

$\$ 21$. We may conclude then that the plasma of blood when shed, or at all events soon after it has been shed, contains fibrinogen; and it also seems probable that the clotting comes about because the fibrinogen is converted into fibrin by the action of fibrin ferment; but we are still far from a definite answer to the question, why blood remains fluid in the body and yet clots when shed?

We have already said that blood or blood plasma, brought up to a temperature of $56^{\circ} \mathrm{C}$. as soon as possible after its removal from the living blood vessels, gives a proteid precipitate and loses its power of clotting. This may be taken to shew that blood, as it circulates in the living blood vessels, contains fibringgen as such, and that when the blood is heated to $56^{\circ} \mathrm{C}$., which is the coagulating point of fibrinogen, the fibrinogen present is coagulated and precipitated, and consequently no fibrin can be formed.

Further, while clotted blood undoubtedly contains an abundance of fibrin ferment, no ferment, or a minimal quantity only, is present in blood as it leaves the blood ressels. If blood be received directly from the blood vessels into alcohol, the aqueous extract prepared as directed above contains no ferment, or merely a trace. Apparently the ferment makes its appearance in the blood as the result of changes taking place in the blood after it has been shed. 
We might from this be inclined to conclude that blood clots when shed but not before, because, fibrinogen being always present, the shedding brings about changes which produce fibrin ferment, not previously existing, and this acting on the fibrinogen gives rise to fibrin. But we meet with the following difficulty. A very considerable quantity of very active ferment may be injected into the blood current of a living animal without necessarily producing any clotting at all. Obviously, either blood within the blood vessels does not contain fibrinogen as such, and the fibrinogen detected by heating the blood to $56^{\circ} \mathrm{C}$. is the result of changes which have already ensued before that temperature is reached; or in the living circulation there are agencies at work which prevent any ferment which may be introduced into the circulation from producing its usual effects on fibrinogen; or there are agencies at work which destroy or do away with the tibrin, little by little, as it is formed.

$\$ 22$. And indeed when we reflect how complex blood is, and of what many and great changes it is susceptible, we shall not wonder that the question we are putting cannot be answered off hand.

The corpuscles with which blood is crowded are living structures, and consequently are continually acting upon and being acted upon by the plasma. The red corpuscles it is true are, as we shall see, peculiar bodies, with a restricted life and a very specialized work, and possibly their influence on the plasma is not very great; but we have reason to think that the relations between the white corpuscles and the plasma are close and important.

Then again the blood is not only acting upon and being acted upon by the several tissues as it flows through the various capillaries, but along the whole of its course, through the heart, arteries, capillaries, and veins, is acting upon and being acted upon by the vascular walls, which like the rest of the body are alive, and being alive are continually undergoing and promoting change.

That relations of some kind, having a direct influence on the clotting of blood, do exist between the blood and the vascular walls is shewn by the following facts.

After death, when all motion of the blood has ceased, the blood remains for a long time fluid. It is not till some time afterwards, at an epoch when post-mortem changes in the blood and in the blood vessels have had time to develope themselves, that elotting begins. Thus, some hours after death the blood in the great veins may be found still perfectly fluid. Yet such blood has not lost its power of clotting; it still clots when removed from the body, and clots too when received over mercury without exposure to air, shewing that, though the blood, being highly venous, is rich in carbonic acid and contains little or no oxygen, its fluidity is not due to any excess of carbonic acid or absence of oxygen. Eventually it does clot even within the vessels, but perhaps 
never so firmly and completely as when shed. It clots first in the larger vessels, but remains fluid in the smaller vessels for a very long time, for many hours in fact, since in these the same bulk of blood is exposed to the influence of, and reciprocally exerts an influence on, a larger surface of the vascular walls, than in the larger vessels. And if it be urged that the result is here due to influences exerted by the body at large, by the tissues as well as by the vascular walls, this objection will not hold good against the following experiment.

If the jugular vein of a large animal, such as an ox or horse, be carefully ligatured when full of blood, and the ligatured portion excised, the blood in many cases remains perfectly fluid, along the greater part of the length of the piece, for twenty-four or even forty-eiglit hours. The piece so ligatured may be suspended in a framework and opened at the top so as to imitate a living test-tube, and yet the blood will often remain long fluid, though a portion removed at any time into a glass or other vessel will clot in a few minutes. If two such living test-tubes be prepared, the blood may be poured from one to the other without clotting taking place.

A similar relation of the fluid to its containing living wall is seen in the case of those serous fluids which clot spontaneously. If, so soon after death as the body is cold and the fat is solidified, the perieardium be carefully removed from a sheep by an incision round the base of the heart, the pericardial fluid (which, as we have already seen, during life, and some little time after death, possesses the power of clotting) may be kept in the pericardial lag as in a living cup for many hours without clotting, and yet a small portion removed with a pipette clots at once.

This relation between the blood and the vascular wall may be disturbed or overridden: clotting may take place or may be induced within the living blood vessel. When the lining membrane is injured, as when an artery or vein is sharply ligatured, or when it is diseased, as for instance in aneurism, a clot is apt to be formed at the injured or diseased spot; and in certain morbid conditions of the body elots are formed in various vascular tracts. Absence of motion, which in shed blood, as we have seen, is unfavourable to clotting, is apt within the body to lead to clotting. Thus when an artery is ligatured, the blood in the tract of artery on the cardiac side of the ligatue, between the ligature and the branch last given off by the artery, ceasing to share in the circulation, remains motionless or nearly so, and along this tract a clot forms, firmest next to the ligature and ending near where the branch is given off; this perhaps may be explained by the fact that the walls of the tract suffer in their nutrition by the stagnation of the blood, and that consequently the normal relation be. tween them and the contained blood is disturbed. 
That the blood within the living blood vessels, though not actually clotting under normal circumstances, may easily be made to clot, that the blood is in fact so to speak always on the point of clotting, is shewn by the fact that a foreign body, such as a needle thrust into the interior of a blood vessel or a thread drawn through and left in a blood vessel, is apt to become covered with fibrin. Some influence exerted by the needle or thread, whatever may be the character of that influence, is sufficient to determine a clotting, which otherwise would not have taken place.

The same instability of the blood as regards clotting is strikingly shewn, in the case of the rabbit at least, by the result of injecting into the blood vessels a small quantity of a solution of a peculiar proteid prepared from certain structures such as the thymus body. Massive clotting of the blood in almost all the blood vessels, small and large, takes place with great rapidity, leading to the sudden death of the animal. In contrast to this effect may be mentioned the result of injecting into the blood vessels of a dog a quantity of a solution of a body called albumose, of which we shall hereafter have to treat as a product of the digestion of proteid substances, to the extent of $3 \mathrm{grm}$. per kilo of body weight. So far from producing clotting, the injected albumose has such an effect on the blood that for several hours after the injection shed blood will refuse to clot of itself and remain quite Huid, though it can be made to clot by special treatment.

\$23. All the foregoing facts tend to shew that the blood as it is flowing through the healthy blood vessels is, so far as clotting is concerned, in a state of unstable equilibrium, which may at any moment be upset, even within the blood ressels, and which is upset directly the blood is shed, with clotting as a result. Our present knowledge does not permit us to make an authoritative statement as to the exact nature of this equilibrium. There are reasons however for thinking that the white corpuseles play an important part in the matter. Whereever clotting occurs naturally, white corpuscles are present; and this is true not only of blood but also of such specimens of pericardial or other serous fluids as clot naturally. And many arguments, which we cannot enter upon here, may be adduced all pointing to the same conclusion, that the white corpuscle; play an important part in the process of clotting. But it would lead us too far into controversial matters to attempt to define what that part is, or to explain the exact nature of the equilibrium of which we have spoken. What we do know is that in blood soon after it has been shed, the body which we have called fibrinogen is present as also the body which we have called fibrin ferment, that the latter acting on the former will produce fibrin, and that the appearance of fibrin is undoubtedly the cause of what is called clotting. We seem justified in concluding that the clotting of shed blood is due to the conversion by ferment of fibrinogen into 
fibrin. The further inference that clotting within the body is the same thing as clotting outside the body and similarly due to the transformation of fibrinogen by ferment into fibrin, though probable, is not proved. We do not yet know the exact nature and condition of the blood within the living blood vessels, and until we know that we cannot satisfactorily explain why blood in the living blood vessels is usually fluid but can at times clot. 


\section{SEC. 2. 'THE CORPUSCLES OF' THE BLOOD.}

\section{The Red Corpuscles.}

\$24. The redness of blood is due exclusively to the red corpuscles. The plasma as seen in thin layers within the living blood vessels appears colourless, as does also a thin layer of serum; but a thick layer of serum (and probably of plasma) has a faint yellowish tinge due as we have said to the presence of a small quantity of a special pigment.

A single red corpuscle seen by itself under the microscope is a fairly homogeneous, imperfectly translucent biconcave disc of a very faint colour, looking yellow rather than red; but when several corpuscles lie one upon the top of the other the mass appears distinctly red; and though a single corpuscle is somewhat translucent, a comparatively thin layer of blood is opaque; type for instance cannot be read through even a thin layer of blood.

When a quantity of whipped blood (or blood otherwise deprived of fibrin) is frozen and thawed several times it changes colour, becoming of a darker hue, and is then found to be much more transparent, so that type can now be easily read through a moderately thin layer. It is then spoken of as laky blood. The same change may be effected by shaking the blood with ether, or by adding a small quantity of bile salts, and in other ways. Upon examination of laky blood it is found that the red corpuscles are 'broken up' or at least altered, and that the redness which previously was confined to them is now diffused through the serum. Normal blood is opaque because each corpuscle while permitting some rays of light (chiefly red) to pass through, reflects many others, and the brightness of the lue of normal blood is due to this reflection of light from the surfaces of the several corpuscles. Laky blood is transparent because there are no longer intact corpuscles to present surfaces for the reflection of light, and the darker hue of laky blood is similarly due to the absence of reflection from the several corpuscles. 
When laky blood is allowed to stand a sediment is formed (and may be separated by the centrifugal machine) which on examination is found to consist of dises, or fragments of dises, of a colourless substance exhibiting under high powers an obscurely spongy or reticular structure. These colourless thin dises seen flat-wise often appear as mere rings. Tlre substance composing them stains with various reagents and may thus be made more evident.

The red corpuscle then consists obviously of a colourless framework, with which in normal conditions a red colouring matter is associated; but by various means the colouring matter may be driven from the framework and dissolved in the serum.

The framework is spoken of as stroma; it is a modified or differentiated protoplasm, and upon chemical analysis yields a proteid substance belonging to the globulin group, and other matters, among which is the peculiar complex fat called lecithin, of which we shall have to speak in treating of nervous tissue.

The red colouring matter which in normal conditions is associated with this stroma may by appropriate means be isolated, and, in the case of the blood of many animals, obtained in a crystalline form. It is called Hamoglobin, and may by proper methods be split up into a proteid belonging to the globulin group, and into a coloured pigment, containing iron, called Homatin. Hæmoglobin is therefore a very complex body. It is found to have remarkable relations to oxygen, and indeed as we shall see the red corpuscles by virtue of their hæmoglobin have a special work in respiration; they carry oxygen from the lungs to the several tissues. We shall therefore defer the further study of hæmoglobin until we have to deal with respiration.

Though the hremoglobin, as is seen in laky blood, is readily soluble in serum (and it is also soluble in plasma), in the intact normal blood it remains confined to the corpuscle; obviously there is some special connection between the stroma and the hæmoglohin; it is not until the stroma is altered, we may perhaps say killed (as by repeated freezing and thawing), that it loses its hold on the hæmoglobin, which thus set free passes into solution in the serum. The disc of stroma when separated from the hæmoglobin has as we have just said an obscurely spongy texture; but we do not know accurately the exact condition of the stroma in the intact corpuscle or how it holds the hrmoglobin. There is certainly no definite membrane or envelope to the corpuscle, for by exposing blood to a high temperature, $60^{\circ} \mathrm{C}$, the corpuscle will break up into more or less spherical pieces, each still consisting of stroma and hæmoglobin.

The quantity of stroma necessary to hold a quantity of hæmoglobin is exceedingly small. Of the total solid matter of a corpuscle more than $90 \mathrm{p}$. c. is hremoglobin. A red corpuscle in fact is a quantity of hæmoglobin held together in the form of a 
disc by a minimal amount of stroma. Hence whatever effect the stroma per se may have upon the plasma, this, in the case of mammals at all events, must be insignificant: the red corpuscle is practically simply a carrier of hæmoglobin.

$\$ 25$. The average number of red corpuscles in human blood may be probably put down at about 5 millions in a cubic millimeter (the range in different mammals is said to be from 3 to 18 millions), but the relation of corpuscle to plasma varies a good deal even in health, and very much in disease. Obviously the relation may be affecter (1) by an increase or decrease of the plasma, (2) by an actual decrease or increase of red corpuscles. Now the former must frequently take place. The blood as we have already urged is always being acted upon by changes in the tissues and indeed is an index of those changes; hence the plasma must be continually changing, though always striving to return to the normal condition. Thus when a large quantity of water is discharged by the kidney, the skin or the bowels, that water comes really from the blood, and the drain of water must tend to diminish the bulk of the plasma, and so to increase the relutive number of red corpuscles, though the effect is probably soon remedied by the passage of water from the tissues into the blood. So again when a large quantity of water is drunk, this passes into the blood and tends temporarily to dilute the plasma (and so to diminish the relative number of red corpuscles), though this condition is in turn soon remedied by the passage of the superfluous fluid to the tissues and excretory organs. The greater or less number of red corpuscles then in a given bulk of blood may be simply due to less or more plasma, but we have reason to think that the actual number of the corpuscles in the blood does vary from time to time. This is especially seen in certain forms of disease, which may be spoken of under the general term of anæmia (there being several kinds of anrmia), in which the number of red corpuscles is distinctly diminished.

The redness of blood may however be influenced not only by the number of red corpuscles in each cubic millimeter of blood but also by the amount of hemoglobin in each corpuscle, and to a less degree by the size of the corpuscles. If we compare, with a common standard, the redness of two specimens of blood unequally red, and then determine the relative number of corpuscles in each, we may find that the less red specimen has as many corpuscles as the redder one, or at least the deficiency in redness is greater than can be accounted for by the paucity of red corpuscles. Obviously in such a case the red corpuscles have too little hæmoglobin. In some cases of anæmia the deficiency of hæmoglobin in each corpuscle is more striking than the scantiness of red corpuscles.

The number of corpuseles in a specimen of blood is determined by mixing a small but carefully measured quantity of the blood with a F. 
large quantity of some indifferent fluid, e.g. a 5 p.c. solution of sodium sulplate, and then actually counting the corpuscles in a known minimal bulk of the mixture.

This perhaps may be most conveniently done by the method generally known as that of Gowers (Hxmacytometer) improved by Malassez. A glass slide, in a metal frame, is ruled into minute rectangles, e.g. $\frac{1}{4} \mathrm{~mm}$. by $\frac{1}{5} \mathrm{~mm}$., so as to give a convenient area of $\frac{1}{20}$ th of a square $\mathrm{mm}$. Three small screws in the frame permit a coverslip to be brought to a fixed distance, e.g. $\frac{1}{5} \mathrm{~mm}$., from the surface of the slide. The blood having been diluted, e.g. to 100 times its volume, a small quantity of the diluted (and thoroughly mixed) blood, sufficient to occupy fully the space between the cuverslip and the glass slide when the former is brought to its proper position, is placed on the slide, and the coverslip brought down. The volume of diluted blood now lying over each of the rectangles will be ${ }_{10}^{1}$ th $\left(\frac{1}{20} \times \frac{1}{5}\right)$ of a cubic $\mathrm{mm}$.; and if, when the corpuseles have subsided, the number of corpuscles lying within a rectangle be counted, the result will give the number of corpuscles previously distributed through ${ }_{10}{ }_{0}$ th of a cubic mm. of the diluted blood. 'This multiplied by 100 will give the number of corpuscles in 1 cubic mm. of the diluted blood, and again multiplied by 100 the number in 1 eubic $\mathrm{mm}$. of the entire blood. It is advisable to count the number of corpuscles in several of the rectangles, and to take the average. For the convenience of counting, each rectangle is suldivided into a number of very small squares, e.g. into 20 , each with a side of $\frac{1}{20}$ th $\mathrm{mm}$., and so an area of $\frac{1}{400}$ th of a square num.

Since the actual number of red corpuscles in a specimen of blood (which may be taken as a sample of the whole blood) is sometimes more, sometimes less, it is obvious that either red corpuscles may be temporarily withdrawn from and returned to the general blood current, or that certain red corpuscles are after a while made away with, and that new ones take their place. We have no satisfactory evidence of the former being the case in normal conditions, whereas we have evidence that old corpuscles do die and that new ones are born.

$\$ 26$. The red corpuscles, we have already said, are continually engaged in carrying oxygen, by means of their hamoglobin, from the lungs to the tissues; they load themselves with oxygen at the lungs and unload at the tissues. It is extremely unlikely that this act should be repeated indefinitely without leading to changes which may be familiarly described as wear and tear, and which would ultimately lead to the death of the corpuscles.

We shall have to state later on that the liver discharges into the alimentary canal, as a constituent of bile, a considerable quantity of a pignent known as bilimubin, and that this substance has remarkable relations with, and indeed may be regarded as a derivative of homatin, which as we have seen (\$24) is a product of the decomposition of hemoglohin. It appears probable in fact that the bilirulin of bile (and this as we shall see is the chief biliary pigment and the source of the other biliary pigments) is 
not formed wholly anew in the body but is manufactured in some way or other out of hæmatin derived from hæmoglobin. This must entail a daily consumption of a considerable quantity of hæmoglobin, and, since we know no other source of hiemoglobin besides the red corpuscles, and have no evidence of red corpuscles continuing to exist after having lost their hemoglobin, must therefore entail a daily destruction of many red corpuseles.

Even in health then a number of red corpuscles must be continually disappearing; and in disease the rapid and great diminution which may take place in the number of red corpuscles shews that large destruction may oceur.

We cannot at present accurately trace out the steps of this disappearance of red corpuscles. In the spleen pulp, red corpuscles have been seen in various stages of disorganisation, some of them lying within the substance of large colourless corpuscles, and as it were being eaten by them. There is also evidence that destruction takes place in the liver itself, and indeed elsewhere

$\$ 27$. This destruction of red corpuscles necessitates the birth of new corpuscles, to keep up the normal supply of hremoglobin; and indeed the cases in which after even great loss of blood by hæmorrhage a healthy ruddiness returns, and that often rapidly, sliewing that the lost corpuscles have been replaced, as well as the cases of recovery from the disease anæmia, prove that red corpuscles are, even in adult life, born somewhere in the body.

In the developing embryo of the mammal the red crrpuscles of the blood are not hiemoglobin-holding non-nucleated dises of stroma, but coloured nucleated cells which have arisen by the development of hæmoglobin and stroma in the "undifferentiated protoplasmic' cell substance of certain cells.

Still later on in the life of the embryo the nucleated red corpuscles are replaced by ordinary red corpuscles, by non-nucleated dises composed almost exclusively of hemoglobin-holding stroma. How the transformation takes place, and especially how the nucleus comes to be absent is at present a matter of considerable dispute.

In the adult as in the embryo the red corpuscles appear to be formed out of preceding coloured nucleated cells. In the interior of bones is a peculiar tissue called marrow, which in most parts, being very full of blood vessels, is called red marrow. In this ret marrow the capillaries and minute veins form an intricate labyrinth of relatively wide passages with very thin walls, and through this labyrinth the flow of blood is comparatively slow. In the passages of this labyrinth are found coloured nucleated cells, that is to say, cells the cell-substance of which has undergone more or less differentiation into hremoglobin and stroma. And there seems to be going on in red marrow a multiplication of such coloured nucleated cells, some of which transformed, in some way or other, into red non-nucleated discs, that is into ordinary 
red corpuscles, pass away into the general blood current. In other words, a formation of red corpuseles, not wholly unlike that which takes place in the embryo, is in the adult continually going on in the red marrow of the bones.

A similar formation of red corpuscles has also been described, though with less evidence, as taking place in the spleen, especially under particular circumstances, such as after great loss of blood.

The formation of red corpuscles is therefore a special process taking place in special regions; we have no satisfactory evidence that the ordinary white corpuscles of the blood are, as they travel in the current of the circulation, transformed into red corpuscles.

The red corpuscles then, to sum up, are useful to the body on account of the hemoglobin which constitutes so nearly the whole of their solid matter. What functions the stroma may have besicles the mere so to speak mechanical one of holding the hiemoglobin in the form of a corpuscle, we do not know. The primary use of the hiemoglobin is to earry oxygen from the lungs to the tissues, and it would appear that it is adrantageous to the economy that the hremoglobiu should be as it were bottled up in corpuscles rather than simply diffused through the plasma. How long a corpuscle may live, fetching and carrying oxygen, we do not exactly know; the red corpuscles of one animal, $\ell . g$. a bird, injected into the vessels of another, $\bullet \cdot g$. a mammal, disappear within a few days; but this affords no measure of the lite of a corpuscle in its own home Eventually however the red corpuscle dies, its place being supplied by a new one. The hamoglobin set free from the dead corpuseles appears to have a secondary use in forming the pigment of the bile and possibly other pigments.

\section{The White or Colourless Corpuseles.}

$\$ 28$. The white corpuscles are far less numerous than the red; a specimen of ordinary healthy blood will contain several hundred red corpuscles to each white corpuscle, though the proportion, even in health, varies considerably under different circumstances, ranging from 1 in 300 to 1 in 700 . But though less numerons, the white corpuscles are probably of greater importance to the blood itself than are the red corpuscles; the latter are chiefly limited to the special work of carrying oxygen from the lungs to the tissues, while the former probably exert a considerable influence on the blood plasma itself, and help to maintain it in a proper condition.

The white corpuscle, which is often taken as the type of ' a cell,' consists of a cell-body or cell-substance and a nuclens. Several varieties or kinds of white corpuscle are found in the blood, differing from each other as to size, as to the characters of the nucleus, as to the characters of the cell-substance, as to the extent to which they exhibit 'amoboid' movements, whereby 
what may be called the normal or resting spherical shape is variously changed (we shall study these annoboid movements later on), and in other respects; but at present we will deal with those features only which they have in common, and speak of the white corpuscle as if all were alike.

The cell body of the white corpuscle may he taken as a good example of what we have called undifferentiated protoplasm. It may perhaps be best considered as consisting of a uniformly transparent but somewhat refractive material forming the ground substance or basis, in which occur vacuoles of varying size but all for the most part minute, and in which are imbedded particles also of varying size but also for the most part minute. Some maintain that the ground substance exists in the form of a network, the interstices of which are filled up either with fluid or with some material different in nature from that of which the bars of the network are composed; but whthout entering into the discussion of a debated question, we may say that the evidence for the natural existence of such a network is not convincing. The imbedded particles are in some cases extremely small, and for the most part distributed uniformly over the cell body, giving it the finely cranular or even hyaline aspect spoken of above; in other cases however the particles are relatively large and obviously discrete, making the corpuscle coarsely granular, the coarse granules being sometimes confined to one or another part of the cell body. These particles or granules, whether coarse or fine, vary in nature: they behave differently towards various staining and other reagents. Some of them, as shewn by their greater refractive power, their staining with osmic acid, and their solution by solvents of fat, are fatty in nature; others may similarly be shewn by their reactions to be proteid in nature; and in certain cases some of the granules are carbohydrate in nature.

The material in which these granules are imbedded, and which forms the greater part of the cell body, has no special optical features; so far as can be ascertained it appears under the microscope to be homogeneous, no definite structure can be detected in it. It must be borne in mind that the whole corpuscle consists largely of water, the total solid matter amounting to not much more than 10 per cent. The transparent material of the cell body must therefore be in a condition which we may call semifluid, or semisolid, without being called upon to define what we exactly mean by these terms. This approath to fluidity appears to be connected with the great mobility of the cell body as shewn in its ancoboid movements.

\$29. When we submit to chemical examination a sufficient mass of white corpuscles separated out from the blood by special means and obtained tolerably free from red corpuscles and plasma (or apply to the white blood corpuscles the chemical results obtained from the more easily procured lymph corpuscles, which 
as we shall see are very similar to and indeed in many ways closely related to the white corpuscles of the blood), we find that this small solid matter of the corpuscle consists largely of certain proteids, or of substances more or less allied to proteids. Our knowledge of these proteids and other substances is as yet imperfect, but we are probably justified in making the following statement.

There is present, in somewhat considerable quantity, a substance of a peculiar nature, which since it is confined to the nuclei of the corpuscles and further seems to be present in all nuclei, has been called nuclein. This nuclein, which though a complex nitrogenous body is different in composition and nature from proteids, is remarkable on the one hand for being a very stable inert body, and on the other for containing a large quantity (acording to some observers nearly 10 p.c.) of phosphorus, which appears to enter in a certain way into the structure of the molecule, whereas in the case of proteids the phosphorus, which is not always present, is, as it were, attached to the molecule.

The substance however which is present in the greatest quantity is one also at present not thoroughly understood, which though it appears to exist in the cell body apart from the nucleus, and indeed to form a large part of the solid matter of the cell body, has since it seens to be a compound of nuclein and albumin (or some other proteid) been called nucleo-albumin. It, like nuclein, contains a considerable quantity of phosphorus, by which as well as by other features it is distinguished from the globulins, though in some respects it seems allied to that class of proteids, and to a somewhat similar proteid, myosin, of which we shall have to speak later on as a constituent of muscle.

Besides these two bodies, the white corpuscles also contain a globulin which, under the name of cell globulin, has been distinguished from the globulin or paraglobulin of blood, as well as a body or bodies like to or identical with serum albumin.

Next in importance to the proteids, as constant constituents of the white corpuscles, come certain fats. Among these the most conspicuous is the complex fatty body lecithin.

In the case of many corpuscles at all events we have evidence of the presence of a member of the large group of carbohydrates, comprising starches and sugar, viz. the starch-like body glyeogen, which we shall have to study more fully hereafter. This glycogen may exist in the living corpuscle as glycogen, but it is very apt after the death of the corpuscle to become changed by hydration into some form of sugar, such as maltose or dextrose.

Lastly, the ash of the white corpuseles is characterised by containing a relatively large quantity of potassium and of phosphates and by heing relatively poor in chlorides and in sodium. But in this respect the corpuscle is merely an example of what seems to be a general rule (to which however there may be 
exceptions), that while the elements of the tissues themselves are rich in potassium and phosphates, the blood plasma or lymph on which they live abounds in chlorides and sodium salts.

$\$ 30$. In the broad features above mentioned, the white blood corpuscle may be taken as a picture and example of all living tissues. If we examine the histological elements of any tissue, whether we take an epithelium cell, or a nerve cell, or a cartilage cell, or a muscular fibre, we meet with very similar features. Studying the element morphologically, we find a nucleus ${ }^{1}$ and a cell hody, the nucleus having the general characters described above with frequently other eharacters introduced, and the cell body consisting of at least more than one kind of material, the materials being sometimes so disposed as to produce the optical effect simply of a transparent mass in which granules are imbedded, in which case we speak of the cell body as protoplasmic, but at other times so arranged that the cell body possesses differentiated structure. Studying the element from a chemical point of view we find proteids always present, and among these bodies identieal with or more or less closely allied to such proteids as globulin and myosin, we generally have evidence of the presence also of fat of some kind and of some member or members of the carbohydrate group, and the ash always contains potassium and phosphates, with sulphates, chlorides, sodium and calcium, to which may be added magnesium and iron.

We stated in the Introduction that living matter is always undergoing chemical change; this continued chemical change we may denote by the term metabolism. We further urged that so long as living matter is alive, the chemical change or metabolism is of a double kind. On the one hand, the living substance is continually breaking down into simpler bodies, with a setting free of energy; this part of the metabolism we may speak of as made up of katabolie changes. On the other hand, the living substance is continually building itself up, embodying energy into itself and so replenishing its store of energy; this part of the metabolism we may speak of as made up of anabolic changes. We also urged that in every piece of living tissue there might be (1) the actual living substance itself, (2) material which is present for the purpose of becoming, and is on the way to become, living substance, that is to say, food undergoing or about to undergo anabolic changes, and (3) material which has resulted from, or is resulting from, the breaking down of the living substance, that is to say, material which has undergone or is undergoing katabolic changes, and which we speak of under the general term 'waste.' In using the word "living substance," however, though we may for convenience sake speak of the really living part as a substance, we may repeat that in reality it is not a substance in the chenical sonse of the word, but material undergoing a series of changes.

1 The existence of multinuclear structures does not affect the present argument. 
If, now, we ask the question, which part of the body of the white corpuscle (or of a similar element of another tissue) is the real living substance, and which part is food or waste, we ask a question which we cannot as yet definitely answer. We have at present no adequate morphological criteria to enable us to judge, by optical characters, what is really living and what is not.

One thing we may perhaps say; the material which appears in the cell body in the form of distinct granules, merely lodged in the more transparent material, eannot be part of the real living substance; it must be either food or waste. Some of these granules are fat, and we have at times an opportunity of observing that they have been introduced into the corpusele from the surrounding plasma. The white corpuscle as we have said lias the power of executing amoboid movements; it can creep round objects, envelope them with its own substance, and so put them inside itself. The granules of fat thus introduced may be subsequently extruded or may disappear within the corpusele; in the latter case they are obviously changed, and apparently made use of by the corpuscle. In other works, these fatty gramules are apparently food material, on their way to be worked up into the living substance of the corpuscle.

But we have also evilence that similar gramules of fat may make their appearance wholly within the corpuscle; they are products of the activity of the corpuscle. We have further reason to think that in some cases, at all events, they arise from the breaking down of the living substance of the corpuscle, that they are what we have called waste products.

But all the granules visible in a corpuscle are not necessarily fatty in nature; some of them may undoubtedly be granules of proteid or allied matter, and it is possible that some of them may at times be of carbohydrate or other nature. In all cases however they are either food material or waste products. And what is true of the easily distinguished granules is also true of other substances, in solution or in a solid form, but so disposed as not to be optically recognised.

Hence a part, and it may be no inconsiderable part, of the body of a white corpuscle may be not living substance at all, but either food or waste. Further, it does not necessarily follow that the whole of any quantity of material, fatty or otherwise, introduced into the corpuscle from without, should actually be built up into and so become part of the living snbstance; the changes from raw food to living substance are as we have already said probably many, and it may be that after a certain number of changes, few or many, part only of the material is accepted as worthy of being made alive, and the rest, being rejected, becomes at once waste matter; or the material may, even after it lias undergone this or that chance, never actually enter into the living substance but all become waste matter. We say waste matter, but this does not 
mean useless matter. The matter so formed may without entering into the living substance be of some subsidiary use to the corpuscle, or as probably more often happens, being discharged from the corpuscle, may be of use to some other part of the body. We do not know how the living substance builds itself up, but we seem compelled to arlmit that, in certain cases at all events, it is able in some way or other to produce changes on material while that material is still outside the living substance as it were, before it enters into and indeed withont its ever actually entering into the composition of the living substance. On the other hand, we must equally admit that some of the waste substances are the direct products of the katabolic ehanges of the living substance itself, and were actually once part of the living substance. Hence we ought perhaps to distinguish the products of the activity of living matter into waste products proper, the direct results of katabolic ehanges, and into by-products which are the results of changes effected by the living matter outside itself and which cannot therefore be considered as necessarily either anabolic or katabolic.

Concening the chenical characters of the living matter itself we cannot at present make any very definite statement. We may say that proteil substance enters in some way into its structure and indeed forms a large part of it, but we are not justified in saying that the living substance consists only of proteid matter in a peculiar condition. And inded the persistency with which some representative of fatty bolies and some representative of carbohydrates always appear in living tissue, woul 1 perhaps rather lead us to suppose that these equally with proteid material were essential to its structure. Again, though the behaviour of the nucleus as contrasted with that of the cell body leads us to suppose that the living substance of the former is a different kind of living substance from that of the latter, we do not know exactly in what the difference consists. The nucleus as we have seen contains nuclein, which perhaps we may regard as a largely nodified proteid; but a body which is remarkable for its stability, for the difficulty with which it is changed by chemical reagents, cannot be regarded as an integral part of the essentially mobile living substance of the nucleus.

In this connection it may be worth while again to call attention to the fact that the corpuscle contains a very large quantity indeed of water, viz. about 90 p.c. Part of this, we do not know how much, probably exists in a more or less definite combination with the protoplasm, somewhat after the manner of, to use what is a mere illustration, the water of crystallization of salts. If we imagine a whole group of different complex salts continually occupied in turn in being crystallized, and being decrystallized, the water thus engaged by the salts will give us a rough image of the water which passes in and out of the substance of the corpuscle as the result of its metabolic activity. We might call this "water of metabolism." 
Another part of the water, carrying in this case substances in solution, probably exists in spaces or interstices too small to be seen with even the highest powers of the microscope. Still another part of the water similarly holding substances in solution exists at times in definite spaces visible under the microscope, more or less regularly spherical, and called racuoles.

We have dwelt thus at length on the white corpuscle in the first place because as we have already said what takes place in it is in a sense a picture of what takes place in all living structures, and in the second place because the facts which we have mentioned help us to understand how the white corpuscle may carry on in the blood a work of no unimportant kind; for from what has been said it is obvious that the white corpuscle is continually acting upon and being acted upon by the plasma.

$\$ 31$. To understand however the work of these white corpuscles we must learn what is known of their history.

In successive drops of blood taken at different times from the same individual, the number of colourless corpuseles will be found to vary very much, not only relatively to the red corpuscles, but also absolutely. They must therefore 'come and go.'

In treating of the lymphatic system we shall have to point out that a very large quantity of thuid called lymph, containing a very considerable number of bodies very similar in their general characters to the white corpuscles of the blood. is being continually poured into the vascular system at the point where the thoracic duct joins the great veins on the left side of the neck, and to a less extent where the other large lymphaties join the venous system on the right side of the neck. These corpuscles of lymph, which, as we have just said, closely resemble, and indeed are with difficulty distinguished from the white corpuscles of the blood, but of which, when they exist outside the vascular system, it will be convenient to speak of as lexcocytes, are found along the whole length of the lymphatic system, but are more numerous in the lymphatic vessels after these have passed through the lymplatic glands. These lymphatic glands are partly composed of what is known as adcnoid tissuc, a special kind of connective tissue arranged as a delicate network. The meshes of this are crowded with colourless nucleated cells, which though varying in size are for the most part small, the nucleus being surrounded by a relatively small quantity of cell-substance. Many of these cells show signs that they are undergoing cell division, and we have reason to think that cells so formed, acquiring a larger amount of cell-substance, become ordinary leucocytes. In other words, leucocytes multiply in the lymphatic glands, and leaving the glands by the lympathic vessels, make their way to the blood. Patches and tracts of similar adenoid tissue, not arranged however as distinct glands but similarly occupied by developing leucocytes and similarly comnected with lymphatic vessels, are found in various parts 
of the body, especially in the mucous membranes. Moreover, the leucocytes appear to multiply by division during their abode in the various lymph passages. Hence we may conclude that from various parts of the body, the lymphatics are continually bringing to the blood an abundant supply of leucocytes, and that these become the ordinary white corpuscles of the blood. This is probably the chief source of the white corpuscles, for though the white corpuscles have been seen dividing in the blood itself, no large increase, so far as we know, takes place in that way.

$\$ 32$. It follows that since white corpuscles are thus continually being added to the blood, white corpuseles must as continually either be destroyed, or be transformed, or escape from the interior of the blood ressels; otherwise the blood would soon be blocked with white corpuscles.

Some do leave the blood vessels. In treating of the circulation we shall have to point ont that white corpuscles are able to pierce the walls of the capillaries and minute veins and thus to make their way from the interior of the blood vessels into spaces filled with lymph, the "lymph spaces," as they are called, of the tissue lying outside the blood ressels. This is spoken of as the "migration of the white corpuscles." In an "inflamed area" large numbers of white corpuscles are thus drained away from the blood into the lymph spaces of the tissue; and it is probable that a similar loss takes place, more or less, under normal conditions. These migrating corpuscles may, by following the devious tracts of the lymph, find their way back into the blood; some of them however may remain, and undergo various changes. Thus, in inflamed areas, when suppuration follows inflammation, the white corpuscles which have migrated may becone 'pus corpuscles,' or, where thickening and growth follow upon inflammation, may, according to many authorities, become transformed into temporary or permanent tissue, especially connective tissue; but this transformation into tissue is disputed. When an inflammation subsides without leaving any effect a few corpuscles only will be found in the tissue; those which had previously migrated must therefore have been disposed of in some way or other.

In speaking of the formation of red corpuscles (\$27) we saw that not only it is not proved that the nucleated corpuscles which give rise to red corpuscles are ordinary white corpuscles, but that in all probability the real hæmatoblasts, the parents of red corpuscles, are special corpuscles developed in the situations where the manufacture of red corpuscles takes place. So far therefore from assuming, as is sometimes done, that the white corpuscles of the blood are all of them on their way to become red corpuscles, it may be donbted whether any of them are. In any case however, even making allowance for those which migrate, a very considerable number of the white corpuscles must 'disappear' in some way or other from the blood stream, and we may perhaps speak of 
their disappearance as being a 'destruction ' or 'dissolution.' We have as yet no exact knowledge tc guide us in this matter, but we can readily imagine that, upon the death of the corpuscle, the substances composmg it, after undergoing ehanges, are dissolved by and become part of the plasma. If so, the corpuseles as they die must repeatedly influence the composition and nature of the plasma.

But if they thus affect the plasma in their death, it is even more probable that they influence it during their life. Being alive they must be continually taking in and giving out. As we have already said they are known to ingest, after the fashion of an anceba, solid particles of various kinds such as fat or carmine, present in the plasma, and probably digest such of these particles as are nutritious. But if they ingest these solid matters they probably also carry out the easier task of ingesting dissolved matters. If however they thus take in, they must also give out; and thus by the removal on the one hand of various sulsstances from the plasma, and by the addition on the other hand of other substances to the plasma, they must be eontinually influencing the plasma. We have already said that the white corpuscles in shed blood as they die are supposed to play an important part in the clotting of blood; similarly they may during their whole life be engaged in carrying out changes in the proteids of the plasma which do not lead to elotting, but which prepare the proteids for their various uses in the body.

Pathological facts afford support to this view. The disease called lencocythemia (or lenkenia) is characterised by an increase of the white corpuscles, both absolute and relative to the red corpuscles, the increase, due to an augmented production or possibly to a retarded destruction, being at times so great as to give the blood a pinkish grey appearance, like that of blood mixed with pus. We aceordingly find that in this disease the plasma is in many ways profoundly affected and fails to nomrish the tissues. As a further illustration of the possibie actions of the white corpuscles we may state that, in certain diseases in which minute organisms, such as bacteria, make their appearance in the blood and tissues, white corpuseles may attack and devour these bacteria, thus acting as "phagocytes," and in this way, or otherwise, by exerting some influence on the bacteria or the products of their activity, modify the course of the disease of which the bacteria are the essential cause.

\section{Blood Platelets.}

§33. In a drop of blood examined with care immediately after removal, may be seen a number of exceedingly small bodies ( $2 \mu$ to $3 \mu$ in diameter) frequently disc-shaped hut sometimes of a romuded or irregular form, homogeneous in appearance when quite 
fresh but apt to assume a faintly granular aspect. They are called blood platelets. They have been supposed by some to become developed into and indeed to be early stages of the red corpuscles, and hence have been called hematoblasts; but this view has not been confirmed, indeed, as we have seen ( $\$ 27$ ), the real hiematoblasts or developing red corpuscles are of quite a different nature.

They speedily undergo change after removal from the body, apparently dissolving in the plasma; they break up, part of their substance disappearing, while the rest becomes granular. Their granular remains are apt to run together, forming in the plasma the shapeless masses which have long been known and described as "lumps of protoplasm." By appropriate reagents, lowever, these platelets may be fixed and stained in the condition in which they appear after leaving the boly.

The substance composing them is peculiar, and though we may perhaps speak of them as consisting of living material, their nature is at present obscure. They may be seen within the living blood vessels, and therefore must be regarded as real parts of the blook and not as products of the changes taking place in blood after it has been shed.

When a neelle or thread or other foreign body is introduced into the interior of a blood vessel, they are apt to collect upon, and indeed are the precursors of the clot which in most cases forms around the needle or threat. They are also found in the thrombi or plugs which sometimes form in the blood vessels as the result of disease or injury. Indeed it has been maintained that what are called white thrombi (to distinguish them from red thrombi, which are plugs of eorpuscles and fibrin) are in reality aggregations of blood platelets; and for various reasons blood platelets have been supposed to play an important part in the clotting of blood, carrying out the work which in this respect is by others attributed to the white corpuscles. But no very definite statement can at present be made about this; and indeed the origin and whole nature of these blood platelets is at present obscure. 


\section{SEC. 3. THE CHEMICAL COMPOSITION OF BLUOD.}

$\$ 34$. We may now pass briefly in review the chief chemical characters of blood, remembering always that, as we have already urged, the chief chemical interests of blood are attached to the changes which it undergoes in the several tissues; these will be considered in connection with each tissue at the appropriate place.

The average specific gravity of human blood is 1055 , varying from 1045 to 1075 within the limits of health.

The reaction of blood as it flows from the blood vessels is found to be distinctly though feebly alkaline. If a drop be placed on a piece of faintly-red highly-glazed litmus paper, and then wiped off, a blue stain will be left.

The whole blood contains a certain quantity of the gases, oxygen, carbonic acid, and nitrogen, which are held in the blood in a peculiar way, and which are given off from blood when exposed to a vacuum or to an atmosphere of suitable composition; the relative amounts differ in different kinds of blood, and so serve especially to distinguish arterial from venous blood. These gases of blood we shall study in connection with respiration.

The normal blood consists of eorpuscles and plasma.

If the corpuscles be supposed to retain the amount of water proper to them, blood may, in general terms, be considered as consisting by weight of from about one-third to somewhat less than one-half of corpuscles, the rest being plasma. As we have already seen, the number of corpuscles in a specimen of blood is found to vary considerably, not only in different animals and in different individuals, but in the same individual at different times.

The plasma is resolved by the clotting of the blood into serum and fibrin.

$\$ 35$. The serum contains in 100 parts Proteid substances

Fats, various extractives, and saline matters Water

about 8 or 9 parts.

" 2 or 1 "


The proteids are paraglobulin and serum albumin (there being probably more than one kind of serum albumin) in varying proportion. We may perhaps, roughly speaking, say that they occur in about equal quantities.

Conspicuous and striking as are the results of clotting, massive as appears to be the clot which is formed, it must be remembered that by far the greater part of the clot consists of corpuseles. The amount by weight of fibrin required to bind together a number of corpuscles in order to form even a large firm clot is exceedingly small. Thus the average quantity by weight of fibrin in human blood is said to be 2 p.c.; the amount however which can be obtained from a given quantity of plasma varies extremely, the variation being due not only to circumstances affecting the blood, but also to the method employed.

The fats, which are scanty, except after a meal or in certain pathological conditions, consist of the neutral fats, stearin, palmitin, and olein, with a certain quantity of their respective alkaline soaps. The peculiar complex fat lecithin occurs in very small quantities only; the amount present of the peculiar alcohol cholesterin which has so fatty an appearance is also small. Among the extractives present in serum may be put down nearly all the nitrogenous and other substances which form the extractives of the body and of food, such as urea, kreatin, sugar, lactic acid, \&c. A very large number of these have been discovered in the blood under various circumstances, the consideration of which must be left for the present. The peculiar odonr of blood or of serum is probably due to the presence of volatile bodies of the fatty acid series. The faint yellow colour of serum is due to a special yellow pigment. The most characteristic and important chemical feature of the saline constitution of the serum is the preponderance, at least in man and most animals, of sodium salts over those of potassium. In this respect the serum offers a marked contrast to the corpuscles. Less marked, but still striking, is the abundance of chlorides and the poverty of phosphates in the serum as compared with the corpuscles. The salts may in fact briefly be described as consisting chiefly of sodium chloride, with some amount of sodium carbonate, or more correctly sodium bicarbonate, and potassium chloride, with small quantities of sodium sulphate, sodium phosphate, calcinm phosphate, and magnesium phosphate. And of even the small quantity of phosphates found in the ash, part of the phosphorus exists in the serum itself, not as a phosphate but as phosphorus in some organic body.

$\$ 36$. The red corpuscles contain less water than the serum, the amount of solid matter being variously estimated at from 30 to 40 or more p.c. The solids are almost entirely organic matter, the inorganic salts amounting to less than 1 p.c. Of the organic matter again by far the larger part consists of hæmoglobin. In 100 parts of the dried organic matter of the corpuscles of human blood, about 
90 parts are hemoglobin, about $S$ parts are proteid substances, and about ? parts are other substances. Of these other substances one of the most important, forming about a quarter of them and apparently being always present, is lecithin. Cholesterin appears also to be normally present. The proteid substances which form the stroma of the red corpuscles appear to belong chictly to the globulin family. As regards the inorganic constituents, the corpuscles are distinguished by the relative abundance of the salts of potassium and of phosphates. This at least is the case in man; the relative quantities of sodium and potassium in the corpuscles and serum respectively appear however to vary in different animals; in some the sodium salts are in excess even in the corpuscles.

$\$ 37$. The proteid matrix of the white corpuscles, we have stated to be composed of nucleo-albumin, globulin, and possibly other proteids. The nuclei contain nuclein. The white corpuscles are found to contain, in addition to proteid material, lecithin and other fats, glycogen, extractives and inorganic salts, there being in the ash as in that of the red corpuscles a preponderance of potassium salts and of phosphates.

The main facts of interest, then, in the chemical composition of the blood are as follows: The red corpuscles consist chiefly of hremoglobin. The organic solids of serum consist partly of serumalbumin, and partly of paraglobulin. The serum or plasma contrasts, in man at least, with the corpuscles, inasmuch as the former contains chiefly chlorides and sodium salts while the latter are richer in phosphates and potassium salts. The extractives of the blood are remarkable rather for their number and variability than for their abundance, the most constant and important being perhaps urea, kreatin, sugar, and lactic acid. 


\section{SEC. 4. THE QUANTITY OF BLOOD, AND ITS}

DISTRIBUTION IN THE BODY.

$\$ 38$. The quantity of blood contained in the whole vascular system is a balance struck between the tissues which give to and those which take away from the blood. Thus the tissues of the alimentary canal largely add to the blood water and the material derived from food, while the excretory organs largely take away water and the other substances constituting the excretions. Other tissues both give and take; and the considerable drain from the blood to the lymph spaces which takes place in the capillaries is met by the flow of lymph into the great veins.

From the result of a few observations on executed criminals it has been concluded that the total quantity of blood in the human body is about $\frac{1}{13}$ th of the body weight. But in various animals, the proportion of the weight of the blood to that of the body has been found to vary very considerably in different individuals, and probably this holds good for man also, - at all events within certain limits.

In the same individual the quantity probably does not vary largely. A sudden drain upon the water of the blood by great activity of the excretory organs, as by profuse sweating, or a sudden addition to the water of the blood, as by drinking large quantities of water or by injecting fluid into the blood vessels, is rapidly compensated by the passage of water from the tissues to the blood or from the blood to the tissues. As we have already said, the tissues are continually striving to keep up an average composition of the blood, and in so doing keep up an average quantity. In starvation the quantity (and quality) of the blood is maintained for a long time at the expense of the tissues, so that after some days deprivation of food and drink, while the fat, the muscles, and other tissues have been largely diminished, the quantity of blood remains nearly the same. 
The total quantity of blood present in an animal body is estimated in the following way: As much blood as possible is allowed to escape from the vessels; this is measured directly. The vessels are then washed out with water or normal saline solution, and the washings carefully collected, mixed, and measured. A known quantity of blood is diluted with water or normal saline solution until it possesses the same tint as a measured specinen of the washings. This gives the amount of blood (or rather of hæmoglobin) in the measured specimen, from which the total quantity in the whole washings is calculated. Lastly, the whole body is carefully minced and waslied free from blood. The washings are collected and filtered, and the amount of blood in them is estimated as before by comparison with a specimen of diluted blood. The quantity of blood, as calculated from the two washings, together with the escaped and directly measured blood, gives the total quantity of blood in the body.

The method is not free from objections, but other methods are open to still graver objections.

The blood is in round numbers distributed as follows: -

About one-fourth in the heart, lungs, large arteries, and veins,

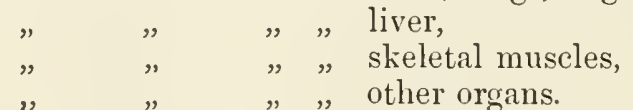

Since in the heart and great blood vessels the blood is simply in transit, without undergoing any great changes (and in the lungs, so far as we know, the changes are limited to respiratory changes), it follows that the changes which take place in the blood passing through the liver and skeletal muscles far exceed those which take place in the rest of the body. 


\section{CHAPTER II.}

\section{THE CONTRACTILE TISSUES.}

$\S 39$. In order that the blood may nourish the several tissues it is carried to and from them by the vascular mechanism ; and this carriage entails active movements. In order that the blood may adequately nourish the tissues, it must be replenished by food from the alimentary canal, and purified from waste by the excretory organs; and both these processes entail movements. Hence before we proceed further we must study some of the general characters of the movements of the body.

Most of the movements of the body are carried out by means of the muscles of the trunk and limbs, which being connected with the skeleton are frequently called skeletal muscles. A skeletal muscle when subjected to certain influences suddenly shortens, bringing its two ends nearer together; and it is the shortening which, by acting upon various bony levers or by help of other mechanical arrangements, produces the movement. Such a temporary shortening, called forth by certain influences and due as we shall see to changes taking place in the muscular tissue forming the chief part of the muscle, is technically called a contraction of the muscle; and the muscular tissue is spoken of as a contractile tissue. The heart is chiefly composed of muscular tissue, differing in certain minor features from the muscular tissue of the skeletal muscles; and the beat of the heart is essentially a contraction of the musclar tissue composing it, a shortening of the peculiar muscular fibres of which the heart is chiefly made up. The movements of the alimentary canal and of many other organs are similarly the results of the contraction of the muscular tissue entering into the composition of those organs, of the shortening of certain muscular fibres built up into those organs. In fact almost all the movements of the body are the results of the contraction of muscular fibres, of various nature and variously disposed. 
Some few movements however are carried ont by structures which cannot be called muscular. Thus in the pulmonary passages and elsewhere movenent is effected by means of cilia attached to epithelium cells; and elsewhere, as in the case of the migrating white corpuscles of the blood, transference from place to place in the body is brought about by amœboid movements. But, as we shall see, the changes in the epithelium cell or white corpuscle which are at the bottom of ciliary or amœboid movements are in all probability fundamentally the same as those which take place in a nuscular fibre when it contracts. They are of the nature of a contraction, and hence we may speak of all these as different forms of contractile tissue.

Of all these various forms of contractile tissue the skeletal muscles, on account of the more complete development of their functions, will be better studied first; the other's, on accomnt of their very simplicity, are in many respects less satisfactorily understood.

All the ordinary skeletal muscles are comnected with nerves. We have no reason for thinking that they are thrown into contraction, under normal conditions, otherwise than by the agency of nerves. Muscles and nerves being thus so closely allied, and having besides so many properties in common, it will conduce to clearness and brevity if we treat them together: 


\section{SEC. 1. THE PHENOMENA OF MUSClE AND NERVE.}

\section{Muscular and Nervous Irritability.}

$\$ 40$. The skeletal muscles of a frog, the brain and spinal cord of which have been destroyed, do not exhibit any spontaneous movements or contractions, even though the nerves be otherwise quite intact. Left undisturbed the whole body may decompose without any contraction of any of the skeletal muscles having been witnessed. Neither the skeletal muscles nor the nerves. distributed to them possess any power of automatic action.

If however a muscle be laid bare and be more or less violently disturbed, - if for instance it be pinched, or touched with a hot. wire, or brought into contact with certain chemical substances, or subjected to the action of galvanic currents, - it will move, that is contract, whenever it is thus disturbed. Though not exhibiting any spontaneous activity, the muscle is (and continues for some time after the general death of the animal to be) irritable. Though it remains quite quiescent when left untouched, its powers are then dormant only, not absent. These require to be roused or 'stimulated' by some change or disturbance in order that they may manifest themselves. The substances or agents which are thus able to evoke the activity of an irritable muscle are spoken of as stimuli.

But to produce a contraction in a muscle the stimulus need not be applied directly to the muscle ; it may be applied indirectly by means of the nerve. Thus, if the trunk of a nerve be pinched, or subjected to sudden heat, or dipped in certain chemical substances, or acted upon by various galvanic currents, contractions are seen in the muscles to which branches of the nerve are distributed.

The nerve like the muscle is irritable; it is thrown into a state of activity by a stimulus; but unlike the muscle it does not itself contract. The stimulus does not give rise in the nerve to any visible change of form; but that changes of some kind or other 
are set up and propagated along the nerve down to the muscle is shewn by the fact that the muscle contracts when a part of the nerve at some distance from itself is stimulated. Both nerve and muscle are irritable, but only the muscle is contractile,-i.e., manifests its irritability by a contraction. The nerve manifests its irritability by transmitting along itself, without any risible alteration of form, certain molecular changes set up by the stimulus. We shall call these changes thus propagated along a nerve, 'nervous impulses.'

$\$ 41$. We have stated above that the muscle may be thrown into contractions by stimuli applied directly to itself. But it might fairly be urged that the contractions so produced are in reality due to the fact that the stimulus, although apparently applied directly to the muscle, is, after all, brought to bear on some or other of the many fine nerve-branches, which as we shall see are abundant in the muscle, passing among and between the muscular fibres in which they finally end. The following facts however go far to prove that the muscular fibres themselves are capable of being directly stimulated without the intervention of any nerves. When a frog (or other animal) is poisoned with urari, the nerves may be subjected to the strongest stimuli withont causing any contractions in the muscles to which they are distributed; yet even ordinary stimuli applied directly to the muscle readily cause contractions. If before introducing the urari into the system, a ligature be passed underneath the sciatic nerve in one leg, - for instance the right, - and drawn tightly round the whole leg to the exclusion of the nerve, it is evident that the urari when injected into the back of the animal, will gain access to the right sciatic nerve above the ligature, but not below, while it will have free access to the rest of the body, including the whole left sciatic. If, as soon as the urari has taken effect, the two sciatic nerres be stimulated, no movement of the left leg will be produced by stimulating the left sciatic, whereas strong contractions of the muscles of the right leg below the ligature will follow stimulation of the right sciatic, whether the nerve be stimulated above or below the ligature. Now, since the upper parts of both sciatics are equally exposed to the action of the poison, it is clear that the failure of the left nerve to cause contraction is not attributable to any change liaving taken place in the upper portion of the nerve, else why should not the right, which has in its upper portion been equally exposed to the action of the poison, also fail ? Evidently the poison acts on some parts of the nerve lower down. If a single muscle be removed from the circulation (by ligaturing its blood vessels), previous to the poisoning with urari, that musele will contract when any part of the nerve going to it is stimulated, though no other muscle in the body will contract when its nerve is stimulated. Here the whole nerve right down to the muscle has been exposed to the action of the poison; and yet it has lost none of its power over the muscle. On 
the other hand, if the muscle be allowed to remain in the body, and so be exposed to the action of the poison, but the nerve be divided high up and the part connected with the musele gently lifted up before the urari is introdnced into the system, so that no blood flows to it and so that it is protected from the influence of the poison, stimulation of the nerve will be found to produce no contractions in the musele, though stimuli applied directly to the muscle at once cause it to contract. From these facts it is clear that urari poisons the ends of the nerve within the musele long before it affects the trunk; and it is exceedingly probable that it is the very extreme ends of the nerves (possibly the end-plates, or peculiar structures in which the nerve fibres end in the muscular fibres, - for urari poisoning, at least when profound, canses a slight but yet distinctly recognisable effect in the microscopic appearance of these structures) which are affected. The phenomena of urari poisoning therefore go far to prove that muscles are capable of being made to contract by stimuli applied directly to the muscular fibres themselves; and there are other facts which support this view.

$\$ 42$. When in a recently killed frog we stimulate by various means and in various ways the muscles and nerves, it will be observed that the movements thus produced, though very various, may be distinguished to be of two kinds. On the one hand, the result may be a mere twitch, as it were, of this or that musele; on the other hand, one or more muscles may remain shortened, contracted for a considerable time, - a limb for instance being raised up or stretched out, and kept raised up or stretched out for many seconds. And we find upon examination that a stimulus may be applied either in such a way as to produce a mere twitch, - a passing, rapid contraction which is over and gone in a fraction of a second, - or in such a way as to keep the muscle shortened or contracted for so long time as, up to certain limits, we may choose. The mere twitch is called a single or simple muscular contraction; the sustained contraction, which as we shall see is really the result of rapidly repeated simple contractions, is called a tetanic contraction.

34. In order to study these contractions adequately, we must have recourse to the 'graphic method' as it is called, and obtain a tracing or other record of the change of form of the muscle. To do this conveniently, it is best to operate with a muscle isolated from the rest of the body of a recently killed animal, and carefully prepared in such a way as to remain irritable for some time. The museles of cold blonded animals remain irritable after removal from the body far longer than those of warm blooded animals, and hence those of the frog are generally made use of. We shall study presently the conditions which determize this maintenance of the irritability of museles and nerves after removal from the body.

A muscle thus isolated, with its nerve left attached to it, is 
called a muscle-nerve preparation. The most convenient muscle for this purpose in the frog is perhaps the gastrocnemius, which should be dissected out so as to leave carefully preserved the attachment to the femur above, some portion of the tendon (tendo achillis) below, and a considerable length of the sciatic nerve with its branches going to the muscle. Fig. 1.

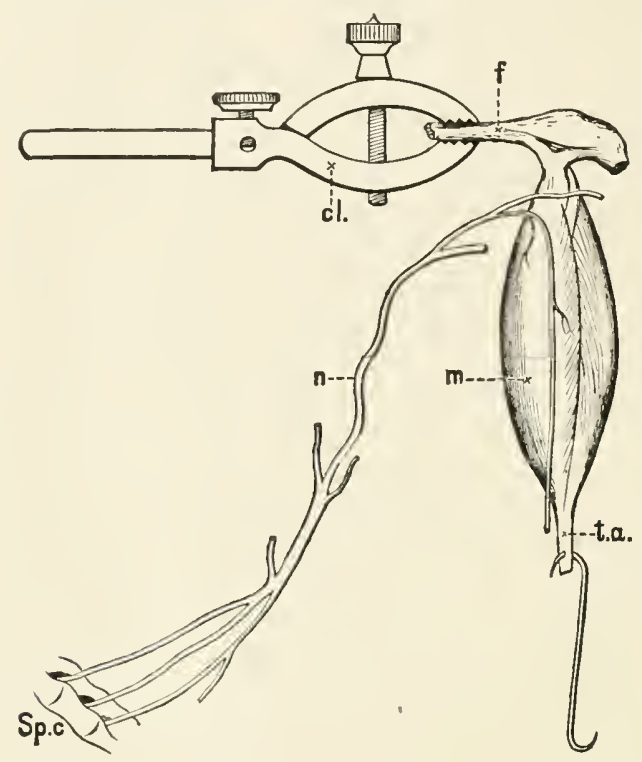

Fig. 1. A Moscle-nerve Preparation.

$m$, the muscle, gastrocnemius of frog; $n$, the sciatic nerve, all the branches being cut away except that supplying the muscle; $f$, femur; $c l$. clamp; $t$. $a$ tendo achillis ; sp. $c$, end of spinal canal.

$\$ 44$. We may apply to such a muscle-nerve preparation the various kinds of stimuli spoken of above, - mechanical, such as pricking or pinching; thermal, such as sudden heating; chemical, such as acids or other active chemical sulistances, or electrical; and these we may apply either to the muscle direetly, or to the nerve, thus affecting the muscle indirectly. Of all these stimuli by far the most convenient for general purposes are electrical stimuli of various kinds; and these, except for special purposes, are best applied to the nerve, and not directly to the muscle.

Of electrical stimuli again, the currents, as they are called, generated by a voltaic cell are most convenient, though the electricity generated by a rotating magnet, or that produced by friction, may be employed. Naking use of a cell or battery of cells, Taniells, Grove's, Leclanche, or any other, we must distinguish between the eurrent produced by the cell itself (the constant 
current as we shall call it) and the induced current obtained from the constant current by means of an induction coil, as it is called; for the physiological effects of the two kinds of current are in many ways different.

It may perhaps be worth while to remind the reader of the following facts : -

In a galvanic battery, the substance (plate of zinc for instance) which is acted upon and used up by the liquid is called the positive element, and the substance which is not so acted upon and used up (plate \&c. of copper, platimum, or carbon, \&c.) is called the negative element. A galvanic action is set up when the positive (zinc) and the negative (copper) elements are comnecterl outside the battery by some conducting material, such as a wire, and the current is said to flow in a circuit or circle from the zinc or positive element to the copper or negative element inside the battery, and then from the copper or negative element back to the zinc or positive element through the wire outside the buttery. If the conducting wire be cut through, the current ceases to flow; but if the cut ends be brought into contact, the current is reestablished and continues to flow so long as the contact is good. The ends of the wires are called 'poles,' or when used for physiological purposes, in which case they may be fashioned in various ways, are spoken of as electrodes. When the poles are brought into contact or are connected by some conducting material, galvanic action is set up, and the current flows through the battery and wires; this is spoken of as "making the current" or " completing or closing the circuit." When the poles are drawn apart from each other, or when some non-conducting miterial is interposed between them, the galvanic action is arresterl; this is spoken of as "breaking the current" or "opening the circuit." The current passes from the wire comnected with the negative (copper) element in the battery to the wire connected with the positive (zinc) element in the battery; hence the pole connected with the copper (negative) element is called the positive pole, and that connecterl with the zinc (positive) element is called the negative pole. When used for physiological purposes the positive pole becomes the positive electrole, and the negative pole the negative electrode. The positive electrode is often spoken of as the anode (ana, up), and the negative electrode as the kathode (kata, dow!n).

A piece of nerve of ordinary length, though not a good conductor, is still a conductor, and when placed on the electrodes, completes the circuit, permitting the current to pass through it; in order to remove the nerve from the influence of the current it must be lifter off from the electrodes. This is obviously inconvenient; and hence it is usual to arrange a means of opening or closing the circuit at some point along one of the two wires. This nay be done in various ways, - by fastening one part of the wire into a cup of mercurs and so by dipping the other part of the wire into the cup to close the circuit and make the current, and by lifting it out of the mereury to open the circuit and break the current; or by arranging, hetween the two parts of the wires, a moveable bridge of good conducting material such as brass, which can be put down to close the circuit or raised up to open the circuit; or in 
other ways. Such a means of closing and opening a circuit and so of making or breaking a current is called a key.

A key which is frequently used by physiologists goes by the name of du Bois-Reymond's key; though undesirable in many respects it has the advantage that it can be used in two different ways. It may be arranged as in A, Fig. 2. In this case, when the brass briclge of $\mathrm{K}$, the key is put down (dotted outline in the figure), so as to form a means of good conduction between the brass plates to which the wires are screwed, the circuit is closed and the current passes from the positive pole (end of the negative - copper - element) to the positive electrode or anole, $A n$. through the nerve, to the negative electrole or kathode $\boldsymbol{K}$ at. and thence back to the negative pole (end of the positive - zinc -

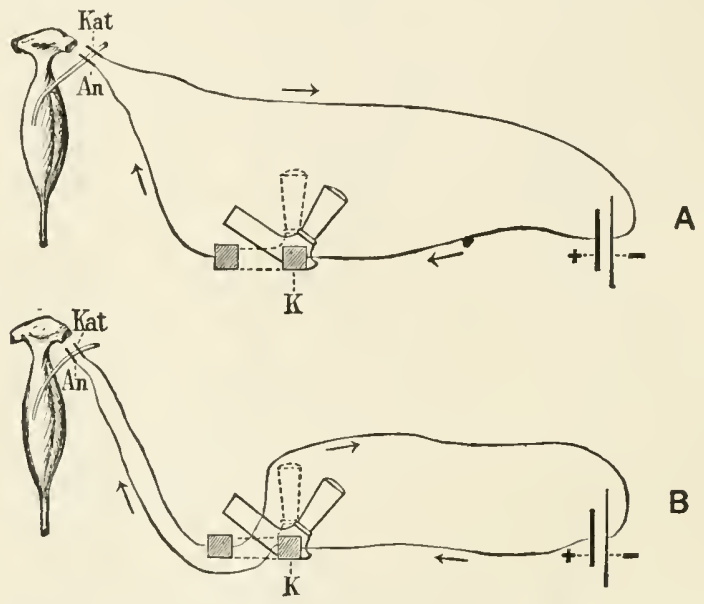

Fig. 2. Diagram of Du Bois-Reymond Key used, A, for Making and Breaking, B, for Short Circuiting.

element) in the hattery; on raising the brass bridge (continuous outline in the figure) the circuit is opened, the current broken, and no current passes through the electrodes. Or it may be arranged as in B. In this case if the brass bridge be 'down,' the resistance offered by it is so small compared with the resistance offered by the nerve between the electrodes, that the whole current from the battery passes through the bridge, back to the battery, and none, or only an infinitesimal portion, passes into the nerve. When on the other hand the bridge is raised, and so the conduction between the two sides suspended, the current is not able to pass directly from one side to the other, but can and does pass along the wire through the nerve back to the battery. Hence in arrangement $A$, 'putting down the key' as it is called makes a current in the nerve, and 'raising' or 'opening the key' breaks the current. In arrangement $\mathrm{B}$, however, putting down the key diverts the eurrent from the nerve by sending it through the bridge, and so back to the battery; the current instead of making the longer circuit through the electrodes makes the shorter circuit through the key; hence this is called 'short circuiting.' When the bridge is raised the current passes through the 
nerve on the electrodes. Thus 'putting down' and 'raising or 'opening' the key have contrary effects in A and B. In B, it will be observed, the battery is always at work. the current, is always flowing either through the electrodes (key up) or through the key (key down); in A, the battery is not at work until the circuit is made by putting down the key. And in many cases it is desirable to take so to speak a sample of the current while the battery is in full swing rather than just as it begins to work. Moreover in $B$ the electrodes are, when the key is down, wholly shut off from the current; whereas in A, when the key is up, one electrode is still in direct connection with the battery; and this comection, leading to what is known as unipolar action, may give rise to stimulation of the nerve. Hence the use of the key in the form $B$.

Other forms of key may be used. Thus in the Morse key ( $F$, Fig. 3) contact is made by pressing down a lever handle (ha); when the pressure is removed, the handle, driven up by a spring, breaks contact. In the arrangement shewn in the figure one wire from the battery being brought to the binling screw $b$, while the binding screw $a$ is connected with the other wire, putting down the handle makes connection between $a$ and $b$, and thus makes a current. By arranging the wires in the several binding screws in a different way, the making contact by depressing the handle may he used to short circuit.

In an "indnction coil," Figs. 3 and 4, the wire connecting the two elements of a battery is twisted at some part of its courso irto a close spiral, called the primary coil. Thus in Fig. 3 the wire $x^{\prime \prime \prime}$ connected with the copper or negative plate c.p. of the battery, $E$, joins the primary coil pr.c., and then passes on as $y^{\prime \prime \prime}$, through the "key" $F$, to the positive (zinc) plate z.p. of the battery. Over this primary coil, but quite nnconnected with it, slides another coil, - the secondary coil, s.c.; the ends of the wire forming this coil, $y^{\prime \prime}$ and $x^{\prime \prime}$, are continued on in the arrangement illustratel in the figure as $y^{\prime}$ and $y$, and as $x^{\prime}$ and $x$, and terminate in electrodes. If these electrodes are in contact or connected with conducting material, the circuit of the secondary coil is said to be closed; otherwise it is open.

In such an arrangement it is found that at the moment when the primary circuit is closel, $-i$. e. when the primary current is "made," a secondary "induced" current is, for an exceedingly brief period of time, set up in the secondary coil. Thus in Fig. 2 when, by moving the "key" $F, y^{\prime \prime \prime}$ and $x^{\prime \prime \prime}$ (previously not in connection with" each other) are put into connection and the primary current thus made, at that instant a current appears in the wires $y^{\prime \prime} x^{\prime \prime}$ \&c., but almost immediately disappears. A similar almost instantaneous current is also developed when the primary current is "broken," but not till then. So long as the primary current flows with uniform intensity, no current is induced in the secondary coil. It is only when the primary current is either made or broken, or suddenly varies in intensity, that a current appears in the seconlary coil. In each case the current is of very brief duration, gone in an instant almost, and may therefore be spoken of as "a shock," an induction shock, - being called a " making shock " when it is caused by the making, and a "breaking shock" when it is caused by the breaking, of the primary circuit. The direction of the current 


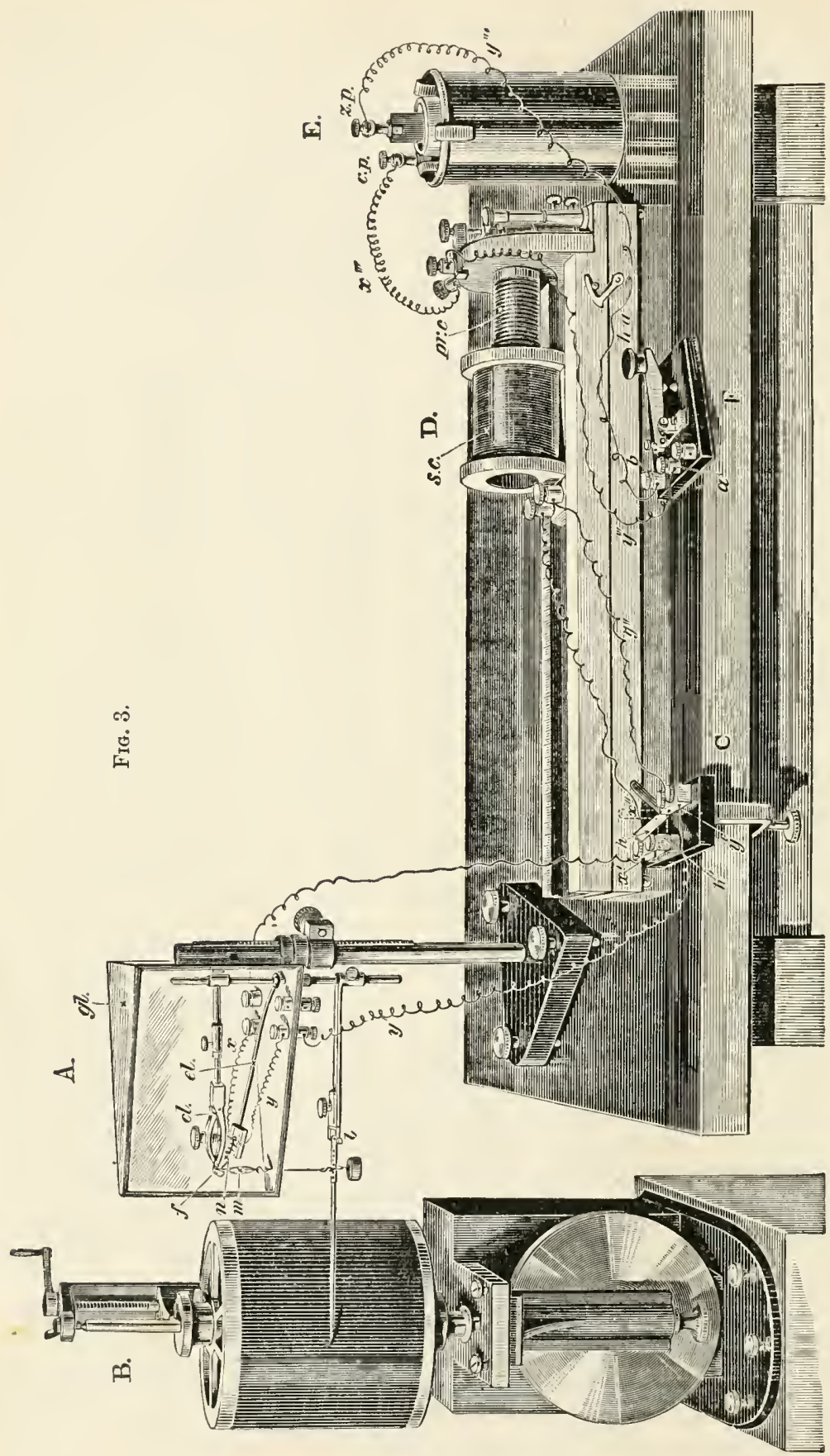


Fig. 3. Diagram illustrating Apparatus arizange for Experiments Witil MUscle AxD Nerve.

A. The noist chamber containing the muscle-nerve preparation. The muscle $m$, supported by the clamp $c l$, which firmly grasps the end of the femur $f$, is connected by nieans of the S hook $s$ and a threal with the lever $l$, placed below the moist chamber. The nerve $n$, with the portiou of the spinal column $n^{\prime}$ still attached to it, is placed on the electrode-liolder $e l$, in contact with the wires $x, y$. The whole of the interior of the glass case $g l$. is kept saturated with moisture, and the electrode-holder is so constructed that a piece of moistened blotting-paper may be placed on it without coming into contact with the nerve.

$B$. The revolving eylinder bearing the smoked paper on which the lever writes.

C. Du Bois-Reymond's key arranged for short-circuiting. The wires $x$ and $y$ of the electrode-holder are connected through binding screws in the floor of the noist chanber with the wires $x^{\prime}, y^{\prime}$, and these are secured in the key, one on either side. 'To the same key are attached the wires $x^{\prime \prime} y^{\prime \prime}$ coming from the secondary coils $s . e$. of the induction-coil $D$. This secondary coil can be made to slide up and down over the primary coil $p r . c$., with which are connected the two wires $x^{\prime \prime \prime}$ and $y^{\prime \prime \prime} . x^{\prime \prime \prime}$ is connected directly with one pole, for instance the copper pole $c . p$. of the battery $E . y^{\prime \prime \prime}$ is carried to a binding screw $a$ of the Morse key $F$, and is continued as $y^{1 \mathrm{~V}}$ from another binding screw $b$ of the key to the zinc pole $z$. p. of the battery.

Supposing everything to be arranged, and the battery charged, on depressing the handle $h a$, of the Morse key $F$, a current will be made in the primary coil pr. $c$, passing from $c . p$. through $x^{\prime \prime \prime}$ to $m$. $c$., and thence through $y^{\prime \prime \prime}$ to $a$, thence to $b$, and so through $y^{\mathrm{IV}}$ to $z p$. On removing the finger from the handle of $F$, a spring thrusts up the handle, and the primary circuit is in consequence immediately brokeu.

At the instant that the primary current is either made or broken, an indnced current is for the instant dereloped in the secondary coil $s c$. If the cross bar $h$ in the du Bois-Rermond's key be raised (as shewn in the thick line in the figure), the wires $x^{\prime \prime} x^{\prime} x$, the nerve between the electrolles and the wires $y, y^{\prime}, y^{\prime \prime}$ form the complete secondary circuit, and the nerve conseqmently experiences a making or breaking induction-shock whenever the primary current is made or broken. If the cross bar of the du Bois-Reymond's key be shut down, as in the dotted line $h^{\prime}$ in the figure, the resistance of the cross bar' is so slight compared with that of the nerve and of the wires going from the key to the nerve, that the whole secondary (induced) current passes from $x^{\prime \prime}$ to $y^{\prime \prime}$ (or from $y^{\prime \prime}$ to $x^{\prime \prime}$ ), along the cross har, and practically none passes into the nerve. The nerve being thus "short-circuited." is not affected by auy changes in the current.

The figure is intended merely to illustrate the general method of studying muscnlar contraction; it is not to be supposed that the details here given are universally adopted or indeed the best for all purposes.

in the making shock is opposed to that of the primary current; thus in the figure while the primary current flows from $x^{\prime \prime \prime}$ to $y^{\prime \prime \prime}$, the induced making shock flows from $y$ to $x$. The current of the breaking shock on the other hand flows in the same direction as the primary current from $x$ to $y$, and is therefore in direction the reverse of the making shock. Compare Fig. 3 , where arrangement is shewn in a diagrammatic manner.

The current from the battery, upon its first entrance into the primary coil, as it passes along each twist of that coil, gives rise in the neighbouring twists of the same coil to a momentary induced current having a direction opposite to its own, and therefore tellding to weaken itself. It is not until this 'self-induction' has passed off that the 
current in the primary coil is established in its full strength. Owing to this delay in the full establishnent of the current in the primary coil, the induced current in the secondary coil is developed more slowly

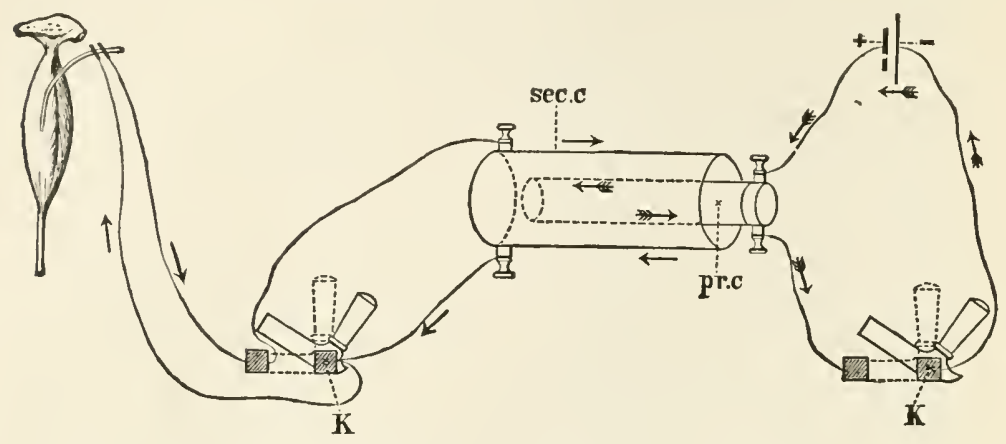

Fig. 4. Diagram of an Induction Coil.

+ positive pole, end of negative element; - negative pole, end of positive element of battery ; $\mathbf{K}$, du Bois-Reymond's key ; pr. c. primary coil, current shewn by feathered arrow ; sc. c. secondary coil, current shewn by unfeathered arrow.

than it would be were no such 'self-induction' present. On the other hand, when the current from the battery is 'broken,' or 'shut off' from the primary coil, no such delay is offered to its disappearance, and consequently the induced current in the secondary coil is dercloped with unimpcded rapidity. We shall see later on that a rapidly developed current is more effective as a stimulus than is a more slowly developed current. Hence the making shock, where rapility of production is interfered with by the self-induction of the primary coil, is less effective as a stimulus than the breaking slock, whose development is not thus interfered with.

The strength of the induced current depends, on the one hand, on the strength of the current passing through the primary coil, - that is, on the strength of the battery. It also depends on the relative position of the two coils. Thus, if a secondary coil is brought nearer and nearer to the primary coil and made to overlap it more and more, the induced current becomes stronger and stronger, though the current from the battery remains the same. With an ordinary battery, the secondary coil may be puslied to some distance away from the primary coil, and yet shocks sufficient to stimulate a muscle will be obtained. For this purpose however the two coils should be in the same line; when the secondary coil is placed cross-wise, at right angles to the primary, no induced current is developed, and at intermediate angles the induced current has intermerliate strengths.

When the primary current is repcatedly and rapidly madc and broken, the secondary current being developerl with each make and with each break, a rapilly recurring series of alternating currents is developed in the secondary coil and passes through its clcctrodes. We shall frequently spcak of this as the intermuted induction current, or more briefly the interrupted current; it is sometimes spoken of as the 
faradaic current, and the application of it to any tissue is spoken of as faradization.

Such a repeated breaking and making of the primary current may be effected in many various ways. In the instrument conmonly used for the purpose, the primary current is male and broken by means of a vibrating steel ship working against a magnet; hence the instrument is called a magnetic interruptor. See Fig. 5 .

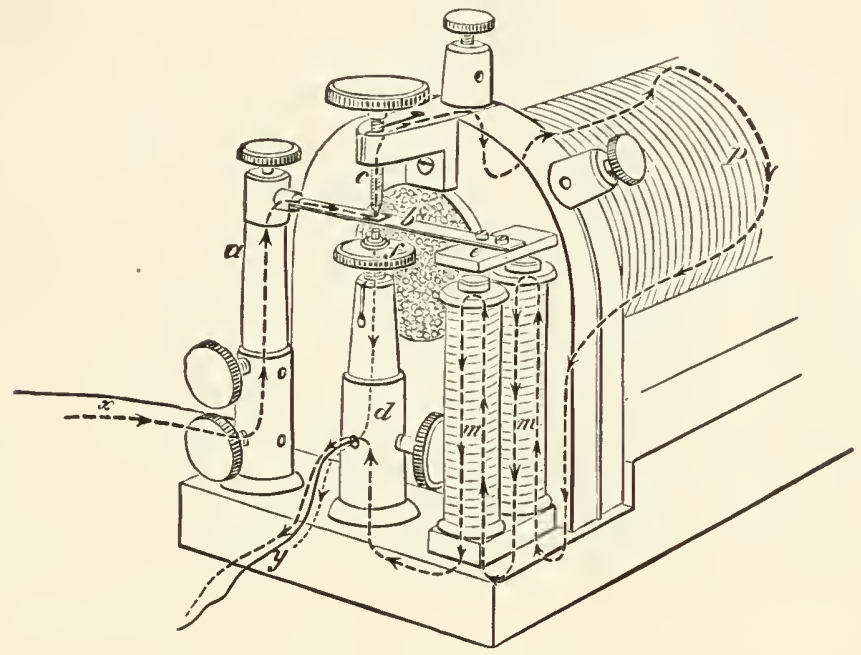

Fig. 5. The Magnetic Interruptor.

The two wires $x$ and $y$ from the battery are connected with the two brass pillars $a$ and $d$ by means of screws. Directly contact is thus made, the current, indicated in the figure by the thick interrupted line, passes in the direction of the arrows, up the pillar $a$, along the steel spring $b$, as far as the screw $c$, the point of which, armed with platinum, is in contact with a small platinum plate on $b$. The current passes from $b$ through $c$ and a connecting wire into the primary coil $p$. Upon its entering into the primary coil, an iuduced (making) current is for the instant developed in the secondary coil (not shewn in the figure). From the primary coil $p$ the current passes, by a connecting wire, through the double spiral $m$, and, did nothing happen, would continue to pass from $n$ by a connecting wire to the pillar $d$, and so by the wire $y$ to the battery. The whole of this course is indicated by the thick interrupted line with its arrows.

As the current however passes through the spirals $m$, the iron cores of these are made magnetic. They in consequence draw down the iron bar $e$, fixed at the end of the spring $b$, the flexibility of the spring allowing this. But when $e$ is drawn down, the platinum plate on the upper surface of $b$ is also drawn away from the screw $c$, and thus the current is "broken" at $b$. (Sometimes the screw $f$ is so arranged that when $e$ is drawn down a platinum plate on the under surface of $b$ is brought into contact with the platinum-armed point of the screw $f$. 
The current then passes from $b$ not to $c$ but to $f$, and so down the pillar $d$, in the airection indicated by the thin interrupted line, and out to the battery by the wire $y$, and is thus cut off from the primary coil. But this arrangement is unnecessary.) At the instant that the current is thus broken and so cut off from the primary coil, an induced (breaking) current is for the moment developed in the secondary coil. But the current is cut off not only from the primary coil, but also from the spirals $m$; in consequence their cores cease to be inagnetised, the bar $e$ ceases to be attracterl by thein, and the spring $b$, by virtue of its elasticity, resumes its former position in contact with the screw $c$. This return of the spring however re-establishes the current in the primary coil and in the spirals, and the spring is drawn down, to be released once more in the same manner as before. Thus as long as the current is passing along $x$, the contact of $b$ with $c$ is alternately being made and broken, and the current is constantly passing into and being shut off from $p$, the periods of alternation being determined by the periods of vibration of the spring $b$. With each passage of the current into, or withdrawal from the primary coil, an induced (naking and, respectively, breaking) current is developed in a secondary coil.

As thus used, each 'making shock,' as explained above, is less powerful than the corresponding 'breaking shock;' and indeed it sometimes happens that instead of each make as well as each break acting as a stimulus, giving rise to a contraction, the 'breaks' only are effective, the several 'makes' giving rise to no contractions.

By what is known as Helmholtz's arrangement, Fig. 6, however,

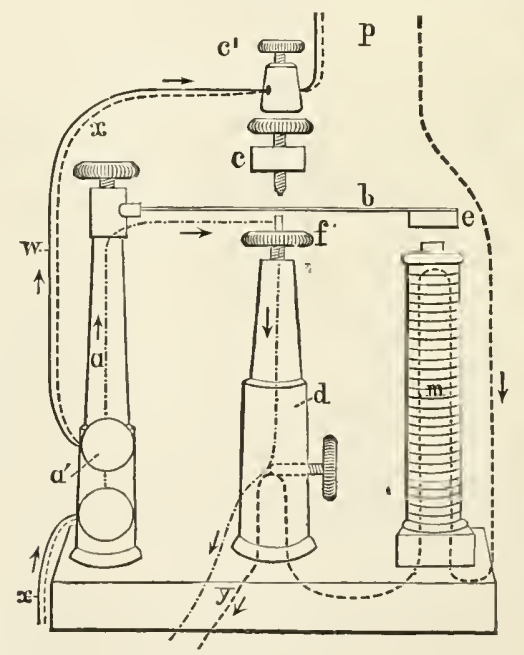

Fig. 6. The Magnetyc Interitipor with Helmholtz arrangement for equalizing the Make and Break Silocks.

the making and breaking shocks may be equalized. For this purpose the screw $c$ is raised out of reach of the excursions of the spring $b$, aud 
a moderately thick wire $w$, offering a certain amount only of resistance, is interposed between the upper binding screw $a^{\prime}$ on the pillar $a$, and the binding screw $c^{\prime}$ leading to the primary coil. Under these arrangewents the current frow the battery passes through $a^{\prime}$, along the interposed wire to $c^{\prime}$, through the primary coil and thus as before to $m$. As before, by the masnetization of $m, e$ is drawn down and $b$ brought in contact with $f$. As the result of this contact, the current from the battery can now pass by $a, f$, and $d$ (shewn by the thin interrupted line) back to the battery; but not the whole of the current, some of it can still pass along the wire $w$ to the primary coil, the relative amount being determined by the relative resistances offered by the two courses. Hence at each successive magnetization of $m$, the current in the primary coil does not entirely disappear when $b$ is brought in contact with $f$; it is only so far diminished that $m$ ceases to attract $e$, and hence by the release of $b$ from $f$ the whole current once more passes along $w$. Since at what corresponds to the 'break' the current in the primary coil is diminished only, not absolutely done away with, self-induction makes its appearance at the 'break' as well as at the 'make;' thus the 'breaking' and 'making' inducel currents or shocks in the secondary coil are equalized. They are both reduced to the lower efficiency of the 'making' shock in the old arrangenent; hence to produce the same strength of stimulus with this arrangement a stronger current must be applied or the seconlary coil pushed over the primary coil to a greater extent than with the otler arrangement.

\section{The Phenomena of a Simple Muscular Contraction.}

$\S 45$. If the far end of the nerve of a muscle-nerve preparation (Figs. 1 and 3 ) be laid on electrodes connected with the secondary coil of an induction-machine, the passage of a single inductionshock, which may be taken as a convenient form of an almost momentary stimulus, will produce no visible change in the nerve, but the muscle will give a twitch, a short, sharp contraction, - i.e., will for an instant shorten itself, becoming thicker the while, and then return to its previous condition. If one end of the muscle be attached to a lever, while the other is fixed, the lever will by its movements indicate the extent and duration of the shortening. If the point of the lever be brought to bear on some rapidly travelling surface, on which it leaves a mark (being for this purpose armed with a pen and ink if the surface be plain paper, or with a bristle or finely pointed piece of platinum foil if the surface be smoked glass or paper), so long as the muscle remains at rest the lever will describe an even line, which we may call the base line. If however the muscle shortens, the lever will rise above the base line and thus describe some sort of curve above the base line. Now, 
it is found that when a single induction-shock is sent through the nerve the twitch which the muscle gives causes the lever to describe some such curve as that shewn in Fig. 7 ; the lever (after a brief interval immediately succeeding the opening or shutting the key, of which we shall speak presently) rises at first rapidly but afterwards more slowly, shewing that the muscle is corlespondingly shortening, then ceases to rise, shewing that the muscle is ceasing

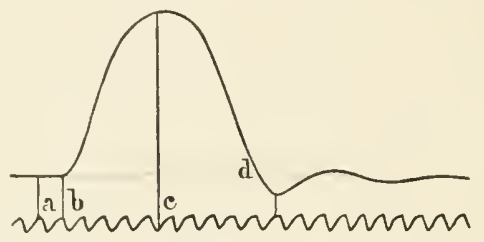

Fig. 7. A Muscle-curve from the Gastrocxemils of the Frog.

This curve, like all succeeding ones, unless otherwise indicated, is to be read from left to right, - that is to say, while the lever and tuning-fork were stationary the recording surface was travelling from right to left.

$a$ indicates the moment at which the induction-shock is sent into the nerve; $b$ the commencement, $c$ the maximum, and $d$ the close of the contraction.

Below the muscle-curve is the curve drawn by a tuning-fork making 100 double vibrations a second, each complete curve representing therefore one-hundredth of a second.

to grow shorter; then descends, shewing that the muscle is lengthening again; and finally, sooner or later, reaches and joins the base line, shewing that the muscle after the shortening has regained its previous natural length. Such a curve described by a muscle during a twitch or simple muscular contraction, caused by a single induction-shock or by any other stimulus producing the same effect, is called a curve of a simple inuscular contraction or, more shortly, a "muscle-curve." It is obvious that the exact form of the curve described by identical contractions of a muscle will depend on the rapidity with which the recording surface is travelling. Thus if the surface be travelling slowly the up-stroke corresponding to the shortening will be very abrupt and the down-stroke also very steep, as in Fig. 8, which is a curve from a gastrocnemius muscle of a frog, taken with a slowly moving drum, the tuning-fork being the same as that used in Fig. 7 ; indeed with a very slow movement, the two may be hardly separable from each other. On the other hand, if the surface travel very rapidly the curve may be immensely long drawn out, as in Fig. 9, which is a curve from a gastrocnemius muscle of a frog, taken with a very

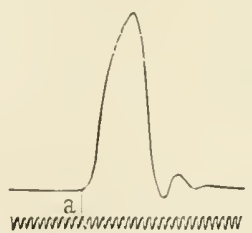

TIG. 8. rapidly moving. pendulum myograph, the tuning-fork marking about 500 vibrations a second. On examination, however, it will 
be found that both these extreme curves are fundamentally the same as the medium one, when account is taken of the different rapidities of the travelling surface in the several cases.

In order to make the 'muscle-curve' complete, it is necessary to mark on the recording surface the exact time at which the induction-shock is sent into the nerve, and also to note the speed at which the recording surface is travelling.

In the pendulum myograph the rate of movement can be calculated from the length of the pendulum; but even in this it is convenient, and in the ease of the spring myograph and revolving cylinder is necessary, to measure the rate of niovement directly by means of a vibrating tuning-fork or of some body vibrating regularly. Indeed it is best to make such a direct measurement with each curve that is taken.

A tuning-fork, as is known, vibrates so many times a second according to its pitch. If a tuningfork, armed with a light marker on one of its prongs and vibrating say 100 a second, - i.e., executing a double vibration, moving forwards and backwards, 100 times a second, - be brought while vibrating to make a tracing on the recording surface inmediately below the lever belonging to the muscle, we can use the curve or rather curves described by the tuning-fork to measure the duration of any part or of the whole of the muscle-curve. It is essential that at starting the point of the marker of the tuning-fork should be exactly underneath the marker of the lever, or rather, since the point of the lever as it moves up and down describes not a straight line but an are of a cirele of which its fulcrum is the centre and itself (from the fulcrum to the tip of the marker) the radius, that the point of the marker of the tuning-fork should be exactly on the arc described by the marker of the lever, either above or below it, as may prove most convenient. If then at starting the tuning-fork marker be thus on the are of the lever marker, and we note on the curve of the tuning-fork the place where the are of the lever cuts it at the beginning and at the end of the muscle-curve, as at Fig. 7 , we can count the number of vibrations of the tuning-fork which have taken place between the two marks, and so ascertain the whole time of the muscle-curve; if for instance there have been 10 double vibrations, each

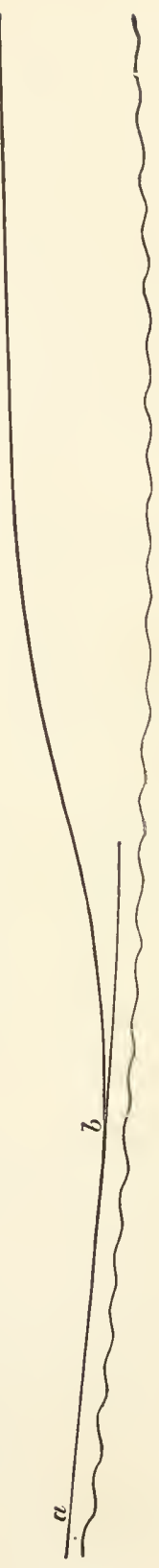

FIG. 9. 


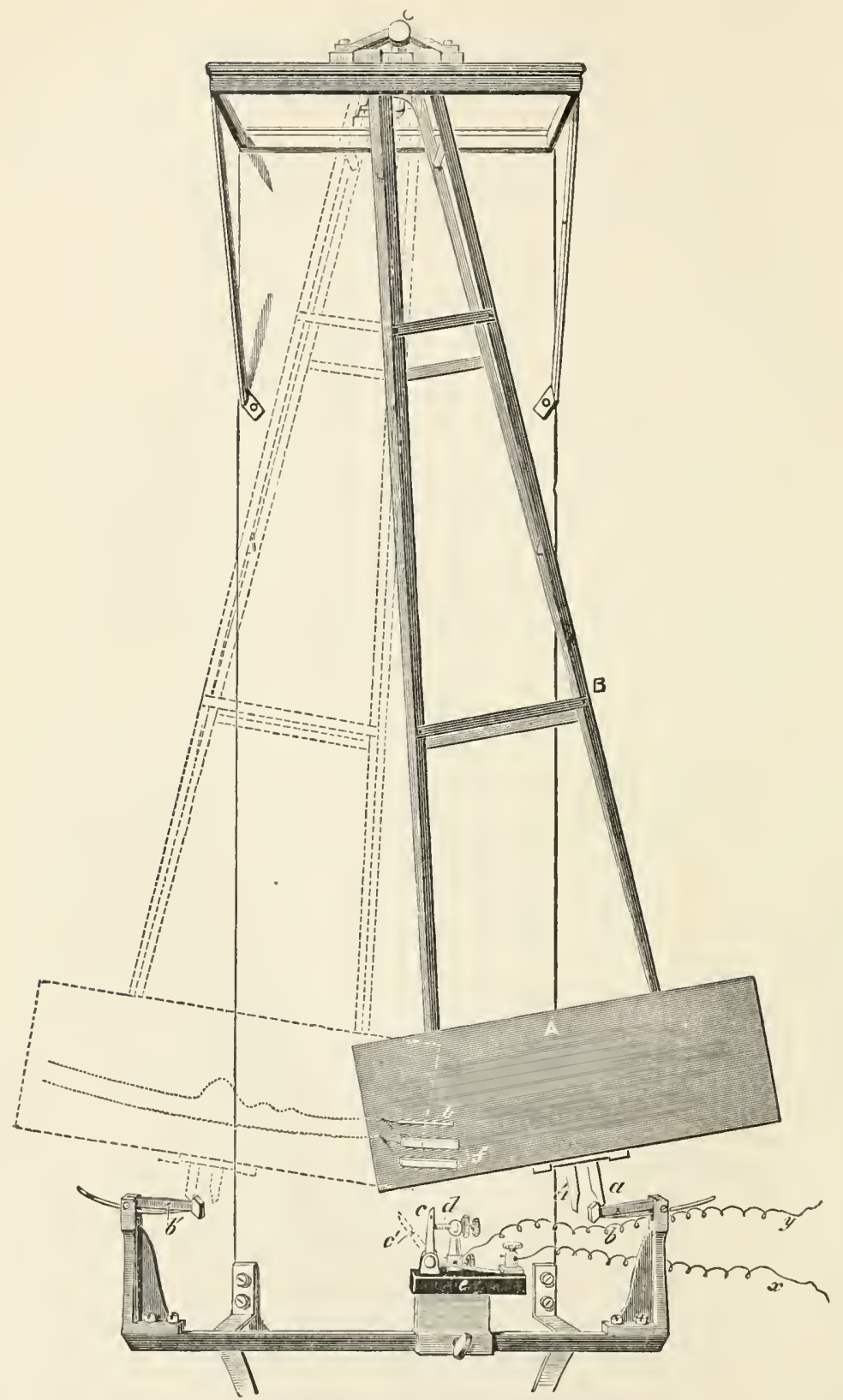

Fig 10. The Pendeluy Mrograpit.

The figure is diagrammatic, the essentials only of the instrument being shewn. The smoked glass plate $A$ swings with the pendulum $B$ on carefully arljusted 
bearings at $C$. The contrivances by which the glass plate can be removed and replaced at pleasure are not shewn. A second glass plate so arranged that the first glass plate may be noved up and down withont altering the swing of the pentulum is also omittert. l3efore commencing an experiment the pendulum is raiserk up (in the figure to the right), and is kept in that position by the tooth $a$ catching on the spring-catch $b$. Un depressing the catch $b$ the glass plate is set free, swings into the new position inclicated by the dotted lines, and is held in that position by the tooth $a^{\prime}$ catching on the catch $b^{\prime}$. In the conrse of its swing the tooth $a^{\prime}$ coming into contact with the projecting steel rork $c$, knocks it on one side into the position indicated by the dotterl line $c^{\prime}$. 'The rod $c$ is in electric continuity with the wire $x$ of the primary coil of an indnction-machine. 'The screw $d$ is similarly in electric continnity with the wire $y$ of the same primary coil. The serew $d$ and the rod $c$ are armed with platinum at the points in which they are in contact, and both are insulated by means of the ebonite block $e$. As long as $c$ and $d$ are in contact the circuit of the primary coil to which $x$ and $y$ belong is closed. When in its swing the tooth $a^{\prime}$ knocks $c$ away from $d$, at that instant the circuit is broken, ant a 'breaking' shock is sent through the electrodes connected with the secondary coil of the machine, and so through the nerve. The lever /, the end only of which is shewn in the figure, is brought to bear on the glass plate, and when at rest describes a straight line, or more exactly an arc of a circle of large radius. 'The tuniug-fork $f$, the ends only of the two limbs of which are shewn in the figure placer immediately below the lever, serves to mark the time.

occupying $\frac{1}{1} \frac{1}{0}$ sec., the whole curve has taken $\frac{1}{10} \mathrm{sec}$. to make. In the same way we can measure the duration of the rise of the curve or of the fall, or of any part of it.

Though the tuning-fork may, by simply striking it, be set going long enough for the purposes of an observation, it is convenient to keep it going by means of an electric current and a magnet, very much as the spring in the 'magnetic interruptor' (Fig. 5) is kept going.

It is not necessary to use an actual tuning-fork; any rod, armed with a marker, which can be made to vibrate regularly, and whose time of vibration is known, may be used for the purpose ; thus a reed, made to vibrate by a blast of air, is sometimes employed.

The exact moment at which the induction-shock is thrown into the nerve may be recorded on the muscle-curve by means of a 'signal,' which may be applied in various ways.

A light steel lever armed with a marker is arranged over a small coil by means of a light spring in such a way that when the coil by the passage of a current through it becomes a magnet it pulls the lever down to itself; on the current being broken, and the magnetization of the coil ceasing, the lever by help of the spring flies up. The marker of such a lever is placed immediately under (i.e., at some point on the arc described $\mathrm{by}$ ) the marker of the muscle (or other) lever. Hence by making a current in the coil and putting the signal lever down, or by breaking an already existing current, and letting the signal lever fly up, we can make at pleasure a mark corresponding to any part we please of the muscle (or other) curve.

If in order to magnetize the coil of the signal, we use, as we may do, the primary current which generates the induction-shock, the breaking or making of the primary current, whichever we use to produce the 
induction-shock, will make the signal lever fly up or come lown. Hence we shall have on the recording surface, under the muscte, a mark indicating the exact moment at which the primary current was broken or made. Now, the time taken up by the generation of the induced current and its passage into the nerve between the electrodes is so infinitesimally small, that we may, without appreciable error, take the moment of the breaking or making of the primary current as the moment of the entrance of the induction-shock into the nerve. Thus we can mark below the muscle-curve, or, by describing the are of the muscle lever, on the uniscle-curve itself, the exact moment at which the induction-shock falls into the nerve between the electrodes, as is done at $a$ in Figs. 7, 8, 9.

In the pendulum inyograph a separate signal is not needed. If, having placed the muscle lever in the position in which we intend to make it record, we allow the glass plate to descend until the tooth $a^{\prime}$ just touches the rod $c$ (so that the rod is just about to be knocked down, and so break the primary cireuit) and make on the base line, which is meanwhile being lescribed by the lever marker, a mark to indicate where the point of the marker is under these circumstances, and then bring back the plate to its proper position, the mark which we have niale will mark the moment of the breaking of the primary eircuit and so of the entrance of the induction-shock into the nerve. For it is just when, as the glass plate swings down, the marker of the lever comes to the mark which we have made that the rod $c$ is knocked back and the primary current is broken.

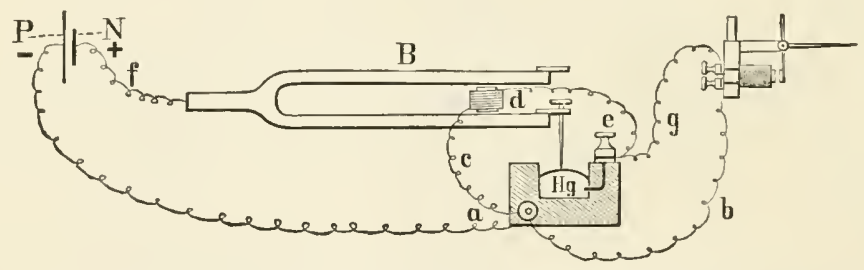

Fig. 11. Diagram of an Arrangement of a Vibrating Tening-fork witil a Degrerez Signal.

The current flows along the wire $f$ connected with the positive $(+)$ pole or end of the negative plate $(N)$ of the lattery, through the tuning-fork, down the pin connected with the end of the lower prong, to the mercury in the cup $H_{g}$, and so by a wire (shewn in the figure as a black line bent at right angles) to the binding screw $e$. From this binding screw part of the current flows through the coil $d$ between the prongs of the tuning-fork, and thence by the wire $c$ to the binding serew $a$, while another part flows through the wire $q$, through the coil of the Desprez signal back by the wire $b$, to the binding screw $a$. From the binding screw $a$ the current passes back to the negative $(-)$ pole or end of the positive element $(P)$ of the battery. As the current flows through the coil of the Desprez signal from $g$ to $b$, the core of coil becoming magnetized draws to it the marker of the signal. As the current flows through the coil $d$, the rore of that coit. also beconing magnetized, draws up the lower prong of the fork. But the pin is so arjusted that the drawing up of the prong lifts the point of the pin ont of the mercury. In consequence, the current, being thus broken at $1 / q$, flows neither through $d$ nor through the Jesprez signal. In consequence, the core of the Desprez thus ceasing to be magnetized, the marker flies back, being usually assisted by a 
spring (not shewn in the figure). But, in consequence of the current ceasing to flow through $d$, the core of $d$ ceases to lift up the prong, and the pin, in the descent of the prong, makes contact once more with the mercury. 'The re-establishment of the enrent, however, once nore acting on the two coils, again pulls upon the marker of the signal, and again, by magnetizing the core of $d$, pulls up the prong and once more breaks the current. 'Thus the current is contiuually made and broken, the rapidity of the interruptions being determined by the vibration periods of the tuning-fork, and the lever of the signal rising and falling synchronously with the movements of the tuning-fork.

A 'signal' like the above, in an improved form known as Desprez's, may be used also to record time, and thus the awkwardness of bringing a large tuning..fork up to the recording surface obviated: For this purpose the signal is introluced into a circuit, the current of which is continually being male and broken by a tuming-fork (Fis. 10). The tuning-fork, once set vibrating, continues to make and break the current at each of its vibrations, and, as stated above, is kept vibrating by the current. But each make or break caused by the tuming-fork affects also the small coil of the signal, causing the lever of the signal to fitl down or fly up. Thus the signal describes vibration curves synehronous with those of the tuning-fork driving it. The signal may similarly be worked by means of vibrating agents other than a tuning-fork.

Various recording surfaces may be used. The form most generally useful is a cylinder covered with smoked paper, and made to revolve by clockwork or otherwise; such a cylinder driven by clockwork is shewn in Fig. 3, B. By using a cylinder of large ralins with adequate gear, a high speer, some inches for instance in a seconcl, can be obtained. In the spring myograph a smoked glass plate is thrust rapilly forward along a groove, by means of a spring suldenly thrown into action. In the pendulum myograph, Fig. 9, a smoked glass plate attached to the lower ent of a long frame, swinging like a pendulum, is suddenly let so at a certain height, and so swings rapilly through an are of a circle. The disadvantage of the last two methods is that the surface travels at a continnally changing rate, whereas, in the revolving eylinder, careful construction and adjustment will secure a very uniform rate.

$\$$ 46. Having thus obtained a time record, and an indication of the exact moment at which the induction-shock falls into the nerve, we may for present purposes consider the muscle-curve complete. The study of such a curve, as for instance that shewn in Fig. 7, taken from the gastrocnemius of a frog, teaches us the following facts :

1. That although the passage of the induced current from electrode to electrode is practically instantaneous, its effect, measured from the entrance of the shock into the nerve to the return of the muscle to its natural length after the shortening, takes an appreciable time. In the figure, the whole curve from $a$ to $d$ takes up about the same time as eleven double vibrations of the tuning-fork. Since each double vibration here represents 100 th of a second, the duration of the whole curve is rather more than $\frac{1}{1} \overline{0}$ sec. 
2. In the first portion of this period, from a to $b$, there is no visible change, no raising of the lever, no shortening of the musele.

3. It is not until $b$ - that is to say, after the lapse of about $\frac{1}{10}$ sec. - that the shortening begins. The shortening as shewn by the curve is at first slow, but soon becomes more rapid, and then slackens again until it reaches a maximum at $c$; the whole shortening occupying rather more than $\frac{4}{10} \mathrm{sec}$.

4. Arrived at the maximum of shortening, the muscle at once begins to relax, the lever descending at first slowly, then more rapidly, and at last more slowly again, until at $d$ the muscle has regained its natural length; the whole return from the maximum of contraction to the natural length occupying rather more than $\overline{1} \frac{5}{0}$ sec.

Thus a simple muscular contraction, a simple spasm or twitch, produced by a momentary stimulus, such as a single inductionshock, consists of three main phases:-

1. A phase antecedent to any visible alteration in the muscle. This phase, dming which invisible preparatory changes are taking place in the nerve and muscle, is called the 'latent period.'

2. A phase of shortening, or, in the more strict meaning of the word, contraction.

3. A phase of relaxation or return to the original length.

In the case we are considering, the electrodes are supposed to be applied to the nerve at some distance from the muscle. Consequently the latent period of the curve comprises not only the preparatory actions which may be going on in the muscle itself, but also the changes necessary to conduct the inmediate effect of the induction-shock, from the part of the nerve between the electrodes along a considerable length of nerve down to the muscle. It is obvious that these latter changes might be eliminated by placing the electrodes on the muscle itself, or on the nerve close to the muscle. If this were done, the muscle and lever being exactly as before, and care were talien that the induction-shock entered into the nerve at the new spot, at the moment when the point of the lever had reached exactly the same point of the travelling surface as before, two curves would be gained having the relations shewn in Fig. 12. The two curves resemble each other in almost all points, except that in the curve taken with the shorter piece of nerve, the latent period, the distance $a$ to $b$ as compared with the distance $a$ to $b^{\prime}$ is shortened: the contraction begins rather earlier. A study of the two curves teaches us the following two facts : -

1. Shifting the electrodes from a point of the nerve at some distance from the muscle to a point of the nerve close to the muscle, has only shortened the latent period a very little. Even when a very long piece of nerve is taken, the difference in the two curves is very small, and, indeed, in order that it may be clearly recognized or measured, the travelling surface must be made to 
travel very rapidly. It is obvious, therefore, that by far the greater part of the latent period is taken up by changes in the muscle

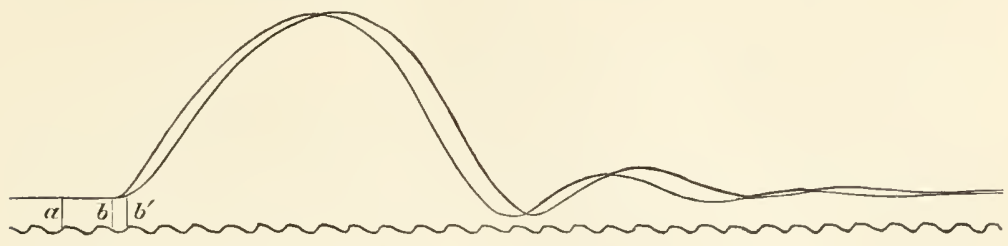

Fig. 12. Curves illestratixg the Meastrenest of the Velocity of a Nervols LMPULSE.

The same muscle-nerve preparation is stimulated ( 1 ) as far as possible from the muscle, (2) as near as possible to the mnscle; both contractions are registered in exactly the same way.

In (1), the stimulus enters the nerve at the time indicated by the line $a$, the contraction begins at $b^{\prime}$; the whole latent period therefore is indicated by the distance from $a$ to $b^{\prime}$.

In (2), the stimulus enters the nerve at exactly the same time $a$; the contraction begins at $b$ : the latent period therefore is indicated by the distance between $a$ and $b$.

The time taken up by the nervous impulse in passing along the length of nerve between 1 and 2 is therefore indicated by the distance hetween $b$ and $b^{\prime}$, which may be measured hy the tuning-fork curve below each double vibration of the tuningfork corresponds to $\frac{1}{1 \frac{1}{2} 0}$ or 0083 sec.

itself, changes antecedent to the shortening becoming actually visible. Of course, even when the electrodes are placed close to the muscle, the latent period includes the changes going on in the short piece of nerve still lying between the electrodes and the muscular fibres. To eliminate this with a view of determining the latent period in the muscle itself, the electrodes might be placed directly on the muscle poisoned with urari. If this were done, it would be found that the latent period remained about the same, - that is to say, that in all cases the latent period is chiefly taken up by changes in the muscular as distinguished from the nervous elements.

2. Such difference as does exist between the two curves in the figure, indicates the time taken up by the propagation, along the piece of nerve, of the changes set up at the far end of the nerve by the induction-shock. These changes we have already spoken of as constituting a nervous impulse; and the above experiment shews that it takes a small but yet distinctly appreciable time for a nervous impulse to travel along a nerve. In the figure the difference between the two latent periods, the distance between $b$ and $b^{\prime}$, seems almost too small to measure accurately; but if a long piece of nerve be used for the experiment, and the recording surface be made to travel very fast, the difference between the duration of the latent period when the induction-shock is sent in at a point close to the muscle, and that when it is sent in at a point as far away as possible from the muscle, may be satisfactorily measured in fractions of a second. If the length of nerve between 
the two points be accurately measured, the rate at which a nervous impulse travels along the nerve to a muscle can thus be easily calculated. This has been found to be in the frog about 28 , and in man about 33 metres per second, but varies considerably, especially in warm-blooded animals.

Thus when a momentary stimulus, such as a single inductionshock, is sent into a nerve connected with a muscle, the following events take place: a nervons impulse is started in the nerve, and this travelling down to the muscle produces in the inuscle first the invisible changes which occupy the latent period, secondly the changes which bring about the visible shortening or contraction proper, and thirdly the changes which bring about the relaxation and return to the original length. The changes taking place in these several phases are changes of living matter: they vary with the condition of the living substance of the muscle, and only take place so long as the muscle is alive. Though the relaxation which brings back the muscle to its original length is assisted by the muscle being loaded with a weight, or otherwise stretched, this is not essential to the actual relaxation, and with the same load the return will vary according to the condition of the muscle; the relaxation must be considered as an essential part of the whole contraction, no less than the shortening itself.

$\$ 47$. Not only, as we shall see later on, does the whole contraction vary in extent and character according to the condition of the muscle, the strength of the indnetion-shock, the load which the muscle is bearing, and various attendant circumstances, but the three phases may vary independently. The latent period may be longer or shorter, the shortening may take a longer or shorter time to reach the same height, and especially the relaxation may be slow or rapid, complete or imperfect. Even when the same strength of induction-shock is used, the contraction may he short and sharp, or very long drawn out, so that the curves described on a recording surface, travelling at the same rate in the two cases, appear very different; and, under certain circumstances, as when a muscle is fatigued, the relaxation, more particularly the last part of it, may be so slow, that it may be several seconds before the muscle really regains its original length. We may add that the latent period, which in an ordinary experiment on a frog's gastrocnemius is so conspicuous, may, under certain circumstances, be so shortened as almost, if not wholly, to disappear. Indeed, it is maintained by some that the occurrence of the latent period is not an essential feature of the whole act.

Hence, if we say that the duration of a simple muscular contraction of the gastrocnemins of a frog under ordinary circumstances is about $\frac{1}{10}$ sec., of which $\frac{1}{10} \overline{0}$ is taken up by the latent period, $\frac{4}{10} \overline{0}$ by the contraction, and $\frac{5}{10}$ by the relaxation, these must be taken as 'round numbers,' stated so as to be easily remembered. The duration of each phise as well as of the whole contraction varies in 
different animals, in different muscles of the same animal, and in the same muscle under different conditious.

The muscle-curve which we have been discussing is a curve of changes in the length only of the muscle; but if the muscle, instead of being suspended, were laid that on a glass plate, and a lever laid over its belly, we should find, upon sending an induction-shock into the nerve, that the lever was raised, shewing that the muscle during the contraction became thicker. And if we took a graphic record of the movements of the lever, we should obtain a curve very similar to the one just discussed; after a latent period the lever would rise, shewing that the muscle was getting thicker, and afterwards wonld fall, shewing that the muscle was becoming thin again. In other words, in contraction the lessening of the muscle lengthwise is accompanied by an increase crosswise; indeed, as we shall see later on, the muscle in contracting is not diminisied in bulk at all (or only to an exceedingly small extent, about $\frac{1}{10} \overline{0} \overline{0}$ of its total bulk), but nakes up for its diminution in length by increasing in its other dianeters.

$\$$ 48. A single iuduction-shock is, as we have said, the most couvenient form of stimulus for producing a simple muscular contraction, but this may also be obtained by other stimuli, provided that these are sufficiently sudden and short in their action, as, for iustance, by a prick of, or sharp blow on, the nerve or muscle. For the production of a single, simple unuscular contraction, the changes in the nerve leading to the muscle must be of such a kind as to constitute what may be called a single nervous impulse, and any stimulus which will evoke a single nervous impulse only may be used to produce a simple muscular contraction.

As a rule, however, most stimuli other than single inductionshocks tend to produce in a nerve several nervous impulses, and, as we shall see, the nervous impulses which issue from the central nervous system, and so pass along nerves to muscles, are, as a rule, not single and simple, but complex. Hence, as a matter of fact, a simple muscular contraction is within the living body a comparatively rare event (at least as far as the skeletal muscles are concerned,) and cannot easily be produced outside the body otherwise than by a'single induction-shock. The ordinary form of muscular contraction is not a simple muscular contraction, but the more complex form known as a tetanic contraction, to the study of which we must now turn.

\section{Tetanic Contructions.}

\$49. If a single induction-shock be followed at a certain interval by a second shock of the same strength, the first simple contraction will be followed by a second simple contraction, both 
contractions being separate and distinct; and, if the shocks be repeated, a series of rhythmically-recurring, separate, simple contractions may be obtained. If, however, the interval between two shocks be made short, - if, for instance, it be made only just long enough to allow the first contraction to have passed its maximum before the latent period of the second is over, - the curves of the two contractions will bear some such relation to each other as that shewn in Fig. 13. It will be observed that the second curve is almost in all respects like the first, except that it starts, so to speak, from the first curve instead of from the base-line. The second nervous impulse has acted on the already contracted muscle, and made it contract again just as it would have done if there had been no first impulse, and the muscle had been at rest. The two contractions are added together, and the lever is raised nearly double the height it would have been by either alone. If in the same way a third shock follows the second at a sufficiently

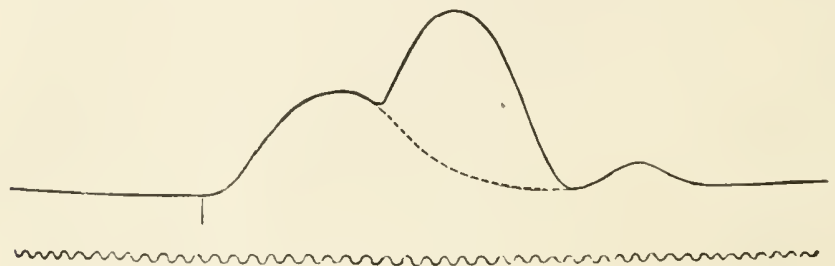

Fig. 13. Tracisg of a Dotble Mrsclectrre.

While the muscle (gastrocuenius of frog) was engaged in the first contraction (whose complete course, had nothing intervenerl, is indirated by the dotted line), a second induction-shock was thrown in, at such a tine that the second contraction began just as the first was beginning to decliue. 'The second curve is seen to start from the first, as does the first from the base-line.

short interval, a third curve is piled on the top of the second; the same with a fourth, and so on. A more or less similar result would occur if the second contraction began at another phase of the first. The combined effect is, of course, greatest when the second contraction begins at the maximum of the first, being less both before and afterwards.

Hence, the result of a repetition of shocks will depend largely on the rate of repetition. If, as in Fig. 14, the shocks follow each other so slowly that one contraction is over, or almost over, before the next begins, each contraction will be distinct, or nearly distinct, and there will be little or no combined effect.

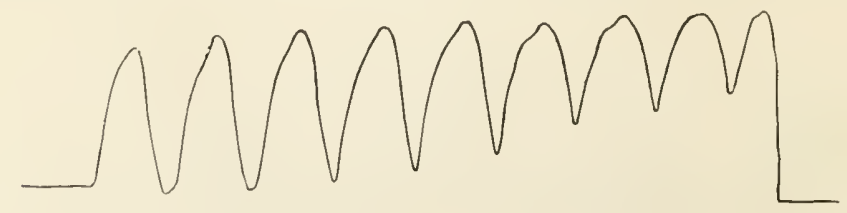

Fig. 14. Musfle-curve. Single Inifethon-shock repeaten showly. 
If, however, the shocks be repeated more rapidly, as in Fig. 15, each succeeding contraction will start from some part of the preceding one, and the lever will be raised to a greater height at each contraction.

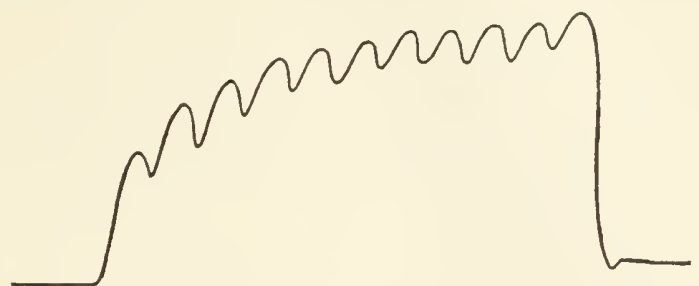

Fig. 15. Mtescle-curve. Single Indection-shock Repeated more Rapidey.

If the frequency of the shocks be still further increased, as in Fig. 16, the rise due to the combination of contraction will be still more rapid, and a snaller part of each contraction will be visible on the curve.

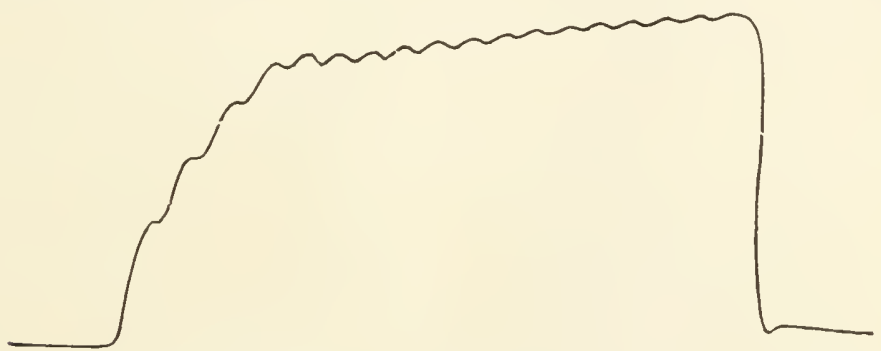

Fig, 16. Muscle-curve. Single Induction-shock Repeated still hore Rapider.

In each of these three curves it will be noticed that the character of the curve changes somewhat during its development. The change is the result of commencing fatigue, caused by the repetition of the contractions, the fatigue manifesting itself by an increasing prolongation of each contraction, shewn especially in a delay of relaxation, and by an increasing diminution in the height of the contraction. Thus in Fig. 14 the contractions, quite distinct at first, become fused later ; the fifth contraction, for instance, is prolonged so that the sixth begins before the lever has reached the base line; yet the summit of the sixth is hardly higher than the summit of the fifth, since the sixth, though starting at a higher level, is a somewhat weaker contraction. So, also, in Fig. 15, the lever rises rapidly at first, but more slowly afterwards, owing to an increasing diminution in the height of the single contractions. In Fig. 16 the increment of rise of the curve due to each contraction diminishes very rapidly, and though the lever does continue to 
rise during the whole series, the ascent, after about the sixth contraction, is very gradual indeed, and the indications of the individual eontractions are much less marked than at first.

Hence, when shocks are repeated with snfficient rapidity, it results that, after a certain number of shoeks, the sueceeding impulses do not cause any further shortening of the muscle, any further raising of the lever, but merely keep up the contraction ahready existing. The curve thus reaches a maximum, which it maintains, subject to the depressing effects of exhaustion, so long as the shocks are repeated. When these ctase to be given, the muscle returns to its natural length.

When the shocks succeed each other still more rapidly than in Fig. 16, the individual contractions, visible at first, may beeome fused together and wholly lost to view in the latter part of the curve. When the shocks succeed each other still more rapidly (the second contraction beginning in the ascending portion of the first), it becomes difficult or impossible to trace out any of the single contractions. ${ }^{1}$ The curve then described by the lever is of the kind shewn in Fig. 17, where the primary current of an

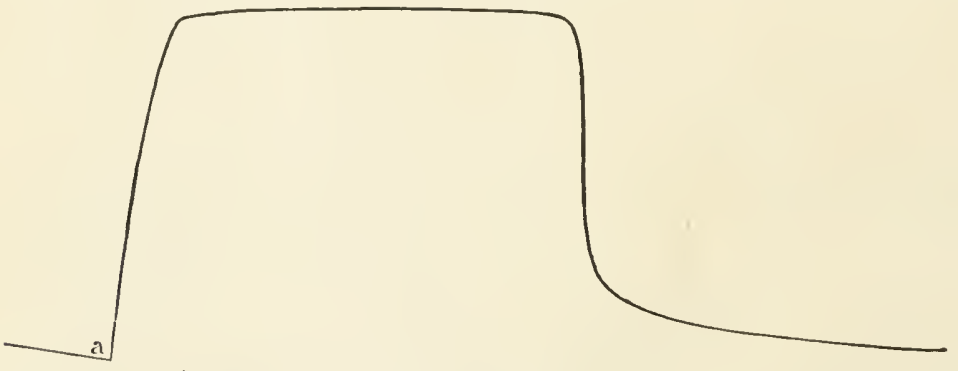

Fig. 17. Tetanus prondeen with the ordinary Magnetic Interruptor of an INIDCTION-M ACInE. (Recording surface travelling slowly.)

The interrupted current is thrown in at $a$.

induction-machine was rapidly made and broken by the magnetic interruptor, Fig. 4. The lever, it will be observed, rises at $t$ (the recording surface is travelling too slowly to allow the latent period to be distinguished), at first very rapidly, — in fact, in an unbroken and almost a vertical line, — and so very speedily reaches the maxi$111 \mathrm{~m}$, which is maintained so long as the shocks continue to be given; when these cease to be given, the curve descends, at first very rapidly, and then more and more gradually towards the base line, which it reaches just at the end of the figure.

This condition of muscle, bronght about by rapidly repeated shocks, this fusion of a number of simple twitches into an

1 The ease with which the individual contractions can be made out depenis in part, it need hardly be said, on the rapidity with which the recording surface travels. 
apparently smooth, continuous effort, is known as tetanus, or tctanic contraction. The above facts are most clearly shewn when induction-shocks, or at least galvanic currents in some, form or other, are employed. They are seen, however, whatever be the form of stimulus employed. Thus, in the case of mechanical stimuli, while a single quick blow may cause a single twitch, a pronounced tetanus may be obtained by rapidly striking successively fresh portions of a nerve. With chemical stimulation, as when a nerve is dipped in acid, it is impossible to secure a monentary application; hence tetanus, generally irregular in character, is the normal result of this mode of stimulation. In the living body, the contractions of the skeletal muscles, brought about either by the will or otherwise, are generally tetanic in character. Even very short, sharp movements, such as a sudden jerk of a limb, or a wink of the eyelid, are, in reality, examples of tetanus of short duration.

If the lever, instead of being fastened to the tendon of a muscle hung vertically, be laid across the belly of a muscle placed in a horizontal position, and the muscle be thrown into tetanus by a repetition of induction-shocks, it will be seen that each shortening of the muscle is accompanied by a corresponding thickening, and that the total shortening of the tetanus is accompanied by a corresponding total thickening. And, indeed, in tetanus we can ohserve more easily than in a single contraction that the muscle in contracting changes in form only, not in bulk. If a living muscle, or group of muscles, be placed in a glass jar, or chamber, the closed top of which is prolonged into a narrow glass tube, and the chamber be so filled with water (or, preferably, with a solution of sodium chloride, 6 p. c. in strength, the " normal saline solution," which is less injurious to the tissue than simple water) that the fluid rises up into the narrow tube. it is obvious that any change in the bulk of the muscle will be easily shewn by a rising or falling of the column of fluid in the narrow tube. It is found that when the muscle is made to contract, even in the most forcible manner, the change of level in the height of the column which can be observed is practically insignificant: there appears to be a fall indicating a diminution of bulk to the extent of about one ten-thousandth of the total bulk of the muscle. So that we may fairly say that in a tetanus, and hence in a simple contraction, the lessening of the length of the muscle causes a corresponding increase in the other directions: the substance of the muscle is displaced not diminished.

50 . So far we have spoken simply of an induction-shock, or of induction-shocks, without any reference to their strength, and of a living or irritable muscle, without any reference to the degree or extent of its irritability; but induction-shocks may vary in strength, and the irritability of the muscle may vary.

If we slide the secondary coil a long way from the primary 
coil, and thus make use of extremely feeble induction-shocks, we shall probobly find that these shocks, applied even to a quite fresh muscle-nerve preparation, produce no contraction. If we then gradually slide the secondary coil nearer and nearer the primary coil, and keep on trying the effects of the shocks, we shall find that, after a while, in a certain position of the coils, a very feeble contraction makes its appearance. As the secondary coil comes still nearer to the primary coil, the contractions grow greater and greater. After a while, however, and that, indeed, in ordinary circumstances very speedily, increasing the strength of the shock no longer increases the height of the contraction; the maximnm contraction of which the muscle is capable with such shocks however strong has been reached.

If we use a tetanizing or interrupted current, we shall obtain the same general results; we may, according to the strength of the current, get no contraction at all, or contractions of various extent up to a maximum, which cannot be exceeded. Under favourable conditions the maximum contraction may be very considerable: the shortening in tetanus may amount to three-fifths of the total length of the muscle.

The amount of contraction then depends on the strength of the stimulus, whatever be the stimulus; bnt this holds good within certain limits only; to this point however we shall return later on.

$\$ 51$. If, having ascertained in a perfectly fresh muscle-nerve preparation the amount of contraction produced by this and that strength of stimulus, we leave the preparation by itself for some time, say for a few hours, and then repeat the observations, we shall find that stronger stimuli, stronger shocks, for instance, are required to produce the same amonnt of contraction as before; that is to say, the irritability of the preparation, the power to respond to stimuli, has in the meanwhile diminished. After a further interval, we should find the irritability still further diminished: even very strong shocks would be unable to evole contractions as large as those previonsly cansed by weak shocks. At last we should find that no shocks, no stimuli, however strong, were able to produce any visible contraction whatever. The amount of contraction, in fact, evoked by a stimulus depends not only on the strength of the stimulns but also on the degree of irritability of the muscle-nerve preparation.

Immediately upon removal from the body, the preparation possesses a certain amount of irritability, not differing very materially from that which the muscle and nerve possess while within, and forming an integral part of the body; bnt after removal from the body the preparation loses irritability, the rate of loss heing dependent on a variety of circumstances; and this goes on until, since no stimulus which we can apply will give rise to a contraction, we say the irritability has wholly disiln!eared. 
We might take this disappearance of irritability as marking the death of the preparation, but it is followed sooner or later by a curious change in the muscle, which is called rigor mortis, and which we shall study presently; and it is convenient to regard this rigor mortis as marking the death of the muscle.

The irritable muscle, then, when stimulated either directly, the stimulus being applied to itself, or indirectly, the stimulus being applied to its nerve, responds to the stimulus by a change of form which is essentially a shortening and thickening. By the shortening (and thickening), the muscle in contracting is able to do work, to move the parts to which it is attached; it thus sets free energy. We have now to study more in detail how this energy is set free and the laws which regulate its expenditure. 


\title{
SEC. 2. ON THE CHANGES WHICH TAKE PLACE IN A MUSCLE DURING A CONTRACTION.
}

\author{
The Change in Form.
}

\$52. An ordinary skeletal muscle consists of elementary muscle fibres, bound together in variously arranged bundles by connective tissue which carries blood vessels, nerves and lymphatics.

The contraction of a muscle is the contraction of all or some of its elementary fibres, the connective tissue being passive; hence while those fibres of the muscle which end directly in the tendon, in contracting pull directly on the tendon, those which do not so end pull indirectly on the tendon by means of the connective tissue between the bundles, which connective tissue is continuous with the tendon.

Each muscle is supplied by one or more branches of nerves which, running in the connective tissue, divide into smaller branches and twigs between the bundles and between the fibres, and eventually end in such a way that every muscular fibre is supplied with at least one nerve fibre, which joins the muscular filme somewhere about the middle between its two ends or sometimes nearer one end, in a special nerve ending called an end-plate. It follows that when a muscular fibre is stimulated by means of a nerve fibre, the nervous impulse travelling down the nerve fibre falls into the muscular fibre not at one end but at about its middle; it is the middle of the fibre which is affected first by the nervous impulse, and the changes in the muscular substance started in the middle of the muscular fibre travel thence to the two ends of the fibre. In an ordinary skeletal muscle however, the fibres and bundles of fibres begin and end at different distances from the ends of the muscle, and the nerve or nerves going to the muscle divide and spread out in the muscle in such a way that the end-plates, in which the indivilual fibres of the nerve end, are distributed widely over the muscle at very different 
distances from the ends of the muscle. Hence, if we suppose a single nervous impulse, such as that generated by a single induction-shock, or a series of such impulses to be started at the same time at some part of the trunk of the nerve in each of the fibres of the nerve going to the muscle, these impulses will reach very different parts of the muscle at about the same time and the contractions which they set going will hegin, so to speak, nearly all over the whole muscle at the same time, and will not all start in any particular zone or area of the muscle.

$\$ 53$. The wave of contraction. We have seen, however, that under the influence of curari the nerve fibre is unahle to excite contractions in a muscular fibre, although the irritability of the muscular fibre itself is retained. Hence, in a muscle poisoned by urari the contraction begins at that part of the muscular substance which is first affected by the stimulus, and we may start a contraction in what part of the muscle we please by properly placing the electrodes.

Some muscles, such for instance as the sartorins of the frog, though of some length are composed of fibres which run parallel to each other from one end of the muscle to the other. If such a muscle be poisoned with urari so as to eliminate the action of the nerves and stimulated at one end (an induction-shoek sent through a pair of electrodes placed at some little distance apart from each other at the end of the muscle may be employed, but better results are obtained if a mode of stimulation, of which we shall have to speak presently, viz. the application of the "constant current," be adopted), the contraction which ensues starts from the end stimulated, and travels thence along the muscle. If two levers be made to rest on, or be suspended from, two parts of such a muscle placed horizontally, the parts being at a known distance from each other and from the part stimulated, the progress of the contraction may be studied.

The movements of the levers indicate in this case the thickening of the fibres which is taking place at the parts on which the levers rest or to which they are attached; and if we take a graphic record of these movements, bringing the two levers to mark, one immediately below the other, we shall find that the lever nearer the part stimulated begins to move earlier, reaches its maximum earlier, and returns to rest earlier than does the farther lever. The contraction, started by the stimulus, in travelling along the muscle from the part stimulated reaches the nearer lever some little time before it reaches the farther lever, and has passed by the nearer lever some little time hefore it has passed by the farther lever; and the farther apart the two levers are the greater will be the difference in time between their movements. In other words the contraction travels along the muscle in the form of a wave, each part of the muscle in succession from the end 
stimulated swelling out and shortening as the contraction reaches it, and then returning to its original state. And what is true of the collection of parallel fibres which we call the muscle is also true of each fibre, for the swelling at any part of the muscle is only the sum of the swelling of the individual fibres; if we were able to take a single long fibre and stimulate it at one end, we should be able under the microscope to see a swelling or bulging accompanied by a corresponding shortening, i.e. to see a contraction sweep along the fibre from end to end.

If in the graphic record of the two levers just mentioned we count the number of vibrations of the tuning-fork which intervene between the mark on the record which indicates the beginning of the rise of the near lever (that is, the arrival of the contraction wave at this lever) and the mark which indicates the beginning of the rise of the far lever, this will give us the time which it has taken the contraction wave to travel from the near to the far lever. Let us suppose this to be 005 sec. Let us suppose the distance between the two levers to be $15 \mathrm{~mm}$. The contraction wave then has taken 005 sec. to travel $15 \mathrm{~mm}$., that is to say it has travelled at the rate of 3 meters per sec. And indeed we find by this, or by other methods, that in the frog's muscles the contraction wave does travel at a rate which may be put down as from 3 to 4 meters a second, though it varies under different conditions. In the warm blooded mammal the rate is somewhat greater, and may probably be put down at 5 meters a second in the excised nuscle, rising possibly to 10 meters in a muscle within the living body.

If again in the graphie record of the two levers we count, in the case of either lever, the number of vibrations of the tuningfork which intervene between the mark where the lever begins to rise and the mark where it has finished its fall and returned to the base line, we can measure the time intervening between the contraction wave reaching the lever, and leaving the lever on its way onward, that is to say, we can measure the time which it has taken the contraction wave to pass over the part of the muscle on which the lever is resting. Let us suppose this time to be say $\cdot 1$ sec. But a wave which is travelling at the rate of $3 \mathrm{~m}$. a second and takes 1 sec. to pass over any point must be $300 \mathrm{~mm}$. long. And indeed we find that in the frog the length of the contraction wave may be put down as varying from 200 to $400 \mathrm{~mm}$; and in the mammal it is not very different.

Now the very longest muscular fibre is stated to be at most only about $40 \mathrm{~mm}$. in length; hence, in an ordinary contraction, during the greater part of the duration of the contraction the whole length of the fibre will be occupied by the contraction wave. Just at the begimning of the contraction there will be a time when the front of the contraction wave has reached for 
instance only half-way down the fibre (supposing the stimulus to be applied, as in the case we have been discussing, at one end only), and just at the end of the contraction there will be a time for instance when the contraction has left the half of the fibre next to the stimulus, but has not yet cleared away from the other half. But nearly all the rest of the time every part of the fibre will be in some phase or other of contraction, though the parts nearer the stimulus will be in more advanced phases than the parts farther from the stimulus.

This is true when a muscle of parallel fibres is stimulated artificially at one end of the muscles, and when therefore each fibre is stimulated at one end. It is of course all the more true when a muscle of ordinary construction is stimulated by means of its nerve. The stimulus of the nervous impulse impinges, in this case, on the muscle fibre at the end-plate which, as we have said, is placed towards the middle of the fibre, and the contraction wave travels from the end-plate in opposite directions toward each end, and has accordingly only about half the length of the fibre to run in. All the more therefore must the whole fibre be in a state of contraction at the same time.

$\S 54$. We may now turn to the question, What takes place in a muscular fibre when a contraction wave sweeps over it?

Optical Changes. Although undoubtedly the optical features of a muscular fibre change while it is contracting, it is very difficult to make an authoritative statement as to what those changes are. In the first place a contraction wave, even when it is travelling with relative slowness, travels so rapidly that the individual features cannot be seized by the eye. We are confined to conclusions drawn from the study of short local contractions, local thickenings and shortenings which may be obtained in the living fibre and fixed by the action of osmic acid vapour or by other means; and it has to be assumed that these local bulgings give a true picture of a normal contraction wave by which, as we have just seen, the whole length of a fibre is occupied at the same time. In the second place the minute structure of a muscular fibre has been and still is the subject of fierce dispute.

If we adopt the view that the fibre is made up of dim bands or discs of dim substance alternating with bright bands or discs of bright substance, with transverse markings in the middle of each bright band forming a line "intermediate" between the two adjacent dim bands, we may, according to some observers, say that during a contraction there seems to be an interchange between the dim and bright bands so that, in ordinary light, at the height of the contraction, in the broadest part of one of the bulgings just spoken of, the previously obscure " intermediate line" becomes a conspicuous dark band, the interval between two such changed intermediate lines becoming relatively and uni- 
formly bright; in other words there is a sort of reversal of the situation, what was bright becoming, in its middle at least, dark, and what was dim becoming relatively bright. When the fibre is examined under polarized light, by which the dim bands are shown to be largely composed of doubly refractive, anisotropic material and the bright bands chiefly of singly refractive, isotropic material, it is found that the above apparent reversal is not based on any reversal of the refractive material, the anisotropic (dim) band remains anisotropic, and the isotropic (bright) band remains isotropic. But while both bands become lroader (across the fibre) and thinner (shorter along the length of the fibre), the anisotropie band does not become so much thinner as does the isotropic band, in other words the dim doubly refractive band or dise increases in bulk at the expense of the bright singly refractive band. And this accords with another feature of the fibre during contraction ; namely, that the sarcolemma, which in the fibre at rest presents a quite even line, is then indented at the middle of the bright band at about the position of the intermediate line, and bulges out opposite the dim band, that is opposite the enlarged anisotropic disc.

It is useless, however, to dwell on these matters until the minute structure of the fibre has been more clearly and satisfactorily made out than it is at present. A contraction is obviously a transloeation of molecules of the muscle substance and may, very roughly, be compared to the movement by which a company, say of one hundred soldiers ten ranks deep, with ten men in each rank, extends out into a donble line of two ranks with fifty men in each rank. The movement of translocation is obviously, in striated muscie, a very complicated one, but how the striation helps the movement we do not at present really know. All we can say is that when swift and rapid contraetion is needed, the contractile tissue employed puts on in nearly all cases the striated structure.

\section{The Chemistry of Muscle.}

$\S 55$. We said, in the Introdnction, that it was difficult to make out with certainty the exact chemical differences between dead and living substance. Muscle however in dying undergoes a remarkahle chemical change, which may be studierl with comparative ease. We have already said that all muscles, within a certain time after removal from the body, or, if still remaining part of the body, within a certain time after 'general' death of the body, lose their irritability, and that the loss of irritability, which even when rapid,.is gradual, is succeeled by an event which is somewhat more sudden, viz. the entrance into the condition known as rigor mortis. The occurrence of rigor mortis, or cadaveric rigid- 
ity, as it is sometimes called, which may be considered as the token of the death of the muscle, is marked by the following features. The living muscle possesses a certain translucency, the rigid muscle is distinctly more opaque. The living muscle is very extensible and elastic, it stretches readily and to a considerable extent when a weight is hung upon it or when any traction is applied to it, but speedily and, under normal circumstances, completely returns to its original length when the weight or traction is removed; as we shall see however the rapidity and completeness of the return depends on the condition of the muscle, a well-nomished, active muscle regaining its normal length much more rapidly and completely than a tired and exhausted muscle. A dead, rigid muscle is much less extensible and at the same time much less elastic; the muscle now requires considerable force to stretch it, and when the force is removed, does not, as lefore, return to its former length. To the touch the rigid muscle has lost much of its former softness, and has become firmer and more resistant. The entrance into rigor mortis is moreover accompanied by a shortening or contraction, which may, under certain circumstances, be considerable. The enelgy of this contraction is not great, so that any actual shortening is easily prevented by the presence of even a slight opposing force.

Now the chemical features of the dead, rigid muscle are also strikingly different from those of the living muscle.

$\$ 56$. If a dead muscle, from which all fat, tendon, fascia, and connective tissue have been as nuch as possible removed, and which has been freed from blood by the injection of 'normal' saline solution, be minced and repeatedly washed with water, the washings will contain certain forms of albmmin and certain extractive bodies, of which we shall speak directly. When the washing has been continued until the wash-water gives no proteid reaction, a large portion of muscle will still remain undissolved. If this be treated with a 10 p. c. solution of a neutral salt, ammonium chloride being the best, a large portion of it will become dissolved; the solution however is more or less imperfect and filters with difficulty. If the filtrate be allowed to fall drop by drop into a large quantity of distilled water, a white floceulent matter will be precipitated. This flocculent precipitate is myosin. Myosin is a proteid, giving the ordinary proteid reactions, and having the same general elementary composition as other proteids. It is soluble in dilute saline solutions, especially those of ammonium chloride, and may be classed in the globulin family, though it is not so soluble as paraglobulin, requiring a stronger solution of a neutral salt to dissolve it; thus while soluble in a 5 or 10 p. c. solution of such a salt, it is far less soluble in a 1 p. c. solution, which as we have seen readily dissolves paraglobulin. From its solutions in neutral saline solution it is precipitated by saturation witl a neutral 
salt, preferably sodium chloride, and may be purified by being washed with a saturated solution, dissolved again in a weaker solution, and reprecipitated by saturation. Dissolved in saline solutions it readily cuagulates when heated, i.e. is converted into coagulated proteid, and it is worthy of notice that it coagulates at a comparatively low temperature, viz. about $56^{\circ} \mathrm{C}$.; this it will be remembered is the temperature at which fibrinogen is coagulated, whereas paraglobulin, serum albumin, and nany other proteids do not coagulate until a higher temperature, $75^{\circ} \mathrm{C}$., is reached. Solutions of myosin are precipitated by alcolıol, and the preeipitate, as in the case of other proteids, becomes by continued action of the alcohol altered into coagulated insoluble proteid.

We have seen that paraglobulin, and indeed any nember of the globulin group, is very readily changed by the action of dilute acils into a boly called acid albumin, characterised by not being soluble either in water or in dilute saline solutions but readily soluble in dilute acids and alkalis, from its solutions in either of which it is precipitated by neutralisation, and by the fact that the solutions in dilute acids and alkalis are not coagulated by heat. When therefore a globulin is dissolved in dilute acid, what takes place is not a mere solution but a chemical change; the globulin cannot be got back from the solution, it has been changed into acid-albumin. Sinilarly when globulin is dissolved in dilute alkalis it is changed into alkali albumin; and broadly speaking alkali albumin precipitated by neutralisation can be changed by solution with dilute acids into acid albumin, and acid albumin by dilute alkalis into alkali albumin.

Now myosin is similarly, and even more readily than is globulin, converted into acid albumin, and by treating a muscle either washed or not, directly with dilute hydrochloric acid, the myosin may be converted into acid albumin and dissolved out. Acid albumin obtained by dissolving muscle in dilute acid used to be called syntonin, and it used to be said that a muscle contained syntonin; the muscle however contains myosin, not syntonin, but it may be useful to retain the word syntonin to denote acid albumin obtained by the action of dilute acid on myosin. By the action of dilute alkalis, myosin may similarly be converted into alkali albumin.

From what has been stated above it is obvious that myosin has many analogies with fibrin, and we have yet to mention other striking analogies; it is however much more soluble than fibrin, and speaking generally it may be said to be intermediate in its character between fibrin and globulin. On keeping, and especially on drying, its solubility is much diminished.

Of the substances which are left in washed muscle, from which all the myosin has been extracted by ammonium chloride solution, little is known. If washed muscle be treated directly with dilute 
hydrochloric acid, a large part of the material of the muscle passes, as we have said, at once into syntonin. The quantity of syntonin thus obtained may be taken as roughly representing the quantity of myosin previously existing in the muscle. A more prolonged action of the acid may dissolve out other proteids, besides myosin, left after the washing. The portion insoluble in dilute liydrochloric acid consists in part of the gelatine yielding and other substances of the sarcolemma and of the connective and other tissues between the bundles, of the nuclei of these tissues and of the fibres themselves, and in part, possibly, of some portions of the muscle substance itself. We are not however at present in a position to make any very definite statement as to the relation of the myosin to the structural features of muscle. Since the dim bands are rendered very indistinet by the action of 10 p.c. sodium chloride solution, we may perhaps infer that myosin enters largely into the composition of the dim bands, and therefore of the fibrille; but it would be hazardous to say much more than this.

$\$ 57$. Living inuscle may be frozen, and yet, after certain precautions will, on being thawed, regain its irritability, or at all events will for a time be found to be still living in the sense that it has not yet passed into rigor mortis. We may therefore take living muscle which has been frozen as still living.

If living contractile muscle, freed as much as possible from blood, be frozen, and while frozen, minced, and rubbed up in a mortar with four times its weight of snow containing 1 p.c. of sodium chloride, a mixture is obtained which at a temperature just below $0^{\circ} \mathrm{C}$. is sufficiently fluid to be filtered, though with difficulty. The slightly opalescent filtrate, or muscle plasma as it is called, is at first quite fluid, but will when exposed to the ordinary temperature become a solid jelly, and afterwards separate into a clot and scrum. It will in fact clot like blood plasma, with this difference, that the clot is not firm and fibrillar, but loose, granular, and flocculent. During the clotting the fluid, which before was neutral or slightly alkaline, becomes distinctly acid.

- The clot is myosin. It gives all the reactions of myosin obtained from dead muscle.

The serum contains an albumin very similar to, if not identical with, serum albumin, a globulin differing somewhat from, and coasulating at a lower temperature than paraglobulin, and which to distinguish it from the globulin of blood has been called myoglobulin, some other proteids which need not be described here, and various 'extractives' of which we shall speak directly. Such muscles as are red also contain a small quantity of hemoglobin and possibly, another allied red pigment.

Thus while dead muscle contains myosin, albumin, and other proteids, extractives, and certain insoluble matters, together with gelatinous and other substances not referable to the muscle 
substance itself, living inuscle contains no myosin, but some substance or substances which bear sumewhat the same relation to myosin that the antecedents of fibrin do to fibrin, and which give rise to myosin upon the death of the muscle. There are indeed reasons for thinking that the myosin arises from the conversion of a previously existing body, which nay be called myosinogen, and that the conversion takes place, or nnay take place, by the action of a special ferment, the conversion of myosinogen into myosin being very analogous to the conversion of tibrinogen into fibrin.

We may in fact speak of rigor mortis as characterised by a clotting of the muscle plasma, comparable to the clotting of blood plasma, but differing from it inasmuch as the product is not fibrin but myosin. 'The rigidity, the loss of suppleness, and the dinninished translucency appear to be at all events largely, though probably not wholly, due to the change from the Huid plasma to the solid myosin. We might compare a living muscle to a number of fine transparent membranous tubes containing blood plasma. When this blood plasma entered into the 'jelly' stage of clotting, the system of tubes would present many of the phenomena of rigor mortis. They would lose much of their suppleness and translucency, and acquire a certain anount of rigidity.

$\$ 58$. There is however one very marked and important difference between the rigor mortis of muscle and the clotting of blood. Blood during its clotting undergoes a slight change only in its reaction; but muscle during the onset of rigor mortis becomes distinctly acid.

A living muscle at rest is in reaction neutral, or, possilly from some remains of lymph adhering to it, faintly alkaline. If on the other hand the reaction of a thoroughly rigid muscle be tested, it will be found to be most distinctly acid. This development of an acid reaction is witnessed not only in the solid untouched fibre but also in expressed muscle plasma; it seems to be associated in some way with the appearance of the myosin.

The exact causation of this acid reaction has not at present been clearly worke l out. Since the coloration of the litmus produced is permanent, carbonic acid, which as we shall immediately state, is set free at the same time, cammot be regarded as the active acid, for the reddening of litmus produced by carbonic acid speedily disappears on exposure. On the other hand, it is possible to extract from rigid muscle a certain quantity of lactic acid, or rather of a variety of lactic acid known as sarcolactic acid ${ }^{1}$; and we may probahly regard the acid rection of rigid muscle as due to a new formation or to an increased formation of this sarcolactic acid. There is reason however to think that the establishment of the

1 There are many varieties of lactic acid, which are isomeric, having the same composition $\mathrm{C}_{3} \mathrm{H}_{6} \mathrm{O}_{3}$, but differ in their reactions and especially in the solubility of their zinc salts. The variety present in muscle is distinguished as sarcolactic acid. 
acid reaction is not a perfectly simple process but a more or less complex one, other substances besides sarcolactic acid intervening.

Coincident with the appearance of this acid reaction, though as we have said, not the direct cause of it, a large development of carbonic acid takes place when muscle becomes rigid. Irritable living muscular substance like all living substance is continually respiring, that is to say, is coutinually consuming oxygen and giving out carbonic acid. In the body, the arterial blood going to the muscle gives up some of its oxyen, and gains a quantity of carbonic acid, thus becoming venous as it passes through the muscle capillaries. Even after removal from the body, the living muscle continues to take up from the surrounding atmosphere a certain quantity of oxygen and to give out a certain quantity of carbonic acid.

At the onset of rigor mortis there is a very large and sudden increase in this production of carbonic acid, in fact an outhurst as it were of that gas. This is a phenomenon deserving special attention. Knowing that the carbonic acid which is the outcome of the respiration of the whole body is the result of the oxidation of carbon-holding substances, we might very naturally suppose that the increased production of carbonic acid attendant on the development of rigor mortis is due to the fact that during that event a certain quantity of the carbon-holding constituents of the muscle are suddenly oxidized. But such a view is negatived by the following facts. In the first place, the increased production of carbonic acid during rigor mortis is not accompanied by a corresponding increase in the consumption of oxygen. In the second place, a muscle (of a frog for instance) contains in itself no free or loosely attached oxygen; when subjected to the action of a mercurial airpump it gives off no oxygen to a vacuum, offering in this respect a marked contrast to blood; and yet; when placed in an atmosphere free from oxygen, it will not only continue to give off carbonic acid while it remains alive, but will also exhibit at the onset of rigor mortis the sane increased production of carbonic acid that is shewn by a muscle placed in an atmosphere containing oxygen. It is obvious that in such a case the carbonic acid does not arise from the direct oxidation of the muscle substance, for there is no oxygen present at the time to carry on that oxidation. We are driven to suppose that during rigor mortis, some complex body, containing in itself ready formed carbonic acid so to speak, is split up, and thus carbonic acid is set free, the process of oxidation by which that carbonic acid was formed out of the carbon-holding constituents of the muscle having taken place at some anterior date.

Living resting muscle, then, is alkaline or neutral in reaction, and the substance of its fibres contains a plasma capable of clotting. Dead rigid muscle on the other hand is acid in reaction, and no longer contains a plasma capable of clotting, but is ladeu with the 
solid myosin. Further, the change from the living irritable condition to that of rigor mortis is accompanied by a large and sudden development of carbonic acid.

It is found moreover that there is a certain amount of parallelism between the intensity of the rigor mortis, the degree of acid reaction and the quantity of carbonic acid given out. If we suppose, as we fairly may do, that the intensity of the rigidity is dependent on the quantity of myosin deposited in the fibres, and the acid reaction to the development if not of lactic acid, at least of some other substance, the parallelism between the three products, myosin, acid-producing substance, and carhonic acid, would suggest the idea that all three are the results of the splitting-up of the same highly complex substance. No one has at present however succeeded in isolating or in otherwise definitely proving the existence of such a body, and though the idea seems tempting, it may in the end prove erroneous.

$\$ 59$. As to the other proteids of muscle, such as the albumin and the globulin, we know as yet nothing definite concerning the parts which they play and the changes which they undergo in the living muscle or in rigor mortis.

Besides the fut which is found, and that not unfrequently in abundance, in the connective tissue between the fibres, there is also present in the muscular substance within the sarcolemma, always some, and at times a great deal, of fat, chiefly ordinary fat, viz. stearin, palmitin, and olein in variable proportion, but with a small quantity of the more complex fat lecithin ; the latter probably is derived from the nerve fibres. As to the function of these several fats in the life of the muscle we know little or nothing.

Carbohydratcs, the third of the three great classes in which we may group the energy-holding substances of which the animal body and its food are alike composed, viz proteids, fat and carbohydrates, are represented in musele by a peenliar body, glycogen, which we shall have to study in detail later on. We must here merely say that glycogen is a body closely allied to starch, having a formula, which may be included under the general formula for starches $n \cdot\left(\mathrm{C}_{6} \mathrm{H}_{10} \mathrm{O}_{5}\right)$, and nuay like it be converted by the action of acids, or by the action of particular ferments known as anylolytic ferments, into some form of sugar, dextrose $\left(\mathrm{C}_{6} \mathrm{H}_{12} \mathrm{O}_{6}\right)$ or some allied sugar. Many, if not all, living muscles contain a certain amount, and some, under certain circumstances, a considerable amount of glycogen. During or after rigor mortis this glycogen is very apt to he converted into dextrose, or an allied sugar. The muscles of the embryo at an early stage contain a relatively enormons quantity of glycogen, a fact which suggests that the glycogen of muscle is carbohydrate food of the muscle about to be wrought up into the living muscnlar substance.

The bodies which we have called extractives are numerous and 
varied. They are especially interesting since it seems probable that they are waste products of the metabolism of the muscular substance, and the study of them may be expected to throw light on the chemical change which muscular substance undergoes during life. Since, as we shall see, muscular substance forms by far the greater part of the nitrogenous - that is, proteid - portion of the body, the nitrogenous extractives of muscle demand peculiar attention. Now, the body urea, which we shall have to study in detail later on, far exceeds in importance all the other nitrogenous extractives of the body as a whole, since it is practically the one form in which nitrogenous waste leaves the body; if we include with urea, the closely allied uric acid (which for present purposes may simply be regarded as a variety of urea), we may say broadly that all the nitrogen taken in as food sooner or later leaves the body as urea; compared with this all other nitrogenous waste thrown out from the body is insignificant. Of the urea which thus leaves the body, a considerable portion must at some time or other have existed, or, to speak nore exactly, its nitrogen must have existed as the nitrogen of the proteids of muscular substance. Nevertheless, no urea at all or an absolutely minimal quantity only is, in normal conditions, present in musenlar substance either living and irritable, or dead and rigid; urea does not arise in muscular substance itself as one of the immediate waste products of muscular substance.

There is, however, always present, in relatively considerable amount, on an average about 25 p.c. of wet muscle, a remarkable body, kreatin. This is in one sense a compound of urea : it may be split up into urea and sarcosin. This latter body is a methyl glycin, that is to say, a glycin in which methyl has been substituted for hydrogen, and glycin itself is amido-acetic acid, a compound of amidogen, that is a representative of ammonia, and acetic acid. Hence kreatin contains urea, which has close relations with ammonia, together with another representative of anmonia, and a surplus of carbon and liydrogen arranged as a body belonging to the fatty acid series. We shall have to return to this kreatin, and to consider its relations to urea and to muscle, when we come to deal with urine.

The other nitrogenous extractives, such as karnin, hypoxanthin (or sarkin), xanthin, taurin, \&c., occur in small quantity, and need not be dwelt on here.

Among non-nitrogenous extractives, the most important is the sarcolactic acid, of which we have already spoken; to this may be added sugar in some form or other, either coming from glycogen or from some other source.

The ash of muscle, like the ash of the blood corpuscles, and, indeed, the ash of the tissues in general, as distinguished from the blood, or plasma, or lymph on which the tissues live, is characterised by the preponderance of potassium salts and of phosphates; these form in fact nearly 80 p.c. of the whole ash. 
$\$ 60$. We may now pass on to the question, What are the chemical changes which take place when a living, resting muscle enters into a contraction? These changes are most evident after the musele has been subjected to a prolonged tetanus; but there can be no doubt that the chenical events of a tetanus are, like the physical events, simply the sum of the results of the constituent single contractions.

In the first place, the muscle becomes acid, not so acid as in rigor mortis, but still sufficiently so, after a vigorous tetanus, to turn blue litmus distinctly red. The cause of the acid reaction, like that of rigor mortis, is not quite clear, but is in all probability the same in both cases.

In the second place, a considerable quantity of carbonic acid is set free; and the production of carbonic acid in muscular contraction resembles the production of carbonic acid during rigor mortis in that it is not accompanied by a corresponding increase in the consumption of oxygen. This is evident even in a muscle through which the circulation of blood is still going on ; for though the blood passing through a contracting muscle gives up more oxygen than the blood passing through a resting muscle, the increase in the amount of oxygen taken up falls below the increase in the carbonic acid given out. But it is still more nuarkedly shewn in a muscle removed from the body; for in such a inuscle both the contraction and the increase in the production of carbonic acid will go on in the absence of oxygen. A frog's muscle, suspended in an atmosphere of nitrogen, will remain irritable for some considerable time, and at each vigorous tetanus an increase in the production of carbonic acid may be readily ascertained.

Moreover, there seems to be a correspondence between the energy of the contraction and the amount of carbonic acid and the degree of acid reaction produced, so that, though we are now treading on somewhat uncertain ground, we are naturally led to the view that the essential chemical process, lying at the bottom of a muscular contraction as of rigor mortis, is the splitting-up of some highly complex substance. But here the resemblance between rigor mortis and contraction ends. We have no satisfactory evidence of the formation during a contraction of any body like myosin. And this difference in chemical results tallies with an important physical difference between rigid muscle and contracting muscle. The rigid muscle, as we have seen, becomes less extensible, less elastic, less translucent; the contracting muscle remains no less transhucent, elastic, and extensible than the resting muscle, - indeed, there are reasons for thinking that the muscle in contracting becomes actually more extensible for the time being.

But if, during a contraction, myosin is not formed, what changes of proteid or nitrogenous matter do take place? We do not know. We have no evidence that kreatin, or any other nitrogenous extractive, is increased by the contraction of muscle; we have no 
satisfactory evidence of any nitrogen waste at all as the result of a contraction; and, indeed, as we shall see later on, the study of the waste products of the body as a whole leads us to believe that the energy of the work done by the muscles of the body comes from the potential energy of carbon compounds, and not of nitrogen compounds at all. But to this point we shall have to return. follows:

61. We may sum up the chemistry of muscle somewhat as

D)uring life the muscular substance is continually taking up from the blood, that is from the lymph, proteid, fatty and carbohydrate material, saline matters and oxygen; these it builds up into itself, how, we do not know, and so forms the peculiar complex living nuscular substance. The exact nature of this living substance is unknown to us. What we do know is that it is largely composed of proteid material, and that such bodies as myosinogen, myoglobulin, and albumin, being always present in it, have probably something to do with the building of it up.

During rest this muscular substance, while taking in and building itself up out of, or by means of, the above-mentioned materials, is continually giving off carbonic acid, and continually forming nitrogenous waste, such as kreatin. It also probably gives off some amount of sarcolactic acid, and possibly other non-nitrogenous waste matters.

During a contraction there is a great increase in the amount of carbonic acid given off, an increased formation of lactic acid, and possibly other changes giving rise to an acid reaction, a greater consumption of oxygen, though the increase is not equal to the increase of carbonic acid, but, as far as we can learn, no increase of nitrogenous waste.

During rigor mortis, there is a similar increased production of carbonic acid and of some other acid-producing substance, accompanied by a remarkable conversion of myosinogen into myosin, by which the rigidity of the dead fibre is brought about.

\section{Thermal Changes.}

$\S 62$. The chemical changes during a contraction set free a quantity of energy, but only a portion of this energy appears in the 'work done;' a considerable portion takes on the form of heat. Though we shall have hereafter to treat this subject more fully, the leading facts may be given here.

Whenever a muscle contracts, its temperature rises, indicating that heat is given out. When a mereury thermometer is plunged into a mass of inuscles, such as those of the thigh of the dog, a rise of the mercury is observed upon the muscles being thrown into a prolonged contraction. More exact results however are obtained by means of a thermopile, by the help of which the rise of tempera- 
ture caused by a few repeated single contractions, or, indeed, by a single contraction, may be observed, and the amount of heat given out approximatively measured.

The thermopile may consist either of a single junction, in the form of a needle plunged into the substance of the muscle; or of several junctions either in the shape of a flat surface carefully opposed to the surface of muscle (the pile being balanced so as to move with the contracting muscle, and thus to keep the contact exact), or in the shape of a thin wedge, the edge of which, comprising the actual junctions, is thrust into a mass of muscles and held in position by them. In all cases the fellow junction or junctions must be kept at a constant temperature.

Another delicate method of determining the changes of temperature of a tissue is based upon the measurement of alterations in electric resistance which a fine wire, in contact with or plunged into the tissue, undergoes as the temperature of the tissue changes.

It has been calculated that the heat given out by the muscles of the thigh of a frog in a single contraction amounts to $3 \cdot 1$ micro-units of heat ${ }^{1}$ for each gramme of musele, the result being obtained by dividing by five the total amount of heat given out in five successive single contractions. It will, however, be safer to regard these figures as illustrative of the fact that the heat given out is considerable rather than as data for elaborate calculations. Moreover, we have no satisfactory quantitative determinations of the heat given out by the muscles of warm blooded animals, though there can be no doubt that it is much greater than that given out by the muscles of the frog.

There can hardly be any doubt that the heat thus set free is the product of chemical changes within the muscle, changes, which, though they cannot, for the reasons given above (\$60), be regarded as simple and direct oxidations, yet, since they are processes dependent on the antecedent entrance of oxygen into the muscle, may be spoken of in general terms as a combustion. So that the muscle may be likened to a stean-engine, in which the combustion of a certain amount of material gives rise to the development of energy in two forms, as heat and as movement, there being certain quantitative relations between the amount of energy set free as heat and that giving rise to movement. We must, however, carefully guard ourselves against pressing this analogy too closely. In the steam-engine, we can distinguish clearly between the fuel which, through its combustion, is the sole source of energy, and the machinery, which is not consumed to provide energy, and only suffers wear and tear. In the muscle we cannot with certainty at present make such a distinction. It may be that the chemical changes at the bottom of a contraction do not involve the real living material of the fibre, but only some substance, manufactured by the living material and lodged in some way, we do not know

1 The micro-unit being a milligramme of water raised one degree centigrade. 
how, in the living material ; it may be that when a fibre contracts it is this substance within the fibre which explodes, and not the fibre itself. If we further suppose that this substance is some complex compound of carbon and hydrogen, into which no nitrogen enters, we shall have an explanation of the difficulty referred to above $(\$ 60)$, namely, that nitrogenous waste is not increased by a contraction. The special contractile, carbon-hydrogen substance may then be compared to the charge of a gun, the products of its explosion being carbonic and sarcolactic acids, while the real, living material of the fibre may be compared to the gun itself ; but to a gun which itself is continually undergoing change, far beyond mere wear and tear, among the products of which change nitrogenous bodies like kreatin are conspicuous. This view will certainly explain why kreatin is not increased during the contraction while the carbonic and lactic acids are. But it must be remembered that such a view is not yet proved; it may be the living material of the fibre as a whole which is continually breaking down in an explosive decomposition, and as continually building itself up again out of the material supplied by the blood.

In a steam-engine only a certain amount of the total potential energy of the fuel issues as work, the rest being lost as heat, the proportion varying, but the work rarely, if ever, exceeding onetenth of the total energy, and generally being less. In the case of the muscle we are not at present in a position to draw up an exact equation between the latent energy on the one hand and the two forms of actual energy on the other. We have reason to think that the proportion between heat and work varies considerably under different circumstances, the work sometimes rising as high as one-fifth, or, according to some, as high even as one-half, sometimes possibly sinking as low as one twenty-fourth of the total energy; and observations seem to shew that the greater the resistance which the muscle has to overcome, the larger the proportion of the total energy expended, which goes out as work done. The muscle, in fact, seems to be so far self-regulating, that the more work it has to do, the greater, within certain limits, is the economy with which it works.

Lastly, it must be remembered that the giving out of heat by the muscle is not confined to the occasions when it is actually contracting. When, at a later period, we treat of the heat of the body generally, evidence will be brought forward that the muscles, even when at rest, are giving rise to heat, so that the heat given out at a contraction is not some wholly new phenomenon, but a temporary exaggeration of what is continually going on at a more feeble rate.

\section{Electrical Changes.}

$\S 63$. Besides chemical and thermal changes a remarkable electric change takes place whenever a muscle contracts. 
IIsclc-currents. If a muscle be removed in an ordinary mamner from the body, and two non-polarisable electrodes, ${ }^{1}$ connected with a delicate galvanometer of many convolutions and

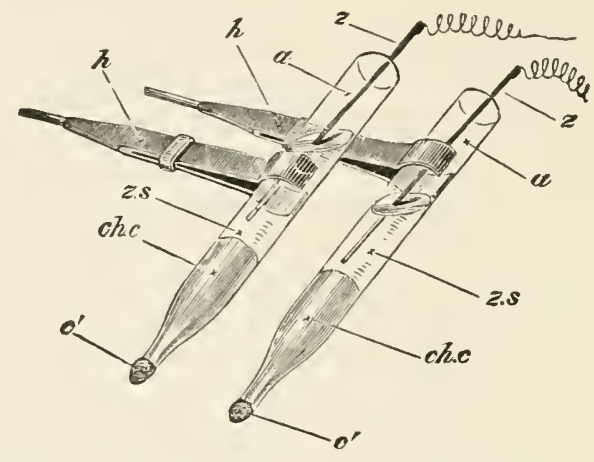

Fig. 18. Non-polarisable Electrones.

$a$, the glass tnbe; $z$, the amalgamated zinc slips connected with their respective wires; z. s., the zine sulphate solntion, $c h . c$, the plug of china clay; $c^{\prime}$, the portion of the china-clay plug projecting from the end of the tube this can be moulded into any required form.

high resistance, be placed on two points of the surface of the muscle, a deflection of the galvanometer will take place, indicating the existence of a current passing through the galvanometer from the one point of the muscle to the other, the direction and amount of the deflection varying according to the position of the points. The 'muscle-currents' thus revealed are seen to the best advantage when the muscle chosen is a cylindrical or prismatic one with parallel fibres, and when the two tendinous ends are cut off by clean incisions at right angles to the long axis of the muscle. The muscle then presents a transverse section (artificial) at each end, and a longitudinal surface. We may speak of the latter as being divided into two equal parts by an imaginary transverse line on its surface called the 'equator,' containing all the points of the surface midway between the two ends. Fig. 19 is a diagrammatic representation of such a muscle, the line $a b$ being the equator. In such a muscle the development of the muscle-currents is found to be as follows.

1 These (Fig. 18) consist essentially of a slip of thoroughly amalgamated zinc dipping into a suturated solntion of zine sulphate, which, in turn, is bronght into connection with the nerre or muscle by means of a plug or brilge of china-clay, moistened with normal sodimn chloride solntion, it is important that the zinc should be thoroughly amalgamated. This form of electrodes gives rise to less polarisation than do simple platinnm or copper electrodes. The clay affords a connection between the zinc and the tissue which neither acts on the tissne nor is acted on by the tissue. Contact of any tissue witl copper or platinum is in itself sufficient to develope a current. 
The greatest deflection is observed when one electrode is placed at the mid-point or equator of the muscle, and the other at either cut end; and the deflection is of such a kind as to shew that positive currents are continually passing from the equator through the galvanometer to the cut end : that is to say, the cut end is negative relatively to the equator. The currents outside the muscle may be considered as completed by currents in the muscle from the cut end to the equator. In the diagram Fig. 19, the arrows indicate the

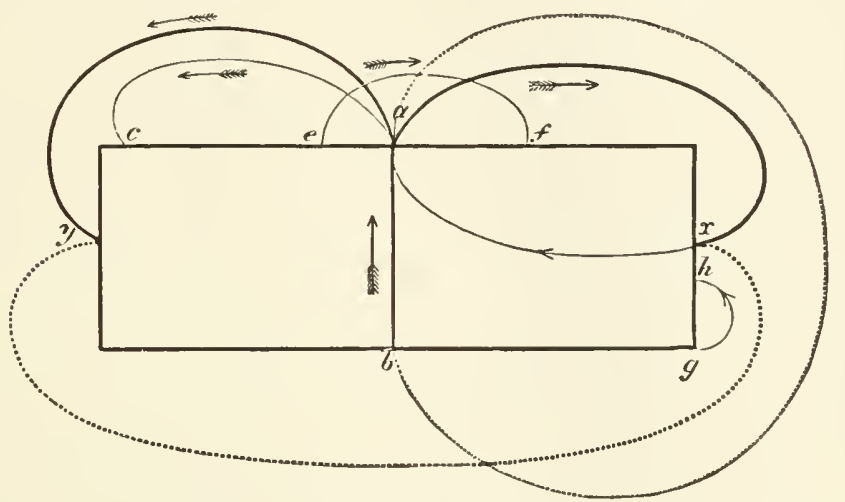

Fig. 19. Diagram illustrating the Electric Currents of Nerve and Muscle.

Being purely diagrammatic, it may serve for a piece either of nerve or of muscle, except that the currents at the transverse section camot be shewn in a nerve. The arrows shew the direction of the current through the galvanometer.

$a b$ the equator. 'The strongest currents are those shewn by the dark lines, as from $a$, at equator, to $x$ or to $y$ at the cut ends. The current from $a$ to $c$ is weaker than from $a$ to $y$, though both, as shewn by the arrows, have the same direction. $A$ current is shewn from $e$, which is near the equator, to $f$, which is farther from the equator. The current (in muscle) from a point in the circumference to a point nearer the centre of the transverse section is shewn at $g h$. From $a$ to $b$ or from $x$ to $y$ there is no current, as indicated by the dotted lines.

direction of the currents. If the one electrode be placed at the equator $a b$, the effect is the same at whichever of the two cut ends $x$ or $y$ the other is placed. If, one electrode remaining at the equator, the other be shifted from the cut end to a spot $e$ nearer to the equator, the current continues to have the same direction, but is of less intensity in proportion to the nearness of the electrodes to each other. If the two electrodes be placed at unequal distances $e$ and $f$, one on either side of the equator, there will be a feeble current from the one nearer the equator to the one farther off, and the current will be the feebler, the more nearly they are equidistant from the equator. If they are quite equidistant, as, for instance, when one is placed on one cut end $x$, and the other on the other cut end $y$, there will be no current at all.

If one electrode be placed at the circumference of the transverse section and the other at the centre of the transverse section, there 
will be a current through the galvanometer from the former to the latter; there will be a current of similar direction but of less intensity when one electrode is at the circumference $g$ of the transverse section, and the other at some point $h_{l}$ nearer the centre of the transverse section. In fact, the points which are relatively most positive and most negative to each other are points on the equator and the two centres of the transverse sections; and the intensity of the current between any two points will depend on the respective distances of those points from the equator and from the centre of the transverse section.

Similar currents may be observed when the longitudinal surface is not the natural but an artificial one; indeed they may be witnessed in even a piece of muscle provided it be of cylindrical shape and composed of parallel fibres.

These 'muscle-currents" are not mere transitory currents disappearing as soon as the circuit is closed; on the contrary, they last a very considerable time. They must, therefore, be maintained by some changes going on in the muscle, by continued chenical action in fact. They disappear as the irritability of the muscle vanishes, and are connected with those nutritive, so-called vital changes which maintain the irritability of the muscle.

Muscle-currents, such as have just been described, may, we repeat, be observed in any cylindrical muscle suitably prepared, and similar currents, with variations which need not be discussed here, may be seen in muscles of irregular shape with obliquely or otherwise arranged fibres. And Du Bois-Reymond, to whom chiefly we are indebted for our knowledge of these currents, has been led to regard them as essential and important properties of living muscle. He has moreover advanced the theory that muscle may be considered as composed of electro-motive particles or molecules, each of which, like the muscle at large, has a positive equator and negative ends, the whole muscle being made up of these nolecules in somewhat the same way (to use an illustration which must not, however, be strained or considered as an exact one) as a magnet may be supposed to be made up of magnetic particles, each with its north and south pole.

There are reasons, however, for thinking that these musclecurrents have no such fundamental origin, that they are in fact of surface and indeed of artificial origin. Without entering into the controversy on this question, the following important facts may be mentioned :-

1 When a muscle is examined while it still retains uninjured its natural tendinous terminations, the currents are much weaker than when artificial transverse sections have been made; the natural tendinous end is less negative than the cut surface. But the tendinous end becomes at once negative when it is dipped in water or acid, indeed, when it is in any way injured. The less roughly, in fact, a muscle is treated the less evident are the 
muscle-currents; and it is maintained that if adequate care be taken to maintain a muscle in an absolutely natural condition, no such currents as those we have been describing exist at all, that natural living muscle is isoelectric, as it is called.

2 . The surface of the uninjured inactive ' ventricle of the frog's heart, which is practically a mass of muscle, is isoelectric, no current is obtained when the electrodes are placed on any two points of the surface. If, however, any part of the surface be injured, or if the ventricle be cut across so as to expose a cut surface, the injured spot or the cut surface becomes at once powerfully negative towards the uninjured surface, a strong current being developed which passes through the galvanometer from the minjured surface to the cut surface or to the injured spot. The negativity thus developed in a cut surface passes off in the course of some hours, but may be restored by making a fresh cut and exposing a fresh surface.

The temporary duration of the negativity after injury, and its renewal upon fresh injury, in the case of the ventricle, in contrast to the more permanent negativity of injured skeletal muscle, is explained by the different structure of the two kinds of muscle. The cardiac muscle, as we shall hereafter see, is composed of short fibre-cells; when a cut is made a certain number of these fibrecells are injured, giving rise to negativity, but the injury done to them stops with them, and is not propagated to the cells with which they are in coutact; hence, upon their death the negativity and the current disappear. A fresh cut involving new cells, produces fresh negativity and a new current. In the long fibres of the skeletal muscle, on the other hand, the effects of the injury are slowly propagated along the fibre from the spot injured.

Now, when a muscle is cut or injured, the substance of the fibres dies at the cut or injured surface. And many physiologists, among whom the most prominent is Hermann, have been led, by the above and other facts, to the conclusion that muscle-currents do not exist naturally in untouched, uninjured inuscles, that the muscular substance is naturally, when living, isoelectric, but that whenever a portion of the muscular substance dies, it becomes while dying negative to the living substance, and thus gives rise to currents. They explain the typical currents (as they might be called) manifested by a muscle with a natural longitudinal surface and artificial transverse sections, by the fact that the dying cut ends are negative relatively to the rest of the muscle.

Du Bois-Reymond and those with him offer special explanations of the above facts and of other objections which have been urged against the theory of naturally existing electro-motive molecules. Into these we cannot enter here. We must rest content with the statement that in an ordinary muscle currents, such as have been described, may be witnessed, but that strong arguments may be 
adduced in favour of the view that these currents are not 'natural' phenomena, but essentially of artifieial origin. It will therefore be best to speak of them as currents of rest.

$\$ 64$. Currents of action. Neyative variation of the Museleeurrent. The controversy whether the 'currents of rest' observable in a muscle be of natural origin or not, does not affect the truth or the importance of the fact that an electrical change takes place, and a current is developed in a musele whenever it enter's into a contraction. When currents of rest are observable in a muscle, these are found to undergo a diminution upon the occurrence of a contraction, and this diminution is spoken of as "the negative variation' of the currents of rest. The negative variation may be seen when a muscle is thrown into a single contraction, but is most readily shewn when the muscle is tetanized. Thus, if a pair of electrodes be placed on a muscle, one at the equator, and the other at or near the transverse section, so that a considerable deflection of the galvanometer needle, indicating a considerable current of rest, be gained, the needle of the galvanometer will, when the muscle is tetanized by an interrupted current sent through its nerve (at a point too far from the muscle to allow of any escape of the current into the electrodes connected with the galvanometer), swing back towards zero; it returns to its original deflection when the tetanizing current is shut off.

Not only may this negative variation be shewn by the galvanometer, but it, as well as the current of rest, may be used as a galvanic shock, and so employed to stimulate a muscle, as in the experiment known as 'the rheoscopic frog.' For this purpose the muscles and nerves need to be in thoroughly good condition, and very irritable. Two muscle-nerve preparations, $A$ and $B$, having been made, and each placed on a glass plate for the sake of insulation, the nerve of the one, $B$, is allowed to fall on the muscle of the other, $A$, in such a way that one point of the nerve comes in contact with the equator of the muscle, and another point with one end of the muscle or with a point at some distance from the equator. At the moment the nerve is let fall and contact made, a current, viz. the 'current of rest' of the musele $A$, passes through the nerve; this acts as a stimulus to the nerve, and so causes a contraction in the muscle connected with a nerve. Thus the muscle $A$ acts as a battery, the completion of the circuit of which by means of the nerve of $B$ serves as a stimulus, causing the muscle $B$ to contract.

If, while the nerve of $B$ is still in contact with the muscle of $\mathrm{A}$, the nerve of the latter is tetanized with an interrupted current, not only is the inuscle of $A$ thrown into tetanus, but also that of $B$; the reason being as follows. At each spasm of which the tetanus of $A$ is male up, there is a negative variation of the muscle current of $A$. Each negative variation of the muscle current of $A$ serves as a stimulus to the nerve of $B$, and is hence 
the cause of a spasm in the muscle of $B$; and the stimuli following each other rapidly, as being produced by the tetanus of $A$, they must do, the spasins in $B$ to which they give rise are also fused into a tetanus in $B$. $\quad B$, in fact, contracts in harmony with $A$. This experiment shews that the negative variation accompanying the tetanus of a muscle, though it causes only a single swing of the galvanometer, is really made up of a series of negative variations, each single negative variation corresponding to the single spasms of which the tetanus is made up.

But an electrical change may be manifested even in cases when no currents of rest exist. We have stated $(\$ 63)$ that the surface of the uninjured inactive ventricle of the frog's heart is isoelectric, no currents being observed when the electrodes of a galvanometer are placed on two points of the surface. Nevertheless, a most distinct current is developed whenever the ventriele contracts. This may be shewn either by the galvanometer or by the rheoscopic frog. If the nerve of an irritable muscle-nerve preparation be laid over a pulsating ventricle, each beat is responded to by a twiteh of the muscle of the preparation. In the case of ordinary muscles, too, instances occur in which it seems impossible to regard the electrical change manifested during the contraction as the mere diminution of a preexisting current.

Accordingly those who deny the existence of 'natural' musclecurrents speak of a muscle as developing during a contraction a 'curreut of action,' occasioned as they believe by the muscular substance as it is entering into the state of contraction, becoming negative towards the muscular substance which is still at rest, or has returned to a state of rest. In fact, they regard the negativity of muscular substance as characteristic alike of beginning death and of a beginning contraction. So that in a muscular contraction a wave of negativity, starting from the end-plate when indirect, or from the point stimulated when direct stimulation is used, passes along the muscular substance to the ends or end of the fibre.

If, for instance, we suppose two electrodes placed on two points (Fig. 20), $A$ and $B$, of a fibre about

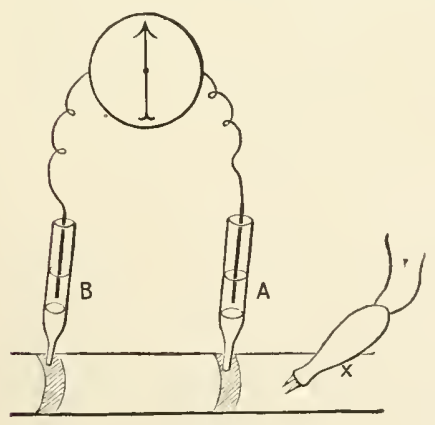

Fig. 20. to be stimulated by a single induction-shock at one end. liefore the stimulation the fibre is isoelectric, and the needle of the galvanometer stands at zero. At a certain time after the shock has been sent through the stimulating electrodes $(x)$, as the wave of contraction is travelling down the fibre, the section of the fibre beneath $A$ will become negative towards the rest of the fibre, and so negative towards the portion of the fibre under $B$, 
i.e. $A$ will be negative relatively to $B$, and this will be shewn by a deflection of the needle. A little later, $E$ will be entering into contraction, and will be becoming negative towards the rest of the fibre, including the part under $A$, whose negativity by this time is passing off; that is to say, $B$ will now be negative towards $A$, and this will be shewn by a deflection of the needle in a direction opposite to that of the deflection which has just previously taken place. Hence, between two electrodes placed along a fibre, a single wave of contraction will give rise to two currents of different phases, to a diphasic change; and this, indeed, is found to be the case.

This being so, it is obvious that the electrical result of tetanizing a muscle when wave after wave follows along each fibre, is a conplex matter; but it is maintained that the apparent negative variation of tetanus can be explained as the net result of a series of currents of action, due to the individual contractions, the second phase of the current in each contraction being less marked than the first phase. We cannot, however, enter more fully here into a discussion of this difficnlt subject.

When we study, as we may do with the help of appropriate apparatus, the rapidity with which the electrical change accompanying a muscular contraction travels, we find it to be the same as that of the contraction wave itself. The older observations seemed to shew that the electrical change fell entirely within the latent period, and might, therefore, be regarded as an outward token of invisible molecular processes, occupying the latent period, and sweeping along the muscular fibre ahead of and preparing for the visible change of form. And, indeed, since we are led to regard the change of form as the result of chemical processes taking place in the inuscular substance, we must suppose that the change of form is preceded by molecular chemical changes. But, as we have said, a latent period of measurable length does not appear to be an essential feature of a muscular contraction; we may, under certain circumstances, fail to detect a latent period. And some recent observations seem to shew that the electrical change and the change of form may begin at the same time. Indeed, some have maintained that the former is the result of the latter, and not, as suggested above, of the forerunning molecular events. The question however is one which cannot at present be regarded as settled.

\section{The Changes in a Nerve during the passage of a Nervous Impulse.}

$\$ 65$. The cliange in the form of a muscle during its contraction is a thing which can be seen and felt; but the changes in a nerve during its activity are invisible and impalpable. We stimulate one end of a nerve going to a muscle, and we see this followed 
by a contraction of the muscle attached to the other end; or we stimulate a nerve still connected with the central nervous system, and we see this followed by certain movements, or by other tokens which shew that disturbances have been set up in the central nervous system. We know therefore that some changes or other, constituting what we have called a nervous impulse, have been propagated along the nerve; but the changes are such as we cannot see. It is possible, however, to learn something about them.

$\$ 66$. The chemistry of a nerve. The medulla of a medullated nerve fibre is usually spoken of as fatty, and yet is in reality very largely compose 1 of a substanes which is not (in the strict sense of the word) a fat. When we examine chemically a quantity of nerve (or what is practically the same thing a quantity of that part of the central nervous system which is called white matter, and which is chielly composed, like a nerve, of medullated nerves, and is to be preferred for chemical examination because it contains a relatively smoll quantity of comnective tissue), we find that a very large proportion, according to some observers about half, of the dried matter consists of the peculiar body cholesterin. Now cholesterin is not a fat but an alcohol; like glycerine, however, which is also an alcohol, it forms compounds with fatty acids; and though we do not know definitely the chemical condition in which cholesterin exists luring life in the medulla, it is more than probable that it exists in some combination with some of the really fatty bodies also present in the medulla, and not in a free isolated state. It is singular that besides being present in such large quantities in nervous tissue, and to a small extent in other tissues and in blood, cholesterin is a normal constituent of bile, and forms the greater part of gall stones when these are present; in gall stones it is undoubtedly present in a free state. Besides cholesterin 'white' nervous matter contains a less but still considerable quantity of a complex fat, whose nature is disputed. According to some authorities rather less than half this complex fat consists of the peculiar body lecithin, which we have already seen to be present also in blood corpuscles and elsewhere. Lecithin contains the radicle of stearic acid (or of oleic, or of palmitic acid) associated not, as in ordinary fats, with simple glycerine, but with the more complex glycerin-phosphoric acid, and further combined with a nitrogenous body, neurin, an ammonia compound of some considerable complexity ; it is therefore of remarkable nature since, though a fat, it contains both nitrogen and phosphorus. According to the same authorities the remainder of the complex fat consists of another fatty body, also apparently containing nitrogen but no phosphorus, called eerebrin. Other authorities regard both these bodies, lecithin and cerebrin. as products of decomposition of a still more complex fat, called protagon. Obviously the fat of the white matter of the central 
nervous system and of spinal nerves (of which fat by far the greater part must exist in the medulla, and form nearly the whole of the medulla) is a rery complex body indeed, especially so if the cholesterin exists in combination with the lecithin, or cerebrin (or protagon). Being so complex it is naturally very unstable, and indeed, in its instability resembles proteid matter. Hence probably the reason why the medulla changes so rapidly and so profoundly after the death of the nerve. lt seems moreover that a certain though small quantity of proteid matter forms part of the medulla, and it is possible that this exists in some kind of combination with the complex fat; but our knowledgè on this point is imperfect.

The presence in such large quantity of this complex fatty medulla renders the chemical examination of the other constituents of a nerve very difficult, and our knowledge of the chemical nature of, and of the chemical clianges going on in, the axis-cylinder, is as yet limited. Examined under the microseope the axis-cylinder gives the xanthoproteic reaction and other indications that it is largely proteid in nature. From nervous matter, and especially from the grey matter of the brain and spinal cord, there may by apjropriate methods be extracted certain proteids similar to those found in leucocytes and other cells $\$ 29$ namely, a nucleo-albumin and one or more globulins; these are probably constituents both of the nerve cells and of the axis-cylinders which are processes of cells. Since kreatin and a lactic acid are present among the 'extractives' of nervous tissue, we may infer that in a broal way the chemical changes in nerves are similar to those in muscles. Beyond this we can say very little.

After the fats of the medulla (and the much smaller quantity of fat present in the axis-cylinder), the proteids of the axis-cylinder, and the other soluble substances present in one or the other, or gathered round the nuclei of the neurilemma, have hy various means been dissolved out of a nerve fibre certain substances still remain. One of these in suall quantity is the nuclein of the nuclei: another in larger quantity is the substance newrokeratin which forms a supporting framework for the medulla, and whose most marked characteristic is perhaps its resistance to solution.

In the ash of nerves there is a preponderance of potassium salts and phosphates but not so marked as in the case of muscle.

\$67. The nerrous impulse. The chemical analogy between the substance of the muscle and that of the axis-cylinder would naturally lead us to suppose that the progress of a nervous impulse along a nerve fibre was accompanied by chemical changes similar to those taking place in a muscle fibre. Whatever changes however do or may take place are too slight to be recognized by the means at our disposal. We have no satisfactory evidence that in a nerve even repeated nervous impulses can give rise to an acid reaction or that the death of a nerve fibre leads to such a reaction. The grey matter of the central nervous system it is true 
is said to be faintly alkaline during life and to become acid after death; but in this grey matter nerve cells are relatively abundant; the white matter, composed chielly of nerve fibres, is and remains, during action as well as rest, and even after death, neutral or slightly alkaline.

Nor have we satisfactory evidence that the progress of a nervous impulse is accompanied by any setting free of energy in the form of heat.

In fact, beyond the terminal resnlts, such as a inuscular contraction in the case of a nerve going to a muscle, or some affection of the central nervous system in the case of a nerve still in connection with its nervous centre, there is one event and one event only which we are able to recognize as the objective token of a nervous impulse, and that is an electric change. For a piece of nerve removed from the body exhibits nearly the same electric phenomena as a piece of muscle It has an equator which is electrically positive relatively to the two cut ends. In fact the diagram Fig. 19, and the description which was given in $\$ 63$ of the electric changes in muscle may be applied almost as well to a nerve, except that the currents are in all cases much more feeble in the case of nerves than of muscles, and the special currents from the circumference to the centre of the transverse sections cannot well be shewn in a slender nerve; indeed it is doubtful if they exist at all.

During the passage of a nervous impulse the 'natural nerve current' indergoes a negative variation, just as the 'natural muscle current' undergoes a negative variation during a contraction. There are moreover reasons in the case of the nerve, as in the case of the muscle, which lead us to douht the pre-existence of any such 'natural' currents. A nerve in an absolutely natural condition appears to be, like a muscle, isoelectric; hence we may say that in a nerve during the passage of a nervous impulse, as in a muscle during' a muscular contraction, a 'current of action' is developed.

'This 'current of action' or 'negative variation ' mar be shewn either by the galvanometer or by the rheoscopic frog. If the nerve of the 'muscle nerve preparation' $B$ (see $\$ 64$ ) be placed in an appropriate manner on a thoroughly irritable nerve $A$ (to which of course no muscle need be attached), touching for instance the equator and one end of the nerve, then single induction-shocks sent into the far end of $A$ will cause single spasms in the muscle of $B$, while tetanization of $A, i$. e. rapidly repeated shocks sent into $A$, will cause trtanus of the muscle of $B$.

That this current, whether it be regarded as an independent 'current of action' or as a negative variation of a 'pre-existing' current, is an essential feature of a nervous impulse is shewn by the fact that the degree or intensity of the one varies with that of the other. They both travel too at the same rate. In describ. 
ing the muscle-curve, and the method of measuring the muscular latent period, we have incidentally shewn $(\$+6)$ how at the same time the velocity of the nervous impulse may be measured, and stated that the rate in the nerves of a frog is about 28 meters a second. Br means of a special and somewhat complicated aplaratus it is ascertained that the current of action travels along an isolated piece of nerve at the same rate. It also, like the contraction, travels in the form of a wave, rising rapilly to a maximum at each point of the nerve and then more gradually declining again. The length of the wave may by special means be measured, and is found to be about $18 \mathrm{~mm}$.

When an isolated piece of nerve is stimulated in the middle, the current of action is propagated equally well in both directions, and that whether the nerve be a chiefly sensory or a chiefly motor nerve, or indeed if it be a nerve-root composed exchusively of motor or of sensory fibres. Taking the current of action as the token of a nervous impulse, we infer from this that when a nerve fibre is stimulated artiticially at any part of its course, the nervous impulse set going travels in both directions.

We used just now the phrase 'tetanization of a nerve,' meaning the application to a nerve of rapidly repeated shocks such as would produce tetanus in the muscle to which the nerve was attached, and we shall have frequent occasion to employ the phrase. It must however be understood that there is in the nerve, in an ordinary way, no summation of nervous impulses comparable to the summation of muscular contractions. Putting asile certain cases which we cannot discuss here we may say that the series of shocks sent in at the far end of the nerve start a series of impulses; these travel down the nerve and reach the musele as a series of distinct impulses; and the first changes in the muscle, the molecular changes which, sweeping along the fibre, initiate the change of form, and which we may perhaps speak of as constituting a muscle impulse, also probably form a series the members of which are distinct. It is not until these molecular changes become transformed into visible changes of form that any fusion or summation takes place.

$\$ 68$. Putting together the facts contained in this and the precerling sections, the following may be taken as a brief approximate history of what takes place in a muscle and nerve when the latter is subjected to a single induction-shock. At the instant that the induced current passes into the nerve, changes occur, of whose nature we know nothing certain except that they cause a 'current of action' or 'negative variation' of the 'natural' nerve current. These changes propagate themselves along the nerve in both directions as a nervous impulse in the form of a wave, having a wave-length of about $18 \mathrm{~mm}$, and a velocity (in frog's nerve) of about $28 \mathrm{~m}$. per sec. Passing down the nerve fibres to the inuscle, flowing along the branching and narrowing tracts, the wave at last 
breaks on the end-plates of the fibres of the muscle. Here it is transmuted into what we have called a muscle inpulse, which with a greatly diminished velocity (about $3 \mathrm{~m}$. per sec.), travels from each end-plate in both directions to the end of the fibre, where it appears to be lost, at all events we do not know what becomes of it. As this impulse wave sweeps along the fibre it initiates an explosive decomposition of material, leading to a discharge of carbonic acid, to the appearance of some substance or substances with an acid reaction, and probably of other unknown things, with a considerable development of heat. This explosive decomposition gives rise to the visible contraction wave; the fibre, as the wave passes over it, swells and shortens and thus brings its two ends nearer together.

When repeated shocks are given, wave follows wave of nervous impulse, muscle impulse, and visible contraction ; but the last do not keep distinct, they are fused into the continued shortening which we call tetanus. 


\section{SEC. 3. THE NATURE OF THE CHANGES THROUGH WHICH AN ELECTRIC CURRENT IS ABLE TO GENE- RATE A NERVUUS IMPULSE.}

\section{Action of the Constant Current.}

$\$ 69$. In the preceding account, the stimulus applied in order to give rise to a nervous impulse has always been supposed to be an induction-shock, single or repeated. This choice of stimulus has been made on account of the almost momentary duration of the induced current. Had we used a current lasting for some considerable time, the problems before us would have become more conplex, in consequence of our having to distinguish between the events taking place while the current was passing through the nerve, from those which occurred at the moment when the current was thrown into the nerve, or at the moment when it was shut off from the nerve. These complications do arise when, instead of employing the induced current as a stimulus, we use a constant current, i.e. when we pass through the nerve (or muscle) a current direct from the battery, without the intervention of any induction-coil.

Before making the actual experiment, we might, perhaps, naturally suppose that the constant current would act as a stimulus throughout the whole time during which it was applied; that, so long as the current passed along the nerve, nervous impulses would be generated, and that these would throw the muscle into something at all events like tetanus. And under certain conditions this does take place; occasionally it does happen that at the noment the current is thrown into the nerve the muscle of the musclenerve preparation falls into a tetanus, which is continued until the current is shut off; but such a result is exceptional. In the vast majority of cases what happens is as follows. At the moment that the circuit is made, the moment that the current is thrown into the nerve, a single twitch, a simple contraction, the so-called making contraction, is witnessed; but after this has passed away 
the muscle remains absolutely quiescent in spite of the current continuing to pass through the nerve, and this quiescence is maintained until the circnit is broken, until the current is shut off from the nerve, when another simple contraction, the socalled breaking contraction, is observed. The mere passage of a constant current of uniform intensity through a nerve does not, under ordinary circumstances, act as a stimulus generating a nervous impulse; such an impulse is only set up when the current either falls into or is shut off from the nerve. It is the entrance or the exit of the current, and not the continuance of the current, which is the stimulus. The quiescence of the nerve and muscle during the passage of the current is, however, dependent on the current remaining uniform in intensity or at least not being surdenly increased or diminished. Any sufficiently sudden and large increase or diminution of the intensity of the current will act like the entrance or exit of a current, and, by generating a nervous impulse, give rise to a contraction. If the intensity of the current, however, be very slowly and gradually increased or diminished, a very wide range of intensity may be passed through without any contraction being seen. It is the sudden change from one condition to another, and not the condition itself, which causes the nervous impulse.

In many cases, both a 'making' and a 'breaking' contraction, each a simple twitch, are observed, and this is perhaps the commonest event; but when the current is very weak, and again when the current is very strong, either the breaking or the making contraction may be absent, i.e. there may be a contraction only when the current is thrown into the nerve, or only when it is shut off from the nerve.

Under ordinary circumstances the contractions witnessed with the constant current, either at the inake or at the break, are of the nature of a 'simple' contraction, but, as has already been said, the application of the current may give rise to a very pronounced tetanus. Such a tetanus is seen sometimes when the current is made, lasting during the application of the current, sometimes when the current is broken, lasting some time after the current has been wholly removed from the nerve. The former is spoken of as a 'making,' the latter as a 'breaking' tetanus. But these exceptional results of the application of the constant current need not detain us now.

The great interest attached to the action of the constant current lies in the fact that during the passage of the current, in spite of the absence of all nervous impulses, and, therefore, of all muscular contractions, the nerve is for the time both between and on each side of the electrodes profoundly modified in a most peculiar manner. This modification, important both for the light it throws on the generation of nervous impulses and for its practical applications, is known under the name of eleetrotonus. 
57. Electrotonus. The marked feature of the electrotonic condition is that the nerve, though apparently quiescent, is changed in respect to its irritability; and that in a different way in the neighbourhood of the two electrodes respectively.

Suppose that on the nerve of a muscle-nerve preparation are placed two (non-polarizable) electrodes (Fig. 21, $u, k$ ), connected with a battery and arranged with a key so that a constant current tan at pleasure be thrown into or shut off from the nerve. This constant current, whose effects we are about to stndy, may be called the 'polarizing current.' Let $a$ be the positive electrode or anode, and $k$ the negative electrode or kathode, both placed at some distance from the muscle, and also with a certain interval between each other. At the point $x$ let there be applied a pair of electrodes connected with an induction-coil. Let the muscle further be connected with a lever, so that its contractions can be recorded, and their amount measured. Before the polarizing current is thrown into the nerve, let a single induction-shock of known intensity (a weak one being chosen, or at least not one which would cause in the muscle a maximum contraction) be thrown in at $x$. A contraction of a certain amount will follow.
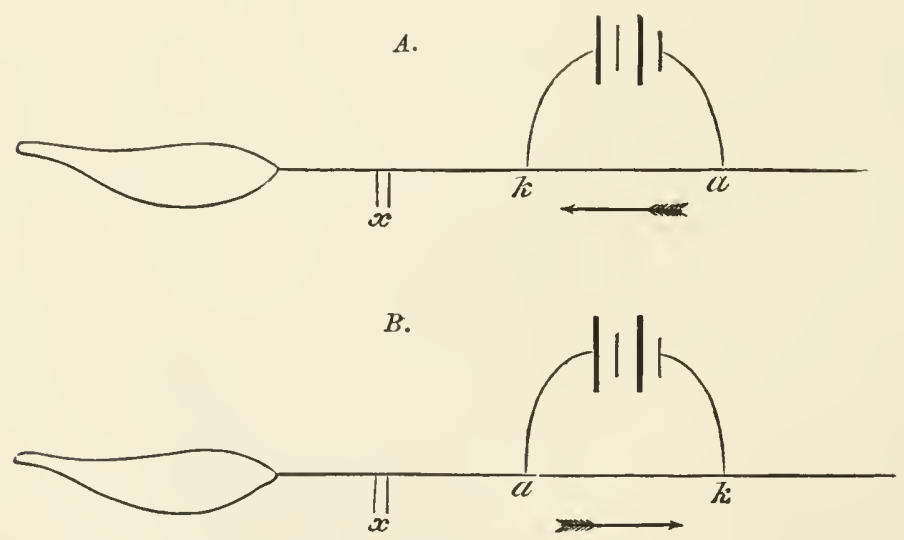

Fig. 21. Muscle-xerve Preparations, with the nerve exposed in $A$ to a descending and in $B$ to an ascending constant current.

In each $a$ is the anode, $k$ the kathode of the constant current. $x$ represents the spot where the induction-shocks used to test the irritability of the nerve are sent in.

That contraction may be taken as a measure of the irritability of the nerve at the point $x$. Now, let the polarizing current be thrown in, and let the kathode or negative pole be nearest the muscle, as in Fig. $21 A$, so that the current passes along the nerve in a direction from the central nervous system towards the muscle; such a current is spoken of as a desccnding one. The entrance of the prolarizing current into the nerve will produce 
a 'making' contraction; this we may neglect. If while the current is passing, the same induction-shock as before be sent through $x$, the contraction which results will be found to be greater than on the former occasion. If the polarizing current be now shut off, a 'breaking' contraction will probably be produced ; this also we may neglect. If now the point $x$, after a short interval, be again tested with the same induction-shock as before, the contraction will be no longer greater, but of the same amount, or perhaps not so great, as at first. During the passage of the polarizing current, therefore, the irritability of the nerve at the point $x$ has been temporarily inereased, since the same shock applied to it causes a greater contraction during the presence than in the absence of the current. But this is only true so long as the polarizing current is a descending one, so long as the point $x$ lies on the side of the kathode. On the otler hand, if the polarizing current had been an ascending one, with the anode or positive pole nearest the nuscle, as in Fig. $21 B$, the irritability of the nerve at $x$ wonld have been found to be diminished instead of increased by the polarizing current; the contraction obtained during the passage of the constant current would be less than before the passage of the current, or might be absent altogether, and the contraction after the current had been shut off would be as great or perhaps greater than before. That is to say, when a constant current is applied to a nerve, the irritability of the nerve between the polarizing electrodes and the muscle is, during the passage of the current, increased when the kathode is nearest the muscle (and the polarizing current descending), and diminished when the anode is nearest the muscle (and the polarizing current ascending). The same result, mutrtis mutandis, and with some qualifications which we need not discuss, would be gained if $x$ were placed not between the muscle and the polarizing current, but on the far side of the latter. Hence, it may be stated generally that during the passage of a constant current through a nerve, the irritability of the nerve is increased in the region of the kathode, and diminished in the region of the anode. The changes in the nerve which give rise to this increase of irritability in the region of the kathode are spoken of as hatelectrotonus, and the nerve is said to be in a katelectrotonic condition. Similarly the changes in the region of the anode are spoken of as anelectrotonus, and the nerve is said to be in an anelectrotonic condition. It is also often usual to speak of the katelectrotonic increase, and anelectrotonic decrease of irritability.

This law remains true whatever be the mode adopted for determining the irritability. The result holds good not only with a single induction-shock, but also with a tetanizing interrupted current, with chemical and with mechanical stimuli. It further appears to hold good not only in a dissected nerve-muscle preparation, but also in the intact nerves of the living body. The 
increase and decrease of irritability are most marked in the immediate neighbourhood of the electrodes, bnt spread for a considerable distance in each direction in the extrapolar regions. The same modification is not confined to the extrapolar region, but exists also in the intrapolar region. In the intrapolar region there must be of course a neutral or indifferent point, where the katelectrotonic increase merges into the anelectrotonic decrease, and where, therefore, the irritability is unclianged. When the polarizing current is a weak one, this indifferent point is nearer the anode than the kathode, but as the polarizing eurrent increases in intensity, draws nearer and nearer the kathode (see Fig. 22).

The amount of increase and decrease is dependent: (1) On the strength of the current, the stronger current up to a certain limit producing the greater effect. (2) On the irritability of the nerve, the more irritable, better conditioned nerve being the more affected by a current of the same intensity.

In the experiments just described the increase or decrease of irritability is taken to mean that the same stimulus starts in the one case a larger or more powerful, and in the other case a smaller or less energetic impulse; but we have reason to think that the mere propagation or conduction of impulses started elsewhere is also affected by the electrotonic condition. At all events anelectrotonus appears to offer an obstacle to the passage of a nervous impulse.

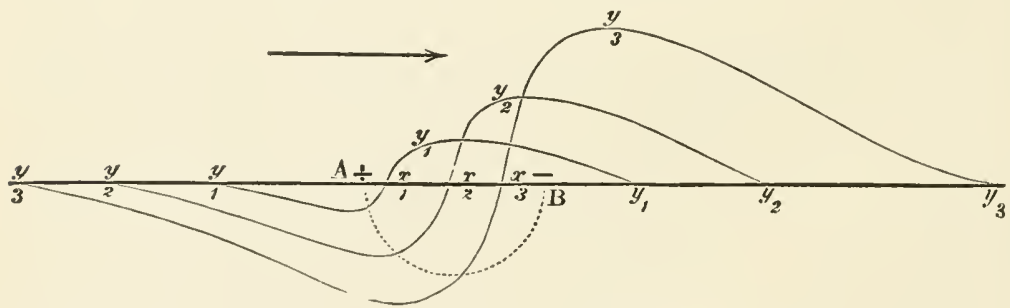

Fig. 22. Diagram illustrating tieVariations of Irritability during Electrotonus, with P'olabizing Currents of Increasing Intexsity (from l'fliger).

The anode is supposed to be placed at $A$, the kathode at $B ; A B$ is consequently the intrapolar district. In each of the three curves, the portion of the curve below the base line represents diminished irritability, that above, increased iritability. $y_{1}$ represents the effect of a weak current; the indifferent point $x_{1}$ is near the anode $\Lambda$. In $y_{2}$, a stronger current, the indifferent point $x_{2}$ is nearer the kathole 13, the diminution of irritability in anelectrotonus and the increase in katelectrotouns being greater than in $y_{1}$; the effert also spreads for a greater distance aloug the extrapolar regions in both directions. In $y_{3}$ the same events are seen to be still nore narked.

\$71. Electrotonic Currents. During the passage of a constant current through a nerve, variations in the electric currents belonging to the nerve itself may be ohserved; and these variations lave certain relations to the variations of the irritability of the nerve. Thus, if a constant current, supplied by the battery $P$ (Fig. 23), be applied 
to a piece of uerve by means of two non-polarizable electrodes $p, p^{\prime}$, the "currents of rest" obtainable from various points of the nerve will be different during the passage of the polarizing current from those which were manifest before or after the current was applied; and, moreover, the changes in the nerve-currents produced by the polarizing current will not be the same in the neighbourhood of the anode $(p)$ as those in the neighbourhoud of the kathode $\left(\gamma^{\prime}\right)$. Thus let $G^{\prime}$ and $I /$ be two galvanometers so connected with the two ends of the nerve as to afford good and clear evidence of the "currents of rest." Before the polarizing current is thrown into the nerve, the needle of $H$ will occupy a position indicating the passage of a current of a certain intensity from $h$ to $h^{\prime}$ through the galvanometer (from the positive longitucinal surface to the negative cut end of the nerve), the circuit being completed by a current in the nerve from $h$ to $h$, i.e. the current

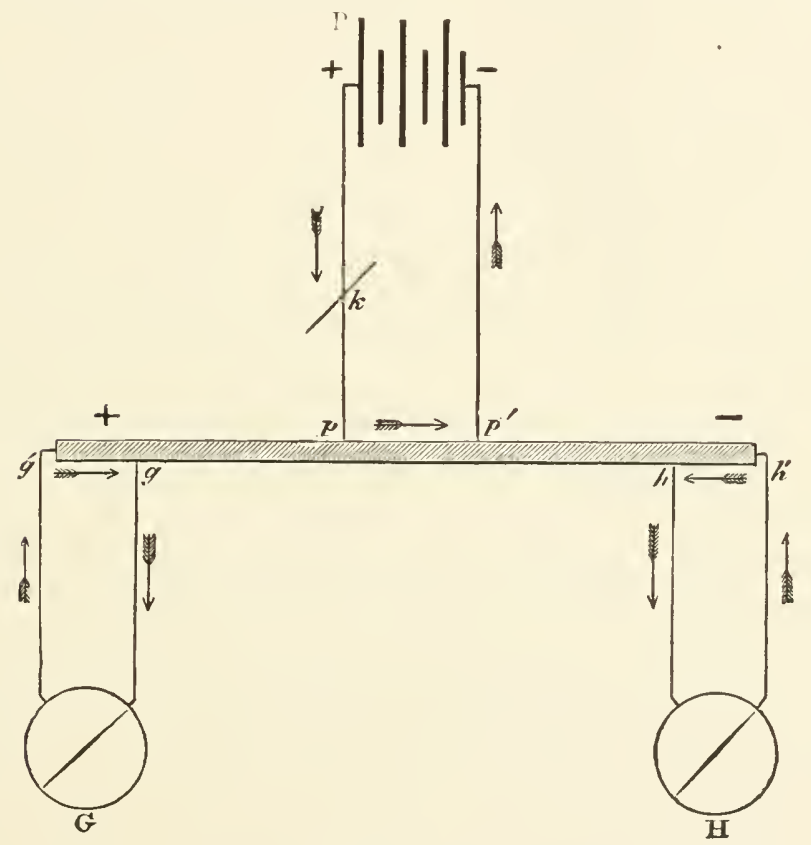

Fig. 23. Diagram illestrativg Electrotonic Currents.

$I$ the polarizing battery, with $k$ a key, $p$ the anode, and $p^{\prime}$ the kathode. At the left end of the piece of nerve the natural current flows throngh the galvanometer $G$ from $g$ to $q^{\prime}$, in the direction of the arrows; its direction, therefore, is the same as that of the polarizing current ; consequently it appears increased, as indicated by the sign + . The current at the other end of the piece of nerve, from $h$ to $h^{\prime}$, through the galvanometer $H$, flows in a contrary direction to the polarizing current; it consequently appears to be diminished, as indicated by the sign -

N. B. For simplicity's sake, the polarizing current is here supposed to be thrown in at the middle of a piece of nerve, and the galvanometer placed at the two ends. Of course it will be understood that the former may be thrown in auywere, and the latter connected with any two pairs of points which will give currents. 
will flow in the direction of the arrow. Similarly the needle of $G$ will by its deflection indicate the existence of a current flowing from $g$ to $g^{\prime}$ through the galvanometer, and from $g^{\prime}$ to $g$ through the nerve, in the direction of the arrow.

At the instant that the polarizing current is thrown into the nerve at $p p^{\prime}$, the currents at $g g^{\prime}, h h^{\prime}$ will unlergo a "negative variation ;" that is, the nerve at each point will exhibit a "current of action" correspond. ing to the nervous impulse, which, at the making of the polarizing current, passes in both directions along the nerve, and may cause a contraction in the attached muscle. The current of action is, as we have' seen, of extremely short duration: it is over and gone in a small fraction of a second. It therefore nust not be confounded with it permanent effect, which, in the case we are dealing with, is observed in both galvanometers. 'This effect, which is dependent on the direction of the polarizing current, is as follows: Supposing that the polarizing current is flowing in the direction of the arrow in the figure, that is, passes in the nerve from the positive electrode or anode $p$ to the negative electrode or kathode $p^{\prime}$, it is found that the current through the galvanometer $G$ is increased, while that through $H$ is diminished. The polarizing current has caused the apjearance in the nerve outsicle the electrodes of a current, having the same direction as itself, called the 'electretonic' current; and this electrotonic current adds to, or takes away from, the natural nerve-current or "current of rest," according as it is flowing in the same direction as that, or in an opposite direction.

The strength of the electrotonic cument is dependent on the strength of the polarizing current, and on the length of the intrapolar recrion, which is exposed to the polarizing current. When a strung polarizing current is used, the electromotive force of the electrotonic current may be much greater than that of the natural nerve-current.

The strength of the electrotonic current varies with the irritability, or vital condition of the nerve, being greater with, the more irritable nerve; and a dead nerve will not manifest electrotonic currents. Moreover, the propagation of the current is stopped by a ligature, or by crushing the nerve.

We may speak of the conditions which give rise to this electrotonic current as a physical electrotonus analogous to that physiological electrotonus, which is made known by variations in irritability. The physical electrotonic current is probably due to the escape of the polarizing current along the nerve under the peculiar conditions of the living nerve; but we must not attempt to enter here into this difficult subject, or into the allied question as to the exact connection between the physical and the pliysiologrical electrotonus, though there can be little doubt that the latter is dependent on the former.

\$ 72. These variations of irritability at the kathode and anode respectively, thus brought about by the action of the constant current, are interesting theoretically, becanse we may trace a connection between them and the nervous impulse which is the result of the making or breaking of a constant current.

For we have evidence that a nervous impulse is generated when a portion of the nerve passes suddenly from a normal 
condition to a state of katelectrotonus, or from a state of anelectrotonus back to a normal condition; but that the passage from a normal condition to anelectrotonus or from katelectrotonus back to a normal condition is unable to generate an impulse. Hence, when a constant current is 'made,' the impulse is generated only at the kathode where the nerve passes suddenly into katelectrotonus; when the current, on the other hand, is 'broken,' the impulse is generated only at the anode where the nerve passes suddenly back from anelectrotonus into a nol'mal condition. We have an indirect proof of this in the facts to which we drew attention a little while back, viz. that a contraction sometimes occurs at the 'breaking' only, sometimes at the 'nnaking' only of the constant current, sometimes at both. For it is found that this depends partly on the strength of the current in relation to the irritability of the nerve, partly on the direction of the current, whether ascending or descending; and the results obtained with strong, medium and weak descending and ascending currents have been stated in the form of a "law of contraction.' We need not enter into the details of this 'law' but will merely say that the results which it formulates are best explained by the hypothesis just stated. We may add that when the constant current is applied to certain structures composed of plain muscular fibres, whose rate of contraction we have seen to be slow, the making contraction may be actually seen to begin at the kathode and travel towards the anode, and the breaking contraction to begin at the anode and travel thence towards the kathode.

Since in katelectrotonus the irritability is increased, and in anelectrotonus decreased, both the entrance from the normal condition into katelectrotonus, and the return from anelectroton us to the normal condition, are instances of a passage from a lower stage of irritability to a higher stage of irritability. Hence, the phenomena of electrotonus would lead us to the conception that a stimulus in provoking a nervous impulse produces its effect by, in some way or other, suddenly raising the irritability to a higher pitch. But what we are exactly to understand by raising the irritability, what molecular change is the cause of the rise, and how either electric or other stimuli can produce this change, are matters which we cannot discuss here.

Besides their theoretical importance, the phenomena of electrotonus have also a practical interest. When an ascending current is passed along a nerve going to a muscle or group of muscles, the region between the electrodes and the muscle is thrown into anelectrotonus, and its irritability is diminished. If the current be of adequate strength, the irritability may be so much lessened that nervous impulses cannot be generated in that part of the llerve, or cannot pass along it. Hence, by this means the irregular contractions of muscles known as 'cramp' may be abolished. Similarly, by bringing into a condition of anelectrotonus a portion 
of a sensory nerve in which violent impulses are being generated, giving rise in the central nervous system to sensations of pain, the impulses are toned down or wholly abolished, and the pain ceases. So on the other hand we may at pleasure heighten the irritability of a part by throwing it into katelectrotonus. In this way the constant current, properly applied, becomes a powerful remedial means.

Lastly, though we are dealing now with nerves going to muscles, that is to say, with motor nerves only, we mar add that what we have said about electrotonus and the development of nervous impulses by it appears to apply equally well to sensory nerves.

s3. In a general way muscular fibres behave towards an electric current very much as do nerve fibres; but there are certain important differences.

In the first place, muscular fibres, devoid of nerve fibres, are much more readily thrown into contractions by the breaking and making of a constant current than by the more transient induction-shock; the muscular substance seems to be more sluggish than the nervous substance and requires to be acted upon for a longer time. This fact may be made use of, and indeed is in medical practice made use of, to determine the condition of the nerves supplying a muscle. If the intramuscular nerves be still in good condition, the muscle as a whole responds readily to single induction-shocks because these can act upon the intramuscular nerves. If these nerves on the other hand have lost their irritability, the muscle does not respond readily to single inductionshocks, or to the interrupted current, but can still easily be thrown into contractions by the constant curent.

In the second place, while in a nerve no impulses are as a rule generated during the passage of a constant current, between the break and the make, provided that it is not too strong, and that it remains uniform in strength, in an urarized muscle on the other hand, even with moderate and perfectly uniform currents, a kind of tetanus or apparently a series of rhythmically rej eated contractions is rery frequently witnessed during the passage of the current. The exact nature and cause of these phenomena in muscle, we nust not however discuss here. 


\section{SEC. 4. THE MUSCLE-NERVE PREPARATION AS A MACHINE.}

$\$ 74$. The facts described in the foregoing sections shew that a muscle with its nerve may be justly regarded as a machine which, when stimulated, will do a certain amount of work. But the actual amount of work which a muscle-nerve preparation will do is found to depend on a large number of circumstances, and consequently tu vary within very wide limits. These variations will be largely determined by the condition of the muscle and nerve in repect to their nutrition; in other words, by the degree of irritability manifested by the muscle or by the nerve or by both. But quite apart from the general influences affecting its nutrition and thus its irritability, a muscle-nerve preparation is affected, as regards the amount of its work, by a variety of other circumstances, which we may briefly consider here, reserving to a succeeding section the study of variations in irritability.

We may here remark that a muscle may be thrown into contraction under two different conditions. In the one case it may be free to shorten: by the lifting of the weight or otherwise, the one end of the muscle may approach the other; and this is the kind of contraction which we have taken, and may take as the ordinary one. But the muscle may be placed under such circumstances that, when it contracts, the one end is not brought nearer to the other, the muscle remains of the same length, and the effect of the contraction is manifested only as an increased strain. In this latter case, the contraction is spoken of as an "isometric," in the former case as an "isotonic" contraction.

The influence of the nature and mode of application of the stimulus. When we apply a weak stimulus, a weak inductionshock, to a nerve, we get a small contraction, a slight shortening of the muscle; when we apply a stronger stimulus, a stronger induction-shock, we get a larger contraction, a greater shortening of the muscle. We take, other things being equal, the amount of contraction of the muscle as a measure of the nervous impulse, and say that in the former case a weak or slight, in the latter case a stronger or larger nervous impulse has been generated. Now the muscle of the muscle-nerve preparation consists of many muscular fibres and the nerve of many nerve fibres; and we may 
fairly suppose that in two experinents we may in the one experiment bring the induction-shock or other stimulus to bear on a few nerve fibres only, and in the other experiment on many or eren all the fibres of the nerve. In the former case only those muscular fibres in which the few nerve fibres stimulated end will be thrown into contraction, the other's remaining quiet, and the shortening of the muscle as a whole, since only a few fibres take part in it, will necessarily be less than when all the fibres of the nerve are stimulated and all the fibres of the muscle contract. 'That is to say, the amount of contraction will depend on the number of fibres stimulated. For simplicity's sake however we will in what follows, except when otherwise indicated, suppose that when a nerve is stimulated, all the fibres are stimulated and all the muscular fibres contract.

This being premised, we may say that, other things being equal, the magnitude of a nervous impulse, and so the magnitude of the ensuing contraction, is directly dependent on what we may call the strength of the stimulus. Thus taking a single inductionshock as the most manageable stimulus, we find that if, before we begin, we place the secondary coil (Fig. 4, sc.) a long way off the primary coil $p r$. c., no visible effect at all follows upon the discharge of the induction-shock. The passage of the momentary weak current is either unable to produce any nervous impulse at all, or the weak nervous impulse to which it gives rise is unable to stir the sluggish muscular substance to a visible contraction. As we slide the secondary coil towards the primary, sending in an induction-shock at each new position, we find that at a certain distance between the secondary and primary coils, the muscle responds to each induction-shock ${ }^{1}$ with a contraction which makes itself visible by the slightest possible rise of the attached lever. This position of the coils, the battery remaining the same and other things being equal, marks the minimul stimulus giving rise to the minimal contraction. As the secondary coil is brought nearer to the primary, the contractions increase in height correspunding to the increase in the intensity of the stimulus. Very soon however an increase in the stimulus caused by further sliding the secondary coil over the primary fails to cause any increase in the contraction. This indicates that the maximal stimulus giving rise to the maximal contraction has been reached; though the shocks increase in intensity as the secondary coil is pushed further and further over the primary, the contractions remain of the same height, until fatigne lowers them.

With single induction-shocks then the mnscular contraction, and by inference the nervous impulse, increases with an increase in the intensity of the stimulus, between the limits of the minimal

1 In these experiments either the breaking or making shock must he used, not sonseimes one and sometimes the other, for, as we have stated, the two kinds of slock differ in efficiency, the breaking being the most potent. 
and maximal stimuli; and this dependence of the nervous impulse, and so of the contraction, on the strength of the stimulus may be observed not only in electric but in all kinds of stimuli.

It may here be remarked that in order for a stimulus to be effective, a certain abruptness in its action is necessary. Thus as we have seen the constant current when it is passing through a nerve with uniform intensity does not give rise to a nervous impulse, and indeed it may le increased or diminished to almost any extent without generating nervous impulses, provided that the change be made gradually enough; it is only when there is a sudden change that the current becomes effective as a stimulus. And the reason why the breaking induction-shock is more potent as a stimulus than the making shock is because as we have seen $(\$ 4)$ the current which is induced in the secondary coil of an induction-machine at the breaking of the prinary circuit, is more rapidly developed, and has a sharper rise than the current which appears when the primary circuit is made. Similarly a sharp tap on a nerve will produce a contraction, when a gradually increasing pressure will fail to do so, and in general the efficiencr of a stimulus of any kind will depend in part on the suddenness or abruptness of its action.

A stimulus, in order that it may be effective, must have an action of a certain duration, the time necessary to produce an effect varying according to the strength of the stimulus and being different in the case of a nerve from what it is in the case of a muscle. It would appear that an electric current applied to a nerve must have a duration of at least about 0015 sec. to cause any contraction at all, and needs a longer time than this to produce its full effect. A muscle fibre apart from its nerve fibre requires a still longer duration of the stimulus, and hence, as we have already stated, a muscle poisoned by urari, or which has otherwise lost the action of its nerves, will not respond as readily to inductionshocks as to the more slowly acting, breaking and making of a constant current.

In the case of electric stimuli, the same current will produce a stronger contraction when it is sent along the nerve than when it is sent across the nerve; indeed it is maintained that a current which passes through a nerve in an absolutely transverse direction is powerless to generate impulses.

$\$ 75$. We have seen that when single stimuli are repeated with sufficient frequency, the individual contractions are fused into tetanus; as the frequency of the repetition is increased, the individual contractions are less obvious on the curve, until at last we get a curve on which they seem to be entirely lost and which we may speak of as a complete tetanus. By such a tetanus a much greater contraction, a much greater shortening of the muscle is of course obtained than by single contractions.

The exact frequency of repetition required to produce com- 
plete tetanus will depend chiefly on the length of the individual contractions, and this varies in different animals, in different muscles of the same animal, and in the same muscle under different conditions. In a cold blooded animal a single contraction is as a rule more prolonged than in a warm blooded animal, and tetanus is consequently produced in the former by a less frequent repetition of the stimulus. A tired muscle has a longer contraction than a fresh musele, and hence in many tetanus eurves the individual contractions, easily recognised at first, disappear later on, owing to the individual contractions being lengthened out by the exhaustion caused by the tetanus itself. In many animals, e.g. the rabbit, some muscles (such as the adductor magnus femoris) are pale, while others (such as the semitendinosus) are red. The red muscles are not only more richly supplied with blood vessels, but the muscle substance of the fibres contains more hamoglobin than the pale, and there are other structural differences. Now the single contraction of one of these red muscles is more prolonged than the single contraction of one of the pale muscles produced by the same stimulus. Hence the red muscles are thrown into complete tetanus with a repetition of much less frequency than that required for the pale muscles. Thus, ten stimuli in a second are quite sufficient to throw the red unscles of the rabbit into complete tetanus, while the pale muscles require at least twenty stimuli in a second.

Su long as signs of the individual contractions are visible on the curve of tetanus it is easy to recognise that each stimulation produces one of the constituent single contractions, and that the number so to spaak of the vibrations of the muscle making up the tetanus corresponds to the number of stimulations; but the question whether, when we increase the number of stimulations beyond that necessary to produce a complete tetanus, we still inerease the number of constituent single contractions is one not so easy to answer. And connected with this question is another difficult one. What is the rate of repetition of single contractions making up those tetanic contractions which as we have said are the kind of contractions by which the voluntary, and indeed other natural, movements of the body are carried out? What is the evidence that these are really tetanic in character?

When a muscle is thrown into tetanus, a more or less musical sound is produced. This may be heard by applying a stethoscope directly over a contracting muscle, and a similar sound but of a more mixed origin and less trustworthy may be heard when the masseter muscles are forcibly contracted or when a finger is placed in the ear, and the muscles of the same arm are contracted.

When the stethoscope is placed over a muscle, the nerve of which is stimulated by induction-shocks repeated with varying frequency, the note heard will vary with the frequency of the shocks, being of ligher pitch with the more frequent shocks. 
How it has been thought that the vibrations of the muscle giving rise to the "muscle sound" are identical with the single contractions making up the tetanus of the muscle. And since, in the human body, when a muscle is thrown into contraction in a voluntary effort, or indeed in any of the ordinary natural movements of the body, the fundamental tone of the sound corresponds to about 19 or 20 vibrations a second, it has been concluded that the contraction taking place in such cases is a tetanus of which the individual contractions follow each other about 19 or 20 times a second. But investigations seem to shew that the vilurations giving rise to the muscle sound do not really correspond to the shortenings and relaxations of the individual contractions, and that the pitch of the note cannot therefore be taken as an indication of the number of single contractions making up the tetanus; indeed, as we shall see in speaking of the sounds of the heart, a single muscular contraction may produce a sound which though differing from the sound given out during tetanus has to a certain extent musical characters. Nevertheless the special characters of the muscle sound given out by muscles in the natural movements of the body may be taken as shewing at least that the contractions of the muscle in these movements are tetanic in nature, and the similarity of the note in all the voluntary efforts of the body and indeed in all movements carried out by the central nervous system is at least consonant with the view that the repetition of single contractions is of about the same frequency in all these movements. What that frequency is, and whether it is exactly identical in all these movements, has not at present leen clearly determined; though certain markings on the myrographic tracings of these movements and other facts seem to indicate that it is about 12 a second.

\$76. The Influenee of the Load. It might be imagined that a mnscle, which, when loaded with a given weight, and stimulated by a current of a given intensity, had contracted to a certain extent, would only contract to half that extent when loaded with twice the weight and stimulated with the same stimulus. Such however is not necessarily the case; the height to which the weight is raised may be in the second instance as great, or even greater, than in the first. That is to say, the resistance offered to the contraction actually angments the contraction, the tension of the muscular fibre increases the facility with which the explosive changes resulting in a contraction take place. And we have other evidence that anything which tends to stretch the muscular fibres, that any tension of the muscular fibres, whether during rest or during contraction, increases the metabolism of the muscle. There is, of course, a limit to this favourable action of the resistance. As the load continues to be increased, the height of the contraction is diminished, and at last a point is reached at which the muscle is mable (even when the stimulus chosen is the strongest possible) to lift the load at all. 
In a muscle viewed as a machine we have to deal not merely with the height of the contraction, that is with the amount of shortening, but with the work done. And this is measured-by multiplying the number of units of height to which the load is raised into the number of units of weight of the load. Hence it is obvious from the foregoing observations that the work done must be largely dependent on the weight itself. Thus there is a certain weight of load with which in any given muscle, stimulated by a given stimulus, the most work will be done; as may be seen from the following example:

$\begin{array}{llrrrrrr}\text { Load, in grammes … . . . . . } & 0 & 50 & 100 & 150 & 200 & 250 \\ \text { Height of contractions in millimeters } & 14 & 9 & 7 & 5 & 2 & 0 \\ \text { Work done, in gram-millimeters . . . } & 0 & 450 & 700 & 750 & 400 & 0\end{array}$

$\$ 77$. The Influcnce of the Size and Form of the Muscle. Since all known muscular fibres are much shorter than the wave-length of a contraction, it is obvious that the longer the fibre, the greater will be the shortening caused by the same contraction wave, the greater will be the height of the contraction with the same stimulus. Hence in a muscle of parallel fibres, the height to which the load is raised as the result of a given stimulus applied to its nerve, will depend on the length of the fibres, while the maximum weight of load capable of being lifted will depend on the number of the fibres, since the load is distributed among them. Of two muscles therefore of equal length (and of the same quality) the most work will be done by that which has the larger number of fibres, that is to say, the fibres being of equal width, which has the greater sectional area; and of two muscles with equal sectional areas, the most work will he done by that which is the longer. If the two muscles are unequal both in length and scctional area, the work done will be the greater in the one which has the larger bulk, which contains the greater number of cubic units. In speaking therefore of the work which can be done by a muscle, we may use as a standard a cubic unit of bulk, or, the specific gravity of the muscle being the same, a unit of weight.

We learn then from the foregoing maragraphs that the work done, by a muscle-nerve preparation, will depend, not only on the activity of the nerve and muscle as determined by their own irritability, but also on the character and mode of application of the stimulus, on the kind of contraction (whether a single spasm, or a slowly repeated tetanus or a rapidly repeated tetanus) on the load itself, and on the size and form of the muscle. Taking the most favourable circumstances, viz. a well-nourished, lively prejaration, a maximum stimulus causing a rapid tetanus and an appropriate load, we may determine the maximum work done by a given weight of muscle, say one gramme. This in the case of the muscles of the frog has been estimated at about four grammeters for one gramme of muscle. 


\section{SEC. 5. THE CIRCUMSTANCES WHICH DETERMINE THE DEGREE OF IRRITABILITY OF MUSCLES AND NERVES.}

\$78. A muscle-nerve preparation, at the time that it is removed from the body, possesses a certain degree of irritability, it responds by a contraction of a certain amount to a stimulus of a certain strength, applied to the nerve or to the muscle. After a while, the exact period depending on a variety of circumstances, the same stimulus produces a smaller contraction, i.e. the irritability of the preparation lias diminished. In other words, the muscle, or nerve, or both, have become partially 'exhausted;' and the exhaustion subsequently increases, the same stimulus producing smaller contractions, until at last all irritability is lost, no stimulus however strong producing any contraction, whether applied to the nerve or directly to the muscle; and eventually the muscle, as we lave seen, becomes rigid. The progress of this exhaustion is more rapid in the nerves than in the muscles; for some time after the nerve trunk has ceased to respond to even the strongest stimulus, contractions may be obtained by applying the stimulus directly to the muscle. It is much more rapid in the warm blooded than in the cold blooded aninuals. The muscles and nerves of the former lose their irritability, when removed from the body, after a period varying according to circumstances from a few minutes to two or three hours; those of cold blooded animals (or at least of an amphibian or a reptile) may, under favourable conditions, remain irritable for two, three, or even more days. The duration of irritability in warm blooded animals may, however, be colisiderably prolonged by reducing the temperature of the body before death.

If with some thin body a sharp blow be struck across a muscle which has entered into the later stages of exhaustion, a wheal lasting for several seconds is developed. This wheal appears to be a contraction wave limited to the part struck, and disappearing very slowly, without extending to the neighbouring muscular substance. It has been called 
an 'idio-muscular' contraction, because it may be brought out even when ordinary stimuli have ceased to produce any effect. It may however be accompanied at its beginning by an ordinary contraction. It is readily produced in the living body on the pectoral and other muscles of persons suffering from phthisis and other exhausting diseases.

This natural exhaustion and diminution of irritability in muscles and nerves removed from the body nay be modified both in the case of the muscle and of the nerve, by a variety of circumstances. Similarly, while the nerve and muscle still remain in the body, the irritability of the one or of the other may be modified either in the way of increase or of decrease by certain general intluences, of which the most important are, severance from the central nervous system, and variations in tenperature, in blood supply, and in functional activity.

The Effects of Scverance from the Central Nervous Systcm. When a nerve, such for instance as the sciatic, is divided in situ, in the living body, there is first of all observed a slight increase of irritability, noticeable especially near the cut end; but after a while the irritahility diminishes, and gradually disappears. both the slight initial increase and the subsequent decrease begin at the cut end and advance centrifugally towards the peripheral terminations. This centrifugal feature of the loss of irritalility is often spoken of as the Ritter-Valli law. In a mammal it may be two or three days, in a frog, as many, or even nore weeks, before irritability has disappeared from the nerve trunk. It is maintained in the small (and especially in the intramuscnlar) branches for still longer periods.

This centrifugal loss of imitability is the forerunner in the peripheral portion of the divided nerve of structural changes which proceed in a similar centrifugal manner. In the central portion of the divided nerve these structural changes may be traced as far only as the next node of Ranvier. Beyond this the nerve usually remains in a normal condition. Such a degeneration may be observed to extend down to the very endings of the nerve in the muscle, including the end-plates, but does not at first affect the muscular substance itself. The muscle, though it has lost all its nervous elements, still remains irritable towards stimuli applied directly to itself: an additional proof of the existence of an independent muscular irritability.

If the muscle thus deprivel of its nervous elements be left to itself its irritability, however tested, sonner or later diminishes; but if the muscle be periodically thrown into contractions by artificial stimulation with the constant current, the decline of irritability and attendant loss of nutritive power may he postponed for some considerable time. But so far as onr experience goes at present the artificial stimulation cannot fully replace the natural one, and sooner or later the muscle like the nerve suffers degeneration, loses 
all irritability and ultimately its place is taken by comnective tissue.

For some time the irritability of the muscle, as well towards stimuli applied directly to itself as towards those applied through the impaired nerve, seems to be diminished; but after a while a peculiar condition (to which we have ahready alluded, \$73) sets in, in which the muscle is found to be not easily stimulated by single induction-shocks but to respond readily to the make or break of a constant eurrent. In fact it is saill to become even more sensitive to the latter mode of stimulation than it was when its nerve was intact and functionally active. At the same time it also becomes more irritable towards direct nechanical stimuli, and very frequently fibrillar contractions, more or less rhythmic and apparently of spontaneous origin, though their causation is obscure, make their appearance. This phase of heightened sensitiveness of a muscle, especially to the constant current, appears to reach its maximum, in man at about the seventh week after nervous impulses have ceased, owing to injury to the nerves or nervous centre, to reach the muscle.

$\$ 79$. The influenee of temperature. We have already seen that sudden lieat (and the same might be said of cold when sufficiently intense), applied to a limited part of a nerve or muscle, as when the nerve or muscle is touched with a hot wire, will act as a stimulus. It is however much more difficult to generate nervous or muscular impulses by exposing a whole motor nerve $^{1}$ or muscle to a gradual rise of temperature.

A muscle may be gradually cooled to $0^{\circ} \mathrm{C}$. or below without any contraction being caused; hut when it is heated to a limit, which in the case of frog's muscles is about $45^{\circ}$, of mammalian muscles about $50^{\circ}$, a sudden change takes place: the muscle falls, at the limiting temperature, into a rigor mortis, which is initiated by a forcible contraction or at least shortening.

Modsrate wamth, $e . g$. in the frog an increase of temperature up to somewhat below $45^{\circ} \mathrm{C}$., favours both museular and nervous irritability. All the molecular processes are hastened and facilitated: the contraction is for a given stimulus greater and more rapid, $i . e$. of shorter duration, and nervous impulses are generated more readily by slight stimuli. Owing to the quickening of the chemic il changes, the supply of new material may prove insufficient, hence muscles and nerves removed from the body lose their irritability more rapidly at a high than at a low temperature.

The gradual application of cold to a nerve produces effects which differ according to the kind of stimulus employed in testing the condition of the nerve, but it may be stated in general that a low temperature, especially one near to $0^{\circ}$, slackens all the molecular processes, so that the wave of nervous impulse is lessened and prolonged, the velocity of its passage being much diminished,

1 The action of cold and heat on sensory nerves will be considered in the later portion of the work. 
e.g. from 28 metres to 1 metre per sec. At about $0^{\circ}$, the irritability of the nerve disappears altogether.

When a muscle is exposed to similar cold, $e . g$. to a temperature very little above zero, the contractions are remarkably prolonged; they are diminished in height at the same time, but not in proportion to the increase of their duration. Exposed to a temperature of zero or below, muscles soon lose their irritability, without however undergoing rigor mortis.

$\$$ 80. The influcnce of blood supply. When a muscle still within the body is deprived by any means of its proper blood supply, as when the blood ressels going to it are ligatured, the same gradual loss of irritability and final appearance of rigor mortis are observed as in muscles removed from the body. Thus if the abdominal aorta be ligatured, the muscles of the lower limbs lose their irritability and finally become rigid. So also in systemic death, when the blood supply to the muscles is cut oft by the cessation of the circulation, loss of irritability ensues, and rigor mortis eventually follows. In a human corpse the muscles of the body enter into rigor mortis in a fixed order: first those of the jaw and neck, then those of the trunk, next those of the arms, and lastly those of the legs. The rapidity with which rigor mortis comes on after death varies considerably, being determined both by external circumstances and by the internal conditions of the body. Thus external warmth hastens and cold retards the onset. After great muscular exertion, as in hunted animals, and when death closes wasting diseases, rigor mortis in most cases comes on rapidly. As a general rule it may be said that the later it is in making its appearance, the more pronounced it is, and the longer it lasts ; but there are many exceptions, and when the state is recogniserl as being fundamentally due to a clotting of the muscle substance, it is easy to understand that the amount of rigidity, i.e. the amount of the clot, and the rapidity of the onset, $i . e$. the quickness with which clotting takes place, may vary independently. When rigor mortis has once hecome thoroughly established in a muscle through deprivation of blood, it cannot be removed by any subsequent supply of blood. Mere loss of irritability, even though complete, if stopping short of the actual clotting of the muscle substance, ma be with care removed.

The influence of blood supply cannot be so satisfactorily studied in the case of nerves as in the case of muscles; there can however be little doubt that the effects are analogous.

$\$ 81$. The influcnec of functional activity. This too is more easily studied in the case of muscles than of nerves.

When a muscle within the body is unused, it wastes; when used, it (within certain limits) grows. Both these facts shew that the nutrition of a muscle is favourably affected by its functional activity. Part of this may be an indirect effect of the increased blood supply which occur's when a muscle contracts. When a 
nerve going to a muscle is stimulated, the blood vessels of the muscle dilate. Hence at the time of the contraction nore blood flows through the muscle, and this increased flow continues for some little while after the contraction of the muscle has ceased. But, apart from the blood supply, it is probable that the exhaustion caused by a contraction is immediately followed by a reaction favourable to the nutrition of the muscle; and this is a reason, possibly the chief reason, why a muscle is increased by use, that is to say, the loss of substance and energy caused by the contraction is subsequently more than made up for by increased metabolism during the following period of rest.

A muscle, even within the body, after prolonged action is fatigued, i.e. a stronger stimulus is required to produce the same contraction; in other words, its irritability may be lessened by functional activity. Whether functional activity therefore is injurious or beneficial depends on its amount in relation to the condition of the muscle.

It is worthy of notice that a motor nerve is far less susceptible of being fatigued by artificial stimulation than is a muscle; in fact it seems extremely difficult to tire a nerve by mere stimulation. In an animal poisoned by urari the sciatic nerve nay be stimulated continuously with powerful currents for even several hours and yet remain irritable. So long as the urari is producing its usual effect, the muscles sheltered by it are not thrown into contraction by the stimnlation of the nerve and so are not fatigued; as the effect of the urari passes off, contractions make their appearance in response to the stimulation of the sciatic nerve, shewing that this, in spite of its having been stimulated for so long a time, has not been exhausted. And other experiments point to a similar conclusion. It would seem that the molecular processes constituting a nervous impulse unlike those constituting a muscular contraction, are of such a nature or take place in such a way, that after the development of one impulse the substance of the nerve fibre is at once ready for the development of a second impulse.

The sense of fatigue of which, after prolongert or unusual exertion, we are conscious in our own bodies, is probably of complex origin, and its nature, like that of the normal muscular sense of which we shall have to speak hereafter, is at present not thoronghly understood. It seems to be in the first place the result of changes in the muscles themselves, but is possibly also caused by changes in the nervous apparatus concerned in muscular action, and especially in those parts of the central nervous system which are concerned in the production of voluntary impulses. In any case it cannot be taken as an adequate measure of the actual fatigue of the muscles; for a man who says he is absolutely exhausted may under excitement perform a very large amount of work with his already weary muscles. The will in fact rarely if ever calls forth the greatest contractions of which the muscles are capable. 
Absolute (temporary) exhaustion of the muscles, so that the strongest stimuli produce no contraction, may be produced even within the body by artificial stimulation: recovery takes place on rest. Out of the body absolute exhaustion takes place readily. Here also recovery may take place. Whether in any given case it does occur or not, is determined by the amount of contraction causing the exhaustion, and by the previous condition of the muscle. In all cases recovery is hastened by renewal (natural or artificial) of the blood stream.

The more rapidly the contractions follow each other, the less the interval between any two contractions, the more rapid the exhaustion. A certain number of single induction-shocks repeated rapidly, say every second or oftener, bring about exhaustive loss of irritability more rapidly than the same number of shocks repeated less rapidly, for instance every 5 or 10 seconds. Hence tetanus is a ready means of producing exhaustion.

In exhausted muscles the elasticity is much diminished; the tired muscle returns less readily to its natural length than does the fresh one.

The exhaustion due to contraction may be the result: - Either of the consumption of the store of really contractile material present in the muscle. Or of the accumulation in the tissue of the products of the act of contraction. Or of both of these causes.

The restorative influence of rest, in the case of a muscle removed from the circulation, may be explained by supposing that during the repose, either the internal changes of the tissue manufacture new explosive material out of the comparatively raw material already present in the fibres, or the directly hurtful products of the act of contraction undergo changes by which they are converted into comparatively inert bodies. A stream of fresh blood may exert its restorative influence not only ly quickening the above two events, but also by carrying off the immediate waste products while at the same time it brings new raw material. It is not known to what extent each of these parts is played. That the products of contraction are exhausting in their effects, is shewn by the facts that the injection of a solution of the muscle-extractives in to the ressels of a muscle produces exhaustion, and that exhausted muscles are recovered by the simple injection of inert saline solutions into their blood vessels. But the matter has not yet been fully worked out.

One important element brought by fresh blood is oxygen. This, as we have seen, is not necessary for the carrying ont of the actual contraction, and yet is essential to the maintenance of irritability. The oxygen absorbed by the muscle apparently enters in some peculiar way in to the formation of that complex explosive material the decomposition of which in the act of contraction, though it gives rise to carbonic acid and other products of oxidation, is not in itself a process of direct oxidation. 


\section{SEC. 6. ON SOME OTHER FORMS OF CONTRACTILE TISSUE.}

\section{Plain, Smooth or Unstriated Muscular Tissue.}

$\$$ 82. This, in vertebrates at all events, rarely occurs in isolated masses or muscles, as does striated muscular tissue, but is usually found taking part in the structure of complex organs, such for instance as the intestines; hence the investigation of its properties is beset with many difficulties.

\$ 83. So far as we know plain muscular tissue in its chemical features resembles striated muscular tissue. It contains albumin, some forms of globulin, and antecedents of myosin which upon the death of the fibres become myosin; for plain muscular tissue after death becomes rigid, losing its extensibility and probably becoming acid, though the acidity is not so narked as in striated muscle. Kreatin has also been found, as well as glycogen, and indeed it seems probable that the whole metabolism of plain muscular tissue is fundamentally the same as that of the striated muscles.

\$ 84. In their general physical features plain muscular fibres also resemble striated fibres, and like them they are irritable and contractile; when stimulated they contract. The fibres vary in natural length in different situations, those of the blood vessels for instance being shorter and stouter than those of the intestine; but in the same situation the fibres may also be found in one of two different conditions. In the one case the fibres are long and thin, in the other case they are reduced in length, it may be to one half or even to one third, and are correspondingly thicker, broader and less pointed at the ends, their total bulk remaining unaltered. In the former case they are relaxed or elongated, in the latter case they are contracted.

The facts of the contraction of plain muscular tissue may be studied in the intestine, the muscular coat of which consists of an outer thin sheet composed of fibres and bundles of fibres disposed longitudinally and of an inner much thicker sheet of fibres disposed circularly ; in the ureter a similar arrangement of two coats obtains. If a mechanical or electrical (or indeed any other) stimulus be 
brought to bear on a part of a fresh living still warm intestine (the small intestine is the best to work with) a circular contraction is seen to take place at the spot stimulated; the intestine seems nipped in ringwise, as if tied romnd with an invisible cord; and the part so constricted, previously vascular and red, becomes pale and bloodless. The individual fibres of the circular coat in the region stimnlated have each become shorter, and the total effect of the shortening of the multitude of fibres all having the same circular disposition is to constrict or narrow the lumen or tube of the intestine. The longitudinally disposed fibres of the outer longitudinal coat in a similar manner contract or shorten in a longitudinal direction, but this coat being relatively much thinner than the circular coat, the longitudinal contraction is altogether overshadowed by the circular contraction. A similar mode of contraction is also seen when the ureter is similarly stimulated.

The contraction thus indnced is preceded by a very long latent period and lasts a very considerable time, in fact several seconds, after which relaxation slowly takes place. We may say then that over the circularly dispersed fibres of the intestine (or ureter) at the spot in question there has passed a contraction-wa ve remarkable for its long latent period and for the slowness of its development, the wave being propagated from fibre to fibre. From the spot so directly stimulated, the contraction may pass also as a wave (with a length of $1 \mathrm{~cm}$. and a velocity of from 20 to 30 millimetres a second in the ureter), along the circular coat both upwards and downwards. The longitudinal fibres at the spot stimulated are as we have said also thrown into contractions of altogether similar character, and a wave of contraction may thus also travel longitudinally along the longitudinal coat both upwards and downwards. It is evident however that the wave of contraction of which we are now speaking is in one respect different from the wave of contraction treated of in dealing with striated muscle. In the latter case the contraction-wave is a simple wave propagated along the individual fibre and starting from the end-plate or, in the case of direct stimulation, from the part of the fibre first affected by the stimulus; we have no evidence that the contraction of one fibre can communicate contraction to neighbouring fibres or indeed in any way influence neighbouring fibres. In the case of the intestine or ureter, the wave is complex, bing the sum of the contractionwaves of several fibres engaced in different phases and is propragated from fibre to fibre, both in the direction of the fibres, as when the whole circumference of the intestine is engaged in the contraction, or when the wave travels longitudinally along the longitudinal coat, and also in a direction at right angles to the axes of the fibres, as when the contraction-wave travels lengthways along the circular coat of the intestine, or when it passes across a breadth of the longitudinal coat; that is to say, the changes leading to contraction are communicated not only in a direct manner across the cement 
substance uniting the fibres of a bundle but also in an indirect manner, probably by means of nerve fibres, from bundle to bundle across the connective tissue between them. Moreover, it is obvious that even the contraction-wave which passes along a single unstriated fibre differs from that passing along a striated tibre, in the very great length both of its latent period and of the duration of its contraction. Hence, much more even than in the case of a striated muscle, the whole of each fibre must be occupied by the contraction-wave, and indeed be in nearly the same phase of the contraction at the same time.

Waves of contraction thus passing along the circular and longitudinal coats of the intestine constitute what is called peristaltic action.

Like the contractions of striated muscle the contractions of plain muscles may be started by stimulation of nerves going to the part, the nerves supplying plain muscular tissue, running for the most part as we have said in the so-called sympathetic system, but being as we shall see ultimately connected with the spinal cord or brain. Here however we come upon an important distinction between the striated skeletal muscles, and the plain muscles of the viscera. As a general rule the skeletal muscles are thrown into contraction only by nervous impulses reaching them along their nerves; spontaneous movements of the skeletal muscles, that is contractions arising out of changes in the muscles themselves are extremely rare, and when they occur are abnormal; so-called 'cramps' for instance, which are prolonged tetanic contractions of skeletal nuscles independent of the will, though their occurrence is largely due to the condition of the muscle itself, generally the result of overwork, are probably actually started by nervous impulses reaching them from without. On the other hand the plain muscles of the viscera, of the intestine, uterus and ureter, for instance, and of the blood vessels very frequently fall into contractions and so carry out movements of the organs to which they belong quite independently of the central nervous system. These organs exhibit 'spontaneous' movements quite apart from the will, quite apart from the central nervous system, and under favourable circumstances continue to do this for some time after they have been entirely isolated and removed from the body. So slight indeed is the connection between the movements of organs and parts supplied with plain muscular fibres, and the will, that these muscular fibres have sometimes been called involuntary muscles; but this name is undesirable since some muscles consisting entirely of plain muscular fibres $(e . g$. the ciliary muscles by which the eye is accommodated for viewing objects at different distances) are directly under the influence of the will, and some muscles composed of striated fibres ( $\bullet . g$. those of the heart) are wholly removed from the influence of the will.

We shall best study however the facts relating to the move- 
ments of parts provided with plain muscular fibres when we come to consider the parts themselves.

Like the skeletal muscles, whose nervous elements have been rendered functionally incapable (\$73), plain muscles are much more sensitive to the making and breaking of a constant current than to induction shocks; a current, when very brief, like that of an induction-shock, produces little or no effect.

The contraction of plain muscular fibres is as we said very slow in its development and very long in its duration. even when started by a momentary stimulus, such as a single induction-shock. The contraction after a stimulation often lasts so long as to raise the question, whether what has been produced is not a single contraction but a tetanus. Tetanus, however, that is the fusion of a series of contractions, seems to be of rare occurrence, though probably it may be induced, in plain muscular tissue; but some of the ends of tetanus are gained by a kind of contraction which, not prominent in skeletal muscle, becomes of great importance in plain muscular tissue, by a kind of contraction called a tonie contraction. The subject is one not without difficulties, but it would appear that a plain muscular fibre may remain for a very considerable time in a state of contraction, the amount of shortening thus maintained being either small or great: it is then said to be in a state of tonic contraction. This is especially seen in the case of the plain muscular tissue of the arteries, and we shall have to return to this matter in dealing with the circulation.

The muscular tissue which enters into the construction of the heart is of a peculiar nature, being on the one hand striated, and on the other in some respects similar to plain muscular tissue, but this we shall consider in dealing with the heart itself.

\section{Ciliary Movement.}

$\$ 85$. Nearly all the movements of the body which are not due to physical causes, such as gravity, the diffusion of liquids \&c., are carried out by muscles, either striated or plain; but some small and yet important effects in the way of movement are produced by the action of cilia, and by those changes of form which are called amœboid. Cilia are generally appendages of epithelial cells.

$\$ 86$. Ciliary action, in the form in which it is most common in mammals and indeed vertebrates, consists in the cilium (i. $\bullet$. the tapering filament spoken of above) being at one moment straight or vertical, at the next moment being bent down suddenly into a hook or sickle form, and then more slowly returning to the straight erect position. When the cilia are vigorous, this double movement is repeated with very great rapidity, so rapidly that the individual movements cannot be seen; it is only when, by reason 
of fatigue, the action becomes slow that the movement itself can be seen; what is seen otherwise is simply the effect of the movement. The movements when slow liave been counted at about eight (double movements) in a second; probably when vigorous they are repeated from twelve to twenty times a second.

The flexion takes place in one direction only, and all the cilia of each cell, and indeed of all the cells of the same epithelium move in the same direction. Moreover the same direction is maintained during the whole life of the epithelium; thus the cilia of the epithelium of the trachea and bronchial passages move during the whole of life in such a way as to drive the flnid lying upon them upwards towards the mouth; so far as we know in vertebrates, or at least in mammals, the direction is not and cannot by any means be reversed.

The flexion is very rapid but the return to the erect position is much slower; hence the total effect of the blow, supposing the cilium and the cell to be fixed, is to drive the thin layer of fluid in which the cilium is working, and which always exists over the epithelium, and any particles which may be floating in that fluid, in the same direction as that in which the blow is given. If the cell be not attached but floating free the effect of the blow may be to drive the cell itself backward; and when perfectly fresh ciliated epithelium is teased out and examined in an inert fluid such as normal saline solution, isolated cells or small groups of cells may be seen rowing themselves abont as it were by the action of their cilia.

All the cilia of a cell move, as we have just said, in the same direction, but not quite at the same time. If we call the side of the cell towards which the cilia bend the front of the cell and the opposite side the back, the cllia at the back move a trifle before those at the front so that the movement runs over the celi in the direction of the movement itself. Similarly, taking any one cell, the cilia of the cells behind it move slightly before, and the cilia of the cells in front of it slightly after, its own cilia move. Hence in this way along a whole stretch of epithelium the movement or bending of the cilia sweeps over the surface in ripples or waves, very much as, when the wind blows, similar waves of bending sweep over a field of corn or tall grass. By this arrangement the efficacy of the movement is secured, and a steady stream of fluid carrying particles is driven over the surface in a uniform continued direction; if the cilia of separate cells, and still more if the separate cilia of each cell, moved independently of the others, all that would be produced would be a series of minute 'wobbles,' of as little use for driving the fluid definitely onwards as the efforts of a boat's crew all rowing out of time are for propelling the boat.

Swift bending and slower straightening is the form of ciliary movement generally met with in the ciliated epithelium of mamwals and indeed of vertebrates; but among the invertebrates we 
find other kinds of movement, such as a to and fro movenent, equally rapid in both directions, a cork-screw movement, a simple undulatory movement, and many others. In each case the kind of movement seems adapted to secure a special end. Thus even in the mamnal while the one-sided blow of the cilia of the epithelial cells secures a flow of fluid over the epithelium, the tail of the spermatozoon, which is practically a single cilium, by moving to and tro in an undulatory fashion drives the head of the spermatozoon onwards in a straight line, like a boat driven by a single oar worked at the stern.

Why and exactly how the cilium of the epithelial cells bends swiftly and straightens slowly, always acting in the same direction, is a problem difficult at present to answer fully. Some have thought that the body of the cell is contractile, or contains contractile mechanisms pulling upon the cilia, which are thus simple passive puppets in the hands of the cells. But there is no satisfactory evidence for such a view. On the whole the evidence is in favour of the view that the action is carried out by the cilium itself, that the bending is a contraction of the cilium, and that the straightenmeg corresponds to the relaxation of a muscular fibre. But even then the exact manner in which the contraction bends and the relaxation straightens the filainent is not fully explained. We have no positive evidence that a longitudinal half, the inside we might say, of the filament is contractile, and the other half, the outside, elastic, a supposition which has been made to explain the bending and straightening. In fact no adequate explanation of the matter has as yet been given, and it is really only on general grounds we conclude that the action is an effect of contractility.

In the vertebrate animal, cilia are, so far as we know, wholly independent of the nervous system, and their movement is probahly ceaseless. In such animals however as Infusoria, Hydrozoa, \&c. the novements in a ciliary tract may of ten be seen to stop and to go on again, to be now fast now slow, according to the needs of the economy, and, as it almost seems, according to the will of the creature; indeed in some of these animals the ciliary movements are clearly under the inflnence of the nervous system.

Observations with galvan ic currents, constant and interrupted, have not led to any satisfactory results, and, so far as we know at present, ciliary action is most affected by changes of temperature and chemical media. Moderate heat quickens the movements, but a rice of temperature heyond a certain limit (about $40^{\circ} \mathrm{C}$. in the case of the pharyngeal membrane of the frog) becomes injurious; cold retards. Very dilute alkalis are favourable, acils are injurious. An excess of carbonic acid or an absence of oxygen diminishes or arrests the movements, either temporarly or permanently, according to the length of the exposure. Chloroform or ether in slight doses diminishes or suspends the action temporarily, in excess kills and disorganises the cells. 


\section{Amoboid Movements.}

$\S 87$. The white blood corpuscles, as we have said ( $\$ 28)$, are able of themselves to change their form and by repeated changes of form to move from place to place. Such movements of the substance of the corpuscles are called amœboid, since they closely resemble and appear to be identical in nature with the movements executed by the amœba and similar organisms. The movement of the endoplasm of the vegetable cell seems also to be of the same kind.

The amcba changes its form (and shifts its place) by throwing out projections of its substance, called pseudopodia, which may be blunt and short, broad bulgings as it were, or may be so long and thin as to be mere filaments, or may be of an intermediate character. As we watch the outline of the hyaline ectosare we may see a pseudopodium beginning by a slight bulging of the outline; the bulging increases by the neighbouring portions of the ectosarc moving into it, the movement under the microscope reminding one of the flowing of melted glass. As the pseudopodium grows larger and engages the whole thickness of the ectosarc at the spot, the granules of the endosare may be seen streaming into it forming a core of endosare in the middle of the bulging of ectosarc. The pseudopodium may continue to grow larger and larger at the expense of the rest of the body, and eventually the whole of the amœba including the nucleus may as it were have passed into the pseudopodium; the body of the amœba will now occupy the place of the pseudopodium instead of its old place; in other words it will in changing its form have also changed its place.

During all these movements, and during all similar amœboid movements, the bulk of the organisin will, so far as can be ascertained, have remained unchanged; the throwing out a pseudopodium in one direction is accompanied by a corresponding retraction of the body in other directions; if as sometimes happens the organism throws out pseudopodia in various directions at the same time, the main body from which the pseudopodia project is reduced in thickness; from being a spherical lump for instance it becomes a branched film. The movement is brought about not hy increase or decrease of substance but by mere translocation of particles; a particle which at one moment was in one position moves into a new position, several particles thus moving towards the same point cause a bulging at that point, and several particles moving away from the same point cause a retraction at that point; but no two particles get nearer to each other so as to occupy together less space and thus lead to condensation of substance, or get farther from each other so as to occupy inore space and thus lead to increase of bulk. 
In this respect, in that there is no change of bulk, but only a shifting of particles in their relative position to each other, the amœboid movement resembles a muscular contraction; but in other respects the two kinds of movement seen different, and the question arises, have we the right to speak of the substance which can only execute amoboid movements as being contractile? We may, if we admit that contractility is at bottom simply the power of shifting the relative position of particles, and that muscular contraction is a specialized form of contraction, the shifting of particles is specialized in the sense that it has always a definite relation to the long axis of the fibre.

The protoplasm of the amœba or of a white corpuscle is, as we have said, of a consistency which we for want of better terms call semi-solid or semi-fluid. Consequently when no internal changes are prompting its particles to move in this or that direction, the influences of the surroundings will tend to give the body, as they will other fluid or semi-thuid drops, a spherical form. Hence the natural form of the white corpuscle is more or less spherical. If under the influence of some stimulus internal or external, some of the particles are stirred to shift their place, amœboid movements follow, and the spherical form is lost. If however all the particles were stirred to move with equal energy, they would neutralize each other's action, no protrusion or retraction would take place at any point of the surface and the body would remain a sphere. Hence in extreme stimulation, in what in the muscle corresponds to complete tetanus, the form of the body is the same as in rest; and the tetanized sphere would not be appreciably smaller than the sphere at rest, for that would imply change of bulk, but this as we have seen does not take place. This result shews strikingly the difference between the general contractility of the amœba, and the special contractility of the muscle. 


\section{CHAPTER III.}

\section{ON THE MORE GENERAL FEATURES OF NERVOUS TISSUES.}

$\S 88$. In the preceding chapter we have dealt with the properties of nerves going to muscles, the nerves which we called motor, and have incidentally spoken of other nerves which we called sensory. Both these kinds of nerves are connected with the brain and spinal cord and form part of the General Nervous System. We shall have to study hereafter in detail the brain and spinal cord; but the nervous system intervenes so repeatedly in the processes carried out by other tissues that it will be desirable, before proceeding further, to discuss some of its more general features.

The Nervous System consists (1) of the Brain and Spinal Cord forming together the cerebrospinal axis, or ecntral nervous system; (2) of the nerves passing from that axis to nearly all parts of the body, those which are connected with the spinal cord being called spinal, and those which are connected with the brain, within the cranium, being called cranial; and (3) of ganglia distributed along the nerves in various parts of the body.

The spinal cord obviously consists of a number of segments or metameres, following in succession along its axis, each metamere giving off on each side a pair of spinal nerves; and a similar division into metameres may be traced in the brain, though less distinctly, since the cranial nerves are arranged in manner somewhat different from that of the spinal nerves. We may take a single spinal metamere, represented diagrammatically in Fig. 24, as illustrating the general features of the nervous system; and since the half on one side of the median line resembles the half on the other side, we may deal with one lateral half only.

Each spinal nerve arises by two roots. The metamere of the central nervous system $C$ consists, as we shall hereafter see, of grey 


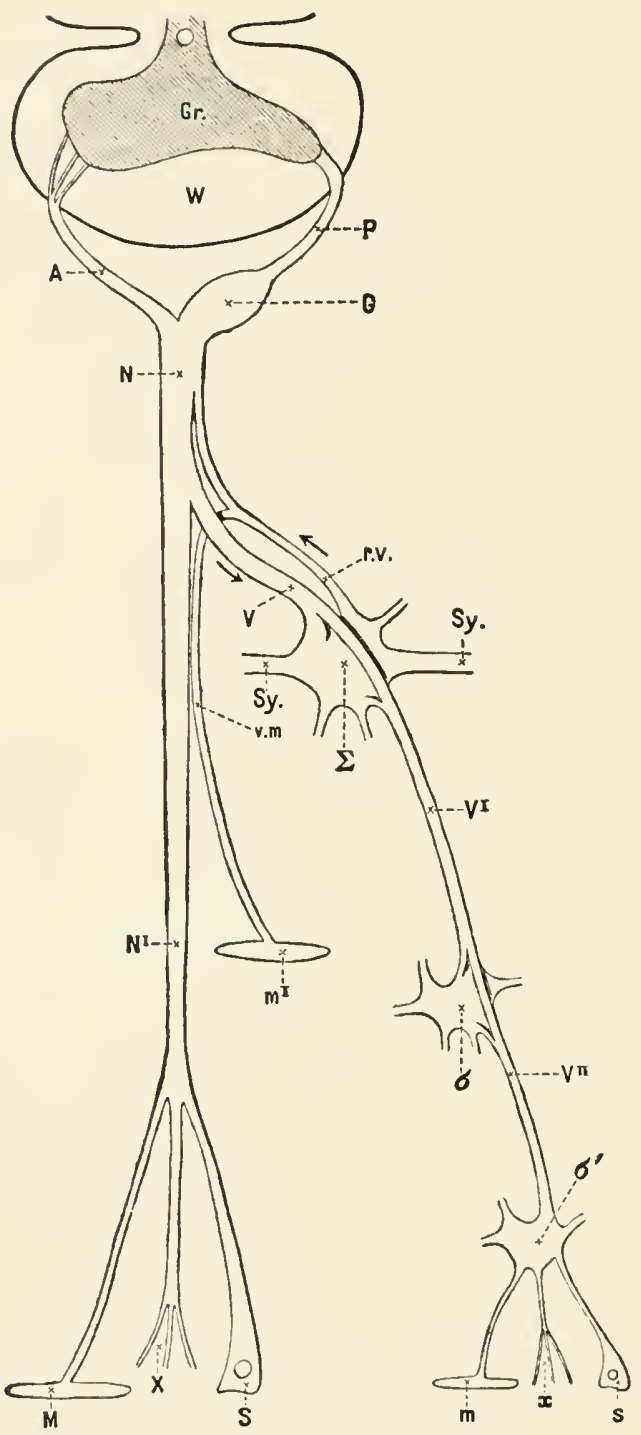

Fig. 24. Scheme of the Nerves of a Segment of the Sinal Cord.

Gr grey, $W$ white matter of spinal cord. $A$ anterior, $P$ posterior root. $G$ ganglion on the posterior root. $V$ whole nerve, $N^{\prime}$ spinal nerve proper, end ing in $M /$ skeletal or somatic muscle, $S$ somatic sensory cell or surface, $X$ in other ways. $V$ visceral nerve (white ramus communicans) jassing to a ganglion of the svmpathetic chain $\Sigma$. and passing on as $V^{\prime \prime}$ to supply the more distant ganglion $\sigma$, then as $I^{\prime \prime}$ to the peripheral ganglion $\sigma^{\prime}$ and ending in $m$ splanchnic muscle, $s$ splauchnic sensory cell or surface, $r$ other possible splanchnic endings.

From $\Sigma$ is given off the rerehent nerve $r$." (grev ramus communicans), which partly passes backward towards the spmal cord, and partly rums is $c . m$, in connection 
with the spinal nerve, to supply vasomotor (constrictor) fibres to the muscles $\left(m^{\prime}\right)$ of blood vessels in certain parts, for example, in the limbs.

$S y$, the sympathetic chain uniting the ganglia of the series $\mathbf{z}$. The terminations of the other nerves arising from $\Sigma, \sigma, \sigma^{\prime}$ are not shewn.

The figure is necessarily schematic, and must not be taken to shew that the visceral branch joins only the ganglion belonging to the same segment as the spinal nerve; the visceral branch joins the sympathetic chain, passing to other ganglia besides the one of the same segment, indeed in some cases does not join this at all.

matter $G r$ in the interior, and white matter $W$ on the outside. From the anterior part of grey matter is given off the anterior nerve root $A$, and from the posterior part the posterior nerve root $P$. The latter passes intu a swelling or ganglion $G$, "the ganglion of the posterior root," or more shortly "the spinal ganglion;" the anterior root does not pass into this ganglion. Beyond the ganglion the roots join to form the nerve trunk $N$. We shall later on give the evidence that the nerve fibres composing the posterior root $P$ are exclusively (or nearly so), occupied in carrying nervous impulses from the tissues of the body to the central nervous system, and that the fibres composing the anterior root $A$ are similarly occupied in carrying impulses from the central nervous system to the several tissues; that is to say the former is made up of sensory fibres, or, (since the impulses passing along them to the central system may give rise to effects other than sensations) afferent fibres, while the latter is made up of motor, or, (since the impulses passing along them from the central nervous system may produce effects other than movements) efferent fibres. The nerve trunk $N$ is consequently a mixed nerve composed of afferent and efferent fibres.

By far the greater part of this mixed nerve, dividing into various branches, is distributed $\left(N^{\prime}\right)$ to the skin and the skeletal muscles, some of the fibres (motor) ending in muscular fibres $(M)$, others (sensory) ending in epithelial cells $(S)$ connected with the skin, which we shall consider hereafter under the name of sensory epithelial cells, while others, $X$, after dividing into minute branches and forming plexuses end, in ways not yet definitely determined, in tissues associated with the skin or skeletal muscles. Morphologists distinguish the parts which go to form the skin, skeletal muscles, \&c. as somatie, from the splanehnie parts which go to form the viscera. We may accordingly call this main part of the spinal nerve the somatic division of the nerve.

Soon after the mixed nerve $N$ leaves the spinal canal it gives off a branch $V$, which under the name of (white) ramus communieans, runs into the longitudinal series of ganglia $(\Sigma)$ conspicuous in the thorax as the main sympathetie chain. This branch is destined to supply the viscera, and might, therefore, be called the splanchnic division of the spinal nerve. We may say at once, without entering into details, that the whole of the sympathetic system with its ganglia, plexuses and nerves is to be regarded as a development or expansion of the visceral or splanchnic divisions of certain spinal nerves. By means of this 
system splanchnic fibres from the central nervous system are distributed to the tissues of the viscera, some of them on their way passing through secondary ganglia $\sigma$, and, it may be, tertiary ganghia. The majority of these splanchnic fibres seem to be efferent in nature, carrying impulses from the central nervous system to the tissues, some ending in plain muscular fibres ( $m$ ) others in other ways $(s)$; but some of the fibres are afferent $(s)$ and conver impulses from the viscera to the central nervous system, and it is possible that some of these begin or end in epithelial cells of the viscera.

All the fibres issuing from the main sympathetic chain do not however pass to the tissues of the viscera; a certain number of fibres turn back ( $r . v$.) from the ganglion to join the spinal nerve and rum for the most part peripherally in the somatic nerve, though some of them pass backwards to the spinal cord, ending probably in the membranes of the cord. In the case of many of the spinal nerves the communicating branch from the spinal nerve consists distinctly of two parts, a 'white ramus' cousisting chiefly of medullated and a 'grey ramus' consisting chiefly of non-medullated fibres; in these cases these backward turning 'revehent' fibres run in the grey ramus; but in the case of some of the spinal nerves it is not possible to distinguish a grey ramus separate from a white ramus.

We shall have occasion in the next chapter to speak of nerves or rather nerve fibres which by influencing the muscles of the blood ressels govern the calibre of those vessels and are spoken of as vaso-motor nerves. Some of these, in their action, constrict or narrow the blood vessels, and are hence called voso-constrictor nerve fibres. All these vaso-constrictor nerve fibres issuing from the central nervous system pass to the splanchnic system, to the sympathetic chain; but while some of them, continuing in the splanchnic system, are distributed to the blood vessels of the viscera, others turning back by revehent branches and running with ordinary somatic fibres in the (somatic) spinal nerves are distributed to the blood vessels certainly of the skin and possibly of other somatic tissues. These are represented in the figure by $\mathrm{m}^{\prime}$. As we shall see, other nerve fibres, having other functions, take a similar course.

A nerve fibre is fundamentally a prolongation of a nerve cell; the axis cylinder which is the essential part of a medullated fibre, and constitutes practically the whole of a non-medullated fibre, is the prolongation of a process, the so-called axis cylinder process of a nerve cell. When we examine a nerve we find that along its course it cousists exclusively of nerve fibres bound together by connective tissue carrying blood vessels and lymphatics, the somatic (spinal and cranial) nerves being composed chiefly of medullated fibres, mixed with which are some non-medullated fibres, and the sympathetic nerves being composed chiefly of non-medullated fibres, some of them containing hardly any medullated fibres. 
Nerve cells are not normal constituents of nerves; they are found only in the central nervous system, from which the nerves issue, or in the ganglia, spinal and sympathetic ganglia, through which they pass or with which they make connections; (we may omit for the present the nerve cells in which, or in comnection with which certain nerve fibres end at the periphery). Hence all the nerve fibres of the body are processes of nerve cells situated in the central nervous system, or in the spinal ganglia and corresponding ganglia on certain cranial nerves, or in sympathetic ganglia.

$\$$ 89. In the central nervous system, the nerve cells are found in the so called 'grey matter.' The 'white matter,' putting aside certain exceptions, consists so far as its nervous elements are concerned exclusively of nerve fibres. Confining ourselves for the present to the spinal cord, we find that the fibres of the anterior root, efferent fibres, are processes of cells, prolongations of the axis cylinder processes of cells, lying in the grey matter of the spinal cord, but that the fibres of the posterior root are (putting aside certain minor exceptions) processes of cells lying in the ganglion of that root. The cell whose axis cylinder process becomes an efferent fibre of an anterior root has other processes, which do not become axis cylinders of nerve fibres but end by a division more or less distinctly arborescent in the grey matter. The fibre of a posterior root whose axis cylinder is a process of a cell in the spinal ganglion ruuning and dividing in the spinal cord, in a manner of which we shall have to speak later on, finally ends in the grey matter also in a more or less distinctly arborescent fashion. The grey matter also contains nerve cells whose axis cylinder and other processes both begin and end in the grey matter; that is to say the body of the nerve cell lies in the grey matter, and all its processes finally end, also in a more or less distinctly arborescent fashion, in the grey matter, without learing the spinal cord or at least the central nervous system, though the fibre which its axis cylinder becomes (and there may be more than one such) may traverse for a while the white matter.

The grey matter of the spinal cord (and the same is true though in a much more complex way of the grey matter of the brain) may therefore be consideted as a centre or a number of centres, nercous eentres, connected on the one hand with afferent and on the other hand with efferent fibres. In some cases the comnection between the afferent and the efferent fibres may be simple and direct; the terminations of the afferent fibre may come into direct comnection with the nerve cell, a process of which is the efferent fibre. In other cases the connection may be an indirect one; between the two intervenes a cell, with certain processes of which the afferent fibre is connected, and another process (or other processes) of which is counected with the cell whose process is the efferent fibre; or more than one such cell may intervene. And recent inquiry shews, that the usual mode of connection of one cell with 
another is that the arborescent terminations of the axis cylind $r$ proce ss of the one cell are applied to the arborescent processes (not of the nature of axis cylinder processes) of the other cell, in such a way that the substances of the two cells are in abundant contact, but not in actual continuity.

The various nervous centres thus supplied by the grey matter of the spinal cord and brain have two important classes of functions calle 1 respectively reflex actions and automatic actions.

$\$ 90$. Reflex actions. In a refiex action afferent impulses reaching the nervous centre give rise to the discharge of efferent impulses, the discharge following so rapidly and in such a way as to leave no doubt that it is caused by the advent at the centre of the afferent impulses. Thus a frog from which the brain has been removed while the rest of the body has been left intact will frequently remain quite motionless (as far at least as the skeletal inuscles are concerned) for an almost indefinite time; but if its skin be pricked, or if in other ways afferent impulses be generated in afferent fibres by adequate stimulation, movements of the limbs or body will immediately follow. Obviously in this instance the stimulation of afferent fibres has been the cause of the discharge of inpulses along efferent fibres.

The machinery involved in such a reflex act consists of three parts: (1) the afferent fibres, (2) the nerve centre, in this case the spinal cord, and (3) the efferent fibres. If any one of these three parts be missing the reflex act cannot take place; if for instance the afferent nerves or the efferent nerves be cut across in their course, or if the centre, the spinal cord, be destroyed, the reflex action cannot take place.

Reflex actions can be carried out by nneans of the brain, as we shall see while studying that organ in detail, but the best and clearest examples of reflex action are manifested by the spinal enrl; in fact, reflex action is one of the most important functions of the spinal cord. We sliall have to study the various reflex actions of the spinal cord in detail hereafter, but it will be desirable to point out here some of their general features.

When we stimulate the nerve of a muscle-nerve preparation the result, though modified in part by the condition of the muscle and nerve, whether fresh and irritable or exhausted for instance, is directly dependent on the nature and strength of the stimulus. If we use a single induction-shock we get a simple contraction, if the interrupted current we get a tetanus, if we use a weak shock we get a slight contraction, if a strong shock a large contraction, and so on; and throughout our study of muscular contractions we assumed that the amount of contraction might be taken as a measure of the magnitude of the nervous impulses generated by the stimulus. And it ne's hardly be said that when we stimulate certain fibres only of a motor nerve, it is only the museular fibres in which those nerve fibres end, which are thrown into contraction. 
In a reflex action on the other hand the movenents called forth by the same stimulus may be in one case insigniticant, and in another violent and excessive, the result depending on the arrangements and condition of the central portion of the reflex mechanism. Thus the mere contact of a hair with the mucous membrane lining the larynx, a contact which can originate only the very slightest afferent impulse; nuay call forth a convulsive fit of conghing, in which a very large number of muscles are thrown into violent contractions; whereas the same contact of the hair with other surfaces of the body may produce no obvious effect at all. Similarly, while in the brainless but o.herwice normal frog, a shight touch on the skin of the flank will produce nothing but a faint flicker of the underlying muscles, the same touch on the same part of a frog poisoned with strychuia will produce violent lasting tetanic contractions of nearly all the muscles of the body. Motor impulses as we have seen travel along motor nerves without any great expenditure of energy and probably without increasing that expenditure as they proceed; and the same is apparently the case witl afferent impulses passing along afferent nerves. When however in a reflex action afferent inpulses reach the nerve centre, a change in the nature and magnitude of the impulses takes place. It is not that in the nerve centre the afferent impulses are simply turned aside or reflected into efferent impulses; and hence the term "reflex" action is a lad one. It is rather that the afferent impulses act afresh as it wele as a stimulus to the nerve centre, producing according to circunistances and conditions either a few weak efferent impulses or a multitude of strong ones. The nerve centre may be reçarded as a collection of explosive charges ready to be discharged and so to start efferent impulses along certain efferent nerves, and these charges are so arranged and so related to certain affelent nerves, that afferent impulses reaching the centre along those nerves may in one case discharge a few only of the charges and so give rise to feeble movements, and in another case discharge a very large number and so give rise to large and violent movements. In a reflex action then the number, intensity, character and distribution of the efferent impulses, and so the kind and amount of movenent, will depend chiefly on what takes place in the centre, and this will in turn depend on the one hand on the condition of the centre and, on the other, on the speeial relations of the centre to the afferent impulses. At the same time we are able to recognize in most reflex actions a certain relation between the strength of the stimulus, that is to say the magnitude of the afferent impulses, and the extent of the movement, that is to say the magnitude of the efferent impulses.

We may add, without going more fully into the subject here, that in most reflex actions a special relation may be observed between the part stimulated and the resulting movement. In the simplest cases of reflex action this relation is merely of such a kind that the muscles thrown into action are those governed by a 
motor nerve which is the fellow of the sensory nerve, the stimulation of which ealls forth the movement. In the more complex reflex actions of the brainless frog, and in other cases, the relation is of such a kind that the resulting movement bears an adaptation to the stimulus: the foot is withdrawn from the stimulus, or the movement is calculated to push or wipe away the stimulus. In other words, a certain purpose is evident in the reflex action.

Thus in all cases, except perhaps the very simplest, the inovements called forth by a retlex action are exceedingly complex compared with those which result from the direct stimulation of a motor trunk.

\$ 91. Automatic actions. Efferent impulses frequently issue from the brain and spinal cord and so give rise to movements without being obviously preceded by any stimulation. Such movements are spoken of as automatic or spontaneous. The efferent impulses in such cases are started by changes in the nerve centre which are not the immediate result of the arrival at the nerve centre of afferent impulses from without, but which appear to arise in the nerve centre itself. Changes of this kind may recur rhythmically ; thus, as we shall see, we have reason to think that in a certain part of the central nervous system called the spinal bulb, or medulla oblongata, changes of the nervous material, recurring rhythmically, lead to the rhythmic discharge along certain nerves of efferent impulses whereby muscles connected with the chest are rhythmically thrown into action and a rlythmically repeated breathing is brought about. And other similar rhythmic automatic movements may be carried out by various parts of the spinal cord.

From the brain itself a much more varied and apparently irregular discharge of efferent impulses, not the obvious result of any immediately foregoing afferent impulses, and therefore not forming part of reflex actions, is very common, constituting what we speak of as volition, efferent impulses thus arising being called volitional or voluntary impulses. The spinal cord apart from the brain does not appear capable of executing these voluntary movements; but to this subject we shall return when we come to speak of the central nervous system in detail.

While reflex and automatic actions are thus frequently carried out by the grey matter of the central nervous system, of which grey matter nerve cells are conspicnous constituents, it is at least not absolutely proved that either kind of action is carried out by the other portions of the nervous system in which nerve cells are found.

As regards the ganglia on the posterior roots of spinal nerves it can be definitely affirmed that these act neither as automatic centres nor as centres of reflex action. The nerve cell of such a ganglion serves to govern the nutrition of the afferent nerve filire to which it is attached by a $\mathrm{T}$ sliaped junction; and a portion of the fibre which is cut away from its nerve cell in the 
ganglion, whether it be the portion which is passing from the skin or other tissue to the ganglion, or the portion which is passing from the ganglion to the spinal cord as part of the posterior root, degenerates and dies on the side of the cut away from the ganglion. This, however, is not a feature confined to these spinal ganglia; an efferent fibre of the anterior root similarly degenerates when it is cut away from the nerve cell in the grey matter, of the axis cylinder process of which it is as we have said a prolongation. Speaking generally, a nerve cell governs the nutrition of, acts as a trophic centre as it is called to, the nerve fibre into which its axis cylinder process is continued.

As regards the sympathetic ganglia, though there are some results which appear to indicate that certain of these ganglia may act in a simple and rudimentary way as centres of reflex action, these cases are by no means clear; and it may be distinctly affirmed that these ganglia do not generally act as centres of reflex action in the same way as does the grey matter of the central nervous system.

Of the fibres rumning in a ramus from a spinal nerve to the ganglion of the same metamere, some may end in connection with the nerve cells of that ganglion. In that case the nerve fibre, which is a medullated one, appears to end in an arborescence in contact with a nerve cell, and that nerve cell gives off one or more processes which become nerve fibres, but non-medullated nerve fibres. Other fibres of the ramus may simply pass through that ganglion, passing by its nerve cells, and end in connection with the nerve cells of some other ganglion, which nerve cells similarly give rise to non-medullated nerve fibres. The nerve fibres leaving a ganglion are more numerous than those reaching it from the central nervous system; and while the latter are medullated, the former are increasingly non-medullated. Hence in the ganglion there is a spreading and distribution of nervous impulses; as to what changes in the nature of the impulses may be effected as they pass through the nerve cells of a ganglion is not at present clear.

There s'ems at first sight evidence of some strength that these sympathetic ganglia, unlike the ganglia on the posterior roots, may serve as centres of rhythmic automatic action. Several organs of the body containing muscular tissue, the most notable being the heart, are during life engaged in rhythmic antomatic movennents, and in many cases contimue these movements after removal from the body. In nearly all these cases ganglia are present in connection with the muscular tissue; and the presence and intact condition of these ganglia seem at all events in many cases in some way essential to the due performance of the rhythmic automatic movements. Indeed it has been thought thiat the movements in question are really due to the rhythmic automatic generation in the cells of these ganglia of efferent impulses which passing down to the appropriate muscular fibres call forth 
the rhythmic movement. When we come to study these movements in detail, we shall find reasons for coming to the conclusion that this view is not supported by adequate evidence.

$\$$ 92. Inhibitor!y nerves. We have said that the fibres of the anterior root should be called efferent rather than motor because though they all carry impulses outward from the central nervous system to the tissues, the impulses which they carry do not in all cases lead to the contraction of muscular fibres. Some of these efferent fibres are distributed to glandular structures, for instance, to the salivary glands, and impulses passing along these lead to changes in epithelial cells and their surroundings whereby, without any muscular contraction necessarily intervening, secretion is brought about: the action of these fibres of secretion we shall study in connection with digestion.

Besides this there are efferent fibres going to muscular tissue or at all events to muscular organs, the impulses passing along which, so far from bringing about muscular contraction, diminish, hinder or stop movements already in progress. Thus if when the heart is beating regularly, that is to say, when the muscular fibres which make up the greater part of the heart are rhythmically contracting, the branches of the pneumogastric nerve going to the heart be adequately stimulated, for instance with the interrupted current, the heart will stop beating; and that not because the muscles of the heart are thrown into a continued tetamus, the rhythmic alternation of contraction and relaxation being replaced by sustained contraction, but because contraction disappears altogether, all the inuscular fibres of the heart remaining for a considerable time in complete relaxation and the whole heart being quite flaccid. If a weaker stimulus be employed the beat may not be actually stopped but slowed or weakened. And, as we shall see, there are many other cases where the stimulation of efferent fibres hinders, weakens, or altogether stops a morement aheady in progress. Such an effect is called an inhibition, and the fibres, stimulation of which produces the effect, are called ' inhibitory' fibres.

The phenomena of inhibition are not, however, confined to such cases as the heart, where the efferent nerves are connected with muscular tissues. In fact it is probable, though not actually proved in every case, that wherever in any tissue, energy is being set free, nervous impulses bronght to bear on the tissue may affect the rate or amount of the energy set free in two different ways; on the one hand, they may increase or quicken the setting free of energy, and on the other hand they may slacken or hinder the setting free of energy. And in at all events a large number of cases it is possible to produce the one effect by means of one set of nerve fibres, and the other effect by another set of nerve fibres. We shall have occasion however to study the several instances of this double action in the appropriate places. 


\title{
CHAP'TER IV.
}

\section{THE VASCULAR MECHANISM.}

\author{
SEC. 1. THE STRUCTURE AND MAIN FEATURES OF \\ THE VASCULAR APPARATUS.
}

$\$$ 93. The blood, as we have said, is the internal medium on which the tissues live; from it these draw their food and oxygen, to it they give up the products or waste matters which they form. The tissues, with some few exceptions, are traversed by, and thus the elements of the tissues surrounded by, networks of minute, thinwalled tubes, the capillary blood vcssels. The elementary striated muscle fibre, for instance, is surrounded by capillaries, running in the commective tissue outside but close to the sarcolemma, arranged in a network with more or less rectangular meshes. These capillaries are closed tubes with continuous walls, and the blood, which, as we shall see, is continually streaming through them, is as a whole confined to their channels, and does not escape from them. The elements of the tissues lie outside the capillaries, and form extra-vascular islcts of different form and size in the different tissues, surromnded by capillary networks. But the walls of the capillaries are so thin and of such a mature that certain of the constituents of the blood pass from the interior of the capillary through the capillary wall to the elements of the tissue outside the capillary, and, similarly, certain of the constituents of the tissue, to wit, certain substances, the result of the metabolism continually going on in the tissue, pass from the tissue outside the capillary through the capillary wall into the blood flowing through the capillary. Thus, as we have already said, $\$ 13$, there 
is a continual interchange of material between the blood in the capillary, and the elements of the tissue ontside the capillary, the lymph acting as middle man. By this interchange the tissue lives on the blood and the blood is affected by its passage through the tissue. In the small arteries which end in, and in the small veins which begin in the capillaries, a similar interchange takes place; but the amount of interchange diminishes as, passing in each direction from the capillaries, the walls of the arteries and veins become thicker; and indeed, in all but the minute veins and arteries, the interchange is so small that it may practically be neglected. It is in the capillaries (and minute arteries and veins) that the business of the blood is done; it is in these that the interchange takes place; and the object of the vascular mechanism is to cause the blood to flow through these in a manner best adapted for carrying on this interchange under varying circumstances. The use of the arteries is in the main simply to carry the blood in a suitable manner from the heart to the capillaries, the use of the veins is in the main simply to carry the blood from the capillaries back to the heart, and the use of the heart is in the main simply to drive the blood in a suitable manner through the arteries into the capillaries and from the capillaries back along the veins to itself again. The structure of these several parts is adapted to these several uses.

\section{Main Features of the Apparatus.}

$\$ 94$. We may pass briefly in review some of the main features of the several parts of the vascular apparatus, heart, arteries, veins and capillaries.

The heart is a muscular pump, that is a pump the force of whose strokes is supplied by the contraction of muscular fibres, working intermittently, the strokes being repeated so many times (in man about 72 times) a minute. It is so constructed and furnished with valves in such a way that at each stroke it drives a certain quantity of blood with a certain force and a certain rapidity from the left ventricle into the aorta and so into the arteries, receiving during the stroke and the interval between that stroke and the next, the same quantity of blood from the veins into the right auricle. We omit for simplicity's sake the pulmonary circulation by which the same quantity of blood is driven at the stroke from the right ventricle into the lungs and received into the left auricle. The rhythm of the beat, that is the frequency of repetition of the strokes, and the characters of each beat or stroke, are determined by changes taking place in the tissues of the heart itself, though they are also influenced by causes working from without.

The arteries are tubes, with relatively stout walls, branching 
from the aorta all over the body. The constitution of their walls, especially of the middle coat, gives the arteries two salient properties. In the first place they are very clastic, in the sense that they will stretch readily, both lengthways and crosswise, when pulled, and returu readily to their former size and shape when the pull is taken off. If thuil be driven into one end of a piece of artery, the other end of which is tied, the artery will swell out to a very great extent, but return immediately to its former calibre when the fluid is let out. This elasticity is chiefly due to the elastic elements in the coats, elastic membranes and feltworks, but the muscular fibres being themselves also elastic contribute to the result. By reason of their possessing such stout elastic walls the arteries when empty do not collapse but remain as open tubes. In the second place the arteries by virtive of their muscular elements are contractile; when stimulated either directly as by applying an electric or mechanical stimulus to the arterial walls or indirectly by means of the so-called vaso-motor nerves, which we shall have to stıdly presently, the arteries shrink in calibre, the circularly disposed muscular fibres contracting and so, in proportion to the amount of their contraction, narrowing the lumen or bore of the vessel. The contraction of these arterial muscular fibres, like that of all plain non-striated muscular fibres, is slow and long continued, wit! a long latent period, as compared with the contraction of skeletal striated muscular fibres. Owing to this muscular element in the arterial walls, the calibre of an artery may be very narrow, or very wide, or in an intermediate condition between the two, neither very narrow nor very wide, according as the muscular fibres are very much contracted, or not contracted at all, or only moderately contracted. Further, while the relative proportion of elastic and muscular elements differs in different arteries, as a general rule the elastic elements predominate in the larger arteries and the muscular elements in the smaller arteries, so that the larger arteries may be spoken of as eminently elastic, or as especially useful on account of their elastic properties, and the smaller arteries as eminently muscular, or as especially useful on account of their muscular properties. Thus in the minute arteries which are just passing into capillaries the muscular coat, though composed often of a single layer, and that sometimes an imperfect one, of muscular fibres, is a much more conspicuous and important part of the arterial wall than that furnished by the elastic elements.

The arteries branching out from a single aorta down to multitudinous capillaries in nearly every part of the body, diminish in bore as they divide. Where an artery divides into two or gives off a branch, though the bore of each division is less than that of the artery before the division or branching, the two together are greater; that is to say, the united sectional area of the branches is greater than the sectional area of the trunk. Hence the 
sectional area of the arterial bed through which the blood flows goes on increasing from the aorta to the capillaries. If all the arterial branches were thrown together into one channel, this would form a hollow cone with its apex at the aorta and its base at the capillaries. The united sectional area of the capillaries may be taken as several hundred times that of the sectional area of the aorta, so greatly does the arterial bed widen out.

The capillaries are channels of variable but exceedingly small size. The thin sheet of cemented epithelioid plates which forms the only wall of a capillary is elastic, permitting the channel offered by the same capillary to differ much in width at different times, to widen when blood plasma and blood corpuscles are being pressed through it and to narrow again when the pressure is lessened or cut off. The same thin sheet permits water and substances, including gases, in solution to pass through itself from the blood to the tissue outside the capillary and from the tissue to the blood, and thus carries on the interchange of material between the blood and the tissue. In certain circumstances at all events white and even red corpuscles may also pass through the wall to the tissue outside.

The minute arteries and veins with which the capillaries are continuous allow of a similar interchange of material, the more so the smaller they are.

The walls of the veins are thinner, weaker and less elastic than those of the arteries, and possess a very variable amount of muscular tissue; they collapse when the veins are empty. Though all veins are more or less elastic and some veins are distinctly muscular, the veins as a whole cannot, like the arteries, be characterized as eminently elastic and contractile tubes; they are rather to be regarded as simple channels for conveying the blood from the capillaries to the heart, having just so much elasticity as will enable them to accommodate themselves to the quantity of blood passing through them, the same vein being at one time full and distended and at another time empty and shrunk, and only gifted with any great amount of muscular contractility in special cases for special reasons. The united sectional area of the veins, like that of the arteries, diminishes from the capillaries to the heart; but the united sectional area of the venæ cavie at their junction with the right auricle is greater than, nearly twice as great as, that of the aorta at its origin. The total capacity aiso of the veins is much greater than that of the arteries. The veins alone can hold the total mass of blood which in life is distributed over both arteries and veins. Indced nearly the whole blood is capable of being received by what is merely a part of the venous system, viz. the vena portic and its branches. 


\section{SEC. 2. THE MAIN FACTS OF THE CIRCULATION.}

$\S$ 95. Before we attempt to study in detail the working of these several parts of the mechanism, it will be well, even at the risk of some future repetition, to take a brief survey of some of the salient features.

At each beat of the heart, which in man is repeated about 72 times a minute, the contraction or systole of the ventricles drives a quantity of blood with very great force into the aorta (and the same quantity of blood with less force into the pulmonary artery); the actual anount varies from time to time, but 180 c.c. (4 to 6 oz.) may be taken as a rather high estimate. The discharge of blood from the ventricle into the aorta is very rapid, and the time taken up by it is, as we shall see, less than the time which intervenes between it and the next discharge of the next beat. So that the flow from the heart into the arteries is most distinctly intermittent, sudden, rapid discharges alternating with relatively longer intervals, during which the arteries receive no blood from the heart.

At each beat of the heart just as much blood flows, as we shall see, from the veins into the right auricle as escapes from the left ventricle into the aorta; but, as we shall also see, this inflow is much slower, takes a longer time, than the discharge from the ventricle.

When the finger is placed on an artery in the living body, a sense of resistance is felt, and this resistance seems to be increased at intervals, corresponding to the heart beats, the artery at each heart beat being felt to rise up or expand under the finger, constituting what we shall study hereafter as the pulse. In certain arteries this pulse may be seen by the eye. When the finger is similarly placed on a corresponding vein, very little resistance is felt, and under ordinary circumstances no pulse can be perceived by the touch or by the eye.

When an artery is severed, the flow of blood from the proximal cut end, that on the heart side, is not equable, but comes in jets, 
corresponding to the heart beats, though the flow does not cease between the jets. 'The blood is ejected with considerable force, and may, in a large artery of a large animal, be spurted out to the distance of some feet. The larger the artery and the nearer to the heart, the greater the force with which the blood issues, and the more marked the intermittence of the flow. The flow from the distal cut end, that away from the heart, may be very slight, or may take place with considerable force and marked intermittence, according to the amount of collateral communication.

When a corresponding vein is severed, the flow of blood, which is chiefly from the distal cut end, that in connection with the capillaries, is not jerked but continuous ; the blood comes out with comparatively little force, and 'wells up' rather than 'spurts out.' The flow from the proximal cut end, that on the heart side, may amount to nothing at all, or may be slight, or may be considerable, depending on the presence or absence of valves and the amount of collateral communication.

When an artery is ligatured, the vessel swells on the proximal side, towards the heart, and the throbbing of the pulse may be felt right up to the ligature. On the distal side, the vessel is empty and shrunk, and no pulse can be felt in it unless there be free collateral communication.

When a vein is ligatured, the vessel swells on the distal side, away from the heart, but no pulse is felt; while on the proximal side, towards the heart, it is empty and collapsed unless there be ton free collateral communication.

$\$ 96$. When the interior of an artery, for instance the carotid, is placed in communication with a long glass tube of not too great a bore, held vertically, the blood, immediately upon the commmication being effected, may be seen to rush into and to fill the tube for a certain distance, forming in it a column of blood of a certain height. The column rises not steadily but by leaps, each leap corresponding to a heart beat, and each leap being less than its predecessor; and this goes on, the increase in the height of the column at each heart beat each time diminishing, until at last the column ceases to rise, and remains for a while at a mean level, above and below which it oscillates with slight excursions at each heart beat.

To introduce such a tube, an artery, say the carotid of a rabbit, is laid bare, ligatured at a convenient spot, $l^{\prime}$ Fig. 25, and further temporarily closed a little distance lower down nearer the heart by a small pair of 'bull-dog' forceps, $b d$, or by a ligature which can be easily slipped. A V-shaped cut is now made in the artery between the forceps, $b d$, and the ligature $l^{\prime}$ (only the drop or two of bloorl which happens to remain enclosed between the two being lost): the end of the tube, representer by $c$ in the figure, is iutroluced into the artery and secured by the ligature $l$. The interior of the tube is now in free communication with the interior of the artery, but the latter 
is, by means of the forceps, at present shut off from the heart. On removing the forceps a clirect communication is at once established between the tube and the artery below; in consequence the blood from the heart flows through the artery into the tube.

This experiment shews that the blood as it is flowing into the carotid is exerting a considerable pressure on the walls of the artery. At the moment when the forceps is removed, there is nothing but the ordinary pressure of the atmosphere to counterbalance this pressure within the artery, and consequently a quantity of blood is pressed out into the tube; and this goes on until the column of blood in the tube reaches such a height that its weight is equal to the pressure within the artery, whereupon no more blood escapes. The whole column continues to be raised a little at each heart beat, but sinks as much during the interval between each two beats, and thus oscillates, as we have said, above and below a mean level. In a rabbit this column of blood will generally have the height of about $90 \mathrm{~cm}$. (3 feet); that is to say, the pressure which the blood exerts on the walls of the carotid of a rabbit is equal to the pressure exerted by a column of rabbit's blood $90 \mathrm{~cm}$. high. This is equal to the pressure of a column of water about $95 \mathrm{~cm}$. high, and to the pressure of a column of mercury about $70 \mathrm{~mm}$. high.

If a like tube be similarly introduced into a corresponding vein, say the jugular vein, it will be found that the column of blood, similarly formed in the tube, will be a very low one, not more than a very few centimeters high; and that while the level of the column may vary a good deal, owing as we shall see later to the influence of the respiratory movements, there will not, as in the artery, be oscillations corresponding to the heart beats.

We learn, then, from this simple experiment, that in the carotid of the rabbit the blood, while it flows through that vessel, is exerting a considerable mean pressure on the arterial walls, equivalent to that of a column of mercury about $70 \mathrm{~mm}$. high, but that in the jugular vein the blood exerts on the venous walls a very slight mean pressure, equivalent to that of a column of blood a few centimeters high, or of a column of mercury three or four millimeters high. We speak of this mean pressure exerted by the blood on the walls of the blood vessels as blood pressure, and we say that the blood pressure in the carotid of the rabbit is very high (70 mm. Hg.), while that in the jugular vein is very low (only 3 or $4 \mathrm{~mm}$. $\mathrm{Hg}$.).

In the normal state of things, the blood flows through the carotid to the arterial branches beyond, and through the jugular vein towards the heart; the pressure exerted by the blood on the artery, or on the vein is a lateral pressure on the walls of the artery and vein respectively. In the above experiment the pressure measured is not exactly this, but the pressure exerted at the end of the artery (or of the vein) where the tube is attached. We 
might directly measure the lateral pressure in the carotid by somewhat modifying the procedure described above. We might connect the carotid with a tube, the end of which was not straight but made in the form of a $\vdash$ piece, and might introduce the $\vdash$ piece in such a way that the blood should flow along one linb (the vertical limb) of the $\vdash$ piece from the proximal to the distal part of the carotid, and at the same time by the other (horizontal) limb of the $r$ piece into the main, upright part of the glass tube. The column of blood in the tube would then be a measure of the pressure which the blood, as it is flowing along the carotid, is exerting on a portion of its walls corresponding to the moutl of the horizontal limb of the $\vdash$ piece. If we were to introduce into the aorta, at the place of origin of the carotid, a similar (larger) $\vdash$ piece, and to connect the glass tube with the horizontal limb of the $\vdash$ piece by a piece of elastic tubing of the same length and bore as the carotid, the column of blood rising up in the tube would be the measure of the lateral pressure exerted by the blood on the walls of the aorta at the origin of the carotid artery, and transmitted to the rigid glass tube through a certain length of elastic tubing. And, indeed, what is measured in the experiment previously described is not the lateral pressure in the carotid itself at the spot where the glass tube is introduced, but the lateral pressure of the aorta at the origin of the carotid, modified by the influences exerted by the length of the carotid between its origin and the spot where the tube is introduced.

$\$ 97$. Snch an experiment as the one described lias the disadvantages that the animal is weakened by the loss of the blood, which goes to form the column in the tube, and that the blood in the tube soon clots, and so brings the experinent to an end. Blood pressure may be more conveniently studied by connecting the interior of the artery (or vein) with a mercury gauge or manoneter, Fig. 25, the proximal, descending limb of which, $m$, is filled above the mercury with some imnocuous fluid, as is also the tube connecting the manometer with the artery. Using such an instrument we should observe very much the same facts as in the more simple experiment.

Immediately that communication is established between the interior of the artery and the manometer, blood rushes from the former into the latter, driving some of the mercury from the descending limb, $m$, into the ascending limb, $m^{\prime}$, and thus causing the level of the merenry in the ascending limb to rise rapidly. This rise is marked by jerks corresponding with the heart beats. Having reached a certain level, the mercnry ceases to rise any more. It does not, however, remain absolutely at rest, but undergoes oscillations; it keeps rising and falling. Each rise, which is very slight compared with the total height to which the mercury has risen, has the same rhythm as the systole of the ventricle. Similarly, each fall corresponds with the diastole. 
Chap. iv.] THE VASCULAR MECHANISM.
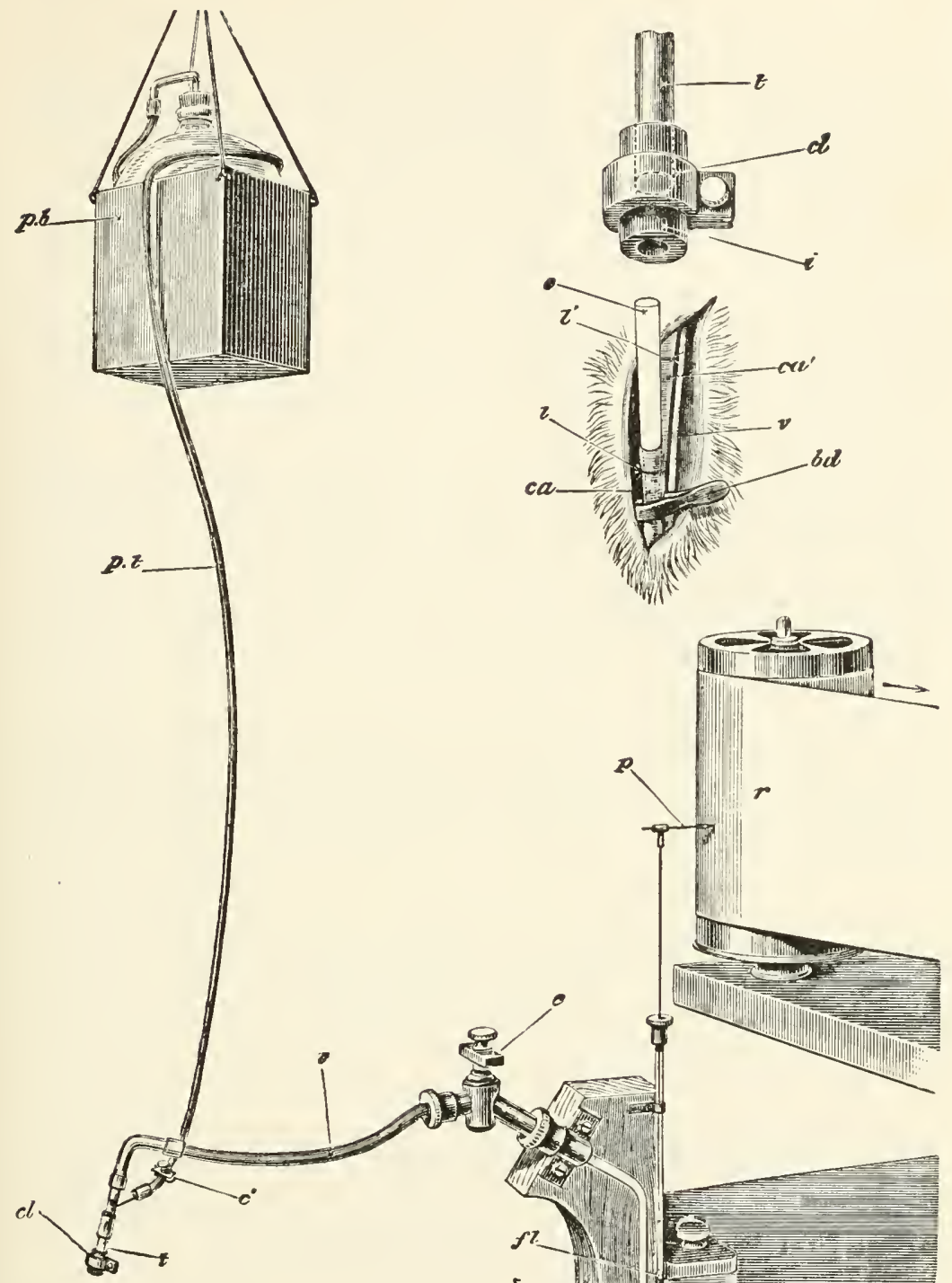

FIG. 25.

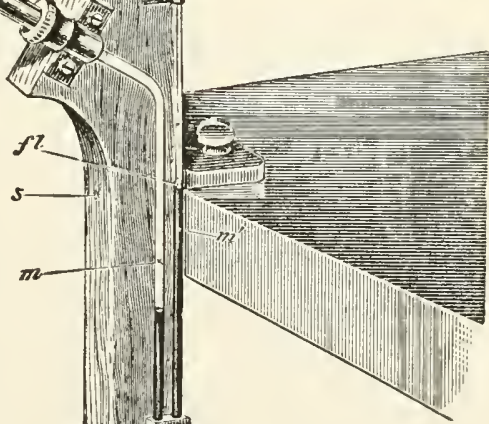




\section{Fig. 25. Apparates for investigating Blood Pressure.}

At the upper right-hand corner is seen, on an enlarged scale, the carotid artery, clamped by the forceps $b d$, with the vagus nerve $v$ lying by its side. 'The artery has been ligatured at $\%$, and the glass caunula $c$ lias been introduced into the artery between the ligature $l^{\prime}$ and the forceps $b d$, and secured in position by the ligature $l$. 'The shrunken artery on the distal side of the canmmla is seen at c $u^{\prime}$.

$p . b$. is a box containing a bottle holding a saturated solution of sodinm carbonate, or of sodium hiearbonate, or a mixture of the two, and capable of being raised or lowered at pleasure. 'The solution flows by the tube p.t. regulated by the clamp $c^{\prime \prime}$ iuto the tube $t$. A syringe, with a stopcock, may be substituted for the bottle, and attached at $c^{\prime \prime}$. This, indeed, is iu many respects a more convenient plan. The tube $t$ is connected with the leaden tube $t$, and the stopcock $c$ with the manometer, of which $m$ is the descending and $m^{\prime}$ the ascending limb, and $s$ the support. The mercury in the ascending limb bears on its surface the float $f l$, a long rod attached to which is fitted with the pen $p$, writing on the recording surface $r$. The clamp $c$. at the end of the tube $t$ lias an arrangement shewn on a larger scale at the right-hand upper corner.

The descending tube $m$ of the manometer and the tube $t$ being completely filled along its whole length with fluid to the exclusion of all air, the cannula $c$ is filled with fluid, slipped into the open eud of the thick-walled india rubber tube $i$, until it meets the tube $t$ (whose position within the india rubber tube is shewn by the dotted lines), and is then securely fixed in this position by the clamp $\mathrm{cl}$.

The stopeocks $c$ and $c^{\prime \prime}$ are now opened, and the pressure-bottle raised or fluid driven in by the syringe until the mercury in the manometer is raised to the required height. The clamp $c^{\prime \prime}$ is then closed and the forceps bd removed from the artery. The pressure of the blood in the carotid ca. is in consequence brought to bear through $t$ upon the mercury in the manometer.

If a float, swimming on the top of the mercury in the ascending limb of the manometer, and bearing a brush or other marker, be brought to bear on a travelling surface, some such tracing as that represented in Fig. 26 will be described. Each of the smaller

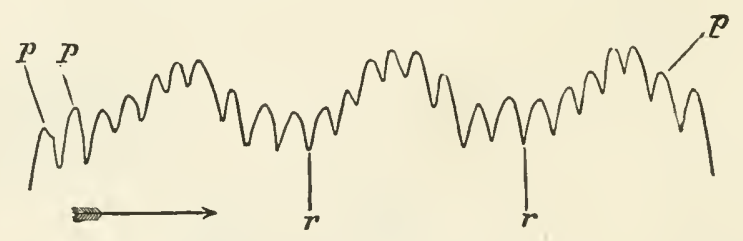

\section{Fig. 26. Tracing of Arterial Pressure with a Mercury Manometer.}

The smaller curves $p p$ are the pulse-curves. The space from $r$ to $r$ embraces a respiratory undulation. The tracing is taken from a dog, and the irregularities visible in it are those frequently met with in this animal.

curves $(p, p)$ corresponds to a heart beat, the rise corresponding to the systole, and the fall to the diastole of the ventricle. The larger undulations $(r, r)$ in the tracing, which are respiratory in origin, will be discussed hereafter. In Fig. 27 are given two tracings taken from the carotid of a rabbit; in the lower curve the recording surface is travelling more rapidly than in the upper curve; otherwise the curves are alike and repeat the general features of the curve from the dog. 

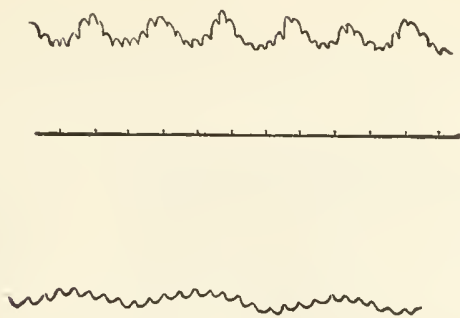

Fig. 27. Bloon Presile Curves from the Carotid of Rabit, the Time MaRKER 1N EaCH CASE MaRKING SECONDS.

Description of Experiment. Into a carotid, or other blood vessel, prepared as explained, a small glass tube, of suitable bore, called a cannula, is introduced by the method described above, and is subsequently connected by means of a short piece of india rubber tubing (Fig. $25 \mathrm{i}$ ), and a leaden or other tube $t$, which is at once flexible and yet not extensible, with the descending limb, $m$, of the manometer or mercury gauge. The cannula, tube, and descending limb of the manometer are all filled with some fluid which tends to prevent clotting of the blood, the one chosen being generally a strong solution of sodium bicarbonate, but other fluids may be chosen. In order to avoid loss of blood, a quantity of fluid is injected into the flexible tube sufficient to raise the mercury in the ascending limb of the manometer to a level a very little below what may be beforehand guessed at as the probable mean pressure. When the forceps $b d$ is removed, the pressure of the blood in the carotid is transmitted through the flexible tube to the manometer, the level of the mercury in the ascending limb of which rises a little, or sinks a little at first, or may do neither, according to the success with which the probable mean pressure has been guessed, and continues to exhibit the characteristic oscillations until the experiment is brought to an end by the blood clotting or otherwise.

Tracings of the movements of the column of mercury in the manometer may be taken either on a smoked surface of a revolving cylinder (Fig. 1), or by means of ink on a continuous roll of paper, as in the more complex kymograph (Fig. 28).

$\S$ 98. By the help of the manometer applied to various arteries and veins we learn the following facts:

(1) The mean blood pressure is high in all the arteries, but is greater in the larger arteries nearer the heart than in the smaller arteries farther from the heart; it diminishes, in fact, along the arterial tract from the heart towards the capillaries.

(2) The mean blood pressure is low in the veins, but is greater in the smaller veins nearer the capillaries than in the larger veins nearer the heart, diminishing, in fact, from the capillaries towards the heart. In the large veins near the heart it may be negative, 
that is to say, the pressure of blood in the vein bearing on the proximal descending limb of the manometer may be less than

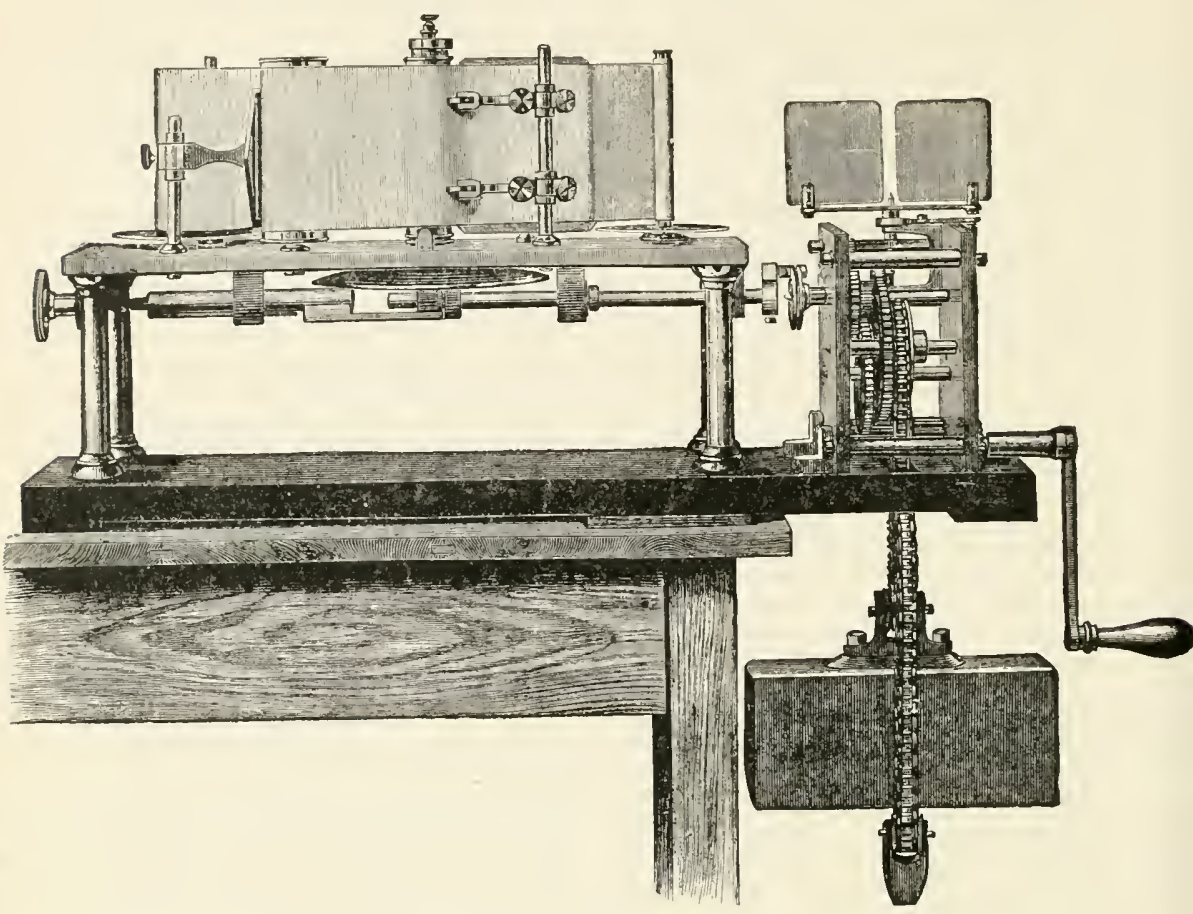

Fig. 28. Lunwig's Kymograph for recording on a continuous roll of paper.

the pressure of the atmosphere on the ascending distal limb, so that when communication is made between the interior of the vein and the manometer, the mercury sinks in the distal and rises in the proximal limb, being sucked up towards the vein.

The manometer cannot well be applied to the capillaries, but we may measure the blood pressure in the capillaries in an indirect way. It is well known that when any portion of the skin is pressed upon, it becomes pale and bloodless; this is due to the pressure driving the blood out of the capillaries and minute vessels, and preventing any fresh blood entering into them. By carefully investigating the amount of pressure necessary to prevent the blood entering the capillaries and minute arteries of the web of the frog's foot, or of the skin beneath the nail or elsewhere in man, the internal pressure which the blood is exercising on the walls of the capillaries and minute arteries and veins may be approximately determined. In the frog's web this has been found to be equal to about 7 or $11 \mathrm{~mm}$. mercury. In the mammal, the capillary blood pressure is naturally higher than this, and may be put down at 
from 15 to $20 \mathrm{~mm}$. It is, therefore, considerable, being greater than that in the veins, though less than that in the arteries.

(3) There is thus a continued decline of blood pressure from the root of the aorta, through the arteries, capillaries and veins to the right auricle. We find, however, on examination, that the most marked fall of pressure takes place between the small arteries on the one side of the capillaries, and the small veins on the other, the curve of pressure being somewhat of the form given in Fig. 29, which is simply intended to shew this fact graphically, and has not been constructed by exact measurements.

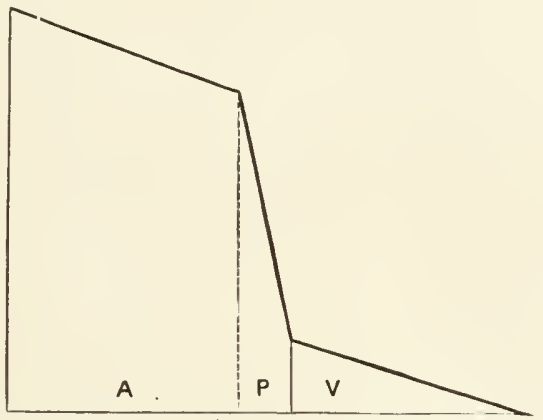

Fig. 29. Diagray of Bloon Pressure.

$A$, Arteries. $P$, Peripheral Region (minute arteries, capillaries and veins). $V$, Veins.

(4) In the arteries this mean pressule is marked by oscillations corresponding to the heart beats, each oscillation consisting of a rise (increase of pressure above the mean) corresponding to the systole of the ventricle, followed by a fall (decrease of pressure below the mean) corresponding to the diastole of the ventricle.

(5) These oscillations, which we may speak of as the pulse, are largest and most conspicuous in the large arteries near the heart, diminish from the heart towards the capillaries, and are, under ordinary circumstances, wholly absent from the veins along their whole extent from the capillaries to the heart.

Obviously a great change takes place in that portion of the circulation which comprises the capillaries, the minute arteries leading to and the minute veins leading away from the capillaries, and which we may speak of as the "peripheral region." It is here that a great drop of pressure takes place; it is here, also, that the pulse disappears.

$\S 99$. If the web of a frog's foot be examined with a microscope, the blood, as judged of by the movements of the corpuscles, is seen to be passing in a continuous stream from the small arteries through the capillaries to the veins. The velocity is greater in the arteries than in the veins, and greater in both than in the capillaries. In the arteries faint pulsations, synchronous 
with the heart's beat, are frequently visible; but these disappear in the capillaries, in which the flow is even ; that is, not broken by pulsations, and this evenness of flow is continued on along the veins so far as we can trace them. Not infrequently variations in velocity and in the distribution of the blood, due to causes which will be hereafter discussed, are witnessed from time to time.

The character of the flow through the smaller capillaries is very variable. Sometimes the corpuscles are seen passing through the channel in single file with great regularity; at other times they may be few and far between. Some of the capillaries, as we have said, are wide enough to permit two or more corpuscles abreast. In all cases the blood, as it passes through the capillary, stretches the walls and expands the tube. Sometimes a corpuscle may remain stationary at the entrance into a capillary, the channel itself being for some little distance entirely free from corpuscles. Sometimes many corpuscles will appear to remain stationary in one or more capillaries for a brief period, and then move on again. Any one of these conditions readily passes into another; and, especially with a somewhat feeble circulation, instances of all of them may be seen in the same field of the microscope. It is only when the vessels of the web are unusually full of blood that all the capillaries can be seen equally filled with corpuscles. The long, oval, red corpuscle moves with its long axis parallel to the stream, occasionally rotating on its long axis, and sometimes, in the larger channels, on its short axis. The flexibility and elasticity of a corpuscle are well seen when it is being driven into a capillary narrower than itself, or when it becomes temporarily lodged at the angle between two diverging channels.

These, and other phenomena on which we shall dwell later on, may be readily seen in the web of the frog's foot or in the stretched-out tongue or in the mesentery of the frog; and essentially similar phenomena may be observed in the mesentery or other transparent tissue of a mammal. All over the body, wherever capillaries are present, the corpuscles and the plasna are being driven in a continuous, and though somewhat irregular, yet, on the whole, steady flow through channels so minute that the passage is manifestly attended with considerable difficulties.

It is obvious that the peculiar characters of the flow through the minute arteries, capillaries, and veins, afford an explanation of the great change, taking place in the peripheral region, between the arterial flow and the venous flow. The united sectional area of the capillaries is, as we have seen, some hundreds of times greater than the sectional area of the aorta; but this united sectional area is made up of thousands of minute passages, varying in man from 5 to $20 \mu$, some of them, therefore, being in an undistended condition, smaller than the diameter of a red corpuscle. Even were the blood a simple liquid free from all 
corpuscles, these extremely minute passages would occasion a very great amount of friction, and thus present a considerable obstacle or resistance to the flow of blood through them. Still greater must be the friction and resistance occasioned by the actual blood with its red and white corpuscles. The blood, in fact, meets with great difficulties in its passage through the peripheral region, and sometimes, as we shall see, the friction and resistance are so great in the peripheral vessels of this or that area that no blood at all passes through them, and an arrest of the flow takes place in the area.

The resistance to the flow of blood thus caused by the friction generated in so many minute passages is one of the most important physical facts in the cireulation. In the large arteries the friction is small; it increases gradually as they divide, but receives its chief and most important addition in the minute arteries and capillaries: it is relatively greater in the minute arteries than in the capillaries on account of the flow being more rapid in the former, for friction diminishes rapidly with a diminution in the rate of flow. We may speak of it as the 'peripheral friction,' and the resistance which it offers as the 'peripheral resistance.' It need, perhaps, hardly be said that this peripheral resistance not unly opposes the flow of blood through the capillaries and minute arteries themselves where it is generated, but, working backwards along the whole arterial system, has to be overcome by the heart at each systole of the ventriele.

\section{Hydraulic Principles of the Circulation.}

100. In the circulation, then, the following three facts of fundamental importance are met with:

1. The systole of the ventricle, driving at intervals a certain quantity of blood, with a cortain force, into the aorta.

2. The peripheral resistance just described.

3. A long stretch of elastic tubing (the arteries), reaching from the ventricle to the region of peripheral resistance.

From these facts we may explain the main phenomena of the circulation, which we have previously sketched, on purely physical principles, without any appeal to the special properties of living tissues, beyond the provision that the ventricle remains capable of good rhythmical contractions, that the arterial walls retain their elasticity, and that the friction between the blood and the lining of the peripheral vessels remains the same; we may thus explain the high pressure and pulsatile flow in the arteries, the steady stream through the capillaries, the low pressure and the uniform pulseless flow in the veins, and, finally, the continued flow of the blood from the aorta to the mouths of the venæ cavæ.

All the above phenomena in fact are the simple results of an 
intermittent force (like that of the systole of the ventricle) working in a closed circuit of branching tubes so arranged that, while the individual tubes first diminish in calibre (from the heart to the capillaries) and then increase (from the capillaries to the heart), the area of the bed first increases and then diminishes, the tubes together thus forming two cones placed base to base at the capiliaries, with their apices converging to the heart, and presenting at their conjoined bases a conspicuous peripheral resistance, the tubing on one side, the arterial, being eminently elastic, and on the other, the venous, affording a free and easy passage for the blood. It is the peripheral resistance (for the resistance offered by the friction in the larger vessels may, when compared with this, be practically neglected), reacting through the elastic walls of the arteries upon the intermittent force of the heart, which gives the circulation of the blood its peculiar features.

$\$$ 101. Circumstances determining the character of the flow. When fluid is driven by an intermittent force, as by a pump. through a perfectly rigid tube, such as a glass one (or a system of such tubes), there escapes at each stroke of the pump from the distal end of the tube (or system of tubes) just as much fluid as enters it at the proximal end. What happens is very like what would happen if, with a wide glass tube completely filled with billiard balls lying in a row, an additional ball were pushed in at one end; each ball would be pushed on in turn a stage further, and the last ball at the further end would tumble out. The escape, moreover, takes place at the same time as the entrance.

This result remains the same when any resistance to the How is introduced into the tube, as, for instance, when the end of the tube is narrowed. The force of the pump remaining the same, the introduction of the resistance undoubtedly lessens the quantity of fluid issuing at the distal end at each stroke, but it at the same time lessens the quantity entering at the proximal end; the inflow and outflow remain equal to each other, and still occur at the same time.

In an elastic tube, such as an india rubber one (or in a system of such tubes), whose sectional area is sufficiently great to offer but little resistance to the progress of the fluid, the flow caused by an intermittent force is also intermittent. The outflow being nearly as easy as the inflow, the elasticity of the walls of the tube is scarcely at all called into play. The tube behaves practically like a rigid tube. When, however, sufficient resistance is introduced into any part of the course, the fluid, being unable to pass by the resistance as rapidly as it enters the tube from the pump, tends to accumulate on the proximal side of the resistance. This it is able to do by expanding the elastic walls of the tube. At each stroke of the pump a certain quantity of fluid enters the tube at the proximal end. Of this only a fraction can pass through the resistance during the stroke. At the moment when 
the stroke ceases, the rest still remains on the proximal side of the resistance, the elastic tube having expanded to receive it. During the interval between this and the next stroke, the distended elastic tube, striving to return to its natural undistended condition, presses on this extra quantity of fluid which it contains and tends to drive it past the resistance.

Thus in the rigid tube (and in the elastic tube without the resistance) there issues, from the distal end of the tube, at each stroke, just as much fluid as enters it at the proximal end, while between the strokes there is perfect quiet. In the elastic tube with resistance, on the contrary, the quantity which passes the resistance is only a fraction of that which enters the tube from the pump at any one stroke, the remainder or a portion of the remainder continuing to pass during the interval between the strokes. In the former case, the tube is no fuller at the end of the stroke than at the beginning; in the latter case there is an accumulation of fluid between the pump and the resistance, and a corresponding distension of that part of the tube, at the close of each stroke, - an accumulation and distension, however, which go on diminishing during the interval between that stroke and the next. The amount of fluid thus remaining after the stroke will depend on the amount of resistance in relation to the force of the stroke, and on the distensibility of the tube; and the amount which passes the resistance before the next stroke will depend on the degree of elastic reaction of which the tube is capable. Thus, if the resistance be very considerable in relation to the force of the stroke, and the tube very distensible, only a small portion of the fluid will pass the resistance, the greater part remaining lodged between the pump and the resistance. If the elastic reaction be great, a large portion of this will be passed on through the resistance before the next stroke comes. In other words, the greater the resistance (in relation to the force of the stroke), and the more the elastic force is brought into play, the less intermittent, the more nearly continuous, will be the How on the far side of the resistance.

If the first stroke be succeeded by a second stroke before its quantity of fluid has all passed by the resistance, there will be an additional accumulation of fluid on the near side of the resistance, an additional distension of the tube, an additional strain on its elastic powers, and, in consequence, the flow between this second stroke and the third will be even more marked than that between the first and the second, though all three strokes were of the same force, the addition being due to the extra amount of elastic force called into play. In fact, it is evident that, if there be a sufficient store of elastic power to fall back upon, by continually repeating the strokes a state of things will be at last arrived at, in which the elastic force, called into play by the continually increasing distension of the tube on the near side of the resistance, will be sufficient to drive through the resistance, between each two strokes, 
just as much fluid as enters the near end of the system at each stroke. In other words, the elastic reaction of the walls of the tube will have converted the intermittent into a continuous flow. The How on the far side of the resistance is in this case not the direct result of the strokes of the pump. The force of the pump is spent, first in getting up, and afterwards in keeping up the distension of the tube on the near side of the resistance; the immediate cause of the continuous flow lies in the distension of the tube, which leads it to empty itself into the far side of the resistance at such a rate that it discharges through the resistance during a stroke and in the succeeding interval just as much as it receives from the pump by the stroke itself.

This is exactly what takes place in the vascular system. The friction in the minute arteries and capillaries presents a considerable resistance to the flow of blood through them into the small veins. In consequence of this resistance, the force of the heart's beat is spent in maintaining the whole of the arterial system in a state of great distension; the arterial walls are put greatly on the stretch by the pressure of the blood thrust into them by the repeated strokes of the heart; this is the pressure which we spoke of above as blood pressure. The greatly distended arterial system is, by the elastic reaction of its elastic walls, continually tending to empty itself by overflowing through the capillaries into the venous system ; and it overflows at such a rate, that just as much blood passes from the arteries to the veins during each systole and its succeeding diastole as enters the aorta at each systole.

$\$ 102$. Indeed, the important facts of the circulation which we have as yet studied may be roughly but successfully imitated on an artificial model, Fig. 30, in which an elastic syringe represents the heart, a long piece of elastic india rubber tubing the arteries, another piece of tubing the veins, and a number of smaller connecting pieces the minute arteries and capillaries. If these connecting pieces be made at first somewhat wide, so as to offer no great resistance to the flow from the artificial arteries to the artificial veins, but be so arranged that they may be made narrow, by the screwing-up of clamps or otherwise, it is possible to illustrate the behaviour of the vascular mechanism wheu the peripheral resistance is less than usual (and as we shall see later on, it is possible in the living organism either to reduce or to increase what may be considered as the normal peripheral resistance), and to compare that behaviour with the behaviour of the mechanism when the peripheral resistance is increased.

The whole apparatus being placed flat on a table, so as to avoid differences in level in different parts of it, and filled with water, but so as not to distend the tubing, the two manometers attached, one, $A$, to the arterial side of the tubing, and the other, $V$, to the venous side, ought to shew the mereury standing at equal heights in both limbs of both instruments, since nothing 
but the pressure of the atmosphere is bearing on the fluid in the tubes, and that equally all over.

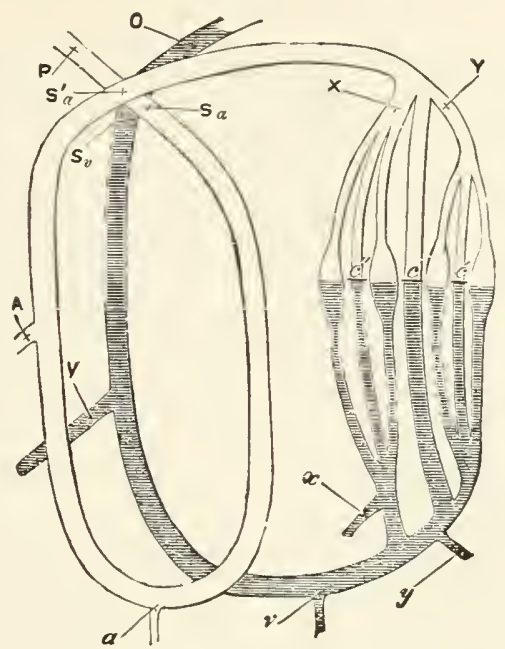

Fig. 30. Arterial Scheme.

$P$, unshaded, is an elastic tube to represent the arterial system branching at $X$ and $Y$, and ending in the region of peripheral resistance, including the capillaries, which are imitated by filling loosely with small pieces of sponge the parts shewn as dilated in the figure. The capillaries are gathered up into the venous system, shaded, which terminates at $O$. Water is driven into the arterial system at $P$ by means of an elastic bag-syringe, or any other form of pump. Clamps are placed on the undilated tubes $c, c^{\prime}, c^{\prime \prime}$. When these clamps are tightened, the only access for the water from the arterial to the renous side is through the dilated parts filled with sponge, which offer a considerable resistance to the flow of fluid through them. When the clamps are unloosed the fluid passes, with much less resistance, through the undilated tubes. Thus by tightening or loosening the clamps the "peripheral" resistance may be increased or diminished at pleasure.

At $A$, on the arterial side, and at $V$, on the venous side, manometers can be attached. At $a$ and $v$ (and also at $x$ and $y$ ) by means of clamps, the flow of fluid from an artery and from a vein, under various conditions, may be observed. At $S a$, $S^{\prime} a$, and $S *$, sphygmographs may be applied.

If now, the connecting pieces being freely open, that is to say, the peripheral resistance being very little, we imitate a ventricular beat by the stroke of the pump, we shall observe the following. Almost immediately after the stroke the mercury in the arterial manometer will rise, but will at once fall again, and very shortly afterwards the mercury in the venous tube will in a similar manner rise and fall. If we repeat the strokes with a not too rapid rhythm, each stroke having the same force, and make, as may by a sinple contrivance be effected, the two manometers write on the same recording surface, we shall obtain curves like those of Fig. 31, $A$ and $V$. At each stroke of the pump the mercury in the arterial manometer rises, but forthwith falls again to or nearly to 
the base line; no mean arterial pressure, or very little, is established. The contents of the ventricle (syringe) thrown into the

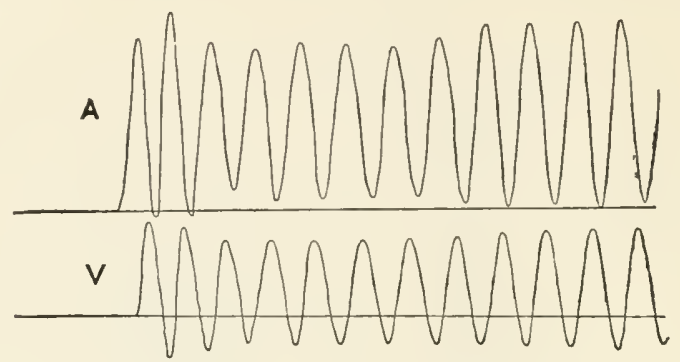

Fig. 31. Tracings taken from an artificial scieme witi tie peripieral RESISTANCE SLIGIIT.

A, Arterial. $\quad V$, Venous Manometer. This figure, to save space, is on a smaller scale than the corresponding Fig. 32 .

arterial system distend it, but the passage through the peripheral region is so free that an equal quantity of fluid passes through to the veins immediately, and hence the mercury at once falls. But the fluid thus passing easily into the veins distends these too, and the mercury in their manometer rises too, but only to fall again, as a corresponding quantity issues from the ends of the veins into the basin, which serves as an artificial auricle. Now introduce 'peripheral resistance' by screwing up the clamps on the connecting tubes, and set the pump to work again as before. With the first stroke the mercury in the arterial nanometer, Fig. 32, $A^{\prime}$, rises as before, but instead of falling rapidly, it falls slowly, because it now takes a longer time for a quantity of fluid equal to that which has been thrust into the arterial system by the ventricular stroke to pass through the narrowed peripheral region. Before the curve has fallen to the base line, before the arterial system has had time to discharge through the narrowed peripheral region as much fluid as it received from the ventricle, a second stroke drives more fluid into the arteries, distending them this time more than it did before, and raising the mercury to a still higher level. A third, a fourth, and succeeding strokes produce the same effect, except that the additional height to which the mercury is raised at each stroke becomes at each stroke less and less, until a state of things is reached in which the mercury, being on the fall when the stroke takes place, is by the stroke raised just as high as it was before, and then beginning to fall again, is again raised just as high, and so on. With each succeeding stroke the arterial system has become more and more distended; but the more distended it is the greater is the elastic reaction brought into play. This greater elastic reaction more and inore overcomes the obstacle presented by the peripheral 
resistance, and drives the fluid more and more rapidily through the peripheral region. At last the arterial system is so distended,
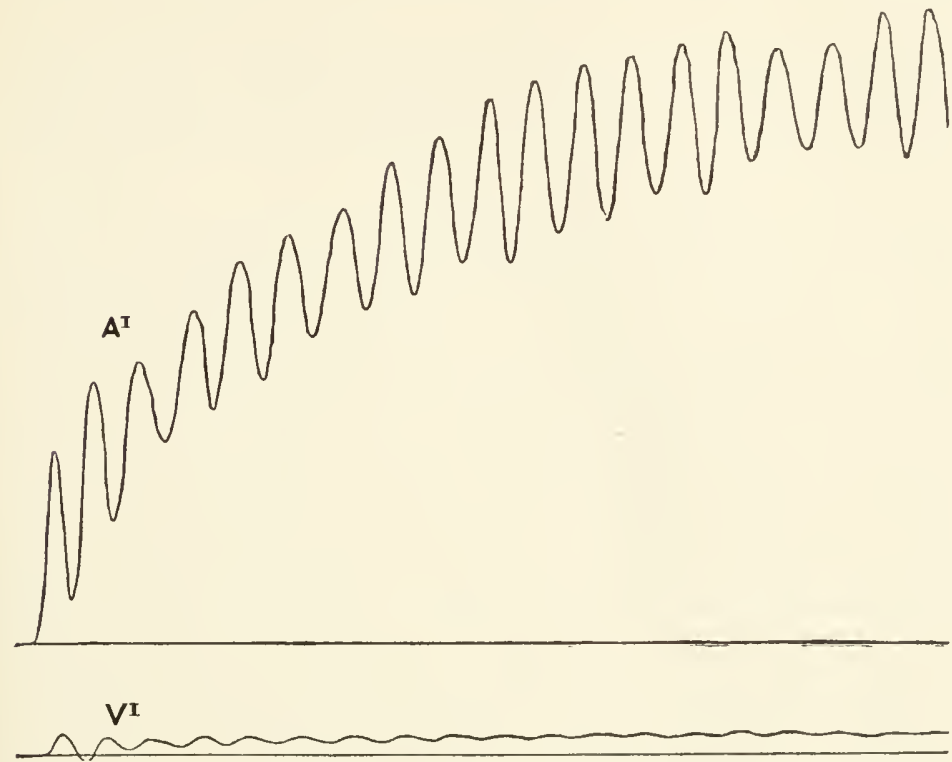

Fig 32. Tracings taken from an artificial schene with tile peripheral RESISTANCE CONSIDERABLE.

$A^{\prime}$, Arterial, $V^{\prime}$, Venous Manometer.

and the force of the elastic reaction so great, that during the stroke and the succeeding interval just as much fluid passes through the peripheral region as enters the arteries at the stroke. In other words, the repeated strokes have established a mean arterial pressure which at the point where the manometer is affixed is raised slightly at each ventricular stroke, and falls equally between the strokes.

'Turning now to the venous manometer, Fig. $32 V^{\prime}$, we observe that each stroke of the pump produces on this much less effect than it did before the introduction of the increased peripheral resistance. The mercury, instead of distinctly rising and falling at each stroke, now shews nothing more than very gentle undulations ; it feels to a very slight degree only the direct effect of the ventricular stroke; it is simply raised slightly above the base line, and remains fairly steady at this level. The slight rise marks the mean pressure exerted by the fluid at the place of attachment of the manometer. This mean 'venous' pressure is a continuation of the mean arterial pressure so obvious in the arterial manometer, but is much less than that because a large part of the arterial mean pressure has been expended in driving the fluid past 
the peripheral resistance. What remains is, however, sufficient to drive the fluid along the wide venous tubing right to the open end.

Thus this artificial model may be made to illustrate how it comes about that the blood flows in the arteries at a relatively high pressure, which at each ventricular systole is raised slightly above, and at each diastole falls slightly below a certain mean level, and flows in the veins at a much lower pressure, which does not shew the immediate effect of each heart beat.

If two manometers, instead of one, were attached to the arterial system, one near the pump and the other farther off, close to the peripheral resistance, the pressure shewn by the near manometer would be found to be greater than that shewn by the far one. The pressure at the far point is less because some of the pressure exerted at the near point has been used to drive the fluid from the near point to the far one. Similarly on the venous side, a manometer placed closed to the peripheral region would shew a higher pressure than that shewn by one farther off, because it is the pressure still remaining in the veins near the capillaries which, assisted as we shall see by other events, drives the blood onward to the larger veins. The blood pressure is at its highest at the root of the aorta, and at its lowest at the mouths of the venæe cavi, and is falling all the way from one point to the other, becuuse all the way it is being used up to move the blood from one point to the other. The great drop of pressure is, as we have said, in the peripheral region, because more work has to be done in driving the blood through this region than in driving the blood from the heart to this region, or from this region to the heart.

The manometer on the arterial side of the model shews, as we have seen, an oscillation of pressure, a pulse due to each heart beat; and the same pulse may be felt by placing a finger or rendered visible by placing a light lever on the arterial tube. It may further be seen that this pulse is most marked nearest the pump and becomes fainter as we pass to the periphery; but we must reserve the featmres of the pulse for a special study. On the venous side of the model no pulse can be detected by the manometer or by the finger, provided that the peripheral resistance be adequate. If the peripheral resistance be diminished, as by unscrewing the clamps, then, as necessarily follows from what has gone before, the pulse passes over on to the venous side; and, as we shall have occasion to point out later on, in the living organism the peripheral resistance in particular areas may be at tines so much lessened that a distinct pulsation appears in the veins.

If in the model, when the pump is in full swing, and arterial pressure well established, the arterial tube be pricked or cut, or the small side tube $a$ be opened, the water will gush out in jets, as does blood from a cut artery in the living body, whereas if the 
venous tube be sinilarly pricked or cut, or the small tube $v$ be opened, the water will simply ooze out or well up, as does blood from a vein in the living body. If the arterial tube be ligatured, it will swell on the pump side, and shrink on the peripheral side; if the venous tube be ligatured, it will swell on the side nearest the capillaries and shrink on the other side. In short, the dead model will shew all the main facts of the circulation which we have as yet described.

$\$$ 103. In the living body, however, there are certain helps to the circulation which cannot be imitated by such a model without introducing great and undesirable complications; but these chiefly affect the flow along the veins.

The veins are in many places provided with valves so constructed as to offer little or no resistance to the flow from the capillaries to the heart, but effectually to block a return towards the capillaries. Hence any external pressure brought to bear upon a vein tends to help the blood to move forward towards the heart. In the various movements carried out by the skeletal muscles, such an external pressure is brought to bear on many of the veins, and hence these movements assist the circulation. Even passive movements of the limbs have a similar effect.

The flow along the large veins of the abdomen is assisted by the pressure rhythmically brought to bear on them through the movements of the diaphragm in breathing, as well as, at times, by the forcible contractions of the abdominal muscles. Again, the movements of the alimentary canal, carried out by means of plain, muscular tissue, promote the flow along the veins coming from that canal, and when we come to study the spleen we shall see that the plain, muscular fibres, which are so abundant in that organ in some animals, serve by rhythmical contractions to pump the blood regularly away from the spleen along the splenic veins.

When we come to deal with respiration, we shall see that each enlargement of the chest constituting an inspiration tends to draw the blood towards the chest, and each return or retraction of the chest walls in expiration has an opposite effect, and, if powerful enough, may drive the blood away from the chest. The arrangement of the valves of the heart causes this action of the respiratory pump to promote the How of blood in the direction of the normal circulation; and, indeed, were the heart perfectly motionless the working of this respiratory pump alone would tend to drive the blood from the venxe cava through the heart into the aorta, and so to keep up the circulation; the force so exerted, however, would, without the aid of the heart, be able to overcome a very small part only of the resistance in the capillaries and small vessels of the lungs, and so would prove actually ineffectual.

There are, then, several helps to the flow along the veins, but it must be remembered that however useful, they are helps only 
and not the real cause of the circulation. The real cause of the flow is the ventricular stroke, and this is sufficient to drive the blood from the left ventricle to the right auricle, even when every muscle of the body is at rest, and breathing is for a while stopped, - when, therefore, all the helps we are speaking of are wanting.

\section{Circumstances determining the Rate of the Flow.}

$\S 104$. We may now pass on to consider briefly the rate at which the blood flows through the vessels, and first the rate of flow in the arteries.

When even a small artery is severed, a considerable quantity of blood escapes from the proximal cut end in a very short space of time. That is to say, the blood moves in the arteries from the heart to the capillaries with a very considerable velocity. By various methods, this velocity of the blood current has been measured at different parts of the arterial system; the results, owing to imperfections in the methods employed, cannot be regarded as satisfactorily exact, but may be accepted as approximately true. They shew that the velocity of the arterial stream is greatest in the largest arteries near the heart, and diminishes from the heart towards the capillaries. Thus in a large artery of a large animal, such as the carotid of a dog or horse, and probably in the carotid of a man, the blood flows at the rate of 300 or $500 \mathrm{~mm}$. a second. In the very small arteries the rate is probably only a few $\mathrm{mm}$. a second.

Methods. The Hæmadromometer of Volkmann. An artery, e.g. a carotid, is clamped in two places, and divided between the clamps. 'Two cannule, of a bore as nearly equal as pussible to that of the artery, or of a known bore, are inserter in the two ends. The two cannulie are connected by means of two stopcocks, which work together, with the two ends of a long glass tube, bent in the shape of a $U$, and filled with normal saline solution, or with a colonred, innocuous Hliit. The clamps on the artery being released, a turn of the stopcocks permits the blood to enter the proximal end of the long $\boldsymbol{U}$ tube, along which it courses, driving the fluid out into the artery through the distal end. Attached to the tube is a graduater scale, by means of which the velocity with which the blood flows along the tube may be read off.

The Rheometer (Stromuhr) of Ludwig. The principle of this consists in measuring the time which it takes the flow through an artery to fill and refill a vessel of known cafocity a certain number of times. The instrument (Fig. 33), which cousists of two glass bulbs, one being of known capacity, is connected, like the foregoing instrument, with two cannulæ fixed in the two ends of a severed artery, and is so arranged that the bulb of known capacity can be 
repeatedly filled and refilled in succession. From the length of time it takes to fill the bulb a certain number of times the flow through the artery is calculated.
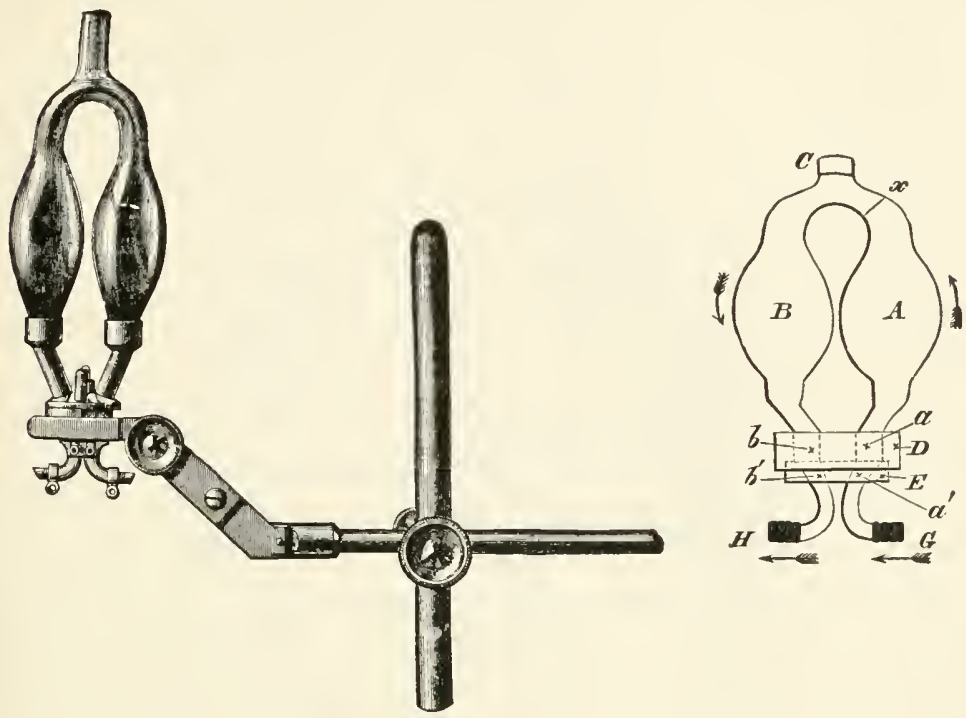

Fig. 33. Ludigig's Stromulir and a Diagamatic representation of tine same.

$G$ and $I I$ fit into the cannulæe placed respectively into the proximal and distal cut ends of the artery under examination. $D$ is a metal disc revolving on a lower similar dise $E$. $A$ and $B$ are glass bulbs (which can be filled through $C$ ) fixed upon $D$; the eapacity of $A$ up to the mark $x$ is known. Hoies are bored through $L$ and $E$ in such a way that in the position shewn in the figure fluid passes from $G$ through $a^{\prime}$ and $a$ into $A$, and so by $B, b$ and $b^{\prime}$ to $I I$. If the dise $D$ be turned through two right angles, fluid passes from $a^{\prime}$ to $b$ and so by $b^{\prime}, A$, and $a$ to $b^{\prime}$ If it be turned through one right angle ouly the fluid passes directly from $G$ to $I I$ withont entering the bulbs at all. $A$ is filled with pure oil up to the mark $x, B$ with lefibrinated blood. The blood is allowed to flow from $G$ into $A$ until the whole of the oil is driven into $B$, the defibrinated blood occupying which is driven into $H$. 'Then, by a rapid turn, the position of $A$ and $B$ is reversed, and the oil driven back into $A$; then again by another turn back from $A$ into $B$, and so on until clotting stops the observation. The time which it takes the flow through $G$ ' to fill $A$ (np to the mark $x$ ) alternately with blood and oil, being thins determined, the sectional area of $G$ and the capacity of $A$ being kuown, the velocity of the flow through $G$ may be calculated.

The Hæmatachometer of Vierordt is constructed on the principle of measuring the velocity of the current by observing the amount of deviation undergone by a pendulum, the free end of which hangs loosely in the stream.

An instrument based on the same principle has been invented by Chauveau and improved by Lortet, Fig. 34. A somewhat wide tuhe, the wall of which is at one point composed of an india rubber membrane, is introduced between the two cut ends of an artery. A long, light lever pierces the india rubber membrane. The short, expanded arm of 
this lever projecting within the tube (and corresponding to the pendulum of Vierordt's instrument) is moved on its fulcrum in the india rubber ring by the current of blood passing through the tube, the greater the velocity of the current, the larger being the excursion of the lever.

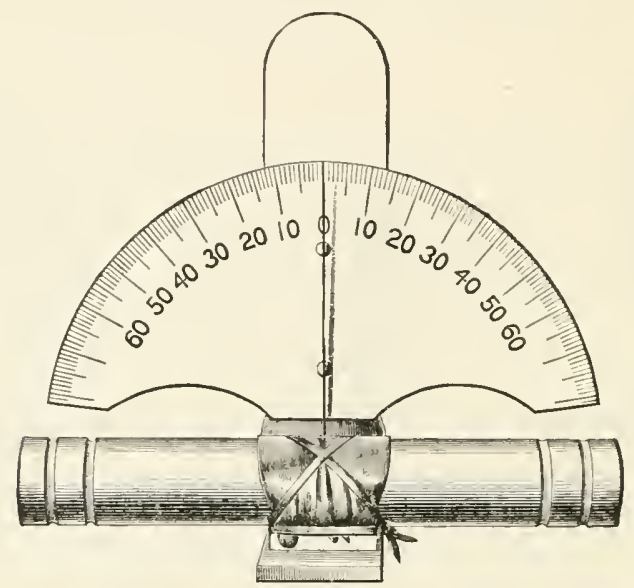

Fig. 34. Hematachometer of Chauveau and Lortet.

The movements of the short arm give rise to corresponding movements in the opposite direction of the long arm outside the tube, and these, by means of a marker attached to the end of the long arm, may be directly inscribed on a recording surface. This instrument is hest arlapted for observing changes in the velocity of the flow. For determining actual velocities it has to be experimentally graduated.

The rapidity of the flow, and especially variations in the rapidity, may also be studied in a more indirect manner by means of the following methorl, called the 'plethysmographic method.'

'The principle of the plethysmograph is that changes in the volume of a part or of an organ of the body, are measured by the displacement of fluid in a chamber with rigid walls surrounding the part or organ. A part of the boly, the arm, for instance, is introduced into a chamber with rigid walls, such as a large glass cylinder, which is filled with fluid, the opening by which the arm is introduced being closed with an india rubber ring or with plaster of Paris. The cavity of the chamber is connected, at one spot, with a narrow glass tuhe, open at the end, in which the fluid, after the introduction of the arm, stands at a certain level. Any change in the volume of the arm manifests itself by a change in the level of the fluid in the tube; when the arm shrinks the level falls, when the arm swells, the level rises. And by means of a piston working in the tube, or by a float bearing a marker and swimming on the top of the fluirl, or by other contrivances, a graphic record of the changes in the level of the fluid in the tube and so of the changes in the volume of the arm may he obtainer. Such an instrument is called a plethysmograph; and, as we shall see it may be applied in various ways to various parts and organs of the body. 
Now, changes in the volume of the arm are mainly caused (we may for the present neglect other causes) by changes in the quantity of blood present in that portion of the arm which lies within the cylinder. Upon examination it is found that besides certain slower changes of volume which take place from time to time, there are changes of volume corresponding to each heart beat. At each heart beat the volume first increases and then decreases again, reaching before the next heart beat the same measure which it had just preceding the beat; there is, we may say, a pulsation of volume like the actual pulse; and we may, by the graphic method, obtain a curve of the changes in volume, a "volume curve." An increase of volume, a rise of the curve, means that the blood is flowing into the arm, within the cylinder, by the (axillary) artery at the level of the rim of the cylinder, more swiftly than it is flowing out by the (axillary) vein or veins at the same level; a decrease of volume, a fall of the curve, means that the blood is flowing in less swiftly than it is flowing out; and a stationary volume, the curve neither rising nor falling, means that the blood is flowing in just as fast as it is flowing ont. The steeper the ascent of the volume curve, the greater is the rapidity of the arterial inflow, and any lessening of the steepness of the ascent means a diminution of that rapility; when the steepness is lessened so much that the curve runs parallel to the base line, then, whatever the actual height of the curve, the inflow by the artery is only just as rapid as the outtlow by the vein. Hence, the limensions of the parts of the apparatus being known, we may calculate how many more or how many less cubic cm. of blood are flowing per second, or per fraction of a second, in by the artery, than are flowing out by the vein. But, as we have seen, the flow in the veins is constant so far as each individual heart beat is concerned; it is not directly influenced by each heart beat. Hence, having obtained by means of the instrument a curve of the change of volume of the arm, we may from that calculate out a curve of the changes in rapidity of the flow in the artery at the level of the mouth of the cylinder. In this way it is ascertained that with each heart beat the rapiclity of the flow at first rises very quickly, then more slowly, then ceases to rise, after which it sinks, and, indeed, sinks to such a degree as to shew that the blood at this moment is flowing less rapilly in the artery than in the vein, but subsequently rises again to fall once more, just before the next heart beat, to the same rate as at the beginning of the beat which is being studied. Noreover, it is possible by help of certain assmmptions to calculate the amount of the whole flow through the artery (and through the vein) in a given time, that is to say, the actual rapidity of the flow.

In the capillaries, the rate is slowest of all. In the web of the frog the flow as judged by the movement of the red corpuscles may be directly measured under the microscope by means of a micrometer, and is found to be about half a millimeter in a second; but this is probably a low estimate, since it is only when the circulation is somewhat slow, slower, perhaps, than what ought to be considered the normal rate, that the red corpuscles can be distinctly seen. In the manmal the rate has been estimated 
at about 75 millimeters a second, but is probably quicker even than this.

As regards the veins, the flow is very slow in the small veins emerging from the capillaries but increases as these join into larger trunks, until in a large vein, such as the jugular of the dog, the rate is about $200 \mathrm{~nm}$. a second.

$\$$ 105. It will be seen, then, that the velocity of the flow is in inverse proportion to the width of the bed, to the united sectional areas of the ressels. It is greatest at the aorta, it diminishes along the arterial system to the capillaries, to the united bases of the cones spoken of in $\$ 94$, where it is least, and from thence increases again along the venous system.

And, indeed, it is this width of the bed and this alone which determines the general velocity of the flow at various parts of the system. The slowness of the flow in the capillaries is not due to there being so much more friction in their narrow channels than in the wider canals of the larger arteries; for the peripheral resistance caused by the friction in the capillaries and small arteries is an obstacle not only to the How of blood through these small vessels, where the resistance is actually generated, but also to the escape of the blood from the large into the small arteries, and, indeed, from the heart into the large arteries. It exerts its influence along the whole arterial tract. And it is obvious that if it were this peripheral resistance which checked the flow in the capillaries, there could be no recovery of velocity along the venous tract.

The blood is flowing through a closed system of tubes, the blood vessels, under the influence of one propelling force, the systole of the ventricle; for this is the force which drives the blood from ventricle to auricle, though, as we have seen, its action is modified in the several parts of the system. In such a system the same quantity of fluid must pass each section of the system at the same time, otherwise there would be a block at one place, and a

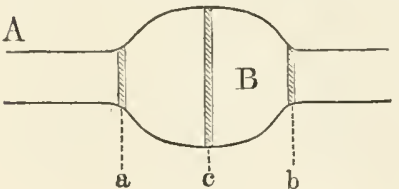

FIG. 35. deficiency at another. If, for instance, a fluid is made to flow by some one force, pressure or gravity, through a tube $A$ (Fig. 35$)$ with an enlargement $B$, it is obvious that the same quantity of fluid must pass through the section $b$ as passes through the section $a$ in the same time, - for instance, in a second. Otherwise, if less passes through $b$ than $a$, the fluid would accumulate in $B$, or if more, $B$ would be emptied. In the same way just as much must pass in the same time through the section $c$ as passes through $\alpha$ or $b$. But if just as many particles of water have to get through the narrow section $a$ in the same time as they have to get through the broader section $c$, they must nove more quickly through a than through $c$, or more slowly through $c$ 
than through $\alpha$. For the same reason, water flowing along a river impelled by one force, viz. that of gravity, rushes rapidly through a 'narrow,' and flows sluggishly when the river widens out into a 'broad.' The flow through $B$ will be similarly slackened if $B$, instead of being simply a single enlargement of the tube $A$, consists of a number of small tubes branching out from $A$, with a united sectional area greater than the sectional area of $A$. In each of such small tubes, at the line $c$, for instance, the flow will be slower than at $\alpha$, where the small tubes branch out from $A$, or at $b$, where they jom again to form a single tube. Hence it is that the blood rushes swiftly through the arteries, flows slowly through the capillaries, but quickens its pace again in the veins.

An apparent contradiction to this principle that the rate of flow is dependent on the width of the bed is seen in the case where, the fluid having alternative routes, one of the rontes is temporarily widened. Suppose that a tube $A$ divides into two branches of equal length $x$ and $y$, which unite again to form the tube $V$. Suppose, to start with, that $x$ and $y$ are of equal diameter: then the resistance offered by each being equal, the How will be equally rapid through the two, being just so rapid that as much fluid passes in a given time through $x$ and $y$ together as passes through $A$ or through $V$. But now suppose $y$ to be widened: the widening will diminish the resistance offered by $y$, and, in consequence, supposing that 110 material change takes place in the pressure or force which is driving the fluid along, more fluid will now pass along $y$ in a given time than did before, that is to say, the rapidity of the flow in $y$ will be increased. It will be increased at the expense of the flow through $x$, since it will still hold good that the flow through $x$ and $y$ together is equal to the flow through $A$ and through $V$ We shall have occasion later on to point out that a small artery, or a set of small arteries, may be more or less suddenly widened, without materially affecting the general blood pressure which is driving the blood through the artery or set of arteries. In such cases the flow of blood through the widened artery or arteries is, for the time, being increased in rapidity, not only in spite of, but actually in consequence of the artery being widened.

It must be understood, in fact, that this dependence of the rapidity of the flow on the width of the bed applies to the general rate of flow of the whole circulation; and that while, on account of the width of the bed, the flow through the capillaries is slower than through the small arteries and veins, that through the small arteries slower than through the larger arteries, and that through the sinall veins slower than through the larger veins, the actual rapidity in any individual capillary, small artery or small vein, or in any individual sets of these, varies largely from time to time, owing to changes of circumstances, prominent among which are changes in the resistance to the flow, - changes which, as we shall 
see, may be brought about in various ways. Hence, any numerical statement as to the rate of flow in these vessels must be regarded as a general statement only.

Moreover, it must be remembered that though we speak of the flow past a point of a large artery as being of a certain rapidity, say $300 \mathrm{~mm}$. a second, that rapidity is continually varying. The cause of the flow through the whole system is the pressure of the ventricular systole manifested as what we have called blood pressmre. At each point along the system nearer the left ventricle, and therefore further from the right auricle, the pressure is greater than at a point further from the left ventricle, and so nearer the right auricle; it is this difference of pressure which is the real cause of the flow from the one point to the other; and other things being equal the rapidity of the flow will depend on the amount of the difference of pressure. But the pressure exerted by the ventricle is not constant; it is intermittent, rhythmically rising and falling. Hence at every point along the arterial system the flow is increased in rapidity during the temporary increase of pressure due to the ventricular systole, and dininished during the subsequent temporary decrease, the increase and decrease being the more marked the nearer the point to the heart; this is shewn in observations made by means of Chauveau and Lortet's instrument or by the plethysinographic method (\$104).

$\$ 106$. Time of the entire circuit. It is obvious from the foregoing that a red corpuscle in performing the whole circuit, in travelling from the left ventricle back to the left ventricle, would spend a large portion of its time in the capillaries, minute arteries, and veins. The entire time taken up in the whole circuit has been approximately estimated by measuring the time it takes for an easily recognized chemical substance, after injection into the jugular vein of one side, to appear in the blood of the jugular vein of the other side.

While small quantities of blood are being drawn at frequently repeated intervals from the jugular vein of one side, or while the blood from the vein is being allowed to fall in a minute stream on an absorbent paper covering some travelling surface, an iron salt such as potassium ferrocyanide (or preferably sodium ferrocyanide as being less injurious) is injected into the jugular vein of the other sile. If the time of the injection be noterl, and the time after the injection into one side at which evidence of the presence of the iron salt can be detected in the sample of blood from the vein of the other side be noted, this gives the time it has taken the salt to perform the circuit; and on the supposition that mere diffusion does not materially affect the result, the time which it takes the blood to perform the same circuit is thereby given.

A modification of this method, doing away with the necessity of withdrawing blood, is based on the fact that the electrical conductivity of the blood may be changed by altering the saline constituents. Two 
(non-polarisable) electrodes are placed one on each side of some part of a blood vessel, artery or vein, say the right jugular or femoral vein (previously laid bare and insulated), and are connected with a Wheatstone bridge and galvanometer, as in the usual way of observing changes in electrical resistance. If a solution of salt be now injected into some other vessel, say the left jugular, the blood laden with the extra quantity of salt, when it reaches the seat of the electrodes will give rise to a change in the electrical resistance through the blool vessel with its contained blood between the electrodes, and this will be indicated by a movement of the galvanometer. If the times of the injection, and of the movement of the galvanometer be noted, the interval between the two will give the time it takes the blood containing the salt to pass from the seat of injection to the seat of the electrodes.

In the horse this time has been experimentally determined at about 30 secs. and in the $\operatorname{dog}$ at about 15 secs. In man it is probably from 20 to 25 secs.

We may arrive at a similar result indirectly by means of a calculation. Taking the quantity of blood as $\frac{1}{13}$ of the body weight, the blood of a man weighing 75 kilos would be about 5,760 grm. If 180 grms. left the ventricle at each beat, a quantity equivalent to the whole blood would pass through the heart in 32 beats, i.e. in less than half a minute.

Taking the rate of flow through the eapillaries at about $1 \mathrm{~mm}$. a sec., it would take a corpuscle as long a time to get through about $20 \mathrm{~mm}$. of capillaries as to perform the whole circuit. Hence, if any corpusele had in its circuit to pass through $10 \mathrm{~mm}$. of capillaries, half the whole time of its journey would be spent in the narrow channels of the capillaries. Inasmuch as the purposes served by the blood are chiefly carried out in the capillaries, it is obviously of advantage that its stay in them should be prolonged. Since, however, the average length of a capillary is about $5 \mathrm{~mm}$., about half a second is spent in the capillaries of the tissues and another half second in the capillaries of the lungs.

$\S 107$. We may now briefly summarise the broad features of the circulation, which we have seen may be explained on purely physical principles, it being assumed that the ventricle delivers a certain quantity of blood with a certain force into the aorta at regular intervals, and that the physical properties of the blood vessels remain the same.

We have seen that, owing to the peripheral resistance offered by the capillaries and small vessels, the direet effect of the ventricular stroke is to establish in the arteries a mean arterial pressure, which is greatest at the root of the aorta and diminishes towards the small arteries; some of it being used up to drive the blood from the aorta to the small arteries, but which retains at the region of the small arteries sufficient power to drive through the small arteries, capillaries and veins just as much blood as is being 
thrown into the aorta by the ventricular stroke. We have seen further that in the large arteries at each stroke the pressure rises and falls a little above and below the mean, thus constituting the pulse, but that this extra distension with its subsequent recoil diminishes along the arterial tract and finally vanishes; it diminishes and vanishes because it, too, like the whole force of the ventricular stroke, of a fraction of which it is the expression, is used up in establishing the mean pressure; we shall, however, consider again later on the special features of this pulse. We liave seen further that the task of driving the blood through the peripheral resistance of the small arteries and capillaries consumes much of this mean pressure, which consequently is much less in the small veins than in the corresponding small arteries, but that sufficient remains to drive the blood, even without the help of the auxiliary agents which are generally in action, from the small veins right back to the auricle. Lastly, we have seen that while the above is the cause of the flow from ventricle to auricle, the changing rate of the flow, the diminishing swiftness in the arteries, the sluggish crawl through the capillaries, the increasing quickness through the veins are determined by the changing width of the vascular 'bed.'

Before we proceed to consider any further details as to the phenomena of the flow through the vessels, we must turn aside to study the heart. 


\section{SEC. 3. THE HEART.}

$\S 108$. The heart is a valvular pump which works on mechanical principles, but the motive power of which is supplied by the contraction of its muscular fibres. Its action consequently presents problems which are partly mechanical, and partly vital. Regarded as a pump, its effects are determined by the frequency of the beats, by the force of each beat, by the character of each beat, - whether, for instance, slow and lingering, or sudden and sharp, and by the quantity of fluid ejected at each beat. Hence, with a given frequency, force, and character of beat, and a given quantity ejected at each beat, the problems which have to be dealt with are for the most part mechanical. The vital problems are chiefly connected with the causes which determine the frequency, force, and character of the beat. The quantity ejected at each beat is governed not only by the action of the heart itself, but also and. indeed more so by what is going on in the rest of the body.

\section{The Phenomena of the Normal Beat.}

The visible movements. When the chest of a mammal is opened, and artificial respiration kept up, the heart may be watched beating. Owing to the removal of the chest-wall, what is seen is not absolutely identical with what takes place within the intact chest, but the main events are the same in both cases. A complete beat of the whole heart, or cardiac cycle, may be observed to take place as follows.

The great veins, inferior and superior venæ cavæ and pulmonary veins, are seen, while full of blood, to contract in the neighbourhood of the heart: the contraction runs in a peristaltic wave towards the auricles, increasing in intensity as it goes. Arrived at the auricles, which are then full of blood, the wave suddenly spreads, at a rate too rapid to be fairly judged by the eye, over the whole of those organs, which accordingly contract with a sudden sharp 
systole. In the systole, the walls of the auricles press towards the auriculo-ventricular orifices, and the auricular appendages are drawn inwards, becoming smaller and paler. During the auricular systole, the ventricles may be seen to become turgid. Then follows, as it were immediately, the ventricular systole, during which the ventricles become more conical. Held between the fingers they are felt to become tense and hard. As the systole progresses, the aorta and pulmonary arteries expand and elongate, the apex is tilted slightly upwards, and the heart twists somewhat on its long axis, moving from the left and behind towards the front and right, so that more of the left ventricle becomes displayed. As the systole gives way to the succeeding diastole, the ventricles resume their previous form and position, the aorta and pulmonary artery shrink and shorten, the heart turns back towards the left, and thus the cycle is completed.

In the normal beat, the two ventricles are perfectly synchronous in aetion; they contract at the same time and relax at the same time, and the two auricles are similarly synchronous in action. It has been maintained, however, that the synchronism may at times not be perfect.

Before we attempt to study in detail the several parts of this complicated series of events, it will be convenient to take a rapid survey of what is taking place within the heart during such a cycle.

$\$ 109$. The cardiuc cycle. We may take as the end of the cycle the moment at which the ventricles having emptied their contents have relaxed and returned to the diastolic or resting position and form. At this moment the blood is flowing freely with a fair rapidity, but, as we have seen, at a very low pressure, through the vense cave into the right auricle (we may confine ourselves at first to the right side), and since there is now nothing to keep the tricuspid valve shut, some of this blood probably finds its way into the ventricle also. This goes on for some little time, and then comes the sharp, short systole of the auricle, which, since it begins, as we have seen, as a wave of contraction rumning forwards along the ends of the venæe cavæ, drives the blood not backwards into the veins, but forwards into the ventricle; this result is further secured by the fact that the systole has behind it on the venous side the pressure of the blood in the veins, increasing as we have seen backwards towards the capillaries, and before it the relatively empty cavity of the ventricle in which the pressure is at first very low. By the complete contraction of the auricular walls the complete or nearly complete emptying of the cavity is ensured. No valves are present in the mouth of the superior vena cava, for they are not needed; and the imperfect Eustachian valve at the mouth of the inferior vena cava cannot be of any great use in the adult, though in its more developed state in the fotus it had an important function in directing the blood of the inferior vena cava through the foramen ovale into the left 
auricle. The valves in the coronary vein are, however, probably of some use in preventing a reflux into that vessel.

As the blood is being driven by the auricular systole into the ventricle, a reflex current is probably set up, by which the blood, passing along the sides of the ventricle, gets between them and the flaps of the tricuspid valve and so tends to float these up. It is further probable that the same reflux current, continuing somewhat later than the flow into the ventricle, is sufficient to bring the Haps into apposition, without any regurgitation into the auricle, at the close of the auricular systole, before the ventricular systole has begun.

The auricular systole is, as we have said, immediately followed by that of the ventricle. Whether the contraction of the ventricular walls (which as we shall see is a simple though prolonged contraction and not a tetanus) begins at one point, and swiftly travels over the rest of the fibres, or begins all over the ventricle at once, is a question not at present definitely settled; but in any case the walls exert on the contents a pressure which is soon brought to bear on the whole contents and very rapidly rises to a maximum. The effect of this increasing intra-ventricular pressure upon the valve is undoubtedly to render the valve more firmly and securely closed; but the exact behaviour of the valve in thus firmly closing is a matter on which observers are not agreed. From the disposition of the flaps of the valve, and their relations to the papillary muscles, the chordae tendinea of a papiltary muscle being attached to the edges of and spreading over the surfaces of two adjacent flaps, we may infer that when the papillary muscles contract, taking their share in the whole ventricular systole, they on the one hand bring at least the edges, if not part of the surfaces of adjacent flaps, into opposition, and, on the other hand, tend to pull down the whole of the valve, more or less in the form of a narrow funnel, into the cavity of the ventricle. If we assume, as some observers do, that the papillary muscles begin their contraction at the same time as the rest of the ventricular wall, we may conclude that the valve is in this manner firmly closed by their action at the very beginning of the systole. Other observers find that a tracing, obtained by attaching a hook to the apex of one of the flaps of the valve, and connecting it with a thread passing through the auriculo-ventricular orifice, and the auricle to a lever, indicates that the apex of the flap does not begin to move downwards until some appreciable time after the beginning of the systole. This they interpret as meaning that the papillary muscles do not begin to contract until some time after the ventricular wall has begun its contraction; (and the tracing in question similarly indicates that the papillary muscle ceases its contraction before the ventricmlar wall does). If we assume this interpretation of the tracing to be correct, we must conclude that, at the first, the pressure exerted by the conmencing systole would 
tend, while bringing the edges of the flaps together, to bulge the whole valve upwards towards the auricle, but that, later, when the papillary muscles contract, these pull the valve in a funnel shape down into the ventricle with the edges of the flaps in complete apposition. On the one view, the papillary muscles serve merely to secure the adequate closure of the valve; on the other view, they add to the pressure exerted by the ventricular wall, by pulling the already closed valve down on the ventricular contents, or, aecording to an old opinion, obviate, by their shortening, the slackening of the chordie which might result from the shortening of ventricle during the systole. Whichever view be taken, it may be worth while to remark that the borders of the valves are excessively thin, so that when the valve is closed, these thin portions are pressed flat together back to back; hence, while the tougher central parts of the valves bear the force of the ventricular systole, the opposed thin, membranous edges. pressed together by the blood, more completely secure the closure of the orifice.

At the commencement of the ventricular systole, the semilunar valves of the pulmonary artery are closed, and are kept closed by the high pressure of the blood in the artery. As, however, the ventricle continues to press with greater and greater force on its contents, making the ventricle hard and tense to the touch, the pressure within the ventricle becomes at length greater than that in the pulmonary artery, and this greater pressure forces open the semilunar valves, and allows the escape of the contents into the artery. The ventricular systole may be seen and felt in the exposed heart to be of some duration; it is strong enough and long enough to empty the ventricle more or less completely, - indeed, in some cases, it may last longer than the discharge of blood, so that there is then a brief period during which the ventricle is empty but yet contracted.

During the ventricular systole the semilunar valves are pressed outwards towards but not close to the arterial walls, reflux currents probably keeping them in an intermediate position, so that their orifice forms an equilateral triangle with curved sides; they offer little obstacle to the escape of blood from the cavity of the ventricle. The exact mode and time of closure of the semilunar valves is a matter which has been and, indeed, is still disputed, and which we shall have to discuss in some detail later on. Meanwhile it will be sufficient to say, after the blood has ceased to flow from the ventricle into the aorta, whether this be due to the cessation of the ventricular systole, or to the whole of the ventricular contents having been already discharged, a reflux of blood in the aorta towards the ventricle at once completely fills and renders tense the pockets, causing their free margins to come into close and firm contact, and thus entirely blocks the way. The corpora Arantii meet in the centre, and the thin, membranous festoons or lunulæ are brought into exact apposition. As in the 
tricuspid valves, so here, while the pressure of the blood is borne by the tougher bodies of the several valves, each two thin, adjacent lunulæ, pressed together by the blood acting on both sides of them, are kept in complete contact, without any strain being put upon them; in this way the orifice is closed in a most efficient manner.

As the ventricular systole passes off, the muscular walls relaxing, the ventricle returns to its previous form and position, and the cycle is once more ended.

What thus takes place in the right side takes place in the left side also. There is the same sudden, sharp, auricular systole beginning at the roots of the puhmonary veins, the sante systole of the ventricle, but, as we shall see, one much more powerful and exerting much more force; the mitral valve with its two flaps acts in the main like the tricuspid valve, and the action of the semilunar valves of the aorta simply repeats that of the valves of the puknonary artery. detail.

We may now proceed to study some of the cardiac events in

$\$ 110$. The change of form. The exact determination of the changes in form and position of the heart, especially of the ventricles, dnring a cardiac cycle is attended with difficulties.

The ventricles, for instance, are continually changing their form; they change while their cavities are being filled from the auricles, they change while the contraction of their walls is getting up the pressure on their contents, they change while under the influence of that pressure their contents are being discharged into the arteries, and they change when, their cavities having been emptied, their muscular walls relax.

With regard to changes in external form, there seems no doubt that the side-to-side diameter is much lessened during the systole. There is also evidence that the front-to-back diameter is greater during the systole than during the diastole, the increase taking place during the first part of the systole. If a light lever be placed so as to press very gently on the surface of the heart of a mammal, the chest having been opened and artificial respiration being kept up, some such curve as that represented in Fig. 36 may be obtained. The rise of the lever in describing such a curve is due to the elevation of the part of the front surface of the heart on which the lever is resting. Such an elevation might be caused, especially if the lever were placed near the apex, by the heart being "tilted" upwards during the systole, but only a small portion at most of the rise can be attributed to this cause; the rise is perhaps best seen when the lever is placed in the middle portion of the ventricle, and must be chiefly due to an increase in the front-to-back diameter of the ventricle during the beat. We shall discuss this curve later on in connection with other curves, and may here simply say that the part of the curve from $b^{\prime}$ to $d$ 
probably corresponds to the actual systole of the ventricle, that is, to the time during which the fibres of the ventricle are undergoing contraction, the sudden fall from $d$ onwards representing the relaxation which forms the first part of the diastole. If this

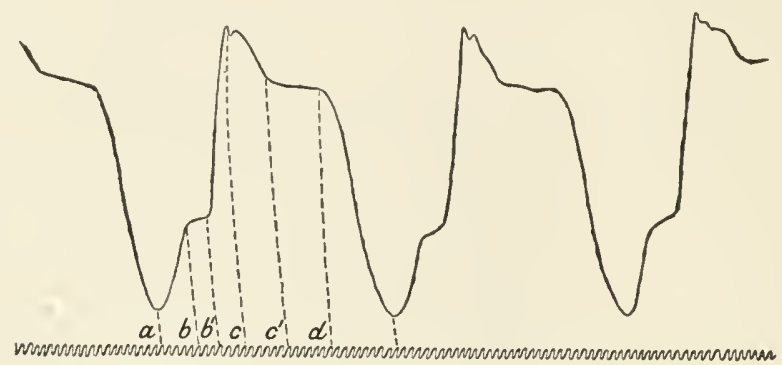

Fig. 36. Tracing from Heapt of Cat, obtaned by placing a light lever

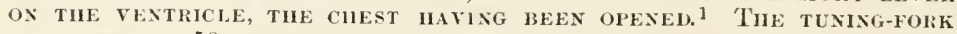
CURVE MARIS 50 VIBRATIONS PER SEC.

interpretation of the curve be correct, it is obvious that the front-to-back diameter is greater during the whole of the systole than it is during diastole, since the lever is raised up all this time. It may, however, be argued that the heart thus exposed is subject to abnormal conditions and is, in diastole, somewhat flattened by the weight of its contents, that this flattening is increased by even slight pressure, and that therefore the above conclusion is not

1 The vertical or rather curved lines (segments of circles) introduced nuto this and many other curves are of use for the purpose of measuring parts of the curve. A complete rurve should exhibit an 'alscissa' line. 'This may be drawn by allowing the lever, arranged for the experiment but remaining at rest, to mark with its point on the recording surface set 11 motion: a straight line, the abscissa line, is thus described, and may be drawn before or after the curve itself is made, and may he placed above or, preferably, below the curve. When a tuming-fork or other time narker is nsed, the liue of the time marker or a line drawn thromgh the curves of the tuning-fork will serve as an abscissa line. After a tracing has been male, the recorting surface should he brought back to such a position that the point of the lever coincides with some point of the curve which it is desired to mark; if the lever be then gently moved up and down, the point of the lever will describe a segment of a circle (the centre of which lies at the axis of the lever), which segment should be made lung enongh to cut both the curve and the abscissa lue (the tmuing-fork curves or other time-marking line) where this is drawn. By moving the recording surface backwards and forwards, similar segments of circles may be drawn through other pounts of the curve. 'The lincs $a, b, c$ in Fig 36 were thrs drawn. The distance between any two of these pouts may thus be measured on the tuning-fork curve or other time curve, or on the abscissit line. Sinilar lines may be drawn on the tracing after its removal from the recording instrument in the following way. Take a pair of compasses, the two points of which are fixed just as far apart as the length of the lever used in the experiment, measured from its axis to its writing point. By means of the compasses find the position on the tracing of the centre of the circle of which any one of the previonsly drawn curved limes forms a segment. Thromgh this centre draw a line parallel to the abscissa. By keeping one point of the compass on this line but moving it aloug the line backwards or forwards, a segment of a circle may be drawn so as to cut any point of the curve that may be desired, and also the abscissa line or the time line. Such a segment of a circle may he used for the same purposes as the original one and any number of snch seguients may be dhawn. 
valid. And, indeed, it is maintained by some that the front-toback diameter does actually diminish during systole.

But it is at least clear that the front-to-back diameter, even if it does not increase, diminishes far less than does the side-to-side diameter; and hence during the systole there is a change in the form of the section of the base of the ventricles. During the diastole this has somewhat the form of an ellipse with the long axis from side to side, but with the front part of the ellipse much more convex than the back, since the back surface of the ventrieles is somewhat flattened. During the systole this ellipse is converted into a figure much more nearly resembling a circle. It is urged, moreover, that the whole of the base is constricted, and that the greater efficiency of the auriculo-ventricular valves is thereby secured.

As to the behaviour of the long diameter from base to apex, observers are not agreed; some maintain that it is shortened, and others that it is practically unchanged. And, in any case, a change in this diameter plays little or no part in the expulsion of the contents of the ventricle; this expulsion is effected by the contraction of the more transversely disposed fibres, whereby the cavity is reduced to an elongated slit. Moreover, if any shortening does take place it must be compensated by the elongation of the great vessels, which, as stated above, nuay be seen in an inspection of the beating heart. For there is evidence that the apex, though, as we have seen, it is somewhat twisted round during the systole, and at the same time brought closer to the chest-wall, does not change its position up or down, i.e. in the long axis of the body. If in a rabbit or dog a needle be thrust through the chest-wall so that its point plunges into the apex of the heart, though the reedle quivers, its head moves neither up nor down, as it would do if its point in the apex moved down or up.

During systole, broadly speaking, the ventricles undergo a diminution of total volume, equal to the volume of contents discharged into the great vessels (for the walls themselves like all muscular structures retain their volume during contraction save for changes which may take place in the quantity of blood contained in their blood vessels, or of lymph in the intermuscular spaces), while they undergo a change of form which may be described as that from a roughly hemispherical figure with an irregularly elliptical section to a more regular cone with a more nearly circular base.

\$11. Cardiac Impulse. If the hand be placed on the chest, a shock or impulse will be felt at each beat, and on examination this impulse, 'cardiac impulse,' will be found to be synchronous with the systole of the ventricle. In man, the cardiac impulse may be most distinctly felt in the fifth costal interspace, about an inch below and a little to the median side of the left nipple. In an animal the same impulse may also be felt in another way, viz. 
by making an incision through the diaphragm from the abdomen, and placing the finger between the chest-wall and the apex. It then can be distinctly recognized as the result of the hardening of the ventricle during the systole. And the impulse which is felt on the outside of the chest is chiefly the effect of the same hardening of the stationary portion of the ventricle in contact with the chest-wall, transmitted through the chestwall to the finger. In its flaccid state, during diastole, the apex is (in a standing position at least) at this point in contact with the chest-wall, lying, somewhat flattened, between it and the tolerably resistant diaphragm. During the systole, while being brought even closer to the chest-wall, by the tilting of the ventricle and by the movement to the front and to the right of which we have already spoken, it suddenly grows tense and hard, and becomes rounder. The ventricles, in executing their systole, have to contract against resistance. They have to produce within their cavities, pressures greater than those in the aorta and pulmonary arteries, respectively. This is, in fact, the object of the systole. Hence, during the swift systole, the ventricular portion of the heart becomes suddenly tense, somewhat in the same way as a bladder full of fluid would become tense and hard when forcibly squeezed. The sudden pressure exerted by the ventricle thus rendered suddenly tense and hard, äided by the closer contact of the apex with the chest-wall (which, however, by itself, without the hardening of contraction, would be insufficient to produce the effect), gives an impulse or shock both to the chest-wall and to the diaphragm. If the modification of the sphygmograph (an instrument of which we shall speak later on, in dealing with the pulse), called the cardiograph, be placed on the spot where the impulse is felt most strongly, the lever is seen to be raised during the systole of the ventricles, and to fall again as the systole passes away, very much as if it were placed on the heart directly. A tracing may thus be obtained, see Fig. 46 , of which we shall have to speak more fully later on, see $\$ 115$. If the button of the lever be placed, not on the exact spot of the impulse, but at a little distance from it, the lever will be depressed during the systole. While at the spot of impulse itself the contact of the ventricle is increased during systole, away from the spot the ventricle (owing to its change of form and subsequently to its diminution in volume) retires from the chest-wall, and hence, by the mediastinal attachments of the pericardium, draws the chest-wall after it.

$\$$ 112. The Sounds of the Heart. When the ear is applied to the chest, either directly or by means of a stethoscope, two sounds are heard, - the first a comparatively long, dull, booming sound, the second a short, sharp, sudden one. Between the first and second sounds the interval of time is very short, too short to he easily measured, but between the second and the succeeding first sound there is a distinct pause. The sounds have been likened 
to the pronunciation of the syllables lūbb dŭp, so that the cardiac cycle, as far as the sonnds are concerned, might be represented by: - lūbb, dūp, pause.

The second sound, which is short and sharp, presents no difficulties. It is coincident in point of time with the closure of the semilunar valves, and is heard to the best advantage over the second right costal cartilage, close to its junction with the sternum, $i . e$. at the point where the aortic arch comes nearest to the surface, and to which sounds generated at the aortic orifice would be best conducted. Its characters are such as would belong to a sonnd generated by membranes like the semilunar valves being suddenly made tense, and so thrown into vibrations. It is obseured and altered, or replaced by 'a murmur,' when the semilunar valves are affected by disease, and may be artificially obliterated, a murmur taking its place, by passing a wire down the arteries, and hooking up the aortic valves. There can be no doubt, in fact, that the second sound is due to the semilunar valves being thrown into vibrations at their sudden closure. The sound heard at the second right costal cartilage is chiefly that generated by the aortic valves, and murmurs or other alterations in the sound caused by changes in the aortic valves are heard most clearly at this spot. But even here the sound is not exclusively of aortic origin, for in certain cases, in which the semilunar valves on the two sides of the heart are not wholly synchronous in action, the sound heard here is double ("reduplicated second sound"), one being due to the aorta, and one to the pulmonary artery. When the sound is listened to on the left side of the sternum at the same level, the pulmonary artery is supposed to have the chief share in producing what is heard, and changes in the sound heard more clearly here than on the right side are taken as indications of mischief in the pulmonary valves.

The first sound, longer, duller, and of a more 'booming" character than the second, heard with greatest distinctness at the spot where the cardiac impulse is felt, presents many difficulties in the way of a complete explanation. It is heard distinctly when the chest-walls are removed. The cardiac impulse, therefore, can have little or nothing to do with it. In point of time, it is coincident with the systole of the ventricles, and may be heard to the greatest advantage at the spot of the cardiac impulse; that is to say, at the place where the ventricles come nearest to the surface, and to which sounds generated in the ventricles would be best conducted.

It is more closely coincident with the closure and consequent vibrations of the auriculo-ventricular valves than with the entire systole; for on the one hand it dies away before the second sound begins, whereas, as we shall see, the actual systole lasts at least up to the closure of the semilunar valves, and on the other hand the auriculo-ventricular valves cease to be tense 
and to vibrate so soon as the contents of the ventricle are driven out. This suggests that the sound is caused by the sudden tension of the auriculo-ventricular valves, and this view is supported by the facts that the sound is obscured, altered or replaced by murnurs when the tricuspid or mitral valves are diseased, and that the sound is also altered or, according to some observers, wholly done away with when blood is prevented from entering the ventricles by ligature of the venæ cavæ. On the other hand, the sound has not that sharp character which one would expect in a sound generated by the vibration of membranes such as the valves in question, but in its booming qualities rather suggests a muscular sound. Further, according to some observers, the sound, though somewhat modified, may still be heard when the large veins are clamped so that no blood enters the ventricle, and, indeed, may be recognized in the few beats given by a mammalian ventricle rapidly cut out of the living body by an incision carried below the auriculo-ventricular ring. Hence the view has been adopted that this first sound is a muscular sound. In discussing tlie muscular sound of skeletal muscle (see $\$ 75$ ), we saw reasons to distrust the view that this sound is generated by the repeated, individual, simple contractions which make up the tetanus, and lience corresponds in tone to the number of those simple contractions repeated in a second, and to adopt the view that the sound is really due to a repetition of unequal tensions occurring in a muscle during the contraction. Now, the ventricular systole is undoubtedly a simple contraction, a prolonged simple contraction, not a tetanus, and, therefore, under the old view of the nature of a muscular sound, could not produce such a sound; but accepting the other view, and reflecting how complex must be the course of the systolic wave of contraction over the twisted fibres of the ventricle, we shall not find great difficulty in supposing that that wave is capable in its progress of producing such repetitions of unequal tensions as might give rise to a 'muscular sound,' and, consequently, in regarding the first sound as mainly so caused. Accepting such a view of the origin of the sound we should expect to find the tension of the muscular fibres, and so the nature of sound, dependent on the quantity of fluid present in the ventricular cavities and hence modified by liga. ture of the great veins, and still more by the total removal of the auricles with the auriculo-ventricular valves. We may add that we should expect to find it morlified by the escape of blood from the ventricles into the arteries during the systole itself, and might regard this as explaining why it dies away before the ventricle has ceased to contract.

Moreover, seeing that the auriculo-ventricular valves must be thrown into sudden tension at the onset of the ventricular systole, which, as we have seen, is developed with considerable rapidity, not far removed at all events from the rapidity with which the 
semilunar valves are closed, a rapidity, therefore, capable of giving rise to vibrations of the valves adequate to produce a sound, it is difficult to escape the conclusion that the closure of these valves must also generate a sound, which in a normally beating heart is mingled with the sound of inuscular origin.

If we accept this view that the sound is of double origin, partly 'muscular,' partly 'valvular,' both causes being dependent on the tension of the ventricular cavities, we can perhaps more easily understand how it is that the normal first sound is at times so largely, indeed, we may say so completely altered and obscured in diseases of the auriculo-ventricular valves, and how it may also be modified in character by changes, such as hypertrophy, of the muscular walls.

Since the left ventricle forms the entire left apex of the heart, the murmurs or other changes of the first sound heard most distinctly at the spot of cardiac impulse belong to the mitral valve of the left ventricle. Murmurs generated in the tricuspid valve of the right ventricle are heard more distinctly in the median line below the end of the sternum.

$\$$ 113. Endocardiac Pressure. Since it is the pressure exerted upon the contents of the ventricle by the contraction of the ventricular walls which drives the blood from the heart into the aorta, and so maintains the circulation, the study of this pressure, endocardiac pressure, is of great importance. The mercurial manometer, so useful in a general way in the study of arterial pressure, is unsuited for the study of endocardiac pressure, since the great inertia of the mercury prevents the instrument responding properly to the exceedingly rapid changes of pressure which take place in the heart. We are obliged to have recourse to other instruments.

One method, having been used by Chauveau and Marey in researches which have become 'classic,' deserves to be noticed, though it is not now employed. It consists in introducing, in a large animal, such as a horse, through a blood vessel into a cavity of the heart, a tube ending in an elastic bag, Fig. $37 \mathrm{~A}$, both tube and bag being filled with air, and the tube being connected with a recording 'tambour.'

A tube of appropriate curvature, A. b. Fig. 37, is furnished at its end with an elastic bag or 'ampulla' $\alpha$. Such an instrument is spoken of as a 'cardiac sound.' When it is desired to explore simultaneously both auricle and ventricle, the somud is furnishel with two anpullix, one at the extreme end and the other at such a distance that when the former as within the cavity of the ventricle the latter is within the cavity of the auricle. 'Each 'ampulla' communicates by a separate air-tight tube with an air-tight tambour (Fig. $37 \mathrm{~B}$ ) on which a lever rests, so that any pressure on the ampulla is communicated to the earity of its respective tambour, the lever of 
which is raised in proportion. When two ampullæ are used, the writing points of both levers are brought to bear on the same recording surface exactly underneath each other. 'The tube is carefully introduced through the right jugular vein into the right side of the heart until the lower (ventricular) ampulla is fairly in the cavity of the right ventricle, and, consequently, the upper (auricular) ampulla in the cavity of the right auricle. Changes of pressure on either ampulla, then, cause movements of the corresponding lever. When the pressure, for instance, on the ampulla in the auricle is increased, the auricular lever is raised and describes on the recording surface an

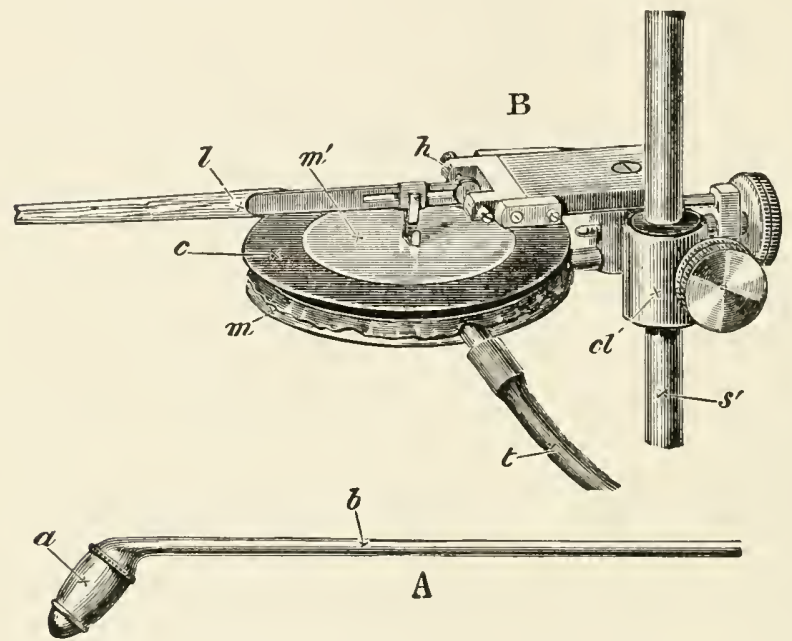

Fig. 37. Marey's Tambovr, with Camdac Sound.

A. A simple cardiac sound such as may be used for exploration of the left ventricle. 'The portion a of the ampulla at the end is of thin india rubber, stretched over an open framework with metallic supports above and below. The long tube $b$ serves to introluce it into the cavity which it is desired to explore.

B. The Tambour. The metal chamber $m$ is covered in an air-tight manner with the ind rubber $c$, bearing a thin, metal plate $m^{\prime}$, to which is attached the lever $/$, noving on the hinge $h$. The whole tambour can be placed by means of the clamp $c l$ at any height on the upright $s$ '. 'The india rubber tube $t$ serves to connect the interior of the tambour either with the eavity of the ampulla of $A$ or with any other cavity. Supposing that the tnbe $t$ were connected witl $b$, any pressure exerted on a would cause the roof of the tambour to rise and the point of the lever wonld be proportionately raised.

ascending curve; when the pressure is taken off, the curve descends, - and so also with the ventricle.

The 'sound' may in a similar manner be introduced through the carotid artery into the left ventricle, being slipped past the aortic valves, and thus the changes taking place in that chamber also may be explored. 
When this instrument is applied to the right auricle and ventricle some such record is obtained as that shewn in Fig. 38, where the upper curve is a tracing taken from the right auricle, and the lower curve from the right ventricle of the horse, both curves being taken simultaneously on the same recording surface. In these curves the rise of the lever indicates pressure exerted upon the corresponding ampulla, and the upper curve, from the right auriele, shews the sudden, brief pressure $b$ exerted by the sudden and brief auricular systole. The lower curve, from the right ventricle, shews that the pressure exerted by the ventricular systole begins almost immediately after the auricular systole, increases very rapidly indeed, so that the lever rises in almost a straight line up to $c^{\prime}$, is continmed for some considerable time, and then falls very rapidly to reach the base line. The figure, it must be understood, does not, by itself, give any information as to the relative amounts of pressure exerted by the auricle and ventricle respectively; indeed, the movements of the auricular lever are much too great compared with those of the ventricular lever. The figure is chiefly useful for giving a graphic general view of the series of events within the cardiac cavities during a cardiac cycle, the short auricular pressure, the long-continued ventricular pressure, lasting nearly half the whole period, and the subsequent pause when both parts are at rest or in

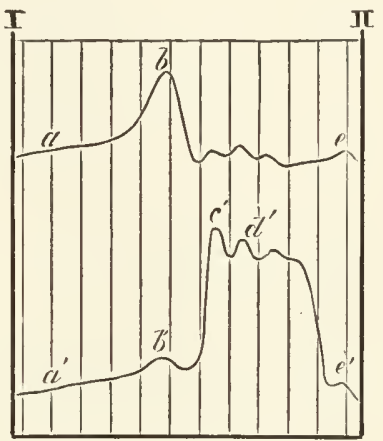
diastole.

Among the more trustworthy methods of recording the changes of endocardiac pressure, we may first mention that of Roy and Rolleston.

By means of a short cannula introduced through a large vessel, or directly, as a trocar, through the walls of the ventricle (or auricle), the blood in the cavity is brought to bear on an easily moving piston. The movements of the piston are recorded by a lever, and the evils of inertia are met by making the piston and lever work against the torsion of a steel ribbon, the length of which, and consequently the resistance offered by which, and hence the excursions of the piston, can be varied at pleasure.

We give as examples of curves obtained by this method two curves from the left ventricle, one (Fig. 39 A) of a rapidly beating, and the other (Fig. 39 B) of a slowly beating heart. 

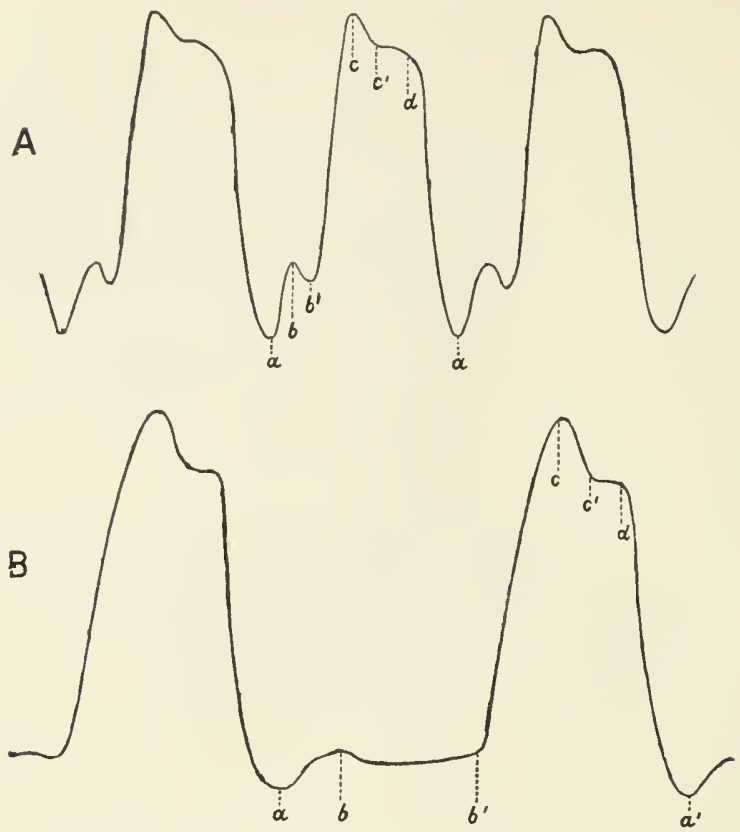

Fig. 39. Curves of Endocardiac Pressure. Fron Left Ventricle of Dog. (Roy and Rolleston.)

A. a quickly beating, B. a more slowly beating heart.

An instrument which has been much used of late, and the use of which has given very valuable results is the "membrane-manometer" of Hiirthle.

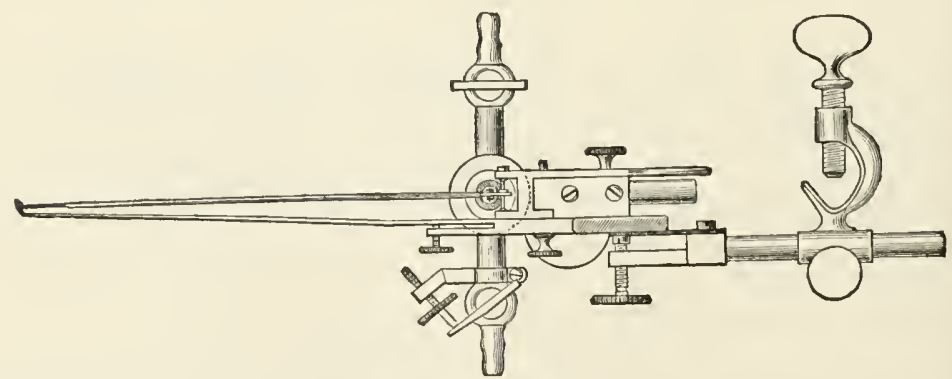

Fig. 40. The Memirane-mavometer of Hüthle. ${ }^{1}$

1 For this figure I am indebted to Mr. Albrecht, the University Instrument maker at Tübingen. 
This consists essentially of a very small netal drum or tambour (Fig. $41 a$ ) somewhat like that of Narey, but hemispherical and not more than $15 \mathrm{~mm}$. in diameter, ending below in a tube $b$. In Fig. 40 the instrument, with its holder, is seen from above. The second lever, which is motionless, is for the purpose of describing the base line. 'The screw-tap on the tube leading, in the figure, up to the tambour, is for the purpose of diminishing the callore of the tube and so of 'damping' the instrument. On the right of the tambour in the figure are seen the arrangements for adjusting the levers. In Fig. 41 the tube b by which the catheter is connected with the tambour, is, for convenience of illustration, shewn as directed parallel to the lever, instead of, as in the instrument itself, at right angles to it. The roof of the tambour is supplied by a care-

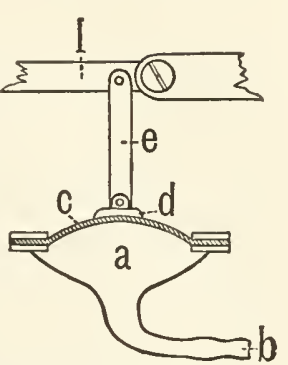

Fig. 41. Diagram to ilLUSTRATE THE ESSEN. TIAL PARTS OF HÜRTHLE'S MEUBRANE MA. NOMETER. fully chosen delicate elastic membrane $c$ which bears at its centre a thin metal disc $d$, comnected by a short upright $e$ with a lever $l$.

A catheter, opened at the end or with a lateral 'eye' and filled with a solution of magnesium sulphate or with some fluid tending to check the clotting of blood, is introduced into the cavity of the leart which it is desired to explore. It may be introduced by the jugular vein into the right auricle, and past the auricle into the right ventricle, or through the carotid artery into the arrta, and so, between the semilunar valves, or piercing one of the flaps (the perforation seems to introduce no error) into the cavity of the left ventricle; or the end of the catheter may be left in the arta above the semilunar valves when it is desired to investigate the pressure at the root of the aorta. The cavity of the tambour also is filled, not with air, as in Marey's tambour, but with the same fluid as is the catheter, or with water; and the tube of the tambour is connected with the catheter.

Variations of pressure within the cavity of the heart are transmitted through the fluid of the catheter to the fluid in the tambour, and thus put into movement the elastic roof of the tambour; the movements of the elastic roof are, in turn, transmitted to the lever, which records, in the usual manner, on some recorling surface. For measuring the amount of the changes of pressure, the instrument must be graduated experimentally. There are many letails in the instrument which need not be described here; but we may state that the instrument may be 'damped,' rendered less sensitive, and thus the features of the curves due to inertia lessened, by narrowing, through a screw-tap, the communication between the catheter and the cavity of the tambour.

The membrane of the tambour may, by means of an ivory button, be brought to bear on one end of a slip of steel, placed horizontally and fastened at the other end, so as to act as a spring. The instrument then becomes a "spring-manometer." "The small movements of the spring caused by the movements of the membrane of the tambour are magnified by a recording lever. 
Fig. 42 gives a curve of endocardiac pressure of the left

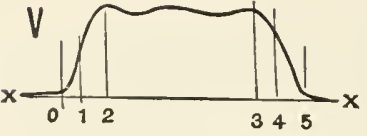

Fig. 42. Curve of Pressure IN TIE LEFT VEXTHICLE OF TIF DOG, HËRTLE'S MEMIBRA XE-MA NUMETER ventricle of the dog obtained by this method. The recording surface is travelling quickly, and the movements of the lever are not great.

The manometer of Gad differs from that of Hirthle in the membrane being replaced by a thin, elastic disc of metal.

In the instrument of Frey and Krehl, which is a modification of one by Fick, the transmission is effected partly by fluid and partly by an air tambour, the batton of which presses against a horizontal steel spring.

A catheter, filled with fluid to prevent clotting and introduced into a cavity of the heart, is connected with a glass cylinder, maintained carefully in a vertical position, the lower half of which is filled with the same fluid as is the catheter. The upper half of the cylinder, containing air only, is conneeted by a very narrow, in fact a capillary tube, with a small tambour. The changes of pressure within the heart are transmitted through the fluid of the catheter to the air in the cylinder, and so to the air in the tambour, the membrane of which moves accordingly in and out. A button on the membrane presses on a horizontal steel spring, and the small movements of the membrane thus transmitted to the spring are recorded by means of a magnifying lever.

Other instruments have been employed by other observers.

When we examine the curves which we have given (Figs. 38, 39,42 ), obtained by three several methods, we find that they agree in the following main features. The curve of pressure in the ventricle, whether right or left, rises at the very beginning of the systole with very great rapidity, very soon reaches its maximum or nearly its maximum, maintains nearly the same height for some time, and then very rapidly descends to the base line (which in these figures indicates the pressure of the atmosphere) or even falls, for a brief space, slightly below it, and remains at or near the base line, until, at the next beat, it repeats the same changes. This means that the contraction of the ventricular walls in the systole acts in such a manner as very suddenly to raise up to a certain height the pressure within the ventricle, which during the diastole was at, or not far removed from that of the atmosphere, that the pressure is maintained without any very great change for a considerable time, and that it then falls back to its original level with great suddemess, almost, if not quite, as suddenly as it was raised. These are the important features of the pressure within the ventricle; in these features all the three curves agree. We may add that the same features are shewn also in curves of pres- 
sure taken by other methods; and, indeed, as shewn in Fig. 36 and in others which we shall give, corresponding features occur in curves of other changes in the heart. All these curves shew a flattening maintained, with smaller variations, during the continuance of the systole; this is so characteristic that it has been called the 'systolic plateau.' It is true that curves of ventricular pressure taken by certain methods, that of Frey and Krehl's for instance, do not shew this 'plateau,' the curve in such cases rising gradually to a maximum and immediately beginning to fall, so that the summit is a simple peak. And it is argued that such a curve is the true curve of ventricular pressure always obtained so long as the blood in the ventricle has free access to the interior of the catheter, and that the plateau is only seen when the end of the catheter is too near the apex, and its opening closed, at the height of the systole, by the ventricular walls coming together; the top of the true curve is thus, as it were, cut off. But the evidence is, on the whole, opposed to this view, and we shall accept the plateau as being a true representation.

Though the curves given above agree in these main features, they differ in many minor features, and other features also of minor value appear in curves of endocardiac pressure according to the various circumstances in which the heart finds itself. Some of these minor features we shall presently find useful in discussing the mechanism of the beat.

$\S 114$. The output. Since the use of the pressure exerted by the ventricle is to drive a quantity of blood out of the ventricle into the aorta (or pulmonary artery) it is important to study the 'output' or quantity of blood so driven out; and since, under normal circumstances, the quantity ejected by the right ventricle is the same as that ejected by the left ventricle, we may confine our attention to the latter.

The normal or average output has been calculated in various ways, by help of certain assumptions; but these we may put on one side since the matter has now been made the subject of direct experimental determination.

Methods. Method of Stolnikow. This consists in allowing the blood to flow from the carotid into a vessel until a certain measured quantity has escaped, and then returning this blond to the right auricle while the blood from the carotid is flowing into a second similar vessel to be similarly returned, and in repeating this manœuvre a certain number of times. One carotid is tier (the animal being a $\operatorname{dog}$ ), and the arch of the aorta plugged beyond (Fig. $43 p$ ). The circulation is thus confined to the lungs and the coronary system. Into the other carotid is tier a tube connected by a forked branching $1 a$ and $2 a$ with two vessels I. and II., which also communicate by a similar forked branching $l v$ and $2 v$ with the right auricle. The blood is allowed to flow through $1 a$ into I. until a certuin quantity has escaped. Then $1 a$ is closed, while $2 a$ and $1 v$ are opened. The blood 
from I. flows back by $1 v$ to the right auricle, while the bluod from the carotid flows into II. by $2 a$. When a certain quantity has escaped into II., the action is reversed, and I. is once more filled; and so on.

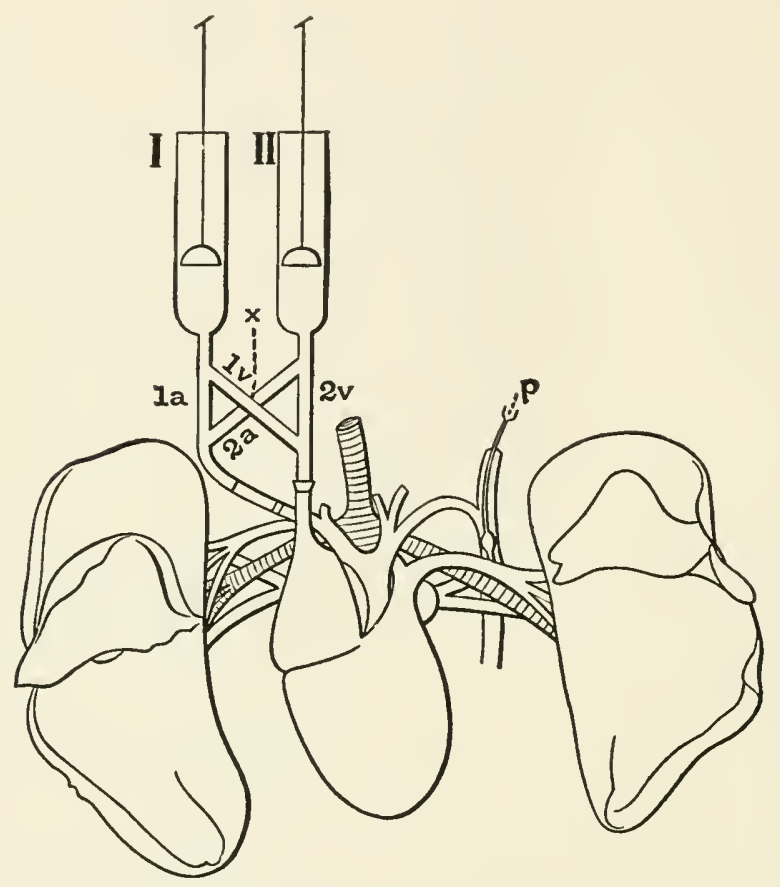

Fig. 43. Diagram of Stolikow's Apparatus.

In this way the quantity of blood which the heart delivers, its 'outpnt' during a given time can be measured; the quantity discharged at a single beat can similarly be determined. $B y$ means of recording floats in I. and II., a graphic record of the output niay also be obtained.

The other methods are plethysmographe ( $(104)$ in nature. The volume of the heart changes only with the volume of its contents, for we may neglect, in the first instance at least, as insignificant the changes of volume due to changes in the amount of blood held by the coronary system, and we may wholly neglect the changes of volume due to changes in the quantity of lymph present in the cardiac tissues. An increase in the volume of the heart means that more blood is flowing into it than is leaving it, a decrease that more is leaving it than is flowing into it. Hence, if we measure the diminution of volume which takes place during the systole, this gives us the volume of bloorl discharged by the two ventricles during that systole, the effect of changes in the amricles being neglected; anil since the two ventricles discharge equal quantities, half this will give ns the quantity of blood discharged by the left ventricle luring the systole.

In the method of Tigerstedt and others the pericardial cavity is 
employed as the plethysmographic chamber, the changes of- volume in it being transmitted by air to the recording apparatus. A cannula is introduced into the pericardium, a little air entering at the same time, and is connected by an air tube with a delicate piston, the movements of which are recorded in the usual way.

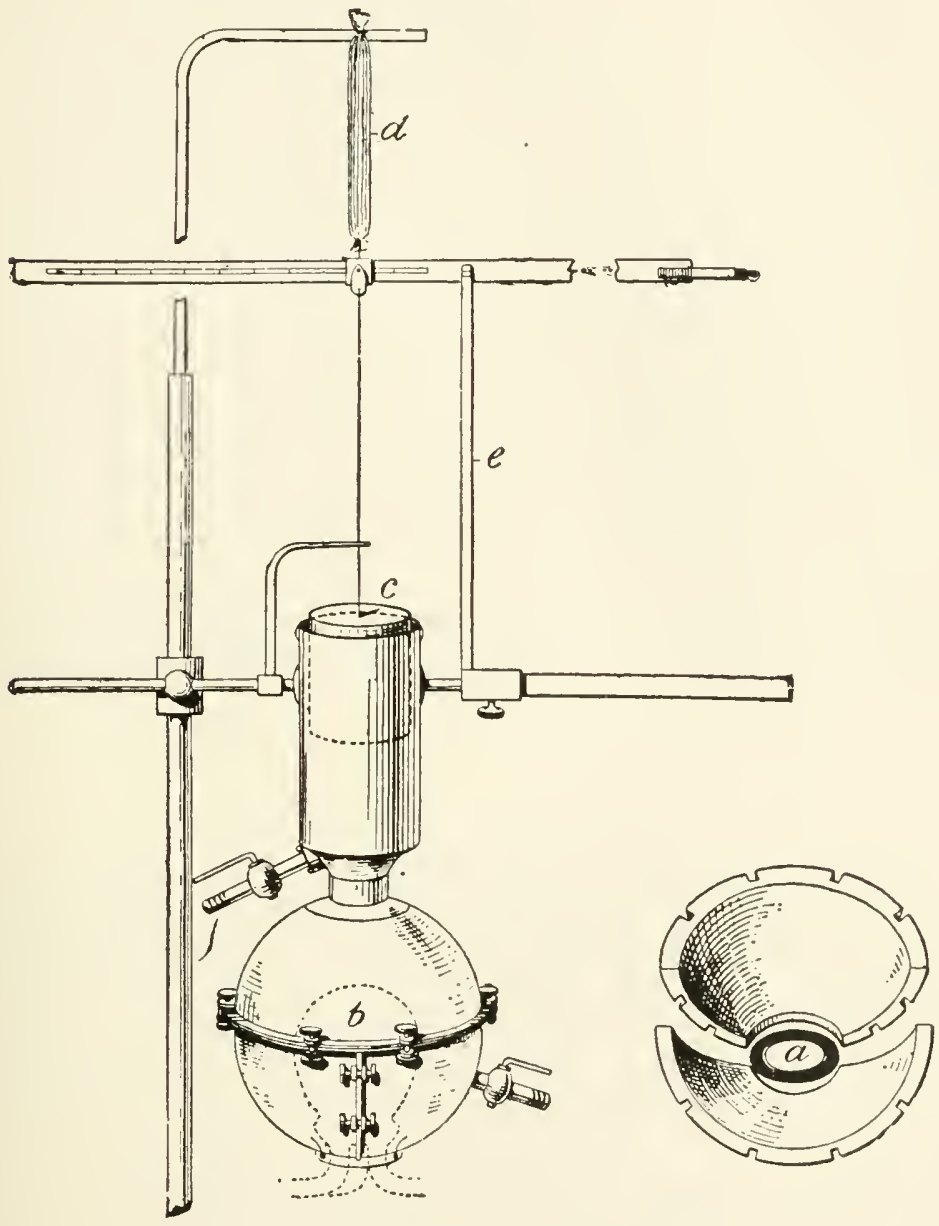

Fig. 44. Cardioneter of Roy and Adam.

In the method of Roy and Adami the heart is placed in a rigid metal box, Fig. $44 b$, the eavity of which, filled with warmed oil, is connected with a light piston $c$ and so with a recording lever. The pericardium being laid open, the two halves of the box are placed round the heart, are securely fixed by means of an india rubber ring $\alpha$, to the parietal pericardium round the roots of the great vessels, and are brought together. The cavity is then filled with oil, and the piston, also filled with oil, is brought into connection with the box, the lever 
and rod of the piston being placed by means of the india rubber spring $d$, in such a position that the pressure within the box is some few mm. Hg below that of the atmosphere.

By these methods it has been determined that the diminution of the volume of the heart at a systule, the "contraction volume" as it has inconveniently been called, that is to say, the quantity of blood discharged at a systole, the output of a systole, or the "pulse-volume" as we may call it, for it is this which causes the pulse, varies very much under various circumstances. We shall have to discuss later on some of the influences bearing on its amount. Meanwhile we merely call attention to the fact that it does vary largely, and that any numerical statement as to a normal pulse-volume has relatively little value.

Another fact of considerable importance brought to light by these methods is that under certain circumstances, at all events, the output by the left ventricle during a number of beats may be less than the intake through the right auricle. This means that under these circumstances the ventricle does not at the systole discharge the whole of its contents; some of the blood remains behind in the cavity of the ventricle at the close of the systole. Hence the assumption that the ventricle, in its systole, always discharges the whole of its contents, so as to be quite empty at the onset of diastole, is not true; the ventricle may completely empty itself but it by no means always does so.

\section{The Mechanism of the Beat.}

$\$ 115$. We may now attempt to consider in rather more detail what we may call the mechanism of the beat, that is to say, the exact manner in which the heart receives and ejects the blood. For this purpose we shall need certain data in addition to those on which we have already dwelt.

In addition to the curve obtained by placing a light lever on the exposed heart (Fig. 45), a method which though useful is open

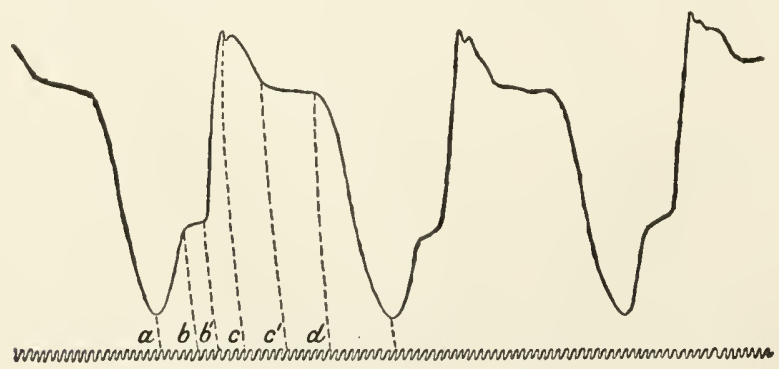

Fig. 45. (Repeated from Fig. 36 ) 
to objection, we may obtain what is very nearly the same thing, viz. a cardiographic tracing (Fig. 46) or cardiogram, that is to say, a tracing of the cardiac impulse, a curve of the changes in the pressure exerted by the apex of the heart on the chest-wall.

Various forms of cardiograph have been used to record the cardiac impulse. In some the pressure of the impulse is transmitted directly to a lever which writes upon a travelling surface. In others the impulse is, by means of an ivory button, brought to bear on an airchamber, connected by a tube with a tambour like that in Fin. 37 ; the pressure of the carlliac impulse compresses the air in the air-chamber, and through this the air in the chamber of the tambour, whereupon the lever is raiserl. In others the impulse, being received by a small, elastic bag filled with fluid and introdnced through an opening marle in the chest-wall, the pleura being left intact, is transmitted through fluid along a tube to a membrane-manometer. Or, to avoid opening the chest-wall, the tube may be marle to begin in a small, trumpetshaped opening or "receiver" covered with an elastic membr"ane, bearing a central button of cork or other material; the button being lightly pressed on the spot where the impulse is felt, the impulse is transmitted along the fluid of the tube from the elastic membrane of the receiver to that of the manometer.

In Fig. 46 we give two such cardiograms obtained by different methods, in Fig. 54 a more diagrammatic curve.

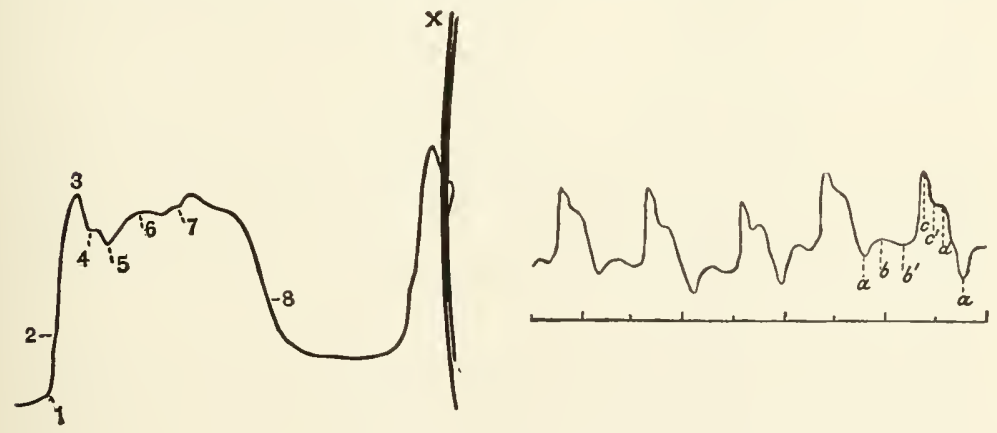

Fig. 46. Cardograms.

The left-hand figure is from Roy and Adami.

Since it is the contraction of the ventricular fibres which is the actual propelling force, an exact record of this contraction, after the manner of a muscle-curve, would serve, could it be obtained, as the basis of discussion. Owing to the intricate arrangement of the cardiac muscular fibres, such a simple record cannot be obtained; the nearest approach to it is the record of the changes in the distance between two points on the surface of the heart brought about during a beat. 
In the instrument of Roy and Adami, by an ingenious arrangement into the details of which we need not go, a delicate rod placed horizontally in connection with two points of the surface of the heart, of the ventricles, for instance, as it glides to and fro, according as the two points approach or recede from each other, records its movements by means of a light lever.

We give in Fig. 47 such a myocardiographic tracing, as it

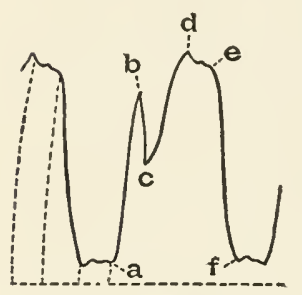

Fig. 47. Mrocardiogram. From the Dog. Roy and Adami. is called; the rise of the lever indicates an approach, the fall a receding of two points taken transversely across the ventricle of a dog.

What conclusions can we draw from the features of the various curves which we have given? We have reproduced in some cases more than one curve representing the same event, for the important reason that certain of the features of almost every curve are due, to some extent at least, to the instrument itself, and must not be taken as exact records of what is actually taking place in the heart; the inertia of one or other part of this or that instrument used plays a more or less important part in determining the form of the curve. It will therefore be readily understood that the interpretation of various heart curves is attended with great difficulties, and has led to much discussion. We must content ourselves here with confining our attention to the more important points, leaving many details, however interesting, on one side.

Let us begin with the beginning of the ventricular systole. All the curves, curve of endocardiac pressure, cardiogram, myocardiogram, and others, shew the important fact that the systole begins suddenly and increases swiftly until it reaches the beginning of what we have called the "systolic plateau," $c$ in Figs. 38, 39, 45, 3 in Fig. 46, $d$ in Fig. 47.

In some curves, as in Figs. $38,39 \mathrm{~B}, 42$, the rise is unbroken; in others, as in Figs. $39 \mathrm{~A}$, 45, the rise is marked with a shoulder. In Fig. 47, this shoulder $b$ has been interpreted, by those who maintain that papillary muscles begin their contraction later than the main ventricular wall, as indicating that event. We will not discuss the question here.

In some of the pressure curres, as in Fig. 38, the rise of pressure in the ventricle due to the actual systole is preceded by a slight temporary rise. This has been interpreted as indicating a slight rise of pressure in the ventricle due to the auricular systole just preceding the ventricular systole; but this interpretation has been debated, and indeed the slight rise in question is not always seen. Similarly, some curves shew a gradual but very slight increase of pressure in the ventricle during the preceding diastole; this has been interpreted as indicating a rise of pressure due to the gradual 
inflow of blood from the auricle and veins, but it, too, is not always present. Both the steady though slight rise of the lever throughout the diastole, with a sudden increase at the end, coincident with the auricular systole, are often seen in cardiograms; see the diagrammatic curve in Fig. 54. The ventricle as a whole enlarges under the venous inflow, and is more suddenly enlarged by the auricular systole.

The feature on which we wish to insist is the rapid rise of the intraventricular pressure, and the sudden change at the commencement of the systolic plateau. What does this sudden change mean? To answer this question we must ascertain what is taking place at the same time in the aorta.

$\$ 116$. If two catheters be introduced at the same time into the left side of the heart of a dog, being so arranged that while the end of one catheter lies in the left ventricle, Fig. 48, V, that of the other lies in the aorta $\mathrm{A}^{0}$ above the semilunar valves, and if each catheter be connected with a membrane-manometer, the two manometers recording on the same surface, one below the other, we obtain some such result as that shewn in Fig. 49.
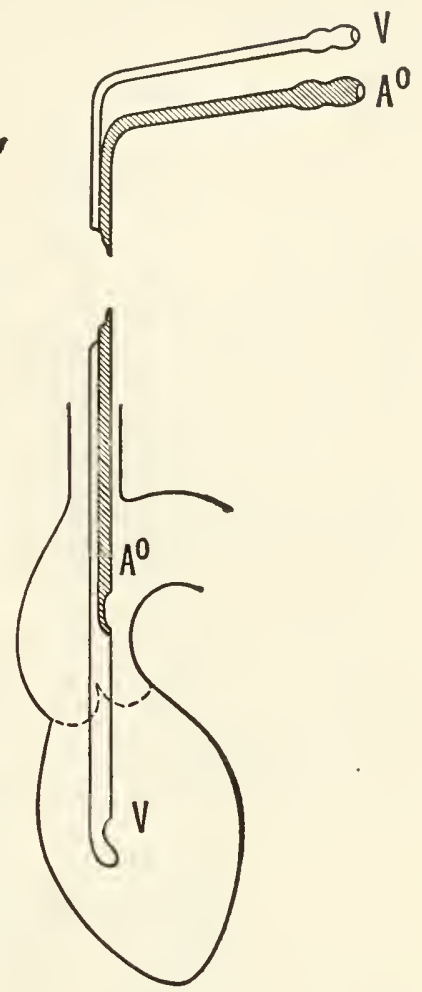

Fig. 48. Diagram illustrating THE METHOD OF RECORDING SImultaneously the Pressure in THE LEFT YeNTRiCLE AND AT THE ROOT OF THE AOrta. HǗthle.

An exanination of the two curves thus obtained shews us the following. At $o$, the beginning of the ventricular systole, or rather the time when the contraction of the ventricular fibres is beginning to raise the pressure within the ventricle, no effect is being produced in the aorta; the blood in the aorta is completely sheltered by the closed aortic valves. A little later, however. at 1, the pressure in the aorta begins to rise. This means that the semilunar valves are now opened, so that the force of the ventricular systole can make itself felt in the aorta. Up to 1 , the pressure in the ventricle, though increasing, is still less than that remaining in the aorta after the last beat, but at 1 the pressure in the ventricle becomes equal to or rather slightly greater than that in the aorta, and the valves are thrown open.

This is also shewn by comparing, as may be done by means 
of the "differential manometer," the changes of pressure in the ventricle and in the aorta at the same time.

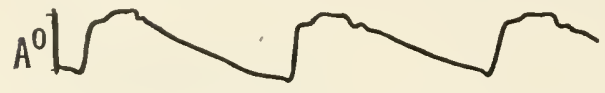

$\mathbf{x}$
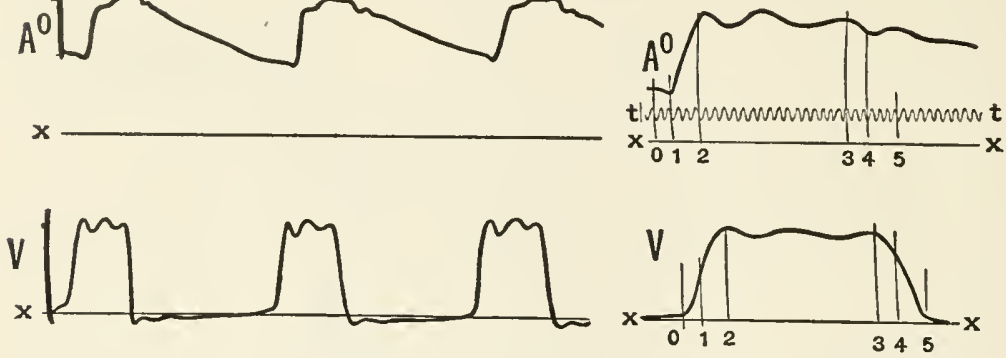

Fig. 49. Simultaneous Tracings of Vextricular and Aortic Pressere. IIÜ RTHLE.

On the left side the recording surface is travelling slowly, on the right more swiftly, the tuning-fork vibrations, $t$, being 100 a second

$A^{0}$. aortic. $I^{r}$ ventricular curve. $x-x$ base line to each. 'The vertical lines $1,2,3,4,5$, cut each curve at exactly the same time.

In the differential manometer, Fig. 50, the two tambours of two membrane manometers ' $\mathrm{T}$ and $\mathrm{T}_{\mathrm{I}}$ (the mouths of the tubes opening into each are seen in section) are arranged so that the central discs of both,

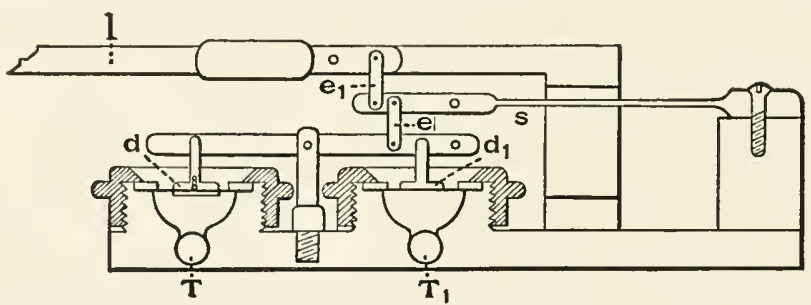

Fig. 50. Diagram of the Differential Maxometer of Hürtille.

$d$ and $d_{1}$, work on a balance above them. When the pressure in the two tambours is equal, the balance is horizontal; any difference of pressure between the two leads to an upward or downward movement of one or other arm, and this working against the light steel spring $s$, by means of $e$ and $e^{\prime}$ moves the lever $l$.

In Figs. 51, 52 we give simultaneous tracings of the pressure in the left ventricle $V$, and in the aorta $A^{0}$, and of the movements of the lever of the balance indicating differences of pressure $D$ between the ventricle and the aorta. At the base line $x-x$ of $D$ the two pressures are equal. The course of the curve below this base line indicates that the pressure in the ventricle is below that of the aorta; as the curve approaches towards the base line the pressure in the ventricle becomes more and more nearly equal to that in the aorta; and such part of the curve as lies above the base line indicates (except in so far as it may be due to the inertia of the 
instrument) that the pressure in the ventricle is for the time being above that in the aorta.

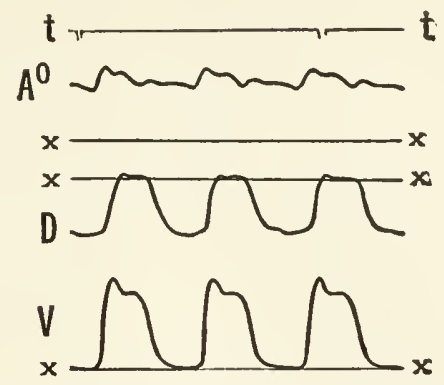

Fig. 51. Simultaneous Curves of Aortic and Ventricular Pressure and of tile Differential Manometer. Hürthle.

A0. aorta. $V$. ventricle. $D$. differential manometer. $x-x$, the base line in each respectively. The recording surface is travelling slowly, the time marker $t, t$ marking seconds.

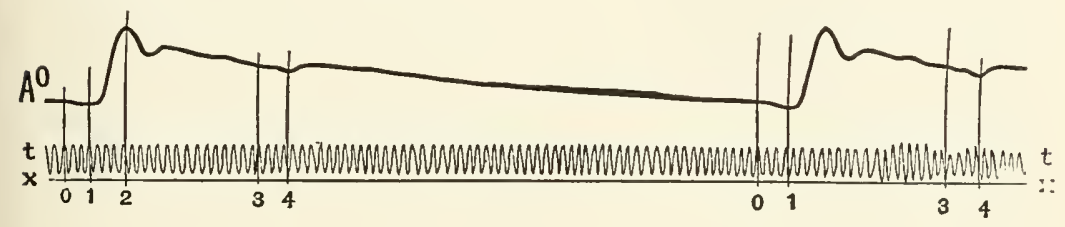

D
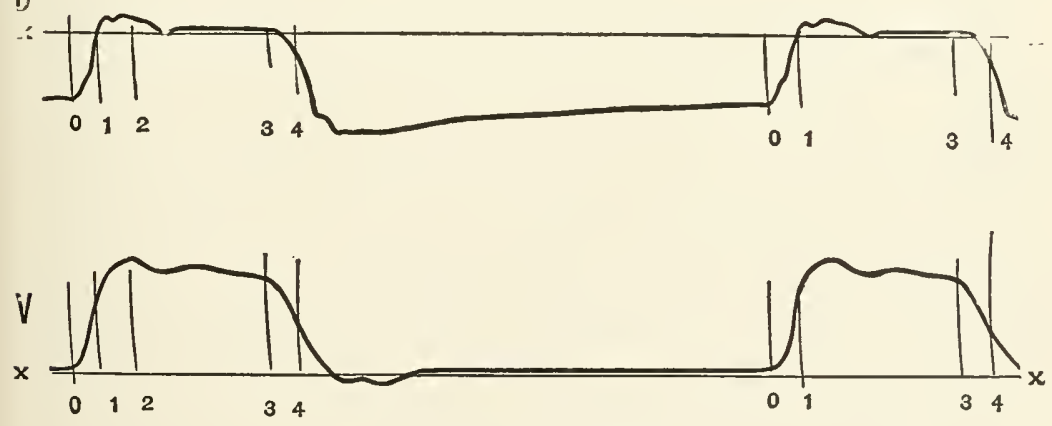

Fig. 52. The SAME.

The recording surface is travelling quickly ; the vibrations of the tuning-fork $t$, $t$, are 100 (double vibrations) a second.

An examination of the figures shews that the pressures in the ventricle and the aorta become equal at the mark (1). Pefore this though the pressure in the ventricle is rising rapidly that in the aorta is not rising, indeed is continuing to sink; the closed 
semilunar valves shelter the blood in the aorta from the ventriculin pressure. But immediately after (1) the pressure in the aorta also begins to rise: this shews that the semilumar valves are now upen, the blood in the ventricle and that in the aorta now forming a continuous column, and allewing the pressure of the ventricle to be felt in the aurtal. A rery slight excess of pressure on the ventrieular side of the valves is sufticient to push aside the thaps of the ralve: so that we may fairly say that the ralves open immediately after (1), which marks the point at which the curre of difference of pressure between the rentricle and the aorta has reached the base line $x-x$ : that is to say, at which the difference between the two has become nil.

It will be observed, however, that the mark (1) ents the ventricular curve not at the summit of its rise but short of this: the pressure in the ventricle continues to rise after the ralves are open, the curve continues after this to ascend rapidy up to ( 2 - $)$. Which marks the begmning of the systolic platean. During the interval between (1) and (2) the pressure is rising in the aorta also. I) uring this interval the pressure in the rentricke, continuing to rise, becomes greater than that in the aorta. the curve of difference rises abore the base line: but the excess of pressure in the ventricle does not become very great. the curve of difference does not rise to any great height, because that rery excess of pressure is used up in driving the contents of the rentricle into the aorta through the open semilunar valves.

During this interval the pressure in the aorta continnes to rise because, until the height of pressure at $(2)$ is reached, the pressure is not yot sufficient to drive the blood on along the arterial system with adequate rapidity.

With the point (2) the systulic platean begins. I) 2 ming this platean the exact course taken by the curve of rentricular pressure differs in different cases. We will take first the perhaps more ordinary case in which the curve with intermediate variations which we may at present pass over gradually declines until the point (3) is reached, when the plateau comes to an end by reason of the sudden fall of the rentricular pressure.

There can be no doulht that the sudden fall after (3) is due to the sudden cessation of the contraction of the ventricular walls. to their sudden relaxation. But what is taking place during the systolic plateau before this point is reached?

It used to be argued. taking count of the distension only of the aorta as indicated by the sphygmograph, an instrument of which we shall speak later on, that the ventricular contents eseape inte the aorta during the period of the distension of the aorta and during this only, having censed to flow by the time that this distension passes away giving place to a sequent shrinking of the aorta. Now when this period of distension is earefully measured it is found to be much shorter than the systole of the 
ventricle, as measured by the length of the systolic plateau. Hence, it being further assumed that the whole of the contents of the ventricle were ejected at each systole, it was inferred that the ventricle remained empty and yet contracted for an appreciable period after the discharge of its contents. And this led, in turn, to a great divergence of opinion as to the exact time at which the semilunar valves were closed.

But when we carefully explore the pressure in the aorta and in the ventricle at the same time, making use of the differential manometer, we come upon facts which seem to disprove this view. Examining Fig. 52 we find that, while during the systolic plateau the pressure is falling in both aorta and ventricle, the curve of difference of pressure $D$ remains above the base line, though not far above it and continually approaching it, up to the mark (3) at the very end of the plateau. At this point, however, at the end of the plateau, at the beginning of relaxation, a very great difference of pressure is established; while the ventricular pressure falls suddenly and soon reaches or even passes the base line (becoming in the latter case negative, $i . e$. below that of the atmosphere), the pressure in the aorta undergoes relatively little change, - indeed, immediately afterwards receives an increase of which we shall have to speak later on as the dicrotic crest of the pulse wave; and the curve of difference $D$ falls with very great abruptness.

The interpretation of this seems to be as follows. During the whole of the systolic plateau up to the mark (3) the semilunar valves are open, the cavity of the ventricle and the root of the aorta form a common cavity which is occupied by a continuous column of blood. Hence the curves of ventricular and aortic pressure, of the pressure at the one end and at the other end of this column, follow the same general course, and, indeed, shew the same secondary variations; this general course is, in the case which we are studying, a descending one by reason, as we have said, of the relatively free escape of blood from the arterial system through the peripheral resistance. But the column of blood in question is a column in motion, the ventricular pressure is driving the blood from the ventricle into the aorta; to effect this the pressure in the ventricle must continue to be higher than that which it is itself generating in the aorta, the curve of difference must remain above the base line. And, since the curve of difference does remain above the base line right up to the mark (3), we may infer that $u$ p to this point blood does pass from the ventricle into the aorta. At (3), however, there is a sudden change. The systole suddenly ceases, and with that the curve of difference suddenly sinks below the base line; the flow from ventricle ceases not because there is no more blood to come, but because the pressure in the ventricle now becomes lower than that in the aorta; and, indeed, the blood would flow back from the aorta to the region of lower pressure, to the ventricle, were it not that the very first effect 
of the reflux is to close the semilunar valves. So soon as these are elosed, the pressures in the ventricle and the aorta, which were previously following sinilar courses, now take separate courses; the latter falls suddenly, the former decreases gradually, and continues to decrease until the next systole once more opens the semilunar valves. We may add that this view is consistent with the conclusion mentioned in $\$ 114$, that not only the pulse-volume may vary, but also, at times at least, the whole contents are not driven out at the systole, some blood remaining behind.

Moreover, the pressure does not always gradually decline during the systolic plateau; sometimes it gradually rises during the whole of the period of the plateau, reaching its highest point just before the final sudden fall. This is shewn in Fig. 53.

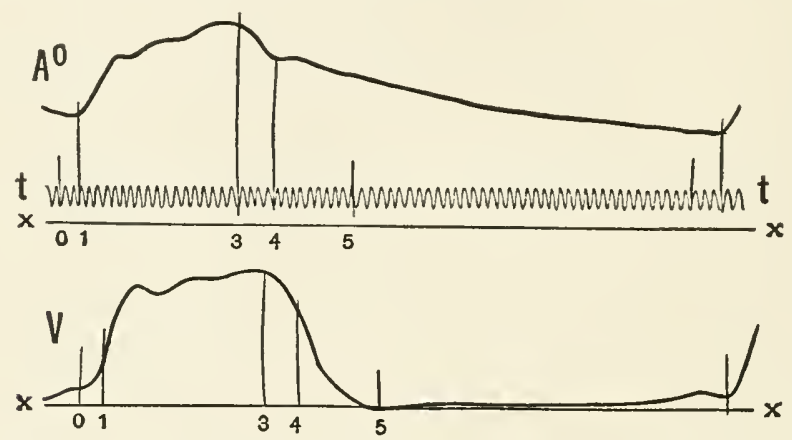

Fig. 53. Curve of Aortic and Ventricular Pressure, with an ascexding Srstolic Platead. IÏ̈rthle.

In this figure the general features are the same as in Fig. 52, save that the curve of ventricular pressure rises during the whole of the systolic plateau. But the curve of aortic pressure also rises in a corresponding manner, and the curve of difference, if shewn, would be the same as in Fig. 52. The explanation of the difference between the two cases is that in Fig. 52 the peripheral resistance in the arterial flow ( $\$ 99)$ is not very great, and the ventricular systole soon overcomes it to sueh an extent as to lead at once to some fall of pressure in the aorta (and in the ventricle). In Fig. 5.3 the peripheral resistance is very great; it is not overcome at first, the ventricle does its best working against it, and produces the most effect, raising the pressure to the highest point, just before its systole comes to an end. We may add that a similar course of the curve may be seen even when the pressure in the aorta is not very high, provided that the pulse-volume, the quantity discharged at the systole is very great; the form of the curve depends on the relative amounts which are entering the arterial system from the heart, and leaving it by the peripheral vessels.

It is possible that under some circumstances the whole of the 
contents may be discharged before the actual systole ends; but the observations and arguments which we have just related, shew that such an event must be regarded as of exceptional, and not, as has been contended, of normal occurrence.

Of the smaller secondary variations visible on the systolic plateau, conspicuous in some curves $(4,5,6,7$ in Fig. 46), various explanations have been given. Into the discussion of these we cannot enter here; we may however say that in many observations, which we may probably regard as correct, these secondary markings are identical in the curves of ventricular pressure, of aortic pressure and of the cardiac impulse, or of the change in the outward form of the heart; the events which cause them tell in the same way on all three.

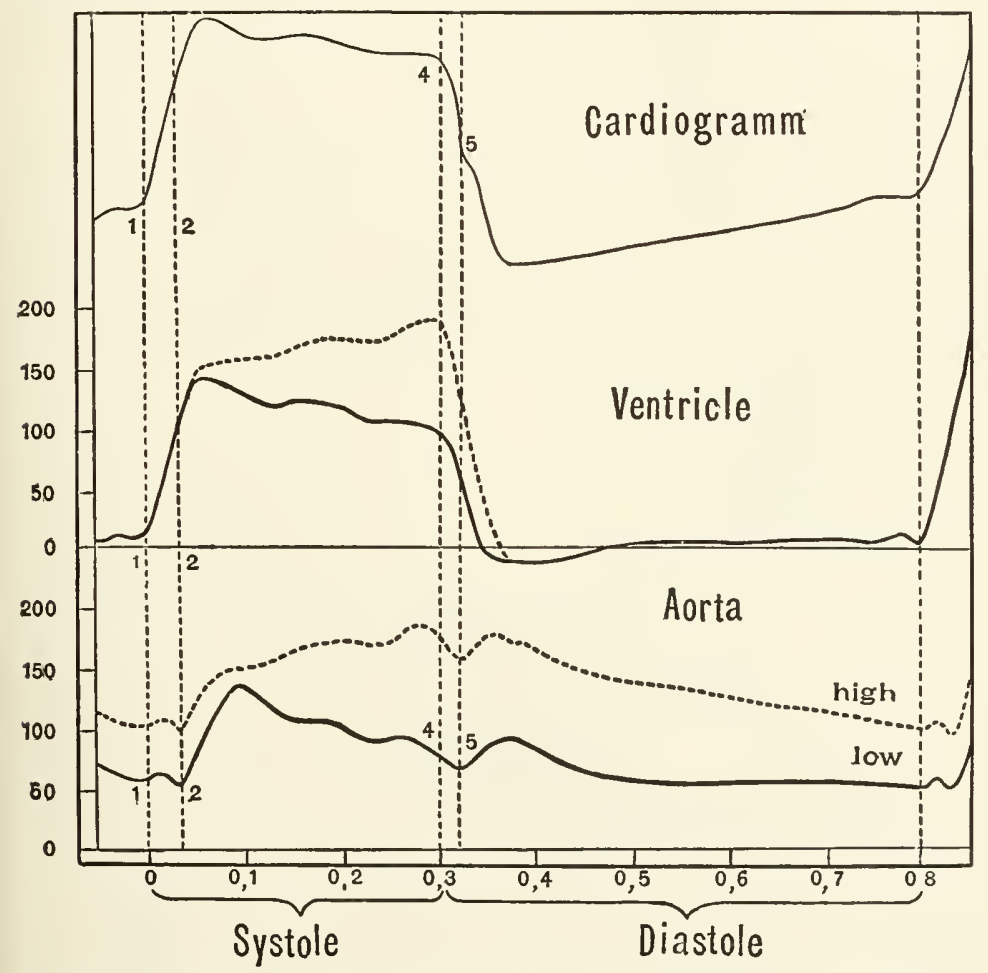

- Fig 54. Diagrau of Ventricular and Aortic Pressure and of the Cardiac Impelse. Hürtille.

We give in Fig. 54 a diagram of the cardiac events according to the exposition which we have just made. The curves previously given were copies of actual curves obtained by experiment; this is a constructed diagram. The upper curve is the curve of the cardiac impulse. The middle curve is the curve of pressure in the 
left ventricle; the unbroken line represents the course of the curve when, the peripheral resistance being small, the pressure needed to drive onward the blood is not very high, in the figure less than $150 \mathrm{~mm}$. Hg. The dotted line represents the course of the curve when, the peripheral resistance being great, the pressure is high, in the figure nearly $200 \mathrm{~mm}$. $\mathrm{Hg}$. The lower curve is the curve of pressure at the root of the aorta, the unbroken and the dotted lines having the same significance as in the ventricular curve. The line 0 marks the commencement of the ventricular systole, the line 1 the opening of the semilunar valves, and 3 the end of the systole. The line $t$ marks the beginning of what in dealing with the pulse, we shall speak of as the dicrotic wave. The semilunar valves are closed between 3 and 4 ; the closure is the result at 3 of the cessation of the systole and as we shall see the cause at 4 of the dicrotic wave of the pulse. The time is given in tenths of a second.

$\$$ 117. In many curves, as in some of those given above, the pressure in the ventricle at the beginning of diastole falls not only to the base line, which is the line of atmospheric pressure, but even below it; that is to say, becomes negative. Such a negative pressure may be shewn by means of a minimum manometer, that is, a manometer arranged so as to shew the lowest pressure which has been reached in a series of events. The mercury manometer, which as we

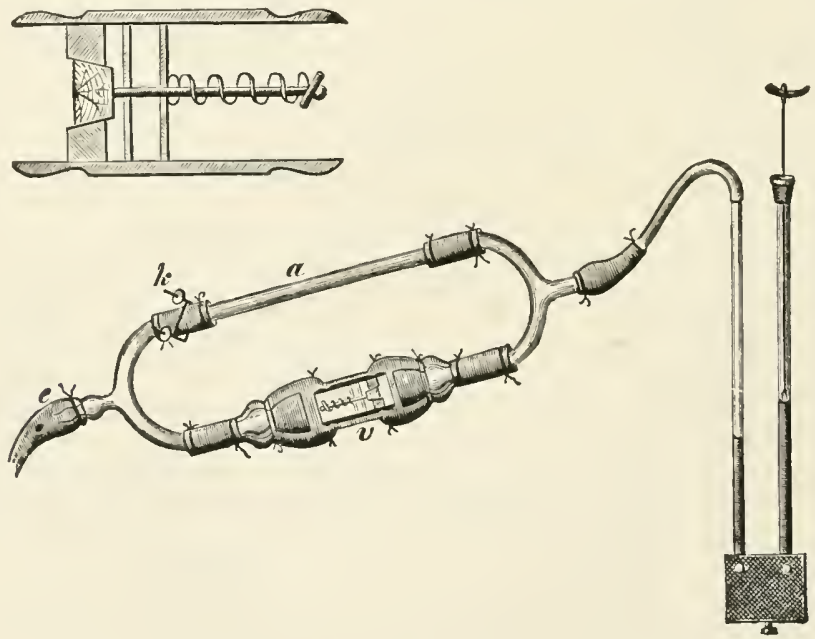

Fig. 55. Tie Maximum Maxometer of Goltz and Gavle.

At $e$ a connection is made with the tube leading to the heart. When the screw clamp $k$ is closed, the valve $r$ comes into action, and the instrmment, in the position of the valve shewn in the figure, is a maximnm manometer. isy reversing the firection of $"$ it is converted into a minimmm manometer. When $k$ is opened, the variations of pressnre are conveyed along $a$, aud the instrument then acts like an ordinary manometer. 
have said, is unsuitable for following the rapid changes constituting a single beat, may be used as a maximum or minimum instrument for determining the highest or lowest pressure reached in one or other of the heart's cavities during a series of beats.

The principle of one form of maxinum manometer, Fig. 55, consists in the introduction into the tuhe leading from the heart to the mercury column, of a (modified cup-and-ball) valve, opening, like the aortic semilunar valves, easily from the heart, but closing tirmly whes fluid attempts to return to the heart. The lighest pressure is that which drives the longest column of fluid past the valve, raising the mercury column to a corresponding height. Since this column, once past the valve, cannot return, the mercury remains at the height to which it was raised by it, and thus records the maximum pressure. By reversing the direction of the valve, the manometer is converted from a maximum into a minimum instrument.

A simpler form of maximum and minimum manometer is that of Hürthle, which consists of a small chamber connected with two manometers, the opening of each manometer into the chamber being armed with a valve of thin membrane, so arranged that it permits in the case of one manometer, the maximum one, the entrance only of the mercury, in the case of the other, the minimum one, the exit only.

By means of the maximum manoneter the pressure in the left ventricle in the $\log$ has been observed to reach a maximum of about $140 \mathrm{~mm}$. (mercury), in the right ventricle of about $60 \mathrm{~mm}$. and in the right auricle of about $20 \mathrm{~mm}$. These figures, however, are given as examples, and not as averages. Similarly negative pressures of from $-50 \mathrm{~mm}$. to -20 in the left ventricle of the $\mathrm{dog}$, of about $-15 \mathrm{~mm}$. in the right ventricle, and of from $-12 \mathrm{~mm}$. to $-7 \mathrm{~mm}$. in the right auricle, have been observed by the minimum manometer. Part of this diminution of pressure in the cardiac cavities is due, as will be explained in a later part of this work, to the aspiration of the thorax in the respiratory movements. But even when the thorax is opened, and artificial respiration kept up, under which circumstances no such aspiration takes place, a negative pressure may be still observed, the pressure in the left ventricle sinking according to some observations as low as $-24 \mathrm{~mm}$. Now, what the instrument actually shews is that at some time or other during the number of beats which took place while the instrument was applied (and these may have been very few), the pressure in the ventricle sank so many $\mathrm{mm}$. below that of the atmosphere. Since, however, the negative pressure nay be observed when the heart is beating quite regularly, each beat being exactly like the others, we may infer that the negative pressure is repeated at some period or other of each cardiac cycle. The instrument itself gives us no information as to the exact phase of the beat in which the negative pressure occurs; but it is clear from what we have already seen that when it occurs, it must take place at the end of the systole, at the beginning of the 
diastole. It is obvious, moreover, from what has gone before, that the semilunar valves are closed before it occurs, and we may dismiss the view which has been put forward that it is of the same nature as the negative pressure which makes its appearance behind a column of fluid moving rapidly and suddenly ceasing, as when a rapid tlow of water through a tube is suddenly stopped by turning a tap. We may probably attribute it to the relaxation of the ventricular walls. This, as all the curves shew, is a rapid process, something quite distinct from the mere filling of the ventricular cavities with blood by the venous inflow; and, though some have objected to the view, it may be urged that this return of the ventricle from its contracted condition to its normal form would develop a negative pressure. This return we may probably regard as simply the total result of the return of each fibre to its natural condition, though some have nrged that the extra quantity of blood thrown into the coronary arteries at the systole helps to unfold the ventricles somewhat in the way that fluid driven between the two walls of a double-walled collapsed ball or cup will unfold it.

We may further conclude that such a negative pressure, when it occurs, will assist the circulation (and it may be remarked that the return of the thick-walled left ventricle naturally exerts a greater negative pressure than the thin-walled right ventricle) by sucking the blood which has meanwhile been accumulated in the auricle from that cavity into the ventricle, the auriculo-ventricular valves easily giving way. At the same time this very flow from the auricle will at once put an end to the negative pressure, which obviously can be of brief duration only.

It should, however, be arded that many observers find the development of a negative pressure to be by no means of such constant occurrence, and not to reach such marked limits as might be inferred from the numbers given above, at least in the unopened chest. If so it cannot be an important factor in the work of the circulation.

$\$ 118$. The duration of the several phases. We may first of all distinguish certain main phases: (1) The systole of the auricles. (2) The systole, proper, of the ventricles, during which their fibres are in a state of contraction. (3) The diastole of the ventricles, that is to say, the time intervening between their fibres ceasing to contract, and commencing to contract again. To these we may add; (4) The pause or rest of the whole heart, comprising the period from the end of the relaxation of the ventricles to the beginning of the systole of the auricles; during this time the walls are undergoing no active changes, neither contracting nor relaxing, their cavities being simply passively filled by the influx of blood.

The mere inspection of almost any series of cardiac curves however taken, those, for instance, which we have just discussed, will shew, apart from any accurate measurements, that the systole 
of the auricles is always very brief, that the systole of the ventricles is always very prolonged, always occupying a considerable portion of the whole cycle, and that the diastole of the whole heart, reckoned from the end either of the systole, or of the relaxation of the veutricle, is very various, being in quickly beating hearts very short and in slowly beating hearts decidedly longer.

When we desire to arrive at more complete measurements, we are obliged to make use of calculations based on various data; and the value of some of these has been debated. Naturally the most interest is attached to the duration of events in the human heart.

A datum which has been very largely used is the interval between the beginning of the first and the occurrence of the second sound. This may be determined with approximative correctness, and is found to vary from 301 to $: 327$ sec., occupying from 40 to 46 p.c. of the whole period, and being fairly constant for different rates of heart beat. That is to say in a rapidly beating heart it is the pauses which are shortened and not the duration of the actual beats.

The observer, listening to the sounds of the heart. makes a signal at each event on a recording surface, the difference in time hetween the marks being measured by means of the vibrations of a tuning-fork recorded on the same surface. By practice it is found possible to reduce the errors of observation within very small limits.

Now whatever be the exact causation of the first sound, it is undoubtedly coincident with the systole of the ventricles, though possibly the actual commencement of its becoming audible may be slightly behind the actual begimning of the muscular contractions. Similarly the occurrence of the second sound, which, as we have seen, is certainly due to the closure of the semilunar valves, may in accordance with the view expounded a little while back, be taken to mark the close of the ventricular systole. And on this view the interval between the beginning of the first and the occurrence of the second sound may be regarded as indicating approximatively the duration of the ventricular systole, i.e. the period during which the ventricular fibres are contracting.

By an ingenious arrangement a microphone attached to a stethoscope may be made to record the heart sounds through the stimulation of a muscle-nerve preparation; and the record so obtained may be compared with the various cardiac curves. When this is done, the first sound is found to begin somewhere on the systolic ascent of the ventricular curve, the exact point varying, and the second sound to occur just as the ventricular curve begins its diastolic descent.

There has been however as we stated above great divergence of 
opinion and much discnssion as to the exact time of the closure of the semilunar valves; the view given in the text above, though it seems to be supported by adequate arguments, is not the only one which is held.

Accepting the view given in the text, we may make the following statement. In a heart beating 72 times a minute, which may be taken as the normal rate, each entire cardiac cycle would last about $0.8 \mathrm{sec}$, and taking $0.3 \mathrm{sec}$. as the duration of the ventricular systole, the deduction of this would leave $0.5 \mathrm{sec}$. for the whole diastole of the ventricle including its relaxation, the latter occupying less than 1 sec. At the end of the diastole of the ventricle there occurs the systole of the auricle, the exact duration of which it is difficult to determine, it being hard to say when it really begins, but which, if the contraction of the great veins be included, may perhaps be taken as lasting on an average $0 \cdot 1$ sec. The 'passive interval,' therefore, during which neither auricle nor ventricle is undergoing contraction, lasts about 4 sec., and the absolute pause or rest, during which neither auricle nor ventricle is contracting or relaxing, about $3 \mathrm{sec}$. The systole of the ventricle follows so immediately upon that of the auricle, that practically no interval exists between the two events. In the systole of the ventricle we may distinguish the phase during which pressure is being got up before the semilunar valves are opened; this is exceedingly short, probably from 02 to 03 sec. During the rest of the $3 \mathrm{sec}$. of the systole, the contents of the ventricle are being pressed into the aorta.

The duration of the several phases may for convenience sake be arranged in a tabular form as follows:

secs. secs.

Systole of ventricle before the opening of the semilunar valves, while pressure is still getting up

Continued contraction of the ventricle, and

Escape of blood into aorta

Total systole of the ventricle

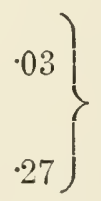

Diastole of both auricle and ventricle, neither contracting, or "passive interval"

Systole of auricle (about or less than)

Diastole of ventricle, including relaxation and filling, $1 p$ to the beginning of the ventricular systole

Total Cardiac Cycle 


\section{Summary.}

$\$ 119$. We may now briefly recapitulate the main facts connected with the passage of blood through the heart. The right auricle during its diastole, by the relaxation of its muscular fibres, and by the fact that all backward pressure from the ventricle is prevented by the closing of the tricuspid valves, offers but little resistance to the ingress of blood from the veins. On the other hand, the blood in the trunks of both the superior and inferior vena cava is under a pressure, which, though diminishing towards the heart, remains higher than the pressure obtaining in the interior of the auricle; the blood in consequence flows into the empty auricle, its progress in the case of the superior vena cava being assisted by gravity. At each inspiration this flow (as we shall see in speaking of respiration) is favoured by the diminution of pressure in the heart and great vessels caused by the respiratory morements. Before this flow has gone on very long, the diastole of the ventricle begins, its cavity dilates, the flaps of the tricuspid valve fall back, and blood for some little time flows in an unbroken stream from the venæ cavæ into the ventricle. How far the entrance of blood from the auricle into the ventricle is, under ordinary circumstances, aided by the negative pressure in the ventricle following the close of the systole, must, as we have said, be left for the present uncertain. In a short time, probably before very much blood has had time to enter the ventricle, the auricle is full; and forthwith its sharp, sudden systole takes place. Partly by reason of the backward pressure in the veins, which increases rapidly from the heart towards the capillaries, and which at some distance from the heart is assisted by the presence of valves in the venous trunks, but still more from the fact that the systole begins at the great veins themselves, and spreads thence over the auricle, the force of the auricular contraction is spent in driving the blood, not back into the veins, but into the ventricle, where the pressure is still exceedingly low. Whether there is any backward flow at all into the great veins, or whether by the progressive character of the systole, the flow of blood continues, so to speak, to follow up the systole without break, so that the stream from the veins into the auricle is really continuous, is at present doubtful; though a slight positive wave of pressure synchronous with the auricular systole, travelling backward along the great veins, has been observed at least in cases where the heart is beating vigorously.

The ventricle thus being filled by the auricular systole, the play of the tricuspid valves described above comes into action, the auricular systole is followed by that of the ventricle, and the pressure within the ventricle, cnt off from the auricle by the tricuspid valves, is brought to bear on the pulmonary semilunar valves, and the column of blood on the other side of those valves. 
As soon as by the rapidly increasing shortening of the ventricular fibres the pressure within the ventricle becomes greater than that in the pulmonary artery, the semilumar valves open, and the still continuing systole discharges the contents of the ventricle into that vessel.

J)uring the whole of this time the left side has with still greater energy been executing the same manœuvre. At the same time that the venæ cavæ are filling the right auricle, the pulmonary veins are filling the left auricle. At the same time that the right auricle is contracting, the left auricle is contracting too. The systole of the left ventricle is synchronous with that of the right ventricle, but executed with greater force; and the flow of blood is guided on the left side by the mitral and aortic valves in the same way that it is on the right by the tricuspid valves and the valves of the pulmonary artery.

As the ventricles become filled with blood, and so increased in volume, the apex begins to press steadily on the chest-wall, as may be often seen in the cardiogram, the curve of the cardiac impulse. The fuller distension due to the auricular systole is more obvious in the same curve; but both these changes are insignificant compared to the effect of the change of form, and of the position of the apex during the ventricular systole, by which the lever of the cardiograph is rapidly and forcibly noved.

With this systole of the ventricles the first sound is heard.

We may more conveniently follow the remaining events in the left ventricle.

The effect of the discharge of the contents of the left ventricle is to raise the pressure at the root of the aorta to nearly the same height as that in the ventricle itself. The ventricular pressure continues for some time, giving rise to the "systolic plateau " of the various cardiac curres. In some cases this pressure soon reaches a maximum, after which it gradually declines, the curve of pressure sloping, with some secondary undulations, gently downwards. In other cases where there is great resistance to the outflow along the arterial system, the pressure may continue to rise during the whole of the ventricular systole. In both cases the curves of the ventricular pressure and of the aortic pressure are similar.

Then comes the sudden cessation of contraction, the sudden relaxation of the ventricular fibres. The pressure in the ventricle becomes less than that which it itself has generated in the aorta, and the semilunar valves suddenly close as the blood flows back from the region of high pressure, the aorta, towards the region of low pressure, the ventricle. At this moment the second sound is heard.

Owing to the semilumar valves being closed, the pressures in the ventricle and in the aorta, which before were following the 
same course, now become different. While the pressure sinks rapidly in the ventricle, falling it may be below that of the atmossphere, and thus becoming a negative pressure, which in some cases may possibly be considerable, that in the aorta does not sink to a corresponding degree; in fact, as we shall see, it is reinforced to a certain extent in a secondary rise, the so-called dicrotic rise.

We have reason to believe not only that the quantity of blood ejected at the systole may vary from time to time, but also that at times at all events if not normally, the whole of the blood present in the ventricle at the systole may fail to leave the ventricle during the systole, more or less remaining behind at the close; the ventricle in such cases does not completely empty itself. On the other hand, we may perhaps admit that, at least under certain circumstances, when, for instance, the contents of the ventricle are small, and the ventricle vigorous or the systole prolonged, the whole of the contents may be discharged in the earlier part of the systole, the ventricle remaining contracted for some little time after it has emptied itself.

\section{The Work done.}

$\S 120$. We have already ( $\$ 114)$ spoken of that most important factor in the determination of the work of the heart, the pulsevolume, or the quantity ejected from the ventricle into the aorta at each systole, and of the various methods by which it may be estimated. We have seen that it probably varies within very considerable limits.

We may here repeat the remark that exactly the same quantity must issue at a beat from each ventricle; for if the right ventricle at each beat gave out rather less than the left, after a certain number of beats the whole of the blood would be gathered in the systemic circulation. Similarly, if the left ventricle gave out less than the right, all the blood would soon be crowded into the lungs. The fact that the pressure in the right ventricle is so much less than that in the left (probably 30 or $40 \mathrm{~mm}$. as compared with $200 \mathrm{~mm}$. of mereury), is due, not to differences in the quantity of blood in the cavities, but to the fact that the peripheral resistance which has to be overcome in the lungs is so much less than that in the rest of the body.

Not only does the amount ejected vary, but the pressure under which it is ejected also varies within very considerable limits. Moreover, the number of times the systole is repeated within a given period may also vary considerably. The work done, therefore, varies very much. But it may be interesting and instructive to note the results of calculating out a very high estimate. Thus if we take 180 grms. as the quantity, in man, ejected at each stroke at a pressure of $250 \mathrm{~mm}$. of mercury, which is 
equivalent to 3.21 meters of blood, this means that the left ventricle is capable at its systole of lifting $180 \mathrm{grms} .3 .21 \mathrm{~m}$. high, $i$. e. it does 578 gram-neters of work at each beat. Supposing the heart to beat $7 \cdot 2$ times a minute, this would give for the day's work of the left ventricle nearly 60,000 kilogram-meters. Calculating the work of the right ventricle at one-fourth that of the left, the work of the whole heart during the day would amount to 75,000 kilogram-meters, which is just about the amount of work done in the ascent of Snowdon by a tolerably heavy man. 


\section{SEC. 4. THE PULSE.}

\$121. We have seen that the arteries, though always distended, undergo, each time that the systole of the ventricle drives the contents of the ventricle into the aorta, a temporary additional expansion so that when the finger is placed on an artery, such as the radial, an intermittent pressure on the finger, coming and going with the beat of the heart, is felt, and when a light lever is placed on the artery, the lever is raised at each beat, falling between.

This intermittent expansion, which we call the pulse, corresponding to the jerking outflow of blood from a severed artery, is present in the arteries only, being, except under particular circumstances, absent from the veins and capillaries. The expansion is frequently visible to the eye, and in some cases, as where an artery has a bend, may cause a certain amount of locomotion of the vessel.

We may, by applying various instruments to the interior of an artery, study the temporary increase of pressure which is the cause of the temporary increase of expansion. This makes itself felt, as we have seen, in the curve of arterial pressure taken by the mercury manometer; but the inertia of the mercury prevents the special characters of each increase becoming visible. In order to obtain an adequate record of these special characters we must have recourse to other instruments.

The nembrane-manometer, of which we have already spoken (§ 113), and on the results gained by which when applied to the root of the aorta by means of a catheter we have dwelt ( $\$ 116)$, may also be applied to other arteries, the tube leading to the tambour of the manometer being connected with the artery by means of a cannula in the ordinary way.

In Fick's spring-manometer, in its original form, Fig. 56, the artery is connected by means of a cannula and a rigid tube containing fluid with the interior of a curved spring; an increase of pressure unfolds the curve of the spring, the movements of the end of which may be recorded by means of a lever. In Fick's improved form the membrane of a small air-tambour works against a horizontal slip of steel which acts as a spring; this instrument, like Frey and Krehl's manometer 
which is only a modification of it (see $\$ 113$ ), can be applied to an artery by a cannula in the ordinary way.

The "sphygmoscope" consists of a small elastic bag, the end of an india rubber tinger, for instance, fitted on to a conical cork, through which passes a tube openius into the bag, and connected by a camnula with the artery; both bag and tube are, before being connected with the artery, filled with fluid of a nature to hinder clotting. The bag, by means of the conical cork, is firmly fitted into the end of a small glass tube, the cavity of which filled with air is connected with a recorling air tambour. The changes of pressure within the artery are transmitted to the elastic bag, and through this to the air of the glass tube and so to the recording tambour.

The taubour-sphygmoscope of Hurthle is a combination of the membrane-manowetel with a tambour. The nembrane of the manometer works not directly on a lever, but on a recording air tambour, the movements of which are recorled in the usual way.

In the sphygnotonometer of Roy, the artery is, by means of a cannula, and rigid tuhe fillerl with fluid, connected with a cylinder in which a light piston works by means of a delicate membrane.

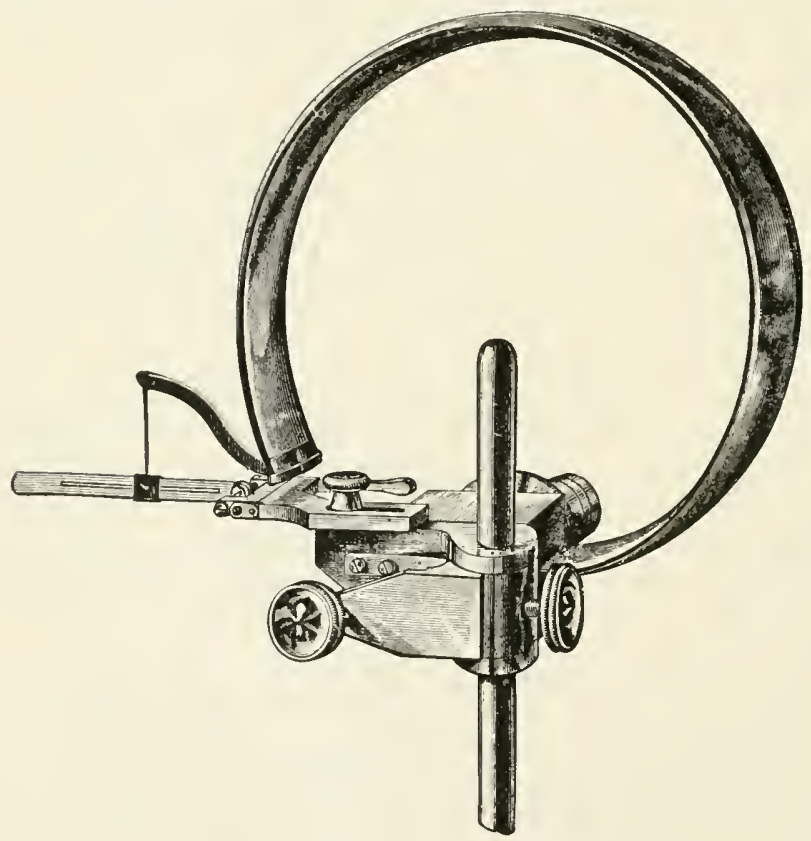

Fig. 56. Fick's Sprixg Maxometer.

The flattened tube in the form of a hoop is firmly fixed at one end, while the other free end is attached to a lever. The interior of the tube, filled with spirit, is brought, by means of a tube contaning sodium carbonate solution, into connection with all artery, in much the same way as in the case of the mercury manometer. The increase of pressure in the artery being transultted to the hollow hoop, tends to straighten it, and correspondingly moves the attached lever. 
And there are still other instruments which may be used in a similar way.

It is not necessary, however, to open the artery; we may study indirectly the changes of pressure by recording the expansions and retractions of the artery, the changes in its diameter, which are produced by the changes of pressure.

The most common method of registcring the expansion of an artery and at the same time one of the simplest, is that of bringing a light lever to bear on the outside of the artery.

A lever specially adapted to record a pulse tracing is caller a sphygmograpl, the instrument generally comprising a small travelling recording surface on which the lever writes. There are many different forms of sphygmograph, but the general plan of structure is the same. Fig. 57 represents in a diagrammatic form the essential parts of the sphygmograph known as Dudgeon's, which we have chosen for representation, not because it is best, but because it is one very largely employed in medical practice. The instrument is generally applied to the radial artery because the arm affords a convenient support to the fulcrum of the lever, and because the position of the artery, near to the

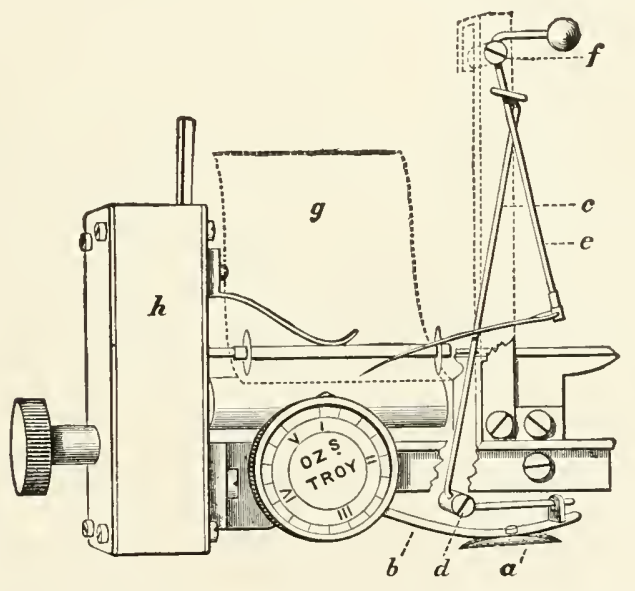

Fig. 57. Diagram of a Sphygmograph (Dudgeon's).

Certain supporting parts are omitted so that the multiplying levers may be displayed.

$a$ is a small metal plate which is kept pressed on the artery by the spring $b$. The vertical movements of $a$ canse to-and-fro movements of the lever $c$ about the fixed point $d$. 'These are communicated to and magnified by the lever $e$, which moves round the fixed point $f$. The free end of this lever carries a light steel marker which rests on a strip of smoked paper $q$. The paper is placed bencath two small wheels, and rests on a roller which can be rotated by means of clock-work contained in the box $h$. The paper is thus caused to travel at a imiform rate. The screw grardnated in onnces Troy is brought to bear on the spring $b$ by means of a camm, and by this the pressure put on the artery can be regulated. 'The levers magnify the pulse morements fifty times. 
surface and with tine support of the radius below so that adequate pressure can be brought to bear by the lever on the artery, is favourable for making observations. It can, of course, be applied to other arteries.

The membrane-manometer of Hürthle may also be applied directly to an unopened artery. The cannula is replaced by a small fummel, the mouth of which is covered by membrane bearing at its centre a small block of cork. If the cork be pressed lightly on an artery, the expansions of the artery move the membrane of the funnel, and the movements of this are transmitted aloug the fluid of a rigid tube to the recording tambour.

A pulse tracing may also be indirectly obtained by the plethysmographic method. If the arm be introduced into a pletliysmograph ( $(104)$, a tracing may be obtained of the rhythmic expansions of the arm, that is, of the rhythmic expansions of the arteries of the arm, due to the heart beats. If the plethysmograph chamber be filled with air insteal of fluid, the changes of pressure in the chamber may be brought to bear on a sensitive flame, the changes of which in turn may be photographed.

If the artery be laid bare, other methods may be adopted. In some cases, in that of the aorta, for instance, it is sufficient to attach a light hook into the outer coat of the artery, and to connect the hook by means of a thread with a carefully balanced lever. The movements of the coat of the artery are then recorded by the lever.

The sphygmotonometer of Roy may also be used without opening the artery. For this purpose a length of the artery is enclosed in a tube with rigid walls, filled with fluid, which acts as a plethysmograph, the movements of the fluid around the artery being recorded by means of a piston working a lever. If the artery be ligatured and diviled, one end may be drawn into the tube for the distance required. The tube may also be made of two halves, one of which is slippecl under the artery simply laid bare, the other placed above it, and the two halves are brought together round the artery, the two ends of the tube being closed with membrane.

And still other methods may he employed.

The several tracings obtained by these several methods differ of course in minor features, but they agree in general features; and from a comparative study of the results obtained by different methods we are ahle, in many cases at all events, to form conclusions as to which of the minor features of a curve are due to the instrument itself, and which represent events actually taking place in the artery. On the whole, the curve obtained by directly recording the pressure within the artery is concordant with that obtained by recording the expansions of the artery; the curve obtained by the manometer or by the sphygmoscope very closely resembles that obtained by the sphygmograph, and the more completely the incidental errors of each instrument are avoided, the more closely do the two curves agree. We may accordingly in treating of the pulse confine ourselves largely to the results obtained by the sphygmograph. Any of the various instruments applied to the radial 
artery would give some such tracing as that shewn in Fig. 58 which is obtained by means of the sphygmograph. At each heart beat the

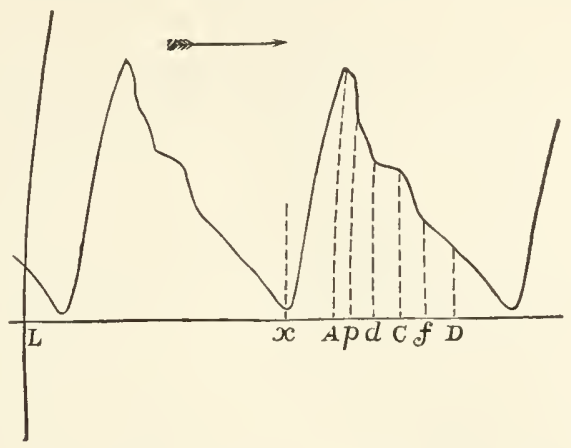

Fig. 58. Pulse tracing from the Radial Artery of Man.

The vertical curved line, $\mathrm{L}$, gives the tracing which the recording lever made when the blackened paper was notionless. 'The curved interrupted lines shew the distance from one arother in time of the chief phases of the pulse-wave, viz. $x=$ commencement, aud $A$ end of expansion of artery. p, predicrotic noteh. $d$, dierotic notch. C, dicrotic erest. D, post-dicrotic erest. $f$, the post-dicrutic notch. These terms are explained in the text later on.

curve rises rapidly, and then falls more gradually in a line which is more or less uneven.

$\S 122$. We have now to study the nature and characters of the pulse in greater detail.

We may say at once, and, indeed, have already incidentally seen, that the pulse is essentially due to physical canses; it is the physical result of the sudden injection of the contents of the ventricle into the elastic tubes called arteries. Its features depend on the one hand on the systole of the ventricle, on the quantity of blood which is thereby discharged into the aorta, and on the manner in which it is discharged, and on the other hand on the elasticity of the arterial walls. The more important of these features may be explained on physical principles, and may be illustrated by means of an artificial model, so far at least as we can imitate the action of the heart.

We may confine ourselves, in the first instance, to the simple expansion of the arterial tube and its return to its previous condition, neglecting for the present all secondary events.

If two levers be placed on the arterial tubes of an artificial model Fig. $30, S . a ., S^{\prime}$. $a$., one near to the pump, and the other near to the peripheral resistance, with a considerable length of tubing between them, and both levers be made to write on a recording surface, one immediately below the other, so that their cnrves can be more easily compared, the following facts may be observed, when the pump is set to work regularly. They are 
perhaps still better seen if a number of levers be similarly arranged at different distances from the pump as in Fig. 59.

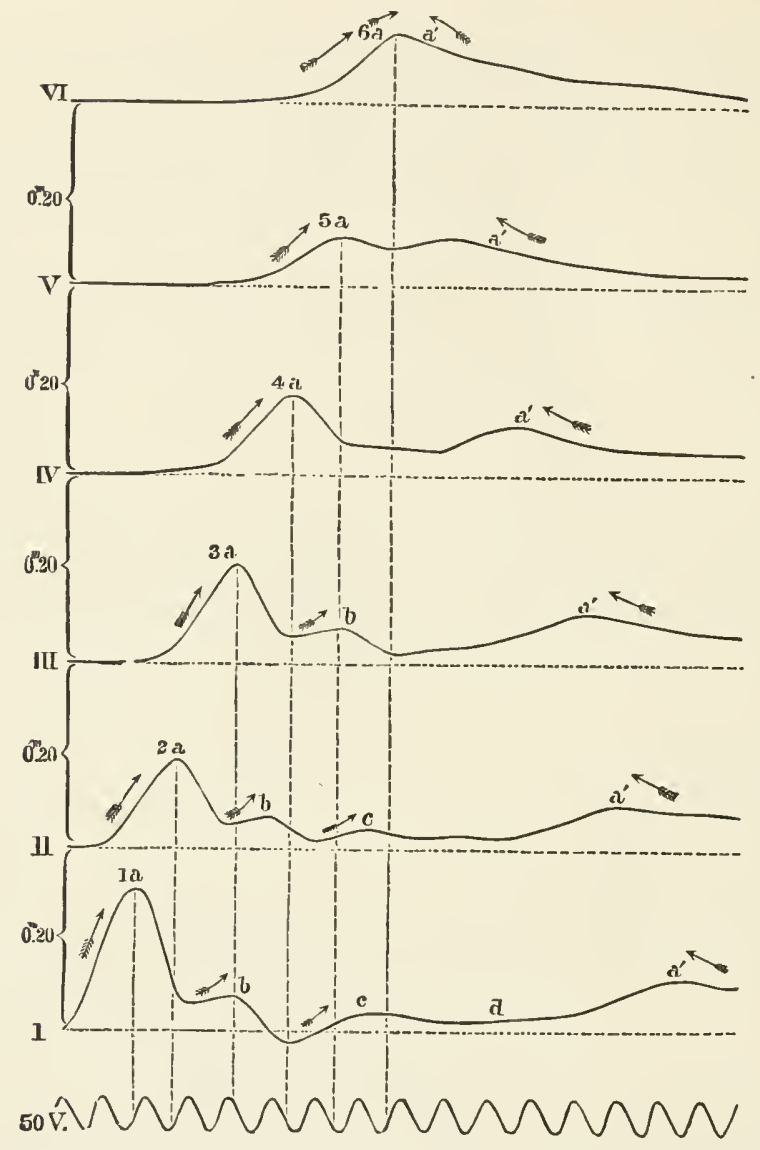

Fir. 59. Pulse-curves described by a series of sphygmographic levers placed at intervals of $20 \mathrm{~cm}$. from each other along an elastic tube, into which fluid is forced by the sudden stroke of a pump. The pulse-wave is travelling from left to right, as indicated by the arrows over the primary $(a)$ and secondary $(b, c)$ pulse-waves. 'T he dotted vertical lines drawn from the sumnit of the several primary waves to the tuning-fork curve below, each complete vibration of which occupies $\frac{1}{50}$ sec., allow the time to be measured which is taken up by the wave in passing along $20 \mathrm{~cm}$. of the tubing. 'The waves $a^{\prime}$ are waves reflerterl from the closed distal end of the tubing; this is inclicated by the direction of the arrows. It will be observed that in the more distant lever VI. the reflected wave, having but a slight distance to travel, becomes fused with the primary wave. (From Marey.)

At each stroke of the pump, each lever rises until it reaches a maximum (Fig. 59, 1a, 2a, \&c.), and then falls again, thus describing a curve. The rise is due to the expansion of the part of the tube under the lever, and the fall is due to that part of the 
tube returning after the expansion to its previous calibre. The curve is therefore the curve of the expansion (and return) of the tube at the point on which the lever rests. We may call it the pulse-curve. It is obvious that the expansion passes by the lever in the form of a wave. At one moment the lever is at rest: the tube beneath it is simply distended to the normal amount indicative of the mean pressure which at the time obtains in the arterial tubes of the model; at the next moment the pulse expansion reaches the lever, and the lever begins to rise; it continues to rise until the top of the wave reaches it, after which it falls again until finally it comes to rest, the wave having completely passed by.

It may perhaps be as well at once to warn the reader that the figure which we call the pulse-curve is not a representation of the pulse-wave itself ; it is simply a representation of the movements, up and down, of the piece of the wall of the tubing at the spot on which the lever rests during the time that the wave is passing over that spot. We may roughly represent the wave by the diagram Fig. 60, in which the wave shewn by the dotted line is

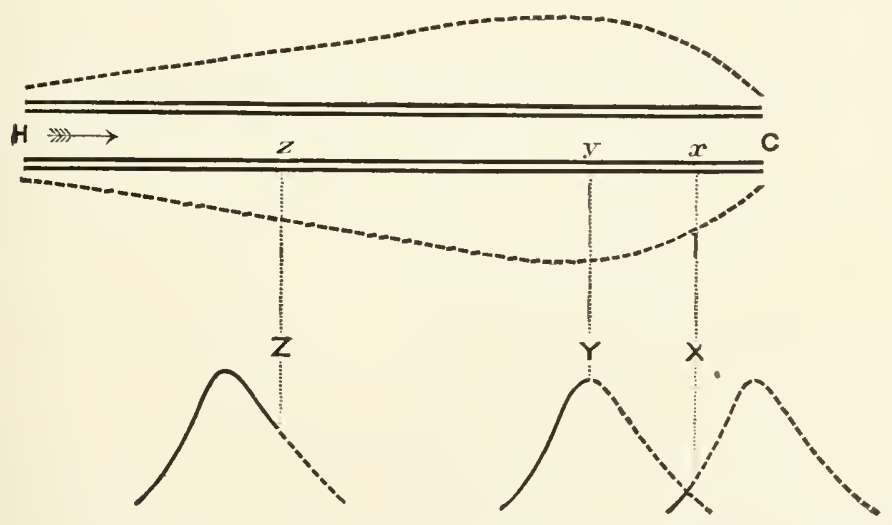

Fig. 60. A rodgit diagrayutic Representation of a Pulse-IVave passing over ax Artery.

passing over the tubs (shewn in a condition of rest by the thick double line) in the direction from $H$ to $C$. It must, however, be remembered that the wave thus figured is a much shorter wave than is the pulse-wave in reality (that being, as we shall see, about 6 meters long), i.e. occupies a smaller length of the arterial system from the heart $H$ towards the capillaries $C$. Moreover, the actual pulse-wave has secondary features, which we are neglecting for the present, and which, therefore, we do not attempt to shew in the figure.

The curves below, $X, Y, Z$, represent, in a similarly diagrammatic fashion, the curves described, during the passage of the wave, 
by levers placed on the points $x, y, z$. At $Z$ the greater part of the wave has already passed under the lever, which, during its passage, has already described the greater part of its curve, shewn by the thick line, and has only now to describe the small part, shewn by the dotted line, corresponding to the remainder of the wave from $Z$ to $I$. At $Y$ the lever is at the summit of the wave. At $X$ the lever has only described a small part of the begiming of the wave, viz. from $C^{\prime}$ to $r$, the rest of the curve, as shewn by the dotted line, having yet to be described.

But to return to the consideration of Fig. 59 .

$\$$ 123. The rise of each lever is somewhat sudden, but the fall is more gradual, and is generally marked with some irregularities which we shall study presently. The rise is sudden because the sharp stroke of the pump suddenly drives a quantity of fluid into the tubing, and so suddenly expands the tube; the fall is more gradual because the elastic reaction of the walls of the tube, which, after the expanding power of the pump has ceased, brings about the return of the tube to its former calibre driving the fluid onwards to the periphery, is more gradual in its action.

These features, the sudilenness of the rise or up-stroke, and the more gradual slope of the fall or down-stroke, are seen also in natural pulse-curves taken from living arteries (Figs. 58, 61 \&c.). We shall see, however, that under certain circumstances this contrast between the up-stroke and the down-stroke is not so marked.

It may here be noted that the actual size of the curve, that is the amount of excursion of the lever, depends in part (as does also to a great extent the form of the curve) on the amount of pressure exerted by the lever on the tube. If the lever only just touches the tube in its expanded state, the rise will be insignificant. If, on the other hand, the lever be pressed down too firmly, the tube beneath will not be able to expand as it otherwise would, and the rise of the lever will be proportionately diminished. There is a certain pressure which must be exerted by the lever on the tube, the exact amount depending on the expansive power of the tubing, and on the pressure exerted by the fluid in the tube, in order that the tracing may be best marked. This is shewn in
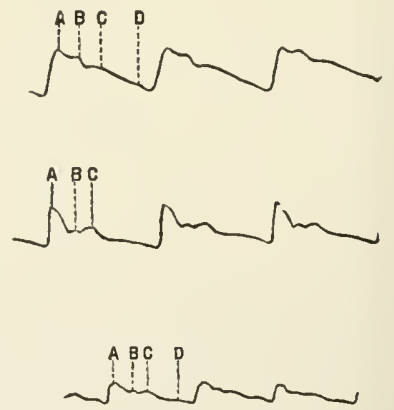

Fig. 61. Pulse tracixgs from tie SAME RADIAL ARTERY U.NDER DIF FERENT PRESSURES OF THE LEVER.

The letters are explained in a later part of the text. 'Taken with Dudgeon's sphygmograph. Fig. 61 , in which are given three tracings taken from the same 
radial artery with the same instrument, in the lower curve the pressure of the lever is too great, in the upper curve too small, to bring out the proper characters of the pulse; these are seen more distinctly in the middle curve with a medium pressure.

$\$ 124$. It will be observed that in Fig. 59, curve I., which is nearer the pump, rises more rapidly and rises higher than curve II., which is farther away from the pump; that is to say, at the lever farther away from the pump the expansion is less and takes place more slowly than at the lever nearer the pump. Similarly in curve IV. the rise is still less, and takes place still less rapidly than in II., and the same change is seen still more marked in $V$. as compared with IV. In fact if a number of levers were placed at equal distances along the arterial tubing of the model, and the model were working properly, with an adequate peripheral resistance, we might trace out step by step how the expansion, as it travelled along the tube, got less and less in amount, and at the same time became more gradual in its development, the curve becoming lower and more flattened out, until, in the neighbourhood of the artificial capillaries, there was hardly any trace of it left. In other words, we might trace out step by step the gradual disappearance of the pulse.

The same changes, the same gradual lowering and flattening of the curve, may be seen in natural pulse tracings; compare, for instance, Fig. 62, which is a tracing from the dorsalis pedis artery, with the tracing from the radial artery Fig. 61, taken from the same individual with the same instrument on the same occasion. This feature is, of course, not obvious in all pulse-curves taken from different individuals with

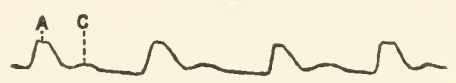
different instruments and under varied circumstances; but if a series of curves from different arteries were carefully taken under the same conditions, it would be found that the aortic tracing is higher and more sudden than the carotid tracing, which again is higher and more sudden than the radial tracing, the tibial tracing being in turn still lower and more flattened. The pulse-curve dies out by becoming lower and lower, and more and inore flattened out.

And a little consideration will shew us that this must be so. The systole of the ventricle drives a quantity of blood into the already full aorta. The sudden injection of this quantity of blood expands the portion of the aorta next to the heart, the part immediately adjacent to the semilnnar valves beginning to expand first, and the expansion travelling thence on to the end of this portion. In the same way the expansion travels on from this portion through all the succeeding portions of the arterial system. 
For the total expansion required to make room for the new quantity of blood is not provided by that portion alone of the aorta into which the blood is actually received; it is supplied by the whole arterial system: the old quantity of blood which is replaced by the new in this first portion has to find room for itself in the rest of the arterial space. As the expansion travels onward, however, the increase of pressure, which each portion transmits to the succeeding portion, will be less than that which it received from the preceding portion. For the whole increase of pressure due to the systole of the ventricle has to be distributed over the whole of the arterial system; the general mean arterial pressure is, as we have seen, maintained by repeated systoles, and any one systole has to make its contribution to that mean pressure; the increase of pressure which starts from the ventricle must therefore leave behind at each stage of its progress a fraction of itself; that is to say, the expansion is continually growing less, as the pulse travels from the heart to the capillaries. Moreover, while the expansion of the aorta next to the heart is, so to speak, the direct effect of the systole of the ventricle, the expansion of the more distant artery is the effect of the systole transmitted by the help of the elastic reaction of the arterial tract between the heart and the distant artery; and since this elastic reaction is slower in development than the actual systole, the expansion of the more distant artery is slower than that of the aorta, the up-stroke of the pulse-curve is less sudden, and the whole pulse-curve is more tlattened.

The object of the systole is to supply a contribution to the mean pressure, and the pulse is an oscillation above and below that mean pressure, an oscillation which diminishes from the heart onwards, being damped by the elastic walls of the arteries, and so, little by little, converted into mean pressure until in the capillaries the mean pressure alone remains, the oscillations having disappeared.

$\$$ 125. If in the model the points of the two levers at different distances from the pump be placed exactly one under the other on the recording surface, it is obvious that, the levers being alike except for their position on the tube, any difference in time between the movenents of the two levers will be shewn by an interval between the beginnings of the curves they describe, the recording surface being made to travel sufficiently rapidly.

If the movenents of the two levers be thus compared, it will be seen that the far lever (Fig. 59, II.) commences later than the near one (Fig. 59, I.); the farther apart the two levers are, the greater is the interval in time between their curves. Compare the series T. to VI. (Fig. 59). In the same way it would be found that the rise of the near lever began some fraction of a second after the stroke of the pump. This means that the wave of expansion, the pulse-wave, takes some time to travel along the tube. 
The velocity with which the pulse-wave travels depends chiefly on the amount of rigidity possessed by the tubing. The more extensible (with corresponding elastic reaction) the tube, the slower is the wave; the more rigid the tube becomes, the faster the wave travels; in a perfectly rigid tube, what in the elastic tube would be the pulse, becomes a mere shock travelling with very great rapidity. The width of the tube is of much less influence, though according to some observers the wave travels more slowly in the wider tubes.

The rate at which the normal pulse-wave travels in the human body has been variously estimated at from 10 to 5 meters per second. In all probability we may take 6 meters as an average rate; but it must be renembered that the rate may vary very considerably under different conditions. According to all observers the velocity of the wave in passing from the groin to the foot is greater than that in passing from the axilla to the wrist $(6 \mathrm{~m}$. against $5 \mathrm{~m}$.). This is probably due to the fact that the femoral artery with its branches is more rigid than the axillary and its branches. So, also, the wave travels more slowly in the arteries of children than in the more rigid arteries of the adult. The velocity is also increased by circumstances which heighten, and decreased by those which lower the mean arterial pressure, since with increasing pressure the arterial walls become more, and with diminishing pressure less rigid. Probably also the velocity of the pulse-wave depends on conditions of the arterial walls, which we cannot adequately describe as mere differences in rigidity. In experimenting with artificial tubes it is found that different qualities of india rubber give rise to very different results.

Care inust be taken not to confound the progress of the pulsewave, i.e. of the expansion of the arterial walls, with the actual onward moveinent of the blood itself. The pulse-wave travels over the moving blood somewhat as a rapidly moving natural wave travels along a sluggishly flowing river. Thus while the velocity of the pulse-wave is 6 or possibly even 10 meters per sec., that of the current of blood is not more than half a meter per sec., even in the large arteries, and is still less in the smaller ones.

$\$ 126$. Referring again to the caution given above, not to regard the pulse-curve as a picture of the pulse-wave, we may now add that the pulse-wave is of very considerable length. If we know how long it takes for the pulse-wave to pass over any point in the arteries and how fast it is travelling, we can easily calculate the length of the wave. In an ordinary pulse-curve the artery, owing to the slow return, is seen not to regain the calibre which it had before the expansion, until just as the next expansion begins, that is to say, the pulse-wave takes the whole time of a cardiac cycle, viz. $\frac{8}{10}$ ths sec., to pass by the lever. Taking the velocity of the pulsewave as 6 meters per sec., the length of the wave will be $\frac{8}{10}$ ths of $6 \mathrm{~m}$., that is, nearly 5 meter And even if we took a smaller 
estimate, by supposing that the real expansion and return of the artery at any point took much less time, say $\frac{4}{10}$ th sec., the length of the pulse-wave would still be more than 2 neters. But even in the tallest man the capillaries farthest from the heart, those in the tips of the toes, are not $2 \mathrm{~m}$. distant from the heart. In other words, the length of the pulse-wave is much greater than the whole length of the arterial system, so that the beginning of each wave has become lost in the small arteries and capillaries some time before the end of it has finally passed away from the beginning of the aorta.

We must now return to the consideration of certain special features in the pulse, which, from the indications they give or suggest of the condition of the vascular system, are often of great interest.

$\$ 127$. Secondary wares. In nearly all pulse tracings, the curve of the expansion and recoil of the artery is broken by two, three, or several smaller elevations and depressions: secondary waves are imposed upon the fundamental or primary wave. In the sphygmographic tracing from the carotid, Fig. 63, and in many of the other tracings given, these secondary elevations are marked

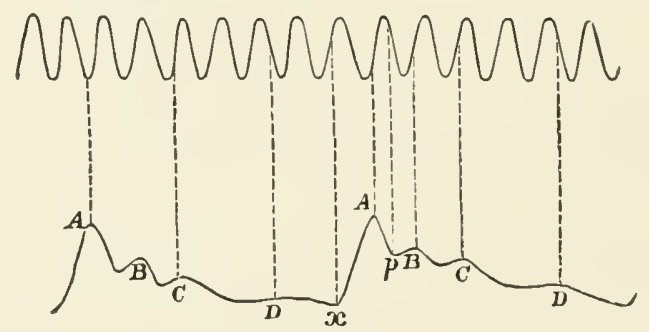

Fig. 63. Pulge tracing from carotid artery of healtir man (Moens).

$\mathrm{x}$, commencement of expansion of the artery. A, summit of the first rise. C, dicrotic secondary wave. B, predicrotic secondary wave; $p$, notch preceding this. 1), succeeding secondary wave. The curve above is that of a tuning fork with ten donble vibrations in a second.

as $\mathrm{B}, \mathrm{C}, \mathrm{D}$. When one such secondary elevation only is conspicuous, so that the pulse-curve presents two notable crests only, the primary crest and a secondary one, the pulse is said to be "dicrotic"; when two secondary crests are prominent, the pulse is often called "tricrotic" ; when several, "polycrotic." As a general rule, the secondary elevations appear only on the descending limb of the primary wave as in most of the curves given, and the curve is then spoken of as "katacrotic." Sometimes, however, the first elevation or crest is not the highest, but appears on the ascending portion of the main curve: such a curve is spoken of as "anacrotic" Fig. 64. As we have alrearly seen $(\$ 116)$ the curve of pressure at the root of the aorta, and, indeed, that of endocardiac pressure may be in like manner "anacrotic" (Figs. 53, 54). 
Of these secondary elevations, the most frequent, conspicuous: and important is the one which appears some way down on the descending limb, and is marked $\mathrm{C}$ on Fig. 63 and on most of the curves here given. It is more or less distinctly visible on all sphygmograms, and may be seen in those of the aorta as well as of other arteries. Sometimes it is so slight as to be hardly discernible; at other times it may be so marked as to give rise to a really double pulse (Fig. 65), i.c. a pulse which can be felt as double by the finger: hence it has been

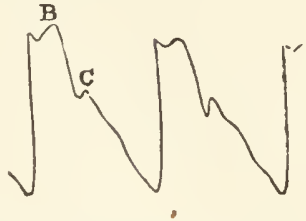

Fig. 64. AxACrotic spuygMOGRAPII TRACIYG FROM TIE AsCENDING AORTA (Aneurism). called the dicrotic elevation or the dicrotic wave, the notch preceding the elevation being spoken of as the "dicrotic notch."
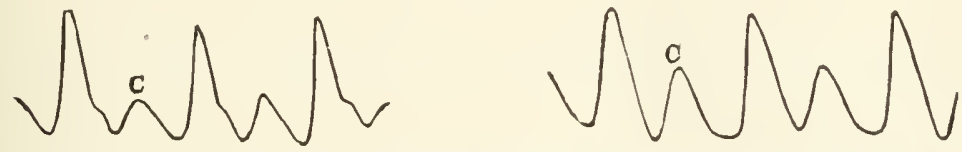

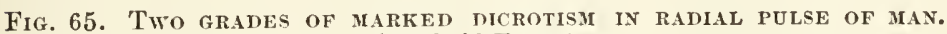
('Typhoid Fever.)

Neither it nor any other secondary elevations can be recognized in the tracings of blood pressure taken with a mercury manometer. This may be explained, as we have said $\$ 121$, by the fact that the movements of the mercury column are too sluggish to reproduce these finer variations. Moreover, when the normal pulse is felt by the finger, most persons find themselves unable to detect any dicrotism. But that it does really exist in the normal pulse is shewn by the fact that it appears, sometimes to a marked extent, sometimes to a less extent, not only in sphygmograms and in curves of arterial pressure taken by adequate instruments, but also and in a most unmistakeable manner in the tracing obtained by allowing the blood to spirt directly from an opened small artery, such as the dorsalis pedis, upon a recording surface.

Less constant and conspicuous than the dicrotic wave, but yet appearing in most sphygmograms, is an elevation which appears higher up on the descending limb of the main wave; it is marked $\mathrm{B}$ in Fig. 63, and on several of the other curves, and is frequently called the predicrotic wave; it may become very prominent. Sometimes other secondary waves, often called 'post-dicrotic,' are seen following the dicrotic wave, as at D in Fig. 63, and some other curves; but these are not often present, and usually even when present inconspicuous.

When tracings are taken from several arteries, or from the same artery under different conditions of the body, these secondary waves are found to vary very considerably, giving rise to many 
characteristic forms of pulse-curve. Were we able with certainty to trace back the several features of the curves to their respective causes, an adeyuate examination of sphygmographic tracings would undoubtedly diselose much valuable information concerning the condition of the body presenting them. The problems, however, of the origin of these secondary waves and of their variations are complex and difficult; so much so that the detailed interpretation of a sphygmographic tracing is still in many cases and in many respects very uncertain.

\$128. The Dicrotic Ware. The chief interest attaches to the nature and meaning of the dicrotic wave. In general the main conditions favouring the dicrotic wave are (1) a highly extensible and elastic arterial wall; (2) a comparatively low mean pressure, leaving the extensible and elastic reaction of the arterial wall free scope to act; and (3) a vigorous and rapid stroke of the ventricle, discharging into the aorta a considerable quantity of blood.

The origin of this dicrotic wave has been and indeed still is much disputed.

In the first place, observers are not agreed as to the part of the arterial system in which it first makes its appearance. In such a system as that of the arteries we liave to deal with two kinds of waves. There are the waves which are generated at the pump, the heart, and travel thence onwards towards the periphery ; the primary pulse-wave due to the discharge of the contents of the ventriele is of this kind. But there may be other waves which, started somewhere in the periphery, travel backwards towards the central pump; such are what are called 'reflected' waves. For instance, when the tube of the artificial model, bearing two levers, is blocked just beyond the far lever, the primary wave is seen to be accompanied by a second wave, which at the far lever is seen close to, and often fused into, the primary wave (Fig. 59, VI. $a^{\prime}$ ), but at the near lever is at some distance from it (Fig. 59, I. $a^{\prime}$ ), being the farther from it the longer the interval between the lever and the block in the tube. The second wave is evidently the primary wave reflected at the block and travelling backwards towarls the pump. It thus, of course, passes the far lever hefore the near one. And it has been argued that the dicrotic wave of the pulse is really such a reflected wave, started either at the minute arteries and capillaries, or at the several points of bifurcation of the arteries, and travelling backwards to the aorta. But if this were the case the distance between the primary crest and the dicrotic crest ought to be less in arteries more distant from, than in those nearer to the heart, just as in the artificial scheme the reflected wave is fused with a primary wave near the block (Fig. 59, VI. $6 a$. $a^{\prime}$ ), but becomes more and more separated from it the farther back towards the pump we trace it (Fig. 59, I. 1. $a . a^{\prime}$ ). Now this is not the case with the dicrotic 
wave; careful measurements sliew that the distance between the primary and dicrotic crests is either the same or certainly not less in the smaller or more distant arteries than in the larger or nearer ones. This feature indeed proves that the dicrotic wave cannot be due to reflection at the periphery or indeed in any way a retrograde wave. Besides the multitudinous peripheral division would probably render one large peripherically reflected wave impossible. Again, the more rapidly the primary wave is obliterated or at least diminished on its way to the periphery the less conspicuous should be the dicrotic wave. Hence increased extensibility and increased elastic reaction of the arterial walls which tend to use up rapidly the primary wave, should also lessen the dicrotic wave. But as a matter of fact these conditions, as we have said, are favourable to the prominence of the dicrotic wave.

We may conclude then that the dicrotic wave like the primary wave begins at the heart, and travels thence towards the periphery. But even if this be admitted observers are not agreed as to the mechanism of its production. The following view is the one which seems the most satisfactory, though it is not accepted by all inquirers.

The simultaneous curves of endocardiac and aortic pressure (Fig. 54 and others) shew us that the dicrotic notch as it is called, the depression mmediately preceding the dicrotic wave is, in a normal heat, coincident with the end of the systole. The curve of the differential manometer further shews us that this is the point at which the pressure in the ventricle begins to becone less than in the aorta. We may therefore reason in the following way. The flow from the ventricle into the aorta ceases because the systole ceases; the cessation takes place while the two cavities are still open to each other, and probably, in nost cases at least, while there is still nore or less hlood in the ventricle. The pressure in the ventricle tends to become less than that in the aorta, and the blood in the aorta tends to flow back into the ventricle. But the first effect of this is to close firmly the semilunar valves. The expansion of the aorta, (which in many cases had been lessening even during the systole owing to the flow through the periphery of the arterial system being more rapid than the flow from the ventricle, but in some cases, in the anacrotic instances, had not,) lessens with the cessation of the flow; the-aorta shrinks, pressing upon its contents. But part of this pressure is spent on the closed semilumar valves, and the resistance offered by these starts a new wave of expansion, the dicrotic wave, which travels thence onwards towards the periphery in the wake of the primary wave. If we ardmit that the blood is flowing from the ventricle during the whole of the systole, we must also admit that the semilunar valves do not close until the end of the systole, and this being, as shewn by the curves, just antecedent to the licrotic wave, we may attribute this wave to the rebound from the closed valves. It is 
not necessary that the valves should act perfectly, and the dicrotic wave may occur in cases where the valves are more or less incompetent; all that is required for its production is an adequate obstacle to the return of blood from the aorta to the ventricle, and without such an obstatle the circulation could not be carried oll.

$\$ 129$. Moreover it must be remembered that though we may thus regard the closed valves as so to speak the determining cause of the dicrotic wave, the wave itself is an oscillation of the arterial walls, and the remarks made a little while back concerning the inertia of the walls hold good for this explanation also. Hence the conditions which determine the prominence or otherwise of the dicrotic wave are conditions relating to the elasticity of the arterial walls, and to the circumstances which call that elasticity into play. For instance, the dicrotic wave is less marked in rigid arteries (such as those of old people) than in healthy elastic ones; the rigid wall neither expands so readily nor shrinks so readily, and hence does not so readily give rise to secondary waves. Again, the dicrotic wave is, other things being equal, more marked when the mean arterial pressure is low than when it is high; indeed it may be induced when absent, or increased when slightly marked, by diminishing, in one way or another, the mean pressure. Now when the pressure is high, the arteries are kept continually much expanded, and are therefore the less capable of further expansion, that is to say, are, so far, more rigid. Hence the additional expansion due to the systole is not very great; there is a less tendency for the arterial walls to swing backwards and forwards, so to speak, and hence a less tendency to the development of secondary waves. When the mean pressure is low, the opposite state of things exists; supposing of course that the ventricular stroke is adequately vigorous (the low pressure being due, not to a diminished cardiac stroke but to diminished peripheral resistance) the relatively empty but highly distensible artery is rapidly expanded, and falling rapidly back enters upon a secondary (dicrotic) expansion, and may even exhibit a third.

Moreover the same principles may be applied to explain why sometimes dicrotism will appear marked in a particular artery while it remains little markel in the rest of the system. In experimenting with an artificial tubing such as the arterial model, the physical characters of which remain the same throughout, both the primary and the secondary waves retain the same characters as they travel along the tuhing save only that both gradually diminish towards the periphery; and in the natural circulation, when the vascular conditions are fairly uniform throughout, the pulse-curve, as a rule, possesses the same general characters throughout, save that it is gralually 'damped off.' But suppose we were to substitute for the first section of the tubing a piece of perfectly rigid tubing, this at the stroke of the 
pump on account of its being rigid would shew neither primary nor secondary expansion, but the expanding force of the pump's stroke would be transmitted through it to the second, elastic section, and here the primary and secondary waves would at once become evident. This is an extreme case, but the same thing would be seen to a less degree in passing from a more rigid, that is less extensible and elastic section, to a less rigid, more extensible and elastic section; the primary and secondary expansions, in spite of the general damping effect, would suddenly increase. Similarly in the living body a pulse-curve which so long as it is travelling along arteries in which the mean pressure is high, and which are therefore practically somewhat rigid, is not markedly dicrotic, may become very markedly dicrotic when it comes to a particular artery, in which the mean pressure is low (we shall see presently that such a case may occur), and the walls of which are therefore for the time being relatively more distensible than the rest.

Lastly we may recall the observation made above $\$ 123$ that the curve of expansion of an elastic tube is modified by the pressure exerted by the lever employed to record it, and that hence, in the same artery, and with the same instrument, the size, form, and even the special features of the curve vary according to the amount of pressure with which the lever is pressed upon the artery. Accordingly the amount of dicrotism apparent in a pulse may be modified by the pressure exerted by the lever. In Fig. 61 for instance the dicrotic wave is more evident in the middle than in the upper tracing.

$\$ 130$. Concerning the other secondary waves on the pulse-curve such as that which has been called the 'predicrotic' wave $(B$ on Fig. 63 and on some of the other pulse-curves) it will not be desirable to say much here. They have been the occasion of much discussion, especially when considered under the view that the ventricle rapidly emptied itself at the earlier part of the systole. We will content ourselves with the following remark. The predicrotic and the other secondary waves in question are, like the dicrotic wave, propagated from the heart towards the periphery, they are seen in sphygmograms taken from the root of the aorta as well as from more peripheral arteries, and some are also seen in the curves of ventricular pressure. Some of the features of these secondary waves may be due to imperfections in the instruments used, to inertia and the like, but the main features undoubtedly represent events taking place in the vascular system itself. When we compare the curve of pressure in the aorta with that in the ventricle, we observe that up to the dicrotic notch, (in what may be called the systolic part of the pulse-curve, the part which corresponds to the systole of the ventricle, in contrast to the diastolic part which follows and which includes the dicrotic wave), the variations seen in the aortic curve, the secondary waves of which we are speaking, 
are exactly reproduced in the ventricular curve. And it has, with considerable reason, been urged that both in the aorta (and so in the other arteries) and in the ventricle they are due to events taking place in the ventricle, the systole for instance not being equally sustained.

We may further call once to mind the fact to which we have already called attention that, while sometimes the curve of ventricular pressure reaches its maximum at the very beginning of the systole, declining more or less slowly afterwards, at other times the maxinum is reached at the end of the systole, the curve of pressure being anacrotic; we may add that the maximum may also occur at any intermediate point. Further, and this is the matter to which we wish to call attention, the curve of aortic pressure follows that of the ventricular pressure, both being katacrotic or anacrotic as the case may be. As we have urged, the anacrotic curve is seen when the peripheral resistance is such that, for some time during the systole, the flow from the aorta towards the periphery is slower than the flow from the ventricle into the aorta. Such a condition is apt to be met with when the arteries are more rigid than normal, and under these circumstances the anacrotic characters of the pulse may become proninent.

$\$$ 131. Venous Pulse. Under certain circumstances the pulse may be carried on from the arteries through the capillaries into the veins. Thus, as we shall see later on, when the salivary gland is actively secreting, the blood may issue from the gland through the veius in a rapid pulsating stream. The nervous events which give rise to the secretion of saliva, lead at the same time, by the agency of vaso-motor nerves, of which we shall presently speak, to a widening of the small arteries of the gland. When the gland is at rest, the minute arteries are, as we shall see, somewhat constricted and narrowed, and thus contribute largely to the peripheral resistance in the part; this peripheral resistance throws into action the elastic properties of the small arteries leading to the gland, and the remmant of the pulse reaching these arteries is, as we before explained, finally destroyed. When the minnte arteries are dilated, their widened channels allow the blood to flow more easily through them and with less friction; the peripheral resistance which they normally offer is thus lessened. In consequence of this the elasticity of the walls of the small arteries is brought into play to a less extent than before, and these small arteries cease to do their share in destroying the pulse which comes down to them from the larger arteries. As in the case of the artificial model, where the 'peripheral' tuling is kept open, not enough elasticity is brought into play to convert the intermittent arterial flow into a continuous one, and the pulse which reaches the arteries of the gland passes on through them and through the capillaries, and is continued on into the veins. A similar venous pulse is also sometimes seen in other organs. 
Careful tracings of the great veins in the neighbourhood of the heart shew elevations and depressions, which appear due to the variations of endocardiac pressure, and which nay perhaps be spoken of as constituting a 'venous pulse,' though they have a quite different origin from the venous pulse just described in the salivary gland. In such a pulse it is the depression of the wave, not the elevation, which corresponds to the systole of the ventricle, the pulse-wave is the negative of the arterial pulse-wave; the matter however needs further study. In cases again of insufficiency of the tricuspid valves, the systole of the ventricle makes itself distinctly felt in the great veins; and an expansion travelling backwards from the heart becomes very visible in the veins of the neck. This, in which the elevation of the wave like that of the arterial pulse-wave corresponds to the ventricular systole, is also spoken of as a venous pulse.

Variations of pressure in the great veins due to the respiratory movements are also sometimes spoken of as a venous pulse; the nature of these variations will be explained in treating of respiration. 


\section{SEC. 5. THE REGULATION AND ADAPTATION OF THE VASCULAR MECHANISM.}

\section{The Regulation of the Beat of the Heart.}

\$132. So far the facts with which we have had to deal, with the exception of the heart's beat itself, have been simply physical facts. All the essential phenomena which we have studied may be reproduced on a dead model. Such an unvarying mechanical vascular system would however be useless to a living body whose actions were at all complicated. The prominent feature of a living mechanism is the power of adapting itself to changes in its internal and external circumstances. And the vascular mechanism in all animals in which it is present is capable of local and general modifications, adapting it to local and general changes of circumstance. These modifications fall into two great classes :

1. Changes in the heart's beat. These, being central, have of course a general effect; they influence or may influence the whole body.

2. Changes in the peripheral resistance, due to variations in the calibre of the minute arteries, brought abont by the agency of their contractile muscular coats. These changes may be either local, affecting a particular vascular area only, or general, affecting all or nearly all the blood vessels of the body.

These two classes of events are chiefly governed by the nervous system. It is by means of the nervous system that the heart's beat and the calibre of the minute arteries are bronght into relation with each other, and with almost every part of the body. It is by means of the nervous system acting either on the heart, or on the small arteries, or on both, that a change of 
circumstances affecting either the whole or a part of the body is met by compensating or regulative changes in the flow of blood. The study of these changes becomes therefore to a large extent a study of nervous actions.

The circulation may also be modified by events not belonging to either of the above two classes. Thus, in this or that periplieral area, changes in the capillary walls and the walls of the minute arteries and veins may lead to an increase of the tendency of the blood corpuscles to adhere to the vascular walls, and so, quite apart from any change in the calibre of the blood vessels, may lead to increase of the peripheral resistance. This is seen in an extreme case in inflammation, but may possibly intervene to a less extent in the ordinary condition of the circulation, and may also be under the influence of the nervous system. Further, any decided change in the quantity of blood actually in circulation must also influence the working of the vascular mechanism. But both these changes are unimportant compared with the other two kinds of clianges. Hence, the two most important problems for us to study are, 1 , how the nervous system regulates the beat of the heart, and 2, how the nervous system regulates the calibre of the blood vessels. We will first consider the former problem.

\section{The Development of the Normal Beat.}

$\$ 133$. The heart of a mammal or of a warm blooded animal generally ceases to beat within a few minutes after being removed from the body in the ordinary way, the hearts of newly-born animals continuing however to beat for a longer time than those of adults. Hence, though by special precautions and by means of an artificial circulation of blood, an isolated mammalian heart may be preserved in a pulsating condition for a much longer time, our knowledge of the exact nature and of the causes of the cardiac beat is as yet very largely based on the study of the hearts of cold blooded animals, which will continue to beat for hours, or under favourable circumstances even for days, after they have been removed from the body with only ordinary care. We have reason to think that the mechanism by which the beat is carried on varies in some of its secondary features in different kinds of animals: that the hearts, for instance, of the eel, the snake, the tortoise and the frog, differ in some minor details of behaviour, both from each other and from those of the bird and of the mammal; but we may, at first at all events, take the heart of the frog as illustrating the main and important truths concerning the causes and mechanism of the beat. 
In studying clusely the phenomena of the beat of the heart it becomes necessary to obtain a graphic recorl of the various movements.

1. In the frog, or other cold blooded animal, a light lever may be placed directly on the ventricle (or on an auricle, icc.), and changes of form, due either to distension by the influx of bloud, or to the systole, will eause movements of the lever, which nay be recorded on a travelling surface. The same method as we have seen may be applied to the m.mmalian heart.

2. Or', as in Gaskell's method, the heart may be fixed by a clamp carefully arljusted round the auriculo-ventricular groove, while the apex of the ventricle and some portion of one auricle are attached by threads to horizontal levers, placed respectively above and below the heart. The auricle and the ventricle each in its systole pulls at the lever attached to it; and the times and extent of the contractions may thus be recorderl. Or the thread may be attached to the apex of the ventricle only, the heart being fixed by the aorta or left in position in the body.

3. A record of endo-cardiac pressure may be taken in the frog or tortoise, as in the mammal, by means of an appropriate manometer. Aul in these animals, at all events, it is easy to keep up an artificial circulation. A camnula is introduced into the sinus venosus, and another into the ventricle through the aorta. Serum or dilute blood (or any other fluid which it may be desired to employ) is driven by moderate pressure through the former; to the latter is attached a tube commected by means of a sile piece with a small mercury or other manometer. So long as the exit-tube is open at the end, fluid flows freely through the heart and apparatus. Upon closing the exit-tube at its far end, the force of the ventricular systole is brought to bear on the manometer, the index of which registers in the usual way. Newell Martin has succerled in applying a modification of this method to the mammalian heart.

4. The movements of the ventricle may be registered by introducing into it, through the auriculoventricular orifice, a so-called 'perfusion' cannula, Figs. 66 and $67 \mathrm{I}$., with a louble tube, one insicle the other, and tying the ventricle on to the cannula at the auriculo-ventricular croove, or at any level below that which may be desired. The blond or other finicl is driven at an adequate pressure through the tube $\alpha$, enters the ventricle, and returns by the tube $b$. If $b$ be connectel with a manometer, as in method 3, the movements of the ventricle may be registered.

5. In the apparatus of Roy, Fig. 67 II., the exit-

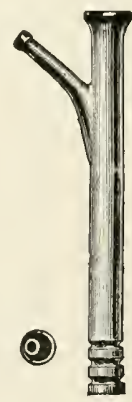

FI. 66. A PERfusion Cannula. tube is free, but the ventricle (the same method may be adopter for the whole leart) is placed in an air-tight chamber, filled with oil, or partly with normal saline solution anr partly with oil. By means of the tube $b$ the interior of the chamber a is continuous with that of a small cylinder $c$, in which a piston $d$, secured by thin, flexible, animal membrane, works 
up and down. The piston again bears on a lever $e$ by means of which its movements may be registered. When the ventricle contracts, and by contracting diminishes in volume, there is a lessening of pressure in
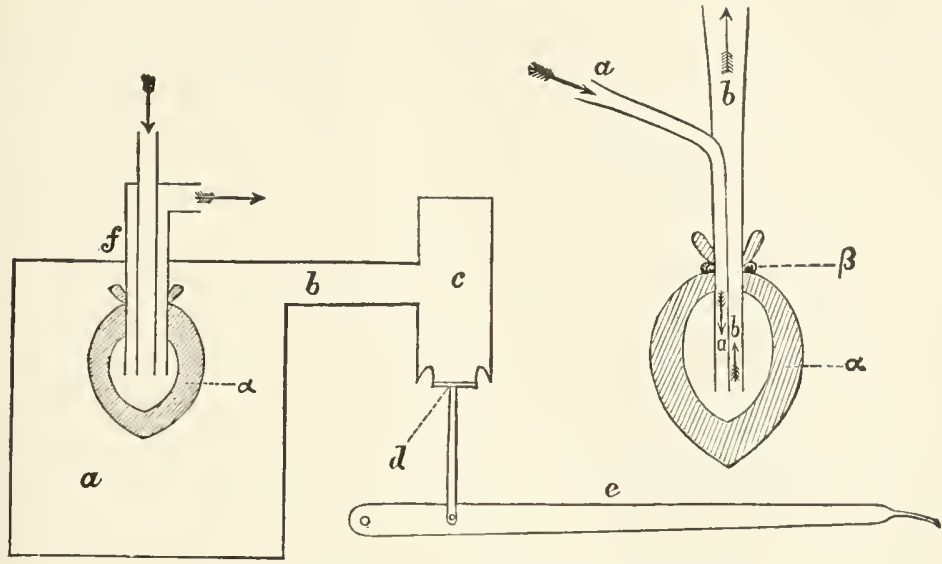

Fig. 67. Purely diagramiatic figures of

I. Perfusion cannula tied into frog's ventricle. $a$, entrance, $b$, exit-tube ; $a$, wall of ventricle; $\beta$, ligature.

II. Roy's apparatus modified by Gaskell. $a$, chamber filled with saline solution and oil, containing the ventricle $a$ tied on to the profusion cannula $f: \quad b$, tube leading to cylinder $c$, in which moves piston $d$, working the lever $e$.

the interior of the chamber; this is transmitted to the cylinder, and the piston correspondingly rises, carrying with it the lever. As the ventricle subsequently beeomes distended, the pressure in the chamber is increaserl, and the piston and lever sink. In this way variations in the volume of the ventricle may be recorded, without any great inter. ference with the flow of blood or fluid through it.

The heart of the frog, as we have just said, will continue to beat for hours after removal from the body, even though the cavities have been cleared of blood, and, indeed, when they are almost empty of all fluid. The beats thus carried out are in all important respects identical with the beats executed by the heart in its normal condition within the living body. Hence we may infer that the beat of the heart is an automatic action: the muscular contractions which constitute the beat are due to causes which arise spontaneously in the heart itself.

In the frog's heart, as in that of the mammal, $\$ 108$, there is a distinct sequence of events which is the same whether the heart he removed from, or be still in its normal condition within the body. First comes the beat of the sinus venosus, preceded by a more or less peristaltic contraction of the large veins leading into it; next follows the sharp beat of the two auricles together; then comes the longer beat of the ventricle; and lastly the cycle is completed by the 
beat of the bulbus arteriosus, which does not, like the mammalian aorta, simply recoil by elastic reaction after distension by the ventricular stroke but carries out a distinct muscular contraction passing in a wave from the ventricle outwards.

When. the heart in dying ceases to beat, the several movements cease, as a rule, in an order the inverse of the above. Omitting the bulbus arteriosus, which sometimes exhibits great rhythmical power, we may say that first the ventricle fails, then the auricles fail, and lastly the sinus venosus fails.

The heart after it has ceased to beat spontaneously remains for some time irritable, that is capable of executing a beat, or a short series of beats, when stimulaterl either mechanically as by touching it with a blunt needle or electrically by an induction shock or in other ways. The artificial beat so called forth may be in its main features identical with the natural beat, all the divisions of the heart taking part in the beat, and the sequence of events being the same as in the natural beat. Thus when the sinus is pricked the beat of the sinus may be followed by a beat of the auricles and of the ventricle; and even when the ventricle is stimulated, the directly following beat of the ventricle may be succeeded by a complete beat of the whole heart. Under certain circumstances however the division directly stimulated is the only one to beat; when the ventricle is pricked for instance it alone may beat, or when the sinus is pricked it alone may beat. The results of stimulation moreover may differ according to the condition of the heart and according to the particular spot to which the stimulus is applied.

With an increasing loss of irritability, the response to stimulation ceases in the several divisions in the same order as that of the failure of the natural beat; the ventricle ceases to respond first, then the auricles, and lastly the sinus venosus, which frequently responds to stimulation long after the other divisions have ceased to make any sign.

It would appear as if the sinus venosus, auricles, and ventricle formed a descending series in respect to their irritalility and to the power they possess of carrying on spontaneous rhythmic beats, the sinus being the most potent. This is also seen in the following experiments.

In order that the frog's heart may beat after removal from the body with tlie nearest approach in rapidity, regularity, and endurance to the normal condition, the removal must be carried out so that the excised heart still retains the sinus venosus intact.

When the incision is carried through the auricles so as to leave the sinus renosus behind in the body, the sinus venosus beats forcibly and regularly, having suffered hardly any interruption from the operation ; but the auricles and ventricle remain motionless, often for a considerable time, and when they do resume spontaneous beats these have a rhythm different from that of the sinus, and are less vigorous and lasting than those of the 
entire heart. If the incision be carried between the auricles and ventricle, the former with the sinus beat regularly and forcibly while the latter often exhibits no spontaneous beats at all, or if these do appear they last for a slort time only. Lastly if the ventricle be cut across leaving the upper third attached to the auricles, this beats regularly with the sinus and auricles, but the detached lower two-thirds do not beat spontaneously at all.

Now, while ganglia are abundant over the sinus, are numerous over the auricles, and, as Bidder's ganglia, are present at the auriculo-ventricular junction, no nerve cells are present in the lower part of the ventricle. Hence the view has suggested itself that the rhythmic spontaneous beating is due to impulses proceeding rlythmically from the nerve cells of the ganglia. But serious objections may be urged against this view. Even in the case of the frog, the lower part of the ventricle, the mere tip almost, may under specially favourable circumstances beat in an apparently spontaneous manner; this occurs when it is tied round the end of a perfusion cannula (Fig. 66), and fed with blood or serum at a somewhat high pressure. And in the case of the tortoise a mere strip of muscle, quite free from nerve cells, cut out of the ventricle may be made to beat, in an apparently spontaneous manner, with great regularity for a considerable time.

Without entering into any lengthy discussion concerning a matter which is and has been much debated, we may say that the cardiac muscular fibre differs in properties, as it does in structure, from the skeletal muscular fibre, that it is not to the same extent as that, a mere instrument, so to speak, in the hands of a motor nerve fibre, but has, itself, largely to do with originating its own contraction. The muscular contraction, we may here observe, of whicl the beat is a development, is not a tetanus, but a somewhat long continued simple contraction. This may' be readily shewn to be the case on the slip of the tortoise ventricle just referred to. Such a slip, when attached to a lever, and stimulated with a single induction shock, gives what is obviously a simple contraction, and a beat of the slip occurring naturally has exactly the same features. And the electric change shewn at any part of the heart during a beat natural or induced by stimulation is that characteristic of a simple contraction. The intact ventricle at rest is as we have already said ( $\$ 63$ ) isoelectric, but each part just as it is entering into a state of contraction becomes negative towards the rest. Hence when the electrodes of a galvanometer are placed on two points $A, B$ of the surface of the ventricle a diphasic variation of the galvanometer needle is seen when a beat, natural or excited, occurs. Supposing that the wave of contraction reaclies $A$ first, this will become negative towards the rest of the ventricle, including $B$, but when the wave sometime afterwards reaches $B, B$ will become negative towards the rest of the ventricle, including $A$. Compare $\$ 64$.

But the contraction of cardiac muscle differs from that of a 
skeletal muscle in the following important feature. When we stimulate a skeletal muscle with a strong stimulus we get a large contraction, when we apply a weak stimulus we get a small contraction; within certain limits (see $\$ 74$ ) the contraction is proportional to the stimulus. This is not the case with the quiescent ventricle or heart. When we apply a strong inductionshock we get a beat of a certain strength; if we now apply a weak shock we get either no beat at all or quite as strong a beat as with a stronger stimulus. That is to say the magnitude of the beat depends on the condition of the ventricle (or heart) and not on the magnitude of the stimulus. If the stimulus can stir the ventricle up to beat at all, the beat is the best which the ventricle can at the time accomplish; the stimulus produces either its maximum effect or none at all. It would seem as if the stimulus does not produce a contraction in the same way that it does when it is brought to bear on a skeletal muscle, but rather stirs up the heart in such a way as to enable it to execute a spontaneous beat which, without the extra stimulus, it could not bring about. And we have reason to think that the normal beat of the heart within the body is the maximum beat of which it is capable at the moment. These and other special features of the contraction of cardiac muscle lead to the conclusion that the rhythmic power does not reside wholly in the ganglia; hut we must not here discuss the question further, nor enter upon the difficult problem of how the remarkable sequence in contraction of the several parts is developed and as a rule maintained.

$\$ 134$. In the above we have dealt chiefly with the heart of the cold blooded animal, but so far as we know the same general conclusions hold good for the mammalian heart also. There is, it is true, in the mammal, no prepotent sinus venosus, but as in the frog the auricles are dominant, and their beat determines the beat of the ventricles. Even more clearly than in the frog however, the ventricles, though they normally follow the auricles in their beat, being initiated as it were by them, possess an independent rhythmic power of their own. By a mechanical contrivance all conduction of nervous or muscular impulses between the auricles and ventricles may be abolished, thongh the blood may continne to flow from the cavities of the former to those of the latter. When this is done the ventricles go on beating rhythmically, but at a rate which is quite independent of that of the auricular beats.

We may now turn to the nervous mechanisms by which the beat of the heart, thus arising spontaneously within the tissnes of the heart itself, is modified and regulated to meet the requirements of the rest of the body.

\section{The Govermment of the Heart Beat by the Nerrous System.}

$\$ 135$. It will be convenient to begin with the heart of the frog. This is connected with the central nervous system through, 
and therefore governed by, the two vagus nerves, each of which though apparently a single nerve contains, as we shall see, fibres of different origin and nature.

If while the beats of the heart of a frog are being carefully registered an interrupted current of moderate strength be sent through the vagus nerve, the heart is seen to stop beating. It remains for a time in diastole, perfectly motionless and flaceid; all the muscular fibres of the several cliambers are for the time being in a state of relaxation. The heart has been inhibited by the impulses descending the vagus from the part of the nerve stimulated.

If the duration of the stimulation be short and the strength of the current great, the standstill may continue after the current has been shut off ; the beats, when they reappear, are generally at first feeble and infrequent, but soon reach or even go beyond their previous vigour and frequency. If the duration of the stimulation be very long, the heart may recommence beating while the stimulation is still going on, but the beats are feeble and infrequent though gradually increasing in strength and frequency. 'The effect of the stimulation is at its maximum at or soon after the commencement of the application of the stimulus, gradually declining afterwards; but even at the end of a very prolonged stimulation the beats may still be less in force or in frequency, or in both, than they were before the nerve was stimulated, and on the removal of the current may shew signs of recovery by an increase in force and frequency. The effect is not produced instantaneously ; if on the curve the point be exactly marked when the current is thrown in, as at on Fig. 68, it will frequently be found that one beat at
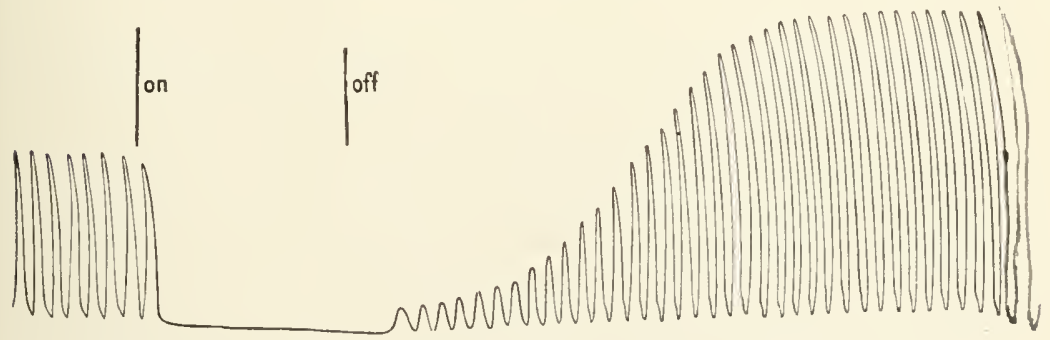

Fig. 68. Ininition of Frog's Heirt by stimulation of Vagus Nerve.

on marks the time at which the interrupted current was thrown into the vagus, off when it was shut off. 'The time marker below marks secouds. 'The beats were registered by suspending the ventricle from a clamp attached to the aorta and attaching a light lever to the tip of the ventricle.

least occurs after the current has passed into the nerve; the development of that beat has taken place before the impulses descending the vagus have had time to affect the heart. 
The stimulus need not necessarily be the interrupted current; mechanieal, chenical or thermal stimulation of the vagus will also produce inhibition; but in order to get a marked effect it is desirable to make use of not a single nervous impulse but a series of nervous impulses; thus it is difficult to obtain any recognisable result by employing a single induction shock of moderate intensity only. As we shall see later on 'natural' nervous impulses descending the vagus from the central nervous system, and started there, by afferent impulses or otherwise, as parts of a reflex act, may produce inhibition.

The stimulus may be applied to any part of the course of the vagus from high up in the neck right down to the sinus; indeed, very marked results are obtained by applying the electrodes directly to the sinus where as we have seen the two nerves plunge into the substance of the heart. The stimulus may also be applied to either vagus, though in the frog, and some other animals, one vagus is sometimes more powerful than the other. Thus it not unfrequently happens that even strong stimulation of the vagus on one side produces no change of the rhythm, while even moderate stimulation of the nerve on the other side of the neck brings the heart to a standstill at once.

If during the inhibition the ventricle or other part of the heart be stimulated directly, for instance mechanically by the prick of a needle, a beat may follow; that is to say, the impulses descending the vagus, while inhibiting the spontaneous beats, have not wholly abolished the actual irritability of the cardiac tissues.

With a current of even moderate intensity, such a current for instance as would produce a marked tetanus of a muscle-nerve preparation, the standstill is complete, that is to say, a certain number of beats are entirely dropped; but with a weak current the inhibition is partial only, the heart does not stand absolutely still but the beats are slowed, the intervals between them being prolonged, or weakened only without much slowing, or both slowed and weakened. Sometimes the slowing and sometimes the weakening is the more conspicuous result.

$\$ 136$. It sometimes happens that, when in the frog the vagus is stimulated in the neck, the effect is very different from that just described; for the beats are increased in frequency, though they may be at first diminished in force. And, occasionally, the beats are increased both in force and in frequency: the result is augmentation, not inhibition. But this is due to the fact that in the frog the vagus along the greater part of its course is a mixed nerve and contains fibres other than those of the vagus proper.

If we examine the vagus nerve closely, tracing it up to the brain, we find that just as the nerve has pierced the cranium, just where it passes through the ganglion ( $G V$, Fig. 69), certain fibres pass into it from the sympathetic nerve of the neck, $S y$, of the further connections of which we shall speak presently. 
This being the case, we may expect that we should get different results according as we stimulated (1) the vagus in the cranium,

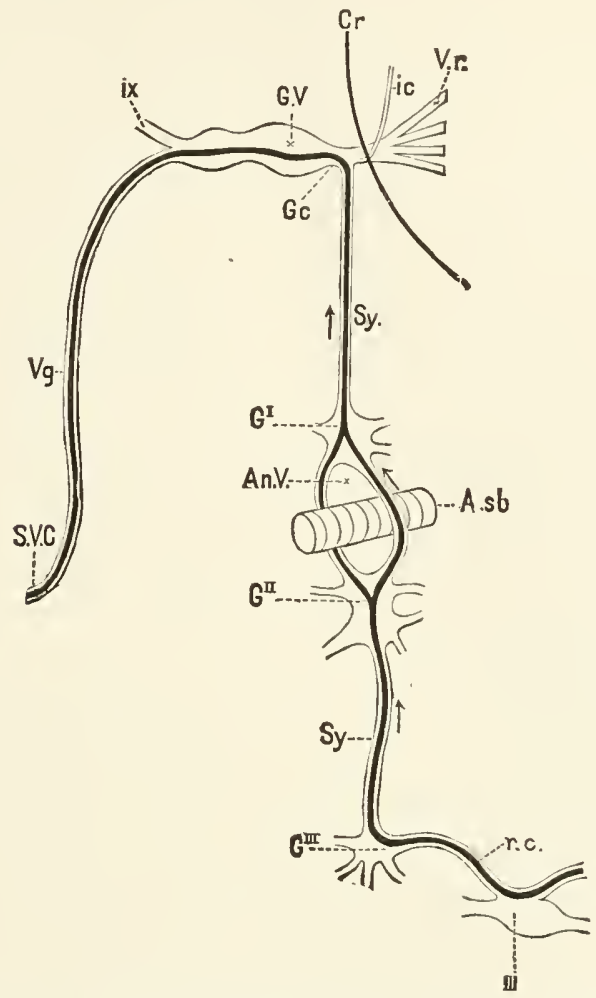

Fig, 69. Diagramutic Representation of the codrse of Cardic Aughextor Fibres in tile Frog.

$V r$. roots of vagus (and $\mathbf{x t h}$ ) nerve. $G V$. ganglion of same. $C r$. line of cranial wall. $V q$, vagus trunk. Ix, ninth, glosso-pharyugeal nerve. $\$ . V . C$. superior veua cava. Sy. sympathetic nerve in neck. G.C. junction of sympathetic ganglion with vagus ganglion, sending 2.c. intracranial fibres passing to Gasserian ganglion. 'The rest of the filres pass along the vagus trunk. Gissmpathetic ganglion connected with the first spinal nerve. GII sympathetic ganglion of the second spinal nerve. $A n . V$. anmulus of Vieussens. A. sb. subclavian artery. GIII sympathetic ganglion of the third spinal nerve. $I I I$, third spinal nerve. r.c. ramus communicans.

The course of the augmentor filres is shewn by the thick black line. 'They may be traced from the spinal cord by the anterior root of the third spinal nerve, through the ramus communicans to the corresponding sympathetic ganglion $G^{I I I}$ and thence by the second ganglion $G^{I I}$, the annulus of Vieussens, and the first ganglion $G^{I}$ to the cervical sympathetic $S y$, and so by the vagus trunk to the superior vena cava S.V.C.

before it was joined by the sympathetic, (2) the sympathetic fibres before they join the vagus, and (3) the vagus trunk, containing both the real vagus and the sympathetic fibres. What we have previously described are the ordinary results of stimulating the mixed 
trunk, and these, as we have said, are not wholly constant, though, usually and in the main, most distinct inhibitory results follow.

If we stimulate the sympathetic in the neck as at $\$ y$, Fig. 69 , cutting the nerve below so as to block all impulses from passing downwards, and only allow impulses to pass up to the vagus and thence down the mixed ragus trunk to the heart, we get very remarkable results. The beat of the heart instead of being inhibited is augmented, the beats are increased either in frequency or in force, or most generally both in frequency and in force. The effect is perhaps best seen when the heart before stimulation is beating slowly and feebly; upon stimulation of the cervical sympathetic the beats at once improve in vigour and frequency ; indeed, a heart which for one reason or another has almost ceased to beat nuay, by proper stimulation of the sympathetic, be called back into vigorous activity.

If, on the other hand, we stimulate the vagus before it has been joined by the sympathetic fibres (and to ensure the result not being marred by any escape of the stimulating current on to the sympathetic fibres it is necessary to stimulate the vagus within the eranium) we get pure and constant inhibitory results, the beats are for a time wholly abolished, or are slowed, or are weakened, or are both slowed and weakened.

Obviously, then, the heart of the frog is supplied through the vagus by two sets of fibres coming from the central nervous system, the one by the vagus proper and the other by the cervical sympathetic nerve, and these two sets have opposite and antagonistic effects upon the heart.

The one set, those belonging to the vagus proper, are inhibitory; they weaken the systole and prolong the diastole, the effect with a strong stimulation being complete, so that the heart is for a time, brought to a standstill. Sometimes the slowing, sometimes the weakening is the more prominent. When the nerve and the heart are in good condition, it needs only a slight stimulus, a weak current, to produce a marked effect, and it may be mentioned that the more vigorous the heart, the more rapidly it is beating, the easier is it to bring about inhibition. Although, as we have said, the effect is at its maximum soon after the beginning of stimulation, a very prolonged inhibition may be produced by prolonged stimulation; indeed, by rhythmical stimulation of the vagus the heart may be kept perfectly quiescent for a very long time and yet beat vigorously upon the cessation of the stimulus. In other words, the instruments of inhibition, that is, the fibres of the vagus and the part or substance of the heart upon which these act to produce inhibition, whatever that part or substance may be, are not readily exhausted. Further, the inhibition when it ceases is, frequently at all events, followed by a period of reaction, during which the heart for a while beats more vigorously and rapidly than before. Indeed the total effect of stimulating the vagus 
fibres is not to exhaust the heart, but rather to strengthen it; and by repeated inhibitions carefully administered, a feebly beating heart nay be nursed into vigorous activity.

The other set, those joining the vagus from the sympathetic, are 'augmentor' or 'accelerating' fibres; the latter name is the more common but the former is more accurate, since the effect of stimulating these fibres is to increase not only the rapidity but the force of the beat; not only is the diastole shortened but the srstole is strengthened, sometimes the one result and sometimes the other heing the more prominent. These augnentor fibres need a somewhat strong stimulation to produce an effect, the time required for the maximum effect to be produced is long, and the eftect, when produced, may last for some time. A slowly or weakly beating heart is more easily augmented than is a strong one. Further, the augmentation is followed by a period of reaction in which the beats are feebler, by a stage of exhaustion; and indeed by repeated stimulation of these sympathetic fibnes a fairly vigorous heart, especially a bloodless one, may be reduced to a very feeble condition.

By watching the effects of stimulating the sympathetic nerve at various points of its course we may trace these augmentor fibres from their junction with the vagus down the short sympathetic of the neck through the sympathetic ganglion connected with the first spinal nerve, GI, Fig. 69, through one or both the loops of the anuulus of Vieussens, $A n$. $V$, through the second ganglion, connected with the second spinal nerve, $G^{I I}$, to the thind ganglion connected with the third spinal nerve, $G^{I I I}$, and thence through the ramus communicans or visceral branch of that ganglion, r.c., to the third spinal nerve, $I I I$, by the anterior root of which ther reach the spinal cord.

$\$ 137$. Both sets of fibres, then, may be traced to the central nervous system; and we find accordingly that the heart may be inhibited or angmented by nervous impulses which are started in the nervous system either by afferent impulses as part of a reflex act or otherwise, and which pass to the heart by the inhibitory or by the augmenting tract.

Thus if the spinal bulb or a particular part of the spinal bulb which is specially connected with the vagus nerve be stimulated, the heart is inhibited; if, for instance, a needle be thrust into this part the heart stands still. This nervous area may be stirred to action, in a 'reflex' manner, by afferent impulses reaching it from various parts of the body. Thus if the abdomen of a frog be laid bare, and the intestine be struck sharply with the handle of a scalpel, the heart will stand still in diastole with all the phenomena of vagus inhibition. If the nervi mesenterici or the connections of these nerves with the spinal cord be stimulated with the interrupted current, cardiac inhibition is similarly produced. If in these two experiments both vagi are divided, or the 
spinal bulb is destroyed, inhibition is not produced, however much either the intestine or the mesenteric nerves be stimulated. This shews that the phenomena are caused by impulses ascending along the mesenteric nerves to the spinal bulb, and so affecting a portion of that organ as to give rise by reflex action to impulses which descend the vagus nerve or nerves as inhibitory impulses. The portion of the spinal bulb thus mediating between the afferent and efferent impulses may be spoken of as the cardio-inhititory centre. This centre may be thrown into activity, and so inhibition produced, by afferent impulses reaching it along various nerves; by means of it reflex inhibition through one vagus may be brought about by stimulation of the central end of the other.

And we have reason to think that in a similar manner augmentor impulses are developed in the central nervous system either as part of a reflex chain or otherwise.

$\$ 138$. So far we have been dealing with the heart of the frog, but the main facts which we lrave stated regarding inhibition and augmentation of the heart beat apply also to other vertebrate animals including mammals, and, indeed, we meet similar phenomena in the hearts of invertebrate animals.

If in a manmal the heart be exposed to view by opening the thorax, and the vagus nerve be stimulated in the neck, the heart may be seen to stand still in diastole, with all the parts flaccid and at rest. If the current employed be too weak, the result, as in the frog, is not an actual arrest but a slowing or weakening of the beats. By placing a light lever on the lieart or by other methods, a graphic record of the standstill, or of the slowing, of the complete or incomplete inhibition may be obtained. The result of stimulating the vagus is also well shewn on the blood
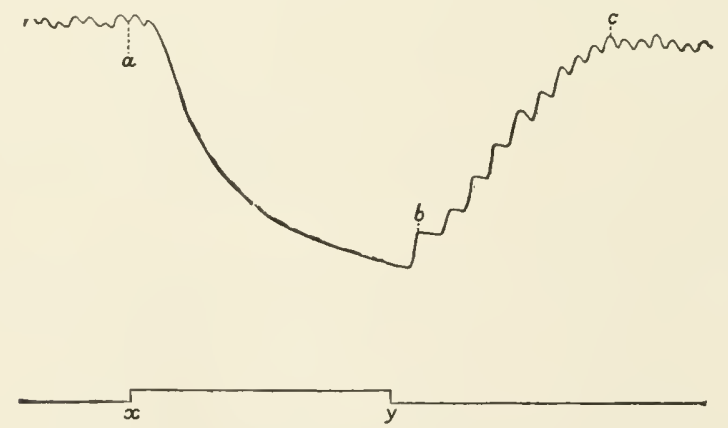

Fig. 70. Tracing, Shewing the influence of Cardiac Inhibition on Blood Pressure. From a Rabbit.

$r$ the marks on the signal line when the current is thrown into, and $y$ shut off from the vagus. The time marker below marks seconds, the heart, as is frequently the case in the rabbit, beating very rapidly. 
pressure curve, the effect of complete cardiac inhibition on blood pressure being most striking. If, while a tracing of arterial pressure is being taken, the beat of the heart be suddenly arrested by vagus stimulation, some such curve as that represented in Fig. 70 will be obtained. It will be observed that two beats follow the application of the current marked by the point $a$, which corresponds to the signal $x$ on the line below. Then for a space of time no beats at all are seen, the next beat $b$ taking place almost immediately after the shutting off the current at $y$. Immediately after the last beat following $a$, there is a sudden fall of the blood pressure. At the pulse due to the last systole, the arterial system is at its maximum of distention; forthwith the elastic reaction of the arterial walls propels the blood forward into the veins, and, there being no fresh fluid injected from the heart, the fall of the mercury is unbroken, being rapid at first, but slower afterwards, as the elastic force of the arterial walls is more and more used up. With the returning beats the pressure correspondingly rises in successive leaps until the normal mean pressure is regained. The size of these returning leaps of the mercury may seem disproportionately large, but it must be remembered that by far the greater part of the force of the first few strokes of the heart is expended in distending the arterial system, a small portion only of the blood which is ejected into the arteries passing on into the veins. As the arterial pressure rises, more and more blood passes at each beat through the capillaries, and the rise of the pressure at each beat becomes less and less, until at last the whole contents of the ventricle pass at each stroke in to the veins, and the mean arterial pressure is established. To this it may be added, that, as we have seen, the force of the individual beats may be somewhat greater after than before inhibition. Besides, when the mercury manometer is used, the inertia of the mercury tends to magnify the effects of the initial beats.

The above is an example of complete inhibition, of a total standstill for a while of the whole heart, such as may be obtained by powerful stimulation of the vagus; both auricles and ventricles remain for a period free from all contractions; and as the previously existing arterial pressure drives the blood onward from the arteries through the capillaries and veins towards the heart, the cavities of the heart become distended with blood, especially on the right side.

A weaker stimulation of the vagus produces an incomplete inhibition, the heart continues to beat but with a different rhythm and stroke, and by careful observation many interesting features may be observed. If a record be obtained, by one or other of the methods mentioned in $\$ 113$ or elsewhere, of the behaviour of the auricles and ventricles respectively, it will be observed that the inhibition tells much more on the auricles than on the ventricles. The extent of the auricular contractions is 
especially affected, more so than that of the ventricles, and it may sometimes be observed that the auricles are brought to complete quiescence while the ventricles still continue to beat; the latter now exhibit that independent rhythm of which we spoke in $\$ 134$. In a somewhat similar manner the stimulation of the vagus, by affecting the rhythm of the auricles more than that of the ventricles, may lead to a want of coordination between the two, the especially slowed auricles beating at one rate, the ventricles at another. It is indeed maintained by some that the vagus acts directly on the auricles only, the changes in the ventricles being of a secondary nature, caused by the changes in the auricles.

When the output from the ventricles during vagus stimulation is measured, by the cardiometer or otherwise, it is found, as might be expected, that this is lessened. The diminution during a given period may be due to the mere slowing of the beat; but the individual pulse volume is in some cases, at least, also lessened. It may by the same method be observed that the quantity remaining in the ventricle at the end of the systole is increased; the ventricle appears to expand more during diastole. Of the effects thus produced on the circulation we shall speak later on.

We may now turn to some further details concerning the course of these inhibitory fibres. They run in the trunk of the vagus; this is clear not only in the case of an animal like the rabbit, in which the vagus runs separate from the cervical sympathetic but also in the case of the dog, in which the two nerves are more or less bound up together. Leaving the vagus by the cardiac branches, they reach the cardiac tissues by the cardiac plexuses. When we trace the fibres in the other direction towards the central nervous system, we have to bear in mind that the fibres which compose the trunk of the vagus have, as we shall see in studying the central nervous system, two distinct central origins. On the one hand, there are the fibres which are the proper vagus fibres which, leaving the spinal bulb, pass through both the jugular ganglion and trunk ganglion (Fig. $71 \mathrm{r}$. GJ. $G$. Tr. $V g$.). On the other hand, there are fibres which, belonging to the spinal accessory nerve $(S p . A c$. ) and to what we shall learn to speak of as the bulbar division of that nerve, pass after leaving the spinal bulb to the trunk ganglion of the vagus, and thence form part of the vagus trunk. Now, it is these fibres of the spinal accessory nerve and not the proper vagus fibres which supply the inhibitory fibres to the heart. Thus, if the bulbar roots of the spinal accessory be divided, those of the vagus proper being left intact, the spinal accessory fibres in the vagus trunk degenerate, and when this has taken place stimulation of the vagus fails to produce the ordinary inhibitory effect.

Within the spinal bulb these inhibitory fibres are connected, in the mammal as in the frog, with a cardio-inhibitory centre; and in the mammal as in the frog inhibition may be brought about 
not only by artificial stimulation of the vagus, but by stimulation in a reflex manner or otherwise of the cardio-inhibitory centre. Thus the fainting which often follows upon a blow on the stomach is a repetition of the result mentioned a little while ago as obtained on the frog by striking the stomach or stimulating the nervi mesenterici. So also the fainting, complete or partial, which accompanies severe pain or mental emotion, is an illustration of cardiac inhibition by the vagus. 'These are familiar examples of more or less complete inhibition ; but simple slowing or weakening of the beat through the inhibitory mechanism is probably an event of much more common occurrence. For instance, a rise of general blood pressure, or, and perhaps more especially, a rise in the blood pressure of the vessels of the brain, sets going inhibitory inpulses by which the work of the heart is lessened, and the ligh blood pressure lowered, the dangers of a too high pressure being thus averted. Again, the inhibition may be brought about in a reflex manner by impulses started in the heart itself and ascending to the central nervous system along afferent fibres which run in the vagus trunk from the heart to the spinal bulb. In this way the heart regulates its own action according to its condition and its needs.

There is also some reason for thinking that, in some animals at least, the central nervous system by means of the cardiac inhibitory fibres keeps, as it were, a continual rein on the heart, for, in the dog for example, section of both vagi causes a quickening of the heart's beat. But we shall have to speak of these matters more than once later on. Mean while we may turn to the augmentor fibres.

So much of our knowledge of the nervous work of the heart and especially of the action of the augmentor fibres has been gained by experiments on dogs that it may be desirable to give a few details concerning the nerves of the heart in this animal.

In the $\log$ the vagus son after it issues from its trunk ganglion (G. Tr. Vg., Fig. 71 ) is joined by the sympathetic nerve proceeding from the superior cervical ganglion, the two forming the vago-sympathetic trunk. As this trunk enters the thorax, the sympathetic portion bears a ganglion (G.C.) usually called the lower cervical ganglion. To this ganglion there pass from the stellate ganglion $(G . S t$ ) of the thoracic sympathetic chim, two nerves, one running ventral to, the other dorsal to the subclavian artery, and thus forming with the two ganglia, the annulus of Vieussens $(\mathrm{A} n . \mathrm{T}$.$) .$

A very large number of the cardiac nerves spring from the lower cervical ganglion and from the vagus trunk lying in contact with it, from the vagus trunk below this ganglion, from the annulus of Vieussens, chiefly at least from the ventral limb, and sometimes from the stellate ganglion. There are besides cardiac branches passing from the vago-sympathetic trunk between the levels of the superior and of the inferior cervical ganglia, cardiac branches of the recurrent laryngeal, a cardiac branch of the superior laryngeal, and a long 


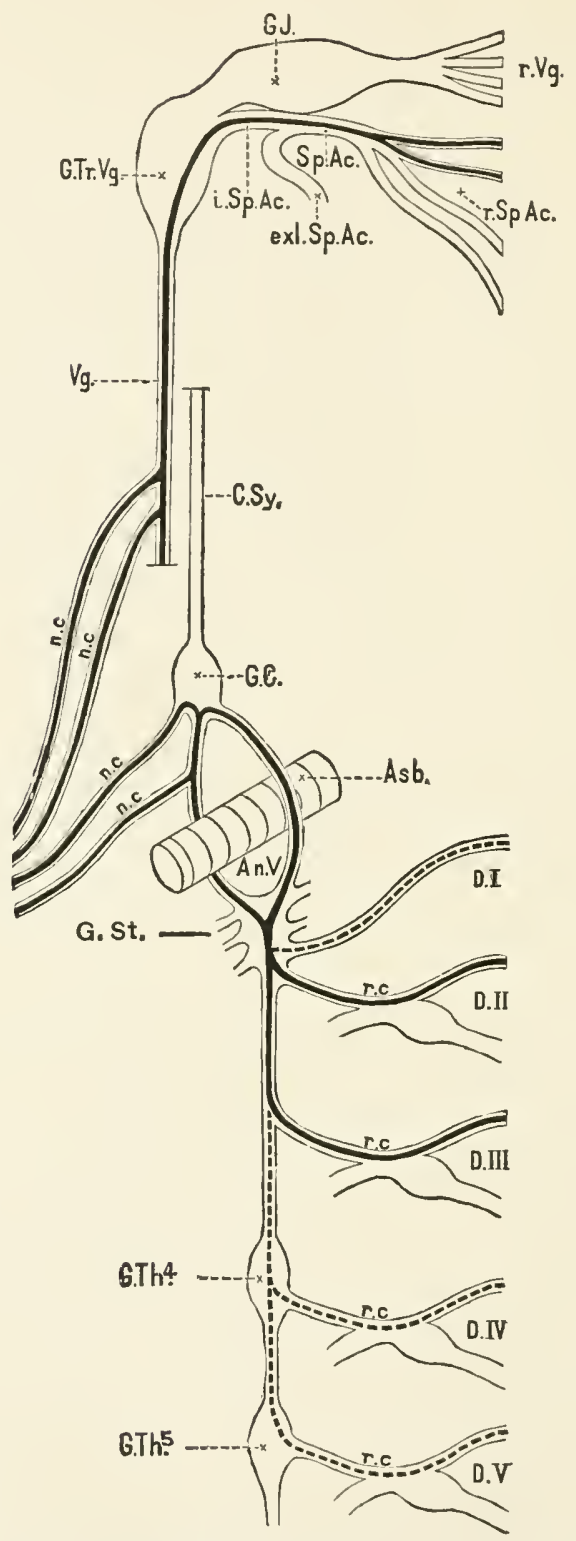

Fig. 71. Diagrammatic Representation of the Cardial Inimbitory and Augmentor Fibies in the Dog. fibres.

The upper portion of the figure represents the inhibitory, the lower the augmentor 
$r . \mathrm{V} g$. roots of the vagus, $r . S p A c$. roots of the spinal accessory; both drawn very diagrammatically. G.J. ganglion jugnlare. G.Tr. I'g ganglion trunci vagi. Sp.Ac. spinal accessory trunk. Ext.Sp.Ac. external spinal accessory. $i$ Sp.Ac. internal spinal accessory. $T_{y}$. trunk of vagus nerve. n.c. branches going to heart C.Sy. cervical sympathetic. G.C. lower cervical gauglion. A.sh. subclavian artery. An.V. Annulus of Vieusens. G.St. stellate ganglion, corresponding to the first, second, anl third ganglia of the thoracic cliain. G.Th.4, G.Th. ${ }^{5}$, fourth and fifth thoracic ganglia D.1., D.11., L.111., D.Iv., L.v., first, second, third, fourth and fifth thoracic spinal nerves. $r$. c ramus communicans. n.r. nerves (cardiac) passing to the heart from the cervical ganglion and from the annulus of Vieussens.

The inhibitory fibres, shewn by black lines, run in the upper (bulbar) roots of the spinal accessory, by the internal branch of the spinal accessory, past the ganglion trunci vagi, along the trunk of the vagus, and so by branches to the heart.

The angmentor fibres, also shewn by black lines, pass from the spinal cord by the anterior roots of the second and thirl thoracic nerves (possilly also from the first. fourth and fiftl as indicated by broken black lines), pass the stellate ganglion by the anumlus of Vienssens to the lower cervical ganglion, from whence, as also from the annulus itself, they pass along the cardiac nerves to the heart. An occasional tract from the stellate ganglion itself is not shewn in the figure.

slender nerve from the superior cervical ganglion passing independently to the heart. The arrangement is not exactly the same on the two sides of the body, and the minor details differ in different individuals. As in other animals the various cardiac nerves mingle in the cardiac plexuses.

In the dog it has been ascertained by separate stimulation of these several cardiac nerves, that augmentor fibres are contained in some or other of the nerves passing from the lower cervical ganglion and the adjoining vagus trunk, from the anuulus of Vieussens, especially the lower, ventral, limb, and sometimes from the stellate ganglion itself. The results differ a good deal in different individuals, and there are reasons for thinking that the nerves in question may contain efferent fibres other than augmentor fibres, by reason of which stimulation of them may give rise to other than pure augmentor effects. Speaking broadly, however, we may say that we may trace the augmentor fibres back from the cardiac plexuses through the lower cervical ganglion and the annulus of Vieussens to the stellate ganglion.

This ganglion is in reality several sympathetic ganglia fused together. It undoubtedly, in the dog, represents the first, second and third thoracic sympathetic ganglia, receiving, as it does, branches, rami communicantes, from the first, second and third thoracic spinal nerves. Since it also receives branches from the eighth and seventh cervical nerves, it has been argued that it represents not only the three thoracic sympathetic ganglia, but also what in man and other animals is called the lower cervical ganglion; if so, what has been called above the lower cervical ganglion should be regarded as the middle cervical ganglion. From the stellate ganglion the sympathetic cord passes to the ganglion, which is connected by a ramus communicans with the 
fourth thoracic spinal nerve, and which is therefore, in reality, the fourth thoracic ganglion, and so on to the rest of the thoracic chain.

Now, when the several rami communicantes, or the anterior roots, of the lower cervical and upper thoracic nerves are separately stimulated, it is found that augmentor effects make their appearance with considerable constancy when the seeond and third thoracic nerves are stimulated; the effects are less constant with the first and fourth thoracic nerves; sometimes some effect may appear with the fifth thoracic nerve, but not with any other thoracic nerves, or with any of the cervical nerves.

We may therefore say that, in the dog, augmentor impulses leave the spinal cord by the anterior roots of the second and third, to some extent the first and fourth, and possibly the fifth thoracic nerves, travel by the several rami communicantes to the stellate ganglion, and pass thence to the cardiac plexuses, and so to the heart, by nerves from the stellate ganglion itself, or from the anmulus of Vieussens, or from the so-called lower cervical ganglion. In the cat the path of the augmentor impulses is very similar, and we may regard the statement just made as representing, in a broad way, the path of these impulses in the mammal generally. They leave the spinal cord by the upper thoracic nerves, and pass to the heart through the lower cervical and upper thoracic sympathetic ganglia.

The effect of stimulating these augmentor fibres is, in some cases, to increase the rapidity of the rhythm. When the heart is beating very slowly this acceleration may be very conspicuous, but when the heart is beating quickly, or even at what may be called a normal rate, the acceleration observed may be very slight. A more constant and striking effect is the increase in the force of the beat. When tracings are taken of the movements of the auricles and ventricles separately, it is observed that in the case both of the auricles and of the ventricles, the extent of the systole is increased; moreover, it would seem also that both cavities undergo a larger expansion: they are filled with a larger quantity of blood during the diastole. 'This means that the output of the heart is increased by the action of the augmentor nerves, and that such is the effect may be directly shewn by the cardiometer. Moreover, this increase of the output may take place in spite of a concomitant rise of arterial pressure, so that the effect of the action of the augmentor nerves is distinctly to increase the work of the heart; and this may take place even though no marked acceleration occurs.

In the nummal as in the case of the frog, when the augmentor fibres are stimulated, some time elapses before the maximmm effect is witnessed and the influence of the stimulation may last some considerable time after the stimulation has ceased.

When records are taken of the behaviour of the heart during the stimulation of afferent nerves, such as the sciatic or the splanchnic, the records shew that the heart may behave very much 
in the same way as when the augmentor fibres are directly stimulated; there is a marked increase in the force of the auricular and of the ventricular systole, and at times an obvious acceleration of the rhythm. We may infer that in such a case the augmentor fibres are thrown into activity through the afferent impulses as part of a reflex act. At the same time it nust be remembered, that afferent impulses may increase the beat of the heart not by exciting the augmentor mechanism, but by depressing, that is by inhibiting a previously existing activity of the cardio-inhibitory centre; to this point we shall again have to refer.

We may however conclude that both the inhibitory and the augmentor mechanisms of the heart can he brought into action by means of the central nervous system. Speaking broadly the effect of the forner is to diminish the work of the heart, and so to lower the blood pressure, and that of the latter to increase the work of the heart, and so to heighten the blood pressure.

$\$ 139$. If, either in a frog or a mammal, or other animal, after the vagus fibres have been proved, by trial, to produce, upon stimulation, the usual inhilitory effects, a small quantity of atropin be introduced into the circulation (when the experiment is conducted on a living animal, or be applied in a weak solution to the heart itself when the experiment is conducted, in the frog for instance, on an excised heart or after the circulation has ceased), it will after a short time be found, not only that the stimulation, the application of a current for instance, which previously when applied to the vagus produced marked inhibition, now produces no inhibition, but even that the strongest stimulus, the strongest current applied to the vagus, will wholly fail to affect the heart, provided that there be no escape of current on to the cardiac tissues themselves; under the influence of even a small dose of atropin, the strongest stimulation of the vagus will not produce standstill or appreciable slowing or weakening of the beat.

Further, this special action of atropin on the heart is so to speak complemented by the action of muscarin, the active principle of many poisonous mushrooms. If a small quantity of muscarin be introduced into the circulation, or applied directly to the heart, the beats become slow and feeble, and if the dose be adequate the heart is brought to a complete standstill. The effect is in some respects like that of powerful stimulation of the vagus. Now if, in a frog, the heart be brought to a standstill by a dose of muscarin, the application of an adequate quantity of atropin will bring back the beats to quite their normal strength and rhythm. The one drug is so far as the heart is concerned (and indeed in many other respects) the antidote of the other.? These and other results have been taken to indicate that there exists in the heart a special inhibitory mechanism, and that it is through this special mechanism that the inhibitory fibres of the vagus produce inhibition, while atropin produces the effect just mentioned by paralys- 
ing, by rendering incapable of activity, and muscarin its effect by ewiting, stimulating into activity, this same inhibitory mechanism. It has further been suggested that some of the ganglia in the heart furnish the mechanism in question. And it has been supposed that there is a corresponding auginenting mechanism. But objections may be urged against this view, and it is safer to leave as an open question the exact manner in which inhibition and augmentation are brought about.

One point is perhaps worthy of mention. We lave seen that inhibition may be followed by a phase of increased activity, and that on the whole the heart is strengthened rather than weakened by the process, while on the other hand augmentation is followed by depression and the process is distinctly an exhansting one. Hence whatever be the exact mechanism of inhibition and of augmentation, whatever be the particular elements of the cardiac structures which are concerned in the one or the other, augmentation means increased expenditure, inhibition means a lessened expenditure, of energy on the part of the inuscular tissue of the heart. Whatever the manner in which the respective fibres act, the effect of the activity of the augmentor fibres is to hurry on the downward, catabolic changes of the cardiac tissue, while that of the inhibitory fibres is an opposite one, and we may probably say that the latter assists the constructive, anabolic, changes.

\section{Other Influences regulating or modifying the Beat of the Heart.}

$\$ 140$. Important as is the regulation of the heart by the nervous system, it must be borne in mind that other influences are or may be at work. The beat of the heart may for instance he modified by influences bearing directly on the nutrition of the heart. The tissues of the heart, like all other tissues, need an adequate supply of blood of a proper quality; if the blood vary in quality or quantity the beat of the heart is correspondingly affected. The excised frog's heart, as we have seen, continues to beat for some considerable time, though apparently empty of blood. After a while however the beats diminish and eventually disappear; and their disappearance is greatly hastened by washing out the heart with normal saline solution, which when allowed to flow through the cavities of the heart readily permentes the tissues on account of the peculiar construction of the ventricular walls. If such a 'washed out' quiescent heart he fed by means of a perfusion cannula, in the manner described (\$ 133), with diluted blood (of the rabbit, sheep, \&c.), it may he restored to functional activity. A similar but less complete restoration may be witnessed if serum be used instead of blood; and a heart fed regularly with fresh supplies of blood or even of serum may be kept beating for a very great length of time. 
Now, serum is as we have seen a very complex fluid containing several proteids, many 'extractives' and various inorganic salts. As regards proteids experiments have shewn that peptone and albumose so far from being beneficial are directly poisonous to the heart, that paraglobulin is without effect, but that serum-albumin will maintain the beats for a long time and will restore the beats of a 'washed-out' heart. We might infer from this that serumalbumin is directly concerned in the nutrition of the cardiac tissue ; but we are met with the striking fact that a frog's heart may be maintained in vigorous pulsation for many hours, and that a 'washed-out' frog's heart may be restored to vigorous pulsation by being fed with normal saline fluid to which a calcium salt with a trace of a potassium salt has been added. ${ }^{1}$ On the other hand, serum from which the calcium salts have been removed by precipitation with sodium oxalate is powerless to maintain or to restore cardiac pulsations. Obriously in the changes, whatever they may be, through which such fluids as serum, milk and the like (for nilk and other fluids have been found efficient in this respect) maintain the beat of the heart, calcium salts play an important part; and it is tempting to connect this with the relation of calcium salts to the clotting of blood $(\$ 20)$. We are not however justified in inferring because serum is ineffective in the absence of calcinm salts, that the serum albumin is useless; and, indeed the beneficial effects of the calcic saline fluid are not so complete as those of serum or of blood; moreover the possible influences of the various extractives, such as sugar for instance, present in the serum have to be considered. We may in addition call to mind, what we said in treating of the skeletal muscles ( $\$ 81$ ), that fatigue or exhaustion may have a double nature, the using up of contractile material on the one hand and on the other hand the accumulation of waste products; and the nutritive or restorative influence over the heart of any material may bear on the one or the other of these. Thus the beneficial effect of alkalies is probably in part due to their antagonizing the acids which as we have seen are being constantly produced during muscular contraction.

In the various experiments which have been made in thus feeding hearts with nutritive and otler fluids two facts worthy of notice have been brought to light.

One is that various substances have an effect on the muscular walls, apart from the direct modification of the contractions. The muscular fibres of the heart over and above their rhythmic contractions are capable of varying in length, so that at one time they are longer, and the chambers when pressure is applied to them internally are dilated beyond the normal, while at another time they are shorter, and the chambers, with the same internal

1 By Ringer's Heart-Fhid, for instance, which is made by saturating in the cold normal saline solıtion ( 65 p. c. sodium chloride) with calcium phosphate, and adding to 100 c.c. of the mixture, 2 c.c. of a 1 p. c. solution of potassium chloride. 
pressure, are contracted beyond the normal. In other words, the heart possesses what we shall speak of in reference to arteries as tonicity or tonic contraction, and the amount of this tonic contraction, and in eonsequence the capacity of the chambers, varies accurding to circumstances. The presence of some substances appears to increase, of others to diminish this tonicity and thus to diminish or increase the capacity of the chambers during diastole. This of course would have an effect, other things being equal, on the output from the heart and so on its work; and indeed there is some evidence that the augmentor and inhibitory impulses may also affect this tonicity, but observers are not ayreed as to the manner in which and extent to which they may thus act.

Another fact worthy of notice is when the heart is thus artificially fed with serum, or other fluids or even with blood, the beats, whether spontaneous or provoked by stimulation, are apt to become intermittent and to arrange themselves into groups. This intermittence is possibly due to the fluid employed being unable to carry on nutrition in a completely normal manner, and to the consequent production of abnormal chemical substances; and it is probable that cardiac intermittences seen during life are in certain cases thus brought abont by some direct interference with the nutrition of the cardiac tissue and not through extrinsic nervous impulses descending to the heart from the central nervous system. Various chemical substances in the blood, arising within the body or introduced as drugs, may thus affect the heart's beat by acting on its muscular fibres, or its nervous elements, or both, and that probably in varions ways, modifying in different directions the rhythm, or the individual contractions, or both.

Concerning the effect on the heart of blood which has not been adequately changed in the lungs we shall speak when we come to treat of respiration.

The physical or mechanical circumstances of the heart also affect its beat; of these perhaps the most important is the amount of the distention of its cavities. The contractions of cardiac muscle, like those of ordinary musele (see $\$ 6$ ), are increased up to a certain limit by the resistance which they have to overcome; a full ventricle will, other things being equal, contract more vigorously than one less full; though, as in ordinary muscle, the limit at which resistance is beneficial may be passed, and an overfull ventricle will fail to beat at all. Hence an increase in the quantity of blood in the rentricle will augment the work done in two ways; the quantity thrown out will, unless antagonistic influences intervene, he greater, and the increased quantity will be ejected with greater force. Further, since the distention of the ventricle at the commencement of the systole at all events is dependent on the auricular systole, the work of the ventricle (and so of the heart as a whole) is in a measure governed by the anricle. 
An interesting combination of direct mechanical effects and indirect nervous effects is seen in the relation of the heart's beat to blood pressure. When the blood pressure is high, not only is the resistance to the ventricular systole increased, but, other things being equal, more blood flows (in the mammalian heart) through the coronary arteries. Both these events would increase the activity of the heart, and we might expect that the increase would be manifest in the rate of the rhythm as well as in the force of the individual beats. As a matter of fact, however, we do not find this. On the contrary, the relation of heart beat to pressure may be put almost in the form of a law, that "the rate of the beat is in inverse ratio to the arterial pressure;" a rise of pressure being accompanied by a climinution, and fall of pressure by an increase of the rate of the rhythm. This however only holds good if the vagus nerves be intact. If these be previously divided, then in whatever way the blood pressure be raised - whether by injecting blood or clamping the aorta, or increasing the peripheral resistance, through an action of the vaso-motor nerves which we shall have to describe directly - or in whatever way it be lowered, no such clear and decided inverse relation between blood pressure and pulse-rate is observed. It is inferred therefore that increased blood pressure causes a slowing of the beat, when the vagus nerves are intact, because the cardio-inhibitory centre in the medulla is stimulated by the high pressure, either directly by the pressure obtaining in the blood vessels of the medulla, or in some indirect manner, and the heart in consequence more or less inhibited. 


\section{SEC. 6. CHANGES IN THE CALIBRE OF THE MINUTE ARTERIES. VASO-HOTOR ACTIONS.}

$\S 141$. All arteries contain plain muscular fibres, for the most part circularly disposed, and most abundant in, or sometimes almost entirely confined to, the middle coat. Further as the arteries become smaller, the muscular element as a rule becomes more and more prominent as compared with the other elements, until, in the minute arteries, the middle coat consists almost entirely of a series of plain muscular fibres wrapped round the internal coat. Nerve fibres, of whose nature and course we shall presently speak, are distributed largely to the arteries, and appear to end chiefly in fine plexuses round the muscular fibres, but their exact terminations have not as yet been clearly made out. By mechanical, electrical, or other stimulation, this muscular coat may, in the living artery, be made to contract. During this contraction, which has the slow character belonging to the contractions of all plain muscle, the calibre of the vessel is diminished. The veins also as we have seen possess muscular elements, but these vary in amount and distribution very much more in the veins than in the arteries. Most veins however are contractile, and may vary in calibre according to the condition of their muscular elements. Veins are also supplied with nerves. It will be of advantage however to consider separately the little we know concerning the changes in the veins and to confine ourselves at present to the changes in the arteries.

If any individual small artery in the web of a frog's foot be watched under the microscope, it will be found to vary considerably in calibre from time to time, being sometimes narrowed and sometimes dilated; and these changes may take place withont any obvious changes either in the heart beat or in the general circulation; they are clearly changes of the artery itself. During the narrowing, which is obviously due to a contraction of the muscular coat of the artery, the capillaries fed by the artery and the veins into which these lead become less filled with blood, and 
therefore paler. During the widening, which corresponds to the relaxation of the muscular coat, the same parts are fuller of blood, and redder. It is obvious that, the pressure at the entrance into any given artery remaining the same, more blood will enter the artery when relaxation takes place, and consequently the resistance offered by the artery is diminished, and less when contraction occurs, and the resistance is consequently increased; the blood flows in the direction of least resistance.

The extent and intensity of the narrowing or widening, of the constriction or dilation which may thus be observed in the frog's web, vary very largely. Variations of slight extent, either more or less regular and rhythmic or irregular, occur even when the animal is apparently subjected to no disturbing causes, and may be spoken of as spontaneous; larger changes may follow events occurring in various parts of the body; while as the result of experimental interference the arteries may become either constricted, in some cases almost to obliteration, or dilated until they acquire double or more than double their normal diameter. This constriction or dilation may be brought about not only by treatment applied directly to the web, but also by changes affecting the nerves of the leg or other parts of the body. Thus section of the nerves of the leg is generally followed by a widening which may be slight or which may be very marked, and which is sometimes preceded by a passing constriction; while stimulation of the peripheral stump of a divided nerve by an interrupted current of moderate intensity gives rise to constriction, often so great as almost to obliterate some of the minute arteries.

Obviously, then, the contractile muscular elements of the minute arteries of the web of the frog's foot are capable by contraction or relaxation of causing decrease or increase of the calibre of the arteries; and this condition of constriction or dilation may be brought about through the agency of nerves. Indeed, not only in the frog, but also, and still more so, in warm blooded animals, have we evidence that in the case of a very large number of, if not all, the arteries of the body, the condition of the muscular coat, and so the calibre of the artery, is governed by means of nerves; these nerves have received the general name of vaso-inotor nerves.

$\$ 142$. If the ear of a rabbit, preferably a light coloured one, be held up before the light, a fairly conspicuous artery will be seen running up the middle line of the ear, accompanied by its broader and more obvious veins. If this artery be carefully watched it will be found, in most instances, to be undergoing rhythmic changes of calibre, constriction alternating with dilation. At one moment the artery appears as a delicate, hardly visible pale streak, the whole ear being at the same time pallid. After a while the artery slowly widens out, becomes broad and red, the whole ear blushing, and many small vessels previously invisible coming into view. Again the artery narrows and the blush fades away; and this may be 
repeated at somewhat irregular intervals of a minute, more or less. The extent and regularity of the rhythm are usually markedly increased if the rabbit be held up by the ears for a short time previous to the observation. Similar rhythmic variations in the calibre of the arteries have been observed in several regions of the body, ex. $y r$. in the vessels of the mesentery and elsewhere; probably they are widely spread.

Sometines no such variations are seen, the artery remains constant in a condition internediate between the nore extreme widening and extrene narrowing just described. In fact, we may speak of an artery as being at any given time in one of three phases. It may be very constricted, in which case its nuscular fibres are very much contracted; or it may be very dilated, in which case its muscular fibres are relaxed; or it may be noderately constricted, the muscular fibres being contracted to a certain extent, and remaining in such a condition that they may on the one hand pass into stronger contraction, leading to marked constriction, or, on the other hand, into distinct relaxation, leading to dilation. We have reason to think, as we sliall see, that many arteries of the body are kept habitually, or at least for long periods together, in this intermediate condition, which is frequently spoken of as tonic contraction or tonus, or arteriai tone.

$\$ 143$. If, now, in a vigorous rabbit, in which the heart is beating with adequate strength, and the whole circulation is in a satisfactory condition, the cervical sympathetic nerve be divided on one side of the neck, remarkable changes may be olserved in the blood vessels of the ear of the same side. The arteries and veins widen, they, together with the small veins and the capillaries, become full of blood, many vessels previously invisible come into view, the whole ear blushes, and if the rhythmic changes described above were previously going on, these now cease; in consequence of the extra supply of warm blood the whole ear becomes distinctly warmer. Now, these changes take place, or may take place, without any alteration in the heart beat or in the general circulation. Obviously the arteries of the ear have, in consequence of the section of the nerve, lost the tonic contraction which previously existed; their muscular coats previously somewhat contracted have become quite relaxed, and whatever rhy thmic contractions were previously going on have ceased. The nore marked the previous tonic contraction, and the more vigorous the heart beats, so that there is an adequate supply of blood to fill the widened channels, the more striking the result. Sometimes, as when the heart is feeble, or the pre-existing tonic contraction is slight, the section of the nerve produces no very obvious change.

If now the upper segment of the divided cervical sympathetic nerve, that is the portion of the nerve passing upwards to the head and ear, be laid upon the electrodes of an induction machine, and a gentle interrupted current be sent through the nerve, fresh changes 
take place in the blood vessels of the ear. A short time after the application of the current, for in this effect there is a latent period of very appreciable duration, the ear grows paler and cooler, many small ressels, previously conspicuous, become again invisible, the main artery shrinks to the thinnest thread, and the main veins become correspondingly small. When the current is shut off from the nerve, these effects still last some time, but eventually pass off; the ear reddens, blushes once more, and, indeed, may become even redder and hotter, with the vessels more filled with blood than before. Obviously the current has generated in the cervical, sympathetic, nerve impulses which, passing upward to the ear and finding their way to the muscular coats of the arteries of the ear, have thrown the muscles of those coats into forcible contractions, and have thus brought about a forcible narrowing of the calibre of the arteries, a forcible constriction. Through the narrowed constricted arteries less blood finds its way, and hence the paleness and coldness of the ear. If the impulses thus generated be very strong, the constriction of the arteries may be so great that the smallest quantity only of blood can make its way through them, and the ear may become almost bloodless. If the impulses be weak the constriction induced may be slight only; and, indeed, by careful manipulation the nerve may be induced to send up to the ear impulses only just sufficiently strong to restore the moderate tonic constriction which existed before the nerve was divided.

We infer from these experiments that among the various nerve fibres making up the cervical sympathetic, there are certain fibres which, passing upwards to the head, become connected with the arteries of the ear, and that these fibres are of such a kind that impulses, generated in them and passing upwards to the ear, lead to marked contraction of the muscular fibres of the arteries, and thus produce constriction. These fibres are vaso-motor fibres for the blood vessels of the ear. From the loss of tone, so frequently following section of the cervical sympathetic, we may further infer, that, normally during life, impulses of a gentle kind are continually passing along these fibres, upwards through the cervical sympathetic, which impulses, reaching the arteries of the ear, maintain the normal tone of those arteries. But, as we said, the existence of this tone is not constant, and the effects of these tonic impulses are not so conspicuous as those of the artificial constrietor impulses generated by stimulation of the nerve.

$\$ 144$. The above results are obtained whatever be the region of the cervical sympathetic which we divide or stimulate between the upper and the lower cervical ganglion. We nay therefore describe these vaso-motor impulses as passing upwards from the lower cervical ganglion along the cervical sympathetic, to the upper cervical ganglion, from which they issue by branches which ultimately find their way to the ear. But these impulses do not start from the lower cervical ganglion; on the contrary, by repeating the experi- 
ments of division and stimulation in a series of animals, we may trace the path of these impulses from the lower cervical ganglion,

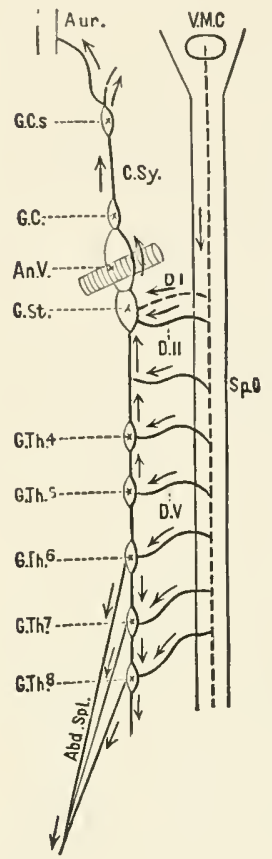
Fig. 72, through the annulus of Vieussens to the ganglion stellatum and upper part of the thoracic sympathetic chain, and thence along the rami communicantes of some or other of the upper thoracic spinal nerves to the anterior roots of those nerves, and so to the spinal cord. In the cat and the $\operatorname{dog}$, and probably in other higher mammals, the chief path of the impulses lies in the second and third thoracic nerves, though some pass by the fourth, and a variable small number by the fifth and the first; in the rabbit the path is more widespread, and reaches lower down, for while the impulses pass chiefly by the fourth and fifth thoracic nerves, some pass by the second and third, and others by the sixth, seventh, and even eighth nerves. The exact path also differs in different individuals of the same species. It will be observed that from the spinal cord up to the annulus of Vieussens, and the lower cervical ganglion, these vaso-motor impulses for the ear, and the augmentor impulses for the heart, (cf. Fig. 71) follow much the same path; but there they part company. We

Fig. 72. Diagram Illustrating the Paths of Vaso-cosstrictor Fibres along the Cervical Sympatietic and (palt of) the Abdominal Splaxchinc.

Aur. artery of ear. G.C.S. superior cervical ganglion. Abd. Spl. upper roots of and part of abdominal splanchnic nerve. V..II.C. vaso-motor centre in spinal bulb. The other references are the same as in Fig. $71, \$ 138$. 'The paths of the constrictor fibres are slewn by the arrows. The dotted line along the middle of the spinal cord, $S p . C$. , is to indicate the passage of constrictor impulses down the cord from the vaso-motor centre in the spinal bulb.

can thus trace these vaso-motor impulses backwards along the cervical sympathetic to the anterior roots of certain thoracic nerves, and through these to the thoracic region of the spinal cord, where we will for the present leave them. We may, accordingly, speak of vaso-motor fibres for the ear as passing from the thoracic spinal cord to the ear along the track just marked ont; stimulation of these fibres at their origin from the spinal cord, or at any part of their course (along the anterior roots of the second, third or other upper thoracic nerves, visceral branches [rami communicantes] of those nerves, ganglion stellatum or upper part of thoracic sympathetic chain, annulus of Vieussens, \&c. \&c.), leads to constriction in the blood vessels of the ear of that side; and section of these fibres at any part of the same conrse tends to abolish any previously 
existing tonic constriction of the blood vessels of the ear, though the effect of section is not so constant or striking as that of stimulation.

$\$ 145$. We must now turn to another case. In dealing with digestion we shall have to study the submaxillary salivary gland. We may for the present simply say that this is a glandular mass well supplied with blood vessels, and possessing a double nervous supply. On the one hand it receives fibres from the cervical sympathetic, Fig. $73 v$. sym. (in the dog, in which the effects which we are about to describe are best seen, the vagus and cervical

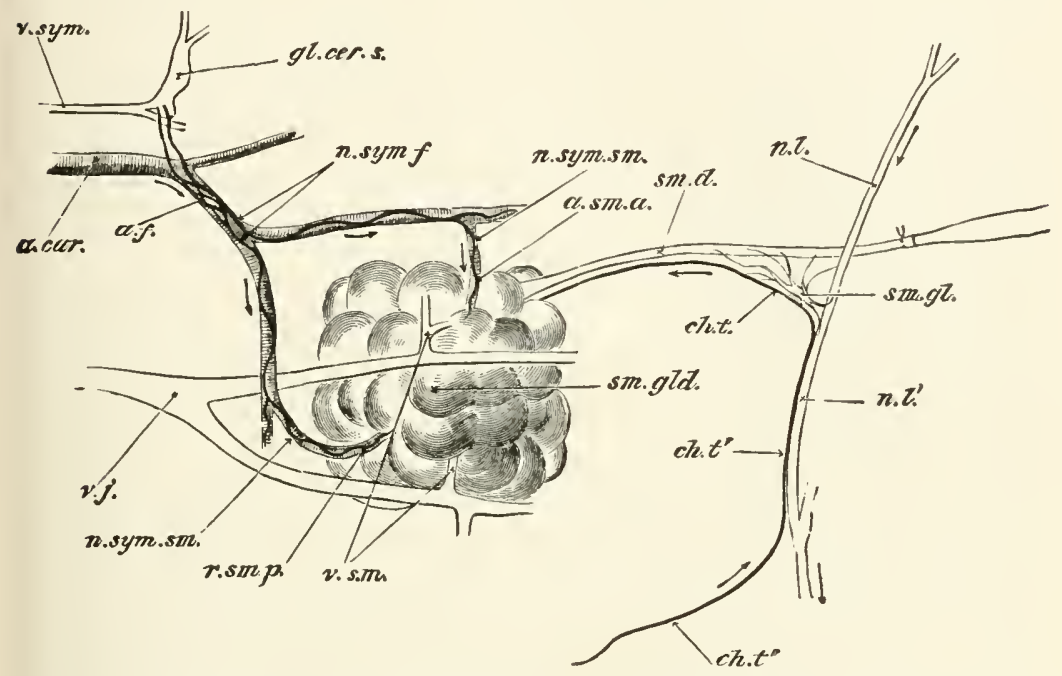

Fig. 73. Diagrammatic Representation of the Submaxillary Gland of the log with its Nerve axd Blood Vesseis.

(The dissection has been made on an animal lying on its back, but since all the parts shewn in the figure cannot be seen from any one point of view, the figure does not give the exact anatomical relations of the several structures.)

$s m$. gld. The submaxillary gland, into the duct $(s m . d$.) of which a cannula has been tied 'The sublingual gland and dnet are not shewn. n.l., n.l'. The lingual branch of the fifth nerve, the part $n . l$. is going to the tongne. ch.t., ch. $t^{\prime}$., ch. $t^{\prime \prime}$. 'The chorda tympani The part $c h$. $t^{\prime \prime}$. is proceeding from the facial nerve; at $c h . t^{\prime}$. it becomes conjoined with the lingual $n t^{\prime}$ and afterwards diverging passes as $c h . t$. to the gland along the duct; the continuation of the nerve in company with the lingual n.l. is not shewn sm. gl. The submaxillary ganglion with its several ro ts. $a$. car. The carotid artery, two small branches of which, $a, s m$, $a$. and $r . s m . p$, pass to the anterior and posterior parts of the glancl. $r . s . m$. The anterior and posterior veins from the gland, falling into $v ; j$. the jugular vein. $v, s y m$. 'The conjoined ragns and sympathetic trunks. g.cer.s. The upper cervical ganglion, two branches of which forming a plexiss $(a, f)$ over the facial artery, are distributed (n.sym.sm.) along the two glandnlar arteries to the anterior and posterior portions of the gland.

The arrows indicate the direction taken by the nervons impulses during reflex stimnlation of the gland. They ascend to the brain by the lingual and descend by the chorda tympani. 
sympathetic are enclosed in a common sheath so as to form what apjears to be a single trunk), which reach the gland in company with the arteries supplying the gland (n. sym. sm.). On the other hand it receives fibres from a small nerve called the chorda tympani (ch. t.), which, springing from the 7 th cranial (facial) nerve, crosses the tympanum of the ear (hence the name), and, joining the lingual branch of the 5 th nerve, runs for some distance in company with that nerve, and then ends partly in the tongue, and partly in a small nerve which, leaving the lingual nerve before reaching the tongue, runs along the duct of the submaxillary gland, and is lost in the substance of tle gland; a small branch is also given off to the sublingual gland.

Now, when the chorda tympani is simply divided, no very remarkable changes take place in the blood vessels of the gland, but if the peripheral segment of the divided nerve, that still in connection with the gland, be stimulated, very marked results follow. 'The small arteries of the gland becomie very much dilated, and the whole gland becomes thushed. (As we shall see later on the gland at the same time secretes saliva copiously, but this does not concern us just now.) Changes in the calibre of the blood vessels are, of course, not so readily seen in a compact gland as in a thin extended ear; but if a fine tube be placed in one of the sinall veins by which the blood returns from the gland, the effects on the blood flow of stimulating the chorda tympani become very obvious. Before stimulation the blood trickles out in a thin, slow stream of a dark venous colour; during stimulation the blood rushes out in a rapid full stream, often with a distinct pulsation, and frequently of a colour which is still scarlet and arterial in spite of the blood having traversed the capillaries of the gland; the blood rushes so rapidly through the widened blood vessels that it has not time to undergo completely that change from arterial to venous which normally occurs while the blood is traversing the capillaries of the gland. This state of things may continue for some time after the stimulation has ceased, but before long the flow from the veins slackens, the issuing blood becomes darker and venous, and eventually the circulation becomes normal.

We shall have occasion later on to speak of the nervi erigentes, the stimulation of which gives rise to the erection of the penis. The erection of the penis is partly due to a widening of the arteries supplying the peculiar erectile tissue of that organ, whereby that tissue becones distended with blood, and the widening is brought about by impulses passing along the nerves in question. Obviously the chorda tympani and the nervi erigentes contain fibres wlich we may speak of as 'vaso-motor' since stimulation of them produces a change in, brings about a movement in the blood vessels; but the change produced is of a character the very opposite to that produced in the blood vessels of the ear by stimulation of the cervical sympathetic. There stimulation of the 
nerve caused contraction of the muscular fibres, constriction of the small arteries; here stiunlation of the nerve causes a widening of the arteries, which widening is undoubtedly due to relaxa. tion of the muscular fibres. Hence we must distinguish between two kinds of raso-motor fibres, fibres the stimulation of which produces constriction, vaso-constrictor fibres, and fibres the stimulation of which causes the arteries to dilate, naso-dilator fibres, the one kind being the antagonist of the other.

\$146. In the chorda tympani, the vaso-motor fibres are exclusively vaso-dilator fibres, and this is true both of the part of the nerve ending in the submaxillary and sublingual glands, and the rest of the ending of the nerve in the tongue. Stimulation of the chorda tympani (so far as the vaso-motor functions of the nerve are concerned, for it has, as we shall see, other functions), at any part of its course from its leaving the facial nerve to its endings in the gland or tongue, produces only vaso-dilator effects, never vaso-constrictor effects. 'The cervical sympathetic on the other hand is not exclusively vaso-constrictor. It contains as we have seen raso-constrictor fibres for the ear. It also contains vaso-constrictor fibres for other regions of the head and face. For instance the branches of the cervical sympathetic going to the submaxillary gland of which we just spoke (Fig. 73 n. sym. sm.), contain vaso-constrictor fibres for the vessels of the gland; stimulation of these filmes produces, on the vessels of the gland, au effect exactly the opposite of that produced by stimnlation of the chorda trmpani; to this point we shall have to return when we deal with the gland in comnection with digestion. And we might give other instances; in fact the dominant effect on the blood ressels of stimulating the cervical sympathetic is a vaso-constrictor effect. There are however certain cases in which the opposite effect, a vaso-dilator effect, in certain regions has been observed as the result of stimulating the cervical sympathetic. And we may now turn to other nerves in which such a double effect, now a vaso-constrictor, now a vaso-dilator effect, may be more readily obtained.

In the frog as we have seen, division of the nerves of the leg leads to a widening of the arteries of the web of the foot of the same side, and stimulation of the peripheral end of the nerve canses a constriction of the vessels, which, if the stimulation be strong, may be so great that the web appears for the time being to he devoid of blood. Also in a mammal division of the sciatic nerve canses a similar widening of the small arteries of the skin of the leg. Where the condition of the circulation can be readily examined, as for instance in the hairless balls of the toes, especially when these are not pigmented, the vessels are seen to be dilated and injected; and a thermometer placed between the toes shews a rise of temperature amounting, it may be, to several degrees. If moreover the peripheral end of the divided nerve he 
stimulated, the vessels of the skin become constricted, the skin grows pale, and the temperature of the foot falls. And very similar results are obtained in the forelimb by division and subsequent stimulation of the nerves of the brachal plexus.

The quantity of blood present in the blood ressels of a part of the body or of an organ of the mammal may sometimes be observed directly by means of the plethysmograph, of which we have already spoken (\$104), but has frequently to be determined indirectly. The temperature of a passive structure subject to cooling influences, such as the skin, is largely dependent on the supply of blood: the more abundant the supply, the warmer the part. Hence in these parts variations in the quantity of blood may be inferred from variations of temperature; but in dealing with nore active structures such as muscles there are obviously sources of error in the possibility of the treatment adopted, such as the stimulation of a nerve, giving rise to an increase of temperature due to increased metabolism, independent of variations in blood supply.

So far the results are quite like those obtained by division and stimulation of the cervical sympathetic, and we might infer that the sciatic nerve and brachial plexus contain vaso-constrictor fibres only for the vessels of the skin of the hind limb and fore limb, vaso-dilator fibres being absent. But sometimes a different result is obtained; on stimulating the divided sciatic nerve the vessels of the foot are not constricted but dilated, perhaps widely dilated. And this vaso-dilator action is almost sure to be manifested when the nerve is livided, and the peripheral stump stimulated some time, two to four days, after division, by which time commencing degeneration has legun to modity the irritability of the nerve. For example, if the sciatic be divided, and some days afterwards, by which time the thushing and increased temperature of the foot, following upon the section, has wholly or largely passed away, the peripheral stump be stimulated with an interrupted current a renewed flushing and rise of temperature is the result. We are led to conclude that the sciatic nerve (and the same holds good for the brachial plexus) contains both vaso-constrictor and vaso-dilator fibres, and to interpret the varying result as due to variations in the relative irritability of the two sets of fibres. The constrictor fibres appear to predominate in these nerves, and hence constriction is the more common result of stimulation; the constrictor fibres also appear to be more readily affected by a tetanizing current than do the dilator fibres. When the nerve after division commences to degenerate the constrictor fibres lose their irritability earlier than the dilator fibres, so that at a certain stage a stimulus, such as the interrupted current, while it fails to affect the constrictor fibres, readily throws into action the dilator filres. The latter, indead, appear to retain their irritability after section of the nerve for a much longer time than 
do ordinary motor nerves ( $\$ 78)$. The result is perhaps even still more striking if a mechanical stimulus, such as that of "crimping" the nerve by repeated snips with the scissors, be employed. Exposure to a low temperature again seems to depress the constrictors more than the dilators; hence when the leg is placed in ice-cold water stimulation of the sciatic, even when the nerve las been but recently divided, throws the dilator only into action and produces flushing of the skin with blood. Slow rhythmical stimulation moreover of even a freshly divided nerve may produce dilation. And there are other facts which support the same view that the sciatic nerve (and brachial plexus) contains both vasoconstrictor and vaso-dilator fibres which are differently affected by different circumstances.

In the splanchnic nerves which supply fibres to the blood vessels of so large a part of the abdominal viscera, there is abundant evidence of the presence of vaso-constrictor fibres. Division of this nerve leads to a widening of the blood vessels of the abdominal viscera, stimulation of the nerve to a constriction; and as we shall see, since the amount of blood vessels thus governed by this nerve is very large indeed, interference either in the one direction or the other with its vaso-motor functions produces very marked results, not only on the circulation in the abdomen but on the whole vascular system. There is some evidence that the splanchnic nerves also contain vaso-dilator fibres, but this evidence is of a more or less indirect character, and in any case, the number of such fibres must be small.

So far as we know, the vaso-motor fibres contained in the sciatic and the like spinal nerves are distributed chiefly at least to the blood vessels of the skin. Though so large a part of the fibres of these nerves end in the muscles, the evidence of vasomotor fibres passing to the blood vessels of the muscles is by no means clear and undisputed. The blood ressels of a muscle mdoubtedly may change in calibre. For instance, when a muscle contracts there is always an increased flow of blood through the muscle; this may be in part a mere mechanical result of the change of form, the shortening and thickening of the fibres opening out the minute blood vessels, but is also, if not chiefly, due to the widening of the arteries by relaxation of their muscular walls. Such a widening may be seen when a thin muscle of a frog is made, in the living body, to contract under the microscope. But this widening has not been proved beyond dispute to be due to the action of vaso-dilator fibres. Indeed it has been argued that when a muscle contracts, some of the chemical products of the metabolism of the muscle may, by direct, local action on the minute blood ressels, lead to a widening of those blood vessels. And in some other organs, the brain and the kidney for instance, w: find functional activity accompanied by a widening of the blond ressels under circumstances which seem to preclude the 
possibility of the widening being due to vaso-dilator impulses reaching the organ from without; in such instances it is suggested that the widening is due to a local effect of the products of the activity of the organ. To this point we shall return. With regard to vaso-constrictor fibres also the evidence that they are supplied to muscles is, in like manner, not beyond dispute. Section or stimulation of the nerves induces it is true changes in the temperature of the muscles as it does in that of the skin. But, as we urged just now, to argue from this that changes in the blood supply have taken place is not wholly safe; moreover the changs in temperature observed are shight. Again, the fact that whe: 1 the nerve of a muscle is divided the blood ressels of the muscle widen, somewhat like the blood vessels of the ear after division of the cervical sympathetic, has been brought forward as indicating the presence of vaso-constrictor tibres carrying the kind of influence which we called tonic, leading to an habitual moderate constriction. Neither arguments can be regarded as absolutely conclusive. The knowledge we possess at present leaves us in fact in doubt whether the blood-How through the muscles, though these form so large a part of the body, is really governed by the central nervous system.

The two parts of the body undoubtedly and pre-eminently supplied by vaso-eonstrictor tibres proceeding from and governed by the central nervous system are on the one hand the skin and on the other hand the abdominal viscera. As we shall see, the variation; in the blood supply to the skin are more strikingly of use to the body at large, in regulating the temperature of the body for instance, than they are to the skin itself. The variations in the blood supply to the abdominal viscera also serve important general purposes; they play their part in the regulation of the temperature of the body, and through them the viscera serve as a reservoir to which blood mav without harm be shunted when occasion demands. It would appear as if the vaso-constrictor mechanism were chiefly used for the general purposes of the economy.

Aceepting the view that the presence of vaso-dilator fibres in the nerves going to muscles is not definitely proved and disregarding the scanty and more or less obscure vaso-dilators of the sciatic and other spinal nerves, we find that in special cases only, in cases where it would seem that special means are needeil to secure an ample flow of blood through a particular part, unmistakably vaso-dilator fibres are present.

\section{The Course of Vaso-motor Fibres.}

$\$$ 147. Both the raso-constrictor and the vaso-dilator fibres have their origin in the central nervous system, the spinal cord 
or the brain, but it will be desirable to speak of the course of the two sets separately.

Vuso-constrictor Fibres. In the manmal, so far as we know at present, all the vaso-constrictor fibres for the whole body take their origin in the middle region of the spinal cord, or rather, leave the spinal cord by the nerves belonging to this middle region. Thus in the dog the vaso-constrictor fibres, not only for the trunk but for the limbs, head, face and tail, leave the spinal cord by the anterior roots of the spinal nerves reaching from about the second thoracic to the fourth lumbar nerve, both inclusive, though some few may pass by the first thoracic and by the fifth lumbar.

Those for the head and neck leave the spinal cord as we have seen, $\$ 144$, chiefly by the second and third thoracic nerves, though some leave by the fourth and a variable small number by the fifth and by the first; those for the fore limbs leave by a number of thoracic nerves reaching from the fourth to the ninth or even the tenth, those by the seventh being the most numerous. Those for the hind limbs leave by the nerves reaching from the eleventh thoracic to the third lumbar, some passing by the tenth thoracic and the fourth lumbar. Those for the tail leave by the first, second and third lumbar. And those for the trunk leave by the successive spinal nerves supplying the trunk. This arrangement may be taken as indicating generally how these fibres leave the spinal cord, bearing in mind that the fourth lumbar nerve of the dog corresponds to about the second lumbar of man, and that the details differ in different kinds of animals and indeed in different individuals.

Rumning in the case of each nerve root to the mixed nerve trunk these vaso-constrictor fibres pass along the visceral branch, white ramus communicans, to the thoracic and abdominal sympathetic ganghia (Fig. 72). From thence they reach their destination in various ways. Thus, those going to the head and neck pass upward throngh the annulus of Vieussens to the lower cervical ganglion and thence, as we have seen, up the cervical sympathetic; many of the fibres for the neck however pass directly from the stellate ganglion. Those for the abdominal viscera pass off in a similar way by the splanchnic nerves, Fig. 72, abd. spl. and by smaller nerves joining the inferior mesenteric ganglion. Those destined for the arm, making their way backwards by grey rami communicantes (Fig. $23 r v$.), join the nerves of the brachial plexus; while those for the hind leg pass in a similar way through some portion of the abdominal sympathetic before they join the nerves of the sciatic plexus. These as we have seen are distributed chiefly to the skin, and the constrictor fibres of the skin of the trunk probably reach the spinal nerves in which they ultimately run in a similar manner. All the vaso-constrictor fibres, whatever their destination, leave the spinal cord by the 
anterior roots of spinal nerves, and then passing through the appropriate visceral branches, join the thoracic or abdominal sympathetic ganglia. In their course the fibres undergo a remarkable change.

Along the anterior root and along the visceral branch they are medullated fibres, but before they reach the blood vessels for which they are destined they become non-medullated fibres; they appear to lose their medulla in sone or other of the ganglia.

We are in many cases able to determine experimentally by the following method, the ganglion or ganglia in which particular fibres end, that is to say in which they become connected with nerve cells. It is found that the drug nicotin abolishes or suspends the action of vaso-motor fibres and of other fibres running in the sympathetic system. Thus in a rabbit, after a certain lose of nicotin has been given, stimulation of the cervical sympathetic nerve in the neck no longer causes constriction of the vessels of the ear. But it is found in such cases that though stimulation of the trunk of the nerve in the neck is without effect, stimulation of the appropriate nerve branches passing off from the superior cervical ganglion on their way to the ear, does produce constriction of the vessels of the ear. Obviously the nicotin does not affect the peripheral fibres and endings of the nerve, but some part of the nerve more central than the branches proceeding from the superior cervical ganglion. Further, if the ganglion itself be cautiously painted with a weak (1 p.c.) solution of nicotin, care being taken to avoid excess, stimulation of the nerve in the neck has no effect on the vessels of the ear, whereas if the nicotin be applied to a corresponding extent to the trunk of the nerve in the neck, none being allowed to have access to the ganglion, stimulation of the trunk in the neck, even if applied to the very spot on which the nicotin has been placed, produces the usual constriction of the vessels of the ear. Obviously the nicotin produces its paralysing effects by acting on the nerve cells, or on the fibres just as they are becoming connected with nerve cells. If the solution of nicotin be applied not to the upper, but to the middle or to the lower cervical ganglion, stimulation of the nerve between the ganglion and the spinal cord prodnces the usual constrictor effects. This shews that the constrictor fibres pass through the lower and the midlle ganglion as fibres, not connected with cells, otherwise they wonld be here affected by nicotin; they are affected by nicotin in the upper ganglion, and we therefore infer that they end in, that is, are connected with cells in that ganglion. In the same way it may be found that the vaso-constrictor fibres of the abdominal splanchnic are connected with cells in the solar plexns. Indeed by this method we may determine in what ganglia the vaso-constrictor and other fibres of the sympathetic system end; and a remarkable distribution, determined by morphological causes among others, has in this way been made out, some fibres 
very speedily becoming connected witl nerve cells, others running a very long course before they so end.

148. Vaso-dilator Fibres. Some of these appear to run much the same cource as the vasu-constrictors. Such are the vaso-dilator fibres ruming in spinal nerves like the sciatic and brachial, those which seem to be present in the splanchnic, and certain fibres of the cervical sympathetic which in some animals at least act as vaso-dilators towards certain parts of the nouth and face. With regard to these, the evidence of whose existence, as we liave seen, is at least in most cases, difficult, special or indirect, we have at present no proof that their general course is essentially different from that of the constrictors.

The more distinct and notable vaso-dilators however do run a different course. These are found in the nerves coming from the cranial and sacral regions of the central nervous system whence, as we have seen, no vaso-constrictor fibres are known to issue. Thus the vaso-dilator fibres for the sub-maxillary gland rumning in the chorda tympani may be traced as we have seen back to the facial or seventh nerve; and the continuation of the chorda tympani along the lingual nerve to the tongue contains vaso-dilator tibres for that organ; when the limgual is stimulated, the blood vessels of the tongue dilate owing to the stimulation of the conjoined chorda tympani fibres. The ramus tympanicus of the glossopharyngeal nerve contains vaso-dilator fibres for the parotid gland, and it appears probable that the trigeminal nerve contains vaso-dilator fibres for the eye and nose and possibly for other parts. The vaso-dilator fibres which pass into the nervi erigentes, leave the sacral region of the cord by the anterior roots of the sacral nerves, the particular nerves differing in different animals; thus in the dog and cat they pass by the first, second and third, in the rabbit by the second, third and fourth, in man by the third, fourth and fifth sacral nerves.

In these instances the vaso-dilator fibres, as they leave the central nervous system, are, like the vaso-constrictor fibres, fine medullated fibres, but unlike the majority at least of the vasoconstrictors they retain their medulla for the greater part of their course and only lose it near their termination in the tissue whose blood ressels they supply.

\section{The Effeets of Vaso-motor Actions.}

$\S 149$. A very little consideration will shew that vaso-motor action is a most important factor in the circulation. In the first place the whole flow of blood in the body is adapted to and governed by what we may call the general tone of the arteries of the body at large. In a normal condition of the body, the muscular fibres of a very large number of the minute arteries 
of the body are in a state of tonic, i.e. of moderate, contraction, and it is the narrowing due to this contraction which forms a large item of that peripheral resistance which we have seen to be one of the great factors of blood pressure. The normal general blood pressure, and therefore the normal flow of blood, is in fact dependent on the 'general tone' of the minute arteries.

In the second place local vaso-motor changes in the condition of the minute arteries, changes, that is to say, of any particular vaseular area, have very decided effects on the circulation. These changes, though local themselves, may have effects which are both local and general, as the following considerations will shew.

Let us suppose that the artery $A$ is in a condition of normal tone, is midway between extreme constriction and dilation. The flow through $A$ is determined by the resistance in $A$ and in the vascular tract which it supplies, in relation to the mean arterial pressure, which again is lependent on the way in which the heart is beating and on the peripheral resistance of all the small arteries and capillaries, $A$ included. If, while the heart and the rest of the arteries remain unchanged, $A$ be constricted, the peripheral resistance in $A$ will increase, and this increase of resistance will lead to an increase of the general arterial pressure. Since, as we have seen, $\$ 101$, it is arterial pressure which is the immediate cause of the flow from the arteries to the veins, this increase of arterial pressure will tend to drive more blood from the arteries into the veins. The constriction of $A$ however, by increasing the resistance, opposes any increase of the flow through $A$ itself, in fact will make the How through $A$ less than before. The whole increase of discharge from the arterial into the venous system will take place through the arteries in which the resistance remains unchanged, that is, through channels other than $A$. Thus, as the result of the constriction of any artery there occur, (1) diminished How through the artery itself, (2) increased general arterial pressure, leading to (3) increased flow through the other arteries. If, on the other hand, $A$ be dilated, while the heart and other arteries remain unchanged, the peripheral resistance in $A$ is diminished. This leads to a lowering of the general arterial pressure, which in turn tends to drive less blood from the arteries into the veins. The dilation of $A$ however, by diminishing the resistance, permits, even with the lowered pressure, more blood to pass through $A$ itself than before. Hence the diminished flow tells all the more on the rest of the arteries in which the resistance remains unchanged. Thus, as the result of the dilation of any artery, there occur (1) increased flow of blood through the artery itself, (2) diminished general pressure, and (3) diminished flow through the other arteries. Where the artery thus constricted or dilated is small, the local effect, the diminution or increase of flow 
through itself, is much more marked than the general effects, the change in blood pressure and the flow through other arteries. When, however, the area the arteries of which are affected is large, the general effects are very striking. Thus if while a tracing of the blood pressure is being taken by means of a manometer connected with the carotid artery, the abdominal splanchnic nerves be divided, a conspicuous but steady fall of pressure is observed, very similar to but more marked than that which is shewn in Fig. 74. The section of the abdominal splanchnic nerves causes the arteries of the abdominal viscera to dilate, and these being very numerous, a large amount of peripheral resistance is taken away, and the blood pressure falls accordingly; a large increase of flow into the portal veins takes place, and the supply of blood to the face, arms, and legs is proportionally diminished. It will be observed that the dilation of the arteries is not instantaneous but somewhat gradual, as shewn by the pressure sinking not abruptly but with a gentle eurve.

The general effects on blood pressure by vaso-motor changes are so marked that the manometer may be used to detect vasomotor actions. Thus, if the stimulation of a particular nerve or any other operation leads to a marked rise of the mean blood pressure, unaccompanied by any notable changes in the heart beat, we may infer that constriction has taken place in the arteries of some considerable vascular area; and similarly, if the effect be a fall of blood pressure, we may infer that constriction has given way to dilation.

\section{Vaso-motor Functions of the Central Nerrous System.}

$\$ 150$. The central nervous system, to which we have traced the vaso-motor nerves, makes use of these nerves to regulate the flow of blood through the various organs and parts of the body; by the local effects thus prodnced it assists or otherwise influences the functional activity of this or that organ or tissue; by the general effects it secures the well being of the body. When the vaso-dilators are brought into play the chief effect is a local one; when a general effect has to be produced the vaso-constrictors are employed, though these of course also bring about local effects. And we may consider the two separately.

The vaso-dilator nerves, the use of which is more simple than that of the vaso-constrictors in so far as it appears not to be complicated by the presence of habitual tonic influences, occur as parts of distinct mechanisms used chiefly at least as reflex mechanisms, with centres placed in different regions of the central nervous system. Thus, when food is placed in the mouth afferent impulses, generated in the nerves of taste, give rise in the central nervous system to efferent impulses, which descend 
the chorda tympani and other nerves to the salivary gltunds and, by dilating the blood vessels, secure a copious flow of blood through the glands while, as we shall see later on, they excite the glands to secrete. The centre of this reflex action appears to lie in the spinal bulb and may be thrown into activity not only by impulses reaching it aloug the specific nerves of taste, but also by impulses passing along other channels; thus, emotions started in the brain by the sight of food or otherwise may give rise to impulses passing down along the central nervous system itself to the spinal bulb, or events in the stomach may send impulses up the vagus nerve, or stimulation of one kind or another may send impulses up almost any sentient nerve, and these various impulses reaching the spinal bulb nuay, by reflex action, throw into activity the vaso-dilator fibres of the chorda tympani and other analogous nerves, and bring about a flushing of the salivary glands, while at the same time they cause the glands to secrete.

The vaso-dilator fibres of the nervi erigentes may be thrown into activity in a similar reflex way, the centre, which is also easily thrown into activity by impulses descending down the spinal cord from the brain, being placed in the sacral and perhaps also in the upper lumbar or lower thoracic region of the spinal cord. That such a centre does exist is shewn by the fact that, when in a $d o g$ the spinal cord is completely dirided in the thoracic region, erection of the penis may readily be brought about by stimulation of appropriate sentient surfaces. And other instances might be quoted in which vaso-dilator fibres appaar as part of a reflex mechanism the centre of which is placed in the central nervous system not far from the origin of the nerves in which the vaso-dilator fibres run.

\$151. Turning now to the vaso-constrictor fibres we find that these form a more coherent system; and this is in accordance with the feature of the vaso-constrictor mechanisms, that they are largely employed to produce general effects Moreover their utility is increased, though at the same time their use becomes somewhat more complicated, by reason of the existence of tonic influences; since the same fibres may, on the one hand, by an increase in the impulses passing along them, be the means of constriction, and on the other hand, by the removal or diminution of the tonic influences passing along them, be the means of dilation. We have already traced all the vaso-constrictor fibres from the middle region of the spinal cord to the sympathetic system in the thorax and abdomen; from thence they pass (1) by the splanchnic, hypogastric, and other nerves to the viscera of the abdomen and pelvis, (concerning the vaso-motor nerves of the thoracic viscera we know at present very little), (2) by the cervical sympathetic to the skin of the head and neck, the salivary glands and month, the eyes and other parts, and possibly the brain including its 
membranes, though the presence of vaso-motor fibres in the brain itself is much disputed, (3) by the brachial and sciatic plexuses to the skin of the fore- and hind-limbs, and by various other nerves to the skin of the trunk. The chief parts of the body supplied by vaso-constrictor fibres appear to be the skin with its appendages, and the alimentary canal with its appendages, glandular and other; the great mass of skeletal muscles appears, as we have seen, to receive a relatively small supply of vaso-constrictor fibres.

If in an animal the spinal cord be divided in the lower thoracic region, the skin of the legs becomes flushed, their temperature frequently rises, and there is a certain amount of fall in the general blood pressure as measured, for instance, in the carotid; and this state of things may last for some considerable time. Obviously the section of the spinal cord has cut off the usual tonic influences descending to the lower limbs; in consequence the blood vessels have become dilated, in consequence the general peripheral resistance has become proportionately diminished, and in consequence the general blood pressure has fallen. The tonic vaso-constrictor impulses for the lower limbs, therefore, have their origin in the central nervous system higher up than the lower thoracic region of the spinal cord.

If the spinal cord be divided higher up, say above the roots of the fifth or sixth thoracic nerves, the cutaneous blood vessels of the lower limbs dilate, as in the former case, and on examination it will be found that the blood vessels of the abdomen are also largely dilated; at the same time the blood pressure undergoes a very marked fall, it may indeed be reduced to a very few millimeters of mercury. Obviously the tonic vaso-constrictor impulses passing to the abdomen and to the lower limbs take origin in the central nervous system higher up than the level of the fifth thoracic nerve.

If the section of the spinal cord be made above the level of the second thoracic nerve, in addition to the abovementioned results the ressels of the head and face also become dilated; but in consequence of the fall of general blood pressure just mentioned, these vessels never become so full of blood, the loss of tone is not so obvious in them as after simple division of the cervical sympathetic, since the latter operation produces little or no effect on the general blood pressure.

Obviously then the tonic vaso-constrictor impulses, which passing to the skin and viscera of the body maintain that tonic narrowing of so many small arteries by which the general peripheral resistance, and so the general blood pressure, is maintained, proceed from some part of the central nervous system higher up than the upper thoracic region of the spinal cord. And, since exactly the same results follow upon section of the spinal cord in the cervical region right up to the lower limit of the spinal 
bulb, we infer that these tonic impulses proceed from the spinal bulb.

On the other hand we may remove the whole of the brain right down to the upper limits of the spinal bulb, and yet produce no flushing, or only a slight transient flushing, of any part of the body and no fall at all, or only a slight transient fall, of the general blood pressure. We therefore seem justified in assuming the existence in the spiual bulb of a nervous centre, which we may speak of as a vaso-motor centre, or the bulbar vaso-motor centre, from which proceed tonic vaso-constrictor impulses, or which regulates the emission and distribution of such tonic vasoconstrictor impulses or influences over various parts of the body.

$\$ 152$. The existence of this vaso-motor centre may, moreover, be shewn in another way. The extent or amount of the tonic constrictor impulses proceeding from it may be increased or diminished, the activity of the centre may be augmented or inhibited, by impulses reaching it along various afferent nerves; and provided no marked changes in the heart beat take place at the same time, a rise or fall of general blood pressure may be taken as a token of an increase or decrease of the activity of the centre.

In the rabbit there is found in the neck, lying side by side with the cervical sympathetic nerve and running for some distance in company with it, a slender nerve which may be ultimately traced down to the heart, and which, if traced upwards, is found to come off somewhat high up from the vagus, by two or more roots, one of which is generally a branch of the superior laryngeal nerve. This nerve (the fibres constitnting which are in the dog bound up with the vagus, and do not form an independent nerve) appears to be exclusively an afferent nerve; when after division of the nerve the peripheral end, the end still in connection with the heart, is stimulated no marked results follow. The beginnings of the nerve in the heart are therefore quite different from the endings of the inhibitory fibres of the vagus, or of the augmentor fibres of the sympathetic system; the nerve has nothing to do with the nervous regulation of the heart treated of in Sec. 5 . If now, while the pressure in an artery such as the carotil is being registered, the central end of the nerve (i.e. the one connected with the brain) be stimulated with the interrupted current, a gradual but marked fall of pressure (Fig. 74) in the carotid is observed, lasting, when the period of stimulation is short, some time after the removal of the stimulus. Since the beat of the heart is not markedly changed, the fall of pressure must be due to the diminution of peripheral resistance occasioned by the dilation of some arteries. And it is probable that the arteries thus dilated are chiefly if not exclusively those arteries of the abdoninal viscera which are governed by the splanchnic nerves; for if these nerves are divided on both sides previous to the experi- 
ment, the fall of pressure when the nerve is stimulated is very small, in fact almost insignificant. The inference we draw is as follows. The atferent impulses passing upwards along the nerve

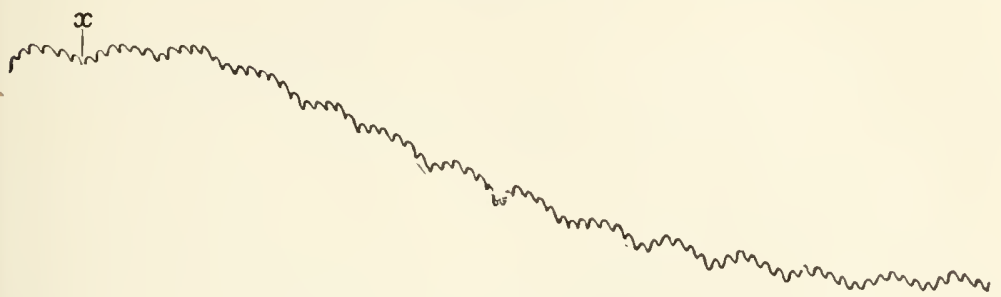

Fig. 74. Tracing, silewing the Effect on Bloon Pressure of stimulating the central end of the Depressor Nerve in tile Rabit.

On the time narker below the intervals correspond to seconds. At $x$ an interrupted current was throwi into the nerve.

in question have so affected some part of the central nervous system that the influences which, in a normal condition of things, passing along the splanchnic nerves keep the minute arteries of the abdominal viscera in a state of moderate tonic constriction, fail altogether, and those arteries in consequence dilate just as they do when the splanchnic nerves are divided, the effect being possibly increased by the similar dilation of other vascular areas. Since stimulation of the nerve of which we are speaking always produces a fall, never a rise of blood pressure, the amount of fall of course being dependent on circumstances, such as the condition of the nervous system, state of blood pressure and the like, the nerve is known by the name of the depressor nerve. As we shall point out later on, by means of this afferent nerve from the heart the peripheral resistance is, in the living body, lowered to suit the weakened powers of a labouring heart.

This gradual lowering of blood pressure by diminution of peripheral resistance affords a marked contrast to the sudden lowering of blood pressure by cardiac inhibition ; compare Fig. 74 with Fig. 70.

$\$ 153$. But the general blood pressure may be modified by afferent impulses passing along other nerves than the depressor, the modification taking on, according to circumstances, the form either of decrease or of increase.

Thus, if in an animal placed under the influence of urari (some anesthetic other than chloral \&c. being used), the central stump of the divided sciatic nerve be stimulated, an increase of blood pressure (Fig. 75) almost exactly the reverse of the 
decrease brought about by stimulating the depressor, is observed. The curve of the blood pressure, after a latent period during which no changes are visible, rises steadily, reaches a maximum and

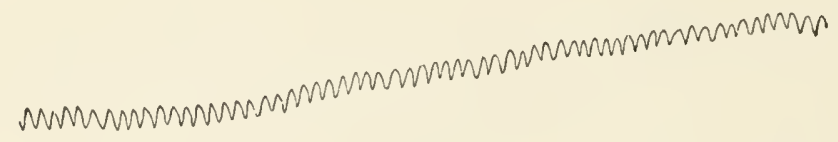

$x$

Fig. 75. Effect on Blood Pressure Curve of stimulating Sciatic Nerve under Urari (Cat).

$x$ marks the moment in which the current was thrown into the nerve. Artificial respiration was carried on, and the usual respiratory undulations are absent.

soon slowly falls again, the fall sometimes beginning to appear before the stimulus has been removed. This rise of pressure, since it may be observed in the absence of any increase in the heart beat, such at least as could give rise to it, must be due to the constriction of certain arteries; the arteries in question being those of the splanchnic area certainly, and possibly those of other vascular areas as well. The effect is not confined to the sciatic; stimulation of any nerve containing afferent fibres may produce the same rise of pressure, and so constant is the result that the experiment has been made use of as a method for determining the existence of afferent fibres in any given nerve and even the paths of centripetal impulses through the spinal cord.

If, on the other hand, the animal be under the influence not of urari but of a large dose of chloral, instead of a rise of blood pressure a fall, very similar to that caused by stimulating the depressor, is observed when an afferent nerve is stimulated. The condition of the central nervous system seems to determine whether the effect of afferent impulses on the central nervous system is one leading to an augmentation of vaso-constrictor impulses, and so to a rise, or one leading to a diminution of vasoconstrictor impulses and so to a fall of blood pressure.

$\$ 154$. We have used the words 'central nervous system' in speaking of the above; we have evidence, however, that the part of the central nervous system acted on by the afferent impulses is the vaso-motor centre in the spinal bulb, and that the effects in the way of diminution (depressor) or of augmentation (pressor) are the results of afferent impulses inhibiting or augmenting the tonic activity of this centre or of a part of this centre especially connected with the splanchnic nerves. The whole brain may be removed right down to the bulb, and yet the effects of stimulation in the direction either of diminution or of augmentation may still be brought about. If the bulb be removed, these effects are no 
longer seen, though all the rest of the nervous system be left intact. Nay, more, by partially interfering with the bulb, we may partially diminish these effects and mark out, so to speak, the limits of the centre in question within the bulb itself. Thus, in an intact animal under urari, stimulation of the sciatic nerve with a stimulus of a certain strength will produce a rise of blood pressure up to a certain extent. After removal of the whole brain right down to the bulb, the same stimulation will produce the same rise as before; the vaso-motor centre has not been interfered with. Proceeding downwards, however, and removing the bulb piecemeal by successive transverse sections a level is soon met with, beyond which removal of the nervous substance causes an obvious diminution in the effect produced by the stimulation of the sciatic; this marks the upper limit of the centre. Proceeding still further downwards with successive slices, stimulation of the sciatic produces less and less rise of blood pressure, until at last a level is reached, at which even strong stimulation of the sciatic or any other afferent nerve produces no effect at all on blood pressure; this marks the lower limit of the centre. In this way the lower limit of the bulbar vaso-motor centre has been deternined in the rabbit at a horizontal line drawn about 4 or $5 \mathrm{~mm}$. above the point of the calamus scriptorius, and the upper limit at about $4 \mathrm{~mm}$. higher up, i.e. about 1 or $2 \mathrm{~mm}$. below the corpora quadrigemina. We may add that the centre appears to be bilateral, the halves being placed not in the middle line but more sideways and rather nearer the anterior than the posterior surface. But we will reserve what we have to say as to the structural features of this centre until we come to study the spinal bulb in detail.

$\$ 155$. The above experiments appear to afford adequate evidence that, in a normal state of the body, the integrity of the bulbar vaso-motor centre is essential to the production and distribution of those continued constrictor impulses by which the general arterial tone of the body is maintained, and that an increase or decrease of vaso-constrictor action in particular arteries, or in the arteries generally, is brought about by neans of the same bulbar vaso-motor centre. But we must not therefore conclude that this small portion of the spinal bulb is the only part of the central nervous system which can act as a centre for vaso-constrictor fibres; and, so we have seen, there is no evidence at present that the vaso-dilator fibres are connected with either this or any other one centre. In the frog reflex vaso-motor effects may be obtained by stimulating various afferent nerves after the whole spinal bulb has been removed, and, indeed, even when only a comparatively small portion of the spinal cord has been left intact, and connected, on the one hand, with the afferent nerve which is being stimulated, and, on the other, with the efferent nerves in which run the vasn-motor fibres, whose action is being studied. In the mammal such effects do not so readily appear, but may with care 
and under special conditions be obtained. Thus in the dog, when the spinal cord is divided in the thoracic region, the arteries of the hind limbs and hinder part of the body, as we liave already said, $\$ 150$, become dilated. This one would naturally expect as the result of their severance from the bulbar vaso-motor centre. But if the animal be kept in good condition for some time, a normal or nearly normal arterial tone is after a while re-established; and the tone thus regained may, by afferent impulses reaching the cord below the section, be modified in the direction certainly of diminution, $i$. $e$. dilation, and possibly, but this is not so certain, of increase, $i$. $e$. constriction ; dilation of various cutaneous vessels of the limbs may be readily produced by stimulation of the central stump of one or another nerve.

These and other results lead to the conclusion that the bulbar vaso-motor centre is not to he regarded as the sole vaso-motor centre, whence alone can issue tonic constrictor impulses or whither afferent impulses from this or that part of the body must always travel before they can affect the vaso-constrictor impulses passing along this or that nerve. We are rather to suppose that the spinal cord along its whole lengtl contains, interlaced with the reflex and other mechanisms by which the skeletal muscles are governed, vaso-motor centres and mechanisms of varied complexity, the details of whose functions and topography have yet largely to be worked out. As in the absence of the sinus venosus the auricles and ventricle of the frog's heart may still continue to beat, so in the absence of the spinal bulb these spinal vaso-motor centres provide for the vascular emergencies which arise.

$\S 156$. We may sum up the history of vaso-motor actions somewhat as follows.

In the case of at least a very large number of the arteries of the body we have direct experimental evidence that these arteries are connected with the central nervous system by nerve fibres, called vaso-motor fibres, the action of which varies the amount of contraction of the muscular coats of the arteries and so leads to changes in calibre. The action of these vaso-motor fibres is more manifest, and probably more important in the case of small and minute arteries than in the case of large ones.

These vaso-motor fibres are of two kinds. The one kind, vasoconstrictor fibres, are of such a nature or have such connections at their peripheral endings that stimulation of them produces narrowing, constriction of the arteries. During life these fibres appear to he the means by which the central nervous system exerts a continued tonic influence on the arteries and maintains an arterial 'tone;' and this arterial tone may be modified by the action of the central nervous system, so as to give place on the one hand to constriction and on the other to widening. The other kind, vaso-dilator fibres, are of such a kind, or have such connections, that stimulation of them produces widening, dilation of the 
arteries. There is no adequate evidence that these vaso-dilator fibres serve as channels for tonic dilating innpulses or influences.

The vaso-constrictor fibres leave the spinal cord by the anterior roots of the nerves coming from the middle region only of the spinal cord. In the $\operatorname{dog}$, this region extends from about the first or second thoracic to the fourth or fifth lumbar nerve; and in other animals is probably of corresponding extent. Leaving the spinal nerves by the respective visceral branches, rami communicantes, the fibres pass into the sympathetic system, the majority joining the main sympathetic chain of ganglia in the thorax and abdomen, but some, for instance those going to certain parts of the intestine and some other viscera, leaving that chain on one side and passing directly to more peripheral ganglia, such as the solar plexus and the inferior mesenteric ganglia. From the sympathetic chain the fibres run to their destination in such nerves as the cervical sympathetic and splanchnic, those allotterl to the skin of the limbs and trunk rumning back again to join the respective spinal nerves. In the ganglia of the sympathetic chain or in other more peripheral ganglia the fibres lose their medulla, and continue their course as non-medullated fibres.

In the intact organism the emission and distribution along these vaso-constrictor fibres of tonic constrictor impulses, by which general and local arterial tone is maintained and regulated, is governed by a limited portion of the spinal bulb known as the bulbar vaso-motor centre; and when some change of conditions or other natural stimulus brings abont a change in the activity of the vaso-constrictor fibres of one or more vascular areas, or of all the arteries supplied with vaso-constrictor fibres, this same bulbar vaso-motor centre appears in such cases to play the part of a centre of reflex action. Nevertheless, in cases where the nervous connections of this bulbar vaso-motor centre with a vascular area are cut off by an operation, as by section of the cord, other parts of the spinal cord may act as centres for the vaso-constrictor fibres of the area, and possibly these subordinate centres may be to a certain extent in action in the intact organisin.

The vaso-dilator fibres of whose existence we have clear and undisputed experimental evidence, are very limited in distribution. In the cases best known, the fibres leave certain regions of the central nervous system and proceed to their destination along certain cerebro-spinal nerves; they do not lose their medulla until they approach their termination. But as we have seen there is evidence of vaso-dilator fibres also running in nerves of the sympathetic system. The vaso-dilator fibres are generally thrown into action as part of a reflex act, and the centre, in the reflex act, appears in each case to lie in the central nervous system not far from the origin of the ordinary motor fibres which the dilator fibres accompany.

The effects of the activity of the vaso-dilator fibres appear to be 
essentially local in nature. When any set of the fibres come into action the vascular area which these govern is dilated; and the vascular areas so governed are relatively so small that changes in them produce little or no effect on the vascular system in general; the fibres are called into play to produce special effects in special organs.

The effects of changes in the activity of the vaso-constrictor fibres are both local and general. They are also double in nature; by an inhibition of tonic constrictor impulses a certain amount of dilation may be effected; by an augmentation of constrictor impulses, constriction, it may be of considerable extent, may be brought about. When the vascular area so affected is small the effects are local, more or less blood is distributed through the area ; when the vascular area affected is large, the inhibition of constriction may lead to a marked fall, and an augmentation of constriction to a marked rise of general blood pressure. Broadly speaking, we may say that whenever a vascular change is needed for the general well-being of the economy, it is this raso-constrictor system which is called into play.

The distribution of clearly proved vaso-dilator fibres is as we have said very limited, and even the vaso-constrictor fibres are most abundant in the nerves going to the skin and to the viscera. In respect to the arteries supplying the numerous skeletal muscles, there is much dispute as to whether they are supplied by vaso-dilator fibres; and the supply of vaso-constrictor fibres to them is at least not large. We may perhaps infer that the vascular changes in the muscles are intended chiefly for the benefit of the muscles themselves, and are not to any great extent, like those of the skin and viscera, utilized for the more general purposes of the economy.

$\$ 157$. We shall have occasion later on again and again to point out instances of the effects of vaso-motor action both local and general, but we may here quote one or two characteristic examples. "Blushing" is one. Nervous impulses started in some parts of the brain by an emotion produce a powerful inhibition of that part of the bulbar vaso-motor centre which governs the vascular areas of the head supplied by the cervical sympathetic, and hence has an effect on the vaso-motor fibres of the cervical sympathetic almost exactly the same as that produced by section of the nerve. In consequence the muscular walls of the arteries of the head and face relax, the arteries dilate and the whole region becomes suffused. Sometimes an emotion gives rise not to blushing, but to the opposite effect, viz. to pallor of the face. In a great number of cases this has quite a different cause, being due to a sudden diminution or even temporary arrest of the heart's heats; but in some cases it may occur without any change in the beat of the heart, and is then due to a condition the very converse of that of blushing, that is, to an increased arterial constriction; 
and this increased constriction, like the dilation of blushing, is effected through the agency of the central nervous system and the cervical sympathetic. Blushing and its opposite pallor are most marked in the face; but other parts of the body may bhush (or grow pale) the change being brought about by appropriate nerves.

The vascular condition of the skin at large affords another instance. When the temperature of the air is low the vessels of the skin are constricted, and the skin is pale; when the temperature of the air is high the vessels of the skin are dilated, and the skin is red and flushed. In both these cases the effect is mainly a reflex one, it being the central nervous system which lrings about augmentation of constriction in the one case and inlibition in the other; though possibly some slight effect is produced by the direct local action of the cold or heat on the vessels of the skin. Moreover the vascular changes in the skin are accompanied by corresponding vascular changes in the viscera (chiefly abdominal) of a reverse kind. When the ressels of the skin are dilated those of the viscera are constricted, and vice versa; so that a considerable portion of the whole blood ebbs and flows, so to speak, according to circumstances from skin to viscera and from viscera to skin. By these changes, as we shall see later on, the maintenance of the normal temperature of the boly is in large measure secured.

We shall see later on that the secretion of urine is in a peculiar way dependent on the flow of blood through the kidney. A very favourable condition for this flow is a dilated condition of the renal arteries coincident with a high general blood pressure, and this condition as we shall see leads to a copious secretion of urine. The high general blood pressure in this case is largely caused by very general arterial constriction, leading to great increase of peripheral resistance, while the dilated state of the renal arteries appears to be due to a lack of the usual tonic constrictor impulses; though these constrictor impulses are increased in respect to other arteries, they are diminished in respect to the renal arteries themselves.

When food is placed in the mouth the blood ressels of the sılivary glands as we have seen are flushed with blood as an adjuvant to the secretion of digestive fluid; and as the food passes along the alimentaly canal each section in turn, with the glandular appendages belonging to it, welcomes its advent by flushing with blood. As we have already said, we have, at present, no satisfactory evidence, except in the case of the salivary glands, that this thushing is carried out by special vaso-dilator nerves. Along the rest of the alimentary canal the widening of the arteries and thus the increased flow seems to be brought about by diminution of vaso-constrictor impulses, so far at least as it is ensured by the intervention of the central nervous system. We say 'so far' because as we shall see we have evidence that the vessels of the kidney may change in calibre independently of any influences 
proceeding from the central nerrous system, after for instance all the nerves going to the kidney have been divided; in such cases the changes in the calibre of the renal vessels seem to be due to some direct local action; and it is possible that the flushing of the alimentary canal when food enters it is similarly, in part or at times, the result of some local action on the blood ressels.

$\$ 158$. Vaso-motor nerves of the Veins. Although the veins are provided with muscular fibres and are distinctly contractile, and although rhythmic variations of calibre due to contractions may be seen in the great veins opening into the heart, in the veins of the bat's wing, and elsewhere, our knowledge as to any nervous arrangements governing the veins is at present very limited. The portal vein, the walls of which are conspicuously muscular, the muscular fibres being arranged both as a circular and as a longitudinal coat, is like the veins just mentioned subject to rhythmic variations of calibre; these might be due to active rhythmic contractions of the portal vein itself or might be of a passive nature, due to a rhythmic rise and fall in the quantity of blood discharged into it from the ressels of the viscera. The former view is supported by the observation that after the aorta has been obstructed, so that no blood can pass into the portal vein from the mesenteric and other arteries, contractions of the portal vein may be obtained by stimulating the splanchnic nerves. The great distention of the venous system with blood which occurs in the frog when the brain and spinal cord are destroyed, and which renders the heart almost bloodless, the greater part of the blood being lodged in the veins, has also been supposed to point to some normal tone of the veins dependent on the central nervous system. 


\section{SEC. 7. THE CAPILLARY CIRCULATION.}

$\S 159$. We have already, some time back ( $\$ 99$ ), mentioned some of the salient features of the circulation through the capillaries, viz. the difficult passage of the corpuscles (generally in single file, though sometimes in the larger channels two or more abreast) and plasma through the narrow channels, in a stream which though more or less irregular is steady and even, not broken by pulsations, and slower than that in either the arteries or the veins. We have further seen $(\$ 94)$ that the capillaries vary very much in width from time to time; and there can be no doubt that the changes in their calibre are chietly of a passive nature. They are expanded when a large supply of blood reaches them through the supplying arteries, and, by virtue of their elasticity, shrink again when the supply is lessened or withdrawn; they may also become expanded by an obstacle to the venous ont tlow.

On the other hand, there is a certain amount of evidence that, in young animals at all events, the calibre of a capillary canal may vary, quite independently of the arterial supply or the venous outflow, in consequence of changes in the form of the epithelioid cells, allied to the changes which in a muscle-fibre or muscle-cell constitute a contraction; and though the matter requires further investigation, it is possible that these active changes play an important part in determining the quantity of blood passing through a capillary area ; but there is as yet no satisfactory evidence that they, like the corresponding changes in the arteries, are governed by the nervous system.

Over and above these changes of form, the capillaries and minute vessels are subject to still other changes and so exert influences by virtue of which they play an important part in the work of the circulation. Their condition determines the amount of resistance offered by their channels to the flow of blood through those channels, and determines the amount and character of that interchange between the blood and the tissues which is the main fact of the circulation. 
If the web of the frog's foot, or, better still, if some transparent tissue of a mammal he watehed under the microscope, it will be observed that, while in the small capillaries the corpuscles are pressed through the channel in single file, one after the other, each corpuscle as it passes occupying the whole bore of the capillary, in the larger capillaries (of the mammal), and especially in the small arteries and veins which permit the passage of more than one corpuscle abreast, the red corpuscles run in the middle of the channel, forming a coloured core, between which and the sides of the vessels all round is a colourless layer, containing no red corpuseles, called the 'plasmatic layer' or 'peripheral zone.' This division into a peripheral zone and an axial stream is due to the fact that in any stream passing through a closed channel the friction is greatest at the sides, and diminishes towards the axis. The corpuscles pass where the friction is least, in the axis. A quite similar axial core is seen when any fine particles are driven with a sufficient velocity in a stream of fluid through a narrow tube. As the velocity is diminished the axial core becomes less marked and disappears.

In the peripheral zone, especially in that of the veins, are frequently seen white corpuscles, sometimes clinging to the sides of the vessel, sometimes rolling slowly along, and in general moving irregularly, stopping for a while and then suddenly moving on. The greater the velocity of the flow of blood, the fewer the white corpuscles in the peripheral zone, and with a very rapid flow they, as well as the red corpuscles, may be all confined to the axial stream. The presence of the white corpuseles in the peripheral zone has been attributed to their being specifically lighter than the red corpuscles, since when fine particles of two kinds, one lighter than the other, are driven through a narrow tube, the heavier particles flow in the axis and the lighter in the more peripheral portions of the stream. But, besides this, the white corpuseles have a greater tendency to adhere to surfaces than have the red, as is seen by the manner in which the former become fixed to the glass slide and cover-slip when a drop of blood is mounted for inicroscopical examination. They probably thus adhere by virtue of the amœboid novements of their protoplasm, so that the adhesion is to be considered not so much a mere physical as a physiological process, and hence may be expected to vary with the varying nutritive conditions of the corpuscles and of the blood vessels. Thus while the appearance of the white corpuscles in the peripheral zone may be due to their lightness, their temporary attachment to the sides of the vessels and characteristic progression is the result of their power to adhere; and as we shall presently see their amœboid movements may carry them on beyond mere aclliesion.

$\$ 160$. These are the phenomena of the normal circulation, and may be regarded as indicating a state of equilibrium between 
the blood on the one hand and the blood vessels with the tissues on the other; but a different state of things sets in when that equilibrium is overthrown by causes leading to what is called inflammation or to allied conditions.

If an irritant, such as a drop of chloroform or a little diluted oil of mistard, be applied to a small portion of a frog's web, tongue, mesentery, or some other transparent tissue, the following changes may be observed under the microscope; they may be still better seen in the mesentery or other transparent tissue of a mammal. The first effect that is noticed is a dilation of the arteries, accompanied by a quickening of the stream. The irritant, probably by a direct action on the muscular fibres of the arteries, has led to a relaxation of the muscular coat, and hence to a widening; and we have already, $\$ 105$, explained how such a widening in a small artery may lead to a temporary quickening of the stream. In consequence of the greater flow through the arteries, the capillaries become filled with corpuscles, and many passages, previously invisible or nearly so on account of their containing no corpuscles, now come into view. The veins at the same time appear enlarged and full. If the stimulus be very slight, this may all pass away, the arteries gaining their normal constriction, and the capillaries and veins returning to their normal condition; in other words, the effect of the stimulus in such a case is simply a temporary blush. Unless, however, the chloroform or mustard be applied with especial care, the effects are much more profound, and a series of remarkable changes set in.

In the normal circulation, as we have just said, white corpuscles may be seen in the peripheral, plasmatic zone, but they are scanty in number, and each one, after staying for a little time in one spot, suddenly gets free, sometimes almost by a jerk as it were, and then rolls on for a greater or less distance. In the area now under consideration a large number of white corpuscles soon gather in the peripheral zones, especially of the veins and venous capillaries (that is of the larger capillaries which are joining to form veins), but also, of the other capillaries, and, to a less extent, of the arteries; and this takes place although the vessels still remain dilated and the stream still continues rapid, though not so rapid as at first. Each white corpuscle appears to exhibit a greater tendency to stick to the sides of the vessels, and though driven away from the arteries by the stronger arterial stream, becomes lodged so to speak in the veins. Since new white corpuscles are continually being brought by the blood stream on to the scene, the number of them in the peripheral zones of the veins increases more and nore, and this may go on until the inner surface of the reins and venous capillaries appears to be lined with a layer of white corpuscles. The small capillaries too contain more white corpuscles than usual, and even in the arteries these are abundant, though not forming the distinct layer seen in the veins. The white cor. 
puscles, however, are not the only bodies present in the peripheral zone. 'Though in the normal circulation blood-platelets (see \$33) cannot be seen in the peripheral zone, and hence (on the view, which has the greater support, that these bodies are really present in (puite normal blood) must be confined to the axial stream, they make their appearance in that zone during the changes which we are now describing. Indeed, in many cases they are far more abundant than the white corpuscles, the latter appearing imbedded at intervals in masses of the former Soon after their appearance the individual platelets lose their outline, and run together into formless masses.

$\$ 161$. This much, the appearance of numerous white corpuscles and platelets in the peripheral zones, may take place while the stream, though less rapid than at the very first, still remains rapid; so rapid at all events that, owing to the increased width of the passages, in spite of the obstruction offered by the adherent white corpuscles, the total quantity of blood flowing in a given time through the inflamed area is greater than normal. But soon, though the vessels still remain dilated, the stream is observed most distinctly to slacken, and then a remarkable phenomenon makes its appearance. The white corpuscles lying in contact with the walls of the veins or of the capillaries are seen to thrust processes through the walls; and, the process of a corpuscle increasing at the expense of the rest of the body of the corpuscle, the whole corpuscle, by what appears to be an example of amoboid movement, makes its way through the wall of the vessel into the lymph space outside; the perforation appears to take place in the cement substance joining the epithelioid plates together. This is the migration of the white corpuscles to which we alluded in $\$ 32$, and takes place chiefly in the veins and capillaries, not at all or to a very slight extent in the arteries. Through this migration the lymph spaces around the ressels in the inflamed area become crowded with white corpuscles. At the same time fluid passes from the interior of the blond vessels through the altered walls into the lymph spaces more rapidly than it escapes from the lymph spaces along the lymphatic channels; these lymph spaces lecome distended with lymph, which also changes somewhat in its chemical characters; it tends to clot more readily and more firmly, and is sometimes spoken of as 'exudation thuid,' or by the older writers as 'coagulable lymph.' This turgescence of the lymph spaces, together with the dilated crowded condition of the blood vessels, gives rise to the swelling which is one of the features of intlammation.

If the inflammation now passes off the white corpuscles cease to emigrate, cease to stick for any length of time to the sides of the vessels, the stream of blood through the vessels quickens again, and the vessels themselves, though they may remain for a long time dilated, eventually regain their calibre, and a normal circulation is 
re-established. The migrated corpuscles move away from the region along the labyrinth of lymph spaces, and the surplus lymph also passes away along the lymph spaces and lymphatic vessels.

A nore powerful action of the irritant may learl to still other events. More and more white corpuscles, arrested in their passage, crowd the channels and block the way, so that though the vessels remain dilated, the stream becomes slower and slower, until at last it stops altogether, and 'stagnation' or 'stasis' sets in. The red corpuseles are driven in, often in masses, among the white corpuscles and platelets, the distinction between axial stream and peripheral zone becoming lost; and arteries, veins and capillaries, all distended, sometimes enormously so, are filled with a mass of mingled red and white corpuscles and platelets. The red corpuseles run together so that their outlines are no longer distinguishable; they appear to become fused into a homogeneous red mass. And it may now be observed that, not only white corpuseles but also red corpuscles, make their way through the distended and altered walls of the capillaries, chiefly, at all events, at the junctions of the epithelioid plates, into the lymph spaces beyond. This is spoken of as the diapedesis of the red corpuscles.

This condition of 'stasis' may be the prelude to further mischief, and, indeed, to the death of the tissue, but it, too, like the earlier stage of inflammation, may pass away. As it passes away the outlines of the corpuscles become once more distinct, those on the venous side of the block gradually drop away into the neighbouring currents, little by little the whole obstruction is removed, and the current through the area is re-established.

$\$$ 162. The slowing or the arrest of the blood current described above is not due to any lessening of the heart's beat; the arterial pulsations, or at least the arterial flow, may be seen to be continued in full force down to the affected area, and there to cease very sudienly. It is not due to the peripheral resistance being increased by any constriction of the small arteries, for these continue dilated, sometimes exceedingly so. It must, therefore, be due to some new and unusual resistance occurring in the area itself, and this we are by many reasons led to attribute to an increased tendency of the corpuscles, especially of the white corpuscles, to stick to the sides of the vessels. The increase of adhesiveness is not caused by any change confined to the corpuseles themselves; for if after a temporary delay one set of corpuscles has managed to pass away from the affected area, the next set of corpuscles brought to the area in the blood stream is subjected to the same delay. The cause of the increased adhesiveness must therefore lie in the walls of the blood vessels, or in the tissue of which these form a part. That the increased adhesion is due to the vascular walls and not primarily to the corpuscles themselves is further shewn by the fact that if, in the frog, an artificial blood of normal saline solution, to which milk has been added, be substitnted for normal blood, a 
stasis may by irritants be induced in which oil-globules play the part of corpuscles, and by their aggregation bring about an arrest of the flow.

We are led to conclude that there exist in health certain relations between the blood on the one hand, and the walls of the vessels on the other, by which the tendency of the corpuscles to adhere to the blood vessels is kept within certain limits; these relations consequently determine the normal flow, with its axial stream and peripheral zone, and the normal amount of peripheral resistance; in inflammation, these relations, in a manner we cannot as yet fully explain, are disturbed so that the tendency of the corpuscles to adhere to the sides of the vessels is largely and progressively increased. Hence the tarrying of the corpuscles in spite of the widening of their path, and finally their agglomeration and fusion in the distended channels.

The changes occurring in the vascular walls at the same time also modify the passage from the blood to the tissue of the fluid parts of the blood, the lymph of inflamed areas being more abundant and richer in proteids than normal lymph. There is a greater outflow from the interior of the blood vessel into the lymph spaces outside, and, indeed, it has been urged that this, carrying the blood corpuscles with it, mechanically promotes the gathering of the white corpuscles at the sides of the vessel and their subsequent passage through the walls.

We must not, however, pursue this subject of inflammation any further. We have said enough to shew that the peripheral resistance (and consequently all that depends on that peripheral resistance) is not wholly determined by the varying width of the minute passages, but is also dependent on the vital condition of the tissue of which the walls of the passages form a part. When the tissue is in health, a certain resistance is offered to the passage of blood through the capillaries and other minute vessels, and the whole vascular mechanism is adapted to overcome this resistance to such an extent that a normal circulation can take place. When the tissue becomes affected, the disturbance of the relations between the tissue and the blood may so augment the resistance that the passage of the blood becomes, as in inflammation, difficult, or, as in stasis, impossible. And it is quite open to us to suppose that under certain eircumstances the reverse of the above may occur in this or that area, that conditions may arise in which the resistance is lowered below the normal, and the circulation in the area quickened. Thus the vital condition of the tissue becomes a factor in the maintenance of the circulation; and it is possible, though not yet proved, that these vital conditions are directly under the dominion of the nervous system.

$\$ 163$. Changes in the peripheral resistance may also be brought about by changes in the character of the blood, especially by changes in the relative amount of gases present. When a 
stream of defibrinated blood is artificially driven through a perfectly fresh excised organ, such as the kidney, it is found that the resistance to the flow of blood through the organ, measured, for instance, by the amount of outflow in relation to the pressure exerted, varies considerably owing to changes taking place in the organ, and may be increased by increasing the venous character, and diminished by increasing the arterial character of the blood. Remarkable changes in the resistance are also brought about by the addition of small quantities of certain drugs such as chloral, atropin \&c. to the blood.

These changes have been attributed to the altered blood acting on the walls of the vessels, inducing, for instance, constriction or witening of the small arteries, or, it may be, affecting the capillaries, for it has been asserted that the epithelioid plates of the capillaries vary in form according to the relative quantities of carbonic acid and oxygen present in the blood. But this is not the whole explanation of the matter, since similar variations in resistance are met with when blood is driven through fine capillary tubes of inert matter. In such experiments it is found that the resistance to the flow increases with a diminution of the oxygen carried by the red corpuscles, and is modified by the addition to the blood of even small quantities of certain drugs.

It is obvious, then, that in the living body the peripheral resistance, being the outcome of complex conditions, may be modified in many ways. Experiment teaches us that, even in dealing with non-living inert matter, the flow of fluid through capillary tubes may be modified on the one hand by changes in the substance of which the tubes are composed, and on the other hand by changes in the chemical nature (even independent of the specific gravity) of the fluid which is used. In the living body both the fluid and the tubes, both the blood and the walls of the minute vessels, are subject to incessant change; the vessels are continually changing because they are living structures, and the blood is continually changing not only because it too is in part at least alive, but also because all the tissues of the body are working upon it. The changes in the one, moreover, are capable of reacting upon and inducing changes in the other; and, lastly, the changes both of the one and of the other may be primarily set going by events taking place in some part of the body far away from the region in which these changes are modifying the resistance to the Hlow. 


\section{SEC. 8. CHANGES IN THE QUANTITY OF BLOOD.}

$\$ 164$. In an artificial scheme, changes in the total quantity of fluid in circulation will have an immediate and direct effect on the arterial pressure, increase of the quantity heightenmg and decrease diminishing it. This effect will be produced partly by the pump being more or less filled at each stroke, and partly by the peripheral resistance being increased or diminished by the greater or less fulness of the small peripheral chamnels. The pressure along the whole system and hence the venous pressure will under all circumstances be raised with the increase of fluid, but an increase of the arterial pressure beyond that of the venous pressure will be observed only so long as the elasticity of the arterial tubes can be brought into play.

In the natural circulation, the direct results of change of quantity are modified by compensatory arrangements. Thus experiment shews the following when an animal with normal blood pressure is bled from one carotid. The pressure in the other carotid sinks so long as the bleeding is going on; this is chiefly because the free opening in the vessel, from which the bleeding is going on, cuts off a great deal of the peripheral resistance, and so leads to a general lowering of the blood pressure. It remains depressed for a brief period after the bleeding has ceased, but in a short time regains or nearly regains the normal heiglit. This recovery of blood pressure, after hiemorrhage, is witnessed so long as the loss of blood does not amount to more than about 3 per cent. of the body-weight. Beyond that, a large and frequently a sudden dangerous permanent depression is observed.

The restoration of the pressure after the cessation of the bleeding is too rapid to permit us to suppose that the quantity of fluid in the blood vessels is replaced by the withdrawal of lymph from the extra-vascular elements of the tissues. In all probability the result is gained by an increased action of the vaso-constrictor nerves increasing the peripheral resistance, the vaso-motor centre being thrown into increased action by the diminution of its blood supply; when the blood by ligature of the arteries in the 
neck is suddenly cut off from the brain and so from the spinal bulb, a rise of blood pressure is observed. When the loss of blood has gone beyond a certain limit, this vaso-constrictor action is insufficient to compensate the diminished quantity (possibly the vaso-motor centre in part becomes exhausted), and a considerable depression takes place; but at this epoch the loss of blood frequently causes anæemic convulsions.

Similarly, when an additional quantity of blood is injected into the vessels, no marked increase of blood pressure is observed so long as the vaso-motor centre in the spinal bulb is intact. If, however, the cervical spinal cord be divided previous to the injection, the pressure, which, on account of the removal of the bulbar vaso-motor centre, is very low, is permanently raised by the injection of blood. At each injection the pressure rises; it falls somewhat afterwards, but eventually remains at a higher level than before. This rise is stated to continue until the amount of blood in the vessels above the normal quantity reaches from 2 to 3 per cent. of the body-weight, beyond which point it is said no further rise of pressure occurs. The absence of any marked rise of blood pressure, so long as the bulbar vaso-motor centre is intact, shews that the addition of the extra quantity of blood stimulates that centre to increased activity. But while a diminution of blood supply seems to affect the centre directly, an increase of blood supply probably acts in an indirect manner. When the arteries in the neck are ligatured, the rise of blood pressure is much more marked if the depressor nerves be divided; so long as these nerves are intact impulses passing along them from the heart withstand the stinulating effects on the vaso-motor centre of the loss of blood. And we may perhaps infer that when an extra quantity of blood is injected, the grcater fulness stimulates the endings of the depressor nerves in the heart, and so by developing depressor impulses lessens the activity of the vasoinotor centre.

The facts stated seem, then, to shew, in the first place, that when the volume of the blood is increased, compensation is effected by a lessening of the peripheral resistance by means of a diminished action of the vaso-motor centre, so that the normal blood pressure remains constant. They further shew that a much greater quantity of blood can be lodged in the blood vessels than is normally present in them. That the additional quantity injected does remain in the vessels is proved by the absence of extravasations, and of any considerable increase of the extra-vascular lymphatic fluids. It has already been insisted that, in health, the veins and capillaries must be regarded as being far from filled; for were they to reccive all the blood which they can, even at a low pressure, hold, the whole quantity of blood in the body would be lodged in them alone. In these cases of large addition of blood, the extra quantity appears to be lodged in the small veins and capillaries (especially 
of the internal organs), which are abnormally distended to contain the surplus.

We learn, also, from these facts the two practical lessons : first, that blood pressure cannot be lowered directly in a mechanical manner by bleeding, unless the quantity removed be dangerously large; and secondly, that there is no necessary connection between a high blood pressure and fulness of blood or plethora, since an enormous quantity of blood may be driven into the vessels without any marked rise of pressure.

When a quantity of blood or, indeed, of fluid is injected into the veins, the output from the heart is increased and observations seem to shew that the increase in the output is out of proportion to the quantity of fluid injected, indicating that the result is of complex origin. In spite of this increased output, the blood pressure is not raised; the effect is compensated by vascular dilation somewhere. Conversely when blood is withdrawn, the output is diminished, but here again the effect on the blood pressure is soon compensated, this time by vascular constriction. 


\section{SEC. 9. A REVIEIV OF SOME OF THE FEATURES OF THE CLRCULATION.}

$\S 165$. The facts dwelt on in the foregoing sections have shewn us that the factors of the vascular mechanism may be regarded as of two kinds: one constant, or approximately constant; the other variable.

The constant factors are supplied by the length, natural bore, and distribution of the blood vessels, by the extensibility and elastic reaction of their walls, and by such mechanical contrivances as the valves. By the natural bore of the various blood vessels is meant the diameter which each would assume if the muscular fibres were wholly at rest, and the pressure of fluid within the vessel were equal to the pressure outside. It is obvious, however, that even these factors are only approximately constant for the life of an individual. The length and distribution of the vessels change with the growth of the whole body or parts of the body, and the physical qualities of the walls, especially of the arterial walls, their extensibility and elastic reaction change continually with the age of the individual. As the body grows older, the once supple and elastic arteries become more and more stiff and rigid, and often in middle life, or it may be earlier, a lessening of arterial resilience which proportionately impairs the value of the vascular mechanism as an agent of nutrition, marks a step towards the grave.

The chief variable factors are on the one hand the beat of the heart, and on the other the peripheral resistance, the variations in the latter being shietly brought about by muscular contraction or relaxation in the minute arteries, but also, though to what extent has not yet been accurately determined, by the condition of the minute vessels according to which the blood can pass through them with less or with greater ease, as well as by the character of the circulating blood.

These two chief variables, the beat of the heart and the width of the minute arteries, are known to be governed and regulated by the central nervous system, which adapts each to the circumstances 
of the moment, and at the same time brings the two into mutual dependence; but the central nervous system is not the only means of government: there are other modes of regulation, and so other safeguards.

$\$ 166$. Let us first consider the heart. The object, if we may use the expression, of the systole of the ventricle is to secure the needed arterial pressure; it is this, as we have seen, which drives the blood through the capillaries back to the heart. To do this the ventricle must deliver at the stroke a certain quantity of blood, exerting the pressure required to lodge the blood in the arteries, and repeating the stroke at appropriate intervals. Hence the work done will, in part, depend on the quantity of blood collected in the ventricle during the diastole, that is, on the inflow from the venous system. If the quantity brought be too small, then though the whole contents of the ventricle be ejected with adequate force at each stroke, and the stroke repeated regularly, the ventricle will fail in its object; speaking generally we may say that a lessened venous inflow will tend to lessen, and an increased venous inflow will tend to increase the work of the heart. This venous inflow is dependent on various causes, and may be variously modified by various events.

The blood in filling the ventricle distends its walls, and this distension, especially the fuller distension resulting from the auricular systole, also influences the ventricular stroke; for the contraction of the cardiac fibre, like that of the skeletal muscular fibre, is increased up to a certain limit by the fibre being put on the stretch $(\$ 140)$. This influence, however, is more distinctly seen on the arterial side. The greater the arterial pressure against which the ventricle has to deliver its contents, the greater the tension of the ventricular walls; and hence, a high arterial pressure tends of itself to enforce the ventricular systole. As in the skeletal nuscle, however, this beneficial influence soon reaches its limit.

$\$ 167$. The spontaneous beat of the heart is the outcome of the nutrition of the cardiac tissues. In the absence of all interference by inhibitory or angmentor fibres, the heart will continue beating with a certain rhythm and force, determined by the metabolism going on in its muscular and nervous elements. The beat therefore will be influenced by anything which affects that metabolism. And the obvious and direct cause of changes in the nutrition and so in the behaviour of the heart lies in changes in the quantity and quality of the blood supplied to the cardiac tissues. In the mammal this means the quantity and quality of the blood flowing through the coronary arteries.

If in a manmal the coronary arteries he tied or otherwise occluded the heart in a few seconds comes to a standstill; this, which always results if both arteries be tied, sometimes if one only be tied, is preceded by an irregularity or by changes in the 
beat and is followed by fibrillar contractions of parts of the ventricles. This is an extreme case, but it illustrates in a striking manner how closely the rhythnic contraction of the cardiac fibres is dependent on the blood supply.

The quantity of blood Howing through the coronary arteries is dependent on the pressure in the aorta, or rather on the difference between that pressure and the pressure in the right auricle into which the coronary veins open, and on the resistance offered by the coronary vessels. Hence with a high aortic pressure, more blood passes to the cardiac tissue. This is at least favourable to the development of the beat, and may be the direct cause of a stronger stroke; indeed observations seem to shew this. Thus a high aortic pressure itself helps the heart to the effort necessary to overcome that high pressure. Conversely a low aortic pressure would similarly tend to spare the heart an unnecessary exertion. As to how the heart may be influenced by changes in the width of the coronary arteries brought about by vaso-motor action, we have at present but little definite knowledge.

More important still than the quantity is the quality of the blood flowing through the coronary vessels. We shall have occasion in treating of respiration to speak of the manner in which blood deficient in oxygen or overladen with carbonic acid affects the beat of the heart; and we may here be content to point out that every change in the constitution of the blood, whether arising from the presence of substances such as drugs and poisons, introduced from without, or of substances manufactured in this or that tissue of the body or resulting from the absence or paucity or from excess of one or more of the normal constituents, may unfavourably or, it may be, favourably affect the heart beat, by directly intluencing the cardiac tissues through the coronary arteries. These changes in the blood may of course also work upon the heart through the central nervous system, and this indirect effect may possibly be different from the direct effect. Thus, when the breathing is interfered with, the too highly venous blood, while it acts clirectly on the cardiac tissues and that unfavourably, also stimulates the cardio-inhibitory centre, whereby the heart is slowed and its expenditure of energy lessened.

$\$ 168$. As is well known, the beat of the heart may become temporarily or permanently irregular. That many hearts go on beating day after day, year after year, without any such irregularity is a striking proof of the complete balance which usually obtains between the several factors of which we are speaking. Sometimes such cardiac irregularities, those of a transient nature and brief duration, are the results of extrinsic nervous influences. Some events taking place in the stomach, for instance, give rise to afferent impulses which ascending from the mucous membrane of the stomach along certain afferent fibres of the vagus to the spinal bulb, so augment the action of the cardio-inhibitory centre 
as to stop the lieart for a beat or two, the stoppage being frequently followed by a temporary increase in the rapidity and force of the beat. Such a passing failure of the heart beat, in its sudden onset, in its brief duration, and in the reaction which follows, very closely resembles the complete but temporary inhibition brought about by artificial stimulation of the vagus. And as we have seen the inhibitory action of the vagus is especially prone to be set going by afferent impulses passing up to the central nervous system from the viscera.

The effects however which we produce by our rough means of direct stimulation of the trunk of the vagus do not afford a true picture of the action of the cardio-iuhibitory mechanism in the living body; we come nearer to this when we obtain inhibition in a retlex manner. From the knowledge gained in this way we may infer that the fainting which comes from pain, emotions and the like, is due to the action of the inhibitory mechanism. Several forms of irregular heart beat are probably brouglit about by the same mechanism; we may in this relation call to mind that one effect of the action of the inhibitory fibres is to produce not merely slowing or weakening but distinct irregularity of the heart beat.

Many observations shew that the cardio-inhibitory mechanism may be affected by afferent impulses or otherwise in two different ways. On the one hand the cardio-inhibitory centre may be thrown into action, or when already in action may have its action increased; on the other hand if already in action, that action may be lessened; the inhibition may itself be inhibited. The division of both vagus nerves in the dog affords an instance of the effect on the heart of arresting previously existing inhibitory impulses. Hence it becomes difficult in the complex living body to distinguish between an augmentation due to activity of the augmentor mechanism and one due to suspension of the previously active inhibitory mechanism. The two may probably be distinguished by studying the details of the behaviour of the heart in the two cases. Failing this it is difficult to say whether a case of that irregularity of the heart which we call 'palpitation' has been brought about positively by the one mechanism or negatively by the other.

We must remember, morenver, that irregularity in the heart beat in at least a large number of cases is the result not of nervous influences from without, but of intrinsic events. For instance, in many cases the irregularity of the heart beat is wholly unaffected by atropin, and therefore cannot be due to vagus action. It is very often the result of what we may call a disordered nutrition of the cardiac substance, though we cannot state the exact nature of the disorder.

$\$ 169$. We may repeat that the effect of inhibitory action is to lessen the expenditure of energy and so to assist the heart for 
future efforts; it saves the heart at the expense of the rest of the economy. The heart, so far as we know, cannot in the working of the living economy be brought to a final arrest by the simple action of the vagus. The effect of the augmentor action on the other hand is to increase the expenditure of energy ; it saves the rest of the econony at the expense of the heart. And probably in some cases augmentor action may bring about the cessation of the heart beat. Disordered cardiac nutrition shews itself frequently in a dilated condition of the ventricles; the systole is inadequate to secure an adequate discharge into the arteries, the residual blood in the ventricles is increased. If the augmentor mechanism be brought to bear on such a weakened and dilated ventricle, it may induce a fruitless expenditure of energy; the beat though increased is still inadequate to secure the needed discharge of the contents, while the fibre is exhausted by the increased metabolism. And the final result of such an effort may be the cessation of the beat.

$\$ 170$. Turning now to the minute arteries and the peripheral resistance which they regulate, we may call to mind the existence of the two kinds of mechanism, the vaso-constrictor mechanism, which, owing to the maintenance by the central nervous system of a tonic influence, can be worked both in a positive constrictor, and in a negative dilator direction, and the vaso-dilator mechanism, which, so far as we know, exerts its influence in one direction only, viz. to dilate the blood vessels. The latter, dilator mechanism seems, as we have seen, to be used in special instances only, as seen in the cases of the chorda tympani and nervi erigentes; the use of the former, constrictor mechanism appears to be more general. Thus the relaxation of the cutaneous arteries of the head and neck, which is the essential feature in blushing, seems due to mere loss of tone, to the removal of constrictor influences previously exerted through the vaso-constrictor fibres of the cervical sympathetic. Though probably dilator fibres pass directly along the roots of the cervical and of certain cranial nerves to the nerves of the head and neck, we have no evidence that these come into play in blushing; as we have seen, blushing may be imitated by mere section of the cervical sympathetic. So also the 'glow' and redness of the skin of the whole body, $i$. e. general dilation of the cutaneous arteries, which is produced by external warmth, is probably another instance of diminished activity of tonic constrictor influences; though the result, that the dilation produced by warming an animal in an oven is greater than that produced by section of nerves, seems to point to the dilator fibres for the cutaneous vessels which, as we have seen, probably exist in the sciatic and brachial plexuses and possibly in all the spinal nerves, also taking part in the action. A similar loss of constrictor action in the cutaneous vessels may be the result of certain emotions, whether going so far as actual blushing of the body, or merely 
producing a 'glow.' The warm and flushed condition of the skin, which follows the drinking of alcoholic fluids, is probably, in a similar manner the result of an inlibition of that part of the vasomotor centre which governs the cutaneous arteries. The effect of cold on the other hand, and of certain emotions, or of emotions under certain conditions, is to increase the constrictor action on the cutaneous vessels, and the skin grows pale. It may be worth while to point out, that in both the above cases, while both the cold and the warnith produce their effects, chiefly at all events throngh the central nervons system, and very slightly, if at all, by direct action on the skin, their action on the central nervous system is not simply a general augmentation or inhibition of the whole vaso-motor centre. On the contrary, the cold, while it constricts the cutaneous vessels, so acts on the vaso-motor centre as to inhibit that portion of the vaso-motor centre which governs the abdominal splanchnic area; while less blood is carried to the colder skin, by the opening up of the splanchnic area more bloot is turned on to the warmer regions of the body, and the rise of blood pressure which the constriction of the cutaneous vessels tended to produce, and which might be undesirable, is hereby prevented. Conversely, when warmth dilates the cutaneous vessels, it at the same time constricts the abdominal splanchnic area, and prevents an undesirable fall of pressure.

$\$ 171$. The influence on the body of exercise illustrates both the manner in which the two vascular factors, the heart beat and the peripheral resistance, are modified by circumstances, and the nutual action of these on each other. This influence is exceedingly complex, and we cannot treat it properly until we have studied several physiological matters to which we sliall come later on. We can here only tonch in a general way on some salient points.

We know from superficial observation that during active exertion the breathing is increased, the heart beats more quickly and apparently with greater vigour, and the skin, flushed with blood, perspires freely.

The repeated strong contractions of the skeletal muscles to which we turn as the ultimate canse of these events affect the body in two main ways, the one chemical, the other physical. When the muscles contract they take from the blood a larger amonnt of oxygen, they give up to the blood a larger amount of carbonic acid, and they discharge into the blood, either directly into the capillaries of the muscles or indirectly througl the lymph stream, a quantity of substances, probably of several kinds, such as sarcolactic acid and the like, which arise from the metabolism of the muscular sulsstance. The blood leaving a muscle at work is chemically different from the blood leaving a muscle at rest. There is also a physical chance. I)uring the contraction of a muscle the blood vessels are dilated; this when many muscles 
are at work would lead unless compensated to a lessening of peripheral resistance, and so to a fall of arterial pressure, for the minute vessels of the muscles form a large part of the whole system of minute vessels of the body; at the same time it would increase the venous inflow into the heart.

Now we shall later on point out that the increased breathing which follows upon exertion is due to the chemical changes thus produced in the blood, and not only to the diminution of oxygen and increase of carbonic acid, but also and perhaps especially to the presence of the other products of metabolism referred to above. Indeed we have reason to think that the increase in breathing is sufficient to maintain the blood in a normal condition so far as oxygen and carbonic acid are concerned; the blood is not more venous during exertion than during rest, it is possibly less venous. The increased breathing however, though it clears the blood of the excess of carbonic acid, leaves behind in the blood the other muscular products, ready to produce their effects on the body before they are got rid of by organs other than the lungs.

This increased breathing promotes mechanically, as we shall point out later on, the flow of blood to the heart and through the lungs. And this together with the increased venous flow from the contracting muscles favours the beat of the heart, supplying the means for a greater output and probably also tending to increase the force of the systole.

But there are other influences at work on the heart. The changes in the blood and probably the presence of the above mentioned metabolic products, no less than the excess of carbonic acid, so affect the vaso-motor centre as to lead to a great widening of the cutaneous vessels; at the same time as we shall see these so affect other parts of the central nervous system as to lead to a great activity of the sweat glands, by means of which the products in question are got rid of or rendered inert. But the widening of the vessels of the skin and of many muscles at the same time must unless compensated lead to a fall of arterial pressure. We have evidence however that the arterial pressure does not fall, in fact may be higher than normal; a very marked compensation must therefore take place. This is probably of a double nature.

On the one hand, the altered blood increases the work of the heart, enabling it by a quicker rhythm or a stronger stroke or by both conbined, to avail itself of the advantages of the greater venous inflow and to increase its output, whereby the arterial pressure increases. We cannot suppose that this increased work is due to the direct effect of the altered blood on the cardiac muscles, for the altered blood, is distinctly injurious to muscular tissue. The increase of the heart's work is gained in spite of this influence of the altered blood, and is due to the intervention of the central nervous system. There are several facts which seem to support the view that the altered blood throws into activity the 
augmentor system, and thus by increasing the work of the heart raises or maintains the arterial pressure.

On the other hand, we have reason to think that while that part of the vaso-motor centre which governs the cutaneous vascular area is being inhibited, that part which governs the abdominal splanchnic area is on the contrary being augmented. In this way a double end is gained. On the one hand, the mean blood pressure is maintained or increased in a more economical manner than by increasing the heart beats, and on the other hand, the blood during the exercise is turned away from the digestive organs which at the time are or ought to be at rest and therefore requiring comparatively little blood. These organs certainly at all events ought not during exercise to be engaged in the task of digesting and absorbing food, and the old saying, "after dinner sit awhile," may serve as an illustration of the working of the vascular mechanism with which we are dealing. The duty which some of the digestive organs have during exercise to carry out in the way of excretion of metabolic waste products is as we have already said probably taken on by the flushed and perspiring skin. It is true that at the beginning of a period of exercise, before the skin so to speak has settled down to its work, an increased flow of urine, dependent on or accompanied by an increased flow of blood through the kidney, may make its appearance; but in this case, as we shall see later on in dealing with the kidney, the flow of blood through the kidney may be increased in spite of constriction of the rest of the splanchnic area, and, besides, such an initial increase of urine speedily gives way to a decrease.

$\$ 172$. The effect of food on the vascular mechanism affords a marked contrast to the effect of bodily lahour. The most prominent result is a widening of the whole abdominal vascular area, accompanied by so much constriction of the cutaneous vascular area and so much increase of the heart's beat as are sufficient to neutralize the tendency of the widening of the abdominal vascular area to lower the mean pressure, or perhaps even sufficient to raise slightly the mean pressure.

The widening of the abdominal vascular area, as we have seen $(\$ 157)$, is probably an inhibition of tonic vaso-constrictor impulses as a reflex act, assisted possibly by some local action due to the presence of the food and similar to that supposed to take place in the skeletal muscles during contraction. We have at present no clear evidence that the absorbed products of digestion play any important part in this splanchnic dilation by acting on the central nervous system; but the concomitant increase of the heart beat is probably due to this cause. We have no exact knowledge of how the absorbed products bring this about, and possibly the mode of action differs with the different constituents of food. With regard to alcohol, which is so often part of a meal, we may perhajs say that the character of its 
effects, the quickening and strengthening of the beats, seems to point to its setting in action the augmentor mechanism, but it also probably acts directly on the cardiac tissues. In any case the effects depend largely on the lose, and if this is large the direct effects become prominent, and the ultimate result is a deleterious weakening.

Any large widening of the cutaneous area, especially if accompanied by muscular labour and the incident widening of the arteries of the muscles, would tend so to lower the general blood pressure (unless met by a wasteful use of cardiac energy) as injuriously to lessen the flow through the active digesting viscera. A moderate constriction of the cutaneous vessels on the other hand, by throwing more blood on the abdominal splanchnic area without tasking the heart, is favourable to digestion, and is probably the physiological explanation of the old saying, "If you eat till you're cold, you 'll live to be old."

In fact during life there seems to be a continual give-and-take between the blood vessels of the somatic and those of the splanchnic divisions of the body: to fill the one the other is proportionately emptied, and vice versa.

$\$ 173$. In the following sections of this work we shall see repeated instances, similar to or even more striking than the above, of the management of the vascular mechanism by means of the nervous system, and we therefore need dwell no longer on the subject. We may simply repeat that at the centre lies the cardiac muscular fibre, and at the periphery the plain muscular fibre of the minute artery. On these two elements the central nervous system, directed by this or that impulse reaching it along afferent nerve fibres, or affected directly by this or that influence, is during life continually playing, now augmenting, now inhibiting, now the one, now the other, and so, by help of the elasticity of the arteries and the mechanism of the valves, directing the blood flow according to the needs of the body. 



\section{BOOK II.}

THE TISSUES OF CHEMICAL ACTION WITH THEIR RESPECTIVE MECHANISMS. NUTRITION. 



\section{CHAPTER I.}

\section{THE TISSUES AND MECHANISMS OF DIGESTION.}

$\$$ 174. THE food in passing along the alimentary canal is subjected to the action of certain juices supplied by the secretory activity of the epithelium cells which line the canal itself or which form part of its glandular appendages. These juices (viz. saliva, gastric juice, bile, pancreatic juice, and the secretions of the small and large intestines), poured upon and mingling with the food, produce in it such changes, that from being largely insoluble it becomes largely soluble, or otherwise modify it in such a way that the larger part of what is eaten passes into the blood, either directly by means of the capillaries of the alimentary canal or indirectly by means of the lacteal system, while the smaller part is discharged as excrement.

Those parts of the food which are thus digested, absorbed and made use of by the body, are spoken of as food-stuffs (they have also been called alimentring principles) and may be conveniently divided into four great classes.

1. Proteids. We have previously (\$15) spoken of the chief characters of this class, and have dealt with several members in treating of blood and muscle. We may here repeat that in general composition they contain in 100 parts by weight "in round numbers" rather more than 15 parts of nitrogen, rather more than 50 parts of carbon, about 7 parts of hydrogen, and rather more than 20 parts of oxygen; though essentially the nitrogenous bodies of food and of the lody, they are made up of carbon to the extent of more than half their weight.

The nitrogenous boly gelatin, which occurs largely in animal food, and some other bodies of less importance, while more closely allied to proteid bodies than to any other class of organic substances, differ considerably from proteids in composition and especially in their behaviour in the body ; they are not of sufficient importance to form a class by themselves. 
2. Fats, frequently but erroneously called Hydrocarbons. These vary very widely in chemical composition, ranging from such a comparatively simple fat as butyrin to the highly complex lecithin ( $\$ 66)$; they all possess, in view of the oxidation of both their carbon and their hydrogen, a large amount of potential eneroy.

3. Curbo-hydiates, or sugars and starches. These possess weight for weight relatively less potential energy than do fats; they already contain in themselves a large amount of combined oxygen and when completely oxidised give out, weight for weight, less heat than do fats.

4. Saline or Mineral Bodies, and Water. These salts are for the most part inorganie salts; and this class diflers from the thiee preceding classes inasmuch as the usefulness of its members to the body lies not so much in the amount of energy which may be given out by their oxidation, as in the various intluences which, liy their presence, they exercise on the metabolic events of the body.

These several food-stuffs are variously acted upon in the several parts of the alimentary canal, and we may distinguish, as the food passes along the digestive tract, three main stages: digestion in the mouth and stomach, digestion in the small intestine, and digestion in the large intestine. In many animals the first stage is, to a large extent, preparatory only to the second which in all animals is the stage in which the food undergoes the greatest change; in the third stage the changes legun in the previous stages are completed, and this stage is especially characterised by the absorption of fluid from the interior of the alimentary canal.

It will be convenient to study these stages, more or less apart, though not wholly so, and it will also be convenient to consider the whole subject of digestion under the following heads:-

First, the characters and properties of the various juices, and the changes which they bring about in the food eaten.

Secondly, the nature of the processes by means of which the epithelium cells of the varions glands and various tracts of the canal are able to manu facture so many various juices out of the common source, the blood, and the manner in which the secretory activity of the cells is regulated and subjected to the needs of the economy.

Thirdly, the mechanisms, here as elsewhere chiefly of a muscular nature, by which the food is passed along the canal, and most efficiently brought into contact with the several juices.

Fourthly and lastly, the means by which the nutritious digested material is separated from the undigested or excremental material, and absorbed into the blood. 


\title{
SEC. 1. THE CHARAC'IERS AND PROPERTIES OF SALIVA AND GASTRIC JUICE.
}

\author{
Saliva.
}

$\S 175$. Mixed saliva, as it appears in the mouth, is a thick, glairy, generally frothy and turbid fluid. Under the microscope it is seen to contain, bcsides the molecular débris of food, bacteria and other organisms (frequently cryptogamic spores), epitheliumscales, mucus-corpuscles and granules, and the so-called salivary corpuscles. Its reaction in a healthy subject is alkaline, especially when the secretion is abundant. When the saliva is scanty. or when the subject suffers from dyspepsia, the reaction of the mouth may be acid. Saliva contrins but little solid matter, on an average probably about 5 p.c., the specific gravity varying from 1.002 to 1.006 . Of these solids, rather less than half, about 2 p.c., are salts (including at times a minute quantity of potassium snlphocyanate). The organic bodies which can be recognised in it are globulin and serum-albumin (see $\$ \$ 16,17$ ) fonnd in small quantities only, other obscure bodies occurring in minute quantity, and mucin; the latter is by far the most conspicnous organic constitıent, the glairincss or ropiness of mixed and other kinds of saliva being due to its presence.

Wucin. If acetic acid be cautiously added to mixed saliva the viscidity of the saliva is increased, and on further addition of the acid a semi-opaque ropy mass separates out, leaving the rest of the saliva limpid. This ropy mass, which is mucin, if stirred carefully with a glass rod, shrinks, becoming opaque, clings to the glass rod and may be thus removed from the flnid If the quantity of mucin be small and the saliva be violently shaken or stirred while the acid is being added, the mucin is apt to be precipitated in flakes, and may then be separated by filtration It may be added that the precipitation of mucin by acid is greatly influenced hy the presence of sodium chloride and other salts: thus after the addition of sodium chloride acetic acid even in considerable excess will not canse a precipitate of mucin. 
Mucin, thus prepared and purified by washing with acet:c acid, swells out in water, without actually dissolving; it will however dissolve into a viscid fluid readily in dilute $(0.1$ p.c.) solutions of potassium hydrate, more slowly in solutions of alkaline salts. In order to filter a mucin solution, great dilution with water is necessary. Mucin is precipitated by strong alcohol and by various metallic salts: it may also be precipitated by dilute mineral acids, but the precipitate is then soluble in excess of the acid. Mucin gives the three proteid reactions mentioned in $\$ 15$, but it is a very complex body, more complex eren than proteids, for by treatment with dilute mineral acids, and in other ways, it may be converted into some form of proteid (acid-albumin when dilute mineral acid is used), while at the same time there is formed a body which appears to be a carbohydrate and resembles a sugar in having the power of reducing cupric sulphate solutions. Several kinds of mucin appear to exist in various animal bodies, but they seem all to agree in the character that they can by appropriate treatment be split up into a proteid of some kind and into a carbohydrate or allied body.

$\$ 176$. The chief purpose served by the saliva in digestion is to moisten and soften the food, and to assist in mastication and deglutition. In some animals this is its only function. In other animals and in man it has a specific solvent action on some of the food-stuffs. Such minerals as are soluble in slightly alkaline fluids are dissolved by it. On fats it has no effect save that of producing a very feeble emulsion. On proteids it has also no specific action, though pieces of meat, cooked or uncooked, a pear greatly alteled after they have been masticated for some time; the chief alteration however which thus takes place is a change in the hremoglobin, and a general soltening of the muscular fibres by aid of the alkalinity of the saliva. Of course when particles of food are retained for a long time in the mouth, as in the interstices, or in cavities of the teeth, the bacteria or other organisms which are always present in the mouth may produce much more profound changes, but these are not the legitimate products of the action of saliva. The charactenistic property of saliva is that of converting starch into some form of sugar.

Action of Saliva on Starch If to a quantity of boiled starch, which is always more or less viscid and somewhat opaque or turbid, a small quantity of saliva be added, it will be found after a short time that an important change has taken place, inasmuch as the mixture has lost its previous viscidity and become thinner and more transparent. In order to understand this change, the reader must bear in mind the existence of the following bodies all belonging to the class of carbohydrates.

1. Starch, which forms with water not a true solution but a more or less viscid mixture, and gives a characteristic blue colour with iodine. The formula is $\mathrm{C}_{6} \mathrm{H}_{10} \mathrm{O}_{5}$ or more correctly $\left(\mathrm{C}_{6} \mathrm{H}_{10} \mathrm{O}_{5}\right)_{n}$ 
since the molecule of star h is some multiple ( $n$ being not less than 5) of the simpler formula. A kind of starch, known as soluble starch, while giving a blue colour with iodine, forms, unlike ordinary starch, a clear solution.

2. Dextrins, differing from starch in forming a clear solution. Of these there are at least two; one erythrodextrin, of ten spoken of simply as destrin, giving a port-wine red colour with iodine, and a second, uchroodertrin, which gives no colour at all with iodine. The formula for dextrin is the same as that for starch, but has a smaller molecule and might be represented by $\left(\mathrm{C}_{6} \mathrm{H}_{10} \mathrm{O}_{5}\right)_{n^{\prime}}$.

3. Dertrose, also called glucose or grape-sugar, giving 110 coloration with iodine, but characterised by the power of reducing cupric anl other metallic salts; thus, when dextrose is boiled with a fluid known as Fehling's fluid, which is a solution of hydrated cupric oxide in an excess of caustic alkali and double tartrate of sodium and potassium, the cupric oxide is reduced and a red or yellow deposit of cuprous oxide is thrown down. This reaction serves with others as a convenient tast for dextrose. $\mathrm{N}$ sither starch nor that commonest form of sugar known as canesugar, give this reaction: what'ler the dextrins do is doubtful. The formula for dextrose is $\mathrm{C}_{6} \mathrm{H}_{12} \mathrm{O}_{6}$; it is more simple than that of starch or dextrin and contains an additional $\mathrm{H}_{2} \mathrm{O}$ for every $\mathrm{C}_{6}$. Unlike starch and dextrin it can be obtained in a crystalline form, either from aqueous solutions (it being readily soluble in water), in which case the crystals contain water of crystallisation, or from its solutions in alcohol (in which it is sparingly soluble), in which case the crystals have no such water of crystallisation. Solutions of dextrose have a marked dextrorotatory power over rays of light.

4. Ir ultose, very similar to dextrose, and like it capable of reducing cupric salts. The formula is somewhat different, being $\mathrm{C}_{12} \mathrm{H}_{22} \mathrm{O}_{11}$. Besides this, it differs from dextrose chiefly in its smaller reducing power, i.e. a given weight will not convert so much cupric oxide into cuprous oxide as will the same weight of dextrose, and in having a stronger rotatory action on rays of light. Like dextrose it can be crystallised, the crystals from aqueous solutions containing water of crystallisation.

Now when a quantity of starch is boiled with water we may recognise in the viscid imperfect solution, on the one hand the presence of starch, by the blue colour which the addition of iodine gives rise to, and on the other hand the absence of sugar (maltose, dextrose), by the fact that when boiled with Fehling's fluid no reduction takes place and̦ no cuprous oxide is precipitated.

If however the boiled starch be submitted for a while to the action of saliva, especially at a somewhat high temperature such as $35^{\circ}$ or $40^{\circ} \mathrm{C}$., it is found that the subsequent addition of iodine gives no blue colour at all, or very much less colour, shewing that the starch has disappeared or diminished; on the other hand the mixture readily gives a precipitate of euprous oxide when boiled 
with Fehling's fluid, shewing that maltose or dextrose is present. That is to say the saliva has converted the starch into maltose or dextrose. The presence of the previously absent sugar may also be shewn by fermentation and by the other tests for sugar Moreover, if an adequately large quantity of starclı be subjecterl to the charge, the sugar formed may be isolated, and its characters determined. When this is done it is found that while some dextrose is formed the greater part of the sugar which appears is in the form of maltose. As is well known, starch may by the action of dilute acid be converted into dextrin, and by further action into sugar; but the sugar thus formed is always wholly dextrose, and not maltose at all. The action of saliva in this respect differs from the action of dilute acid.

While the conversion of the starch by the saliva is going on the addition of iodine frequently gives rise to a red or violet colour instead of a pure blue, but when the conversion is complete no coloration at all is observed. The appearance of this red colour indicates the presence of dextrin (erythrodextrin); the violet colour is due to the red being mixed with the blue of still unchanged starch.

The appearance of dextrin shews that the action of the saliva on the starch is somewhat complex; and this is still further proved by the fact that even when the saliva has completed its work the whole of the starch does not reappear as maltose or dextıose. A considerable quantity of the other dextrin (achroodextrin) always appears and remains unchanged to the end; and there are probably several other bodies also formed out of the starch, the relative proportions varying according to circumstances The change therefore, though perhaps we may speak of it in a general way as one of hydration, cannot be exhibited under a simple formula, and we may rest content for the present with the statement that starch when subjected to the action of saliva is converted chiefly into the sugar known as maltose with a comparatively small quantity of dextrose and to some extent into achroodextrin (erythrodextrin appearing temporarily only in the process), other bodies on which we need not dwell being formed at the same time.

Raw unboiled starch undergoes a similar change but at a much slower rate. This is due to the fact that in the euriously formed starch grain the true starch, or granulose, is invested with coats of cellulose. This latter material, which requires previous tieatment with sulphuric acid before it will give the blue reaction on the addition of iodine, is apparently not acted upon by saliva. Hence the saliva can only get at the granulose by traversing the coats of cellulose, and the conversion of the former is thereby much hindered and delayed.

$\$ 177$. The conversion of stach into sugar, and this we may speak of as the amylolytic action of saliva. will go on at the ordinary 
temperature of the atınosphere. The lower the temperature the slower the change, and at about $0^{\circ} \mathrm{C}$. the conversion is indefinitely prolonged. After exposure to this cold for even a consideralle time the action recommences when the temperature is again raised. Increase of temperature up to about $35^{\circ}-40^{\circ}$, or even a little higher, favours the change, the greatest activity being said to be manifested at about $40^{\circ}$. Much beyond this point, however, increase of temperature becomes injurious, markedly so at $60^{\circ}$ or $70^{\circ}$; and saliva which has been boiled for a few minutes not only has no action on starch while at that temperature, but does not regain its powers on cooling. By being boiled, the amylolytic activity of saliva is parm mently destroyed.

The action of saliva on starch is most rapid when the reaction of the mixture is neutral or nearly so; it is hindered or arrested by a distinctly acirl reaction. Indeed the presence of even a very small quantity of free acid, at all events of hydrochloric acid, at the temperature of the body not only suspends the action but. speedily leads to permanent abolition of the activity of the juice. The bearing of this will be seen later on.

The action of saliva is hampered by the presence in a concentrated state of the product of its own action, that is, of sugar. If a small quantity of saliva be added to a thick mass of boiled stareh, the action will after a while slacken, and eventually come to almost a stand-still long before all the starch has been converted. On diluting the mixture with water, the action will recommence. If the products of action be removed as soon as they are formed, by dialysis for example, a small quantity of saliva will, if sufficient time be allowed, convert into sugar a very large, one might almost say an indefinite, quantity of starch. Whether the particular constituent on which the activity of saliva depends is at all consumed in its action has not at present been definitely settled.

On what constituent do the anylolytic virtues of saliva depend?

If saliva, filtered and thus freed from much of its mucin and from other formed constituents, be treated with ten or fifteen times its bulk of alcohol, a precipitate is formed containing besides other substances all the proteid matters. Upon standing under the alcohol for some time (several (lays), the proteids thus precipitated become coagulated and insoluble in water. Hence, an aqueous extract of the precipitate, made after this interval, contains very little proteid material; yet it is exceedingly active. Moreover by other more elaborate methods there may be obtained from saliva solutions which appear to be almost entirely free from proteids and yet are intencely amylolytic. But even these probably contain other bolie; hesides the really active constituent. Whatever the active substance be in itself, it exists in such extremely small quantities that it has never yet been satisfactorily isolated; and indeed the only clear evidence we have of its existence is the manifestation of its peculiar powers. 
The salient features of this body, this amylolytic agent, which we may call ptyalin, are then :- 1 st, its presence in minute and ahmost inappreciable quantity. 2nd, the close dependence of its activity on temperature. 3rd, its permanent and total destruction by a high temperature and by various chemical reagents. $4 t h$, the want of any clear proof that it itself undergoes any change during the manifestation of its jower's ; that is to say, the energy necessary for the transformation which it effects does not come out of itself; if it is all used up in its action, the loss is rather that of simple wear and tear of a machine than that of a substance expended to do work. 5th, the action which it induces is probably of such a kind (splitting up of a molecule with assumption of water) as is affected by that particular class of agents called "hydrolytic."

These features mark out the amylolytic active body of saliva as belonging to the class of ferments; 1 and we may henceforward speak of the amylolytic ferment of saliva. The fibrin-ferment (\$ 20) is so called because its action in many ways resembles that of the ferment of which we are now speaking.

$\$ 178$. Mixed saliva, whose properties we have just discussed, is the result of the mingling in various proportions of saliva from the parotid, submaxillary, and sublingual glands with the secretion from the buccal glands. These constituent juices have their own special characters, and these are not the same in all animals. Moreover in the same individual the secretion differs in composition and properties according to circunstances; thus, as we shall see in detail hereafter, the saliva from the submaxillary gland secreted under the influence of the chorda tympani nerve is different from that which is obtained from the same gland by stimulating the sympathetic nerve.

In man pure parotid saliva may easily be obtained by introducing a fine cannula into the opening of the Stenonian duct, and submaxillary saliva, or rather a mixture of submaxillary and sublingual saliva, by similar catheterisation of the Whartonian duct. In animals the duct may be dissected ont and a cannula introduced.

Parotid saliva in man is clear and limpid, not viscid; the reaction of the first drops secreted is often acid, the succeeding portions,

1 Ferments may, for the present at least, be divided into two classes, commonly called moanised and unorqamsed. () the former, yeast may be taken as a wellknown example The fermentative activity of veast which leads to the conversion of sugar into alcohol, is dependent on the life of the yeast-cell. Unless the yeastcell he living and functional, fermentation does not take place; when the yeastcell dies fermentation ceases: and no substance obtained from the fluid parts of yeast, by precipitation with alcohol or otherwise, will give rise to alcololic fermentation. The salivary ferment helongs to the later class; it is a substance, not a living organison like yeast. It mav he added however that nossibly the organised ferment, the yeast for instance, produces its effect by means of an ordinary unorganised ferment which it generates, hut which is immediately made away with. 
at all events when the flow is at all copious, are alkaline; that is to say the natural secretion is alkaline, but this may be obscured by acid changes taking place in the fluid which has been retained in the duct, possibly by the formation of an excess of carbonic acid. On standing, the clear thuid becomes turbid from a precipitate of calcic carbonate, due to an escape of carbonic acid. It contains globulin and some other forms of albumin, with little or no mucin. Potassium sulphocyanate may also sometimes be detected, but structural elements are absent.

Submaxillary saliva, in man and in most animals, differs from parotid saliva in being more alkaline and, from the presence of mucin, more viscid; it contains salivary corpuseles, that is bodies closely rusembling if not identical with leucocytes, and, often in abundance, amorphous masses. The so-called chorda saliva in the dog, that is to say saliva obtained by stimulating the chorda tympani nerve, (of which we shall presently speak), is under ordinary circumstances thinner and less viscid, contains less mucin, and fewer structural elements, than the so-callerl sympathatic saliva, which is remarkable for its viscidity, its structural elements, and for its larger total of solids.

Sublingual saliva is more viscid, and contains more salts (in the dog about 1 p.c.), than the submaxillary saliva.

The action of saliva varies in intensity in different animals. Thus in man, the pig, the guinea-pig, and the rat, both parotid and submaxillary and mixed saliva are amylolytic; the submaxillary saliva being in most cases more active than the parotid In the rabbit, while the submaxillary saliva has scarcely any action, that of the parotid is energetic. The saliva of the cat is much less active than the above; that of the dog is still less active, indeed is almost inert. In the horse, sheep, and ox, the amylolytic powers of either mixed saliva, or of any one of the constituent jnices, are extremely feeble.

Where the saliva of any gland is active, an aqueous infusion of the same gland is also active. The importance and bearing of this statement will be seen later on. From the aqueous infusion of the gland, as from saliva itself, the ferment may be approximately isolated. In some cases at least some ferment may be extracted from the gland even when the secretion is itself inactive. In fact a ready method of preparing a highly amylolytic liquid tolerably free from proteid and other impurities, is to mince finely a gland known to have an active secretion, such for instance as that of a rat, to dehydrate it by allowing it to stand under absolute alcohol for some days, and then, having poured off most of the alcohol, and removed the remainder by evaporation at a low temperature, to cover the pieces of gland with strong glycerine. Though some of the ferment appears to be destroyed by the alcohol a mere drop of such a glycerine extract rapidly converts starch into sugar. 


\section{Gastric Juice.}

$\$$ 179. There is no difficulty in obtaining what may fairly be considered as a normal saliva; but there are many obstacles in the way of determining the normal characters of the secretion of the stomach. When no food is taken the stomach is at rest and no secretion takes place. When food is taken, the characters of the gastric juice secreted are obscured by the food with which it is mingled. The yastrie nembrane may it is true be artificiall: stimulated, by touch for instance, and a secretion obtained. This we may speak of as gastric juice, but it may be dombted whether it ought to be considered as normal gastric juice. And indeed as we shall see even the juice, which is poured into the stomach during a meal, varies in composition as digestion is going on. Hence the characters which we shall give of gastric juice nust be considered as having a general value only.

Gastric juice, obtained in as normal a condition as possible from the healthy stomach of a fasting dog, by means of a gastric fistula, is a thin almost colourless Huid with a sour taste and odour.

In the operation for gastric fistula, an incision is made through the abdominal walls, along the linea alla, the stomach is opened, and the lips of the gastric wound securely sewn to those of the incision in the abdominal walls. Union soon takes place, so that a permanent opening from the exterior into the inside of the stomach is established. A tube of proper construction, introduced at the time of the operation, becomes firmly secured in place by the contraction of healing. Through the tube the contents of the stomach can be received, and the mucous membrane stimulated at pleasure.

When oltained from a natural fistula in man, its specific gravity has been found to differ little from that of water, varying from 1.001 to 1.010 , and the amount of solids present to be correspondingly small. In animals, pure gastric juice seems to be equally poor in solids, the higher estimates which some observers have ohtained being probably due to admixture with food, \&c.

of the solid matters present about half are inorganic salts, chiefly alkaline (sodimm) chlorides, with small quantities of phospluates. The organic material consists of pepsin, a body to be described immediately, mixed with other substances of undetermined nature. In a healthy stomach gastric juice contains a very small quantity only of mucin, unless some submaxillary saliva has been swallowed.

The reaction is distinctly acid, and the acidity is nomally due to free hydrochloric acid. This is shewn by varions proofs, among which we may mention the conclusive fact that the amount of chlorine present in gastric juice is more than wonld suffice to form "lllorides with all the bases present, and that the excess if regarded as existing in the form of hydrochloric acid 
corresponds exactly to the quantity of free acid present. Lactic and butyric and other acids when present are secondary products, arising either by their respective fermentations from articles of food, or from the decomposition of their alkaline or other salts. In man the amount of free hydrochloric acid in healthy juice may be stated to be about 2 per cent., but in some animals it is probably higher.

$\$$ 180. On starch gastric juice has no amylolytic action; on the contrary when saliva is mixed with gastric juice any anylolytic ferment which may be present in the former is at once prevented from acting by the acidity of the mixture. Moreover in a very short time, especially at the temperature of the body, the amylolytic ferment is destroyed by the acid so that even on neutralisation the mixture is unable to convert starch into sugar.

On dextrose healthy gastric juice has no effect. And its power of inverting cane-sugar seems to be less than that of hydrochloric acid diluted to the same degree of acidity as itself. In an unhealthy stomach however containing much mucus, the gastric juice is very active in converting cane-sugar into dextrose. This power seems to be due to the presence in the mucus of a special ferment, analogous to, but quite distinct from, the ptyalin of saliva. An excessive quantity of cane-sugar introduced into the stomach causes a secretion of mucus, and hence provides for its own conversion.

On fats gastric juice has at most a limited action. When adipose tissue is eaten, the chief change which takes place in the stomach is that the proteid and gelatiniferous envelopes of the fat-cells are dissolved, and the fats set free. Though there is experimental evidence that emulsion of fats to a certain extent does take place in the stomach, the great mass of the fat of a meal is not so changed.

Such minerals as are soluble in free hydrochloric acid are for the most part dissolved; though there is a difference in this and in some other respects between gastric juice and simple free hydrochloric acid diluted with water to the same degree of acidity as the juice, the presence either of the pepsin or of other bodies apparently modifying the solvent action of the acid.

The essential property of gastric juice is the power of dissolving proteid matters, and of converting them into a substance called peptone.

Action of gastric juice on proteids. The results are essentially the same whether natural juice obtained hy means of a fistula or artificial juice, i.e. an acid infusion of the mucous membrane of the stomach, be used.

Artificial gastric juice may be prepared in any of the following ways.

1. The mucous membrane of a pig's or dog's stomach is removed from the muscular coat, finely minced, rubbed in a mortar with 
pounded glass and extracted with water. The aqueous extract filtered and acilnlated (it is in itsolf somewhat acid) until it has a free acidity corresponding to 2 p.c. of hydrochloric acid, contains but little of the products of digestion such as peptone, but is fairly potent.

2 . The mucous membrane similarly prepared and minced is allowed to digest at $35^{\circ} \mathrm{C}$. in a large quantity of hydrochloric acid diluted to 2 p.c. The greater part of the membrane disappears, shreds only being left, and the somewhat opalescent liquid can be decanted and filtered. The filtrate has powerful digestive (peptic) properties, but contains a considerable amount of the products of digestion (peptone, Sc.), arising from the digestion of the mucous membrane itself. ${ }^{1}$

3. The mucous membrane, similarly prepared and minced, is thrown into a coniparatively large quantity of concentrated glycerine, and allowed to stand. The membrane may be previously dehydrated by being allowed to stand under alcohol, but this is not necessary, and a too prolonged action of the aleohol injures or even destroys the activity of the product. The decanted clear glycerine, in which a comparatively small quantity of the ordinary proteids of the mucous membrane are dissolved, if acked to hydrochloric acid of 2 p.c. (about 1 c.c. of the glycerine to 100 c.c. of the dilute acid is sufficient), makes an artificial juice tolerably free from ordinary proteids and peptone, and of remarkable potency, the presence of the glycerine not interfering with the results.

Before proceeding to study the action of gastric juice on proteids it will be useful to review very briefly the chief characters of the more important members of the group.

The more important proteids which we have thus far studied are: 1. Fibrin, insoluble in water and not really soluble (i.e. without change) in saline solutions. 2. Myosin, insoluble in water but soluble in saline solutions, provided these are not too dilute or too concentrater. 3. Globnlin (including paraglobulin, filringgen \&c.), insoluble in water, but readily soluble in even very dilute saline solutions. 4. Albumin, serum-albumin, soluble in water in the absence of all salts. 5. Acid-albumin, into which glolunlins and myosin are rapidly comverted by the action of dilute acids, the particular acid-albunin into which the myosin of muscle is clanged being sometimes called syntonin. If the reagent used be not dilute acid but dilute alkali, the product is called alkalialbumin. The two hodies, acid-albumin and alliali-allumin, ane very parallel in their characters, and may readily be converted the one into the other by the use of dilute alkali or dilute acid respectively. Their most important common characters are insolulility in water and in saline solutions and ready solubility in dilute acids and alkalis. 6. Congulated proteids. As we have seen, when fibrin suspended in water, serum-albumin in solution,

1 These however may be removed by concentration at $40^{\circ} \mathrm{C}$. and subsequent dialysis. 
acid-albumin or alkali-albumin suspended in water, or paraglobulin suspended in water or dissolved in a dilute saline solution, are heated to a temperature, which for the whole group may be put down at about $75^{\circ}-80^{\circ} \mathrm{C}$., each of them becomes coagulated, and after the change is insoluble in water, saline solutions, dilute acids \&c., in fact in everything but very strong acids. Myosin and fibrinogen undergo a similar change at a lower temperature, viz. about $56^{\circ} \mathrm{C}$. IVe may, for present purposes, speak of all these proteids thus changed under the one term of coagulated proteids.

To the above list we may now add two other proteids, viz.: 7. A kind of albumin which forms the great bulk of the proteid matter present in raw ' white of egg,' and which, since it differs in minor characters from the albumin of blood and of the tissues, is called egg-albumin. 8. The peculiar proteid casein, an important constituent of milk. This may perhaps be regarded as a naturally occurring alkali-albumin since it has many resemblances to the artificial alkali-albumin ; but for several reasons it is desirable to consider it as an independent body.

Egrr-albumin like serum-albumin becomes coagulated at a temperature of about $75^{\circ}-80^{\circ} \mathrm{C}$., and though casein as it naturally exists in milk is not coagulatel on boiling, when separated out in a special way, and suspended in water in which it is insoluble, it becomes coagulated at about $75^{\circ}-80^{\circ} \mathrm{C}$.

It will be observed that all these proteids form, as regards their solubilities, a descending series, in the following order. Coagulated Proteids. Fibrin. Acil-albumin with Alkali-albumiı, and Cåsein. Myosin, Globulins. Serum-albumin with Eggalbumin.

We must now return to the action of gastric juice.

If a few shreds of fibrin, obtained by whipping blood, after being thoroughly washed and boiled and thus by the boiling coagulated, be thrown into a quantity of gastric juice, and the mixture be exposed to a temperature of from $35^{\circ}$ to $40^{\circ}$ C., the fibrin will speedily, in some cases in a few minutes, be dissolved. The shreds first swell up and become transparent, then gradually dissolve, and finally disappear with the exception of some granular débris, the amount of which, though generally small, varies according to circumstances. If raw, that is unboiled, uncoagulated fibrin be employed the same changes may be observed, but they take place much more rapidly.

If small morsels of coagulated albumin, such as white of egg, be treated in the same way, the same solution is observed. The pieces become transparent at their surfaces; this is especially seen at the edges, which gradually become rounded down; and solution steadily progresses from the outside of the piece inwards.

If any other form of coagulated albumin (e.g. precipitated acid- or alkahi-albumin. suspenderl in water and boiled) be treated 
in the same way, a similar solution takes place. The readiness with which the solution is effected, will depend, cateris paribus, on the smallness of the pieces, or rather on the amount of surface as compared with bulk, which is presented to the action of the juice.

Gastric juice then readily dissolves coagulated proteids, which otherwise are insoluble, or soluble only, and that with difficulty, ill very strong atids.

When proteids, which are soluble in water or in dilute acid, are treated with gastric juice, no visible ehange takes place; but nevertheless, it is found on examination that the solutions have undergone a remarkable change, the natue of which is easily seen by contrasting it with the change effected by dilute acid alone. If raw white of egg, largely diluted with water and strained, be treated with a sufficient quantity of dilute hydrochloric acid, the opalescence or turbidity which appeared in the white of egr on dilution (and which is due to the precipitation of various forms of globulin accompanying the ego-albumin in the raw white) disappears, and a clear mixture results. If a portion of the mixtmre be at once boiled, a large deposit of coagulated albumin occurs. If, however, the mixture be exposed to $50^{\circ}$ or $55^{\circ} \mathrm{C}$. for some time, the amount of coagulation which is produced by boiling a specimen becomes less, and, finally, boiling produces no coagulation whatever. By neutralisation, however, the whole of the albumin (with such restrictions as the presence of certain nentral salts may cause) may be obtained in the form of acid-albumin, the filtrate after neutralisation containing no proteids at all (or a very small quantity). Thus the whole of the albumin present in the white of egg may be, in time, converted, by the simple action of dilute hydrochloric acid, into acid-albumin. Serum-albumin similarly treated nudergoes, in course of time, a similar conversion into acid-albumin, and we have already seen (\$56) that solutions of myosin or of any of the globulins are with remarkable rapidity converted into acid-albumin. Thus simple dilute hydrochloric of the same degree of acidity as gastric juice, merely converts these proteids into acid-allumin, the rapidity of the change differing with the different proteids, being in some cases very slow, and requiring a relatively high temperatmre.

If the same white of egg or serum-albumin be treated with gastric juice instead of simple dilute hydrochloric acid, the events for some time seem the same. Thus after a while boiling causes no coasulation, while neutralisation gives a considerable precipitate of a proteid body, which, being insoluble in water and in sodium chloride solutions, and soluble in dilute alkali and acirls, at least closely resembles acid-albumin. But it is found that only a portion of the proteid originally present in the white of egg or serum-albumin can thus be regained by precipitation. Though the neutralisation be carriel out with the greatest care it will bs 
found, on filtering off the neutralisation precipitate, that the filtrate, as shewn on employing the various tests for proteid (see $\$ 15)$ or on adding an adequate quantity of strong alcohol, still contains a very considerable quantity of proteid matter; and, on the whole, the longer the digestion is carried on, the greater is the proportion borne by the proteid remaining in solution to the precipitate thrown down on neutralisation; indeed, in some cases at all events, all the proteid matter originally present remains in solution, and there is no neutralisation precipitation at all, or at most a wholly insignificant one.

$\$ 181$. The proteid matter, thus remaining in solution after neutralisation, differs from all the proteids which we lave hitherto studied in as much as, though existing in a neutral solution, it is not coagulated by heat, like the ego-albumin or serum-albumin from which it has been produced; the solution, after the neutralisation precipitate has been filtered off, remains quite clear when boiled. The only other solutions of proteids which do not coagulate on boiling are solutions of acid or alkali-albumin; but these solutions must be acid or alkali respectively; the acid-albumin or alkali-albumin is insoluble in a neutral solution, and when simply suspended in water is readily coagulated at a temperature of $75^{\circ}$. This new proteid matter of which we are sperking is soluble in neutral solutions, indeed in distilled water, and can under no circumstances be coagulated by heat.

Upon examination we find that the new proteid matter thus left in solution consists of at least two distinct proteid bodies. If to the solution neutral ammonium sulphate be added to saturation, part of the proteid matter is precipitated while part is still left in solution. The proteid body thus thrown down is ealled albumose. The bolly which is not thrown down by ammonium sulphate is called peptone. Now peptone is characterised by being diffusible; it will pass through membranes. The diffusion is not nearly so rapid as that of salts, sugar, and other similar substances; indeed solutions of peptones may be freed from salts by dialysis. But it is very marked as compared with that of other proteids; these pass through membranes with the greatest difficulty, if at all. Peptone is insoluble in alcohol, and may be precipitated from its solutions by the addition of an adequate quantity of this reagent; but for this purpose a very large excess of alcohol is needed, otherwise much of the peptone remains in solution. It may be kept under alcohol for a long time without undergoing change, whereas other proteids are more or less slowly coagulated by alcohol. A useful test for peptone is furnished by the fact that a solution of peptone, mixed with a strong solution of caustic potash, gives on addition of a mere trace of cupric sulphate in the cold a pink colour, whereas other proteids give a violet colour. In applying this test, known as the 'biuret' test, nowever, care must be taken not to add too much cupric sulphate 
since in that case a violet colour, deepening on boiling, that is the ordinary proteid reaction, is obtained. There are reasons for thinking that there are several kinds or at least more than one kind of peptone; but we may for the present speak of the substance as one.

Albunnose differs from peptone, not only in being precipitated by ammonium sulpliate but also in being much less difíusible, and in other minor characters. Albumose like peptone gives the biuret reaction. Te are able to distinguish several kinds of albumose, but into the details of these we need not enter. The amount of albumose appearing in a digestion experiment, relative to the amount of true peptone, depends on the activity of the juice, and other circunstances. We may regard albumose as a less complete product of digestion than peptone. For a long time albumose was confounded with peptone, and many of the commercial forms of "peptone" consist largely of albumose.

When fibrin, either raw or boiled, or any form of coagulated proteid is dissolved and seems to disappear under the influence of gastric juice, the same products, albumose and peptone, make their appearance. The same borlies result when myosin or any one of the globulins or acid-albumin or alkali-albumin is subjected to the action of the juice.

liesides albumose other bodies, which may also be regarded as less complete products of digestion, make their appearance, to a variable extent under different circumstances when proteid is digested with gastric juice. On these bodies however, known as parapeptone and by other names, we need not dwell.

It is obvious that the effect of the action of the gastric juice is to change the less soluble proteid into a more soluble form, the change being either completed up to the stage of peptone, the most soluble of all proteids, or being left in part incomplete. This will be seen from the following tabular arrangement of proteids according to their solubilities.

\section{Soluble in distilled water.}

Aquenus solutions not coagulated on boiling. Diffusible . . . . . . . . . Peptone.

Much less diffusible . . . . . . Albumose. Aqueous solutions coagulated on boiling . Albumin.

\section{Insoluble in distilled water.}

Rearlily soluble in dilute saline solutions

( $\mathrm{NaCl} 1$ per cent.)

Globulins.

Soluble only in stronger saline solutions ( $\mathrm{NaCl} 5$ to 10 p.c.) . . . . . . Myosin. 


\section{Insoluble in dilute saline solutions.}

Readily soluble in dilute acid ( $\mathrm{HCl} \cdot 1$ p.c.) in the cold

Soluble with difficulty in dilute acid, that is at high temperature $\left(60^{\circ}\right.$ C. $)$ and after prolonged treatment only .

Insoluble in dilute acids, soluble only in strong acids $\left\{\begin{array}{l}\text { Acid-albumin. } \\ \text { Alkali-albumin. } \\ \text { Casein. }\end{array}\right.$

Fibrin.

Milk when treated with gastric juice is first of all "curdled." This is the result partly of the action of the free acid but chiefly of the special action of a particular constituent of gastric juice, of which we shall speak hereafter. The curd consists of a particular proteid matter mixed with fat; and this proteid matter is subsequently dissolved with the same appearance of peptore, albumose and other bodies as in the case of other proteids. In fact, the digestion by gastric juice of all the varieties of proteids consists in the conversion of the proteid into peptone, with the concomitant appearance of a certain variable amount of albumose and other bodies.

$\$$ 182. Circumstances affecting gastric digestion. The solvent action of gastric juice on proteids is modified by a variety of circunstances. The nature of the proteid itself makes a difference, though this is determined as well by physical as by chemical characters. Hence in making a series of comparative trials the same proteid should be used, and the form of proteid most convenient for the purpose is fibrin. If it be desired simply to ascertain whether any given specimen has any digestive powers at all, it is best to use boiled fibrin, since raw fibrin is eventually dissolved by dilute hydrochloric acid alone, probably on accomnt of some pepsin previously present in the blond becoming entangled with the fibrin during clotting. Bnt in estimating quantitatively the peptic power of two specimens of gastric juice under different conditions, raw fibrin prepared by Grutzner's method is the most convenient.

Portions of well washed fibrin are stained with carmine and again washed to remore the superfluous colouring matter. A fragment of this coloured fibrin thrown into an active juice on becoming dissolved, gives up its colour to the fluid. Hence if the same stock of coloured fibrin be used in a series of experiments, and the same bulks of fibrin and of fluid be used in each case, the amount of fibrin dissolved may be fairly estimated by the depth of tint given to the fluid. Fibrin thus coloured with earmine may be preserved in ether.

Since, if sufficient time be allowed, even a small quantity of gastric juice will dissolve at least a very large if not an indefinite 
quantity of fibrin, we are led to take, as a measure of the activity of a specimen of gastric juice, not the quantity of fibrin which it will ultimately dissolve, but the rapidity with which it dissolves a given quantity.

The greater the surface presented to the action of the juice, the more rapid the solution ; hence minute division and constant movement favour digestion. And this is probably, in part at least, the reason why a fragment of spongy filamentous fibrin is more readily dissolved than a solid chump of boiled white of egg of the same size. Neutralisation of the juice wholly arrests digestion; fibrin may be submitted for an almost indefinite time to the action of neutralised gastric juice without being digested. If the neutralised juice be jroperly acidified, it may again become active; when gastric juice however has been made alkaline, and kept for some time at a temperature of $35^{\circ}$, its solvent jowers are not only suspended but actually destroyed. Digestion is most rapid with dilute hydrochloric acid of 2 p.c. (the acidity of natural gastric juice). If the juice contains much more or much less free acid than this, its activity is distinctly impaired. Other acids, lactic, phosphoric, \&c. may be substituted for hydrochloric; but they are not so effectual, and the degree of acidity most useful varies with the different acids. The presence of neutral salts, such as sodiun chloride, in excess is injurious. The action of mammalian gastric juice is most rapid at $35^{\circ}-40^{\circ} \mathrm{C}$.; at the ordinary temperature it is much slower, and at about $0^{\circ} \mathrm{C}$. ceases altogether. The juice may be kept however at $0^{\circ} \mathrm{C}$. for an indefinite period without injury to its powers. The gastric juice of cold-blooded vertebrates is relatively more active at low temperatures than that of warmblooded mammals or birds.

At temperatures much above $40^{\circ}$ or $45^{\circ}$ the action of the juice is impaired. By boiling for a few minutes the activity of the most powerful juice is irrevocably destroyed. The presence in a concentrated form of the prodncts of digestion hinders the process of solution. If a large quantity of fibrin be placed in a sniall quantity of juice, digestion is soon arrested ; on dilution with the normal hydrochloric acid ( $\cdot 2$ p.c.), or if the mixture be submitted to dialysis to remove the peptones formed, and its acidity be kept up to the normal, the action recommences. By removing the products of digestion as fast as they are formed, and by keeping the acidity il) to the normal, a given amount of gastric juice may be made to digest a very large quantity of proteid material. Whether the quantity is really unlimited is disputed; but in any case the energies of the juice are not rapidly exhansted by the act of digestion.

\$183. Nature of the action. All these facts go to shew that the digestive action of gastric juice on proteids, like that of saliva on starch, is a ferment-action; in other words, that the solvent action of gastric juice is essentially due to the presence in it of a 
ferment-body. To this ferment-body, which as yet has been only approximately isolated, the name of pepsin has been given. It is present not only in gastric juice but also in the glands of the gastric nucous nembrane, especially in certain parts and under certain conditions which we shall stndy presently. Concerning its exact nature we cannot make any definite statement; we have no absolute proof that it is a proteid, probable as this may seem. We are as yet unable to define it by any chemical characters. At present the manifestation of peptic powers is our only safe test of the presence of pepsin.

In one impurtant respect pepsin, the ferment of gastric juice, differs from ptyalin, the ferment of saliva. Saliva is active in a perfectly neutral medium, and there seems to be no special connection between the ferment and any alkali or acid. In gastric juice, however, there is a strong tie between the acid and the ferment, so strong that some writers speak of pepsin and hydrochloric acid as forming together a componnd, pepto-hydrochloric acid.

In the absence of exact knowledge of the constitution of proteids, we camnot state distinctly what is the precise nature of the change into peptone; the various proteids differ from each other in elementary composition quite as widely as does peptone from any of them. Judging from the analogy with the action of saliva on starch, we may fairly suppose that the process is at bottom one of hydration; and this view is further suggested by the fact that peptone closely resembling, if not identical with, that obtained by gastric digestion, may be obtained by the action of strong acids, by the prolonged action of clilute acids especially at a high temperature, or simply by digestion with super-heated water in a Papin's digester, that is to say by means of agents which, in other cases, produce their effects by bringing about hydrolytic changes.

$\$ 184$. All proteids, so far as we know, are converted by pepsin into peptone. Concerning the action of gastric juice on other nitrogenous substances more or less allied to proteids but not truly proteid in nature our knowledge is at present imperfect. Mucin, nuclein, and the chemical basis of horny tissues are wholly unaffected by gastric juice. The gelatiniferous tissues are dissolved by it; and the bundles and membranes of connective tissue are very speedily so far affected by it, that at a very early stage of digestion, the bundles and elementary fibres of muscle which are bound together by connective tissue fall asunder; moreover both prepared gelatine and the gelatiniferous basis of connective tissue in its natural condition, that is without being previously heated with water, are by it changed into a substance so far analogous with peptone, that the characteristic property of gelatinisation is entirely lost. Chondrin and the elastic tissues undergo a similar change.

\$185. Action of gastric juice on milk. It has long been 
known that an infusion of ealves' stomach, called rennet, has a remarkable effect in rapidly curdling milk, and this property is made use of in the manufacture of cheese. Gastric juice has a similar effect; milk when subjected to the action of gastric juice is first curdled and then digested. If a few drops of gastric juice be added to a little milk in a test-tube, and the mixture exposed to a temperature of $40^{\circ}$, the milk will curdle into a complete clot in a very short time. If the action be continued the curd or clot will be ultimately dissolved and digested. Milk contains, lesides a peculiar form, or peculiar forms of albumin, fats, milk-sugar and various salines, the peculiar proteid casein. In natural milk casein is present in solution, and 'curdling' consists essentially in the soluble casein being converted (or more probably as we shall see presently, split up) into an insoluble modification of casein, which as it is being precipitated carries down with it a great deal of the fat and so forms the 'curd.' Now casein is readily precipitated from milk upon the addition of a small quantity of acid, and it might le supposed that the curdling eflect of gastric juice was due to its acid reaction. But this is not the case, for neutrahsed gastric juice, or neutral rennet, is equally efficacious.

The curdling action of rennet is closely dependent on temperature, being like the peptic action of gastric juice favoured by a ise of temperature up to about $40^{\circ}$. Mioreover the curdling action is destroyed by previous boiling of the juice or rennet. These facts suggest that a ferment is at the bottom of the matter; and indeed, all the features of the action support this view. Moreover, as a matter of fact, a curdling ferment may be extracted by glycerme and by the other methods used for preparing ferments. The ferment, however, is not pepsin, but some other body; and the two may be separated from each other.

It might be thought that the rennet-ferment, rennin we may call it, acted by inducing a fermentation in the sugar of milk, giving rise to lactic acid which precipitated the casein by virtue of its being an acid. But this view is disproved by the following facts which shew that the ferment produces its curdling effect by acting. directly on the natural casein itself. Casein may be precipitated unchanged, that is, capable of redissolving in water (the presence of calcic phosphate being assumed) by saturating milk with neutral saline bodies (such as sodium chloride or magnesium sulphate); and by being precipitated and redissolved more than once may be obtained largely free from fat and wholly free from milk-sugar. Such solutions of isolated casein freed from milksugar may be made to curdle like natural milk by the addition of remnin, shewing that the milk-sugar has nothing to do with the matter. Morenver the precipitate thrown down from milk by dilute acids, lactic acid included, is itself unaltered or very slightly altered casein not curd, and with care may be so prepared as to be redissolved into solutions which curdle 
with rennin, like solutions of casein prepared by means of neutral salts.

When isolated casein is curdled by means of rennin two proteids, it is stated, make their appearance, one of which is soluble and allied to albumin, and another, which is insoluble and forms the curd. Curdling, therefore, according to this result appears to be the splitting up by a ferment of a more complex body; and it is interesting to observe, as perhaps throwing light on the somewhat analogous formation of fibrin, that this curdling action will not take place if calcic phosphate be wholly absent from the mixture. The calcic phosphate appears to play a peculiar part in determining the insolubility of the curd, for there is evidence that in the absence of calcic phosphate the ferment has power to attack the casein and split it up, but that both products remain in solution; if calcic phosphate be present, the one, viz. the curd, becomes insoluble.

Remnin is abundant in the gastric juice and in the gastric mucous membrane of ruminants, but is also found in the gastric juice of other animals, and either it, or what we shall presently have occasion to speak of as the antecedent of the ferment or zymogen, is present also in the mucous membrane of the stomach of most animals. A very similar if not identical ferment has also been found in many plants. 


\section{SEC. 2. THE ACT OF SECRETION OF SALIVA AND GASTRIC JUICE AND THE NERVOUS MECHANISMS WHICH REGULA'TE IT.}

$\$$ 186. The saliva and gastric juice whose jroperties we have studied, though so different from each other, are both drawn ultimately from one common source, the blood, and they are poured into the alimentary canal, not in a continuous flow, but intermittently as occasion may demand. The epithelimm cells which supply them have their periods of rest and of activity, and the amount and quality of the fluids which these cells secrete are determined by the needs of the economy as the food passes along the canal. We have now to consider low the epithelium cell manufactures its special secretion out of the materials supplied to it by the blood, and how the cell is called into activity by the presence of food, it may be as in the case of saliva at some distance from itself, or by circumstances which do not bear directly on itself. In dealing with these matters in connection with the digestive juices, we shall have to enter at some length into the physiology of secretion in general.

The question which presents itself first is: By what mechanism is the activity of the secreting cells bronght into play?

While fasting, a small quantity only of saliva is poured into the mouth; the buceal cavity is just moist and nothing more. When food is taken, or when any sapid or stimulating substance, or indeed a bolly of any kind, is introduced into the mouth, a flow is induced which may be very copious. Indeed the quantity secreted in ordinary life during 24 hours has been roughly calculated at as much as from 1 to 2 litres. An abundant secretion in the absence of food in the mouth may be called forth by an emotion, as when the mouth waters at the sight of food, or ly a smell, or by events occurring in the stomach, as in some cases of nansea. Evidently in these instances some nervous mechanism is at work. In studying the action of this nervous mechanism, it will be of advantage to confine our attention at first to the submaxillary gland. 
$\$ 187$. The submaxillary gland is supplied with two sets of nerves. These are represented in Fig. 76, which is a very diagrammatic rendering of the appearances presented when the submaxillary gland is prepared for an experiment in a dog, the animal being placed on its back and the gland exposed from the neck. The one set, and that the more important, belongs to the chorda tympani nerve $\left(c h . t^{\prime \prime}\right)$. This is a small nerve, which branches off from the facial or seventh cranial nerve in the Fallopian canal before the nerve issues from the skull. Whether it really belongs to the facial proper has been doubted; in man the fibres which form it are either fibres coming not from the roots of the facial proper, but from the portio intermedia Wrisbergi, or, according to some, fibres which though joining the facial in the Fallopian canal are ultimately derived from another (the fifth) cranial nerve. Leaving the facial nerve the chorda tympani passes through the tympanic cavity or drum of the ear (hence the name) and joins or rather runs in company (ch.t') with the lingual or gustatory branch of the fifth nerve. Some of the fibres run on with the lingual right down to the tongue (these are not shewn in the figure), but many leave the lingual as a slender nerve $(c h . t)$, which reaching Wharton's duct or duct of the submaxillary gland $(s m . d)$ runs along the duct to the gland. As the nerve courses along the duet nerve cells make their appearance among the tibres, and these are especially abundant just after the duct enters the hilus of the gland. The fibres may be traced into the gland for some distance, but as we have said their ultimate ending has not yet been definitely made out. Along its whole course up to the gl ind, the tibres of the chorda are very fine medullated fibres, but they lose their medulla in the gland.

The other set of nerve-fibres reaches the gland along the small arteries of the gland. These are non-medullated fibres mixed with a few medullated fibres and may be traced back to the superior cervical ganglion. From thence they may be traced still further back down the cervical sympathetic to the spinal cord, following apparently the same tract as the vaso-constrictor fibres, treated of in $\$ 144$.

$\$ 188$. If a tube be placed in the duct, it is seen that when sapid substances are placed on the tongue, or the tongue is stimulated in any other way, or the lingual nerve is laid bare and stimulated with an interrupted current, a copious flow of saliva takes place. If the sympathetic be divided, stimulation of the tongue or lingual nerve still produces a flow. But if the small chorta nerve be divided, stimulation of the tongue or lingual nerve produces no flow.

Evidently the flow of saliva is a nervous reflex action, the lingual nerve serving as the channel for the afferent and the small chorda nerve for the efferent impulses. If the trunk of the lingual be divided above the point where the chorda leaves 
it, as at $n . l^{\prime}$, Fig. 76 , stimulation of the (front part of) tongue produces, under ordinary circumstances, no flow. This shews that the centre of the reflex action is higher up than the point of section; it lies in fact in the brain.

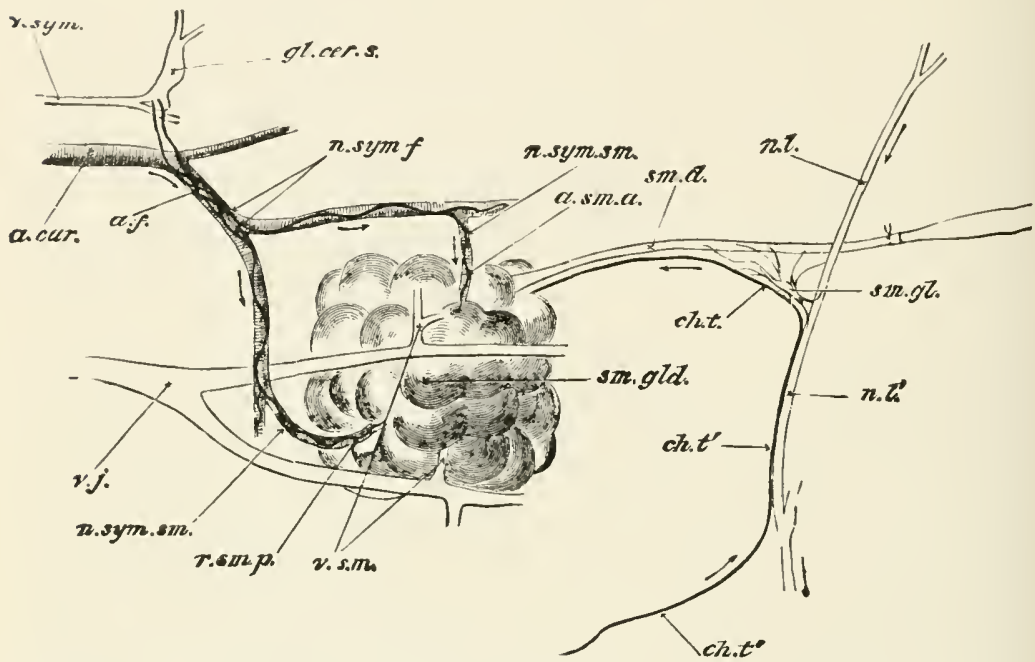

Fig. 76. Diagramatic Representation of the Submaxillary Gland of tine Dug Witi its Nerves and Blood Vessels.

(The dissection has been made on an animal lying on its back, but since all the parts shewn in the figure cannot be seen from any one point of view, the figure does not give the exact auatomical relations of the several structures.)

$s m . g / d$. The submaxillary gland, iuto the duct $(s m . d)$ of which a cannula las been tied. The sublingual gland and duct are not shewn. $n . l, n l^{\prime}$. 'The lingual branch of the fifth nerve, the part n.l. is going to the tongue. cht., ch.t'., ch.t'. "The chorda tympani. 'The part ch.t". is proceeding from the facial nerve; at ch.t'. jt becomes conjoined with the lingual n.l'. and afterwards divergiug passes as cht.t. to the gland along the duct; the continuation of the nerve in company with the lingual n.l. is not shewn. sm.gl. The submaxillary gangliou with its several roots. a.rar. 'The carotid artery, two small branches of which, $a$ sm.a. and $r^{*} . s m . p$. . pass to the anterior and posterior parts of the gland. r.s.m. The anterior aud posterior veins from the gland, falling into $v, j$. the jugular vein. $r$ sym. The conjoined vagus and sympathetic trunks. gl.cer.s. 'The upper cervical ganglion, two branches of which forming a plexus $(n . f$.$) over the facial artery, are distributed (n.sym.sm.)$ along the two glandular arteries to the anterior and posterior portions of the gland.

The arrows indicate the direction taken by the nervous impulses during reflex stimulation of the gland. They ascend to the brain by the liugual and descend by the chorda tympani.

In the angle between the lingual and the chorla, where the latter leaves the former to pass to the gland, lies the small submaxillary ganglion (represented diagrammatically in Fig. $76 \mathrm{sm} . g l$.). This consists of small masses of nerve cells lying on the small bundles of nerve-fibres which spread ont like a fan from the lingual and chorda tympani 
nerves (ch.t.) towards the ducts of the submaxillary and sublingual glands. It has been much debated whether this ganglion can act as a centre of reflex action in connection with the submaxillary gland, but no conclusive evidence that it does so act has as yet been shewn; it probably belongs in reality to the sublingual gland.

Stimulation of the glossopharyngeal is even more effectual than that of the lingual. Probably this indeed is the chi-1 afferent nerve in ordinary secretion. Stimulation of the mucous membrane of the stomach (as by food introduced through a gastric fistula) or of the vagus may also produce a flow of saliva, as indeed may stimulation of the sciatic, and probably of many other afferent nerves. All these cases are instances of reflex action, the cerebro-spinal system acting as a centre. We may further define the centre as a part of the medulla oblongata, apparently not far removed from the vaso-motor centre. When the brain is renoved down to the medulla oblongata, that organ being left intact, a flow of saliva may still be obtained by adequate stimulation of various afferent nerves; when the medulla is destroyed no such action is possible. And a flow of saliva may be produced by direct stimulation of the medulla itself. When a flow of saliva is excited by ideas, or by emotions, the nervous processes begin in the higher parts of the brain, and descend thence to the medulla before they give rise to distinctly efferent impulses; and it would appear that these higlier parts of the brain are called into action when a flow of saliva is excited by distinct sensations of taste.

Considering then the flow of saliva as a reflex act the centre of which lies in the medulla oblongata, we may imagine the efferent impulses passing from that centre to the gland either by the chorda tympani or by the sympathetic nerve. Although it would perhaps be rash to say that in this relation the sympathetic nerve never acts as an efferent channel, as a matter of fact we have no satisfactory experimental evidence that it does so; and we may therefore state that, practically, the chorda tympani is the sole efferent nerve. Section of that nerve, either where the fibres pass from the lingual nerve and the submaxillary ganglion to the gland, or where it rums in the same sheath as the lingual, or in any part of its course from the main facial trunk to the lingual, puts an end, as far as we know, to the possibility of any flow being excited by stimuli applied to the sensory nerves, or to the sentient surfaces of the mouth or of other parts of the body.

The natural reflex act of secretion may be inhibited, like the reflex action of the vaso-motor nerves, at its centre. Thus when, as in the old rice ordeal, fear parches the mouth, it is probable that the afferent impulses caused by the presence of food in the mouth cease, through emotional inhibition of their reflex centre, to give rise to efferent impulses.

$\$$ 189. In life, then, the flow of saliva is brought about by the 
advent in the gland along the chorda tympani of efferent impulses, started chiefly by reflex actions. The inquiry thus narrows itself to the question: In what manner do these efferent impulses cause the increase of flow?

If in a dog a tube be introduced into Wharton's duct, and the chorda be divided, the How, if any be going on, is from the lack of efferent impulses arrested. On passing an interrupted current through the peripheral portion of the chorda, a copious secretion at once takes place, and the saliva begins to rise rapidly in the tube; a very short time after the application of the current the flow reaches a maximum which is maintained for some time, and then, if the current be long continued, gradually lessens. If the current be applied for a short time only, the secretion may last for some time after the current has been shut off. The saliva thus obtained is but slightly viscid, and under the microscope a very few salivary corpuseles, and, occasionally only, amorphous lum s of peculiar material, probably mucous in nature, are seen. If the gland itself be watched, while its activity is thus roused, it will be seen (as we have already said, $\$ 145$ ) that its arteries are dilated, and its capillaries filled, and that the blood flows rapidly through the veins in a full stream and of bright arterial hue, frequentiy with pulsating movements. If a vein of the gland be opened, this large increase of flow, and the lessening of the ordinary deoxygenation of the blood consequent upon the rapid stream, will be still more evident. It is clear that excitation of the chorda largely dilates the arteries; the nerve acts energetically as a vaso-dilator nerve.

Thus stimulation of the chorda brings about two events: a dilation of the blood ressels of the gland, and a flow of saliva. The question at once arises, Is the latter simply the result of the former or is the flow caused by some direct action on the secreting cells, apart from the increased blood-supply? In support of the former view we night argue that the activity of the epithelial secreting cell, like that of any other form of protoplasm, is dependent on blood-supply. When the small arteries of the gland dilate, while the pressure in the arteries on the side towards the heart is (as we have previously seen when treating generally of blood-pressure $\$ 102$ ) correspondingly diminished, the pressure on the far side in the capillaries and veins is increased; hence the capillaries become fuller, and innre blood passes through them in a given time. From this we might infer that a larger amount of nutritive material would pass away from the capillaries into the surrounding lymph-spaces, and so into the epithelium cells, the result of which would naturally be to quicken the processes going on in the cells, and to stir these up to greater activity. But even admitting all this it does not necessarily follow that the activity thus excited should take on the form of secretion. It is quite possible to conceive that the increased blood-supply should lead 
only to the accumulation in the cell of the constituents of the saliva, or of the raw materials for their construction, and not to a discharge of the secretion. A man works better for being fed, but feeding does not make him work in the absence of any stimulus. The increased blood-supply therefore, while favourable to active secretion, need not necessarily bring it about. Moreover, the following facts distinctly shew that it need not. When a cannula is tied into the duct and the chorda is energetically stimulated, the pressure acquired by the saliva accumulated in the cannula and in the duct may exceed for the time being the arterial blood-pressure, even that of the carotid artery; that is to say, the pressure of fluid in the gland outside the blood ves:els is greater than that of the blood inside the blood vessels. This must, whatever be the exact mode of transit of nutritive material through the vascular walls, tend to check that transit. Again, if the head of an animal be rapidly cut off, and the chorda imm sliately stimulated, a flow of saliva takes place far too copious to be accounted for by the emptying of the salivary channels through any supposed contraction of their walls. In this case secretion is excited in the gland though the blood-supply is limited to the small quantity still remaining in the blood vessels. Lastly, if a small quantity of atropin be injected into the veins, stimulation of the chorda produces no secretion of saliva at all, though the dilation of the blood vessels takes place as usual; in spite of the greatly increased blood-supply no secretion at all takes place. These facts prove that the secretory activity is not simply the result of vascular changes, but may be called forth independently; they further lead us to suppose that the chorda contains two sets of fibres, one which we may call se sretory fibres, acting directly on the secreting structures only, and the other vaso-dilator fibres, acting on the blood vessels only, and further that atropin, while it has no effect on the latter, paralyses the former just as it paralyses the inhibitory fibres of the vagus. Hence when the chorda is stimulated, there pass down the nerve, in addition to impulses affecting the blood-supply, impulses affecting directly the secreting cells, and calling them into action, just as similar impulses call into action the contractility of the substance of a muscular fibre. Indeed the two things, secreting activity and contracting activity, are very parallel.

Since the chorda acts thus directly on the secreting cells, we should expect to find an anatomical connection between the colls and the nerve; but concerning this our knowledge is as yet imperfect.

$\$ 190$. When the cervical sympathetic is stimulated, the vascular effects, as we have already said, $\S 146$, are the exact contrary of those seen when the chorda is stimulated. The small arteries are constricted, and a small quantity of dark venous blood escapes by the veins. Sometimes, indeed, the flow through the 
gland is almost arrested. The sympathetic therefore acts as a vaso-constrictor nerve, and in this sense is antagonistic to the chorda.

As concerns the flow of saliva brought about by stimulation of the sympathetic, in the case of the submaxillary gland of the dog the effects are very peculiar. A slight flow results, and the saliva so secreted is remarkably viscid, of higher specific gravity, and richer in corpuscles and in the above-mentioned amorphous lum s than is the chorda saliva. This action of the sympathetic is little or not at all affected by atropin.

In the submaxillary gland of the dog then the contrast hetween the effects of chorda stimulation and those of sympathetic stimtilation are very marked: the former gives rise to vascular dilation with a copious How of fairly limpid saliva poor in solids, the latter to vascular constriction with a scanty flow of riseid saliva richer in solids. And in other animals a similar contrast prevails, though with minor differences. We shall return again presently to these different actions of the two nerves; meanwhile we have seen enough of the history of the submaxillary gland to learn that secretion in this instance is a reflex action, the efferent impulses of which directly affect the secreting cells, and that the vascular phenomena may assist, but are not the direct cause of, the flow.

$\$ 191$. We have dwelt long on this gland because it has been more fruitfully studied than any other But the nervous mechanisms of the other salivary glands are in their main features similar. Thus the secretion of the parotid gland, like that of the submaxillary, is governed by two sets of fibres: one of cerebrospinal origin, running along the auriculo-temporal branch of the fifth nerve but originating possibly in the glossopharyngeal, and the other of sympathetic origin coming from the cervical sympathetic. Stimulation of the cerebro-spinal fibres produces a copious flow of limpid saliva, free from mucus; stimulation of the cervical sympathetic gives rise in the rabbit to a secretion also fiee from mucns but rich in proteids and of greater amylolytic power than the cerebro-spinal secretion; in the dog little or no secretion is produced, though, as we shall see later on, certain changes are brought about in the gland itself. In both animals the cerebrospinal fibres are vaso-dilator, and the sympathetic fibres vasoconstrictor in action.

$\$ 192$. The secretion of gastrie juice. Though a certain amount of gastric juice may sometimes be found in the stomachs of fasting animals, it may be stated generally that the stomach, like the salivary glands, remains inactive, yielding no secretion, so long as it is not stimulated by food or otherwise. The advent of food into the stomach lowever at once causes a copious flow of gastric juice; and the quantity secreted in the twenty-four hours is probably very considerable, but we lave no trustworthy data for calculating the 
exact amount. So also when the gastric mucous membrane is stimulated mechanically, as with a feather, secretion is excited; but to a very small amount even when the whole interior surface of the stomach is thus repeatedly stimulated. The most efficient stimulus is the natural stimulus, viz. food; though dilute alkalis seem to have unusually powerful stimulating effects; thus the swallowing of saliva at once provokes a flow of gastric juice. Iluring fasting the gastric membrane is of a pale grey colour somewhat dry, covered with a thin layer of mucus, and thrown into folds; during digestion it becomes red, flushed, and tumid. the folds disappear, and minute drops of fluid appearing at the mouths of the glands, speedily run together into small streams. When the secretion is very active, the blood flows from the capillaries into the veins in a rapid stream without losing its bright arterial hue. The secretion of gastric juice is in fact accompanied by vascular dilation in the same way as is the seeretion of saliva.

$\$ 193$. Seeing that, unlike the case of the salivary secretion, food is brought into the immediate neighbourhood of the secreting cells, it is exceedingly probable that a great deal of the secretion is the result of some direct local action; and this view is supported by the fact that when a mechanical stimulus is applied to one spot of the gastric membrane the secretion is limited to the neighbourhood of that spot and is not excited in distant parts.

The stomach is supplied with nerve-fibres from the two vagi nerves and from the solar plexus of the splanchnic system. Our knowledge however of the action of the nervous system upon the stomach by means of these two sets of fibres is very imperfect. There are many facts which shew that the central nervous system may affect the secretion of gastric juice. On the other hand a secretion of quite normal gastric juice will go on after both vagi, or the nerves from the solar plexus going to the stomach have been divided, and indeed when all the nervous connections of the stomach are so far as possible severed.

$\$ 194$. The contrast presented between the scanty secretion resulting from mechanical stimulation and the copious flow which ac ual food induces is interesting hecause it seems to shew that the secretory activity of the cells is heightened by the absorption of certain products derived from the portions of food first digested. This is well illustrated by the following experiment of Heidenhain. This observer, adopting the method employed for the intestine, of which we shall speak later on, succeeded in isolating a portion of the fundus from the rest of the stomach; that is to say, he cut out a portion of the fundus, sewed together the cut edges of the main stomach, so as to form a smaller but otherwise complete organ, while hy sutures he converted the excised piece of fundus into a small independent stomach opening on to the exterior by a fistulous 
orifice. When food was introduced into the main stomach secretion also took place in the isolated fundus. This at first sight might seem the result of a nervous reflex act; but it was observed that the secondary secretion in the fundus was dependent on actual digestion taking place in the main stomach. If the material introduced into the main stomach were indigestible or digested with difficulty, so that little or no products of digestion were formed and absorbed into the blood, such ex. gr. as pieces of ligamentum nuchr, very little secretion took place in the isolated fundus. We quote this now as bearing on the question of a possible nervous mechanism of gastric secretion, but we shall have to return to it under another aspect.

\section{The changcs in a gland constituting the act of sceretion.}

$\S 195$. We have now to consider what are the changes in the glandular cells and their surroundings which cause this flow of fluid possessing specific characters into the lumen of an alveolus, and so into a duct. It will be convenient to begin with the pancreas.

The thin extended pancreas of a rabbit may, by means of special precautions, be spread out on the stage of a microscope and examined with even high powers, while the animal is not only alive but under such conditions that the gland remains in a nearly normal state, capable of secreting vigorously. It is possible under these circumstances to olserve even minutely the appearances presented by the gland when at rest and loaded, and to watch the changes which take place during secretion.

When the animal has not been digesting for some little time, the outlines of the individual cells lining the alveolus are very indistinct, the lumen is invisible or very inconspicuous, and each cell is crowded with small, refractive spherical granules, forming an irregular granular mass which hides the nucleus and leaves only a very narrow clear outer zone next to the basement membrane, or it may be hardly any such zone at all. Fig $77 \mathrm{~A}$. The gland is said to be 'loaded' or at rest.

The blood-supply moreover is scanty, the small arteries being constricted and the capillaries imperfectly filled with corpuscles.

If, however, the same pancreas be examined while it is in a state of activity, either from the presence of food in the stomach, or from the injection of some stimulating drug, such as pilocarpin, a very different state of things is seen. The individual cells (Fig. $77 B$ ) have become smaller and much more distinct in outline, and the contour of the alveolus which previously was even is now wavy, the basement membrane being indented at the junctions of the cells; also the lumen of the alveolus is now wider and more conspicuous. In each cell the granules have become 
much fewer in number and as it were have retreated to the inmer margin, so that the inner granular zone is much namower and the outer transparent zone much broader than before; the latter too is frequently marked at its inner part by delicate strice rumning into the inner zone. At the same time the blood vessels ale

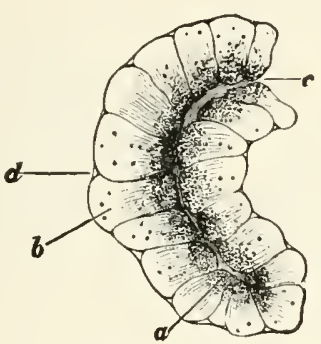

B.

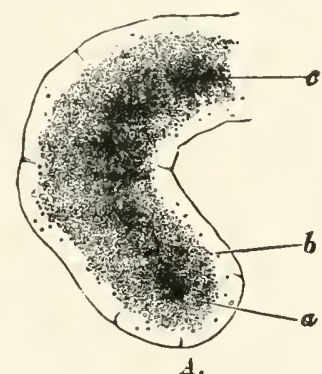

d.

Fig. 77. A Portion of the Paxcreas of the Rabit. (Kühne and Sheridan Lea) $A$ at rest, $B$ in a state of activity.

$a$ the inner granular zone, which in $A$ is larger, and more closely studded with fine gramules, than in $B$, in which the granules are fewer and coarser

$b$ the outer transparent zone, small in $A$, larger in $B$, and in the latter marked with faint strix.

$c$ the lumen, very obvious in $B$, but indistinct in $A$.

$d$ an indentation at the junction of two cells, seen in B, but not occurring in $A$.

largely dilated and the stream of blood through the capillaries is full and rapid.

With care the change from the one state of things to the other may be watched under the microscope. The vascular changes can of course be easily appreciated, but the granules may also be seen to diminish in number. Those at the inner margin seem to be discharged into t'le lumen, and those nearer the outer margin to travel inwards through the cell-substance towards the lumen, the faint strice spoken of above, apparently at all events, being the marks of their paths. Obviously during secretion, the granules with which the cell-substance was 'loaded' are 'discharged ' from the cell into the lumen of the alveolus. What changes these granules may undergo during the discharge we shall consider presently.

Sections of the prepared and hardened pancreas of any animal tell nearly the same tale as that thus told by the living pancreas of the rabbit. In sections for instance of the pancreas of a dog which has not been fed, and therefore has not been digesting, for some hours (24 or 30 ), the cells are seen to be crowd with granules (which however are usually shrunken and irregnlar owing to the influence of the hardening agent), leaving a very narrow outer zone. In similar sections of the pancreas of a dog which has been recently fed, six hours before for example, and in which 
therefore the gland has been for some time actively secreting, the granules are far less numerous, and the clear outer zone accordingly much broader and more conspicnous. With osmic acid these granules stain well, and are preserved in their spherical form, so that the cell thus stained maintains much of the appearance of a living cell. But with carmine, hematoxylin \&c. the granules do not stain nearly so readily as does the cell-substance of the cells, so that a discharged cell stains more deeply than does a loaded cell beeause the staining of the 'protoplasmic' cell-substance is not so much obscured by the unstained granules; besides which however the actual cell-substance stains probably somewhat more deeply in the discharged cell. It may be added that in the discharged cell the nucleus is conspicuous and well formed; in the loaded cell it is generally in prejared sections, more or less irregular, possibly because in these it is less dense and more watery than in the discharged cell, and so shrinks under the influence of the reagents employed.

These several observations suggest the conclusion that in a gland at rest the cell is occupied in forming by means of the metabolism of its cell-substance and lodging in itself $(\$ 30)$ certain granules of peculiar substance intended to be a part and probably an important part of the secretion. This goes on until the cell is more or less completely 'loaded.' In such a cell the amount of actual living cell-substance is relatively small, its place is largely occupied by granules, and it itself has been partly consumed in forming the granules. During the act of secretion the granules are discharged to form part of the secretion, other matters including water, as we shall see, making up the whole secretion; and the cell would be proportionately reduced in size were it not that the act of the discharge seems to stimulate the cellsubstance to a new activity of growth, so that new cell-substance is formed; this however is in turn soon in part consumed in order to form new granules. And what is thus seen with considerable distinctness and ease in the pancreas, is seen with more or less distinctness in other glands.

$\S 196$. When we study an 'albuminous,' or 'serous' salivary gland, the parotid gland for instance, in a living state, we find that the changes which take place during activity are quite comparable to those of the pancreas. During rest (Fig. $78 \mathrm{~A}$ ), the cells are large, their outlines very indistinct, in fact almost invisible, and the cell-substance is studded with granules. During activity (Fig. $78 \mathrm{~L}$ ), the cells become smaller, their ontlines more distinct, and the granules disappear, especially from the outer portions of each cell. After prolonged activity, as in Fig. $78 C$, the cells are still smaller with their outlines still more distinct, and the granules have disappeared almost entirely, a few only being left at the extreme inner margin of each cell, abutting upon the conspicuous, ahmost gaping lumen of the alveolus. And 
upon special examination it is found that the nuclei are large and round. In fact we might almost take the parotid, as thus studied, to be more truly typical of secretory changes than even the pancreas. For, the demarcation of an inner and outer zone is not a necessary feature of a secreting cell at rest. What is essential
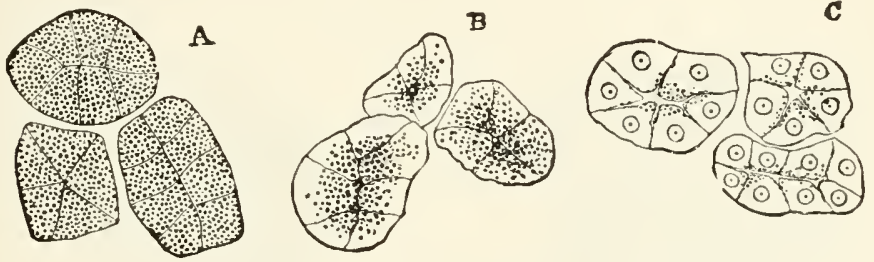

Fig. 78. Changes in the Parotin during Secretion. (Langley.)

The figure, which is somewhat diagrammatic, represents the microscopic changes which may be observed in the living glaud. A. Durmg rest. 'The obscure outlines of the cells are introduced to shew the relative size of the cells, they could not be readily seen in the specimen itself. $B$. After moderate stimulation. $C$. After prolonged stimulation. The nuclei are diagrammatic, and introduced to shew their appearance and position.

is that the cell-substance manufactures material, which for a while, that is during rest, is deposited in the cell, generally in the form of granules but not necessarily so, and that during activity this material is used up, the disappearance of the granules, when these are visible, being naturally earliest and nost marked at the outer portions of each cell, and progressing inwards towards the lumen, the whole cell becoming smaller and as it were shrunken.

In the cells of the parotid gland and other albuminous cells the granules seen in the living or fresh cell differ from the granules seen in the pancreatic cell, inasmuch as they are easily dissolved or broken up by the action of alcohol, chromic acid, and the other usual hardening reagents, and hence in hardened specimens have disappeared. In consequence, in sections of hardened and prepared albuminous glands the differences between resting or loaded and active or discharged cells are not so conspicuous as in the pancreas. The difference however even in hardened specimens between the parotid of the rabbit at rest, and that excited by stimulation of the sympathetic is well marked. During rest, the cells (Fig. $79 \mathrm{~A}$ ) are pale, transparent, staining with difficulty, and the nuclei possess irregular outlines as if shrunken by the reagents employed. After stimulation of the sympathetic, the cell-substance becomes turbid ( Fig. $79 \mathrm{~B}$ ), and stains much more readily, while the nuclei are no longer irregular in outline but round and large, with conspicuous nucleoli, the whole cell at the 
same time, at least after prolonged stimulation, becoming distinctly smaller.

$\$ 197$. In a mucous salivary gland the changes which take place are of a like kind, though apparently somewhat more complicated, owing probably to the peculiar characters of the mucin which is so conspicuous a constituent of the secretion.

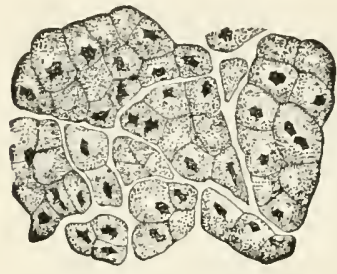

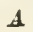

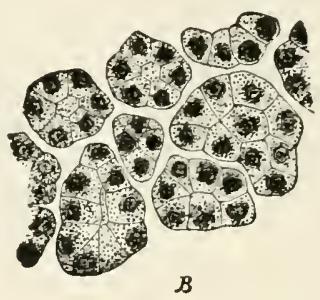

$B$

Fig. 79. Sections of the Parotid of the Rabbit. $A$ at rest, $B$ after stimulation of the cervical sympathetic. Both sections are from hardened gland. (After Heidenhain.)

If a piece of resting, loaded submaxillary gland be teased out, while fresh and warm from the body, in normal saline solution, the cell-substance of the mucous cells (Fig. $80 a$ ) is seen to be crowded
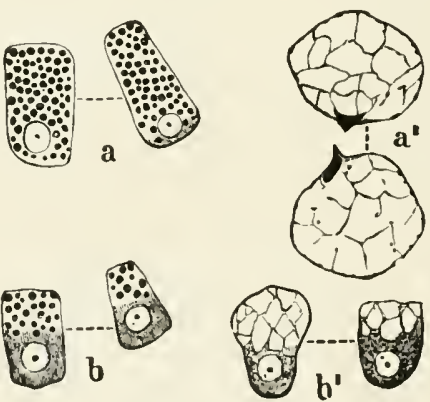

Fig. 80. Mucou's Celis from a fresh Stbmaxillary Gland of Dog. (Langley.)

$a$ and $b$ isolated in 2 p.c. salt solution; $a$, from loaded gland, $b$ from discharged gland (the nuclei are usually more obscured by gramules than is here represented).

(On teasing ont a fragment of fresh in 2 to 5 p.c. salt solution, the cells usually become broken up so that isolated cells are rarely obtained entire; isolated cells are common if the gland be left in the body for a day after death.)

$a^{\prime}, b^{\prime}$, treated with dilute acid; $a^{\prime}$ from loaded, $b^{\prime}$ from discharged gland.

with granules or spherules which may fairly be compared with the granules of the pancreas, though perhaps less dense and solid than these. 
If a piece of a gland which has been secreting for some time, and is therefore a discharged gland, be examined in the same way (Fig. $80 \mathrm{~b}$ ) the granules are far less numerous and largely confined to the part of the cell nearer the lumen, the outer part of the cell around the nucleus consisting of ordinary 'protoplasmic' cellsubstance. The distinction however between an inner 'granular zone' next to the lumen and an outer 'clear zone' next to the basement membrane is less distinct than in the pancreas, partly because the granules do not disappear in so regular a manner as in the pancreas and partly because the outer zone of the mucous cell, as it forms, is less homogeneous than that of the pancreatic cell.

The 'mranules' or 'spherules' of the mucous cell are moreover of a peculiar nature. If the fresh cell, shewing granules, (either many as in the case of a loaded or few as in the case of a discharged cell) be irrigated with water or with dilute acids or dilute alkalis the granules swell up (Fig. $80 a^{\prime} b^{\prime}$ ) into a transparent mass, giving the reactions of mucin, traversed by a network of 'protoplasmic' cell-substance. In this way is produced an appearance very similar to that shewn in sections of mucous glands hardened and stained in the ordinary way.

In the loaded mucous cell in such hardened and stained preparations (Fig. $81 a$ ) there is seen a small quantity of protoplasmic
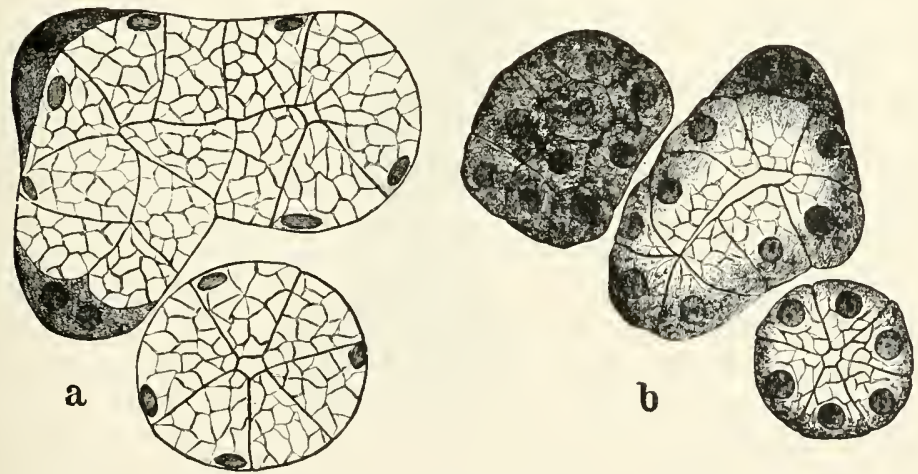

Fig. 81. Alveoli of Dog's Submaxillary Gland hardenen in alcohol AND STAINed With Carmine. (Langley.) (The network is diagrammatic.)

$a$, from a loaded gland.

$b$, from a discharged gland; the chorda tympani having been stimulated at short intervals during five hours.

cell-substance gathered round the nucleus at the outer part of the cell next to the hasement membrane; the rest of the cell consists of a network of cell-substance, the interstices being filled with 
transparent material, which, unlike the network itself and the mass of cell-substance round the nucleus, does not stain with carnine or with certain other dyes. The discharged cell in similar preparations (Fig. 81 b) diflers from the loaded cell in the anount of transparent non-staining material being much less and chiefly confined to the inner part of the cell, while the protoplasmic cell-substance around the now large and well-formed mucleus is not only, both relatively and absolutely, greater in amount, but stains still more deeply than in the loaded cell.

It would alpear therefore that in the mucous cell, as in the pancreatic cell, the cell-substance forms and deposits in itself

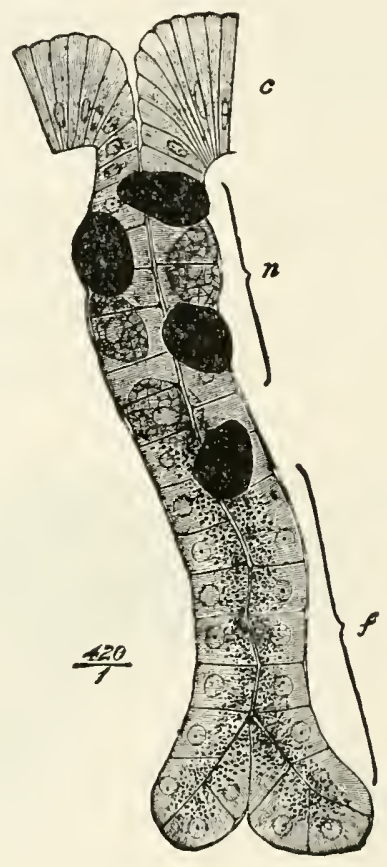

Fig. 82. Gastric Gland of Mammal (Bat) during Activity. (Langley.)

$c$, the month of the gland with its cylindrical cells.

$n$, the neck, containing conspicuous ovoid cells, with their coarse protoplasmic network.

$f$, the body of the gland. The grannles are seen in the central cells to be limited to the inner portions of each cell, the round nucleus of which is conspicuous.

certain material in the form of granules. During secretion these granules disappear and presumably form part of the secretion.

$\$$ 198. The 'central' or 'chief' cells of the gastric glands also exhilit similar changes. In such an animal as the newt 
these cells may, though with difficulty, be examined in the living state. They are then found to be studded with granules when the stomach is at rest. During digestion these granules become much less numerous and are chiefly gathered near the lumen, leaving in each cell a clear outer zone. And in many mammals the same abundance of granules in the loaded cell, the same paucity of granules for the most part restricted to an immer zone in the discharged cell, may be demonstrated by the use of osmic acid, Fig. 82.

When the stomach is hardened by alcohol these changes, like the similar changes in an albuminous cell, are obscured by the shrinking of the 'granules' or by their swelling up and becoming diffused through the rest of the cell-substance; so that though, in sections so preparel, very striking differences are seen between loaded and discharged cells, these are unlike those seen in living glands. In specimens taken from an animal which has not been fed for some time, the central cells of the gastric glands are pale, finely granular, and do not stain readily with carmine and other dyes. During the early stages of gastric digestion, the same cells are found somewhat swollen, but turbid and more coarsely granular; they stain much more readily. At a later stage they become smaller and shrunken, but are even more turbid and granular than before, and stain still more deeply. This is true, not only of the central cells in the cardiac glands, but also of the cells of which the pyloric glands are built up. In the loaded cell very little staining takes place, brcause the amount of living staining callsubstance is small relatively to the amount of material with which it is loaded and which does not stain readily. In the cell which after great activity has discharged itself, the cell is smaller, but what remains is largely living cell-substance, some of it new, and all staining readily. It would appear also that during the activity of the cell some substances, capable of being precipitated by alcohol, make their appearance, and the presence of this material adds to the turbid and granular aspect of the cell; possibly also this material contributes to the staining. A similar material seems to make its appearance in the cells of albuminous glands.

In the ovoid or border cells no very characteristic changes make their appearance. During digestion they become larger, more swollen as it were, and in consequence bulge out the basement membrane, but no characteristic disappearance of granules can be observed. In the living state, the cell-substance of these ovoid cells appears finely granular, but in hardened and prepared sections has a coarsely granular, "reticulate" look which is perhaps less marked in the swollen, active cells than in the resting cells.

$\$ 199$. All these various secreting cells then, pancreatic cell, mucous cell, albuminous cell, and central gastric cell, exhihit the same series of events, modified to a certain extent in the several 
cases. In each case the 'protoplasmic' cell-substance manufactures and lodges in itself material destined to form part of the juice secreted. In the fresh cell this material may generally be recognized under the microscope by its optical characters as granules, these however are apt to become altered by reagents. But we must guard ourselves against the assumption that the material which can thus be recognized is the only material thus stored up; we may, in future, by chemical or other means be able to differentiate other parts of the cell-body as being also material similarly stored up.

During activity, while the gland is secreting, this material, either unchanged or after undergoing change, is wholly or partially discharged from the cell. The cell in consequence of having thus got rid of more or less of its load consists to a larger extent of actual living cell-substance, this being in many cases increased by rapid new growth, though the bulk of the discharged cell may be less than that of the loaded cell.

This activity of growth continues after the act of secretion, but the discharged cell soon begins again the task of loading itself with new secretion material for the next act of secretion.

Thus in most cases there is, corresponding to the intermittence of secretion, an alternation of discharge and loading; but it must be borne in mind that such an alternation is not absolutely necessary even in the case of intermittent secretion. We can easily imagine that the discharge, say, of 'granules' during secretion should stir up the cell to an increased activity in forming granules, and that the formative activity should cease when the secretory activity ceased. In such a case the number of new granules formed might always be equal to the number of old granules used ıp, and the active cell in spite of its discharge would possess as many granules, that is to say, as large a load, as the cell at rest. And in the central gastric cells of some animals it would appear that such a continued balancing of load and discharge does actually take place, so that no distinction in granules can be observed between resting and active cells.

$\$ 200$. We spoke just now of the material stored up in the cell and destined to form part of the secretion as undergoing change before it was discharged. In the mucous cell we have seen that the material deposited in the living cell has at first the form of granules. These granules however are easily converted into a transparent material lodger in the spaces of the cellsubstance, which material even if not exactly identical with at least closely resembles the mucin found in the secretion; and apparently, in the act of secretion the granules do undergo some such change. In the case of some other glands moreover we have chemical as well as optical evidence that the material stored up in the cell is, in part at least, not the actual substance appearing in the secretion but an antecedent of that substance. 
An important constituent of pancreatic juice is; as we shall see later on, a body called trypsin, a ferment very similar to pepsin, acting on proteid bodies and converting them into peptone and other substances. Though in many respects alike, pepsin and trypsin are quite distinct bodies, and differ markedly in this, that while an acid medium is necessary for the action of pepsin, an alkaline medium is necessary for the action of trypsin; and accordingly the pancreatic juice is alkaline in contrast to the acidity of gastric juice. Trypsin, can, like pepsin (\$183), be extracted with glycerine from substances in which it occurs; glycerine extracts of trypsin however need for the manifestation of their powers the presence of a weak alkali, such as a 1 p.c. solution of sodium carbonate.

Now trypsin is present in abundance in normal pancreatic juice; but a loaded pancreas, one which is ripe for secretion, and which if excited to secrete would immediately pour out a juice rich in trypsin, contains no trypsin or a mere trace of it; nay even a pancreas which is engaged in the act of secreting contains in its actual cells an insignificant quantity only of trypsin, as is shewn by the following experiment.

If the pancreas of an animal, even of one in full digestion, be treated, while still warm from the body, with glycerine, the glycerine extract, as judged of by its action on fibrin in the presence of sodium carbonate, is inert or nearly so as regards proteid bodies. If, however, the same pancreas be kept for 24 hours before being treated with glycerine, the glycerine extract readily digests fibrin and other proteids in the presence of an alkali. If the pancreas, while still warm, be rubbed up in a mortar for a few minutes with dilute acetic acid, and then treated with glycerine, the glycerine extract is strongly proteolytic. If the glycerine extract obtained without acid from the warm pancreas, and therefore inert, be diluted largely with water, and kept at $35^{\circ} \mathrm{C}$. for some time, it becomes active. If treated with acidulated instead of distilled water, its activity is much sooner developed. If the inert glycerine extract of warm pancreas be precipitated with alcohol in excess, the precipitate, inert as a proteolytic ferment when fresh, becomes active when exposed for some time in an aqueous solution, rapidly so when treated with acidulated water. These facts shew that a pancreas taken fresh from the body, even during full digestion, contains but little ready-made ferment, though there is present in it a body which, by some kinds of decomposition, gives birth to the ferment. We may remark incidentally that though the presence of an alkali is essential to the proteolytic action of the actual ferment, the formation of the ferment out of its forerunner is iavoured by the presence of a small quantity of acid; the acid must be used with care, since the trypsin, once formed, is destroyed by acids. To this body, this mother of the ferment, which has not at present been satisfactorily isolated, but which 
appears to be a complex body, splitting up into the ferment, which as we have seen is at all events not certainly a proteid body, and into an undeniably proteid body, the name of zymogen has been applied. But it is better to reserve the term zymogen as a generic name for all such bodies as, not being themselves actual ferments, may by internal changes give rise to ferments, for all 'mothers of ferment' in fact; and to give to the particular mother of the pancreatic proteolytic ferment, the name trypsinogen.

Evidence of a similar kind shews that the gastric glands, both the cardiac and the pyloric glands, while they contain comparatively little actual pepsin, contain a considerable quantity of a zymogen of pepsin, or pepsinogen; and there can be little doubt but that this pepsinogen is lodged in the central cells of the cardiac glands and in the somewhat similar cells which lime the whole of the pyloric glands.

$\$ 201$. The act of secretion itsclf. The above discussion prepares us at once for the statement that the old view of secretion according to which the gland picks out, separates, secretes (hence the name secretion) and so filters as it were from the common store of the blood the several constituents of the juice, is untenable. According to that view the specific activity of any one gland was confined to the task of letting certain constituents of the blood pass from the capillaries surrounding the alveolus through the cells to the channels of the ducts, while refusing a passage to others. We now know that certain important constituents of each juice, the pepsin of gastric juice, the mucin of saliva and the like are formed in the cell, and not obtained ready made from the blood. A minute quantity of pepsin does exist it is true in the blood, but there are reasons for thinking that this has made its way back into the blood, either being absorbed from the interior of the stomach or, as seems more probable, picked up directly from the gastric glands, and so with some of the other constituents of other juices. The chief or specific constituents of each juice are formed in the cell itself.

But the juice secreted by any gland consists not only of the specific substances such as mucin, pepsin or other ferment, or other bodies, found in it alone, but also of a large quantity of water, and of various other substances, chicfly salines, common to it, to other juices and to the blood. And the question arises, Is the water, are the salts and other common substances furnished by the same act as that which supplies the specific constituents?

Certain facts suggest that they are not. For instance, as mentioned some time ago, in the snbmaxillary gland of the dog, stimulation of the chorda tympani produces a copious flow of saliva, which is usually thin and limpid, while stimulation of the cervical sympathetic prodnces a scanty flow of thick viscid saliva. That is to say, stimulation of the chorda has a marked effect in 
promoting the discharge of water, while stimulation of the sym. pathetic has a marked effect in promoting the discharge of mucin. To this we may add the case of the parotid of the dog. In this gland stimulation of a cerebro-spinal nerve, the auriculo-temporal, produces a copious How of limpid saliva, while stimulation of the sympathetic produces itself lit tle or no secretion at all; but when the sympathetic and cerebro-spinal nerves are stimulated at the same time, the saliva which Hows is much richer in solid and especially in organic matter than when the cerebro-spinal nerve is stimulated alone. And we have already seen that in this gland the microscopic ehanges following upon sympathetic stimulation are more conspicuous than those which follow upon cerebro-spinal stimulation.

These and other facts have led to the conception that the act of secretion consists of two parts, which in one case may coincide, in another may take place apart or in different proportions. On the one hand, there is the discharge of water carrying with it common soluble substances, chiefly salines, derived from the blood; on the other hand, a metabolic activity of the cellsubstance gives rise to the specific constituents of the juice. To put the matter broadly, the latter process produces the specific constituents, the former washes these and other matters into the duct. It has been further supposed that two kinds of nerve fibres exist: one governing the former process and, in the case of the submaxillary gland for instance, preponderating, though not to the total exclusion of the other kind, in the chorda tympani; the other governing the latter process and preponderating in the branches of the cervical sympathetic. These have been called respectively 'secretory' and 'trophic' fibres; but these terms are not desirable. It may be here remarked that even the former process is a distinct activity of the gland, and not a mere filtration. For, as we have seen in the case of the salivary glands, when atropin is given, not only do the specific constituents cease to be ejected as a consequence of stimulation of the chorda, but the discharge of water, in spite of the blood vessels becoming dilated, is also arrested: no saliva at all leaves the gland. And what is true of the salivary glands as regards the dependence of the flow of water on something else besides the mere pressure of the blood in the blood ressels, appears to hold good with other gland; also. The whole act of secretion is a very complicated one, probably too complicated to be described as consisting merely of the two processes mentioned above.

$\$ 202$. Throughout the above we have spoken as if the secretion were furnished exclusively by the cells of the alveoli or secreting portion of the gland, as if the epithelium cells lining the ducts, or conducting portion of the gland, contributed nothing to the act. In the gastric glands the slender cells lining the mouths of the glands (which correspond to ducts) and covering the ridges 
hetween, are mucous cells secreting into the stomach generally a small, but under abnormal conditions a large, amount of mucus, which has its uses but is not an essential part of the gastric juice. In the salivary glands we cau hardly suppose that the long stretch of characteristic colnmmar epithelium which reaches from the alveoli to the mouth of the long main duct serves simply to furnish a smooth lining to the conducting passages; but we have as yet no clear indications of what the function of this epithelium can be.

$\$ 203$. Before we leave the mechanism of secretion there are one or more accessory points which deserve attention.

In treating just now of the gastric glands we spoke as if pepsin were the only important constituent of gastric juice, whereas, as we have previously seen, the acid is equally essential. The formation of the free acid of the gastric juice is very obscure, and many ingenious but unsatisfactory views have been put forward to explain it. It seems natural to suppose that it arises in some way from the decomposition of sodium chloride drawn from the blood; and this is supported by the fact that when the secretion of gastric juice is atively going on, the amount of chlorides leaving the blood by the kidney is proportionately diminished; but nothing certain can at present be stated as to the mechanism of that decomposition.

In the frog, while pepsin free from acid is secreted by the glands in the lower portion of the osophagus, an acid juice is afforded by glands in the stomach itself, which have accordingly been called oxyntic (óvivesv to sharpen, acidulate) glands; but these oxyntic glands appear also to secrete pepsin. In the mammal the isolated pylorus secretes an alkaline juice; in fact, the appearance of an acil juice is limited to those portions of the stomach in which the glands eontain both 'chief ' or 'central,' and 'ovoid 'or 'border' cells Now from what has been previously said there ean be no doubt that the chief cells do secrete pepsin. On the other hand there is no evidence whatever of the formation of pepsin by the 'border' or 'ovoid' cells, though this was once supposed to be the case and these cells were unfortunately formerly called 'peptic' cells. Hence it has been inferred that the border cells secrete acid; but the argmment is at present one of exchnsion only, there being no direct proof that these cells actually manufacture the acid.

The remin appears to be formed by the same cells which manufacture the pepsin, that is, by the chief cells of the fundus generally and to some extent by the cells of the pyloric glands. We may add that we have evidence of the existence of a zymogen of remin analogous to the zymogen of pepsin or of trypsin.

$\$ 204$. Seemg the great solvent power of both gastric and pancreatic juice, the question is naturally suggested, Why does not the stomach digest itself? After death, the stomach is 
irequently found partially digested, viz. in cases when death has taken place suddenly on a full stomach. In an ordinary death, the membrane censes to secrete before the circulation is at an end. That there is no special rirtue in living things which prevents their being digested is shewn by the fact, that the leg of a living frog or the ear of a living raboit introduced into the stomach of a dog through a gastric fistula is readily digested. It has been sugrested that the bloul-current heeps up an alkalinity sutticient to neutralize the acidity of the juice in the region of the glands themselves; but this will not explain why the pancreatic juice, which is active in an alkaline medium, does not digest the proteids of the pancrens itself, or why the digestive cells of the bloodless actinozoon or hydrozoon do not digest themselves. We might add, it does not explain why the amceba, while dissolving the protoplasm of the swallowed diatom, does not dissolve its own protoplasm. We camnot answer this question at all at present, any more than the similar one. why the delicate protoplasm of the amoba resists during life the entrance into itself by osmosis of more water than it requires to carry on its work, while a few moments after it is dead water enters freely by osmosis, and the effects of that entrance become abundantly evident by the formation of bulle and the breaking up of the protoplasm. 


\section{SEC. 3. THE PROPERTIES AND CHARACTERS OF BILE, PANCREATIC JUICE AND SUCCUS ENTERICUS.}

$\S 205$. In the living body the food, subjected to the action first of the saliva and then of the gastric juice, undergoes in the stomach changes which we shall presently consider in detail, and the food so changed is passed on into the small intestine, where it is further subjected to the action of the bile secreted by the liver, of pancreatic juice secreted by the pancreas, and possibly to some extent, though this is by no means certain, of a juice secreted by the intestine itself, and called succus cntcricus. It will be convenient to study the minute structure of the liver in connection with other functions of the liver more important perhaps than that of the secretion of lile, namely the formation of glycogen, and other metabolic events occurring in the hepatic cells; we have already studied the structure of the pancreas; and the structure of the intestine will best be considered by itself. We therefore turn at once to the properties and characters of the above-named juices.

\section{Bile.}

Though bile, after secretion in the lobules of the liver, is passed on along the hepatic duct, it is in the case of most animals not poured at once into the duodenum but taken by the cystic duct to the reservoir of the gall-bladder. Here it remains, until such time as it is needed, when a quantity is poured along the common bile duct into the intestine.

The quality of bile varies much, not only in different animals, but in the same animal at different times. It is moreover affected by the length of the sojourn in the gall-bladder; bile taken direct from the hepatic duct, especially when secreted rapidly, contains little or no mueus; that taken from the gall-bladder, as of slaughtered oxen or sheep, is loaded with mucus. The colour of the bile of carnivorous and omnivorous animals, and of man, is generally a bright golden red, sometimes a greenish yellow: of 
herbivorous animals, a yellowish green, or a bright green, or a dirty green, according to circumstances, being much modified by retention in the gall-bladder. The reaction is neutral or alkaline. The following may be taken as the average composition of human bile taken from the gall-bladder, and therefore containing much more mucus as well as, relatively to the solids, more water than bile from the hepatic duct.

In 1000 parts.

\begin{tabular}{|c|c|c|c|c|c|c|c|c|c|}
\hline \multirow{2}{*}{\multicolumn{10}{|c|}{$\begin{array}{l}\text { Water } \\
\text { Solids : - }\end{array}$}} \\
\hline & & & & & & & & & \\
\hline Bile Salts & . & & & & & & & & $91 \cdot 4$ \\
\hline Fats, \&c. . & - & . & • & • & - & & & & $9 \cdot 2$ \\
\hline Cholesterin & & . & - & . & - & & & & $2 \cdot 6$ \\
\hline Mucus and & Pigmen & & . & . & . & & & & $29 \cdot 8$ \\
\hline Inorganic Sa & & & & & . & & & & $7 \cdot 8$ \\
\hline
\end{tabular}

$859 \cdot 2$

The entire absence of proteids is a marked feature of bile; pancreatic juice, as we shall see, contains a considerable quantity, saliva, as we have seen, a small quantity, normal gastric juice probably still less and bile none at all. Even the bile which has been retained some time in the gall-bladder, though rich in mucus, contains no proteids.

The constituents which form, apart from the mucus, the great bulk of the solids of bile and which deserve chief attention, are the pigments and the bile-salts; of these we shall speak immediately.

With regard to the inorganic salts actually present as such sodium salts are conspicuous, sodium chloride amounting to 2 or more per cent., sodium phosphate to nearly as much, the rest being earthy phospliates and other matters in small quantity. The presence of iron, to the extent of about 006 p.c., is interesting, since, as we shall see, there are reasons for thinking that the pigment of bile, itself free from iron, is derived from iron-holding hæmoglobin ; some, at least, of the iron set free during the conversion of hæmoglobin into bile pigment, which probably takes place in the liver, finds its way into the bile. Bile also appears to contain a small quantity, at all events occasionally, of other metals, such as manganese and copper; metals introduced into the body are apt to be retained in the liver and eventually leave it by the bile.

The small quantity of fat present consists in part of the complex body lecithin.

The peculiar body cholesterin, which though fatty looking (hence the name 'bile fat') is really an alcohol with the composition $\mathrm{C}_{26} \mathrm{H}_{44} \mathrm{O}$, is conspicuous by its quantity and constancy. It forms the greater part of most gall-stones, though some are composed chiefly of pigment. Insoluble in water and cold alcohol, 
though soluble in hot alcohol and readily soluble in ether, chloroform \&c., it is dissolved ly the bile-salts in aqueous solution and hence is present in solution in bile Its physiological functions are obscure.

'The ash of bile consists largely of soda, derived partly from the sodium chloride and partly from the bile-salts, of sulphates derived ehielly if not wholly from the latter, and of phosphates partly ready formed, and in part derived from the lecithin.

\$206. Pigments of Bile. The natural golden red colour of normal human or carnivorous bile, is due to the presence of Bilirubin. This, which is also the chief pigmentary constitnent of gall-stones, and occurs largely in the urine of jaundice, may he obtained in the form either of an orange-coloured amorphous powder, or of well-formed rhombic tablets and prisms. Insoluble in water, and but little soluble in ether and alcohol, it is readily soluble in chloroform, and in alkaline fluids. Its composition is $\mathrm{C}_{16} \mathrm{H}_{18} \mathrm{~N}_{2} \mathrm{O}_{3}$. Treated with oxidizing agents, such as nitric acid yellow with nitrous acid, it displays a succession of colours in the order of the spectrum. The yellowish golden red becomes green, this a greenish blue, then blue, next violet, afterwards a dirty red, and finally a pale yellow. This characteristic reaction of bilirubin is the basis of the so-called Gmelin's test for bile-pigments. Each of these stages represents a chistinct pigmentary substance. As alkaline solution of bilirubin, exposed in a shallow vessel to the action of the air, turns green, becoming converted into Biliverdin $\left(\mathrm{C}_{16} \mathrm{H}_{18} \mathrm{~N} \mathrm{O}_{4}\right)$, the green pigment of herbivorous bile. Biliverdin is also found at times in the urine of jaundice, and is probably the body which gives to bile which has been exposed to the action of gastric juice, as in biliary vomits, its characteristic green hue. It is the first stage of the oxidation of bilirubin in Gmelin's test. Treated with oxidizing agents biliverdin runs through the same series of colours as bilirubin, with the exception of the initial golden red.

$\$ 207$. The Bile-solts. These consist, in man and many animals, of sodium glycocholate and taurocholate, the proportion of the two varying in different animals. In man both the total quantity of bile-salts and the proportion of the one bile salt to the other seem to vary a good deal, but the glycocholate is said to be always the more abundant. In ox-gall, sodium glycocholate is abundant, and tanrocholate scanty. The bile-salts of the dog, cat, bear, and other camivora, consist exclusively of the latter.

Insoluble in ether but soluhle in alcohol and in water, the

- aqueous solutions having a decided alkaline reaction, both salts may be obtained lyy crystallisation in fine acicular needles They are exceedingly delinnescent The solutions of both acids have a dextro-rotatory action on polarized light.

Preporetion. Bile, mixed with animal charcoal, is evaporated to dryness and extracted with alcohol. If not colourless, the alcoholic 
filtrate must be further decolourized with animal charcoal, and the alcohol distilled off. The dry residue is treated with absolute alcohol, and to the alcoholic filtrate anlydrous ether is added as long as any precipitate is formed. On standing the cloudy precipitate becomes transformed into a crystalline mass at the bottom of the vessel. If the alcohol be not absolute, the crystals are very apt to be changed into a thick syrupy fluid. This mass of erystals has been often spoken of as bilin. Both salts are thus precipitated, so that in such a bile as that of the ox or man bilin consists both of sodium glycocholate and sodium taurocholate. The two may be separated by precipitation from their aqueous solutions with sugar of lead, which throws dow the former much more readily than the latter. The acids may be separated from their respective salts by dilute sulphuric acid, or by the action of leadacetate and sulphydric acid.

On boiling with dilute acids (sulphuric, hydrochloric) or canstic potash, or haryta water, glycocholic acid is split up into cholalic (cholic) aeid and glycin. Taurocholic acid may similarly be split up into cholalic acid and taurin. Thus

$$
\begin{aligned}
& \text { glycocholic acid } \\
& \mathrm{C}_{26} \mathrm{H}_{43} \mathrm{NO}_{6}+\mathrm{H}_{2} \mathrm{O}=\mathrm{C}_{24} \mathrm{H}_{40} \mathrm{O}_{5}+\mathrm{CH}_{2} \cdot \mathrm{NH}_{2}(\mathrm{CO} . \mathrm{OH}) \\
& \begin{array}{cc}
\text { cholalic acid } \\
\text { taurocholic acid }
\end{array} \\
& \mathrm{C}_{26} \mathrm{H}_{45} \mathrm{NSO}_{7}+\mathrm{H}_{2} \mathrm{O}=\mathrm{C}_{24} \mathrm{H}_{40} \mathrm{O}_{5}+\mathrm{C}_{2} \mathrm{H}_{4} \cdot \mathrm{NH}_{2} \cdot \mathrm{SO}_{3} \mathrm{H} .
\end{aligned}
$$

Both acids contain the same nou-nitrogenous acid, cholalic acid ; but this acid is in the first case assoeiated or conjugated with the important nitrogenous body glycin, or amido-acetic acid, which is a compound formed from ammonia and one of the "fatty acid" series, viz. acetic; and in the second case with taurin, or amidoisethionic acid, that is a compound into which representatives of ammonia, of the ethyl group, and of sulphuric acid enter. The decomposition of the bile acids into cholalic acid and taurin or glycin respectively takes place naturally in the intestine, the glycin and taurin being probably absorbed, so that from the two acids, after they have served their purpose in digestion, the two ammonia compounds are retmrned into the blood. Each of the two acids, or cholalic acid alone, when treated with sulphuric acid and canesugar, gives a magnificent purple colour (Pettenkofer's test) with a characteristic spectrum. A similar colour may however often be produced by the action of the same bodies on albumin, amyl alcohol, and some other organic hodies.

$\$ 208$. Action of Bile on Food. In some animals at least bile contains a ferment capable of converting starch into sugar; but its action in this respect is wholly subordinate.

On proteids bile has no direct digestive action wliatever, but being, generally at least, alkaline, and often strongly so, tends to neutralise the acid contents of the stomach as they pass into the duodenum and as we shall see so prepares the way for the action 
of the pancreatic juice. To peptic action it is distinctly antagonistic; the presence of a suttieient quantity of bile renders gastrie jnice inert towards proteids. Moreover when bile, or a solution of bile-salts, is added to a flnid containing the products of gastric digestion, a precipitate takes place, consisting of parapeptone (when present), peptone, pepsin and bile salts. The precipitate is redissolved in an exeess of hile or solntion of lile-salts; but the pejsin though redissolved remains inert towards proteils. This precipitation actmally does take place in the duodenum, and we shall speak of it again later on.

With regard to the action of bile on fats, the following statements may be made. Bile has a slight solvent action on fats, as seen in its use by painters. It has by itself a slight but only slight emulsif ying power : a mixture of oil and bile separate after shaking rather less rapidly than a mixture of oil and water. With fatty acids bile forms soaps. It is moreover a solvent of solid soaps, and it wonld appear that the emulsion of fats is under eertain circumstances at all events facilitated by the presence of soaps in solntion. Hence bile is probably of mich greater nse as an emmlsion agent when nixed with pancreatic juice than when acting by itself alone. To this point we shall return. Lastly, the passage of fats through membranes is assisted by wetting the membranes with bile, or with a solution of hile-salts. Oil will pass to a certain extent through a filter-paper kept wet with a solution of bile-salts, whereas it will not pass or passes with extreme diftieulty through one kept constantly wet with distilled water.

Bile possesses some antiseptic qualities. Out of the body its presence hinders various putrefactive processes; and when it is prevented from flowing into the alimentary canal, the contents of the intestine undergo changes different from those which take place under normal conditions, and leading to the appearance of varions products, especially of ill-smelling gases.

These various actions of bile seem to be dependent on the bile salts and not on the pigmentary or other constituents.

\section{Panereatic Juice.}

\$209. Natural healthy pancreatic juice ohtained by means of a temporary pancreatic fistula differs from the digestive juices of which we have already spoken, in the comparatively large quantity of proteids which it contains. Its composition varies according to the rate of secretion, for, with the more rapid flow, the increase of total solids does not keep pace with that of the water, though the ash remains remarkably constant.

By an incision through the linea alba the pancreatic duct or (ducts) can easily be fonnd either in the rabbit or in the dog, and a cammla secured in it. There is no difficulty about a temporary fistnla; but 
with permanent fistulat the secretion is apt to beenme altered in mature, amd to lose many of its eharateristie properties. some. howerer, have sucereted in obtaining permanent fistula without any impaiment of the secretion.

Healthy pancreatic juice is a elear, somewhat riscid thiul, frothing when shaken. - It has a very dechled alkiline reaction, and eomtains few or no structural comstituents.

The averace anount of solids in the pancreatic juice (of the dog) obtained from a temporary fistula is about $\$$ to 10 p.e? : but in even thoromghly ative juice obtained from a permanent fistula, is not nore than about of to i p.e. is being inorganic matter: and this is probably the normal amount. The important constitnents of quite tresh juice are albumin, a peculiar form of protad allied to mosiu. viving rise to a sort of clotting. a small amount of fats and soaps, and a complatively lange quantity of sodium carbonate, to which the alkaline reaction of the juice is due, and which seems to be peeuliary associated with the proteids.

since, as we shall presently sere, panteratic juice contains a ferment ating eneretieally on proteid nuters in an allatine medimu, it rapidly digests its own proteid constituents, and, when kept. specedily elianges in elaracter. The nyosin-like elot is dissolved, and the juice soon comtains a peenliar form of alkalialbumin (precipitable hy saturation with magnesium sulphate) as well as small quantities of lemein, tyosin and peptome, whieh seem to be the products of self-digestion and are entilely absent from the perfectly fresh juice.

\$210. Letion on Fool-stutis. (1) stereh, pancreatic juice

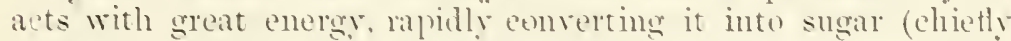
maltose). All that has beens said in this respeet toncerning saliva might be repeated in the ease of pancreatic juiee, exerpt that the activity of the latter is fiar vreater than that of the former. Panceatic juice and the agueons infusion of the gland are always capable of converting starel into sugar. Whether the animal from which they were taken be staring or well fed. From the juice, or, by the dyexpine method, from the gland itself, an amylolytic ferment may be approximately isolated.

On meteils paucreatic juice also exereises a solvent action, so far similar to that of wastric juice that by it proteids are comverted into peptome. If a few slupds of fibrin are thrown into a small quantity of paureatie juica, they speedily disappear, especially at a temperature of $35^{\circ} \mathrm{C}$. and the mixtme is fonnd to contain peptone. 'The activity of the juice in thus comberting potoils into peptome is faroured by inerease of temperature up to $40^{\circ}$ or thereabouts, and hindered biy low temperatures : it is permanentlydestroyed by boiling. The digestive powers of the juice in firet depend, like those of anstric juies. on the presence of a ferment whieh, as we have alreaty said, may be isolated much in the 
same way as pepsin is isolated, and to which the name trypsin has been given.

The appearance of fibrin undergoing pancreatic digestion is however different from that undergoing peptic digestion. In the former case the fibin does not swell up, but remains as opaqne as before, and appears to suffer corrosion rather than solution. But there is a still more important distinction between pancreatic and peptic digestion of proteids. Peptic digestion is essentially an acid digestion; we have senn that the action only takes place in the presence of an acid, and is arrested by neutralisation. I'ancreatic digestion, on the other hand, may be regarded as an alkaline digestion; the action is most energetic when some alkali is present; and the activity of an alkaline juice is hinciered or delayed by neutralication and arrested by acidification at least with mineral acids. The glycenine extract of pancreas is mnder all circumstances as inert in the presence of free mineral acid as that of the stomach in the presence of alkalis. If the digestive mixture be supplied with codium carlonate to the extent of 1 p.c., digestion proceeds rapidly, just as does a peptic mixture when acidulated with hydrochloric acid to the extent of 2 p.c. Sodium carbonate of 1 p.c. seems in fact to play in tryptic digestion a part altogether comparable to that of hydrochloric acid of $\cdot 2$ p.c. in gastric digestion. And just as pepsin is rapidly destroyed by Feing heated to ahout $40^{\circ}$ with a 1 p.c. solution of sodium carbonate, so trypsin is rapidly destroyed by being similarly heated with dilute hydrochloric acid of " 2 p.c. Alkaline bile, which arrests peptic digestion, seems, if anything, farourable to tryptic digestion.

Pancreatic digestion and gastric digestion agree in that by both proteids are converted into peptones. Naturally in the alkaline pancreatic digestion no bye products allied to acid-albumin, such as parapeptone, make their appearance; there are however various bye products on which we need not dwell. Albumoses are not conspicuons in pancreatic digestion, they are very rapidly carried on to the further stage of peptone.

In one respect there is an essential difference between gastric and pancreatic digestion. In gastric digestion the products are not carried beyond the proteid stage ; in panceatic digestion part if the proteid is changed into something which is no longer proteid

During the pancreatic digestion of proteids, two remarkab'e nitrogenous crystalline bodies, leucin and tyrosin make their appearance. When fibrin (or other proteid) is submitted to the action of pancreatic juice, the amount of peptone which can be recovered from the mixture falls far short of the original amount of proteids; and the longer the digestive action, the greater up to a certain point is this apparent loss. If a pancreatic digestion mixture be freed from the bye products by neutralisation and filtration, the filtrate yields, when concentrat d by evaporation, a crop of crystals of 
tyrosin. If these be removed the peptone may be precipitated from the concentrated filtrate by the addition of a large excess of alcohol and separated by filtration. The second filtrate upon being concentrated by evaporation yields abundant crystals of leucin and traces of tyrosin. Thus by the action of the pancreatic juics a considerable amount of the proteid, which is being digested, is so broken up as to give rise to products which are no longer proteid in nature. From this breaking up of the proteid there arise leucin, tyrosin, and probably several other bodies, such as fatty acids and volatile substances. We said that in gastric digastion more than one kind of peptone was probably formed, and the same may be said of panereatic digestion. We may now add that in both gastric and pancreatic digestion two kinds of peptone are probably formed, one of which resists the action of trypsin, and undergoes no further change. but the other of which, whether arising from gastric or puncreatic digestion undergoes further change by the action of trypsin and it is this which is the source of the leucin and other bodies of which we are speaking.

As is well known, leucin and tyrosin are the hodies which nake their appearance when proteids or gelatin are acted on by dilute acids, alkalis, or various oxidising agents. Leucin is a body, which in an impure state crystallizes in minute round lumps witl an obscure radiate striation, but when pure, forms thin glittering Hat erystals. It has the formula $\mathrm{C}_{6} \mathrm{H}_{13} \mathrm{NO}_{2}$ or $\mathrm{C}_{5} \mathrm{H}_{10} \cdot \mathrm{NH}_{2}(\mathrm{CO} . \mathrm{OH})$ and is amido-caproic acid. Now caproic acid is one of the "fatty acid" series, so that leucin may be regarded as a compound of ammonia with a fatty acid. Tyrosin, $\mathrm{C}_{9} \mathrm{H}_{11} \mathrm{NO}$, on the other hand, belongs to the "aromatic" series; it is a phenyl compound, and hence allied to benzoic acid and hippuric acid. So that in pancreatic digastion the large complex proteid molecule is split up into fatty acid and aromatic molecules, some other bodies of less importance making their appearance at the same time. We infer that the proteid molecules are in some way built up out of "fatty acid" and "aromatic" molecules, together with other components, and we shall later on see additional reasons for this view.

Among the supplementary products of pancreatic digestion may bu mentionel the body in lol $\left(\mathrm{C}_{8} \mathrm{H}_{7} \mathrm{~N}\right)$, to which apparently the strong and peculiarly fecal odour which sometimes makes its appearance during pancreatic digestion is due. Indol, however, unlike the leucin and tyrosin, is not a product of pure pancreatic digastion, but of an accompanying decomposition due to the action of organised ferments. A pancr atic digestive mixture soon becomes swarming with bacteria, in spite of ordinary precautions, when natural juice or an infusion of the gland is used. When isolated ferment is used, and atmospheric germs are excluded, or when pancreatic digestion is carried on in the presence of salicylic 
acid, or thymol, which prevent the development of bacteria and like organisms but permit the action of the trypsin, no odour is perceived, and no indol is produced.

On the gelatiniferous elements of the tissues in the condition in which they actually exist in the tissue previous to any treatment pancreatic juice appears to have no solvent action. The fibrillite and bundles of fibrillie of ordinary untouched connectivetissue are not digested by pancreatic juice, which in this respect affords a striking contrast to gastric juice. But when they have been previously treated with acid or boiled so as to become converted into actual gelatine, trypsin is able to dissolve them, apparently changing them much in the same way as does pepsin. Trypsin unlike pepsin, will dissolve mucin. Like pepsin, it is inert towards nuclein, horny tissues, and the so-called amyloid matter.

On futs pancreatic juice has a twofold action. In the first place it emulsifies fats. If hog's lard be gently heated until it melts and be then mixed with pancreatic juice before it solidifies on cooling, a creamy emulsion, lasting for almost an indefinite time, is formed. So also when olive oil is shaken up with pancreatic juice, the separation of the two fluids takes place very slowly, and a drop of the mixture under the microscope sherrs that the division of the fat is very minute. An alkaline aqueous infusion of the gland has similar emulsifying powers. In the second place pancreatic juice splits up neutral fats into their respective acids and glycerine. Thus palmitin (or tripalmitin) $\left(\mathrm{C}_{15} \mathrm{H}_{31} \cdot \mathrm{CO} \cdot \mathrm{O}\right)_{3} \cdot \mathrm{C}_{5} \mathrm{H}_{3}$ is with the assumption of $3 \mathrm{H}_{2} \mathrm{O}$ split up into three molecules of palmitic acid $3\left(\mathrm{C}_{15} \mathrm{H}_{31}\right.$. $\left.\mathrm{CO} . \mathrm{OH}\right)$ and one of glycerine $\left(\mathrm{C}_{3} \mathrm{H}_{6}\right)(\mathrm{OH})_{3}$; and so with the other neutral fats. If perfectly neutral fat be treated with pancreatic juice, especially at the body-temperature, the emulsion which is formed speedily takes on an acid reaction, and by appropriate means not only the corresponding fatty acids but glycerine may be obtained from the mixture. When alkali is present, the fatty acids thus set free form their corresponding soaps. Pancreatic juice contains fats, and is consequently apt after collection to have its alkalinity reduced; and an aqueous infusion of a pancreatic gland (which always contains a considerable amount of fat) very speedily becomes acid.

Thus pancreatic juice is remarkable for the power it possesses of acting on all the food-stuffs, on starch, fats and proteids.

The action on starch, the action on proteids, and the splitting up of neutral fats appear to be due to the presence of three distinct ferments, and methods have been suggested for isolating them. The emulsifying power, on the other hand, is connected with the general composition of the juice (or of the aqueous infusion of the gland), being probably in large measure dependent on the alkali and the alkali-albumin present. The proteolytic ferment trypsin as ordinarily prepared seems to be proteid in nature and capai.c 
of giving rise, by digestion, to peptone; but it may be doubted, as in the case of pepsin and other ferments, whether the pure ferment has yet been isolated. There are no neans of distinguishing the amylolytic ferment of the pancreas from ptyalin. The term pancreatin has been variously applied to many different preparations from the gland, and its use liad perhaps better be avoided.

The action of pancreatic juice, or of the infusion or extract of the gland, on starch, is seen under all circumstances, whether the animal be fasting or not. The same may probably be said of the action on fats. On proteids the natural juice, when secreted in a normal state, is always active. The glycerine extract or aqueous infusion of the gland, on the contrary, as we have already explained, $\$ 200$, is active in proportion as the trypsinogen has been converted into trypsin.

\section{Succus Entericus.}

\$211. When, in a living animal, a portion of the small intestine is ligatured, so that the secretions coming down from above cannot enter its canal, while yet the blood-supply is maintained as usual, a small amount of secretion collects in its interior. This is spoken of as the suceus enterieus, and is supposed to be furnished by the glands of Lieberkühn, of which we shall presently speak.

Succus entericus may be obtained by the following method, known as that of Thiry modified by Vella. The small intestine is divided in two places at some distance $(30$ to $50 \mathrm{~cm}$.) apart. By fine sutures the lower end of the upper section is carefully united with the upper end of the lower section, thus as it were cutting out a whole piece of the small intestine from the alimentary tract. In successful cases, union between the cut surfaces takes place, and a shortened but otherwise satisfactory canal is re-established. Of the isolated piece the two ends are separately brought through incisions in the abdominal wall and their mouths carefully fastened in such a manner that each mouth of the piece opens on to the exterior. During the process of healing two fistulie are thus established, one leading to the beginning of and the other to the end of a short piece of intestine quite isolated from the rest of the alimentary canal; by means of these openings a small quantity of fluid can be obtained.

The quantity secreted is said to be considerably increased by the administration of pilocarpin.

Succus entericus obtained from the dog by the above method is a clear yellowish fluid having a faintly alkaline reaction and containing a certain quantity of mucus. It is said to convert starch into sugar, and proteids into peptone (the action being very similar to that of pancreatic juice), to split up neutral fats, to emulsify fats and to curdle milk. It is also said to invert canesugar rapidly, and by a fermentative action to convert cane-sugar 
into lactic acid, and this again into butyric acid with the evolution of carbonic acid and free hydrogen.

According to the above results, succus entericus is to be regarded as an important secretion acting on all kinds of food. But even at the best, its actions are slow and feeble. Moreover many observers have obtained negative results, so that the various statements are contlicting. Besides, we have no exact knowledge as to the amount to which such a secretion takes place under normal circumstances in the living body. We may therefore conclude that, at present at all events, we have no satisfactory reasons for supposing that the actual digestion of food in the intestine is, to any great extent, aided by such a juice.

Of the possible action of other secretions of the alimentary canal, as of the cæeum and large intestine, we shall speak whin we come to consider the changes in the alimentary canal.

$\$$ 212. Gallstones. Concretions, often of considerable size, known as gallstones are not unfrequently formed in the gall bladder, and smaller concretions are sometimes formed in the lile passages. In man two kinds of gallstones are common. One kind consists almost entirely of cholesterin, sometimes nearly free from any admixture with pigment, sometimes more or less discoloured with pigment. Gallstones of this kind have a crystalline structure, and when broken or cut shew frequently radiate and concentric markings. The other kind consists chiefly of bilirubin in combination with calcium. Gallstones of this kind are dark coluured and amorphous. Less common than the above are small dask coloured stones, having often a mulberry shape, consisting not of bilirubin itself, but of one or other derivative of bilirubin. Gallstones consisting almost entirely of inorganic salts, calcic carbonates and phosphates, are also occasionally met with. In the lower animals, in oxen for instance, bilirubin gallstones are not uncommon, but cholesterin gallstones are rare.

A gallstone appears always to contain a more or less obvious 'nucleus,' around which the material of the stone has been deposited, and which may be regarded as the origin of the stone; the real cause of the formation of the stone lies however in certain changes in the bile, by which the cholesterin, or bilirubin, or other constituent ceases to remain dissolved in the bile. But we cannot discuss this matter here. 


\section{SEC. 4. THE SECRETION OF PANCREATIC JUICE AND OF BILE.}

\$213. The Secretion of Pancreatic Juice. Although in some cases, as that of the parotid of the sheep, the flow of saliva is contimuous or nearly so, in most animals, as in man, the intermittence of the secretion is very nearly absolute. While food is in the mouth saliva flows freely, but between meals only just sufficient is secreted to keep the mouth moist, and probably the greater part of this is supplied not by the larger salivary but by the small buccal glands. The flow of pancreatic juice, on the other hand, is much more prolonged, being in the rabbit continuous, and in the dog lasting for twenty hours after food. But this contrast between the secretion of saliva and that of pancreatic juice is natural, since the stay of food in the mouth even during a protracted feast is relatively short, whereas the time during which the material of a meal is able in some way or other to affect the pancreas is very prolonged.

The flow though continuous, or nearly so, is not uniform. In the dog the flow of pancreatic juice begins immediately after food has been taken, and rises to a maximum which may be reached within the first, or as in the case furnishing the diagram given in Fig. 83 the second hour, but which more commonly is not reached until the third or fourth hour. This rise is then followed by a fall, after which there is a secondary rise, reaching a second maximum at a very variable time but generally between the fifth and seventh hours. 'This second maximum, however, is never so high as the first.

The second rise may be due to material alsorbed from the intestines being carried in the circulation to the pancreas and so directly exciting the gland to activity, much in the same way aa, in the case of the stomach, the absorption of digested material promotes the flow of gastric juice, see $\$ 194$; and a similar abcorp- 
tion may contribute to the first rise also, but it is more probable that so marked and sudden a rise as this is carried out by some

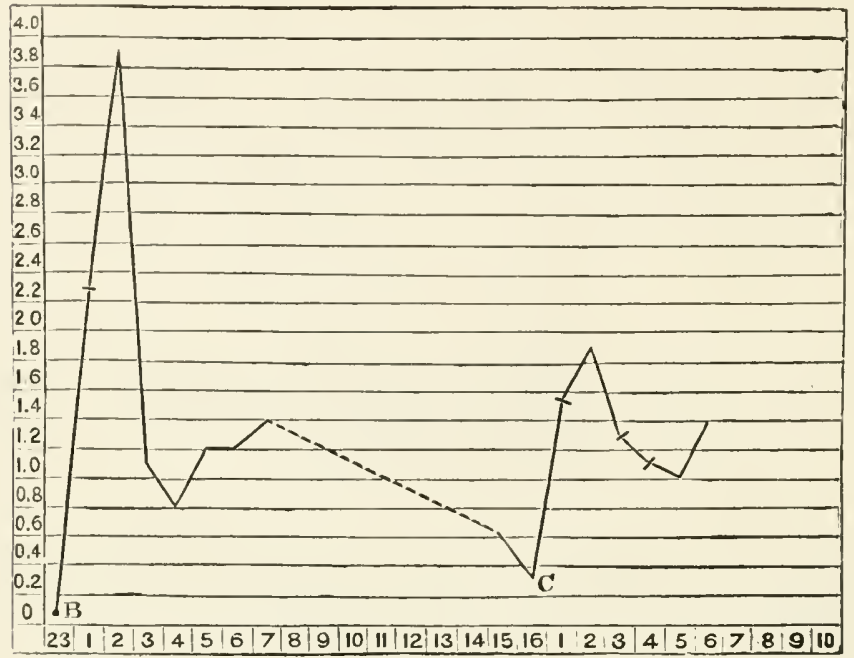

Fig. 83. Diagray illestrating the influence of Food on the Secretion of l'axcreatic Juice. (N. O. Bernstein.)

The abscissa represent hours after taking food; the ordinates represent in e.e. the amount of secretion in $10 \mathrm{~min}$. A marked rise is seen at $\mathrm{B}$ immediately after food was taken, with a secondary rise between the 4 th and 5 th honrs afterwards. Where the line is dotted the observation was interrupted. On food being again given at (', another rise is seen, followed in turn by a depression and a secondary rise at the 5th hour. A very similar curve would represent the secretion of bile.

nervous mechanism. The details of this mechanism have however not as yet been satisfactorily worked out.

Stimulation of the medulla oblongata, or of the spinal cord, will call forth secretion in a quiescent pancreas, or increase a secretion already going on. On the other hand a secretion already going on may be arrested by stimulation of the central end of the vagus, and the stoppage of the secretion which has been observed as occurring during and after vomiting is probably brought about in this way. This effect however is not confined to the vagus, it occurs also after stimulation of other afferent nerves, such as the sciatic.

$\$ 214$. The Secretion of Bile. The act of secretion of bile br the liver must not be confounded with the discharge of bile from the hile-duct into the duodenum. When the acid contents of the stomach are poured over the orifice of the biliary duct, a gush of bile takes place. Indeed, stimulation of this region of the duodenum with a dilute acid at nnce calls forth a flow, though alkaline fluids so applied have little or no effect. When no such 
acid thuid is passing into the duodenum no bile is, under normal circumstances, discharged into the intestine. The discharge is due to a contraction of the muscular walls of the gall-bladder and ducts, accompanied by a relaxation of the sphincter of the orifice; both acts are probably of a reflex nature, but the details of the mechanism have not been worked out.

The secretion of bile on the other liand, as shewn by the results of biliary fistula, is continuous; it appears never to cease. When no food is taken the bile passes from the liver along the hepatic and then back along the cystic luct (the flow being aided probably by peristaltic contractions of the museular fibres of the duct) to the gall-bladder, where it is temporarily stored; hence in starving animals, when no discharge is excited by food, the gallbladder becomes greatly distended with bile. But the secretion, though continuous, is not uniform. The rate of secretion varies, and is especially intluenced by food; it is seen to rise rapidly after meals, reaching its maximum, in dogs, in from four to eight hours. There seems to be an immediate, sudden rise when food is taken, then a fall, followed subsequently by a more gradual rise up to the maximum, and ending in a final fall to the lowest point. The curve of secretion, in fact, resembles that of the secretion of pancreatic juice in having a double rise; and as in that case so in this, it is very probable that the first rise is in part the result of nervous action, and it is also possible that nervous influences intervene in the second more lasting rise; but, as we shall see presently, even nervous influences may affect the liver in a very indirect manner, and our knowledge as to any direct action of the nervous system on the liver is at present very imperfect.

$\$ 215$. It must be remembered, however, that the liver is so peculiarly related to the other organs of digestion, and its vaseular arrangements so special that, with regard to it, as compared with many other organs, an intrinsic nervous mechanism must occupy a more or less subordinate position. The blood-supply of the pancreas for instance is dependent chiefly on the width for the time being of the pancreatic arteries; it will be affected of course by the general arterial pressure and by any circumstances which affect the outflow by the pancreatic veins, and therefore by the condition of the portal venous system of which those veins form a part; but in the main, the amount of blood bathing the alveoli of the pancreas will depend on whether the pancreatic arteries are constricted or dilated. The quality of the blood reaching the pancreas, being arterial blood drawn direct from the arterial foundation, will be modified only by such circumstances as modify the general mass of the blood.

Very different is the case of the liver. The supply of arterial blood coming direct through the hepatic artery is small compared with the mass pouring through the vena porta; it moreover, as we shall see, is distributed in capillaries among the small inter- 
lobular branches of the rena portie and has become venous, indeed merged with the portal blood, before it reaches the actual lobules. 'The supply of blood for the liver is mainly that throrgin the vena portie; and this supply is not, like an arterial supply, it fairly uniform one, modified chiefly by the vaso-motor events of the organ itself, but is dependent on what happens to be taking place in the alimentary canal and in abdominal organs other than the liver itself. When no food is being digested and the alimentary canal is at rest, the ressels of that canal, as we have already said in speaking of the stomach, are like those of the pancreas and salivary glands, in a state of tonic constriction; a relatively small quantity if blood passes through them ; hence the flow through the vena portite is relatively inconsiderable, and the pressure in that vessel is low. When digestion is going on all the minute arteries of the stomach, intestine, spleen and panereas are dilated, and general arterial pressure being by some means or other maintained (see $\$ 17 \cdot 2$ ), a relatively large quantity of blood rushes into the vena portie and the pressure in that vessel becomes much increased, though of course remaining lower than the general arterial pressue. Moreover, during digestion, peristaltic movements of the muscular coats of the alimentary canal are, as we have seen, active; and these movements, serving as aids to the circulation (see $\$ 103$ ), hell' to increase the portal flow. Further the spleen, as we shall see in speaking of that organ, is in many animals richly provided with plain muscular fibres, and in such cases seems, especially during digestion, to act as a muscular pump diving the blood onwards, with increased vigour, along the splenic veins to the liver. So that even were the liver not connected with the central nervous system by a single nervous tie, the tide of blood through the liver would ebb and flow according to the absence or presence of food in the alimentary canal.

An increase of bloot-supply does not of course necessarily mean an increase of secretory activity. As we have seen, $§ 189$, in the presence of atropine, the secretion of saliva may stand still in spite of dilated blood vessels and the consequent rush of blood; but we may safely assert that, other things being equal, a fuller blood-supply is favourable to activity. Apparently a mere change in the quantity of blood bathing an alveolus will not start in the cells the changes which constitute the act of secretion, any more than an increase in the blond bathing a muscular fibre will necessarily set going a contraction; but unless there be some counteracting intluence at work, a fuller and richer lymph around a cell will naturally lead to the cell taking up more material from the lymph, and so will increase the cell's store of energy. Hence, especially in the hepatic cell, which appears to he always at work, always undergoing metabolism of such a kind as to give rise to bile, we might fairly expect the greater flow through the portal vein to yuicken the flow through the bile duct. 
And as a matter of fact we do find vaso-motor action dominant over the secretion. In the various experiments which have been made to ascertain the action of the nervous system on the secretion of bile, it has always been found that stimulation of the medulla oblongata, or of the spinal cord, or of the abd minal splanchnic nerves, stops or at least checks the flow of bile. Now the effect of these stimulations is, as we have already seen more than once, a powerful constricting action on the abdominal blood vessels; by such stimulation the blood-supply of the liver is materially diminished, and in consequence the secretory activity is slackened or arrested.

But there is something bsides the mere quantity of blood to be considered in this relation. 'The blood which passes from the alimentary canal at rest is ordinary venous blood, laden simply with carbonic acid and the ordinary products of the metabolism of the muscular and mucous coats of the canal. When digestion is going on the portal blood is laden, as we shall see, with soms at all events of the products of digestion, with sugar probably and with various proteir borlies. And it is quite possible or even probable that some of these bodies in the portal blood reaching the hepatic cells stir them up to secretory activity; indeed this view may be regarded as supported by the facts that proteid food increases the quantity of bile secreted, whereas fatty foot, which as we shall see passes, chietly if not wholly, not by the portal vein but by the lymphatics and which is probably largely disposed of in some way or other before it can reach the liver, has no such effect.

Hence we may infer that at all events the second increase of the flow of bile which occurs during the later stages of digestion may be to a large extent the direct effect of blood, laden with digestive products, passing from the stomach and intestines, especially the latter, to the liver by the portal vein, quite independent of any direct nervous action on the liver itself; and indeed it is posible that the first rise also may be partly due to the increased How of blood from the stomach, aided by the absorption from that organ of a certain amount of digested material. Since, however, there is no evidence of any decrease in hlood-supply, or in the rate of absorption, corresponding to the fall between the two rises, some inthuences other than those which we are discussing must be at work in the matter.

$\$ 216$. It is interesting to observe that the pressure under which the bile is secreted is relatively low, not high like that of the saliva; it is much lower than the arterial pressure in the same animal, whereas in the case of saliva $(\$ 189)$ the pressure is greater than the blood-pressure in the carotid artery. But, in the case of bile, since the blood which flows through the hepatic lobules is, mainly, venous portal blood, we have to compare the pressure of the secretion not with arterial pressure but with the venous 
pressure in the portal system; and in the dog it has been found that while the pressure of the bile secreted stood at about $200 \mathrm{~mm}$. of a solution of sodium carbonate, that is, about $15 \mathrm{~mm}$. mercury, the blood-pressure in a branch of the superior mesenteric vein stood only at about $90 \mathrm{~mm}$. of the same solution, that is, about $7 \mathrm{~mm}$. mercury. Now the venous pressure in the mesenteric veins is higher, though only slightly higher, than that in the portal vein into which these pour their blood (the difference of pressure being the main cause why the blood flows from the one into the other), and is therefore certainly higher than the pressure in the portal capillaries of the hepatic lobules. So that what is true of the salivary gland is also true, on a different scale, of the liver, viz. that the pressure exerted by the secretion is higher than the pressure of the blood in the vessels feeding the secreting cells.

$\$ 217$. If the pressure in the bile duct be artificially increased, as by pouring fluid into the glass tube or manometer with which the cannula in the duct is connected, a resorption of the secreted bile takes place; and resorption will also take place within the body, when the pressure generated by the act of secretion itself reaches and is maintained at a sufficiently high level. Thus when in the living body the bile duct is ligatured, or becomes obstructed by gallstones or otherwise, fluid is accumulated on the near side of the ligature at a pressure which goes on increasing until resorption of bile takes place, bile salts and biliary pigments are thrown back upon the system, and "jaundice" results. It would appear that in these cases resorption takes place through the interlobular bile ducts and not through the hepatic cells or other structures within the lobules. The high pressure in the ducts does not lead to a reversal of the current in the hepatic cells (at most it slackens or possibly stops the current) but the bile secreted into the interlobular ducts escapes from these. It further appears that the escape is not into the blood ressels but into the lymphaties; the bile salts, pigments and other constituents are carried into the thoracic duct, and in an indirect manner only find their way into the blood stream.

To complete the history of the secretion of bile we ought now to turn to the manufacture of the biliary constituents within the cells. But since the hepatic cells are also engaged in labours other and more important perhaps than that of secreting bile, it will be convenient to defer what we have to say on this point until we come to speak of the formation of glycogen and of the general metabolic events taking place in the liver. 
SEC. 5. THE MUSCULAR MECHANISMS OF DIGESTION.

§ 218. From its entrance into the mouth until such remnant of it as is undigested leaves the body, the food is continually subjected to movements having for their object the trituration of the food as in mastication, or its more complete mixture with the digestive juices, or its forward progress through the alimentary canal.

Peristaltic Movements. The dominant movement in the alimentary canal is of the kind called peristaltic, carried out by means of the circular and longitudinal muscular coats. This is seen in its simplest form in the small intestine, is somewhat modified in other parts as in the stomach, and at the beginning and end of the canal is replaced or assisted by complicated movements carried out by various muscles.

The main part of a peristaltic movement, as seen in the small intestine, is a wave of contraction progressing longitudinally over the circular coat ( $(84)$. A contraction of the circular coat takes place at some level or other, narrowing the intestine at this level. From thence, the circularly disposed bundles contracting in sequence, the contraction travels as a wave downwards or upwards or both downwards and upwards. As a rule the wave, when started naturally, travels downwards from a part nearer the mouth to a part nearer the rectum. Thus a narrowing or constriction of the tube travels on wards as a wave driving the contents of the tube before it; when a butcher empties the contents of the intestine of a slaughtered animal by squeezing it high up with his hand or his thumb or forefinger, and then carrying the squeezing action downwards along the length of the intestine, he makes the passive intestine do very much what the circular coat does, actively, by contraction, in the living animal.

This action of the circular coat is further aided by a corresponding contraction of the longitudinal coat. When a length of the longitudinal coat is thrown into contraction, that length of the tube is shortened and widened; the effect is the antagonist of that produced by the contraction of the circular coat. Hence 
the two coats must contract at different times, otherwise they would neutralise each other's action. Most probably a section of the longitudinal coat contracts in front of the section of the circular coat which is about to contract, thus affording room for the contents which are about to be driven on, or even itself drawing them forward; but a contraction of the longitudinal coat, even if it followed after that of the circular coat, might still be useful in helping to bring back the tube to its normal width.

In the small intestine the tube is hung loosely and much twisted so that many loops are formed; the contents moreover are largely fluid. Hence the steady onward movement, such as is seen when more solid contents pass along the straight and somewhat firmily attached oesophagus, is complicated by movements due to a loop being projected forward by the entrance of fluid from above, or being dragged down by the weight of its new contents, or, on the other hand, due to a loop being retracted by the driving onward of its contents and the emptying of itself, and the like. In this way a peculiar writhing movement of the bowel is brought about, and the phrase 'peristaltic movement' is generally used to denote this total effect of the contraction of the muscular coats; it will however be best to restrict the meaning to the progressive contraction of the circular coat assisted, in most cases, by a similar progressive contraction of the longitudinal coat. We may consider the several special movements of the different parts of the canal.

Mastication. This in man consists chiefly of an up and down movement of the lower jaw, combined, in the grinding action of the molar teetl, with a certain amount of lateral and fore-and-aft movement. The lower jaw is raised by means of the temporal, masseter, and internal pterygoid muscles. The slighter effort of depression brings into action chiefly the digastric muscle, though the mylohyoid and geniohyoid probably share in the matter. Contraction of the external pterygoids pulls forward the condyles, and thrusts the lower teeth in front of the upper. Contraction of the pterygoids on one side will also throw the teeth on to the opposite side. The lower horizontally placed fibres of the temporal serve to retract the jaw.

Dnring mastication the food is moved to and fro, and rolled about by the movements of the tongue. These are effected by the muscles of that organ governed by the hypoglossal nerve.

The act of mastication is a voluntary one, guided, as are so many voluntary acts, not only by muscnlar sense but also by contact sensations. The motor filires of the fifth cranial nerve convey motor impulses from the brain to the above-mentioned muscles; but paralysis of the sensory fibres of the same nerve renders mastication difficult by depriving the will of the aid of the usual sensations.

$\$ 219$. Deglutition. The food when sufficiently masticated is, 
by the movements of the tongue, gathered up into a bolus on the middle of the upper surface of tnat urgan. The front of the tongue being raised - partly by its intrinsic muscles, and partly by the styloglossus - the bolus is thrust back between the tongue and the palate through the anterior pillars of the fauces or isth:nus faucinm. Inmmediately before it arrives there, the soft palate is raised by the levator palati, and so brought to touch the posterior wall of the pharynx, wnich, by the contraction of the upper margin of the superior constrictor of the pharynx, bulges somewhat forward. The elevation of the soft palate causes a distinct rise of pressure in the nasal chambers; this can be shewn by introducing a water manometer into one nostril, and closing the other jut previous to swallowing. By the contraction of the palatopharyng al muscles which lie in the posterior pillars of the fauces, the curved edges of those pillars are made straight, and thus tend to meet in the mid lle line, the small gap between them being tillel up by the uvula. Through these manouvres, the entrance into the posterior nares is blocked, while the soft palate is formed into a sloping roof, guiaing the bolus down the pharynx. By the contraction of the stylo-pharyngeus and palato-pharyngeus, the funnel-shaped bag of the pharynx is brought up to meet the descending morsel, very much as a glove may be drawn up over the finger.

Memwhile in the larynx, as shewn by the laryngoscope, the arytenoid cartilages and vocal cords are approximated, the latter being also raised so that they come very near to the false vocal cords; and the cushion at the base of the epiglottis covers the rima glottidis, while the epiglottis itself is depressed over the larynx. 'The thyroid cartilage is now, by the action of the laryngeal muscles, suddenly raised up behind the hyoid bone, and thus assists the epiglottis to cover the glottis. This movement of the thyroid can easily be falt on the outside. Thus, both the entrance into the posterior nare; and that into the larynx being closed, the impulse given to the bolus by the tongue can have no oth r effect than to propel it beneath the sloping soft palate, over the incline formed by the root of the tongue and the epiglottis. The palato-glossi or constrictores isthmi faucium, which lie in the anterior pillars of the fauces, by contracting, close the door behind the food which has passed them.

When the bolus of food is large, it is received by the middle and lower constrictors of the pharynx, which, contracting in sequence from above downwards, thrust it into the osophagus, along which it is driven by a similar series of sucessive contractions, that is to sav, by peristaltic action. This comparatively slow descent of the food from the pharynx into the stomach, may be readily seen if animals with long necks such as horses and dogs be watched while swallowing. When however the morsel is not large or when tie substance swallowed is liquid, the move- 
ment of the back part of the tongune may be sufficient not merely to introduce the food into the grasp of the constrictors of the pharynx, but even to propel it rapidly, to shoot it in fact, along the lax oesophagus before the muscles of that organ have time to contract. In such a mode of swallowing the middle and lower constrictors take little or no part in driving the food onward, though they and the osophagus appear to contract from above downwards after the food has passed by them, as if to complete the act and to ensure that nothing has been left behind. Deglutition in this fashion still remains possible after these constrictors have become paralysed by section of their motor nerves.

When a second act of deglutition succeeds a first with sufficient rapidity, the nervous changes which start the pharyngeal movements of the second act appear to inhibit the oesophageal movements of the first act; and when swallowing is repeated rapidly several times in succession, the ossophagus remains quiet and lax during the whole time, until immediately after the last swallow, when a peristaltic movement closes the series.

When the stethoscope is applied over the œesophagus, at different regions, a sound is heard during deglutition; sometimes two sounds are heard. The first and most constant is coincident with the passage of the bolus, and is due to this and to the muscular sound of the contracting muscles. The later and less constant sound appears to be caused by a quantity of air-bubbles with which the bolus was entangled, lodged at the cardiac end of the csophagus, being forced into the stomach by the sequent peristaltic contraction of the osophagus.

It will be seen, from what has been said, that deglutition, though a continuous act, may be regarded as divided into three stages. The first stage is the thrusting of the food through the isthmus faucium; this may be either of long or short duration. The second stage is the passage through the upper part of the pharynx. Here the food traverses a region common both to the food and to respiration, and in consequence the movement is as rapid as possible. The third stage is the descent through the grasp of the constrictors. Here the food has passed the respiratory orifice, and in consequence its passage again becomes comparatively slow, except in case of fluids and small morsels, when, as we have seen, it may continue to be rapid. The passage along the oesophagus may perhaps be regarded as constituting a fourth stage; but it will be more convenient to consider the oesophageal movements by themselves.

The first stage in this complicated process is undoubtedly a voluntary act. The raising of the soft palate and the approximation of the posterior pillars may also be, at times, voluntary, since they have been seen, in a case where the pharynx was laid bare by an operation, to take place before the food had touched these parts; but the movement may take place without any exer- 
cise of the will and in the absence of consciousness. Indeed the second stage taken as a whole, though some of the earlier component movements are, as it were, on the borderland between the voluntary and involuntary kingdoms, must be regarded as a reflex act. The third and last stage, whatever be the exact form which it takes, is undoubtedly reflex; the will has no power whatever over it, and can neither originate, stop, nor modify it.

Deglutition in fact as a whole is a reflex act; it cannot take place unless some stimulus be applied to the mucous membrane of the fauces. When we voluntarily bring about swallowing movements with the mouth empty, we supply the necessary stimulus by forcing with the tongue a small quantity of saliva into the fauces, or by touching the fauces with the tongue itself.

In the reflex act of deghtition, caused in the ordinary way by the food coming in contact with the fauces, the afferent impulses originated in the fauces are carried up to the nervous centre by the glosso-pharyngeal nerve, by branches of the fifth, and by the pharyngeal branches of the superior laryngeal division of the vagus. The latter seem of special importance, since the act of swallowing, quite apart from the presence of food in the mouth, may be brought out by centripetal stimulation of the superior laryngeal nerve. The efferent impulses descend the hypoglossal to the muscles of the tongue, and pass down the glosso-pharyngeal, the vagus through the pharyngeal plexus, the fifth, and the spinal accessory, to the muscles of the fauces and pharynx: their exact paths being as yet not fully known, and probably varying in different animals. The laryngeal muscles are governed by the laryngeal branches of the vagus.

The centre of the reflex act lies in the medulla oblongata. Deglutition can be excited, by tickling the fauces, in an animal rendered unconscious by removal of the brain, provided the medulla be left. If the medulla be destroyed, deglutition is impossible. The centre for deglutition lies higher up than that of respiration, so that in diseases or injuries involving the upper part of the medulla oblongata the former act may be impaired or rendered impossible while the latter remains untouched. It has been said to form part of the superior olivary bodies, but this view is based on anatomical grounds only. We shall have to deal with this and similar matters in treating of the central nervous system. It is probable that, as is the case in so many other reflex acts, the whole movement can be called forth by stimuli affecting the centre directly, and not acting on the usual afferent nerves.

$\$ 220$. Movements of the Esophagus. These as we have just said are fairly simple. The circular contraction hegun by the constrictors of the pharynx is continued along the circular coat of the œsophagus and assisted by an accompanying contraction of the longitudinal coat, the direction being always, save in the abnormal action of vomiting, from above downwards. 
It will be remembered that the muscular bundles of the oesophagus are composed of striated fibres in the upper part, and of plain unstriated fibre-cells in the lower part, the transition oceupying a different level in different animals. Nevertheless, as far as the peristaltic novement is concerned, the two kinds of fibres behave in the same way except that the peristaltic ware if we may so call it travels nore rapidly in the striated region.

These peristaltic movements of the wsophagus may, like those of the intestine, be seen after removal of the organ from the body; and indeed may continue to appear upon stimulation, for an unusual length of time. They may therefore be carried out by the museular elements, with or without the help of the nervous elenents embedded in then, apart from any action of the central nervous system. Nevertheless, in the living body, the novenents of the oesophagus seem to be in a special way dependent on the central nervous system; the contractions are not started and carried out by the walls of the tube alone and so transmitted from section to section in the walls of the tube itself; but afferent impulses started in the pharynx and passing to the medulla oblongata, give rise to reflex efferent impulses which descend along nervous tracts to successive portions of the organ. If the cesophagus le cut across some way down, or if a portion of the mitdle region be excised, stimulation of the pharynx will produce a peristaltic contraction, which travelling downwards will not stop at the cut or excision but will be continued on into the lower disconnected portion by means of the central nervous system. And it is stated that ordinary peristaltic contractions of the lower part of the osophagus can be readily excited by stimulation of the pharyux, but not by stimuli applied to its own mucous membrane. In the reflex act which thus brings about the peristaltic contraction of the cesophagus the afferent nerves are those of the pharynx, viz. the superior laryngeal nerve and pharyngeal branches of the vagus, branches of the fifth, and in some animals at least branches of the glossopharyngeal, but chiefly the first; and oesophageal movements can easily be excited by centripetal stimulation of the superior laryngeal. The centre lies in the medulla oblongata, being a part of the general deglutition centre; and the efferent impulses pass along fibres of the vagus, reaching the upper part of the ossophagus by the recurrent laryngeal nerves and the lower part through the oesophageal plexuses of the vagus (Fig. 8t). Section of the trunk of the vagus renders difficult the passage of food along the œsophagus, and stimulation of the peripheral stump causes œesophageal contractions.

The force of this movement in the oesophagus is considerable; thus in the dog a ball pulling by means of a pulley against a weight. of 250 grammes has been found to be readily carried down from the pharynx to the stomach.

At the junction of the cesophagus with the stomach the circular 
fibres usually remain in a more or less permanent condition of tonic or obscurely rhythmic contraction, nore particularly when the stomach is full of food, and thus serve as a sphincter to prevent the return of ford from the stomacli into the osophagus. Upon the arrival of the bolus of food at the end of the oesophagus, the centre for this sphincter is inhibited and the orifice is thus opened up. Possibly the patency of the orifice is still further secured by a contraction of the longitudinal muscular fibres which radiate from the end of the oesophagus over the stomach.

$\$ 221$. Movements of the Stomach. While the object of the oesophageal novement is simply to carry the swallowed bolus with all due speed to the stomach, and while the intestinal movement has, in like manner, simply to carry the intestinal contents onward, the twisted course of the looped path ensuring all the mixing of the constituents of the contents that may be necessary, the movements of the stomach have a double object: on the one hand to provide an adequate exposure of the contents of the dilated chamber to the influence of the gastric juice, and on the other to propel the partially digested food, when ready, into the duodenum. We may accordingly distinguish between what we may call the "churning" and the "propulsive" movements of the stomach.

When the stomach is empty all the muscular fibres as we have said, longitudinal, circular and oblique, fall into a condition which we may perhaps speak of as an obscure tonic contraction. The whole stomach is small and contracted, its cavity is nearly obliterated, and the mucous membrane, owing to the predominance of the circular coat, is like the lining membrane of an empty artery, thrown into longitudinal folds. As more and more food enters the stomach all the coats hecome relaxed, with the exception of the pyloric sphincter, which remains at first permanently closed, and the less marked cardiac sphincter, which merely relaxes from time to time at each act of swallowing. No sooner however do the conts thus become relaxed than they set up obscure rhythmical peristaltic contractions, giving rise to the "churning" movenents. These movements have been described as of such a kind that the contents How in a main current from the cardia along the greater curvature to the pylorus, and back to the cardia along the lesser curvature, subsidiary currents mixing the peripheral portions of the contents with the more central; it may be doubted however whether any snch regularity of flow is marked or constant, and it is not easy to see by what combination and sequence of contractions in the three coats, longitudinal, circular and olliqne, such a regular How can be produced. But in any case, by such rhythmical contractions the food and gastric juice are rolled about and mixed together. These churning movements are feeble at first, even thongh the stomach be filled and distended by a large meal rapilly eaton; they become more and more pronounced as digestion- proceeds. 
Before digestion has proceeded very far the 'propulsive' movements begin. These occur at intervals, and are repeated at first slowly but afterwards more rapidly. Each movement consists in a contraction of the circular muscular fibres more powerful than any taking part in the churning movements, and leading to a circular constriction which, beginning apparently at about the obscurely defined groove which marks the beginning of the antrum pylori, travels down towards the pylorus, propelling the food onward. This movement is accompanied or rather preceded by a relaxation of, that is to say in all probability an inhibition of the permanent contraction of, the sphincter pylori itself, in order that the gastric contents may pass into the duodenum. But the occurrence of this relaxation is determined by the nature of the gastric contents; for if the propulsive movement drives large undigested pieces towards the pylorus, the sphincter is apt to close again, the result of which is that the undigested morsels are carried back into the main body of the stomach.

The combined effect then of the churning and of the propulsive movements is, after a certain part of the meal has been reduced to a thick fluid condition somewhat resembling pea soup and often called chyme, to strain off this more fluid part into the duodenum, and to submit the remaining still solid pieces to the further action of the gastric juice.

As digestion proceeds, more and more material leaves the stomach, which is thus gradually emptied, the last portions which are carried through being those parts of the food which are least digestible, and any wholly indigestible foreign bodies which happen to have been swallowed; the latter may perhaps never leave the stomach at all. The presence of food leads to the development of the movements; but evidently it is not the mere mechanical repletion of the organ which is the cause of the movements, since the stomach is fullest at the beginning when the movements are slight, and becomes emptier as they grow more forcible. The one thing which does increase pari pussu with the movements is the acidity, which is at a minimum when the (generally alkaline) food has been swallowed, and increases steadily onwards. It has not however been definitely shewn that the increasing acidity is the efficient stimulus, giving rise to the movements.

The movements of even a full stomach are said to cease during sleep. The nervous mechanism of the gastric movements had better be considered in connection with that of the intestinal movements.

$\S 222$. Vomiting. In a conscious individual this act is preceded by feelings of nausea, during which a copious flow of saliva into the mouth takes place. This being swallowed carries down with it a certain quantity of air, the presence of which in the stomach, by assisting in the opening of the cardiac sphincter, subsequently facilitates the discharge of the gastric contents. The nausea is 
generally succeeded at first by ineffectual retching in which a deep inspiratory effort is made, so that the diaphragm is thrust down as low as possible against the stomach, the lower ribs being at the same time forcibly drawn in; since during this inspiratory effort the glottis is kept closed, no air can enter into the lungs; but some is drawn into the pharynx, and thence probably descends hy a swallowing action into the stomach. When retching passes on to actual romiting this mspiratory effort is succeeded by a sudden violent expiratory contraction of the abdominal walls, the glottis still being closed, so that the whole force of the effort is spent, as we shall see it is in defæcation, in pressure on the abdominal contents. The stomach is therefore forcibly compressed from without. At the same time, or rather immediately before the expiratory effort, by a contraction of its longitudinal fibres the œsophagus is shortened and the cardiac orifice of the stomach brought close under the diaphragm, while apparently by an inhibition of the circular sphincter, aided perhaps by a contraction of the fibres which radiate from the end of the cesophagus over the stomach, the cardiac orifice, which is normally closed, is somewhat suddenly dilated. This dilation opens a way for the contents of the stomach, which, pressed upon by the contraction of the abdomen, and to a certain but probably only to a slight extent by the contraction of the gastric walls, are driven forcibly up the cesophagus. The mouth being widely open, and the neck stretched to afford as straight a course as possible, the vomit is ejected from the body. At this moment there is an additional expiratory effort which serves to prevent the vomit passing into the larynx. In most cases too the posterior pillars of the fauces are approximated, in order to close the nasal passage against the ascending stream. This however in severe vomiting is frequently ineffectual.

Thus in vomiting there are two distinct acts: the dilation of the cardiac orifice and the extrinsic pressure of the abdominal walls in an expiratory effort. Without the former the latter, even when distressingly vigorous, is ineffectual. Without the latter, as in urari poisoning, the intrinsic movements of the stomach itself are rarely sufficient to do more than eject gas, and, it may be, a very small quantity of food or fluid. Pyrosis or waterbrash is however probably brought about by this intrinsic action of the stomach.

During vomiting the pylorus is generally closed, so that but little material escapes into the duodenum. When the gall-hladder is full, a copious flow of bile into the duodenum accompanies the act of vomiting. Part of this may find its way into the stomach, as in bilious vomiting, the pylorus then having evidently been opened.

The nervous mechanisin of yomiting is complicated and in many aspects obscure. The efferent impulses which cause the 
expiratory effort must come from the respiratory centre in the medulla, with these we shall deal in speaking of respiration. 'The dilation of the cardiac orifice is caused, in part at least, by impulses deseending the vagi, since when these are cut real voniting with discharge of the gastric contents, if it takes place at all, becomes difficult through want of readiness in the dilation. Such intrinsic movements of the stomach as do take place, and the movements of the oesophagus appear to be carried out by the usual nerves. The elferent impulses which cause the flow of saliva in the introduct ory nausea also descend along the usual nerves such as the chorda tympani. These various impulses may best be consilered as starting from a vomiting centre in the medulla, having close relations with the respiratory centre. This centre may be excited, may be thrown into action, in a reflex manner, by stimuli applied to p eripheral nerves, as when vomiting is induced by tickling the fauces, or by irritation of the gastric membrane, or by obstruction of the intestine due to ligature, hernia, etc. That the vomiting in the last instance is due to nervous action, and not to any regurgitation of the intestinal contents, is shewn by the fact that it will take place when the intestine is perfectly empty and may be prevented by section of the mesenteric nerves. The vomiting attending renal and biliary calculi is apjarently also reflex in origin. $\checkmark$ omiting in fact as a rule is a reflex action, the afferent impulses passing along one or other nerves, but most frequently along those connected with the alimentary canal, that is along afferent fibres running in the vagus or in the splanchnic nerves. The centre however may be affected directly, as probably in the cases of some poisons, and in some instances of vomiting from disease of the medulla oblongata. Lastly, it may be thrown into action by impulses reaching it from parts of the brain higher up than itself, as in cases of romiting produced by smells, tastes or emotions, or by the recollection of past events, and in some cases of vomiting due to cerebral disease.

Many emetics, such as tartar emetic, appear to act directly on the centre, since, introduced into the blood, they will produce romiting after a bladder has been substituted for the whole stomach. Others again, such as mustard and water, act in a reflex manner by irritation of the gastric mucous membrane. With others, again, which cause vomiting by developing a nauseous taste, the action involves parts of the brain higher than the centre itself.

$\$ 223$. Morements of the Small Intestine. These, as we have already said, are the typical peristaltic movements, simple except in so far as ther are complicated by the existence of the pendent loops, the peculiar oscillating movements of which appear to be produced chiefly by the longitudinal fibres.

The peristaltic movements, as a rule, take place from above downwards, and a wave heginning at the pylorus may ke traced a long way down. But contractions may, and in all probability 
occasionally do, begin at various points along the length of the intestine. A movement started by artificial stimulation some way down the intestine, may travel not only downwards but also upwards ; it has leen disputed however, whether in the living body any natural backward peristaltic movement really takes place. In the living body the intestines have periods of rest, alternating with periods of activity, the occurrence of the periods depending on various circumstances; the intensity of the movements also varies very considerably.

$\$ 224$. Morements of the Large Intestine These are fundamentally the same as those of the small intestine, but distinct in so far as the latter cease at the ileo-crecal valve, at which spot the former normally begin; they are simpler, in as much as the pendent loops are absent, and not so vigorous, since relatively to the diameter of the tube, the amount of muscular fibre is less. Along the colon where the sacculi are well developed the movement may perhaps be described as almost intermittent from sacculus to sacculus, the contents of one sacculus being driven by the peristaltic contractions of its circular fibres into the next sacculus, which prepares to receive them by a relaxation of its circular and a contraction of its longitudinal fibres

Since the lips of the ileo-creal valve are placed transversely across the cxeum, not only does distention of the cæcum. by stretching the valve along the line of the lips, bring them into apposition, but the pressure exerted by the peristaltic movement has the same effect. In this way any return of the contents from the large to the small intestine is prevented.

Arrived at the sigmoid flexure, the contents, now more or less solid fæces, are supported by the bladder and the sacrum, so that they do not press on the sphincter ani.

$\$ 225$. Deforertion. This is a mixed act, heing superficially the result of an effort of the will, and yet carried out by means of an involuntary mechanism. Part of the voluntary effort consists in producing a pressure-effect, by means of the abdominal muscles. These are contracted forcibly as in expiration, but the glottis being closed and the escape of air from the lungs prevented, the whole force of the pressure is brought to bear on the abdomen itself, and so drives the contents of the descending colon onward towards the rectum. The sigmoid flexure is by its position sheltered from this pressure; a body introduced per anum into the empty rectum is not affected by even forcible contractions of the abdominal walls.

The anus is guarded by the sphincter ani, which is habitually in a state of normal tonic contraction, capable of heing increased or diminished by a stimulus applied, either internally or externally, to the anus. The tonic contraction is in part at least due to the action of a nervous centre situated in the lumbar spinal cord. If the nervous connection of the sphincter with the spinal cord be 
broken, relaxation takes place. If the spinal cord be divided somewhat higher up, for instance in the dorsal region, the sphincter, after the depressing effect of the operation, which may last several days, has passed off, regains and subsequently maintains its tonicity, shewing that the centre is not placed higher up than the lumbar region of the cord. The increased or diminished contraction following on local stinulation is probably due to reflex augmentation or inhibition of the action of this centre. The centre is also subject to influences proceeding from higher regions of the cord, and from the brain. By the action of the will, by emotions, or by other nervous events, the lumbar sphincter centre may be inhibited, and thus the sphincter itself relaxed; or augmented, and thus the sphincter tightened. A second item therefore of the voluntary process in defrecation is the inhibition of the lumbar sphincter centre, and consequent relaxation of the sphincter muscle. Since the lumbar centre may remain wholly efficient when separated from the brain, the paralysis of the sphincter which occurs in certain cerebral diseases is probably due to inhibition of this lumbar centre, and not to paralysis of any cerebral centre.

Thus a voluntary contraction of the abdominal walls, accompanied by a relaxation of the sphincter, might press the contents of the descending colon into the rectum and out at the anus. Since however, as we have seen, the pressure of the abdominal walls is warded off the sigmoid flexure, such a mode of defæcation would always end in leaving the sigmoid flexure full. Hence the necessity for these more or less voluntary acts being accompanied by an involuntary augmentation of the peristaltic action of the large intestine, sigmoid flexure and rectum.

In the movenents of the rectum we can trace out more distinctly than in other regions of the alimentary canal the separate actions of the longitudinal and circular fibres. The former, by means of contractions travelling from above downwards, shorten the rectum, and since the anus affords a more or less fixed support pull the rectum and its contents down; the latter, by means of contractions travelling from above downwards bnt taking place somewhat later, narrow the rectum and so squeeze the contents onwards and outwards.

Defrcation then appears to take place in the following manner. The large intestine and sigmoid flexure becoming more and more full. stronger and stronger peristaltic action is excited in their walls. By this means the fieces are driven into the rectum and so, by a continuance of the movements increasing in vigour. against the sphincter. Through a volnntary act, or sometimes at least by a simple reflex action, the lumbar sphincter centre is inhibited and the sphincter relaxed. At the same time the contraction of the abdominal muscles presses firmly on the descending colon, and thus, contractions of the levator ani assisting, the contents of the rectum are ejected. 
It must however be remembered that, while in appealing to our own consciousness, the contraction of the abdominal walls and the relaxation of the sphincter seem purely voluntary efforts, the whole act of defæcation, including both of these seemingly so voluntary components, may take place in the absence of consciousness, and indeed, in the case of the dog at least, after the complete severance of the lumbar from the thoracic cord. In such cases the whole act must be purely reflex, excited by the presence of faces in the rectum.

$\$ 226$. The nervous mechanisms of gastric and intestinal movements. Both the stomach and intestines when removed from the body and thus wholly separated from the central nervous system may, by direct stimulation, be readily excited to movements; and indeed in the absence of all obvious stimuli, movements which seem to be spontaneous may at times be observed. The movements of which we are speaking are orderly movements of a peristaltic nature, not mere local contractions of a few bundles of plain muscular fibres. The alimentary canal therefore, like the heart, though to a less degree, possesses within itself such mechanisms as are requisite for carrying out its own movements; and, as in the case of the heart, there is no adequate evidence that the ganglia scattered in its muscular walls, those nanely forming the plexus of Auerbach, play any prime part in developing these movements.

On the other hand, powerful movements of a peristaltic kind may be induced, not only as we have already seen in the osophagus but also in the stomach, in the small intestine, and even in the large intestine by stimulation of the vagus nerve.

The chief and usual cause of the movements of the stomach and intestines is the presence of food in their interior. But we do not know definitely the exact manner in which the food produces the movement. It may be that the food, by stimulating the mucous membrane, sends up afferent impulses, and that these give rise by reflex action to efferent inıpulses which descend the vagus fibres to successive portions of the canal, in a manner similar to that already described in reference to the osophagus. If this be so the efferent impulses reach the stomach and upper part of the duodenum by the terminal portions of the two vagi, Fig. 84, $R$. V. L. V., and reach the intestines by the portion of the right or posterior vagus, Fig. $84, R^{\prime}$. $V^{\prime}$, which passes into the solar plexus and thence by the mesenteric nerves. The afferent impulses from the stomach travel also apparently by the vagus; the paths of those from the intestines have not yet been determined.

But that such a reflex action through vagus fibres is not the only means by which the presence of food brings about the movements in question, is shewn by the fact that these continue to be developed after section of both vagus nerves. Probably the whole action is a mixed one which we may picture to ourselves somewhat 
as follows. The alimentary canal possesses a power of spontaneous movement, feeble it is true, very inferior to that of the heart, and very apt to be latent, but still existing. The presence of food

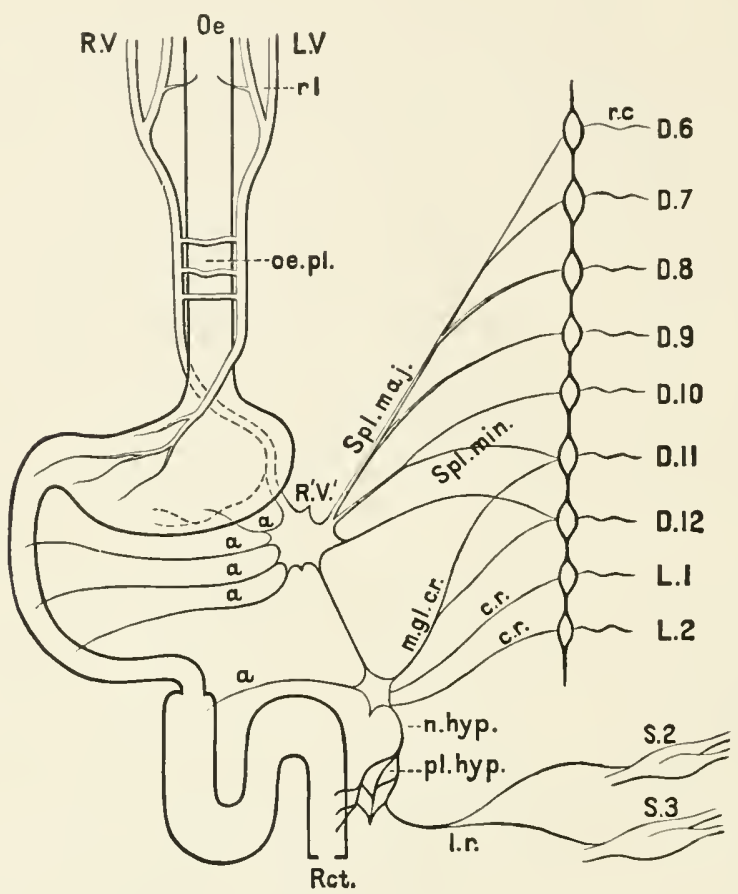

Fig. 84. Diagram to illustrate the Nerveg of the Almextary Caxal in THE DOG.

'The figure is for the sake of simplicity made as diagrammatic as possible, and does not represent the antomical relations.

Oe to Ret.-The alimentary canal, oesophagus, stomach, small intestine, large intestine, rectum.

$L V$. I.eft vagus nerve, ending on frout of stomacl. r.l. recurrent larvngeal nerve supplying upper part of cesophagus. R.I. right vagus, joining left vagus in œsophageal plexus, oe. pl., supplying the posterior part of stomach and con. tinued as $R^{\prime} . V^{\prime}$. to join the solar plexus, here represented by a single ganglion and connected with the inferior mesenteric ganglion (or plexus) m. gl.- $u$. branches from the solar plexus to stomach and small intestine, and from the mesenteric ganglion to the large intestine.

Spl. maj. Large splanchnic nerve arising from the thoracic ganglia and rami com. mmicantes r.r. belonging to dorsal nerves from the 6th to the 9 th (or 10 th).

Spl. min. Small splanchnic nerve similarly arising from 10 th and 11 th dorsal nerves. These both join the solar plexus and thence make their way to the alimentary canal.

C.r. Nerves from the ganglia \&c. belonging to 11 th and 12th dorsal and 1st and 2nd lumbar nerves, proceeding to the inferior mesenteric ganglia (or plexus) $m$. $g l$. and thence br the hypogastric nerve $n$. hyp. and the hypogastric plexus $\mathrm{pl}$. hup. to the circular muscles of the rectum.

l.r. Nerves from the $2 n d$ and $3 r d$ sacral nerves, $S_{2}, S_{3}$ (nervi erigentes), proceeding by the hypogastric plexns to the longitudinal muscles of the rectum. 
in some way or other, by some direct action quite apart from the central nervous system, is able to increase this power so that, without any aid from the central nervous system, as after section of the vagi, adequate peristaltic movements can, under favourable circumstances, be carried out. Nevertheless in the normal course of events satisfactory movements are still further secured by the reflex action through vagus fibres just described. Thus, in the dog, the act of swallowing food or even the mere smell of food has been observed to increase the movements of a piece of intestine isolated from the rest of the alimentary canal but retaining its connections with the central nervous system. Under this view the peristaltic movements produced by centrifugal stimulation of the vagus in the neck are comparable not so much with the contraction of a skeletal muscle when its motor nerve is stimulated as with the beats which may be called forth in an inhibited or otherwise quiescent heart by stimulation of the cardiac augmentor fibres.

Indeed we may perhaps call the vagus fibres which pass to the stomach and intestines augmentor fibres rather than motor fibres. We have all the more reason to do so since there exist companion but antagonistic inhibitory fibres. If while lively peristaltic action is going on in the bowels, the splanchnic nerves be stimulated the bowels are brought to rest, often in a very abrupt and marked manner. Inhibitory fibres therefore run in the splanchnic nerves, Fig. 84, Spl. mag. and min., passing along them from the spinal cord to the abdominal plexuses, and thence to the alimentary canal.

This view however that the movements of the alimentary canal are of a spontaneous nature, simply augmented on the one hand and inhibited on the other by the central nervous system, can only be applied to the middle regions, to the stomach and intestines in which peristaltic action is seen in its simple form. At the beginuing of the alimentary canal, at the mouth and pharynx and also at the cesophagus, the central nervous system intervenes in a decided manner : the movements of these parts, as we have seen, are carried out directly by the central nervous system. Something similar is also seen at the end of the canal, at the rectum and sigmoid flexure. These parts are governed on the one hand by fibres reaching them from the lover regions of the cord by the sympathetic system, by the liypogastric nerves and hypogastric plexus, and on the other hand by fibres reaching them along certain cerebro-spinal, namely sacral, nerves (in the dog the second and third sacral nerves) by the branches of these nerves, called nervi erigentes (Fig. 84). And the govermment by these nerves is one in which the movenents are directly carried out by means of the central nervous system.

Hence this is the part of intestinal movement which fails in diseases of the central nervous system; the failure leadiug to 
obstinate constipation if not to actual difficulty of defrecation. The presence of frees in the sigmoid flexure no longer stirs up the reflex mechanism for their discharge; meanwhile the more independent movements of the higher parts of the canal continue to drive the contents onward; and hence the freces accumulate in the sigmoid flexure and colon awaiting the delayed action of the imperfect reflex mechanism.

With regard to the exact manner in which the presence of food acts as a stimulus it may be worth while to remark, that. though in the stomach as we have seen mere fulness is not the efficient cause of the movements, since these become more active as digestion proceeds and the bulk of the contents diminishes, yet in the intestine distension of the bowel up to certain limits most distinctly increases the vigour of the movements just as distension of the cardiac cavities within certain limits improves the cardiac stroke. This is well seen in obstruction of the bowels, in which cases the bowel distended above the obstruetion is frequently thrown into violent peristaltic movements.

$\$ 227$. Next to the presence of food in the interior of the alimentary canal, a deficient oxygenation of the blood supplied to the walls of the canal or the sudden cutting off of the supply of blood, may be regarded as the most powerful provocatives of peristaltic action. When the aorta is clamped or when the respiration is seriously interfered with, peristaltic movements become very pronounced. Thus, in death by asphyxia or suffocation, an involuntary discharge of freces, which is in part at least the result of increased peristaltic action, is not an unfrequent result; and the marked peristaltic movements which are so frequently seen in an animal when the abdomen is laid open immediately after death, appear to be due to the cessation of the circulation and the consequent failure in the supply of blood to the walls of the alimentary canal and not, as has been suggested, to the contact with air of the peritoneal surface. Since it is blood which brings oxygen to the tissues, failure in the supply of blood is tantamount to failure in the supply of oxygen; but the blood current brings other things besides oxygen and also takes things away; and the failure of this aetion also probably, as well as failure in the supply of oxygen, provolie the movements in question.

The movements thus produced are to some extent the result of the defieient supply of blood acting directly on the walls of the canal, though in asphyxia at all events this effect may be increased by the too venous blood stimulating the ecntral nervous system and thus sending augmentor impulses down the vagus.

With regard to the mode of action of the drugs which promote peristaltic action it will be suffieicnt here to say that while some such as nicotine appear to act directly on the walls of the canal, others such as strychinia produce their effect chiefly by acting through the eentral nervous system. 


\section{SEC. 6. THE CHANGES WHICH THE FOOD UNDER- GOES IN THE ALIMENTARY CANAL.}

$\$ 228$. Having studied the properties of the digestive juices as exhibited outside the body, and the various mechanisms by means of which the food introduced into the body is brought under the influence of those juices, we have now to consider what, as matters of fact, are the actual changes which the food does undergo in passing along the alimentary canal, what are the steps by which the contents of the canal are gradually converted into freces. The events which lead to this conversion are twofold. On the one hand the digestive juices do bring about, inside the alimentary canal, changes which in the main are the same as those observed in laboratory experiments outside the body and described in previous sections, though the results are somewhat modified by the special conditions which obtain within the body. On the other hand absorption, that is to say, the passage from the interior of the canal into the blood vessels and lymphatics, of digested material in company with water, is going on along the whole length of the canal, and especially in the small and large intestines. It will be convenient to confine ourselves at present to the study of the first class of events, the changes effected in the canal, merely noting the disappearance of this or that product, and deferring the difficult problem of how absorption takes place to a subsequent and separate discussion.

In the mouth the presence of the food, assisted by the movements of the jaw, causes, as we have seen, a flow of saliva. By mastication, and by the addition of mucous saliva, the food is broken into small pieces, moistened, and gathered into a convenient bolus for deglutition. In man some of the starch is, even during the short stay of the food in the mouth, converted into sugar ; for if boiled starch free from sugar be even momentarily held in the mouth, and then ejected into water (kept boiling to destroy the ferment) it will be found to contain a decided amount of sugar. In many animals no such change takes place. The viscid saliva of the dog serves almost solely to assist in deglutition; and even the longer stay which food makes in the 
mouth of the horse is insufficient to produce any marked conversion of the starch it may contain. During the rapid transit through the cesophagus no appreciable change takes place.

The amount of absorption of digested material, or even of simple water from the mouth or cesophagus, must always be insignificant.

\section{The Changes in the Stomach.}

$\$ 229$. The arrival of the food, the reaction of which is either naturally alkaline, or is made alkaline, or at least is reduced in acidity, by the addition of saliva, causes a flow of gastric juice. This, already commencing while the food is as yet in the mouth, increases as the food accumulates in the stomach, and as, by the churning gastric movements, one part after another of the food is brought into contact with the mucous membrane.

'The characters of the juice appear to change somewhat as the act of digestion proceeds. The amount of pepsin in the gastric contents increases for some time after food is taken, and probably the actual secretion increases also. 'The acidity of the gastric contents is at first very feeble ; indeed in man, in some cases at least, for some little time after the beginning of a meal no free acid is present, and during this period the conversion of starch into sugar may continue. This condition however is temporary only; very soon the contents become acid, arresting the action of and ultimately destroying the amylolytic ferment; and, since the rate of the secretion of acid appears to be fairly constant, the contents of the stomach, unless fresh alkaline food be taken, become more acid as digestion goes on.

'The gross effect of gastric digestion is to break up and partly to dissolve the larger lumps of masticated food into a thick greyish soup-like liquid called chyme, with which are still mixed in variable quantity larger and smaller masses of less changed food. This is the result, partly of the solution of proteid matters. partly of the solution of the gelatiniferous connective-tissue holding the proteid elements together. In a fragment of meat, for instance, the muscular fibres, through the solution of the connective-tissue binding them together, fall asunder, the sarcolemma is dissolved, and the fibres themselves split up sometimes longitudinally but most frequently by transverse cleavage into discs, and are ultimately more or less reduced partly into a granular mass, partly to actual solution. In a piece of tissue containing fat, the connective-tissue binding the fat cells together and the envelopes of the fat cells are dissolved, so that the fat, fluid at the temperature of the body, is set free from the individual cells and runs together into larger and smaller masses. In vegetable tissue the proteid elements are in part dissolved and, though there is no evidence that in man cellulose 
is dissolved in the stomach, the whole tissue is softened and to a certain extent disintegrated. Milk is curdled and the curd subsequently more or less dissolved.

The thick soup-like acid chyme consists accordingly partly of substances which have entered into actual solution, partly of mere particles or droplets of proteid, fatty or other nature and partly of masses small or great, which may be recognized under the microscope as more or less changed portions of animal or vegetable tissue. The amount of material actually dissolved is in most specimens of chyme exceedingly small. When the solid parts are removed by filtration the clear filtrate eontains besides salts, pepsin and free hydrochloric acid (the constituents of the gastric juice), a small amount of sugar, of some of the bye products of proteid digestion, and of albumose and peptone. The sugar is often absent, and the amount of peptone (or albumose) is always small.

During gastric digestion the chyme thus formed is from time to time ejected through the pylorus, accompanied by even large morsels of solid less-digested matter. 'This may oceur within a few minutes of food having been taken; but the larger escape from the stomach probably does not in man begin till from one to two, and lasts from four to five hours, after the mcal, becoming more rapid towards the end, and such pieces as are the least broken up by the gastric juice and movements being the last to leave the stomach. Water taken by itself appears to be passed on at once into the small intestine.

'The time taken up in gastric digestion probably varies in the same animal not only with different articles of food but also with varying conditions of the stomach and of the body at large. In different animals it varies very considerably, being from 12 to 24 hours in the dog after a full meal, while the stomachs of rabbits are never empty but always remain largely filled with food, even during starvation. In man the stomach probably becomes empty between the usual meals.

The total amount of change which the food undergoes in the stomach, that is the share taken by the stomach in the whole work of digestion, seems to vary largely in different animals, and in the same animal differs according to the nature of the meal. In a dog fed on an exclusively meat diet, a very large part of the digestion is said to be carried out by the stomach, very little work apparently being left for the intestines; that is to say, the larger part of the meal is reduced in the stomach to actual solution and a considerable quantity is probably absorbed directly from the stomach. In such cases the amount of peptone found in the stomach during the digestion of the meal is found to be fairly constant, from which it may be inferred that the peptone is absorbed so soon as it is formed. There is also evidence that fat may to a certain extent undergo in the stom- 
ach changes leading to emulsion, similar to those which, as we shall see, are carried out in the small intestine.

But such cases as these cannot be regarded as typical cases of gastric digestion, and in man, at all events, living on a mixed diet the work of the stomach appears to be to a large extent preparatory only to the subsequent labours of the intestine. It is true that our information on this matter is imperfect, being chiefly drawn from the study of cases of gastric or duodenal fistula, in which probably the order of things is not normal, or being in large measure deductions from experiments on animals, whose economy in this respect must be largely different from our own; but we are probably safe in concluding that, in ourselves, the chief effect of gastric digestion is by means of the disintegration spoken of above to reduce the lumps of food to the more uniform chyme and so to facilitate the changes which take place in the small intestine. During that disintegration some of the proteid in the meal is converted into peptone; and the peptone so formed is probably absorbed at once; but much proteid remains unchanged or at least is not converted into peptone, and the fats and starches undergo in themselves very little change indeed.

In the act of swallowing, no inconsiderable quantity of air is carried down into the stomach, entangled in the saliva, or in the food. This is returned in eructations. When the gas of eructation or that obtained directly from the stomach is examined, it is found to consist chiefly of nitrogen and carbonic acid, the oxygen of the atmospheric air having been largely absorbed. In most cases the carbonic acid is derived by simple diffusion from the blood, or from the tissues of the stomach, which similarly take up the oxygen. In many cases of flatulency, however, it may arise from a fermentative decomposition of the sugar which has been taken as such in food or which has been produced from the starch, the gas being either formed in the stomach or passing upwards from the intestine through the pylorus.

The enormous quantity of gas which is discharged through the mouth in cases of hysterical flatulency, even on a perfectly empty stomach, and which seems to consist largely of carbonic acid, presents difficulties in the way of explanation; it is possible that it may be simply diffused from the blood, but it is also possible that in many cases it is derived from air which the patient has hysterically swallowed, the oxygen having been removed, in the stomach, by absorption and replaced by carbonic acid.

\section{In the Small Intestine.}

$\$ 230$. The semi-digested acid food, or chyme, as it passes over the biliary orifice, causes as we have seen $(\$ 215)$ gushes of bile, and at the same time the pancreatic juice flows into the 
intestine freely. These two alkaline fluids, especially the more strongly and constantly alkaline pancreatic juice, tend to neutralize the acidity of the chyme, but the contents of the duodenum do not become distinctly alkaline until some distance from the pylorus is reached. The rapidity with which the change in the reaction is completed is not the same in all animals, and in the same animal appears to vary according to the nature of the food, and various circumstances. In man, living on a mixed diet, the contents have probably become distinctly alkaline before they have passed far down the duodenum. On the other hand in dogs, the contents of the small intestine have been observed to be acid thronghout, and that, not only when fed on starch and fat, which might, by an acid fermentation of which we shall presently speak, give rise to an acid reaction, but even when fed on meat.

The conversion of starch into sugar, which as we have seen is sooner or later arrested in the stomach, is resumed with great activity and indeed completed by the pancreatic juice, possibly assisted by the succus entericus, the presence of bile being said to increase the activity of the pancreatic amylolytic ferment. 'The conversion begins as soon as the acidity of the chyme is sufficiently reduced and continues along the intestine; portions however of still undigested starch may be found in the large intestine, and even at times in the freces.

The pancreatic juice, as we have seen, emulsifies fats, and also splits them into their respective fatty acids and glycerin. The fatty acids thus set free bceome converted by means of the alkaline contents of the intestine into soaps; but to what extent saponification thus takes place is not exactly known. Undoubtedly soaps have to a small extent been found both in portal blood and in the thoracic duct after a meal; but there is no proof that any large quantity of fat is introduced in this form into the circulation. On the other hand, the presence of neutral fats in the lacteals, and to a slight extent in portal blood, is a conspicuous result of the digestion of fatty matters; and in all probability saponification in the intestine is a subsidiary process, the effect of which is rather to facilitate the emulsion of neutral fats than to introduce soaps as such into the blood. For the presence of soluble soaps favours the emulsion of neutral fats. Hence a rancid fat, i.e. a fat containing a certain amount of free fatty acid, forms an emulsion with an alkaline fluid more readily than does a quite neutral fat. A drop of rancid oil let fall on the surface of an alkaline fluid, such as a solution of sodium carbonate of suitable strength, rapidly forms a broad ring of emulsion, and that even without the least agitation. As saponification takes place at the junction of the oil and alkaline fluid currents are set up, by which globules of oil are detached from the main drop and driven out in a cen- 
trifugal direction; the intensity of the currents and the consequent amount of emulsion depend on the concentration of the alkaline medium and on the solubility of the soaps which are formed. Now the bile and pancreatie juice supply just such conditions as the above for emmlsionizing fats : they both together afford an alkaline medimm, the panereatic juice gives rise to an adequate amount of free fatty acid, and the bile in addition brings into solution the soaps as they are formed. So that we may speak of the emmlsion of fats in the small intestine as being earried on by the bile and pancreatic juice acting in conjunction; and as a matter of fact the bile and pancreatic juice do largely emulsify the contents of the small intestine, so that the greyish turbid chyme is changed into a creamy-looking fluid, which has been sometimes called chyle. It is advisable howerer to reserve this name for the contents of the lacteals. Many of the fats present in food, for instance, hutter, alrearly contain some fatty acids when eaten ; for these fats the initial action of the pancreatic juice is less necessary.

Fats we may therefore say are digested, for this emulsification is the main digestion of fats, by both bile and pancreatic juice working together. Hence if either bile or pancreatic juice be prevented from gaining access to the small intestine, fat is not digested, is not absorbed, and appears in the freces. 'This is true at least of ordinary fat; milk in which the fat is already emulsified nay be digested and absorbed, in the absence of these secretions.

$\S 231$. We have seen, $\S 208$, that the addition of bile to a digesting mixture gives rise to a precipitate. This is partly a coarse flocenlent precipitate, consisting of undigested or partially digested proteids with some amount of bile acids, and partly of a finer more gramular precipitate, which is longer in falling down, and consists chiefly of bile acids with a variable amount of peptone; the latter is re-dissolved on the further addition of bile even though the reaction of the mixture remain acid. In the upper part of the duodenum the inner surface, if cxamined while digestion is going on, is found to be lined by a coloured floceulent and granular material, which is probably a precipitate thus formed; the purpose of this precipitation is probably to delay the passage of the undigested parapeptone along the duodenum. Morcover, apart from this precipitation, bile arrests the action of pepsin, even while the reaction of the mixtmre still remains acid; and as soon as an alkaline reaction is estahlished the pepsin is apparently destroyed by the trypsin, so that with the flow of bile ancl pancreatic juice into the chuodenum the processes which have been going on in the stomach come to an end. In fact it would seem that the juices of the various districts of the alimentary canal are mutually destructive; thus, while pepsin in an acid solution destroys the active 
constituents of saliva and of pancreatic juice (probably also those of the succus entericus), it is in its turn antagonized or destroyed by the bile and the other alkaline juices of the intestine. Ilence pancreatic juice introduced through the mouth must lose its powers in the stomach and can only be of use as an alkaline medium containing certain proteid matters. On the other hand if, as we have reason to believe, the contents of the stomach as they issue from the pylorus still contain a large quantity of undigested proteids, these must be digested by the pancreatic juice (with or without the assistance of the suceus entericus), the action of which seems to be assisted or at least not hindered by bile. And in dogs fed through a duodenal fistula, so that all gastric digestion is excluded, proteids are completely digested and give rise to quite normal faces. 'To what stage the pancreatic digestion is carried, whether peptone is, practically, the only product, or whether the pancreatic juice in the body, as out of the body, carries on its work in the more destructive form, whereby the proteid material subjected to it is so broken down as to give rise to appreciable quantities of leucin and tyrosin, is at present not exactly known. Ieucin and tyrosin have been found in the intestinal contents, and may therefore be formed during normal digestion, but whether an insignificant quantity or a considerablo quantity of the proteid material of food is thus hurried into a crystalline form cannot be definitely stated. 'The extent to which the action is carried is probably different in different animals, and probably varies also according to the nature of the meal and the condition of the body. Possibly when a large and mmecessary quantity of proteid material is taken at a meal together with other substances, no inconsiderable amount of the proteids undergo this profound change, and, as we shall see, rapidly leave the body as urea, without having been used by the tissues, their contribution to the energy of the body being limited to the heat given out during the changes by which they are converted into urea. To this apparently wasteful use of proteids we shall return in speaking of what is called the 'luxus consumption' of food.

$\S 232$. In dealing with the action of pancreatic juice wc drew attention, $\$ 210$, to the difference between the results of pure tryptic digestion and those obtained when bacteria or other micro-organisms were allowed to be present. We saw that indol, for example, was the product of the action of these organisms, not of trypsin. Now indol is formed, in varying quantity, during the digestion which actually takes place in the intestine, some of it at times appearing in the urine as indigo-yielding substance (indican). Moreover bacteria and other micro-organisms are present in the intestinal contents. Hence we must regard the changes taking place in the intestine not as the pure results of the action of the several digestive juices, but as these 
results modified by or mixed with the results of the action of micro-organisms. We spoke above, $\$ 208$, of bile as being antiseptic, but this must be understood as meaning not that the presence of bile arrests the action of all micro-organisms within the intestine, but that it modifies their action, keeping it within certain limits and along certain lines.

Concerning the exact nature and extent of the changes thus due to micro-organisms our knowlerge is at present very imperfect. The proteids and the carbohydrates seem to be the food stuffs on which these organisms produce their chief effect. Out of the proteids they give rise not only to indol but to several other compounds, among which may be mentioned phenol $\left(\mathrm{C}_{6} \mathrm{H}_{6} \mathrm{O}\right)$, of which a small quantity may be recognized in the freces, the rest being absorbed and appearing in the urine in the form of certain phenol-compounds, such as phenyl-sulphuric acid. Out of proteids they may also form the peculiar poisonous bodies called ptomaines, which appear in the ordinary putrefaction of proteids. But their most conspicuous effects are those on the carbohydrates. As the food descends the intestine, the prescnce of lactic acid becomes more and more obvious; indeed in some cases the naturally alkaline reaction of the intestinal contents may in the lower part of the intestine be changed into an acid one by the presence of lactic acid. Now lactic acid may be formed out of sugar by means of a special organism inducing what is spoken of as the lactic acid fermentation. And we have every reason to believe that in even normal digestion, a certain quantity of sugar, either eaten as such, or arising from the amylolytic conversion of starch, does not pass away from the intestine into the blood as sugar, but undergoes this fermentation into lactic acid. To what extent this change takes place we do not know; the amount probably varies according to the amount of carbohydrates eaten, the condition of the alimentary canal, and other circumstances. It may be under certain circumstances simply a part of normal digestion; under other circumstances it may be excessive and give rise to troubles.

That fermentative changes may occur in the small intestine is further indicated by the facts that the gas there present may contain free hydrogen, and that chyme after removal from the intestine continues at the temperature of the body to produce carbonic acid and hydrogen in equal volumes. This suggests the possibility of the sugar of the intestinal contents undergoing the butyric acid fermentation during which, as is well known, carbonic anhydride and hydrogen are evolved. By this change the sugar is removed from the carbohydrate group into the fatty acid group; it is thus, so to speak, put on its way to become fat. We shall sec hercafter that sugar may be some...no in the borly converted into fat; this conversion however 
takes place chiefly if not wholly in the tissues, and such change as may take place in the alimentary canal is to be regarded as suggestive rather than as important.

The hydrogen thus occurring in the intestine may also arise from the proteid decompositions spoken of above. However arising, it may act as a reducing agent, reducing sulphates for instance, and thus giving rise to sulphides and to sulphuretted hydrogen; as a reducing agent it assists in the formation of the fiecal and urinary pigments.

Thus during the transit of the food through the small intestine, by the action of the bile and pancreatic juice, and possibly to somc extent of the succus entericus, assisted by various microorganisms, the proteids are largely dissolved and converted into peptone and other products, the starch is changed into sugar, the sugar possibly being in part furtler converted into lactic or other acids, and the fats are largely emulsified, and to some extent saponified. 'These products, as they are formed, pass into either the lacteals or the portal blood vessels, so that the contents of the small intestine, by the time they reach the ileocacal valve, are largely but by no means wholly deprived of their nutritious constituents. So far as water is concerned, the secretion of water into the small intestine maintains such a relation to the absorption from it that the intestinal contents at the end of the ileum, though much changed, are about as fluid as in the duodenum.

\section{In the Large Intestine.}

$\$ 233$. The contents, whether alkaline or not in the ileum, now become once more distinctly acid. This, however, is not caused by any acid secretion from the mucous membrane: the reaction of the intestinal walls in the large as in the small intestine is alkaline. It must therefore arise from acid fermentations going on in the contents themselves, and that fermentations do go on is shewn by the appearance of marsh gas as well as hydrogen in this portion of the alimentary canal. The character and amount of fermentation probably depend largely on the nature of the food, and probably also vary in different animals.

Of the particular changes which take place in the large intestine we have no very definite knowledge; but since such secretions as are afforded by the walls of the intestine itself do not seem to contain any ferments, we may conclude that the changes which do take place are effected by micro-organisms. It is excecdingly probable that in the voluminous cæcum of the herbivora a large amount of digestion of a peculiar kind goes on. We know that in herbivora a considerable quantity of cellulose disappears in passing through the alimentary canal, and cren in 
man some is digested. It seems probable that this cellulose digestion takes place in the large intestine, and is the result of fermentative changes carried out by means of micro-organisms, marsh gas being one of the products formed at the same time.

Be this as it may, whether digestion, properly so called, is all but complete at the ileo-cecal valve, or whether important changes still await the chyme in the large intestine, one great characteristic of the work done in the colon is absorption. By the abstraction of all the soluble constituents, and especially by the withdrawal of water, the liquid chyme becomes as it approaches the rectum converted into the firm solid fieces, and the colour shifts from the bright orange, which the grey chyme gradually assumes after admixture with bile, into a darker and dirtier brown.

\section{The Freces.}

$\$ 234$. These consist in the first place of the indigestible and undigested constituents of the meal: shreds of elastic tissue, hairs and other horny elements, much cellulose and chlorophyll from vegetable, and some comnective-tissue from animal food, fragments of disintegrated muscular fibre, fat-cells, and not unfrequently undigested starch-corpuscles. The amount of each must of course vary very largely according to the nature of the food, and the digestive powers, temporary or permanent, of the individual. In the second place, to these must be added substances not distinetly recognizable as parts of the food but derived for the most part from the secretions of the alimentary canal; when a portion of the intestine is isolated from the rest so that no food enters into it, a quantity of material accumulates in the interior and this in the course of time assumes a facal appearance.

The fieces contain mucus in variable amount, sometimes albumin, cholesterin, butyric and other fatty acids, lime and magnesia soaps, colouring matters, and inorganic salts, especially eartlyy phosphates, crystals of ammonio-magnesia phosphates being very conspicuous. The reaction is generally but not always acid. They also contain a ferment similar in its action to pepsin, and an amylolytic ferment similar to that of saliva or pancreatic juice. 'The bile salts are represented by a small quantity of cholalic acid, or some product of that body, and sometimes a very small quantity of taurin. The glyein and most or all of the taurin have been absorbed from the intestine, and the cholalic acid has been partly absorbed and partly decomposed. The fact that the fieces becomes 'clay-coloured' when the bile is cut off from the intestine shews that the bile-pigment is at least the mother of the facal pigment; and a special pig111.nt, which has been isolated and called stercobilin, is said to l, i lontieal with the substance called urobilin, which may be 
formed from bilirubin. As other special constituents of the faces may be mentioned excretin, a somewhat complex nitrog. enous body, whose exact chemical nature is at present uncertain, and skatol $\left(\mathrm{C}_{9} \mathrm{H}_{9} \mathrm{~N}\right)$, a nitrogenous body which like indol is derived from the decomposition of proteids by means of micro-organisms, and which is the chief cause of the frecal odour, since only a small quantity of indol remains in the freces. These odoriferous bodies are derived directly from the food; at the same time it is quite possible that other specific odoriferous substances may be secreted directly from the intestinal wall, especially from that of the large intestine. 


\section{SEC. 7. THE LACTEALS AND THE LYMPHATIC SYSTEM.}

$\S 235$. We have seen that absorption does, or at least may, take place from the stomach. We have also stated that a large absorption, especially of water, occurs along the whole large intestine. Nevertheless it is during the transit of food along the small intestine that the largest and most important part of the digested material passes away from the canal, partly into the lacteals, partly into the portal vessels. The portal vessels are simply parts of the general vascular system; the lacteals, into which we may at once say the greater part of the fat passes, are similarly parts of the general lymphatic system, being in fact the lymphatic vessels of the alimentary canal, and especially of the small intestine. The only reason for the special name of lacteals is that, unlike the lymphatic vessels of other parts of the body, the lymphaties of the intestine contain at times a fluid of a milky white appearance. Hence for the better understanding of absorption by the lacteals it will be desirable to study at some length the whole subject of the lymphatic system.

The lymphatic vessels may be said to begin in minute passages, possessing special characters, known as lymph-capillaries. Broadly speaking these lymph-capillaries are found, in the mammal, in all parts of the body in which connective tissue is found; and they have special connections with those minute spaces in connective tissue which we have already more than once spoken of as lymph-spaces. These lymph-capillaries, which are frequently arranged in plexuses, are continuous with other passages also minute but of a different and more regular structure, the lymphatic vessels proper, which are gathered into larger and larger vessels, all rumning like the blood vessels in a bed of connective tissue, until at last all the lymphatic vessels of the body join either the great thoracic duct which opens by a valvular orifice into the venous system at the junction of the left jugular and subelavian veins, or the small lymphatic trunk which similarly opens into the junction of the right jugular and subclavian veins. The large 'serous cavities, peritoneal and the like, are also connected with the lymphatics, and may be regarded as part of the lymphatic system. 
SEC. 8. THE NATURE AND MOVEMENTS OF LYMPH (INCLUDING CHYLE).

$\S 236$. We are thus led to regard the multitudinous spaces, both small and great, of connective tissue all over the body, including among these the "serous cavities," as forming the beginning or roots of the lymphatic system. Into these spaces certain parts of the plasma of the blood transude and so become lymph; (how far the epithelioid lining of the large serous cavities plays a distinct part in regulating the transudation of serous fluid, i.e. of lymph, into those cavities we do not know ;) from these spaces the lymph is continually flowing through the lymph-capillaries into the lymphatic vessels, and so by the thoracic duct and right lymphatic trunk back into the blood system.

The amount of lymph occupying the lymph-spaces, lymphcapillaries, and minute lymphatic vessels of any region varies from time to time according to circumstances. A hand for instance which has been kept hanging down for some time becomes swollen and the skin tense; if it be raised the swelling lessens and the skin becomes loose; and a similar temporary swelling of the skin of the limbs, and of the skin generally, is frequently the result of active exercise. Such a swelling is partly due to the blood vessels being dilated, or to the return flow along the veins being retarded so that the blood capillaries become distended with blood, but is much more largely owing to the lymph-spaces and lymphatic vessels of the skin and underlying structures being unusually filled with lymph. On the other hand the skin may become shrivelled and dry from a deficiency of lymph in the lymph-spaces and vessels. Under even normal cireumstances the quantity of lymph in the tissues may vary considerably, and under abnormal circumstances a very large amount of lymph may greatly distend the spaces of the connective tissue of the skin and other structures, giving rise to cedema or dropsy. Obviously there are agencies at work in the body by which the appearance of lymph in the spaces or its removal thence along the lymph-channels, or both, may be either increased or diminished. 


\section{The Characters of Lymph.}

$\$ 237$. As it slowly flows from its origin in the tissues to the month of the thoracic dnct (we may for simplicity's sake omit the right lymphatic trunk) the lymph is subjected to the influence of the lymphatic glands, and is possibly affected by the walls of the lymph-vessels. Moreover the lymph coming from one tissuc differs more or less in certain characters from the lymph arising in another tissue, just as the renous blood of one organ differs from the venous blood of another organ; and these differences may be exaggerated by the activity of the one or other tissue. Of these differences by far the most striking is that between the lymph coming from the alimentary canal during active digestion and known as chyle, and the lymph coming from other parts of the body. When digestion is not going on, and when consequently no considerable absorption of material from the alimentary canal into the lacteals is taking place, the fluid flowing along the lacteals is lymph, not differing from the lymph of other regions to any marked degree.

The fluid accordingly which flows along the thoracic duct in an animal which has not been fed for some considerable time may be taken as illustrating the general characters of lympl. The contents of the thoracic luct may be obtained by laying bare the junction of the subclavian and jugular (in the dog the junction of the axillary and jugular) veins, and introducing a cannula into the duct as it enter's into the venous system at that point. The operation is not unattended with difficulties.

Lymph, so obtained, is a clear transparent or slightly opalescent fluid, which left to itself soon elots. The clotting is not so pronounced as that of blood, but clotting is caused as in blood by the appearance of fibrin. The fibrin which is formed though scanty, .05 p.c., is identical apparently with that of blood, and as far as we know, all that has been said previously, $\$ 514-23$, concerning the nature of clotting in blood applies equally well to lymph.

Examined with the microseope lymph contains a number of corpuscles, lyuph-corpuscles, which in all their characters so far as is at present known are identical with white blood corpuscles; they vary in size from $5 \mu$ to $15 \mu$, and the smaller corpuseles are much more abundant in lympli than in blood. Like the white blood corpuscles of blood they exhibit amoboid movements. Their number varies in different animals, and, alparently, in the same animal, according to circumstances, on the whole perhaps it may be said that lymph corpuscles are about as numerous in lymph as white corpuscles in blood. Even when every care is taken to avoid admixture with blood, lympl, and especially chyle, not unfrequently contains a cer- 
tain number of red blood corpuscles; sometimes these are sufficient to give the lymph (or chyle) a reddish tinge. 'They have been observed within the living lymphatic vessels, even within small ones, and have probably in some manner or other made their way from the blood into the lymph-chammels.

$\$ 238$. The chenical composition of lymph, even when taken in each case from the thoracic duct, varies a good deal. The total solids are much less than in blood, amounting in general to not more than 5 or 6 p.c. Hence the venous blood of a vascular area contains rather more solids than the arterial blood of the same area, since the blood in giving rise to the lymph during its passage through the capillaries from the arteries to the veins has parted with relatively more water than solid matter.

'The proteids amount on the average to about 3 or 4 p.e., that is to say, to about half as much as in blood, the particular proteids present being the same as in blood, viz. albumin, paraglobulin and antecedents of fibrin. In lymph, as distinguished from chyle, the quantity of fat is small, and consists of the usual neutral fats and the soaps of their fatty acids, together with lecithin; cholesterin may also be present. A certain amount of sugar (dextrose) appears to be always present, and several observers have found an appreciable quantity of urea. The ash of lymph like that of blood serum contains a considerable quantity of sodium chloride, while phosphates and potash are scanty; it also contains iron, apparently in too great a quantity to be accounted for by the few red corpuscles which may be present. From lymph a certain amount of gas can be extracted, consisting shietly or almost exclusively of carbonic acid, with a small quantity of nitrogen, the amount of oxygen present being cxceedingly small. The importance of this we shall see when we come to study respiration.

Broadly speaking we may say that all the substances present in blood-plasma are present also in lymph, but are accompanied by a larger quantity of water.

$\$ 239$. Lymph may also be obtaincd from separate regions of the body, as from the lower or upper limbs, for instance, by introducing a fine cannula into a lymphatic vessel. In its general features the lymph so obtained resembles that taken from the thoracic duct. Analyses of the lymph distending the subcutaneous connective tissue in cases of dropsy shew that this contains much less solid matter than normal lymph taken from the thoracic duct or larger lymphatic vessels. From this it has been inferred that the lymph normally existing in the lymphspaces, lympli-capillaries and minute vessels contains an excess of water; and indced it has been asserted that the percentage of solids increases in passing from the smaller to the larger vessels; but this cannot be regarded as distinctly proved. 'T'he number of corpuscles however, as we have already said, appears 
to be increased in passing through the lymphatic glands. It has also been stated that the lymph in the finer lymph-vessels clots even less firmly than that in the thoracic duct. From this we may infer that some of the leucocytes in the adenoid tissue of the follicles of a lymphatie gland find their way into the lymph-sinus, and so into the efferent lymphaties, and that some of the fibrin factor's are added to the lymph, or at least that some changes favourable to elotting are brought about.

$\$ 240$. We said that the large scrous cavities of the peritoneum, pericardium, \&c., are to be considered as parts of the lymphatic system; indeed pericardial or other serous fluid has all the general characters of lymph. We have alrcady said, $\$ 20$, that these fluids when taken fresh from the body, clot (this is, at least, the casc in most animals); the clot when examined microscopically is found to consist of colourless corpuscles like those of lymph or of blood entangled in the meshes of fibrin. Both in their proteid and other chemical constituents these serous fluids resemble lymph. Analyses of the accumulations of fluid occasionally occurring in these cavities shew that they contain sometimes less and sometimes more solid matter than ordinary lymph. The aqueous humour of the eye contains very little solid matter; and the cerebrospinal fluid is so peculiar that it had better be considered by itself in connection with the nervous system.

$\$ 241$. Chyle. In fasting animals the fluid flowing along the lacteals, as may be seen by inspection of the mesentery, is clear and transparent; it is lymph, differing, as we have said, in no essential respects from the lymph flowing along other lymphatic vessels. Shortly after a meal containing fat (and every meal does contain some fat), the lymph becomes white and opaque like milk, the more so the richer the meal is in fat; it is then called chyle. Owing to the relatively large quantity of this milky fluid which for some time after a meal continues to be poured into the thoracic duct, the contents of that duct also become milky, and are also called chyle. In the thoracic duct the chyle of the lacteals is more or less mixed with lymph from other lymphatic vessels, but the former is so preponderating that the contents of the duct may be taken as illustrating the nature of chyle.

Chyle differ's from lymph in one important respect, and onc only; whereas lymph ordinarily contains a small quantity only of fat, chyle contains a very large amount. The actual amount of fat present in the chyle of the thoracic duct varies, as may be expected, very considerably, according to the nature of the meal, the stage of digestion, and various circumstances. Five per cent. is a very common amount; in the dog it has been found to vary from 2 to 15 per cent. The increase in fat is chiefly if not exclusively due to an increase in the ncutral fats; 
though whether the small quantity of soaps and of lecithin present is greater than in lymph has not been distinctly ascertained. Cholesterin is probably present in greater amount than in lymph, since it probably comes from the bile poured into the intestine during digestion; but this is not certain. How far the nature of the fat, that is, the proportion of the various kinds of fat, of stearin, \&c., varies with the fats present in the meal has not been definitely ascertained.

The condition of the fat in chyle is peculiar. Some of it exists, like the fat in milk, in the form of fat globules of various sizes, but all small. A very considerable quantity however is present in the form of exceedingly minute spherules or granules, far smaller than any globules to be seen in milk; these exhibit active 'Brownian movements.' The fat present in this form is spoken of as the 'molecular basis' of chyle, and is very distinctive of chyle. In the emulsified contents of the intestine, often called chyle, the fat is finely divided, and to a large extent into small globules, but there is nothing corresponding to this molecular basis; the fat does not assume this condition until it has passed out of the intestine into the lacteals. Lymph examined with the microscope sliews besides the white corpuscles only very few oil-globules, and nothing of this molecular basis. Just as in fact lymph is, broadly speaking, blood minus its red corpuscles, so chyle is lymph plus a very large quantity of minutely divided neutral fat.

The total amount of lymph or of chyle which enters the blood system through the thoracic duct, though it probably varies considerably, is probably also always very large. It has been calculated that in a well-fed animal a quantity equal at least to that of the whole blood may pass through the thoracic duet in 24 hours, and of this it is supposed that about half comes through the lacteals from the abdominal viscera, and therefore to a large extent from food, and the remainder from the body at large. These calculations are based on uncertain data, and cannot therefore be taken as of exact value, but we may use them for the sake of an illustration. Thus in a man of average weight, that is, about 70 kilos, the quantity of blood (\$38) being $\frac{1}{13}$ of the body weight is about 6 kilos. The quantity of lymph or chyle therefore discharged into the blood in an hour would be according to this calculation a quarter of a kilo, or something less than a quarter of a litre; and since the flow must vary considerably in the 24 hours, would be therefore sometimes less and sometimes even more than this.

\section{The Movements of Lymph.}

$\$ 242$. Making every allowance for the uncertainty of the calculation detailed in the preceding paragraph, it is obvious that 
the lymph must flow with a not inconsiderable rapidity (if we take about half the above estimate, the rate will be about 1 or 2 c.c. per minute) through the thoracic duct, and therefore must also be continually streaming into that duct, along the various lymphatic channels from the manifold lymph-spaces of the body. This onward progress of the lymph is determined by a variety of circumstances. In the first place, the remarkably wide-spread presence of valves in the lymphatic vessels causes every pressure exerted on the tissues in which they lie to assist in the propulsion forward of the lympl. Hence all muscular movements increase the flow. If a cannula be inserted in one of the larger lymphatic trunks of the limb of a dog, the discharge of lymph from the cannula will be more distinctly increased by movements, even passive movements, of the limb than by anything elsc. When we come to speak of the entrance of chyle into the lacteal radicles of the villi, we shall see that the muscular fibres of the villus act as a kind of muscular pump, driving the chyle past the valved end of the lacteal radicle into the lymphatic eanals below. In addition to the presence of valves along the course of the vessels, the opening of the thoracic duct into the venous system is guarded by a valve, so that every escape of lymph or chyle from the duct into the veins becomes itself a help to the flow. In the second place, we have already seen that the blood-pressure in the capillaries and minute vessels is considerably greater than that in the large veins, such as the jugular; in fact this difference of pressure is the cause of the flow of blood from the capillaries to the heart. Now even assuming that the lymph in the lymphatic spaces outside the capillaries and minute vessels necessarily stands at a lower pressure than the blood inside the capillaries, on the ground that otherwise the transudation from the blood into the tissues would be checked, we must still admit that the difference is less, probably much less, than the difference between the pressure in the capillaries and that in the large venous trumks. So that the lymph in the lymph-spaces of the tissues may be considered as standing at a higher pressure than the blood in the venous trunks, for instance in the jugular vein. That is to say, the lymphatic vesscls as a whole form a system of channels leading from a region of higher pressure, viz. the lymph-spaces of the tissues, to a region of lower pressure, viz. the interior of the jugular and subclavian veins. This difference of pressure will, as in the case of the blood vessels, cause the lymph to flow onward in a continuous stream. Further, this flow, caused by the lowness of the mean venous pressure at the subclavian vein, will be assisted at every respiratory movement, since at cvery inspiration the pressure in the venous trunks beconics, as we slall see in dealing with respiration, negative, and thus lymph will be sucked in from the thoracic luct, while the increase of pressure in the 
great veins during expiration is warded off from the duet by the valve at its opening. In the third place, the flow may be increased by rhythmieal contractions of the walls of the lymphatics themselves, which are remarkably muscular; and the peculiar interlacing of the muscular fibres above each valve suggests that the walls here act after the fashion of a tiny heart and by a rhythmical systole drive on the fluid, which by the action of the valve below collects at the spot. We have however no cxperimental proof of this; for, though rhythmic variations have been observed in the lacteals of the mesentery, it is maintained that these are simply passive, i.e. caused by the rhythmic peristaltic action of the intestine, each contraction of the intestine filling the lymph-channels more fully, and are not due to contractions of the walls of the lacteal vessels themselves. In some of the lower animals, for instance in the frog, the museular walls of the vessels are developed at places into distinetly contractile propulsive-organs, spoken of as lymph-hearts. Lastly, if the very processes which give rise to the appearance of lymph in the lymph-spaces of the tissues are, as we shall see we have some reason to think, analogous to the process of secretion, then remembering the pressure which is developed by the secretion of a secreting gland, such as a salivary gland (\$189), we may regard these very processes as tending themselves to promote the flow of lymph. WVe have at least, under all circumstances, one or other of these causes at work, promoting a continual flow from the lymphatic roots to the great veins. 'They are together sufficient to drive, in man, the lymph from the lower limbs and trunk, against the effects of gravity, into the veins of the neck. In the upper limb, the influences of gravity owing to the varied movements of the limb, are as often favourable to, as opposed to, the natural flow of the lymph; but as we have already said, a long-continued unfavourable action of gravity, especially in the absence of the aid of movements in the skeletal muscles, as when the arm hangs down motionless for some time, leads to accumulation of lymph at its origin in the lymph-spaces. 'The strength of the causes combining to drive on the lymph is strikingly shewn in animals when the thoracic duct is ligatured; in such cases a very great distension of the lymphatic vessels below the ligature is observed.

$\$ 243$. Although the phenomena of disease and, perhaps, general considerations render it probable that the nervous system governs in some way the stream of lymph, regulating it may be not only the flow along the definite lymph-canals but also the transit of plasma into the lymph-spaces and the escape of lymph thence into the definite canals, our knowledge on these points is very imperfect. We have as yet at least no proof that the muscular fibres in the walls of the lymphatic vessels are governed by nerves, or that the lymph-spaces are influenced directly by 
nervous action; attempts to demonstrate any direct action of the nervous system on the lymphatics have hitherto failed.

$\$ 244$. The passage of material, namely, of water containing certain substances in solution, from the interior of the blood vessel where they form part of the plasma into the lymph-capillary where they are called lymph consists of two steps: the passage from the blood ressel into the lymph-space, and the passage from the lymph-space into the lymph-capillary; for it is only in particular places that the lymph-capillary immediately surrounds the blood ressel. Once arrived in the lymphcapillary the lymph finds an open path along the rest of the lymphatic system, but the connection between the lymph-space and the lymph-capillary is peculiar and at least not a free and open one.

The passage of material from the blood vessel into the lymphspace we speak of as transudation. What can we say as to the nature of this process? There are two known physical processes with which we may compare it: diffusion through a membranous or other porous partition, and filtration through a similar partition. Diffusion, though influenced by fluid pressure, is not the direct result of fluid pressure but may on the contrary be the cause of differences of pressure on the two sides of a partition, and may work against fluid pressure. When a strong solution and a weak solution of salt are separated by a diffusion septum, diffusion takes place whether the columns of fluid be at the same level on the two sides of the septum or at different levels; and if the columns be at the same level to start with, that of the stronger solution soon comes to exceed the other in height, on account of the osmotic flow of water from the weaker into the stronger solution. Filtration on the other hand is the direct result of pressure; without difference of pressure filtration does not take place; and, the filter remaining of the same nature and in the same condition, the amount of filtrate is dependent on the amount of pressure. May we speak of the process of transudation as a simple process of diffusion or a simple process of filtration, that is to say, can all the phenomena of transudation be explained as simply the results of one or other of these physical processes? Diffusion by itself will not account for the results; for the proteids of the blood-plasma are indiffusible or very nearly so and yet the lymph contains a considerable quantity of these proteids. We have no satisfactory knowledge of the exact composition of lymph as it exists in the lymph-spaces. In the lymph of the larger lymph-trunks the diffusible saline substances are present in about the same proportion, and the indiffusible proteids to about or less than half as much as in blood-serum; and we may perhaps assume that the lympl in the lymph-spaces contains relatively less proteids but has otherwise the same composition as blood-plasm. Mere 
diff asion would not give rise to a fluid of such a nature. Can we speak of transudation then as a filtration? The blood is undoubtedly flowing through the capillaries and other small vessels under a certain pressure; we liave seen ( $\$ 98)$ that the pressure is roughly speaking about $20 \mathrm{~mm}$. $\mathrm{Hg}$.; and it would be possible to select such a filter or porous partition as would at about this pressure permit the passage of a certain quantity of the inorganic and crystalline constituents of blood-plasma to pass through in company with a relatively smaller quantity of the proteids and a large quantity of the water, the red and white corpuscles being excluderl. Such a filtrate would be more or less of the nature of lynph; and so far we might be justified in speaking of the transudation of lymph as a process of filtration. But the transit through the living wall of the blood vessel is affected by circumstances in a manner so different from the manner in which the same circumstances affect the transit through an ordinary lifeless filter, that we gain but little, and may be led into error by speaking of the process as a filtration. Substances in solution or otherwise pass through a filter when the pressure is sufficient to drive them through the passages furnished by the interstices existing in the substance of the filter. In the case of an ordinary filter the substance of the filter is within limits permanent, and the passages correspondingly constant. The living wall of a capillary however is not a constant unchanging thing. The epithelioid plates and other elements which constitute it are alive, and being alive are continually undergoing change and are especially subject to change; moreover, as we have seen (\$\$ 22, 23), the vascular walls appear to be continually acting upon and being acted upon by the blood. Hence a change in the blood tends to cause changes in them; and these changes may materially affect in one direction or another their action as filters. In an ordinary filter increase of pressure necessarily entails increase of filtration; in a living filter we should expect to find that it may or may not, that variations of pressure may according to circumstances produce very different results as regards the transudation of lymph, and that the latter may vary independently of the former.

Observations seem to confirm this view. In the first place an increase of blood-pressure does not necessarily increase the transudation of lymph. It is true that when a small artery dilates, by which the pressure in the still smaller branches and capillaries of that artery is, as we have more than once pointer out, increased, more lymph as a rule appears in the lymphspaces; indeed it is one of the main purposes of the widening of small arteries to supply the elements of the tissue with more lymph, that is, with more food. But it does not therefore follow that under all circumstances widening of the artery should 
increase the passage of lymph; something may occur to counteract the natural effect of the increased pressure in the blood vessels. An instance of this seems to be afforded by the case of the submaxillary gland, when the chorda nerve is stimulated while the gland is under the influence of atropin. As we have seen, though the arteries dilate, no secretion takes place; and we cannot explain the absence of a flow into the alveoli by supposing that the extra amount of lymph which would in normaI circumstances form part of the secretion, and in the case of a fairly copious secretion would be considerable, now passes away by the lymphaties without reaching the cells of the alveoli, for in such cases 110 extra flow in the lymphatics leading from the gland has been observed, and there is no accunulation of lymph in the connective tissuc of the gland. Apparently, for some reason or other, in spite of the increased pressure in the blood vessels more lymph than usual does not pass into the lymphspaces.

In the sceond place, increase of pressure does not always produce the same amount of transudation. For instance, as we shall prescntly have occasion to point out, an increase of pressure in the blood vessels produced by obstruction to the venous outflow is much more efficient in promoting an increase of transudation, at all events an abnormal increase, than is an increase of arterial pressure; and the difference between the two cases appears to be too great to be accounted for on the ground that an obstruction to the venous outflow raises the pressure within the capillaries and small vessels more readily and to a higher degree than does the widening of the arteries. Further the same amount of venous obstruction giving rise to the same amount of capillary pressure may or may not give rise to excessive transudation according to the condition of the blood or other cireumstances. For instance, though the obstruction produced by ligaturing a vein frequently causes excessive transudation, it does not always cause it, and the femoral vein of a dog may be ligatured without any excessive transudation taking place; yet if, after the ligature, certain changes be induced in the blood excessive transudation occurs in the leg, the vein of which has been ligatured but not elsewhere. Pointing towards the same conclusion is the fact that excessive transudation more readily occurs when a vein is plugged by a thrombus arising from abnormal conditions of the vascular system than when a vein is simply ligatured.

In the third place if we measure the flow of lymph along the duct (by introducing a cannula into the thoracic luct at its end near the great veins), and we may take this as a measure of the transudation going on, we find that this flow may be very sreatly inereased, without any change of blood-pressure necessarily taking place, by the introduction into the blood of certain 
substances, such as leech extract or extract of crabs' muscles. 'The presence of these substances in the blood appears to produce such a change in the vascular walls, that without any change of pressure, a larger amount of lymph and that richer in solids, especially in proteids, makes its way into the lymph-spaces, and so into the thoracic duct. 'The result has an obvious resemblance to the act of secretion, and the substances in question have been called lymphagogues. We may therefore conclude that two things chiefly determine the amount of transudation : the pressure of the blood in the blood vessels, and the condition of the vascular walls in relation to the blood, the latter being at least as important as the former.

We said just now that we may take the flow of lymph along the thoracic duct as a measure of the transudation from the blood vessels. But this is not strictly true. The lymph in the lymph-spaces, which is the source of the lympli in the thoracic duct, is not simply the result of the transudation from the blood vessels, but the result of the combined action of the blood vessels on the one hand and the tissues on the other. The lymph in the lymph-space is the middleman; the tissues take from and give to it in some such manner as the blood gives to and, we may add, takes from it. We remarked in $\$ 30$ on the peculiar relations of living tissue to water, and there are reasons for thinking that the very substance of a cell or a fibre (a muscular fibre for instance) may hold in itself a larger quantity of water at one time than at another. The water thus taken up or given out, and other substances carried by that water, come from and go to the lymph. The condition of the tissue determines by itself the amount of lymph in the lymph-spaces, and thus the flow of lymph along the thoracic duct. For instance, a certain quantity of sugar introduced into the blood gives rise to a very rapid flow of somewhat dilute lymph along the thoracic duct; and similar results are produced by much smaller quantities of sodium chloride and other substances. Since the blood is found to be, at the same time, more watery, in spite of a copious secretion of urine, we may conclude that the excess of lymph in the lymph-spaces is drawn from the tissues.

$\$ 245$. Under the influence of all these several actions the lymph in the various lymph-spaces of the body varies in amount from time to time, but under normal circumstances never exceeds certain limits. Under pathological conditions those limits may be exceeded, and the result is known as oedema or dropsy. Similar excessive accumulations of lymph may occur not in the ordinary lymph-spaces, but in those larger lymph-spaces, the serous cavities, any large excess of fluid in the peritoneal cavity being known as ascites.

'The possible causes of odema are on the one hand an obstruc- 
tion to the flow of lymph from the lymph-spaces, and on the other hand an excessive transudation, the lymph gathering in the lymph-spaces faster than it can be carried away by a normal flow; with the former the lymphatic system itself, with the latter chiefly the vascular system is concerned. As a matter of fact, however, cedema is almost always, if not always, due to abnormal conditions of the vascular system, and is the result not of hindered outflow but of excessive transudation. Owing to the numerous anastomoses of the lymph-ressels and the consequent establishment of collateral streams, obstruction in the lymph-passages themselves rarely if ever gives rise to redema; and it may be here remarked that owing to the same free collateral communication between the lymph-vessels the labyrinthine passages of the lymphatic glands do not offer the serious obstacle to the onward flow of the general lymph-stream as might at first sight be supposed. Nor have we at present any knowledge which would lead us to suppose that any physiological changes in the walls of the lymphatic vessels or of the lymph-capillaries, or in the lymph-spaces, by giving rise in some way to obstacles to the flow of lymph, ever lead to an accumulation of lymph in the latter.

One kind of cedema we have already touched upon in speaking of the capillary circulation ( $\$ 161)$, viz. the "inflammatory" oedema. In this kind of cedema owing to changes in the vascular walls a larger amount of transudation passes into the lymph-spaces, and that transudation is richer in proteid matters, and contains a larger amount of the fibrin factors, or at all events is much more distinctly coagulable than ordinary lymph, as well as crowded with migrating corpuscles. Allied to this inflammatory adema is the increase of lymph, also apparently changed somewhat in character, which appears as "effusion" in the serous cavities when these are inflamed, as in pleurisy and peritonitis.

One of the most common forms of cedema is an œedema of primarily, though not wholly, mechanical origin, œedema arising from obstruction to the venous flow; under these circumstances more lymph passes into the lymph-spaces than the lymph-vessels are able to carry. If the femoral vein be ticd the leg may become oedematous, and, as we have said, cedema is a common result of the plugging or obstruction of veins through disease; the aedema which is so common an accompaniment of heart discasc involving obstruction to the return of venous blood to the right side of the heart, and the ascites which follows upon hindrance to the portal flow are instances of adema of this kind. We have already remarked on the relation of transudation to blood-pressure; and in venous obstruction the rise of pressure within the small blood vessels is distinguished from that due to arterial dilation by being ac- 
companied with a want of adequate renewal of the blood; this probably affects the epithelioid lining of the blood vessels in such a way as to increase the transudation. And indeed, as is seen in cases of heart disease with prolonged or repeated venous obstruction, the odema, as time goes on and the tissues become impaired, is more easily excited and with greater difficulty removed, though the actual amount of obstruction, the actual increase of pressure in the small vessels, remains the same, or at least is not proportionally increased.

Still another kind of ordema is one due to changes taking place in the blood, quite apart from variations of blood-pressure. 'This kind of cedema is seen in some diseases of the kidney, in "Bright's disease" for instance. In such cases the blood contains less proteids, and indeed less solids, is more watery and of lower specific gravity than is normal. But the œdema is not in these cases to be explained on the view that the more watery blood passes more readily through the capillary walls, for it may be shewn experimentally that the mere thinning of the blood, as by the injection of normal saline solution into the blood vessels, will not at once lead to oedema, at least in the limbs and trunk, and it is these which in Bright's disease especially become cedematous. In all probability the œedema of Bright's disease if it be really due to the abnormal character of the blood, is produced by the abnormal blood so acting on the blood vessels that these allow a transudation greater than the normal. Lastly, calling to mind what we said just now as to the relations of the tissue to the lymph, we must remember that the cause of œdema may also lie in changes in the tissue itself.

But these are pathological questions into which we must not enter here. We have touched upon them because they illustrate the important processes taking place in the lymph-spaces, and as we have more than once insisted the lymph in the lymphspaces is the middleman of all the tissues, and hence facts illustrating the laws which govern the flow of lymph into and out of the lymph-spaces are of fundamental physiological importance. 


\section{SEC. 9. ABSORPTION FROM THE ALIMENTARY CANAL.}

$\S 246$. We may now return to consider the absorption of the products of digestion, that is to say, the passage of these bodies from the interior of the alimentary canal, where they are really outside the body proper, into the body itself. For simplicity's sake we may consider digestion in a broad way as the conversion of practically non-diffusible proteids and starch into more diffusible peptone and highly diffusible sugar, and as the emulsifying, or division into minute particles, of fats. We have seen reason to believe that some of the sugar may be changed into lactic acid or even into butyric or other acids, that some of the proteids are carried beyond the peptone condition into leucin and other bodies, and that some of the fat may be saponified; and it may be that some of the proteid material of the food passes into the body as albumose or even as parapeptone, or in some other little changed condition. But we may probably with safety, for present purposes, assume that the greater part of the proteid is absorbed as peptone, that carbohydrates are mainly absorbed as sugar, and that the greater part of the fat passes into the body as emulsified but otherwise unchanged neutral fat; and we may neglect the other conditions of digested food as subsidiary, and as far as absorption is concerned, unimportant.

We have seen that two paths are open for these products of digestion, one by the capillaries of the portal system, the other by the lacteals. It cannot be a matter of indifference which course is taken. For if the products pass by the lacteals they fall into the general blood-current after having undergone only such changes as they may expericnce in the lymphatic system; while if they pass into the portal vein they are subjected to certain powerful influences of the liver (which we shall study in a future chapter) before they find their way to the right side of the heart. It has been possible, in the dog, so to commect the portal vein with the inferior vena cava, that the portal blood is diverted into the latter, and so is thrown on the general eir- 
culation without having passed through the liver. When this is done very grave troubles result. IV may therefore consider first which of the two paths is, as a matter of fact, taken by the several products, and subsequently study the mechanism of absorption in the two cases.

\section{The Course taken by the Several Products of Digestion.}

\$247. From what has already been said we have been led to regard the villi as the most active organs of absorption, and the structure of a villus leads us further to conclude that the diffusible peptones and sugar pass, together with the water in which they are dissolved, into the superficially placed capillary network of the villus and so into the portal system, while the merely emulsified fat, unable to traverse the wall of the capillary, passes on to the deap-seated lacteal radicle, and so finds its way into the lymphatic system. And the results of observation and experiment, as far as they go, support this view.

Fats. After a meal containing fat the lymph of the lacteals contains fat, and is now called chyle; and the richer the meal in fat the more conspicuous is the fat in the lymph-vessels. We cannot however prove that all the fat of a meal absorbed from the alimentary is poured by the thoracic duct into the venous system. If a meal containing a known quantity of fat be given to a dog and the small quantity of fat present in the faces corresponding to the meal be subtracted from that amount, we can determine the amount of fat absorbed, for we have no evidence whatever that any appreciable amount of fat undergoes a destructive decomposition in the alimentary canal. Collecting by means of a cammula inserted into the thoracic duct the whole of the chyle during and after the meal so long as it remains milky, showing that fat is being absorbed, we can ascertain the quantity of absorbed fat, which would, but for the operation, have passed into the venous system. When this has been done, a very remarkable deficit, amounting it may be to 40 or 50 p.c. has been observed; that is to say, of every 100 parts of fat which disappear from the alimentary canal only about 60 parts find their way through the thoracic duct into the venous system.

Are we then to conclude that the missing quantity finds its way into the portal system? Now the portal blood does. during digestion, contain a certain quantity of fat; indeed the serum is said at times to appear milky from the presence of fat. But the whole circulating blood during the digestion of a fatty meal contains, for a while, the fat poured into it by the thoracic duct; and it has been ascertained in the dog that the blood of the portal vein during digestion contains not more but less fat than the blood of the carotid artery, so that the fat which 
appears in the portal blood during digestion is, for the most part at least, not fat absorbed by the capillaries of the alimentary canal but fat absorbed by the lacteals. Noreover, when the chyle of the thoracic duct is diverted through a cannula, and not allowed to flow into the blood, the quantity of fat in the portal blood as in the blood at large is very small indeed. Lastly, when a villus of an intestine in full digestion of fat is treated with osmic acid, fat cannot be recognized by the microscope within the capillaries or other blood vessels, though it abounds outside them in the substance of the villus and in the lacteal radicle.

We may probably therefore infer with safety that all or at least very nearly all the fat absorbed from the intestine takes the path of the lacteals. As to the deficit mentioned above, that is as yet without explanation. It may be that in some way, on its course, in the lymphatic glands, for instance, the fat is taken away from the chyle, hidden so to speak somewhere away from both chyle and blood; but on this point we have no exact information.

$\$ 248$. Water and Salts. If, in an animal, the rate of flow of lymph or chyle through a cannula placed in the thoracic duct be watched, and water or, to a void the injurious effect of simple water on the mucous membrane, normal saline solution be then injected in not too great quantity into the intestine, no marked increase in the flow of chyle through the cannula is observed. From this we may infer that the water of the intestinal contents is absorbed not into the lacteals but into the portal system. If however a very large quantity of the normal saline solution be injected so as to distend the intestine, then the flow of chyle is increased to some extent. It would appear therefore that while under normal conditions the water passes from the intestine mainly into the portal blood, some of it may under circumstances pass into the lacteals.

With regard to the course taken by ordinary saline matters we possess no detailed information. When special salts such as potassium iodide and others, easily recognized by appropriate tests, are introduced into the intestine, they may be speedily detected both in the blood and in the contents of the thoracic duct; but whether, in such cases, these salts find their way into the thoracic duct by the lacteal radicle of the villi, or pass into the lymph stream at some later part of its course, we do not know. Nor can we with regard to such a salt as sodium chloride, state absolutely that it passes mainly with the water into the portal blood, though we may fairly suppose this to be the case.

$\$ 249$. Sugar. Both blood and chyle contain, normally, a certain small amount of sugar; and careful inquiries shew that the percentage of sugar in chyle and in general blood is fairly 
constant, neither being to any marked extent increased by even amylaceous meals; on the other hand, a meal containing sugar or starch does temporarily increase the quantity of sugar in the portal blood. From this we may infer that such portions of the sugar of the intestinal contents as are absorbed as sugar pass exclusively by the portal vein. We may however here call attention to the difficulties attending an argument of this kind. In the first place the quantitative determination of a small amount of sugar in so complex a fluid as blood is attended with great difficulties and uncertainties. In the second place a very large quantity of blood is at any one moment streaming through the capillaries of the alimentary canal; and we may perhaps speak of the quantity which passes through them during the whole period of digestion as being enormous. Hence though each 100 c.c. in passing through the capillaries might take up a quantity of sugar so small as to fall almost within the limits of errors of observation, yet the whole quantity absorbed during the hours of digestion might be considerable; or to put it in another way, an error of observation, mavoidable with our present means of analysis, on a sample of blood taken from the portal vessels might lead to a wholly unwarranted conclusion that sugar was or was not being absorbed. Making every allowance however for these difficulties, the increase of sugar which has been observed in the portal blood during digestion seems too great to permit of any other conclusion than that sugar is really absorbed from the alimentary canal by the blood vessels.

When however a large quantity of sugar dissolved in a large quantity of water is present in the intestine, the sugar in the chyle is said to be increased. In such a case the excess of water, as stated above, passes into the lacteals, and in so doing appears to carry some of the sugar with it.

In thus speaking of the sugar as passing into the portal blood, it should be remembered, that while the greater part of the sugar of a meal, that formed from starch, is maltose, the sugar in the portal blood is dextrose; either within the alimentary or more probably in passing tlirough the epithelium of the wall of the alimentary canal, the maltose is changed into dextrose.

$\$ 250$. Proteids. The difficulties attending the experimental determination of the path taken by proteids are greater even than in the case of sugar; since the quantitative determination of peptone in portal blood and chyle respectively, itself a task still more difficult than the quantitative determination of sugar in the same fluids, gives us no clue. For we have evidence that though peptone may be the form, or chief form, in which proteid material leaves the interior of the alimentary, it is not the form in which it reaches its destination be that destination 
the portal blood or the chyle; during its transit through the epithelium of the walls of the alimentary it is transformed from peptone back again into some or other of the natural proteids of the blood or lymph. That peptone is so changed before it can get into the blood is shewn, among other ways, by the following observation. If an artificial circulation of blood be kept up in the mesenteric arteries supplying a loop of intestine removed from the body, the loop may be kept alive for some considerable time. During this survival a considerable quantity of peptone placed in the cavity of the loop will disappear, i.e. will be absorbed, but cannot be recovered from the blood which is being used for the artificial circulation, and which escapes from the veins after traversing the intestinal capillaries. The disappearance is not due to any action of the blood itself, for peptone introduced into the blood before it is driven through the mesenteric arteries in the experiment may be recovered from the blood as it escapes from the mesenteric veins. That if it did pass into the chyle it would undergo a similar change by some action of the epithelium is indicated by the fact that when peptone is introduced into the lymph-spaces of the connective tissue, its presence may soon be recognized in the lymph of the thoracic duct, but that no peptone can be detected in the lymph of the thoracic duct when peptone, even in large quantity, is introduced into the alimentary cavity provided that the epithelium be intact and healthy.

We are therefore guided in deciding this question by indirect evidence; and this, though pointing to the probability that the proteids pass into the portal blood and not into chyle, cannot be regarded as conclusive. One argument in this direction may be drawn from the fact that when the portal blood is experimentally diverted from the liver into the vena cava, the grave troubles which result seem to be chiefly caused by proteid food.

But, if this view be provisionally accepted, it must be on the understanding that it is probable only; and it may be that proteids do not take the same paths and are not absorbed in the same condition in all animals. In carnivorous animals whose (natural) food contains a considerable quantity of fat, the lacteals might be considered as preoccupied in the absorption of fat. The food of herbivora on the other hand contains a relatively small amount of fat; and if in these animals all the proteids and carbohychates are absorbed by the blood vessels, there is comparatively little left for the lacteals to do. Yet in these animals the lacteals and the lymphaties are well developed. In the villus of a herbirorous guinea-pig or rabbit, though the reticular tissue is very scanty as compared with that present in the villus of a dog, the lacteal chamber is, relatively to the diameter of the villus, not merely as large as but much larger than in the 
dog. It is difficult to suppose that this wide chamber is intended solely for the absorption of the relatively small amount of fat present in vegetable food. The question which we are discussing is clearly at present to be regarded as by no means settled.

\section{The Mechanism of Absorption.}

\$251. The Absorption of Fats. We have now to consider the manner in which these several substances pass into either the lacteal radicle or the capillary blood vessel. It will be convenient to begin with the absorption of the fats.

We have scen reason $(\$ 230)$ to think that the fats, remaining chiefly as neutral fats, are emulsified in the intestine, by means of the bile and pancreatic juice, the small quantity of soap which is formed probably serving simply the purpose of facilitating the emulsification.

The neutral fats so cmulsified pass in the first instance into the bodics of the columnar cells of the villi. It has, it is true, bcen maintained by some that they pass between the cells and not into them; but the evidence is against this view. Since no collections of fat globules are seen in the cubical cells of the glands of Lieberkihhn we infer that these have nothing to do with the absorption of fat.

How the fat enters into the substance of the cell we do not know. We may presume that the striated border plays some part, but what part we do not know. Though the rods making up the border appear able to move so far, at least, as to change their form, we have no evidence that the fat is introduced into the cells by means of any movements of these rods. We may imagine that the globules pass into the cell substance by help in some way of these rods, through amoboid movements comparable with the ingestive movements of the body of an amoba; but we have no positive evidence to support this view. We said (\$208) that bile promotes the passage of fat through membranes, possibly by in some way promoting a closer contact between the particles of fat and the substance of the membrane; but even if bile las this effect on the surface of the cells, its action in this respect can be subsidiary only. When an animal is fed not on neutral fats but on fatty acids, the chyle in the thoracic duct contains a large quantity of nentral fat. We may infer that a synthesis of the fatty acids into neutral fats has in such a case somewhere taken place. And indeed it has been urged by some that even the nentral fats are not absorbed by the epithelium cells as merely emulsified fat, but that they are split up within the canal, absorbed by the cells as fatty acids, and immediately synthesized again into neutral fats. 
Within the columnar cell the fat may be seen, both in fresh living cells, and in osmic acid preparations, to be disposed in globules of various sizes, some large and some small, each globule placed in a space of the protoplasmic cell substance. It does not follow that the fat actually entered the cell exactly in the form of these globules; it may be that the fat passes the striated border in very minute spherules which, reaching the body of the cell, run together into larger globules; but whether this is so or not we do not know.

From the columnar cell the fat passes into the spaces of the reticular tissue of the villus. It has, it is true, been contended that it passes along the substance of the bars of the reticulum; but in earefully prepared osmic acid specimens of a villus in active digestion of fatty food, the fat may be distinctly recognized as largely filling up, still in the form of globules of various sizes, the spaces in the meshes of the reticulum which are not occupied by the leucocytes or allied wandering cells. The bases of the columnar cells, through the gaps in the basement membrane, directly abut upon the labyrinth of spaces; and the fat once out of the base of the cell is free in the spaces of this labyrinth. How it issues from the cell we do not exactly know: possibly by a process analogous to the excretion of solid matters by an amoeba.

From the labyrinth of spaces of the reticulum of the villus the fat passes into the cavity of the lacteal radicle; and it is worthy of note that in the passage it undergoes a change. In the interior of the intestine, in the substance of the columnar cell, and apparently in the labyrinth of the reticulum it is simply emulsified fat consisting of globules small and large; within the lacteal radicle it eonsists partly of the same casily recognized globules but partly of the extremely divided ' molecular basis' (\$ 241); it is now no longer emulsified fat but chyle. How and by what means this extremely minute division of the globular fat into the 'molecular basis' takes place we do not know; nor do we know the exact manner in which the fat passes from the spaces of the reticulum into the intcrior of the radicle.

We may here perhaps remark that the eontents of the lacteal radicle consist not exclusively of fat, but of fat accompanied by the proteid and other substances which go to make up the chyle. Proteid and other substances besides fat are also present in the lymph which occupies in part the labyrinth of the body of the villus, and are derived, like the lymph elsewhere, from the blood of adjacent capillaries; at least, they are in part so deriverl, though it may be not wholly, for as we have just seen the passage of proteid material from the intestine into the substance of the villus past the eapillaries, though not proved, must still be considered as possible. 
The spaces of the reticulum of the villus are more or less occupied by wandering cells of which we spoke inder the general term of leucocytes. These do not all present the same appearances and most probably are not of all the same kind. Some of these lencocytes wander not only through the labyrinth of the reticulum but pass into the epithelium between the cells, and may project processes into, or even make their way eventually into the interior of the intestine; or following the reverse course may wander from the inside of the canal between the epithelium cells into the body of the villus; some of them moreover undoubtedly contain fat. Hence the vicw has been suggested that these lencocytes are important agents, indeed the chief agents in the absorption of fat. It has been supposed that they, receiving the globules of fat into their cell substance, in fact eating the fat exactly after the manner of an amoba, either while projecting between the columnar cells, in which they carry their burden of fat through the epithelium into the villus, or while wandering in the labyrinth of the villus, bear it away bodily into the lymphatic system. But the number of leucocytes really containing any appreciable quantity of fat is too small to account for the amount of fat absorbed. Nor is the abundance of leucocytes in the mucous membrane during the period of digestion a sure proof that they are concerned in absorption, but rather an indication only that active changes of some kind are going on, since after the administration of a saline such as magnesium sulphate, which produces effects the very reverse of absorption, these leucocytes are present in unusual numbers. Noreover under some circumstances, as in the villi of a new-born puppy after a meal of milk, they are absent even when digestion of fat is rapidly going on and the lacteals are filling with fat. In fact, what we stated above concerning the presence of fat in the bodies of the columnar cells shews that leucocytes can have little to do in transferring fat from the interior of the intestine into the body of villus; and there are no adequate reasons for attributing to them any real share in the transference of fat from the body of the villus into the lacteal chamber.

$\$ 252$. The lacteal chamber opens at the base of the villus into the valved lymphatic vessels lying below, and in these the flow of lymph (chyle) is being promoted by the various causes detailed in $\$ 242$. The pressure, for instance, exerted by the peristaltic contractions of the intestine helps to empty the lymphatic vessel into which a lacteal chamber opens and so promotes the emptying of the latter. In addition to this the plain muscular fibres of the villus supply a special muscular pump for the emptying and filling of the lacteal chamber. These fibres and small bundles of fibres running through in various dircetions and varying in number and arrangement in 
different animals, take on the whole a longitudinal direction parallel to the long axis of the villus. It has been supposed that in contrating and shortening the villus they compress the lacteal and thus empty it, and that when they relax and the villus elongates again, the emptied chamber fills once more. But a different interpretation of their action has been offered somewhat as follows. When the muscular fibres contract they shorten the villus. In thus becoming shorter the body of the villus becomes proportionately broader. since probably no great change of bulk in the reticulum takes place; in this broadening the part to give way will be the lacteal chamber, which thus becomes broader and larger. When the muscular fibres relax, the retieulum. the bars of which have been put on the stretch in a lateral direction, by elastic reaction brings back the villus to its former length, and the lacteal chamber elongates and narrows. On this view the musenlar contraction expands and so fills, while the relaxation narrows and so empties the lacteal chamber. Whichever view we adopt, we may at least conclude that contractions and relaxations of the muscular fibres in some way or other alternately fill and empty the lacteal chamber, and in all probability, at all events during digestion, rhythmical contractions of these fibres are continually going on. When the villus is shortened by the contraction of the muscular fibres, the columnar cells are compressed, becoming longer and narrower; when the museular fibres relax and the villus elongates, the columnar cells return to their previous form. The alternating changes of form to which the columnar cells are thus subjected, and the alternating changes of pressure taking place in the reticulum, may also serve to promote the passage of material through the one and through the other.

$\$ 253$. The Absorption of Diffusible Substances and of Water. On the provisional assumption which we have made that the proteids are converted into peptone, we may consider, for the present at all events, peptone, sugar, and soluble salts as together forming a class distinguished from fats by their being diffusible, some more so than others. And we have made the further provisional assmmption that these pass into the blood vessels and not into the lacteals.

'The network of eapillary blood vessels is spread immediately beneath the basement membrame, and all the material which enters the lacteal chamber has to run the gauntlet of the meshes of this network. During digestion the capillaries of the intestine are filled and distended, so that at a time when absorption is taking place these meshes between the capillaries are unusually narrow. From the interior of these capillaries, here as elsewhere, transudation is taking place; these capillaries supply the lymph which helps to fill up the labyrinth of the reticulum and the lacteal elamber. But to a much greater extent 
than elsewhere (cf. $\$ 244$ ) this current of transudation from within the capillary to without is accompanied by a reverse current from without to within. 'The diffusible substances in question pass from the intestine through the layer of epithelium cells, through the attenuated reticular lyuph-space between the basement nembrane and the eapillary wall, and through the capillary wall into the blood eurrent. 'Their passage consists of two stages; that through the epithelium cells from the intestine to the lymph-space, and that from the lymph-space into the blood vessels. These two stages may be expected to differ, seeing that the structures concerned are different; but we may at first consider them as one, and speak of the passage from the intestine into the blood as a single event.

In speaking of these substances as diffusible we are using the terms in reference to the well-known passage of sueh substances through thin membranes or porous partitions. When a strong solution of sugar or of common salt is separated by a thin membrane (vegetable parchment, dead urinary bladder, dead intestine, \&c.) from a weak solution of sugar or of salt, the sugar or salt passes with a certain rapidity from the stronger to the weaker solution, and water passes from the weaker solution to the stronger; if, to begin with, simple water be substituted for the weaker solution the effect is at first still nore striking. Peptone passes in the same manner but as we have seen much more slowly. The process is spoken of as a physical one since it is not aceompanied, necessarily, by any chemical change in the diffusing substance, nor is there any necessary change in the membrane or partition. The rate at which a substance diffuses, and the total amount of diffusion which can take place, are determined by certain qualities of the substance (which we nuay call physical though they depend on the chenlical nature of the substance) in relation to certain qualities of the menbrane; thus two salts may diffuse through the same membrane at different rates, with different rates in the associated current of water, the osmotic current as it is called, from the weaker to the stronger solution; and the same substance may pass at different rates through different membranes. By a number of observations, in which various substances in solution and several known membranes or partitions have been employed, a certain number of "laws of diffusion" liave been establisherl.

Now if by the statement that diffusible substances pass by diffusion into the blood-capillaries of the intestine we are led to expect that the passage takes place exactly according to the laws established by observations on ordinary membranes we should be led into error ; for the disappearance of these substanees from the interior of the intestine does not take place according to the laws which regulate their disappearance from 
one side of an ordinary diffusion septum. This can be ascertained by introducing solutions of the substances, of various strength, into a loop of intestine, isolated in the living animal hy the method described in \$ $\$ 11$, and watching their disappearance by analysis of the contents of the loop. For instance, sodium sulphate passes through an ordinary diffusion septum with a rapiclity rather greater than that of dextrose, whereas dextrose disappears from the intestine distinctly more rapidly than solium sulphate, peptone which diffuses very slowly indeed through an ordinary diffusion septum disappears rapidly (though not so rapidly as dextrose) from the intestine, and when the details of the disappearance from the intestine of weak solutions of two salts which diffuse through an ordinary membrawe at different rates, which have as it is said different osmotic equivalents, are studied, these details are quite different from those of ordinary diffusion. The more the matter is studied the more decidedly apparent becomes the difference between ordinary diffusion and the absorption of diffusible substances from the intestine.

Two opposite processes are carried on by the wall of the alimentary canal: on the one hand material is transferred from the blood stream to the inside of the canal in the form of the several digestible juices, and on the other hand digested material is transferred from the inside of the canal to the blood stream. The former process we without hesitation regard as the work of the epithelium cells forming the lining of the canal, whether the cells lie as in the gastric and lieberkiihn's glands in the thickness of the wall of the canal, or as in the pancreas are removed to some distance from it; we call the process 'secretion.' And the evidence goes increasingly to show that the other process is also the work of epithelium cells, that the two processes are in the main alike save that the eurrent resulting from the activity of the cells is in opposite direc. tions in the two cases. Wre might in fact venture to speak of absorption from the canal as an inverted secretion. And we may regard as wholly secondary the fact that in the small intestine the cells of Lieberkühn appear at least to be chiefly devoted to ordinary secretion, and those of the villi to the inverted secretion. Wre may further consider the conversion of food into diffusible substances as in the main a means by which the material of the food enter's more readily into the substance of the eprithelial cell and so is placed more easily within its grasp; and we have seen the material, having thus entered, appears in certain cases to unclergo an immediate change, the maltose being converted into dextrose, and the peptone into some other proteid, diffusibility being in the latter case lost.

Such a view, howerer, of absorption as a kind of secretion must in the first instance be confined to the first step of the 
whole process, namely the transference through the epithelium cell from the inside of the canal to the lymph-space of the villus. And we may perhaps be inclined still to regard the second step the transference from the lymph-space to the blood stream as more strictly an act of diffusion. But the considerations which we urged ( $(236)$ in regard to translation from the blood vessel to the lymph-space may also be applied to the reverse current; we may look upon this as something much much complex than, and so different from, ordinary physical diffusion. 


\section{CHAPTER II.}

\section{RESPIRATION.}

$\$ 254$. OxE particular item of the body's income, viz. oxygen, is peculiarly associated with one particular item of the body's waste, viz. carbonic acid, inasmuch as the means which are applied for the introduction of the former are also used for the getting rid of the latter. Both are gases, and the ingress of the one as well as the egress of the other seems to be more directly dependent on the simple physical process of diffusion than on any active vital processes carried on by means of tissues. Oxygen passes from the air into the blood mainly by diffusion, and mainly by diffusion also from the blood into the tissues; in the same way carbonic acid passes mainly by diffusion from the tissues into the blood, and from the blood into the air. Whereas, as we have seen, in the secretion of the digestive juices the epithelium-cell plays an all-important part, in respiration the entrance of oxygen from the lungs into the blood, and from the blood into the tissue, and the passage of carbonie acid in the contrary direction, appear to be affected, if at all, in a wholly subordinate mamner, by the behaviour of the pulmonary, or of the capillary epithelium. What we have to deal with in respiration then is not so much the vital activities of any particular tissue, as the various mechanisms by which a rapid interchange between the air and the blood is effected, the means by which the blood is enabled to carry oxygen and carbonic acid to and from the tissues, and the mamer in which the several tissues take oxygen from and give carbonic acid np to the blood. We have reasons for thinking that oxygen can be taken into the blood, not only from the lungs, but also to a certain small extent from the skin, and, as we have seen, from the alimentary canal also ; and carbonic acid certainly passes away from the skin, and through the various secretions, as well as by the lungs. Still the lungs are so eminently the channel of the interchange of gases between the body and the air, that in dealing at the present with respiration, we shall confine ourselves entirely to pulmonary respiration, leaving the consideration of the subsiliary respiratory processes till we come to study the secretions of which they respectively form part. 
SEC. 1. THE MECHANICS OF PULMONARY RESPIRATION.

$\$ 255$. The lungs are placed, in a state which is always one of distension, sometimes greater, sometimes less, in the airtight thorax, the cavity of which they, together with the heart, great blood vessels and other organs, completely fill. By the contraction of certain muscles the cavity of the thorax is enlarged. The lungs must follow this enlargement and be themselves enlarged, otherwise the pleural cavities would be enlarged, but this is impossible so long as the thoracic walls are intact 'The cnlargement of the lung consists chiefly in an enlargement or expansion of the pulmonary alveoli, the air in which becomes by the expansion rarefied. That is to say the pressure of the air within the lungs becomes less than that of the air outsidc the body, and this difference of pressure causes a rush of air through the trachea into the lungs until an equilibrium of pressure is established between the air inside the lungs and that outside. This constitutes inspiration. ¿ ${ }^{\top}$ pon the relaxation of the inspiratory muscles (the muscles whose contractions have brought about the thoracic expansion), the elasticity of the lungs and chest-walls, aided perhaps to some extent by the contraction of certain muscles, causcs the chest to return to its original size; in consequence of this the pressure within the lungs now becomes greater than that outside, and thus air rushes out of the trachea until equilibrium is once more established. This constitutes expiration; the inspiratory and expiratory act together form a respiration. 'The fresh air introduced into the upper part of the pulmonary passages by the inspiratory movement contains more oxygen and less carbonic acid than the old air previously present in the lungs. By diffusion the new or tidal air, as it is frequently called, gives up its oxygen to, and takes carbonic acid from, the old or stationary air, as it has been called, and thus when it leaves the chest in expiration has been the means of both introducing oxygen into the chest and of removing carbonic acid from it. In this way, by the ebb and flow of the tidal air, and by diffusion between it and the stationary air, the whole air in the 
lungs is being eonstantly renewed through the alternate expansion and contraetion of the ehest.

$\$ 256$. In ordinary respiration, the expansion of the ehest never reaehes its maximum; by more forcible museular eontraetions, by what is ealled laboured inspiration, an additional thoracic expansion ean be brought about, leading to the inrush of a eertain additional quantity of air before equilibrium is established. 'This additional quantity is often spoken of as complemental air. In the same way, in ordinary respiration, the contraetion of the ehest never reaehes its maximum. By ealling into use additional museles, by a laboured expiration, an additional quantity of air, the so-ealled reserve or supplemental air, may be driven out. But even after the most foreible expiration, a eonsiderable quantity of air, the residual air, still remains in the lungs. The natural condition of the lungs in the ehest is in faet one of partial distension. The elastie pulmonary tissue is always to a eertain extent on the stretelı; it is always, so to speak, striving to pull asunder the pulmonary from the parietal pleura; but this it eannot do, beeause the air ean have no aecess to the pleural eavity. When, however, the ehest eeases to be air-tight, when by a puneture of the ehestwall or diaphragm, air is freely introdueed into the pleural ehamber, the elastieity of the lungs pulls the pulmonary away from the parietal pleura, and the lungs shrink, driving out by the windpipe a eonsiderable quantity of the residual air. Even then, however, the lungs are not eompletely emptier, some air still remaining in them; this is probably air imprisoned in the infundibula by eollapse of the bronehioles, the walls of which are not rigid but flaeeid. If in a living animal the pressure of the atmosphere eontinue to have aecess to the outside of a lung the air thus imprisoned is gradually absorbed and the lung beeomes solid. 'The same result may oceur from the pressure of fluid aeeumulated in the pleural carity.

It need hardly be added that when the pleura is punetured, and air can gain free admittanee from the exterior into the pleural ehamber, since the resistanee to the entranee of the air into the pleural ehamber is far less than the resistance to the entranee into the lungs, the effeet of the respiratory movements is simply to drive air in and out of that chamber, instead of in and out of the lung. There is in consequence no renewal of the air within the lungs under those circumstanees. If there be a suffieient obstaele to the entrance of air into the pleural chamber, sueh as a fold of tissue blocking up the opening, the expansion of the ehest may still lead to a distension of the lungs; and in this way in some cases puncture of the chestwalls has not seriously interfered with respiration. The parietal and pulmonary pleura are, in normal cireumstances, separated by a very thin layer only of fluid. so that we may perhaps 
speak of them as being in a state of 'adhesion,' such as obtains between two wet membranes superimposed. And it has been suggested that this adhesion, having to be overcome before the two surfaces can separate, assists in preventing the entrance of air into the pleural cavity after puncture of the thorax; but it has not been clcarly shewn that this is really of importance in the matter.

\$25\%. Before birth the lungs contain no air ; they are in the condition called atelectatic. The walls of the alveoli, the epithelial lining of which is at that time well developed, consisting of distinctly nucleated cells with granular cell-substance, are in contact, the cavity of the alveolus not having as yet come into existence ; the walls of the bronchioles are similarly in a eollapsed condition, with their walls touching; the more rigid bronchia, like the trachea, possess some amount of lumen which, however, is occupied by fluid. When the chest expands with the first breath taken, the pressure of the inspired air has to overcome the "adhesion," obtaining between the walls of the alveoli thus in contact with each other and also those of the bronchiolcs. The force spent in thus opening out and unfolding, so to speak, the alveoli and bronchioles is considerable, and in the expiration succeeding the first inspiration most of the air thus introduced remains, the force exerted by the chest in returning to its previous dimensions after the breathing in, and the elastic action of the alveoli being insufficient to bring the walls of the alveoli again into contact. Succeeding breaths unfold the lungs norc and more until all the alveoli and bronchioles are opencd up, and then the whole force of the expiratory act is directed to driving out the previously inspired air.

It is not, however, until sometime after birth that the lungs pass into that further distended state of which we spoke above. In a newly-born animal there is no negative pressure obtaining in the pleural cavities, the lungs when at rest are not on the stretch, and opening the thorax does not lead to collapse of the lungs. The state of things obtaining later on is established, not at once but gradually, and is apparently brought about by the thorax growing more rapidly, and so becoming relatively more capacious than the lungs. The distension of the lungs in the adult may be familiarly described as being due to the chest being too large for the lungs.

$\$ 258$. In man the pressure exerted by the clasticity of the lungs alone amounts to about 5 or $7 \mathrm{~mm}$. of mereury. This is estimated by tying a manometer into the windpipe of a clead subject and observing the rise of mcrcury which takes place when the chest-walls are punctured. If we took $7.6 \mathrm{~mm}$. as the pressure, this would be just $1 / 100$ of the pressure of the atmospherc. If the chest be forcibly distended bcforehand, a much larger rise of the mercury is observed, amounting, in the 
case of a distension corresponding to a very forcible inspiration, to $30 \mathrm{~mm}$. In the living body this mechanical elastic force of the lungs may be assisted by the contraction of the plain muscular fibres of the bronchi ; the pressure, however, which can be exerted by these probably does not exceed 1 or $2 \mathrm{~mm}$.

When a manometer is introduced into a lateral opening of the windpipe of an animal, the mercury will fall, indicating a negative pressure as it is called, during inspiration, and rise, indicating a positive pressure, during expiration, both fall and rise being slight and varying accorling to the freedom with which the ail passes in and out of the chest. When a manometer is fitted with air-tight closure into the mouth, or better, in order to avoid the suction-action of the mouth, into one nostril, the other nostril and the mouth being closed, and efforts of inspiration and expiration are made, the mercury falls or undergoes negative pressure with inspiration, and rises, or undergoes positive pressure during expiration. It has been foumd in this way that the negative pressure of a strong inspiratory effort may vary from 30 to $7 t \mathrm{~mm}$., and the positive pressure of a strong expilation from 62 to $100 \mathrm{~mm}$.

The total amount of air which can be given out by the most forcible expiration following upon a most forcible inspiration, that is, the sum of the complemental, tidal and reserve airs. has been called 'the vital capacity;' 'extreme differential capacity' is a better phrase. It may be measured by a modification of a gas-meter called a Spirometer; and though it varies largely, the average may be put down at $3-1000$ c.c. (200 to 250 cubic inches).

Of the whole measure of vital capacity, about 500 c.c. (30 c. inch) may be put down as the average amount of tidal air, the remainder being nearly equally divided between the complemental and reserve airs. The quantity left in the lungs after the decpest expiration amounts to about 1400 or 2000 c.c.

Since the respiratory movements are so easily affected by various eircumstances, the simple fact of attention being directed to the breathing being sutficient to cause modifications both of the rate and depth of the respiration, it becomes very difticult to fix the volume of an average breath. 'Thus various anthol's have given figures varying from 53 c.c. to 792 c.c. The statement made above is the mean of observations varying from 177 to 695 c.e.

\$259. Graphic Records of Respiratory Movements. These may be obtained in many various ways.

The simplest, readiest and perhaps the most generally useful method is that of recording the movements of the column of air. This may be reffected hy introrlucing a $T$ piece into the trachea, one cross piece being left open, and the other connected with a Marey's 
Chap. II.]

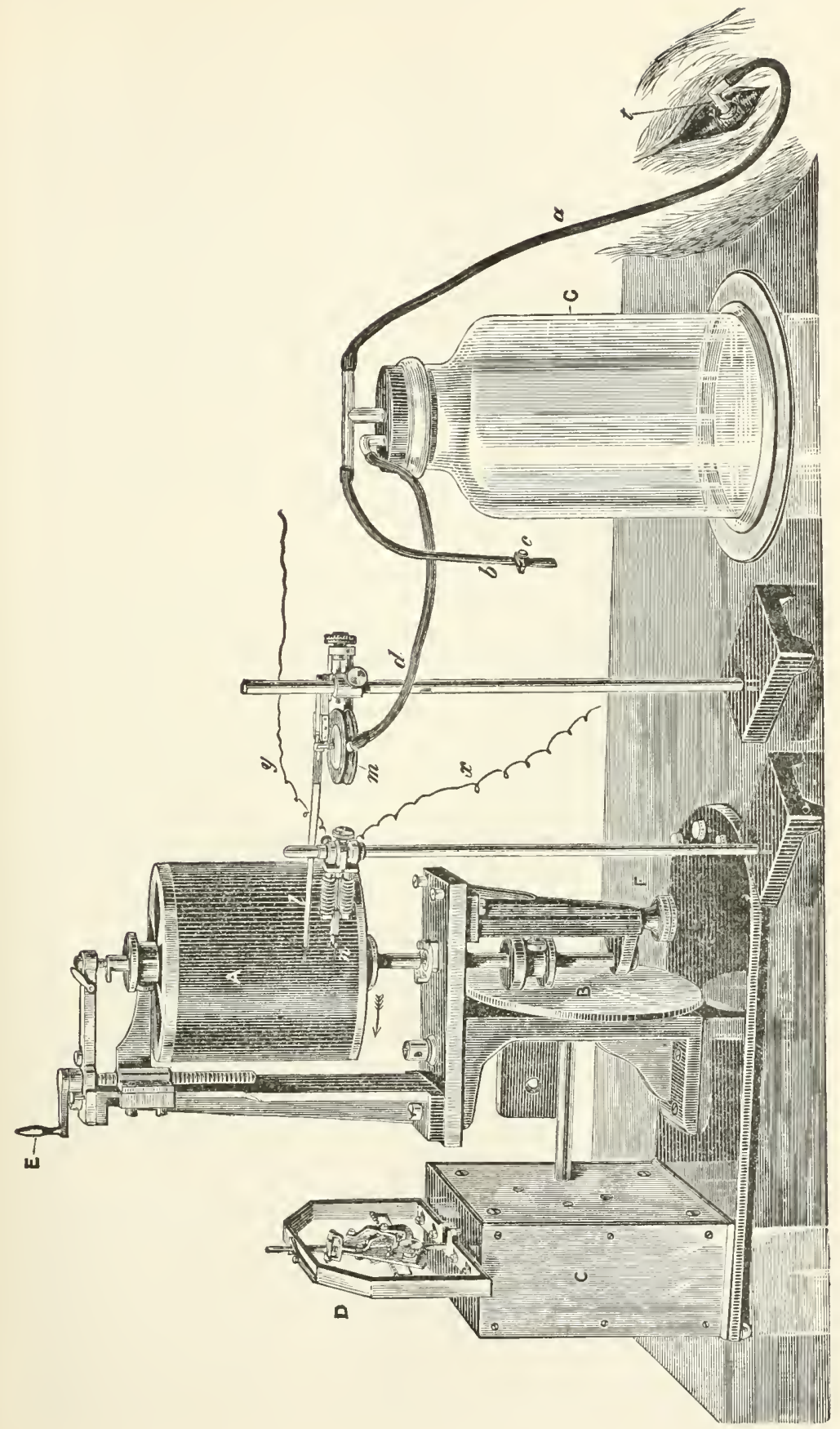


Fig. 85. Apparatus for takivg Tracisgs of the Movements of the Colum of Air in Respiration.

The recording apparatus shewn is the ordinary cylinder recording apparatus. The cylinder A covered with smoked paper is by means of the friction-plate B put into revolution by the spring clock-work in C regulated by Foucault's regulator I). By means of the screw E, the cylinder can be raised or lowered, and by means of the screw $\mathrm{F}$ its speed may be increased or diminished.

The tracheotomy tube $t$ fixed in the trachea of an animal is connected by india-rubber tubing $a$ with a glass $T$ piece inserted into the large jar G. From the other end of the $T$ piece proceeds a second piece of tubing $b$, the end of which can be either closed or partially obstructed at pleasure by means of the screw clamp $c$. From the jar proceeds a third piece of tubing $d$, connected with a Marey's tambour $m$ (see Fig. 36), the lever of which $l$ writes on the recording surface. When the tube $b$ is open the animal breathes freely through this, and the movements in the air of $G$ and consequently in the tambour are slight. On closing the clamp $c$, the animal breathes only the air contained in the jar, and the movements of the lever of the tambour become consequently much more marked.

Below the lever is seen a small time-marker $n$ connected with an electromagnet, the current through which coming from a battery by the wires $x$ and $y$ is made and broken by a clock-work or metronome.

tambour or with a receiver which in turn is connected with a tambour, see Fig. 36, and Fig. 85. The movements of the column of air in the trachea are transmitted to the tambour, the consequent expansions and contractions of which are transmitted to the recording drum by means of a lever resting on it.

If, a receiver being used, the open end of the $\vdash$ be closed, the animal breathes into and out of the receiver, and the movements of the tambour are greatly increased. This has the disadvantage that the air in the receiver soon becoines unfit for further respiration. A similar increase of the movements of the lever of the tambour may be obtained by connecting a piece of india-rubber tubing to the open end of the $\vdash$. By increasing the length of this tube, or slightly, constricting it, the movements of the lever may be increased without very seriously interfering with the breathing of the animal.

In another method the movements of the chest are recorded. When a small animal such as a rabbit is used, the whole animal may be placed in an air-tight box, breathing being carried on by means of a tube inserted in the trachea and carried through an air-tight orifice in the wall of the box. By another orifice and tube the air in the box is brought into connection with a tambour, which accordingly registers the changes of pressure in the air of the box produced by the movements of the chest (and body) and thus indirectly the movements of the chest. In man and larger animals the changes in the girth of the chest may be conveniently recorded by means of Marey's pneunograph. This consists of a hollow elastic cylinder, or a cylinder with elastic ends, the interior of which is connected with a tambour. By means of a strap attached to each end of the cylinder the instrument can be buckled round the chest like a girdle. When the chest expands, the ends of the cylinder are pulled out, and the air within the chamber rarefied; in consequence the lever of the tambour connected with its interior is depressed; conversely, when the chest contracts, the lever is elevated. 'The pneumatograph 
of Fick is somewhat similar. Or changes in one or other diameter of the chest may be recorded by what may be called the 'callipers' method, as in the recording stethometer of Burdon-Sanderson. This consists of a rectangular framework constructed of two rigid parallel bars joined at right angles to a cross piece. 'The free ends of the bars, the distance between which can be regulated at pleasure, are armed, the one with a tambour, the other simply with an ivory button. The tambour bears on the metal plate of its membrane ( $m$ ' Fig. 36) a small ivory button in place of the lever. When it is desired to record the changes occurring in any diameter of the chest, e.g. an antero-posterior diameter from a point in the sternum to a point in the back, the instrument is made to encircle the chest somewhat after the fashion of a pair of callipers, the ivory button at one free end being placed on the spine of a vertebra behind and the tambour at the other on the sternum in front in the line of the diameter which is being studied. The distance between the free ends of the instrument being carefully adjusted so that the button of the tambour presses lightly on the sternum, any variations in the length of the diameter in question will, since the framework of the tambour is immobile, give rise to variations of pressure within the tambour. These variations of the 'receiving' tambour as it is called are conveyed by a flexible tube containing air to a second or 'recording' tambour, the lever of which records the variations on a travelling surface. For the purpose of measuring the extent of the movements the instrument must be experimentally graduated. Other forms of callipers may of course be used.

By still another method the variations in intra-thoracic pressure, by means of which the movements of the chest-walls produce the movement of air in the lungs, may be recorded. 'This may be effected by introducing carefully, to the total exclusion of air, into a pleural cavity, or into the pericardial cavity, a cannula connected by a rigid tube with a manometer. With each inspiration a negative pressure, or rather an increase of the existing negative pressure, is produced, the mercury, or fluid, in the manometer returning at each expiration. An easier method of recording this intra-thoracic pressure is to introduce into the oesophagus an elastic sound (similar to the cardiac sound Fig. 36) connected with a tambour. The œsophagus within the thorax like the heart and great vessels, as we shall see, is affected as well as the lungs by the variations of intra-thoracic pressure brought about by the respiratory movements.

In yet another method the movements of the diaphragm which, as we shall see, serve as the prime agent in bringing about the enlargement of the thoracic cavity are recorded. This may be done by inserting, through an incision in the abdominal wall, a flat elastic bag between the diaphragm and abdominal organs. When in inspiration the diaphragm descends it exerts on the bag a pressure which, by means of a tube, may be communicated to a tambour. Or a needle may be thrust through the chest-wall so as to rest upon or transfix the diaphragm, and the head of the needle outside the body connected by a thread or otherwise with a lever; each upward and downward movement of the head of the needle, corresponding to the 
downwarl and upward movements of the diaphragm, is registered by the lever.

Tarious molifications of these several methods have been adopted by various observers. They all, however, leave much to be desired. A vely ingenions method of registering the contractions of the diaphragin has recently been introduced. In the rabbit two slips of muscular fibres forming part of the diaphragm, one on each side of the ensiform cartilage, are so disposed and possess such attachments that one, or both of them, may be isolated. without injury to either nerves or blood vessels, and arranged so that while one end of the slip is securely fixed to the chest-wall as a fixed point, the other end can by a thread be brought to bear on a lever. The slip, even when thus arranged, appears to contract rhythmically in complete unison with the contractions of the whole rest of the diaphragm; it serves so to speak as a sample of the diaphragm; and hence its contractions like those of the whole diaphragm may be taken as a record of respiratory movements. The record has to be corrected for variations in the position of the fixed point.

$\$ 260$. In these various ways curves are obtained, which, while differing in detail, exhibit the same general features, and more or less resemble the curve shewn in Fig. 86.

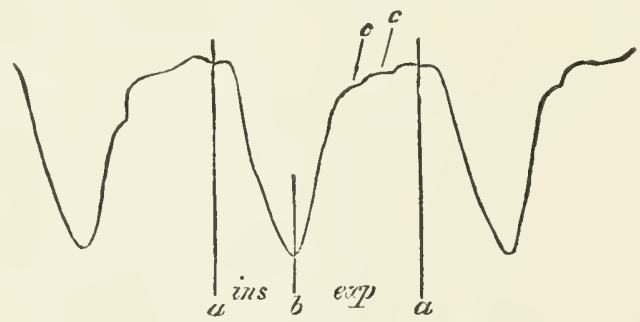

Fig. 86. Tracivg of Thoracic Respiratory Movements obtained b Meaxs OF Marey's PXeumograpil.

A whole respiratory phase is comprised between $a$ and $a$; inspiration, during which the lever descends, extending from $a$ to $b$, and expiration from $b$ to $a$. The undulations at $c$ are caused by the heart's beat.

As the figure shews, inspiration begins somewhat suddenly and arlvances rapidly, being followed immediately by expiration, which is calried out at first rapidly, but afterwards more and more slowly. Such pauses as are seen usually ocent between the end of expiration and the beginning of inspiration. In normal breathing, hardly any such pause exists, but in eases where the respiration becomes infrequent, panses of considerable length may be observed. Is we shall see in detail hereafter, the several parts of the whole act vary much, under various circunstances, in relation to each other. Sometimes expination. sometines inspiration is prolonged; and either inspiration 
or expiration may be slow or rapid in its development. At times the chest may remain for a while at the height of inspiration, thus making a pausc between inspiration and expiration.

In what may be considered as normal breathing, the respiratory act is repeated about 17 times a minute, the duration of the inspiration as compared with that of the expiration (and such pause as may exist) being about as ten to twelve; but the rate varies very largely; and in this as in the volume of each breath it is very difficult to fix a satisfactory average, the figures given varying from 20 to $1: 3$ a minute. It varies according to age and sex. It is influenced by the position of the body, being quicker in standing than in lying, and in lying than in sitting. Muscular exertion and emotional conditions affect it deeply. In fact, amost every event which occurs in the body may influence it. IVe shall have to consider in detail hereafter the manner in which these influences are brought to bear.

When the ordinary respiratory movements prove insufficient to effect the necessary changes in the blood, their rhythm and character become changer. Normal respiration gives place to laboured respiration, and this in turn to dyspnoea, which, unless some restorative event occurs, terminates in asphyxia. 'These abnor'mal conditions we shall study more fully hereafter.

\section{The Respiratory Movements.}

\$261. When the movements of the chest during normal breathing are watched, or when a graphic record is taken by one or other of the methods just described, it is seen that during inspiration an enlargement takes place in the antero-posterior diameter, the sternum being thrown forwards, and at the same time moving upward. The lateral width of the chest is also increased. 'The vertical increase of the cavity is not so obvious from the outside, though when the movements of the diaphragm are watched by means of an inserted needle or otherwise, it is clear that the upper surface of that organ descends at each inspiration, the anterior walls of the abdomen bulging out at the same time. In the female human subject, the movement of the upper part of the chest is usually conspicuous, the breast rising and falling with every respiration; while in the male, the movements of the lower part of the chest are more marked. In laboured respiration all parts of the chest are alternately expanded and contracted, the breast rising and falling as well in the male as in the female. We have now to consicler these several movements in greater detail, and to study the means by which they are carried out.

\$262. Inspiration. There are two chief means by which the chest is enlarged in normal inspiration, viz. the descent of the diaphragm and the elevation of the ribs. The former callses 
that movement in the lower part of the chest and abdomen largely characteristic of male breathing, which is hence called diaphragmatic; the latter causes the movement of the upper chest largely characteristic of female breathing, which is called costal. These two main factors are assisted by less important and subsidiary events.

Even in the female human subject, the share taken in respiration by the diaphragm is an important one, in the male the diaphragm must be regarded as the chief respiratory agent, and in some animals its use, for this purpose, is so prominent that the movements of the ribs may in normal breathing be almost neglected. In the rabbit for instance, in normal breathing, ahmost all the respiratory work is done by the contractions of the diaphragm.

The descent of the diaphragm is effected by means of the contraction of its muscular fibres. When at rest the diaphragm presents a convex surface to the thorax; when contracted it becomes much flatter, and in consequence the level of the chestfloor is lowered, the vertical diameter of the chest being proportionatcly enlarged. In descending, the diaphragm presses on the abdominal viscera, and so causes a projection of the flaccid abdominal walls. From its attachments to the sternum and the false ribs, the diaphragm, while contracting, naturally tends to pull the sternum and the upper false ribs downwards and inwards, and the lower false ribs upwards and inwards, towards the lumbar spine. In normal breathing, this tendency produces little effect, being counteracted by the accompanying general costal elevation, and by certain special muscles to be mentioned presently. In forced inspiration, however, and especially where there is any obstruction to the entrance of air into the lungs, the lower ribs may be so much drawn in by the contraction of the diaphragm, that the girth of the trunk at this point is obviously diminished.

$\$ 263$. The elevation of the ribs is a much more complex matter than the descent of the diaphragm. If we examine any one rib, such as the fifth, we find that while it moves frcely on its vertehral articulation, it inclines when in the position of rest in an oblique direction from the spine to the sternum; hence it is obvious that when the rib is raised, its sternal attachment must not only be carried upward, but also thrown forward. The rib may in fact be regarded as a radius, moving on the vertebral articulation as a centre, and causing the sternal attachment to describe an are of a circle in the vertical plane of the body; as the rib is carried upwards from an oblique to a more horizontal position, the sternal attachment must of necessity be carried farther away in front of the spine. Since all the ribs have a downward slanting direction, they must all tend, when raised towards the horizontal position, to thrust the sternum 
forward, some more than others according to their slope and length. The elasticity of the sternum and costal cartilages, assisted by the articulation of the sternum to the clavicle above, permits the front surface of the chest to be thus thrust forwards as well as upwards, when the ribs are raised. By this action, the antero-posterior diameter of the chest is enlarged.

Since the ribs form arches which increase in their sweep as one proceeds from the first downwards as far at least as the seventh, it is evident that when a lower rib such as the fifth is elevated so as to occupy or to approach towards the position of the one above it, the chest at that level will become wider from side to side, in proportion as the fifth arch is wider than the fourth. Thus the elevation of the rib increases not only the antero-posterior but also the transverse diameter of the chest. Further, on account of the resistance of the sternum, the angles between the ribs and their cartilages are, in the elevation of the ribs, somewhat opened ont, and thus also the transverse as well as the antero-posterior diameter, somewhat increased. In more than one way, then, the elevation of the ribs enlarges the dimensions of the chest.

$\$ 264$. The ribs are raised by the contraction of certain muscles. Of these the external intercostals are perhaps the most important. Even in the case where two ribs, sucl as the fifth and sixth, are isolated from the rest of the thoracic cage, by section of the structures occupying the intercostal spaces above and below, the contraction of the external intercostal muscle of the intervening space raises the two ribs, thms bringing them towards the position in which the fibres of the muscle have the shortest length, viz. the horizontal one. 'This elevating action is, in the entire chest, further favoured by the fact that the first rib is less moveable than the second, and so affords a comparatively fixed base for the action of the muscles between the two, the second in turn supporting the third, and so on, while the scaleni muscles in addition serve to render fixed, or to raise, the first two ribs. So that in normal respiration, the act may probably be described as beginning by a contraction of the scaleni. The first two ribs being thus raised or at least fixerl, the contraction of the series of external intercostal muscles acts at a great advantage.

IThile the elevating, i.e. inspiratory action of the external intercostals is admitted by nearly all authors, the function of the internal intercostals has been much disputed. Some regard their action as wholly inspiratory; others maintain, what is perhaps the more commonly adopted view, that while those parts of them which lie between the sternal cartilages act like the external intercostals as elevators, i.e. as inspiratory in function, those parts which lie between the osseous ribs act as depressors, i.e. as expiratory in function. 
In the well-known model consisting of two rigid bars, representing the ribs, moving vertically by means of their articulations with an upright representing the spine, and connected at their free cnds by a piece representing the sternum, it is undoubtedly true that stretched elastic bands attached to the bars in such a way as to represent respectively the external and internal intercostals, viz. sloping in the one case downwards and forwards and in the other downwards and backwards, do, on being left free to contract, in the former case elevate and in the latter depress the ribs. Such a model however does not fairly represent the natural conditions of the ribs, which are not straight and rigid, but peculiarly curved and of varying elasticity, capable moreover of rotation on their own axes, and having their movements determined by the characters of their vertebral articulations. The mechanical conditions in fact of these muscles are so complex, that a deduction of their actions from simple mechanical principles, or from the direction of the fibres, must be exceedingly difficult and dangerous. Actual experiments on the eat and dog tend to shew that in these animals the contraction of the internal intercostals, along their whole length, takes place, in point of time, alternatcly with that of the diaphragm, and thus afford an argument in favour of these muscles being expiratory in function.

Next in importance to the external intercostals come the levatores costarum, which, though small muscles, are able, from the ncarness of their costal insertions to the fulcrum, to produce considerable movement of the sternal ends of the ribs. 'The external intercostals and the levatores costarum with the scaleni may fairly be said to be the elevators of the ribs, i.e. the chief muscles of costal inspiration in normal breathing.

It must be added however that some observers deny that cither set of intercostal muscles take any important part in raising the ribs. They hold that the chief if not the only use of these muscles is by their contraction to render the intercostal spaces firm and the whole thoracic cage rigid, so that the thorax is moved as a whole by the other museles mentioned, and the intercostal spaces do not give way during the respiratory movements.

Additional space in the transverse diameter is afforded probably by the rotation of the ribs on an antero-posterior axis ; but this movement is quite subsidiary and unimportant. When the chest is at rest, the ribs are somewhat inclined with their lower borders directed inwards as well as downwards. When they are drawn up by the action of the intercostal muscles, their lower borders are everted. 'Thus their flat sides are presented to the thoratic eavity, which is thereby slightly increased in width.

$\$ 265$. Laboured Inspiration. When respiration becomes laboured, other museles are brought into plary. The scaleni are 
strongly contracted, so as distinctly to raise or at least give a very fixed smpport to the first and seeond ribs. In the same way the scrratus posticus superior, which descends from the fixed spine in the lower cervical and upper dorsal regions to the sccond, third, fourth, and fifth ribs, by its contractions raises those ribs. In laboured breathing a function of the lower false ribs, not very noticeable in easy breathing, comes into play. 'They are depressed, retracted, and fixed, thereby griving increased support to the diaphragm, and directing the whole encrgies of that muscle to the vertical enlargement of the chest. In this way the serratus posticus inferior, which passes upward from the lumbar aponeurosis to the last four ribs, by depressing and fixing those libs becomes an adjuvant inspiratory muscle. 'The quadratus lumborum and lower portions of the sacro-lumbalis may have a similar function.

All these mnscles may come into action eren in breathing which, though deeper than usual, can hardly perhaps be called laboured. When, however, the need for greater inspiratory efforts becomes urgent, all the muscles which can, from any fixed point, act in enlarging the chest, come into play. 'Thins the arms and shoulder being fixed, the serratus magnus passing from the scapula to the middle of the first eight or nine ribs, the pectoralis minor passing from the coracoid to the front parts of the third, fourth, and fifth ribs, the pectoralis major passing from the humerus to the costal eartilages, from the second to the sixth, and that portion of the latissimus dorsi which passes from the humerus to the last three ribs, all serve to elevate the ribs and thus to enlarge the chest. The sterno-mastoid and other muscles passing from the neck to the sternum, are also talled into action. In fact, every muscle which by its contraction can cither elevate the ribs or contribute to the fixed support of muscles which do elevate the ribs, such as the trapezius, levator anguli scapulce and rhomboidei by fixing the scapula. may, in the inspiratory cfforts which accompany dyspncea, be brought into play.

\$266. Expiration. In normal easy breathing, expiration is in the main a simple effect of elastic reaction. By the inspiratory effort the elastic tissue of the lungs is put on the stretch ; so long as the inspiratory muscles continue contracting, the tissue remains stretcherl, but directly those muscles relax, the elasticity of the lungs comes into play and drives out a portion of the air contained in thenn. Similarly the elastic sternum and costal cartilages are by the elcvation of the ribs put on the stretch : they are driven into a position which is unnatural to them. When the intereostal and other elevator muscles eease to contract, the elasticity of the sternum and costal cartilages causes them to return to their previous position, thus depressing the ribs, and diminishing the dimensions of the chest. When 
the diaphragm descends, in pushing down the abdominal viscera, it puts the abdominal walls on the stretch : and hence, when at the cnd of inspiration the diaphragm relaxes, the abdominal walls return to their place, and by pressing on the abdominal viscera, push the diaphragm up again into its position of rest. Expiration then during easy breathing is, in the main, simple elastic reaction : but there is probably some, though possibly in most cases, a very slight, expenditure of muscular energy to bring the chest more rapidly to its former condition. This is, as we have seen, supposed by many to be afforded by the internal intercostals acting as depressors of the ribs. If these do not act in this way, we may suppose that the elastic return of the abdominal walls is accompanied and assisted by a contraction of the abdominal muscles. The triangularis sterni, the effect of whose contraction is to pull down the costal cartilages, may also be regarded as an expiratory muscle.

When expiration becomes laboured, the abdominal muscles become important expiratory agents. By pressing on the contents of the abdomen, they thrust them and therefore the diaphragm also up towards the chest, the vertical diameter of which is thereby lessened, while by pulling down the sternum and the middle and lower ribs they lessen also the cavity of the chest in its antero-posterior and transverse diameters. They are, in fact, the chief expiratory muscles, though they are doubtless assisted by the serratus posticus inferior and portions of the sacro-lumbalis, since when the diaphragm is not contracting, the depression of the lower ribs which the contraction of these muscles causes, serves only to narrow the chest. As expiration becomes more and more forced, every muscle in the body which can either by contracting depress the ribs, or press on the abdominal viscera, or afford fixed support to muscles having those actions, is called into play.

\$267. Facial and Laryngeal Respiration. The thoracic respiratory movements are accompanied by associated respiratory movements of other parts of the body, more particularly of the face and of the glottis.

In normal healthy respiration, the current of air which passes in and out of the lungs, travels, not through the mouth but through the nose, chiefly through the lower nasal meatus. The ingoing air, by exposure to the rascular mucous membrane of the narrow and winding nasal passages. is more efficiently warmed than it would be if it passed through the mouth; and at the same time the mouth is thereby protected from the desiccating effect of the continual inroad of comparatively dry air.

D) uring each inspiratory effort the nostrils are expanded, probably by the action of the dilatores naris, and thus the entrance of air facilitated. The return to their previous condition during expiration is effected by the elasticity of the nasal 
cartilages, assisted perhaps by the compressores naris. This movement of the nostrils, perceptible in many people even during tranquil breathing, becomes very obvious in laboured respiration.

When the mouth is closed, the soft palate which is held somewhat tense, is swayed by the respiratory current, but entirely in a passive manner, and it is not until the larynx is reached by the ingoing air that any active movements are met with. When the larynx (the details of which we shall have to deal with at a later part of this work) is examined with the laryngoscope, it is frequently seen that, while during inspiration the glottis is widely open, with each expiration the arytenoid cartilages approach each other so as to narrow the glottis, the cartilages of Santorini projecting inwards at the same time. Thus, synchronous with the respiratory expansion and contraction of the chest, and the respiratory elevation and depression of the alie nasi, there is a rlythmic widening and narrowing of the glottis. Like the movements of the nostril, this respiratory action of the glottis is much more evident in laboured than in tranquil breathing. Indeed in the latter case it is frequently absent. The manner in which this rhythmic opening and narrowing is effected will be described when we come to study the produetion of the voice. Whether there exists a rhythmic contraction and expansion of the trachea and bronchial passages, especially the smaller and more exclusively muscular ones, effected by means of the plain muscular tissue of those organs and synchronous with the respiratory movements of the chest, is uncertain. 


\section{SEC. 2. CHANGES OF THE AIR IN RESPIRATION.}

\$268. During its stay in the lungs, or rather during its stay in the bronehial passages, the tidal air (by means of diffusion chiefly) effects exchanges with the stationary air; in consequence the expired air differs from inspired air in several important particulars.

The temperature of expired air is variable, but under ordinary circumstances is higher than that of the inspired air. At an average temperature of the atmosphere, for instanee at about $20^{\circ} \mathrm{C}$., the temperature of expired air is, in the mouth $33.9^{\circ}$, in the nose $35.3^{\circ}$. When the external temperature is low, that of the expired air sinks somewhat, but not to any great extent, thus at $-6 \cdot 3^{\circ} \mathrm{C}$. it is $29 \cdot 8^{\circ} \mathrm{C}$. When the external temperature is high, the expired air may become eooler than the inspired, thus at $41 \cdot 9^{\circ}$ it has been found to be $38 \cdot 1^{\circ}$. The expired air takes its temperature from that of the body, that is, of the blood, and this as we shall see later on while generally higher may, at times, be lower than that of the atmosphere. The exaet temperature of the expired air in faet depends on the relative temperatures of the blood and inspired air, and on the depth and rate of breathing. The change in temperature takes place not in the lungs but in the upper passages, and chiefly in the nose and pharynx.

$\$ 269$. The expired air is loaderl with aqueous vapour. The point of saturation of any gas, that is, the utmost quantity of water which any given volume of gas can take up as aqueous vapour, varies with its temperature, being higher with the higher temperature. For its own temperature expired air is, aecording to most observers, saturated with aqueous vapour. 'The moisture, like the warmth, is imparted not in the depths of the lung but in the upper passages. The inspired air as it passes into the bronehia is already saturated with moisture.

$\$ 270$. The expired air contains about 4 or 5 p.c. less oxygen, and about 4 p.c. more carbonic aeid than the inspired air, the quantity of nitrogen suffering but little change. 'Thus

$\begin{array}{llcc} & \text { oxygen. } & \text { nitrogen. } & \text { carbonic acid. } \\ \text { Inspired air eontains } 20.81 & 79.15 & .04 \\ \text { Expired " " } & 16.033 & 79.587 & 4.38 \\ & 440 & & \end{array}$


The quantity of nitrogen in the expired air is sometimes found to be slightly greater than, as in the table above, but sometimes equal to, and sometimes less than, that of the inspired air.

In a single breath the air is richer in carbonic acid (and poorer in oxygen) at the end than at the beginning of the breath. Hence the longer the breath is held, the greater the (artificial) pause between inspiration and expiration, the higher the percentage of carbonic acid in the expired air. Thus by increasing the interval between two expirations to 100 seconds, the percentage may be raised to $7 \cdot 5$. When the rate of breathing remains the same, by increasing the depth of the breathing the percentage of carbonic acid in each breath is lowered, but the total quantity of carbonic aeid expired in a given time is increased. Similarly, when the depth of breath remains the same, by quickening the rate the percentage of carbonic acid in each breath is lowered, but the quantity expired in a given time is increased.

Taking, as we have done, the amount of tidal air passing in and out of the chest of an average man at $500 \mathrm{c} . \mathrm{c}$. , such a person will expire about 22 c.c. of carbonic acid at each breath ; this, reckoning the rate of breathing at 17 a minute, would give over 500 litres of carbonic acid for the day's production. Actual determinations however give a rather smaller total than this; thus in a series of experiments of which we shall have to speak hereafter, the total daily excretion of carbonic acid in an average man was found to be $800 \mathrm{gr} m s$. , i.e. rather more than 400 litres (406), containing $218.1 \mathrm{grms}$. carbon, and 581.9 grms. oxygen, the oxygen which actually disappeared from the inspired air at the same time being about 700 grms. This amount it should be said represents, owing to the manner in which the experiment was conducted, the gases given out and taken in, not by the lungs only, but by the whole body; but the amount of carbonic acid given out by other chamnels than the lungs is, as we shall see, very slight (10 grms. or even less), so that 800 grms. may be taken as the average production of carbonic acid by an average man. The quantity however, both of oxygen consumed and of carbonic acid given out, is subject to very wide variations; thus in the observations of which we are speaking the daily quantity of carbonic acid varied from 686 to 1285 grms., and that of the oxygen from 594 to 1072 grms. These variations and their causes will be diseussed when we come to deal with the problems of nutrition.

$\$ 271$. When the total quantity of tidal air given out at any expiration is compared with that taken in at the corresponding inspiration, it is found that, both being dried and measured at the same temperature and pressire, the expired air is less in volume than the inspired air, the difference amounting to about $\frac{1}{40}$ th or $\frac{1}{50}$ th of the volume of the latter. 
Hence, when an animal is made to breathe in a confined space, the air is absolutely diminished in volume. The approximate equivalence in volume between inspired and expired air arises from the fact that the volume of any given quantity of carbonic acid is equal to the volume of the oxygen consumed to produce it : the slight falling short of the expired air is due to the circumstance that all the oxygen inspired does not reappear in the carlonic acid expired, some having formed within the body other combinations.

$\$ 272$. Besides carbonic acid, expired air contains various substances which may be spoken of as impurities. many of an unknown nature, and all in small amounts. Traces of ammonia have been detected in expired air, even in that taken directly from the trachea, in which case its presence could not be due to decomposing food lingering in the mouth. When the expired air is condensed by being convered into a cooled receiver, the aqueous product is found to contain organic matter, which, from the presence of micro-organisms, introduced in the inspired air, is very apt rapidly to putrefy. The organic substances thus shewn to be present in the expired air are the cause in part of the odour of breath. It is probable that some of them are of a poisonous nature, either poisonous in themselves as coming direct from and produced in some way or other in the pulmonary apparatus, or poisonous as being the products of putrefactive decomposition; for various animal substances and fluids give rise by decomposition to distinct poisonous products, known as ptomaines, and it is possible that some of the constituents of expired air are of an allied nature. In any case the substances present have a deleterious action, for an atmosplere containing simply 1 p.c. of carbonic acid (with a corresponding diminution of oxygen) has very little effect on the animal economy, whereas an atmosphere in which the carbonic acid has been raised to 1 p.c. by breathing, is highly injurious. In fact, air rendered so far impure by breathing that the carbonic acid amounts to .08 p.c. is distinctly unwholesome. not so much on account of the carbonic acid, as of the accompanying impurities. Since these impurities are of unknown nature and camnot be estimated. the easily determined carbonic acid is usually taken as an indirect measure of their presence. We have seen that the average man loads, at each breath, 500 c.c. of air with carbonic acid to the extent of 4 p.c. He will accordingly at each breath load 2 litres to the extent of 1 p.c.; and in one hour, if he breathes 17 times a minute. will load rather more than 2000 litres to the same extent. At the very least then a man ought to be supplied with this quantity of air hourly; and if the air is to be liept fairly wholesome, that is with the carbonic acid reduced considerably below $\cdot 1$ p.c., he should have even more than ten times as much. 


\section{SEC. 3. THE RESPIRATORY CHANGES IN THE BLOOD.}

$\$ 273$. While the air in passing in and out of the lungs is thus robbed of a portion of its oxygen, and loaded with a certain quantity of carbonic acid, the blood as it streams along the pulmonary capillaries undergoes important correlative changes. As it leaves the right ventricle it is renous blood of a dark purple or maroon colour; when it falls into the left auricle it is arterial blood of a bright scarlet hue. In passing through the capillaries of the body from the left to the right side of the heart, it is again changed from the arterial to the venous condition. We have to inquire, What are the essential differences between arterial and venous blood, by what means is the venous blood changed into arterial in the lungs, and the arterial into venous in the rest of the body, and what relations do these changes in the blood bear to the changes in the air which we have already studied?

The facts, that venous blood at once becomes arterial in appearance on being exposed to or shaken up with air or oxygen, and that arterial blood becomes venous in appearance when kept for some little time in a closed vessel, or when submitted to a current of some indifferent gas such as nitrogen or hydrogen, prepare us for the statement that the fundamental difference between venous and arterial blood is in the relative proportion of the oxygen and carbonic acid gases contained in each. From both, a certain quantity of gas can be extracted by means which do not otherwise materially alter the constitution of the blood; and this gas when obtained from arterial blood is found to contain mole oxygen and less carbonic acid than that obtained from venous blood. 'This is the real differential character of the two bloods; all other differences are either, as we shall see to be the case with the colour, dependent on this, or are unimportant and fluctuating.

If the quantity of gas which can be extracted by the mercurial air-pump from 100 vols. of blood be measured at $0^{\circ} \mathrm{C}$., and a pressure of $760 \mathrm{~mm}$., it is found to amount, in round numbers, to 60 vols. 


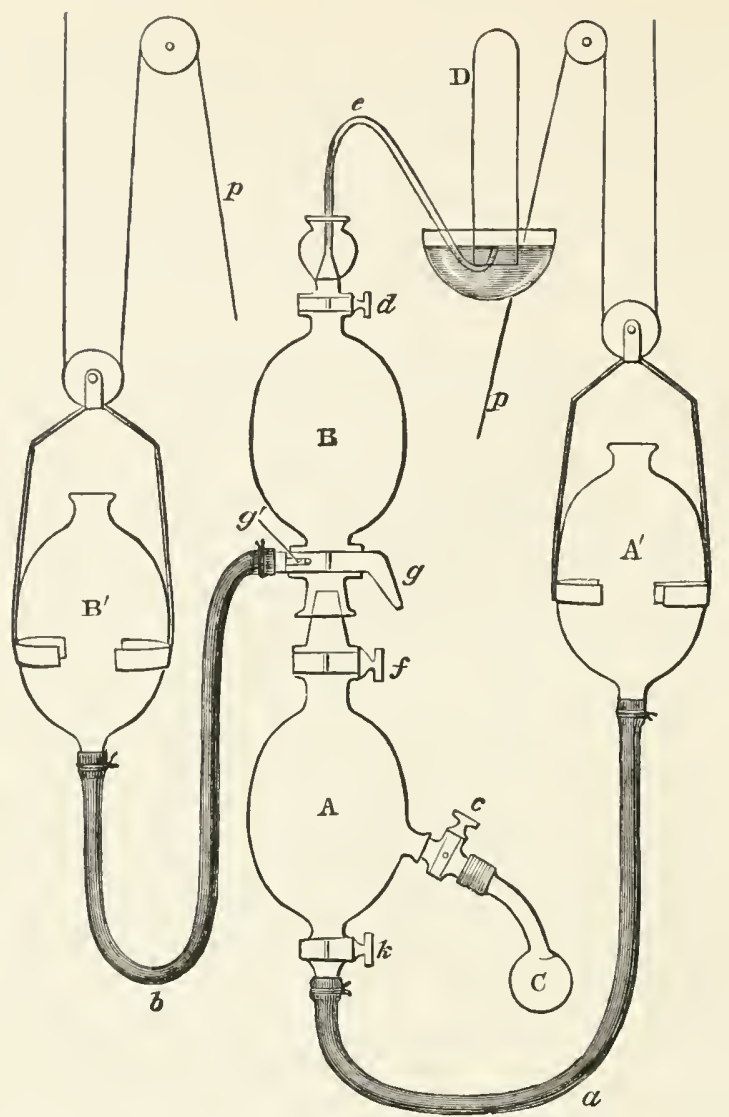

Fig. 87. Diagramatic Illustration of Ludwig's Mercurial Gas-Pump.

$\mathrm{A}$ and $\mathrm{B}$ are two glass globes, connected by strong india-rubber tubes, $a$ and $b$, with two similar glass globes $A^{\prime}$ and $B^{\prime}$. A is further connected by means of the stopcock $c$ with the receiver $\mathrm{C}$ containing the blood (or other fluid) to be analyzed, and $\mathrm{B}$ by means of the stopcock $d$ and the tube $e$ with the receiver $\mathrm{D}$ for recciving the gases. $A$ and $B$ are also comnected with each other by ineans of the stopcocks $f$ and $g$, the latter being so arranged that $B$ also communicates with $\mathrm{B}^{\prime}$ by the passage $g^{\prime}$. $\quad \mathrm{A}^{\prime}$ and $\mathrm{B}^{\prime}$ being full of mercury and the cocks $k, f$, $g$, and $d$ being open but $e$ and $g^{\prime}$ closed, on raising $\Lambda^{\prime}$ by means of the pulley $p$ the mercury of $A^{\prime}$ fills $A$, driving out the air contained in it, into $B$, and so out through $e$. When the mercury has risen above $f, f$ is closed, and $g^{\prime}$ being opened, $\mathrm{B}^{\prime}$ is in turn raised till $\mathrm{B}^{3}$ is completely filled with mercury, all the air previonsly in it being driven out through $e$. Upon closing $d$, and lowering $\mathrm{B}^{\prime}$, the whole of the mercury in B falls in $\mathbf{B}^{\prime}$, and a vacum consequently is established in B. On closing $g^{\prime}$, but opening $g, f$, and $k$ and lowering $A^{\prime}$, a vacuum is similarly established in $A$ and in the junction betwcen $A$ and $B$. If the cock $c$ be now opened the gases of the blood in $\mathrm{C}$ escape into the vacuum of $\mathrm{A}$ and $\mathrm{B}$. By raising $\Lambda^{\prime}$, after the closure of $c$, and opening of $d$, the gases so set free are driven from $A$ into $\mathrm{B}$, and by the raising of $\mathrm{B}^{\prime}$ from $\mathrm{B}$, through $e$ into the receiver $\mathrm{D}$, standing over mercury. 
The vacuum produced by the ordinary mechanical air-pump is insufficient to extract all the gas from blood. Hence it becomes necessary to use a mercury pump capable of producing a large Torricellian vacuum. In the form of mereurial pump which bears Ludwig's name (Fig. 87) two large globes of glass, one fixed and the other moveable, are connected by a flexible tube; the fixed globe is made to communicate by means of air-tight stopeocks alternately with a receiver containing the blood, and with a receiver to collect the gas. When the moveable globe filled with mercury is raised above the fixed one, the mercury from the former runs into and completely fills the latter, the air previously present being driven out. After adjusting the cocks, the moveable globe is then depressed thirty inches below the fixed one, in which the consequent fall of the mereury produces an almost complete vacuum. By turning the proper cock this vacuum is put into connection with the receiver containing the blood, which thereupon becomes proportionately exhausted. By again adjusting the cocks and once more elevating the moveable globe, the gas thus extracted is driven out of the fixed globe into a receiver. The vacuum is then once more established and the operation repeated as long as gas continues to be given off from the blood.

A modified form of pump working on the same principles as that of Ludwig, but involving the use of only one globe to be made vacuous and one moveable reservoir for mercury, has been constructed by Pflüger. It presents several advantages over the one just described, the chief being that (i) non-defibrinated blood may be used for the extraction of its gases. (ii) the vacuum into which the gases are evolved is large, (iii) this vacuum is kept dry by being connected laterally with a vacuous chamber containing sulphuric acid. The details of its construction are however complicated. and the greatest care is required in its use to avoid breakage. Of later years a simplified form of pump has been introduced for laboratory work. It was first used by Gréhant and Paul Bert, and is now frequently called an Alvergniat's pump, from the name of its present maker. Fig. $S S$ gives a diagrammatic representation of its construction.

$A$ is a glass bulb some five inches in diameter, blown on to a glass tube $a$ below and on to a vertical tube $b$ above. The lower end of $a$ is connected by a thick-walled india-rubber, tube with a reservoir for mercury $B$, which ean be raised and lowered by means of a string passing over a pulley $c$. The vertical tube $b$ is thickened at one place. and into this thickened portion a three-way tap $d$ is ground. The upper end of $b$ is prolonged (above the three-way tap) into a fine point. This point passes by a tight joint through the bottom of a vessel $e$, which can be partly filled with mercury, and over which a receiver $f$. filled with mercury for the collection of the gases, can be inverted. A tube $g$ fused on laterally to one opening of the three-way tap $d$ places the latter in connection with a thickwalled Woulff's bottle $C$ containing a layer of strong sulphuric acid. The second tubulure of this bottle is similarly connected by an elastic tube with the vessel $D$, into which blood or other fluid may be introduced by means of the tap $h$. All the moveable joints of the apparatus are protected by india-rubber tubes into which water 
can be poured, and a metal casing round the tap $d$, which may also be filled with water, similarly prevents the possibility of any leakage here.

The pump is used as follows. By placing the tap $d$ in the position shewn in the figure and raising $B$, the bulb $A$ may be filled with

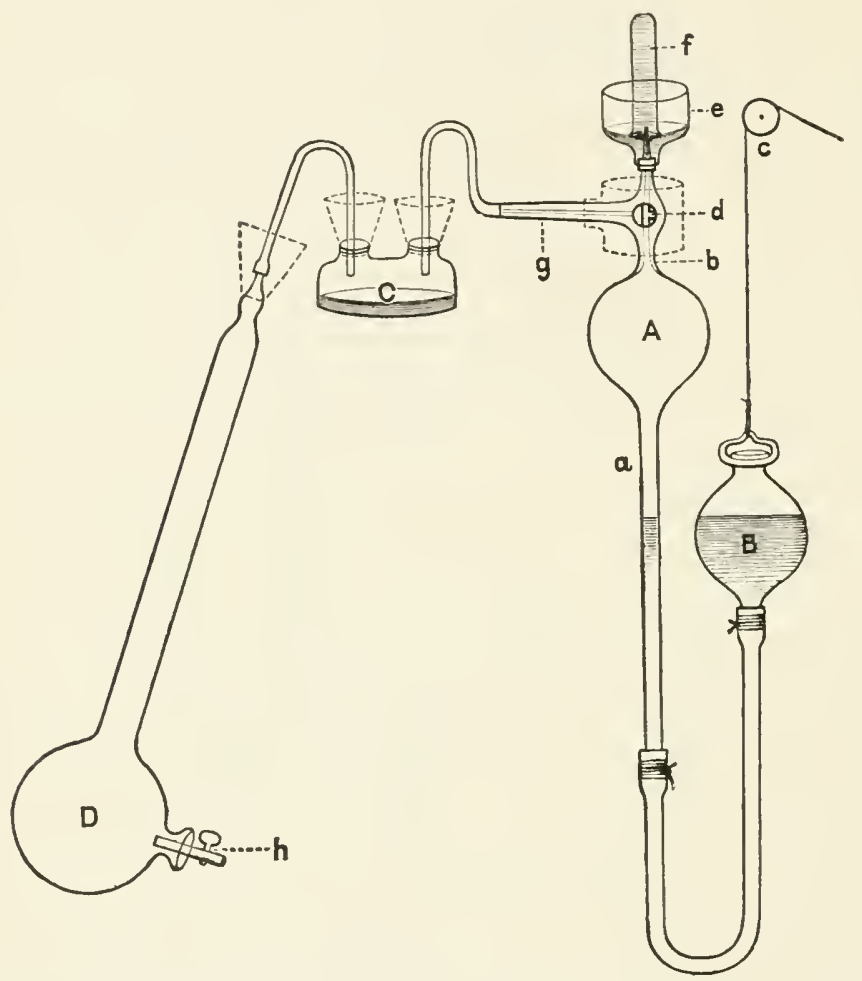

Fig. 88. Diagram of Alvergniat's Pump.

mercury up to the top, the contained air being expelled through the upper end of $b$. By a slight turn of the tap all connection between $A$ and either the tube $g$ or the upper part of $b$ may be cut off, and on lowering $B$ a vacuum is established in the bulb $A$ and part of the tube $a$. A may now be comnected by the tap $d$ with the tube $g$, and hence with $C$ and $D$, and, $h$ being closed. a partial vacuum is established in $C$ and $D$. By ineans of the tap $d$ the air in $A$ may be cut off from $g$, and on raising $B$ and placing the plug of $d$ as shewn in the figure this air may be expelled through the upper end of $b$. By slightly turning $d$ and lowering $B$ a vacuum is again established in $A$, and as before a further portion of air in $C$ and $D$ may be allowed to pass over into $A$ and the racuum in $D$ and $C$ increased. In this way all the air in $D$ can be extracted, the final stages being facili- 
tated by the admission of a little water into $D$, the last traces of air being driven over into $A$ by the rush of vapour from the water. A known volume of blood having been collected over mercury in a small tube is now allowed to enter $D$ through the tap $h$ and yields up its gases to the vacuum. A repetition of the processes by which the air in $D$ was originally extracted will now remove the gases which have been given off from the known volume of blood, the only difference being that now the tube $f$ filled with mercury is inverted in the trongh $e$ over the upper end of the tube $b$. In this way the gases originally in $D$ are not allowed to escape into the air, as was the case when the apparatus was being originally made vacuous, but are collected in $f$ for subsequent analysis. During the extraction of the gases from the blood the bulb $D$ is immersed in a vessel of warm water, to facilitate the exit of the gases and, by causing the formation of large quantities of aqueous vapour, to sweep the gases rapidly over into $A$. The sulphuric acid chamber $C$ dries the vacuum before the admission of the blood into $D$, and hence makes it more perfect and causes the most complete and rapid evolution of gases from the blood.

The average composition of the gas thus obtained from each of the two kinds of blood (the arterial blood being taken from a large artery, and the venous blood from the right side of the heart) is, stated in round numbers, as follows:

From 100 vols.

Of Arterial Blood, Of Venous Blood,

\section{may be obtained}

Of oxygen, of carbonic acid, 20 vols. $\quad 40$ vols. 8 to 12 vols. 46 vols. all measured at $760 \mathrm{~mm}$. and $0^{\circ} \mathrm{C}$.

of nitrogen.

1 to 2 vols.

1 to 2 vols.

That is to say, venous blood, as compared with arterial blood, contains 8 to 12 p.c. less oxygen and 6 p.c. more carbonic acid. It must be remembered, however, that while arterial blood from whatever artery taken has always nearly the same proportion of gases, or at all events the same amount of oxygen, the amount of oxygen in venous blood, even when taken from the same vein, may vary a good deal, still more so when it is taken from different veins. The reason of this we shall see hereafter.

It will be convenient to consider the relations of each of these gases separately.

\section{The relations of Oxygen in the Blood.}

$\$ 274$. When a liquid such as water is exposed to an atmosphere containing a gas such as oxygen, some of the oxygen will be dissolved in the water, that is to say, will be absorbed from the atmosphere. The quantity which is so absorbed will depend on the pressure of the oxygen in the 
atmosphere above; the greater the pressure of the oxygen, the larger the amount which will be absorbed. If the pressure of the whole atmosphere remain the same, at $760 \mathrm{~mm}$. of mercury for instance (the ordinary atmospheric pressure), the pressure of the oxygen may be increased or diminished by increasing or diminishing the proportion of oxygen in the atmosphere. So that with an atmosphere remaining at any given pressure the quantity of oxygen absorbed will depend on the quantity present in that atmosphere. If on the other hand water, alreaty containing a good cleal of oxygen dissolved in it, be exposed to an atmosphere containing little or no oxygen, the oxygen will escape from the water into the atmosphere. The oxygen, in fact, which is dissolved in the water, like the oxygen in the atmosphere above, stands at a certain pressure, the amount of pressure depending on the quantity dissolved; and when water containing oxygen dissolved in it is exposed to any atmosphere, the result, that is, whether the oxygen escapes from the water into the atmosphere, or passes from the atmosphere into the water, depends on whether the pressure of the oxygen in the water is greater or less than the pressure of the oxygen in the atmosphere. Hence when water is exposed to oxygen, the oxygen either escapes or is absorbed until equilibrium is established between the pressure of the oxygen in the atmosphere above and the pressure of the oxygen in the water below. This result is, as far as mere absorption and escape are concerned, quite independent of what other gases are present in the water or in the atmosphere. Suppose a half-litre of water was lying at the bottom of a two-litre flask, and that the atmosphere in the flask above the water was one-third oxygen; it would make no difference, as far as the absorption of oxygen by the water was concerned, whether the remaining two-thirds of the atmosphere was carbonic acid, or nitrogen, or hydrogen, or whether the space above the water was a vacuum filled to one-third with pure oxygen. Hence it is said that the absorption of any gas depends on the partial pressure of that gas in the atmosphere to which the liquid is exposed. This is true not only of oxygen and water, but of all gases and liquids which do not enter into chemical combination with each other. Different liquids will of course absorb different gases with differing readiness; but, with the same gas and the same liquid, the amount absorbed will depend directly on the partial pressure of the gas in the overlying space. It should be added that the process is much influenced by temperature. Hence, to state the matter generally, the absorption of any gas by any liquid will depend on the nature of the gas, the nature of the liquid, the pressure of the gas, and the temperature at which both stancl.

Now it might be supposed, and indeed was once supposed, 
that the oxygen in the blood was simply dissolved by the blood. If this were so, then the amount of oxygen present in any given quantity of blood exposed to any given atmosphere, ought to rise and fall steadily and regularly as the partial pressure of oxygen in that atmosphere is increased or diminished; the absorption (or escape) of oxygen ought to follow what is known as the Henry-Dalton law of pressures. But this is found not to be the case. If we expose blood containing little or no oxygen to a succession of atmospheres containing increasing quantities of oxygen, we find that at first there is a very rapid absorption of the available oxygen, and then this somewhat suddenly ceases or becomes very small; and if on the other hand we submit arterial blood to successively diminishing pressures, we find that for a long time very little oxygen is given off, and then suddenly the escape becomes very rapid. The absorption of oxygen by blood does not follow the general law of absorption according to pressure. 'The phenomena on the other hand suggest the idea that the oxygen in the blood is in some particular combination with a substance or some substances present in the blood, the combination being of such a kind that it holds good during a lowering of pressure down to a certain limit, and that then dissociation readily occurs; we may add that this limit is very closely dependent on temperature. It is, however, not to be supposed that as the pressure is lowered, no oxygen whatever is given off from the substance until a certain point is reached, and that at that point the whole store is in an instant dissociated, no more remaining to be given off. The case is rather that while pressure is being lowered down to a certain point, no appreciable dissociation takes place, and that then having begun it increases rapidly with each further lowering of pressure until the whole of the oxygen is given off. During this narrow range, between the first beginning to give off oxygen and the completion of the giving off, the compound of the oxygen with the substance or substances may be spoken of as partly, that is more or less, dissociated. What is the substance or what are the substances with which the oxygen is thus peculiarly combined?

If serum, free from red corpuscles, be used in such absorption experiments, it is found that, as compared with the entire blood, very little oxygen is absorbed, about as much as would be absorbed by the same quantity of water; and such as is absorbed does follow the law of pressures. In natural arterial blood the quantity of oxygen which can be obtained from serum is exceedingly small; it does not amount to half a volume in one hundred volumes of the entire blood to which the serum belonged. It is evident that the oxygen which is present in blood is in some way or other peculiarly connected with the red corpuscles. Now the distinguishing feature of the red corpuscles is the 
presence of hamoglobin. We have already seen $(\$ 24)$ that this constitutes 90 per cent. of the dried red corpuscles. 'There can be a priori little doubt that this must be the substance with which the oxygen is associated; and to the properties of this body we must therefore direct our attention.

$\$ 275$. Homoglobin. When separated from the other constituents of the serum, hamoglobin appears as a substance, either amorphous or erystalline, readily soluble in water (especially in warm water) and in serum.

Since hæmoglobin is soluble in serum, and since the identity of the crystals observed occasionally within the corpuscles with those obtained in other ways shews that the hæmoglobin as it exists in the corpuscle is the same thing as that which is artificially prepared from blood, it is evident that some peculiar relationship between the stroma and the hæmoglobin must, in natural blood, keep the latter from being dissolved by the serum. Hence in preparing hæmoglobin it is necessary first of all to break up this connection and to set the hæmoglobin free from the corpuscles. This may be done by the addition of water, of ether, of chloroform or of bile salts, or by repeatedly freezing and thawing; blood so treated becomes 'laky,' cf. $\$ 2 t$. It is also of advantage previously to remove the alkaline serum as much as possible so as to operate only on the red corpuseles. The stroma and hæmoglobin being thus separated, a solution of hæmoglobin is the result. The alkalinity of the solution, when present, being reduced by the cautious addition of dilute acetic acid, and the solvent power of the aqueous medium being diminished by the addition of one-fourth its bulk of alcohol, the mixture, set aside in a temperature of $0^{\circ} \mathrm{C}$. in order still further to reduce the solubility of the hæmoglobin, readily crystallizes, when the blood used is that of the dog, cat, horse, rat, guinea-pig, \&c. In the case of the dog indeed it is simply sufficient to add ether carefully to the blood until it just becomes 'laky,' and then to let it stand in a cool place; the mixture soon becomes a mass of crystals. The crystals may be separated by filtration, redissolved in water and recrystallized.

Hremoglobin from the blood of the rat, guinea-pig, squirrel, hedgehog, horse, cat, dog, goose, and some other animals, crystallizes readily, the crystals being generally slender four-sided prisms, belonging to the rhombic system, and often appearing quite acicular. The crystals from the blood of the guinea-pig are octahedral, but also belong to the rhombic system; those of the squirrel are six-sided plates. The blood of the ox, sheep, rabbit, pig, and man, erystallizes with difficulty. Why these differences exist is not known; but the crystals obtained from different animals differ both in percentage composition and in the amount of water of crystallization. In the dog, the percentage composition of the erystals has been determined as C. 53.85, H. 7.32, N. $16 \cdot 17$, O. $21 \cdot 84$, S. 0.39, Fe. $\cdot 43$, with 3 
to 4 per cent. of water of crystallization. It will thus be seen that hæmoglobin contains, in addition to the other elements usually present in proteid substances, a certain amount of iron ; that is to say, the element iron is a distinct part of the hemoglobin molecule: a fact which of itself renders hæmoglobin remarkable among the chemical substances present in the animal body.

$\$ 276$. The crystals, when seen in a sufficiently thick layer under the microscope, have the same bright scarlet colour as arterial blood has to the naked eye; when seen in a mass they naturally appear darker. An aqueous solution of hemoglobin, obtained by dissolving purified erystals in distilled water, has also the same bright arterial colour. A tolerably dilute solution placed before the spectroscope is found to absorb certain rays of light in a peculiar and characteristic manner. A portion of the red end of the spectrum is absorbed, as is also a much larger portion of the blue end ; but what is most striking is the presence of two strongly marked absorption bands, lying between the solar lines D and E. (See Fig. 89.) Of these the one towards the red side, sometimes spoken of as the band $a$, is the thinnest, but the most intense, and in extremely dilute solutions (Fig. 89, 1) is the only one visible; its middle lies at some little distance to the blue side of $\mathrm{D}$. Its position may be more exactly defined by expressing it in wave-lengths. As is well known the rays of light which make up the spectrum differ in the length of their waves, diminishing from the red end, where the waves are longest, to the blue end, where they are shortest. Thus Fraunhofer's line D corresponds to rays having a wavelength of 589.4 millionths of a millimeter. Using the same unit, the centre of this absorption band $a$ of hæmoglobin corresponds to the wave-line 578; as may be seen in Fig. 89, where however the numbers of the divisions of the scale indicate only 100,000 of a millimeter. The other, sometimes called $\beta$, much broader, lies a little to the red side of $\mathrm{E}$, its blueward edge, even in moderately dilute solutions (Fig. 89, 2) coming close up to that line; its centre corresponds to about wave-length 539. Each band is thickest in the middle, and gradually thins away at the edges. These two absorption bands are extremely characteristic of a solution of hemoglobin. Even in very dilute solutions both bands are visible (they may be seen in a thickness of $1 \mathrm{c.m}$. in a solution containing $1 \mathrm{grm}$. of hamoglobin in 10 litres of water), and that when scarcely any of the extreme red end, and very little of the blue end, is eut off. They then appear not only faint but narrow. As the strength of the solution is increased, the bands broaden, and become more intense ; at the same time both the red end, and still more the blue end, of the whole spectrum, are encroached upon (Fig. 89, 3). This may go on until the two absorption bands become fused together 


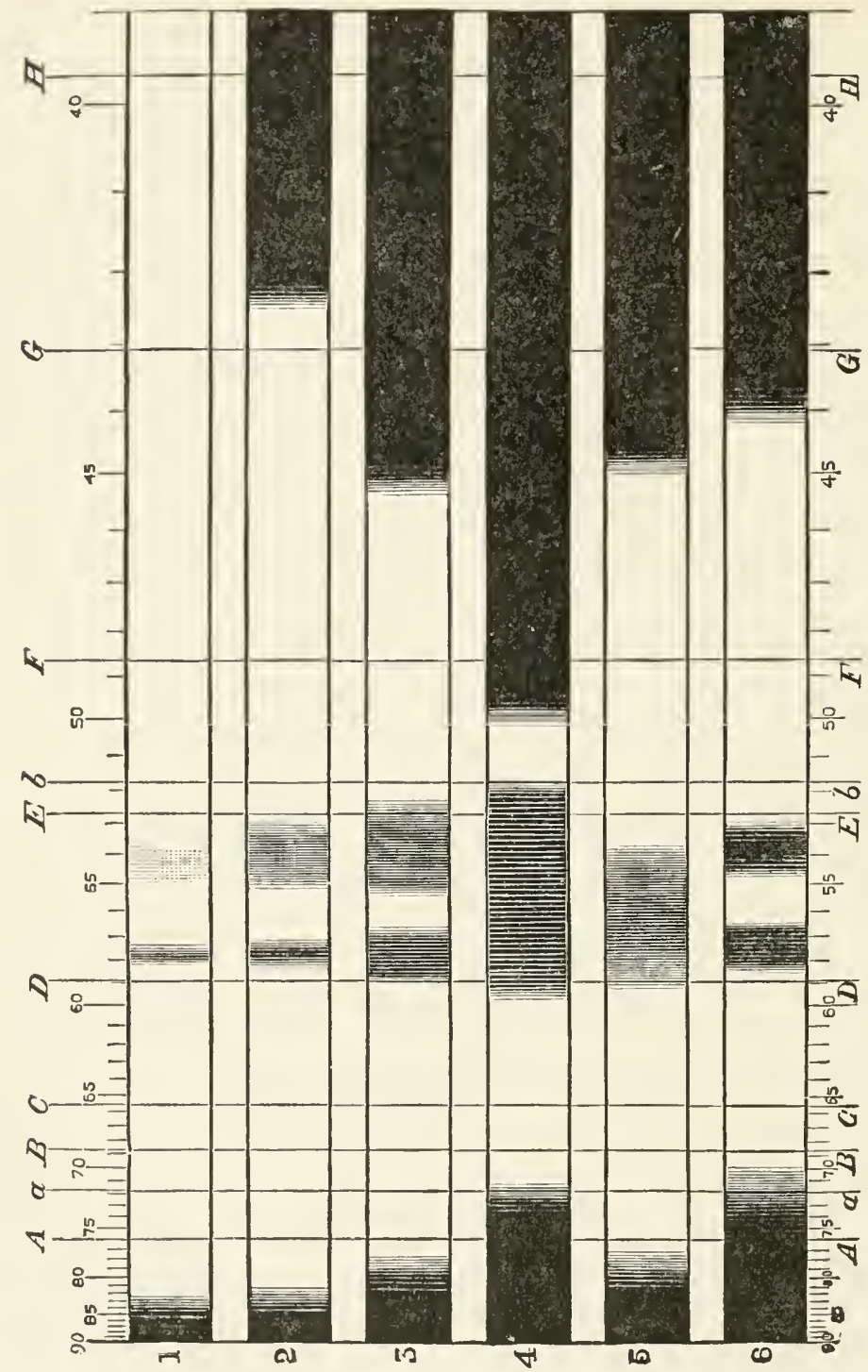

Fig. 89. (After Preyer and Gamgee.) The Spectra of Oxy-Hamoglobin in DIFFERENT (aRADES OF CONCENTRATION, OF (REDUCED) HAMOgLOBIN AND OF Carbonic-Oxide-HLmoglobin.

1 to 4. Solution of Oxy-Hæmoglobin containing (1) less than .01 p.c., (2) 09 p.c., (3) .37 p.c., (4) .8 p.c.

5. $\quad " \quad$ (reduced) Hremnglobin containing about $\cdot 2$ p.c.

6. " " carbonic-oxide-Hæmoglobin.

In each of the six cases the layer brought before the spectroscope was $1 \mathrm{c.m}$. in thickness. The letters ( $A, a$ \&c.) inclicate Fraunhofer's lines, and the figures wave-lengths expressed in 100,000 th of a millimeter. 
into one broad band (Fig. 89, 4). 'The only rays of light which then pass through the hamoglobin solution are those in the green between the blueward edge of the mnited bands and the general absorption which is now rapidly advancing from the blue end, and those in the red between the united bands and the general absorption at the red end. If the solution be still further increased in strength, the interval on the blue side of the united bands becomes absorbed also, so that the only rays which pass through are the red rays lying to the red side of D; these are the last to disappear, and hence the natural red colour of the solution as seen by transmitted light. Exactly the same appearances are seen when crystals of hemoglobin are examined with a microspectroscope. 'They are also seen when arterial blood itself (diluted with saline solutions so that the corpuscles remain in as natural a condition as possible) is examined with the spectroscope, as well as when a drop of blood, which from the necessary exposure to air is always arterial, is examined with the microspectroscope. In fact, the spectrum of hrmoglobin is the spectrum of normal arterial blood.

$\$ 277$. When crystals of hremoglobin, prepared in the way described above, are subjected to the vacuum of the mercurial air-pump, they give off a certain quantity of oxygen, and at the same time they change in colour. In other words, the crystals of hremoglobin, over and above the oxygen which enter's intimately into the composition of the molecule (and which alone is given in the elementary composition previously stated), contain another quantity of oxygen, which is in loose combination only, and which may be dissociated fiom them by subjecting them to a sufficiently low pressure. The change of colour which ensues when this loosely combined oxygen is removed, is characteristic; the crystals become darker and more of a purple hue, and at the same time dichroic, so that while the thicker ridges are purple, the thin edges appear greenish. The quantity of oxygen given off is said to be definite; thus $1 \mathrm{grm}$. of the crystals of dog's blood gives off $1.59 \mathrm{c.cm}$. of oxygen measured at $760 \mathrm{~mm}$. Hg and $0^{\circ} \mathrm{C}$.; but there are some reasons for thinking that even in the same blood the quantity may vary.

An ordinary solution of hamoglobin, like the erystals from which it is formed, contains a definite quantity of oxygen in a similarly peculiar loose combination; this oxygen it also gives up when subjected in the air-pump to a sufficiently low pressure, becoming at the same time of a purplish hue. This loosely combined oxygen may also be removed by passing a stream of hydrogen or other indifferent gas through the solution; the stream of hydrogen acts like an oxygen-vacumm to the hamoglobin and thus dissociation is effected. Carbonic acid gas is unsuitable for this purpose. since, as we shall see, being an aeid it acts in another way on the hiemoglobin. 'The oxygen may 
also be removed from the hæmoglobin not only by physical but also by chemical means, as by the use of reducing agents. 'Thus if a few drops of ammonium sulphide or of an alkaline solution of ferrous sulphate, kept from precipitation by the presence of tartaric acid, be added to a solution of hemoglobin, or even to an unpurified solution of blood corpuscles such as is afforded by the washings from a blood clot, the oxygen in loose combination with the hrmoglobin is immediately seized upon by the reducing agent. This may be recognized at once, by the characteristic change of colour; from a bright scarlet the solution becomes of a purplish claret colour, when seen in any thickness, but greenish when sufficiently thin: the colour of the reduced solution is exactly like that of the crystals from which the loose oxygen has been removed by the air-pump.

Examined by the spectroscope, this reduced solution, or solution of reduced homoglobin, as we may now call it, offers a spectrum (Fig. 89, 5) very different from that of the unreduced solution.

The two absorption bands have disappeared, and in their place there is seen a single, much broader, but at the same time much fainter band, whose middlc occupies a position about midway between the two absorption bands of the unreduced solution, though the redward edge of the band shades away rather farther towards the red than does the other edge towards the blue; its centre corresponds to about wave-length 555. At the same time the general absorption of the spectrum is different from that of the unreduced solution; less of the blue end is absorbed. Even when the solutions become tolerably concentrated, many of the bluish-green rays to the blue side of the single band still pass through. Hence the difference in colour between hremoglobin which retains the loosely combined oxygen, ${ }^{1}$ and hamoglobin which has lost its oxygen and become reduced. In tolerably concentrated solutions, or tolerably thick layers, the former lets through the red and the orange-yellow rays, the latter the red and the bluish-green rays. Accordingly, the one appears scarlet, the other purple. In dilute solutions, or in a thin layer, the reduced hamoglobin lets through so much of the green rays that they preponderatc over the red, and the resulting impression is one of green. In the unreduced hemoglobin or oxyhremoglobin, the potent yellow which is blocked out in the reduced hremoglobin, makes itself felt, so that a very thin laycr of oxyhemoglobin, as in a single corpriscle seen under the microscope, appears yellow rather than red.

It must be remembered that when we speak of reduced

1 For brevity's sake we may call the hrmoglobin containing oxygen in loose combination, oxyhernoglobin, and the hrmoghobin from which this loosely combined oxygen has been removed, reduced hæmoglobin or simply hemoglobin. 
hamoglobin (or more briefly hæmoglobin), with a purple colour and a characteristic onebanded spectrum, we mean hemoglobin which has lost all its loosely associated oxygen. If a quantity of oxyhamoglobin be exposed to an insufficiently low pressure, or to the action of an insufficient quantity of the reducing action, it gives up a part only of its oxygen; it is only partly reduced. Such a partly reduced solution still shews the two bands of oxyhremoglobin.

$\$ 278$. When the hemoglobin solution (or erystal) which has lost its oxygen by the action either of the air-pump or of a reducing agent or by the passage of an indifferent gas, is exposed to air containing oxygen, an absorption of oxygen at once takes place. If sufficient oxygen be present, the hamoglobin seizes upon sufficient oxygen to obtain its full complement, each gramme taking up in combination $1.59 \mathrm{c.cm}$. of oxygen; if there be an insufficient quantity of oxygen the hæmoglobin still remains partly reduced; or perhaps we may say that a part only of the hæmoglobin gets its allowance while the remainder continues reduced. If the amount of oxygen be sufficient, the solution (or crystal), as it takes up the oxygen, regains its bright scarlet colour and its characteristic absorption spectrum, the single band being replaced by the two. Thus if a solution of oxyhæmoglobin in a test-tube, after being reduced by the action of a drop or two of ammonium sulphide solution and thus shewing the purple colour and the single band, be shaken up with air, the bright scarlet colour at once returns, and when the fluid is placed before the spectroseope, it is seen that the single faint broad band of the reduced hrmoglobin has wholly disappeared, and that in its place are the two sharp thimner bands of the oxyhæmoglobin. If left to stand in the test-tube the quantity of reducing agent still present is generally sufficient again to rob the hemoglobin of the oxygen thus newly acquired, and soon the scarlet hue fades back again into the purple, the two bands giving place to the one. Another shake and exposure to air will however again bring back the scarlet hue and the two bands; and once more these may disappear. In fact, a few drops of the reducing fluid will allow this game of hremoglobin taking oxygen from the air and giving it up to the reducer to be played over and over again; at each turn of the game the colour shifts from scarlet to purple, and from purple to searlet, while the two bands exchange for the one, and the one for the two.

$\$ 279$. Colour of Tenous and Arterial Blood. Evidently we have in these properties of hamoglobin an explanation of at least one-half of the great respiratory process, and they teach us the meaning of the change of colour which takes place when venous blood becomes arterial or arterial venous.

In venous blood, as it issues from the right ventricle, the 
oxvgen present is insuffieient to satisfy wholly the himoglobin of the red corpuseles; the hiemoglobin is, to a large extent, rednced. hence the purple colour of venous blood. W' hen ordinary venous blood, diluted without aecess of oxygen, is bronght before the spectroscope, the two bands of oxyhiemoglobin are seen. This is explained by the fact that in partly reduced hemoglobin, which we may conveniently regard as a mixture of oxyhemoglobin and (reduced) haemoglobin, the two sharp bands of the former are always much more readily seen than the much fainter band of the latter. Now in ordinary renous blood there is always some loose oxygen, removable by diminished pressure or otherwise; the hiemoglobin is only partly reduced. there is always some, indeed a considerable quantity, of oxyhamoglobin as well as (rednced) hemoglobin. It is only under special circumstances, as for instance after death by what we shall presently speak of as asphyxia, that all the loose oxygen of the blood disappears; and then the two bands of the oxyhamoglobin vanish too. If even only a small quantity of oxygen be present so distinct are the two bands that a solution of completely reduced hemoglobin may be used as a test for the presence of oxygen: if oxygen be present in any fluid to which the reduced liemoglobin is added, the single band immediately gives way to the two bands of oxyhremoglobin.

As the venous blood passes through the capillaries of the lungs, this reduced hremoglobin takes from the pulmonary air its complement of oxygen, all or nearly all the hamoglobin of the red eorpuscles becomes oxyhæmoglobin, and the purple colour forthwith shifts into searlet. For careful observitions shew that the hremoglobin of arterial blood is saturated or nearly saturated with oxygen, it probably falls short of complete saturation by about 1 rol. of oxygen in 100 vols. of blood. by inereasing the pressure of the oxygen, an additional quantity mary be driven into the blood, but this, after the hamoglobin has become completely saturated, is effected by simple alsorption. The quantity so added is extremely small compared with the total quantity combined with the hromoglobin.

Passing from the left ventricle to the capillaries of the tis. snes the oxyhremoglobin gives up some of its oxygen to the tissues, becoming, in part, reduced hemoglobin, and the blood in eonsequence becomes once more venons, with a purple hue. 'Thus the red corpuseles by virtue of their hamoglobin are em phatically oxygen-carriers. Undergoing no intrinsic change in itself, the hamoglolin combines in the lungs with oxygen, which it earries to the tissues; these, more greedy of oxygen than itself, rob it of its charge, and the rednced hiemoglobin hurries back to the lungs in the venous blood for another portion. 'The ehange from venous to arterial blood is then in part (for as we shall see there are other events as well) a peeulian combination 
of hamoglobin with oxygen, while the change from arterial to venous is, in part also, a reduction of oxyhæmoglobin : and the difference of colour between venous and arterial blood depends almost entirely on the fact that the reduced hrmoglobin of the former is of purple colour, while the oxyhiemoglobin of the latter is of a scarlet colour.

There may be other causes of the change of colour, but these are wholly subsidiary and unimportant. When a corpuscle swells, its refractive power is diminished, and in consequence the number of rays which pass into and are absorbed by it are increased at the expense of those reflected from its surface; anything therefore which swells the corpuscles, such as the addition of water, tends to darken blood, and anything, such as a concentrated saline solution. which causes the corpuscles to shrink, tends to brighten blood. Carbonic acid has apparently some influence in swelling the corpuscles, and therefore may aid in darkening the venous blood.

$\$ 280$. We have spoken of the combination of hrmoglobin with oxygen as being a peculiar one. The peculiarity consists in the facts that the oxygen may be associated and dissociated, without any general disturbance of the molecule of hæmoglobin, and that dissociation may be brought about very readily. Hæmoglobin combines in a wholly similar manner with other gases. If carbonic oxide (monoxicle) be passed through a solution of hamoglobin, a change of colour takes place, a peculiar bluish tinge making its appearance. At the same time the spectrum is altered; two bands are still visible, but on accurate measurement it is seen that they are placed more towards the blue end than are the otherwise similar bands of oxyhemoglobin (see Fig. 89,6), their centres corresponding respectively to about wave-lengths 572 and 533, while those of oxyhrmoglobin as we have seen correspond to 578 and 539 . When a known quantity of carbonic oxide gas is sent through a hemoglobin solution, it will be found on examination that a certain amount of the gas has been retained, an equal volume of oxygen appear. ing in its place in the gas which issues from the solution. If the solution so treated be crystallized, the crystals will have the same characteristic colour, and give the same absorption spec. trum as the solution; when subjected to the action of the mercurial pump, they will give off a definite quantity of earbonic oxide, $1 \mathrm{grm}$. of the erystals yielding $1.59 \mathrm{c} . \mathrm{cm}$. of the gas. In fact, hemoglobin combines loosely with carbonic oxide just as it does with oxygen; but its affinity with the former is greater than with the latter. While carbonic oxide readily turns out oxygen, oxygen cannot so readily turn out carbonic oxide. Indeed, carbonic oxide has been used as a means of driving out and measuring the quantity of oxygen present in any given blood. This property of carbonic oxide explains its poisonous 
nature. When the gas is breathed, the reduced and the unreduced hrmoglobin of the venous blood unite with the carbonic oxide, and hence the peculiar bright cherry-red colour observable in the blood and tissues in cases of poisoning by this gas. The carbonic oxide hæmoglobin, however, is of no use in respiration; it is not an oxygen-carrier, nay more, it will not readily, though it does so slowly and eventually, give up its carbonic oxide for oxygen, when the poisonous gas ceases to enter the chest and is replaced by pure air. The organism is killed by suffocation, by want of oxygen, in spite of the blood not assuming any dark venous colour ; to adopt a phrase which has been used, the corpuscles are paralyzed.

Hemoglobin similarly forms a compound, having a characteristic spectrum, with nitric oxide, more stable even than that with carbonic oxide.

It has been supposed by some that the oxygen thus associated with hæmoglobin is in the condition known as ozone; but the arguments urged in support of this view are not as yet conclusive.

\section{Products of the decomposition of Homoglobin.}

$\S 281$. Although a crystalline body, hemoglobin diffuses with great difficulty. This arises from the fact that it is in part a proteid body ; it consistns of a colourless proteid, associated with a coloured substance, which may be separated out from the hæmoglobin, though not in the exact condition in which it naturally exists in the compound ; this substance when separated out appears as a brownish-red body known as hoematin. All the iron belonging to the hæmoglobin is in reality attached to the hiematin. A solution of hremoglobin, when heated, coagulates, the exact degree at which the coagulation takes place depending on the amount of dilution; at the same time it turns brown from the setting free of the hæmatin. If a strong solution of hamoglobin be treated with acetic (or other) acid, the same brown colour, from the appearance of hamatin, is observed. The proteid constituent however is not coagulated, but by the action of the acid passes into the state of acid-albumin. On adding ether to the mixture, and shaking, the hematin is dissolved in the supernatant acid ether, which it colours a dark red, and which, examined with the spectroscope, is found to possess a well-marked spcetrum, the spectrum of the so-called acid hematin of Stokes (Fig. 90,6). The protcid in the water below the ether appear's in a coagulated form owing to the action of the ether. In a somewhat similar manner alkalis split up humoglobin into a proteid constituent and hematin.

The exact nature of the proteid constituent of hemoglobin has not as yet been clearly determined. It was supposed to be globulin (hence the name hiematoglobulin, contracted into 
Chap. II.]

RESPIRATION.

459
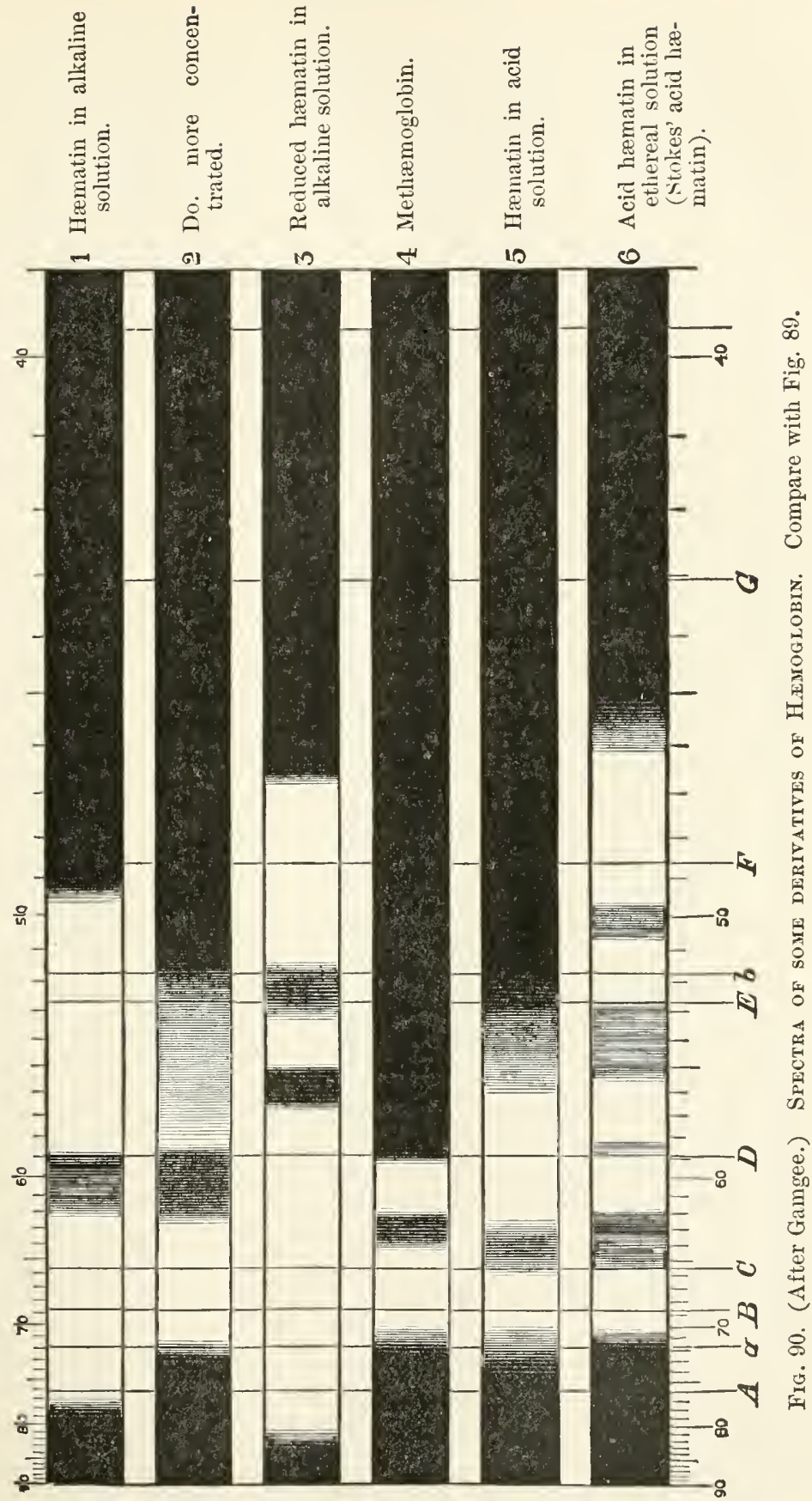
hremoglobin), but though belonging to the globulin family, has characters of its own ; it is possibly a mixture of two or more distinct proteids. It has been provisionally named globin and is said to be free from ash.

$\$ 282$. Hiematin when separated from its proteid fellow, and purified, appears as a dark-brown amorphous powder, or as a sealy mass with a metallic lustre, having the probable composition of $\mathrm{C}_{32}, \mathrm{H}_{34}, \mathrm{~N}_{4}, \mathrm{Fe}, \mathrm{O}_{5}$. It is fairly soluble in dilute acid or alkaline solutions, and then gives characteristic spectra (Fig. 90, 1, 2, 5).

An interesting feature in hrmatin is that its alkaline solution is capable of being reduced by reducing agents, the spectrum changing at the same time (Fig. 90, 3), and that the reduced solution will. like the hamoglobin, take up oxygen again on being brought into contact with air or oxygen. This would seem to indicate that the oxygen-holding power of hiemoglobin is connected exclusively with its hamatin constituent.

By the action of strong sulphuric acid hematin may be robbed of all its iron. It still retains the feature of possessing colour, the solution of iron-free hematin bcing a dark rich brownish red; but is no longer capable of combining loosely witl oxygen. This indicates that the iron is in some way associated with the peculiar respiratory functions of hamoglobin; though it is obviously an error to suppose, as was once supposed, that the change from venous to arterial blood consists essentially in a change from a ferrous to a ferric salt.

'Though not crystallizable itself, hematin forms with hychrochloric acid a compound, occurring in minute rhombic crystals, known as hemin crystals.

When blood is left until it decomposes, the hremoglobin is very apt to become changed into a peculiar body known as methemoglobin, in the spectrum of which a very conspicuous band is seen in the red between $C$ and D (sce Fig. 90,4). The same change may be brought about by the action of weak acids, such as carbonic acil, by ozone, and by other agents such as nitrites and potassinm permanganate. When a stream of carbonic acid is driven through blood or through a solution of hamoglobin the band in the red characteristic of methamoglobin soon makes its appcarance. Methemoglobin differs but little if at all in elementary composition from hæmoglobin ; it is maintained that it eontains the same quantity of oxygen as oxyhamoglobin but in a more stable condition, more intimatcly associated with the molceule.

In conchnsion, the condition of oxygen in the blood is as follows. Of the whole quantity of oxygen in the blood, only a minute fraction is simply absorbed or dissolved according to the law of pressures (the Henry-Dalton law). The great mass is in a state of combination with the hemoglobin, the connection 
being of such a kind that while the hremoglobin readily combines with the oxygen of the air to which it is exposed, clissociation readily occurs at low pressures, or in the presence of indifferent gases, or by the action of substances having a greater affinity for oxygen than has liemoglobin itself. The difference between venous and arterial blood, as far as oxygen is concerned, is that while in arterial blood the hamoglobin holds nearly its full complement of oxygen and may be spoken of as nearly wholly oxyhrmoglobin, in venous blood the hemoglobin is to a large but variable extent, reduced; and the characteristic colours of venous and arterial blood are in the main due to the fact that the colour of reduced hæmoglobin is purple, while that of oxyhæmoglobin is scarlet.

\section{The relations of the Carbonic Acid in the Blood.}

$\$ 283$. The presence of carbonic acid in the blood appears to be determined by conditions more complex in their nature and at present not so well understood as those which determine the presence of oxygen. The carbonic acid is not simply dissolved in the blood; its absorption by blood does not follow the law of pressures. It exists in association with some substance or substances in the blood, and its escape from the blood is a process of dissociation. We cannot however speak of it as being associated, in the same definite and clear way as is the oxygen, with the hremoglobin of the red corpuseles.

Scveral facts seem to support the view that the carbonic acid exists associated with some substance or substances in the plasma, but at the same time indicate that the conditions of its association (and therefore of its dissociation) are determined by the action of some substance or substances present in the corpuseles. It has been suggested that the association of the carbonic acid in the plasma is with one or other of the proteids of the plasma; but it has also been suggested that the association is one with sodium as sodium bicarbonate, and further that the hamoglobin of the corpuscles plays a part in promoting the dissociation of the sodium bicarbonate or even the carbonate, and thus keeping up the carbonic acid of the entire blood. Other observers however maintain that the plasma does not hold this exclusive possession of the carbonic acid, but that a considerable quantity at least of this gas is in some definite way associated with the red corpuscles. Further investigations are necessary before the matter can be said to have been placed on a satisfactory footing.

\section{The relations of Nitrogen in the Blood.}

$\$ 284$. The small quantity of this gas which is present in both arterial and venous blood seems to exist in a state of simole solution. 


\section{SEC. 4. THE RESPIRATORY CHANGES IN THE LUNGS.}

\$285. The Entrance of Oxygen. We have already seen that the blood in passing through the lungs takes up a certain variable quantity (from 8 to 12 vols. p.c.) of oxygen. W'e have further seen that the quantity so taken up, putting aside the insignificant fraction simply absorbed, enters into direct but loose combination with the limoglobin. In drawing a distinction between the oxygen simply absorbed and that entering into combination with the hremoglobin, it must not be understood that the latter is wholly independent of pressure. On the contrary, all chemical compounds are in various degrees subject to dissociation at certain pressures and temperatures; and the existence of the somewhat loose compound of oxygen and hemoglobin is dependent on the partial pressure of oxygen in the atmosphere to which the hæmoglobin is exposed. Not only will a solution of hæmoglobin or a quantity of blood either absorb oxygen and thus undergo association or undergo dissociation and give off oxygen according as the partial pressure of oxygen in the atmosphere to which it is exposed is high or low, but also the amount taken up or given off will depend on the degree of the partial pressure; the hæmoglobin as we have seen may be either partially or wholly reduced. The law however according to which absorption or escape thus takes place is quite different from that observed in the simple absorption of oxygen by liquids. The association or dissociation is further especially dependent on temperature, a ligh temperature favouring dissociation, so that at a high temperature less oxygen is taken up than would be taken up (or, as the case may be, more given off than would be given off) at a lower temperature, the partial pressure of the oxygen in the atmosphere remaining the same.

Moreover in the blood we have to deal not with hæmoglobin in simple solution, in which the molecules are dispersed uniformly through the solvent, but with the hamoglobin segregated into minute isolated masses, bottled up as it were in the individual corpuscles. The lomoglobin of each corpuscle is 
separated from its fellows by a layer, thin it may be but still a distinct layer, of colourless, hamoglobinless plasma. As the corpuscle makes its way through the narrow capillary paths of a pulmonary alveolus, it is separated from the air of the alveolus by a thin layer of plasma as well as by the film of the conjoined capillary and alveolar walls; and a like layer of plasma separates it from its fellows as it journeys in company with them through the wider passages of the arteries and veins. Through this layer of plasma, which containing no liemoglobin can hold oxygen in simple solution only, the oxygen has to pass on its way to and from the corpuscle; and every corpuscle may be considered as governing, as far as oxygen is concerned, a zone of plasma immediately surrounding itself. The corpuscle takes its oxygen directly from this zone and gives up its oxygen directly to this zone; and the pressure at which at any moment the oxygen exists in this zone will depend on the pressure of oxygen outside the zone, in the air of the pulmonary alveolus for instance, and on the smaller or greater amount of oxygen associated with the hemoglobin of the corpuscle.

The evidence, however, afforded by various experiments, so far as it goes, seems to shew that blood absorbs oxygen in the same way as an aqueous solution of hamoglobin of the same concentration; the zone of plasma spoken of above as surrounding each corpusele seems to behave as far as regards the passage of oxygen to and from the corpuscles in no essentially different respect from the way in which the molecules of water, belonging to a molecule of dissolved hamoglobin, behave in regard to the absorption or the giving-off of oxygen by an aqueous solution of hemoglobin.

The film of the conjoined capillary and alveolar wall is a thin membrane soaked with lymph and wet; we cannot speak of it as actually secreting a liquid secretion into the alveolus, for the cavity of the alreolus is filled with air which, though satmrated with moisture, is air, not a liquid; still enough passes through the film to keep the film continually moist. 'Through this film the oxygen has to make its way in order to gain access to the plasma and so to the corpuscle; it makes its way dissolved in the fluid, that is the lymph, which keeps the film moist. This film moreover is composed of living matter, and the considerations which a little while back (\$253) we urged concerning the diffusion through a living membrane of solic substances in solution, hold good also for the diffusion of gases in solution.

If now we ask the question, Are the conditions in which hamoglobin and oxygen exist in ordinary venous blood as it flows to the lungs, of such a kind that the venous blood in passing through the pulmonary capillaries will find the partial pressure of the oxygen in the pulmonary alveoli sufficient 
through the action of simple physical causes to bring about the association of the additional quantity of oxygen whereby the venous is converted into arterial blood? The reply is as follows.

$\$ 286$. In man, as we have seen, expired air contains abont 16 p.c. of oxygen. The air in the pulmonary alveoli must contain less than this, since the expired air consists of tidal air mixed by diffusion with the stationary air. How much less it contains we do not exactly know, but probably the difference is not very great. At the ordinary atmospheric pressure of $760 \mathrm{~mm} .16$ p.c. is equivalent to a partial pressure of $12.2 \mathrm{~mm}$. The question therefore stands thus, Will venous blood, exposed at the temperature of the body to a partial pressure of less than $122 \mathrm{~mm}$. (less than 16 p.c.) of oxygen take up sufficient oxygen (from 8 to 12 vols. p.c.) to convert it into arterial blood? Numerous experiments have been made (chiefly but not exclusively on the dog) to determine on the one hand the oxygen-pressure of both arterial and venous blood (i.e. the partial pressure of oxygen in an atmosphere exposed to which the arterial blood neither gives up nor takes in oxygen, and the same for venous blood), and on the other hand the behaviour, at the temperature of the body or at ordinary temperatures, of blood towards an atmosphere in which the partial pressure of oxygen is made to vary. Without going into detail, we may state that these experiments seem to shew that the partial pressure of oxygen in the lungs is amply suffieient to bring about, at the temperature of the body, the association of that additional amount of oxygen by which venous blood becomes arterial. When blood is successively exposed to inereasing oxygen pressures, as the partial pressure of oxygen is gradually increased, the curve of absorption rises at first very rapidly but afterwards more slowly; that is to say, the later additions of oxygen at the higher pressures are proportionately less than the earlier ones at the lower pressures. And this is consonant with what appears to be the fact that the hamoglobin of arterial blood though nearly saturated with oxygen, i.e. associated with almost its full complement of oxygen, is not quite saturated. When arterial blood is thoroughly exposed to air it takes up rather more than 1 vol. p.e. of oxygen; and that appears to represent the difference between exposing blood to pure air, such as enters or ought to enter the mouth in inspiration, and exposing blood to the air as it exists in the pulmonary alveoli. 'The greater relative absorption at the lower pressures has a beneficial effect in as much as it still permits a considerable quantity of oxygen to be absorbed even when the partial pressure of oxygen in the air in the lungs is largely rednced, as in ascending to great heights.

Similar observations seem to shew that arterial blood ceases 
to take up oxygen and begins to give off oxygen, in other words, that dissociation begins to take place, when the partial pressure of the oxygen in the atmosphere to which it is exposed sinks to about $60 \mathrm{~mm}$. of mercury, that is to say, when the whole atmospheric pressure is reduced from $760 \mathrm{~mm}$. to about $300 \mathrm{~mm}$. or when the percentage of oxygen in the atmosphere is reduced by decidedly more than half. And this accords with the observation that, in man, when the oxygen of inspired air is gradually diminished, without any other change in the air, symptoms of dyspnoea do not make their appearance until the oxygen sinks to 10 p.c. in the inspired air and must therefore be less than this in the pulmonary alveoli. We may remark that at ordinary altitudes, even taking into account the diminution the oxygen undergoes before it reaches the pulmonary alveoli, the partial pressure of the oxygen in the atmosphere leaves a wide margin of safety. But at an altitude of 5500 metres (1700 feet) at which the pressure of the whole atmosphere stands at about the limit given above of $300 \mathrm{~mm}$., the partial pressure of the oxygen will be such that the venous blood cannot take up the quantity of oxygen proper to convert it into arterial blood, since at this limit arterial blood begins to give off oxygen. We may add that it is at this altitude that breathing becomes especially difficult, but to this we shall return.

$\S 287$. The statements made so far refer to ordinary breathing, but the question may be asked, What happens when the renewal of the air in the pulmonary alveoli ceases, as when the trachea is obstructed? In such a case the oxygen in the alveoli is found to diminish rapidly, so that the partial pressure of oxygen in them soon falls below the oxygen-pressure of ordinary venous blood. But in such a case the blood is no longer ordinary venous blood; instead of being moderately, it is largely and increasingly reduced ; instead of containing a comparatively small amount, it contains a large and gradually increasing amount, of reduced hæmoglobin. And as the reduction continues to increase, the oxygen-pressure of the venous blood also continues to decrease; it thus keeps below that of the air in the lungs. Hence apparently even the last traces of oxygen in the lungs may be taken up by the blood, and carried away to the tissues.

Guided by these observations then, we should be led to conclude that the film of the conjoined pulmonary and capillary wall does not exert any influence, by virtue of its being a living structure, upon the entrance of oxygen into the blood, or indeed exert any influence at all even as a mere membrane or septum; the oxygen appears to pass into the blood in the same way that it would if the blood were freely exposed to the alveolar air without any intervening partition. Nevertheless there are facts which seem to throw doubt on the validity of this conclusion. 
The partial pressure of the oxygen in the gas in the swim-bladder of fishes for instance far exceeds that of the fishes' blood; and if the gas be drawn off, it is soon replaced by gas having the like high partial pressure of oxygen. Hence we are led to conclude that oxygen makes its appearance in the swim-bladder by a kind of secretion. And other facts might be brought forward, strong enough at least to support the doubt, whether the purely physical explanation given above of the entrance of oxygen into the blood, adequate as it at first sight seems, is really the true one.

$\$ 288$. The Exit of Carbonic Acid. In a similar manner analogous experiments appear to support the view that the escape of carbonic acid from the blood into the pulmonary alveolus is the result of ordinary diffusion; observations seem to shew that the difference obtained between the pressure of the carbonic acid in the venous blood and the partial pressure of carbonic acid in the air of the pulmonary alveolus (which is of course greater than that of the expired air) is sufficient to account for the loss of carbonic acid, whereby arterial blood is distinguished from venous blood. But in respect to this as in respect to the entrance of oxygen, doubts have been raised, and it has been urged that the escape of carbonic acid into the pulmonary alveoli is carried out by some action of the walls of the alveoli comparable to the act of secretion. 


\section{SEC. 5. THE RESPIRATORY CHANGES IN THE TISSUES.}

$\$ 289$. In passing through the several tissues the arterial blood becomes once more venous. The oxyhiemoglobin becomes considerably reduced, and a quantity of earbonic acid passes from the tissues into the blood. The amount of change varies in the various tissues, and in the same tissue may vary at different times. Thus in a gland at rest, as we have seen, the venous blood is dark, shewing that the hemoglobin is to a large extent in the reduced condition; when the gland is active, the venous blood in its colour, and in the extent to which the hemoglobin is in the condition of oxyhremoglobin, resembles closely arterial blood. The blood therefore which issues from a gland at rest is more 'venous' than that from an active gland; though owing to the more rapid flow of blood which, as we saw in an earlier section, accompanies the activity of the gland, the total quantity of oxygen taken up from and of carbonic acid discharged into the blood from the gland in a given time may be greater in the latter. The blood, on the other hand, which comes from an active, i.e. a contracting muscle, is, in spite of the more rapid flow, not only richer in carbonic acid, but also, though not to a corresponding amount, poorer in oxygen than the blood which flows from a muscle at rest.

In all these cases the question which first comes up for our consideration is this: Does the oxygen pass from the blood into the tissues, and does the oxidation take place in the tissues, giving rise to carbonic acid, which passes in turn away from the tissues into the blood? or do certain oxidizable reducing substances pass from the tissues into the blood, and there become oxidized into carbonic acid and other products, so that the chief oxidation takes place in the blood itself?

There are. it is true, reducing oxidizable substances in the blood, but these are small in amount, and the quantity of carbonic acid to which they give rise when the blood containing them is agitated with air or oxygen, is so small as scarcely to exceed the errors of observation.

We may add, that the oxidative power which the blood itself removed from the body is able to exert on substances 
which are undoubtedly oxidized in the body is so small that it may be neglected in the present considerations. If grape-sugar be added to blood, or to a solution of hæmoglobin, the mixture may be kept for a long time at the temperature of the body, without undergoing oxidation. Even within the body an even slight excess of sugar in the blood over a certain percentage wholly escapes oxidation, and is discharged unchanged.

On the other hand, it will be remembered that in speaking of muscle, we drew attention $(\$ 58)$ to the fact that a frog's muscle removed from the body (and the same is true of the muscles of other animals) contains no free oxygen whatever; none can be obtained from it by the mercurial air-pump. Yet such a muscle will not only when at rest go on producing and discharging a certain quantity, but also when it contracts evolve a very considerable quantity, of carbonic acid. Moreover this discharge of carbonic acid will go on for a certain time in muscles under circumstances in which it is impossible for them to obtain oxygen from without. Oxygen, it is true, is necessary for the life of the muscle: when venous instead of arterial blood is sent through the blood vessels of a muscle, the irritability speedily disappears, and unless fresh oxygen be administered the nuscle soon dies. The muscle may however, during the interval in which irritability is still retained after the supply of oxygen has been cut off, continue to contract vigorously. The supply of oxygen, though necessary for the maintenance of irritability, is not necessary for the manifestation of that irritability, is not necessary for that explosive decomposition which develops a contraction. A frog's muscle will continue to contract and to produce carbonic acid in an atmosphere of hydrogen or nitrogen, that is, in the total absence of free oxygen both from itself and from the medium in which it is placed.

Thus on the one hand the muscle seems to have the property of taking up and fixing in some way or other the oxygen to which it is exposed, of storing it up in its own substance in such a condition that it cannot be removed by simple diminished pressure (so that the pressure of oxygen in the muscular substance may be considered as always nil), and yet has not entered into any distinct combination which we can speak of as an oxidation, but is still available for such a purpose. On the other hand the muscular substance is always undergoing a decomposition of such a kind that carbonic acid is set free, sometimes, as when the muscle is at rest, in small, sometimes, as during a contraction, in large quantities. The oxygen present in this carbonic acid, as an oxidation product, comes from the previously existing store of which we have just spoken. 'The oxygen taken in by the muscle, whatcver be its exact condition immediately upon its entrance into the muscular substance, sooner or later enters into a combination, or perhaps we should rather say, 
enters into a series of combinations. We have previously urged $(\$ 30)$ that all living substance may be regarded as incessantly undergoing changes of a double kind, changes of building up and changes of breaking down. In the end-products of the breaking down, in the carbonic acid given out by muscle for instance, we can recognize an oxidation rroduct; but we do not know exactly at what stage or exactly in what way the oxygen is combined with the carbon. IVe may imagine that the oxygen, as it comes from the blood, is caught up so to speak by, and disappears in, the building up processes, and that through those processes it is made part of complex decomposable substances whose decomposition ultimately gives rise to the carbonic acid; but, so far as actual knowledge goes, we cannot as yet trace out the steps taken by the oxygen from the moment it slips from the blood into the muscular substance to the moment when it issues united with carbon as carbonic acid.

But if the oxygen-pressure of the muscular tissue be thus always nil, oxygen will be always passing over from the bloodcorpuscles, in which it is at a comparatively high pressure, through the plasma, through the capillary walls, the lymphspaces and the sarcolemma, into the nuscular substance, and as soon as it arrives there will be in some manner or other hidden away, leaving the oxygen-pressure of the muscular substance once more nil. Conversely, the carbonic acid produced by the decomposition of the muscular substance will tend to raise the carbonic acid pressure of the muscle until it exceeds that of the blood; whereupon carbonic acid will pass from the muscle into the blood, its place in the muscular substance being supplied by freshly generated supplies. There will always in fact be a stream of oxygen from the blood to the muscle and of carbonic acid from the muscle to the blood. The respiration of the muscle then does not consist in throwing into the blood oxidizable substances, there to be oxidized into carbonic acid and other matters; but it does consist in the assumption and storing up of oxygen somehow or other in its substance, in the building up by help of that oxygen of explosive decomposable substances, and in the carrying out of decompositions whereby carbonic acid and other matters are discharged first into the substance of the muscle and subsequently into the blood.

$\$ 290$. Our knowledge of the respiratory changes in muscle is more complete than in the case of any other tissue; but we have no reason to suppose that the phenomena of muscle are exceptional. On the contrary, all the available evidence goes to shew that in all tissues the oxidation takes place in the tissue, and not in the adjoining blood. It is a remarkable fact, that lymph, serous fluids, bile, urine, and milk contain a mere trace of free or loosely combined oxygen, but a very consider- 
able quantity of carbonic acid. And we may probably assert with safety with regard to all the tissues that in the tissues themselves, in the lymph which bathes their lymph-spaces, and in the secretions which some of them pour forth free oxygen is either wholly absent or so seanty that their oxygen-pressure may be regarded as nil, while carbonic acid is so abundant that the pressure of carbonic acid in them may be regarded as exceeding that of renous blood. An exception seems to be presented by the case of the lymph flowing along the larger lymphatic vessels, for in this the amount of carbonic acid, while usually higher than that of arterial blood, is lower than that of the general venous blood; but this probably is due to the fact that the lymph in its passage onwards is largely exposed to arterial blood in the connective tissues and in the lymphatic glands, where the production of carbonic acid is slight as compared to that going on in mnseles. All the facts point to the conclusion, that it is the tissues, and not the blood, which become primarily loaded with carbonic acid, the latter simply receiving the gas from the former by diffusion, except the (probably) small quantity which results from the metabolism of the bloodcorpuscles; and that the oxygen which passes from the blood into the tissues is at once taken up and placed under such conditions that it is no longer removable by diminished pressure.

It was shewn long ago that animals might continue to breathe out carbonic acid in an atmosphere of nitrogen or hydrogen; and this is further illustrated by the experiment, that a frog kept at a low temperature will live for several hours, and continue to produce carbonic acid, in an atmosphere absolutely free from oxygen. The carbonic acid produced during this period was made by help of the oxygen inspired in the hours anterior to the commencement of the experiment. 'The oxygen then absorbed was stowed away from the hemoglobin into the tissues, it was matle use of to build up the explosive compounds, whose explosions later on gave rise to the earbonic acid. Or, to arlopt a simile which has been suggesterl. the oxygen helps to wind up the vital clock; but once wound up the clock will go on for a period without further winding. The frog will continue to live, to move, to produce carbonic acid for a while without any fresh oxygen, as we know of old it will without any fresh food; it will continue to do so till the explosive compounds which the oxygen built 1 p are exhatsted ; it will go on till the vital elock has rum down.

$\$ 291$. To sum up, then, the results of respiration in its chemical aspects. As the blood passes through the lungs, the low oxygen-pressure of the venous blood permits the entrance of oxygen from the air of the pulmonary alveolus. through the thin alveolar wall, through the thin capillary sheath, through the thin layer of blood-platsmat. to the red corpusele. and the 
reduced hamoglobin of the venous blood becomes wholly, or all but wholly, oxyhremoglobin. Hurried to the tissues, the oxygen, at comparatively high pressure in the arterial blood, passes largely into them. In the tissues, the oxygen-pressure is always kept at an exceedingly low pitch, by the fact that they, in some way at present unknown to us, pack away at every moment into some stable combination each molecule of oxygen which they receive from the blood. With its oxyhremoglobin largely but not wholly reduced, the blood passes on as venous blood. To what extent the hemoglobin is reduced will depend on the activity of the tissue itself. The quantity of hiemoglobin in the blood is the measure of limit of the oxidizing power of the body at large; but within that limit the amount of oxidation is determined by the tissue, and by the tissue alone.

We cannot trace the oxygen through its sojourn in the tissue. We only know that sooner or later it comes back combined in carbonic acid (and other matters not now under consideration). Owing to the continual production of carbonic acid, the pressure of that gas in the extravascular elements of the tissue is always higher than that in the blood; the gas accordingly passes from the tissue into the blood, and the venous blood passes on not only with its hremoglobin more or less reduced, i.e. with its oxygen-pressure decreased, but also with its carbonic acid pressure increased. Arrived at the lungs, the blood finds the pulmomary air at a lower carbonic acid pressure than itsclf. The gas accordingly streams through the thin vascular and alveolar walls until the pressure without the blood. vessel is equal to the pressure within. At the same time the blood finds in the air of the pulmonary alveoli a supply of oxygen, more than adequate to convert, not entirely but nearly so, the reduced hemoglobin back again to oxyhanoglobin. Thus the air of the pulmonary alveoli, having given up oxygen to the blood and taken up carbonic acid from the blood, having in consequence a higher carbonic acid pressure and a lower oxygenpressure than the tidal air in the bronchial passages, mixes rapidly with this by diffusion. The mixture is further assisted by ascending and descending currents; and the tidal air issues from the chest at the breathing ont poorer in oxygen and richer in carbonic acid than the tidal air which entered at the breathing in. 


\section{SEC. 6. THE NERVOUS MECHANISM OF RESPIRATION.}

$\S 292$. Breathing is an involuntary act. Though the diaphragm and all the other muscles employed in respiration are voluntary muscles, i.e. muscles which can be called into action by a direct effort of the will, and though respiration may be modified within very wide limits by the will, yet we habitually breathe without the intervention of the will : the normal breathing may continue, not only in the absence of consciousness, but even after the removal of all the parts of the brain above the spinal bulb (medulla oblongata).

We have already seen how complicated is even a simple respiratory act. A very large number of muscles are called into play. Many of these are very far apart from each other, such as the diaphragm and the nasal muscles; yet they act in harmonious sequence in point of time. If the lower intercostal muscles contracted before the scaleni, or if the diaphragm contracted alternately with the other chest-muscles, the satisfactory entrance and exit of air would be impossible. These muscles moreover are coordinated also in respect of the amount of their several contractions; a gentle and ordinary contraction of the diaphragm is accompanied by gentle and ordinary contractions of the intercostals, and these are preceded by gentle and ordinary contractions of the scaleni. A forcible contraction of the scaleni, followed by simply a gentle contraction of the intercostals, would perhaps hinder rather than assist inspiration, and at all events would be waste of power. Further, the whole complex inspiratory effort is often followed by a less marked but still complex expiratory action. It is impossible that all these so carefully coordinated muscular contractions should be brought about in any other way than by coordinate nervous impulses descending along effcrent nerves from a coordinating nervous centre. By experiment we find this to be the case.

When in a rabbit the trunk of a phrenic nerve is cut, the diaphragm on that side remains motionless, and respiration goes on without it. When both nerves are eut, the whole diaphragm remains quicscent, though the costal respiration becomes excessively laboured. 
When an intercostal nerve is cut, no active respiratory movements are seen in the intercostal muscles of the corresponding space, and when the spinal cord is divided below the origin of the seventh cervical spinal nerve, that is below the exits of the roots of the phrenic nerves, costal respiration ceases, though the diaphragm continues to act, and that with increased vigour. When the cord is divided just below the spinal bulb, all thoracic movements cease, but the respiratory actions of the nostrils and glottis still continue. These however disappear when the facial and recurrent laryngeal nerves are divided. We have already stated that after removal of the brain above the spinal bulb, respiration still continues very much as usual, the modifications which ensue from the loss of the brain being unessential. Hence, putting all these facts together, it is clear that the respiratory movements are, as we suggested, brought about by coordinated impulses which, developed in the central nervous system and starting in the first instance in the spinal bulb, find their way along the several efferent nerves. "The proof is completed by the fact that the removal of or extensive injury to the spinal bulb alone is, save in exceptional cases which we will discuss presently, at once followed by the cessation of all respiratory movements, even though the rest of the nervous system including every muscle and every nerve concerned be left intact. Nay more, if only a small portion of the spinal bulb, a tract whose limits have not been clearly defined, but which may be described as lying below the vaso-motor centre in the immediate neighbourhood of the nuclei of the vagus nerves, be removed or injured, respiration ceases, and death at once ensues. Hence this portion of the nervous system was called by Flourens the vital knot, or ganglion of life, 'noeud vital.' We shall speak of it as the respiratory centre.

$\S 293$. The nature of this centre must be exceedingly complex; for while even in ordinary respiration it gives rise to a whole group of coordinate nervous impulses of inspiration followed in due sequence by a smaller but still coordinate group of expiratory impulses of an antagonistic nature, in laboured respiration fresh and larger impulses are generated, though still in coordination with the normal ones, the expiratory events being especially augmented; and in the cases of more extreme dyspnoea and asphyxia impulses overflow, so to speak, from it in all directions, though only gradually losing their coordination, until almost every muscle in the body is thrown into contractions.

We must not however conceive of this centre as one of such a kind that the impulses leave it fully coordinated and equipped so that nothing remains for them but to travel, unchanged, along the several efferent nerve-fibres to their several muscular 
destinations. On the contrary we have reason to think that the respuiratory motor nerves, like other motor nerves, are connected, just as they are about to issue from the spinal cord, with a nervous machinery, in which nerve cells play a part a point which we shall consider more fully in treating of the spinal cord; we have reason to think that the respiratory impulses starting from the respiratory centre pass into and are modified by secondary spinal nervous mechanisms before they issue along the motor nerve-roots. Indeed observations shew that under particular conditions, and especially in young animals, respiratory movements may be carried ont in the entire absence of the spinal bulb. Thus if in a kitten or puppy, or young rabbit, after division of the spinal cord below the bulb, artificial respiration be kept up, and then pauses be made in the artificial respiration, during these pauses not only may what appear to be respiratory movements be induced, in a reflex manncr, by pinching or by blowing on the skin, but, especially if the excitability of the spinal cord be heightened by small doses of strychnia, even spontancous efforts of breathing may occasionally be observed. These are the exceptional instances mentioned above. We shall probably not greatly err in regarding the respiratory nervous system as in many ways analogous to the vaso-motor nervous system, with its head centre in the spinal bulb, and secondary centres elsewhere, and in continuing to speak of the centre in the spinal bulb as being "the respiratory centre" while admitting that it works through other nervous machinery placed lower down in the spinal cord, and that this subordinate machinery may, in exceptional eases, carry out, though inadequately, the work of the chief centre.

$\$ 294$. Admitting then the existence of this bulbar respiratory centre the question naturally arises, Are we to regard its rhythmic action as due essentially to changes taking place in itself, or as due to afferent nervous impulses or other stimuli which affect it in a rhythmic manner from without? In other words, Is the action of the centre automatic or purely reflex? Wc know that the centre may be influenced by impulses proceeding from without, and that the breathing may be affected by the action of the will, or by an emotion, or by a dash of cold water on the skin, or in a hundred other ways; but the fact that the action of the centre may be thus modified from without, is no proof that the continuance of its activity is dependent on extrinsic causes.

In attempting to decide this question we naturally turn to the pneumogastric as being the nerve most likely to serve as the channel of afferent impulses setting in action the respiratory centre. If both vagus nerves be divided, respiration still continues, though in a modified form. This proves distinctly that afferent impulses ascending those nerves are not the efficient 
cause of the respiratory movements. We have seen that when the spinal cord is divided below the spinal bull, the facial and laryngeal movements still continue. 'This proves that the respiratory centre is still in action, though its activity is unable to manifest itself in any thoracic movement. But when the eord is thus divided, the respiratory centre is cut off from all sensory impulses, save those which may pass into it from the cranial nerves of sensory function; and that these sensory cranial nerves are not specially concerned in developing the activity of the respiratory centre is shewn by the fact that the division of these cranial nerves by themselves, when the bulb and spinal cord are left intact, does not do away with the continuance of respiration. One cranial nerve, as we shall see, is especially concerned in respiration, viz. the vagus nerve; but if after removal of the brain above the bulb botll vagus nerves be divided, respiration still goes on; indeed the respiratory impulses proceeding from the centre are, though in a peculiar way, exaggerated. Hence though we camnot put the matter to an experimental test by dividing every sensory nerve in the body, while leaving the motor nerves of respiration intact, such an operation being practically impossible, we may infer that the respiratory impulses proceeding from the respiratory centre are not simply afferent impulses reaching the centre along afferent nerves and transformed by reflex action in that centre. 'They evidently start de novo from the centre itself, however much their characters may be affected by afferent impulses, reaching that eentre at the time of their being generated. 'The action of the centre is automatic, not simply reflex.

$\$ 295$. We find, on inquiry, that the activity of the centre is profoundly influenced by two classes of events. These, as we might expect, are on the one hand events producing changes in the quality of the blood distributed to the spinal bulb through the arteries, especially as regards its gases, that is to say, events modifying the interchange taking place in the lungs; and on the other hand nervous impulses, started in various ways and reaching the centre along various nerves or nervous tracts. It will be convenient to consider the latter first.

Afferent nervous impulses may affect the centre in many various ways. The whole act of breathing or of taking a breath is a double act consisting of an inspiration and an expilation, and nervous impulses may especially affect the one or the other. One mole of breathing may differ from another in the depth of the individual breath, in the volume of air taken in and given out; and nervous impulses may increase or may diminish the depth of a breath, the volume of air respired. One mode of breathing again differs from anotler in the rapidity with which one breath succeeds another, that is, in the rate of rhythm; and nervous impulses may slow or nay quicken the 
rate of rhythm. Then, again, combinations of effects so numerous and varied as almost to baffle description may result from the influence of various nervous impulses. Emotions may affect a single breath or a long series of breaths, may quicken the rhythm while making each breath more shallow or may at the same time make each breath deeper, or may slow the rhythm in either the one or the other manner, and may bear chiefly on inspiration or on expiration. Moreover there is not an afferent nerve in the body which, by means of afferent impulses passing along it, may not be the instrument of influencing the respiratory centre. Of all the automatic centres in the body the respiratory centre is the one whose independence is most obscured by the repeated effects of afferent nervous impulses.

Certain afferent nerves however appear to be more closely connected with it than others; and of these the most conspicuous and important are the two vagus nerves, which we have already mentioned in this comnection. Their importance is well illustrated by the following experiments. If one vagus be divided in an ordinary way, without any special precautions,

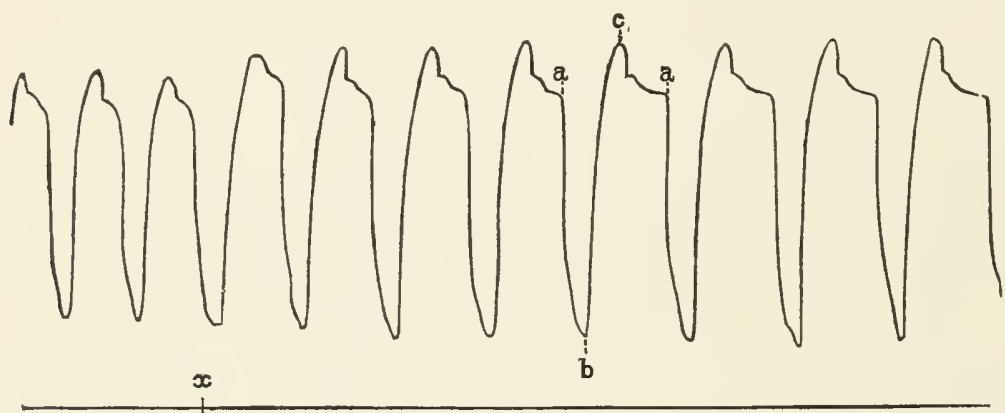

A

Fig. 91. Effect on Respiration of section of one Vagus.

The vagus was divided at the point marked $x$. The curve was obtained by means of a tambour connected with a receiver into which the animal (rabbit) breathed as shewn in Fig. 85, the lever falling in inspiration as air is sucked out of the tambour, and rising in expiration as the air returns. Inspiration begins at $a$ and ends at $b$. Expiration begins at $b$ and ends at $c$. The lever gradually falls between $c$ and $a$ owing to the escape of air from the apparatus.

the respiration is either not materially changed, or if affected becomes slower (Fig. 91). If both be divided (Fig. 92) it becomes very slow, the pauses between expiration and inspiration being markedly prolonged. The character of the respiratory movement too is markedly changed; each respiration is fuller and deeper, so much so indeed that, according to some observers, what is lost in rate is gained in extent, the amount 
of carbonic acid produced and oxygen consumed in a given period remaining after division of the nerves about the same as when these were intact; but it is undesirable to insist too much on the exactness of this compensation.

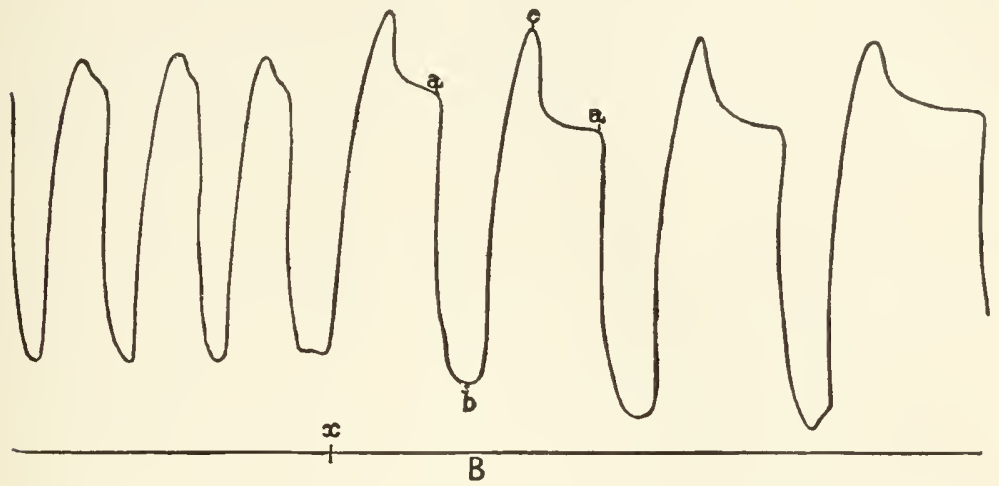

Fig. 92. Effect on Respiration of section of both Vagus nerves.

The curve was obtained in the same way as Fig. 91. The second vagus nerve was divided at $x$.

When after division of both vagus nerves in the neck, the medulla being intact, the central stump, that connected with the central nervous system, of one of them is stimulated with a gentle interrupted current, the effects are not always the same; one of two results may follow and that whichever of the two nerves be used. In a certain number of cases, and these may perhaps be regarded as the more typical ones, the respiration, which from the division of the nerves had become slow, is quickened again (Fig. 93); and with care, by a proper application of the stimulus, the normal respiratory rhythm may for

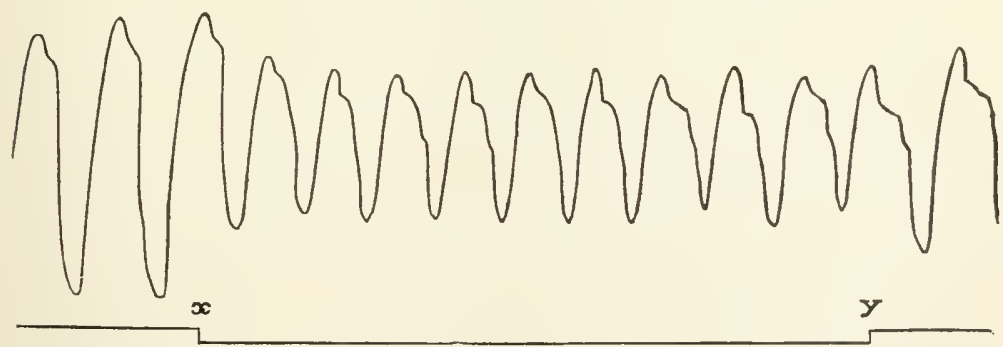

Fig. 93. Quickening of Respiration by gentle stimulation of the Central End of the Vagus trunk.

The curve was obtained in the same way as Figs. 91, 92. Stimulation of the vagus began at $x$, and ended at $y$. 
a time be restored. Upon the cessation of the stimulus, the slower rhythm returns. If the current be increased in strength, the rhythm may in some cases be so accelerated that inspiration begins before the expiration of the preceding breath is completed, Fig. 94; and this may go on until at last the diaphragm is brought into a condition of prolonged tetanus, and a standstill of respiration in an extreme inspiratory phase is the result. On the other hand in a certain number of cases the result is of an opposite character. Even though the respiration be already

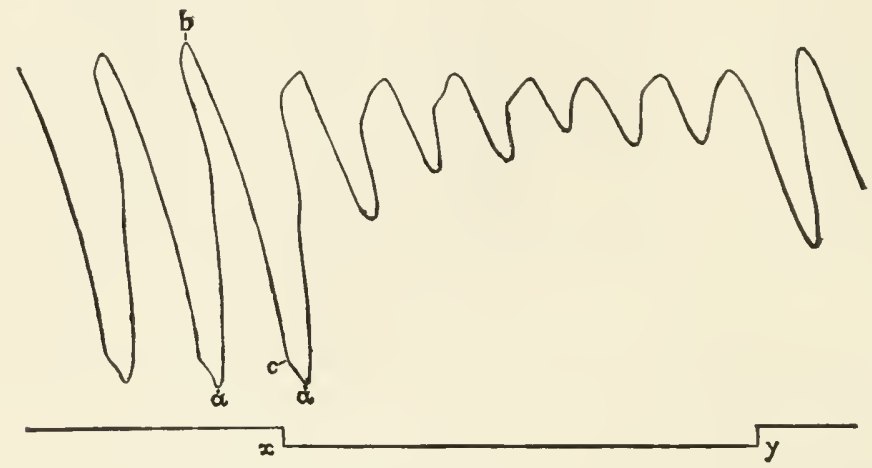

Fig. 94. Stimulation of Vages leading to Inspiratory increase.

This curve, unlike the preceding, was obtained by inserting a needle through the body wall so as to rest on the diaphragm and attaching a lever to the needle; see $\S 251$. The lever rises with each contraction of the diaphragm so that inspiration begins at $a$ and ends at $b$, expiration begins at $b$ and ends at $c$, the interval between $c$ and $a$ corresponding to the pause.

Stimulation of the vagus begins at $x$. It will be seen that upon stimulation the inspiratory rises of the lever begin long before the preceding expirations are complete.

slowed by division of the nerves, stimulation produces a still further slowing, the pauses between each expiration and the succeeding inspiration are prolonged (cf. Fig. 95), and in a certain number of cases, actual standstill is brought about, but a standstill of a kind the opposite of the one just described, since the diaphragm which in that case was in prolonged tetanus is, in this case, completely relaxed, and remains for some time in the condition in which it is at the close of an ordinary breath. In a certain number of cases, and these are not uncommon, the result is intermediate between the two above extremes; the diaphragm stands still in a prolonged contraction in a position which is intermediate between the height of inspiration and expiration.

These results suggest the conclusion that the vagus nerve (we are dealing now with the main trunk of the nerve) tontains 
afferent fibres of two kinds connected with the respiratory centre : one kind algmenting the action of the centre somewhat in the same way as the augmentor cardiac fibres augment the beat of the heart, and the other kind laving an inhibitory effect. Apparently sometimes the one and sometimes the other kind is, according to circumstances, most provoked by the stimulation, much in the same way as stimulation of the vagus in the frog, which as we have secm, $\$$ 136, is the channel for both inhibitory and augmentor cardiac impulses, produces, sometimes inlibition. sometimes augmentation of the heart beat. 'lo affect the heart of course the stimulation of the vagus must be centrifugal, directed towards the periphery, whereas to affect the respiration it must be centripetal, applied to the part of the nerve connected with the brain; and while the usual effect on the heart of ordinary stimulation of the vagus is inhibition, augmentation only occurring in special cases, the most common effect on respiration is augmentation, though inhibition is not unfrequently seen. When the experiment is conducted on an animal under the full influence of chloral stimulation of the vagus generally produces inhibition of respiration, probably because the chloral renders the respiratory centre more susceptible to inhibitory influence.

$\$ 296$. We said just now "the action of the centre;" but the respiratory centre is a double one ; it gives rise to inspiratory and to expiratory efferent impulses, and thesc are antagonistic the one to the other. If inspiratory and expiratory impulses issued from the centre at the same time and in equal potency, there could be no breathing at all, they would neutralize each other's effects ; and indeed any amount of inspiratory impulse is antagonistic to a simultaneous expiratory impulse, and vice versa. Hence for the adequate services of the respiratory centre we might expect to find that each kind of afferent impulse ascending the vagus affected the centre in a double and opposite way, inhibiting expiration while augmenting inspiration, or inhibiting inspiration while augmenting expiration. If we allow ourselves to speak of the whole respiratory centre as consisting of two parts, one the inspiratory part, or inspiratory centre concerned in the issue of inspiratory impulses, and the other the expiratory part, or expiratory centre concerned in the issue of expiratory impulses, we may suppose that these centres are so related to each other that afferent impulses, reaching the spinal bulb, which augment or inhibit the one, necessarily inhibit or augment the other. We need perhaps hardly add that of these two centres we should expect to find the inspiratory centre the dominant and the most responsive one ; in normal breathing it comes almost alone into obvious use, since as we have scen the expiratory muscles have then a very slight task only, the chest being emptied chiefly by elastic reaction ; and, 
speaking generally, breathing in is the first consideration, we breathe out mostly becausc we have already breathed in.

There are many facts which support this view of the double antagonistic action of afferent respiratory impulses. If the central end of the superior laryngeal branch of the vagus be stimulated the effects are much more constant than those of stimulating the main vagus trunk. Whether the main trunk of the nerve be previously severed or not, the result of centripetal stimulation of the supcrior laryngeal branch is always in the direction of a slowing of the respiration (Fig. 95); and

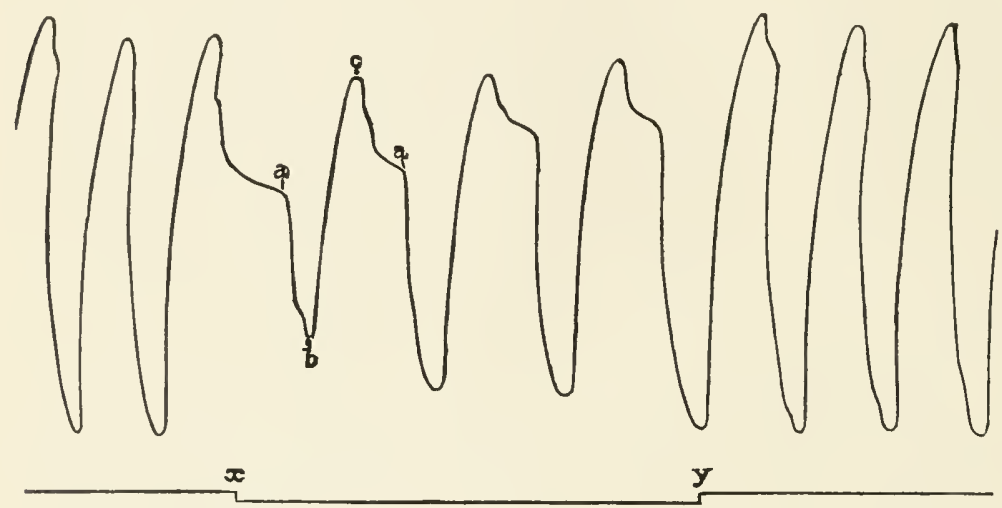

Fig. 95. Slowing of Respiration by stimulation of superior LaryeGEAL NERVE.

This curve was obtained in the same way as Figs. 91, 2, 3 and the letters have the same meaning as in those figures. Stimulation begins at $x$, and ends at $y$.

this may by proper stimulation be carried so far that a complete standstill of respiration in the phase of rest, is brought about. While the main trunk of the vagus contains fibres of two kinds, both augmentor and inlibitory of inspiration, the superior laryngeal branch appears to contain one kind only, those which inhibit inspiration. If now while this experiment is being conducted on a rabbit the abdomen be watehed it will be seen that the inhibition of inspiration is accompanied by a contraction of the abdominal muscles, that is by an effort at expiration; the stimulation of the nerve while inhibiting respiration provokes, to a certain cxtent, expiration.

$\$ 297$. That the trunk of the vagus is the channel of these two kinds of impulses, of a mutually antagonistic character, is further shewn by applying what may be considered as natural stimuli to the endings of the nerve in the lungs; and the results so obtained liave an especial value since the artificial 
stimulation of a nerve-fibre at a part of its course by means of an electric eurrent is at best a rough process, by which we cannot hope to do more than approximate to the results actually taking place in the living body when the nerve is stimulated at its endings by natural stimuli; and the approximation is perhaps less in the case of the exquisitely sensitive respiratory centre than in many other cases.

If in an animal in which a careful graphic record of the respiratory movements is being taken, the trachea be suddenly closed at the summit of an inspiration, the result is a pause before the succeeding inspiration follows, that is to say, a partial or temporary inhibition of inspiration; and if during such an experiment on a rabbit a curve be taken by means of the isolated slip of the diaphragm, $\$ 259$, it will be seen (Fig. $96 \mathrm{~A}$ ) that the slip elongates somewhat; that is to say, previously in a state of slight tonic contraction, it changes in the direction of expiration. If on the other hand the trachea be suddenly closed at the end of an expiration (Fig. 96 B), when
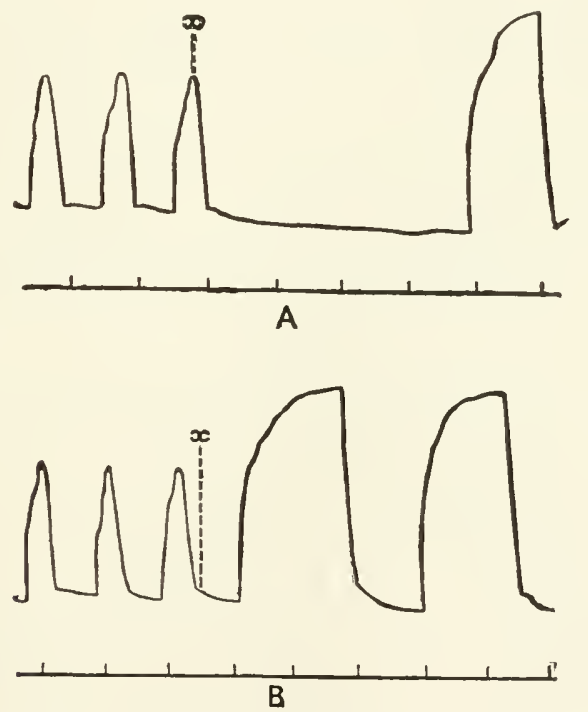

Fig. 96. Effects of Distension and Collapse of Lung. (Head.)

Both curves are described by a lever attached, as stated in $\S 259$, to a slip of the diaphragm of a rabbit. A contraction of the diaphragm (inspiration) raises the lever; during relaxation of the diaphragm, the lever falls.

In $\mathbf{A}$, the trachea is closed at $x$, the height of inspiration ; a pause follows during which the lever gradually sinks until an inspiration (a very powerful one) sets in.

In $\mathrm{B}$, the trachea is closed at the end of expiration, $x$; there follow powerful inspirations. 
the lungs have returned to their emptied condition, the result is an increase of the sequent inspirations, that is to say, an augmentation of inspiratory impulses. If the chest or if the lung only be gently inflated a temporary cessation of all inspiration may be produced, accompanied sometimes by an attempt at expiration. If on the other hand air be sucked out of the chest, or if one lung be made to collapse by puncture of
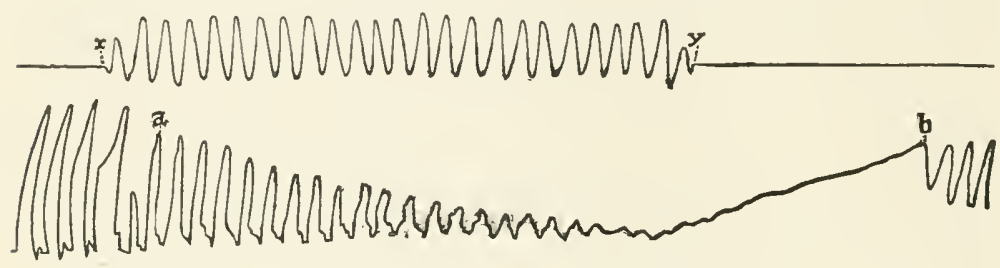

Fig. 97. Effects of repeated Inflations. Positive ventulation. (Head.)

The lower curve is described, as in Fig. 96, by a lever attached to a slip of the diaphragm. The upper curve shews the intlations from $x$ to $y$, which were made without any attempt to draw the air out at each inflation; each rise on this curve denotes an inflation. It will be observed that as the inflations are continued the respiratory movements of the diaphragm are gradually "knocked down."

one pleural chanber, a prolonged inspiration is the frequent result, the diaphragm being thrown into a prolonged inspiratory tetanus. If the lungs are repeatedly inflated, without any means being taken to draw out the air after each inflation (Fig. 97), a procedure which we may speak of as positive

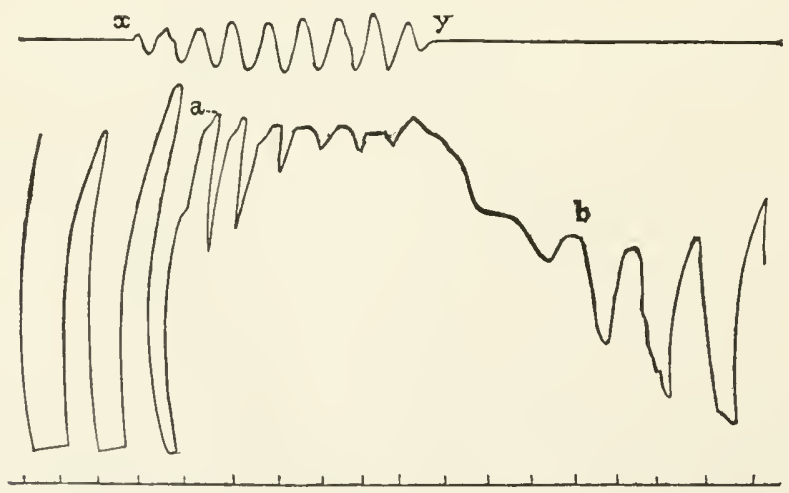

Fig. 98. Effects of repeated Suctions of the Lungs. Negative ventilation. (Head.)

The curve corresponds exactly to Fig. 97, except that the lungs are subjected to repeated suctions without corresponding inflations. The result is that the inspirations are repeated in such a way as to be led almost to an inspiratory tetanus of the diaphragm. 
ventilation, the result is that the inspiratory efforts are diminished, and if the ventilation is continued may cease altogether. If on the other hand air is repeatedly sucked out of the lungs, without any corresponding inflations, negative ventilation, the inspiratory efforts are increased (Fig. 98) and the increase may be such as to bring the diaphragm to a state of tetanus. And in general, though several complications occur which we cannot discuss here, the results of inflation of the lungs on the one hand and of suction or collapse of the hungs on the other hand, shew that the mere inflation or perhaps rather the mere distension of the lung tends to inhibit inspiratory and usher in expiratory impulses, while collapse of the lung tends to inhibit expiratory and to develop inspiratory impulses, the effect on the inspiratory impulses, as might be expected from the dominance of the inspiratory portion of the centre being more marked than the effect on the expiratory impulses. That the instrument by which these effects are produced is the vagus nerve is shewn by the fact that they are no longer distinctly recognizable when both vagus nerves are divided. And that the results are due to the mere mechanical expansion and collapse of the lung in insufflation and collapse, and not to any chemical influences exerted by the larger amount or smaller amount of air present in the lung in the two cases increasing or diminishing the absorption of oxygen and escape of carbonic acid, is shewn by the fact that the results remain in their main features the same when some indifferent gas such as hydrogen is used for inflation instead of air or oxygen. We infer therefore that the expansion of the pulmonary alveoli in some way or other so stimulates the endings in the lung of the pulmonary branches of the vagus, that impulses are generated which ascending the vagus trunk inhibit the inspiratory processes in the respiratory centre ; and that conversely collapse of the lung similarly generates impulses which are augmentative of inspiratory impulses. And, assuming on the strength of analogy the existence in the vagus of two sets of fibres we may say that expansion stimulates the endings of the fibres which inhibit inspiration and concurrently tend to augment expiration, while collapse stimulates the fibres which inhibit expiration and augment inspiration. The respiratory pump may thus be looked upon as a self-regulating mechanism : the expanision of the lungs which is the result of the efferent inspiratory impulses tends to check the issue of these impulses and to inaugurate the sequent expiration; and the return of the lungs in expiration tends to set going the succeeding inspiration.

$\S 298$. The double or alternate respiratory action of the vagus nerves on which we have dwelt above may be taken as in a general way illustrative of the manner in which other afferent nerves and various parts of the cerebrum are enabled to influence respiration. As we have already said, and indeed 
know from daily experience, of all the apsychical nervous centres, the respiratory centre is the one which is most frequently and most deeply affected by nervons impulses from various quarters. Besides the rhanges brought about by the will (and when we breathe voluntarily we probably make use to some extent of the normal nervons machinery of respiration, working through this, rather than sending independent volitional impulses direct to the diaphragm and other respiratory muscles), we find that cmotions and painful sensations alter profoundly the character of the respiratory movements. And though these effects may be partly indirect (the emotion modifying the heartbeat or the tonus of the arteries, and so influencing the flow of blood through the respiratory centre), they are chiefly due to the direct action of nervous impulses reaching that centre from higher parts of the brain. So also impulses from almost every sentient surface, or passing along almost evcry sensory nerve, may modify respiration in one direction or another. The influence in this way of stimuli applied to the skin is well known to all; but perhaps next to the vagus the nerve most closely connected with the respiratory centre is the fifth nerve, branches of which guard the nasal respiratory channels; the slightest stimulation of the nostrils at once affects the brcathing and most frequently arrests it. The effects of stimuli of various strengths brought to bear on various nerves are very varied. Sometimes the result is an increase of inspiration; and that either by a quickening of the rhythm or by an increase of the individual breaths or by a combination of the two. Sometimes the result is an inhibition of inspiration accompanied or not by an increase of expiration, and sometimes, as when the stimulation causes a cough, the expiratory results may be out of all proportion to the modifications of inspiration.

$\$ 299$. The complicated nature of the respiratory contre is further shewn by the fact that it appears to consist of two lateral halves which normally work in unison and yet may be made to work independently. If the spinal bulb be carefully divided in the middle line respiration may continue to go on in quite a normal fashion. If, however, one vagus be then divided, the respiratory movements, both costal and diaphragmatic, on the side of the body on which division of the vagus has taken place, become slower than those on the other side, so that the two sides are no longer synchronous; and a stimulus confined to one vagus affects the respiratory movements of that side of the body only. So also a section of a lateral half of the cord below the bulb stops the respiratory movements on that side alone.

$\$ 300$. Besides these nervous influences, however, there is another circumstance which perhaps above all others affects the respiratory centre, and that is the condition of the blood in respect to its respiratory changes; the more venous (less arte- 
rial) the blood, the greater is the activity of the respiratory centre. When by reason either of any hindrance to the entrance of air into the chest, or other interference with the due interchange between the blood and the pulmonary air or of a greater respiratory activity of the tissues, as during muscular exertion, the bloor becomes less arterial, more venous, i.e. with a smaller charge of oxygen and more heavily laden with carbonic acid, the respiration from being normal becomes laboured. We may speak of normal breathing as eupnoea, and say that this, when the blood is insufficiently arterialized, passes into dyspnoea, an intermediate stage in which the respiratory movements are simply exaggerated being known as hyperpnoe. The modifications of breathing thus caused by deficient arterialization of blood are especially characterized by an increase in the total energy of the respiratory impulses generated, and in this respect differ from the modifications resulting from interference with the nervous arrangements such as those following upon section of the vagus nerves, in which case as we have seen the rhythm is much more profoundly affected than the amount. In dyspnoa the breathing is frequently quicker as well as deeper, there is an increase in the sum of efferent respiratory impulses, and the expiratory impulses, which in normal respiration are very slight, acquire a pronounced importance. As the blood becomes, in cases of obstruction, less and less arterial, more and more venous, the discharge from the respiratory centre becomes more and more vehement, and instead of confining itself to the usual tracts, and passing down to the ordinary respiratory muscles, overflows into other tracts and puts into action other muscles, until there is perhaps hardly a muscle in the body which is not made to feel its effeets. The muscles which are thus more and more thrown into action are especially those tending to earry out or to assist expiration; and at last, if no relief is afforded, the violent but still definite respiratory movements give way to general convulsions of the whole body, which however have, to a certain extent, an expiratory character. With the onset of these convulsions dyspncea is said to have passed into asphyxia. By the violence of these convulsions the whole nervous system becomes exhausted, the convulsions cease and death is ushered in through a few infrequent and long-drawn breaths; but to this matter we shall return. The effect of venous blood then is to augment all those natural explosive decompositions of the substance of the central nervous system which give rise to respiratory impulses ; it increases their amount, and also quickens their rhythm. The latter change, however, is much less marked than the former, the respiration being much more deepened than hurried, and the several respiratory acts are never so much hastened as to eatch each other up, and so to produce an inspiratory tetanus like that resulting from stimulation of the vagus. On 
the contrary, especially as exhaustion begins to set in, the rhythm becomes slower out of proportion to the weakening of the individual movements.

$\S 301$. The question naturally arises, Does this condition of the blood affect the substance of the central nervous system, that is to say, of the respiratory centre in the spinal bulb (and the subsidiary spinal nervous mechanisms) directly, or does it produce its effect by stimulating the peripheral ends of afferent nerves in various parts of the body, and, by the generation there of afferent impulses, indirectly modify the action of the central nervous system? Without denying the possibility that the latter mode of action may help in the matter, as regards not only the vagus, but all afferent nerves, the following facts seem to shew that the main effect is produced by the direct action of the blood on the central nervous system and indeed on the bulbary respiratory centre itself. If the spinal cord be divided below the bulb, and both vagi be cut, want of proper aeration of the blood still produces an increased activity of the respiratory centre, as shewn by the increased vigour of the facial respiratory movements; in such a case, it must act directly on the respiratory centre, for all afferent paths along the nerves, except the few cranial ones, have been blocked by the operation. The same direct action is further shewn by the following "cross circulation " experiment. In two animals the peripheral portion of one carotid of one animal is connected by a tube with the central portion of one carotid of the other animal, the other carotid in each animal being tied. Hence the brain and the brain only of one animal is supplied by the blood of the other animal, the rest of its body being supplied by its own blood. If now respiration be stopped in one animal the other becomes dyspnœic, while it in itself shews no dyspnoa; it is the animal to whose brain (spinal bulb) alone too venous blood is brought, not the animal the whole of whose body is supplied with the too venous blood, which manifests disturbance of the respiratory centre. Again, if in an animal the supply of blood be cut off from the spinal bulb by ligature of the carotid and intervertcbral arteries dyspnœea is produced, though the operation produces at first no change in the blood generally, but simply affects the respiratory condition of the medulla itself by cutting off its blood-supply, the immediate result of which is an aceumulation of carbonic acid and a paucity of available oxygen in the nervous substance of that region. If the blood in the carotid artery in an animal be warmed above the normal, a dyspnoea is produced which, though apparently not quite identical with the dyspnoea caused by imperfect arterialization of the blood, shews that the too high temperature of the blood directly affects the activity of the respiratory centre. We may conclude therefore that the condition of the blood affects respiration by acting directly on the respiratory centre. 
While the respiratory centre is thus being affected by the too venous blood, it is, until exhaustion begins to set in, more irritable,'more easily and largely affected by afferent impulses than in its normal condition. During dyspncea a stimulus which applied to the vagus or to some other sensory nerve under normal conditions would produce little or no effect, may start very powerful respiratory movements.

$\$ 302$. Deficient aeration produces two effects in blood: it diminishes the oxygen, and increases the carbonic acid. Do both of thesc changes affect the respiratory centre, or only one, and if so, which? When an animal is made to breathe an atmosphere containing nitrogen only, the exit of carbonic acid by diffusion is not affected, and the blood, as is proved by actual analysis, contains no excess of carbonic acid. Yet all the phenomena of dyspncea are present, and if the experiment be continued, convulsions ensue and the animal dies in asphyxia. In this case the result can only be attributed to the deficiency of oxygen. On the other hand, if an animal be made to breathe an atmosphere rich in carbonic acid, but at the same time containing abundance of oxygen, though the breathing becomes markedly deeper and also somewhat more frequent, there is no culmination in a convulsive asphyxia, even when the quantity of carbonic acid in the blood, as shewn by direct analysis, is very largely increased. On the contrary, the increase in the respiratory movements may after 'a while pass off, the animal becoming unconscious, and appearing to be suffering rather from a narcotic poison than from simple dyspnoea; the excess of carbonic acid in the blood appears to affect other parts of the central nervous system, and especially portions of the brain, more profoundly than it does the respiratory centre. It has been maintained by some that while a deficiency of oxygen promotes inspiratory movements, an excess of carbonic acid stimulates the expiratory movements, the nervous mechanisms being so arranged that a lack of oxygen leads to an effort to get more of it and a too great load of carbonic acid to an effort to get rid of it; but the facts are opposed to the existence of any such teleological adaptation. It is obvious however that a lack of oxygen and an excess of carbonic acid affect the respiratory centre in very different ways, and that in ordinary cases of interference with the interchange in the lungs, as in deficient aeration, it is the lack of oxygen which plays the principal part in developing the abnormal respiratory movements. We may infer that it too is chiefly concerned in regulating the more normal respiration, but cannot as yet say what is the exact share to be attributed to the carbonic acid.

We may here point out that it is not to be supposed that each breath is determined by the condition of the blood flowing through the capillaries of the medulla at the moment preceding 
tlat breath, it is not to be imagined that each breath is the result of the lack of oxygen felt immediately before. On the contrary, the condition of blood merely modifies the natural automatic action of the centre.

$\$ 303$. There are reasons for thinking that conditions of the blood, other than variations in the amount of oxygen and carbonic acid, may also materially affect the working of the respiratory centre. It is a matter of common experience that muscular exertion, especially if at all cxcessive, increases the respiratory movements ; violent exercise soon puts a man " out of breath." 'This increased activity of the respiratory centre is in large measure at all events caused by the character of the blood which during and for some little time after the movements is carried to the spinal bulb, and not by any nervous impulses sent up to the bulb from the contracting muscles. 'This is shewn by the fact that if in an animal the spinal cord be divided in the dorsal or lumbar region and the hind limbs be powerfully tetanized, the respiratory movements are increased ; the animal pants as it would do if it had been rumning. In such a case the only connection between the hind limbs and the respiratory centre is through the blood; it must be some change in the blood caused by the muscular contractions which affects the respiratory centre when the blood passes from the hind limbs to be distributed by the heart to the bulb. Now when a muscle contracts its consumption of oxygen and production of carbonic acid, especially the latter ( $\$ 60)$, are increased; the blood leaving the muscle is more venous than usual. Hence when many muscles are contracting powerfully the blood carried to the right side of the heart is more venous than usual; and we might expect that it is this unusually venous blood failing to be adequately arterialized in the lumgs and hence reaching the bulb from the left side of the heart in a more venous. less completely arterialized condition than usual, which stirs up the respiratory centre to increased activity.

On exannination however it is found that the blood leaving the left side of the heart in such cases, is not less arterialized but if anything more arterialized than usual. The increased respiratory movements induced by the clianged blood soon prove sufficient or even more than sufficient to give the blood the extra quantity of oxygen and to remove the cxtra quantity of carbonic acid. Obviously the blood coming from the tetanized muscles affects the respiratory centre by virtue of some quality which, unlike that due to the deficiency of oxygen or excess of carbonic acid, is not immediately affected by the passage through the lungs. Whether the quality in question be dependent on an excess of sarcolactic acid, or on some other product or produr.ts of muscular netabolism, we do not as yet know. But the fact that substances in the blood may so affect the respiratory 
centre is interesting since it shews by how many safeguards the working of the respiratory centre is carefully adapted to the needs of the economy.

$\S 304$. Apnoea. When we attempt to hold our breath, we find that we can do this for a limited time only; sooner or later a breath must come; but, as is well known, the time during which we can remain without breathing may on occasion be much prolonged, if we first of all take a series of deep breaths. The breath sooner or later inevitably follows because at last the natural impulses proceeding from the respiratory centre become too imperious to be any longer held in check by the impulses of volition passing down to the centre from the brain. The fact that a series of deep breaths, a thorough ventilation of the lungs, postpones the victory of the unconscious centre, shews that such a ventilation in some way delays the development of the natural respiratory impulses. A similar but still more marked delay may often be seen in an animal under artificial respiration. If in a rabbit artificial respiration is carried on very vigorously for a while, and then suddenly stopped, the animal does not immediately begin to breathe. For a variable period $n o$ respiratory movements at all take place, and breathing when it does begin occurs gently and normally, only passing into dyspnoea if the animal is mable to breathe of itself ; and even then the transition is quite gradual. Evidently during this period the respiratory centre is in a state of complete rest, no explosions are taking place, no respiratory impulses are being generated, and the quiet transition from this condition to that of normal respiration shews that the subsequent generation of impulses is attended by no great disturbance. Not only is the centre at rest, lut it is less irritable than the normal; impulses along the vagus or other nerves which otherwise would produce respiratory explosions are now ineffectual. This state of things is known as that of apnoea, the converse of dyspnoea; and the longer pause in breathing mentioned above as possible after unusual ventilation of the lungs may be regarded as a brief apnoea.

Now it seemed natural to suppose that such a state of rest of the respiratory centre was brought about by the more than necessarily ample supply of oxygen afforded by the previous increased inspiratory movements ; and indeed it was maintained that apnca was the result of too great, just as dyspncea is the result of too little arterialization of the blood reaching the respiratory eentre. It was argued that owing to the increased vigour of the artiticial respiratory movements the hamoglobin of the arterial blood, which in normal breathing is not quite saturated with oxygen, became almost completely so, and that at the same time the quantity of oxygen simply dissolved in the blood became largely increased and its tension largely 
augmented. But there are reasons which render such a view untenable. In the first place there is no direct and satisfactory proof that in apnoa the arterial blood is overloaded with oxygen as supposed; indeed during the course of apnoa before it has come to an end the blood becomes distinctly less arterial, more venous than usual. In the second place apnoea, if not entirely impossible, is much more difficult to bring about when both vagus nerves are divided, and if it does occur after section of the vagus nerves has not the same characters as ordinary apnoea. Now, when artificial respiration is being carried on section of the vagus nerves can have no effect on the quantity of oxygen taken up by the blood in the lungs. But the vagus nerves are the channel of impulses affecting the respiratory centre, and this relation of the apnœa to the vagus nerves suggests another and different interpretation of apnœea. As we have seen, expansion of the lung by acting in some way or other on the pulmonary terminations of the vagus nerve sends up along that nerve impulses which inhibit inspiration. And it is argued that repeated forcible inflations of the lungs produce apnea by gencrating potent inhibitory impulses, which by a kind of summation of their effects in the spinal bulb stop for a while the generation of respiratory impulses in the respiratory centre. This conclusion moreover is strongly supported by the fact that an apnœa may be produced, so long as the vagus nerves are intact, by forcible artificial respiration with hydrogen instead of atmospheric air ; in other words, the inhibitory impulses generated in the vagus nerves by the inflation are sufficient wholly to neutralize the development of respiratory impulses which the deficient arterialization of the blood would otherwise have produced.

$\S$ 305. Secondary Respiratory Rhythm. Cheyne-Stokes Respiration. A remarkable abnormal rhythm of respiration, first observed by Cheyne but afterwards more fully studied by Stokes, and hence called by their combined names, occurs in certain pathological cases. The respiratory movements gradually decrease both in extent and rapidity until they cease altogether, and a condition of apnea, lasting it may be for several seconds, ensues. This is followed by a feeble respiration, succeeded in turn by a somewhat stronger one, and thus the respiration returns gradually to the normal, or may even rise to hyperpnœa or slight dyspnœa, after which it again declines in a similar mamner. A secondary rhythm of respiration is thus developed, periods of normal or slightly dyspnoic respiration alternating by gradual transitions with periods of apnoea. The cause of the phenomena is not thoroughly understood. 
SEC. 7. THE EFFECTS OF CHANGES IN THE COMPOSITION AND PRESSURE OF THE AIR BREATHED.

$\S$ 306. The preceding sections have shewn us that the respiratory mechanism is arranged to work satisfactorily when the lungs are adequately supplied with air of the ordinary composition of, and at the ordinary pressure of the atmosphere. We have further seen that the mechanism can adapt itself within certain limits to changes in the composition and pressure of the air supplied. We may now consider briefly what takes place when those limits are overstepped. The most striking effects are seen, when, on account of occlusion of the trachea, or by breathing in a confined space, or for other reasons, a due supply of air not being obtained, normal respiration gives place, through an intermediate phase of dyspnoea, to the condition known as asphyxia; this, unless remedial measures be taken, rapidly proves fatal.

Asphyxia. As soon as the blood becomes less arterial, more venous than normal, the respiratory movements become deeper and at the same time more frequent; both the inspiratory and expiratory phases are exaggerated, the supplementary muscles spoken of $\$ 265$ are brought into play, and the rate of the rhythm is hurried. These effects, as we have seen, are chiefly to be ascribed to the deficiency of oxygen in the blood.

As the blood continues to become more and more venous the respiratory movements continue to increase both in force and frequency, a larger number of muscles being called into action and that to an increasing extent. Very soon, however, it may be observed that the expiratory movements are becoming more marked than the inspiratory. Every muscle which can in any way assist in expiration is in turn brought into play; and at last almost all the muscles of the body are involved in the struggle. The orderly expiratory movements culminate in expiratory convulsions, the order and sequence of which are obscured by their violence and extent. 'That these convulsions, through which dyspnoea merges into asphyxia, are due to a stimulation (by the venous blood) of the spinal bulb, is proved 
by the fact that they fail to make their appearance when the spinal cord has been previously divided below the bulb, though they still occur after those portions of the brain which lie above the bulb have been removed. It is usual to speak of a convulsive centre' in the bulb, the stimulation of which gives rise to these convulsions; but if we accept the existence of such a centre we must at the same time admit that it is commected by the closest ties with the normal expiratory division of the respiratory centre, since every intervening step may be observed between a simple slight expiratory movement of normal respiration and the most violent convulsion of asphyxia. An additional proof that these convulsions are carricd out by the agency of the bulb is afforded by the fact that convulsions of a wholly similar character are witnessed when the supply of blood to the bulb is suddenly eut off by ligaturing the blood vessels of the head. In this case the nervous centres, being no longer furnished with fresh blood, become rapidly asphyxiated through lack of oxygen, and expiratory convulsions quite similar to those of ordinary asphyxia, and preceded like them by a passing phase of dyspnea, make their appearance. Similar 'anæmic' convulsions are seen after a sudden and large loss of blood from the body at large, the bulb being similarly stimulated by the lack of arterial blood. In ordinary fainting, which is loss of consciousness due to an insufficient supply of blood to the brain, the diminution of blood supply is not great enough to produce these convulsions.

Such violent efforts speedily exhaust the nervous system; and the convulsions after being maintained for a brief period suddenly cease and are followed by a period of calm. The calm is one of exhaustion; the pupils, dilated to the utmost, are unaffected by light; touching the cornca calls forth no movement of the eyclids, and indeed no reflex actions can anywhere be produced by the stimulation of sentient surfaces. All expiratory active movements have ceased; the muscles of the body are flaceid and quiet; and though from time to time the respiratory centre gathers sufficient energy to develop respiratory movements, these resemble those of quiet normal breathing, in being, as far as muscular actions are concerned, almost entircly inspiratory. They oceur at long intervals, like those after section of the vagi; and like them are deep and slow. The exhausted respiratory centre takes some time to develop an inspiratory explosion; but the impulse when it is generated is proportionately strong. It scems as if the resistance which har in each case to be overcome was considerable, and the effort in consequence, when sncecssful, productive of a large effect.

Very soon, these inspiratory efforts become less frequent; their rhythm becomes irregular; long pauses, cach one of which seems a final one, are succeeded by several somewhat rapidly 
repeated inspirations. The pauses become longer, and the inspiratory movements shallower. Each inspiration is accompanied by the contraction of accessory muscles, especially of the face, so that each breatl becomes nore and more a prolonged gasp. 'The inspiratory gasps spread into a convulsive stretching of the whole body; and with extended limbs, and a straightcned trunk, with the head thrown back, the nouth widely open, the face diawn, and the nostrils dilated, the last breatli is taken in.

'Thus we are able to distinguish three stages in the phenomena which result from a continued deficiency of air: (1) A stage of dyspncea, characterized by an increase of the respiratory movements both of inspiration and expiration. (2) A convulsive stage, characterized by the dominance of the expiratory efforts, and culminating in general convulsions. (3) A stage of exhaustion, in which lingering and long-drawn inspirations gradually die out. When brought about by sudden occlusion of the trachea these events run through their course in about 4 or 5 minutes in the $\log$, and in about 3 or 4 minutes in the rabbit. The first stage passes gradually into the second, convulsions appearing at the end of the first minute. 'The transition from the second stage to the third is somewhat abrupt, the convulsions suddenly ceasing early in the second minute. The remaining time is occupied in the third stage.

The duration of asphyxia varies not only in different animals but in the same animal under different circumstances. Newly bor'n and young animals need much longer immersion in water before deatl by asphyxia occur's than do adults. 'Thus while in a full-grown dog recovery from drowning is unusual after

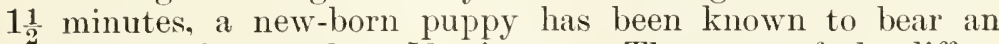
immersion of as much as 50 minutes. The cause of the difference lies in the fact that in the quite young or rather just born animal the respiratory changes of the tissues are much less active. These consume less oxygen, and the general store of oxygen in the blood has a less rapid demand made upon it. The respiratory activity of the tissues may also be lessened by a deficiency in the circulation; hence bodies in a state of syncope at the time when the deprivation of oxygen begins can endure the loss for a much longer period than can bodies in which the circulation is in full swing. There being the same store of oxygen in the blood in each case, the quicker circulation must of necessity bring about the speedier exhaustion of the store. So also anæesthetics may diminish the effects and delay the final results; large doses of anasthetics may prevent the exaggerated and convulsive movements. In many cases of drowning, death is hastened by the entrance of water into the lungs.

By training, the respiratory centre may be accustomed to 
bear a scanty supply of oxygen for a much longer time than usual before dyspnoea sets in, as is seen in the case of divers.

The phenomena of slow asphyxia, where the supply of air is gradually diminished, are fundanentally the same as those resulting from a sudden and total deprivation. The same stages are seen, but their development takes place more slowly.

$\$ 307$. The composition of the atmosphere, the pressure remaining the same, may be modified by the introduction of foreign gases. 'To some of these the respiratory mcchanism is indifferent; for instance, hydrogen may be substituted for nitrogen without any change in the respiration, provided of course that the oxygen is not diminished. Other gases may produce poisonous effects, either by interfering with some of the respiratory processes or in other ways. Thus carbon monoxide, by combining with the hrmoglobin of the red corpuscles, and so preventing the corpuscles from acting as oxygen-carriers, produces asphyxia through deficiency of oxygen. Sulphuretted hydrogen interferes with the oxygenation of the blood by acting as a reducing agent. Some gases while allowing the ordinary respiratory changes of the blood to go on as usual produce toxic effect by acting on one or other of the tissues. 'Thus, as we have seen, an excess of carbonic acid in the blood seems to have a special effect on the central nervous system and so acts as a narcotic poison. The peculiar effects of nitrous oxide (laughing gas) are similarly due to the direct action of the gas in the blood on the central nervous system. Some gases are irrespirable and may interfere with respiration, even causing suffocation, on account of their causing spasm of the glottis, and this is said to be, to a certain extent, the case with an atmosphere which is wholly or largely composed of carbonic acid.

\$308. The Effects of Changes in Atmospheric Pressure. Diminution of pressure. The partial pressure of the oxygen in the inspired air may be changed, not only by altering the composition of the air entering at the ordinary atmospheric pressure, but also by altering the total pressure of the atmosphere without changing its composition. The results of the latter are however complicated; we have then to deal not merely with the effects on the interchange of gases in the lungs but with the effects on the whole organism. All the complicated machinery of the body is adapted and arranged to work under what we may call ordinary atmospheric pressure, that is to say, within the limits of 760 mm. merenry at the sea level and about $500 \mathrm{~mm}$., corresponding to an altitude of 6000 feet, this being the range of ordinary human dwellings. Any great increase or decrease of pressure beyond these limits will affect not only the exit of carbonic acid from and the entrance of oxygen into the blood, but, in varying degree, all the physical and chemical processes of the body. A gross instance of this is seen when an animal is suddenly sub- 
jected to a great diminution of pressure, as when it is placed in the receiver of an air-pump and the receiver rapidly exhausted. The animal is socn thrown into fatal convulsions, which are in part, but only in part, due to the liberation of gas from the blood within the blood vessels; the gas so set free mechanically interferes with the circulation, as by obstructing the play of the cardiac valves, or by plugging the smaller blood vessels, and thus helps to bring the machine to a standstill. The free gas found in the vessels upon examination after death is said to be composed chiefly of nitrogen, the carbonic acid and the oxygen, which probably were also set free, having been reabsorbed before the examination was made.

But, quite apart from gross effects of this kind, it is very obvious that the organism must in many ways suffer from a diminution of pressure. The complex and delicately balanced vascular system is constructed to work at the ordinary atmospheric pressure. The force of the heart-beat and the tonic contraction of the small arteries are, so to speak, pitched to meet the influence exerted on the outside of the blood vessels by the ordinary pressure of the atmosphere; and any great diminution of that pressure must produce a greater or less disarrangement of the vascular mechanism until it is counterbalanced by some compensating changes. And a little reflection will supply many other instances.

We have already called attention ( $\$ 285)$ to the fact that, the total pressure of the atmosphere remaining the same, the partial pressure of the oxygen in the inspired air may be reduced as low as about $76 \mathrm{~mm}$. (10 p.c.) without seriously modifying the respiration. In order to attain this diminution of the partial pressure of the oxygen without changing the composition of the atmosphere, the total pressure of the atmosphere must be reduced to the limit of $300 \mathrm{~mm}$., corresponding to an altitude of 17,000 feet. Now it is a matter of common experience that in ascending a mountain "distress" is felt long before such an altitude is reached. The distress felt on such occasions is probably due not so much, if indeed at all directly, to the diminution of oxygen as to a general disarrangement of the organism and perhaps more particularly of the vascular system. The nose-bleeding which is so frequent an occurrence under the circumstances shews that the minnte blood vessels more directly exposed to the diminution of pressure are profoundly affected by it; and what is true of them is, probably, in various ways and to different degrees true of the whole vascular system. The breathlessness whith is so marked a feature on these occasions seems due not so much to the fact that the blood which reaches the respiratory nervous centres is deficient in oxygen, as to the fact that the troubled vascular system fails to deliver to those centres their blood in an adequate fashion. 
$\$$ 309. The Effects of Increase of Atmospheric Pressure. These are in many ways remarkable. Up to a pressure of several atmospheres of air, the only symptoms which present themselves are those somewhat resembling narcotic poisoning. The animal becomes sleepy and stupid, the result probably not so much of respiratory changes, as of the effects of the increased pressure on the whole organism to which we have just alluded. At a pressure however of 15 atmospheres of air, or what amounts to the same thing, of 3 atmospheres of oxygen, and upwards, a very remarkable phenomenon presents itself. The animals die of asphyxia and convulsions, exactly in the same way as when oxygen is deficient. Corresponding with this it is found that the production of carbonic acid is diminished. 'That is to say, when the pressure of the oxygen is increased beyond a certain limit, the oxidations of the body are diminished, and with a still further increase of the oxygen are arrested altogether. The oxidation of phosphorus is perhaps analogous; at a high pressure of oxygen phosphorus will not burn. Not only animals but plants, bacteria, and organized ferments, are similarly killed by a too great pressure of oxygen. 
SEC. 8. THE RELATIONS OF THE RESPIRATORY SYSTEM TO THE VASCULAR AND OTHER SYSTEMS.

$\$ 310$. Many events in the body shew the influence which the respiratory movements exert on the circulation. When the brain of a living mammal is exposed by the removal of the skull, a rhythmic rise and fall of the cerebral mass, a pulsation of the brain, quite distinct from the movements caused by the pulse in the arteries of the brain, is observed; and upon examination it will be found that these movements are synchronous with the respiratory movements, the brain rising up during expiration and sinking during inspiration. They disappear when the arteries going to the brain are ligatured, or when the venous sinuses of the dura mater are laid open so as to admit of a free escape of the venous blood. They evidently arise from the expiratory movements in some way hindering and the inspiratory movements assisting the return of blood from the brain. We have already ( $\$ 98)$ stated that during inspiration the pressure of blood in the great veins may become negative, i.e. may sink below the pressure of the atmosphere; and a puncture of one of these veins may cause death by air being actually drawn into the vein and thus into the heart during an inspiratory movement. When the veins of an animal are laid bare in the neck and watched, the so-called pulsus venosus may be observed in them, that is, they swell up during expiration and diminish again during inspiration. And indeed a little consideration will shew that the expansion and contraction of the chest must have a decided effect on the flow of blood through the thoracic portion of, and thus indirectly on that through the whole of, the vascular system.

This is well illustrated by the effects of respiration on arterial blood-pressure. We have seen, while treating of the circulation, that the arterial blood-pressure curves are marked by undulations, which, since their rhythm is synchronous with that of the respiratory movements, are evidently in some way connected with respiration. Similar undulations may be observed in the pulse tracings taken from man. 


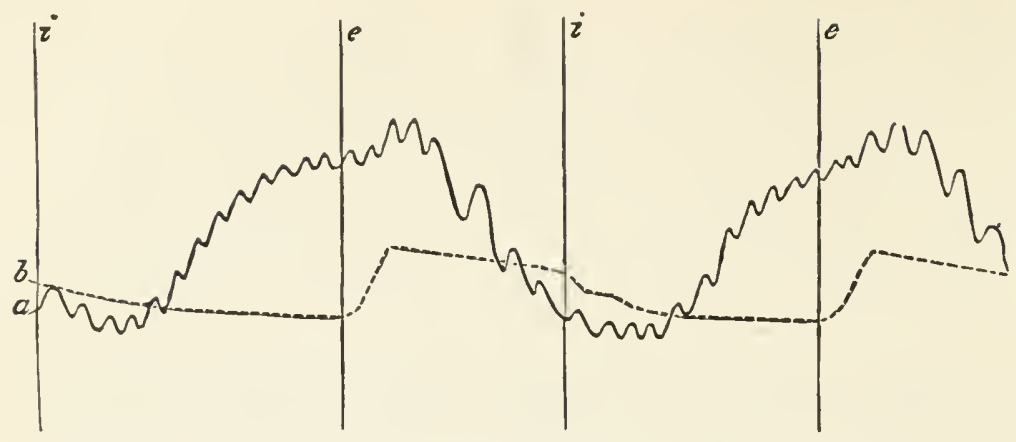

Fig. 99. Comparison of Blood-Presscre Crrve with Curve of Intra-thoracic Pressure. (Dog.)

$a$ is the blood-pressure curve taken by means of a mercury manometer; it shews the respiratory undulations, the slower beats on the descent being very marked. $b$ is the curve of intra-thoracic pressure obtained by connecting one limb of a manometer with the pleural cavity. Inspiration begins at $i$, expiration at $e$. With the beginning of inspiration $(i)$ the expansion of the chest causes a marked fall of the inercury in the intra-thoracic manometer; but the effect soon diminishes, since the lessening of intra-thoracic pressure does not bear on the manometer alone but on the lungs also; and as the lungs expand more and more the fall in the mercury becones less and less until towards the end of inspiration the curve becomes very nearly a straight line. Conversely, the return of the chest at the beginning of expiration (e) produces at first a marked rise of the mercury in the manometer; but this soon ceases as the air leaves the chest and the lungs shrink, whereupon the mercury falls slowly.

When these undulations of the blood-pressure curve are compared carefully with the respiratory movements or with the variations of intra-thoracic pressure, what is most commonly observed is that while the blood-pressure, on the whole, rises during inspiration and falls during expiration neither the rise nor the fall is exactly synchronous with either inspiration or expiration. Fig. 99 shews two tracings from a dog taken at the same time, one, $a$, being the ordinary blood-pressure curve from the carotid, and the other, $b$, representing the condition of the intra-thoracic pressure as obtained by carcfully bringing a manometer into connection with the pleural cavity. On comparing the two curves it is evident that neither the rise nor the fall of arterial pressure coincides exactly either with inspiration or with expiration. At the beginning of inspiration $(i)$ the arterial pressure is seen to be falling; it soon however begins to rise, but does not reach the maximum until some time after expiration (e) has begun; the fall continues during the remainder of expiration, and passes on into the succeeding inspiration. 'This suggests the iclea that, while inspiration tends to increase and expiration to diminish the blood-pressure, there are causes at work which in each case delay the effect.

Extended observations however shew that such a relation as 
that shewn in the figure though frequent is not constant. In fact the effects of the respiratory movements on blood-pressure are found to vary very widely according as the respiration is quick or slow, easy and shallow, or laboured and deep, and especially as the air enters into the chest readily or with difficulty. Moreover, respiratory undulations of blood-pressure are seen not only with natural but also with artificial respiration; in the latter the mechanical conditions are to a large extent the reverse of those of the former, and might fairly be expected to affect the circulation in a different way. The causation of these respiratory undulations is in fact complex. 'The respiratory act affects the vascular system in several different ways, and the general effect varies according as one or other influence is predominant. These several actions are sufficiently intcresting and important to deserve discussion.

$\S 311$. The heart and great blood vessels are, like the lungs, placed in the air-tight thoracic cavity, and are subject like the lungs to the pumping action of the respiratory movements. Were there no lungs present in the chest, the whole force of the expansion of the thorax in inspiration would be directed to drawing blood from the extra-thoracic vessels towards the heart, and conversely in expiration the effect of the retur'n of the thorax to its previous dimensions would be to drive the blood thus drawn in back again from the heart towards the extra-thoracic vessels. And, even in the presence of the lungs, some of this effect is still felt. 'The main purpose and the main result of the expansion of the chest in inspiration is of course to draw air into the lungs; by that expansion the air in the pulmonary alveoli is rarified and brought to a lower pressure than that of the atmosphere outside the chest; and the difference of pressure thus set up leads to an inrush of inspired air until an equilibrium of pressure is established between the air in the lungs and that outside the chest. Before however the inspired air can fill a pulmonary alveolus the elastic walls of the alveolus have to be distended, and that distension is effected by means of the pressure which causes the inspired air to enter. Part of the atmospheric pressure in fact which causes the entrance of the air into the lung is spent in overcoming the elasticity of the pulmonary passages and cells. So that while by the inrush of inspired air the difference of pressure between the air inside the pulmonary alveoli and that outside the chest, brought about by the thoracic expansion, is completely neutralized, the difference between the pressure to which the parts lying within the thorax but outside the lungs are exposed and that outside the chest is not so completely neutralized. The pressure on these parts always falls short of the pressure of the atmosphere by the amount of pressure necessary to counterbalance the elasticity of the pulmonary passages and alveoli. Consequently, any struct- 
ure lying within the thorax but outside the lungs, is never, even at the conchusion of an inspiration when the lungs are filled with air, subject to a pressure as great as that of the atmosphere. And, since the fraction of the atmospheric pressure which is thus spent in distending the lungs increases as the lungs become more and more stretched, it follows that the fuller the inspiration the greater is the difference between the pressure on structures within the thorax but outside the lungs and the ordinary pressure of the atmosphere. Now we have seen that the pressure necessary to counterbalance the elasticity of the lungs, when they are completely at rest (in the pause between expiration and inspiration), is in man about 5 to $7 \mathrm{~mm}$. of mercury, and that when the lungs are fully distended, as at the end of a forcible inspiration, the pressure rises to as much as $30 \mathrm{~mm}$. of mercury. Hence at the height of a forcible inspiration the pressure exerted on the heart and great vessels within the thorax is $30 \mathrm{~mm}$. less than the ordinary atmospheric pressure of $760 \mathrm{~mm}$., and even when the chest is completely at rest, at the end of an expiration, the pressure on the heart and great vessels is slightly (by about $5 \mathrm{~mm}$. mercury) below that of the atmosphere. We may add that any obstacle to the free ingress of the inspired air, any difficulty in the full expansion of the pulmonary alveoli, of course increases the negative pressure to which the thoracic structures outside the lungs are subjected by the expansion of the chest. Hence when the trachea is closed a very large part of the thoracic expansion is directed to increasing the negative pressure around the heart and great blood vessels.

During an inspiration then the pressure around the heart and great blood vessels becomes considerably less than that of the atmosphere on the vessels outside the thorax. During expiration this pressure returns towards that of the atmosphere, but in ordinary breathing never quite reaches it. It is only in forcible expiration that the pressure on the thoracic vascular organs reaches or exceeds that of the atmosphere. But if during inspiration the pressure bearing on the right auricle and the venæe cave becomes less than the pressure which is bearing on the jugular, subclavian, and other veins outside the thorax, this must result in an increased flow from the latter into the former. Hence during each inspiration a larger quantity of blood enters the right side of the heart. This probably leads to a stronger stroke of the heart, and at all events causes a larger quantity to be ejected by the right ventricle ; this causes a larger quantity to escape from the left ventricle, and thus more blood is thrown in to the aorta, and the arterial pressure proportionately increased. During expiration the converse takes place. The pressure on the intra-thoracic blood vessels returns to the normal, the flow of blood from the veins outside the thorax into the vene cava and right auricle is no longer assisted, and in consequence less 
blood passes through the heart into the aorta, and arterial pressure falls again. During forced expiration, the intra-thoracic pressure may be so great as to afford a distinct obstacle to the flow from the veins into the heart.

The cffect of the respiratory movements on the arteries is naturally different from that on the veins. During inspiration the diminution of pressure in the thorax around the aortic arch tends to expand the aortic arch, and thus to check the onward flow of blood, and to diminish the pressure of blood within the aorta. During expiration, the increase of pressure outside the aortic arch of course tends to increase also the blood-pressure within the aorta, acting in fact just in the same way as if the coats of the aorta themselves contracted. Thus as far as arterial blood-pressure is concerned the effects of the respiratory movements on the great veins and great arterics respectively are antagonistic to each other; the effect on the brains being to increase arterial pressure during inspiration and to diminish it during expiration, while the effect on the arteries is to diminish arterial pressure during inspiration and to increase it during expiration. But we should natmally expect the effect on the thin-walled veins to be greater than that on the stout thickwalled arteries, so much so that the direct effect on the arteries may be neglected. That is to say, we should expect the bloodpressure to rise during inspiration and to fall during expiration. 'This as we have seen is frequently the case, and indeed when the breathing is deep and laboured, and especially during violent and sudden respiratory movements, the influence in this direction on the blood-pressure curve of the pumping action of the chest is unmistakeable.

In attcmpting however to estimate the effect of the respiratory movements on blood-pressure we must bear in mind what is taking place in the abdomen. In inspiration the descent of the diaphragm compresses the abdominal viscera, and so, while at the very first it drives a quantity of blood onward along the inferior vena cava, subsequently hinders the upward flow from the abdomen and lower limbs; at the same time by compressing the abdominal aorta, it tends to raise the pressure in the thoracic aorta and its branches, while lowering that of the abdominal aorta and its branches. The effect of easy expiration would be the converse of this; but in forced expiration the pressure of the contracting abdominal muscles would, as an inspiration, first tend to drive the blood onward along the vena cava but subsequently to hinder the flow both along the vena cava and the aorta. The effect of the abdominal movements therefore is mixed and variable, and their influence on the blood-pressure in the femoral artery must be different from that on the radial artery or other branch of the thoracic aorta. It is difficult to predict what in all cases the effect would be; and the matter 
cannot be settled by eliminating the movements of the diaphragm through section of the phrenic nerves, since in such a case the whole working of the respiratory pump is materially affected.

$\$ 312$. In addition to the influence thus exerted by the thoracic movements on the great veins leading to, and the great arteries leading from the heart, we have to consider the bchaviour of the pulmonary vessels themselves under the varying thoracic pressure. These, like the venæ cave and aorta, tend to expand under the influence of the inspiratory expansion of the chest, and thus to become fuller of blood, very much as they would if the whole lung were placed under a large cuppingglass. The first effect of this increased filling of the pulmonary vessels would be to retain for a while a certain quantity of blood in the lungs and thus to lessen the amount falling into the left auricle. But this would be temporary only; and the widening of the pulmonary vessels would speedily produce an exactly contrary effect, namely, an increased flow through the lumgs due to the diminished resistance offered by the widened passages. Conversely, the first effect of expiration would be an increased flow into the left auricle due to the additional quantity of blood driven onwards by the partial collapse of the pulmonary vessels, followed by a more significant diminished flow caused by the greater resistance now offered by the narrower vascular channels. Thus the effect of inspiration in this way would be first to diminish the flow into the left auricle and so into the left ventricle, but afterwards, for the rest of the inspiration until the begimning of expiration, to increase the flow into the ventricle; while converscly the effect of expiration would be first, for a brief period, to increase and afterwards, during the rest of the movement, to diminish the flow of blood into the left ventricle. Further, while this may be considered as the effect on the pulmonary vessels, large and small taken altogether, the influence both of the thoracic negative pressure during inspiration, and the return in a positive direction during expiration, will bear more on the thin-walled pulmonary veins than on the stouter pulmonary artery; that is to say, as inspiration becomes established, there will be a diminution of pressure in the pulmonary veins greater than that in the pulmonary artery, and this will be an additional influence favouring the flow into the left ventricle; during expiration a similar difference of effect will be felt in the contrary direction.

During the increase of flow into the ventricle, the quantity of blood ejected at each stroke will increase, and each stroke will (\$140) be increased in vigour, in consequence of which the arterial pressure will rise. Conversely, during the decrease of flow into the ventricle, the arterial pressure will fall. Hence the general effect of the movements of the clicst on the pulnonary ressels will be during the beginning of in- 
spiration to continue the lowering of arterial pressure which was taking place cluring expiration but subsequently to raise the arterial pressure; and conversely at the beginning of expiration to continue the rise of arterial pressure which was taking place during inspiration but subsequently to lower arterial pressure. In ordinary breathing, as we have seen, what may be considered as the normal relations of blood-pressure to the respiratory movements are precisely of this kind.

$\S 313$. Effects of the respiratory movements, however, are seen not only in natural but also in artificial respiration. When, for instance, in an animal under urari, artificial is substituted for natural respiration, undulations of the blood-pressure curve, synchronous with the respiratory movements, are still observed (Fig. 100), though generally less in extent than those seen under natural conditions.

Now in artificial respiration, the mechanical conditions under which the thoracic viscera are placed as regards pressure are the exact opposite of those existing cluring natural respiration, for when air is blown into the trachea to distend the lungs, the pressure within the chest is increased instead of diminished. Under these circumstances, applying the considerations laid down in the preceding paragraph with regard to natural respiration, we should expect to find that while the first effect of an artificial inspiration would be to drive an additional quantity of blood out of the lungs into the left ventricle, and thus to raise arterial pressure, this would be in turn followed by a fall of arterial pressure due to the increased resistance offered both to the passage of blood through the lungs and to the entrance of blood through the venæ cavie into the right auricle. Conversely, the effect of the succeeding expiration would be an initial continuance of the fall of arterial pressure succeeded by a rise. In other words, we should expect to find in artificial respiration effects exactly the reverse of those which we find in normal respiration; and indeed in many curves of blood-pressure taken during artificial respiration this is the case.

According to the explanation given above, the total effect of each respiratory movement, both of inspiration and expiration, whether natural or artificial, being the result of two factors acting in contrary directions, one an initial one acting only at the mere establishment of inspiration or expiration, the other sequent and acting during the continuance of the inspiratory or expiratory phase, ought to differ according to the character of the respiratory movement. If, for instance, the respiration is rapid, and each movement brief, the first factor will be more prominent than the second; on the contrary the second factor will be prominent if the respiration be slow and each phase be prolonged; and the total effects will differ in the two cases. We should expect therefore to find, what we do find, that both 
in natural and in artificial respiration, the features of the bloodpressure eurve vary according as the breathing is hurried or slow, shallow or deep, and aecording to the facility with whieh air cnters the chest. So much so indeed is this the ease that at times the blood-pressure eurves of natural and artifieial respiration may closely resemble each other.

$\S 314$. We have even in normal quiet breathing indieations of anther kind of influence of respiration on the beat of the heart. One striking feature of the respiratory undulation in the blood-pressure curve of the $\log$ is the faet that the pulserate is quiekened during the rise of the undulation and becomes slower cluring the fall; see Fig. 99. A similar influence may be seen in the blood-pressure eurves of some other animals, but is slight or absent in others, sueh as the rabbit; it may be recognized in pulse-traeings taken from man. Now this influenee is at once done away with, without any other essential change in the undulations, by section of both vagus nerves. Evidently the slower pulse during the fall is eaused by a eoineident stimulation of the cardio-inhibitory centre in the spinal bulb, the quicker pulse during the rise being due to the faet that, during that interval, the centre is eomparatively at rest. IVe have here indieations that, while the respiratory eentre in the spinal bulb is at work, sending out rhythmie impulses of inspiration and expiration, the neighbouring eardio-inhibitory eentre is, as it were by sympathy, thrown into an aetivity of sueh a kind that its influenee over the heart waxes with eaeh expiration and wanes with each inspiration.

$\$ 315$. Besides the mechanical effeets of the respiratory movements the vascular system is influeneed by respiration through the ehanges in the gases of the blood. The many and varied changes whieh take plaee in the vaseular system when the blood fails to be duly arterialized are well brought out by a study of asphyxia. The exaggerated respiratory movements and convulsive struggles whieh are characteristie of this condition, introduce meehanieal and other complieations whieh it may be well in the first instanee to eliminate; this can readily be done by plaeing the animal under urari. If in an animal (dog) under urari the artificial respiration, neeessary under the cireumstances for the due arterialization of the blood, be stopped the blood-pressure curve soon shews striking changes, ef. Fig. 100. The mean pressure after a brief period, the length of which depends on the character of the previous artifieial respiration, begins to rise, and eontinues to rise, at first slowly, afterwards more rapidly, until finally it may reach the double or even more of its previous height. On the curve of pressure the indications of the heart-beats are conspieuous. This is due on the one hand to the rlyythm of the heart being slowed, and on the other hand to the output at each beat, as shewn by direct observation 
with the cardiometer, being increased. The slowing of the rhythm is in part due to vagus inhibitory action, the too venous blood exciting the bulbar cardio-inhibitory centre; for the effect is much less when both vagus nerves are divided. But as illustrated by Fig. 100, which is a curve of blood-pressure
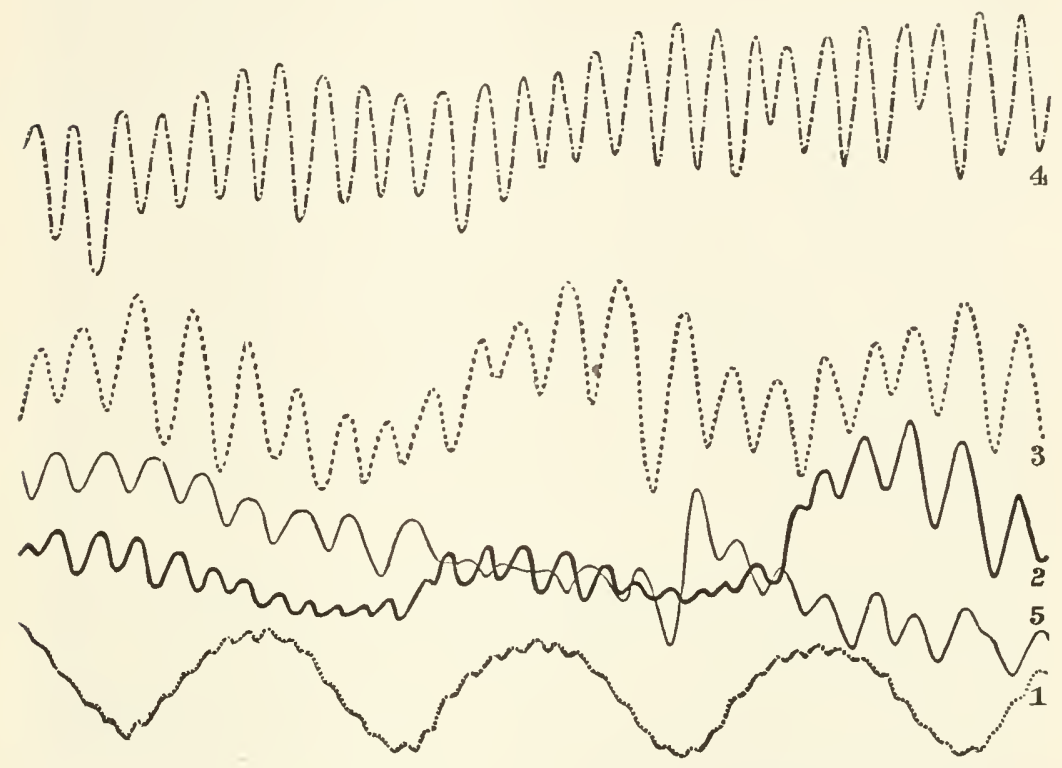

Fig. 100. Blood-Pressure Curves dering a Suspension of Breathing. Traube-Hering Curves.

The curves $1,2,3,4,5$ are portions selected from one long continuous tracing forming the record of a prolonged observation, so that the several curves represent successive stages of the same experiment. Each curve is placed in its proper position relative to the base line, which, to save space, is omitted ; and it is obvious that, starting from the stage represented by 1 , the blood-pressure rises in stages 2, 3, and 4, but falls again in stage 5. Curve 1 is taken from a period when artificial respiration was being kept up, and the undulations visible are those the nature of which has been discussed; the vagus nerves having been cut the pulsations on the ascent and descent of the undulations do not differ. When the artificial respiration was suspended these undulations disappeared, and the blood-pressure rose steadily while the heart-beats became slower. Soon, as shewn in curve 2, new undulations appeared. A little later, the blood-pressure was still rising, the heart-beats still slower, but the undulations still more obvious (curve 3). Still later (curve 4), the pressure was still higher, but the heart-beats were quicker, and the undulations flatter. The pressure then began to fall rapidly (curve 5), and continued to fall until some time later artificial respiration was resumed.

during asphyxia after division of both vagus nerves, the effect is not wholly done away; the slowing is in part due to other causes, but what these are is not very clear.

These changes in the heart in no way explain the rise of 
pressure; that is obviously due to a very great increase of peripheral resistance, the heart contributing to the result only so far that its output does not diminish as the peripheral resistance increases, or indeed may at first, at least, somewhat increase. That the peripheral resistance is due to a large vaso-constriction brought about by the too venous blood stimulating the bulbar vaso-motor centre is shewn by the fact that the rise of pressure is far less, indeed very small, if the cord be divided below the bulb; only a small part, at most, of the peripheral resistance can be attributed to the difficulty which the blood, on account of its increasing venosity, finds in passing through the capillaries ( $\$ 163)$.

If a limb be placed in a plethysmograph during this rise of pressure, its volume is found to increase ; and the same is true of the brain. This shews that the vaso-constriction does not take place to any great extęnt in the skin (or the muscles) of the limb or in the brain. No such increase of volume is seen in the kidney or other abdominal organs. Hence we may conclude that the vaso-constriction is, in the main, one of the splanchnic area and not of the skin, or indeed of the rest of the body.

If the pressure in the pulmonary artery be examined this is found to increase, even out of proportion to the increase of the systemic pressure. We may infer that the peripheral resistance in the lungs is very largely increased; and we may also probably infer that the resistance is due to vaso-constriction, though possibly the too venous blood may find increased difficulty in traversing the pulmonary capillaries.

The high arterial pressure both on the left and right sides leads to great distension of the ventricles, and this is still further inereased on the right side by the large quantity of blood which the high systemic pressure is able to discharge into the venæe cavæ through the vascular areas in which no vaso-constriction is taking place.

These then are the main features of the circulation during asphyxia (under urari): high systemic pressure due chiefly to vaso-constriction in the splanchnic area; high pulmonary pressure due to high pulmonary resistance, working against an ample supply of venous blood to the right ventricle; a heart beating slowly, but with increased output, and increasing distension of both ventricles (leading to distension of the auricles) but greater perhaps on the right side.

This state of things however lasts for a certain time only; the blood-pressure soon begins to fall, and falling rapidly soon becomes very low. The diminished energy of the heart-beats, the output at the systole diminishing greatly though the diastolic distension remains, is sufficient to account for this fall; and indeed that the fall is not due to lessening of the periph- 
eral resistance by slackening of the vaso-constriction is shewn by the fact that if the artificial respiration be resumed while this fall is taking place, or when it has taken place, the pressure at once rises again very rapidly as the heart recovers its power, shewing that the vaso-constriction is still at work. 'The diminished energy of the heart beat is due to the nutrition of the cardiac tissue suffering under the increasing venosity of the blood, and if the air continue to fail to get access to the blood in the lungs, the heart finally ceases to beat. The right side, by virtue of what appears to be an inherent quality, continues to beat rather longer than the left; but the pulmonary peripheral resistance continuing, the efforts of the right ventricle to empty itself are ineffectual; and at death it is the right side which is especially distended.

In an animal, not under urari, and dying by asphyxia in an ordinary way, the phenomena are in the main the same as those of which we have just given a sketch; but as we have said the exaggerated respiratory movements, and especially the convulsive struggles, in which these culminate, introduce complications. Perhaps the most marked of these is the increased venous inflow to the right side of the heart, of which these movements are the cause, for as we have seen all the movements in question augment the flow along the veins to the heart. But the pulmonary peripheral resistance is a bar to the progress to the left side, and hence the right side becomes increasingly distended.

During asphyxia, under urari, the blood-pressure curve shews other certain interesting features deserving of attention.

Upon the cessation of the artificial respiration, the respiratory undulations of course cease also, so that the blood-pressure curve rises at first steadily broken only by the heart-beats; yet after a while new undulations, the so-called Traube or Traube-Hering curves, make their appearance (Fig. 100, 2, 3), similar to the previous ones, except that their curves are larger and of a more sweeping character. These new undulations, since they appear in the absence of all thoracic or pulmonary movements, passive or active, and are witnessed even when both vagi are cut, must be of vaso-motorial origin; the rhythmic rise must be due to a rhythmic constriction of the small arteries; and this probably is caused by a rhythmic discharge from vaso-motor centres, and especially from the bulbar vaso-motor centre. The undulations are maintained so long as the blood-pressure continues to rise. With the increasing venosity of the blood, the vaso-motor centres become enfeebled and the undulations disappear.

We may here incidentally remark that the occurrence of long slow undulations is not dependent on the cessation of the respiratory movements, and on an abnormally venous condition of the blood. They are sometimes (Fig. 101) seen in an animal 
whose breathing is fairly normal. We need not discuss them any further now, and have introduced them chiefly to illustrate the fact that the vaso-motor nervous system is apt to fall into a condition of rhythmic activity.

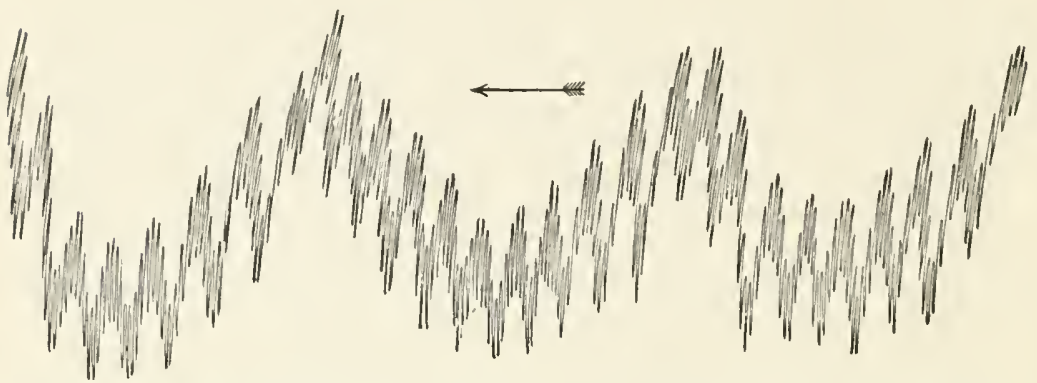

Fig. 101. Bloon-Pressure Curve of a Rabit, recorded on a slowly moving surface, to shew Traube-Hering Curves.

('The curve was described not by means of a mercury manometer, but by an instrument similar to but not identical with Fick's spring-kymograph.) In each heart-beat the upward and downward stroke are very close together but may be easily distinguished by the help of a lens. The undulations of the next order are those of respiration. The wider sweeps are the Traube-Hering curves, of which two complete curves and portions of two others are shewn. Fach TraubeHering curve comprises about nine respiratory curves, and each respiratory curve about the same number of heart-beats.

\$ 316. While changes occurring primarily in the respiratory system thits affect the vascular system, conversely changes oceuring primarily in the vascular system affect the respiratory system.

Of these the most common and important however are changes in the circulation through the lungs. In the normal organism an adequate stuply of arterial blood to the tissues is seeured by an adequate renewal of the air in the pulmonary alveoli and an adequately rapid flow of blood through the pulmonary capillaries. When, as by obstruction in the pulmonary arteries, or by failure of the cardiac valves, or, and perhaps especially, by an insufficient cardiac stroke, the stream of blood from the lungs into the left ventricle is lessened either in amount or in rapidity, less oxygen is carried to the tissues, including the nervous tissue of the spinal bulb, and dyspncea or "want of breath" follows. When the circulation through the lungs is in full healthy swing, the hamoglobin of the red corpuscles is as we have scen saturated or nearly saturated with oxygen. If owing to a slower stream the red corpuseles tarry longer in their passage along the walls of the pulmonary alveoli they cannot thereby take up a compensating addition of oxygen, indeed it is doubtful if they can take up any additional oxygen at all. The blood falling under these circumstances 
into the left ventricle and sent thence over the body is not more arterial than usual; at the same time the amount of blood sent out at each heart stroke is less, often much less, than the normal; and the spinal bulb as well as the other tissues suffer in consequence from a deficiency of oxygen. The deficient supply to the bulb manifests itself in dyspnoeic or at least in laboured breathing, which sometimes through the mechanieal influences discussed above has the happy result of improving the pulmonary circulation and so produces compensating effects. When the pulmonary artery is suddenly plugged with a clot the primary and urgent symptom is "want of breath," though air enters freely into the chest; and "cardiac dyspnoea" is a common symptom of cardiac disease.

$\$ 317$. Other systems of the body are also related to the respiratory system, though by ties less striking than those which bind to it the vascular system. We have seen that deficient arterialization of the blood stirs up the museles of the alimentary canal to increased activity, and we shall presently see that the same condition has a notable effect in promoting the perspiration; it probably has a similar influence over other secretions. On the other hand, as we have seen $\$ 303$, there are reasons for thinking that the activity of the respiratory centre and so the energy of the whole respiratory act is influenced by chemical changes, other than the decrcase of oxygen and increase of carbonic acid, brought about in the blood by the activity of the skeletal muscles.

The closeness and the intricacy of the ties which thus connect the respiratory system with almost all parts of the body may be illustrated by considering the effects of muscular work on the body, and the conditions which, apart from the eapacity of the muscles themselves and of the motor nervous apparatus which puts them to work, deternine the power of the body to do work. During work, especially arduous work, the muscular contractions rob the blood of much oxygen and load it with much carbonic acid. This change in the blood would itself increase the activity of the respiratory centre and the energy of the respiratory movements, and might be sufficient to secure such an increase of these movements that the deficiency of oxygen and increase of carbonic acid should never overstep certain limits. But, as we have said, apparently other products of muscular metabolism act so potently in stimulating the respiratory centre that the respiratory movements are more than sufficient to compensate the changes in the gases of the blood. The efficacy of the augmented respiratory movements is much increased by a concomitant increase in cardiac activity and a swifter or fuller stream of blood through the lungs; indeed unless backed up by the cardiac increase the mere incrcase of the pulmonary ventilation might prove inadequate. 
Hence the capacity for arduous muscular labour is determined not by the respiratory mechanism alone, nor by the vascular system alone, but by both, and especially by both working together in harmony and concert. The increased rentilation would be idle unless it were accompanied by a quicker circulation, and the quicker circulation would similarly be of comparatively little use unless accompanicd by increased ventilation. 'To a bystander the working of the respiratory pump is much more obvious than that of the vascular system, and indeed the subject himself is much more directly conscious of changes in the former than of changes in the latter. Hence when the organism ceases to be able to meet the demands which the labour is making upon it, the subject is said to be "out of breath," though in a large number of cases the failure lies much more at the door of the vascular than of the respiratory system. And, as a rule, it may perhajs be said that when two men differ in their capacity for strenuous work. such as rumning a race, the difference, though it is often familiarly spoken of as one of "wind" or power of breathing, is in reality not a difference in ventilating capacity but a difference in the power of the heart to keep up to and work in harmony with the increased respiratory movements.

Thus there arc two main factors in respiration, the respiratory mechanism proper, and the circulation, the one bringing the air to the blood, and the other the blood to the air. We may remind the reader that there is also a third factor, and that one of great moment, the amount of hemoglobin, that is, the number of red corpuscles, in the blood. The amount of oxygen taken up from the lungs depends not only on the strokes of the respiratory and the vascular pumps but also on the richness of the blood in red corpuscles. A body which from loss of blood or from disease is anæmic is thrown out of breath by very slight exertion, not so much because the respiratory or the vascular pump is weak, but because, through lack of oxygen-carriers, with their best efforts the combined pumps can only deliver to the tissues, including the medulla, an inadequate supply of oxygen. And fat persons, whose store of hremoglobin in proportion to their body weight is always below par, are proverbially "scant of breath." 


\section{SEC. 9. MODIFIED RESPIRATORY MOVEMENTS.}

$\S 318$. The respiratory mechanism with its adjuncts, in addition to its respiratory function, becomes of service, especially in the case of man, as a means of expressing emotions. The respiratory column of air, moreover, in its exit from the chest, is frequently made use of in a mechanical way to expel bodies from the upper air-passages. Hence arise a number of peculiarly modified and more or less complicated respiratory movements, sighing, coughing, laughter, \&c. adapted to secure special ends which are not distinctly respiratory. They are all essentially reflex in character, the stimulus determining each movement, sometimes affecting a peripheral afferent nerve as in the case of coughing, sometimes working through the higher parts of the brain as in laughter and erying, sometimes possibly, as in yawning and sighing, acting on the respiratory centre itself. Like the simple respiratory act, they may with more or less success be carried out by a direct effort of the will.

Sighing is a deep and long-drawn inspiration, chiefly through the nose, followed by a somewhat shorter, but correspondingly large expiration.

Yawning is similarly a deep inspiration, deeper and longer continued than a sigh, drawn through the widely open mouth, and accompanied by a peculiar depression of the lower jaw and frequently by an elevation of the shoulders.

Hiccough consists in a sudden inspiratory contraction of the diaphragm, in the course of which the glottis suddenly closes, so that the further entrance of air into the chest is prevented, while the impulse of the column of air just entering, as it strikes upon the closed glottis, gives rise to a well-known accompanying sound. The afferent impulses of the reflex act are conveyed by the gastric branches of the vagus. The closure of the glottis is carried out by means of the inferior laryngeal nerve. See Voice.

In sobbing a series of similar convulsive inspirations follow each other slowly, the glottis being closed earlier than in the case of hiccough so that little or no air enters into the chest. 
Coughing consists in the first place of a deep and long-drawn inspiration by which the lungs are well filled with air. This is followed by a complete closure of the glottis. and then comes a sudden and forcible expiration. in the midst of which the glottis suddenly opens, and thus a blast of air is dricen through the upper respiratory passages. The afferent impulses of this reflex act are in most cases, as when a foreign bodr is lodged in the larrnx or by the side of the epiglottis. convered br the superior larrngeal nerve; but the movement mav arise from stimuli applied to other afferent branches of the ragus. such as those supplying the bronchial passages and stomach and the auricular branch distributed to the meatus externus. Stimulation of other nerves also, such as those of the skin by a draught of cold air. may develop a cough.

In sneezing the morement is the same, in so far that it consists of a deep inspiration followed by a sudden and forcible expiration. But the mouth. instead of being widely open as in coughing. is partly. or at first even wholly closed. and the buccal carity with the pharrnx is so disposed that the blast of air in being driven out through the mouth produces the characteristic sound. If the obstruction. the sudden remoral of which initiates the expiratory blast. is caused br closure of the glottis. and this is not clear. the glottis is so disposed as not to give rise to a rocal sound as is the case in coughing. Though the movement is accompanied by secretion from the nasal passages. the outgoing blast appears not to pass through the nose. being cut off from that passage by eleration and pressing back of the soft palate. The afferent impulses here usually come from the nasal branches of the fifth. When sneezing howerer is produced by a bright light. the optic nerve would seem to be the afferent nerve.

Luughing consists essentially in an inspiration succeeded. not by one. but br a whole series. often long continued. of short spasmodic expirations. the glottis being freely open during the whole time. and the rocal cords being thrown into characteristic vibrations.

In crying. the respiratorr morements are modified in the same way as in laughing: the rhythm and the accompanying facial expressions are howerer different. though laughing and (rring frequently become indistinguishable. 


\section{CHAPTER III.}

\section{THE ELIMIATION OF WASTE PRODUCTS.}

\$ 319. WE have traced the food from the alimentary canal into the blood. and. did the state of our knowledge permit. the natural course of our studr would be to trace the food from the blood into the tissues. and then to follow the products of the activity of the tissues back into the blood and so out of the body. This howerer we cannot as ret satisfactorily do: and it will be more convenient to study first the final products of the metabolism of the bodr. and the manner in which they are eliminated. and afterwards to return to the discussion of the intervening steps.

Our food consists of certain food-stuffs. riz. proteids. fats. and carbohrdrates. of rarious salts. and of water. In their passage through the blood and tissues of the body. the proteids. fats. and carbohrdrates are converted into urea (or some closely allied bodr). carbonic acid and water. the nitrogen of the urea being furnished by the proteids alone. Many of the proteids contain sulphur. and also have phosphorus attached to them in some combination or other. and some of the fats taken as food contain phosphorus: these elements ultimately undergo oxidation into plosphates and sulphates. and leave the body in that form in company with the other salts.

Broadly speaking then. the waste products of the animal economr are urea. carbonic acid. salts and water. These leave the bodr by one or other of three main channels. the lungs. the skin. and the kidner. Some part. it is true. leaves the bodr br the bowels. for. as we have seen. the fieces contain. besides undigested portions of food. substances which have been secreted into the bowel. and are therefore waste products; but the amount of these is so small that ther mar be neglected.

The lungs serre as the channel for the discharge of the greater part of the carbonic acid. and a considerable quantity of water: this discharge we have just studied. Through the skin 
there leave the body a comparatively small quantity of salts, a little carbonic acid, and a variable but on the whole large quantity of water.

The kidneys discharge all or nearly all the urea and allied bodies, the greater portion of the salts, and a large amount of water, with an insignificant quantity of carbonic acid. They are especially important since by them practically all the nitrogenous waste leaves the body, and to them we will turn first. 


\section{SEC. 1. THE COMPOSITION AND CHARACTERS OF URINE.}

$\$ 320$. These are so fully dwelt upon in special works that we may confine ourselves here to salient points. The healthy urine of man is a clear yellowish slightly fluorescent fluid, of a peculiar odour, saline taste, and acid reaction, having a mean specific gravity of 1.020 , and generally holding in suspense a little mucus. The mucus, when present, comes from the urinary passages, as do also the occasional epithelial cells. All the rest of the urine may be considered as the secretion of the kidney.

The urine as we have said is the chief channel by which solid matters leave the body, a small quantity only passing by the skin and practically none by the lungs. Hence, neglecting for the present the skin, we may say that all the substances taken into the body sooner or later leave the body by the urine, save the few substances which may be retained permanently within the body and the substances which make up the body at the moment of its death. We accordingly find that the urine contains a large number of substances, the exact amount of each substance present in a given quantity of urine varying, in the case of every substance somewhat, and in the cases of many substances very largely, from time to time. The composition of urine is not only complex but extremely variable.

Moreover a little consideration will shew that the several substances present in urine must have very different histories. Some of the constituents of urine appear in it in the exact form in which they were introduced into the mouth; they have been simply absorbed from the alimentary canal into the blood and excreted by the kidney without undergoing change; they are derived directly and without change from the food.

Others again are the products of changes which the food has undergone in the body; and these clanges may be slight or may be extensive, and may take place on the one hand in the alimentary canal, or during a brief transit of the substance in the blood-stream, or even in the urine itself, may so to speak be 
superficial; or on the other hand may take place in the very depths of the tissues and be closely associated with the very life of the tissues. Wr shall, however. have to return to these matter's later on. and may here briefly consider what substances are. normally and abnorinally, present in urine, and the chief features of the fluid itsclf.

$\$ 321$. Besides water, the constituents of urine are:-

Nitrogenous Crystalline Bodies. Neglecting the small proportion of these bodies which, espccially in the case of flesh eaters, are introdnced into the economy with the food, as kreatin and the like, and so pass into the urine with no or with comparatively little change, we may on the whole regard the substances of this class as the products of the changes which the proteid matter's (and allied substances such as gelatin and the like) present in food have undergone either while the food was simply food, still in the alimentary canal for instance, or after the food had been built up into the tissues of the body.

Of these by far the most important, in the urine of man and mammalia, is the body urea $\left(\mathrm{N}_{2} \mathrm{H}_{4} \mathrm{CO}\right)$. It is the chief form in which, in these animals, nitrogen leaves the body. WV shall have to discuss the relations and formation of urea later on, but meanwhile we will simply state that it has remarkable double connections with two great groups. On the one hand it is related to the ammonia group, and by hydration is readily converted into ammonium carbonate $\left(\mathrm{N}_{2} \mathrm{H}_{4} \mathrm{CO}+2 \mathrm{H}_{2} \mathrm{O}=\right.$ $\left.\left(\mathrm{NH}_{4}\right)_{2} \mathrm{CO}_{3}\right)$. On the other hand it is related to the great cyanogen group, ammonium cyanate and urea being isomeric, and the former by simple heating being converted into the latter $\left(\mathrm{NH}_{4} \cdot \mathrm{CNO}=\mathrm{N}_{2} \mathrm{H}_{4} \mathrm{CO}\right)$.

'Though a base, forming salts with acids, such as nitrates, oxalates, \&c. urea occurs in urine in a free and independent condition.

Closely allied to urea, occurring apparently as a bye product of the same line of metabolism, is uric acid $\left(\mathrm{C}_{5} \mathrm{H}_{4} \mathrm{~N}_{4} \mathrm{O}_{3}\right)$, which is found always in the urine of man, oceurring in small but variable quantity. In the urine of some animals such as birds and reptiles it occurs in abundance, and indeed in these replaces urea as the chicf nitrogenous exeretion. Uric acid is a more complex body than urea, one molecule of uric acid splitting up, moler the influence of certain reagents, into two molecules of urea and a componnd of oxalic acid. Its decomposition products however, under different reagents, are very numerous and complex though urea occurs among them frequently and characteristically. Uric acid may be synthetically produced out of urea and glycin (glycocoll).

It is a weak dibasic acid, and ocenrs in normal human urine, not as a free acid but as an acid salt, being combined with potassium and solium, and to a less cxtent with calcium and am- 
monium. In quite normal urine these salts are soluble in the urine, even after the fluid has cooled down to the ordinary temperature of the air; but not infrequently the urates, soluble in the urine at the temperature at which it leaves the body, are precipitated when the fluid cools, forming the well-known " deposit of urates." On further standing the salts are apt to be decomposed and thus to give rise to erystals of uric acid.

Besides urea and uric acid the urine contains small but variable quantities of more or less nearly allied bodies such as kreatinin, xanthin, hypoxanthin, and guanin. Concerning these we will at present only say that kreatinin is a dehydrated form of the body kreatin which we spoke of $(\$ 59)$ as a constituent of muscles. Kreatin by dehydration is readily converted into kreatinin, and kreatinin by hydration into kreatin; kreatin introduced into the alimentary canal or into the blood appears in the urine as kreatinin; and in flesh eaters some at least of the kreatinin of the urine is derived directly from the kreatin present in the meat eaten as food; but we shall discuss the subject of kreatin later on.

Besides the above, such bodies as leucin, taurin, cystin, allantoin and ammonium oxalurate are occasionally found in urine, but cannot be regarded as constituents of normal urine.

In the mine of man hiphuric acid appears to be always present in small quantities, and in the urine of herbivora occurs in large quantities. In these latter it is derived more or less directly, by changes of which we shall have to speak in a succeeding chapter, from constituents of the food containing bodies belonging to the aromatic group (benzoic acid series); but the small quantity present in man and other carnivora appears to come from the metabolism of proteid matter which, as we have already seen, contains an aromatic constituent. Another member of the aromatic group, tyrosin, is oceasionally present in urine.

A special intcrest belongs to certain compounds, which may be regarded as, in small quantities, normal constituents of urine, but which may oceur in much larger quantities; these are certain phenol compounds such as phenyl-sulphuric acid, certain indigo compounds, the so-called indican, and others. These arise from bodies appearing in the alimentary canal as products of the decomposition of proteids, effected not by natural juices, but by micro-organisms. $\$ \$ 210,232$. Their amount in the urine may be taken as a measure of the extent to which proteids are being changed by these agents in the alimentary canal.

322. Inorganic Salts. These for the most part exist in urine in natural solution. the composition of the ash almost exactly corresponding with the results of the direct analysis of the fluid ; in this respect urine contrasts forcibly with blood, the ash of which is largely composed of inorganic substances, which previous to the incineration existed in peculiar combination with 
proteid and other complex bodies. In the ash of urine there is rather more sulphur than corresponds to the sulphuric acid directly determined; this indicates the existence in urine of some sulphur-holding complex body. And there are traces of iron, pointing to some similar iron-holding substance. But otherwise, all the substances found in the ash exist as salts in the natural fluid.

The chief bases are sodium, potassium, calcium and magnesium in the form of chlorides, phosphates and sulphates. The exact way in which the several bases and acids are combined is to some extent a matter of uncertainty; but sodium chloride is certainly present and in considerable quantity; it is the most abundant and important inorganic constituent. A large portion of the phosphoric acid seems to exist as acid sodium phosphate, the rest as soluble calcium and magnesium phosphates. The remaining chief salts, occurring however in smaller quantity, are potassium and sodium sulphate, and calcium chloride.

Ammonia occurs in small quantity, alkaline carbonates are frequently found, traces of nitrates are at all events occasionally present, as also indications of silicates and of sulphocyanates.

The phosphates are derived partly from the phosphates taken as such in food, partly from the phosphorus or phosphates peculiarly associated with the proteids, and partly from the phosphorus of certain complex fats such as lecithin. When urine becomes alkaline (and, as we shall presently see, it may do so by changes taking place in itself) the calcic and magnesic phosphates are converted into basic salts which, being insoluble, are precipitated, the sodium phosphate remaining in solution. When the alkalinity, as is frequently the case, is due to ammonia, ammonio-magnesium phosphate is formed and is apt to appear in crystals. The sulphates are derived partly from the sulphates taken as such in food and partly from the sulphur of the proteids. 'The carbonates, when occurring in large quantity, generally lave their origin in the oxidation of such salts as citrates, tartrates, \&c. The bases present depend largely on the nature of the food taken. 'Thus with a vegetable diet, the excess of the alkalis in the food reappears in the urine; with an animal diet, the earthy bases in a similar way come to the front.

\$323. Non-nitrogenous Bodies. These exist in very small quantities, and many of them are probably of uncertain occurrence. Some of these are organic acids, the most constant perhaps being oxalic acid; to this may be added glycerinphosphoric, lactic, formic, acetic, butyric and possibly succinic acids. Inosit has also been said to occur normally. It has been maintained that minute quantities of sugar (dextrose) are 
invariably present in even healthy urine; this however has not as yet been placed beyond all doubt. 'The nature of the substances which give to urine its characteristic odour has not been male out; probably there are more such bodies than one.

$\$ 324$. Pigments. Urine is always coloured, the tint varying from a light to a dark yellow with an admixture of brown. In the course of twenty-four hours, a not inconsiderable quantity of pigment must leave the body by the urine; but the nature of the normal pigment or pigments of urine is at present obscure and the subject of much controversy. 'The matter is apparently further complicated by the presence in urine of what have been called 'chromogens', that is to say, bodies which are not coloured themselves but which readily give rise to pigments upon oxidation; and it is probable that some of these 'chromogens' of the urine are reduction products of the respective pigments, the reduction taking place in the urine after secretion, or during or even before secretion. 'There is frequently present in urine, especially in cases of fever, a pigment which has been isolated and determined, which has a characteristic spectrum, and which being maintained by some to be a derivative of bilirubin, has been called urobilin. It is not this urobilin however which gives to urine its ordinary colour. Some observers, on the other hand, maintain that normal urine does contain and, in part at least, owes its normal colour to a somewhat similar but different body, which in consequence they have called 'normal' urobilin. It is in fact not possible, at the present moment, to make definite and satisfactory statements as to whether urine contains one or more than one normal pigment, as to its or their nature, as to whether they are derived from bile-pigment or directly from the hrmatin of hamoglobin or in other ways, or as to the several steps by which they are produced. There are also abnormal colouring matters present on occasion, such for instance as the peculiar red colouring matter occurring sometimes in the urine of acute rheumatism, which has been called uroerythrin ; but our knowledge concerning these is very imperfect.

$\$ 325$. Ferments and other bodies. Even normal urine has frequently been found to contain a small quantity, hardly amounting to more than a trace, of proteid material, apparently an albumin ; but the normal presence of even this small quantity has been disputed. Urine, however, certainly contains ferment bodies.

When urine is treated with many times its volume of alcohol, a granular or floceulent precipitate is thrown down, consisting chicfly of phosphates, together with some other substance or probably several other substances, in very small quantities. An aqueous solution of the precipitate, which may be freed from the phosphates, is both amylolytic and proteolytic. Ferments 
mas also and more readily be extrated from urine by allowing shreds of fibrin to soak in the urine for a few hours, and then removing and washing them. The ferments become entangled in the fibrin in such a way as not to be easily removed by washing. 'The washed shreds will convert starch into sugar ; and when treated with dilute hydrochloric acid digest themselves, shewing the presence of pepsin. By this method it has bcen ascertained that an anylolytic ferment and pepsin are present in quantities which vary in the twenty-four hours according to the meals. Remnin has also been found, and at times at least, trypsin. From this it appears that some of the ferments of the alimentary canal cscape from the body by the urine, being probably re-absorbed directly from the respeetive glands; the quantity moreover which thus eseapes is insignificant.

A small quantity of gas, about 15 vols. p.c., can be extracted by the mercurial pump from urine received direct from the body without exposure to air. The gas so obtained consists chiefly of carbonic acid, nitrogen being very scanty, and oxygen oceurring in very small quantities or being wholly absent. The meaning of this we have already touched upon in speaking of respliration, see \$290.

$\$ 326$. The quantities in which these multifarious bodies, all of which as we have seen we may perhaps regard as constituents of normal urine, are present in different specimens of urine, vary within very wide limits, being dependent on the nature of the food taken, and on the conditions of the body. The amount not of water only, but of many of the other several constituents, varies widely and indeed rapidly, so that the percentage eomposition of urine will vary from hour to hour if not from minute to minute. The causes which determine these variations in the nature and amount of urine we shall study later on. Meanwhile what may be called the average composition of human urine is shewn in the following table in which the acids and bases are put down separately.

AMOUNTS OF THE SEVERAL URINARY CONSTITUENTS PASSED IN TWENTY-FOUR HOURS. (After PARKEs.)

By an average man of 66 kilos.

Water

Total Solids

Urea

Uric Acid

$33 \cdot 180$

.555

Hippuric Acid

Kreatinin

$\cdot 400$

.910

Pigment, and

other substances $10 \cdot 000$
Per 1 kilo

of body weight.

$1500 \cdot 000$ grammes 23.0000 grammes

$1 \cdot 1000$

$\cdot 5000$

.0084

.0060

$\cdot 0140$ 
By an average

man of 66 kilos.

Total Solids (continued)

$\begin{array}{lr}\text { Sulphuric Acid } & 2 \cdot 012 \\ \text { Phosphoric Acid } & 3.164 \\ \text { Chlorine } & 7 \cdot 000 \\ & (8 \cdot 21) \\ \text { Ammonia } & .770 \\ \text { Potassium } & 2 \cdot 500 \\ \text { Sodium } & 11 \cdot 090 \\ \text { Calcium } & \cdot 260 \\ \text { Magnesium } & \cdot 207\end{array}$

Per 1 kilo

of body weight.

$\cdot 0305$

$\cdot 0480$

$\cdot 1260$

\section{$72 \cdot 000$}

\$27. The Acidity of Urine. The healthy urine of man is acid, owing to the presence of acid sodium phosphate, the absence of free acid being shewn by the fact that sodium hyposulphite gives no precipitate. The amount of acidity is about equivalent to 2 grms. of oxalic acid in twenty-four hours, but the degree of acidity at any one time varies much during the day, being in an inversc ratio to the amount of acid secreted by the stomach; thus it decreases after food is taken, and increases again as gastric digestion comes to an end. It varies with the naturc of the food; with a vegetable diet the excess of alkalis in the food, being scereted by the urine, leads to alkalinity, or at least to diminished acidity, whereas this effect is wanting with an animal diet, in which the alkalis are less abundant, earthy bases preponderating. Hence the urine of carnivora is generally very acid, while that of herbivora is alkaline. The latter, when fasting, are for the time being carnivorous, living entirely on their own bodies, and hence their urine becomes under these circumstances acid.

The natural acidity increases for some time after the urine has been discharged, owing to the formation of fresh acid, apparently by some kind of fermentation. This increase of acid frequently causes a precipitation of urates, which the previous acidity, even after the cooling of the urine, had been insufficient to throw down. After a while however the acid reaction gives way to alkalinity. This is caused by a conversion of the urea into anmonium carbonate through the agency of a specific 'organized' ferment. 'This ferment as a general rule does not make its appearance except in urine exposed to the air ; it is only in unhealthy conditions that the fermentation takes place within the bladder, and in such cases is due either to micro-organisms introduced into the bladder from without, during the use of instruments for instance, or to the action of an unorganized ferment, secreted apparently by the walls of the bladder. 
\$ 328. Abnormal Constituents of Urine. The structural elements found in the urine under various circumstances are blood, pus and mucus corpuscles, epithelium from the bladder and kidney, and spermatozoa. To these may be added the so-called ' casts ' which are either 'epithelial casts,' that is to say cylinders of more or less altered epithelial cells shed from the tubules, or structureless 'fibrinous' casts, which are cylinders of peculiar material moulded in the lumina of the tubules; the exact nature of this material is at present a matter of doubt; it is not always the same but appears not to be fibrin.

The most common and important abnormal constituents of urine are albumin, giving rise to albuminuria, and sugar, giving rise to glycosuria or diabetes. The soluble proteids generally spoken of as 'albumin' in the urine differ in different cases. The exact determination of their nature is a matter of some diffi culty, since, as we have seen, we have in differentiating the various proteids to trust largely to their behaviour as regards precipitation upon the addition of certain saline bodies; and the presence of saline bodies in the natural urine introduces complications. It would appear, however, that the proteids usually present are serum-albumin and globulin; these are not however as a rule, if ever, present in the same relative proportions as in blood-plasma; and either the one or the other may be present by itself. A form of albumose ( $\$ 181)$ called hemialbumose, is sometimes found, and indeed probably very many distinct kinds of proteids are from time to time present. If eggalbumin be injected into the blood it appears in the urine as egg-albumin, and peptone similarly injected appears as peptone.

The sugar which is found in the urine of diabetes is undistinguishable from ordinary dextrose; but whether it is absolutely identical with that body, or whether the sugar in all cases of diabetic urine is exactly the same, cannot perhaps as yet be regarded as definitely settled.

When blood is mingled with urine in the kidney and in the urinary passages the constituents of the former are of course added to those of the latter ; and when, as sometimes happens, chyle from the lacteals makes its way into the kidneys the urine contains the fats and other constituents of chyle. Fats, however, may be present without the urine being distinctly 'chylous.'

Cholesterin, bilc-acids, bile-pigments, and one or other of a large number of bodies arising from a disordered metabolism of the body, such as leucin, tyrosin, acetone (in cases of diabetes), oxalic acid, taurin, cystin and many others are also found more or less frequently; some of these indeed have been regarded as normal constituents. Besides these the urine serves as the chief channel of elinination for various bodies, not proper constituents of food, which nay happen to have been taken into 
the system. Thus various minerals, alkaloids, salts, pigmentary and odoriferous matters, may be passed unchanged. Many substances thus occasionally taken undergo, however, changes in passing through the body ; the most important of these, since the changes which they undergo throw light on the metabolic processes of the body, will be considered in a succeeding chapter. 


\section{SEC. 2. THE SECRETION OF URINE.}

$\S 329$. The kidney, unlike the other secreting organs which we have hitherto studied, consists of two parts, so distinct in structure that it seems impossible to resist the conclusion that the functions of the two parts are different, and that the mechanism by which the urine is secreted is of a double kind. On the one hand the tubuli uriniferi with their characteristic epithelium seem obviously to be actively secreting structures comparable to the secreting alveoli of the salivary and other glands. On the other hand the Malpighian capsules with their glomeruli are organs of a peculiar nature with an almost insignificant epithelium, and their structure irresistibly suggests that they act rather as what may be called in a general way a filtering than as a truly secreting mechanism. Hence has arisen the view, which frequently bears the name of Bowman since he was the first to put it forward, that certain constituents only of the urine are secreted after the fashion of other secreting glands by the tubuli uriniferi, and that the rest of the constituents, including a great deal of the water with such highly soluble and diffusible salts as pre-exist in adequate quantity in the blood, are as it were filtered off by the glomeruli of the Malpighian capsules. We shall see later on reason to doubt whether we are justified in applying the term 'filtration,' which has a definite physical meaning, to the process by which water and other substances pass from the blood vessels of the glomerulus into the lumen of the tubule; for that process is as we shall find peculiar and complex. But snch a doubt need not prevent us from recognizing that the whole act of secretion of urine consists of two parts, one of which is much more closely dependent on the flow of blood through the kidney than is the ordinary process of secretion such as has hitherto come before us, and another part which seems to bear the same relation to the flow of blood as does ordinary secretion.

That the work of the kirlncy is to an unmsual degree dependent on the flow of blood through it seems suggested by the vascular arrangements ; for these are extremely favourable to a full and 
rapid stream of blood through the organ. The short and relatively broad renal artery comes off direct from the abdominal aorta, where the blood-pressure is extremely high; the renal vein opens directly into the vena cava, where the blood-pressure is extremely low. Between the mouth of the renal artery and the mouth of the renal vein the difference of pressure is very great indeed; and as we have seen in treating of the vascular system it is the difference of pressure between two points of the vascular tract which is the actual cause of the flow of blood from the one point to the other. The difference of pressure indeed which drives the blood through the limited area of the kidney is the same difference of pressure which drives the blood along the abdominal aorta down to the foot and back again to the vena cava.

'This free and abundant supply of blood is regulated, is either increased or diminished, according to the needs of the moment, by the vaso-motor system; this is shewn by experimental and other results, which it will be profitable to study in some detail. Before entering into these details, however, it will be well to call attention to the fact that when vaso-motor events modify the flow of blood through an organ they produce their effects in one direction or another by working on arterial blood-pressure. Thus, as we shall see, when stimulation or section of a nerve increases the flow of blood through the kirlney it does so by increasing the pressure in the small vessels of the kidney, including the capillary loops of the glomeruli. In such a case the walls of the glomerular loops, through which the passage of materials to form (part of) the urine takes place, are subjected to two influences; on the one hand to a fuller, more rapid flow of blood past them, and on the other to an increase of the pressure which that blood as it passes along exerts on them. We shall have subsequently to discuss the share taken by these two influences in determining and modifying the passage of material through the walls of the glomerular loops; and this will bear on the question of filtration to which we have above alluded; but for the present it will be convenient to deal with the effects of variation in blood-pressure apart from this secondary question.

$\$ 330$. The vaso-motor mechanisms of the kidney. It may be shewn experimentally that the kidney is supplied with a vasomotor mechanism as well developed perhaps as that of any other part of the body. By means of a modification of the plethysmograph (Figs. 102, 103), we can readily observe the variations which take place in the volume of the kidney.

The instrument ronsists of two parts, one of which (Fig. 102), called the oncometer, ${ }^{1}$ is applied to the organ about to be studied, while the other (Fig. 103), called the oncograph, is the recording part

1 From oncos, bulk. 
of the apparatus. Any diminution in the rolume of the organ (Fig. $\left.102, K^{\prime}\right)$, kidney, spleen, etc. as the case may be, diminishes the pressure on the fluid in the chamber $a$; some of the fluid in the chamber $M$ (Fig. 103) accordingly passes through the tube $K$ (Fig. 103 ) and the tube $T$ (Fig. 102) to the chamber $a$; the piston $D$ accordingly falls and with it the lever $H$. Similarly an increase in the volume of the organ causes the lever to rise.

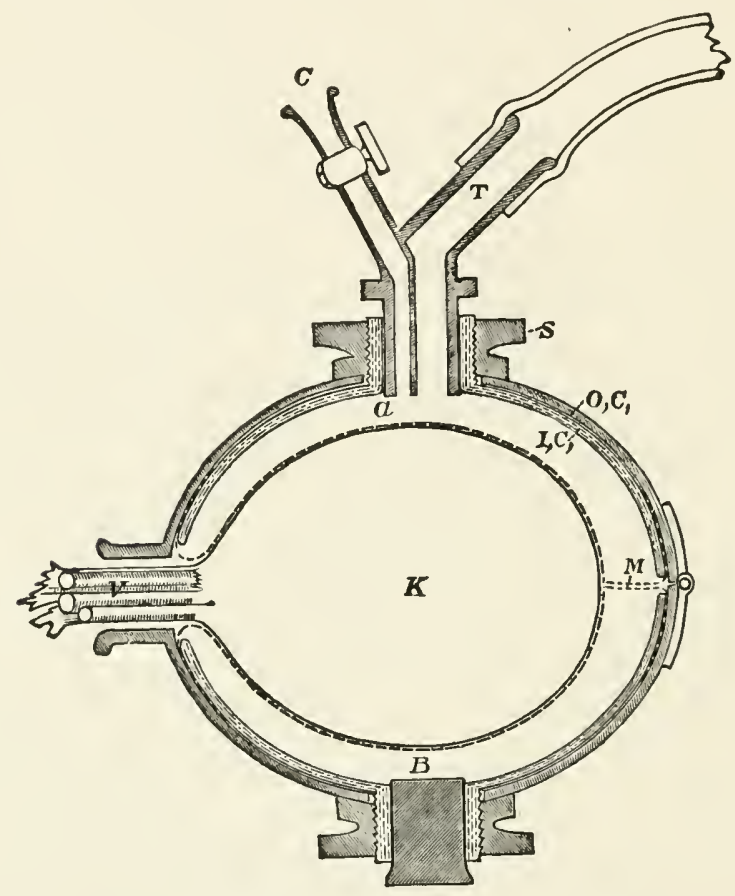

Fig. 102. Renal Oncometer. Seen in section (semi-diagrammatic). $K$. kidney, $V$. vessels and nerves imbedded in fat, \&c. entering hilus of organ, $O . C$. and $I . C$. outer and inner metal capsules screwed together by the screw $S$, and holding between them the edge of the membrane $\boldsymbol{M}$ which applies itself to the surface of the kidney, and forms with the metal capsule two chambers $a$ and $B$, one of which $(B)$ is closed by a plug filling the opening $B$, while the other $(a)$ communicates by a tube $T$ with the recording instrument. The other opening $C$ (which is closed by a small tap) is for the purpose of filling the chamber $a$ with warm oil, after the kidney has been placed in the box, the other chamber $\boldsymbol{b}$ having been previously partly filled, the quantity introduced into it depending upon the size of the kidney.

The volume of the kidney may be increased by a swelling of its constituent cells and other structural elements, by an accumulation of lymph in its lymph-spaces, and by a distension of its blood vessels. Compared with the third, the two former causes are in health so insignificant and problematical that they may be disregarded. Further, the distension of the blood vessels will 
in general depend on the constriction or dilation of the renal arteries and their ramifications, for distension due to venous obstruction will only oceur in special cases. Hence variations in the volume of the kidney may be taken as a measure of varia-

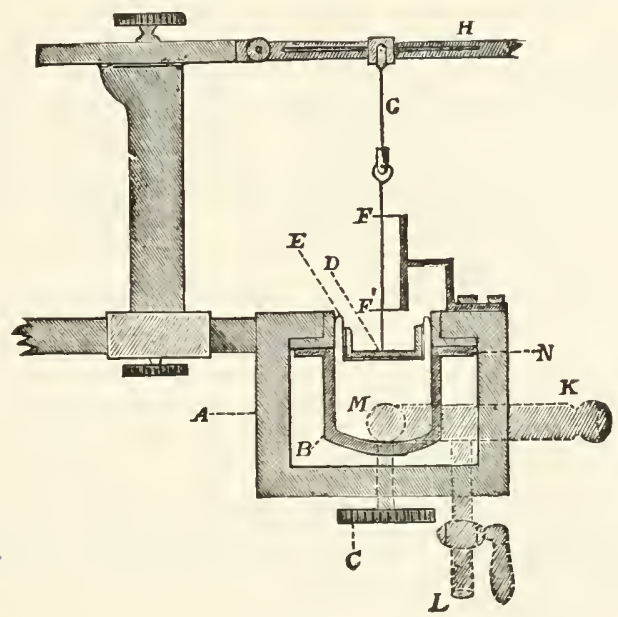

Fig. 103. Semi-diagramatic Sectional view of Oncograpit. Half natural size. $\boldsymbol{K}$, tube connecting instrument with oncometer. $D$, piston floating on oil contained in the cavity $M$; the oil is prevented from escaping by the side of the piston by the delicate flexible nembrane $E$, which does not interfere with the movements of the piston. $H$, recording lever connected with the piston by a needle $G$ passing through the guides $F, F^{\prime}$. 'The screw $C$ is for the purpose of clamping the edge of the membrane between the two ring-shaped surfaces at $N$, while the side tube $L$ is for the purpose of filling the instrument.

tions in its vascular supply, increase of volume indicating dilated renal vessels, and decrease of volume indicating constriction of the renal vessels.

When by means of the instrument just described a tracing is taken of the volume of a kidney in what may be considered a normal condition, some such result as that shewn in Fig. 104 is obtainerl.

The rolume of the kidney is seen to be so delicately responsive to (hanges in the mean arterial pressure that the curve reproduces almost exactly a blood-pressure curve, shewing not only the respiratory undulations, but even the rise and fall due to the individual heart-beats. With each rise of mean arterial pressure more blood is driven into the renal vessels and the kidney swells : with each fall of pressure less bloor enter's and the kidney shrinks. On other tracings taken in the same way may often be seen (not shewn in Fig. 104) the wider variations corresponding to the Traube-Hering curves; but it will be observed that in these the kidney shrinks with the rise of pressure and 
swells with the fall. For as we have seen (\$315) the rise in the Traube-Hering undulation is due to an augmentation of peripheral resistance caused by the constriction of minute arteries; and this constriction occurs in the kidney as elsewhere; the renal arterioles take their share in producing the result,

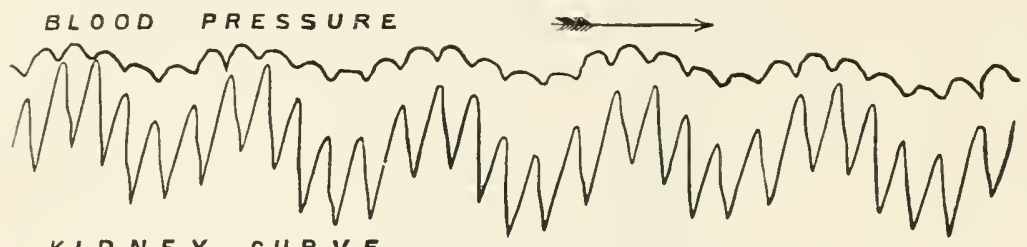

Fig. 104. Blood-pressure tracing, and Curve from Renal Oncometer. Natural size. The blood-pressure abscissa line has been raised $2.75 \mathrm{~cm}$. (the actual medium blood-pressure having been $115 \mathrm{~mm}$. Hg.). The time-curve gives interruptions recurring every three seconds.

and in consequence of their constriction the kidney shrinks. Similarly the relaxation of the renal vessels contributes to bring about the sequent fall.

331. In the course of a discussion in an earlier part of this work ( $\$ 149)$ on the local and general effects of arterial constriction and dilation, we saw that the local blood-pressure in and flow of blood through the capillaries and other minute vessels of this or that vascular area nay be increased -

1. By an increase of the general blood-pressure, brought about - $(\alpha)$ by an increased force, frequency, \&c. of the heart's beat, $(b)$ by the constriction of the small arteries supplying areas other than the area in question.

2. By a relaxation of the artery (or arteries) supplying the area itself, which, while diminishing the pressure in the artery itself, increases the pressure in the capillaries and small veins which the artery supplies. It need hardly be added that this local relaxation must not be accompanied by a too great dilation elsewhere.

The same local blood-pressure and flow of blood may similarly be diminished -

1. By a constriction of the artery of the area itself (and its branches), which, while increasing the pressure on the ardiac side of the artery, diminishes the pressure in the capillaries and veins which are supplied by the artery. This again must not be accompanied by a too great constriction elsewhere. 
2. By a lowering of the general blood-pressure, brought about - $(a)$ by diminished force, \&e. of the heart's beat, $(b)$ by a general dilation of the small arteries of the body at large, or by a dilation of vascular areas other than the area in question.

Applying these considerations to the blood vessels of the kidney, we should expect to find the following.

A rise in general blood-pressure, and that means a rise of pressure in the abdominal aorta at the mouth of the renal artery, will cause a greater flow of blood through, and so an expansion of the kidney, provided that the renal arteries themselves are not unduly constricted at the same time. This is well shewn, as we have seen, in the curve given above, where the incrcase of pressure due to each heart-beat, as well as that due to cach respiratory movement, being of central origin and not due to arterial constriction and being maccompanied by any compensating constriction of the renal artery, leads to expansion of the kidney, that is, to a greater flow of blood through the kidney.

If, however, the rise of general blood-pressure be due to events which at the same time cause a constriction of the renal arteries, the flow through the kidney may not only not be increased but even be diminished; the kidney may shrink instead of expanding. Thus if dyspnoea be brought about, as by stopping artificial respiration during an experiment, the kidney at once shrinks; the too venous blood stimulates the vaso-motor centre, and probably also by direct action on the blood vessels leads to a general arterial constriction and so to a rise of blood-pressure; but the renal vessels are involved in this constriction, so much so that their constricted condition more than counterbalances the general rise of blood-pressure, and less blood flows through the renal vessels. So also when the medulla or spinal cord is directly stimulated by induction shocks (the animal being under urari so as to eliminate the complications due to contractions of the slieletal muscles) the renal vessels share so fully in the arterial constriction which results that, in spite of the great rise of mean pressure which is induced, less blood than normal passes through the renal vessels, and the kidney shrinks. Or if the abdominal splanchnic nerves be stimulated, since as we shall sec these carry vaso-constrictor fibres for the kidney, in spite of the rise of blood-pressure which follows, the kidney shrinks on account of the great constriction of the renal vessels.

On the other hand if a rise of blood-pressure be for any reason not accompanied by a compensating constriction of the renal arteries, that rise, whether it be bronght about by general constriction of arteries other than the renai or by an increase of the cardiac delivery, causes the kidney to swell, shewing 
a greater flow of blood. Such a condition of things may be induced by section of the nerves of the renal plexus, whereby the paths of all vasco-constrictor impulses to the kidney are bloeked. After this has been done a rise of general pressure whether by dyspnoea, or by direct stimulation of the spinal cord, or by stimulation of the abdominal splanchnic nerves, leads to a greater flow through the renal vessels and an increased expansion of the kidney.

A rise of general blood-pressure then may be accompanied by either a shrinking or a swelling of the kidney, by either a greater or a less flow of blood through the kidney, according to the concomitant condition of the renal vessels; or indeed may under certain circumstances be accompanied by no change at all in the renal cirenlation, the local effects exactly counterbalancing the general ones.

Conversely, in a similar way, a fall of blood-pressure leads to a lesser flow through the renal vessels and a shrinking of the kidney unless it be accompanied by a dilation of the renal vessels out of proportion to the general fall. Thus when the spinal cord is divided below the medulla the fall of general blood-pressure is, as we have seen (\$151), very marked, being due to an abolition for the time being of wonted constrictor impulses. The pressure in the aorta falls rapidly, and at the same time, owing to the more open pathway through the region of peripheral resistance in the body generally, the pressure in the vena cava is increased; the difference of pressure between the mouth of the renal artery in the aorta and the mouth of the renal vein in the vena cava is so largely rednced that in spite of the concomitant relaxed condition of the renal vessels themselves the flow of blood through the kidney is largely diminished.

It will of course be understood that, the general bloodpressure remaining the same, the flow through the kidney will at once be on the one hand increased by dilation and on the other decreased by constriction of the renal vesscls themselves. The constricted or dilated condition of the renal vessels can by themselves produce but little effect on the pressure either in the aorta or in the vena cava; and the difference between the pressure at the mouth of the renal artery and that at the mouth of the renal vein remaining the same, the more open passages of the dilated renal vessels must lead to a fuller, and the narrower passages of the constricted renal vessels to a scantier flow, through the kilney.

\$332. By means of the oncometer, watching the shrinking and swelling of the kidney and thus judging of the flow of blood through it, the results being always interpreted with reference to the general blood-pressure on the lines of the above discussion, the paths of vaso-motor impulses to the kidney have been approximately made out. Vaso-constrictor tibres for the kidney 
are supplied from what we have previously ( $\$ 147$ and elsewhere) spoken of as the vaso-constrictor region of the spinal cord. They issue from the spinal cord by the anterior roots of a large number of the spinal nerves taking origin from this region, and may be traced (in the $d o g$ ) as high up as the 6th thoracic, a few perhaps even to the th thoracic, and as low down as the 2nd lumbar ( 4 th lumbar if only 13 nerves be counted as thoracic); but most seem to pass by the 11th, 12th and 13th thoracic nerves. Passing through the corresponding ganglia of the sympathetic chain, these fibres reach the solar plexus and thus the renal plexus by the splanchnic nerve; those however coming from some of the lower nerves apparently do not contribute to the splanchnic nerve, but take a separate course. Centrifugal stimulation of these anterior roots produces shrinking of the kidney, all the more marked and distinct in the case of the 11th, 12 th and 13 th thoracic roots because the effect on the kidney is then not so much masked by vaso-motor effects on other organs. Stimulation of the higher roots also produces shrinking of the kidney but less marked, since in these cases the stimulation bears at the same time largely on vaso-constrictor fibres for other abdominal organs, and so by raising the general blood-pressure tends to neutralize the local effect on the kidney. And even the very decided shrinking of the kilney which results from the stimulation of the splanchnic trunk itself is less than would take place if the stimulation affected the vessels of the kidney only.

$\$ 333$. There is also some evidence gained by the method of slowly repeated rhythmical stimulation (\$146) that some of the higher (anterior) roots also contain renal vaso-dilator fibres; but the matter is not at present beyond dispute.

$\S 334$. It is obvious then that by means of this vaso-motor mechanism the flow of blood through the kidney is governed by the central nervous system in such a way that afferent impulses, started in this or that region or surface, and passing up to the central nervous system, may lead either to constriction or to dilation of the renal vessels; and to such actions of this kind we shall presently return. Meanwhile, we wish to call attention to the fact that changes in the flow of blood through the kidney, as shewn by changes of volume, may be brought about quite apart from the central nervous system. For instance after all the nerves going to the kidney have been severed, the kidney, as shewn by the oncometer, swells when substances such as urea, which cause an increase in the secretion of urine, are injected into the blood. The substance reaching the kidney by the blood stimulates the kidney to activity, and this is accompanied by a dilation of the blood vessels, which, since the nerves have been severed, must be brought about by some local action. The event seen is similar to the greater flow of blood through a muscle when it contracts. Cf. $\S 146$. 
\$ 335. If, while the kidney is in the oncometer, and the various experiments on section and stimulation of nerves and the like are being carried on, a cannula be tied in the ureter, the secretion of urine may be watched at the same time. It will then be seen that the flow of urine through the end of the cannula is not equable, and does not either increase or decrease in an even manner. On the contrary, it will frequently be found that a sort of gush of urine takes place, several drops following each other in rapid succession, followed by a cessation of flow; and if the ureter be watched it will be seen that the gushes of urine are synchronous with waves of peristaltic contraction sweeping down the ureter. Obviously the urine collects to a certain extent in the pelvis of the kidney and is driven thence by muscular action from time to time; to this point we shall return later on.

Making every allowance, however, for these irregularities of flow, we may take the rate of flow from the end of the cannula as a measure of the rate of secretion; and it is found that as a general rule increased flow of urine is coincident with swelling of the kidney, that is with a greater flow of blood through it, and diminished or arrested flow of urine is coincident with shrinking of the kidney, that is with a diminished flow of blood through it.

A striking instance of this is afforded by the experiment of dividing in the dog the spinal cord below the spinal bulb. The blood-pressure then, as we know, falls rapidly, owing to the removal of constrictor impulses from the small arteries and the great diminution of peripheral resistance which follows upon so many small arteries becoming dilated; and thougl the renal arteries probably share in the general relaxation yet, owing to the fall of pressure in the aorta conjoined as this is by a corresponding rise of pressure in the vena cava, the flow of blood through the kidney is largely diminished. We find that after the operation the secretion of urine is greatly diminished; indeed, in most cases, the flow from the end of a cannula is almost arrested. In fact we may almost make the general assertion that, when in the dog the blood-pressure falls to about $30 \mathrm{~mm}$. $\mathrm{Hg}$ or less, the secretion of urine is for the time stopped. These and other results support the view stated above that the secretion of urine is in quite a special way dependent on the flow of blood through the kidney; and we may further conclude that the secretion which is so particularly influenced by the flow of blood is that special kind of secretion, allied to filtration, which takes place through the glomeruli, and not the more ordinary kind of secretion by means of the epithelium of the tubuli uriniferi. But before we proceed to discuss how the increased flow of blood increases the glomerular flow of urine, we must turn to consider the functions of the epithelium of the tubuli. 


\section{Secretion by the Renal Epithelium.}

\$336. The glomerular mechanism is after all a small portion only of the whole kidney, and the epithelium over a large part of the course of the tubuli uriniferi bear's most distinetly the characters of an active secreting epithelium. These facts would lead us $a$ priori to suppose that the flow of urine is in part the result of an active secretion eomparable to that of the salivary or other glands which we have already studied. And we have experimental and other evidence that such is the case.

In the first place a flow of urine may be artificially excited even when the natural flow has been arrested by dinimution of blood-pressure. Thus if, when the urine has ceased to flow in consequence of a section of the spinal bulb, certain substances, such as urea, urates, sodium acetate, and the like, be injected into the blood, a more or less copious secretion is at once set up. This secretion is, or at least may be, unacempanied by any rise of general blood-pressure sufficient to account for the increased secretion as the mere result of an increaserl flow of blood. It is true (as we have seen $\$ 334$ ) that the injection of these substances leads to an expansion of the kidney, to a fuller flow of blood through it; but this is the effect rather than the cause of the secretory activity. We may infer that the presence of the above substances in the blood excites the renal epithelium cells to an unwonted activity, causing them to pour into the interior of the tubules a copious secretion, just as the presence of pilocarpin in the blood will cause the salivary cells to pour forth their secretion into the lumen of their ducts; and that this activity of the epithelium cells is accompanied, also as in the case of the submaxillary and other glands, by a vascular dilation, which, though adjuvant and beneficial, is not the distinct cause of the activity. This view is further supported by the following remarkable experiment, which goes far to shew that of the varions substances which having found their way into the blood are thrown ont by the kidney, some pass into the urine through the glomeruli while others are distinctly secreted by the tubuli uriniferi, the discharge of the latter being accompanied by a general activity of the seereting cells, as shewn hy the flow of water taking place at the same time.

In the amphibia, the kidney has a double vascular supply: it receives arterial blood from the renal artery, but there is also poured into it venous blood from another source. 'The femoral vein divides at the top of the thigh into two branches, one of which runs along the front of the abdomen to meet its fellow in the middle line and form the anterior abdominal vein, while the other passes to the outer border of the kidney and branches in the substance of that organ, forming the so-called renal portal 
system. Now the glomeruli, in some species at least of these animals, are supplied exclusively by the branches of the renal artery, the renal vena portae only serving to for'm the capillary plexus around the tubuli uriniferi, which is also supplied by the efferent vessels of the glomeruli. From this it is obvious that if the renal artery be tied, the blood is shut off entirely from the glomeruli; and actual observation of the kidney has, in the animals in question, shewn that under these circumstances there is no reflux from the capillary network surrounding the tubules back to the glomeruli; thus the kidney by this simple operation is transformed into an ordinary secreting gland devoid of any special filtering mechanism. Such a kidney may be used to ascertain what substances are cxcreted by the glomeruli, and what by the tubules in some other part of their course. It is found that urea injected into the blood gives rise to a secretion of urine when the renal arteries are tied; this substance therefore is secreted by the epithelium of the tubules, and in being so secreted gives rise at the same time to a flow of water through the cells into the interior of the tubules. Sugar and peptones, on the other hand, which injected into the blood readily pass through the untouched kidney and appear in the urine, do not pass through a lidney the renal arteries of which have been tied, even when a diuretic such as urea is given at the same time in order to secure a flow of urine. These substances therefore are excreted by the glomeruli.

The validity of this experiment, which may be accepted as indicating a marked difference between glomerular secretion on the one hand and epithelial or tubular secretion on the other, depends on the absence of any collateral circulation whereby the glomeruli may be supplied with blood after ligature of the renal artery. In these animals anastomoses occur between the renal arteries and the arteries of the generative organs; and unless the renal artery be so tied as to avoid these collateral communications the results of the experiment are different.

Additional evidence in favour of the secretory activity of the epithelium cells is afforded by the following observation. Into the veins of animals in which the urinary flow had been arrested by section of the spinal cord below the medulla a quantity of the blue colouring material known as sodium sulphindigotate ${ }^{1}$ is injected. This substance is rapidly excreted on the one hand by the liver in the bilc, and on the other hand by the kidney. By varying the quantity injected, killing the animals at appropriate times after the injection of the material, and examining the kidneys microscopically and otherwise, it may be ascertained that the pigment so injected passes from the blood into the renal

1 Sometimes called indigo-carmine, though this name is more properly applied to a crude impure preparation of potassium sulphindigotate. 
epithelium, and from thence into the channels of the tubules. There being no stream of fluid through the tubules, owing to the arrest of urinary flow by means of the preliminary operation, the pigment travels very little way down the interior of the tubules, and remains very much where it was cast out by the epithelium cells. There are no traces whatever of the pigment having passed by the glomeruli; and the cells which appear most distinctly to take up and eject it, are those lining such portions of the tubules (viz. the first and second convoluted tubules, zigzag tubules and ascending limbs of the loops of Henle) as from their microscopic features have been supposed to be the actively secreting portions of the entire tubules.

The above observation may be objected to on the ground that this colouring matter does not occur as a constituent of the blood either in health or disease, and especially that the absence of any concomitant discharge of fluid from the cells excites suspicion that the process observed was not really one of secretion; for the injection of such substances as urea or urates into the blood does cause a copious flow of fluid, and incleed thus prevents the microscopic tracking out of their passage, which in the case of urates might otherwise be done much in the same way as with the sodium sulphindigotate. Still in birds, the urine of which contains little water, urates may be detected in the epithelium of the tubules though not in the capsules. Without insisting too much on the value of the sodium sulphindigotate experiments, they may be taken as fairly supporting the view which we are considering. IVe may, for the present, conclude that the secretion of urine does consist of two separate and distinct acts: secretion by the glomeruli, which we may for brevity's sake speak of as glomerular secretion, and secretion by the epithelium of the tubuli, which we may speak of similarly as tubular secretion. Both these forms of secretion, especially the former but to a certain extent the latter also, differ from the secretion of such a gland as the salivary, and both deserve some special consideration.

$\$ 337$. The nature of glomerular secretion. We have seen that the expansion of the kichey which has for its accompaniment an increased flow of urine is one brought about by the renal artery and its various branches becoming dilated, under such circumstances that the difference between the blood-pressure in the aorta at the mouth of the renal artery and the blood-pressure at the vena cava at the mouth of the renal vein is at the same time increased, or at all events is not diminished.

In dealing with the vascular system we saw that relaxation of a small artery, taking place without any marked change in the general blood-pressure and in neighbouring arteries, leads to a fuller and more rapid stream of blood through the capillaries supplied by the artery, and that at the same time the pressure in 
the capillaries themselves is increased; owing to the decrease of peripheral resistance through the widening of the artery, the great fall of pressure (see $\$ 98$ ) so characteristic of the peripheral region is shifted from the arterial side of the capillaries towards the vellus side and to the capillaries themselves.

Hence, as we have already said, when the renal artery dilates two things happen in the loops of the glomeruli: a fuller, more rapid stream of blood passes through them, and that blood as it flows through them is exerting a grcater pressure than before on their walls. How does each of the events stand towards the secretion of urine?

We have not at present the means of inducing a fuller and more rapid flow without increasing the pressure; but we may easily obtain increase of pressure without the fuller and more rapid flow. If we hinder or obstruct the outflow through the renal vein we at once increase the pressure in the glomerular loops as in the other capillaries of the kidney. Now, when the blood-pressure in the glomeruli is thus raised by partial obstruction to the venous outflow, the flow of urine so far from being increased is diminished. Obviously then the passage of water and material through the walls of the glomerular loops, to go to for'm the urine, is not the result of mere pressure, and camnot therefore be spoken of properly as a process of filtration. (Cf. \$244.) And we may here draw a comparison between the passage of water and material through the wall of a capillary in an ordinary situation to form lymph and the passage through the wall of the glomerular loop to form urine or part of urine. The former' as we have seen ( $\$ 244)$ appears to be dependent on pressure, though influenced as we have also seen in a very material way by the condition of the vascular wall; and hindrance to venous outflow, so inefficient in promoting a flow of urine, is as we have seen especially favourable to the transudation of lymph. Moreover, the substances which pass through the capillary wall to form lymph may be clescribed as the constituents of the blood generally, proteids as well as salts and other soluble and diffusible matters. Through the wall of the glomerular loop there pass, so long as that wall is sound and intact, neither albumin nor globulin nor fibrin factor, but only water accompanied by some, and apparently a selection of some. of the soluble diffusible constituents of the blood; for, as we have said, the presence of proteids in normal urine is contested, and, at most, there is present an insignificant quantity only (which moreover may come from the tubular epithelium). This difference in the material which passes through may be referred to the differences in the nature of the partition. The transidation of lymph takes place through the capillary wall; between the blood on one side and the lymph in the lymph-spacc on the other is only the thin film of conjoined epithelioid plates. But the corresponding wall 
of the glomerular loop is covered over and wrapped round so to speak by an adherent layer of cells, which tholigh reduced and thin are still epithelial cells; the materials which go to form urine have to pass through these cells as well as through the film of epithelioid plates. It seems to be this layer of cells which determines what shall pass and what shall not.

Obviously the passage through this epithelium is of a peculiar nature. The necessary condition for the due accomplishment of the passage is as we have seen a full and rapid stream of (arterial) blood; the high pressure which accompanies that full and rapid stream, though probably under normal circumstances an adjuvant, is by itself helpless. 'Thus when the pressure is raised by venous obstruction, in which case the high pressure is accompanied by a slow stream or by actual arrest of the flow, even the passage of mere water is retarded. Seeing that many of the constituents of urine are diffusible substances certainly preexisting in the blood, inorganic salines for instance, and seeing that, if we may trust the experiments on the amphibian kidney spoken of above, diffusible abnormal constituents of blood, such as peptone and sugar, pass into the urine not by the tubular epithelium but by the glomeruli, we might expect that diffusion, in contrast to filtration (see $\$ 253$ ) played an important part in the passage; and a full rapid stream would undonbtedly favour diffusion. But diffusion by itself will not explain matters. Egg-albumin differ's very shightly as regards diffusibility from serum-albumin, and yet while at the most a minute quantity only of the latter passes into the urine in normal cireumstances, the former when injected into the blood at. once makes its way into the urine, and there is evidence that it passes by the glomeruli. On the other hand urea is an eminently diffusible body, and yet if we can trust the experiments on the amphibian kidney, the main mass at all events of the urea of the urine passes by the epithelium of the tubules.

The important part played by the epithelium is shewn when the epithelium is deranged. If the renal artery be temporarily ligatured or otherwise obstructed, so that the glomeruli are for some little time shut off from their blood-supply, the secretion of urine is stopped; on reestablishment of the circulation the secretion of urine slowly returns, and the urine is then found to be albuminous, remaining so for some little time. The serumalbumin and globulin which could not pass through the intact epithelium, can pass through when the epithelium has been damaged by interference with its nutrition. The appearance of albumin in the urine (albuminuria) is a not infrequent symptom of kidney disease, and its presence in other than minute quantities indicates imperfections in the glomerular epithelium. But even under unhealthy conditions that epithelium still governs to a certain extent the passage of material; for the proteids of the 
blood-plasma do not pass through bodily or in a proportion which corresponds either to the relative proportion in which they exist in the plasma or to the relative ease (or difficulty) with which they pass through membranes. Though the "albumin" of albuminous urine frequently consists of both serumalbumin and globulin, these do not necessarily occur in the same proportion as in blood; they vary in urine much more than they do in blood; and indeed the one or the other may be absent; moreover fibrin factors are very rarely found.

Hremoglobinuria, or the presence of hamoglobin in urine, may be brought about by injecting into the blood vessels laky blood, or some substance such as pyrogallic acid, which will "break up" the corpuscles of the blood. Now in such cases there is evidence that the hamoglobin passes through the glomeruli; minute disc-like masses of hæmoglobin, the so-called 'menisci,' are, by appropriate methods of preparation, found in situ in the capsules. Such a passage is very far removed from being a process of diffusion.

We may conclude then that the passage of material through the glomeruli, like the transudation of lymph and even to a more marked extent, is a complex affair in which the ordinary physical processes of diffusion and filtration may play their part, but are not masters of the situation.

$\S 338$. The work of the epithelium of the tubules. As we have said the structural features of the epithelium cells of the tubules seem to justify the conclusion that they exercise a secretory activity comparable with that of a salivary or a gastric gland. But their work is in many ways peculiar. In the case of the salivary, gastric, and pancreatic glands there can be no doubt that the specific constituents of the several secretions, mucin, pepsin, trypsin and the like, are manufactured in the alveolar cells out of antecedents of some nature or other. The evidence, as we have seen, is all against the view that these glands merely withdraw, secrete in the old sense of the word, from the blood these substances preexisting in the blood. When the salivary glands are extirpated or the pancreas or the stomach removed there is no accumulation in the blood of the specific constituents of the corresponding secretions. So also when the liver is extirpated there is no accumulation in the blood of either bile acids or bile pigment. With regard to the kidney in relation to the most important constituent of urine, namely urea, the case is different. If the kidneys in a mammal be extirpated, or if the kidneys by disease or by ligature of the ureters be so damaged as to be unable to carry on their work, an accumulation takes place in blood, not as was once thought of some antecedent of urea such as kreatin, but of urea itself. In the case of birds and reptiles which excrete not urea but chiefly uric acid the accumulation is one of uric acid. Obviously in secreting 
urea the work of the epithelium of the tubules is largely if not exchusively confined to simply picking the urea out of the blood and pushing it so to speak into the lumina of the tubules. We might perhaps say exclusively, for there is no evidence that any urea at all is actually manufactured in the kidney.

How the urea, which is in this peculiar manner taken out of the blood, comes to make its appearance in the blood is a problem in which the kidney is not concerned and with which we shall deal in treating of the metabolic events of the body generally.

$\$ 339$. In the case of some other constituents of the urine we have evidence that the cells do something more than simply pick the constituent out of the blood. Hippuric acid, as we have seen, occurs in small quantity in the urine of man, and in larger amount in the urine of herbivora. Now hippuric acid may be formed by the combination, with deliydration, of benzoic acid and glycin $\left(\mathrm{C}_{7} \mathrm{H}_{6} \mathrm{O}_{2}+\mathrm{C}_{2} \mathrm{H}_{5} \mathrm{NO}_{2}-\mathrm{II}_{2} \mathrm{O}=\mathrm{C}_{9} \mathrm{H}_{9} \mathrm{NO}_{3}\right)$; and benzoic acid introduced into the alinentary canal or injected into the blood, reappears in large measure in the urine as hippuric acid. Somewhere in the body the benzoic acid meets with and combines with glycin. And we have experimental proof that the combination may and probably does take place in the kidney.

If a circulation of blood be kept up through the blood vessels of the kidney freshly removed from a living animal, and benzoic acid and glycin be added to the blood as it is about to enter into the kidney, hippuric acid will be found in the blood issuing from the kidney, especially if the same blood be passed through the kidney several times; the blood used must be blood containing oxyhæmoglobin, carbonic-oxide-hæmoglobin not producing the effect. The mere mixing with the blood itself is insufficient; and if the blood be sent not through a kidney just removed from the living body but through one taken from a dead body or one which has been left to itself for some time after removal from a living body, the synthesis will not be effected. To carry out the combination by means of the kidney which has been removed from the body the kidney must retain for a while its own life, it must be a "surviving" kidney. Nor is it absolutely necessary to bring the benzoic acid and glycin to the kidney by means of a blood-stream. If a "surviving" kidney be divided rapidly into small pieces and the benzoic acid rapidly mixed with the pieces, hippuric acid is formed. Nor is it necessary to furnish the glycin. If benzoic acid alone be used, hippuric acid is formed all the same. Glycin, as we have previously said, cannot be recognized as a normal constituent of any of the tissues; nevertheless, as we have seen in speaking of glycocholic acid in the bile and as we shall see later on, glycin must make a momentary appearance in various metabolic processes of the body, being immediately on its appearance converted into something else, so that it never remains as glycin. It apparently is formed in the 
kidney, and is thus momentarily available for the conversion of benzoic into hippuric acid.

It seems probable therefore that, with regard to this particular constituent of urine, hippuric acid, the cells of the tubules have the jower of effecting a combination between the benzoic acid brought to them by the blood and the glycin which they furnish by means of their own metabolism, and in this way produce hippuric acid.

Not only benzoic acid but many other bodies taken in to the system reappear in the urine combined with glycin, and in their cases also the combination probably takes place through the activity of the cells of the tubules of the kidney. Moreover, other changes than the assumption of glycin, the various changes which many chemical substances taken into the system undergo before reappearing in the urine, probably also take place to a large extent in the kidney, and are also carried out by means of the epithelium of the tubules.

What other constituents of normal urine are prodnced in this or a similar manner we do not as yet definitely know. The pigment urobilin, which as we have seen is supposed to be a derivative from bilirubin, may be brought ready formed from the liver or may have the finishing touches given to it in the kidney itself; and the other normal or abnormal trinary pigments possibly arise either directly from hæmoglobin or indirectly from that body through the biliary pigment by a transformation taking place in the cells of the tubules. There is also evidence in frogs that acid sodium phosphate is furnished by the cells of the tubules.

In conclusion then we may say that the activity of the epithelium of the kidney appear's especially modified, as compared with other secreting glands, to meet the special object which the kidney has to secure. The purpose of the kidney is not to provide a fluid, urine, which can be made use of for the needs of the body, but to cast out waste matters from the body. Hence its secretory activity is limited largely to the mere discharge of matters which reach it preexistent in the blood, though in several cases it gives the final shape to the excreted substance before this passes in to the ureter.

$\$ 340$. We may illustrate the preceding discussions by briefly passing in review some of the more usual ways in which the secretion of urine is in ordinary life modified.

In the preceding section the composition of urine was illustrated by the daily output of the several constituents rather than by a percentage account of any specimen of urine, for the reason that the composition of urine varies within extremely wide limits. This is especially the case as regards the proportion of water to solids. One urine may be of high specific gravity with a small amount of water relatively to the solids, 
while another may have so little colour and such a low specific gravity as to appear hardly more than water. The reason of these extreme differences lies in the fact that the kidney is not only the channel by which waste solids leave the body but also an important outlet for the discharge of the stream of water which, in order that the various processes of the body may be duly carried on, is continually passing through the system. It is frequently of advantage to the body to discharge through the kidney a large amount of water, more or less irrespective of the solid matters which are so to speak washed away with it; and hence the advantage of the glomerular mechanism so specially adapted for the special discharge of water.

As we shall see presently, to the skin also falls the duty of discharging large quantities of water. The respiratory organs also, as we have seen, serve for the discharge of water; but the amount which the latter put out can only be varied by the inconvenient method of increasing or diminishing the whole act of breathing. Hence we find special relations between the skin and the kidneys correlating the work of the one to that of the other as regards this particular work of the discharge of water.

When the body is exposed to cold the discharge of water from the skin in the form of sweat is checked, and the cutaneous vessels are constricted. At the same time the blood vessels of the abdominal viscera, including the kidneys, are dilated, but not out of proportion to the constriction of the cutaneous vessels, for the general blood-pressure does not fall but if anything rises somewhat. Thus there is established just the state of things which is favourable to a full and rapid stream of blood through the renal glomeruli; and an increased flow of urine results.

Conversely, when the body is exposed to warmth the skin perspires freely and the cutaneous vessels are widely dilated; and conversely also the renal and other abdominal vessels are constricted, so that a slow and small stream of blood trickles through the glomeruli, and the urine which is secreted is scanty.

$\$ 341$. Even more important than its relations to the skin are the relations of the kidney to the water absorbed by the alimentary canal; this is especially seen when large quantities of fluid are drunk. The whole of the water thus introduced into the alimentary canal passes into the blood, for in a healthy organism no amount of fluid drunk, unless it throws the economy out of order, can affect the amount of water present in the faces. But the addition to the blood of even a very large quantity of fluid does not, as we lave seen, by its nere quantity (\$164), increase the general blood-pressure, and therefore cannot in this way produce what it undoubtedly does produce, an increased flow of urine.

Since a kidney, all the nerves of which have been severed, dilates, as shewn by the oncometer, that is has a fuller supply 
of blood and at the same time yields a fuller flow of urine when water is injected into the blood, we may infer that the blood diluted by the absorption of water acts directly on the kidney. We may further suppose that it is the glomerular mechanism which is thus especially increased in activity, though it may be that the epithelial secretion is also augmented.

When however fluid is taken simply as a proper accompaniment of solid food, the increase of urine which results has probably another origin. As we have already said, and as we shall point out more fully later on, the absorption of proteid material, which is a constituent and generally a conspicuous constituent of every meal, leads to a formation of urea; and urea, as we have seen reason to believe, directly stimulates the epithelium of the tubules to secretory activity. And what scems prominently true of urea is probably true of many other products of digestion; so that the increased flow of urine which follows an ordinary meal accompanied with not more than the ordinary amount of fluid, is the result of the labours of the epithelium of. the tribules as well as of the fuller stream of blood through the glomeruli.

$\$ 342$. What has just been said concerning the influence on the kidney of food and water may be applied also to the action of substances which being especially efficacious in promoting a flow of urine when taken into the body are called "diuretics." The several actions of various diureties are very varied, and it would be out of place to discuss them fully. We may however say that while the action of some appears simple that of others is complex.

Such agents as sodium acetate and potassium nitrate appear to produce their effect chiefly by acting directly on the kidney. They induce, as we have seen, $\$ 334$, local vascular dilation and probably act by stirring up, after the fashion of urea, the epithelium of the tubules to secretory activity, the accompanying fuller stream of blood through the whole kidney being, as in the case of the salivary and other glands, a useful adjuvant, though it may also increase the glomerular secretion.

The diuretic effect of such an agent as digitalis is probably more complex. By increasing the cardiac stroke, and at the same time constricting many small vessels, digitalis raises the general blood-pressure; but the tendency of the increased bloodpressure to increase the flow of urine may be counterbalanced by the constriction of the renal vessels themselves. And while it is a matter of common experience that digitalis is very effective as a diuretic in cardiac discase, there is great doubt whether it really acts as a diuretic in health; in cardiac disease it probably raises the blood-pressure by improving the cardiac stroke and not by constriction of the blood vessels. But even in the absence of cardiac disease, digitalis has been found in particular 
cases to act as a powerful diuretic, and in these cases either it must act directly on the tubular epithelium or its effects in constricting the renal arteries must be less than its effects on other small arteries or must pass off before the influcnce of the heightened blood-pressure has disappeared.

$\$$ 343. Quite removed from the intervention of chemical substances in the blood and yet most striking is the influence on the kidney of the central nervous system. 'The potent influcnce of emotions in promoting the secretion of urine is proverbial, and the general features of 'nervous' urine, the water' increased out of proportion to the solid constituents, especially seen in the "urina hysterica," which is hardly more than simple water, often discharged in enormous quantity, at once suggests the view that impulses originating in the brain and passing down to the kidney along the vaso-dilator fibres, of whose existence evidence was given in $\$ 333$, lead to dilated blood vessels and great play of glomerular activity, without perhaps producing any other direct effect on the economy; though possibly the same emotions by constricting the cutaneous and, it may be, other vessels may raise the general blood-pressure and so help the dilated renal vessels. 


\section{SEC. 3. THE DISCHARGE OF URINE.}

$\S 344$. The urine, like the bile, is secreted continuously; the flow may rise and fall, but, in health, never absolutely ceases for any length of time. 'The cessation of renal activity, the so-called suppression of urine, entails speedy death. The minute streams passing continuously, now more rapidly now more slowly, along the collecting and discharging tubules, are gathered into the renal pelvis, whence the fluid is carried along the nreters into the bladder by pressure and gravity aided by the peristaltic contractions of the muscular walls of the ureter.

If in a living animal a ureter be laid bare and stimulated, mechanically or otherwise, at a part of its course, waves of peristaltic contraction may be seen to pass in both directions from the spot stimulated, upwards towards the kidney and downwards towards the bladder. In the absence of artiticial stimulation spontaneous waves of contraction make their appearance, sometimes repeated with tolerable regularity (about cvery 20 seconds in the rabbit), sometimes occurring in groups with longer pauses between. These spontaneous contractions invariably pass in one direction, from the kidney to the bladder; and their frequency and vigonr seem to be determined by the activity of the secretion of urine. But they are not directly called forth by the urine either mechanically distending the tube or chemically stimnlating the inner surface, for regularly recurring contractions may be obscrved in a kidney and ureter removed from the body, or even in an isolated excised piece of the ureter.

The rhythmically repeated contractions arise spontaneonsly in the muscular coat of the ureter much in the same way as the similar cardiac contractions arise in the muscular substance of the heart; and it may here be mentioned, in support of what was urged in $\$ 154$ with regard to the heart-beats not being started by nerve-cells, that ihythmically repeated spontaneous peristaltic contractions have been observed in isolated pieces of ureter taken from the middle of its course, in which no ncrvecells could be observed. 
In the living body these spontaneous movements, beats they might be called, are subordinated to the flow of urine into the pelvis; the more active the secretion of urine the more frequent and vigorous are the beats of the pelvis and ureter; but the exact mechanism by which the secretion and the movements are maintained in harmony has not yet been cleared up.

\section{Micturition.}

$\$ 345$. In the urinary bladder, the urine is collected, its return into the ureters being prevented by the oblique entrance into the bladder and valvular nature of the orifices of those tubes; and its discharge from thence in considerable quantity is effected from time to time by a somewhat complex muscular mechanism, of the nature and working of which the following is a brief account.

The involuntary muscular fibres forming the greater part of the vesical walls are arranged partly in a more or less longitudinal, and partly in a circular manner. The bladder after it has been emptied is contracted and thrown into folds; as the urine gradually collects, the bladder becomes more and more distended. The escape of the fluid is in part prevented by the resistance offered by the elastic fibres in the walls of the urethra which help to keep the urethral channel closed. But this is not all; for observation shews that fluid is retained within the bladder up to a pressure of 20 inches of water so long as the bladder is governed by an intact spinal cord, but gives way to a pressure of 6 inches only when the lumbar spinal cord is destroyed or the vesical nerves are severed. 'This affords very strong evidence that the obstruction at the neck of the bladder to the exit of urine depends on some tonic muscular contraction maintained by a reflex or automatic action of the lumbar spinal cord. It has been maintained that the circularly disposed fibres specially developed around the neck of the bladder are the subjects of this tonic contraction and thus the chief instrument of the retention; hence the name sphincter vesicæ. The continuity of these fibres, however, with the rest of the circular fibres of the bladder suggests that they probably do not act as a sphincter, but that their use lies in their contracting after the rest of the vesical fibres, and thus finishing the evacuation of the bladder. The resistance in question is supplied by a tonic contraction not of these circular fibres of the bladder itself but of the muscular fibres, partly plain, partly striated, surronnding the prostatic portion of the urethra, and constituting the sphincter vesico externus or prostaticus or sphineter of Henle. It is stated that artificially excited contractions of these fibres will resist a pressure of fluid in the bladder. 
When the bladder has bccome full, we feel the need of making water, the sensation being heightened if not caused by the trickling of a few drops of urine from the full bladder into the urethra. We are then conscious of an effort; during this effort the bladder is thrown into a long-continued contraction of an obscurely peristaltic nature, the force of which is more than suffieient to overeome the resistance offered by the urethra, and the urine issnes in a stream, the sphinctcr vesica cxternus being at the same time either relaxed after the fashion of the sphincter ani, or at least overcome. In its passage along the urethra, the exit of the urine, at all events of the last portions, is forwarded by irregularly rhythmic contractions of the bulbo-cavernosos or ejaculator urince muscle, the contractions of which compress the urethra: and the whole act is further assisted by pressure on the bladder exerted by means of the abdominal muscles, very much the same as in defecation.

Experiments on cats, dogs and other animals shew that contractions of the bladder can be brought about by stimulation of the anterior roots of certain lumbar nerves chiefly the third and fonrth, and of the first three sacral nerves; stimulation of the anterior roots of the nerves between these two scts does not give contractions of the bladder. The sacral roots seem to have more powerful and more distinctly unilateral effects than have the lumbar roots, and the movements brought about have not exactly the same character in the two cases, though it cannot be said that the contraction is in the former case strictly longitudinal and in the lattcr case circular. The nerve fibres issuing by the lumbar nerves pass into the sympathetie chain and thence by the inferior mesenteric ganglion and hypogastrie nerves to the hypogastric plexus; the nerve fibres issuing by the sacral pass more directly to the hypogastric plexus.

$\$ 346$. We said just now "when the bladder has become full," but this must not be understood to mean, "when the bladder has received a certain quantity of fluid." On the contrary, it is a matter of common experience that we feel the desire to make water sometimes when a large quantity and sometimes when a small quantity of urine has accumulated in the bladder. We have evidence that the bladder possesses to a very high degree that obscure continuous contraction which we speak of as 'tone'; and further that the amount of its tone is exceedingly variable, the organ, quite independently of distinct efforts at micturition, being at one time contracted and at another flaccid and distended. When it is in a contracted state, a small quantity of fluid may exert the same effect on the vesical walls as a larger quantity when the bladder is flaccid. Hence while the determining cause of the desire to make water is the pressure of the urine upon the vesical walls, the quantity needed to produce the necessary fulness is dependent on the amount of tonic 
contraction of the muscular fibres existing at the time. And we have evidence that this tone is regulated by the nervous system.

$\$ 347$. Micturition as sketehed above seems at first sight, and especially when we appeal to our own consciousness, a purely voluntary act. A voluntary effort throws the muscular fibres of the bladder into contractions, an accompanying voluntary effort lessens the tone of the sphincter externus, probably by inhibiting its centre in the spinal cord, while other voluntary efforts throw the ejaculator and abdominal muscles into contractions, and, the resistance of the urethra being thereby overcome, the exit of the urine naturally follows.

'There are facts, however, which prevent the acceptance of so simple a view. In the first place, in cases of urethral obstruction, where the bladder camnot be emptied when it reaches its accustomed fulness, the increasing distension sets up fruitless but powerful contractions of the vesical walls, contractions which are clearly involuntary in nature, which wane or disappear, and return again and again in a rhythmic manner, and which may be so strong and powerful as to cause great suffering. It scems that the fibres of the bladder, like all other muscular fibres, have their contractions augmented in proportion as they are subjected to tension. Just as a previously quiescent ventricle of a frog's heart may be excited to a rhythmic beat by distending its eavity with blood, so the quiescent bladder may, quite independent of the will, be excited by the distension of its cavity, to a peristaltic action which in normal cases is nercr carried beyond a first effort, since with that the bladder is emptied and the stimulus is removed, but which in cases of obstruction is enabled clearly to manifest its rhythmic nature.

In the second place it has been shewn that quite normal micturition may take place in a $\log$ in which the lumbar region of the spinal cord has been completely and permanently separated by section from the upper dorsal region. In such a case there can be no exercise of volition, and the whole process appears as a reflex action. When under these circumstances the bladder becomes full (and otherwise apparently the act fails) any slight stimulus, such as sponging the anus or slight pressure on the abdominal walls, causes a complete act of micturition: the bladder is entirely emptied, and the strean of urine towards the end of the act undergoes rhythmical augmentations due to contractions of the ejaculator urine. 'These facts can only be interpreted on the view that there exists in the lower spinal cord (of the $(\mathrm{log})$ what we may speak of as a micturition centre capable of being thrown into action by appropriate afferent impulses, the action of the centre being such as to cause a contraction of the walls of the bladder and of the ejaculator urinie, and at the same time to suspend the tone of the sphineter vesice externus. Clinical experience also goes to shew the 
existence of a similar micturition centre in man, placed higher up in the cord than the corresponding 'genital' centre governing the genital organs.

Moreover we have, in the case both of man and of other animals, experimental and other evidence that contraction of the bladder is frequently brought about by reflex action. Thus the pressure within the bladder when observed for any length of time is found to be subject to considerable and manifold variations. Over and above passive changes in pressure due to the respiratory movements, through which the bladder is pressed upon at each descent of the diaphragm, active contractions, of a strength inadequate to bring abont micturition, are from time to time observed. These in some instances appear to be spontaneous, or to be the result of emotions, but they may be readily induced in a reflex manner, by stimulating various sentient surfaces or sensory nerves. And common experience affords many instances where vesical contractions thus bronght about in a reflex manner acquire strength adequate to empty the bladder.

Observations of vesical pressure may be most conveniently carried out by introducing into the bladder a catheter connected with a water manometer and a registering apparatus, and so arranged as to allow fluid to be driven into or received from the bladder at pleasure.

$\$ 348$. Involuntary micturition obviously of reflex nature has frequently been observed in cases of paralysis from disease of or injury to the spinal cord; and the involuntary micturition which is common in children, as the result of irritation of the penis and genital organs, and which sometimes occurs in the adult as the result of emotions, or at least sensory impressions, appears to be the result of reflex action. In these several cases we may fairly suppose that the centre in the spinal cord is affected by afferent impulses reaching it along varions sensory nerves or descending from the brain. Hence we are led to the conception that when we make water by a conscious effort of the will, what occurs is not a direct action of the will on the muscular walls of the bladder, but that impulses started by the will descend from the brain after the fashion of afferent impulses and thus in a reflex manner throw into action the micturition centre in the spinal cord. Nor is this view negatived by the fact that paralysis of the bladder, or rather inability to make water either voluntarily or in a reflex manner, is a common symptom of cerebral or spinal discase or injury. Putting aside the cases in which the reflex act is not called forth because the appropriate stimulus has not been applied, the failure in mictnrition muder these circumstances may be explained by supposing that the shoek of the spinal injury or 
some extension of the disease has rendered the spinal centre unable to act.

The so-called incontinence of urine in children is simply an easily exeited and frequently repeated reflex micturition. In cases of cerebral or spinal disease a form of incontinence is frequently met with which seems to be of a different nature. The bladder becoming full, but, owing to a failure in the mechanism of voluntary or reflex micturition, being unable to empty itself by a complete contraction, a continual clribbling of urine takes place through the urethra, the fulness of the bladder being sufficient to overcome the resistance at the neck of the urethra. It is probable, lowever, that even in these cases the flow is partly caused by obscure, unfelt, intrinsic contractions of the bladder.

\$34. Whether, under normal conditions, the urine undergoes any notable change during its stay in the bladder has been much debated. Experiments shew that poisonous substances injected into the bladder with all due care to aroid any abrasion of the epithelium are absorbed and produce their usual effects. It has also been stated that if a solution of urea be injected into the bladder after ligature of both ureters, and allowed to stay for some hours, part of the urea disappears. But at present there is no very decided proof that under ordinary conditions either the water or other constituents of urine are to any appreciable extent absorbed by the bladder.

Under abnormal conditions, as in inflammation or irritation of the bladder, the urine may lave undergone marked changes during its stay in the bladder, one of the most common being a change of some of the urea into ammonium carbonate, by which the urine becomes alkaline. Under abnormal conditions also, the mucus of the urine, which in a healthy man is insignificant, though in some animals, for instance the horse, it occurs in considerable quantity, is largely increased during the stay in the bladder. Since there are in man no goblet cells in the vesical epithelium (in the frog they are present) or mucus glands in the walls of the bladder, this mucus must be supplied by an abnormal metabolism of the ordinary epithelial cells. 


\section{SEC. 4. THE NATURE AND AMOUNT OF PERSPIRATION.}

$\S 350$. The quantity of matter which leaves the human body by way of the skin is very considerable. Thus it has been estimated that while 5 gram passes away through the lungs per minute, as much as .8 gram passes througl the skin. The amount, however, varies extremely; it has bcen calculated, from data gained by enclosing the arm in a caoutchouc bag, that the total amount of perspiration from the whole body in 24 hours might range from 2 to 20 kilos; but such a mode of calculation is obviously open to many sources of error.

Of the whole amount thus discharged, part passes away at once as watery vapour mixed with volatile matters, while part may remain for a time as a fluid on the skin; the former is frequently spoken of as insensible, the latter as sensible perspiration or sweat. The proportion of the insensible to the sensible perspiration will depend on the rapidity of the secretion in reference to the dryness, temperature and amount of movement of the surrounding atmosphere. Thus, supposing the rate of secretion to remain constant, the drier and hotter the air, and the more rapidly the strata of air in contact with the body are renewer, the greater is the amount of sensible perspiration which is by evaporation converted into the insensible condition; and conversely when the air is cool, moist, and stagnant, a large amount of the total perspiration may remain on the skin as sensible sweat. Since, as the name implies, we are ourselves aware of the sensible perspiration only, it may and frequently does happen that we seem to ourselves to be perspiring largely, when in reality it is not so much the total perspiration which is being increased as the relative proportion of the sensible perspiration. The rate of secretion may, however, be so much increased, that no amount of dryness, or heat, or movement of the atmosphere, is sufticient to carry out the necessary evaporation, and thus the sensible perspiration may become abundant in a hot, dry air. And practically this is the usual occurrence, since certainly a high temperature conduces, as we shall point out presently, to an increase of the secretion, and it is possible that mere dryness of the air has a similar effect. 
The amount of perspiration given off is affected not only by the condition of the atmosphere, but also by the circumstances of the body. Thus it is influenced by the nature and quantity of food eaten, by the amount of fluid drunk, by the character of exercise taken, by the relative activity of the other excreting organs, more particularly of the kidney, by mental conditions and the like. Variations may also be induced by drugs and by diseased conditions. How these various influences produce their effects we shall study immediately.

The fluid perspiration, or sweat, when collected, is found to be a clear colourless fluid of a distinctly salt taste, with a strong and distinctive odour varying according to the part of the body from which it is taken. Besides accidental epidermic scales, it contains no structural elements.

Sweat, as a whole, is furnished partly by the sweat-glands and partly by the sebaceous glands, for as we shall see the small amount which simply transudes through the epidermis, apart from the glands, may be neglected. Now the secretions from these two kinds of glands differ widely in nature, and the characters of the sweat as a whole will vary according to the relative proportion of the two kinds of secretion. The amount of secretion of the sebaceous glands appears to be fairly constant, the larger variations of the total sweat depending chiefly on the varying activity of the sweat-glands. Hence when sweat is scanty, the constituents of the sebum influence largely the characters of the sweat; when on the contrary the sweat is very abundant, these may be disregarded and the sweat may be considered as the product of the sweat-glands.

We are not able, at present, to make a complete statement as to what bodies occur exclusively in the sebum and what in the secretion of the sweat-glands. The former consists very largely of fats and fatty acids, and appears to contain some form or forms of proteids; but we have reason to think that the sweatglands secrete in small quantity some forms of fat, and especially volatile fatty acids.

When sweat is scanty, the reaction is generally acid, but when abundant, is alkaline; and when a portion of the skin is well washed the sweat which is collected immediately afterwards is usually alkaline. From this we may infer that the secretion of the sweat-glands is naturally alkaline, but that when mixed sweat is acid, the acidity is due to fatty (or other) acids of the sebum. In the horse, which is singular among hair-covered animals for its frequent profuse sweating, the sweat is sairl to be always alkaline, and to contain a considerable quantity of some form of proteid.

Taking ordinary sweat, such as may be obtained by enclosing the arm in a bag, we may say that, in man, the average amount of solids is from 1 to 2 p.c., of which about two-thirds 
consist of organic substances. The chief normal constituents are: (1) Sodium chloride, with small quantities of other inorganie salts. () $V^{r}$ arious acids of the fatty series, such as formic, acetic, butyric, with probably propionic, caproic, and caprylic. 'The presence of these latter is inferred from the odour; it is probable that many various volatile acids are present in small quantities. Iactic acid, which has been reckoned as a normal constituent, is stated not to be present in licaltl. (3) Neutral fats, and cholesterin; these have been letected even in places, such as the palms of the hand, where sebaceous glands are absent. (t) 'The evidence goes to shew that neither' urea nor any ammonia compound exists in the normal sceretion to any extent, though some observers have found a considerable quantity of urea (calculated at 10 grms. in the 24 hours for the whole body). Apparently some small amount of nitrogen leaves the body by the skin as a whole, but this is probably supplied by the sebum or by the epidermis.

In various forms of disease the swcat has been found to contain, sometimes in considerable quantities, blood, albumin, urea (particularly in cholera), uric acid, ealcium oxalate, sugar (in diabetic patients), lactic acid, indigo (or indigo-vielding bodies giving rise to 'blue' sweat), bile and other piginents. Iodine and potassium iodide, suceinic, tartaric, and benzoic (partly as hipponric) acids have been found in the sweat when taken internally as medicines.

\section{Cutaneous Respiration.}

\$351. A frog, whose lungs have bcen removed, will continue to live for some time; and during that period will continue not only to prodnce carbonic acid, but also to consume oxygen. In other words, the frog is able to breathe without lungs, respiration being carried on efficiently by means of the skin. In mammals and in man this cutaneous respiration is, by reason of the thickness of the epidcrmis, restricted to within very narrow limits: and indecd it has becn questioned whether it can be spoken of at all as a true respiration. W'hen the body renains for some time in a closed chamber to which the air passing in and ont of the lungs has no access (as when the body is enclosed in a large air-tight bag fitting tightly round the neck, or where a tule in the trachea carries air to and from the lungs of an animal placed in an air-tight box), it is found that the air in the chamber loses oxygen and gains carbonic acid. The amount of carbonic acirl which is thus thrown off by the skin of an a velage man in 24 hours amounts to abont $10 \mathrm{grms}$, or according to some obscrvers to (no more than) about tgrns.., increasing witl a rise of temperature, and being very markedly augmented by bodily exercise. It is stated that the amonnt of oxygen con- 
sumed is about equal in volume to that of the carbonic acid given off, but some observers make it rather less. It may be doubted, however, whether the carbonic acid comes direct from the blood; it may come from decompositions taking place in the sweat, of carbonates for instance. Similarly the oxygen which disappears may be simply used in oxydizing some of the constituents of the sweat. It is evident that the loss which the body suffers through the skin consists, besides a small quantity of sodium chloride, chiefly of water.

When an animal, a rabbit for instance, is covered over with an impermeable varnish such as gelatin, so that all exit or entrance of gases or liquids by the skin is prevented, death shortly ensues. This result camnot be due, as was once thought, to arrest of cutaneous respiration, seeing how insignificant and doubtful is the gaseous interchange by the skin as compared with that by the lungs. Nor are the symptoms at all those of asphyxia, but rather of some kind of poisoning, marked by a very great fall of temperature, which however seems to be the result not of diminished production of heat, but of an increase of the discharge of heat from the surface. Owing to the dilated condition of the cutaneous vessels, caused by the application of varnish, the loss of heat through the skin is abnormally large, even though the varnish may not be a good conductor. The animal may be restored, or at all events its life may be prolonged with abatement of the symptoms, if the great loss of heat which is evidently taking place be prevented by covering the body thickly with cotton wool, or keeping it in a warm atmosphere. The symptoms have not as yet been clearly analyzed, but they seem to be due in part to a pyrexia or fever possibly caused by the retention within or reabsorption into the blood of some of the constituents of the sweat, or by the products of some abnormal metabolism.

\$352. Absorption by the skin. Although under normal circumstances the skin serves only as a channel of loss to the body, it has been maintained that it may, under particular circumstances, be a means of gain; and the little which we have to say on this matter may perhaps be said here. Cases are on record where bodies are said to have gained in weight by immersion in a bath, or by exposure to a moist atmosphere during a given period, in which no food or drink was taken, or to have gained more than the weight of the food or drink taken; the gain in such cases must have been due to the absorption of water by the skin. Direct experiments, however, throw doubt on these statements, for they shew that under ordinary circumstances such a gain by the skin is slight, being apparently due to mere imbibition of water by the upper layers of the epidermis.

Absorption of various substances takes place very readily 
by abraded surfaces where the dermis is laid bare or covered only by the lowest layers of epidermis, but it has been debated whether substances in aqueous solution can be absorbed by the skin when the epidermis is intact, the evidence on this point being contradictory. In the case of the skin of the frog an absorption of water and of various soluble substances certainly takes place. In the case of the sound human skin there are no a priori reasons why water carrying substances dissolved in it should not pass inwards through the corneous as well as the other layers of the epidermis, the amount so passing depending, among other things, upon the condition of the skin; and common experience seems to shew that it does. Nevertheless the results of actual experiment are conflicting. Some observers maintain that soluble non-volatile substances are not absorbed, and that volatile substances such as iodine which may be detected in the system after a bath containing them are absorbed not by the skin but by the mucous membrane of the respiratory organs, the substance making its way to the latter by volatilization from the surface of the bath. Others again have found evidence of absorption, especially with volatile substances, even when care has been taken to avoid all errors; and the greater weight may perhaps be given to these since they accord with common experience. The conflict of experimental results, however, at least shews that we do not fully understand the conditions under which such absorption takes place.

There is moreover evidence that even solid particles can pass through an intact skin. The lymphaties in the skin of a newborn infant have been found crowded with the particles of the peculiar fatty secretion which covers the skin at birth; and solid particles rubbed into even the sound skin may, especially when applied in a fatty vehicle, as ex. gr. in the wellknown mercury-ointment, find their way into the underlying lymphatics. The wandering leucocytes which are at times found among the epidermic cells may perhaps take part in this transport. 


\section{SEC. 5. THE MECHANISM OF THE SECRETION OF SIVEAT.}

$\$ 353$. In dealing with the manner in which various circumstances affect the amount of sweat secreted we may, as we have already said, consider the sweat as a whole to be supplied by the sweat-glands alone. For though it seems evident that some amount of fluid must pass by simple transudation through the ordinary epidermis of the portions of skin intervening between the mouths of the glands, yet on the whole it is probable that the portion which so passes is a small fraction only of the total quantity secreted by the skin; and direct experiment shews that even the simple evaporation of water is much greater from those parts of the skin in which the glands are abundant than from those in which they are scanty. IVe have as yet no evidence that the sebaceous glands vary in activity; their very peculiar form of secretion, if we may speak of it as a secretion, is not adapted to sudden changes, and at all events we have as yet no evidence that circumstances rapidly and largely modify the amount of sebum discharged by healthy sebaceous glands.

The secreting activity of the skin, like that of the other glands, is usually accompanied and aided by vascular dilation. In one of the early experiments on division of the cervical sympathetic, it was observed that in the ease of the horse, the vascular dilation of the face on the side operated on was accompanied by increased perspiration. Indeed the connection between the state of the cutaneous blood vessels and the amount of perspiration is a matter of daily observation. When the vessels of the skin are constricted, the secretion of the skin is diminished; when they are dilated, it becomes abundant. In this way, as we shall later on point out, the temperature of the body is largely regulated. When the surrounding atmosphere is warm, the cutaneous vessels are dilated, the amount of sweat secreted is increased, and the consequently augmented evaporation tends to cool down the body. On the other hand, when the atmosphere is cold, the cutaneous vessels are constricted, perspiration is scanty, and less heat is lost to the body by evaporation. 
The analogy with the other secreting organs which we have already studied leads us, however, to infer that there are special nerves directly governing the activity of the sudoriparous glands, independent of variations in the vascular supply. And not only is this view suggested by many facts, such as the profuse perspiration of the death agony, of various crises of disease, and of certain mental emotions, and the cold sweats occurring in phthisis and other maladies, in all of which the skin is anæmic rather than hyperemic, but we have direct experimental evidence of a nervous mechanism of perspiration as complete as the vaso-motor mechanism.

If in the cat ${ }^{1}$ the peripheral stump of the divided sciatic nerve be stimulated with the interrupted current, drops of sweat may readily be observed to gather on the hairless sole of the foot of that side. 'The sweating is not due to any increase of blood-supply, for it may be observed when the cutaneous vessels are thrown into a state of constriction by the stimulus, or even when the aorta or crural artery is clamped previous to the stimulation, and indced may be obtained by stimulating the sciatic nerve of a recently amputated leg. Moreover when atropin has been injected, the stimulation produces no sweat, though vaso-motor effects follow as usual. The analogy between the sweat-glands of the foot and such a gland as the submaxillary is in fact very close, and we are justified in speaking of the sciatic nerve as containing secretory fibres distributed to the sudoriparous glands of the foot. Similar results may be obtained with the nerves of the fore limb. And in ourselves a copious secretion of sweat may be induced by tetanizing through the skin the nerves of the limbs or the face.

If a cat in which the sciatic nerve has been divided on one side be exposed to a high temperature in a heated chamber, the limb the nerve of which has been divided remains dry, while the feet of the other limbs sweat freely. This result shews that the sweating which is eaused by exposure of the body to high temperatures is brought about by the agency of the central nervous system, and not by a local action on the sweat-glands; for the foot of the limb whose nerve has been divided is equally exposed to the high temperature. A high temperature it is true increases up to a certain limit the irritability of the epithelium of the sweat-glands and predisposes it to secrete, just as it promotes action in the case of a muscle or nerve or other forms of living substance. Thus stimulation of the sciatic in the eat

1 The cat sweats freely in the hairless soles of the feet but not on any part of the body covered with hairs. The dog also sweats in the same regions but not so freely as the cat ; indeed sweating is often absent, the ducts being stopped by growth of the corneous epidermis. Rabbits and other rodents appear not to sweat at all. The snout of the pig sweats freely; and the often profuse sweating of the horse, a singular event among hair-covered animals, is known to all. 
produces a much more abundant secretion in a limb exposed to a temperature of $35^{\circ}$ or somewhat above, than in one which has been exposed to a distinctly lower temperature, and in a limb which has been placed in ice-cold water hardly any secretion at all can be gained; but apparently mere rise of temperature without nerve-stimulation will not give rise to a secretory activity of the glands. The sweating caused by a dyspnoeic condition of blood, and such appears to be the sweat of the death agony, is similarly brought about by the agency of the central nervous system. When an animal with the sciatic nerve divided on one side is made dyspnoeic, no sweat appears in the hind limb of that side, though abundance is seen in the other feet.

Sweating may be brought about as a reflex act. Thus when the central stump of the divided sciatic is stimulated sweating is induced in the other limbs, and in ourselves the introduction of pungent substances into the mouth will frequently give rise to a copious perspiration over the side of the face. We are thus led to speak of sweat centres, analogous to the vaso-motor centres, as existing in the central nervous system; and as in the case of vaso-motor centres, a dispute has arisen as to whether there is a dominant sweat centre in the medulla oblongata or whether such centres are more generally distributed over the whole of the spinal cord.

It does not at present appear certain whether the sweating caused by heat is carried out by direct action of the heated blood on the sweat centres, or by the higher temperature stimulating the skin and so sending up afferent impulses which produce the effect in a reflcx manner; but in the case of dyspnora at least we may fairly suppose that the action of the venous blood is chiefly if not exclusively on the nerve centres. Some drugs, such as pilocarpin, which cause sweating, appear to produce their effect chiefly by a local action on the glands, since the action continues after the division of the nerves (though pilocarpin apparently has as well some slight action on the nerve centres), and the antagonistic action of atropin is similarly local. Picrotoxin and strychnia appear to produce their sweating action chicfly if not exclusively by acting on the central nervous system, while nicotin seems to act both centrally and peripherally.

354. In the eat (in which animal the matter has been most studied), the sweat fibres for the hind-foot leave the spinal cord by the anterior roots of the first and second lumbar nerves, but also to a less extent by the two thoracic nerves above these and the third lumbar nerve below. Passing to the sympathetic chain, and rumning in it for a certain distance, they leave that chain by the grey rami of the sixth and seventh lumbar and first and second sacral ganglia, thus reaching the spinal nerves 
corresponding to these ganglia and so the sciatic nerve. Along their course the fibres are connected with nerve-cells in these ganglia, the fibres in a grey ramus starting from cells in the ganglion from which the ramus run, or in the ganglion above it. In the same animal, the sweat-fibres for the fore-feet leave the spinal cord by the anterior roots of the sixth, seventh, and eighth thoracie nerves, but also, to a less extent, by the nerves alove and below. Passing into the sympathetic chain, they ascend to the ganglion stellatum, with the nerve-cells of which alone they are comnected, and by the branches of this ganglion reach the branchial plexus and so the median and ulnar nerves. The course of the sweat-fibres in other animals is probably very similar to the above. In the horse the sweat-frbres for the side of face and in the pig those for the snout appear to run in branches of the fifth nerve and not in the facial; in the latter animal at least some of these fibres reach the fifth nerve from the cervical sympathetic, but apparently not all.

$\$ 355$. The fact mentioned above that in the horse, after section of the cervical sympathetic nerve on one side of the neck, profuse sweating is apt to break out on that side of the face, has suggested the idea that this nerve conveys inhibitory impulses to the sweat-glands of the head and face, and that when it is divided the sweat-fibres rumning in the fifth nerve, having nothing to counteract them, set up sweating. But it is probably sufficient in this case to suppose that the glands predisposed to activity by the higher temperature brought about by the section of the sympathetic dilating the blood vessels, are more easily excited by any stimulus working upon them through the fifth nerve. And though the idea of a double nervous mechanism, augmenting and inhibitory, governing the activity of the sweat-glands, is a tempting one, there are at present no satisfactory reasons for adopting it. 


\section{CHAPTER IV.}

\section{THE METABOLIC PROCESSES OF THE BODY.}

$\$$ 356. We have followed the food through its changes in the alimentary canal, and have seen it enter into the blood, either directly or by the intermediate channel of the lacteals, in the form of peptone (or otherwise modified albumin), sugar (lactic acid), and fats, accompanied by various salts and water. We have further seen that the waste products which leave the body are urea, carbonic acid, salts and water. We have now to attempt to connect together the food and the waste products; to trace out as far as we are able the various steps by which the one is transformed into the other. There remains the further task to inquire into the manner in which the energy set free in this transformation is distributed and made use of.

The master tissues of the body are the muscular and nervous tissues; all the other tissues may be regarded as the servants of these. And we may fairly presume that, besides the digestive and excretory tissues which we have already studied, many parts of the body are engaged either in further elaborating the comparatively raw food which enters the blood, in order that it may be assimilated with the least possible labour by the master tissues, or in so modifying the waste products which arise from the activity of the master tissues that they may be removed from the body as speedily as possible. There can be no doubt that manifold intermediate changes of this kind do take place in the body ; but our knowledge of the matter is at present very imperfect. In a few instances only can we localize these metabolic actions and speak of distinct metabolic tissues. In the majority of cases we can only trace out or infer chemical changes, without being able to say more than that they do take place somewhere ; and in consequence, perhaps somewhat loosely, speak of them as taking place in the blood.

How little we know concerning the metabolism of the master tissues themselves was shewn when we were dealing with these 
tissues in an earlier part of this work; but success in the study of these can hardly be expected until our knowledge is increased as regards the changes which the blood undergoes before it reaches and after it leaves the muscle or the nerve. 'The fact that a large part of the absorbed food is carried through the liver before it is thrown on the general circulation leads us to suppose that in this large organ important metabolic processes are carried on ; and observation with experiment confirms this view. Important as the secretions of bile may be the other metabolic functions of the liver are of still greater importance. 


\section{SEC. 1. THE HISTORY OF GLYCOGEN.}

$\S 357$. If the liver of a well-fed animal be removed immediately after death, rapidly divided into small pieces, thrown into boiling water, rubbed up and boiled, a decoction may be obtained which after careful neutralization and filtration will be tolerably free from proteid matter. Such a decoction is remarkably opalescent, milky in fact in appearance, much more so than a similar decoction from muscle or other tissue, and remains opalescent even after repeated filtration. Treated with iodine, the solution turns a brownish red, port-wine red colour, not unlike that given by dextrine when iodine is added; the colour disappears on warming, but reappears on cooling provided that not too much proteid matter has been left in the solution. Treated with Fehling's fluid or other tests for sugar, the solution is found to contain a small and variable, but only a small, quantity of sugar.

If the solution be exposed, preferably in the warm, to the action of saliva or of some other amylolytic ferment, or be boiled with dilute acid, the opalescence disappears; and the now clear transparent solution gives no longer the port-wine reaction with iodine. 'Tested moreover with Fehling's fluid or by other means it is now found to contain a considerable quantity of sugar.

If alcohol be added to the opalescent solution until the mixture contains 60 p.c. of the alcohol (previous concentration by evaporation being desirable) a white amorphous precipitate is thrown down. This precipitate, removed by filtration, boiled with an alcoholic solution of potash in which it is insoluble, but which dissolves and destroys any proteids which may be present, treated with ether to remove fatty impurities, and washed with alcohol may be obtained in a pure condition. It then appears as a white amorphous powder, fairly soluble in water, but always giving rise to a milky opalescent solution unless an excess of alkali be present, in which case the opalescence may be slight or absent.

The opalescent solution of this purified material gives a port-wine reaction with iodine, but no reaction whatever with Fehling's fluid or the other sugar tests. 'Treated with an 
amylolytic ferment or boiled with dilute acid, the solution, like the raw decoction of liver, loses its opalescence and its portwine reaction with iodine but now gires abundant evidence of the presence of sugar, dextrose, if boiling with acid has been employed, maltose chiefly, if an amylolytic ferment has been used. If quantitative determinations be employed it will be found that the amount of sugar obtained is proportionate to the amount of the white powder acted upon; in other words the substance forming an opalescent solution is converted into sugar, the solution of which is elear. Obviously the substance is a body allied to stareh; and this is confirmed by its elementary composition, which is found to be $\mathrm{C}_{6} \mathrm{H}_{10} \mathrm{O}_{5}$ or some multiple of this.

Hence this body is called glycogen. And it is obvious from what has been stated above, that the liver of a well-fed animal at the moment of death contains a eonsiderable quantity of glyeogen either in a free state or in such a condition that it is set free by subjecting the liver to the action of boiling water. We may add that it oceurs in the liver in the hepatic cells, for these when glyeogen is present in the liver give, when properly tested with iodine, the characteristic port-wine reaction.

$\$ 358$. If the liver, instead of being treated immediately upon the death of the animal, is allowed to remain in the body of the dead animal for several hours, especially in a warm place, before a decoction is made of it, the decoction will be found to have little or no opalescence, to be quite or nearly quite elear, to give little or no port-wine reaction with iodine, but to contain a very considerable quantity of sugar. As we said above, the decoction even of a liver taken immediately after death generally contains some little sugar, and the quantity of sugar in the liver appears as a rule to increase after death, the amount of glycogen diminishing at the same time. We may infer from this that the glycogen present in the liver at the moment of death is gradually after death by some action or other converted into sugar.

The action is that of some agency whose activity is destroyed by the temperature of boiling water; hence the directions repeatedly given above to throw the liver into boiling water. This naturally suggests the presence in the liver of an anylolytic ferment. But, not only have attempts to isolate from the liver an amylolytic ferment failed, in the hands of most observers at least, but the exact nature of the sugar which appears shews that the change is not effected by an ordinary anylolytic ferment. In the case of the amylolytic ferment of saliva, pancreatic juice, intestinal juice, and indeed of all other amylolytic animal fluids, the sugar into whieh starch or glycogen is converted is maltose. Now the sugar which appears in the liver after deatl is dextrose, identical, so far at least as can at present he male out, with ordinary dextrose. We are led therefore to infer that the elhange of glycogen into suger which appears to 
go on after death is carried out by some action of the liver, probably of the hepatic cell itself, which is done away with by a temperature of $100^{\circ} \mathrm{C}$., but which is not the action of a ferment capable of being isolated.

$\$ 359$. We have used above the phrase 'well-fed' animal because the amount of glycogen present in the liver of an aminal at any one time is very variable, and especially dependent on the amount and nature of the food previously taken. When all food is withheld from an animal, the glycogen in the liver diminishes, rapidly at first, but more slowly afterwards. Even after some days' starvation a small quantity is frequently still found: but in rabbits, at all events, the whole may eventually disappear.

If an animal, after having been starved until its liver may be assumed to be free or ahmost free from glycogen, he fed on a diet rich in earbohydrates or on one consisting exclusively of carbohychates, the liver will in a short time be found to contain a very large quantity of glycogen. Obviously the presence of carbohydrates in food leads to an accumulation of glycogen in the liver; and this is true both of stareh and of dextrin and of the various forms of sugar, eane, grape and milk sugar. 'The effect may be quite a rapid one, for glyeogen has been found in the liver in considerable quantity within a few hours after the introduction of sugar into the alimentary canal of a starving aminal.

If an animal, similarly starved, be fed on an exclusively meat diet a certain amount of glyeogen is found in the liver. This appears to be especially the case with dogs (probably with other carnivorous animals also); and in earlier works on the subject the constant presence of glycogen in the livers of dogs fed on meat was regarded as an important indication of the formation within the body of non-nitrogenous from nitrogenous material. But in the first place, the quantity of glycogen thus stored up in the liver as the result of a meat diet, is much less than that which follows upon a carbohydrate diet; and in the second place, ordinary meat, especially horse-flesh on which dogs in such experiments are usually fed, contains in itself (\$59) a certain amount either of glycogen or some form of sugar. Moreover when animals are fed not on meat but on purified proteid, such as fibrin, easein or albumin, the quantity of glycogen in the liver becomes still smaller, though according to most observers remaining greater than during starvation. We may infer therefore that part of the glycogen which appears in the liver after a meat diet is really due to carbohydrate materials present in the meat. Part, however, would appear to be the result of the actual proteid food; and we have similar evidence that gelatine taken as food leads to the formation of some glycogen in the liver. But in this respect these nitrogenous substances fall far short of earbohydrate material.

With regard to fats, all observers ane arreed that these lead 
to no accumulation of glycogen in the liver; an animal fed on an exclusively fatty diet las no more glycogen in its liver than a starving animal.

Hence of the three rreat classes of food-stuffs, the carbohydrates stand out prominently as the substances which taken as food leal to an aecunulation of glycogen in the liver. We may remark that the greatest accumulation of glycogen is effected not by a pure carboliydrate diet, but by a mixed diet rich in carbohydrates. A quantity of carbohydrate mixed with a certain proportion of proteid gives rise to a larger anount of glycogen in the liver than the same quantity of carbolydrate given by itself; and it is possible that the presence of an appropriate quantity of fat still further assists the accumulation. But this result probably depends, in part at least, on the fact that, though differences may be met with in different animals, a mixture of the several classes of food-stuffs is more readily digested resulting in more nutritive material being thrown upon the blood, than is a meal consisting exclusively of one kind of food-stuff alone.

So far as we know at present the glycogen which thus appears in the liver as the result of feeding either with any of

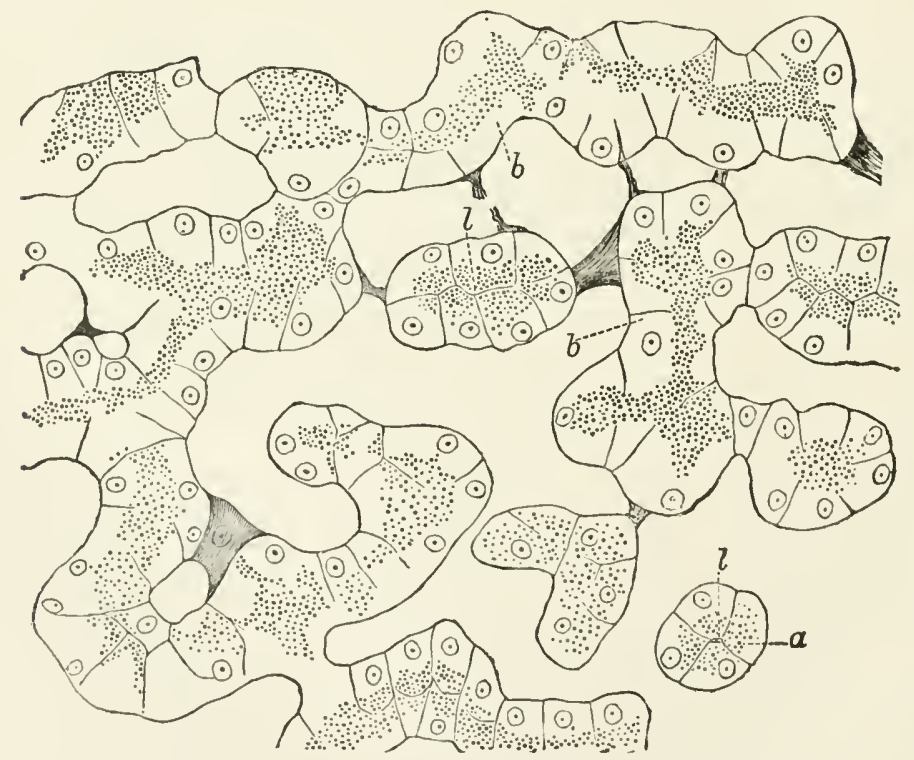

Fig. 105. Section of Liver of Frog. (Langley.)

The Figure shews the tubular structure of the liver. At (a) a tubule is seen in transverse, at (b) in longituhinal section. l, lumen of tubule.

'The liver was that of a winter frog, and the cells shew an imner zone of proteid granules; the outer zone was chietly occupied by glycogen. 
the varions forms of earbohydrates, or with proteids, or with other substantes, is of the same kind and presents the same characters; at least we have no evidence to the contrary.

The storing-up of glyeogen in the liver is also influenced by other circumstances than the taking of food. For instance in the frog an increase of glyeogen takes place during the winter montlis. In the summer months the liver of a frog will be found to contain very little glycogen, Fig. $106 \mathrm{c}$, unless the animal has been unusually well fed; whereas a liver examined in mid winter, Figs. 105, $106 \mathrm{~A}$, will be found to tontain a considerable quantity, even though no food has been taken for months. In such a ease the material for the formation of the glycogen in the liver must have been furnished by some part of the body of the frog, and could not, as may be the case when a meal leads immediately to an increase of glyeogen, be supplied

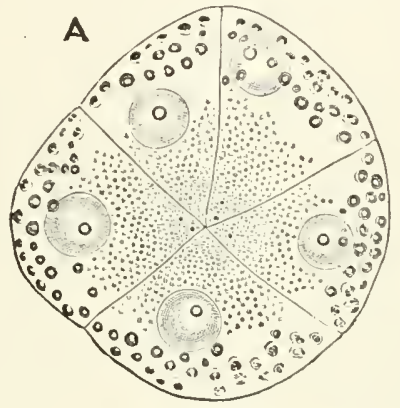

B
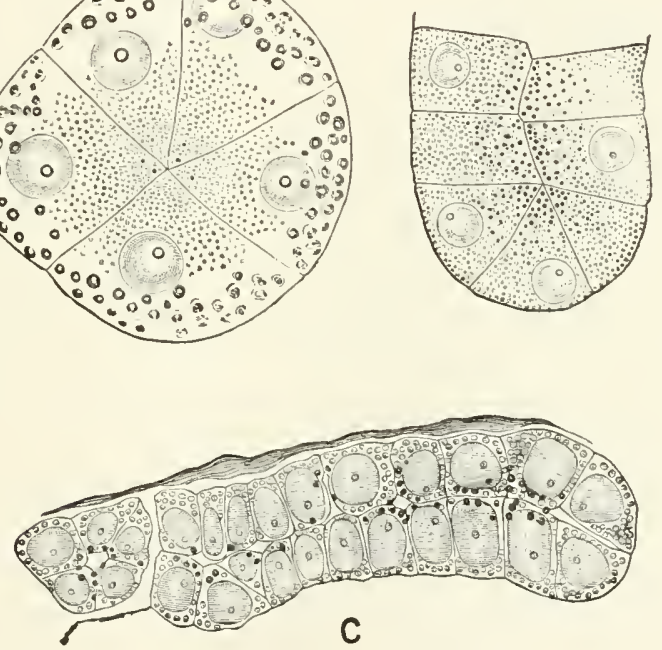

Fig. 106. Three phases of the Hepatic Cells of the Frog. (Langley.)

A. Cells rich in glycogen. Taken from a frog during winter. The cells are large, and proteid granules are massed round the lumen, the homogeneons outer zones of the cells being largely composed of glycogen which was iresent in considerable abundance. The outer zones contained numerous fat globules, shewn as dark dots; but as stated in the text these fat globules vary much.

B. Cells poor in glycogen. Taken from a winter frog which had been kept at $22^{\circ} \mathrm{C}$. for 10 days. The cells contain very little glycogen and the proteid granules are dispersed throughout the cell. In a summer frog well fed on proteids the cells would present a very similar appearance.

C. Starved cells. Taken from a summer frog after a long fast. The cells are small and almost free from glycogen. The proteid granules are dispersed throughout the cell.

All the specimens were hardened in 1 p.c. osmic acid, and are drawn to the same or nearly to the same scale. 
directly from the foorl. It seems as if in the summer the frog lives up to its capital of hepatic glycogen. spending it as fast almost as it is made, but that during the winter a quantity is funded to provide for the demands of late winter and early spring.

This winter storage of hepatic glycogen in the frog seems closely dependent on temperature. If a winter frog, whose liver is presumably morc or less loarled with glycogen, be exposed for some time to a temperature of $20^{\circ}$ or a little higher, the liver will afterwards be found to contain little or no glycogen, Fig. $106 \mathrm{I}$; and conversely if a summer frog be cxposed to untimely cold, glycogen, though not in any great quantity, begins to be stored up in the liver.

$\$ 360$. Before we attempt to discuss further how food and other circumstances thus affect the glycogen in the liver, it will be desirable to consider certain histological changes occurring in the hepatic cells, under various conditions. It will be convenient to begin with the cells of the more distinctly tubular gland of the frog.

In a frog which has not been subjected to any special treatment the cell-substance of the hepatic cell (cf. Fig. 106 A) will generally be found to contain lodged in itself three kinds of material, the presence of which, if not directly recognizable in the fresh cell, may be demonstrated by the use of various reagents. In the first place, oil globules of variable size and in variable anount are scattcred throughout the cell; sometimes, as we have already said, these are extremely abundant; but there is otherwise nothing very special about these fat globules in the hepatic cell to demand any discussion concerning them apart from the general discussion on the formation of fat, into which we shall enter later on.

In the second place, a number of small discrete granules may be seen lodged in the cell-substance. These appear to be of a proteid nature and are generally most abundant on the inner side of the cell near the lumen of the bile passage. The presence of these granules is closely dependent on the activity of the digestive processes. They diminish when digestion is going on and accumulate again afterwards. Putting aside certain details we may say that these granules behave very much like the granules in an albuminous salivary cell, a pancreatic cell or a chief gastric cell; and we may probably safely conclutle that they, like the granules in these cells, are in some way concerned in the formation of the secretion; that is, in their case, bile.

In the third place, the cell contains more especially in its onter parts, ncarer the blood vessel, away from the lumen of the bile passage, a variable quantity of material which differs from the ordinary cell-substance in being hyaline and refractive and lience glassy looking, and in staining port-wine red with 
iodine instead of brownish yellow as docs ordinary cell-sub. stance. 'This material is, though with some little diffieulty, soluble in water, and by this means may be dissolved out from the cell. When this is done the places which it occupied appear as vacuoles or gaps of various sizes limited by bar's of the cell-substance, which thus takes on the form of a network, the meshes of which are wider and more conspicuous in the outer part of the cell, in which the lyaline material was previously most abundant. In the inner part of the cell where the hyaline material was seanty the cell-substance is more dense, and even in the outer part a shell of more dense, less reticulate cell-substance affords a definite outline to the cell. There can be no doubt that this hyaline material is either actual glycogen such as may be extracted from the liver, or, as seems more probable from its deficient solubility, glycogen in some more or less loose combination with some other body, a combination, however, of such a kind that the iodine reaction makes itself felt.

$\$ 361$. The above may be taken as a general description of a cell in an ordinary condition. The question now comes before us, What changes are brought about by various foods or by the absence of food?

If a frog be largely fed on a diet containing large quantities of carbohydrates, the liver will be found rich in glyeogen and the cells will present the following characters. The cell is relatively large (ef. Fig. $106 \mathrm{~A}$ ) and as it were swollen; the cell-substance is largely oecupied by the hyaline material just spoken of, especially in its outer parts, so that in sections prepared and mounted in the ordinary way in which the glycogen has been dissolved out the greater part of the cell consists of a loose open network of bars of stained cell-substance, with wide meshes; a certain quantity of more solid, generally granular looking cell-substance occupies the part of the cell nearest the lumen, and a thin shell of cell-substance forms an envelope for the rest of the cell. The nucleus is large and distinct. When such a cell is seen in a perfectly fresh state, the hyaline refractive material (giving the reaction with iodine) often hides the nucleus and the greater part of the cell-substance proper.

If on the other hand the frog be fed on a proteid diet free from carbohydrates, for instance on fibrin, the liver contains little or no glycogen, and the hepatic cells are not only much smaller but present an appearance very different from the above (cf. Fig. $106 \mathrm{~B}$ ). Little or no hyaline material is visible, the cells give little or no port-wine reaction with iodine, but only the usual brown yellow proteid reaction, and in specimcns prepared and mounted in the ordinary way the cell-substance appears densely granular throughout.

Lastly, if the frog be starved, and if to the effects of starvation there be added those of exposure to a high temperature 
$\left(25^{\circ}\right)$, by which as we have seen the hepatic cells are markedly affected, the liver is found to be free from glycogen, and the hepatic cells to be extremely small (cf. Fig. $106 \mathrm{C}$ ), only half the size or even less, of those of the well-fed frog, but otherwise much like the cells in a frog fed on proteid material.

$\$ 362$. In the mammal changes in the hepatic cells similar to those just described as oceurring in the frog have also been

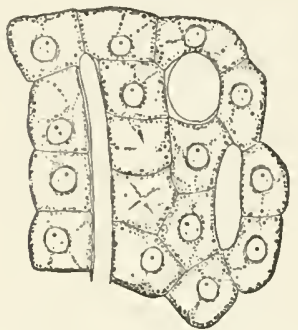

Fig. 107. SECTION OF Mamilatax Liver Rich in GLYCOGEN, (Langley.)

Osmic acid specimen, glycogen not dissolved out. observed. When the animal is fed on a diet rich in carbohydrates, and when therefore as we have seen the liver abounds in glyeogen, the hepatic colls (Fig. 107) are larger (so large that they liave by some authors been described as compressing the lobular capillaries) and loaded with the same refractive lyyaline material staining port-wine red with iodine. When this material, which is disposed more centrally in the coll than is the case in the frog, is dissolved out a coarse open network of cell-substance is displayed. We may add that in an animal thus fed the whole liver is very large and as it were swollen; it is also soft and tears easily.

In an animal fed on proteids alone, for instance on fibrin, the liver frequently contains some glycogen and the hepatic cells contain a small quantity of hyaline glycogenic material. As in the corresponding case in the frog, the cells are comparatively small, and the cell-substance appears finely and uniformly granular.

In a starved mammal, the liver is small, dense to the touch and tough; it contains a trace only of glycogen or none at all; the cells (Fig. 108) are small, as it were shrunken, and

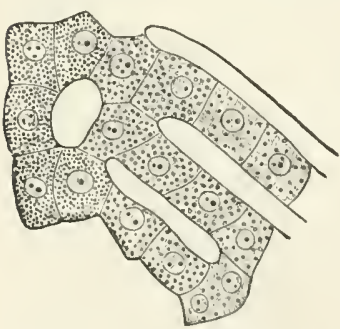

Fig. 108. Section of MAMMALIAN LIVER, CONTAINING LITTLE OR NO GLYCOGEN. (Langley.)

Osmic acid specimen. The gramules are not well preserved in some of the cells. the cell-substance, which gives no portwine reaction, or a mere trace only, with iodine, is still more finely granular.

$\S 363$. The microscopic appearances just described shew, and indeed general considerations lead us to the same conclusion, that the processes taking place in a hepatic cell are very complex. In the first place, the constituents of bile arc being formed and discharged into the bile passages after the fashion of an ordinary secreting gland. In the second place, a formation of glycogen is also taking place, and we shall have pres- 
ently to consider briefly the relations of the one process to the other. In the third place, as is especially indicated by the somewhat peculiar effects on the hepatic cell of food exclusively proteid in nature, other processes, similar perhaps to the formation of glycogen but not resulting in the storage of my carbohydrate material and dealing possibly with proteid substances, also take place. Hence the exact interpretation of all the changes which may be observed becomes exceedingly diffieult.

Leaving the processes of the first and third kind wholly on one side for the present, and confining our attention entirely to the glycogen, it is obvious that the hepatie cell manufactures the glycogen in some way or other, and lodges it in its own substance for the time very much in the way that a secreting cell manufactures and lodges in itself for a time material for the secretion which it is about to pour forth. 'There is this difference, that in the one case the material of the secretion, after undergoing as we have seen more or less change, is cast out into the lumen of the alveolus, whereas in the other case the glycogen, which must undergo change since it may be made to disappear rapidly from the hepatic cell, is not when changed cast out into the bile passages ; it must therefore be sent back again to the blood.

$\$ 364$. We say " manufactures the glycogen in some way or" other," and we have now to inquire what we know concerning the nature and the several steps of this manufacture.

We have already seen that the presence of glycogen in the liver is especially favoured by a carbohydrate diet; and in our studies on digestion we have seen reason to think that a very large part at all events of the carbohydrate material of a meal is absorbed as sugar by the capillaries of the intestine and carried as sugar to the liver in the portal blood. Hence, it seems only reasonable to conclude that the glycogen which makes its appearance in the liver after an amylaceous meal arises from a direct conversion of the sugar earried to the liver by the portal vein, the sugar becoming through some action of the hepatic cell-substance dehydrated into glycogen, or animal starch as it has been called, the process being a reverse of that by which in the alimentary canal starch is hydrated into sugar through the action of the salivary and pancreatic ferments. Vegetable cells can undoubtedly convert both starch into sugar and sugar into starch; and there are no $\dot{a}$ priori arguments or positive facts which would lead us to suppose that the activity of animal living substance cannot accomplish the latter as well as the former of these changes. We are quite ignorant it is true of the exact way in which either the hydration or the dehydration is effected by living substance; but we are equally ignorant of the exact way in which an amylolytie ferment effects the hydration of starch into sugar, which it carries out with so much apparent ease. It is not a great assumption to suppose that the continually changing living 
substance, which in its changes is continually giving out energy, has the power of acting on molecules of starch or of sugar in contact with, or even only near to itself, and so of hydrating starch into the sugar or of dehydrating sugar into starch. The latter process may be a more difficult one than the former, but not one beyond the power of the living substance. We may fairly suppose that a quantity of sugar in solution present in a vacuole, for instance, of the hepatic cell-substance can be, by some action of the cell-substance, converted into glycogen in a solid form, filling up the vacuole. Again, as we have incidentally mentioned, sugar injected into the jugular vein readily gives rise to sugar in the urine; but a very considerable quantity can be slowly injected into the portal vein without any appearing in the urine. This suggests the idea that the liver, so to speak, catches the sugar as it is passing through the hepatic capillaries and at once dehydrates it into glycogen.

Similar considerations may also be applied to the case men tioned above of the appearance of glycogen in the hepatic cells of winter (fasting) frogs. We have reason to think that sugar makes its appearance as a product of the metabolism of various tissues. The sugar thus arising finding its way into blood may be made use of at once elsewhere, converted speedily for instance into carbonic acid and so got rid of. But we can readily imagine that under certain circumstances, as, for instance when the activities of the animal were lessened by a low temperature, it was not so made use of and remained in the blood. If so it would in the course of the circulation be carried to the liver, and might be at once taken up by the hepatic cells and converted into glycogen; and these might be so active that the blood was never at any time allowed to remain loaded with sugar to such an extent as to permit a loss through the urine.

$\$ 365$. Upon such a view, the carbohydrate taken as food would be converted into glycogen by the agency of the hepatic cell, without at any time becoming an integral part of the living substance of the cell. Such a view may be the true one; but it is open for us to look at the matter in another light. $W_{c}$ may push still further the analogy between the glycogen of the hepatic cell and the material with which a secreting cell is loaded. In dealing with secretion we saw reasons for regarding such a body as mucin to be a product of the metabolism of the cell-substance of the mucous cell, and we may similarly regard glycogen, or sugar readily convertible into glycogen, or at least some or other carbohydrate material, as a normal prodnct of the metabolism of the hepatic cell. We may thus conceive of the hepatic cells as being continually engaged in giving rise to carbohydrate material in the form either of sugar or of some other body; and we may suppose that under 
certain circumstances, as in the absence of adequate food, the carbohyrlate material thus formed is at once discharged into the blood of the hepatic vein for the general use of the body, but that under other circumstances, as when an amylaceous meal has been taken, the immediate wants of the economy being covered by the earbohydrates of the meal, the carbohydrate products of the hepatic metabolism are stored up as glyeogen. Under such a view the sugar of the meal is used up somewhere in the body, and the glycogen to the storage of which in the liver it gives rise comes direct from the hepatic substance. And a similar cxplanation may be given of the storing-up of glyeogen in the liver under such cireumstances as those of the winter frog previously mentioned.

WV do not possess at present experimental or other evidence of so clear a kind as to enable us to decide dogmatically between these two views. It may be that both views are true, or rather that the true conception embraces both views. It may be that the normal metabolism of the hepatic cell does produce a certain amount of carbohydrate material; but if so the probability is that the exact form in which that carbohydrate appears in the first instance in the laboratory of the cell is not that of glycogen but of sugar of some kind or other, and that the conversion into glyeogen is a subsidiary act for the purpose of retaining the carbohydrate material in the grasp of the cell. If this be the case, then until it has been shewn that there is something peculiar about the sugar thus produced by the cell itself, by virtue of which it alone can be converted by the cell into glycogen, we may fairly infer that the cell might also convert into glycogen sugar passing into the interstices of the cell-substance from the portal capillaries.

$\$ 366$. We may now turn to another question, the answer of which is in a measure dependent on the one which we have just discussed. What is the use and purpose of this hepatic glycogen? What ultimately becomes of the glycogen thus for a while stored up in the liver?

One view which has been put forward is as follows. We have cvidence, as we shall presently learn, that a great deal of the fat of the body is not taken as such in the food, but is constructed anew in the body out of other substances. Both carbohydrates and proteids, taken in excess or under certain circumstances, lead to an accumulation of fat; and we have reason to believe that carbohydrates on the one hand and the carbon-holding portions of various proteids on the other, may by some process or other be converted into fat. And it has beên suggested that the glycogen in the liver is a phase of a constructive fatty metabolism, that it is material on its way to become fat. There is, however, no positive evidence in favour of this view. 
Another view. whieh has already been suggested while we were dealing with the manner of formation of glyeogen, makes use of the formation of fat for the purposes of analogy only. Seeing that adipose tissue serves as a storehouse of fat whieh is not wanted by the body at the moment but may be wanted presently, the question readily presents itself, May not the hepatic glyeogen have an analogous function? May we not regard the presenee of glyeogen in the liver as in large measure due to the faet that it is deposited there simply as a store of earbohydrate material, being aceumulated whenever amylaeeous material is abundant in the alimentary eanal, and being eonverted into sugar and so drawn upon by the body at large to meet the general demands for carbohydrate material during the intervals when fool is not being takeu? And we ean aeeept this view without being able to say definitely what beeomes of the sugar thus thrown into the hepatic blood. It was formerly believed that this sugar underwent an inmediate and direct oxidation as it was circulating in the blood, but we have alrearly dwelt ( $\$ 290)$ on the objeetions to such a view. It is sufficient for us at the present to admit that the sugar is made use of in some way or other.

Now, many considerations read us to believe that a certain average eomposition is neeessary for that great internal medium the blood, in order that the several tissues may thrive upon it to the best advantage, one element of that composition being a certain percentage of sugar. It would appear that some at least if not all of the tissues are eontinually drawing upon the blood for sugar, and that henee a certain supply must be kept up to meet this demand. On the other hand an exeess of sugar in the blood itself would be injurious to the tissues. And as a matter of faet we find that the quantity of sugar in blood is small but constant; it remains about the same when food is being taken as in the intervals between meals. If sugar be injeeted into the jugular vein in too large quantities or too rapidly, a eertain quantity appears in the urine, indieating an effort of the system to throw off the exeess and so bring baek the blood to its average eondition. The maintenance of such a eonstant percentage of sugar would obviously be provided for or at least largely assisted by the liver aeting as a strueture where the sugar might at once and without mueh labour be paeked away in the form of the less soluble glycogen, at those times when, as during an anylaceous meal, sugar is rapidly passing into the blood, and there is a danger of the blood becoming loaded with far more sugar than is needed for the time being; and it may be ineidentally noted that a larger quantity of sugar may be injected into the portal than into the jigular vein without any reappearing in the urine, apparently beeause a large portion of it is in sueh a case retained in the 
liver as glycogen. At those times, on the other hand, when we may suppose that sugar ceases to pass into the blood from the alimentary canal, the average percentage in the blood is maintained by the glycogen previously stored up becoming reconverted into sugar, and being slowly discharged into the hepatic blood.

Moreover, this view, that the glycogen of the liver is a reserve fund of carbohydrate material, is strongly supported by the analogy of the migration of starch in the vegetable kingdom. We know that the starch of the leaves of a plant, whether itself having previously passed through a glucose stage or not, is normally converted into sugar, and carried down to the roots or other parts, where it frequently becomes once more changed back again into starch.

367. Glycogen is found in other parts of the body than the liver, and a study of the facts relating to the presence of glycogen in other tissues will help us to a true conception of the purpose of the hepatic glycogen. Next to the liver, the skeletal muscles are perhaps the most conspicuous glycogen holders. So frequently is glycogen found in muscle that it may be regarded as an ordinary though not an invariable constituent of that tissue; indeed it may almost be considered as a constituent of all contractile tissues. The quantity varies very largely both in the different muscles of the same animal and corresponding muscles of different animals. It disappears, according to some observers, readily upon starvation, even before the hepatic glycogen is exhausted; but all observers are not agreed on this point, and in some muscles, at least, it appears to be retained for a very long time. It is said to be increased in quantity when the nerve of the muscle is divided, and the muscle thus brought into a state of quiescence. On the other hand it diminishes or even disappear's, being apparently converted into dextrose, when the muscle enters into rigor mortis. Some observers have found that it diminishes during tetanus, and maintain that it, after conversion into dextrose, is used up in the act of contraction, forming through its oxidation the immediate supply of the energy set free in the contraction. But even granting that the glycogen in a muscle may be diminished during prolonged labour, it cannot be admitted that the oxidation or other chemical change of glycogen is a necessary part of the ordinary metabolism of a muscular contraction, since many muscles wholly free from glycogen are perfectly well able to carry on long-continued contractions.

What is probably the use of glyeogen in muscle is suggested by the fact that undeveloped embryonic muscles are peculiarly rich in glycogen. In a young embryo, at the time when the muscular substance, though undergoing striation, is still largely 'protoplasmic' in nature, the quantity of 
glycogen present is enormous; it frequently amounts to 40 p.c. of the dry material. At this period the hepatic cells are immature and very little glycogen is present in them. Later on, as the muscles become more wholly striated, the glycogen largely disappcars from the muscle, and very soon afterwards begins to be stored up in the liver. 'The meaning of this can hardly be mistaken. The glycogen in the immature muscle is a store of carbohydrate material, laid down on the spot, and ready at once to be used in what we may probably call the fierce metabolic struggle by which the simple protoplasmic cellsubstance of the rudiment of the muscular fibre is transformed into the highly differentiated striated contractile substance. And we shall probably not err in considering the glycogen of the mature muscle to hold a similar position ; it is carbohydrate material stored up on the spot, a local branch so to speak of the great carbohydrate bank. It is destined to become part of the contractile substance, and as such will contribute to the energy set free in a muscular contraction; but its energy is only available in this way after it has undergone the necessary metabolism and become part of muscular substance; it cannot be fired off in a contraction while it lies as raw glycogen in the interstices of the muscular fibre.

$\$ 368$. Glycogen may also be found in considerable quantity in the placenta. Here, as we shall see in a later part of this work, it is laid down in epithelial cells which lie on the boundary between the maternal and the fotal tissues. And here too there can be little doubt that it serves as a store of carbohydrate material for the nourishment of the foetus.

It has also been found in leucocytes, and in cartilage corpuscles, especially in those large rapidly growing and rapidly multiplying cartilage corpuscles which lie in the outer zone of endochondral ossification, and in other situations. In cases of diabetes, where the body is overloaded with carbohychate material, it has been found in considerable quantity in the testis, in the brain and elsewhere. Its occurrence in these situations, and under these circumstances, may be regarded as additional evidence of the truth of the view which we have expounded above that the main purpose of the deposition of glycogen is to afford a store, either general or local, of carbohydrate material, which can be packed away without much trouble so long as it remains glycogen, but which can be drawn upon as a source of solnble circulating sugar whenever the needs of this or that tissue demand it. It thus forms a very complete analogue to the vegetable starch, and fitly earns the name of animal starch.

We have some reasons for thinking that there are sevcral varieties of glycogen, and that the glycogen which exists in muscle is not quite identical with that which occurs in the liver. Indeed there seem to be intermediate stages between glycogen 
and starch or dextrin. The physiological value of these differences has not yet however been clearly determined, and, with this caution, we may continue to speak of glycogen as a single substance.

\section{Diabetes.}

$\$$ 369. Natural diabetes is a disease characterized by the appearance of a large quantity of sugar in the urine. This is due, as we have already said, to the presence of an abnormal quantity of sugar in the blood. The system can only dispose (either by oxidation, or as seems more probable in other ways) of a certain quantity of sugar in a certain time. Sugar injected into the jugular vein reappears in the urine whenever the injection becones so rapid that the percentage of sugar in the blood reaches a certain (low) limit. Sugar in the mine means an excess of sugar in the blood. Into the pathology of the various forms of this disease it is impossible to enter here; but a temporary diabetes, the appearance for a while of a large quantity of sugar in the urinc, may be artificially produced in animals in several ways.

If the spinal bulb of a well-fed rabbit be punctured in the region which we have previously described (\$ 154) as that of the vaso-motor centre (the area marked out as the "diabetic area" agreeing very closely with that defincd as the vasonotor area), though the animal need not necessarily be in any other way obviously affected by the operation, its urine will be found, in an hour or two, or even less, to be increased in amount and to contain a considerable quantity of sugar. A little later the quantity of sugar will have reached a maximum, after which it declines, and in a day or two, or even less, the urine will be again perfectly normal. The better fed the animal, or, more exactly, the richer in glycogen the liver, at the time of the operation, the greater the amount of sugar. If the animal be previously starved so that the liver contains little or no glycogen, the urine will after the operation contain little or no sugar. It is clear that the urinary sugar of this form of artificial diabetes comes from the glycogen of the liver. The puncturc of the bulb causes such a change in the liver that the previously stored-up glycogen disappears, and the blood becomes loaded with sugar, much if not all of which passes away by the mrine. In the absence of any proof to the contrary, we nay assume that in this form of artificial diabetes the glycogen previously present in the liver becomes converted into sugar, just as we know that it does becone so converted by post-mortem changes. 'The glycogenic function of the liver is therefore subject to the influence of the nervous system, and in particular to the influence of a region of the cerebro-spinal centre which we already 
know as the vaso-motor centre, or at least of a part of that region.

$\$ 370$. With regard to the exact nature of the influence started by the puncture of the spinal bulb, and the path by which that influence reaches the liver, our information is at present very imperfect. One thing seems clear, viz. that the influence in question is not carried down by the main vagus trunks; for not only has the section of both these nerves in the neck no marked effect in the way of producing diabetes; but the 'diabetic puncture' of the spinal bulb is as efficient after division of both vagus nerves as before. 'The influence appears to reach the lines by way of the sympathetic system; but no authoritative statement as to the exact path can as yet be made. As to the nature of the influence we can perhaps at present only say that most probably the normal actions of the hepatic cells arc in some way directly interfered with, for we have no satisfactory evidence that vaso-motor changes, such as dilation of the hepatic artery, and consequent increase of the supply of arterial blood relatively to the supply of venous blood by the portal vein, bring alout the result in question.

$\$ 371$. A temporary diabetes may be brought about by the administration of the substance phloridzin. This however is a glucoside, and part of the sugar which appears in the urine, after a close of it, may come direct from the drug itself; but the quantity of sugar discharged is too great to be accounted for in this way, and similar diabetic effects are produced by the administration of phloretin, a derivate of phloridzin, not a glucoside, and not giving rise to sugar by its own decomposition. 'The sugar which appears in the urine after a close of eitlier of these substances seems to come in part at least from the liepatic store of glycogen when that is present; but the drug will give rise to sligir in the urine of starving animals, from whose livers (and otler tissues) glycogen is presumably absent.

Artificial diabetes is also a prominent symptom of urari poisoning. 'This is not due to the artificial respiration, which is had recourse to in order to keep the urarized animals alive; because, though disturbance of the respiratory functions sufficient to interfere with the hepatic circulation mat produce sugar in the urine, artificial respiration may with care be carried on without any sugar making its appearance. Moreover, urari causes diabetes in frogs, although in these animals respiration can be satisfactorily carried on without any pulmonary respiratory movements. 'The exact way in which this form of diabetes is brought about has not yet been clearly made out.

A very similar diabetes is seen in carbonic oxide poisoning; and is one of the results of a sufficient dose of morphia, or amylnitrite and of some other drugs. 
A diabetes of a permanent character, much more closely resembling the disease as occurring naturally, may be brought about in the following remarkable manner. If in a dog (and the same result may be obtained in many other animals) the whole of the pancreas be removed, sugar makes its appearance in the urine, and the animal soon becomes emaciated, with all the symptoms of ordinary diabetes. The gland must be removed; mere ligature or blocking of the duct does not produce the effect. And the whole gland must be removed; if only a small portion be left, the symptoms do not appear or are slight and temporary. Moreover, it has been found possible to transplant a portion of the gland, removing it from its normal surroundings and grafting it in some other situation. In such a case the whole of the rest of the gland may be removed without causing diabetes; but the symptoms immediately appcar if the transplanted portion be subsequently removed. We may infer that the pancreas, besides secreting pancreatic juice, produces some effect on the blood circulating through it, probably discharges into the blood some substance, and that this effect, this substance, has to do with the regulation of the sugar in the blood. So long as even a small portion of the gland is left, adequate effect is produced, and sugar does not accumulate in the blood; but if the whole gland is wanting, then in consequence of the lack of the normal effect, sugar does accumulate in the blood and the condition of diabetes is set up. How this result comes about, whether by reason of a failure to get rid of the sugar which is normally produced or by an abnormal production of sugar, has not yet been clearly made out. The salivary glands, in many respects so like the pancreas, have no such action.

The diabetes thus set up by extirpation of the pancreas has further the following resemblance to ordinary diabetes. In mild forms of the natural disease, sugar only makes its appearance in the urine when carbohydrate food is taken; but in severer forms a large quantity of sugar may be present in the urine even though no carbohydrate food at all be taken. The sugar in such a case probably comes from the splitting up of proteid matter, and this view is supported by the fact that a certain relation may be observed between the sugar and the urea secreted in the urine. So also after extirpation of the pancreas, especially if some of the pancreas be left behind, a mild effect may be produced, in which sugar appears in the urine only after carbohychate food. On the other hand severer forms are also met with in which sugar passes away by the urine, though carbohydrates be rigidly excluded from the food.

As a sort of converse to diabetes we may nention that the administration of arsenic in sufficient doses or for an adequate time prevents an accumulation of glycogen in the liver and 
apparently in the body generally, whatever be the diet used. The presence of the metal in the hepatic cell seems to prevent the cell-substance from manufacturing glycogen either from carbohydrate material brought to it, or out of its own substance. As another kind of converse we may also state that the arlministration of glycerine, espeeially through the alimentary canal, diminishes the effect of the diabetic puncture, or of morphia or other poisoning, in hurrying on the hepatic store of glycogen into sugar, and thus diminishes the sugar in the urine; the presence of the glycerine in the hepatic cell appears to be in some way a hindrance to the conversion of the glycogen into sugar. Now glycerine injected into the alinentary canal of a normal animal leads to an increase of glycogen in the liver ; and the view very naturally suggests itself that this increase arising from the glycerine is to be explained by the glycerine inhibiting in some way a normal conversion of the glycogen store into sugar which is continually going on, and thus increasing for the time that store. 
$\S$ 372. The Movements of the Spleen. A salient structural feature of the spleen is, that many of the minute arteries open out into the labyrinths of the coarse reticulum which occupy the irregular chambers marked off by the trabeculie; blood passes bodily into the spaces between the branched cells of the reticulum. The amount of blood which thus travels slowly through or even for a while tarries in the meshes of the reticulum, forming the so-called "spleen-pulp," as compared with the amount which traverses the spleen in the ordinary way confined to the closed channels of the capillaries, varies from time to time according to the condition of the organ. For the spleen is subject to changes leading to considerable variations in its volume.

After a meal the spleen increases in size, reaching its maximum about five hours after the taking of food; it remains swollen for some time, and then returns to its normal bulk. In certain diseases, such as in the pyrexia attendant on certain fevers or inflammations, and more especially in ague, a somewhat similar temporary enlargement takes place. In prolonged ague a permanent hypertrophy of the spleen, the so-called ague-cake, occurs.

The turgescence of the spleen seems to be due to a relaxation both of the small arteries and of the muscular tissue of the capsule and of the trabecule; to be, in fact, a vascular dilation accompanied by a local inhibition of the tonic contraction of the other plain muscular fibres entering into the structure of the organ, the latter, at all events in some animals, being probably the more important of the two. And the condition of the spleen, like that of other vascular areas, appears to be regulated by the central nervous system, the digestive turgescence being fairly comparable to the flushed condition of the pancreas and of the gastric membrane during their phases of activity.

The application of the plethysmographic method to the spleen, carried out in the way which we described in speaking of the kidney ( $\$ 330)$, enables us to study more exactly the variations in volume which the organ undergoes. 
A 'spleen curve" (Fig. 109) taken in the same way as a "kidney curve" does not, in the dog at all events, shew variations in the volume of the spleen corresponding with the pulse wares. The kidney curve, as we have seen (\$330), gives clear indications of each heart-beat, but the spleen eurve shews, besides the larger waves of which we shall speak directly, only undulations due to the respiratory movements; and these, always very slight, are sometimes not visible. In other words, the spleen does not expand with the increase of blood-pressure occurring in the splenie arteries after each heart-beat. Moreover when the supply of blood to the spleen is wholly and suddenly eut off, as by clamping the aorta, the splcen curve sinks very slowly, shewing that the spleen is diminishing in volume not suddenly but very slowly. The pathway of the blood through the splenic retieulum is peeuliar; and inerease or decrease in the volume of the spleen means more or less blood held in the splcen pulp, not neeessarily a greater or less flow of blood through the organ.

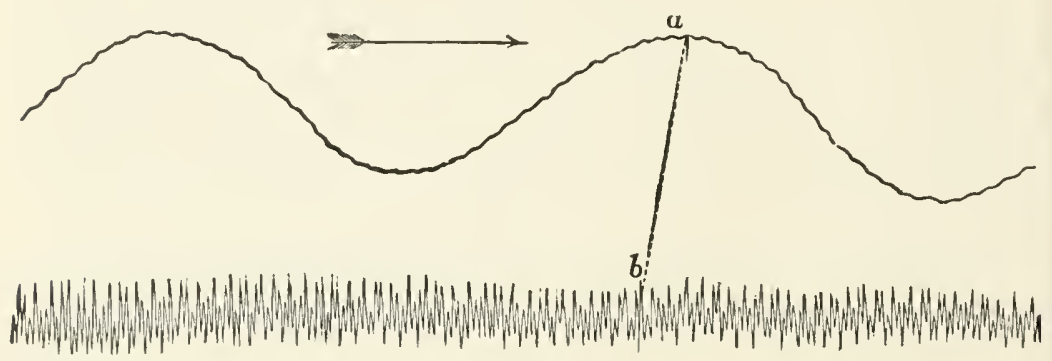

Fig. 109. Normal Spleen Curve from Dog. (Roy.)

The upper curve is the spleen curve shewing the rhythmic contractions and expansions; the smaller waves are due to the respiratory movements. The lower curve is the blood-pressure curve, and the point $a$ of the spleen curve corresponds in time to the point $b$ of the blood-pressure curve. The marks on the time curve below indicate seconds.

Of special interest are the large slow variations of volume which, besides the respiratory undulations, the spleen curve usually shews, as seen in the figure. Rhythmic contractions and expansions, though not always present, frequently make their appearance, each contraetion with its fellow expansion lasting in the eat and dog about a minute, and rceurring with great regularity for a long time; and besides these the volume varies widely from time to time. There ean be little doubt but that the rhythmic variations in volume are due in these animals to rhythmic contraetions, with intervening relaxations, 
of the muscular trabecula and capsule; the slower variations are also probably due to the same cause. In many animals the contractility of the splenic tissue is shewn by the white lines of constriction which appear when the electrodes of an induction machine in action are drawn over its surface; and similar lines may be produced by mechanical stimulation with the point of a needle. So that the spleen in these animals may be considered as a muscular organ, now expanding to receive a larger quantity of blood and now contracting to drive the blood on to the liver. When the muscular elements are scanty in or absent from the capsule and trabecula, the expansion and contraction of the whole organ must depend alone or chiefly on variations in the width of the supplying arteries. We have evidence moreover that the muscular activity of the spleen, whether of the muscular capsule and trabeculie and arteries combined or of the lattcr alone, is under the dominion of the nervous system. A rapid contraction of the spleen may be brought about in a direct manner by stimulation of the splanchnic or vagus nerves, or in a reflex manner by stimulation of the central end of a sensory nerve; it may also be caused by stimulation of the medulla oblongata with a galvanic current or by means of asphyxia. Though the matter has not yet been fully worked out, we have already sufficiently clear indications that the flow of blood through the spleen is, through the agency of the nervous system, varied to meet changing needs. At one time a small quantity of blood is passing through or is being held by the organ, and the metabolic changes which it undergoes in the transit are comparatively slight. At another time a larger quantity of blood enters the organ, and is let loose, so to speak, into the splenic pulp, there to undergo more profound changes, and afterwards to be ejected by the rhythmic contractions of the muscular trabeculie.

It is further obvious that these changes going on in the spleen must have an important influence on the changes going on in the liver; it cannot be of indifference to the latter organ whether a relatively small quantity of blood, relatively little changed, reaches it from the spleen, or whether it receives a relatively large quantity of blood, profoundly altered by the changes which it has undergone in the spleen pulp. Some of the changes taking place in the spleen are histological in nature.

$\$ 373$. When the so-called spleen pulp is examined under the microscope, it is found to consist, besides the branched cells and fibres constituting the reticulum, of cells which may be described as partly red corpuscles and partly white corpuscles or leucocytes. We spoke of the meshes of the reticulum as being filled with blood; but it is obvious that the corpuseles of the blood must move less readily through the labyrinth than 
does the fluid plasma, and that hence a concentration of the corpuscles as compared with the plasma must take place in the meshes. The contents of the meshes cannot, properly speaking, be called blood, but are rather aggregations of corpuscles with a relatively small quantity of fluid.

The white corpuscles or leucocytes are of various kinds. Some are small, like the leucocytes of a lymphatic gland, the cell-substance being seanty relatively to the nucleus. Others are indistinguishable from the kinds of white corpuseles present in the blood. Others again are large, twice as large as an ordinary white corpuscle or even larger than this, possess more than one nucleus, and contain in their cell-substance numerous refractive, pale yellow or colourless granules. Some of these larger forms, which like the others exhibit amoeboid movements, and are often irregular in form, are characterized by the presence in their cell-substance of red corpuscles, sometimes in almost a natural condition, sometimes more or less irregular in shape with their red hæmoglobin changing into the browner hrematin, and sometimes disintegrated into a mass of brown granules. The fluid or plasma in which these cells float also contains besides normal red corpuscles a certain number of red corpuscles in various stages of change, as well as pigment granules which appear to be derived from hæmoglobin. Obviously a certain number of red corpuscles do undergo change in the spleen, but whether the change is mainly effected in the cell-substance of the cells just mentioned, or takes place in the plasma, the products of disintegration being subsequently taken up, in amoeboid fashion, by the cells in question, is not as yet clear. Besides the above, in the spleen of young animals, nucleated cells with hæmoglobin holding cell-substance, hæmatoblasts (see $\$ 27$ ), have been described; these are said to appear also in the spleen of adults after very great loss of blood.

\$374. The Chemical Constituents of the Spleen. Besides the chemical bodies which one would expect to find in a vascular, muscular organ full of blood, the spleen contains bodies, lodged apparently in the spleen pulp, which give it special chemical characters. One of the most important of these is a special proteid of the nature of alkali-albumin, holding iron in some way peeuliarly associated with it. The occurrence of this ferruginous proteid, accompanied as it is by several peculiar but at present little understood pigments, rich in carbon, which are partly present in the cells spoken of above and partly deposited in the branched cells of the reticulum, appears to be connected with the changes undergone by the hrmoglobin which we shall presently discuss. The inorganic salts of the spleen, or at least those of its ash, are remarkable for the large amount of both soda and phosphates, and the small amount of potash and chlorides which they contain, thus dif- 
fering from those of blood-corpuscles on the one hand, and from those of blood-serum on the other. But perhaps the most striking feature of the spleen-pulp is its richness in the so-called extractives. Of these the most common and plentiful are succinic, formic, acetic, butyric and lactic acids, inosit, lencin, xanthin, hypoxanthin, and uric acid. Tyrosin apparently is not present in the perfectly fresh spleen, though leucin is: both are found when decomposition has set in. The constant presence of uric acid is remarkable, especially since it has been found even in the spleen of animals, such as the herbivora, whose urine contains none.

The richness of the spleen in these extractives is an indication of the importance of the metabolic events with which the organ has to do; but it will be more profitable to discuss what goes on in the spleen in comnection with the metabolic changes in other parts of the body, in the liver for instance, than to attempt to lay down any so-called 'functions' of the spleen. When we confine our attention to the spleen itself we learn very little; thus the whole organ may be successfully removed without any very obvious changes in the economy resulting. We may return therefore to the discussion of the formation of the bilirubin of bile, and of the changes undergone by hæmoglobin, with which as we shall see the spleen is connected, and which moreover has to do with the formation of other pigments. 


\section{SEC. 3. THE FORMATION OF THE CONSTITUENTS OF BILE.}

$\S 375$. Bile Pigments. After extirpation of the liver no accumulation of bile pigment or bile salts takes place in the blood. This is well shewn in frogs, which survive the operation for some considerable time; but the same results have been obtained in birds (geese and ducks). There can be no doubt therefore that these substances are formed in the liver and not simply withdrawn from the blood by the liver in some such way as we have seen reason to think urea is withdrawn from the blood by the kidney.

When the plasma of circulating blood is made to contain hamoglobin detached from the corpuscles, bile pigment frequently makes its appearance in the urine. The presence of free hæmoglobin may be secured by injecting into the veins a solution of hæmoglobin or blood made "laky" by freezing and thawing or by the addition of a small quantity of bile salts, or by simply injecting into the veins a quantity of distilled water or a small quantity of ether or chloroform or of bile salts, all of which tend to 'break up' red corpuscles and set free hremoglobin. A similar resnlt occurs in poisoning by certain drugs, such as toluylendiamine. Under these circumstances not only does bile pigment, bilirubin, make its appearance in the urinc, but the quantity of bilirubin secreted by the liver is increased. Obviously the presence of dissolved hæmoglobin in the plasina of the blood, and, presumably more especially of the blood reaching the liver by the portal vein, leads to an increased formation of bilirubin, which takes place in such a manner that the whole of the bilirubin so formed does not pass into the bile but part is retained in or thrown back into the circulation and appears in the urine.

We have already mentioned the chemical comnection between hamoglobin and bilirnbin. Hamoglobin, after the detachment of its proteid component becomes hematin $\left(\mathrm{C}_{32} \mathrm{H}_{32} \mathrm{~N}_{4} \mathrm{FeO}_{4}\right)$. By treatment with sulphuric acid or otherwise (\$ $28 \cdot z)$, hamatin may be deprived of its iron; and this iron-free hematin (some- 
times called hæmatoporphyrin) is said to have the composition $\mathrm{C}_{32} \mathrm{H}_{32} \mathrm{~N}_{4} \mathrm{O}_{5}$, differing from bilirubin only in its oxygen and hydrogen $\left(\mathrm{C}_{32} \mathrm{H}_{32} \mathrm{~N}_{4} \mathrm{O}_{5}+2 \mathrm{H}_{2} \mathrm{O}-\mathrm{O}=\mathrm{C}_{32} \mathrm{H}_{36} \mathrm{~N}_{4} \mathrm{O}_{6}\right){ }^{1}$ Moreover in old blood clots in the body the hrmoglobin of the clot becomes in time transformed in to an iron-free body which has been called hæmatoidin, but which both in composition and in reactions appears to be identical with bilirubin.

These several facts lead us to the conclusion that the bilirubin of the bile is simply some of the hæmoglobin of the blood transformed by the throwing off of its proteid and its iron components. It is natural to suppose that the transformation takes place in and is effected by the agency of the hepatic cells; and this view is supported by the fact that the hepatic cells are characterized by containing certain peculiar iron compounds. When all the blood is carefully washed out of the liver by injection through the blood vessels, by which means the remaining bile is got rid of at the same time, the hepatic substance is found to contain a small quantity of iron, sufficient to give the cells a diffused dark colour when treated with ammonium sulphide; the exact amount appears to vary largely, but the causes of the variation have not been determined. 'That this iron is in organic combination is indicated by the fact that with potassium ferrocyanide and sulphocyanide the blue or red reaction is not observed until after treatment with hydrochloric acid. Apparently there are several such compounds, of a proteid or of a nuclein ( $\$ 29)$ nature, from some of which the iron is more easily removed than others, and these compounds appear to be present in both the cell-substance and the nucleus. It will be remembered ( $\$ 205)$ that bile contains a distinct quantity of iron, which probably has its origin in the iron thus set free from hrmoglobin and retained in the hepatic cell; but it does not follow that all the iron thus set free makes its way into the bile; and indeed the quantity of irou discharged in the bile in 24 hours is much smaller than the quantity calculated to be set free in the formation out of hæmoglobin of the quantity of bilirubin discharged during the same period. Apparently the iron compounds of the hepatic cell have some other work than the simple discharge of iron into the bile.

$\S 376$. We may assume then that the hepatic cell has the power of splitting up the hrmoglobin brought to it, and of discharging part as bilirubin while it retains for a time the iron component in some organic combination. But are we justified in assuming that the whole work is done by the hepatic cells? Are we to conclude that bilirubin is manufactured by some act of the hepatic cells which includes not only the conversion of hæmoglobin into bilirubin, but also the extraction of the

1 Doubling the formula for bilirubin given in $\S 206$. 
hemoglobin from the red corpuscles as these are streaming slowly through the lobular hepatic capillaries in close contact with the hepatic cells? Now, as far as we know at present, hæmoglobin can only be set free by means of a disintegration of the corpuscles; we have no instances of a corpuscle parting with some of its hæmoglobin and proceeding on its way otherwise unchanged; and we have no histologieal evidence of any disintegration of red corpuscles in the liver corresponding to the formation of bile. Nor can we draw any conclusion from the results of a comparative enumeration of red corpuscles in the portal and hepatic blood, for these are too insecure to rest any conclusion upon. On the other hand, as we have just seen, the presence in the plasma of the blood of hæmoglobin in a free condition is peculiarly potent in exciting the formation of bilirubin. The evidence therefore is very strong for the view that, as far as the formation of the greater part at least of the bilirubin is concerned, the action of the hepatic cell is limited to converting into bilirubin the free hæmoglobin offered to it by the portal blood.

By what means, under normal conditions, is the presence of that free hæmoglobin secured? We have seen reason ( $\$ 373$ ) to conclude from histological appearances that a certain number of red corpuscles undergo change in the spleen pulp; and it seems natural to infer that one duty of the spleen is to set free hemoglobin from the corpuscles and thus, through the splenic veins and so the portal vein, to supply the liver with material for bilirubin. But this cannot be the only source, since the secretion of bile continues after extirpation of the spleen. There must therefore be other regions of the body in which a similar change of red corpuscles is going on; it has been suggested that the red marrow of bones is one of these; but further information on these points is needed.

We may then go so far as to say that the bilirubin of the bile is derived from the hæmoglobin of the blood, and that the later stages of the transformation, including the discharge of the iron of the hæmatin component, take place in and by means of the hepatic cell; but much beyond this is at present uncertain. It must be remembered too that, though after extirpation of the liver no accumulation of bilirubin takes place, shewing that the bilirubin is formed by the liver and not elsewhere; yet the whole change from red corpuscle to bilirubin may occasionally take place quite apart from the liver, as shewn by the presence of hematoidin in old blood-clots.

$\S 377$. The formation of the bile acids. About this we know still less. Taking glycocholic and taurocholic acids as the typical bile acids, recognizing ( $\$ 207$ ) that these arise from the union of cholalic acid with glycin and taurin respectively, and remembering that taurin is found in several tissues, and that glycin 
(see $\$ 339$ ) though not an actual constituent of any of the tissues must certainly arise in tissue metabolism, we may conclude that the chief work in this respect of the hepatic cell is to provide the cholalic acid, and to effect the combination with glycin and taurin, though possibly some amount of either one ol the other of these bodies may be furnished by the hepatic substance itself. As to how cholalic acid arises out of the metabolism of the hepatic cell we know no more than we do about the formation of kreatin in muscle or of pepsin in a gastric cell. We are equally ignorant about the origin of glycin and taurin, and cannot explain why in one animal glycocholic, and in another taurocholic acid is prominent in the bile, though the two bodies, as shewn especially by the presence of sulphur in the taurin, are widely different. It has been observed that the presence of bile in the intestine seems to excite the liver to increased biliary action; since the bile acids are rapidly changed in the intestine and the cholalic acid speedily altered, it seems probable that the increased biliary activity is due to the absorption of the glycin and taurin respectively. From which we may conclude that the presence of these bodies stirs up the hepatic cell to an increased formation of cholalic acid.

$\S 378$. As a general rule the formation of bile acids runs parallel with the formation of bile pigment, an increase or decrease of bile meaning an increase or decrease of both constituents. But there are some facts which seem to shew that the two actions may be dissociated.

'The condition or symptom known as 'jaundice' is essentially an excess of bilirubin in the blood, whereby the tissues such as the skin, and the fluids such as the urine are coloured with the yellow pigment. In most of the maladies of which jaundice is a symptom, there is evidence of an obstruction to the flow of bile through the bile passages; and the presence of bile in the blood and hence in the tissues at large is in such cases due to the fact that the bile after secretion by the hepatic cells is reabsorbed from the bile ducts, see $\S 217$. But in certain cases where jaundice is a prominent symptom, no evidence of any obstruction whatever to the flow of bile can be obtained. This is the case in the jaundice of yellow ferer and of a peculiar allied malady known as 'acute yellow atrophy of the liver.' Now in these cases there is no evidence of an accumulation in the blood or elsewhere of bile acids though there is of bile pigment. And in the obscure malady known as simple or idiopathic jaundice, in which though the anatomical conditions are unknown there is at least no sign of obstruction, the urine though loaded with bile pigment is said to contain no bile acids.

$\S 379$. The question may be aslied, Is the secretion of bile independent of or in some way or other connected with the glycogenic activity of the cells? 'To this we cannot at present 
give a definite answer. In some of the invertebrata the cells in the organ, called a liver, which manufacture glycogen, are distinct from those which secrete bile or other digestive juices; and it might be inferred that in the vertebrate the two actions though taking place, as they certainly do, in the same cell, take place apart and distinct. 'There are facts which seem to indicate that the two are intimately comnected; but we have as yet no exact knowledge concerning the matter. It has been urged that the portal blood is chiefly concerned with the formation of glycogen, and the blood of the hepatic artery with the secretion of bile, but there is no adequate support of this view. It must be remembered moreover that, in addition to the formation of glycogen and the secretion of bile, other metabolic events, especially affecting proteid or at least nitrogenous constituents of the body, are also taking place; and to these we must now turn. 


\section{SEC. 4. ON UREA AND ON NITROGENOUS METABO- LISM IN GENERAL.}

$\S 380$. We have seen that nitrogenous proteid material in some form or other enters into the composition of all the tissues of the body, and we have further seen that it is so conspicuously and constantly present wherever living substances are manifesting vital energies as to justify the conclusion that the changes which it undergoes are in some way essential to the manifestation of those energies. We have seen, it is true, reason to think that in some tissues at least, in muscle for instance, a large part of the energy set free during activity preexisted as latent energy and had its immediate source not in proteid (nitrogenous) but in some other constituents of muscle; and indeed, as we shall see later on, the greater part of the whole energy of the body must be regarded as the energy of carbon compounds and not of nitrogen compounds; but this is quite consistent with the view that proteid material in some way or other essentially intervenes in, we may perhaps go so far as to say directs, the changes by which in the body energy is set free in the peculiar way which we speak of as living.

We have seen that at all events the greater part of the proteid material of the food enters the blood as proteid material either as peptone or in some other form, and is carried as proteid material to the tissues.

We have seen that the nitrogen of proteid material leaves the body so largely in the form of urea, that the other nitrogenous excretions may for the time be left out of consideration.

And lastly we have seen reason to think that this urea which leaves the body in urine is brought to the kidneys as urea in the blood, the kidneys themselves apparently having no special power of forming urea out of something which is not urea, but only contributing to the general stock of urea by virtue of their own proteid metabolism. We have now to study the little we know concerning the steps by which the proteid material of the food and of the body is converted into this urea of the blood which is the source of the urea of the urine. 
$\$ 381$. In the first place we may take it for granted that the unea carried to the kidney in the blood lad an antecedent in something which was not urea. We can hardly suppose that the proteid constituent of living substance, when in the course of its netabolism it ceases to be proteid, breaks mp at once into urea and into non-nitrogenons bodics. All we lave learnt goes to shew that what we call metabolism is not a single abrupt change, but consists essentially in a series of changes; and we may safely conchnde that proteid material in becoming urea passes through phases in which the nitrogen exists in chemical combinations distinet from proteid material on the one hand and urea on the other.

In the second place it is extremely probable that the series of changes by which proteid material becomes urea is not the same in all the tissues and on all oceasions. We shonld naturally expect to find the proteid material following different lines of metabolism in different places or under different circumstances, the different lines all converging to the same body urea, decause for some reasons or other urea appears to be, in the main, the most convenient form in which the nitrogen can leave the blood and the body.

We should accordingly expect to find, on the one hand, varions nitrogenous bodies resulting from proteid metabolism in various parts of the body, and, on the other hand, arrangements by means of which these various bodies were reduced to the common form urea, preparatory to their discharge from the body by the kidney. And actual observation as far as it goes supports this view, though our knowledge of the whole matter is very imperfect.

$\$ 382$. We may turn our attention first to the metabolism of the skeletal muscles, since these represent, as far as mere quantity is concerned, by far the greater part of the proteid capital of the body. We may safely infer that they furnish a large part of the urea of the urine; though undonbtedly a small mass of tissue might by reason of its more rapid metabolism work over a greater quantity of proteid material than a much larger mass witl a slower metabolism; yet we have no reason to think that the proteid metabolism of skeletal muscle, obseure though it is in its nature, is so slow as to nentralize the probable effect of the great bnlk of muscle existing in the body.

In dealing with the chemistry of muscle ( $\$ 59)$ we saw that urea was conspicuous by its absence from the extract of muscle, whereas a very appreciable quantity of kreatin was invariably present, and indeed was the prominent nitrogenous crystalline constituent of that extract. It seems difficnlt to resist the conclusion that kreatin is the main normal nitrogenous product of the metabolism of skelctal muscles. If we accept this vicw, then upon the fact of the presence of kreatin in, and the absence of 
urca from, the muscle itself, we may base the conclusion that while the muscle produces kreatin as an antecedent of urea, the kreatin so produced is converted into urea in some part of the body other than the muscle itself. Kreatin as we have alrcady scen may be easily split up, and we may probably with safety assume is split up, somewhere in the body, into urea and sareosin. But sareosin does not appear in the mine as such ; hence the conversion of kreatin into (part of) the urea of the mrine entails as well the further conversion of sareosin into urea. Now sareosin as we have seen is methyl-glycin; we may regard it for omr present purposes as simple glycin, and hence the total conversion of kreatin into urea entails the conversion of glyein into urea. This however does not offer any additional diffienlty, since we know from direct olservation that glycin introduced into the alimentary canal does not reappear as such in the urine but produces a correspronding increase in the urea of the urine; from which we infer that glycin absorbed from the alimentary canal is somewhere in the body converted into urea. We shall speak of this conversion later on, and shall then see that, so far as urea is conecrned, glyein (amido-acetic acid) and sarcosin (methyl-glyein, methyl-amido-acetic acid) undergo the same change, the anide moiety in each case being converted into urea, while the non-nitrogenous moiety is oxidized and thrown off. Meanwhile we may state the conclusion at which we have provisionally arrived, namely that the nitrogenous metabolism of muscle probably gives rise to kreatin, which in some part of the body other than musele is probably split up into urea, ready for excretion, and into sarcosin which also, somewhere in the body, is further converted into urea. And bearing in mind the large mass of the skeletal muscles, we may further conclude that a large portion of the urea leaving the body by the urine is formed in this way.

383. We must not however leave this statement without referring to a difficulty. Kreatinin as we have scen is so frequently found in urine as to be regarded as a normal constitnent, at all events of human urine; and kreatinin is as we have seen the minary form so to speak of kreatin; the one body easily ehanges into the other by the assumption or removal of $\mathrm{I}_{2} \mathrm{O}$. This suggests the question, Is not the kreatinin of urine the represencative of the kreatin of the museles, which is thus excreted directly without undergoing the change into urea just discussed? In answer to this we may say in the first place that the quantity of kreatinin in urine, though variable, is small; we may put the average at about $1 \mathrm{grm}$. in 24 hours. Now muscle contains from $\cdot 2$ to 4 p.e. of kreatin; and this, taking the total muscle of the body (to say nothing of other sourecs of kreatin which we shall mention presently) at about 30 kilos would give 60 to 120 grms. kreatin as present in the muscles of the body at 
any one moment. We can hardly suppose that the metabolism of muscle is so slow as out of this stock only to provide the 1 grm. of kreatinin in 24 hours. Moreover the kreatinin in urine vanishes during starvation, is very markedly increased by a diet of flesh which contains kreatin, and is not increased either by muscnlar exercise (which however would only indirectly affect the nitrogenous metabolism of muscle) or by such conditions, fever for instance, as notably increase the urea of urine by increasing the nitrogrenous metabolism of muscle. We infer therefore that the normal presence of kreatinin in urine is due to the direct administration of kreatin present in a (normal) flesh diet and has nothing to do with the muscular metabolism of the individual who is secreting the kreatinin in his urine.

The fact however that the kreatin present in the muscle of the food and absorbed from the alimentary canal does not undergo a change into urea but is excreted as kreatinin, that is virtually as kreatin, warns us to be careful in adopting the conclusion arrived at above that the kreatin produced by muscular metabolism in the living body is a conspicuous antecedent of the urea of the urine. It is difficult to see why kreatin passing into the blood of the eapillaries of the muscle shonld be changed into urea while that which passes into the capillaries of the portal system is not; for reasons which will be apparent presently we should rather expect that the latter being more directly exposed to the influence of the liver would be more readily and more completely converted than the former. Inrleed the question forces itself upon us, Is kreatin after all the natural main product of the nitrogenons metabolism of muscle? Is it possible that in the normal metabolism of the living muselc the nitrogen leaves the muscular substance and passes into the blood in another form, as some substance not kreatin, and that it is as the musele dies that kreatin is formed, just as the solid myosin is unknown to the living fibre but makes its appearance in a dying one? We have no positive evidence however that this is so, and meanwhile may continue to suppose that kreatin is formed, and that in consequence kreatin is a conspienous antecedent of the urea of the urine; but we must not regard this as proved.

$\$ 384$. Our knowledge of the metabolism of the nervous tissues is, as we have seen, very imperfect ( $\$ 67$ ), but the presence of kreatin in the central nervons system leads us to infer that the nitrogenous metabolism of the living substance of nerve cells and of the axis cylinder of nerve fibres, is in its broad features identical with that of muscle substance. The mass however of the nerve cells and axis cylinders of the body, all put together, is small compared with the mass of skeletal musele; moreover, the energy set free by the metabolism of a mass of nervous matter thomgh · higher' in quality is less in quantity 
than that set free by the metabolism of an equal mass of muscle, or in other words its metabolism is less rapid. Hence we may probably consider the nletabolism of the nervous system as a mere addition to that of the muscular system, at least as regards the point on which we are now dwelling. The amount of nitrogenous metabolism taking place in connective tissue, cartilage, bone, and the skin is probably still less, and for our present purposes needs no special discussion.

$\S 385$. The nitrogenous metabolism of the glands however, more particularly that of the liver, does deserve special consideration; and we may at once turn to a quite different aspect of the question in hand.

When the rate of discharge of urea from the body is observed during a period of some length, especially under varied circumstances, the direct effect of nitrogenous food becomes most striking. We have already said, and shall again return to the point, that muscular contraction does not directly increase the output of urea; the discharge of urea for instance is not necessarily increased by even great bodily labour. The introduction however of even a small quantity of proteid material into the alimentary canal at once increases the urea of the urine; and in the curve of the discharge of urea in the twenty-four hours each meal is followed by a conspicuous rise. The absorption of proteid material from the alimentary canal is followed by an immediate proportionate increase in the quantity of urea which is secreted by the kidneys, and that as we have seen means an increase in the urea brought to the kiclney by the renal artery. What is the origin of this additional urea?

Two views present themselves. On the one hand since some portion of the proteid material of every meal, at all events of every necessary meal, goes to repair the proteid waste continually going on in the parts of the body where proteid metabolism is taking place, we nuay suppose that the presence of an extra quantity of proteid material thrown upon the blood from the food acts as a stimulus to the tissues, to the muscles for instance as well as others, stirs them up to increased nitrogenous metabolism and thus produces an increase of energy, chiefly if not exclusively in the form of heat, accompanied by an increase of the antecedents of urea and so of urea. In other words the increase of urea in question is the result of an increase in the general nitrogenous metabolism of the body.

On the other hand we may suppose that in order to prevent the whole body being encumbered with it, this excess of proteid food material is, in some special part of the body, split up into a nitrogenous and a non-nitrogenous moiety, and that, while the latter is stored up as fat or glycogen, the former is at once converted into urea and got rid of. We have already ( $\$ 210$ ) seen that a step in this direction may take place while the food is as 
yet in the alimentary canal; we have seen that pancreatic juice may carry part of the proteids on which it acts beyond the stage of albumose and peptone, and reduce that part into leucin, tyrosin, and other bodies. We do not know, as we have already said, to what extent this more profound digestion by pancreatic juice does actually take place in the living body ; it may under certain circumstances take place to a very slight extent and under other's to a considerable extent. But in any case it illustrates the way in which a somewhat similar disruption of proteid material, a disruption which may be broadly described as a splitting up of the proteid into a nitrogenous and a non-nitrogenous moiety, may take place somewhere in the body and so lead to the sudden formation of some antecedent of urea. The antecedent may be leucin or may be some other body or bodies.

In support of this view may be urged the fact that such bodies as leucin, glycin, asparagin and mary others when introduced into the alimentary canal are transformed into urea. When these bodies are administered in not too great quantities they do not reappear in the urine but the urea is proportionately increased.

$\S 386$. We have seen reason to think that proteids of a meal are absorbed not by the lacteals but by the portal blood vessels, and such bodies as leucin probably take the same course. This being so, all these bodies pass through the liver and are subjected to such influences as may be exerted by the hepatic cells. Now we have no positive evidence that the liver does or can exert such an action on proteid material itself as to separate a relatively simple nitrogen compound from the remaining constituents, leaving these to form a body rich in carbon; we have no positive proof that the incrcase of proteid metabolism just spoken of as leading tc an increase of urea takes place in the liver rather than in the tissues at large; we may perhaps suspect that it is so but we have no convineing demonstration. We have however a convergence of evidence that the last stage of the process, namely the conversion into urea of some or other product of proteid metabolism which though allied to is not exactly urea does occur in the liver. In the first place, a large quantity of urea seems to be present in the liver of mammals; in this respect the liver presents a strong contrast to the muscles; in the liver of birds the urea is represented by urates. In the second place, in eertain cases of a form of disease of the liver known as acute yellow atrophy in which the hepatic cells are so changed that their functional activity is largely diminished, the urea of the urine not only undergoes a very marked decrease but appears to be replaced to a very large extent by leucin. This fact suggests that leucin (and not for instance kreatin) is the chicf immediate product of the nitrogenous metabolism of the body. and that the leucin thus produced is 
in a normal state of things converted into urea by the liver. And in this connection it may be remarked that not only is leucin found in nearly all the tissues after death, especially in the glandular tissues, but also appears with striking readiness in almost all decompositions of proteids, and is moreover a product of decomposition of gelatiniferous substances. Without going however so far as to conclude that leucin is the chief antecedent of urca, we may take the above observation as indicating that the normal liver has, in some way or other, the power of converting leucin into urea. If this be so then we may also venture to suppose that when such bodies as leucin, glycin, \&c., introduced into the alimentaly canal appear in the urine as urea the transformation has taken place in the liver. The body tyrosin which so often accompanies leucin, belonging as it does to the aromatic series, stands on a different footing from leucin and the like.

$\$$ 387. The trainsformation however of leucin into urea raises a new point of view. Leucin, as we know, is amido-caproic acid; and, with our present chemical knowledge, we can conceive of no other way in which leucin can be converted into urea than by the complete reduction of the former to the ammonia condition (the caproic acid residue being either elaborated into a fat or oxidized into carbonic acid) and by a reconstruction of the latter out of the ammonia so formed. We have a somewhat parallel case in glycin, which is amidoacetic acid; here too a reconstruction of urea out of an ammonia phase must take place. Moreover when ammonium chloride is given to a dog a very large portion reapjears as urea, i.e. there is an increase in the urea of the urine corresponding to a large portion of the nitrogen contained in the ammonium chloride. And in the case of other animals also, indeed of man himself, there is evidence that somewhere in the body ammonia may be converted into urea. Hence in all these cases where ammonia or ammonia compounds are changed into urea the last step at all events is one of synthesis; and this suggests the possibility that in the ordinary proteid metabolism also, the downward katabolic series of changes may finish off with a synthetic effort, the last stage of the former being the appearance of an ammonia compound which is subsequently reconstructed into urea.

This synthesis, like the transformation of leucin and other bodies, probably takes place in the liver; and in support of this view we have a certain amount of experimental evidence. Birds may be kept alive after total extirpation of the liver for a longer time than can mammals; and when in geese the liver is removed the uric acid (representing in these animals the urea of the mammal) is largely decreased, while the amnonia of the mine is largely increased. After the removal of the liver also, 
leucin, glycin, and other amides or amido-acids administered by the alimentary canal no longer increase the uric acid of the urine, as they do in the intact animal. In these animals, the synthesis of ammonia compounds into uric acid, which is parallel to the synthesis into urea occurring in the mammal, seems to take place in the liver, and we may infer is in some way or other effected by the hepatic cells.

As to the exact way in which ammonia either as such or in form of an amide or amido-acid elianges into urea we have no certain knowledge. Ammonium carbonate, we know, is readily formed out of urea by simple hydration, and we may imagine that the living organism can carry out the reverse process and dehydrate ammonium carbonate into urea. There is, however, a certain amount of evidence that not ammonium carbonate but ammonium carbamate is the immediate antecedent of urea ; and indeed, out of the body, by electrolyzing a solution of ammonium carbamate with alternating currents, a certain amount of urea may be artificially produced. But this is a matter too obscure to be discussed here.

$\$ 388$. Uric Acid. This, like urea, is a normal constituent of liuman urine, and, like urea, has been found in the blood, in the liver and in the spleen; it is a conspicuous constituent of an extract of the latter organ. In various diseases the quantity in the urine is increased; and at times, as in gout, uric acid accumulates in the blood, and a deposit of urates takes place in the tissues. In some animals, such as birds and most reptiles, uric acid takes the place of urea. Since by oxidation a molecule of uric acid can be split up into two molecules of urea, and a molecule of some carbon acid, uric acid is commonly spoken of as a less oxidized product of proteid metabolism than urea. But there is no evidence whaterer to shew that the former is a necessary antecedent of the latter; on the contrary, all the facts known go to shew that the appearance of uric acid is the result of a metabolism slightly diverging from that leading to urea; indeed it is probable that the divergence occurs towards the end of the scries of changes, for urea given by the mouth to birds appears in the urine as uric acid, and, conversely, uric acid given to mammals appears in the urine as urea. $\mathrm{W}$ c have no evidence to prove that the cause of the divergence lies in an insufficient supply of oxygen to the organism at large; on the contrary, uric acid occurs in the rapidly breathing birds as well as in the more torpid reptiles. Nor can the fact that in the frog again urea replaces uric acid be explained by reference to that animal having so large a cutaneous in addition to its pulmonary respiration. 'The final causcs of the divergence are to be sought rather in the fact that urea is the form adapted to a fluid, and uric acid to a more solid excrement. Nor is there in man or the mam- 
mal any satisfactory physiologieal or clinical evidente that an increase of uric acid is the result of deficient oxidation. The absolute amount of uric acid discharged by man and its proportion to the urea passed at the same time varies a good deal. There is no positive evidence that the quantity excreted is necessarily increased by nitrogenous diet, unless some disorder supervenes; indeed it is asserted that both absolutely and relatively to the urea the quantity excreted is greatcr upon a mixed diet than upon a highly proteid one. Alkalis in the food seem undoubtedly to diminish it, and alcohol, at least in excess, to increase it.

So far from considering uric acid as a less oxidized antecedent of urea we ought perhaps rather to regard its appearance as a result of a synthesis in which urea or some allied body takes part. As we have said uric acid may be formed synthetically by heating together urea and glycin; and it has more recently been similarly prepared from various allied bodies. As to where or how such a synthesis is effected in the living body, we know little or nothing for certain, and can only make conjectures. 'The constant presence of uric acid in the spleen however, and the frequently noted comnection between a rise and fall of uric acid in the urine and variations in the volume and therefore presumably in the activity of the spleen, suggest that the change may be brought about in this organ; but it must be remembered that in birds and reptiles the formation of uric acid seems to be effected in the same organs as that of urea and in an analogous manner; and the arguments which we have used concerning the formation of urea in the liver of mammals may be applied to the formation of uric acid in the livers of birds and reptiles. It is more probable therefore that in the mammal the turn to uric acid rather than mrea is given in the liver, the spleen however possibly playing its part also in the matter.

389. Of the meaning of the appearance in the tissues of such bodies as xanthin, hypoxanthin, guanin and the like, and of the exact nature of the metabolism which gives rise to them or which they themselves undergo, we know little or nothing. The presence of these several bodies may be taken as illustrating the complex and varied nature of proteid metabolism to which we referred above. Urea is the chief end-product of proteid metabolism, but that end is probably reached in several ways; so that probably a very large number of nitrogenous chemical substances make a momentary appearance in the body. Some of these fail to become urea, and either without or after further change make their appearance in the urine. But we do not know whether their appearance is aceidental, the result of imperfect chemical machinery; or whether they, though small in quantity, serve some special ends in the economy. Perhaps 
sometimes or with some of them it is the one case, at other times or with other's it is the other case.

When proteirl material undergoes outside the body, either by the action of trypsin or as the result of decomposition or nnder the influence of chemical agents, that change by which it is converted into leucin, the lencin, which appears in some considerable quantities, is accompanied by tyrosin, which appear's in smaller quantities, as well as by other bodies. The almost constant appearance of tyrosin as a result of the decomposition of proteid material leads one, as we have previously said, to the conception that some representative of the aromatic series enters into the constitution of proteid substance ; and it is possible that the hippmric acid of flesh-eating animals derives its benzoic acid constituent from this aromatic radicle of proteid matter. Tyrosin itself does not appear in the body as a normal product of proteid metabolism, and we are therefore led to infer that in proteid metabolism the aromatic radicle takes on some other form. Whether as in tyrosin the aromatic (phenyl) nucleus is associated with an ammonia representative or no, we do not know. But if it is then, since neither tyrosin nor any similar body is a constituent of normal urime, the anmonia constituent is somewhere dissociated from the phenyl one; and while the former contributes to the stock of urea, the latter is either discharged by the urine as hippuric acid, having as we have seen effected in the kidney a new association with the ammonia representative glycin, or leaves the body as one or other of the urinary phenyl compounds, or possibly may be oxidized somewhere into carbonic acid and water. Our knowledge on this point is limited, but we have ventured to refer to the point since it further illustrates the complexity of proteid metabolism.

$\$ 390$. In speaking of urea $(\$ 321)$ we alluded to its relations to the cyanogen compounds. Bearing in mind the peculiarly large amount of energy set free as heat during the isomeric transformation of many cyanogen compounds, as well as the large store of potential energy existing in cyanogen itself, the heat of combustion of which is very large, and contrasting these properties with those of ammonia and the anmonia componnds, we camnot help being tempted towards the view that in the actual living structure the nitrogen exists in the form of eyanogen compounds, and that in the passage to dead nitrogenous waste, during which energy is set free, the cyanogen compound changes to the anide or other ammonia representative. And there are several facts which lend support to such a view, such as the presence of sulphocyanates in saliva and urine, which we may look upon as a sort of leakage of cyanogen factors, the artificial production of kreatinin out of cyanamide and sarcosin, and other facts. But the matter, 
though it deserves to be borne in mind, is too obscure to be dwelt on here.

$\$ 391$. We may now briefly sum up the varied discussions which have occupied us in the present section.

Urea is the main end-product of proteid metabolism. Unlike hippuric acid and some other constituents of urine, urea is simply excreted by the kidneys, being brought to them in the blood, they apparently, beyond the simple act of excretion, doing no more than merely contributing to the stock of urea in so far as they arc masses of proteid matcrial undergoing proteid metabolism as part of their general life. What are the immediate antecedents of urea we do not clearly know ; but it is probable that they are not one but several and indeed possibly many. We have reason to think that urea may be formed out of amides or amido-acids, or ont of ammonia itself by a synthetic process; and we have indications that this synthesis is effected in the liver by the agency of the hepatic cells. But we do not know whether this synthesis bears only on particular nitrogen-holding substances of food or of the body, or whether it comes into play in the normal metabolism of proteid material. If the kreatin which is so conspicuous a constituent of muscular and nervous structures is a stage in the direct line to urea, then the synthesis would affect only the surcosin which the kreatin in becoming urea sets free. But we have seen that it is by no means clear that kreatin is such a stage.

The evidence as far as it goes tends to shew that the metabolism of proteid is very complex and varied, that a large number of nitrogen-holding substances make a momentary appearance in the body, taking origin at this or that step in the downward stairs of katabolic metabolism and changing into something else at the next step, and that the presence in various parts of the body and even in the urine, in small quantities, of so many varied nitrogenous crystalline substances, forming a large part of what are known as extractives, has to do with this varied metabolism. Possibly the transformations by which nitrogen thus passes downwards take place to a certain extent in such organs as the liver and the spleen which are remarkably rich in these extractives. 


\section{SEC. 5. ON SOME STRUCTURES AND PRUCESSES OF OBSCURE NA'TURE.}

\$ 392. The Thyroid Body. Certain struetures whieh, though they differ in many ways, we may conveniently treat of together, such as the thyroid and pituitary bodies, the suprarenal capsules, and the thymus, appear to play not unimportant parts in the metabolic processes of the body.

In regard to the thyroid we have clinieal and experimental evidence pointing distinetly in this direction. In certain animals (such as monkeys and $\log s$ ) the removal of the thyroid gives rise to various symptoms of disorder. Among the earlier of these are muscular tremors, spasms or even tetanic convulsions, accompanied or snceeeded by irregularity or failure of voluntary movements, all indicating mischief in the eentral nervous system, in which indeed histological changes may be detected. Subsequently there ensue other varied symptoms which may be deseribed under the general term of those of disordered nutrition, and which eventually end in death. In order to obtain these results the whole of the thyroid gland, including the small so-called accessory thyroids, when these are present, inust be removed; if a part only of the body be left behind the symptoms do not appear, or are slight and transient. Mere injury either to the thyroid body itself, or to the surrounding nervous and other struetures, is insufficient to produce the characteristie results. Moreover, if the thyroid, after the removal from its natural position and attachment, be inserted as a whole or in part in some other part of the body, so as to live and thus be "grafted," the symptoms do not appear. 'The story in fact is very similar to that of the panereas in relation to sugar in the blood (see $\$ 374$ ). And we may infer that in these animals the blood in passing through the thyroid undergoes some special ehange, some thing or things being taken away from it or added to it, by which it is fitted for the nutrition of the rest of or at least of other parts of the body. We may add that in other animals, herbivora for instance, these symptoms are not so easily produced. The reason may be the greater 
difficulty in ensuring the removal of the whole gland; but this is not wholly clear.

The view that the thyroid effects some change on the blood passing through it is further confirmed by clinical experience. 'The disease known as myxoedema, charactcrized by disordered nutrition, notably of the skin and of the nervous system, but also of other parts of the body, is elosely associated with morbid changes of the thyroid body, and thus is allied to goitre and cretinism. The symptoms in many respects resemble those produced in animals by removal of the thyroid. Now in such cases the symptoms are in a most remarkable way lessened or even removed by the systematic subcutaneous injection of the extract of the fresh thyroid body of an animal, or even by the extract being taken regularly by the mouth. The small quantity of substance thus introduced into the blood is sufficient to modify the altered nutrition of the body, and to bring it back to its normal condition. 'The inference is that under normal conditions the thyroid gives up in some way to the blood the substance or substances which in the above instance are artificially administered in the thyroid extract, and the presence of which is in some way essential to the normal nutrition of the body.

What that substance is or those substances are, and how they act, we are not yet in a position to say. 'The characteristic presence in the alveoli of the thyroid of mucin, or of a substance, the so-called "colloid" having at least a superficial resemblance to mucin, and the fact that in myxoedema a mucin-like body is in excess in the tissues, hence the name, have led to speculations as to the comnection of the thyroid and mucin. But our knowledge is not at present such as to justify any definite statement.

393. The Pituitary Body. 'The lower', posterior, lobe of this organ in many respects resembles the thyroid body (the upper, anterior, lobe is of quite distinct nature, being really a part of the central nervous system), but concerning the processes which take place in this lobe and the purposes of the organ as a whole we know absolutely nothing.

$\$ 394$. The Suprarenal Bodies. These differ wholly in structure from the thyroid body. The two parts of which the body consists, cortex and medulla, are not, like the cortex and medulla of a lymphatic gland, different arrangements of the same material, but are of essentially different nature and indeed are of different origin. The medulla is derived from, is a modification of, sympathetic ganglia, while the cortex is derived from masses of mesoblastic cells surrounding the great blood vessels; and in sone animals the two form wholly separate bodies.

Some of the histological features of the suprarenal bodies, namely the groups of cells and their abontant bloot supply, 
suggest on the one hand that important metabolic processes take place in them, some of which are probably connected with the history of the pigments of the body at large. On the other hand the unusually large nerve supply, and the derivation of part of the body from the sympathetic ganglia, suggest peculiar nerrous connections. And the organ has often served as a starting point for speculations in these two directions; but our exact knowledge concerning them is very limited. By experiment we learn that removal of the whole of both suprarenal bodies entails speedy death, the symptoms having a general resemblance to those due to the removal of the thyroid. 'The removal of one suprarenal alone is inadequate to produce this result; and the symptoms following removal of both may be at least mitigated by injection of an extract prepared from the organ. This suggests a function of the suprarenals analogous to that of the thyroid. Injection of the extract of suprarenals in adequate doses also produces distinct physiological effects, notably constriction of the blood vessels and inhibition of the heart.

One fact, gained by clinical experience, pointing in the same direction is of great interest. Disease of the suprarenal bodies, apparently tubercular in nature and beginning in the medulla, is so often associated with a change in the colour of, with an increase of the pigment of the skin, 'bronzed skin,' 'Addison's disease,' that some comnection between the two must exist; but the several links of the chain are as yet unknown. It is tempting to associate the increase of pigment in the bronzed skin with the fact that the suprarenal body contains some substance or substances, possessing striking colour reactions, giving a dark blue or dark green colour with ferric chloride, and a carmine red tint with various oxidizing agents; but we have no exact knowledge at present.

$\$ 395$. The Thymus. This, again, is essentially a lymphatic structure, and indeed might be regarded as a part of the lymphatic system.

From the thymus there may be extracted by means of saline solution a form of a peculiar proteid, a so-called nucleo-albumin which, like the corresponding bodics from lymphatic glands or from leucocytes, seems to have some special relations to the formation of fibrin. Thus, as has already been said (\$22), a solution of this body from the thymus, injected into the veins, will give rise to extensive intravascular clotting.

The thymus, like the other bodies on which we are now dwelling, is also rich in extractives. Thus xanthin, hypoxanthin, leucin, lactic, succinic and other acids have been found in it.

But of what really takes place in the body we have no exact knowledge. Since the thymus is best developed before birth, 
Chap. iv.] METABOLIC PROCESSES OF THE BODY. 603

disappearing after birth at a rate which varies much in different individuals and still more in different kinds of animals, and being eventually replaced by fat and connective tissue, it is obvious that its chief functions are in some way associated with events taking place before birth or in early life. 


\section{SEC. 6. THE HISTORY OF FAT. ADIPOSE TISSUE.}

$\S 396$. Globules of fat of various sizes make their appearance in the very elements of most of the tissues, in muscular fibres, in epithelial cells, in nerve cells, in lencocytes, and so on ; and the medulla of medullated nerves consists largely of a peculiar fatty material. Besides this, certain cells of connective tissue at various times, and in various places, become so loaded with fat that groups of the cells become practically masses of fat. Connective tissue thus loaded with fat is called adipose tissue; and masses of adipose tissue of all manner of sizes and of shapes adapted to the several situations are found in various parts of the body. Many of the internal organs, more especially the kidneys, are wrapped in adipose tissue; but the largest deposit is one lying in the subcutaneous comnective tissue, sometimes called the "pamiculus adiposus;" and a 'fat' body is distinguished from a 'lean' body chiefly, though by no means exclusively, by the amount of subcutaneous adipose tissue.

Of all the tissues of the body adipose tissue is the most fluctuating in bulk; within a very short space of time a large amount of adipose tissue may disappear, and within a very short space of time the quantity present in a body may be several times multiplied. When too much or too little food is given it is the subcutaneous adipose tissue which first and most rapidly increases or decreases in bulk.

$\$ 397$. A fat-cell is a cell, belonging to connective tissue, in the cell-substance of which fat has been collected to such an extent that the cell, which increases largely in bulk during the proeess, is almost wholly transformed into a large vacuole filled with fat, the eell-substance being reduced to a thin envelope of the vaeuole, thickened at one part where the nucleus, thrust on one side by the gathering fat, is placed. Adipose tissue is a collection of such fat-cells held together by a meagre quautity of vascular connective tissue.

By studying the development of adipose tissue in the embryo or elsewhere, we may trace out the steps of the formation of the 
fat-cells. In the embryo, in a situation where adipose tissuc is about to be formed, the connective tissue is seen to contain a number of small nucleated colls, rounded or somewhat irregular in form, the cell-substance of which at first presents no special characters, and contains not more than what may be called the ordinary amount of fat globules or spherules. Very soon however these minute drops or specks increase in number, the cellsubstance at the same time increasing in bulk while remaining round or becoming more distinctly so, and the smaller drops run together into larger ones. This goes on ; the fat increasing in quantity coalesces more and more, and the cell, as a whole, becomes larger and larger, the cell-substance at first keeping up in bulk with the increasing fat, but subsequently ceasing to increase, being apparently used up in the formation of the fat. Thus the original small 'protoplasmic' cell is at last transformed into the larger fat-cell, all the fat having run together into a vesicle the envelope of which, thickened on one side to carry the nucleus, is furnished by the remnant of the cell-substance. In some cases, the nucleus instead of being pushed early on one side, remains central though the collection of fat has become considerable; it is however eventually displaced. The whole process appears very similar to the deposition of mucin in the cells of a mucous gland, $\$ 197$; and we may by analogy infer that the fat-cell becomes a fat-cell by the cell manufacturing fat in some way or other, and depositing the fat so formed in the interstices of its substance. The most striking superficial distinctions seem to be that in the mucous cell the granules or spherules remain discrete within the cell, being separated by bars of cell-substance, whereas in the fat-cell the globules, as they form, run together until at last they unite into a single inass; and further that while in the mucous cell, cven when most heavily loaded, a relatively large amount of active cell-substance still remains, in the fat-cell a mere remnant is left and that chiefly surrounding the displaced nucleus.

The fat in the interior of bones forming the yellow marrow appears to have the same general structure and to be formed in the same way as the rest of the adipose tissue.

$\S 398$. The fat thus deposited in a fat-cell sooner or later disappears. It is not ejected bodily into the surrounding lympli-spaces of the connective tissue, but passes away gradually either into the lymphatics or into the blood stream by some processes not as yet fully understood. During the disappearance of the fat the cell behaves in one of two different ways. On the one hand, as the fat gradually disappears, little by little, the rounded distended vesicle gradually lessening assumes the characters of a connective tissue corpuscle, even of a branched one. On the other hand, especially when the disappearance is rapid and total, the space previously occupied 
by fat becomes filled with a clear fluid resembling lymph, the fat vesicle being transformed into a lymph resicle. 'This condition however is temporary only, the lymph is subsequently absorbed and the vesicle shrinks. Or the cell-substance may shrink round the lessening fat, but in doing so deposits on its outside a mucous substance. At timcs, the emptying of the eell, whether by the onc method or the other, is followed by a rejurenescence of the cell, the nucleus by division gives rise to several nuclei, and the cell divides into new cells, each of which may, under appropriate conditions, develope again into a fat-cell.

399. 'The fat thins lodged in adipose tissue varies somewhat in composition in various animals, but is chiefly composed of olein, palmitin and stearin in varying proportions, with small quantities of the glycerine compounds of such fatty acids as butyric, capronic, caprylic, \&c., together with a little lecithin and cholesterin. 'The 'fat' of one animal, that is the fat thus contained in adipose tissue, differs from the fat of another animal partly by the presence of more or less of one or more of these less abundant fats, but chicfly by the proportion in which the three main fats, olein, palmitin, and stearin, are respectively present in the mixed fat. 'The melting points of these three fats being different, the melting point of the fat of the body will differ according to the relative proportions in which the three are present. Thus the subcutaneous fat of man melts at from $15^{\circ}$ to $22^{\circ}$ or higher, the fat round the kidney being firmer and not melting until $25^{\circ}$; the fat of the dog melts at about $22^{\circ}$, that of the goose at about $25^{\circ}$, of the ox at about $40^{\circ}$, and of the sheep at $50^{\circ}$, the less resistant fat of the man and $\operatorname{dog}$ containing relatively more olein than that of the ox or of the sheep.

$\$ 400$. When we come to consider the question, By what processes does the fat make its appearance in the fat-cell? we are brought face to face with much the same kind of problem as that which occupied us in dealing with glycogen. On the one hand we may suppose that the fat is brought to the fat-cell as fat and is in some way taken up by the cell and deposited in the cell-substance with little or no change. On the other hand, we may suppose that the fat is manufactured by the fatcell in some such way as mucin or pepsin is manufactured by a mucous or a gastric cell, out of and by means of its cellsubstance, and that the process of fattening, or of producing fat in fat-cells, consists essentially in feeding and so building up the cell-substance which subsequently breaks down into fat, and does not consist merely in bringing fat within reach of the cell. Which of these views is the true onc, or how far are both these operations carried on in the animal body?

In support of the latter view it may be urged that, not only the more eomplex living substance, but, as we have more than 
once urged, the simpler proteid constituent of living substance obviously contains what we may call a fatty radicle, so that we might expect fat to be formed out of its metabolism. And as a matter of fact not only in adipose tissue, but in every part of the body, living substance is continuously giving rise to and temporarily depositing in itself some amount of fat, and in what is known as fatty degeneration there seems to be evidence of the formation of fat out of proteid material.

On the other hand, we have traced the fats taken as food, and found that they pass with comparatively little cliange from the alimentary canal, chiefly througin the intermediate jassage of the lacteals, into the blood, from which they rapidly disappear after a meal. We might infer from this that an excess of fat thus entering the blood would naturally be clisposed of by being simply stored up in the available adipose tissue without any further change; we can inagine that the fat, not immediately wanted by the economy, passes in some way from the blood to the connective tissue (the white blood corpuscles which appear loaded with fat after a meal possibly acting as intermediaries), and that the comnective tissue corpuscles swallow the fat brought to them after the fashion of an amceba, not digesting it but simply keeping it in store until it was wanted elsewhere.

What do experiments teach on this matter?

In the first place, it is evident that in an animal fattened on ordinary fattening food, only a small fraction of the fat stored up in the body can possibly come direct from the fat of the food. Long ago, in opposition to the views of Dumas and his school, who taught that all construction of organic material, that all actual manufacture of living substance or even of its organic constituents, was confined to vegetables and mknown in animals, Liebig shewed that the butter present in the milk of a cow was much greater than could be accounted for by the scanty fat present in the grass or other folder she consumed. He also urged, as an argument in the same direction, that the wax produced by bees, which though having a different composition from fat may be used as an analogy, is out of all proportion to the wax or allied bodies contained in their food, consisting as this does chiefly of sugar. And it has since been shewn in many ways that, in fattening animals, the fat accumulated in the body cannot be accounted for by the fat which has been taken in the food. It has been proved by direct analysis. Thus of two young pigs, as much alike as possible, of the same litter, one was killed and analyzed, the amount of fat in the body being among other things determined. The other was fattened for a certain length of time on food whose composition was known, and then killed and analyzed. It was found that for every 100 parts of fat in the food 472 parts of fat were stored up in the body during the 
fattening period. It is clear that fat may be formed in the body out of something which is not fat.

$\$ 401$. There are two possible sources of this manufactured fat. 'The carbohydrates of the food form one source. In treating of digestion ( $\$ 292$ ), we referred to the possibility of carbohydrates during digestion in the alimentary canal becoming by fermentation converted into butyric acid; and we suggested that higher and more complex members of the same fatty acid series might be obtained out of carbohydrates by somewhat analogous changes, carried on however not in the alimentary canal by means of foreign organized ferments, but in the tissues througl the activity of the tissues themselves. We cannot as yet trace ont the steps nor can we definitcly point to any particular tissues other than the fat-cells themselves as the seats of any such changes; though it is possible that the fat may be manufactured in this or that tissue and subsequently transferred, for storage, to the fat-cells. But there can be no doubt that carbohydrate material does in some way or other give rise to fat. A carbohydrate diet is the kind of diet most efficacious in producing an accumulation of fat in the body: sugar or starch, in some form or other, is always a large constituent of ordinary fattening foods.

Another source of fat is to be found in the proteids. We have seen that the urea of the urine practically represents the whole of the nitrogen which passes through the body. Now in any given quantity of urea the amount of carbon is far less than that found in the quantity of proteid containing the same amount of nitrogen. 'Thus the percentage composition of the two being respectively,

$\begin{array}{lccccc} & \text { Carbon. } & \text { Hydrogen. } & \text { Oxygen. } & \text { Nitrogen. } & \text { Sulphur. } \\ \text { Urea } & 20 \cdot 00 & 6 \cdot 66 & 26 \cdot 67 & 46 \cdot 67 & \\ \text { Proteid } & 53 & 7 \cdot 30 & 23 \cdot 04 & 15 \cdot 53 & 1 \cdot 13\end{array}$

100 grms. of urca contain about as much nitrogen as 300 grms. of proteid; but the 300 grms. of proteid contain 139 grms. $(159-20)$ more carbon than do the 100 grms. urea. Hence the 300 grms. of proteid in passing through the body and giving rise to 100 grms. of urea, would leave behind 139 grms. of carbon, in some combination or other: and this surplus of carbon, if the needs of the economy did not demand that it should be immediately converted into carbonic acid and thrown off from the body, might be deposited somewhere in the form of fat. It has been calculated that in this way $100 \mathrm{grms}$. of proteid food might furnish 42 grms. of fat. We have already seen, in treating of the action of the pancreatic juice (\$210), that there is evidence of a fatty elcment (viz. lencin, which is amido-caproic acid, and so belongs to the fatty acid series) being thrown off from the complex proteid compound in the very pro- 
cess of digestion ; and though, as we have said, we have no proof that this action of pancreatic juice takes place largely in the normal body, its value as an example is none the less important.

Some observers have pushed this view of the production of fat out of proteids so far as to insist that all the fat formed in the body arises in this way out of proteid material, and that when carbohydrate food gives rise to the formation of fat it does so by shielding from oxidation the carbon moiety of the proteid food taken at the same time and thus permitting it to be stored up as fat. 'The carbohydrate itself, they argue, never becomes fat but its presence allows fat to be formed out of proteid material. This view has obviously a very important economical bearing, since, if it were true, it would be useless to increase the carbohydrate material of food for the purpose of fattening, unless a sufficient proportion of proteid material be given at the same timc. It has however been proved to be untenable.

$\$ 402$. It is clear then that a construction of fat does occur in the body somewhere. What limits can we place on the degree to which this construction is carried? When the food contains sufficient actual fat to account for the fat stored up in the body, does any construction of fat take place? In the first place we find that when the food contains abnormal fats such as are not present in the body, spermaceti for instance, or erucin (from rape-seed oil), these fats are not to be found, or are found in very small quantity only, in the fat which is stored up in the body as a consequence of a large supply of that food. In the second place we may call to mind the statement previously made, that the composition of fat varies in different animals. 'The fat of a man differs from the fat of a dog, even if both feed on exactly the same food, fatty or otherwise. Were the fat which is taken as food stored up as adipose tissue directly and without change, recourse being had to other sources of food for the construction of fat only in cases where the fat in the food was deficient, we should expect to find that the nature of the fat of the body would vary greatly with the food. So far from this being the case, direct experiment shews that the fat of the $\log$ is, as far as composition is concerned, very largely independent of the food, that the normal eonstituents of fat make their appearance very much as usual and in very much their appropriate proportion, though their proportion in the food may largely vary, and though some of them may be wholly absent. Thus in one experiment the fat of the body contained considerable quantities of stearin after a diet free from stearin, and in another preserved the normal amount of olein after a diet free from olein. This shews that the constructive power of the economy is, as regards fat, very great; indeed it is even possible that all the fat stored up in the body is fat formed ancw. 


\section{SEC. 7. THE MAMMARY GLAND.}

$\S 403$. Since milk is a secretion, and indeed an excretion, the mammary gland ought not to be classed as a metabolic tissue, in the linited meaning we are now attaching to those words. Yet the metabolic phenomena giving rise to the secretion of milk are so marked and distinct, have so many analogies with the purely metabolic events which take place in adipose tissue, and so strikingly illustrate metabolic events in general, that it will be more convenient to consider the matter here, rather than in any other connection.

The mammary gland, formed like a sweat gland, of which it may be considered an extreme development, by an ingrowth of the Malpighian layer of the epidermis, is a compound racemose gland, constructed after the general plan of such a gland and thus composed of branching ducts ending in secreting alveoli.

$\$ 404$. The appearances presented by the alveoli differ widely according as the gland is one which is being used for suckling or is onc in a resting or dormant condition, that is to say before any pregnancy at all has taken place or in the interval between two suckling periods. In the suckling gland each alveolus consists of a basement membrane, presenting the usual characters, lined with a single layer of cells leaving a wide lumen; but the appearances presented by the cells differ from time to time according to circmmstances and are not the same in all the alveoli at the same time. We may however distinguish two conditions which, since they seem to correspond to the loaded and discharged conditions of an ordinary gland, we may call the loaded and the discharged phase respectively, conditions intermediate between the two being met with.

In the discharged plase the alveolus is lined by a layer of low cubical or even flattened cells, so that the relatively large area of the alveolus is almost wholly occupied by the lumen in which some of the constitnents of the milk may still be retained. Each cell consists of granular cell-substance in which is placed a rounded or oval nucleus. Sometimes the free edge of the 
cell is jagged and uneven as if a portion of the free border had been torn away.

In a fully loaded phase the appearances are very different. The alveolus is now lined with a layer of tall columnar cells projecting unevenly into the lumen, the outline of which is correspondingly irregular and the area of which is much reduced. While the broader base of each cell rests on the basement membrane, the other end, conical or irregular, stretches towards the centre of the lumen. Instead of one nucleus, two or even more are now present. one well formed and normal being placed nearer the base, and the others, often shewing signs of breaking up or degeneration, nearer the free end. Sometimes constrictions are seen whereby the free peripheral portion of the cell, including one or more of the nuclei, is apparently being separated from the basal portion in which the remaining nucleus is lodged; and occasionally portions or fragments of cells, nucleated or nucleusless, may be seen lying in the cavity of the alveolus. In the cell-substance, especially towards the free border of the cell, are numerous oil globules of various sizes as well as granules or particles of other nature; some of the larger oil globules may be seen projecting from the surface as if about to be extruded from the cell; and in the cavity of the alveolus oil globules with a thimmer or thicker coating of cellsubstance are frequently present.

Between such a fully loaded phase, and a completely discharged phase, various intermediate conditions may be observed. the cells being of greater or less height, containing one nucleus only or more than one, the cell-substance occupied with few or with many oil globules and other granules, and the free border more or less jagged.

$\$$ 405. The dormant resting mammary gland, that for instance of an animal which has never been pregnant, is much smaller than a suckling gland, owing to the alveoli being both smaller and less numerous. Each alveolus moreover is not a cavity lined with a single layer of epithelium, but a solid cylinder or mass of comparatively small, rounded or polyhedral cells. So long as pregnancy does not occur the growth of these is exceedingly slow, and the products of such metabolism as goes on in them are carried away by the blood, so that under normal circumstances no secretion takes place.

When pregnancy occurs rapid growth of the mamma takes place, numerous new alveoli being formed by budding, but all for a time remaining solid cylinders of cells. At the approach of the birth of the offspring, the central cells undergo metabolic changes, especially a fatty transformation, and either before or after birth are cast off, leaving a single layer to line the alveoli and to carry on the work of secretion as described above. It is generally supposed that these shed cells supply the so-called 
'colostrum corpuseles' characteristic of the first milk, of which we shall speak presently.

At the end of lactation an absorption of some of the alrcoli takes place; and in old age still further absorption goes on with great climinution of the lumina.

$\$ 406$. In the lymphatic spaces of the connective tissuc which joins together the lobules of various sizes, surrounds the lobules and runs in between the projecting blind ends of the alveoli within the lobules leucocytes are numerous, and some of these may make their way through the basement membrane and between the secreting cells into the cavities of the alveoli and so appear in the milk.

$\$ 407$. The nuture of milk. Human milk has a specific gravity of from 1.028 to 1.034 , and when quite fresh possesses a slightly alkaline reaction. It speedily becones acid; and cow's milk, even when quite fresh, is sometimes slightly acid, the change of reaction taking place during the stagnation of the milk in the mammary ducts.

The constituents of milk are:

1. Proteids, viz. casein, and an albumin, agreeing in its general features with ordinary serum-albumin, but which, since it is said to differ somewhat in its solubilities and rotatory power from serum-allomin, has been called lactalbumin. The casein, as we have seen, $\$ 185$, undergoes through the action of rennin a change whereby insoluble casein (tyrein) makes its appearance and the milk is curdled. Casein may however be precipitated in an unchanged form by saturating milk with neutral salts, or by the careful addition of acetic acid to diluted milk, or by first adding to the diluted milk a slight quantity of acetic acid and then passing through it a strean of carbonic acid. In the filtrate the presence of the lactalbumin, which oceurs in small and variable quantities, may be shewn by coagulation with heat, or by precipitation with potassium ferrocyanide, \&c. In the process of curdling the casein, as stated in $\$ 185$, appears to be not simply changed into tyrein but to be split up into tyrein and into another proteid, which unlike the lactalbumin is not coagulated by heat and which appears to be allied to albumose. 'This or a similar albumose-like body has also been found in small quantities even in milk which has not curdled; it has been called lactoprotein. The lactalbumin, though eoagulated by heat when isolited, is not so coagulated as it exists in the natural milk, the alkalinity of the milk, which is increased by boiling, preventing this. Similarly casein, though coagulated by heat when simply suspended in water after being precipitated, is not coagulated by heat when it exists in a natural condition in milk; in these respects casein behaves like alkali-albumin, which it resembles in other features also. Hence milk when boiled does not coagulate as a whole, though 
in the superficial layers exposed to the air changes take place by which a film or skin, derived chiefly from the albumin but partly from the casein, appears on the surface; if this be removed a fresh portion undergoes the same change.

2. Fats. 'These are, in the main, palmitin, stearin, and olein; but other fats, supplied by butyric and other fatty acids in combination with glycerine, accompany the above in small quantities. In this respect the fat of milk resembles that of adipose tissue. Lecithin and cholesterin are also present in very small quantity, as well as a yellow colouring matter. 'The fat present in milk differ's in different animals as to the relative proportion of olein, palmitin and stearin, and as to the kinds and relative amount of the other suantier fats.

'The mixturc of these fats, fluid at ordinary temperatures, is present in natural milk in the form of globules of various sizes but for the most part exceedingly small (in man from $2 \mu$ to $5 \mu$ ). Milk is in fact a typical emulsion, and it is the presence of the casein in the milk which brings about the emulsion.

On standing a great deal of the fat collects on the top of the milk in the form of cream, but in this, as in the butter which is formed from it, the globules are still discrete, so long at least as the buttcr is 'fresh.' By the use of a centrifugal machine nearly the whole of the fat may be separated from the plasma.

3. Milk sugar or lactose. This is very apt to undergo fermentation into lactic acid, through the agency of an organized ferment; the milk thus becomes sour, and the casein is precipitated in a flocculent form when the acid is produced in sufficient quantity. Since the change will take place even when every care is taken to exclude germs from the atmosphere having access to the milk, the organized ferments must be present in the milk in the ducts of the gland.

4. Salts. Though traces of urea and kreatinin have been noted by some observers, the extractives of milk, beyond the lecithin and cholesterin already mentioned, are insignificant. 'The salts are of more importance; these are chiefly calcic phosphate, of whose function in the process of curdling we spoke in $\$ 185$, and potassic and sodic chlorides, with a small quantity of magnesic phosphate. Sulphates appear to be absent. A small quantity of an iron salt is present, and traces of sulpho-cyanide have been observed. Besides the phosphorus in the actual form of phosphates, milk contains a further considerable quantity of phosphorus in the proteids and in the nuclein, as well as some sulphur in the former. 'The inorganic constituents of milk may, broadly speaking, be said to differ distinctly from those of blood, and to much more nearly re. semble those of the entire body.

The composition of milk in the same animal varies widely 
from time to time, and besides undergoes marked changes during the period of lactation. The relative general composition of human milk and that of the cow, the mare, and the bitch may perhaps be shewn by the following table: - but it is difficult to draw an arerage since the individual analyses given differ so much; the figures given for casein and fat in the milk of the bitch may be unusually high.

\section{Average Composition of Milk in Different Animals.}

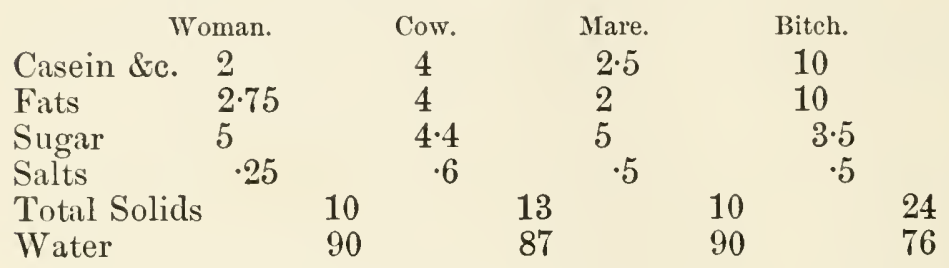

The quantity of milk secreted by a woman in twenty hours at the height of lactation has been calculated at 700 to $800 \mathrm{cc}$. A good milch cow will yield about 10 litres of milk per diem.

$\$$ 408. Colostrum. This is the name given to the milk secreted at the beginning of a period of lactation, just before and for some days after parturition. This milk differs from the subsequent milk in microscopical characters and in chemical composition.

When ordinary milk is examined under the microscope hardly anything is seen besides the fat globules except a very few imperfect cells or portions of cells consisting of cell-substance more or less loaded with fat and containing sometimes a more or less altered nucleus. A few minute granules, thought by some to be particles of suspended casein or nuclein, are however also visible.

Colostrum on the other hand contains a large number of cells or' corpuseles, which have been called 'colostrum corpuscles.' Some of these closely resemble lencocytes, others are either cells of about the same size, round or irregular, and possessing a nucleus, often misshapen, or are merely portions of cell-substance without a nucleus. In all of them the cell-substance may be loaded with fat globules or may be fairly free from fat. Some of these cells appear to be undergoing disintegration; some may at a favourable temperature exhibit slow amoboid movements, and must then at least be regarded as living.

Colostrum also differs from ordinary milk in containing not only a large quant it y of albumin (lactalbunin) but also a decided amount of globulin. In consequence of this colostrum differs from milk inasmuch as it is distinctly coagulated by heat.

As stated above, during the rapid growth by which the gland 
is enlarged preparatory to lactation, the alveoli are at first solid masses of cells with little or no lumen, and a lumen is established subsequently by the discharge of the central cells. It is usually supposed that the cells so discharged, some undergoing much, other's comparatively little change, supply the colostrum corpuscles just spoken of, and at the same time furnish the globulin and excess of albumin also characteristic of colostrum. But this is not certain. The alveoli at this time contain peculiar cells resembling colostrum corpuscles except that they are free from fat; and it is suggested that these being discharged and taking up fat in amoboid fashion become colostrum corpuscles. Some regard the colostrum corpuscles as simply leucocytes which have similarly taken up fat.

$\$ 409$. The mammary gland is present both in the female and the male child at birth; and in both sexes at and for a few days after birth is thrown, in common with all the other secreting glands, into secretory activity, and a small quantity of milk, the "witches" milk" so called by the Germans, is discharged from the nipple. This milk resembles in all essential features the milk of lactation. In both sexes this initial activity soon passes off, the gland in the female further developing at puberty, but in the male remaining, save in exceptional cases, in its infantile condition or somewhat retrograding.

$\$ 410$. The secretion of milk. From what has been already said it is obvious that the secretion of milk, while resembling the secretion of the other secreting glands which we have studied in being essentially an activity of the cpithelium cells lining the alveoli, nevertheless presents certain interesting features special to itsclf. If the account given in $\$ 40+$ be a true one, morphological changes in the cells are more prominent than in the case of other glands; and we may interpret the appearances there related somewhat as follows. When the discharged gland with its low epithelium begins the work of loading, the cells distinctly 'grow.' Their cell-substance increases in bulk, and elongating projects into the lumen of the alveolus. At the same time the nucleus divides as if the cell were about to give birth to new cells; but at first at all events no division of the cell-substance takes place, and the new nuclei lie imbedded in a common cell body. The cell-substance meanwhile puts on secretory activity; it deposits in itself material to form milk. The deposit of fat is conspicuous and easily recognized, but we may fairly infer that the other less easily distinguished proteid and carbohydrate materials are deposited in the cell-substance in a similar fashion. Then follows the ejection of the prepared material; and this may take place in one of two ways. The oil globules of fat may be protruded from the cell-substance much in the same way that an amoeba cxtrudes its excrement, and possibly other constituents of milk may be ejected by a similar method. 
But besides this, the deferred cell division now takes place in a somewhat imperfect fashion, so that portions of the old cell carrying nuclei with them come asunder from the rest of the cell in which a nueleus is left, and lie loose in the lumen of the alveolus; portions of cell-substance free from nuclei appear also to be cast off. Here, in the lumen of the alveolus, they rapidly undergo change; the cell-substance is altered and dissolved, and its load of prepared material, probably undergoing in the act some further change, is set free, the nuclei also undergoing change and becoming ultimately broken up. Hence the constituents of milk are provided for, not only as in other glands by the material with which the cell loads itself and subsequently discharges into the lumen of the alveolus, but also by the actual substanee of part of the eell itself. The characteristie nuclein of the milk has thus its origin in all probability in the shed nuclei of the secreting cells, and we may perhaps infer that the still more characteristic casein exists in milk in the form of tasein and not of some other proteid in consequence of this intervention of the actual cell-substance in the formation of the millk.

The secretion of milk differs from such a secretion as that of saliva, and approaches the formation of sebum inasmuch as the transformed cell-substance is shed bodily to form part of the milk. IVe say form part of the milk because this gross mode of secretion is accompanied by the more ordinary morle. The cells are at the same time in the more ordinary way discharging into the lumen water holding saline and other constituents in solution. And the peculiar features of milk, as we shall see presently, correspond to this double mode of secretion. Perhaps however we ought not to call it a double mode, for the one method really passes insensibly into the other. The discharge of sodium chloride in solution from every kind of gland, of mucin from a mucons gland, of oil globules with a proteid envelope from a mammary gland, and lastly of nucleated loaded cell-substance from the mammary gland, present so many different phases of the same act of secretion.

$\$ 411$. The secretion of milk then would appear to illustrate, even more fully and clearly than do other glands, the truth on which we have so often insisted, that a secretion is eminently the result of the metabolic activity of the secreting cell. The blood is the ultimate source of milk, but it becomes milk only through the activity of the cell, and that activity consists largely in a metabolic manufacture by the cell and in the cell of the common things brought by the blood into the special things present in the milk. Experimental results tell the same tale. 'Thus the quantity of fat present in milk is largely and directly increased by proteid, but not increased, on the contrary dininished, by fatty food. 'This effect on the 
mammary gland in particular is in accordance with what we shall presently learn to be the general effect on the body of proteid in contrast to that of fatty food; proteid food seems to increase the general metabolic activity of the body while fatty food tends to lessen it. Moreover the proteid food seems actually to furnish the fat; and we have already suggested a manner in which proteids may give rise to fat. That the fat of the milk need not necessarily come from the fat of the food is shewn by the following experiment. A bitel fed on meat for a given period gave off more fat in her milk than she could possibly have taken in her food ; and this moreover took place while she was gaining in weight and 'laying on fat,' so that she could not have supplied the mammary gland with fat by simply transferring fat from the store previously existing in the adipose tissue of her body; she apparently obtained the fat ultimately from the proteids of her food. And the histological facts given above favour the view that the formation of fat out of proteids in such cases takes place in the cells of the alveoli. 'The experimental then as well as the histological evidence goes to shew that the fat of milk is formed in the cell and by the cell, and is not simply gathered out of the blood.

The casein in a similar way seems to be formed by the action of the cell. It cannot be gathered out of the blood since the blood contains no real cascin; it must be formed in the gland. Some observers have maintained that when milk is kept at $35^{\circ}$, the casein is increased through some ferment action taking place in the milk itself; but this seems not to be the case, and the formation of casein must be regarled as the result of the action of the cell. Even the albumin present appears to be not the ordinary serum-albumin simply passed from the blood through the cell into the lumen of the alveolus, but the slightly different lactalbumin. We may perhaps regard the albumin as less difficult to manufacture than the casein; and we may explain the fact that relatively to the albumin the casein is less at the very beginning and especially toward the end of lactation, by supposing that the cell has in the first case not got into full working order and in the second case is waning in power. The peptone-like body in milk though small in quantity is a further indication of the proteid metabolism taking place in the cell.

'That the milk-sugar, lactose, also is formed in and by the cell, is indicated by the facts that it is found in no other part of the body, and that its presence in milk is not dependent on carbohydrate food, for it is maintained in abundance in the milk of carnivora when these are fed exclusively on meat, as free as possible from any kind of sugar or glycogen. A glycogen-like body has moreover been described as existing in the cells, and it is suggested that this body is the antecedent of the lactose. We thus have evidence in the mammary gland 
of the formation, by the metabolic activity of the secreting cell, of the representatives of the three great classes of food-stuffs, proteids, fats, and carbohydrates.

$\$ 412$. That both the secretion and ejection of milk are under the control of the nervous system is shewn by common experience, but the exact nervous mechanism has not yet been fully worked out. While erection of the nipple ceases when the spinal nerves which supply the breast are divided, the secretion continues, and is not arrested even when the sympathetic as well as the spinal nerves are cut. 


\title{
CHAPTER V.
}

\author{
NUTRITION.
}

\section{SEC. 1. THE STATISTICS OF NUTRITION.}

§ 413. THE preceding chapter has shewn us how wholly impossible it is at present to master the metabolic phenomena of the body by attempting to trace out forwards or backwards the several changes undergone by the individual constituents of the food, the body, or the waste products. Another method is however open to us, the statistical method. We may ascertain the total income and the total expenditure of the body during a givell period, and by comparing the two may be able to draw conclusions concerning the changes which must have taken place in the body while the income was being converted into the output. Miany researches have been carried out by this method; but valuable as are the results which have been thereby gained, they must be received with caution, since in this method of inquiry a small error in the data may, in the process of calculation and inference, lead to most wrong conclusions. The great use of such inquiries is to suggest ideas, but the views to which they give rise need to be verified in other ways before they can acquire real worth.

Composition of the Animal Body. The first datum we require is a knowledge of the composition of the body, as far as the relative proportion of the various tissues is concerned. In the human body the proportions by weight of the chief tissues, in the fresh state, are probably somewhat as follows:

\begin{tabular}{|c|c|}
\hline Adult Man. & Newborn Baby. \\
\hline 15.9 p.c. & $17 \cdot 7$ p.c. \\
\hline $\begin{array}{r}41 \cdot 8 \quad " \\
1.7\end{array}$ & $\begin{array}{r}22 \cdot 9 \quad ", \\
3 \cdot 0\end{array}$ \\
\hline $7 \cdot 2 "$ & 11.5, \\
\hline $18 \cdot 2$, & $90 \cdot 0$ \\
\hline $6 \cdot 9$, & 200 \\
\hline 1.9, & $15 \cdot 8$, \\
\hline
\end{tabular}

Skeleton
Muscles

Thoracic viscera

Abdominal viscera

Fat

Skin

Brain

Adult Man.

15 p.c.

$41 \cdot 8$,

18.2

6.9 ,

619 
An analysis of a cat has given the following result:

$\begin{array}{lr}\text { Muscles and tendons } & 45 \cdot 0 \text { p.c. } \\ \text { Bones } & 14 \cdot 7 ", \\ \text { Skin } & 12 \cdot 0 ", \\ \text { Mesentery and adipose tissue } & 3 \cdot 8, \\ \text { Liver } & 4 \cdot 8, \\ \text { Blood (escaping at death) } & 6 \cdot 0 " \\ \text { Other organs and tissues } & 13 \cdot 7,\end{array}$

One point of importance to be noticed in these analyses is that the skeletal muscles form nearly half the body; we have already seen (\$38) that about a quarter of the total blood in the body is contained in them, and have already ( $\$ 382)$ insisted that a large part of the metabolism of the body is carried on in the muscles. Next to the muscles we must place the liver, for though far less in bulk than them, it is subject to a very active metabolism; this is suggested by the fact that it alone may hold about a quarter of the whole blood, and is also indicated by the numerous facts brought before us in the preceding chapter.

$\$$ 414. The Starving Body. Before attempting to study the influence of food, it will be useful to asecrtain what changes occur in a bodly when all food is withheld. A cat of known weight was starved for 13 days. At the beginning of the period the body was presumed to have the composition given above; at the close of the period a direct analysis of the body was made. From this it appeared that during the hunger period the cat had lost 734 grammes of solid material, of which $248 \cdot 8$ were fat and 118.2 muscle, the remainder being derived from the other tissucs. The percentages of dry solid matter lost by the more important tissues during the period were as follows:

$\begin{array}{lr}\text { Adipose tissue } & 97 \cdot 0 \text { p.c. } \\ \text { Spleen } & 63 \cdot 1, \\ \text { Liver } & 56 \cdot 6 ", \\ 30 \cdot 2, \\ \text { Muscles } & 17 \cdot 6, \\ \text { Blood } & 0 \cdot 0, \\ \text { Brain and spinal cord } & \end{array}$

Thus the loss during starvation fell most heavily on the fat, indeed nearly the whole of this disappeared. Next to the fat, the glandular organs, the tissues which we have seen to be eminently metabolic, suffered most. 'Then come the muscles, that is to say, the skeletal muscles, for the loss in the heart was very trifling; obviously this organ, on account of its importance in carrying on the work of the economy, was spared as much as possible: it was in fact fed on the rest of the body. 'The same 
remark applies to the brain and spinal cord; in order that life might be prolonged as much as possible, these important organs were nourished by material drawn from less noble organs and tissues. The blood suffered proportionally to the general bodywaste, becoming gradually less in bulk but retaining the same specific gravity; of the total dry proteid constituents of the body $17 \cdot 3$ p.c. was lost, which agrees very closely with the $17 \cdot 6$ p.c. dry material (almost wholly proteid) lost by the blood. It is worthy of remark that the tissues in general became more watery than in health. Similar observations on other animals have led to similar results, the chief discordance being that in some cases the bones have suffered considerable loss, in others comparatively little. We might be inclined to infer from these data the conclusions that metabolism is most active in the adipose tissue, next in such metabolic tissues as the hepatic cells and spleen-pulp, then in the muscles, and so on; but we have no warrant for these conclusions. Because the loss of cardiac and nervous tissue was so small, we must not therefore infer. that their metabolism was feeble; they may have undergone rapid metabolism, and yet have been preserved from loss of substance by their drawing upon other tissues for their material. 'The great loss of adipose tissue is obviously to be explained by the fact that that tissue is essentially a storehouse of material, and the similarly great though less loss in the spleen and liver indicates, as indeed the facts recorded in the previous chapter suggest, that these organs too serve in part as storehouses.

During this starvation period, the urine contained in the form of urea (and that plactically represents all the nitrogen of the urine) $27 \cdot 7$ grammes of nitrogen. Now the amount of muscle which was lost during the period contained about $15 \cdot 2$ of nitrogen. 'Thus, more than half the nitrogen of the output during the starvation period must lrave come ultimately from the metabolism of muscular tissue. This fact we have alleady used in discussing the history of urea and shall have occasion to nake further use of it hereafter. The amount of urea excreted per diem has been observed in some cases to fall very rapidly during the first day or two of starvation, and then to diminish gradually, though often shewing considerable irregularities. In other cases no such large initial fall has been observed. It is most marked in animals which have been well fed before the beginning of the starvation, especially in those which have had a rich nitrogenous diet: and the discharge in these cases of an extra quantity of urea in the first day or two is obviously connected with that immediate effect of food on the excretion of urea to which we have already ( $\$ 385)$ referred and to which we shall have to return in speaking of what is known as "luxus-consumption." 


\section{Comparison of Income and Output of Material.}

\$415. Method. We have now to inquire how the elements of food are distributed in the excreta, in order that, from the manner of the distribution, we may infer the nature of the intermediate stages which take place within the body. By eomparing the ingesta witl the exereta, we shall learn what elements lave been retained in the body, and what elements appear in the excreta whieh were not present in the food ; from these we may infer the elanges which the body has undergone through the influenee of the food.

In the first plaec, the real income must be distinguished from the apparent one by the subtraction of the frees. We have seen that by far the greater part of the frees is undigested matter, i.e. food which, though plaeed in the alimentary eanal, has not really entered into the body. The sliare in the frees taken up by matter which has been exereted from the blood into the alimentary canal, is so small that it may be negleeted; certainly with regard to nitrogen, the whole quantity of this element, which is present in the freces, may be regarded as indieating simply undigested nitrogenous matter.

The ineome, thus eorrected, will eonsist of so mueh nitrogen, earbon, hydrogen, oxygen, sulphur, phosphorus, saline matter's, and water, eontained in the proteids, fats, carbohydrates, salts, and water of the food, together with the oxygen absorbed by the lungs, skin, and alimentary canal. The output may be regarded as eonsisting of (1) the respiratory products of the lungs, skin, and alimentary eanal, consisting chiefly of earbonic acid and water, with small quantities of hydrogen and earburetted hydrogen, these two latter coming exelusively from the alimentary canal; (2) of perspiration, consisting chiefly of water and salts, for the dubious exeretion (see $\$ 350$ ) of urea by the skin may be neglected, and the other organic eonstituents of sweat amount to very little; and (3) of the urine, which is assumed to contain all the nitrogen really exereted by the body, hesides a large quantity of saline matters and of water. Where great aceulacy is required the total nitrogen of the urine ought to be determined; it is maintained, however, that no errors of serions importance arise when the urea alone, as determined by Liebig's method (whieh was largely used in the researehes forming the basis of the present discussion), is taken as the measure of the total quantity of nitrogen in the urine, sinee, in this method, other nitrogenous bodies besides urea are preeipitated, and so eontribnte to the quantitative result. It has been and indeed still is debated whether the body may not suffer loss of nitrogen by other ehannels than by the urine and ficees, whether nitrogen may not leave the body by the skin or indeed in a gaseous state by the lungs. 'The balanee of the conflieting 
evidence seems however in favour of the view that no such loss takes place. It would appear that though nitrogen, the pivot, so to speak, of the chemical changes of living beings, forms so large a portion of the atmosphere and moreover is physically diffused through the bodies of both plants and animals, free nitrogen is of no chemical use to either of them. It enters into and remains in their bodies as an inert substance, and the nitrogen which leaves a plant or animal, in a gaseous state, is simply a part of the same inert supply and does not come from the breaking up of the nitrogenous substances of the body or of the food.

Of these elements of the income and output, the nitrogen, the carbon, and the free oxygen of respiration are by far the most important. Since water is of use to the body for merely mechanical purposes, and not solely as food in the strict sense of the word, the hydrogen element becomes a dubious one, the sulphur of the proteids and the phosphorus of the fats are insignifieant in amount; while the saline matters stand on a wholly different footing from the other parts of food, inasmuch as they are not sources of energy, and pass through the body with comparatively little change. The body-weight must of course be carefully ascertained at the beginning and at the end of the period, correction being made where possible for the frees.

It will be seen that the labour of such inquiries is considerable. The urine, which must be carefully kept separate trom the faces, requires daily measurement and analysis. Any loss by the skin, either in the form of sweat, or, in the case of woolly animals, of hair, must be estimated or accounted for. The food of the period must be as far as possible uniform in character, in order that the analyses of specimens may serve faithfully for calculations involving the whole quantity of food taken; and this is especially the case when the diet is a meat one, since portions of meat differ so much from each other. But the greatest difficulty of all lies in the estimation of the carbonic acid produced and the oxygen consumed. In some of the earlier researches this factor was neglceted and the variations occurring were simply guessed at, through which very serious errors were introduced. No comparison of income and output can be considered satisfactory unless at least the carbonic acid produced be directly measured by means of a respiration chamber. And in order that the comparison should be really complete, the water given off by the skin and lungs must be directly measured also; but this seems to be more difficult than the determination of the carbonic acid.

In the plan originally adopted by Regnault and Reiset and followed by some other observers, the animal experimented on is allowed to breathe a limited and measured atmosphere. The carbonic acid, as fast as it is formed, is fixell and removed by a strong 
solution of caustic potash, and the normal percentage of oxygen in the atmosuhere is maintained by a supply of this gas from a gasholder. In this way both the oxygen consumed and the carbouic acid produced are directly determined, while the continual smpply of fresh oxygen prevents any evil effects due to breathing a confined portion of air. In orler however to avoid all possible errors arising from a too restricted atmosphere a different method has been adopted by lettenkofer and Voit. Their apparatus consists essentially of a large chamber, capable of holding a man comfortably. By means of a stean-engine a current of pure air, measured by a gasometer, is drawn through the chamber. Measured portions of the outgoing air are from time to time withdrawn and analyzed; and from the data afforded by these analyses, the amounts of tarbonic acid (and other gases) and of water given off by the occupant of the chamber during a given time are determined. The oxygen consumed is not determined directly; but if the total amounts of carbonic acid and of water given out by the lungs and skin are ascertained and the amount of nrine and freces known, the quantity of oxygen consumed may be arrived at by a simple calculation. For evidently the difference between the terminal weight plus all the egesta and the initial weight plus all the ingesta ean be nothing else than the weight of the oxygen absorber dmring the period. This method in turn however is also oper to objections, since minnte errors in the analyses of the small samples of air employed for the determinations attain consilerable dimensions when these are multiplied so as to give the changes in the whole mass of air passed throngh the apparatus. It seems moreover undesirable to leave the quantity used of so important an element as oxygen to be determined by indirect calculations.

Let us imagine, then, an experiment of this kind to have been completely carried out, that the animal's initial and terminal weights have been accurately determined, the composition of the food satisfactorily known to consist of so much proteid, fat, carbohydrates, salts, and water, and to contain so much nitrogen and carbon, the wcight of the faces and the nitrogen they contain ascertained, the nitrogen of the urine determined, the earbonic acid and water given off by the whole body carefully measured, and the amount of oxygen absorbed calculated - what interpretation can be placed on the results?

Let us suppose that the animal has gained $w$ in weight during the period. Of what does $w$ consist? Is it fat or proteid material which has been laid on, or simply water which has been retained, or some of one and some of the other? Let us further suppose that the nitrogen of the mine passed during the period is less, say by $x$ grammes, than the nitrogen in the foorl taken, after deduction of course of the nitrogen in the fieces. 'I'his means that $x$ grammes of nitrogen have been retained in the body; and we may with reason infer that they have been retained in the form of proteid material. We may even go farther and say that they are retained in the form of 
flesh, i.e. of muscle. In this inference we are going somewhat beyond our tether, for the nitrogen might be stored up as some proteid constituent of the hepatic cells or of some other tissue; indeed it might be for the while retained in the form of some nitrogenous crystalline body. But this last event is unlikely; and if we use the word 'flesh' to mean nitrogen (proteid) holding living substance of any kind, we may without fear of any great error reckon the deficiency of $x$ grammes nitrogen as indicating the storing up of a grammes flesh. There still remain $w-a$ grammes of increase to be accounted for. Let us suppose that the total carbon of the egesta has been found to be $y$ grammes less than that of the ingesta; in other words, that $y$ grammes of carbon have been stored up. Some carbon has been stored up in the flesh with the nitrogen just considered; this we must deduct from $y$, and we shall then have $y^{\prime}$ grammes of carbon to account for. Now there are only two principal forms in which carbon can be stored up in the body: as glycogen or as fat. The former is even in most favourable cases inconsiderable, and we therefore cannot err greatly if we consider the retention of $y^{\prime}$ grammes carbon as indicating the laying on of $b$ grammes fat. If $a+b$ are found equal to $w$, then the whole change in the economy is known; if $w-(a+b)$ leaves a residue $c$, we infer that in addition to the laying on of flesh and fat some water has been retained in the system. If $w-(a+b)$ gives a negative quantity, then water must have been given off at the same time that flesh and fat were laid on. In a similar way the nature of a loss of weight can be ascertained, whether of flesh, or fat, or of water, and to what extent of each. The careful comparison, the debtor and creditor account of income and output, enables us, with the cautions rendered necessary by the assumptions just now mentioned, to infer the nature and extent of the bodily changes. The results thus gained ought of course, if an account is kept of the water taken in and given out, to agree with the amount of oxygen consumed, and also to tally with the conclusions arrived at concerning the retention or the reverse of water.

Having thus studied the method and seen its weakness as well as its strength, we may briefly review the results whith have been obtained by its means.

\$416. Nitrogenous Metabolism. When a meal of lean meat, as free as possible from fat, is given to a dog, which has previously been deprived of food for some time, and whose body therefore is greatly deficient in flesh, it might be expected that the larger part of the food would be at once stored up to supply pressing deficiencies, and that only the smaller part would be immediately worked off as urea corresponding to the nitrogenous metabolism going on in the body at the time, increased somewhat by the labour thrown on the economy by the very 
presence of the food. This howerer is not the case as far as the nitrogen of the meal is concerned; the larger portion passes off as urea at once, and only a comparatively small quantity is retained. If the diet be continued, and we are supposing the meals giver to be large ones, the proportion of the nitrogen which is given off in the form of urea goes on increasing until at last a condition is established in which the nitrogen of the egesta exactly equals that of the ingesta. This condition, which is spoken of as "nitrogenous equilibrium" is attained in dogs with an exclusively meat diet only when large quantities of food are given, and is not easily maintained for any length of time. The exact quantity of meat required to attain nitrogenous equilibrium varies with the previous condition of the log; equilibrium is frequently attained when 1500 or 1800 g'rms. of meat are given daily.

'Thus the most striking effect of a purely nitrogenous diet is largely to increase the nitrogenous metabolism of the body; and we shall see later on that it increases the metabolism not only of the nitrogenous but also of the other constituents of the body.

The establishment of nitrogenous equilibrium does not mean that a body-equilibrium is established, that the body-weight neither increases nor diminishes. On the contrary, when the meal necessary to balance the nitrogen is a large onc, the body though it is neither gaining nor losing nitrogen may gain in total weight; and the increase is proved by calculation from the income and output, and indeed by actual examination of the body, to be due to the laying on of fat. The amount so stored up may be far greater than can possibly be accounted for by any fat still adhering to the meat given as food. We are therefore driven to the conclusion that the proteid food is split into a urea moiety and a fatty moiety, that the urea moiety is at once discharged, and that such of the fatty moiety as is not made use of directly by the body is stored up as adipose tissue. And this disruption of the proteid, as we have already ( $\$ 385)$ suggested, explains at the same time why the meat diet so largely and immerliately increases the urca of the egesta.

This characteristic effeet of proteid food to increase the metabolism of the body is shewn on other animals besides the dog, and not only by means of calculations of what is supposed to take plaec in the body, but also by direct analysis. Thus the analysis of the body of a pig, which had been fed on a known diet, compared with the analy, wis with that of another pig of the same litter, killed at the time when the first was put on the fixed diet, gave as a result that of the dry nitrogenons material of the food only about 7 p.c. was laid up as dry proteid material during the fattening period, though the amonnt of proteid food was low. This contrasts strongly with the amount of fat stored up during the same period (see $\$ 400$ ). Similar obscrvations carried out 
on sheep shewed that in these animals the storing up of nitrogenous material was even less, only about 4 p.c. of that given in the food.

Every quantity of proteid material taken into the alimentary canal thus appears to affect proteid metabolism in two ways. On the one hand it excites a rapid proteid metabolism giving rise to an immediate, and generally large, increase of urea; on the other hand, it serves to maintain the more regular normal proteid metabolism continually taking place in the body, and so contributes to the normal regular discharge of urea. It seems very natural to suppose that the proteid which plays the first of these two parts is not really built up into the tissues, does not become actual living substance, but undergoes the changes which give rise to urea outside the actual living substance in the blood or elsewhere; and we have seen that under the influence of the pancreatic juice some of the proteid food may undergo the greater part of such a change while it is as yet within the alimentary canal. Hence has arisen the very natural distinction to which we have already alluded between "tissue proteids" or" "morphotic proteids" which are actually built up into the living substance of the tissues and give rise to urea through the metabolism of living substance, and "circulating proteids" or "floating proteids" which do not at any period of their eareer within the body become an integral part of the living substance and by their metabolism set free energy not in the way of vital manifestations but in the form of heat only. We shall later on consider what is the exact meaning which we ought to attach to the words "becoming part of the living substance;" and hence shall defer until then any discussion of the appropriateness of these phrases and of the validity of the distinction which they formulate.

It was once thought, as we shall presently see erroneously, that the exclusive purpose of proteid food was to supply the proteid tissues, and that all the energy set free in the bouy in vital manifestations, such as movement and the like as distinguished from heat, had its origin in proteid metabolism, the metabolism of fats and carbohydrates giving rise to heat only. Hence when it first became known that a certain proportion of proteid food apparently underwent a metabolism giving rise to heat only, without becoming part of the tissues, this seemed to be a wasteful expenditure of precious material; and the metabolism of this portion of proteid food was accordingly spoken of as a "luxus-consumption," a wasteful consumption.

$\$$ 417. The Effects of Fatty and of Carbohydrate Food. Unlike those of proteid food, the effects of fats and carbohydrates camnot be studied alone. When an animal is fed simply on non-nitrogenous food, death soon takes place; the food rapidly ceases to be digested, and starvation ensues. We can there- 
fore only stıdy the nutritive effects of these substances when they are taken together with proteid material.

When a small quantity of fat is taken, in company with a fixed moderate quantity of protcid material, the whole of the carbon of the food reappear's in the cgesta. No fat is stored up; some even of the previously existing fat of the body may be consumed. As the fat of the meal is increased, a point is soon reached at which carbon is retained in the body as fat. So also with starch or sugar; when the quantity of this is small, there is no retention of carbon; as soon however as it is increased beyond a certain limit, carbon is stored up in the form of fat or, to a smaller extent, as glycogen. Fats and carbohydrates therefore differ markedly from proteid food in that they are not so distinctly provocative of metabolism. This is exceedingly well shewn in the results obtained on the pig previously mentioned. It was found that 472 units of fat were laid on for every 100 units of fat taken as such in the food (which consisting of barley-meal, \&c. contained a very small amount of actual fat), while for every 100 units of the total dry non-nitrogenous food including fat, starch, cellulose, \&c., no less than 21 units were retained in the body in the form of fat. No clearer proof than this could be afforded that fat is formed in the body out of something which is not fat. In $\$ 401$ we have already discussed this formation of fat out of carbohydrates.

As one might imagine, the presence of fat or carbohyclrates in the food is found to decrease the amount of proteid material necessary to establish nitrogenous equilibrium. For instance, with a diet of $800 \mathrm{grms}$. meat and $160 \mathrm{grms}$. fat, the nitrogen in the egesta became equal to that in the ingesta in a dog, in whose case 1800 grms. meat had to be given to produce the same result in the absence of fats or carbohydrates.

On the other hand, it was found that, with a fixed quantity of fatty or carbohydrate food, an increase of the accompanying proteid led not to a storing up of the surplus carbon contained in the extra quantity of proteid, but to an increase in the consumption of carbon. Proteid food increases not only proteid but also non-nitrogenous metabolism. 'This explains how an excess of proteid food may, by the increase of general metabolism, actually reduce the fat of the, body.

We have at present no exact information concerning the nutritive differences between fats and carbohydrates, beyond the fact that in the final combustion of the two, while carbohydrates require sufficient oxygen to combine with their carbon only, there being alrearly sufficient oxygen in the carbohydrate itself to form water with the hydrogen present, fats require in arddition oxygen to combine with some of their hydrogen. Hence in herbivora, living largely on carbohydrates, a larger 
portion of the oxygen consumed reappears in the carbonic acid of the egesta than in carnivora, in which animals, living chiefly on proteids and fats, more of it leaves the body combined with hydrogen to form water. This relation of the oxygen to the carbonic acid is often expressed as the quotient of the volume of the carbonic acid expired divided by the volume of the oxygen consumed, the 'respiratory quotient, $\frac{\mathrm{CO}^{2}}{\mathrm{O}^{2}}$, which is in herbivora about 9 and in carnivora about $\cdot 6$ or $\cdot 7$. When a herbivorous animal starves, it feeds on its own fat, and under these circumstances the respiratory quotient falls to the carnivorous standard; and indeed many circumstances affect this respiratory quotient. The carbohydrates are notably more digestible than the fats, but on the other hand the fats contain more potential energy in a given weight. As to the nutritive difference between starch and sugar, we know nothing very definite; it has been thought however that cane-sugar is rather more fattening than starch.

$\$$ 418. The Effects of Gelatin as Food. It is a matter of common experience that gelatin will not supply the place of proteids as a constituent of food. Animals fed on gelatin together with fat or carbohydrates die very much in the same way as when they are fed on non-nitrogenous material alone. -Nevertheless it would appear, as might be expected, that the presence of gelatin in food is not without effect. 'Thus nitrogenous equilibrium is established at a lower level of real proteid food when gelatin is added. In a dog, moreover, fed on a riet of gelatin and fat, the excess of nitrogen in the excreta over that in the ingesta is less than when the same dog is fed on a diet of fat alone; that is to say, the gelatin lias sheltered from metabolism some proteid constituents of the boly; and the consumption of fat seems also to be lessened by the presence of gelatin. These facts become intelligible if we suppose that gelatin is rapidly split up into a urea and a fat moiety, in the same way that we have seen a certain quantity of proteid material to be. It is this direct destructive metabolism of proteid matter which gelatin can take up; it seems however unable to imitate the other function of proteid matter, and to take part in the formation of living substance; or in the phraseology of a preceding paragraph (\$ $\$ 16)$, it can take the place of circulating but not of tissue proteid. What is the cause of this difference, we cannot at present say.

419. Peptone as Food. Since proteids are at least largely, as we have seen $(\$ 250)$, converted into and absorbed as peptone, and since as we have also seen the peptone appears during the very act of absorption to be reconverted into some other form of proteid matter, possibly serum-albumin, it might seem natural to suppose that peptone given as food would as far as 
metabolism is concerned play the same part as other proteids. Nevertheless, some observers have maintained with regard to both peptones and the allied albumoses that, like gelatin, these bodies "can take the place of circulating but not of tissue proteid." On the whole, however, the evidence goes to shew that animals can 'lay on flesh' when the proteid in their food consists entirely of peptone or albumose. A difficulty, appertaining to digestion, prevents any large substitution of peptone for ordinary proteids, since as might be expected diarrhuea is apt to be set up.

$\$$ 420. The Effects of Salts as Food. All food contains, besides the substances possessing potential energy, which we have just studied, certain saline matters, organic and inorganic, having in themselves little or no such potential energy, but yet either absolutely necessary or highly beneficial to the body. These must have important functions in directing the metabolism of the body : the striking distribution of them in the tissues, the preponderance of sodium and chlorides in blood-serum and of potassium and phosphates in the red corpuscles for instance, must have some meaning; but at present we are in the dark concerning it. The element phosphorus seems no less important from a biological point of view than carbon or nitrogen; it is as absolutely essential for the growth of a lowly being like Penicillium as for man himself. We find it probably playing an important part as the conspicuous constituent of lecithin and other complex fats belonging to the nervous system, we find it prominent in the peculiar body nuclein, we find it peculiarly associated with the proteids; but we cannot explain its rôle. The element sulphur, again, is only second to phosphorus, and we find it as a constituent of nearly all proteids; but we cannot foretell the exact clianges which would take place in the economy if all the sulphur of the food were withdrawn. In the keratin of the epidermis and its appendages, hairs, \&c., it is probably undergoing excretion, though its presence in this body may have to do with the peculiar physical characters of corneous epithelium.

We know that the various saline matters are essential to health, that when they are not present in proper proportions nutrition is affected. Dogs fed on food, freed as mucl as possible from all saline matters, but otherwise abundant, with a proper proportion of the food-stuffs, soon exhibit symptoms shewing that the metabolism of their tissues, especially of their central nervous system, is going wrong; they suffer from weakness, soon amounting to paralysis, and are often carried off by convulsions. And more or less similar derangements of nutrition follow the absence or a deficiency of individual salts. During starvation these various salts continue to be discharged from the body; in some way or other they are carried along 
in the metabolic stream, and their presence is in some way essential to the various metabolic processes; hence they need to be always present in daily food. In what way it is that they thus direct metabolism we do not know; we are aware that the properties and reaetions of various proteid substances are closely dependent on the presence of certain salts; but beyond this we know very little. The inorganic salts are those, the nutritive value of which has been chiefly studied by experiment; but we have reason to believe that the organic salts, or extractives, which are present in greater or less quantity in all food of both vegetable and animal origin, are no less essential to the proper metabolic activities of the body. The undoubted connection of scurvy with the lack of fresh vegetable food, other conditions helping, may perhaps turn in part on this, for the evidence that the disease is due to the deficiency of potash alone is not conclusive.

Lastly, water has an effect on metabolism, as shewn, among other things, by the fact that when the water of a diet is increased, the urea is increased to an extent beyond that which can be explained by the increase of fluid increasing the facilities of mere excretion. 


\section{SEC. 2. THE ENERGY OF THE BODY.}

\section{The Income of Energy.}

\$421. Broadly speaking, the animal body is a maehine for converting potential into actual energy. The potential energy is supplied by food; this the metabolism of the body eonverts into the aetual energy of heat and mechanieal labour. We have in the present section to study what is known of the laws of this eonversion, and of the distribution of the energy set free.

Neglecting all subsidiary and unimportant sources of energy, we may say that the income of animal energy eonsists in the oxilation of food into its waste products, viz. the oxidation of proteids, fats and carbohydrates into urea, earbonie aeid and water. A principle laid down by the chemist teaehes that the potential energy of any body, eonsidered in relation to any chemical change which it may undergo, is the same when the final result is the same, whether that result be gained at one leap or by a series of steps; that, for instance, the energy set free by the oxidation of 1 grill. of fat into earbonie acid and water is the same, whatever the changes forwards or backwards which the fat undergoes before it finally reaches the stage of carbonic aeid and water; and similarly, that the energy available for the body in $1 \mathrm{grm}$. of dry proteid is the energy given out by the complete eombustion of that $1 \mathrm{grm}$., less the energy given out by the complete eombustion of that quantity of urea to which the 1 grm. of proteid gives rise in the body. Taking this as our guide we can readily calculate the amount of potential energy contained in an average 24 hours' diet, and thus obtain the average daily income of energy. For the potential energy of most of the substanees used as food has been determined by direct calorimetric observations; and the several detcrminations, though they vary somewhat, agree suffieiently closely to serve as data for the calculations in question.

The total combustion of the following substances has given for onc gramme of each substance the following results cxpressed in calories, that is in gramme-degree units of heat. 
Meat, free from fat, 5103, and 5324. Fibrin 5511. Eggalbumin 5579. 'Thus, taking round numbers we may say that 1 grm. of proteid material contains 5000 or 5500 calories of potential energy, according as we use the lower or higher ileterminations.

Fat of beef or mutton 9069, 9365, 9423. Butter 7267 or 9192. Again in round numbers we may say that $1 \mathrm{grm}$. of fat. contains about 9000 calories.

Arrowroot (nearly pure starch) 3912. Starch 4123. Cellnlose 4146. Dextrose 3692. Cane Sugar 3866. Here again, taking round numbers, we shall not be far wrong in saying that the potential energy of $1 \mathrm{grm}$. of carbohychate material is about 4000 calories.

The combustion of $1 \mathrm{grm}$. of urea sets free an amount of energy which has been determined by one observer at 2206 , by another as 2465 calories. We have seen (\$ $\$$ 401) that $1 \mathrm{grm}$. of proteid gives rise in the body to $\frac{1}{3}$ grm. urea. Hence, to obtain the energy of $1 \mathrm{grm}$. proteid nuaterial available for the economy, we must deduct from its total potential energy, one third the potential energy of $1 \mathrm{grm}$. urea, that is, in round numbers 700 or 800 calories. This will give us $5000-700$, or $5500-800$, that is 4300 or 4700 calories, according as we take the lower or ligher data; or we may take as a mean 4500 calories. The data then so far are as follows,

1 grm. proteid

1 grm. fat

1 grm. carbohydrate
4500 calories.

9000,

4000,

The average diet of an average man, that is the average amount of each food-stuff respectively taken daily, may be determined experimentally or statistically. 'Thus a man may determine by a series of trials the diet on which, while neither losing nor gaining weight and maintaining "nitrogenous equilibrium,'s 416 , he enjoys good health. Or an average may be struck of a large number of diets used by various people. We shall have something to say of this latter statistical method when we come to speak of diet. For the present purpose we may use one arrived at experimentally which we will speak of as Ranke's diet, since it was determined by a physiologist of that name from observations on himself. It was composed of $100 \mathrm{grm}$. proteid, $100 \mathrm{grm}$. fat, $240 \mathrm{grm}$. carbohydrate. Such a diet would give

100 grm. proteid (4500)

$100 \mathrm{grm}$. fat $(9000)$

240 grm. carbohydrate (4000)
450,000 calories.

$900,000 \quad "$
$2,360,000$


If we translate the units of heat into units of rork, the 2.310,000 gramme-degree, or 2.310 kilogramme-degree ealories will give us about 150,000 , or, in round number's, somewhere about one million kilogramme-meters.

IV e may, in passing, call attention to the fact that the proteids supply a relatively small part of the total energy, and that the share contributed by the large mass of carbohydrates is not mueh greater than that belonging to the mueh smaller quantity of fat. In the average diet obtained by the statistical method, in whieh the data are largely drawn from public institutions, the (eheaper) earbohydrates are still further increased at the expense of the (dearer) fats, a ehange which may tend to reduee somewhat the total energy; but this does not materially affect the broad result just given.

\section{The Expenditure.}

\$ 422. There are two ways only in which energy is set free from the body: mechanical labour and heat. The body loses energy in producing muscular work, as in locomotion and in other kinds of labour, in the movements of the air in respiration and speeeh, and, though to a hardly reeognizable extent, in the movements of the air or contiguous bodies by the pulsations of the vascular system. The body loses energy in the form of heat by conduction and radiation, by respiration and perspiration, and by the warming of the urine and freces. All the internal work of the body, all the mechanieal labour of the internal museular meehanisms with their aeeompanying friction, all the molecular labour of the nervous and other tissues, is eonverted into heat before it leaves the body. The most intense mental aetion, unaceompanied by any museular manifestations, the most energetie action of the heart or of the bowels, with the slight exceptions mentioncd above, the busiest activity of the seereting or metabolic tissues, all these end simply in augmenting the expenditure in the form of heat.

A normal daily expenditure in the way of mechanieal labour ean be easily determined by observation. Whether the work take on the form of walking, or of driving a machine, or of any kind of museular toil, a good day's work may be put down at about 150,000 kilogramme-meters.

The normal daily expenditure in the way of heat eannot be so readily determined. Direet calorimetric observations on living structures are in all eases attended with many diffieulties and subject to many sourees of error. 'These are very great when the observations are made on the whole body, even in the case of small animals; and olservations made by plaeing a part only of the body, an arm or leg for example, in the calo- 
rimeter, and from the data thus gained calculating the heat produced by the whole body, are subject to additional sources of error. Improved methods, however, especially of recent years, have so far eliminated many sources of error that the results obtained by observations on the whole body may be received with increasing confidence.

The calorimeters usually employed in chemical operations, in measuring for instance the heat given out in chemical changes, are unsuitable for experiments on living animals. Such are the mercury-calorimeter, in which the chenical action to be studied is made to take place in the midst of a mass of mercury, from the consequent expansion of which through the heat taken up the amount of heat given out is calculated, or the ice-calorimeter in which in a similar way the heat given out is calculated from the amount of ice melted. 'The latter has been used for pliysiological purposes, but an animal surrounded by ice is under such abnormal conditions that the results are of little value. The methods usually adopted by physiologists are as follows.

In one method, the water-calorimeter, the animal is placed in a metal chamber surrounded by a jacket filled with water. The heat given out by the animal warms the water in the jacket, and the amount given out is calculated upon the increase of the temperature of the water. By supplying the animal with air through a long spiral tube passing through the water-jacket, the heat given out in the expired air is prevented from being lost.

This method may be employed in a simpler form, when the heat given out by a part of the body, the arm or leg for instance, is all that has to be determined. The part is then merely placed in a bath of water, from the changes of temperature of which the amount given out is calculated. And this modification of the method may with due precautions be employed for the whole body.

In Rosenthal's calorimeter the chamber in which the body or part of the body is placed is surrounded by, not a water-jacket, but an air-jacket, which thus serves as an air-calorimeter. The instrument consists essentially of three concentric copper cylinders; the inner one contains the animal (or other source of heat); the outer one serves merely as a casing to protect those inside from changes of temperature due to currents of air and the like; and the mirldle one encloses an air space between itself and the inner one. There are special arrangements for elosing the cylinders after the introduction of the animal, and for supplying the animal with air for breathing purposes. With the air-jacket, or space between the inner and middle cylinders, are connected a manometer and a thermometer. When an animal (or other source of heat) is placed in the inner cylinder, the temperature and the pressure of the air in the air-jacket are increased; and from the amounts of increase measured by the thermometer and the manometer the amount of heat given out from the animal is calculated.

The calorimeters of D'Arsonval and Rubner are constructed on very similar principles. 
Various attempts have been made to ascertain the amount of heat given out by the body in an indirect manner, as for instance by calculating the heat given out by the oxidation of the food. As trustworthy as any is the plan of simply subtracting the normal daily mechanical expenditure from the normal daily income. 'Thus, $150,000 \mathrm{k}$.-m. subtracted from one million k.-m. gives $850,000 \mathrm{k} .-\mathrm{m}$. as the daily expenditure in the form of heat; i.e. between one-fifth and one-sixth of the total income is expended as mechanical labour, the remaining four-fifths or five-sixths leaving the body in the form of heat. The results given by direct calorimetric observations and by other calculations give somewhat higher figures than these; and indeed these may probably be taken as under rather than over the true amount. In any case they are to be regarded as furnishing hardly more than a rough average estimate for a man of average build and weight, taking an average amount of average food and doing an average amount of work.

\$ 423. The Energy of Mechanical Work. We have already in treating of muscle and elsewhere partly discussed this subject, but may here say the rest that has to be said.

'The older writers, even after it had been proved that the animal body was constructive so far as the formation of fat was concerned, still held to the distinction between nitrogenous or plastic and non-nitrogenous or respiratory food. Put broadly, this view was that all the nitrogenous food went to build up the proteid tissues, the muscular flesh and the like, and that the nitrogenous egesta arose solely from the functional metabolism of these tissues, while the non-nitrogenous food was used with equal exclusiveness for respiratory or calorific purposes, being either directly oxidized in the blood or, if present in excess, stored up as fatty tissue. According to this view the two classes of income corresponded exactly to the two forms of expenditure. We have already urged several objeetions against this view. We have seen that in the blood itself very little oxidation takes place, that it is the active tissue, and not the passive bloodplasma, which is the seat of oxidation. We have further seen that proteid food may undoubtedly be, in the above sense, respiratory and incidentally give rise to the storing-up of fat. One division of the view is thereby overthrown. We have now to inquire whether the other division holds good, whether muscle and the other proteid tissues arc fed exclusively on the proteid matcrial of food, and whether muscular energy comes exclusively from the metabolism of the proteid constituents of muscle. We have already seen ( $\$ 60)$ that when the muscle itself is examined, we find no proof of nitrogenous waste, but, on the other land, clear evidence of the production of nonnitrogenous bodies, such as carbonic acid. And when we ask the question, Does muscular exercise proportionately inerease 
the urea given off by the body as a whole? for this, according to the theory in question it certainly ought to do, the evidence we can obtain, though somewhat varying, gives on the whole a decidedly negative answer.

In the majority of observations no marked change at all in the amount was met with; indeed in some cases there was a distinct decrease, followed by an increase on the following days. Some observers however found a very marked increase, and this was especially the case when the subject under observation took a large amount of food and performed very severe labour. On the whole the various results obtained by different observers justify the conclusion that exercise by itself, even when severe, does not necessarily increase the amount of urea excreted, but that conditions may obtain in which such an increase undeniably occurs. We may draw the further conclusion that experiments of this kind do not supply the right method for determining the point at issue. It must be remembered that it is not the muscles alone which feel the influence of the labour; the circulation and indeed the whole body are affected by it. If we suppose a large part or even only some part of the urea to come from other than muscular metabolism, from changes in the hejatic cells for instance, we should expect that these changes, and with them the amount of urea discharged, would be influenced by labour, especially by severe labour.

In no case las a direct relation between the amount of labour and amount of urea been observed. More than this, the following experience lands us in an absurdity if we suppose the whole energy of muscular work to arise from proteid metabolism. Two observer's performed a certain amount of work (an ascent of a mountain) on a non-nitrogenous diet, and estimated the amount of urea passed during the period. Assuming the urea to represent the oxidation of so much proteid matter, which oxidation represented in turn so much energy set free, they found that whereas the actual work done amounted to $129 \cdot 026^{\circ}$ and $148 \cdot 656$ kilogram.-kilometers, for each observer respectively, the total energy available from proteid metabolism during the period was in the ease of the first $68 \cdot 69$, and of the second 68.376 kilogram.-kilometers. That is to say, the energy set free by the proteid metabolism of the muscles engaged in the work was far less than the amount necessary to accomplish the work actually done, to say nothing of its having to provide as well for the movements of respiration and circulation. 'Their muscular energy therefore must have had other sources than proteid metabolism.

That on the contrary muscular exercise at once and largely increases the production of carbonic acid is beyond all doubt. One hour's liard labour will increase fivefold the quantity of carbonic acid given off within the hour. And in an experiment 
directed to this point it was found that a man in 24 hours constmed 954 grms. oxygen and produced 1284 grms. carbonic acid when doing work, as against 708 grms. oxygen consumed and 911 grms. carbonic aeid produced when remaining at rest, the quantity of urea secreted being in the first case 37 grms., in the second $37 \cdot 2$ grms.

It is evident that the conclusions arrived at by the statistical method entirely corroborate those gained by an examination of muscle itself, viz. that during muscular contraction the explosive decomposition which takes place bears chiefly, if not exclusively, on the non-nitrogenous constituents of the muscle, and that it is the non-nitrogenous products which alone escape from the muscle and from the body, any nitrogenous products which result being retained within the muscle, or at least within the body. We must therefore reject the second as well as the first division of the views under discussion; not only is the muscle not fed exclusively on proteid material, but also its energy does not arise from an exclusively proteid metabolism.

\section{Animal Heat.}

$\S 424$. The Sources and Distribution of Heat. We have already seen that the conception of the non-nitrogenous portions of food being solely calorifacient or respiratory proves to be unfounded when we attempt to trace the history of the food on its way through the body. The same vicw is still more strikingly shewn to be inalequate when we study the mamner in which the heat of the body is produced. We may indeed at once affirm that the heat of the body is generated by the chemical changes, which we may speak of generally as those of oxiclation, undergone not by any particular substances, but by the tissues at large. Wherever metabolism is going on, or to be more exact wherever lestructive metabolism, katabolism, is going on, heat is being set free. In growth and in repair, in the deposition of new material, in the transformation of lifcless pabulum into living tissue, in the constructive metabolism, the anabolism of the body, and in the smaller synthetic processes of which we spoke in dealing with urea ( $\$ 387$ ), heat is undoubtedly to a certain extent being absorbed and rendered latent: the energy of the construction may be, in part at least, supplied by the heat present. But all this, and more than this, viz. the heat present in a potential form in the substances themselves so built up into the tissue, is lost to the tissue during its destructive metabolism; so that the whole metabolism, the whole cycle of changes from the lifeless pabulum through the living tissue back to the lifeless products of vital action, is eminently a source of heat. 
Of all the tissues of the body the muscles, not only from their bulk, forming as they do so large a portion of the whole frame, but also from the characters of their metabolism, must be regarded as the chief somrees of heat.

In treating ( $\$ 62)$ of the thermal changes in muscle we have seen that in the total energy expended in a muscular contraction, the ratio of that which appears as heat to that which appears as external work is variable. If we assume that the energy appearing as work done in a muscular contraction is on the average about one-tenth of the total energy expended, the rest going out as heat, then, upon the calculation that the total cxternal work of the body is about one-fifth of the total energy set free in the body, it is clear that the heat given out by the muscles, even if we consider only the heat given out when they are contracting, must form a very large part of the total heat given out by the boly. And even if, as recent researehes indicate, the museular machine works more economically than we have hitherto supposed, the amount of heat given out by the skeletal muscles must still remain very large. Moreover to the skeletal muscle we must add the heart which, never resting, does in the twenty-four hours as we have seen, $\$ 120$, no inconsiderable amount of work, and must give rise to no ineonsiderable amount of heat. But the skeletal museles, though frequently, are not continually contrating; they have periods, at times long periods, of rest; and during these periods of rest, metabolism, of a subdued kind it is true, but still a metabolism involving an expenditure of energy, is going on. 'This quiescent metabolisn must also give rise to a certain amount of heat; and if we add this amount, which in the present state of our knowledge we cannot exactly gauge, to that given out during the movements of the body, it is very elear, even in the absence of exact data, that the metabolism of the museles must supply a very large proportion of the total heat of the body. They are par excellence the thermogenic tissues.

Next to the muscles in importance come the various secreting glands. In these the secreting elements, at the periods of secretion at all events, are in a state of metabolic activity, which activity as elsewhere will naturally give rise to heat. In the case of the salivary gland a rise of temperature has been actually observed; but objections have been brought against the observation. Of all these various glands, the liver deserves special attention on account of its size and large supply of blood, and because it appears to be continually at work. If there be any truth in the views urged in the preceding ehapter touching the large and varied metabolic work of the liver, we must conclude that a very large amount of heat is set free in this organ; and that holds good even if we make a large allowance for the various synthetic anabolic processes which may take place and 
by which heat would be absorbed and made latent. We find indeed that the blood in the hepatic vein is the warmest in the body. Thus in the $\log$ a temperature of $40.73^{\circ} \mathrm{C}$. has been observerl in the hepatic vein, while that of the vena cava inferior was $38 \cdot 35^{\circ}$ to $39 \cdot 58$, and that of the right heart $37 \cdot 7$. The fact that the blood of the hepatic vein is warmer than that of either the portal vein or the aorta, shews that the increased temperature is not due simply to the liver being far removed from the surface of the body.

The brain too may be regarded as a source of heat, since its temperature is higher than that of the arterial blood with which it is supplied; though from the smaller quantity of blood passing through its vessels as well as from the changes in it being less massive, it cannot in this respect compare with either the liver or the muscles as a source of heat to the body.

The blood itself camnot be regarded as a source of any considerable amount of heat, since, as we have so frequently urged, the oxidations or other metabolic changes taking place in it are comparatively slight. The heat evolved by the indifferent tissues such as bone, cartilage and connective tissue, may be passed over as insignificant; and we cannot even regard the adipose tissue as a seat of the production of heat, since the fat of the fat-cells is in all probability not oxidized in situ but simply carried away from its place of storage to the tissue which stands in need of it, and it is in the tissue that it undergoes the metabolism by which its latent energy is set free. Some amount of heat is also produced by the changes which the food undergoes in the alimentary canal before it really enters the body.

Hence, taking a survey of the whole body, we may conclude that since metabolism is going on to a greater or less extent everywhere, heat is everywhere being generated; but that, looked at from a quantitative point of view, the muscles and the glandular organs must be regarded as the main sources of the heat of the body, the muscles being the more important of the two.

$\$ 425$. But heat. while being thus continually produced, is as continually being lost, by the skin, the lungs, the urine and the fieces. 'The blood passing from one part of the body to the other, and carrying warmth from the tissues where heat is bcing rapidly generated, to the tissues or organs where heat is being lost by radiation, conduction or cvaporation, tends to equalize the temperature of the various parts, and thus maintains a "constant bodily temperature."

When the production of heat is not great as compared with the loss there is no great accumulation of heat within the body, the temperature of which consequently is but slightly raised above that of surrounding oljects. 'Thus the temperature of 
the frog, for instance, is rarely more than $.04^{\circ}$ to $.05^{\circ}$ above that of the atmospliere, though in the breeding season the difference may amount to $1^{\circ}$. Such animals, and they comprise all classes except birds and mammals, are spoken of as coldblooded; they have been also called poikilothermic, that is, of varied temperature. Exceptions among them are not uncommon. Some fish, such as the tunny, are warmer than the water in which they live, and in a species of Python ( $P$. bivittatus) a difference of as much as $12^{\circ}$ has been observed. In a beelive the temperature may rise at times to as much as $40^{\circ}$. In the so-called warm-blooded animals, birds and mammals, the loss and production of heat are so balanced that the temperature of the body remains constant at, in round numbers, $35^{\circ}$ or $40^{\circ}$, whatever bc the temperature of the air; hence these have been called homoiothermic, of constant temperature. 'The temperature of man is about $37^{\circ}$; in some birds it is as high as $44^{\circ}$ (Hirundo) and in the wolf it is said to be as low as $35 \cdot 24^{\circ}$.

This temperature is with slight variations maintained throughout life. After death the generation of heat rapidly diminishes, and the body speedily becomes cold; but for some short time immediately following upon systemic death, a rise of temperature may be observed, due to tlie fact that, while the metabolism of the tissues is still going on, the loss of heat is somewhat checked by the cessation of the circulation. The onset of pronounced rigor mortis causes a marked accession of heat, and when occurring after certain discases may give rise to a very considerable elevation of temperature.

This mean bodily tempcrature of warm-blooded animals is, during health, maintained, with small variations of which we shall presently speak, within a very narrow margin, a rise or indeed a fall of much more than a degree above or below thc limit given above being indicative of some failure in the organism, or of some unusual influence being at work. It is eviclent, therefore, that the mechanisms which coordinate the loss with the production of heat must be exceedingly scnsitive. It is obvious, moreover, that these mechanisms may act when the bodily temperature is tending to rise, by eitlier checking the prodnction or by augmenting the loss of heat; conversely when the bodily temperature is tending to fall, they may act by either increasing the production or by diminishing the loss of heat. As the regulation of tempcrature by variations in the loss of heat is better known than regulation by variations in production, it will be best to consider this first.

\$226. Regulation by variations in loss. Heat is lost to the body by the warming of the freces and of the urine, by the warming of the expired air, by the evaporation of the water of respiration, by conduction and radiation from the skin, and 
by the evaporation of the water of perspiration. It has been calculated that the relative amounts of the loss by these several channels are as follows: In warming the fieces and urine about 3, or according to others 6 per cent. By respiration about 20, or according to other's about 9 only per cent., leaving 77 , or alternatively 85, per cent. for conduction and radiation and evaporation by the skin.

The two chief means of loss then, which are at all susceptible of any great amount of variation, and which ean be used to regulate the temperature of the body, are the skin and the lungs.

'The more air passes in and out of the lungs in a given time, the greater will be the loss in warming the expired air, and in evaporating the water of respiration. In such animals as the dog, which do not perspire frecly by the skin, respiration is a most important means of regulating the temperature; and in the $\log$ a very close connection may be observed between the production of heat and respiratory activity. The changes which give rise to this loss take place before the inspired air reaches the pulmonary alveoli; both the warming and the evaporation are effected in the nasal and pharyngeal, and to some extent in the bronchial passages. Some observers have maintained that the left side of the heart is warmer than the right, and hence have argued that chemical changes leading to a considerable development of heat take place in the pulmonary capillaries. It would appear however that the right rentricle, owing to its lying nearer to the liver, the high tempcrature of which has already been mentioned, is in reality rather hotter than the left. And indeed we have no satisfactory evidence of any large amount of heat being produced by any pulmonary metabolism.

The great regulator however is undoubtedly the skin; and this has a more or less double action. In the first place it regulates the loss of heat by means of the vaso-motor mechanism. The more blood passes throngh the skin the greater will be the loss of heat by conduction, radiation, and e vaporation. Hence, any action of the vaso-motor mechanism which, by causing dilation of the cutaneous vascular areas, leads to a larger flow of blood through the skin, will tend to cool the body; and conversely, any vaso-motor action which, by constricting the cutaneous vascular areas, or by dilating the splanchnic vascular areas, causes a smaller flow through the skin, and a larger flow of blood through the abdominal viscera, will tend to heat the body. In the second place, besides this, the special nerves of perspiration will act directly as regulators of temperature, increasing the loss of heat when they promote, and lessening the loss when they cease to promote, the secretion of the skin. The working of this heat-regulating mechanism is well seen in the case of exercise. Since every muscular contraction gives 
rise to heat, exercise must increase for the time being the production of hicat; yet the bodily temperature rarely rises so much as a degree centigrade, if at all. By exercise the respiration is quickened, and the loss of heat by the lungs increased. The circulation of blood is also quickened, and the cutaneous vascular areas becoming dilated, a larger amount of blood passes through the skin. Added to this, the skin perspires freely. 'Thus a large amount of heat is lost to the body, sufficient to neutralize the addition caused by the muscular contraction, the increase which the more rapid flow of blood through the abdominal organs might tend to bring about being more than sufficiently comteracted by their smaller supply for the time. The sense of warmth which is felt during exercise in consequence of the flushing of the skin, is in itself a token that a regulative cooling is being carried on. In a similar way the application of external cold or heat defeats its own ends, either partially or completely. Under the influence of external cold the cutaneous vessels are constricted, and the splanchnic vascular areas dilated, so that the blood is withdrawn from the colder and cooling regions to the hotter and heat-producing organs. 'This vascular change may be used to explain the fact that stripping naked in a cold atmosphere often gives rise to a distinct increase in the mean temperature of the blood, as indicated by a thermometer placed in the mouth, though possibly the effect may be partly due to an actual increase of the production of heat. Under the influcnce of external warmth, on the other hand, the cutancous vessels are dilated, a rapid discharge of heat takes place; and if the circumstances be such that the body can perspire freely, and the perspiration be readily evaporated, the temperature of the body may remain very near to the normal, even in an excessively hot atmosphere. Thus, more than a century ago, two observers were able to remain with impunity in a chamber heated even to $127^{\circ}$ ( $260^{\circ} \mathrm{Fahr}$.), and with ease in one so hot, that it became painful for them to touch the metal buttons of their clothing. It is unnecessary to give any more examples of this regulation of temperature by variations in the loss of heat; they all readily explain themselves.

$\$ 427$. The production of heat, its variations and regulation. As we have already said the exact determination of the amount of heat produced in the living body is attended with great difficulties; still certain conclusions have been arrived at based partly on direct calorimetric observations, the more recent ones with improved calorimeters being especially valuable, and partly on what seem to be trustworthy deductions from observed chemical changes.

The rate of production of heat in a living body is determined by a variety of circumstances. In the first place what 
may be called the general rate of metabolism. and so of the production of heat, varies in different kinds of animals. Of two animals of the same bulk and weight placed under the same circumstances one 'lives faster' than the other, metabolizes its living substance more rapidly, and so produces heat more rapidly. Thus direct calorimetric observations, as far as they at present go, shew that a man on the arerage produces more heat, per kilo, per hour, than does a dog, and a dog more than a rabbit. Probably every species has what may be called its specitic coefficient, and every individual his personal coefficient of heat-production, the coefficient being the expression of the inborn qualities proper to the living substance of the species and of the indivichal.

A larger living body will naturally produce more heat than a smaller living body of the same nature, since the larger body possesses so to spealk a greater number of heat-producing units. But this is neutralized by an opposing tendency. The smaller body, having relatively to its bulk a larger amount of surface, loses heat at a more rapid rate than does the larger body; and therefore. to maintain the balance between loss and production, so as to secure the same constant bodily temperature (and as we have just seen the bodily temperature of warm-blooded animals is remarkably uniform), it must produce heat, per unit of its body at a more rapid rate. As a rule the greater loss of heat owiing to the relatively greater surface is so marked that of two animals having the same constant bodily temperature, of two species of mammals, or of two individuals of the same race. we should expect the smaller one to produce a relatively larger amount of heat. And direct calorimetric observations shew that this is so. The struggle for existence has raised what we have just called the specific or personal coefficient of the smaller animal.

From what we have seen concerning the immediate effects of a meal, we should be inclined to expect that food would temporarily increase the production of heat; and not only is this view confirmed by conmon experience and by our own sensations, but clirect calorimetric observations afford experimental proof of its truth. In the dog it has been found that the rate of production increases after a meal, reaching its maximum from the 6 th to the 9th hour, and then declining to a level which may be regarded as that secured by the general metabolism of the body, and which appears to be maintained with remarkable constancy until after long starvation the economy begins to break down.

Labour, muscular work, has a powerful influence in increasing the production of heat. As we have seen, of the total heat produced in the body, a certain portion must always be attributed to muscular contractions which even in the most quiet body 
are always going on; in an ordinary active body a considerable quantity of heat must be thus generated. Hence the more active the body the greater the production of heat. As we stated before, in a contraction the proportion of the energs set free to do work to that set free as heat appears to vary under different circumstances: and the increase of heat due to labour probably varies in a corresponding way. The details of this relation have yet to be worked out. but we may at least conclude that, wheu a man pushes his daily labour beyond the 150,000 k.m.. the additional energy thus learing his body as work done is not taken ont of the $8.00 .000 \mathrm{k} . \mathrm{m}$. giren in $\$ 2.2$ as the average daily output of heat. but the total setting free of energy and the total production of heat is at the same time increased.

$\$$ 428. The production of heat thus determined by these sereral influences, some of which are themselves regulated by the nervous system. is further regulated in a remarkable manner. For it is not solely br rariations in the loss of heat that the constant temperature of the warm-blooded animal is maintained. Variations in the amount of heat actually generated in the body constitute an important factor not only in the maintenance of the normal temperature. but also in the production of the abnormally high or low temperatures of various diseases. Many considerations have long led physiologists to suspect the existence of a nervous mechanism by which afferent impulses arising in the skin or elsewhere might through the central nervous system originate efferent impulses whose effect would be to increase or to diminish the netabolism of the muscles or other organs, and thus to increase or diminish the amount of heat generated for the time being in the body. And we have experimental evidence that such metabolic or thermogenic nervous mechanism. comparable in many respects to the raso-motor mechanism or to the various secreting nerrous mechanisms. does really exist.

The warm-blooded animal is distinguished from the coldblooded animal by the fact that when it is exposed to cold or heat. it does not like the latter become colder or hotter, as the case may be. but, within certain limits. maintains its normal temperature. If the maintenance of the temperature of the warmblooded animal during exposure to cold is assisted by an increased production of heat and is not due simply to a diminished loss. we ought to find evidence of an increased metabolism during that exposure. We ought to find under these circumstances an increased production of carbonic acid, and an increased consumption of oxygen, since it is to these products, rather than to the nitrogenous factors. on the peculiarities of which as uncertain signs of metabolism we have already insisted. we must look for indications of the rise or fall of metabolic activity. 
Taking then the consumption of oxygen, and the production of carbonic acid, as a measure of metabolic activity and so of heat-production, it has been shewn that a marked contrast in this respect exists between cold-blooded and warm-blooded animals exposed to changes of temperature. In the cold-blooded animal, cold diminishes and heat increases the metabolic activity of the body; as the temperature to which the animal is subjected rises or falls, so the consumption of oxygen and production of carbonic acid is increased or lessened. 'The body of a cold-blooded animal behaves in this respect like a mixture of dead substances in a chemist's retort: heat promotes and cold retaris chemical action in both cases. Very different is the bchaviour of a warm-blooded animal. In this case, within a lower and a higher limit, cold increases and heat diminishes the bodily metabolism, as shewn by the increased or diminished consumption of oxygen and production of carbonic acid as the temperature falls or rises. In these animals there is obviously a mechanism of some kind, counteracting and indeed overcoming those more direct effects which alone obtain in cold-blooded animals. And that this mechanism is of a nervous nature, is indicated by the following facts.

When a warm-blooded animal is poisoned by urari, the temperature falls and the metabolism, measured by the consumption of oxygen and the production of carbonic acid, sinks also; and that the latter is the cause, not the effect, of the former is shcwn by the fact that the metabolism continues to fall though loss of heat be prevented by surrounding the animal with wrappings of cotton wool. In such a urarized animal, exposure to higher temperatures augments and exposure to lower temperatures diminishes metabolism; the urarized warm-blooded animal in fact behaves like a cold-blooded animal. Similar but perhaps not such striking or so constant results are gained by division of the spinal bulb. After this operation the temperature of the body sinks, and the fall, though partly due to incrcased loss of heat by the skin, caused by the dilated condition of the cutaneous ressels, is also accompanied by diminished metabolism and is therefore in part due to diminished production of heat. And when an animal is in this condition, exposure to higher temperatures increases and exposure to lower temperatures diminishes the bodily metabolism. We can best cxplain these results by supposing that, under normal conditions, the muscles, which as we have seen contribute so largely to the total heat of the body, are placed, by means of their motor nerves and the central nervous system, in some special connection with the skin, so that a lowering of the temperature of the skin leads to an increase, while a heightening of the temperature of the skin leads to a decrease, of the muscular metabolism. Further, the centre of this thermotaxic reflex 
mechanism appears to be placed somewhere in the nervous system above the spinal cord. When urari is given, the reflex chain is broken at its muscular end; when the spinal cord is divided the break is nearer the centre.

We may add that the muscular metabolism which thus helps to regulate temperature need not involve visible muscular contractions. At the same time the heat given out by the museles will be temporarily increased at every contraction which may occur. Thus, the shivering which follows exposure to cold distinctly helps to warm the body; indeed some observers have been led to think that, in man, this visible effect of cold plays a more important part in his heat regulation than the invisible actions which we have just described. We may also add that the regulative nervous mechanism may apparently be overborne by an exposure to too great heat or cold. When for instance the cold to which the animal is exposed becomes excessive, the reaction of the thermotaxic nervous system is powerless against the direct action on the tissues of the depressing influences, and the metabolism, together with the temperature, sinks.

$\S 429$. In a number of experiments it has been shewn that injuries to, such as those caused by puncture or galvanie cautery, or electrical stimulation of limited portions of the more central portions of the brain may give rise to a great increase of the temperature of the body without producing any other marked symptom. The increase is shewn, by the increase of metabolism, increased production of carbonic acid and increased consumption of oxygen, as well as by direct calorimetric observations, to be due to an increased production of heat. 'This naturally suggests that the portions of the brain in question contain the hypothetical heat centre just mentioned, the lesion on stimulation exciting the centre to activity by direct action on it, instead of in the usual reflex manner. The matter has not however as yet been clearly worked out; and indeed observers are not agreed as to the exact parts of the brain injury to which, or stimulation of which, produces the effect.

$\S 430$. By regulative mechanisms of the kind just discussed the temperature of the warm-blooded animal is maintained within very narrow limits. In ordinary health the temperature of man varies between $36^{\circ}$ and $38^{\circ}$, the narrower limits being $36.25^{\circ}$ and $37.5^{\circ}$, when the thermometer is placed in the axilla. In the mouth the reading of the thermometer is somewhat $\left(25^{\circ}\right.$ to $\left.1.5^{\circ}\right)$ higher; in the rectum it is still higher (about $\cdot 9^{\circ}$ ) than in the mouth. The temperature of infants and children is slightly higher and much more susceptible of variation than that of adults, and after 40 years of age the average maximum temperature (of health) is somewhat lower than before that epoch. A diumal variation, independent of food or other circumstances, has been observed, the 
maximum ranging from $9 \mathrm{~A}$... to $6 \mathrm{r}$. $\mathrm{x}$. and the minimum from 11 P.M. to 3 A.M. Meals cause sometimes a slight elevation, sometimes a slight depression, the direction of the influence depending on the nature of the food: alcohol seems always to produce a fall. Exereise and variations of external temperature, within ordinary limits, cause very slight change, on account of the compensating influences which have been discussed above. 'The rise from even active exercise does not amount to $1^{\circ}$; when labour is carried to exhaustion a depression of temperature may be observed. In travelling from very cold to very hot regions a variation of less than a degree occurs, and the temperatme of inhabitants of the tropics is practically the same as of those dwelling in arctic regions.

$\$ 431$. Many of the maladies of the boly are characterized by an increase of the bodily temperaturc known as "fever" or" "pyrexia," the thermometer very frequently rising to $39^{\circ}$ or $40^{\circ}$, not unfrequently to $41^{\circ}$, and at times reaching $43^{\circ}$ or even $44^{\circ}$; but these higher temperatures cannot long bc borne without the organism failing. And as we have said, any increase in man of the bodily temperature beyond $38^{\circ}$, or even beyond $37 \cdot 5^{\circ}$, indicates some distmbance. In most cases the rise of temperature has a definite objective cause, some local inflammation or suppuration, or, as in specific fevers, the presence in the economy of some "materies morbi," of the nature of an organized germ or of some other nature. We cannot here discuss the connection between the local inflammation or the specific poison and the high temperature, but we have increasing evidence that the high temperature of fever is due, not merely to a diminution of the loss of heat, though this may be a factor, but also, and indeed chiefly, to an increased production of heat. In fever, the production of carbonic acid, and the consumption of oxygen, that is to say, the metabolic changes of the tissues, are increased. The urea also is increased, and that in such a way as to confirm the view already expressed that much of the heat comes from such a metabolism of the skcletal muscles as, unlike an ordinary contraction, directly involves the nitrogenous elements. 'Tle inordinate metabolism of the body at large thus characteristic of fever is shewn by the wasting which it entails. Calorimetric observations also shew in a direet manner that the production of heat is increased. Of course mere incraased production alone would be insufficient to raise the temperature of the body, for it unight be met, up to a very high limit, by a compensating increase of loss of heat; but in fever this compensation is wanting, and it is perhaps this absence of due regulation which is most characteristic of the febrile condition.

In some maladies the bodily tempcrature falls distinctly below the normal average, reaching for instance $35^{\circ}$ or even 
lower. In such cases there can be little doubt that the condition is due to diminished metabolism and diminished heat production.

One of the most marked phenomena of starvation is the fall of temperature, which becomes very rapid during the last days of life. The lowered metabolism diminishes the production of heat, and the lowered temperature in turn still further diminishes the metabolism. Indeed the low temperature is a powerful factor in bringing about death, for life may be much prolonged by wrapping a starving animal in some bad conductor so as to economize the bodily heat.

$\$$ 432. Etfects of Great Heat. As we said above, the regulative heat mechanism is unable to withstand the strain of too great an external heat or too prolonged an exposure to a great but less degree of heat. The temperature of the body then rises above the normal; and it has been observed that the temperature is more easily raised by warmth than depressed by cold, at least when neither are very intense. When either in this way by external warmeth or through pyrexia the temperature of the body is raised some $6^{\circ}$ or $7^{\circ}$ above the normal. to $45^{\circ}$ or thereabouts, death speedily ensues. The chain of events thus leading to death has not been as yet clearly made out, and most likely the events do not take exactly the same course in all cases; but we shall probably not go far wrong in attributing death to the fact that the high temperature hurries on the metąbolism of the several tissues, of some more than others, at such a spendthrift rate that their capital is soon exhausted. We have seen, \$301, that too wam blood produces dyspnora, and soon exhausts the metabolie capital of the respiratory centre. 'Too warm blood similarly hurries on the beats of the heart: an explosion of the contractile substance is each time prematurely brought on before a sufficient quantity of explosive substance is aceumulated, each stroke becomes more and more feeble as the rate is quickened, the heats become irregular, and finally cease. Either of these two events alone and certainly both together are enough to bring the working of the bodily mechanism to an end; but other tissues beside the heart and the respiratory centre are suffering in the same way, notably the rest of the central nervous system. 'This too is being hurried on unduly in its inner changes, so that not only consciousness is lost and other objective manifestations of nervous action go wrong or fail, but that regulative grasp of the central nervous system on the tissues of the body at large is loosened, and tumult takes the place of order. Whether this or that sign of disorder comes to the front, whether for instance convulsions take place, would appear to depend upon the exact turn taken by the abnormal events. In heat-stroke, more commonly known as sun-stroke, the essential 
condition of which seems to be a rapid rise of the temperature of the borly owing to a sudden failure of the thermotaxic mechanism, the symptoms vary. Sometimes the heart suddenly gives way, at other times the respiratory centre seems to be more directly affected; sometimes convulsions make their appearance, but more commonly death takes place through a comatose condition of the brain, an initial phase of excitement of the centrial nervous system being not unfrequently witnessed.

Mammalian muscle, it will be remembered, $\$ 79$, becomes rigid at about $50^{\circ}$; but death probably always occurs before that higher temperature is reached by the blood, so that a sudden rigor mortis from heat (rigor caloris) cannot be regarded as a factor in death from exposure to too great heat.

$\$$ 433. Effects of Great Cold. The effects of a too great lowering of the temperature of body, which is generally the result of too great external cold and rarely if ever arises from internal causes lowering the metabolism and thus the production of heat, are in their origin the reverse of those of a too high temperature. The metabolism of the tissues is lowered; and not only are the katabolic changes which lead to the setting free of energy thus affected, but the anabolic changes also share in the depression. The "living substance" falls to pieces less readily, but is also made up less readily; and could this slackening of metabolism be carried on in the several tissues at a rate proportionate to the rate at which each tissue lives, life might thus be brought to a peaceful end by gradual arrest of the life of each part of the whole body. And indeed in some cases, where the lowering of the temperature takes place gradually, something like this does occur even in warm-blooded animals. The diminished metabolism tells first and chiefly on the central nervous system, especially on the brain and more particularly on those parts of that organ which are concerned in consciousness. The intrinsic lowering of the cerebral metabolism is further assisted by a slowing of the heart-beat and of the breath, drowsiness is succeeded by a condition very like to, if not identical with that known as sleep, which we shall study later on, but by a sleep which insensibly passes into the sleep of death. In some cases, however, especially those in which the lowering of the temperature is sudden and rapid, disorders of the nervous system intervene, and convulsions like those of asphyxia are produced. 


\section{SEC. 3. ON NUTRITION IN GENERAL.}

$\$ 434$. It may now be profitable to take a brief survey of the various conclusions at which we have arrived concerning the problems of nutrition.

We have seen that the several tissues, using lymph as a medium, live upon the blood, taking up from the blood the materials for, and returning to the blood the products of, their metabolism. The blood itself we have also seen to be replenished with food from the alimentary canal and with oxygen from the lungs, and to be freed from waste products by means of the excretory organs. In this double action the raw material of the food on the one hand undergoes, between its being placed in the mouth and its taking part in the metabolism of the tissue which ultimately uses it, many intermediate changes carried on in various parts of the body, and the waste products similarly undergo intermediate changes between leaving the tissue and appearing in the urine, the sweat or the expired air.

We have further seen reason to think that the metabolic events of the body take place in the main in the tissues, not in the blood stream on its way between the heart and the tissues. Changes, proper to the blood itself, take place in the blood; the corpuseles, red and white, with the plasma undergo like the rest of the body, their proper metabolic cycles, and in this sense blood may be called a tissue if there is any advantage in using the phrase; but, apart from these intrinsic blood changes, as far as we can see at present, the metabolism undergone during their transit along the blood channels, by the substances which are merely carried in the blood from place to place, is an insignificant part of the total metabolism of the body.

By metabolism of a tissue we understand the total chemical changes taking place in the tissue; and we divide these changes into those which either directly or indirectly are concerned in the building up (anabolic) and those which are in like manner concerned in the breaking down (katabolic) of the living substance. We shall explain presently what we mean by the 
words 'dircetly' and 'indirectly' used in this conncetion. And we may here repeat the caution ( $\$ 30$ ) that though for convenience sake we use the phrase 'living substance,' what is really meant by the words is not a thing or body of a particular chemical composition but matter undergoing a scries of chamges.

435. We know more about the chemical changes of muscle than perhaps of any other tissue, though this even at the most is not much, and we may perhaps take the nutrition of muscle as a type of nutrition in general. The muscle in a normal state of things lives ultimately on the proteids, fats, carbohydrates, salts and water of the food, and on the oxygen of the inspired air, but lives directly on the blood which brings these things to it.

Concerning the relation of proteids to muscle, we know little more than was stated in $\$ 140$ in speaking of the heart. We can do no more than infer, and that doubtfully, that serumalbumin is the form in which the muscle takes up proteids. Concerning carbohydrates we have apparently a definite fact. Dextrose is, as we have repeatedly said, always present in the blood in small quantity, and appear's to be the ouly carbohydrate constituent of blood-plasma. Experiments carried out on a large animal, such as the horse or cow, have shewn that the venous blood coming from a muscle contains less dextrose than the arterial blood going to the muscle, and that the difference is much increased by throwing the muscle into contraction. From this we may provisionally conclude that dextrose is an essential part of the food of the muscle.

Concerning fats we have little or no knowledge, but we may perhaps infer that the body has power to transform fits into carbohydrates as it has the power to transform carbohydrates into fats, and that the carbon whether of the fat or of the carbohydrates of food is presented to the muscle in the form of carbohydrate, namely of dextrose. But we have no distinct proof of this.

The various salts brought to the muscle by the plasma, though they supply no energy, are as essential to the life of muscle as the energy-holding proteid or carbon compound; and experiments made with regard to some of them, calcic salts for instance, shew that their presence or absence materially affects the maintentnce or restoration of irritability. Some of these probably play the part only of securing by their presence favourable conditions for the die metabolic processes, somewhat after the vay in which the presence of a calcic salt determines the clotting of blood and the eurdling of milk; but some we probably ought to regard as actually entering into the processes themselves. Of these matters however we know very little. 
$\$ 436$. The products of muscular metabolism pass into the lymph bathing the fibre and so, either by a direct path into the capillaries or by a more circuitous course through the general lymphatic system, into the blood. 'The fate of the carbonic acid we have fully treated of in dealing with respiration; the little we know concerning the nitrogenous product or products has been stated in dealing with urea; the third recognized product is lactic acid, sarcolactic acid. Did any considerable amount of oxidation take place in the blood stream while the blood is flowing along the larger channels, subject only to the influence of the vascular walls, we might fairly expect that the lactic acid discharged from the muscles would be subjected to oxidizing influences while still within the blood stream of the larger channels. We have however no satisfactory evidence of any lactic acid being oxidized in this way. On the contrary, there is a certain amount of experimental and other evidence that lactic acid present in the blood is somehow or other disposed of by the liver; and that if the liver fails to do its duty lactic acid may appear in the urine.

$\$ 437$. We may here ask the question, What is the relation of these various metabolic processes to the structural elements of the tissue? When we say that the muscular fibre is continually undergoing metabolism do we mean that every jot and tittle of the fibre is undergoing change and that at the same rate? We can hardly suppose this. It seems unlikely, for instance, that the metabolism of the fibrillar substance is identical with that of the interfibrillar substance, whaterer be the view we take as to the properties or meaning of the two substances. W'e should thus be led to regard the metabolic events occurring in muscle as falling into two classes at least; those taking place in the living more permanent framework, and those bearing on the formation and destruction of the contractile substance lodged in that living framework. These of course are at present matters of speculation; but on the whole the evidence we can gather tends and perhaps increasingly tends to shew that in muscle there does exist such a framework of what we may call more distinctly living substance which rules the histological features of the fibre, and whose metabolism though high in quality does not give rise to massive discharges of energy, and that the interstices so to speak of this framework are occupied by various kinds of material related in different degrees to the framework and therefore deserving to be spoken of as more or less living, the chief part of the energy set free by muscle coming directly from the metabolism of some or other of this material. And the same view may be extcnded to other tissues. Both the framework and the intercalated material undergo metabolism, and have, in different degrees, their anabolic and katabolic changes; both are concerned in the life of the living 
substance, but one more directly than the other, and this is what was meant by the terms 'directly ' and 'indirectly,' used in $\$ 434$.

$\$ 438$. Whether the chief product of the metabolism of any tissuc be a proteid sulsstance, or a fat, or a carbohydrate, proteid substance is the pivot so to speak of the metabolism, and nitrogenous bodies alway appear as the products of metabolism. This is strikingly seen in the nutrition of plants where, as far as mere bulk or weight is concerned, the active metabolizing tissue is insignificant compared with the mass of products of mctabolism heaped up in the form of starch or cellulose or some allied carbohydrate. The protoplasm of a vegetable cell soon becomes a mere film bearing a heavy burden of heaped up metabolic products and eventually disappears; and of that film only a part corresponds to what we spoke of above as the living framework of the muscle. Yct that scanty protcid-built framework is more or less directly concerned in the production of the carbolydrate material and the various conversions which that material undergoes. Proteid, nitrogen, changes are entangled with the carbon changes; and since the products of metabolism in the plant are not as in the animal cast out of the organism, but for the most part heaped up within it, we find the plant storing up in parts, where if they serve no useful purpose they at least do not harm, nitrogenous products of metabolism, such as those known as vegetable alkaloids, many of which by their amide nature betray their kinship to the animal nitrogenous product urea.

$\$ 439$. In the preceding chapters of this work we have had abundant evidence that the metabolism of the tissues is subject to the government of the central nervous system; the contraction of a muscle, the secretory activity of a gland, the increased or diminished production of heat all afford instances of nervous impulses affecting metabolism. In most of these instances the changes induced fall within the downward, katabolic, phase and liave a downward character; thus when a muscle contracts, the result is a conversion of more complex bodies into simpler bodies; and the same as far as we can see is true of most other cases. But it is open for us to suppose that nervous impulses might affect the upward, anabolic, phase and have a constructive influence.

At all events we are not justified in assuming that a nervous impulse can only produce disruptive katabolic changes such as are scen in muscular contraction or in secretion. The effects of stimulating a nerve going to a muscle or a salivary gland are striking and obvious and the behaviour of a muscle or a gland as far as contraction and secretion are concerned is, within certain limits, under experimental control. But there arc certain phenomena, secn chiefly in the course of discase, and lying, to a very small extent only, witlin the control of experiment, 
which seem to shew that the central nerrous system governs the metabolic changes, the nutrition, not only of muscle and gland, but of various other tissues in a deeper and more general way than that of simply promoting (or hindering) contraction or secretion. Thus as we have seen $(\$ 78)$ when the connection between a muscle and the central nervous system is severed, the muscle eventually wastes and loses its vitality; when all the nerves going to the sub-maxillary gland are severed, the gland instead of being as in the normal condition intermittently actire and quiescent, pours forth a continuous "paralytic" secretion and eventually degenerates and wastes. When in a rabbit the fifth nerve is divided in the skull the loss of sensation in those parts of the face of which it is the sensory nerve is followed by nutritive changes. V'ery soon, within twenty-four hours, the cornea becomes cloudy ; and this is the precursor of an inflammation which may involve the whole eye and end in its total disorganization. At the same time the nasal chambers of the side operated on are inflamed, and very frequently ulcers make their appearance on the lips and gums. And similar results have been seen in other animals including man. If the operation be conducted in a young animal, which subsequently lives to maturity, the head may become bilaterally unsymmetrical, as shewn especially by the skull. Again division of both vagus nerves is very apt to be followed by inflammation of both lungs, by fatty degeneration of the heart, and so by death.

In several of these instances the effect is a mixed one and the problem complicated. Thus, in the case of division of the fifth nerve, seeing how delicate a structure the eve is, and how carefully it is protected by the mechanisms of the eyelids and tears, it seems reasonable to suppose that the inflammation in question might simply be the result of the irritation caused by dust and contact with foreign bodies, to which the eye, no longer guided and protected by sensations, these being destroyed by the section of the nerre, became subject. In the same way the ulcers on the lips and gums might be explained as injuries inflicted by the teeth on those structures in their insensitive condition. And some observers maintain that the inflammation of the eye may be greatly lessened or altogether prevented if the organ be carefully covered up and in all possible ways protected from the irritating influences of foreign bodies. Other observers however have failed to prevent the inflammation in spite of every care. So also the inflammation of the lungs following upon division of both vagus nerves seems to be due not to any direct nutritive action of the pulmonary branches of the vagus on the pulmonary tissue, but to food accumulating in the pharynx owing to the paralysis of the oesophagus and larynx, and then passing into the air passages and so setting up inflammation. Death in these cases is moreover often the 
simple result of inanition cansed by the paralysis of the assophagus allowing no food to reach the stomach. The phenomena of the paralytic secretion of saliva are also of a complicated nature.

But even without insisting on such instances as the above, various other phenomena of disease seem to indicate such an influence of the nervous system on nutrition as we are discussing. As examples we might mention the rapid and peculiar degeneration of and loss of contractility in the skeletal muscles in certain affections of the spinal cord, the changes in the muscles being more rapid and profound than in the nerves; the phenomena of bed-sores, especially the so-called acute bed-sores of cerebral apoplexy; some at least of the cases of vesical affections attendant on spinal or cerebral diseases or injuries; the more rapid atrophy and loss of contractility in muscles which follow upon contusions of nerves as compared with the effects of simple section of nerves; the occurrence of certain eruptions, snch as lichen, zona, cethyma, ic., in variotis spinal or cerebral diseases, and indeed the gencral phenemona, and especially the topography of the eruption, of a large number of cutaneous diseases. Lastly but not least we might quote the general process of inflammation. These are cximples of clisordered nutrition. To them we might add as instances of altered but yet orderly nutrition the remarkable eonnections observed between changes in the form of the fingers and growth of the nails and hairs, and certain internal malarlies, such for instance as the 'clubbed fingers' of phthisical and other patients, and the likc. We might also call attention to the influence of light on the nutrition of animals. The experience of blind people and blind animals indicates some special connection between visual scnsations and the nutrition of the skin; and this can hardly be other than a nervous connection. 'The effects of prolonged darkness on nutrition in general and the experimental results which shew that the total metabolism of the body is inflnenced by light, also snggest some nervons action. 'The influence of cold again in determining the growth of hair points in the same direction.

Making every allowance for the intervention as factors in the production of the phenomena quoted above of such common actions of the nervous system as are alrcady well known to us, such as vaso-motor changes, making every allowance for the eonsequences of the failure or blnntness of sensation and the absence of those beneficial after results of muscular activity which we pointed out in $\$ 81$, recognizing moreover that changes in one organ may affect the condition of other distant organs by ehanges induced in the composition or qualities of the blood. there still remains a resilue which seems distinctly to point to the conclusion that the inflnence of the nervous system is not limited to such changes of the miscles as belong to the produc- 
tion of contractions or the generation of heat, but bears on the whole nutrition of the muscle. Similar considerations lead us also to conclude that the influence of the nervous system bears on the whole nutrition of the glands, of the blood vessels, of the skin and the connective tissues in general, in fact of nearly the whole body. 


\section{SEC. 4. ON DIET.}

$\$ 440$. An ordinary man living an ordinary life will need for the maintenance of vigorous health a certain amount of food of a certain kind; this we may take as a normal diet.

P'resuming that the experience of man has led him to adopt what is good for him, we may ascertain approximately the normal diet by means of the statistical method, by examining the nature and amount of the daily food of a very large number of individuals. The most valuable data for this purpose are those gained by inquiries among persons who choose their own food; the results gained from the diets used in prisons or other institutions, or among bodies of men such as the army, though more readily arrived at, are open to the objection that the diets in question are determined in part by the theoretical opinions of those whose duty it is to fix the diet. Putting together the various statistical results thus obtained, and selecting the quantities which seem to be most commonly used rather than attempting to strike a strict average or take a strict mean, we find that in an ordinary diet for the twenty-four hours the several food-stuffs are

$$
\begin{array}{lrr}
\text { Proteids from } 100 \text { to } 130 \text { grms. } \\
\text { Fats } 40,80 \% " \\
\text { Carbohydiates } 450,550 "
\end{array}
$$

to these we must add

$$
\begin{aligned}
& \text { Salts } 30 \text { grms. } \\
& \text { Water } 2800 \text {, }
\end{aligned}
$$

The total (available) potential energy of the lower estimate is 2610, of the higher 3505 (kilogramme-dlegrce) calories, calculated, in round numbers, on the data of $\$ 21$. With such a statistical diet we may compare an experimental diet, that is to say a diet arrived at through a series of trials on an individual man whose body might be taken to be an average one, that diet being considered a normal one in which the body, maintaining vigorous health, neither gained nor lost in weight, and remained moreover in nitrogenous equilibrium with the nitrogen of the 
egesta equal to that of the ingesta. To make sure that under such a diet the body was remaining of the same composition there ought to be evirlence of a carbon equilibrinm also, otherwise during the period of the experiment fat might be being replaced by water (see $\$ 415$ ); but this is unlikely, and we may therefore accept the method as a fair one. It has given in the hands of two different observers the following somewhat different results, the diet $A$ being that already quoted in $\$+21$ :

A

B

$\begin{array}{lrcr}\text { Proteids } & 100 & \text { grms. } & 118 \\ \text { Fats } & 100 & , & 56 \\ \text { Carbohydrates } & 240 & , & 500 \\ \text { Salts } & 25 & , & - \\ \text { Water } & 2600 & , & -\end{array}$

The total (available) potential energy is respectively 2310, and 3035 calories.

On the whole the diets gained by the two methods agree very largely. 'To put down a single column of figures as "the normal diet" would be to affect a vain and delusive accuracy. If we desire, for theoretical purposes, to select some one set of figures rather than others, we might be influenced by the considerations that the lower amount of proteids in the experimental diet was nearer the mark than the higher amount of some of the statistical diets, and further that, where cost is not of moment, the substitution of fat for an excess of carbohydrates is desirable. We should be thus led to take the experimental diet $A$ as on the whole the best or most 'normal' one, and that is the one which we employed in the calculations of $\$ 421$. It will be observed that the potential energy of this diet is less than that of any of the others, and, as we said while then speaking of it, may be considered low; but there was no evirlence that it was insufficient. Still it must be remembered that neither it nor any of the other's is to be regarded as distinctly proved to be the real normal diet. Against the experimental diet we may urge that the number of experiments have been few and conducted on a few individuals only at most, and that a larger number of experiments, with a variety of combinations of different amounts of the several food-stuffs, might lead to a different result; that for instance with certain amounts of fats and carbohydrates, the amount of proteid needed to maintain healthy bodily equilibrium, including nitrogenous equilibrium. might be reduced much below the 100 grammes. especially if particular kinds of proteids, fat or carbohydrates were used, and especial attention (see $\$ 40$ ) were paid to the salts. And indeed a considerable number of observations have been made tending to shew that a man of average size and weight 
may eontinue in nitrogenous equilibrium and in good health with a daily ration of much less than 100 grm. proteid, with as little as $40 \mathrm{grm}$. for example. 'To this we shall have to refer in speaking of a vegetable diet. Against the statistieal diet on the other hand we may urge that instinet is not an nnerring guide, and that the choice of a diet is determined by many other circumstances than the physiologieal value of the food.

$\$ 441$. Taking however some such diet as the above to be the approximately true normal diet, we may call attention to the fact that the normal diet is made up of eaeh of the three great food-stuffs, carbohydrates being in excess. We may here remark ineidently that the diets of both the earnivora and herbivora agree with that of ommivora in eontaining all three food-stuffs: they differ from eaeh other as to the relative proportions only. As we have seen, the body may be maintained in equilibrium on proteid food alone; but an exclusively proteid diet is not only bought dearly in the market, but also paid for dearly within the economy; we are of course now speaking of man. To obtain the necessary earbon out of the earbon moiety of proteid umneeessary labour is thrown on the economy, and the system tends to become blocked with the amides and other nitrogenous waste arising out of the nitrogen moiety simply thrown off to secure the carbon.

Fats and earbohyclates are much more akin to each other than is either to proteid; and if on the one hand, as ( $\$ 435$ ) seems possible or even probable, the fat of the food and of the body is converted into sugar either on its way to become built up into the tissue or in the course of the changes taking place outsicle the real living framework of the tissue by which it is reduced to earbonic acid, and that on the other hand carbohydrates can furnish the fat whose prescnee in the body is necessary, we might expect that earbohydrate alone without fat might, with proteid, form a normal diet. But on this point experience is probably to be trusted; and we may infer that in cvery normal diet some fat at least must be added to the starehes and the sugars.

The advantage of this mixture is probably felt while the food is as yet within the alimentary eanal. What we have learnt eoneerning digestion leads us to regard it as a compiieated process, and we eamnot readily imagine that the proteolytic, amylolytie and adipolytic changes run their sereral courses, especially in the small and large intestinc, apart from and irrespective of eaeh other. We are rather led to suppose that the aceompaniment of one set of changes, in some indirect manner, favours the others; and it is for that reason probably that we take our food-stuffs not separately but mixed in the same meal, often on the same plate and even in the same mouth- 
ful. But apart from this the two food-stuffs, fats and carboliydrates, must play different parts in the economy, so that the one camnot be wholly substituted for the other; and though, beyond the fact that the one seems to be a source of energy and the other not, we do not as yet know the true physiological function of the hydrogen of the fat as compared with that of the differently disposed hydrogen of the carbohydrate, we may perhaps infer that the difference of use within the body of the two kinds of food-stuffs bears not so much on their ultimate consumption to supply energy as on the various complicated processes which they indergo and arrangements in which they take part before the end of their work is reached. We have had a hint that the carbohydrate more rapidly supplies the heatgiving metabolism than does the fat; and this suggests an advantage to the economy in receiving daily a certain portion of the more tardy material, while at the same time it may be taken to mean that the fat before it is used to give rise to energy has first to be converted into sugar, and so takes more time in its work.

The main carbohydrate of every diet is starch, and as far as we can learn at present, the starch which is so large a part of the cereals and regetables consumed by man is the same body in all of them ; for the use of such bodies as inulin is so insignificant that it may be neglected. Man howerer consumes no inconsiderable quantity of sugar, chiefly cane sugar. Since the starch of a meal does not become available for the economy until it has been converted into sugar, we might be inclined to infer that it was a matter of indifference whether the carbohydrate of a diet were supplied as starch or as sugar. Our knowledge of sugars and of their fate in the economy is too imperfect for us to be able to state the effects on the body of digested starch as compared with those of cane sugar or milk sugar; but that these are or may be different is shewn by the experience of medical practice. In many cases the total effect on the body of a diet from which cane sugar is as much as possible eliminated, though stareh be allowed, is very different from that of one of which eane sugar forms an appreciable part.

Concerning cellulose, which in herbivora appears certainly to serve as a source of energy and to be a real food-stuff, our knowledge will not allow us to decide whether it has any special uses of its own, or whether the body is simply led to utilize and make the best of what is a necessary accompaniment of the starch of regetable food.

Concerning the salts present in a diet we need only repeat what was said in $\$ 420$ that these, though affording of themselves little or no energy, are as essential a part of a diet as the energy giving food-stuffs, in as much as they in some way or other direct metabolism and the distribution of energy. And 
this is true not only of the inorganic salines such as chlorides and phosphates but also of the so-ealled extractives. As we have seen, the presence of these bodies, both the simpler inorganic and the more complex organic salts, in the blood or in the extravasenlar juices or lymph of the tissues is essential to or directs or modifies the metabolic activity of the several tissues. 'The beneficial effects, as components of special diets, of such things as beef-tea and meat-cxtract, which consist chiefly of salts and extractives with a very small quantity of albumose or other forms of proteid, and the effects either bencficial or deleterious of drugs both turn in common upon their taking a part of some kind or other in, it may be upon their interference with metabolic processes. The salts and extractives of a diet may be looked upon as necessary daily medicines, and a medicine as a more or less extraordinary variation in these elements of a diet.

Alcohol, to the use of whieh as a component of an ordinary diet special interest for various reasons attaches, comes in this class. For though observations shew that the greater part of a moderate dose of alcohol is oxidized within the body and so serves as a source of energy, man has recourse to alcohol not for the minute quantity of energy which is supplied by itsclf, but for its powerful influence on the distribution of the energy furnished by other things. That influence is a very complex one and cannot be fully discussed here. We may add that the physiological action of aleoholic drinks is still further complicated by the fact that most such drinks contain besides ethylic aleohol, various other allied substances, whose action is even more potent than that of the ethylic alcohol itself, and whose presence very markedly determines the total effect of the drink. Such articles of diet as tea and coffee stand upon very much the same footing as alcohol.

The quantity of fluid which a man drinks or should drink daily, or more correctly the quantity of water which he should daily arld to the dry solids of his diet, must vary widely aecording to circumstance. It will differ according as he is perspiring greatly or not, according to the nature of the dry solids of the diet, whether largely carbohydrate or not, and so on. A lower limit, below which excretion is impeded, and a higher limit, above which digestion and metabolism are injuriously affected, probably exist; but we have as yet no adequate data which will enable us to fix either of them.

$\$ 442$. In the selection of articles of food to supply the food-stuffs and other constituents of a normal diet, regard must of eourse be had in the first place to the amount of potential energy present in the material. The articles chosen for the daily fare must enntain between them so much proteid, fat, and carbohydrate representing so much available energy. But 
it is no less important to secure that the energy potential in the material should be really available for the economy. The material must have such qualities that it is digested within the alimentary canal, and further that its digestion and absorption do not give rise to trouble either in the alimentary canal or in that secondary digestion carried on by means of the various metabolic events which we have discussed in preceding sections. A really nutritious substance is one which not only contains in itself an adequate supply of energy, but is of such a nature that its energy can be appropriated by the economy with ease or at least with as little trouble as possible. We have approximate data for detcrmining how far an estimate of the relative usefulness of varions articles of food must be corrected by allowing for the proportion of each which after an ordinary meal merely passes through the alimentary canal and the energy of which is not in any way available for the body's use. Thus a number of observations carried out on healthy individuals gave in the case of the following articles of food, the following figures as the percentage, reckoned in each case on dry material, which could be recovered from the freces, and was therefore not digested and not used by the body: - Meat 5 p.c., Eggs 5 p.c., Milk 9 p.c., Bread (white) \pm p.c., Black Bread 15 p.c., Rice 4 p.c., Maccaroni 4 p.c., Maize 7 p.c., Peas 9 p.c., Potatoes 11 p.c. It must however be remembered that the actual correction to be made in any case will depend on the mode of cooking of the material, on the character of the meal of which it forms part and on the individual capabilities of the consumer, the latter too varying under different circumstances.

The above refers to what may be called rough digestibility, but besides this there are other circumstances to be considered. The same food-stuff in two articles of food, though actually digested, that is to say taken up by the alimentary canal, may, even while still within the alimentary canal, undergo changes in the one case differing from those in the other. A proteid may for instance in one case tend to be entirely converted into peptone, or to break up into leucin, \&c., or in other cases to undergo other changes; and a carbohydrate may in one case be absorbed as sugar, and in another give rise to lactic acid. Indeed, when we speak of the digestibility or the indigestibility of this or that article of food, we do not in many cases so much mean the relative amount of the substance taken up in some way or other by the alimentary canal, as the characters advantageous or otherwise of the changes which it undcrgoes in being so taken up.

Hence the purely chemical statement of the amount of potential energy present in an article of food is no safe guide of the physiological value of the substance. A chunk of cheese 
stancls very high on, generally at the top of, a table of the nutritive value of articles of food drawn up on exclusively chemical principles, aecording to the units of energy present in a unit of the material; but it is very low down in a corresponding physiological table. And similarly a dish of old peas has a very different physiological function from a plate of fresh meat even when both contain the same amount of nitrogen.

In thus correcting for digestion the nutritive value of a diet it must also be borne in mind that the alimentary canal, while chiefly a receptive organ, is also to some extent. $\$ 234$, an excretory organ: a free passage through the canal is needed not only for carrying off undigested matter but also for getting rid of excreted matter; and the presence of the former, up to certain limits, assists the discharge of the latter. Were it possible to prepare a diet every jot and tittle of which could be digested and absorbed, the use of such a diet would probably bring about disorder in the economy, through the absence of a sufficiently rapid discharge of the matters excreted into the alimentary canal. Hence cellulose and like substances even when unutilized through absorption, are not without their use, and experience shews that digestion may be promoted by eating undigestible things.

$\$ 443$. The several food-stuffs of a diet may be drawn from the animal or from the vegetable kingdom. Vegetable proteids appear to undergo the same changes in the alimentary canal as do animal proteids, and the main effects on the body of proteids from the two sources seem to be the same. Our knowledge at present however is too imperfect to enable us to decide whether the functions of the two are exactly the same, whether the borly behaves exactly the same upon a diet in which the proteids are exclusively of vegetable origin, as upon a diet in which, otherwise the same, the proteids are partly of animal origin also. Nor have we much better knowledge of the relative nutritive value of vegetable and animal fats. And as we have already said, we possess little or no exact knowledge as to the part played by those extractives in respect to the amount and nature of which animal food strikingly differs fron vegetable food. In attempting therefore a judgment from a purely physiological point of view as to the valuc of an exchusively vegetarian diet compared with a diet of both animal and vegetable origin, we can do little more at present than inquire whether the former supplies the several food-stuffs in adequate quantity, in proper proportion, and in such a form as to be economically utilized by the body.

The careful examination cluring three separate periods of several dlays each of the ingesta and egesta of a man, 28 years old, weighing 57 kilos, who har for three years lived on an exchusively vegetable diet, viz. bread, fruit and oil, gave the following results. 
The daily diet consisted on the average of $719 \mathrm{grm}$. solid matter and 1084 grm. water. It contained

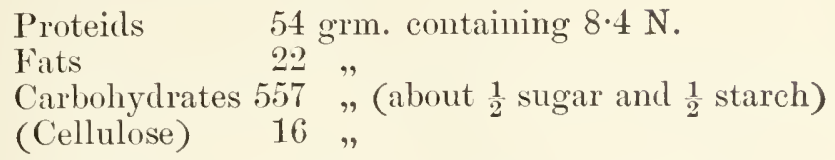

The daily freces weighed, when fresh, $333 \mathrm{grm}$. containing 75 grm. solid matter, and were therefore both bulky and watery. 'There were present in the fieces fat $7 \mathrm{grm}$., starch 17 grm. and cellulose 9 grm. shewing that 30 p.c. of the fat, 6 p.c. of the starch and 56 p.c. of the cellulose had not been utilized by the body. The subject had really lived on fat 15 grm., carbohydrates 540 grm. (and cellulose 7 grm.). The faeces contained no less than $3 \cdot 46$ nitrogen. If we reckon the whole of this as proteid, this would give $22 \mathrm{grm}$. of undigested proteid, so that there had been a waste of 41 p.c. of the proteids. leaving only $32 \mathrm{grm}$. available for real use in the body; and indeed a very small portion only of this nitrogen can be regarderl as really discharged from the borly itself. The total solids of the fieces must be reckoned as partly excreta but chiefly undigested food. If we regard the $75 \mathrm{grm}$. of solid faces as entirely undigested food, the whole solid food available for the body must be reduced from $701 \mathrm{grm}$. to $644 \mathrm{grm}$.

The urine of the day contained 5.33 grm. nitrogen; this added to the 3.46 grm. nitrogen in the freces gives $8.79 \mathrm{grm}$. nitrogen in the total egesta as compared with the $8.4 \mathrm{~g}$ rm. nitrogen of the food, indicating a slight loss of nitrogenous material from the body; but if we suppose that all the nitrogen in the freces was not in the form of undigested food we may neglect this; and indeed the subject of the observation was in apparently good health and stationary weight.

Compared with either of the normal diets given in $\$ 440$ the above diet is striking for the low amount of proteids and of fats and the relative excess of carbohydrates. But though such a diet may be taken as perhaps fairly typical of the daily food of a rigid vegetarian, a much more richly proteid diet may be obtained from sources still strictly vegetable. Thus the diet, entirely vegetable in nature, of an average Japanese labourer of about the same weight as the individual whose data we have just given has been estimated to consist of Proteids $102 \mathrm{grm}$. , Fat 17 grm., Carbolrydrates $578 \mathrm{grm}$. And the diet of a Roumanian peasant, living chiefly on beans and maize with the addition of fat of some kind, has been calculated to furnish no less than Proteids 182 grm., Fat 93 grm., Carbohydrates $968 \mathrm{grm}$.; but the real nutritive value of such a diet must need very large correction indeed. Cf. $\$ 4+2$. 
The examination of the diet of an individual living with a fair nitrogenous equilibrium and apparently good health on a moditied vegetable diet, that is to say one which included milk and eggs, gave the following: Proteids 74 grım., Fat 58 grm., Carbohydrates $490 \mathrm{grm}$., a diet which differ's from the normal diet almost solely in the lesser amount of proteids, one third of which by the bye was smplied by the amimal material, eggs and milk. In another instance, nitrogenous equilibrium and fairly good health were secured, for some weeks at all events, on a vegetable diet yielding Proteids about 100 grm., Fats 70 grm., Carbohydrates $400 \mathrm{grm}$; but in this nearly the whole of the fat was furnished by the animal produet butter, and Liebig"s extract was freely used.

Confining ourselves however to the more strictly vegetarian diet, we may conclude in the first place that, mless the daily food be very large in amount, the proteid element of such a diet falls considerably below the 100 or more grm. given in the normal diet. But we cannot authoritatively say that such a reduction is necessarily an evil; for as we stated above, $\$ 440$, our knowledge will not at present pernit us to make an authoritative exact statement as to the extent to which the proteid may be reduced without disadvantage to the body when atecompanied by adequate provision of the other elements of food; and this statement holds good whether the body be undertaking a small or large amount of labour. A second feature of such a diet is the marked reduction of the fat and its replacement by carbohydrates. Although here again we camnot make a distinctly authoritative statement, the evidence wlich we possess bears elearly in the direction that such a reduction is a marked disadvantage. A third and very characteristic feature of the strictly vegetarian diet is the relatively large amount of undigested food lost to the body and discharged as fieces. Even when the diet is scanty, so that the proteid elenent is low, the amount of fieces relatively to the total food is high; and when a more normal proteid contribution is secured by ample meals the fieces become exceedingly voluminous. Indeed when, leaving man, we compare the herbivorous with the carnivorous mammal, we find that the former is almost as clearly distinguished from the latter by its frequent and abundant fitces as by the anatomieal features of its organization. We have already urged that, since the faces serve as a means of excretion of the real waste products of metabolism, a certain amount of vehicle to carry these away is of advantage or even necessary; but there are no facts at present known to us, which shew that the larger intestinal current of the purely vegetable diet effects any such good as can compensate for the obvious waste of labour incurred in its transport and managenent, to say nothing of the opportumities of mischief offered by a mass of material more subject 
to the dominion of foreign organisms than even to that of the body itself, though these opportunities are less than with a corresponding mass of animal origin. With respect to these three features then, the strictly vegetarian diet scems, on physiological grounds, inferior to one of a mixed nature. There are as we said other aspects, still of a strictly physiological kind, to be considered, such as the relative digestibility of vegetable articles of food, the relative metabolic value of the food-stuff's of vegetable origin, and the influence of animal extractives; but any fuller discussion of these points would be out of place here.

$\$ 444$. We have treated the diet discussed above as a normal diet, suitable for man under ordinary or general circumstances. Ought such a diet to be modified for the various exigences of life such as labour, age, climate, and the like?

We shall discuss the influence of age in the concluding portions of this work.

We may be inclined at first sight to assume that the total amount of the diet should vary with the weight. that is the size of the individual; and indeed in discussions on nutrition, statements concerning metabolism and amount of food are often given in terms of per kilo of body weight. In a broad scnse it may be true that a small man needs less food than a large one; but it must be remembered that, as we saw in speaking of animal heat, the smaller organism, having the relatively larger surface, carries on a more rapid metabolism per unit of body weight, and so needs relatively more food. And moreover the influence of size is probably far less than the influence exerted by the inborn individual characters of the organism, giving rise to what we may call the personal equation of metabolism. The smaller metabolism of woman, leading to the use of a scantier diet, as compared with that of man, is to be regarded in this light rather than with reference to the average lesser weight of woman. The relative metabolism of the two sexes may be illustrated by the case of an active man and his wife, both of about the same age and weight, the man being rather the heavier and the woman rather the older, who, in earrying out together an experiment on the relative values of vegetable and animal food, both lived for some time on the same kind of diet, and found that nutritive equilibrium was, in the one case and in the other, maintained when

$\begin{array}{lccc} & \text { Proteids. } & \text { Fats. } & \text { Carbohydrates. } \\ \text { The man consumed daily about } 100 & 70 & 400 \\ \text { The wife , , , } & 60 & 67 & 340\end{array}$

The most striking difference is in the proteids.

$\$ 445$. With regard to elimate the chief considerations attach to temperature. When the body is exposed to a low temperature the general metabolism of the body is increased 
owing to a regulative action of the nervous system, $\$ 428$. We miglit infer from this that more food is necessary in cold climates ; and, since the increase in the metabolism appears to manifest itself chiefly in a greater discharge of carbonic acid and therefore to be especially a carbon metabolism, we might infer that the carbon elements of food should be especially increased. When the body is exposed to high temperatures the same reflex mechanism tends to lower the metabolism; but the effects in this direction are much less clear than those of cold, and soon reach their limits; the bodily temperature is maintaincd constant under the influence of surrounding warmth not so much by diminished production as by increased loss. We may infer from this that in warm climates not less but if anything rather more food than in temperate climates is necessary in order to supply the perspiration needed for the greater evaporation and discharge of heat by the skin.

In both cold and warm climates however man trusts much more to variations in his elothings and immediate surroundings to protect him against cold or to guard him from heat than to any marked variations in his normal diet. In the former he may perhaps bc expected to eat somewhat more, since, in spite of wrappings, his skin still feels in part the cold, and thus the nervous mechanism for the increase of metabolism is to a certain extent set to work. And since the metabolism thus increased appears to affect especially the carbon of the body, he may further be expected to increase the fats rather than the carbohydrates of his food seeing that the former supply him with the most energy for their weight. But it is very doubtful whether what he might thus be expected to gain over a corresponding increase in carbohydrates is not more than counterbalanced by the increased labour of digestion; and the habits of the dwellers in arctic climates cammot safely be taken as guides in this matter, for their reputed love of fat is probably the result of that being their most available form of carbon. Indecd the evidence that the increase of metabolism provoked by cold bears exclusively on carbon constituents is so uncertain that it may be doubted whether any change in the normal diet, beyond some increase in the whole, should be made to meet a cold climate. Similar reasons would lead one to infer that man in the warmer cliniate would maintain on the whole the same normal diet, the only change perhaps being to increase it slightly, possibly throwing the increase chiefly on the carbohydrates with the special view of furthering perspiration.

$\$ 446$. A special diet for the purpose of fattening, that is to say for the accumulation of adipose tissue out of proportion to the rest of the body, is not needed in the case of man. 'The power to store up fat in adipose tissue is much more dependent on certain inborn qualities of the organism which we camnot at 
present define than on the kind of food; of two bodies living on the same diet, and under the same circumstances, one will become fat while the other will remain lean ; and it is an object of the agriculturalist to develope by breeding and selection a "constitution" which will store up the most fat on the cheapest diet. In fattening animals, the chief care, when the selection of the kind of animal has been made, is to provide adequate carbohydrate food, which as we have seen is the chief fattener ; and the object of the farmer in rearing stock for the butcher is mainly to convert cheap vegetable carbohydrate into dear animal fat. Further aids in fattening may be found in providing repose for the body of such a kind that, while sufficient energy is expended to secure adequate digestion and absorption of food, all causes leading to an increase of metabolism, by which energy is set free and leaves the body, are avoided as much as possible.

To avoid fat rather than to increase it is often an object of human care. This may be effected by diminishing fats and carbohydrates, but also, in a very marked manner, by relatively increasing the proteids. Proteid food as we have seen augments the whole metabolism of the body, hurrying on the destruction not only of proteid but of carbon food; and a tendency to corpulency may be counteracted by a diet in which fats and carboliydrates are much restricted, and proteids are largely increased. When, as in what is known as the Banting method, the diet is almost exclusively proteid, the nitrogenous overwork entails dangers on organisms which do not possess the power of ridding themselves freely of the large amount of nitrogenous waste which such a diet produces. A less severe method in which the fats and carbohydrates are diminished only, not entirely done arvay with, and the proteids only moderately increased, is less open to objection; and such a diet, assisted by other hygienic conditions, has proved successful.

An increase of daily food, largely proteid in nature, given under circumstances, such as a large amount of passive exercise and skin stimulation, known as 'massage,' which will not only favour digestion but also promote metabolism in general, nay be given, with favourable results. In this way, an enormous metabolism may be cxcited, and yet so carried on that the body gains both in flesh and in fat. Thus, in one case, the patient with an initial weight of 45 kilos, and a daily nitrogenous metabolism, calculated as $28 \mathrm{grm}$. proteid, reached in the course of about 50 days a weight of 60 kilos, the daily nitrogenous metabolism being raised on one occasion to $182 \mathrm{grm}$. protcid, with an average on the whole period of $150 \mathrm{grm}$. During the treatment no less than $8420 \mathrm{grm}$. of proteid were taken as foorl.

$\$ 447$. With regard to labour, since as we have seen the energy expended as work done is not taken out of and away 
from the amount set free as heat, the two forms of energy being so related that an increase of work done is accompanied by a greater or less increase of heat set free, it is obvious that a man who is doing a hard day's muscular work needs a larger income of energy for the day than does an idle man. What we have learnt concerning muscular metabolism further shews us that the additional energy nceded is not necessarily to be supplied by an increase in the proteid components of the diet; the energy of muscular contraction does not come as was once thought from proteid metabolism ( $\$ 423)$. The fact that it is the earbon metabolism which is augmented in muscular work may suggest that the extra food for extra work should be exclusively carbon compounds; and if, as we have seen to be probable, the carbohydrates are more readily and directly available for the functional metabolism of muscle than are the fats, we might be further led to recommend an increase in carbohydrates to form a diet especially suited for labour. This view seems directly supported by the experimental result that even a small quantity of sugar taken by the mouth has an immediate favourable effect on the power of the muscles. But several considerations have to be taken into account in this matter. A muscle is not a machine within the body which can be loaded and fired off irrespective of the rest of the body. In the performance of muscular labour, the condition of the muscle, the amount of energy available in the muscle itself, is of course of prime importance; but, and this perhaps especially holds good in severe labour, of great importance also, we might almost say of no less importance, is as we have urged $(\$ 317)$ the power of the body as a whole to avail itself of the energy latent in the muscle. 'The power of doing work hangs not on the muscle alone, but on the heart, the lungs, the nervous system and indeed on the whole body. It is very doubtful whether we ever, even in supreme efforts, draw upon more than a portion of the capital of energy lodged in the muscle itself; fatigue is far more a nervous than a muscular condition, and even the distinctly muscular fatigue is as we have seen ( $\$ 81)$ partly at least the result of the accumulation of products and not alone the using up of available energy. In choosing a diet for muscular labour we must have in view not the muscle itself but the whole organism. And though it is possible that future research may suggest minor changes in the various components of a normal diet such as would lessen the strain during labour on this or that part of the body, on the muscles as well as on other organs, our present knowledge would rather lead us to conclude that what is good for the organism in comparative rest is good also for the organism in arduous work, that the dict, normal for the former condition, would need for the latter a limited total increase but no striking change in its composi- 
tion. In preparing the body for some coming arduous labour in "training" as it is called, an increase of proteid food, for the purpose of hurrying on the general metabolism of the body, and thus of making 'new flesh' and renovating the body, so to speak, in view of the strain to be put upon it, may perhaps suggest itself; but even this is doubtful.

The principles of such a conclusion with regard to muscular work may be applied with still greater confidence to nervous or mental work. The actual expenditure of energy in nervous work is relatively small, but the indirect influence on the economy is very great. The closeness and intricacies of the ties which bind all parts of the body together is very clearly shewn by the well-known tendency of so-called brain work to derange the digestive and metabolic activities of the body; and if there be any diet especially suited for intellectual labour it is one directed not in any way towards the brain, but entirely towards lightening the labours of and smoothing the way for such parts of the body as the stomach and the liver. 



\section{B00K III.}

THE CENTRAL NERVOUS SYSTEM AND ITS INSTRUMENTS. 



\section{CHAPTER I.}

\section{THE SPINAL CORD.}

\section{SEC. 1. ON SOME FEATURES OF THE SPINAL NERVES.}

\$448. WE have called the muscular and nervous tissues the master tissues of the body; but a special part of the nervous system, that which we know as the central nervous system, the brain and spinal cord, is supreme among the nervous tissues and is master of the skeletal muscles as well as of the rest of the body. We have already (Book I. Chap. III.) touched on some of the general features of the nervous system, and have now to study in detail the working of the brain and spinal cord. We have to inquire what we know concerning the laws which regulate the discharge of efferent impulses from the brain or from the cord, and to learn how that discharge is determined on the one hand by intrinsic changes originating, apparently, in the substance of the brain or of the cord, and on the other hand by the nature and amount of the afferent impulses which reach them along afferent nerves.

As we shall see, the study of the spinal cord cannot be wholly separated from that of the brain, the two being very closely related. Nevertheless it will be of advantage to deal with the spinal cord by itself as far as we can. The medulla oblongata or spinal bulb ${ }^{1}$ we shall consider as part of the brain. But before we speak of the spinal cord itself, it will be desirable to say a few words concerning the spinal nerves, that is to say the nerves which issue from the spinal cord.

We have already seen $(\$ 88)$ that each of the spinal nerves arises by two loots, an anterior root attached to the ventral or

1 The term medulla oblongata is not only long, but presents difficulties, since the word medulla is now rarely used to denote the whole spinal cord (medulla spinalis) but is generally used to denote the peculiar coat of a nerve fibre, the white substance of Schwann. In using instead the word bulb or if necessary, spinal bulb there is little fear of confusion with any other kind of bulb. The adjective is in not uncommon use, in such phrases as 'bulbar paralysis.' 
anterior surface, and a posterior root attached to the dorsal or posterior surface of the cord. We have further seen that the latter bears a ganglion, a 'ganglion of the posterior root' or 'spinal ganglion.' We stated at the same time that while the trunk of a spinal nerve contained both efferent and afferent libres, the efferent fibres were gathered up into the anterior root and the afferent fibres into the posterior root; but we gave 110 proof of this statement.

$\S 449$. Before we proceed to do so, it will be as well to say a few words on the terms 'efferent' and 'afferent.' By efferent nerve fibres we mean nerve fibres which in the body usually carry impulses from the central nervous system to peripheral organs. Most efferent nerve fibres carry impulses to muscles, striated or plain, and the impulses passing along them give rise to movements ; hence they are frequently spoken of as 'motor' fibres. But all efferent fibres do not end in or carry impulses to muscular fibres; we have seen for instance that some efferent fibres are secretory. Moreover all the nerve fibres going to muscular fibres do not serve to produce movement; some of them, as in the case of certain vagus fibres going to the heart, are inhibitory and may serve to stop movement.

By 'afferent' nerve fibres we mean nerve fibres which in the body usually carry impulses from peripheral organs to the central nervous system. A very common effect of the arrival at the central nervous system of impulses passing along afferent fibres is that change in consciousness which we call a 'sensation'; hence afferent fibres or impulses are often called 'sensory' fibres or impulses. But as we have already in part seen, and as we shall shortly see in greater detail, the central nervous system may be affected by afferent impulses, and that in several ways, quite apart from the development of any such change of consciousness as may be fairly called a sensation. We shall see reason for thinking that afferent impulses reaching the spinal cord, and indeed other parts of the central nervous system, may modify reflex or automatic or other activity without necessarily giving rise to a "sensation." Hence it is advisable to reserve the terms 'efferent' and 'afferent' as more general modes of expression than 'motor' or 'sensory.'

We have seen in treating of muscle and nerve, that the changes produced in the muscle serve as our best guide for determining the changes taking place in a motor nerve; when a motor nerve is separated from its muscle (\$67) the only change which we can appreciate in it is an electrical change. Similarly in the case of an afferent nerve, the central system is our chief teacher; in a bundle of afferent fibres isolated from the central nervous system, in a posterior root of a spinal nerve for instance, the only change which we can appreciate is an electrical change. To learn the characters of afferent impulses 
we must employ the central nervous system. But in this we meet with difficulties. In studying the phenomena of motor nerves we are greatly assisted by two facts. First, the muscular contraction by which we judge of what is going on in the nerve is a comparatively simple thing, one contraction differing from another only by such features as extent or amount, duration, frequency of repetition and the like, and all such differences are capable of exact measurement. Secondly, when we apply a stimulus directly to the nerve itself, the effects differ in degree only from those which result when the nerve is set in action by natural stimuli, such as the will. When we come, on the other hand, to investigate the phenomena of afferent nerves, our labours are for the time rendered heavier, but in the end more fruitful, by the following circumstances:- First, when we judge of what is going on in an afferent nerve by the effects which stimulation of the nerve produces in some central nervous organ, in the way of exciting or modifying reflex action, or modifying automatic action, or affecting consciousness, we are met on the very threshold of every inquiry by the difficulty of clearly distinguishing the events which belong exclusively to the afferent nerve from those which belong to the central organ. Secondly, the effects of applying a stimulus to the peripheral end-organ of an afferent nerve are very different from those of applying the same stimulus directly to the nerve-trunk. This may be shewn by the simple experience of comparing the sensation caused by bringing any sharp body into contact with a nerve laid bare in a wound with that caused by contact of an intact skin with the same body. These and like differences reveal to us a complexity of impulses, of which the phenomena of motor nerves gave us hardly a hint.

We shall further see in detail later on that our consciousness may be affected in many different ways by afferent impulses; we must distinguish not only sensory from other afferent impulses, but also different kinds of sensory impulses from each other. Certain afferent nerves are spoken of as nerves of special sense, and the nature of the afferent impulses passing along these special nerves together with the modifications of consciousness caused by arrival of these impulses at the central nervous system constitute by themselves a complex and difficult branch of study. In some of the problems connected with the central nervous system we shall have to appeal to the results of a study of these special senses; but, on the other liand, a knowledge of the central nervous system is necessary to a proper understanding of the special senses; and on the whole it will be more convenient to study the former before the latter.

$\$$ 450. 'The proof that the afferent and efferent fibres which are both present in the trunk of a spinal nerve are parted at the roots, the efferent fibres running exclusively in 
the ventral or anterior root and the afferent fibres exclusively in the dorsal or posterior root, is as follows.

When the anterior root is divided, the muscles supplied by the nerve cease to be thrown into contractions either by the will, or by reflex action, while the structures to which the nerve is distributed retain their sensibility. During the section of the root, or when the proxinnal stump, that connected with the spinal cord, is stimulated, no sensory effects are produced. When the distal stump is stimulated. the muscles supplied by the nerve are thrown into contractions. When the posterior root is divided, the muscles supplied by the nerve continue to be thrown into action by an exercise of the will or as part of a reflex action, but the structures to which the nerve is distributed lose the sensibility which they previously possessed. During the section of the root, and when the proximal stump is stimulated, sensory effects are produced. When the distal stump is stimulated no movements are called forth. These facts demonstrate that sensory impulses pass exclusively by the posterior root from the peripheral to the central organs. and that motor impulses pass exclusively by the anterior root from the central to the peripheral organs; and so far as our knowledge goes the same holds good not only for sensory and motor but also for afferent and efferent impulses.

An exception must be made to the above general statement. on account of the so-called "recurrent sensibility" which is witnessed in conscious mammals, under certain circumstances. It sometimes happens that when the distal stump of the divided anterior root is stimulated, signs of pain are witnessed. These are not caused by the concurrent muscular contractions or cramp which the stimulation occasions, for they persist after the whole trunk of the nerve has been divided some little way below the union of the roots above the origins of the muscular branches, so that no contractions take place. They disappear when the posterior root is subsequently divided, and they are not seen if the mixed nerve-trunk be divided close to the union of the roots. The phenomena are probably due to the fact, that bundles of sensory fibres of the posterior root after running a short distance down the mixed trunk turn back and run upwards in the anterior root, (being distributed probably to the pia mater.) and by this recurrent course give rise to the recurrent sensibility.

$\$ 451$. Concerning the ganglion on the posterior root, we have already said that we have no evidence either that it can act as a centre of reflex action. or that it can spontaneously give origin to efferent impulses and thus act as an antomatic centre, as can the central nervous system itself. 'The bodies of the nerve-cells behave somewhat differently from the axiscylinders at some distance from the cells, though, as we have 
seen, these are in reality processes of the nerve-cells; thus the nerve-cells in the ganglion appear to be more sensitive to certain poisons than are the nerve-fibres of the nerve-trunk. But beyond this, our knowledge concerning the function of the ganglion is almost limited to the fact that it is in some way intimately connected with the nutrition of the nerve. As we have already (\$8) said, when a mixed nerve-trunk is divided the peripheral portion degenerates from the point of section downwards towards the periphery. 'The central portion does not so degenerate, and if the length of nerve removed be not too great, the central portion may grow downwards along the course of the degenerating peripheral portion, and thus regenerate the nerve. This degeneration is observed when the mixed trunk is divided in any part of its course from the periphery to close up to the ganglion. When the posterior root is divided between the ganglion and the spinal cord, the portion attached to the spinal cord degenerates, but that attached to the ganglion remains intact. When the anterior root is divided, the proximal portion in comnection with the spinal cord remains intact, but the distal portion between the section and the junction with the other root degenerates; and in the mixed nerve-trunk many degenerated fibres are seen, which, if they be carefully traced out, are found to be motor (efferent) fibres. If the posterior root be divided carefully between the ganglion and the junction with the anterior root, the small portion of the posterior root left attached to the peripheral side of the ganglion above the section remains intact, as does also the rest of the root from the ganglion to the spinal cord, but in the mixed nerve-trunk are seen numerous degenerated fibres, which when examined are found to have the distribution of sensory (afferent) tibres. Lastly, if the posterior ganglion be excised, the whole posterior root degenerates, as do also the sensory (afferent) fibres of the mixed nerve-trunk. Putting all these facts together, it would seem that the growth of the efferent and afferent fibres takes place in opposite directions, and starts from different nutritive or 'trophic' centres. 'The afferent fibres grow away from the ganglion either towards the periphery, or towards the spinal cord. The efferent fibres grow outwards from the spinal cord towards the periphery. This difference in their mode of nutrition is frequently of great lrelp in investigating the relative distribution of efferent and afferent fibres. When a posterior root is eut beyond the ganglion, or the ganglion excised, all the afferent nerves degenerate, and in the mixed nerve branches these afferent fibres, by their altered condition, can readily be traced. Conversely, when the anterior roots are cut, the efferent fibres alone degenerate, and can be similarly recognized in a mixed nerve tract. When the anterior root is divided 
some few fibres in it do not, like the rest, degenerate, and when the posterior root is livided, a few fibres in the anterior root are seen to degenerate like those of the posterior root; these appear to be the fibres which give to the anterior root its "recurrent sensibility." In the case of certain spinal nerves at all events, it has also been ascertained that when the posterior root is divided, while most of the fibres in the part of the root thus cut off from the ganglion but left attached to the cord degenerate, some few do not. These few appear to have their trophic centre not in the ganglion, but in some part of the spinal cord itself; we shall refer to these later on.

This method of distinguishing nerve fibres by the features of their degeneration, called the "degeneration method," or" sometimes from the name of the physiologist who introduced it, the "Wallerian method," has proved of great utility. Thus in the vagus nerve which is composed not only of fibres which spring from the real vagus root but also of fibres procceding from the spinal accessory roots, the two may be distinguished by section of the vagus and spinal accessory roots respectively. We shall presently see that this method may be applied to the differentiation of tracts of fibres in the brain and spinal cord. 


\section{SEC. 2. THE STRUCTURE OF THE SPINAL CORD.}

$\$ 452$. Before we proceed to discuss the functions of the spinal cord it may be as well to call to mind some of the main featurcs of its minute structure. (Figs. 110,111, 112.) 'The greater number of the fibres of the anterior root are the clothed axis-cylinder processes of cells lying in the anterior horn of the same side, placed not far from the origin of the root and belonging to the segment of the cord indicated by the root. 'The rest of the fibres of the root are the clothed axis-cylinder processes of cells lying in other parts of the grey matter, in the anterior horn of the opposite side and elsewhere. In the case of all the fibres of the root, the axis cylinder is the continuation of a process of a cell lying in the grey matter not far from the origin of the root. IVe may infer that changes started in these cells issue as efferent impulses along the fibres of the root; those started in the cells of anterior horn are motor impulses passing to the skeletal muscles, the smaller number started in other cells are probably efferent impulses destined for other structures, vasoconstrictor impulsés and the like.

The fibres of the posterior root, coming from and dependent for their nutrition on the ganglion of the root (we may neglect the few exceptional fibres of a different nature and having different relations), passing for the most part into the posterior column so as to form the "root-zone" lying close to the median side of the posterior horn, take the following course. Each fibre, soon after its entrance into the cord, bifurcates, one division rumning forwards towards the head, the other backwards towards the hind extremity. Each division gives off "collateral" fibres, and the end of each collateral as well as the final termination of each division is furnished by an arborescence which embraces and is in contact with but not in continuity with, the body of, and generally with the branches of the body of some nerve-cell in the grey matter. But the course of thc fibres is not the same in all cases. Many of the fibres (and apparently all the backward travelling divisions of all the fibres) are thus by means of the collaterals or the main terminations brought 


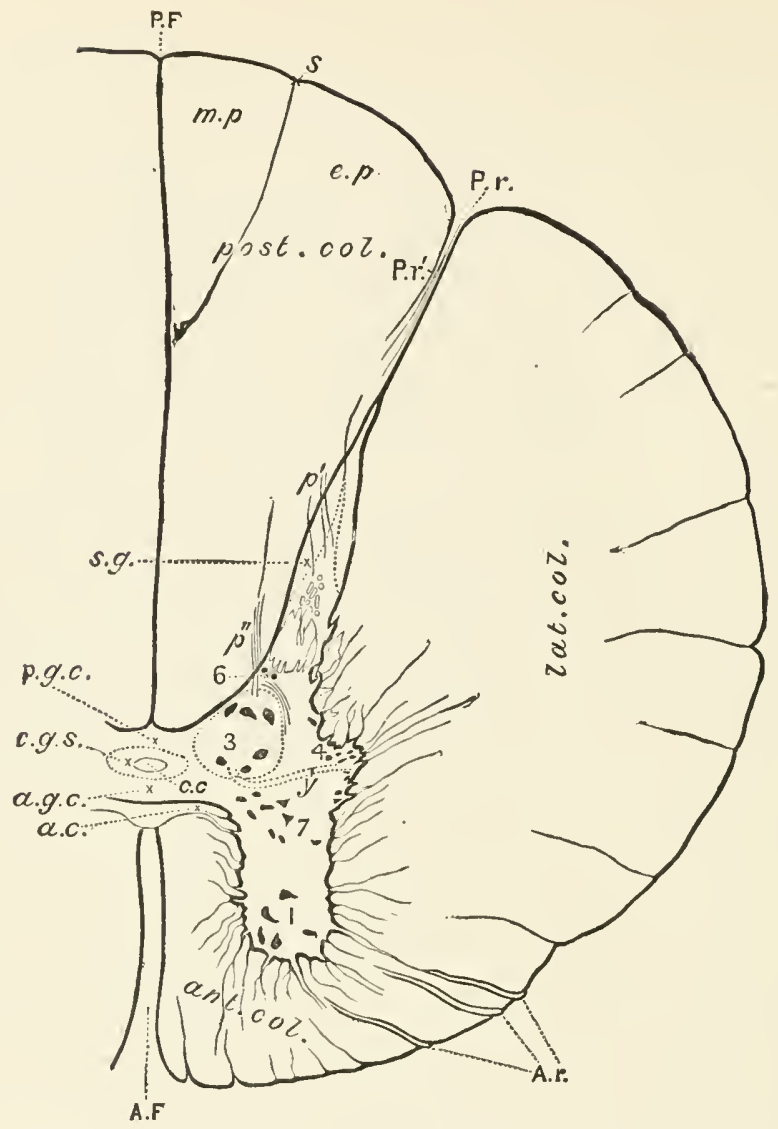

Fig. 110. A Transverse Dorsoventral Section of the Spinal Cord (Human) at the Level of the Sixtir Thorlcic (Dorsil) Nerve. (Sherrington.)I

Magnified 15 times. One lateral half only is slrewn. The large conspicuous nerve-cells (drawn from actual specimens) are shaded black to render their relative size, shape anıl position more obvious; the outline of the grey matter has been made thick and dark in order to render it conspicuous.

A.F. anterior fissure. P.F. posterior fissure. c.c. central canal. c.g.s. central gelatinous substance. A.r. anterior root, P.r. lateral (or intermediate) bundle, $I^{\prime} \cdot r^{\prime}$. median bundle of posterior root of spinal nerve, $p^{\prime}, p^{\prime \prime}$ fibres of posterior root passing $p^{\prime}$, indirectly through the substance of Rolando, $p^{\prime \prime}$, directly into grey matter. a.g.e. anterior grey commissure. p.g.c. posterior grey commissure. a.c. anterior white commissure. ant. col. anterior column. lat. col. lateral column. post. col. posterior column. s.g. the substance of Rolando. s. septum inarking out the external posterior column or column of Burdach, e.p., from the median posterior column or column of Goll, m.p.

1. cells of the anterior horn. 3. posterior vesicular column or vesicular cylinder, or columm of Clarke; the area of the cyliniler is defined by a dotted line. 4. cells of the intermedio-lateral tract or lateral horn. 6. cclls of the posterior horn. 7. cells of the anterior ccrvix. $y$ a tract of fibres passing from the vesicular cylinder to the lateral column.

1 For this and many succeeding firures I am deeply indebted to my friend and former pupil Dr. Sherrington who has kindly prepared the figures for me from his original drawings. 


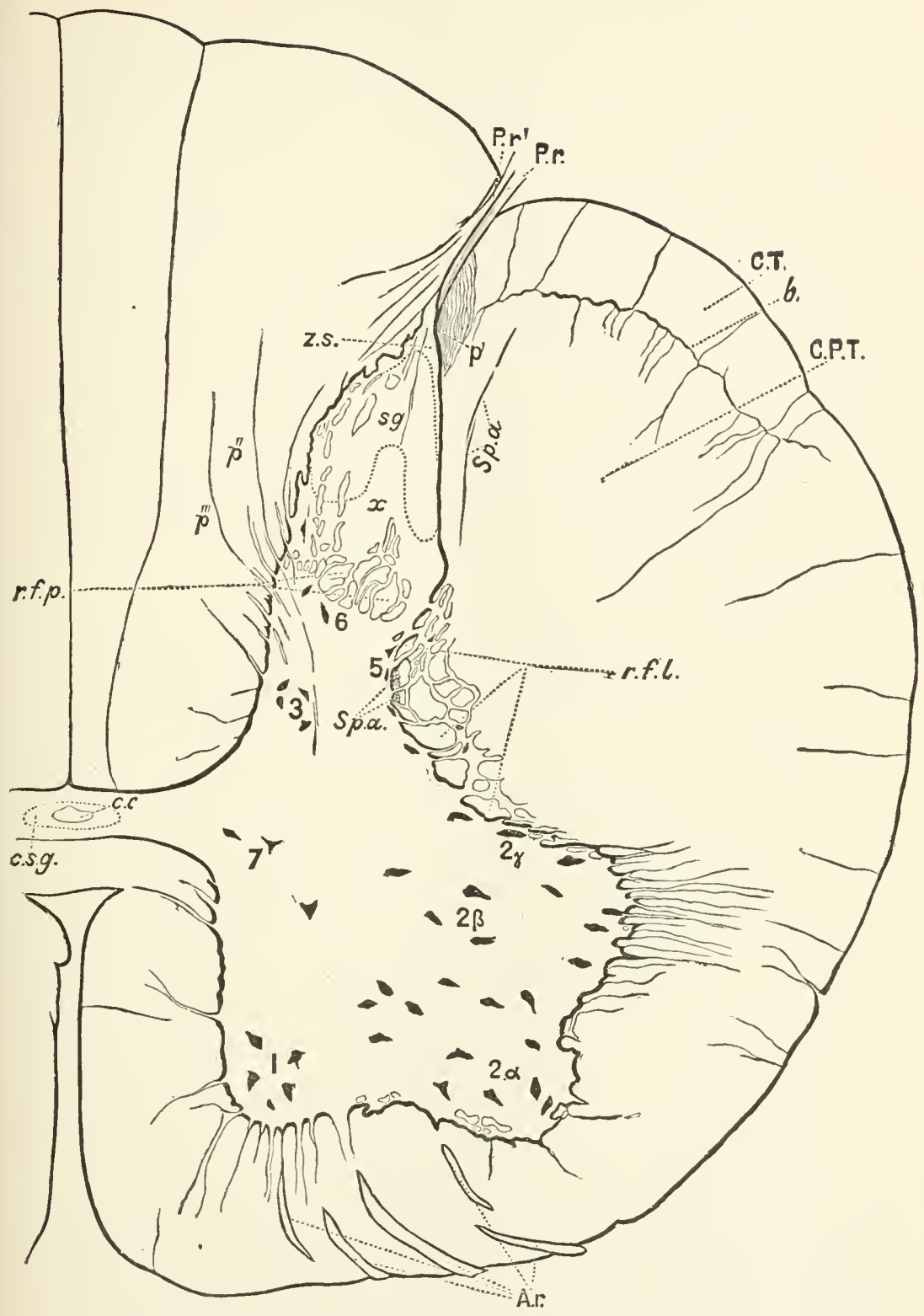

Fig. 111. Transverse Dorsoventral Section of Spinal Cord (Hrmay) at the Level of the Sixti Cervical Nerve. (Sherrington.)

This is drawn on the same scale as Fig. 110, that is magnified 15 times.

r.f.l. lateral reticular formation. r.f.p. posterior reticular formation. $p^{\prime}$. fine fibres of lateral bundle of the posterior root; $p^{\prime \prime}, p^{\prime \prime \prime}$ fibres of median bundle 
of posterior root, entering grey matter from external posterior column. $x$. grey matter of posterior horn. Sp. $a$. bundles of fibres belonging to the spinal accessory nerve; in the lateral reticular formation they are seen cut transversely. $b$. is a natural septum of connective tissue marking out the cerebellar tract C.T. from the crossed pyramidal tract C.P.T. $z$. s. zona spongiosa. $2 \alpha, \beta, \gamma$, lateral cells of the anterior horn. 5 . Cells in the region of the lateral reticular formation. 'The other letters of reference are the same as in Fig. 110.

into connection with cells not far from the entrance of the root. The cells with which the fibres are thus connected are situated in various parts of the grey matter, on one or the other side of the cord. Among these, we have reason to believe, are the cells of the anterior hom giving off axis-cylinder processes to anterior roots, and in this way a direct chain between afferent and efferent fibres seems to be established. Others of these cells give off axis-cylinder processes, which run upwards. headwards, forming strands in the white matter of the cord and end in parts, lying in many cases at least in the brain above the cord. Such cells form relays in the transmission of afferent impulses; the posterior vesicular eylinder, the column of Clarke, is a group of such cells.

In a certain number of cases however the (anterior divisions of the) fibres do not make such speedy connections, but run upwards in the median posterior column, column of Goll, which indeed they for the most part form. Hence when a posterior root is cut, degenerating fibres are found in the median posterior column above the entrance of the root, and may be traced as an 'ascending' degeneration right up to the spinal bulb. The fibres coming from successive roots take up definite positions in the column as is illustrated in Fig. 114. The contribution to the column furnished by each root diminishes however upwards, as shewn by the diminishing area of degeneration; that is to say, some of the fibres of the posterior root after running in the median posterior column for a greater or less distance, and the distance may be considerable, eventually turn aside, and enter the grey matter of the cord; here they end in connection with nerve cells. The contribution to the column, though thus diminished, may be traced to the spinal bulb; here the remaining fibres of the root end in connection witl the cells of the gracile mucleus.

Thus the fibres forming the posterior root of each spinal nerve have a wide and manifold grip upon the central nervous system. Some stretch right beyond the spinal cord and lay hold of the spinal bulb; and these, it will be remarked, keep entirely to the side on which they enter. Others, rumning for variable distance in the posterior median column of the same side, lay hold of the grey matter, it may be far above the entrance of the root, while yet others lay hold of the grey matter soon after their entrance into the cord, and that either above 
or below the level of the entrance, on the same side or on the opposite side. In other words, an afferent impulse passing along the posterior root may, according to the course which it

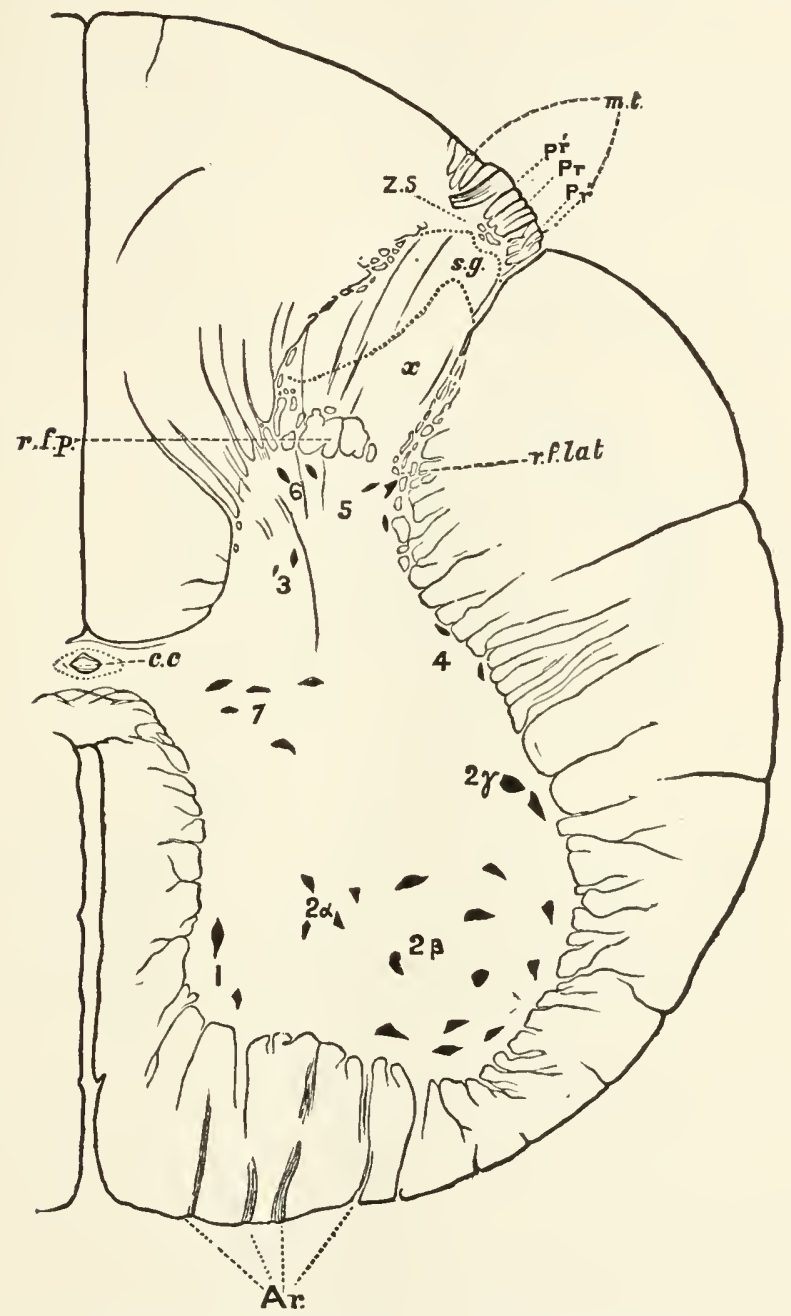

Fig. 112. Transverse Dorsoventral Section of the Spinal Cord (Human) at the Level of the Third Lumbar Nerve. (Sherrington.)

This is drawn to the same scale as Fig. 110, and in the same way except that the outline of the grey matter is not exaggerated. $\operatorname{Pr}^{\prime}$. median, $\mathrm{Pr}$. intermediate, $P r^{\prime \prime}$. lateral bundles of posterior roots. The region comprised under m.t. is the marginal zone or Lissauer's zone. The other letters of reference are the same as in 110 and 111.

'The three figures $110,111,112$ are intended to illustrate the main differential features of the thoracic, cervical, and lumbar cord. 
takes, produce effects almost immcdiately above or below, on the same or on the other side, or may have to travel some length upwards, on the same side, before it produces its effects, or may produce no effect on the spinal cord itself, but spend its whole strength on the spinal bulb.

The method of degeneration, confirmed by the method of development, has shewn that certain fibres, starting as axiscylinder processes of certain cells in the region of the cerebral cortex which we shall speak of as the motor area, finding their way to the spinal bulb through the crus cerebri, form the anterior pyramids of the bulb, cross at the decussation of the pyramids to the lateral columm of the cord and there form a definite strand, the "crossed pyramidal tract" (Figs. 113, 114). 'This

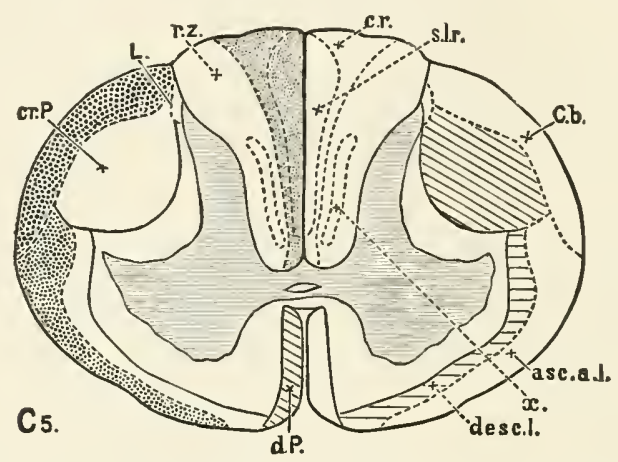

Fig. 113. Diagray to illustrate tile general arrangement of the several Tracts of Winte Matter in tile Spinal Cord. (Sherrington.)

The section is taken at the level of the fifth cervical nerve. The relations of the tracts in different regions of the cord are shewn in Fig. 114.

'The ascending tracts, tracts of ascending degeneration, are shaded with dots, the descending tracts, tracts of descending degeneration, are shaded with lines: the shading is in each case put on one side of the cord only, the reference letters being placed on the other side.

cr.P. crossed pyramidal tract, or more shortly pyramidal tract. $\quad$ d.P. direct pyramidal tract, shaded on the side opposite to that on which $\mathrm{cr} . P$. is shaded, in order to indicate the difference of the two as to crossing. C.b. cerebellar tract. s.lr. and c.r. together indicate the median posterior tract or tract of fibres of the posterior roots, c.r. representing, as is explained more fully in the text, the cervical and s.lr. the sacral, lumbar and dorsal roots. asc.a.l. the antero-lateral ascending tract. desc.l. the antero-lateral descending tract. The area, not shaded, marked $x$, is the small descending tract or rather patch mentioned in the text as observed, in certain regions of the cord, in the external posterior column $r z$. The small area at the tip of the posterior horn, marked $L$, is the posterior marginal zone or Lissauer's zone.

tract lying in the dorsal region of the lateral column, lateral to the posterior horn, and marked out by a 'descending' degeneration, may be traced down the whole length of the cord, diminishing as it goes. It diminishes because fibres successively leave it to make connections, also of contact merely not 
of continuity, with the cells of the anterior horn of the same side which give off axis-cylinder processes to form fibres of an anterior root. By means of it, impulses leaving the cells of the cortex of one side of the brain and crossing over in the spinal bulb are able to give rise to efferent impulses in the spinal nerves of the opposite side. Some of the fibres starting from like cells in the cortex do not take exactly this course; they do not cross over at the decussation of the pyramids, but continue along the same side of the cord, forming in the median part of the anterior column, the direct pyramid tract (Figs. 113, 114)。
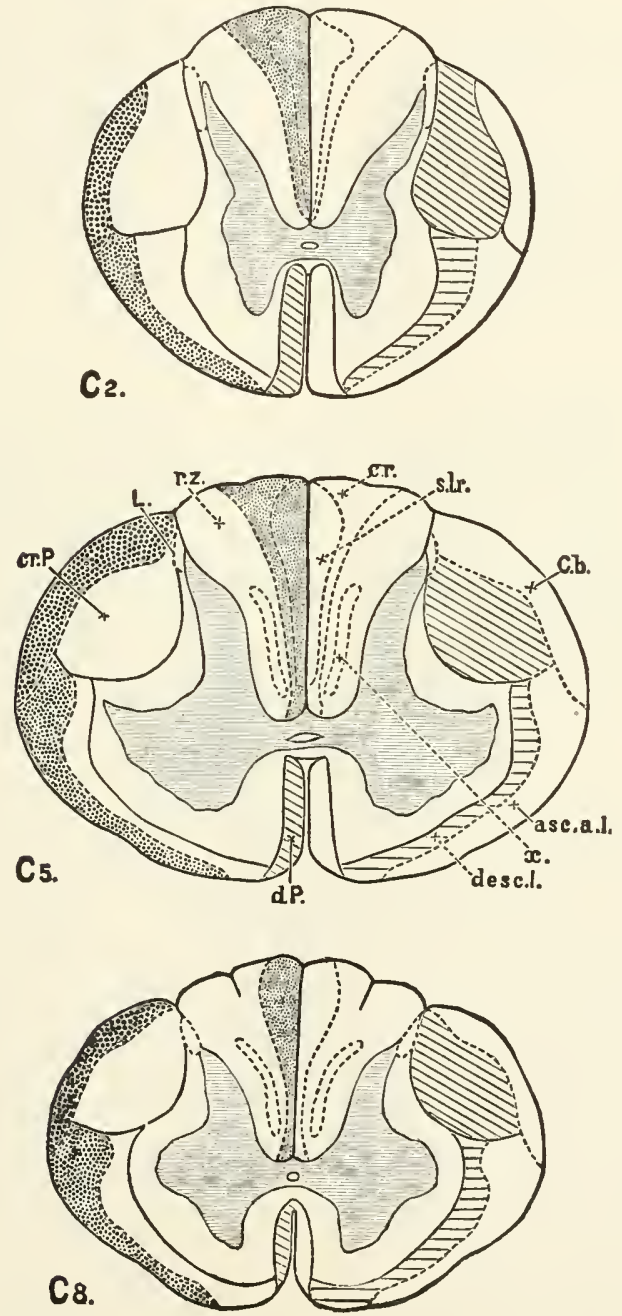

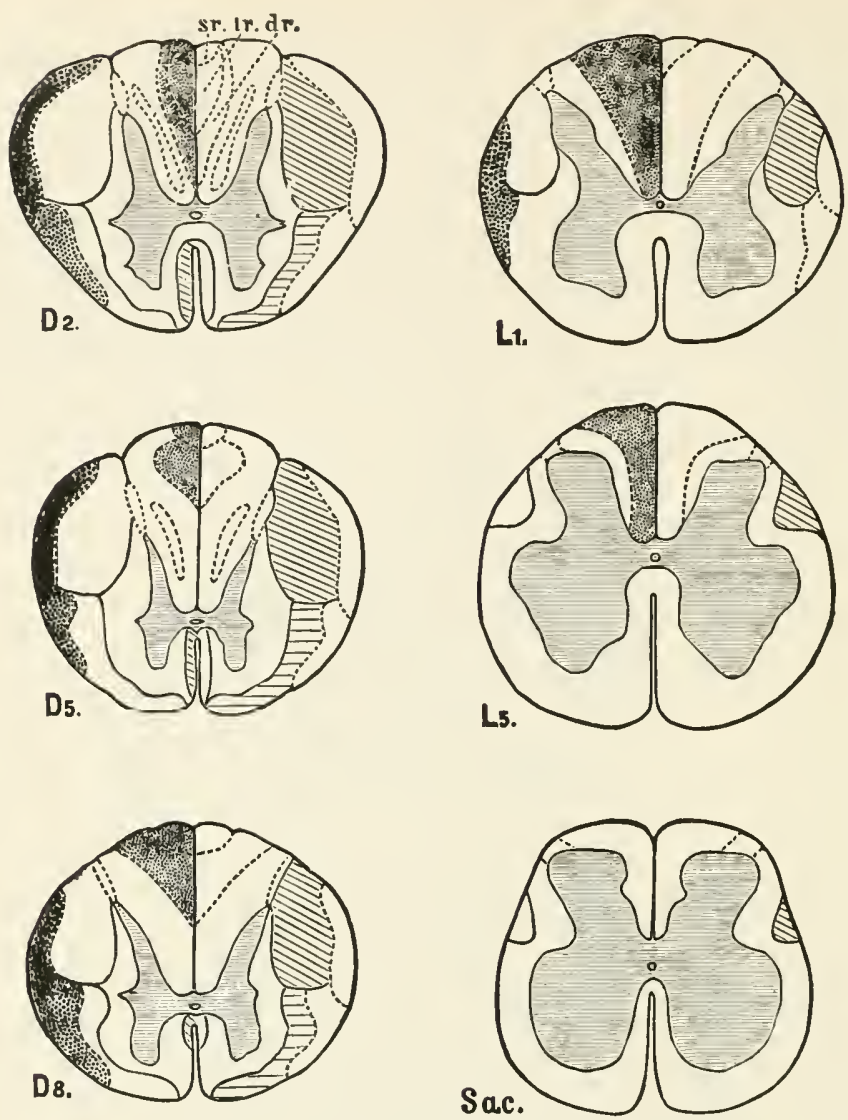

Fig. 114. Diagram illustrating some of the features of the Spinat Cord at DIFFERENT levels. (Sherrington.)

All the figures are drawn to scale, and represent the cord magnified four times. They shew the differences at different levels in the shape and size of the cord, in the outline of the grey matter, and in the relative position of the anterior and posterior fissures, and also shew the variations at different levels of the several 'tracts' of the white matter.

$\mathrm{C}_{2}$ at the level of the second cervical nerve, $\mathrm{C}_{5}$ of the fifth cervical, $\mathrm{C}_{8}$ of the eighth cervical. $D_{2}$ of the second thoracic, $D_{5}$ of the fifth thoracic, $I_{1}$ of the first lumbar, $\mathrm{L}_{5}$ of the fifth lumbar, and Sac. of the second sacral nerve.

The shading of the tracts is the same as in Fig. 113; but in the median posterior column of $\mathrm{D}_{2}$ the areas of fibres coming from the sacral nerves $s . r$, and lumbar nerves l.r. are distinguished from the area, d.r. of fibres belonging to the thoracic nerves. In C 8, no distinction is made between any of these sets of fibres; in L 5 only fibres of sacral nerves are represented ; in $\mathrm{L}_{1} \mathrm{D}_{8}$ $\mathrm{D}_{5}$, the more dorsal small portion corresponds to sacral fibres and the next to lumbar, or lumbar thoracic nerves.

This tract, very variable in different animals, is only found in the upper part of the cord; it diminishes rapidly and soon dis- 
appears, the fibres forming it, crossing over in the anterior commissure, pass to the nerve-cells of the anterior horn of the opposite side with which, like the brother-fibres of the crossed pyramidal tract, they make commections by contact.

A conspicuous tract in the white matter of the lateral column is the cerebellar tract (Figs. 113, 114) marked out by a descending degeneration. 'This, starting in the lower parts of the cord, and as a whole increasing as it goes, may be traced through the restiform body of the same side to the cerebellum. We have reason to believe that the cells, the axis-cylinder processes of cells which supply the fibres composing the tract, are those forming the posterior vesicular cylinder. The tract, we may conclude, carries to the cerebellum afferent impulses furnished by the posterior roots, but modified, we may presume, at the relay in the posterior vesicular cylinder.

Other tracts have also been made out in the white matter of the cord, but these are not so conspicuous as the above. It is inore important to remember that a large number of the fibres, both of the lateral and anterior (ventral) columns are axiscylinder processes of cells of one part of the cord, and end by arborescences making contact with cells in another part of the cord. Some of these fibres run their whole course on the same side of the cord; others cross over to the opposite side; these may be regarded as commissural fibres, longitudinal and transverse. Lastly, some of the cells in the grey matter are such that all their processes end as they begin, within the grey matter, and do not contribute at all to the white matter.

\$453. The Special Features of the several regions of the Spinal Cord. The cord begins below in the slender filament called the filum terminale, which lying in the vertebral canal, in the midst of the mass of nerve roots called the cauda equina, rapidly enlarges at about the level of the first lumbar vertebra into the conus medullaris. 'This may be regarded as the beginning of the lower portion of a fusiform enlargement of the cord known as the lumbar swelling, which reaches as high as about the attachment of the roots of the twelfth or eleventh thoracic nerve at the level of the eighth thoracic vertebra, the broadest part of the swelling being about opposite the third lumbar nerve. Above the lumbar swelling, through the thoracic region the somewhat narrowed cord retains about the same diameter until it reaches the level of the first or second thoracic nerve opposite the seventh cervical vertebra where a second fusiform enlargement, the cervical swelling. broader and longer than the lumbar swelling, begins. 'The broadest part of the cervical swelling is about opposite to the fifth or sixth cervical nerve; from thence the diameter of the cord becomes gradually somewhat less until it begins to expand into the bulb, but even in the highest part is greater than in the thoracic region. 'The sectional area of 
the cord increases therefore from below upwards, but not regularly, the irregularity being due to the lumbar and cervical swellings.

The cxtremity of the filum terminale is said to consist entirely of neuroglia closely invested by the membranes, even the central canal being alssent. A little higher up the central canal begins, and nerve-cells with nerve-fibres make their appearance in the neuroglia; thus a kind of grey matter covered by a thin superficial layer of white matter is established. We have elsewhere referred to the peculiar features of the lower end of the conus; but higher up the canal becomes central and small, the posterior columns are developed, and the grey matter contains more nervous elements and relatively less neuroglia, becomes in fact ordinary grey matter. From thence onward to very near the junction with the bulb, where transitional features begin to come in, the spinal cord may be said to have the general structure previously described.

The sectional area of the white matter increases in absolute size and on the whole in a steady manner from below upwards.

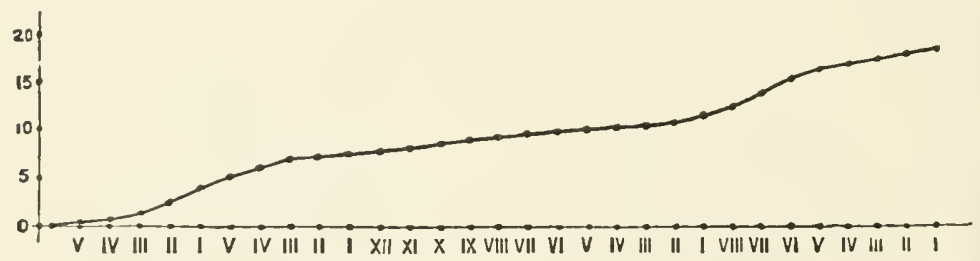

Fig. 115. Diagram shewing the united sectional areas of the Spinat, Nerves, proceediNg From Below upwards.

In this as in the succeeding figures $116-17,-18,-19,-20$, all of which refer to man, the left-hand side represents the bottom of the cord and the right-hand the top of the cord, the numerals indicating sucessfully the sacral, lumbar, thoracic and cervical nerves. The several figures are not drawn to the same scale.

In other words, in a section at any level, the number of longitudinal fibres forming the white matter is greater than the number at a lower level, and less than the number at a ligher level; for any difference which may exist in the diameter of the individual fibres is insufficient to explain the differences in the total sectional area of the white matter. If we were to measure in man the sectional area of each of the spinal nerves as it joins the cord, and to ard them together, passing along the cord from below npwards the results put in the form of a curve would give us some such figure as that shewn in Fig. 115; the area gained by adding together the sectional areas of the ncrves increases in a fairly steady manner from below upwards. The curve of the sectional area of the white matter of the cord taken from below upwards would be very similar, but if anything more 
regular. It must be understood however that the dimensions of the areas would not be the same in the two cases. The sectional area of the white matter at the top of the cervical region, though greater than anywhere lower down, is far less than the united sectional area of all the nerves below that level. The white matter is not formed by all the fibres from the nerves which join the spinal cord continuing to run along the cord up to the brain; as we have seen, some at least of the fibres end in the grey matter. Nevertheless the white matter in passing up the cord appears to receive a permanent addition at the entrance of each nerve. We may infer that each nerve has a representative of itself starting from the level of its entrance and running up to some part of the brain.

$\$ 454$. The grey matter in contrast to the white matter shews great variations in area along the length of the cord (Fig. 116). From the entrance of the coceygeal nerve upvards the

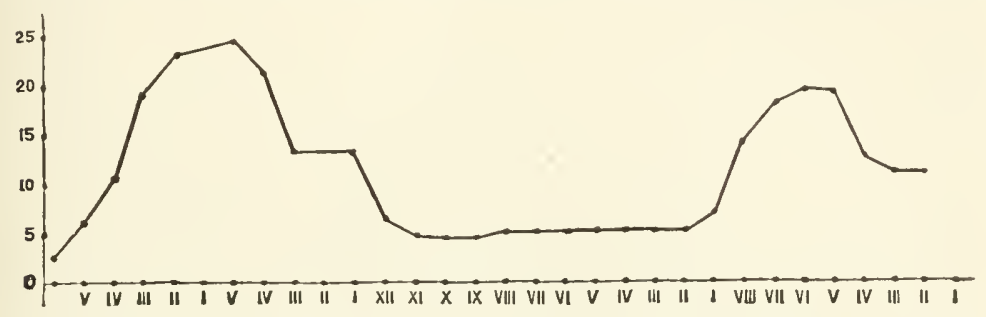

Fig. 116. Diagram shewing the variations in the sectional area of the grey matter of the Spinal Cord, along its lengti.

area increases very rapidly, reaching a maximum at about the level of the 5th lumbar nerve. It then rapidly decreases to about the level of the 11th thoracic nerve, maintains about the same dimensions all through the thoracic region, and begins to increase again at about the level of the 2nd thoracic nerve. Its second maximum is reached at about the level of the 5th or 6th cervical

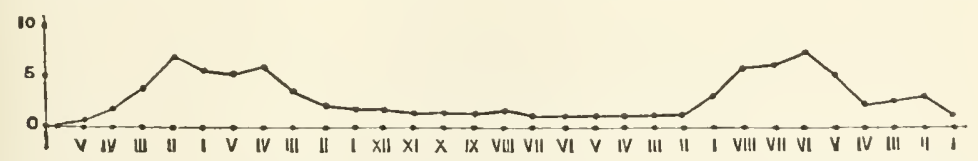

Fig. 117. Diagram shewing the relative sectional areas of tile Spinal Nerves, as tiney Joix tire Sphic Cord.

nerve, after which the area again becomes smaller, remaining however at the upper cervical region much larger than in the thoracic region.

The meaning of these variations becomes clear when we turn to Fig. 117, whieh shews in a similar diagrammatic manner the 
sectional areas of the several spinal nerves. It will be observed that the increase and decrease of the sectional area of the grey matter follow very closely the increase and decrease of the quantity of nerve, that is to say, neglecting differences in the diameter of the fibres, in the number of nerve-fibres passing into the cord. The sectional areas of the 1st and 2 nd sacral, 4 th and 5 th lumbar nerves are very large, and opposite to these the sectional area of the grey matter of the cord is very large also ; the enlargement of grey matter which is the essential cause of the lumbar swelling is correlated to the large number of fibres which enter and leave the cord at this region to supply chiefly the lower limbs. Similarly the enlargement of grey matter which is the essential cause of the cervical swelling is correlated to the large number of fibres which enter and leave this region of the cord to supply chiefly the upper limbs. In the thoracic region, where the number of fibres entering and leaving the cord is relatively less, the scetional area of the grey matter is also less. Since the attachments of the several spinal nerves are not exactly equidistant from each other along the length of the cord, the sectional area is not an exact measure of bulk; the total bulk of grey matter for instance belonging to two nerves which enter the cord close together is less than that of two nerves giving rise to the same sectional area of grey matter as the former two but entering the cord far apart from each other. Still the error which may be introduced by taking sectional area to mean bulk is, for the present purposes at all events, so small that we may permit oursclves to say that in the successive regions of the spinal cord the bulk of grey matter in any segment is greater or less according to the size of the nerve (or pair of nerves, right and left) belonging to that segment.

From this anatomical fact we appear justified in drawing the conclusion that at all events a great deal of the grey matter of the spinal cord may be considered as furnishing a nervous mechanism, with which the efferent fibres of each spinal ncrve just before they leave the cord, and the afferent fibres soon after they join the cord are more immediately connected. It may be that the whole of the grey matter is thus directly connected with and thus rises and falls with the fibres of the nerves; or it may be that there is a sort of core of grey matter, which maintains a uniform bulk along the whole length of the cord and serves as a basis which is here more and there less swollen by the addition of the grey matter more immediatcly connected with the fibres of the nerves. This question the method which we are now using cannot settle.

$\$ 455$. Owing to these different rates of increase of the grey and white matter respectively along the length of the cord, we find that in sections of the cord taken at different levels the appearances presented vary in a very distinct man- 
ner. This is strikingly shewn by comparing Figs. 110, 111 and 112. At the level of the third lumbar nerve (Fig. 112) the grey matter is very large, reaching, as we have seen, its maximal sectional area at about this point, so that although the area of white matter is not very great the whole area of the cord is considerable.

At the level of the sixth thoracic nerve (Fig. 110), in spite of the white matter having very deciledly increased, the grey matter has shrunk to such very small dimensions, that the total sectional area of the cord has markedly diminished.

At the level of the sixth eervical (Fig. 111) the grey matter has again increased, reaching here as we have seen its second maximum; the white matter has also furtler increased, and that indeed very considerably, so that the total area of the cord is much greater than in any of the lower regions.

Further details of the varying size of the white matter and of the grey matter at different levels are also shewn in the series given in Fig. 114. In these, combined with the three figures just referred to. it will be observed that the serial increase and decrease of the grey matter does not affect all parts of the grey matter alike, so that the outline of the grey matter changes very markedly in passing from below upwards. In the coccygeal region each lateral half is a somewhat irregular oval, and in the sacral region, Fig. 114, Sae, the differentiation into anterior and posterior horns is still very indistinct. In the lumbar region the two horns are sharply marked out, though both the posterior and anterior horns are broad and more or less quadrate. In the thoracic region the decrease of grey matter has affected both horns, so that both are pointed and slender, while the junction between them has not undergone so much diminution, so that what has been called the lateral horn is relatively conspicuous. In the cervical region the returning increase bears much more on the anterior horn which again becomes large and broad, than on the posterior horn which still remains slender and pointed. Taking the form of the grey matter in the thoracic region as the more typical form of the grey matter we may say that while the increase on the lumbar swelling bears equally on the anterior and posterior horns, that in the cervical region bears chiefly on the anterior horns.

$\$ 456$. The white matter as we have seen increases in sectional area with considerable regularity from below upwards. If instead of a diagram of the increase of the whole white matter, we construct in a similar way diagrams of the anterior, posterior and lateral columns respectively we find that while the sectional area of the lateral column (Fig. 118) increases with some considerable regularity from below upwards, though not so regularly as does the whole area of white matter, both 
the anterior (Fig. 119) and the posterior (Fig. 120) columns agrce to a certain extent with the grey matter in shewing a decided increase in both the lumbar and the cervical swellings. We may, provisionally at least, infer from this that, while considerable portions of both the anterior and the posterior columms are like the arljoining grey matter in sone way or other concerned in the exit and entrance of cfferent and afferent fibres, the larger portion of the lateral column is concerned in the transmission of impulses to and fro, betwecn the local mechanisms below, immediately connected with the several

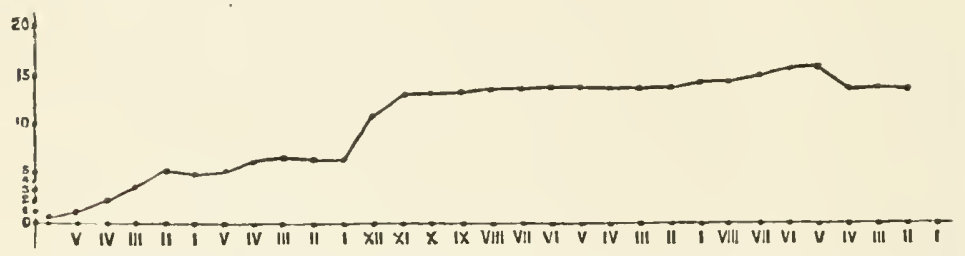

Fig. 118. Diagrat Siewing the variations in time sectional area of the lateral colums of tile Spinal Cord, along its lengtil.

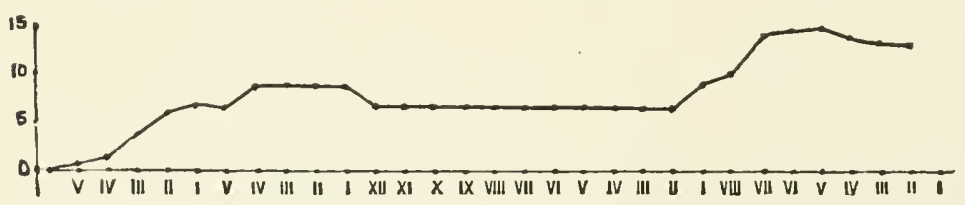

Fig. 119. Diagrai SHEWING THE VARIATIONS IN THE SECTIONAL AREA OF the anterior Columins of the Spinal Cord, along its length.

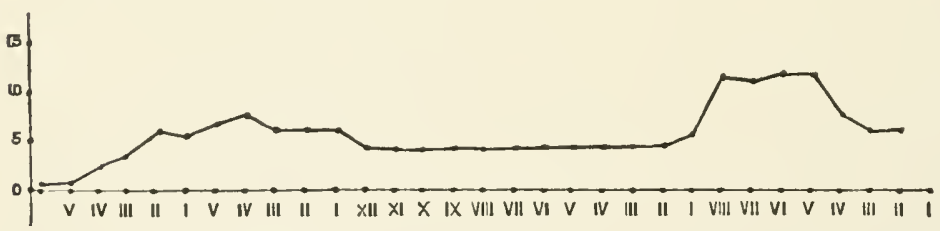

Fig. 120. Dhagray silewing tile variations in tile sectional area of tile posterior colums of the Srinal Cord, along its length.

spinal nerves, and the brain above. This conchusion seems incidentally confirmed, (though these diagrams must not be strained to carry detailed inferences,) by the sudden increase of the lateral column above the lumbar swelling, as if the large mass of nervous mechanism for the lower limbs concentrated in this region demanded a sudden increase in the number of fibres connecting it with the brain above.

'This more or less continuous increase of the lateral column partly explains the change of form in the general outline of 
the transverse section of the cord which is observed in passing upwards from the lower to the higher regions. In the coceygeal, sacral and lumbar regions the outline, though varying somewhat ehiefly owing to the disposition of the grey matter, is on the whole circular. In the thoracic region especially in the upper part the increase of the lateral columns increases the side to side diameter so much that the section becomes oval, and in the cervical region this increase of the side to side diameter out of proportion to the dorso-ventral diameter is very marked. The actual outline of the whole transverse section is however determined also to a certain extent by the changes of for'm of the grey matter.

The cord morcover undergoes along its length a change which is not very clearly indicated in the diagrams Figs. 119, 120. By comparing the series of transverse sections given in Fig. 114 it will be seen that the relative position of the central canal shifts along the length of the cord. In the sacral and lumbar regions the central canal is nearly at the centre of the circle of outline, and the posterior and anterior fissures are nearly of equal depth. Even in the upper lumbar region, and still more in the thoracic region, the position of the central canal is shifted nearer to the rentral surface so that the posterior fissure becomes relatively longer, deeper, than the anterior. This shifting goes on through the cervical region up to about the level of the 2nd cervical nerve, where it is arrested by the beginning of the changes through which the spinal cord is transformed into the far more complicated bulb.

This lengthening of the posterior fissure indicates an increase in the dorso-ventral diameter of the postcrior columns, and this, not being accompanied by a compensating diminution of the side to side diameter, shews in turn that the posterior columns undergo an increase in passing upwards. From this we may add to the provisional conclusion just arrived at with regard to the lateral columns, the further conclusion that some part of the posterior columns also is concerned in transmitting impulses, in a more or less direct manner, between the various regions of the cord below and the brain above. The anterior columns do not increase in the same marked manner, though over and above the increase due to the lumbar and cervical swellings, a continued increase may be observed especially in the upper cervical region; it is in this upper region that the direct pyramidal tract is best developed. 


\section{SEC. 3. THE REFLEX ACTIONS OF THE SPINAL CORD.}

$\S 457$. In the preceding portions of this work we have repeatedly seen that though we can learn much concerning the working of an organ, or tissue or part of the body by studying its behaviour when isolated from the rest of the body, all the conclusions thus gained have to be checked by a study of the behaviour of the same organ or part, while it is still an integral part of the intact body. All the several organs and tissues are so bound together by various ties, that the actions of each depend on the actions of the rest; and to say that the life of each part is a function of the life of the whole, is no less true than to say that the life of the whole is a function of the life of each part. This is especially borne in upon us, when we come to study the actions of the central nervous system. $\mathrm{We}$ may, on anatomical grounds, separate the spinal cord from the brain; but when we come to consider the respective functions of the two, we are brought face to face with the fact that in actual life a large part of the work of the brain is carried out by means of the spinal cord, and conversely the spinal cord does its work habitually under the influence of, if not at the direct bidding of the brain. We may gain certain conclusions by studying the behaviour of the spinal cord isolated from the brain, or of parts of the spinal cord isolated from each other; but we must be even more cautious than when we were dealing with other parts of the body, and must greatly hesitate to take it for granted that the work which we can make the spinal cord or a part of the spinal cord do, when isolated from the brain, is the work which is actually done in the intact body when the brain and spinal eord form an unbroken whole. Moreover this caution becomes increasingly necessary, when in our studies we pass from the simpler nervous system of one animal to the more complex nervous system of another ; for it is by the complexity of their central nervous systems more than by anything else, that the 'highest' animals are differentiated from those 'below' them. When we compare a rabbit, a dog, a monkey and a man, the differences in the vascular, 
digestive and respiratory systems of the four, striking as they may appear, sink into insignificance compared with the differences exhibited by their respective central nervous systems. We nced caution when from the rcsults of experiments on dogs or rabbits, we draw conclusions as to the digestion or circulation of man, but we need far greater caution when from the behaviour of the isolated spinal cord of one of these animals we infer the behaviour of the intact spinal cord of man.

A further difficulty meets us when an experimental investigation entails operative interference with the central nervous system. Removal or section of, or other injury to parts of the brain or spinal cord is very apt to give rise in varying degree to what is known as 'shock.' 'The cutting or tearing or other lesion of any considerable mass of nervous substance affects the activity, not only of the structures inmediately injurcd, but of other, it may be far distant, structures. 'The nature of 'shock' is not as yet thoroughly understood, but may perhaps, in part at all events, be explained by regarding the lesion as a very powerful stimulus, which, partly by way of inhibition but still more by way of exhaustion, depresses or suspends for a while normal functions, and thus gives rise to temporary diminution or loss of consciousness, or volition, of reflex movements and other nervous actions. 'Thus a section through the spinal cord, even when made with the sharpest instrument and with the utmost skill, so as to avoid all bruising as much as possible, may for a while suspend all reflex activity of the cord, or indeed all the obvious activities of the whole central nervous system. We may add that such a "shock' of the central nervous system may also be produced by sudden lesions not bearing directly on the central nervous system, as for instance by extensive injury to a limb.

Moreover in many cases in which the effects of experimental interference have been watched for some considerable time, days, months or years after the operation, it has been observed, on the one hand, that phenomena which are conspicuous in the early period may eventually disappear, and, on the other hand, that activities which are at first absent may later on make their appearance; movements for instance which are at first frequent after a while die away, and conversely, movements which at first seemed impossible are later on easily achieved. We have to distinguish or to attempt to distinguish between the temporary and the lasting effects of the operation, including anong the former not only those of ordinary 'shock,' but others of slower development or longer duration. In many instances where a part of the central nervous system is by section or otherwise suddenly separated from the rest, the phenomena suggest that the separated part is at first profoundly influenced as to its activities by the withdrawal of various influences which 
previously were being exerted upon it by the rest of the system, but later on accommodates itself to its new eonditions, and learns, so to speak, to aet without the help of those influences. And indeed it is possible that some of the effects of even immediate 'shoek' may be due, not, as suggested above, to the action of an inhibitory or exhausting stimulus, but to the sudden eessation of habitual influenees.

Still, in spite of all these diffieulties, it is possible not only to ascertain the working of an isolated portion of the central nervons system, but even to infer from the results some ennelusions as to the share taken by that portion in the working of the entire and intaet system. There ean be no doubt, for instance, that the spinal cord ean, quite apart from the brain, carry ont various reflex aetions, and that moreover it does carry out aetions of this kind when in the intaet organism it is working in eoncert with the brain. Indeed the earrying out of various reflex aetions seems to be one of the most important functions of the spinal cord, so much so that, though the brain or, at least, parts of the brain ean also and do develope reflex actions, the spinal cord offers the best field for the study of these actions. We have already $(\$ 90)$ touehed on the general features of reflex aetions, and elsewhere have ineidentally dwelt on particular instanees; we may therefore confine ourselves now to certain points of special interest.

$\$ 458$. Many of the features of reflex movements are best studied in the frog and other cold-blooded animals, sinee in these the aetions of the cord are less dependent on, and henee less obscured by the working of, the other parts of the eentral nervous system. In these animals moreover the shoek, which as we have said follows upon division of the spinal cord, and for a while suspends reflex aetivity, soon passes away; within a very short time after the bulb for instance has been divided the most eomplieated reflex movements ean be earried on by the frog's spinal eord when the appropriate stimuli are applied. In the frog reflex actions may be earried out by a very small portion of the cord, by a single segment corresponding to a pair of nerves, isolated by two transverse seetions from the parts above and below. Stimulation of the sensory fibres of the nerve belonging to the segment will give rise to eontraetions in the museles supplied by the motor fibres of the nerve. The movements thus evoked are naturally simple in eharacter. When a larger portion of the eord is experimented upon, and especially when the whole eord is employed, there are brought to light what are perhaps the most notable features of reflex movements, their eomplexity and purposeful eharaeter; a few afferent impulses developed in a few afferent fibres may lead to many muscles being thrown into eontractions, the time, order and vigour of the several contractions being so related 
to each other, being so "coordinated" that the movement, the result of the contractions, secures some end, either apparent or real. Thus when the afferent fibres distribnted to a small, it may be a very small, area of the skin of the flank are stimulated by placing on the area a small piece of paper soakerl in dilute acid, the hind leg of the same side, by a series of flexions and extensions, repeatedly sweeps over the spot with the foot, the purpose of the movement, namely, to brush off the cause of irritation, being obvious. For the carrying out of any reflex movement, simple or complex, some portion of the grey matter of the cord must intervene between the afferent and efferent fibres. In the simpler movement, spoken of above, as carried out by a single segment of the cord, we may perhaps suppose that the arborescent terminations of the afferent fibres impinging on the nerve-cells giving off the efferent fibres, supply all the nervous mechanism which is needed. In the more complex and more ordinary movements, the mechanism must be proportionately more complex; and we may perhaps conclude that in these other cells of the grey matter intervene between the termination of the afferent fibre, and the cell whose axis-cylinder process is the efferent fibre. In any case we may venture to speak of a nervous mechanism within the cord as the means by which a reflex movement is carried out.

The exact working of such a mechanism in each particular case, and so the character of the movement carried out, is determined by various causes. It is in part determined by the characters of the afferent impulses. Simple nervous impulses generated by the direct stimulation of afferent nerve fibres generally evoke as reflex movements merely irregular spasms in a few muscles; whereas the more complicated differentiated sensory impulses generated by the application of the stimulus to the skin, leadily give rise to large and purposeful movements. It is easier to produce a complex reflex action by a slight pressure on or other stimulation of the skin than by even strong induction-shocks applied directly to a nerve trunk. If, in a brainless frog, the area of skin supplied by one of the dorsal cutaneous nerves be separated by section from the rest of the skin of the back, the nerve being left attached to the piece of skin and carefully protected from injury, it will be found that slight stimuli applied to the surface of the piece of skin easily evoke reflex actions, whereas the trunk of the nerve may be stimulated with even strong currents without producing anything more than irregular movements.

$\S 459$. The character of the movement forming part of a reflex action is also influenced by the intensity of the stimulus. A slight stimulus, such as gentle contact of the skin with some body, will produce one kind of movement; and a strong stimulus, such as a sharp prick applied to the same spot of skin, will 
call forth quite a different movement. When a decapitated snake or newt is suspended and the skin of the tail lightly touched with the finger, the tail bends towards the finger; when the skin is pricked or burnt, the tail is turned away from the offending object. And so in many other instances. It must be remembered of course that a difference in the intensity of the stimulus entails a difference in the characters of the afferent impulses; gentle contact gives rise to what we call a sensation of touch, while a sharp prick gives rise to pain, consciousness being differently affected in the two cases because the afferent impulses are different. Hence the instances in question are in reality fuller illustrations of the dependence of the characters of a reflex movement on the characters of the afferent impulses.

$\S 460$. Further, the movement, forming part of a reflex action, varies in character according to the particular part of the body to which the stimulus is applied. The reflex actions developed by stimulation of the internal viscera are different from those excited by stimulation of the skin. We have reason to think that the contraction of or other changes in a skeletal muscle may produce, by reflex action, contractions of other muscles; and such reflex actions also differ from those started by stimulation of the skin. In reflex actions started by applying a stimulus to the skin the movements vary largely according to the particular area of the skin which is affected. Thus, pinching the folds of skin surrounding the anus of the frog produces different effects from those witnessed when the flank or the toe is pinched; and, speaking generally, the stimulation of a particular spot calls forth particular movements. In the case of the simpler reflex movements, it appears to be a general rule that a movement started by the stimulation of a sensory surface or region on one side of the body, is dereloped on the same side of the body, and if it spreads to the other side, still remains most intense on the same side; the movement on the other side moreover is symmetrical with that on the same side.

$\S 461$. From these and similar phenomena it is obvious that when we allow ourselves to speak of the grey matter of the cord as supplying central mechanisms for carrying out reflex movements we must not regard these several mechanisms as rigidly separate from each other or indeed as anatomically distinct. On the contrary without any anatomical change, as the result simply of physiological changes, the afferent impulses reaching the cord along a set of afferent fibres, and it may be any set, will under certain conditions give rise to efferent impulses, not restricted to a definite set of efferent fibres and so leading to a definite purposeful movement, but overflowing along almost all the efferent fibres leaving the cord, and bringing about contractions of almost all the skeletal muscles. Thus when a frog is poisoned with strychnia, a mere touch of the skin at almost 
any point will bring out tetanic convulsions of the whole body. In such a case the strychnia has produced no anatomical change, it has only modified the molecular condition of the substance of the cord; and that has led to the apparent disappearance of all the central mechanisms directing the paths of impulses; these now seem to travel in all directions all over the cord. In other words the mechanisms of which we are speaking are mapped out, not by anatomical relations, but by the physiological condition of the cord. Hence we cannot always predict exactly the nature of the movement which will result from the stimulation of any particular spot, because the result will vary according to the condition of the spinal cord, especially in relation to the strength and character of the stimulus. Indeed, under a change of circumstances a movement quite different from the normal one may make its appearance. 'Thus when a drop of acid is placed on the right flank of a brainless frog, the right foot is almost invariably used to rub off the acid; in this there appears nothing more than a mere 'mechanical' reflex action. If however the right leg be cut off, or the right foot be otherwise hindered from rubbing off the acid, the left foot is, under the exceptional circumstances, used for the purpose. This at first sight looks like an intelligent choice. A choice it evidently is ; and were there many instances of choice, and were there any evidence of a variable automatisn, like that which we call 'volition.' being manifested by the spinal cord of the frog. we should be justified in supposing that the choice was determined by an intelligence. But, as we shall have occasion later on to point out, a frog, deprived of its brain so that the spinal cord only is left, makes no spontaneous movements at all. Such an entire absence of spontaneity is wholly inconsistent with the possession of intelligence. Then again the above experiment, if not the only instance. is at all events by far the most striking instance of choice on the part of a brainless frog. We are therefore led to conclude that the phenomena must be explained in some other way than by being referred to the working of an intelligence. Moreover this conclusion is supported by the behaviour of other animals. Thus similar vicarious reflex movements may be witnessed in mammals, though not perhaps to such a striking extent as in frogs. In dogs, in which partial removal of the cerebral hemispheres has apparently heightened the reflex excitability of the spinal cord, the remarkable scratching movements by means of the hind leg which are called forth by stimulating a particular spot on the loins or side of the body, are executed by the leg of the opposite side, if the leg of the same side be gently held. In this case the vicarious movements are ineffectual, the leg not being. as in the case of the frog, crossed over so as to bear on the spot stimulated, but simply made to scratch the corresponding but unstimulated spot on its own side, and cannot be considered as 
betokening intelligence. Again the 'mechanical' nature of reflex actions is well illustrated by the bchaviour of a decapitated snake. When the body of the animal in this condition is brought into contact at several places at once with an arm or a stick, complex reflex movements are excited, the obvious purpose as well as effect of which is to twine the body round the object. A decapitated snake will however with equal and fatal readiness twine itself round a red-hot bar of iron, which is made to touch its skin in sereral places at the same time.

$\$ 462$. The explanation of the above-quoted instance of apparent choice on the part of the frog's spinal cord is to be sought for in the modifications of the activity of the central mechanism, resulting from afferent impulses other than those which supply the exciting cause of the reflex movement. The existence and importance of these other afferent impulses will be seen if we bear in mind that most reflex movements are, as we have said, 'coordinated;' that is to say not only are many distinct muscles brought into play but certain relations are maintained between the amount, duration and exact time of occurrence of the contraction of each muscle and those of the contractions of its fellow muscles sharing in the movement. In the absence of such coordination the movement would become irregular and ineffectual. We shall have occasion later on in dealing with voluntary movements to point out that the coordination and hence the due accomplishment of a voluntary movement is dependent on certain afferent impulses passing up from the contracting muscies to the central nervous system, and guiding the discharge of the efferent impulses which call forth the contractions. When these afferent impulses affect consciousness we speak of them as constituting a 'muscular' sense;' it is, as we shall see, by the 'muscular sense' that we become aware of and can appreciate the condition of our muscles. But we have reason to think that the afferent impulses which constitute the basis of the muscular sense, whatever be their exact nature, in order to play their part in bringing about the coordination of a voluntary movement need not pass right up to the brain and develope a distinct muscular 'sense,' but may produce their effect by working on the nervous mechanisms of the spinal cord with which the motor fibres carrying out the movement are connected. In other words, the coordination of a voluntary movement may take place in the part of the spinal cord which earries out the movement.

But if the spinal cord possesses mechanisms for carrying ont coordinated movements, which in the case of voluntary movements are discharged by nervons impulses descending from the brain, we may infer that in reflex actions the same mechanisms are brought into action though they are discharged by afferent impulses coming along afferent nerves instead of by impulses 
descending from the brain. The movements of reflex origin, in all their features except their exciting cause, appear identical with voluntary movements; the two can only be distinguished from each other by a knowledge of the exciting cause. And it seems unreasonable to suppose that the spinal cord should possess two sets of mechanisms in all respects identical save that the one is discharged by volitional impulses from the brain and the other by afferent impulses from afferent nerves. $\quad \mathrm{W}$ e are led therefore to the conclusion that in a reflex action two kinds of afferent impulses are concerned: the ordinary afferent impulses which discharge the nervous mechanism within the cord and so provoke the movement, and the afferent impulses which connect that nervous mechanism with the muscles about to be called into play, and which take part in the coordination of the movement provoked. The nature of these latter afferent impulses is at present obscure; but if we admit. as we seem compelled to do, that the character of a reflex action is determined by them as well as by the afferent impulses which actually discharge the mechanism, the way is opened for an explanation of the fact that when, as in the case of the frog in question, the usual set of muscles cannot be employed by the nervous mechanism, recourse is had to another set.

$\$ 463$. Lastly, the characters of a reflex movement are, as we need hardly say, dependent on the intrinsic condition of the cord. The action of strychnia just alluded to is an instance of an augmentation of reflex action. Conversely, by various influences of a depressing character, as by various ancesthetics or other poisons, reflex action may be lessened or prevented. So also, various diseases may so affect the spinal cord as to produce on the one hand increased reflex excitability so that a mere touch may produce a violent movement, and on the other hand diminished reflex excitability so that it becomes difficult or impossible to call forth a reflex action.

In the mammal the study of reflex action is rendered somewhat difficult by the effects of shock to which we referred above. For days even after division of the spinal cord the parts of the body supplied by nerves springing from the cord below the section may exhibit very feeble reactions only. In the dog, for instance, after division of the spinal cord in the lower thoracic region, the hind limbs hang flaceid and motionless, and pinching the hind foot evokes as a response either slight irregular movements or none at all. Indeed were our observations limited to this period we might infer that the reflex actions of the spinal cord in the mammal were but feeble and insignificant. If however the animal be kept alive for a longer period, for weeks or better still for months, though no union or regeneration of the spinal cord takes place, reflex movements of a powerful, varied and complex character mani- 
fest themselves in the hind limbs and hinder parts of the body ; a very feeble stimulus applied to the skin of these regions promptly gives rise to extensive and yet coordinate movements. Indeed the more the matter is studied, the stronger is the evidence that the reflex movements carried out by isolated portions of the spinal cord of the mammal are hardly less definite, complete and purposeful, than those witnessed in the frog. And the main points on which we have dwelt above in relation to the frog hold good for the mammal. It is worthy of attention, as bcaring out the remarks made above on the great differentiation of the central nervous system in the higher animals, that the reflex phenomena in mammals vary very much not only in different species but also in different individuals and in the same individual under different circumstances. Race, age, and previous training, seem to have a marked effect in determining the extent and character of the reflex actions which the spinal cord is capable of carrying out; and these seem also to be largely influenced by passing circumstances, such as whether food has been recently taken or no. It has been asserted that the isolated spinal cord of the rabbit, which has been the subject of so many experiments, is, as compared with that of the dog and many other mammals, singularly deficient in the power of carrying out complex reflex movements.

$\$ 464$. When we come to study the reflex actions of man we should at first perhaps be inclined to infer that, since in him the spinal cord is so largely used as the instrument of the brain. the independent reflex actions of the cord, at least such as affect skelctal muscles, are in him of much less importance than they appear to be in animals; and experience seems to support this view. But it must be remembered that in his case, as we have already stated (\$458), we lack the guidance of experimental results; we are obliged to trust to the entangled phenomena of discasc or to a study of the behaviour of the cord while it is still a part of an intact nervous system; and each of these methods presents difficulties of its own. The movements, which in the intact human body we can recognize as indubitable reflex actions, are as a rule simple and unimportant. They are, in by far the greater number of instances, occasioned by stimulation of the skin or of the mucous membrane, for the most part involve a few muscles only, and rarely indicate any very complex coordination. The flexion, followed by extension, of the leg which is called forth by tickling the sole of the foot, may perhaps be regarded as the type of these movements. A very common form of reflex action is that in which a muscle or group of muscles is thrown into contraction by stimulation of the overlying or neighbouring skin, as when the abdominal muscles contract upon stroking the skin of the 
abdomen or the testicle is retracted upon stroking the inside of the thigh. A special form of reflex action, or at least an action maintained by many to be a reflex action, is called forth by sharply striking certain tendons. It is well known for instance that when the leg is placed in an easy position, resting for instance on the other leg, a sharp blow on the patellar tendon will cause a sudden jerk forward of the leg, brought about by a contraction of part of the quadriceps femoris ; it is necessary or at least desirable for a good development of the jerk that the tendon (and muscle) should be somewhat on the stretch. Similarly the muscles of the calf may be thrown into action by tapping the tendo Achillis put somewhat on the stretch by flexion of the foot; and in some cases the same muscles may be made to execute a series of regular rhythmic contractions, called 'clonic' contractions. by suddenly pressing back the sole of the foot so as to put them on the stretch. 'The "knee-jerk," as the sudden extension of the leg when the patella is struck is familiarly called, has acquired great interest in medical practice, and has been carefully studied not only in man but in various animals. It has been contended by some that the act is not truly a reflex one, but the evidence on the whole goes to shew that it is. From various experiments we learn that in it as in an ordinary reflex movement there are three factors, namely, an afferent limb, a spinal centre and an efferent limb; if any one of these three factors fails, the whole act fails. The efferent limb is furnished by fibres in the anterior crural nerve which reaching that nerve by the anterior roots of (in man) the $3 d$ and th lumbar nerves, and passing to the vastus internus muscle and adjoining portions of the crureus muscle, these being the parts of the great quadriceps concerned. The spinal centre lies in the lumbar portion of the cord, in what in man corresponds to the $3 d$ and 4 th lumbar segments. The afferent limb is furnished by fibres starting in the patellar tendon, rumning in the anterior crural nerve, and reaching the cord by the posterior root of (in man) the th lumbar nerve. It is further worthy of notice as an illustration of what we were urging a little while back that the vigour of the knee-jerk is influenced by the antagonistic hamstring muscles, and that not merely in a mechanical way but by nervous action. The knee-jerk is reinforced by cutting the nerves supplying the hamstring muscles, or by simple division of certain posterior roots, fibres of which take origin in those muscles or their tendons; it is depressed by central stimulation of those nerves, or by simply stretching the hamstring muscles. It would appear that these flexor muscles antagonistic to the extensor muscles, send up to the spinal cord, according to their condition, afferent impulses which influence the spinal centre for the knee-jerk. The activity of the same centre may also be 
influenced, in the way of angmentation or depression, by nerrous impulses reaching it from other parts of the nervous system.

When we turn to the teaching of disease, we again find that reflex movements carried out by the cord or by parts of the cord are, on the whole, scanty and simple.

In some stages of certain diseases of the spinal cord extensive reflex movements it is true are witnessed; but these are not purposeful coordinated movements, such as have been described above as occurring in frogs and mammals after experimental interference, but rather mere exaggerations of the simpler reflex movements witnessed when the nervous system is intact. In cases of paraplegia (such being the term generally used when disease or injury has cut off the cord, generally the lower part of the cord, from the brain so that the will cannot bring about movements in, and the mind derives no sensations from, the parts below the lesion, the legs for instance), it sometimes happens that contact with the bedclothes, or other external objects, sets up from time to time rhythmically repeated movements, the legs being alternately drawn up and thrust out again. And an exaggeration of the 'knee-jerk' or other 'tendon reflexes' is a very common symptom in certain spinal diseases. It is rarely if ever that reflex movements of a really complicated character are observed. Moreover clinical experience shews that in man, when a portion of the cord is isolated, reflex actions carried out by means of that portion so far from being exaggerated are much more commonly exceeding feeble or absent altogether. In the cases in which the physiological continuity of the lower with the upper part of the cord has been broken by disease, by some growth invading the nervous structures or by some changes of the nervous structures themselves, we may attempt to explain the absence from the lower part of coordinate reflex activity, such as is seen in the lower animals, as due to the disease not only affecting the powers of the actually diseased part, but influencing the whole cord below, and either by inhibition. of which we shall speak presently, or in some other way depressing its functions. But the same absence of complex reflex movements is also often observed in cases in which the cord has been severed by accident, and indeed, though accidental injuries to the human cord generally produce more profound and extensive mischief than that which results in animals from skilful experimental interference, clinical experience tends, on the whole, to support the view that in man the more complete subordination of the spinal cord to the brain has led to the dying out of the complex reflex actions which are so conspicuous in the lower animals. This however cannot be regarded as distinctly proved.

$\$ 465$. We have dwelt above chiefly on reflex actions, 
in which the efferent impulses cause contractions of skeletal muscles since these are undoubtedly the most common and the most prominent forms of reflex action; but it must not be forgotten that the efferent impulses of reflex origin may produce contractions of other muscles, as well as other effects, such as secretion for instance. On several of these we have dwelt, from time to time in previous parts of this work, and it will be unnecessary to repeat them here. But it may be worth while to point out that the spinal cord by serving as a reflex centre for innumerable ties which correlate the nutritive or metabolic activities of the several tissues to events taking place in other parts of the body, plays a conspicuous part in securing the welfare of the whole body. In dealing (\$ 539$)$ with the general problems of nutrition, we stated that an orderly nutrition appears to be in some way dependent on nervous influences. Many of these nervous influences appear to issue from the spinal cord, either as parts of a reflex act. or as the outcome of some automatic processes. In man, extensive injuries to the spinal cord are followed by bed sores and other results of impaired nutrition; and indeed death is generally brought about in this way, in cases of paraplegia caused by acciclental crushing or severance of the cord.

\$466. Inhibition of Reflex Action. The reflex actions of the spinal cord, like other nervous actions, may be totally or partially inhibited, that is to say may be arrested or hindered in their development by impulses reaching the centre while it is already in action. Thus if the body of a decapitated snake be allowed to hang down, slow rhythmic pendulous movements, which appear to be reflex in nature, soon make their appearance, and these may be for a while arrested by slight stimulation, as by gently stroking the tail. We have already seen that the action of such nervous centres as the respiratory and vaso-motor centres, which frequently at all events is of a reflex nature, may be either inhibited or augmented by afferent impulses. 'The micturition centre in the mammal, which is also largely a reflex centre, may be easily inhibited by impulses passing downward to the lumbar cord from the brain, or upward along the sciatic nerves. In the case of dogs, whose spinal cord has been divided in the thoracic region, nicturition set $u$ ) as a reflex act by simple pressure on the abdomen or by sponging the anus, is at once stopped by sharply pinching the skin of the leg. And it is a matter of common experience that in man micturition may be suddenly checked by an emotion or other cerebral event. The erection centre in the lumbar cord, also in large measure a reflex centre, is similarly susceptible of being inhibited by impulses reaching it from various sources. And indeed many similar instances of the inhibition of reflex movements might readily be quoted. 
Several apparent instances of the inhibition of reflex acts are not really such: in these cases all the nervous processes of the act may take place in their entirety and yet fail to produce their effect on account of a failure in the muscular part of the act. Thus when we ourselves by an effort of the will stop the reflex movements which otherwise would be produced by tickling the soles of the feet, we achieve this to a large extent by throwing voluntarily into action certain muscles, the contractions of which antagonize the action of the muscles engaged in carrying out the reflex movements. But it may be doubted even in these cases, whether inhibition is always or wholly to be explained in this way; and certainly in very many instances of reflex inhibition, no such muscular antagonism is present, and the reflex act is checked at its nervous centre.

When the brain of a frog is removed, and the effects of shock have passed away, reflex actions are developed much more readily and to a much greater degree than in the entire animal, and in mammals also reflex excitability has been observed to be increased by removal of the cerebral hemispheres. This suggests the idea that in the intact nervous system the brain is habitually exerting some influence on the spinal cord tending to prevent the normal development of the spinal reflex actions. And we learn by experiment that stimulation of certain parts of the brain has a remarkable effect on reflex action. If a frog, from which the cerebral hemispheres have been removed (the optic lobes, bulb and spinal cord being left intact), be suspended by the jaw, and the toes of the pendent legs be from time to time dipped into very dilute sulphurie acid, a certain average time will be found to elapse between the dipping of the toe and the resulting withdrawal of the foot. If, however, the optic lobes or optic thalami be stimulated, as by putting a crystal of sodium chloride on them, it will be found on repeating the experiment while these structures are still under the influence of the stimulation, that the time intervening between the action of the acid on the toe and the withdrawal of the foot is very much prolonged. 'That is to say, the stimulation of the optic lobes has caused impulses to descend to the cord, which have there so interfered with the nervous processes engaged in carrying out reflex actions as greatly to retarl the generation of efferent implnlses, or in other words, has inhibited the reflex action of the cord. And similar results may be obtained in mammals by stimulating certain parts of the corpora quadrigemina. which bodies are homologons to the optic lobes of frogs. From this it has been inferred that there is present in this part of the brain a special mechanism for inhibiting the reflex actions of the spinal cord, the impulses descending from this methanism to the various centres of reflex action being of a specific inhibitory nature. 
But, as we have already seen, impulses of an ordinary kind, passing along ordinary sensory nerves, may inhibit reflex action. We have quoted instances where a slight stimulus, as in the pendulous movements of the snake, and where a stronger stimulus as in the case of the micturition of the dog, may produce an inhibitory result; we may adll that in the frog adequately strong stimuli applied to any afferent nerve will inhibit, i.e. will retard or even wholly prevent reflex action. If the toes of one leg are dipped into dilute sulphuric acid at a time when the sciatic of the other leg is being powerfully stimulated with an interrupted current the period of incubation of the reflex act will be found to be much prolonged, and in some cases the reflex withdrawal of the foot will not take place at all. And this holds good, not only in the complete absence of the optic lobes and bulb, but also when only a portion of the spinal cord, sufficient to carry out the reflex action in the usual way, is left. There can be no question here of any specific inhibitory centres, such as have been supposed to exist in the optic lobes. But if it is clear that inhibition of reflex action may be brought about by impulses which are not in themselves of a specific inhibitory nature, we may hesitate to accept the view that a special inhibitory mechanism in the sense of one giving rise to nothing but inhibitory impulses is present in the optic lobes of frogs, and after removal of the brain that the exaltation of reflex actions which is manifest is due to the withdrawal of such a specific inhibitory mechanism.

\$ 467. The Time required for Reflex Actions. W'Ten one eyelid is stimulated with a sharp electirical shock, both eyelids blink. Hence, if the length of time intervening between the stimulation of the right eyelid and the movement of the left eyelid be measured, this will give the total time required for the various processes which make up a reflex action. It has been found to be from $\cdot 0662$ to $\cdot 0578$ sec. Deducting from these figures the time required for the passage of afferent and efferent impulses along the fifth and facial nerves to and from the bulb, and for the latent period of the contraction of the orbiscularis muscle, there would remain $\cdot 0555$ to $\cdot 0471$ sec. for the time consumed in the central operations of the reflex act. 'The calculations, however, necessary for this reduction, it need not be said, are open to sources of error; moreover the reflex act in question is carried out by the bulb and not by the spinal cord proper. Blinking thus produced is a reflex act of the very simplest kind; but as we have seen in the preceding pages, reflex acts differ very widely in nature and character; and we accordingly find, as indeed we have incidentally mentioned, that the time taken up by a reflex movement varies very largely. This indeed is seen in blinking itself. When 
the blinking is cansed not by an electric shock applied to the eyelid, but by a flash of light falling on the retina, in which case complex risual processes are involved, the time is distinctly prolonged; moreover the results in different experiments in which light serves as the stimulus are not nearly so uniform as when the blinking is caused by stimulation of the eyelid. In the "knee-jerk" the time is very short, it may be not nore than 03 sec.; this is one of the reasons which have led some to regard the act as not truly a reflex one.

In general it may be said that the time required for any reflex act varies very considerably with the strength of the stimulus employed, being less for the stronger stimuli; this we should expect, seeing that the efferent impulses of the reflex act are not simply afferent impulses transmitted through the central organ, but result from internal changes in the central organ started by the afferent impulse or impulses ; and these internal changes will naturally be more intense and nore rapidly effected when the afferent impulses are strong. It is stated that when the movement inducerl is on the same side of the body as the surface stimulation of which starts the act, the time taken ul, is less than when the movement is on the other side of the borly, allowance being made for the length of central nervous matter involved in the two cases; that is to say the central operations of a reflex act are propagated more rapidly along the cord than across the cord. 'The rapidity of the act varies of course with the condition of the spinal cord, the act being greatly prolonged when the cord becomes exhausted; and a similar delay has been observed in cases of disease. The time thus occupied by purely reflex actions must not be confounded with the interval required when the changes taking place in the central nervous system are of a more complicated nature, and more or less distinctly involve mental operations; of the latter we shall speak later on. 


\section{SEC. 4. THE AUTOMATIC ACTIONS OF THE SPINAL CORD.}

$\S 468$. We speak of an action of an organ or of a living body as being spontaneous or automatic when it appears to be not immediately due to any changes in the circumstances in which the organ or body is placed, but to be the result of changes arising in the organ or body itself and determined by causes other than the influences of the circumstances of the moment. Some automatic actions are of a continued character; others, like the beat of the heart, are repeated in regular rhythm ; but the most striking automatic actions of the living body, those which we attribute to the working of the will and which we call voluntary or volitional, are characterized by their apparent irregularity and variableness. Such variable automatic actions form the most striking features of an intact nervous system, but are conspicuously absent from a spinal cord when the brain has been removed.

A brainless frog placed in a condition of complete equilibrium in which no stimulus is brought to bear on it, protected for instance from sudden passing changes in temperature, from a too rapid evaporation by the skin and the like, remains perfectly motionless until it dies. Such apparently spontaneous movements as are occasionally witnessed are so few and seldom, that we can hardly do otherwise than attribute them to some stimulus, internal or external, which has escaped observation. In the mammal ( $\log$ ) after division of the spinal cord in the dorsal region regular and apparently spontaneous movements may be observed in the parts governed by the lumbar cord. When the animal has thoroughly recovered from the operation the hind limbs rarely remain quiet for any long period; they move restlessly in various ways; and when the animal is suspended by the upper part of the body, the pendent hind limbs are continually being drawn up and let down again with a monotonous rhythmic regularity, suggestive of automatic rhythmic discharges from the central mechanisms of the cord. In the newly born nammal too, after removal of the brain, movements apparently spontaneous in nature are frequently observed. 
But all these movements, even when most highly developerl, are very different from the movements, irregular and variable in their occurrence though orderly and purposeful in their character, which we recognize as distinctly voluntary. Even admitting that some of the movements of the brainless mammal may resemble voluntary movements in so far as they are due to changes taking place in the spinal cord itself independent of the immediate influence of any stimulus. we are not thereby justified in speaking of the spinal cord as developing a will in the scnse that we attribute a will to the brain.

$\$ 469$. In the case of the beat of the heart, the antomatic rhythmic discharge of energy appears to be exchusively the outcome of the molecular nutritive changes taking place in the cardiac substance. 'The beat may be modified, as we have seen, by nervous impulses reaching the cardiac substance along certain nerves; but the actual existence of the beat is wholly independent of these extraneous influences; the rhythmic discharge continues when they are entirely absent. 'The automatic rhythmic discharge of respiratory impulses from the respiratory centre is also dependent on the intrinsic molecular changes of the centre, these bcing, as we have seen, largely determined by the character of the blood streaming through it; but in this case extrinsic nervous impulses, reaching the centre along the vagus and other nerves, play a much more important part than do similar impulses in the ease of the heart. They act so continually on the centre and enter so largely into its working, that we are compclled to regard the activity of the centre as fed, if we may use the word, not only by the intrinsic molecular nutritive processes of the centre itself, but also by the extrinsic nervous influences which flow into the centre from without. The automatism of the spinal cord as a whole resembles, in this aspect, that of the respiratory centre rather than that of the heart. It has for its basis doubtless the intrinsic molecular changes of the grey matter; the metabolic events of this substance are so ordered as to give rise to discharges of energy; but the discharge appears to be also intimately dependent on the inflow into the grey matter of afferent impulses and influences. The normal discharge of efferent impulses from the cord undoubtedly takes place under the influence of these incoming impulses; and it may be doubted whether the grey matter of the cord would be able, in the absence of all afferent impulses, to generate any sustained series of discharges out of its merely nutritive intrinsic changes. The automatic activity of the cord is fed not only by intrinsic nutritive events, but also by extrinsic influences.

In this feature we may, moreover, find perhaps the reason why the automatic activity of the spinal cord is so limited, as compared with that of the brain. In spite of certain striking 
but superficial characters of which we shall speak later on, the grey matter of the brain presents no histological features so different from those of the grey matter of the cord, as to justify us in concluding that the one is capable and the other incapable of developing the impulses, which we call volitional, out of the molecular nutritive changes of its substance. We are, therefore, led to the conclusion that the fuller automatic activity of the brain is due to the intrinsic changes of its substance being so much more largely assisted by the influx of varions afferent impulses and influences, notably those of the special senses. 'To this question, however, we shall have to return later on.

$\$ 470$. In treating of the vascular system we saw that the central nervous system exercised through the vaso-motor nerves such an influence on the muscular coats of the blood vessels as to maintain. what we spoke of as 'tone,' section of vaso-constrictor fibres leading to "loss of tone." We saw further, that arterial tone, though normally dependent on the general vaso-motor centre in the bulb, could be kept up by the cord itself, that for instance a tone of the blood vessels of the hind limbs could be maintained by the isolated dorso-lumbar cord. This maintenance of arterial tone may be spoken of as one of the "antomatic" functions of the spinal cord. We have also seen that plain muscular fibres, other than those of the arteries, notably the fibres forming sphincters, such as the cardiac and pyloric sphincters of the stomach, the sphincter of the bladder, and especially the sphincter of the anus, also possess tone. and that the tone of these sphincters is also dependent on the spinal cord, or on some part of the central nervons system. We need not repeat the discussions concerning these mechanisms and other instances of the spinal cord exercising an automatic influence over various viscera; we have referred to them here, since they serve as an introluction to a question which has been much debated, and which has many collateral and important bearings, namely the question whether the spinal cord exercises an automatic function in maintaining a tone of the skeletal muscles.

The question is not one which, like the case of arterial tone, can be settled off hand by a simple experiment. Most observers agree that the section of a motor nerve does not produce any clearly recognizable immediate lengthening of a muscle supplied by the nerve, in the same way that section of a vaso-constrictor nerve undoubtedly gives rise to a relaxation of the muscular fibres in the arteries governed by it; and it has been inferred from this that skeletal tone does not exist. But there are several facts to be taken into consideration before we can come to a just decision.

The skeletal muscles have been described as being placed "on the stretch" in the living borly. If a muscle be cut away from its attachments at each end, it shortens; if it be cut across, it 
gapes. In other words, the muscle in the living body possesses a latent tendency to shorten, which is continually being counteracted by its disposition and attachments. In studying muscular contraction we saw ( $\$ 81)$ that the shortening of a contraction is followed by a relaxition or return to the former length, both the contraction and relaxation being the result of molecular changes in the living muscular substance. IV have now to extend our view and to recognize that, apart from the occurrence of ordinary contractions, molecular changes are by means of nutritive processes continually going on in the muscle in such a way that the muscle, though continually on the stretch, loes not permanently lengthen, but retains the power to shorten upon removal or lessening of the stretch, and conversely though possessing this power of shortening permits itself to lengthen when the stretch is increaseil. In this way the muscle is able to accommodate itself to rariations in the amount of stretch to which it is from time to time subjected. When a flexor muscle for instance contracts, the antagonistic extensor muscle is put on an increased stretch and is correspondingly lengthened; when the contraction of the flexor passes off the extensor returns to its previous length; and so in other instances. 'Thus by virtue of certain changes within itself a muscle maintains what may be called its natural length in the body, always returning to that natural length both after heing shortened and after being stretched. In this the muscle does no more than to the other tissues of the body which, within limits, retain their natural form under the varied stress and strain of life; but the property is conspicuous in the muscle; and its effects in skeletal muscles correspond so closely to those of arterial tome, that we may venture to speak of it as skeletal tone. Indeed, the molecular changes at the bottom of both are probahly the same.

These changes are an expression of the life of the muscle; they disappear when the muscle dies and enters into rigor mort is; and moreover, during life they vary in intensity so that the 'tone" varies in amonnt accolding to the nutritive changes going on. We have seen reason to believe that the nutrition of a muscle as of other tissues is governed in some way by the central nervons system. We saw, in treating of muscle and nerve (S 78), that the irritability of a muscle is markerlly affected by the section of its nerve, i.e. by severance from the central nervous system; ant again (\$ 5.39 ) in speaking of the so-called trophic action of the nervous system, we referred to changes in the nutrition of muscles occasionerl by diseases of the nervons system. And experience, especially clinical experienee, shews that the nutritive changes which determine tone are very closely dependent on a due action of the central nervous system. When we handle the limb of a healthy man, we find that it offers a certain amount of resistance to passive move- 
ments. This resistance, which is quite independent of, that is to say, which may be clearly recognized in the abscnce of all distinct muscular contractions of volitional or other origin, is an expression of muscular tone, of the effort of the various muscles to maintain their 'natural' length. In many cases of disease this resistance is felt to be obvionsly less than normal; the limb is spoken of as "limp" or "flabby"; or as having "a want of tone.' In other cases of disease, on the other hand, this resistance is markedly increased; the limb is felt to be stiff or rigid; more or less force is necded to change it from a flexed to an extended, or from an extended to a flexed condition; and, in the range of disease, we may meet with very varying anounts of increased resistance, from a condition which is only slightly above the normal to one of extreme rigidity. In some cases the condition of the muscle is such as at first sight seems much more comparable to a permanent ordinary contraction than to a mere exaggeration of normal tone; but all intermerliate stages are met with; and indeed these extreme cases may be taken as inclicating that the molecular processes which maintain what we are now calling tone, are at bottom, of the same nature as those which carry out a contraction; they serve to shew the funclamental identity of the skeletal tone with the more obvious arterial tone.

Clinical experience then shews that the central nervous system does exert on the skeletal muscles such an influence as to give rise to what we may speak of as skeletal tone, changes in the central nervous system, leading in some cases to diminution or loss of tone, in other cases to exaggeration of tone, manifested often as conspicuous rigidity. The question why the changes take one direction in one case and another in another is onc of great difficulty (the occurrence of extreme rigidity being especially obscure), and cannot be discussed here. W' liave called attcntion to the facts simply because they shew the existence of skeletal tone and its dependence on the central nervous system. This conclusion is confirmed by experiments on animals, and these also afford proof that in animals the spinal cord can by itself, apart from the brain, maintain the existence of such a tone. In a frog, after division of the cord below the brain, the limbs during the period of shock are flabby and toneless; but after a while, as the shock passes off, tone returns to the muscles, and the limbs offer when handled a resistance like that of the limbs of an entire frog. When the animal is suspended the hind limbs do not hang perfectly limp and helpless, but assume a definite position; and that this position is due to some influence proceeding from the spinal cord is shewn by dividing the sciatic nerve on one side; the hind limb on that side now hangs quite helpless. This more pendent position shews that some of the flexors have lengthened in consequence of the section of the 
nerve, and this result may be taken as refuting the argument, quoted above, against the existence of tone, which is based on the statement that a musele eannot be observed to lengthen after section of its nerve. It may be here remarked that if the brainless frog, whose hind limbs are nore or less pendent when the body is suspended, be placed on its belly the hind limbs are brought into a flexed position under the body by means of obvious museular contraction; and from this it might be inferred that the maintenance of the position of the pendent limb was also the result of a fecble contraction. But no obvious contractions can be observed in the latter case, as in the former; and when in the former the limb has once been brought into the flexed position, that position, like the pendent position. is maintained without obvious eontractions. As we sair above t tone" may pass into something which appears to be identical with a contraetion, but where no obvious contractions are observed it seems preferable to speak of the state of the muscle as one of tone.

In the dog, after division of the cord in the thoracic region, the hind limbs during the period of shoek are limp and toneless. In the warm-blooded animal, as we have said. the effects of shock are much more lasting than in the eold-blooded animal; and in the dog the tone of the skeletal musele returns much more slowly than in the frog. Indecd when the division of the cord has taken place low down the skeletal tone returns very slowly, and may be manifested very feebly, or even be absent altogether. But under favourable circumstances, when a sufficient length of cord has been left, a fairly normal tone is reestablished. In man, in accordance with the facts previously mentioned ( $\$ 46 t)$ skelctal tone, which has been lost through the continuity of the eord being broken by disease or accident. appears rarely if ever to return fully in the regions below the lesion.

We may therefore on the whole of the evidence conclude that the maintenance of skeletal tone is one of the functions of the cord; but we may here repeat that the eondition of the cord, on which depends the issue from the cord along efferent nerves of the influences, whatever their nature. which produce tone in the muscle, may be, and indeed is, in its turn dependent on afferent impulses. In the case of the frog quoted above the tone of the pendent limbs disappears or is greatly lessened when the posterior roots of the seiatie nerves are divided. though the anterior roots be left intaet. In the absence of the usual stream of afferent impulses passing into it, the cord ceases to send forth the influences which maintain the tone. Hence the maintenance of tone presents many analogies with a reflex action espeeially when we remember that, as stated above, tone passes insensibly into contraction; and it may seem a mere 
matter of words whether we speak of the maintenance of tone as an automatic or as a reflex action of the cord. We may. however, distinguish the part played by the afferent impulses in assisting the cord to a condition in which it is capable of maintaining tone from the part played by an afferent impulse in causing a reflex action; in the former the action of the afferent impulses seems analogous to that of a supply of arterial blood in maintaining an adequate irritability of the nervous substance, in the latter the afferent impulses lead directly to a discharge of energy. And it is convenient to distinguish the two things by different names.

$\$ 471$. The close connection between tone and reflex action is shewn by the fact that some observers contend that the 'knee-jerk' and similar 'tendon-phenomena' are not instances of reflex action. 'They maintain that the contraction of the muscle is an example of the direct stimulation of the muscle by the vibrations set up in the tense tendon when it is sharply struck or suddenly pulled; and they explain the dependence of the act on the spinal cord by attributing rariations in the response of the muscle to variations in the tone of the muscle, the tone being dependent on the spinal cord.

$\$ 472$. Disease in man reveals other actions of the spinal cord which bear features different from those of an ordinary reflex movement, and yet have been described as reflex in nature. For instance certain affections of the cord are characterized by the legs becoming rigid in extreme extension, the rigidity of the straightened limbs being often so great, that when a bystander lifts up one leg from the bed, the other leg is raised at the same time. The rigidity is due to the extensor' muscles being thrown into a state of contraction, which is so uniform and long continued that it may be spoken of as a "tonic" contraction; such a tonic rigidity may however be replaced by a series of rhythmic "clonic" contractions. It has sometimes been observed that the limbs when flexed are supple and free from rigidity, but that rigidity sets in so soon as they are brought into the position of extension, the leg becoming suddenly fixed and straight somewhat in the way that a claspknife springs back when opened. It seems clear that the peculiar contraction is carried out by means of the spinal cord, but the whole action, though' it is often spoken of as a 'musclereflex,' is very unlike an ordinary reflex movement. In an ordinary movement an extensor is brought into action when a limb is flexed, not when it is already extended; and if in a reflex act the condition of the muscle about to be thrown into action determines in any way the discharge of impulses from the reflex centre, we should expect that the stretching of an extensor muscle by flexion, not its relaxation by extension, would determine the discharge of extensor impulses. In the 
case of the diseases in question just the opposite seems to take place; the position which appears to determine the development of the remarkable contraction is precisely that in which the strain upon the extensors is at its mininnm. It may be doubted, therefore, whether the word reflex should be used to denote such phenomena; but the phenomena themselves deserve attention, especially perhaps as shewing how in the disorders of the grey matter of the cord due to disease impulses or influences which are latent only in health become actual and effective.

It remains for us to speak of the part played by the spinal cord, as the instrument of the brain, in the execution of voluntary movements and in the development of conscious sensations; but it will be best to consider these matters in connection with the brain itself, to the study of which we must now turn. 


\title{
CHAPTER II.
}

\author{
THE BRAIN.
}

SEC. 1. ON THE PHENOMENA EXHIBITED BY AN ANIMAL DEPRIVED OF ITS CEREBRAL HEMISPHERES.

\$473. THE cerebral hemispheres, as we have more than once insisted, seem to stand apart from the rest of the brain. In the case of some animals it is possible to remove the cerebral hemispheres and to keep the animal not only alive, but in good health for a long time, days, weeks or even months after the operation. In such case we are able to study the behaviour of an animal possessing no cerebral hemispheres and to compare it with that of an intact animal. Such an experiment is best carried out on a frog. In this animal it is comparatively easy to remove the cerebral hemispheres, including the parts corresponding to the corpora striata, leaving behind intact and uninjured the optic thalami with the optic nerves, the optic lobes (or representatives of the corpora quadrigemina), the small cerebellum and the bulb. If the animal be carefully fed and attended to, it may be kept alive for a very long time, for more than a year for instance.

The salient fact about a frog lacking the cerebral hemispheres, is that, as in the case of a frog deprived of its whole brain, the signs of the working of an intelligent volition are either wholly absent or extremely rare. The presence of the bulb and the middle parts of the brain (for so we may conveniently call the cerebral structures lying between the cerebral hemispheres and the bulb) ensures the healthy action of the vaseular, respiratory and other nutritive systems; food placed in the mouth is readily and easily swallowed; the animal when stimulater executes various movements; but if it be left entirely to itself, and care be taken to shield it from adventitious stimuli, either it remains perfectly and permanently quiescent, or the apparently spontaneous movements which it carries out are so 
few and so limited as to raise the question whether they can fairly be called volitional. Such a frog, for instance, after being kept alive for some time and made to exhibit the phenomena of which we are about to speak, has been placed on a table with a line drawn in chalk around the area covered by its body, and left to itself has subsequently been found dead without having stirred outside the chalked circle.

We must here however repeat the caution laid down in $\S 45 \pi$, as to the ultimate effects of an operation on the central nervous system. The longer the frog is kept alive and in gond health after the removal of the cerebral hemispheres, the greater is the tendency for apparently spontaneous movements to slew themselves. For days or even weeks after the operation there maty be no signs whatever of the working of any volition; but after the lapsc of months, movements, previously absent, of such a character as to suggest that they ought to be called voluntary, may make their appearance. Still even in their most complete development such novements do not absolutely negative the view that the frog in the absence of the cerebral hemispheres is wanting in what we ordinarily call a 'will.'

$\$ 474$. We have seen that a frog from which the whole brain has been removed and the spinal cord alone left appears similarly devoid of a 'will:' but the plienomena presented by a frog possessing the middle portions of the brain differ widely from those presented by a frog possessing. it spinal. cord only. We may perlaaps broadly describe the behaviour of a frog from which the cerebral hemispheres only have been removed, by saying that such an animal, though exhibiting no spontaneous movements, can by the application of appropriate stimuli be induced to perform all or nearly all the movements which an entire frog is capable of executing. It can be made to swim, to leap, and to crawl. Left to itself it assumes what may be called the natural posture of a frog, with the fore limbs erect, and the hind limbs flexed, so that the line of the body makes an angle with the surface on which it is resting. When placed on its back, it immediately regains this natural posturc. When placed on a board, it does not fall from the board when the latter is tilted up so as to displace the animal's centre of gravity: it crawls up the board until it gains a new position in which its centre of gravity is restored to its proper place. Its movenents are exactly those of an entire frog except thạt they need an external stimulus to call them forth. They differ moreover fundamentally from those of an entire frog in the following important feature; they inevitably follow when the stimulus is applied; they come to an end when the stimulus ceases to act. By continually varying the inclination of a board on which it is placed, the frog may be made to continue crawling almost indefinitely; but directly the board is made to assune such a position that 
the body of the frog is in equilibrium, the crawling ceases; and if the position be not disturbed the animal will remain impassive and quiet for an almost indefinite time. When thrown into water, the creature begins at once to swim about in the most regular manner, and will continue to swim until it is exhausted, if there be nothing present on which it can come to rest. If a small piece of wood be placed on the water the frog will, when it comes in contact with the wood, erawl upon it, and so come to rest. If disturbed from its natural posture, as by being placed on its back, it immediately struggles to regain that posture; only by the application of continued force can it be kept lying on its back. Such a frog, if its flanks be gently stroked, will croak; and the eroaks follow so regularly and surely upon the strokes that the animal may almost be played upon like a musical, or at least an acoustic instrument. Moreover, provided that the optic nerves and their arrangements have not been injured by the operation, the movements of the animal appear to be influenced by light; if it be urged to move in any particular direction, it seems in its progress to avoid obstacles, at least such as cast a strong shadow; it turns its course to the right or left or sometimes leaps over the obstacle. In fact, even to a careful observer the differences between such a from and an entire frog which was simply very stupid or very inert, would appear slight and unimportant except in this, that the animal without its cerebral hemispheres is obedient to every stimulus, and that each stimulus evokes an appropriate movement, whereas with the entire animal it is impossible to predict whether any result at all, and if so what result, will follow the application of this or that stimulus. Both may be regarded as machines; but the one is a machine and nothing more, the other is a machine governed and checked by a dominant volition.

Now such movements as crawling, leaping, swimming, and indeed, as we have already urged, to a greater or less extent, all bodily movements, are carried out by means of coordinate nervous motor impulses, influeneed, arranged, and governed by coincident sensory or afferent impulses. Museular movements are determined by afferent influences proceeding from the muscles and constituting the foundation of the muscular sense ; they are also directed by means of afferent impulses passing centripetally along the sensory nerves of the skin, the eye, the ear, and other organs. Independently of the particular afferent impulses, which acting as a stimulus call forth the movement, very many other afferent impulses are concerned in the generation and coordination of the resultant motor impulses. Every bodily movement such as those of which we are speaking is the work of a more or less complicated nervous mechanism, in which there are not only central and efferent, but also afferent factors. And, putting aside the question of consciousness, with which 
we have here no occasion to deal, it is evident that in the frog depriver of its cerabral hemispheres all these factors are present, the afferent no less than the central and the efferent. The machinery for all the necessary and usual bodily movements is present in all its completeness. We may regard the share therefore which the cerebril hemispheres take in executing the novements of which the entire animal is eapable, as that of putting this machinery into action or of limiting its previous activity. The relation which the higher nervous changes concerned in volition bear to this machinery may be compared to that of a stimulus, always bearing in mind that the effect of a stimulus on a nervous centre may be either to start activity, or to increase, or to curb, or to stop aetivity already present. IT e might almost speak of the will as an intrinsic stimulus. Its operations are limited by the maehinery at its eommand. We may infer that in the frog, the action of the eerebral hemispheres in giving shape to a bodily movement is that of throwing into activity particular parts of the nervous machinery situated in the lower parts of the brain and in the spinal cold; precisely the same movement may be initiated in the absence of the cerebral hemispheres by applying such stimuli as shall throw precisely the same parts of that machinery into the same aetivity.

Very marked is the contrast between the behaviour of such a frog which, though deprived of its eerebral hemispheres, still retains the other parts of the brain, and that of a frog which possesses a spinal cord only. The latter wlien placed on its back makes no attempt to regain its normal posture; in fact, it may be said to have eompletely lost its normal posture, for even when placed on its belly it does not stand with its fore feet erect, as does the other animal, but lies flat on the ground. When thrown into water, instead of swimming, it sinks like a lump of lead. When pinched, or otherwise stimulated, it does not crawl or leap forwards; it simply throws out its limbs in valious ways. When its flanks are stroked it does not eloak; and when a board on which it is placed is inclined sufficiently to displace its centre of gravity it makes no effort to regain its balance, but falls off the board like a lifeless mass. Though, as we have seen, the various parts of the spinal cord of the frog contain a large amount of coorlinating machinery, so that the brainless frog may, by appropriate stimuli, be made to execute various purposeful coorlinate movements, yet these are very limited compared with those which ean be similarly earried out by a frog possessing the middle and lower parts of the brain in addition to the spinal cord. It is evident that a great deal of the more complex machinery of this kind, especially all that which has to deal with the body as a whole, and all that which is concerned with equilibrim and is suecially governed by the higher senses, is seated not in the spinal cord but in the brain. 
We do not wish now to discuss the details of this machinery; all we desire to insist upon at present is that, in the frog the nervous machinery required for the execution, as distinguished from the origination, of bodily movements even of the most complicated kind, is present after complete removal of the cerebral hemispheres, though these movements are such as to require the cooperation of highly differentiated afferent impulses.

$\$ 475$. In warm-blooded animals the removal of the cerebral hemispheres is attended with much greater difficulties than in the case of the frog. Nevertheless, in the bird the operation may be carried out with approximate success. Pigeons for instance have been kept alive for five or six weeks after complete removal of the cerebral hemispheres, with the exception of portions of the crura and corpora striata immediately surrounding the optic thalami; these parts were left in order to ensure the intact condition of the latter bodies.

When the immediate effects of the operation have passed off, and for some time afterwards, the appearance and behaviour of the bird are strikingly similar to those of a bird exceedingly sleepy and stupid. It is able to maintain what appears to be a completely normal posture, and can balance itself on one leg. after the fashion of a bird which has in a natural way gone to sleep. Left alone in perfect quiet, it will remain impassive and motionless for a long time. When stirred it moves. shifts its position; and then, on being left alone, returns to a natural, easy posture. Placed on its side or its back it will regain its feet; thrown into the air, it flies with considerable precision for some distance before it returns to rest. It frequently tucks its head under its wings, and at times may be seen to clean its feathers; when its beak is plunged into corn, it eats. It may be induced to move not only by ordinary stimuli applied to the skin. but also by sudden loud sounds, or by flashes of light; in its flight it will, though imperfectly, aroid obstacles, and its various movements appear to be to a certain extent guided not only by touch but also by visual impressions.

In a certain number of cases this sleepy, drowsy condition passes off and is succeeded by a phase in which the bird. apprarently spontaneously, without the intervention of any obvious stimulus. moves rapidly about. It does not $f l y$, that is to say, it does not raise itself from the ground in flight, but walks about incessantly for a long while at a time, periods of activity alternating with periods of repose. It seems, from time to time, to wake up and move about, and then to go to sleep again; and it has been observed that during the night it appears to be always asleep. It is obvious, therefore, that the sleepy, quiescent condition observed at first is not due simply to the absence of the cerebral hemispheres, but is a temporary effect of the operation, and that spontaneous movements, that 
is to say, movements not started by any obvious stimulus, may occur after removal of the cerebral hemispheres. But the morements so witnessed differ from those of an intact bird. They are, it is true, varied; and the variations are in part dependent on external circumstances, the bird being guided by tactile, and. as we have said, visual sensations, or, to be more exact, by impressions mate mpon the sensory nerves of the skin and on the retina; but they do not shew the wide variations of voluntary movements. 'The bird for instance never flies mp from the ground, never spontaneously picks up corn, and its aimless, monotonoms, restless walks, resembling the continned swimming of the frog thrown into the water after being deprived of its cerebral hemispheres, forcibly suggest that the activity is the outcome of some intrinsic impulse generated in the nervous machinery in some way or other, but not by the working of a conscious intelligence as in the impulse which we call the will.

Still we must not shut our eyes to the fact that spontaneous morements, whaterer their cxact nature, are manifested by a bird in the absence of the cerebral hemispheres, and become the more striking the more complete the recovery from the passing effects of the mere operation. Could such birds be kept alive for any consilerable time, possibly further developments might be witnessed, and indced cases are on record where birds have been kept alive for months after the operation, and have shewn spontaneous movements of a still more varied character than those just lescribed; but in such cases the removal of the hemispheres has not been complete, portions of the ventral regions being left behind; and, though a mere remnant left around the optic thalani can hardly be regarded as a sufficient cause for the spontaneity of which we are speaking, a larger mass, still more or less retaining its normal structure, might have a marked effect. And we may here perhaps remark that all these facts seem to point to the conclusion that what may be called mechanical spontaneity, somctimes spoken of as 'automatism, differs from the spontaneity of the 'will' in degree rather than in kind. Looking at the matter from a purely physiological point of view (the only one which has a right to be $\mathrm{cmployed}$ in these pages), the real difference between an automatic act and a voluntary act is that the chain of physiological events between the aet and its physiological cause is in the one case short and simple, in the other long and complex. We have seen that a forg lacking its cerebral henispheres, viewed from one standpoint, appears in the light of a mechanical apparatus, on which each change of cireumstances produces a direct, unvarying, inevitable effect. And yot it is on record that such a frog, if kept alive loug enough for the most complete disappearance of the direct effects of the opera- 
tion, will bury itself in the earth at the approach of winter, and is able to catch and swallow flies and other food eoning in its neighbourhood, although in other respeets it shews no signs of an intelligent volition, and answers with unerring mechanical certainty to the play of stimuli. We may add that in some fishes the removal of their cereoral hemispheres, which in these animals form a relatively small part of the whole brain, produees cxceedingly little change in their general behaviour.

These however are not the considerations on which we wish here to dwell; we have quoted the behaviour of the bird deprived of its cerebral hemisphere mainly to shew that in this warm-blooded animal, as in the more lowly cold-blooded frog, the parts of the brain below or behind the cerebral hemispheres constitute a nervous machinery by which all the ordinary bodily movements may be carried out. 'The bird. like the frog, suffers no paralysis when the cerebral hemispheres are remored; on the contrary, though its movements have not been studied so closely as those of the frog, the bird without its cerebral hemispheres scens capable of exeenting at all events all the ordinary bodily movements of a bird. And in the bird as in the frog, the afferent impulses passing into the central nervous system, whether they give rise to consciousness or no. play an important part not only in originating but in guirling and coordinating the efferent impulses which stir the muscles to contract, the coordination being effected partly in the spinal cord, but largely and indeed eliefly in the parts of the brain lying behind the cerebral hemispheres. It is further worthy of notice that spontaneity of movement of the kind which we have described, is much more prominent in the more highly developed bird, than in the more lowly frog. 'The cerebral hemispheres are not the only part of the central nervous system which has undergone a greater development in the bird; the other parts of the brain have also aequired a far greater complexity than in the frog.

$\$ 476$. In the mammal the removal of the cerebral hemisperes is still more difficult than in the bird; the animal camnot be kept alive for more than a few hours; but in some mammals it is possible to observe during those few hours phenomena kindred to those witnessed in the bird and in the frog. The rabbit or rat, from which the whole of both hemispheres has been removed with the exception of the parts immediately surrounding the optic thalami, can stand, run and leap. P'laced on its side or back it at once regains its feet. Left alone it generally remains as motionless and impassive as a statue, save now and then when a passing impulse seems to stir it to a sudden but brief movement; but sometimes it seems subject to a more continued impulse to move, in which case death usually follows very speedily. Such a rabbit will remain for minutes 
together utterly heedless of a carrot or cabbage-leaf placed just before its nose, though if a morsel be placed within its mouth it at once begins to eat. When stirred it will with ease and steadiness run or leap forward; and obstacles in its course are very frequently, with more or less success, avoided. In some cases the animal (rat) has been described as following by movements of the head a bright light held in front of it (provided that the optic nerves and tracts lave not been injured during the operation), as starting when a shrill and loud noise is made near it, and as erying when pinched, often with a long and seeningly plaintive scream. So plaintive is the ery which it thus gives forth as to suggest to the olsserver the existence of passion ; this, however, is probably a wrong interpretation of a voeal action; the ery appears plaintive sinply because, in consequence of the completeness of the reflex nervous machinery and the absence of the usual restraints, it is prolonged.

Without iusisting too much on such results as these, and allowing full weight to the objection which may be urged, that in some of these cases parts of the cerebral hemispheres surrounding the optic thalami were left, there still remains adequate evidence to shew that a mammal such as a rabbit, in the same way as a frog and a bird, may in the complete or all but complete absence of the cerebral hemispheres maintain a natural posture, free from all signs of disturbance of equilibrium, and is able to carry out with success, at all events all the usual and common bodily movements. And as in the bird and frog, the evidence also shews that these movements not only may be started by, but in their carrying out are guided by and coordinated by afferent impulses along afferent nerves, includiug those of the special senses. But in the case of the rabbit it is even still clearer than in the case of the bird that the effects of these afferent impulses are different from those which result when the impulses gain access to an intact brain. The movements of the animal seem guided by impressions made on its retina, as well as on other sensory nerves; we may perhaps speak of the animal as the subject of sensations; but there is no satisfactory evidence that it possesses either visual or other pereeptions, or that the sensations which it experiences give rise to ideas. Its avoidance of ohjects depends not so much on the form of these as on their interference with light. No image, whether pleasant or terrible, whether of food or of an enemy, produces an effect on it, other than that of an object reflecting more or less light. And we may infer that it lacks the possession of an intelligent will. But it must always be remembered that some of the phenomena are due to the operation producing other results than the mere absence of the part removed. We must bear in mind that in all the above experiments while the positive phenomena, the things which the animal continues able 
to do, are of great value, the negative phenomena, the things which the animal can no longer do, are of much less, indeed of doubtful value. 'The more carefully and successfully the experiments are carried out, the narrower become what we may call the 'leficiency phenomena,' the phenomena which are alone and directly due to something having been taken away. Were it possible to keep the rabbit alive long enough for the mere effects of the operation to pass completely away, we should not only probably witness, as in the case of the bird, a greater scope of movement and more frequent spontaneity, but possibly find a difticulty in describing the exact condition of the animal.

$\$ 477$. Hitherto most attempts to witness similar phenomena in more highly organized mammals such as the dog have failed; these animals do not recover from the operation of removing the whole of hoth their hemispheres sufficiently to enable us to judge whether they, like the frog, the bird and the rabbit, can carry out coordinate bodily movements in the absence of the hemispheres, or whether in them this part of the brain, so largely developed, has usurped functions which in the lower animals belong to other parts. W'hen however in a $\log$ the cerebral hemispheres are removed not all at once but piecemeal at several operations, the animal may be kept alive and in good health for a long time, many months at least, even after the hemispheres have been reluced to a mere fragment; and it is on record that under these circumstances, the animal is not only able to carry out with some limitations his ordinary bodily movements, but also exhibits a spontaneity of movement and a varied responsiveness to stimuli suggestive, at least, of the possession of a conscious volition. If we can thus say little about the condition of a $\operatorname{dog}$ without the cerebral hemispheres we can say still less about the monkey, which in all matters touching the cerebral nervous system serves as our best, indeed our only guide for drawing inferences concerning man; but in all probability the monkey in this respect bears somewhat the same relation to the $\log$ that the dog bears to the bird. In short, the more we study the phenomena exhibited by animals posscssing a part only of their brain, the closer we are pushed to the conclusion that no sharp line can be drawn between volition and the lack of volition, or between the possession and absence of intelligence. Between the muscle-nerve preparation at the one limit, and our conscious willing selves at the other, there is a continuous gradation without a break; we camnot fix on any linear barrier in the brain or in the general nervous system, and say 'beyond this there is volition and intelligence but up to this there is none.'

'This however is not the question with which we- are now dealing. What we want to point out is that in the higher animals, including at least some mammals, as in the frog, after 
the removal of the cerebral hemispheres, even though conscious volition and intelligence appear to be largely, if not entirely, lost, the body is still capable of executing all the ordinary movements which the animal in its natural life is wont to perform, in spite of these movements necessitating the cooperation of various afferent impulses; and that therefore the nervous machinery for the execution of these movements lies in some part of the brain other than the cerebral hemispheres. We have reasons for thinking that it is situated in the structures forming the middle and hind brain; as we shall see, interference with these parts produces at once remarkable disorders of movement. 


\section{SEC. 2. THE MACHINERY OF COORDINATED MOVEMENTS.}

$\$ 478$. We may now direct our attention for a while to some considerations concerning the nature of this complex nervous machinery for the coordination of bodily movements, and especially concerning the part played by afferent impulses. Most of our knowledge on this point has been gained by a study of animals not deprived of, but still possessing their cerebral hemispheres, or by deductions from the data of our own experience; but it is possible in most cases to eliminate from the total results the phenomena which are due to the working of a conscious intelligence.

Let us first of all turn aside to ourselves and examine the coordination of the movements of our own bodies. When we appeal to our own consciousness we find that our movements are governed and guided by what we may call a sense of equilibrium, by an appreciation of the position of our body and its relations to space. When this sense of equilibrium is disturbed we say we are dizzy, and we then stagger and reel, being no longer able to coordinate the movements of our bodies or to adapt them to the position of things around us. What is the origin of this sense of equilibrium? By what means are we able to appreciate the position of our body? There can be no doubt that this appreciation is in large measure the product of visual and tactile sensations; we recognize the relations of our body to the things around us in great measure by sight and touch; we also learn much by our muscular sense. But there is something besides these. Neither sight nor touch nor muscular sense can help us when, placed perfectly flat and at rest on a horizontal rotating table, with the eyes shut and not a muscle stirring, we attempt to determine whether or no the table and we with it are being moved, or to ascertain how much it and we are turned to the right or to the left. Yet under such circumstances we are conscious of a change in our position, and some observers have been even able to pass a tolerably successful judgment as to the angle through which they have 
been moved. There ean be no doubt that such a judgment is based upon the interpretation by consciousness of afferent impnlses which are dependent on the position of the body, but which are not afferent impulses belonging to sensations of touch or sight, or taking part in the museular sense. It is by help of these special afferent impulses that we are aware on the one hand of the position, of the relation to space, in which our body may at one time happen to be, standing upright, lying down, and the like, and on the other hand, of the nature and cxtent of any change of position which our movements may bring about. It is by help of these afferent impulses that we are able to coordinate our movements so as to bring our body into the position we desire; and hence when these afferent impulses are disordered and abnormal, the coordination of our movements, the maintenance of equilibrium, is imperfect. Can we say anything as to the exact nature and origin of these special afferent impulses?

We learn much in this respect by studying the effects of operative interference with certain parts of the internal ear. When in a pigeon the horizontal membranous semicircular canal is cut through, the bird is observed to be continually moving its head from sicle to side. Injury to the bony canal alone is insufficient to produce the symptoms; the membranous canal itself must be divided or injured. If one of the vertical canals be cut through, the movements are up and down. The peculiar novements may not be witnessed when the bird is perfectly quiet, but they make their appearance whenever it is disturbed, or attempts in any way to stir. When the injury is confined to one canal only or even to the canals of one side of the head only, the condition after a while passes away; when the canals of both sides have been divided, it becomes much exaggerated, lasts much longer, and in some cases is said to remain permanently. After such injuries it is found that these peculiar movements of the head are associated with what appears to be a great want of coordination of bodily movements. If the bird be thrown into the air, it flutters and falls down in a helpless and confused manner; it appears to have lost the power of orderly flight. If placed in a balanced position, it may remain for some time quiet, generally with its head in a pecnliar posture; but directly it is disturbed, the movements which it attempts to execute are irregular and fall short of their purpose. It has great difficulty in picking up food and in drinking; and in general its behaviour very much rescmbles that of a person who is exceedingly dizzy.

It can hear perfectly well, and therefore the symptoms cannot be regarded as the result of any abnormal auditory sensations, such as 'a roaring' in the ears. Besides, any such stimulation of the auditory nerve as the result of the section would speedily 
die away, whereas these phenomena may last for at least a very considcrable time. The movements are not occasioned by any partial paralysis, by any want of power in particular muscles or group of muscles; though removal of the canals of one side has been described as leading to diminished museular tonc, especially on the same side of the body, the mere diminution of force is insufficient to explain the phenomena. Nor on the other liand are the movements due to any uncontrollable impulse; a very gentle pressure of the hand suffices to stop the movements of the head, and the hand in doing so experiences no strain. The assistance of a very slight support enables movements otherwise impossible or most difficult, to be easily executed. 'Thus, though when left alone the bird has great difficulty in drinking or picking up corn, it will continue to drink or eat with ease if its beak be plunged into water, or into a heap of barley; the slight support of the water or of the grain seems sufficient to steady its movements. In the same way it can, even without assistance, clean its feathers and seratch its head, its beak and foot being in these operations guided by contact with its own body.

The amount of disorder thus induced differs in different birds; and some movements are more affected than others. As a general rule it may be said that the more complex and intricate a movement, the fuller and more delicate the coordination needed to carry it out successfully, the more markedly is it disordered by the operation; thus after injury to the canals, while a pigeon cannot $\mathrm{Hy}$, a goose is still able to swim.

Similar phenomena have been observed in other classes of animals than birds, in mammals, in frogs and in fishes. The results, which are more striking in some species of animals than in others, may be described by the following general statements. When one canal is severed the head or the eyes, and, in certain cases, parts of the body, in a fish for instance the fins, make certain definite movements, or take up certain definite positions, or tend to do so, the exact characters of the movement or of the position taken up depending upon which of the canals is the one operated on. After the operation, a certain deficiency, a certain want of coordination, in the movements of the animal may be observed, the exact nature of the loss differing with the different canals; but this is generally slight and after a while is overcome by accommodation. Section of all three canals on one side is followed by effects in the way of movements and the like which may be regarded as the sum of the effects of the severance of each canal; the incoordination is made more pronounced, but may in time apparently disappear. When all canals on both sides are severed, the resulting special movements are not so striking or may be absent, but the loss of coordination is still more pronounced and may be permanent. The like results have been obtained, at least by some observers, by 
section of the eighth nerve or of its vestibular portion (the cighth nerve consists really of two distinct nerves, the vestibular nerve and the cochlear nerve) the semicircular canals being left intact; and in cases where the experiment has been possible, the effect of dividing one semicircular canal has been reproduced by the section of the nerve branch distributed to its ampulla, without any injury to the canal itself.

Now in all animals the three canals are placed in the three planes of space in relation to each other, though not necessarily so that one of them lies exactly in the median plane of the head. Hence whenever the head is turned the cristæ of the ampullæ are unequally affected by the changes of pressure or of flow which the turning brings about in the endolymph of the canals, and when the head is at rest the rclations of the endolymph to the several canals are different in the different positions of the head. And these facts naturally suggest the view that according to the relations of the endolymph to the ampullse, impulses are generated in the cristre, which impulses passt ing up to the brain supply the data by. which the animal becomes aware of the position of his head and so of his body, and enter into the coordination of his movements; these impulses in fact are the special afferent impulses spoken of a little while back. This view is further supported by the following experimental results. Characteristic movements of the head or eyes may be obtained by carefully laying bare a canal and gently blowing over the contained endolymph with a fine glass cannula, the movements differing according as the current of air drives the endolymph towards or away from the ampulla. Similar characteristic movements may be brought about by stimulating the cristie in various ways, as by passing a fine hair into the ampulla, or by suddenly heating or cooling the canal, or, in certain cases at least, by passing an electric current through the ampulla, the last two operations not necessitating the opening of the bony canal.

Without entering into any discussion as to the exact way in which the impulses are generated, as to whether the results for instance of the section of a canal are due to the lack of normal impulses or to the introduction of abnormal ones, we may say that the evidence seems to shew beyond doubt that the cristre of the ampullae are organs through which are generated, according to the position of the head, afferent impulses which form the basis of the sense of equilibrium and enter into the coordination of movements affecting that equilibrium.

The thrce canals however are only affected by a turning of the head; when the head otherwise unmoved is carried dircetly forwards or backwards, or directly upwards or downwards, the effect on all the ampullæ is the same. Yet we are as aware of these movements as of tuming movements, and we may con- 
clude that in them too afferent impulses supply the means of coordination. Evidence of a nature similar to that detailed in reference to the cristæ of the ampullæ may be brought forward, shewing that the macule of the utricle and saccule play a part analogous to that of the cristæ. Indeed it is urged that the otoliths are important agents in this matter, a view which is supported by the result of removing in various invertebrata the bodies called otoliths; for this is a want of coordination very similar to that which we are discussing. No effects on the coordination of bodily movements have, so far as is at present known, been seen to follow removal of the cochlea alone, or section of the cochlear nerve alone. We are thus led to the view that the whole vestibular nerve (apart from the sense of hearing which we shall discuss later on) is the agent of the special afferent impulses so essential to the coordination of the movements affecting the equilibrium of the body, and we may speak of those impulses as "vestibular'; the name "labyrinthine, (from the 'labyrintlı' of the ear) has also been suggested for them.

$\$ 479$. We may here say a few words on the interpretation of the subjective condition which we speak of as giddiness or dizziness or vertigo. We compared the condition of the pigeon after an injury to the semicircular canals to that of a person who is giddy or dizzy, and indeed vertigo is the subjective expression of a disarrangement of the coordination machinery, concerned in the maintenance of bodily equilibrium. It may be brought about in many ways. When a constant current of adequate strength is sent through the head from ear to ear, we experience a sense of vertigo; our movements then appear to a bystander to fail in coordination, in fact to resemble those of a pigeon whose semicircular canals have been injured; and indeed the effects are probably produced in the same way in the two cases. In what is called Menière's disease attacks of vertigo seem to be associated with disease in the ear, being attributed by many to disorder of the semicircular canals, and cases have been recorded of giddiness as well as deafness resulting from disease of the eighth nerve. Visual sensations are very potent in producing vertigo. Many persons feel giddy when they look at a waterfall; and this is a case in which both the sense of giddiness and the disarrangement of coordination is the result of the action of a pure sensation and nothing else. In the wellknown intense vertigo which is caused by rapid rotation of the body visual sensations play a part when the rotation is carried on with the eyes open, but only a part; for vertigo may be induced, though not so readily, by rotation with the eyes completely shut. In the latter case the vertigo is in part caused by abnormal vestibular impulses, and in the case of some deaf persons, that is, persons whose eighth nerve is diseased, is brought 
about with difficulty or not at all, when the eyes are shut; nevertheless there are reasons for thinking that it is in part caused by dircet disturbance of the brain. When the rotation is carried out with the eyes open, the vertigo which is felt when the rotation ceases is partly caused by the visual sensations, on account of the behaviour of the eyeballs, ceasing to be in harmony with the rest of the sensations and afferent impulses which help to make up the coordination. The rotation sets up peculiar oscillating movements of the eyeballs, which continue for some time after the rotation has ceased; owing to these movements of the eyeballs the visual scnsations excited are such as would be excited if external objects were rapidly moving, whereas all the other sensations and impulses which are affecting the central nervons system are such as are exeited by objects at rest. In a normal state of things the visual and the other sensations and impulses, which go to make up the coordinating machinery, are in accord with each other in reference to the events in the external world which are giving rise to them; after rotation they are for a time in disaccord, and the coordinating machinery is in consequence disarranged.

When we interrogate our own consciousness, we find that we are not distinctly conscious of this disaccord; the visual sensations are so prepotent in consciousness, that we really think the external world is rapidly whirling round; all that we are further conscious of is the feeling of giddiness and our inability to make our bodily movements harmonize with our visual sensations. So that even in the cases where the loss of coordination is brought about by distinct sensations, what we really appreciate by means of our consciousness is the disarrangement of the coordinating machinery. It is the appreciation of this disorder which constitutes the feeling of vertigo; both the feeling of giddliness and the disordered movements are the outcome, one subjective and the other objective, of the same thing. It is not because we feel giddy that we stagger and reel; our movements are wrong because the machinery is at fault, and it is the faulty action of the machinery which also makes us feel giddy.

IV e may here perhaps remark that it is an actually disordered condition of the coordinating mechanism which gives rise to the affection of consciousness which we call giddiness, not a mere curtailing of the mechanism or any failure on its part to make itself effective. Complete blindness limits the range of activity of the machinery but leaves the remainder intact, and no giddiness is felt. So again in certain diseases of the nervous system the muscular sense is interfered with over considerable regions of the body, and in these regions condination fails or is imperfect, but the central machinery is not thereby affected, though its area of usefulness is limited, and no giddiness is cxperienced; and so in other instances. 
§480. Forced Movements. So far we have dwelt on disorders of the coordinating machinery brought about by the action of various afferent impulses. Whe have now to call attention to some peculiar phenomena which result from operative interference with parts of the brain, and which in some instances at least may be taken to illustrate how this complex machinery works when some of its inner wheels are broken.

All investigator's who have performed experiments on the brain have observed, as the result of injury to various parts of it, remarkable movements which have the appearance of being irresistible, eompulsory, forced. They vary much in the extent to which they are developed; some are so slight as hardly to deserve the name, while others are strikingly intensc. One of the most eommon forms is that in which the animal rolls incessantly round the longitudinal axis of its own body. This is especially common after section of one of the crura cerebri, or of the middle and inferior peduncles of the cerebellum, or after unilateral section of the pons, but has also been witnessed after injury to the bulb and corpora quadrigemina. Sometimes the animal rotates towards and sometimes away from the side operated on. Another form is that in which the animal executes circus movements, i.e. continually moves round and round in a circle of longer or shorter radius, sometimes towards and someGimes away from the injured side. 'This may be seen after several of the above-mentioned operations, and in one form or another is not uncommon after various unilateral injuries to the brain. 'There is a varicty of the circus movement, "the clockhand movement, said to occur frequently after lesions of the posterior corpora quadrigemina, in which the animal moves in a circle, with the longitudinal axis of its body as a radius, and the end of its tail for a centre. And this form again may easily pass into a simple rolling movement. In yet another form the animal rotates over the transverse axis of its body, tumbles head over heels in a series of somersaults; or it may run incessantly in a straight line backwards or forwards until it is stopped by some obstacle. These latter forms of forced movements are sometimes seen after injury to the corpus striatum, even when a very limited portion of the grey matter is affected. And many of these forcer movements may result from injuries which appear to be confined to the cerebral cortex.

When the phenomena are well developed, every effort of the animal brings on a movement of this forced character. Left to itself and at rest the animal may present nothing abnormal, its posture and attitude may be quite natural; but when it is excited to move or when it attempts of itself to move, it executes not a natural movement but a forced one, turning round or rolling over as the case may be. In severe cases the movement is continued until the animal is exhausted; when the 
exhaustion passes off the animal may remain for some little time quiet, but some stimulus, intrinsic or extrinsic, soon inaugurates a fresh outbreak, to be again followed by exhaustion.

In some of the milder forms, that for instance of the circus movement with a long radius, the curved character of the progression appears simply due to the fact that in the effort of locomotion volitional impulses do not gain such ready access to one side of the body as to the other, the injury having caused some obstacle or other. Hence the contractions of the muscles of one sidc (the left for instance) of the body are more powerful than the other, and in consequence the body is continually thrust towards the other (the right) side. As is well known, we ourselves, when our walk is not guided by visual sensations, tend to describe a circle of somewhat wide radius, the deviation being due to a want of bilateral symmetry in our limbs; and the above circus movement is only an exaggeration of this.

But the other more intense forms of forced movements are more complicated in their nature. No mere blocking of volitional impulses will explain why an animal whenever it attempts to move rolls rapidly over, or rushes irresistibly forwards or backwards. It is not possible with our present knowledge to explain how each particular kind of movement is brought about; and indeed the several kinds are probably brought about in different ways, for they differ so greatly from each other that we only elass them together because it is difficult to know where to draw the line between them. But we may regard the more intense forms as illustrating the complex nature of what we have called the coordinating machinery, the capabilitics of which are, so to speak, disclosed by its being damaged. Such gross injuries as are involved in dividing cerebral structures or in injecting corrosive substances into this or that part of the brain, must, of necessity, partly by blocking the way to the impulses which in a normal state of things are continually passing from one part of the brain to another, partly by generating new unusual impulses, seriously affect the due working of the general coordinating machinery. The fact that an animal can, at any monent, by an effort of its own will, rotate on its axis or run straight forwards, shews that the nervous mechanism for the execution of those movements is ready at hand in the brain, waiting only to be discharged; and it is easy to conceive how such a discharge might be affected cither by the substitution for the will of some potent intrinsic afferent impulse or by some misdirection of rolitional impulses. Persons who have experienced similar forced movements as the result of disease report that they are frequently accompanied, and seem to be cansed, by disturbed visual or other sensations; thus they attribute their suddenly falling forward to the occurrence of the sensation that the ground in front of them is suddenly sinking away beneath 
their feet. Without trusting too closely to the interpretations the subjects of these disorders give of their own feelings, and remembering what was said above concerning vertigo, we may at least conclude that the unusual movements are in many cases due to a disorder of the coordinating mechanism, brought about by strange or disordered sensory impulses. And this view is supported by the fact that many of these forced movements are accompanied by a peculiar and wholly abnormal position of the eyes, which alone might perhaps explain many of the phenomena.

$\$ 481$. The phenomena presented by animals deprived of their cerebral hemispheres shew that this machinery of coordination is supplied by cerebral structures lying between the cerebral hemisphere above and the top of the spinal cord below. But when we ask the further question, how is this machinery related to the various elements which go to make up this part of the brain? the only answers which we receive are of the most imperfect kind.

In the case of the frog we can, after removal of the cerebral hemispheres, make an experimental distinction in the parts left between the optic thalami with the optic nerves and tracts, the optic lobes, and the bulb with the rudimentary cerebellum. When the optic thalami are remover, as might be expected, the evidence of visual impressions modifying the movements of the animal disappears; and it is stated that apparently spontaneous movements are much more rare than when the thalami are intact. When the optic lobes as well as the cerebral hemispheres are removed, the power of balancing is lost; when such a frog is thrown off its balance by inclining the plane on which it is placed, it slips back or falls down; the special coordinating mechanism for balancing must therefore in this animal have a special connection with the optic lobes. But after removal of these organs the animal is still capable of a great variety of coordinate movements: unlike a frog retaining its spinal cord only, it can swim and leap, it maintains a normal posture, and when placed on its back immediately regains the normal posture. The cerebellum of the frog is so small, and in removing it injury is so likely to be done to the underlying parts, that it becomes difficult to say how much of the coordination apparent in a frog possessing cerebellum and bulb is to be attributed to the former or to the latter; probably, however, the part played by the former is small.

In the case neither of the bird nor of the mammal have we any exact information as to the behaviour of the animal after removal of the parts behind the hemispheres, in addition to the hemispheres themselves. Our knowledge is confined to the results of the ablation, or of the stimulation of parts, the cerebellum for instance, in animals in which the rest of the brain has been left intact. Observations of this kind have disclosed many interesting facts, besides the forcerl movements just referred 
to, but they have not led to, and indeed could hardly be expected to lead to, any clear views as to the point which we are now discussing. It does not follow that every part, injury or stimulation of which interferes with coordinated movements, or gives rise to definite, forced, or other movements, is to be considered as part of the machinery under consideration. The corpora striata and cercbral hemispheres form, as we have seen, no part of the machinery, yet injury to them may disorder the machinery; and the fact that removal of, or injury to the cerebellum, disorders the machinery is no proof by itself that the cerebellum is an essential part of the machinery.

If we may trust to deductions from structural arrangements, we might be inclined to infer that the anatomical relations of the tegmental region from the bulb upwards point to its serving as the foundation of the machinery in question. Behind, it has full connections with various parts of the cord, while in front by means of the optic thalami and anterior corpora quadrigemina, if not by other ways as well, it is so far associated with the optic nerves that the path seems open for visual impulses to gain access to it. To this foundation, however, we must add the cerebellum, on account of its relations to it, to the cord and to the bulb through the restiform bodies, including its ties with the auditory nerve. And if we add the cerebellum we must also probally add the pons. We may exclude the pes of the crus, since this is composed exclusively of fibres bringing the cerebral hemispheres, including the corpora striata, into connection with the pons, bulb and cord, and so with the coordinating machinery itself, as well as with other parts of the nervous system. And observation as far as it goes supports this deduction from anatomical relationships. WVe will, however, defer what else we have to say on this point until after we have discussed the carrying out of voluntary movements. 


\section{SEC. 3. ON VOLUNTARY MOVEMENTS.}

$\S$ 482. When we examine ourselves we recognize certain of our movements as 'voluntary'; we say that we carry them out by an effort of the 'will.' And when we witness the movements of other people or of animals we regard as also voluntary such of those movements as by their characters and by the circumstances of their occurrence seem to be carried out in the same way as our own voluntary movements. Even in the case of some of our own movements we are not always clear whether they are really voluntary or no; and in the case of other people and of animals it is still more difficult to decide the question. It would be out of place to attempt to discuss here how voluntary movements really differ from involuntary movements, or in other words, what is the nature of the will; we must be content to take a somewhat rough use of the words 'voluntary,' 'volitional,' and 'will' as a basis for physiological discussion. We may however remark that so far as the muscular side of the act, if we may use such an expression, is concerned, a voluntary movement does not differ in kind from an involuntary movement. It is perfectly true that a skilled man may by practice learn to execute muscular manœuvres which he would not have learnt to execute had not an intelligent volition been operative within him; but our own experience teaches us that many more or less intricate movements which have undoubtedly been learnt by help of the will may be carried out under circumstances of such a kind that we feel compelled to regard them as, at the time, involuntary; and it may at least be debated whether every movement which we can carry out, by an effort of the will, may not appear under appropriate circumstances as part of an involuntary act. In the case of the lower animals, in the frog deprived of its cerebral hemispheres for instance, we have seen that voluntary differ from involuutary movements, not by their essential nature but by the relation which their occurrence bears to circumstances. We have therefore to seek for the distinction between voluntary and involuntary, not in the coordination of the muscular and nervous components of a movement, but in the nature of the process which starts the whole act. 
The histories, related in a preceding section, of various animals deprived of their cerebral hemispheres, while they have further shewn the difficulty of drawing a sharp line between the presence and absence of volition, such as when we appeal to our own consciousness we seem able to draw, have taught us that in a broad sense the presence of volition is, in the higher vertebrata, dependent on the possession of the cerebral hemispheres: and we have now to inquire what we know concerning the way in which the cerebral cortex, for this, as we have seen, is the important part of the cerebral hemisphere, by the help of other parts of the nervous system carries out a voluntary movement.

$\$ 483$. With this view we may at once tum to the results of experimental interference with the cortex. When the surface of the brain is laid bare by removal of the skull and dura mater, mechanical stimulation of the cortex produces little or no effect, thus affording a contrast with the results of mechanically stimulating other portions of the brain, or other nervous structures. And for a long time the cortex was spoken of as insensible to stimulation. When, however, the electric current is employed, either the make and break of the constant current, or the more manageable interrupted current, very marked results follow. It is found that certain movements follow upon electric stimulation of certain regions or areas. The results, moreover, differ in different animals. It will be convenient to begin with the $\log$, on which animal the observations of this kind were first conducted.

When the surface of the dog's brain is viewed from the dorsal surface a short but deep sulcus is seen towards the front, rumning outwards almost at right angles from the great longitudinal fissure; this is ealled the crucial sulcus (Fig. 121), the gyrus or convolution in front and behind it, and sweeping round its end being called the sigmoid gyrus. It will hardly be profitable to discuss here cither the homology of this sulcus or the names of the other sulci and convolutions of the dog's braim. We mention this sulcus because it is found that stimulation of the cortex in a region which may be broadly described as that of the neighbourhood of this erucial sulcus gives rise to movements of various parts of the body, whereas no such movements result from stimulation of the extreme frontal region in front of the area around the crucial sulcus, or from stimulation of the occipital region behind this area. Certain exceptions may be marle to this broad statement, but these it will be best to discuss in reference to the more highly developed monkey.

The region of the cortex in the neighbourhood of the crucial sulcus may then be termed an 'cxcitable' or 'motor' region, inasmuch as stimulation of this region leads to movements carried out by skeletal muscles, while stimulation of other regions does not. Further, stimulation of particular districts or areas 
of the region leads to particular movements carried out by particular muscles. For instanee, stimulation of the more median parts of the gyrus behind the crucial sulcus (Fig. 121 ff) leads to movements of the hind limb, whereas stimulation of the lateral part or outer end of the same gyrus leads to movements of the fore limb, and we may here distinguish between an area stimulation of which (Fig. 121 + ) leads to flexion of the fore limb, and an area (Fig. 121 +) stimulation of which leads to extension of the same limb. In a similar way stimulation of other

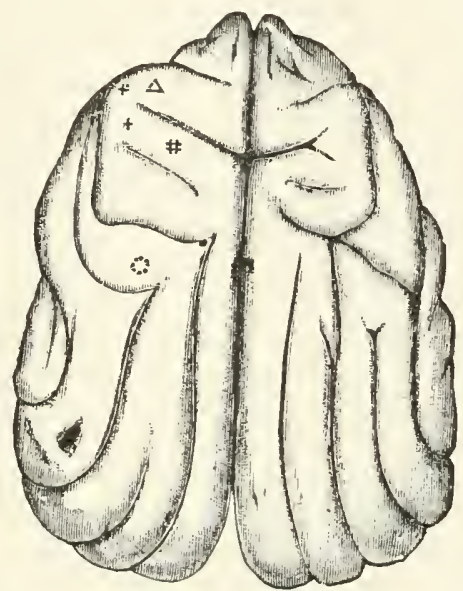

Fig. 121. The Areas of tile Cerebral Coxvolutions of tife Dog, accorde ing to Hitzig and Fritsch.

(1) $\triangle$ The area for the muscles of the neck. (2) + The area for the extension and adduction of the fore limb. (3) + The area for the flexion and rotation of the fore limb. (4) $\ddagger \ddagger$ The area for the hind limb. Running transversely towards and separating (1) and (2) from (3) and (4) is seen the crucial sulcus. (5) $\odot$ The facial area.

areas within the 'motor' region leads to movements of this kind or of that kind of the tail, of the eyes, of the mouth, of other parts of the face, of the tongue, and so on. Obviously in the dog this region of the cortex has connections with the skeletal muscles which do not obtain between other regions of the cortex and those muscles; and further, the region in question is topographically differentiated, so that certain areas or districts of the region are specially connected with certain skeletal muscles or groups of muscles. We may speak of a 'localisation of function' in this region as compared with other regions of the cortex, and in the several areas within the region as compared with each other.

The muscles which are thus thrown into contraction are the muscles of the opposite side of the body. When 'the fore limb 
area,' as we may call it, of the right hemisphere is stimulated, it is the left fore limb which is moved; and so with the other areas; it is only in exceptional cases, as in certain movenents of the eyes, that the effect is bilateral; a movement confined to the same side as that stimulated is never witnessed.

The results are most clear when the current employed as a stimulus is not stronger than is just sufficient to produce the appropriate movement (roughly speaking an interupted current just perceptible to the tongue of the operator is in ordinary cases a useful one), and when the cortex is in good nutritive condition. In any experiment the results obtained by the earlier stimulations, soon after the cortex has been exposed, are the best; after repeated stimulations the surface is apt to become hyperamic, and it is then freguently observed that the movements resulting from the stimulation of a particular area are not confined to the appropriate muscles, but spread to the corresponding muscles of the opposite side, then to muscles comnected with other cortical areas, and at last to the muscles of the body generally: at the same time the movements lose their distinctive purposeful character and the animal is thrown into convulsions of an epileptiform kind. It not unfrequently happens that an experiment has to be stopped in consequence of the onset of these epileptiform convulsions. The response of movement to stimulation may be observed while the animal is under the moderate influence of an anresthetic, but a too profound ancesthesia lessens or annuls the effects.

In order to carry out a closer analysis of the phenomena it is desirable to watch or record the contraction of a particular group of muscles, or perhaps better still a particular muscle, $e$. $g r$. the area for extcnsion of the hind limb may be studied by help of the extensor digitorum communis of the limb. IVhen this is done the following important facts may be observed. The area of cortex having been found which gives the best movements, and the stimulus being no stronger than is necessary, isolation of the area from its lateral surroundings by a circular incision carried to some little depth will not prevent the development of contractions in the muscle; but these do cease, even without the circular incision, if by a horizontal section the grey cortex is separated from the subjacent white matter. After removal of the cortex, stimulation of the white matter underlying the area proluces the appropriate contraction; not only however is a stronger stimulus necessary, but also the latent period, that is the time intervening between the beginning of the application of the stimnlating current and the beginning of the muscular contraction is appreciably shortened. The appropriate contractions not only appear whien the white matter immediately below the cortex is stimulated, but by making successive horizontal sections and stimulating each in turn, the cffect may, so to 
speak, be traced through the central white matter of the hemisphere down to the internal capsule. We may conclude from these results, that when the current is applied to the surface of the cortex, certain parts of certain structures in the grey matter are stimulated, the process having a marked latent period, and that as the outcome of the changes induced in the grey matter, impulses pass along the fibres leading down from the grey matter to the internal capsule, and so by the pedal fibres of the crus to the spinal cord and motor spinal roots. Anatomical considerations lead us to suppose that the fibres in question belong to the great pyramidal tract; and as we shall see, all our knowledge confirms this view.

It must not, however, be supposed that the several areas stimulation of which produces each its distinctive movenent, are in the dog sharply defined from each other; when the term area for extension of the hind limb is used it must not be supposed that the area can be defined by an outline, within which stimulation produces nothing but extension of the hind limb, and outside which stimulation never produces extension of the hind limb. All that is meant in that extension of the hind limb is the salient and striking result of stimulating the area. When we study the various movements, and especially perhaps when we study, by help of a graphic record, the contractions of various individual muscles resulting from the stinulation of various parts of the motor region, we find not only that the areas for particular movements or particular muscles are very diffuse, but that the several areas largely overlap each other. If for instance we were to map out on the same diagram the several areas belonging to four or five muscles of different parts of the body, such as the extensors of the digits of the fore and of the hind limb, the flexors of the same, and the orbicular muscle of the eyelid, that is to say, the several areas within which in turn stimulation of the cortex produced contraction of the particular muscle, the overlapping would be so great that the whole figure would appear highly confused. In a simular way the excitable motor region as a whole would gradually merge into, be broken up into, the unexcitable frontal, occipital and temporal regions, in front, behind and below. In other words, the localization in the cortex of the dog is to a marked degree imperfect.

In this respect the $\mathrm{dog}$, corresponding to its position in the animal hierarchy, is intermediate between such animals as the rabbit, the bird, and the frog, on the one hand, and the more highly developed monkey on the other; and that is one reason why we have taken the dog first and dwelt so long 11 pon it. In the rabbit, a similar localization may be observed, but far less definite, far more diffuse; it becomes still less in the bird, and is hardly recognizalle in the frog. It will not be profitable to dwell on the details of these lower animals; but the phenomena 
of the monkey, leading up as they do to those of man, call for special notice.

484. When in a monkey, in an individual for instance belonging to the genus Macacus, the surface of the cerebrum is explored with reference to the effects of electrie stinulation, it is found that when the eurrent is applied to the precentral or ascending frontal and the post-central or ascending parietal convolutions which lic respectively in front of and behind the important central fissure or tissure of Rolando (cf. Fig. 122),
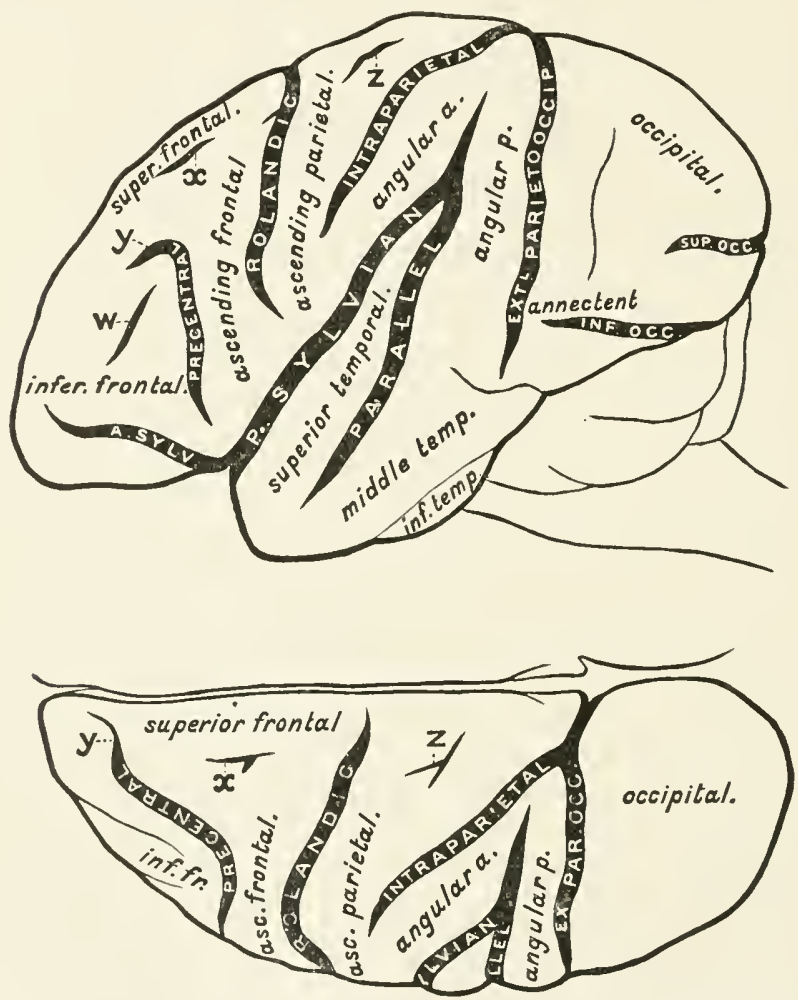

Fig, 122. Outline of Brain of Moniey (Macacus) to shew principal Sulci (Fissures) ani Gris (Convolutions). (Natural size.) (Sherrington after Horsley and Schäfer.)

The brain fignred is the same as that in Fig. 123, and the two figures should be consulted together. Over each sulcus, purposely printed very thick, the name is written in small capitals, over each gyrus in italics. $x$ indicates the small depression, hardly to be called a sulcus, which is supposed to be homologous with the superior frontal sulcus of man; and $w, y, z$ similarly indicate sulci whose homologies are not certain. For some synonyms see Figs. 1:34, 135.

movements of the forc limb follow. The 'motor area for the fore limb' thus discovered is more circumseribed and definite 
than is the corresponding area in the dog. Its outline (Fig. 123 ) is roughly that of a truncated triangle bisected by the central fissure, with the broad base at some distance from the mesial line, and the truncated apex reaching on the lateral surface of the hemisphere to a well-marked bend in the lower part
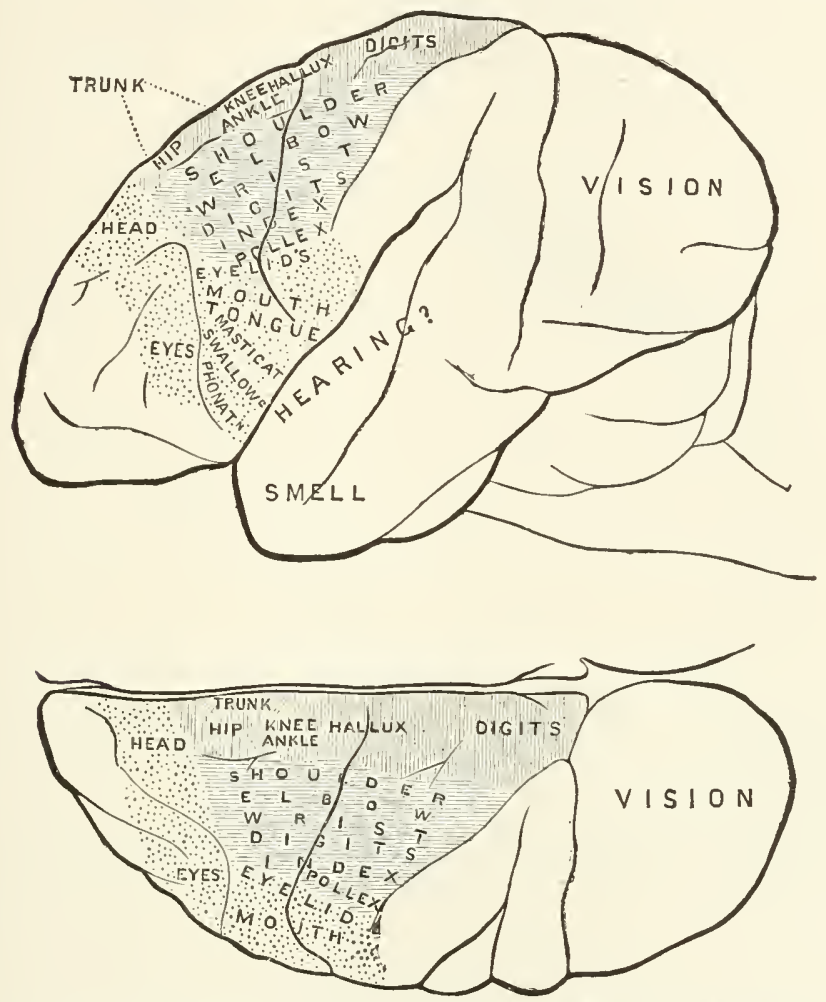

Fig. 123. Left hemispiere of the Cerebrum of Macacus Monkey viewed FROM ITS LEFT SIDE, AND FROM ABOVE. (Natural size.) (Sherrington after Horsley and Beevor.)

The figure shews the positions of the portions of the cortex concerned with movement of various parts, and with the senses of sight, smell, and hearing. The cortical area connected with the novements of the leg is shaded rertically across, that with the movements of the arm horizontally, and that with the movements of the trunk in a slanting direction; the area connected with movements of the head (neck), face, and eyes is dotted. The course of the clief fissures is indicated by single lines.

of the central fissure. Behind, it reaches as far as the intraparietal fissure which somewhat sharply defines its hind border, and in front it ceases no less definitely at some little distance behind the precentral fissure. Further examination shews that the whole area is divided into areas corresponding to movements 
of particular parts of the fore arm, and that these are arranged in a definite relation to each other. In the more dorsal part of the area, at the base of the triangle, stimulation produces movements of the shoulder (Fig. 12:3); if the electrodes be shifted ventrally movements of the elbow make their appearance; if still more ventrally, movements of the wrist come in, and these are in turn succeeded ventrally by movements of the digits generally, of the forefinger, and lastly of the thumb. A very striking experiment may be made by applying a current of suitable strength, first at the lower, ventral horder of the area, and then gradually advancing upwards towards the mesial line; the thumb is moved first, then the forefinger, then the rest of the digits, then the wrist, next the elbow, and lastly the shoulder. Further, in certain parts of the arca the resulting morement is flexion of the appropriate segment of the limb, in other parts extension, in certain parts abduction, in other parts adduction, and so on.

Similar exploration shews that the 'area for the hind limb,' lies on the median side of the area for the fore limb, stretching besides on to the mesial surface along the marginal convolution which forms the dorsal portion of the wall of the great longitudinal fissure; it reaches as far back as tho intra-parietal sulcus, and is suceeeded in front by the 'area for the trunk' (Fig. 124). Within this general area for the hind limb we may similarly distinguish special areas for the hip (Figs. 123, 124) in the front portion, for the knee and ankle behind this, and for the digits still farther backwards, the area for the great toe being however in front of the area for the other digits.

In front of the areas for the limbs and trunk, on the median dorsal surface, dipping down into the mesial surfacc along the marginal convolution (Fig. 124) and reaching laterally on the dorsal lateral surface to the dorsal cxtremity of the precentral sulcus (Fig. 123), is the 'area for the head," that is to say for' movements of the head brought about by contractions of the muscles of the neck.

Ventral to this again, in front of the precentral sulcus is the 'area for the cyes,' that is to say, for contractions of the ocular muscles; and behind the precentral sulcus, ventral to the arm area, lies a small area for movements of the eyelids, brought ahout by contractions of the orbicularis muscle. Ventral to this again is the 'area for the face,' in which we may distingruish an area for the mouth, that is an area stimulation of which produces changes in the buecal orifice, opening, shutting, drawing to one side fe., and an area for movements of the tongue. These two areas reach downwards to the fissure of Sylvius, and backwards to the line of the intra-parietal sulcus. In front of them, occupying all the ventral part of the precentral convolution and reaching forwarks as far as the precentral sulcus, where 
it meets the area for the eyes, lies an area stimulation of which produces movements of the pharynx or larynx as well as the mouth or face, and which may be divider into areas for mastication, for swallowing, and for the production of the voice.

We might speak of these several areas in another way by referring to the nerves concerned in carrying out the several movements, though in doing so we must remember that there is not an exact correspondenee between the relative position of a

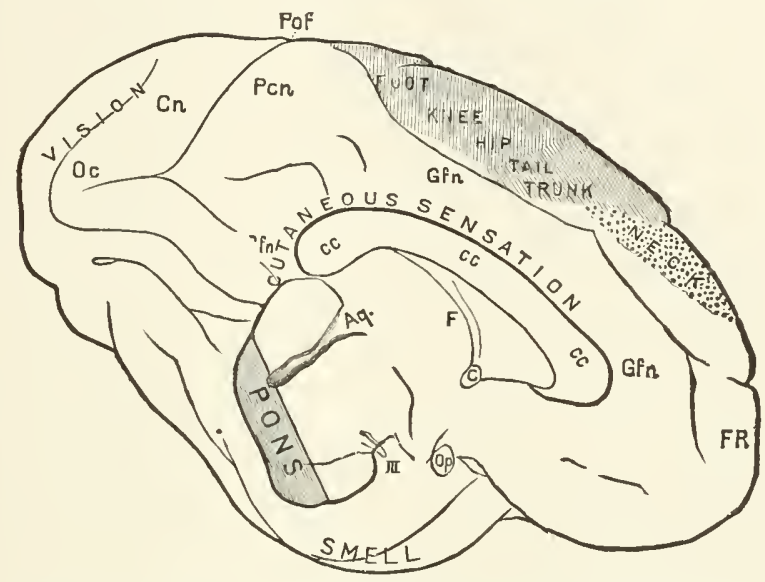

Fig. 124. Mesial aspect of the left inalf of the Brain of Magacus, MISPLAYED BY SECTION IN TIIE MEDIAN SAGITTAL PLANE AND REMOVAL OF the Cerebellum. Natural size. (Sherrington after Horsley and Beevor.)

The hatched and stippled parts of the surface shew the regions of the cortex connected with movements of the foot, knee, hip, tuil, trunk, and neck respectively. The sereral positions of the areas of cortex comnected with vision and smell and with cutaneous sensation are indicated by the appropriate words.

The plane of section has passed through the corpus callosum, $c c, c c, c c$, and through the anterior commissure, $c$, sparing the left pillar of the fornix, $F$; behind it has bisected the anterior part of the pons, laying open the aqueduct, $\mathrm{A} q$. (iter a tertio ad quartum ventriculum). Pons, the left half of the pons in frontal section. Op. the optic commissure cut across.

III. the root of the third cranial nerve.

$F R$. the frontal pole, $O C$. the occipital pole; $C n$. the cuneus, $P c n$. the precuneus; $G$. $f n$. $G_{\text {r. }} f n$. Fr. fn. the gyrus fornicatus; the unlettered fissure seen to form the upper boundary of this gyrus in its supra-callosal part is the callaso-marginal, Po. $f$. the parieto-occipital fissure.

muscle along the axis of the body or along the axis of a limb and the relative position along the cerebrospinal axis of the nerve or nerves governing the muscle. We may however, adopting this method, note that the sacral and lumbar nerves are represented by the most mesial portion of the whole motor area and by the hind division of this mesial portion; that the lumbar and thoracic nerves are represented by the front division of the same 
mesial portion; that the upper thoracic with the lower cervical nerves belong to a region lying lateral to, and the upper cervical nerves to one lying in front of the preceding area; and lastly that the remaining lateral and ventral portions of the whole motor region appertain to the cranial nerves. But the topographical differentiation does not come out so clearly by this method, as by that of taking for our guide distinctive movements of the several parts of the body.

It will be observed that all these areas taken together, represented by the portion of Figs. 123, 124 shaded in one way or another, occupy chiefly the parietal region of the cerebral surface though they also reach into the frontal region. Stimulation of the frontal region in front of this motor area or of the occipital region behind, whether on the lateral or on the mesial surface, or of the temporal region, whether also on the lateral or on the mesial surface, or of the gyrus fornicatus (Fig. 124) connecting the frontal and occipital regions on the mesial surface, and running ventral to the marginal gyrus, does not give rise to movements; or to be more exact, does not give rise to movements comparable to those just described as resulting from stimulation of various parts of the motor region. Movements do take place when certain parts of the occipital or of the temporal region are stimulated, but these are not only less striking and experimentally less certain than, but appear to be of a different nature from those resulting from stimulation of the motor region; it will be eonvenient to speak of the nature and meaning of this kind of movement when we come to discuss the development of sensations.

$\$ 485$. It is obvious from the foregoing that the mechanisms for the development of these movements of cerebral origin are far more highly differentiated in the monkey than in the dog. But even in the monkey (Macacus and allied forms) the differentiation is still very incomplete. If we explore for instance the area for the wrist we find that its limits are ill-defined. In some parts of the area we obtain movements of the wrist only, but in other parts of the area stimulation produces not only movements of the wrist, but also of the shonlder or of the digits, or of the neck; and so with the other areas.

If. however, not a Macacus or other ordinary monkey, but the more highly developed onrang otang be taken as the subject of experiments, the differentiation is found to be distinctly advancerl; the several areas are more sharply defined, and what is important to note, the respective areas tend to be separated from each by portions of cortex, stimulation of which gives rise to no movement at all.

The opportunities of stimulating the cortex of man himself have been few and far between, and have for the most part been conducted under unfaromable circumstances; but so far as the results obtained go, they shew that the topographical distribu- 
tion of areas for the several movements is carried out on the sanle plan as in the monkey (we are purposely confining ourselves now to the results of artificial stimulation); and moreover, justify the conclusion, which a priori reasons would lead us to adopt, that in man the differentiation is advanced still farther than in the monkey.

Thus when we survey a series of brains in succession, from the more lowly frog, through the bird, the rabbit, the dog, and other lower mammals up to the monkey, the anthropoid ape, and so to man himself, we find an increasing differentiation of the cerebral cortex, by which certain areas of the cortex are brought into special connection with certain skeletal or other muscles in such a way that stimulation of a particular portion of the grey matter gives rise to a particular movement and to that alone.

$\$$ 486. Before proceeding further, it will be perhaps advantageous to call to mind some of the important features of the great strand of fibres known as the pyramidal tract. These fibres start from the cerebral cortex of the motor region which we are studying; they are the axis cylinder processes of some of the large pyramidal cells which are so conspicuous in this region of the cortex. Passing downwards from the cortex through the white matter of the hemisphere and the corona radiata they are gathered up into the interual capsule between the nucleus lenticularis on the one side and the nucleus caudatus with the optic thalamus on the other side (Figs. 125, 126, 127). "The internal capsule has the form of a fan, the handlc of which passes into the crus cerebri. while the expansion stretehes into the hemisphere; it is further bent into a rounded angle (Fig. 125, G), the 'knee,' which separates a front from a hind limb. The fibres of the pyramidal tract occupy the knee, a small adjoining portion of the front limb and a large part of the hind limb; but it must be remembered that when we examine the internal capsule by horizontal sections taken in succession from the dorsal to the ventral regions, we find that the knee shifts in position and changes in the width of its angle, that the two limbs vary in direction, in size and in shape, and that at last the bent flattened capsule passes into the more or less rounded crus by the rapid disappearance of the fore limb and the consequent extinction of the angle. When the capsule has thus passed into the crus, or rather into the pes of the crus, the pyramidal tract is found occupying the median region of the pes (Fig. 128). Farther batckward the fibres of the tract are found running in the pons (Fig. 129, 130, 131), in strands in. terwoven with the transverse fibres of that structure, and then issuing from the pons are found concentrated again into the anterior pyramids of the bulb (Fig. 132). At the decussation of the pyramids, while most of the fibres cross over to form the crossed pyramidal tract of the spinal cord some are continued on as the direct pyramidal tract, which however speedily dimin- 


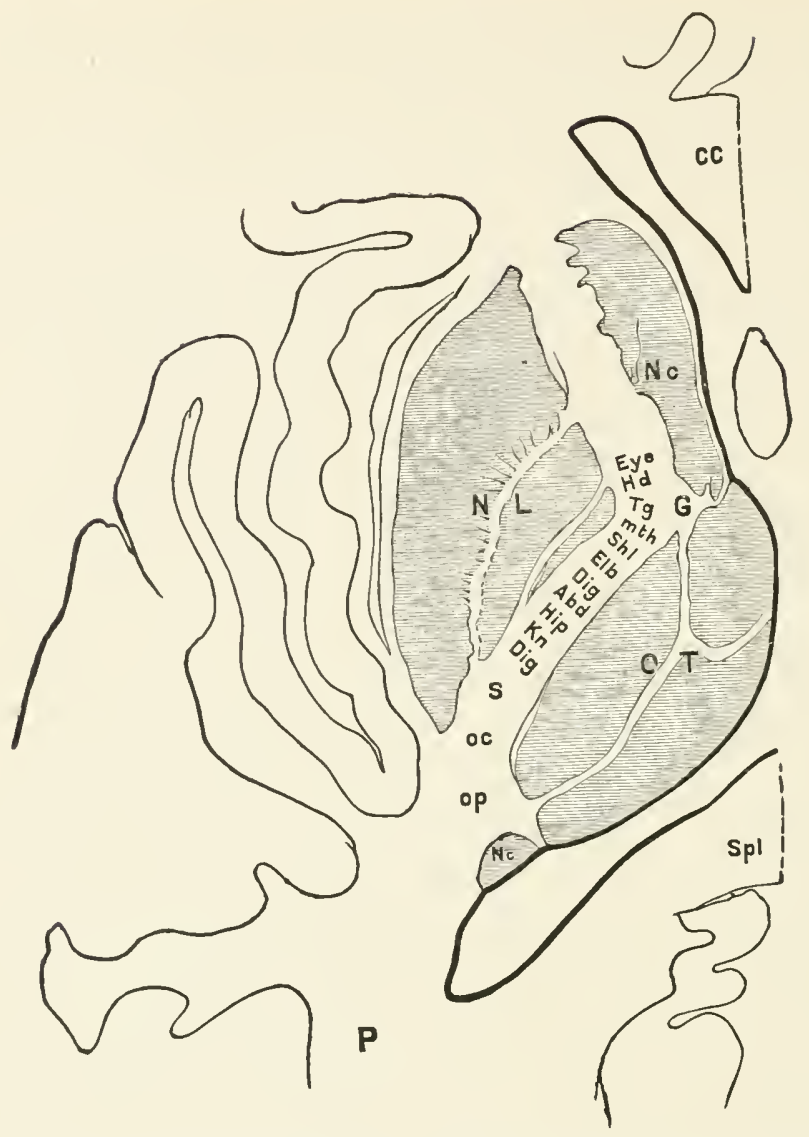

Fig. 125. Outline of Horizontal section of Brain, to shew the interial CAPSUle. (Natural size.)

The section is taken at a level more ventral than shewn in Fig. 115. The grey matter of the cortex and claustrum is left unshaded, but that of the corpus striatum and optic thalamus is shaded.

OT. optic thalamus, shewing the median, lateral, and anterior nuclei. $N L$. nucleus lenticularis, shewing the putamen large, and the inner division of the globus pallidus very small. $N C$. nuclens caudatus, the large head in front of, and the diminishing tail behind, the thalamus.

G. the knee of the internal capsule. From 'Eye' to 'Dig,' marks the position of the pyramidal tract as a whole, and the several letters indicate broadly the relative positions of the several constituents of the tract, named according to the movements with which they are concerned; thus Eye movements of the eyes; $I I l$. of the head ; $T g$. of the tongue ; mlh. of the mouth ; shl. of the shoulder; elb. of the elbow; Dig. of the hand; Abl. of the abdomen; Irip, of the hip; hin. of the knee; Dig. of the foot:

S. the temporo-occipital tract. oc. fibres to the occipital lobe. Op. optic radiation. At this level the fibres of the frontal tract, in the fore limb of the capsule in front of the pyramidal tract, run almost horizontally, parallel with the plane of the section, Cf. Fig. 126, Fron. 
$c c$. the rostrum of the corpus callosum, Spl. the splenium of the same, both cut across horizontally. The thick dark lime indicates the boundary of the cavities of the anterior and descending horns of the lateral ventricle and of the third ventricle, the two ventricles being laid open into one by the removal of the velum and choroid plexus\&e. The oval outline in the fore part of this cavity indicates the fornix.

Lateral to the nucleus lenticularis is seen in outline the claustrum, the cortex of the island of Reil and the operculum or convolution overlapping the island of Reil.

$P$ is inserted to shew which is the hind part of the section.

ishes and disappears, through fibres leaving it to cross over to the other side by the anterior commissure. During its progress along the spinal cord, the fibres both of the crossed and the direct tract end by coming into comnection with the cells of the anterior cornu, either by means of their collaterals or by their actual terminations. In the bulb and higher up, the traet gives off fibres which crossing over to the other side make connections in a similar way with nerve cells whose axis cylinder processes are motor fibres in the cranial nerves. From these relations of the pyramidal tract it is obvious that the fibres of this tract must be concerned in the development of the movements which we have

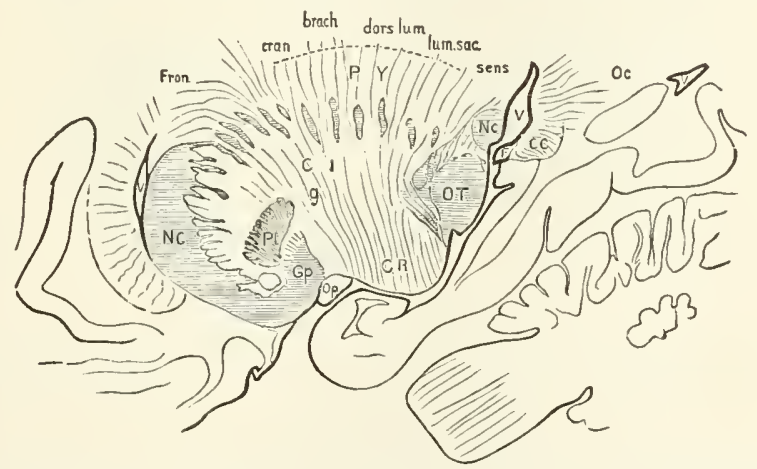

Fig. 126. Outhine of a Sagittal section throvgin the iiemisphere. Man. (Sherrington.)

The section is taken not far to the right of the median plane and is one half linear of natural size. The grey matter of the corpus striatum and thalamus is sharled.

$N c, V_{c}$, the caudate nuclens; $P t$, the putamen and $G p$, the globus pallidus of the lenticular nucleus ; $O T$. the optic thalamus; $C I$, the internal capsule with a streaked appearance revealing approximately the direction taken by fibrebundles passing into it from the portion of corona radiata over it. In these sets of bundles may be broadly distinguished a frontal system, fion., a pyramidal system, $P Y$ (sub-divisible into cranial [cran.], brachial [brach.], dorso-lumbar [dors. lum.], and lumbo-sacral [lum. sac.], parts) and a temporo-oceipital system. sens.; the situation of the genu of the internal capsule is indicated by $g . C R$, the crus cerebri; $O c$, the so-called optic radiations passing into the occipital lobe ; $c c$, the splenial end of the corpus callosum; $v, v, v$, the lateral ventricle cut across in three different places; $F$, the fornix in cross-section; $O p$, the optic tract in cross-section. I'art of the cerebellum is seen in outline to the right. 


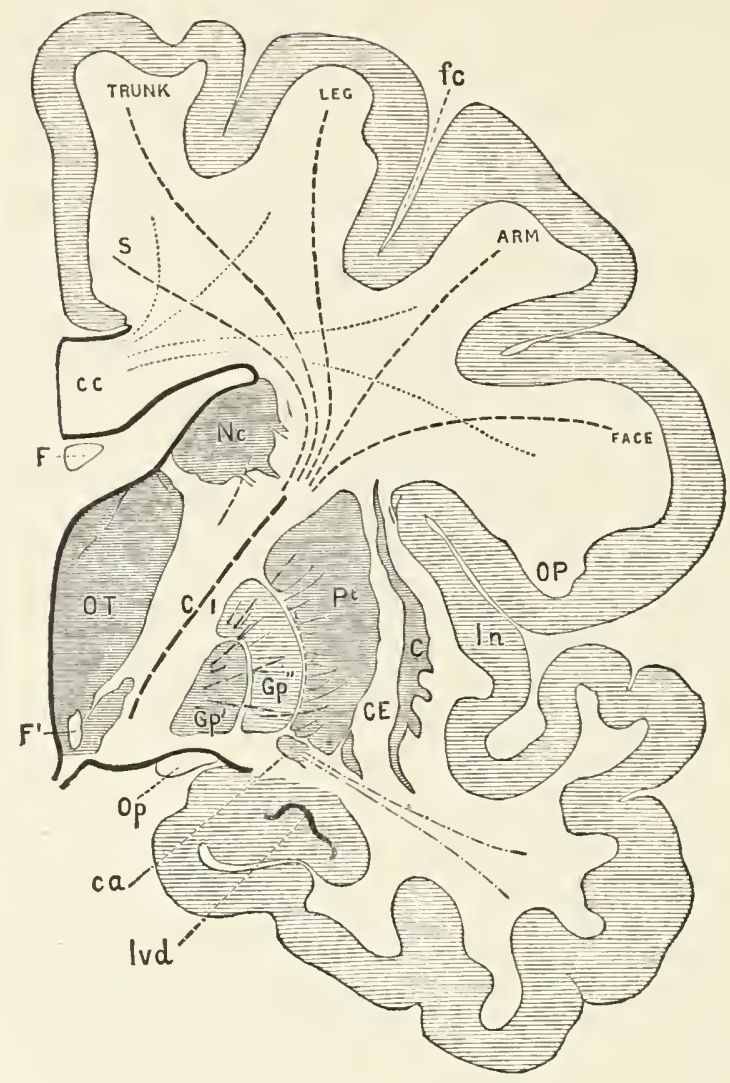

Fig. 127. Outline of a transterse dorso-tentral section of the right HALF OF THE BRAIX. (Natural size.) (Sherrington.)

The section which is taken at the level of the knee of the capsule and is therefore intermediate between those shewn in Figs. 116-117 is introduced to illustrate the course of the constituents of the pyramidal tract.

$O . T$. optic thalamus; N.c. nucleus caudatus, the head only appears in this section. Pt. putamen, $G p^{\prime \prime}, G p^{\prime}$ the two parts of the globus pallidus of the nucleus lenticularis; $C$. the claustrum ; C.E. the external capsule; $I n$. the island of Reil. c.a., the anterior commissure shaded to render it distinct and the fibres from the temporo-sphenoidal lobe which pass into it being indicated by broken lines. Op. the optic tract; lvd. the end of the descending horn of the lateral ventricle; $F$. the fornix; $F^{\prime}$. the end of the anterior pillal of the fornix in the base of the thalamus; c.c. corpus callosuin; O.P. anterior part of the occipital lobe.

f.c. is the central fissure or fissure of Rolando. The course of the fibres of the pyramidal tract connected respectively with the trunk, leg and arm, and hence with spinal nerves, and of those connected with the face and hence with cranial nerves, is shewn by broken lines. These are all secn converging into the internal capsule C.I. This figure should in respect to the course of these fibres be compared with the horizontal section shewn in Fig. 125, and the sagittal figure shewn in Fig. 126. 
just described. When the movements are brought about by stimulation of the fibres in some part of their course, in the internal capsule for instance, there can be no doubt that the stimulation starts impulses which, travelling down the tract to the origins of certain cranial or spinal nerves, in some way give rise to coordinate motor impulses along the motor fibres of the nerves; and we may with reason speak of the impulses then passing along the tract as motor or efferent in nature. When the stimulus is applied direct to the cortex, we may assume that processes, started in the grey matter, eventuate in similar efferent impulses along the fibres of the tract. All the evidence leads us to regard this tract as an efferent tract.

When the spinal cord is divided in the lower dorsal region and the electrodes of an electrometer are brought into connec-

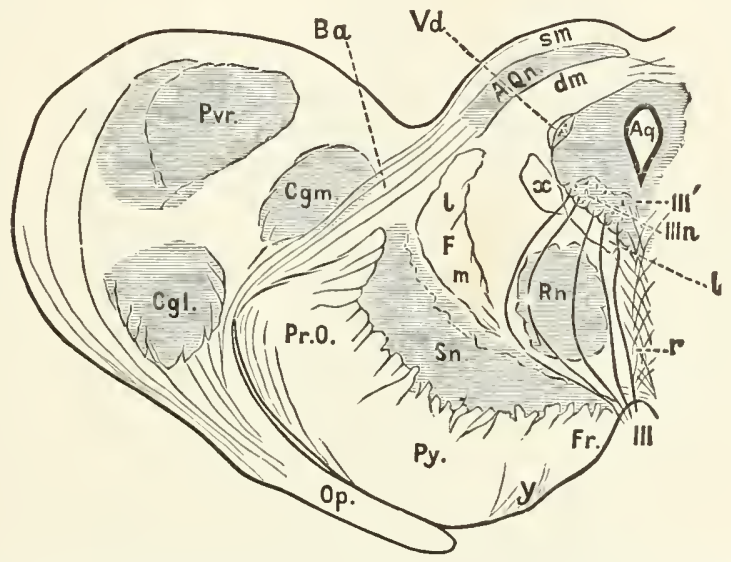

Fig. 128. Through the Crus and Anterior Corpora Quadrigemina.

(One half only is shewn.) (Sherrington.)

(In the line 114, Fig. 108.)

$P y$. the pyramidal portion of the pes. $F r$. the region of the pes occupied by fibres from the frontal portion of the cortex. $P r$. $O$. the region occupied by fibres coming from the occipital portion of the cortex. $y$. fibres coming from the fillet. Op. the optic tract. $F$. the fillet, $l$. the lateral portion, $m$. the median portion. $l$. the posterior longitudinal bundle. B. $a$. the brachium of the anterior corpus quadrigeminum. $x$. fibres from the posterior commissure of the cerebrum. $r$. raphe. S. n. substantia nigra. $R . n$. red nucleus. $C . g . l$. lateral, and $C . g . m$. median corpus geniculatum. $P v r$. pulvinar of optic thalamus. A. Q.n. nucleus or grey matter of anterior corpus quadrigeminum. III. $n$. nucleus of III. third nerve. III'. rootlets from the dorsal part of III. $n$. the nucleus of the third nerve which cross the median line to emerge with rootlets derived from the nucleus of the opposite side. $s . m$. superficial layer of fibres of the ant. corp. quad. $d$. m. deep layer. $V$. $d$. descending root of the fifth nerve. Aq. aqueduct surrounded by cerebial grey matter.

tion with the transverse cut surface and with some point of the longitudinal surface above, the electrometer gives evidence of currents of action (manifested as negative variations of a demar- 
cation current or current of rest, $\$$ 64) whenever the motor area of the hind limb is stimulated, but not when other parts of the cortex are stimulated. We have already said that stimulation of any part of the motor region may under abnormal conditions give rise to general epileptiform convulsions; when these occur during such an experiment as the above, eurrents of action manifest themselves in the lower dorsal cord. whether the stimulation giving rise to the convulsions be applied to the area for the hind limb or to any part of the motor region. It has been further observed that the currents of action developed within the spinal cord tally in a very exact manner with the muscular movements. 'The convulsions begin with a sustained 'tonic' contraction of the muscles, and the electrometer shews a similar' sustained current of action; this is followed by rhythmic movements of the

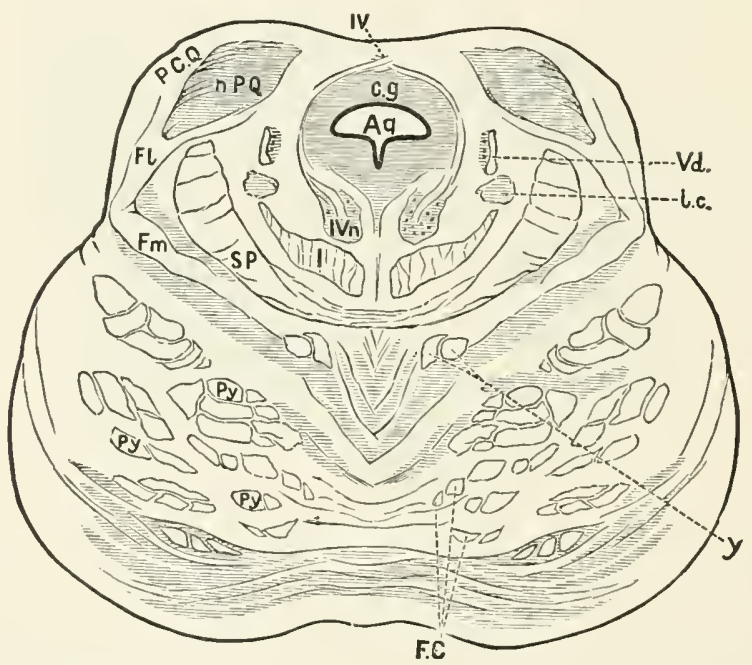

Fig. 129. Throvgh the Fore Part of the Poss. (Sherrington.)

(In the line 113, Fig. 108.)

Py. Pyramidal fibres. F. C. Fibres from the frontal cortex. S. P. Superior Peduncle of the cerebellum. $F$. m. median portion, $F$. $l$. lateral portion of the Fillet. l. posterior longitudinal bundles. P. C. Q. Posterior corpora quadrigemina. $y$. Fibres which become detached from the Fillet, and further forward from (the innermost) part of the Pes of the Crus. l. c. locus caemleus. n. P.Q. nucleus of the posterior corpora quadrigemina; the outline is made too sharp. IV. bundles of the fourth nerve decussating, IV. $n$. its nucleus. $V$. r. descending root of the fifth nerve. Aq. the aqueduct. $c . g$. the region of central grey matter.

muscles, accompanied by corresponding rhythmic movements of the mercury of the electrometer. Withont insisting ton much on the exact interpretation of these results we may take them as at least shewing that, when the motor region of the cortex is 
excited, nervous impulses accompanied by 'currents of action' pass downward along the fibres of the pyramidal tract.

The results of stimulating the fibres of the tract in their course through the corona radiata and the internal capsule and

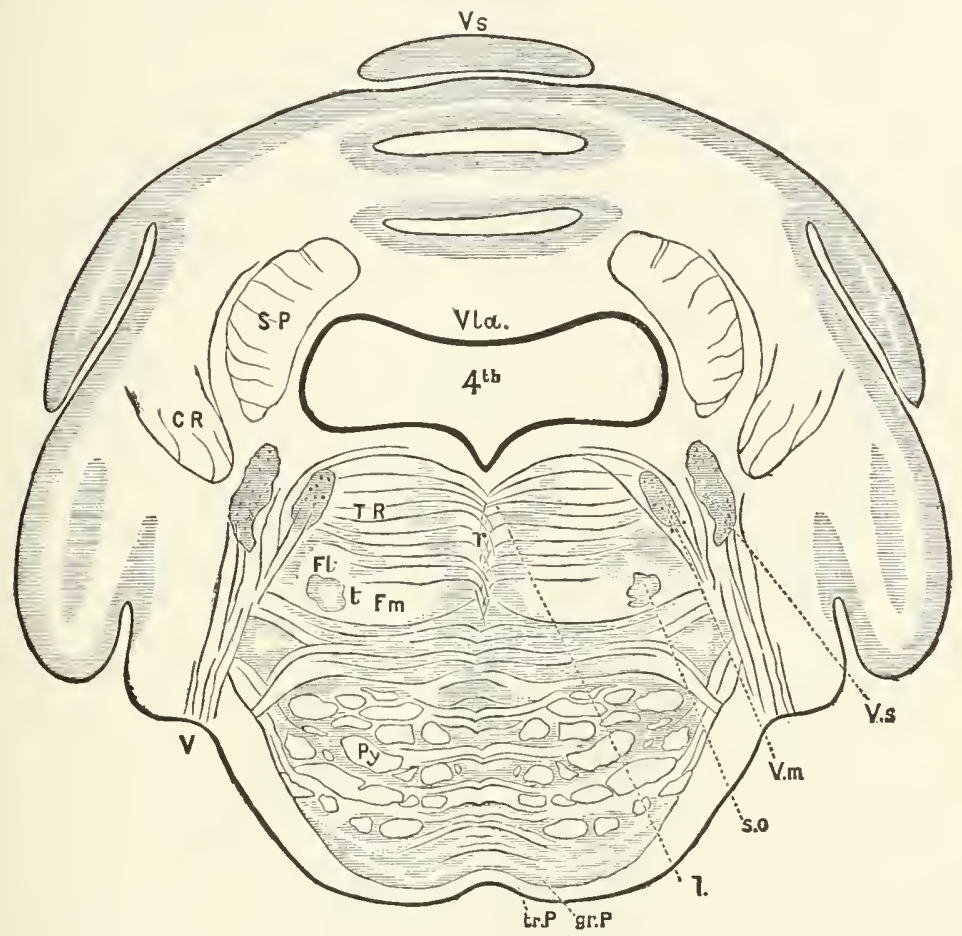

Fig. 130. Throdgh the Pons at the exit of the Fifth Nerve. (Sherrington.)

(In the line 112, Fig. 108.)

C. R. Remains of restiform body. S. P. superior peduncle of the cerebellum. $F$. m. median, F. l. lateral Fillet. $T$. $R$. tegmental reticular formation, $t r . P$. superficial transverse fibres of the Pons, $l$. posterior longitudinal bundles. I. $s$. superior vermix; seetions of three folia are shewn, one being detached; between them the intervening sulci laid open by the section are seen. $V 7$. $\alpha$. valve of Vieussens or anterior velum. $r$. raphe. Py. Pyramidal fibres, $g r$. P. grey matter of the Pons. $s . o$. superior olive. $t$. placed on the left side indicates the position of a bundle of longitudinal fibres which may be traced forward into the subthalamic regions. $\boldsymbol{V} . m$. motor nucleus, $V$. s. sensory nucleus, and $V^{r}$. roots of the fifth nerve.

4th. fourth ventricle; shading of central grey matter omitted as in Fig. 131.

the results obtained by studying the degenerations following upon injury to or removal of the several parts of the cortical motor region, agree in marking out the paths taken by the several constituents of the tract through the central white mat- 
ter of the hemisphere, the corona radiata and the capsule. Comparing Figs. 123, 124 with Figs. 125, 126 and 127 it will be seen that the portion of the tract destined for the cranial nerves, and so for the movements of the eyes, the mouth, face, tongue, pharynx and larynx, starting from the ventral parts of the more

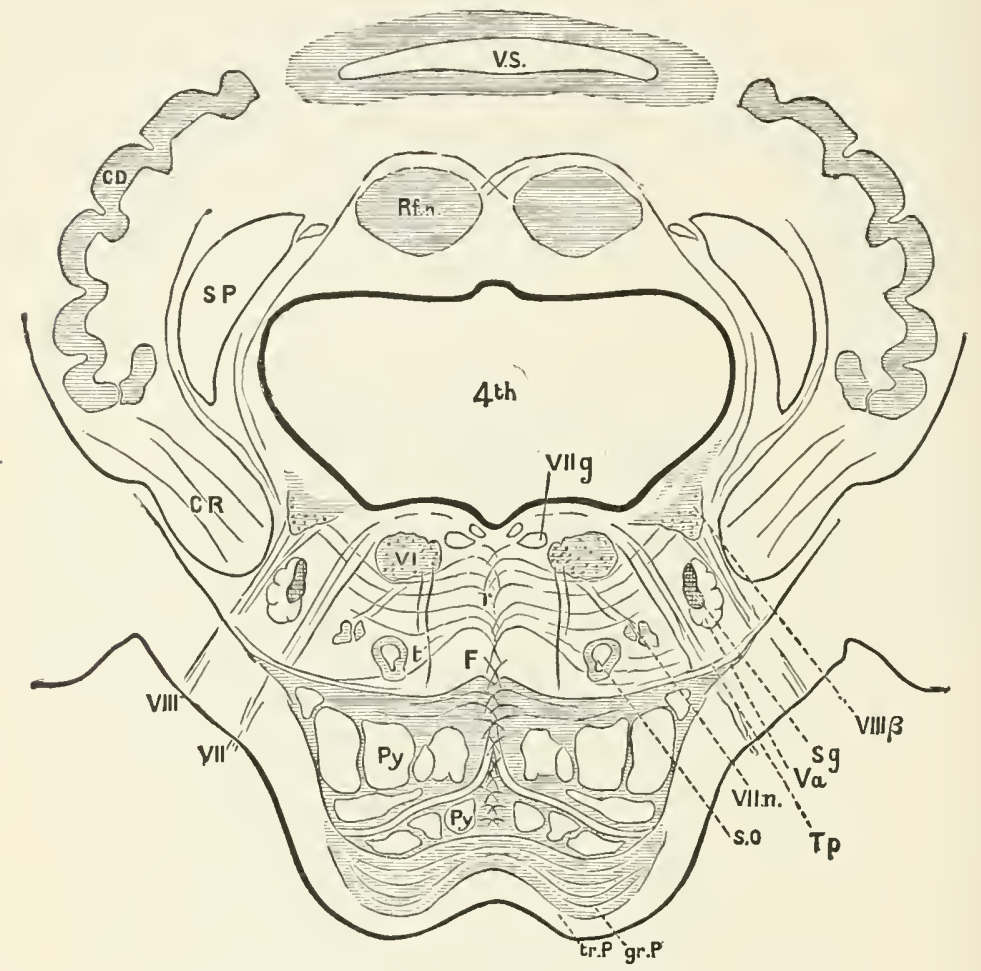

Fig. 131. Throvgit the widest part of the Fourth Ventricle. (Sherrington.)

Taken in the line 111, Fig. 108.

$P y$. Pyramidal fibres cut transversely. $t r . P$. the superficial (ventral) transverse fibres of the pons. The shaded part of the pons ( $g r . P$.) indicates grey matter mingled with the deeper transverse fibres. $F$. the fillet. $T p$. the trapezium. $C . R$. the restiform body or inferior peduncle of the cerebellum, cut across obliquely. $S$. $P$. the superior peduncles of the cerebellum. $r$. raphe. s. o. superior olive. C. D. corpus dentatum of the cerebellum. R. $n$. the nuclens of the roof. $s . g$. tubercle of Rolando. $V$. S. section through sulcus in the vermis superior of the cerebellum. $t$. bundle from the olive to the lenticular nucleus.

VIII. the eighth or auditory nerve, its ventral or vestibular root, proceeding from VIII. $\beta$. the front part of the lateral auditory nucleus. VII. $n$. the nucleus of the seventh or facial nerve. VI. the nueleus of the sixth nerve. VII. $g$. fibres of the seventh nerve cut across as they sweep round the nucleus of the sixth before issuing from the pons as VII.

4th. the fourth ventricle, here ronfed in by the cerebellum; the shading of the central grey matter immediately surrounding the ventricle is, for the sake of simpiicity, omitted. 
frontal district of the motor region, take up their position at the knee of the internal capsule; and the portion destined for those upper cervical nerves which carry out movements of the head through the muscles of the neek, starting from the extreme frontal and dorsal parts of the area, is also apparently directed to the knee of the capsule. 'The rest of the tract, starting from the part of the area lying at once behind and mesial to the above, occupies in the capsule a position posterior to them in the hind limb of the capsule ; and it will be observed that the tract for the fore limb which begins on the surface lateral of the tracts for the trunk and hind limb, shifts its course in relation to theirs, so that in the capsule it is in front of them, not lateral to them.

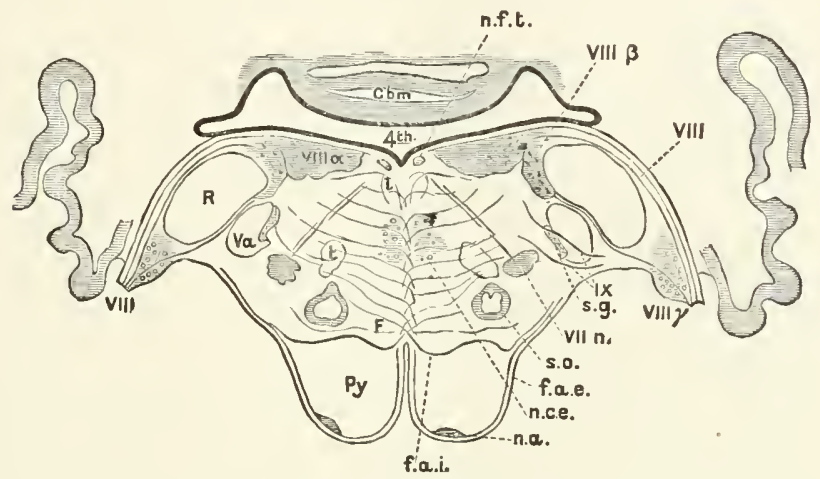

Fig. 132. Throdgin the Bulb jest behixd the Pons. (Sherrington.) Taken in the line 110, Fig. 108.

Py. Pyramids. R. Restiform Body. Cbm. cerebellum. F. Fillet. f. a. e. external, $f . a$. $i$. internal arcnate fibres. $t$. bundle of fibres from olive to the lenticular nucleus. 7 . posterior longitudinal bundles. n.f. $t$. nucleus of fasciculus teres. s. o. superior oìve. n. c. e. nucleus centralis (the marks within it are sections of bundles of fibres by which it is traversed). $s . g$. substance of Rolando.

V. $a$. ascending root of fifth nerve. VII. $n$. nucleus of the 7 th nerve. VIII. auditory nerve, chiefly the dorsal or cochlear root; VIII. $a$. medium nucleus, VIII. $\beta$. lateral nucleus, ViII. $\gamma$ accessory nucleus of auditory nerve. IX. fibres of root of ninth nerve passing through ascending root of fifth nerve.

It may further be observed that while in the tracts for the trunk and hind limb the same fore and aft order which obtains on the surface is reprodnced in the capsule, even apparently to the strange precedence of the ankle over the knee, the order of the several elements in the fore limb tract which is lateral on the surface becomes regularly fore and aft in the capsule. In the capsule the several elements are arranged in a linear order, corresponding broadly to that of the distribution of the muscles along the longitudinal axis of the body; on the cortex they are disposed in an order the cause of which is at present not very 
clcar, but which is probably determined by the respective relations of the several parts of the motor region to the funetional activity of the other parts of the cortex. In the shifting from the one order to the other, the several constituent fibres, as we have said, describe a somewhat peculiar course; and when we remember, that the order shewn in Fig. 125 is only the order obtaining at one particular level of the capsule, and that from the dor'sal beginnings of the capsule in the corona radiata to its ventral end in the pes, the capsule is continually changing in form, and its fibres therefore are continually shifting their relations to each other, the whole course of the several fibres of the tract from their origin in the cortex until they are gathered up into the central portion of the pes (Fig. $128 P^{\prime} y$ ) must be a very complicated one.

When the area of one hemisphere is stimulated, the movement which results is in most cases seen on the other side of the body, and on that other side alone. Thus when the area for the fore limb is stimulated on the left hemisphere it is the right fore limb which is moved. This is in accordance with what we have learnt of the pyramidal tract and its ultimate entire decussation before it reaches the motor nerves, the decussation either occurring massively as in the case of the crossed pyramidal tract, or in a more scattered manner along the upper part of the spinal cord in the case of the direct pyramidal tract; and, as we have seen, there is a similar decussation for such part of the pyramidal tract as is connected with the cranial nerves above the decussation of the pyramids. Except in the case of certain areas for movements naturally bilateral of which we shall speak presently, the movement is normally on the crossed side, and on the crossed side only. Under abnormal conditions however the limb of the other side, that is of the same sicle as the hemisphere stimulated, may move also. But such an abnormal movement of the same side has not the same characters as the proper movement of the crossed limb. Instead of being an orderly coordinate movement, it is a mole simple, either tetanic or perhaps tonic, or rhythmic, clonic, eontraction of the muscles. Obviously its mechanism is of a different nature from that by which the proper movement of the erossed limb is effected; but it is important to bear in mind that a movement of the uncrossed limb may take place; and further that, the abnormal conditions continuing, similar movements of an uncoordinated character may spread to the hind limb and other parts of the crossed side, though the stimulation be still confined to the arm area, then to other parts of the uncrossed side, until as we have said the whole body is thrown into epileptiform convulsions. This feature must not be forgotten. In fact it may be fairly insisted upon that while we may speak of a partieular coordinate movement as being the 
normal outeome of an ordinary careful stimulation of a particular area in a normal condition, it is no less true that diffuse uncoordinated movements, culminating in general epileptiform convulsions, are the natural outcome of the stimulation of any area in an abnormal condition. And in attempting to form any opinion of the nature of the first act, we must bear the second in mind.

As we said above, the movements resulting from cortical stimulation are most conveniently deseribed in terms of parts of the body, of the arm, of the thumb, of the tongue, Sc. The movencnts of the same part ma be further distinguished by means of the nomenclature usually adopted in speaking of muscular movements, such as flexion, extension, abduction, adduction, \&c.; so that, within the area bearing the name of some particular part, such as the wrist for instance, we have to distinguish an area for the flexion, and another for the extension of that joint; and in like mamner in reference to other parts. But it will be readily understood that it is easier to mip out the area for a particular part than to distinguish the areas corresponding to the several movements of that part. Hence the nomenclature usually adopted in speaking of the motor region is one based on the parts of the body moved rather than on the character of the movements. 'The more closely however the movements in question are studied, the more probable it appear's that the localisation which obtains in the cortex is essentially a localisation corresponding not to parts of the body, or to nerves, or to muscles, but to movements. In considering this point it must be remcmbered how rude and barbarous a method of stimulation is that of applying electrodes to the surface of the grey matter compared with the natural stimulation which takes place during cerebral action; the one probably is about as much alike the other, as is striking the keys of a piano at a distance with a broomstick to the execution of a skilled musician. Were it in our power to stimulate the cortex in any way at all approaching the natural method, we should in all probability arrive at two results; on the one hand we should be able to produce at will a variety of movements of different degrees of complexity, some very simple, others very complex, and for these we should have to use names suggested by the characters and pulpose of each movement, and by these alone; on the other hand we should find very decided limits to the number and kind of movements which we could evoke, limits fixed in the case of each subject partly by inherited organisation, partly by the training of the individual.

Some such results of refined experimentation are indeed already foreshadowed by the rude results of our present rough methods. The movements which usually follow stimulation of the notor region, and which we have described as flexion, fe., 
are, so to speak, the elementary factors of ordinary bodily movements, the detached and imperfect ehords of a musical piece; and in the following facts relating to their production we can recognize the influences of organisation and habit. As we have said, stimulation of the motor area of one hemisphere produces movements, as a rule, which are limited to one side of the body, and that the opposite side. Now both in ourselves and in the higher animals a large number of bodily movements, especially of the limbs, are habitually unilateral; and, putting aside the question why there should be two halves of the brain, and why the one half of the brain should be associated with the cross half of the body, we may recognize in them unilateral crossed morements resulting from stimulation of the cortex in accordance with natural habits. But some movements of the body are ordinarily bilateral; the two eyes, for instance, are ordinarily moved together, and the two sides of the trunk move together very much more frequently than do the two fore limbs or the two hind limbs. And in accordance with this we find that stimulation of the motor area for the eyes on either hemisphere produces movements of both eyes, and stimulation of the trunk area of one hemisphere is also very apt to produce bilateral action of the trunk muscles; in such instances the movements on both sides are quite normal movements. We may incidentally remark that removal of the trunk area leads to a good deal of bilateral degeneration, that is, to degeneration of strands in the pyramidal tracts of both sides, whereas such a bilateral degeneration is comparatively scanty after removal of the leg or arm area.

That it is the movement and not the part moved which is, so to speak, represented on the cortex is further shewn by the relative magnitudes of the several cortical areas when they are mapped ont according to parts of the borly. The area for the arm, for instance, ef. Figs. 123, 124, is, so to speak, enormous compared to that of the trunk when the relative bulks of these two parts of the body are considered; and within the arm arca itself the space occupied by the thumb and forefinger and digits is, bulk for bulk, out of proportion to the space allotted to the shoulder; so also the area for the eyes or for the mouth is out of proportion to the size of those organs. But these relative sizes of the respective areas become intelligible when we bear in mind relative mobility, nimbleness and delicacy of exceution; in these respects the shoulder is far behind the thumb, while the eyes and mouth surpass most other parts of the body.

We are brought yet a step further when we compare, in respect of the cortical motor region, animals of different grades of organisation; and the results thus obtained lead us to the conclusion that the motor region is correlated not to movements in general, but to movements of a particular kind. 'Taking in series the rabbit, the dog, the monkey and man, we find in pass- 
ing from one to the other, an increase in prominence and in differentiation of the motor region accompanied by an increase in the bulk of the pyramidal tract; anong the many striking differences between the brains of these several animals, these two features, the increasing complexity of the motor region, and the increasing size of the pyramidal tract, are among the most striking. The size of the pyramidal tract is itself correlated to the complexity of the motor region, and, being the more easily determined, may be used as indicating both; the difference in the size of the pyramidal tract in these animals is seen all along the whole length of the cord (Fig. 133). Now as regards mere quantity of movement, if we may use such an expression, the differences between these animals are of no great moment. If we were to take the amount of energy expended as movement in twenty-four hours per gramme of muscle present in the body in each of the four cases, we should certainly not find any cor-

A

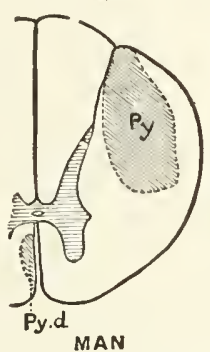

B

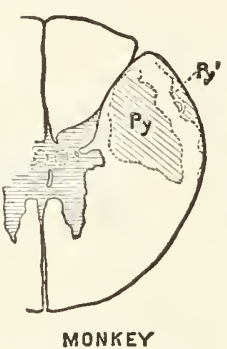

C

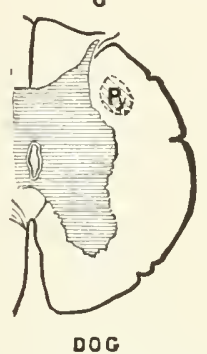

Fig. 133. Diagram to illugtrate the Relative Size of the Pyramidal Tract in the Dog, Moxkey and Max. (Sherrington.)

The figure shews in outline the lateral half of the cord, at the level of the fifth thoracic nerve, in $A$. Man, $B$. Monkey, $C$. Dog; $A$ is a reproduction of $D^{5}$ in Fig. $114 ; B$ and $C$ are drawn of the same size as $A$. $P y$., shaded obliquely, the pyramidal tract; the depth of shading indicates that the tract is more crowded with true pyramidal fibres as well as larger in $A$ than in $B$, and in $B$ than in $C$. In $B, P y^{\prime}$ is an outlying portion of the pyramidal tract separated from the rest by the cerebellar tract. Py.l. the direct pyramidal tract, present in man only. The grey matter seems relatively large in $\dot{C}$ because the section was taken from a very young puppy.

respondence between that and the size of the pyramidal tract. If however we take a particular kind of movement, what we may perhaps call skilled movement, that is movement carried out by means of intricate clianges in the central nervous system, we do find a remarkable parallelism in the above cases between the amount of such skilled movement entering into the daily life of the individual and the size of the pyramidal tract. In these two respects man is much above the monkey, and the monkey far above the dog. We may conclude then that the cortical motor region is in some way especially concerned with the kind of movement which we have called 'skilled.' 
$\$ 48 \%$ Thesc skilled movements are to a large extent, though not exclusively, voluntary morements. IVe have in a previous section seen reason to believe that the cerebral cortcx is in some way especially associated with the devclopment of voluntary movenents. Putting together this conclusion and the conclusions just arrived at we are naturally led to the further conclusion that the cortical motor region, with the pyramidal tract belonging to it, plays an important part in carrying out voluntary movements. Do other facts support this view, and if so, what light do they throw on the question as to what part and what kind of part the motor region thus plays?

In this connection we naturally desire to know what are the results of removing from an otherwise intact animal the whole motor region, and more especially this or that particular portion of it. Before proceeding further, howerer, we may once more call attention to the caution given in $\$ 457$, and repeated in $\$ 476$; indeed when we consider the high organization and complex functions which obviously belong to the cortex, when we bear in mind that it appears to govern, and must therefore be bound by close ties to almost all the rest of the central nervous system, we must be prepared to find after removing a portion of cortex that the pure 'deficiency' phenomena, those which result from the mere absence of a piece of the cortex, are largely obscured by the other effects of the operation.

In the rabbit the results have been almost purely negative. When in this animal the part of the cortex which may be considered as the motor region is removed, nothing remarkable is observed in the movements of the animal. W' ean hardly suppose that the operations of the central nervous system are the same in an operated as in an intact animal, and the differences induced ought to be betrayed by the morements of the body; but at present they have escaped observation.

In the dog the removal of an area is followed by a loss or diminution of voluntary movement in the corresponding part of the body. When, for instance, the area for the fore limb is removed from the left hemisphere, the right fore limb is completely or partially 'paralyzed.' In carrying out its ordinary movements the operated animal makes little or no use of its right fore limb. Bnt this state of things is temporary only. After a while the animal regains power over the limb, and in successful cascs recovery is so complete that it is impossible to point out in the limb any appreciable deviation from the normal use. And careful examination after death has shewn not only that the area had been wholly removed, but also that there was no regeneration of the lost parts; the removal of the cortex leads in such cases, as usual, to degeneration of the corresponding strand in the pyramidal tract right away from the 
cerebral surface to the endings of the strand in the cervical and dorsal spinal cord. Nor can it be urged in such eases that diffused remnants of the arm area had been left in the remaining parts of the motor region; for the whole motor region has been removed, and yet the animal has recovered to such an extent that a casual observer could detect no differences between the movements of the two sides of the body. Closer examination did disclose certain imperfections of movement; but the operation had involved injury to or produced changes in struetures other than the motor region, and the imperfections might have been due to the additional damage. Nor can it be urged that, in such a case, where one side is removed, the remaining hemisphere takes on double functions; for the greater part of the motor areas have been removed on both sides, and yet the animal's movements have been so far apparently complete that a casual observer would see nothing strange in them. Again, the whole motor region has been removed from one hemisphere in a young puppy, and some time later when the movements seemed to have recovered their normal condition, the removal of the motor region of the other hemisphere has produced merely a paralysis of the crossed side of the body, and that as before only of a temporary character.

Two things have to be noted here. In the first place the removal of an area does affect the movements which are brought about by stimulating that area, it leads to their disappearance or at least to great diminution of them; and this affords an additional argument that the connection between the area and the movement is a real and important one. In the second place, the physiological effect is temporary only, though the anatomical results of the operation are permanent, for the cortex is never renewed, and the pyramidal tract degenerates along its whole length, never to be restored; this shews that we have to deal here with events of a very complex character. When a particular movement results from stimulation of the appropriate cortical area, we may be sure that whatever takes place in the cortex and along the pyramidal tract, motor impulses, duly coordinated, pass along certain anterior roots to certain muscles; and we know that if we removed a sufficient length of each of those anterior roots that particular movement would be lost for the rest of the life of the individual. We may therefore infer that the events which, whatever be their exact nature, taking place in the cortex and along the pyramidal tract lead ultimately to the issue of motor impulses along the anterior roots, differ essentially from the events attending the transmission of ordinary motor impulses.

In the case of the monkey, the results of removing parts of the cortical motor region have not been so accordant as in the case of the dog. The two animals agree perfectly in so far that 
the removal of a particular area leads, as an immediate result, to the loss of the corresponding movement; but whilc in some instances recovery of the movement has in the monkey as in the $\log$ after a while taken place, in other instances the "paralysis' has appeared to be permanent. As a rule the paralysis caused by a large lesion is not only more extensive, but also of longer duration than that caused by a small one; and natural bilateral movements, as of the eyes, reappear earlier than unilateral movements. 'The facts however' within our' knowledge relating to the permanence of the effect ale neither numerous nor exact enough to justify at present a definite conclusion. On the one hand the positive cases where recovery has taken place are of more value than the negative ones, since in the latter the recovery may liave been hindered by concomitant events of a nature which we may call accidental; and it is at least a priori most unlikely that the pyramidal tiact mechanisn, if we may use the expression, though it may differ in the monkey and the $\log$ in degree of development, differ's so essentially in kind that damage of it leads in the one case to permanent, and in the other to mere temporary loss of function. We may add that we should further expect to meet in the monkey with more prominent and more lasting complications due to the subsidiary effects of the operation, and it may be doubted whether in any of the recorded experiments the animal has been allowed to live a sufficient time for these subsidiary events to have wholly cleared away, leaving only what we have called the 'deficiency' phenomena, due to the loss of the cortical area alone. On the other hand it must be remembered that the movements of the monkey are more intricate in origin, more 'skilled' than those of the $\log$; it may be that differences in the characters of movements determine the possibility of their recovery; and undoubtedly the coarser movements return first, the finer, nore skilled movements reappear later or not at all. Thus, after the removal of an arm area in the monkey, a certain awkwarduess in the movements of the thumb is one of the lasting effects of the operation.

$\$ 488$. So far we have spoken of changes in movements as if these were the only effects produced by removal of the motor area or of parts of it. But as a matter of fact changes in sensations are as prominent results of such operations as changes in movements, and this fact opens up a different view of the matter. Before however we proceed any further in the discussion, it will be of advantage to turn aside to what is known concerning the cortical motor region in man. As we lave already said, theoretical considerations lead us to believe that the cortical motor region in man is disposed in accordance with the plan of the anthropoid ape as ascertained experimentally, but with the differentiation carried still further; and observa- 
tion supports this view. On the one hand in certain cases (and the number of these is increasing) it has been possible to apply an electric current to the human brain laid bare or covered only with the membranes; the results obtained distinctly corroborate the above view. We may note in passing that in such cases it has been found necessary to apply a relatively strong current. On the other hand corroboration is also afforded by cases of disease, by the phenomena attending circumscribed affections of the cortex, such as tumours and the like, and that in spite of the advantages of dealing with one of ourselves being counterbalanced by the disadvantages due to disease being so often anatomically diffuse and physiologically changeful and progressive.

We said above that during experiments on animals stimulation of any part of the motor region may under abnormal conditions lead to general epileptiform convulsions. Now clinical study has shewn that in man certain kinds of epileptic attacks are of similar cortical origin. In these cases it has been observed that the attack begins in a particular movement, by contractions of particular muscles, or of the muscles of a particular region of the body, of the hand, foot, toe, thumb, \&c., and then spreads in a definite order or 'march' over the muscles of other regions until the whole body is involved. When in an experiment on an animal epileptiform convulsions supervene, they similarly start from the region of the body, the motor area of which is beneath the electrodes at the time, and similarly spread by a definite 'march' over the whole body. Hence in the human epileptiform attacks of which we are speaking, it has been inferred that the immediate exciting cause of the attack is to be sought in events taking place in that part of the cortex which serves as the area for the movement which ushers in the attack. Further inquiry has not only confirmed this view, but has also shewn that the topography of the cortical areas in man, as thus determined, very closely follows that of the monkey.

Other diseases of the cortex have been marked, among other symptoms, by loss or impairment of particular movements. In most of such cases, the cortical lesion has been of such an extent as to involve a number of special areas at the same time, and so to lead to loss or impairment of movement over relatively considerable regions of the body, such as the whole of one arm ; and in general the teaching of these cases of disease, while confirming the deductions from the monkey, and giving us some general idea of the topography of the human motor cortical region, has at present given us approximate results only. Figs. 136 and 137 shew in broad diagrammatic manner the position and relative extent of the motor areas for the leg, arm and face in man, so far as has yet been ascertained. To assist the reader 
we give at the same time diagrams Figs. 134, 135 illustrating the nomenclature of the surface of the liuman brain.

One area is of special and instructive interest. Speech is an eminently 'skilled' movement. We have seen that in the monkey the area for the mouth and tongue lies at the ventral end of the eentral fissure or tissure of Rolando, ventral to the arm arca, and that the extreme ventral and front part of the motor region just above the fissure of Sylvius supplies an area which we marked as that of phonation (Fig. 123). In the monkey the area of phonation is determined by experimental stimulation; in man, in a similar position, on the third or lowest frontal convolution, sometimes called Broea's convolution, ventral to and in front of, and probably overlapping backwards the area which in Fig. 136 is marked 'face' and which includes the month and tongue, clinical study has disclosed the existence of an area which may be spoken of as the area of 'spcech.' Lesions of the cortex in this area cause a loss of or interference with speech, the condition being known as aphasia; to this we shall presently return. In Fig. 136 this area is shewn in an approximate manner.

The movements of speech are essentially bilateral movements. In the $\log$ and monkey various bilateral movements may be excited by stimulation of the appropriate area in either hemisphere; and analogy would lead us to suppose that in man, the movements of speech would be connected with the speech area in both one and the other hemisphere. The results of lesions however shew that it is in most eases especially the left hemisphere which is connected with speech; it is a lesion in the third frontal convolution of the left hemisphere, often associated with other lesions of the same hemisphere leating to paralysis of the right side of the body and face, which causes aphasia, it being only in exceptional cases that the condition results from a lesion of the corresponding area of cortex on the right hemisphere.

In man, then, clinical study corroborates the conclusions deduced from the experimental investigation of the dog and of the monkcy, but still leaves us in uncertainty as to the question what, and what alone are the absolutely permanent effects of the loss of a cortical area and of nothing else. On the one hand, in the cases in which recovery of a movement follows upon its loss or impaiment, it is open for us to suppose that the lesion itself was temporary, and that with the cure of the malady the cortical area regained its normal condition. On the other hand, where the disease continues, the permanency of the loss of any movement may be attributed to the diseasc doing mole than merely suspend the function of the cortical area. Aphasia, especially in young persons, hiss heen followed by recovery, but in such cases it has been supposed that the dormant area on the right sille has been awakener to activity by the loss of the left area; and in support of this view cisses have been recorded in 


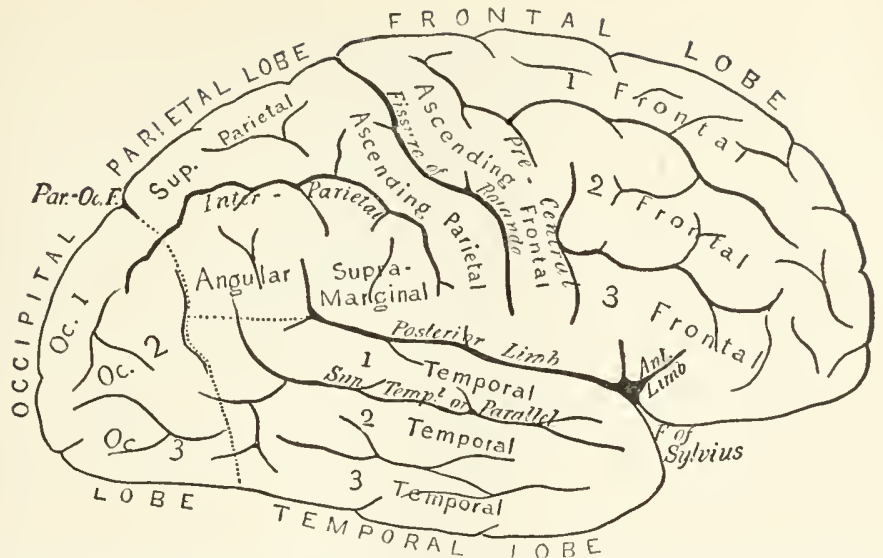

Fig. 134. Diagram of the Gyri (convolutiovs) sulci, (Fissures on the lateral surface of the Right llemispiere of Man. (Gowers.)

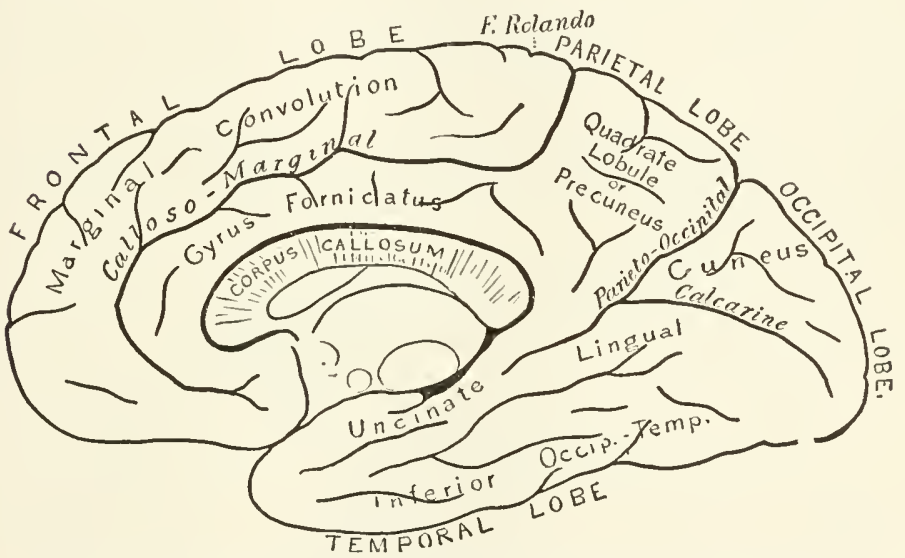

Fic. 135. The same on the Mesial Surface. (Gowers.)

In both figures the sulci are indicated by italic and the convolutions by roman type.

The following list of some synonyms may perhaps be of use in connection with these figures and those of the brain of the monkey, Figs. 12:, 124.

Gyri, or Comelutions. Precentral or anterior central $=$ ascending frontal. Postcentral or posterior central $=$ ascending parietal. Superior temporal $=$ inframarginal $=$ first temporal. Triangular lobule $=$ cuneus. $\quad$ Central lobe $=$ Island of Reil. Paracentral lobule $=$ the mesial face of the ascending frontal, within the marginal gyrus. Cingulum = the part of the gyrus fornicatus which adjoins the Corpus callosum. Gyrus Hippocampi = uneinate gyrus, though the latter name is sometimes restricted to the front part of the hippocampal grrus; the two may be considered as a continuation of the gyrus fornicatus, and the three together, forming a series, have been called 'the great limbic lobe.'

Sulci or Fissures. Central = Rolandic, or of Rolando. Perpendicular $=$ parieto-oceipital. Parietal = intraparietal or sometimes interparietal.

Temporo-sphenoidal lobe $=$ temporal lobe. 


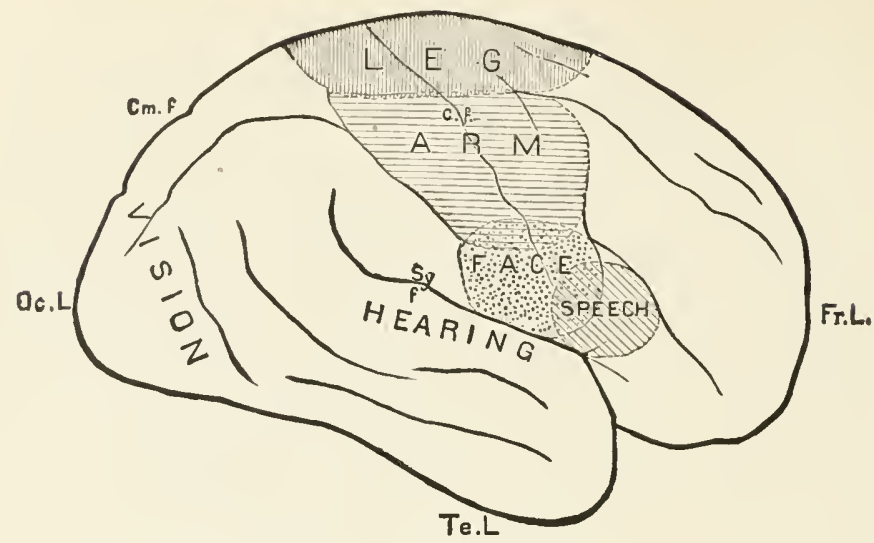

Fig. 136. The Lateral Surface of tile Right Cerebral Hemisphere of Man in outline, to illustrate the Cortical $A$ reas. Reduced from nature.

'The position of the areas of the cortex concerned with movements of the face, arm, and leg, and with the senses of sight and hearing are approximately shewn. 'The position of the area connected with speech (Broca's centre) is also shewn for the sake of comparison of it with the position of the other areas; the representation of speech in the cortex cerebri lies however in the left hemisphere chiefly.

Oc. L. Occipital lobe; Fr. L. Frontal lobe; Te. L. Temporal lobe; Sy. $f$. the fissure of Sylvius ; C.f. the central fissure (Rolandic); $C m$. $f$. indicates the position of the posterior end of the calloso-marginal fissure.

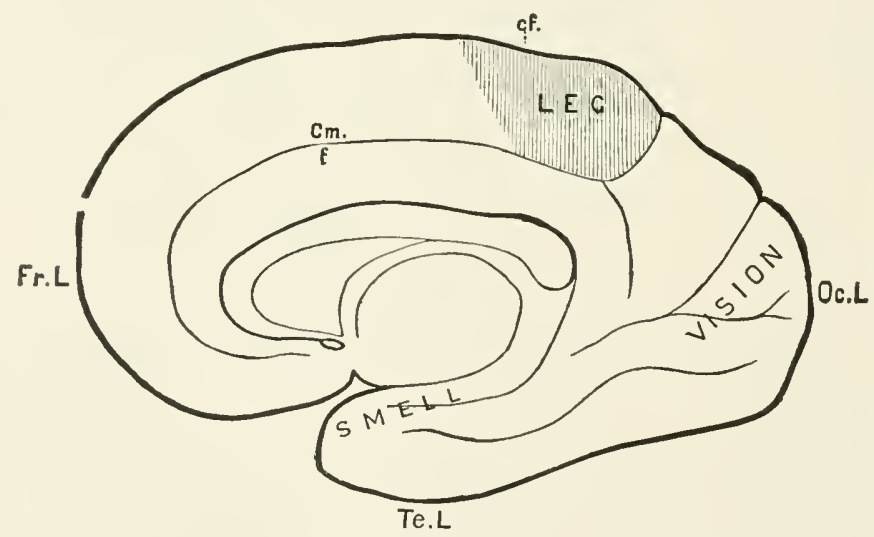

Fig. 137. Tine Mesial Surface of tie Rigit Cerebral Hemisphere of Man in outline, to illustrate the Cortical Areas.

The areas shewn are those connected with the movements of the leg, and with the senses of sight and smell.

$F r$. L. the frontal pole of the hemisphere; Oc. L. the occipital pole, Te. $L$. the temporal pole. Cm. $f$. the calloso-marginal fissure separating the marginal gyrus above from the gyrus fornicatus below. $C f$. marks the situation of the central fissure, the fissure itself not being apparent on the mesial aspect of the hemisphere. The corpus callosum and the anterior commissure are seen in cross section. 
which a first aphasia, due to a lesion on the left side, has been followed by a second aphasia due to a sequent lesion occurring on the right side. On the whole perhaps the evidence of clinical study tends to shew that in man the loss of movement due to the destruction by disease of an area is a permanent one, though actual demonstration of this is wanting.

$\$ 489$. We may now return to the discussion of the question, what is the part played by a motor area, and by the contribution from that area to the pyramidal tract in carrying out the movements with which the area is associated?

We may premise that the evidence points very distinctly to the conclusion that whatever be the nature of the whole chain of events of which the cortical area seems to be a sort of centre, the fibres of the prramidal tract serve as the channel of processes which we must regard as efferent in nature. The characters of the fibres, axis cylinder processes terminating, so far as we can ascertain, in connection with motor cclls, the fact that the degeneration of the fibres is a descending one, though this cannot be trusted by itself to prove that the direction in which the fibres carry impulses is only that from the cortex downwards, and above all the fact that when the fibres of the tract are stimulated at any part of their course, movements, the signs of the occurrence of efferent centrifugal impulses, are produced, these things together, leave no doubt as to the tract being one of efferent fibres. Hence we may infer that whatever be the nature of the events taking place in a motor area during the carrying out of a movement, the part played by the fibres of the pyramidal tract is that of carrying efferent impulses from the area to the muscles concerned.

Let us consider first the movements of speech in man, the evidence touching the connection of which with an area on the third frontal convolution appears so very clear. Speech is eminently a 'skilled' movenent; it involves the most delicate coordination of several muscular contractions, and we may certainly say of it that it has to be 'learut.' The whole chain of coordinated events by which the utterance of a sentence, a word, or any vocal sign is accomplished consists of many links, the breaking of any of which will lead to failure of one kind or another in the act. Something may go wrong in the glossal or other muscles, in the nerve endings in those muscles, or in the fibres of the nerves, hypoglossal and others, between the central nervous system and the muscles, or something may go wrong in that part of the central nervous system, the bull to wit, in which a certain amount of coordination is carried out just previous to the issue of the motor impulses. Damage done to any of these parts of the mechanism may lead to dumbness or to imperfect speech. In the latter case the imperfections have a certain character; if we are at all able to gather the wish of the speaker, 
we recognize that he is attempting to utter the right words in the right sequence, but that his efforts are frustrated by imperfect coordination or imperfect muscular action; his speech is -thick, the syllables are blurred and the like. Disease of the bulb at times leads to imperfect speech of this kind in which the imperfection may be recognized as due to the lack of proper coordination of motor impulses. The affection of speech. known as 'aphasia,' which is caused by lesions of the cortex is of a different character, and the forms of imperfect speech cansed by bulbar disease have justly been distinguished from true aphasia by the use of other terms. Cases of eomplete aphasia in which all power of speech is lost, do little more than help us to ascertain the topographical position in the cortex of the 'speech' area, but cases of partial aphasia are especially instructive. Without attempting to go into the details of the subject and into the many considerations which have to be had in mind in dealing with it, for there are different kinds of aphasia, we may venture to say that the striking feature of partial aphasia is the failure to say certain words or syllables, and the tendency to substitute some wrong word or syllable for the right one. The words or syllables which are uttered are rightly pronounced withont defect of articulation; and in many cases, though the right word cannot be produced as a direct effort of the will, it may be uttered under the influence of an emotion, or indeed sometimes as the result of some psychical processes more complex than those involved in the mere volitional effort to say the word. An instructive case is recorded of a man suffering from slight aphasia, who after' several failures to say the word 'no' by itself, at last said, - I can't say no, sir.'

From the phenomena of partial aphasia we may on the one hand draw the deduction that the cortical speech area does not carry out the whole of the coordination of the impulses involved in articulation. That coordination is exceedingly complex, and we ought perhaps to recognize in it more than one degree or kind of coordination. We must of course admit that a great deal of coordination of a certain kind takes place in the cortex, for the bulb cammot by itself be made to speak. But the failure of articulation in disease of the bulb shews that a certain amount of coordination takes place there also; for the affections of speech due to bulbar disease are not the same as those resulting from the mere loss of this or that muscle or nerve. The word spoken does not start, so to spcak, ready made in the cortex; it is not that a group of impulses start from the cortex with their coordination fully achieved, and pass along certain nerve fibres to certain muscles making their way withont change through the tangle of the bulb, as if this were merely a bundle of lines offering paths for, but exercising no influence over the impulses. We must rather suppose that something takes place in the cortex of the 
third frontal convolution, as the result of which efferent impulses pass along the appropriate fibres of the pyramidal tract to the bulb, and there start a series of events leading to the issue of the coordinated impulses by which the word is spoken. And, since we have no reason to think that the cortical area for speecl differs in its fundamental characters from other divisions of the motor region, we may apply the same reasoning to other motor areas.

On the other hand, the phenomena of aphasia illustrate another view of the nature of the motor areas to which we must now turn. We said that there are different kinds of apluasia. We may in a broad way distinguish two classes. In considering speech we have to deal on the one hand, with the efferent motor factors, the framing and utter'ance of the word, and on the other hand with the afferent, in a broad sense, sensory factors which lead to and guide the framing of the word. And in aphasia we may recognize a class in which the failure is on the notor side of the business so to speak and a class in which the failure is on the afferent side. The dumbress of those who are born deaf is an extreme illustration of the latter class. Now as we said above, when a so-called motor area is removed from the cortex, the results are not purely notor, that is to say the loss or inpairment of movement is not the only effect; a loss or impairment of sensation is also produced in the part of the body, the movement of which is affected. When, for instance, in the monkey the area for the ar'm is removed, not only is the arm, for the time being, paralyzed, but also the animal does not shew signs of sensation, or shews only feeble signs of sensation when the skin of the arm is pricked or otherwise stimulated. And so with other areas. In alì cases the loss of movement is acconpanied by a corresponding loss of sensations, of tactile sensations, of sensations of heat and cold, and even of pain. And the loss or impairment of sensation runs more or less parallel in point of time to the loss or impairment of movement, and shews the same tendency to be in part lasting. Moreover in the cases mentioned above where it has been possible to stimulate the cortex of the human brain, and where this has been done while the subject was conscious, the production of sensations, often described as tingling, in the part of the body corresponding to the particnlar. area, has been at least as striking a result as the production of movement in that part. And this is in harmony witl the fact that in epileptic attacks which, as we said, illustrate the action of cortical areas, the movements which are the objective factors of an attack are preceded by peculiar sensations, the so-called 'aura,' and these equally with the movement have definite relations to the area of the cortex, disease of which causes the attack. In fact there is increasing evidence that the region of the cortex which we have called the motor area, is connected not only with the movements carried out by, but also with the 
sensations derived from the several parts of the body; we seem justified in speaking of a topographical distribution of cortical "sensory" areas, if we may so call them, following very closely that of the motor areas; and we know that by the numerous fibres passing from the cortex to the optic thalamus, if not in other ways, an anatomical path appears to be afforded for sensory impulses. But to this point we shall return later on.

Mean while we may conclude that the loss of movement which follows the removal of a motor area is not due merely to the loss of motor elements, it may be as much due to the loss of sensory elements. Indeed it has been maintained by some that the loss or impairment of movement in question is not a motor business at all, but is simply due to a loss of the muscular sense. We have seen, however, reasons for thinking that the pyramidal tract is certainly an efferent tract, and injury to it in its beginning in the cortex must lead to failure of efferent impulses. Moreover, though removal of the cortex does appear to interfere with muscular sense, it also, and even more clearly, interferes with cutancous and other sensations. The conclusion which we ought to draw from the above facts is perhaps rather this, that the relations of the cereloral cortex are manifold, and that the carrying out even of a simple voluntary movement is a very complicated matter; even if we assume that the cell in the cor tex giving origin to a fibre of the pylamidal tract is in nature a motor cell, we must also recognize that its work is determined by ties which bind it to other elements of the cortex and through them to other parts of the nervous system and indeed of the body. 'The connections of a sensory nature between a motor' area and the part to whose movements it is related is strikingly shewn by results which may make their appearance when stimulation of the cortex is carried on while the animal (dog) is in a particular stage of the influence of morphia. If a subminimal stinulus be found, that is a current of such intensity that applied to a motor area it will produce no movement, but if increased ever so slightly will give a feeble contraction of the appropriate muscles, it may be observed that a slight stimulus, such as gently stroking the skin over the muscles in question, will render the previous subminimal stimulus effective and so call forth a movement. 'Thus if the area experimented on be that connected with the lifting of the forepaw, and the subminimal stimulus be applied to the area at intervals, after sevcral applications followed by no movements, a gentle stroke or two over the slin of the paw will lead to the paw being lifted the next time the stimulns is applied to the area. A similar result, but less sure and striking, may follow upon the stimulation of parts of the body other than the part corresponding to the arca stimulater. Then again it has been observed that in certain other stages of the influence of morphia, the cortex and the rest of the nervous system are in such a con. 
dition that the application of cven a momentary stimulus to an area leads not to a simple movement but to a long-continued tonic contraction of the appropriate muscles. Under these circumstances, a gentle stimulus, such as stroking the skin, or blowing on the face, applied immediately after the application of the electric stimulus to the area, suddenly cuts short the contraction, and brings the muscles at once to rest and normal flaceidity.

$\$ 490$. The carrying out of a voluntary movement is in fact a very complex proceeding, and the motor cortex with the pyramidal tract is only one part of the whole mechanism. 'This complexity is illustrated by the fact that after removing of a motor area not only purely voluntary but also reflex and other movements are for a while abolished or impaired; and even making every allowance for the effects of 'shock' (\$457) we cammot account for the latter to the exclusion of the former, by appealing to such effects. It is further shewn by the fact that in the case of most voluntary movements at least, after removal of an area recovery is after a while complete, though there is no regeneration either of the area or the strand of the pyramidal tract belonging to it; the will finds some other way to the muscles and to mechanism coordinating the movements of those muscles. By the following reflection the complexity of the matter is also shewn in a different direction. When a gymmast executes a skilled voluntary movement in which all his four limbs and other parts as well perhaps of his body are involved, it is probably the case that changes of the nature of efferent impulses sweep down his pyramidal tract, and that these impulses, starting in a definite order from his cortex, that is to say having undergone a certain amount of initial coordination at their very origin, meet with further coordination in the spinal grey matter, which-serves as a set of nuclei of origin for the motor nelves concerned in the movement, before they issue as ordinary motor impulses along the anterior roots. But this is not all. Should the gymnast's semicircular canals happen to be injured and his cerebellum thereby be troubled, or mischief fall on some other part of the brain which like this has no direct connection with either the pyramidal tract or the motor cortex, the movement fails through lack of coordination, though both the cortex, the pyramidal tract, and the spinal motor mechanisms remain as they were before.

Lastly we may note that in the above discussion we have used the word 'will' in a general sense only. A man may be brought into a condition, for instance in certain hypnotic phases, in which he can carry out all the various skilled movements which he has inherited or which he has learnt; and yet, according to some definitions of the word 'will,' those movements could not be said to be initiated by his will. It can hardly be doubted that in such cases the motor cortex and pyramidal tract play 
their usual part. But we may pass from such cases as these through others, until we come to cases where a skilled movement which has been learnt and practised by the working of an intelligent will, may continue to be carried out under circumstances which seem to preclucle the intervention of any conscious will at all; and the transition from one case to another is so gradual, that it is impossible to suppose that there has been any shifting of the machinery employed for carrying out the morement. So that a volitional origin is not an essential feature of these so-called voluntary movements, and the machinery of the motor cortex and pyranidal tract is available for other things than pure volitional impulses.

$\S 491$. The preceding discussion will enable us to be very brief concerning a question which has from time to time been much discussed, and which has acquired perhaps factitious importance, viz. the question as to how volitional impulses leading to voluntary movements travel along the spinal cord. The conclusion at which we have arrived, namely, that in the normal carrying out of voluntary movements the chief part is played by efferent impulses passing along the pyramidal tract, carries with it the answer that volitional impulses travel in the spinal cord along the pyramidal tract.

In the dog, in which the whole pyramiclal tract crosses at the decussation of the pyramids, we should expect to find that a break in the pyramidal tract of one side of the cord at any point along its length caused loss of voluntary movement on the same sicle below the level of the break. And experiments as far as - they go support this view. No one it is true has so far succeeded in dividing or otherwise causing to break in the pyramidal tract alone, leaving the rest of the cord intact; and indeed, cven if an injury were limited to the area marked out as the pyramidal tract, fibres other than pyramidal fibres would be injured at the same timc, since the tract is never a 'pure' one. But it has been found that a section of a lateral half of the cord, a lateral hemisection, or a section limited to the lateral column of one side has for one of its principal effcets loss of voluntary movement on the same side in the parts supplied by motor nerves leaving the cord below the level of the section. We say 'one of its principal effects' because, besides the concomitant interference with sensations concerning which we shall speak presently, the loss of voluntary movement is not absolutely confined to the same sicle; there is some loss of power on the crossed side, at least in a large number of cases. We must not lay stress on this crossed paralysis because it is possibly one of the effects of the mere operation, not a pure 'deficiency' phenomenon, and indeed appears soon to pass away. But taking into consideration what was said above concerning the effects of removing cortical areas, it is important to note that in the experience of 
many experimenters the loss of voluntary power on the operated side diminishes after a while, and that the animal if kept alive and in good health long enough appears to regain almost full voluntary power over the affected parts. In such cases, as in other operations on the central nervous system, there is no regeneration of nervous tissue; the two surfaces of the section unite by connective not nervous tissue, and the tracts which as the result of the section degenerate downwards or upwards are permanently lost. Hence even if we admit that in the intact animal a voluntary movement is chiefly carried out by means of efferent impulses passing along the pyramidal tract right down to the motor mechanisms of the cord immediately connected with the motor nerves, we must also admit that the 'will' under' changed circumstances can find other chamnels for gaining access to the same mechanisms.

It has been further observed that if in the dog a hemisection be made at one level, for instance in the lower thoracic region of the cord, and then, after waiting until the voluntary power over the hind limb of that side has returned, a second hemisection, this time on the other side, be made at a higher level, this second operation is followed by results similar to those of the first; there is loss of voluntary power on the side operated on, with some loss of power on the crossed side, and as in the first case this loss of power not only on the crossed but also on the same side may eventually disappear. 'This shews among other things that the recovery after the first operation was not due to the remaining pyramidal tract doing the work of both. Further, the hemisection may be repeated a third time, the third hemisection being on the same side as the first, and in this case also there may be at least very considerable return of power over both limbs. 'That is to say, under such abnolmal circumstances voluntary impulses may, so to speak, thread their way in a zigzag manner from side to side along the mutilated cord until they reach the appropriate spinal motor mechanisms. Such an abnormal state of things does not however really militate against the view that under normal circumstances volitional impulses normally travel along the pyramidal tract; but it does shew, what indeed has already been shewn by the phenomena of strychnia poisoning, $\$ 461$, that in the central nervous system the passage of nervous impulses (using those words in the general sense of changes propagated along nervous material) is not rigidly and unalterably fixed by the anatomical distribution of tracts of fibres; in all such discussions as those in which we are engaged we must bear in mind that physiological conditions as well as anatomical connections are potent in determining the passage of these impulses.

$\$$ 492. When we reflect on the great prominence of the pyramidal tract in the spinal cord of man as compared with that of the dog, we may justly infer not only that the pyramidal tract 
is under normal circumstances more exclusively the channel of volitional impulses in man than in such lower animals, but also, bearing in mind the discussion in a previous chapter, $\$ 464$, concerning the activities of the spinal cord of man, that the potential alternatives presented by the spinal cord of the dog are greatly reduced in that of man. And such clinical histories of disease or accidental injury in man as we possess support this conclusion. Lesions confined to one half of the cord, or even lesions confined to the lateral column of one half, appear to lead to loss of voluntary power on the same side, and the same side only, in the parts below the level of the lesion: and the same symptoms have been observed to accompany disease limited apparently to the pyramidal tract of one side. Moreover, though cases of recovery of power have been recorded, we have not such satisfactory evidence as in animals of the volitional impulses ultimately making their way along an alternative ronte; but here the same donbts may be entertained as were expressed in discussing the reflex acts of the cord in man.

When we say that the loss of voluntary power is seen on the side of the lesion only, we should add that this statement appear's to apply chiefly to the thoracic and lower parts of the cord. We have seen that in man, in the upper regions of the cord, the pyramidal tract is only partly crossed; a variable but not inconsiderable number of the pyramidal fibres do not cross at the decussation of pyramids, but rumning straight down as the direct pyramidal tract effect their crossing lower down in the cervical and upper thoracic regions. Hence we should infer that a hemisection of, or a lesion confined to one side of the cervical cord, would affect the voluntary movements of the crossed side as well as of the same side, though not to the same extent. But we have no exact information as to this point. And indeed the purpose of the direct tract is not elear; there is no adcquate evidence for the view which has been held that these direct fibres are destined for the upper limbs and upper part of the body; since they are the last to cross we should a priori be inclined to suppose that they were distributed to lower rather than higher parts.

$\$ 493$. We may now briefly summarize what we know concerning voluntary movements. And it will be convenient to trace the events in order backwards.

Certain muscles are thrown into a contraction which even in the briefest movements is probably of the nature of a tetanus. In almost every movement more than one muscle as defined by the anatomists is engaged, and in many movements a part of several muscles is employed, and not the whole of each. It is perhaps partly owing to the latter fact that a muscle which has become tired in one kind of movement, may shew little or no fatigue when employed for another movement, though we must 
bear in mind that in a voluntary movement fatigue is much more of nervous than of muscular origin.

Besides the active muscles, if we may so call them, which directly carry out the movement, the metabolism of which supplies the energy given out as work done, other muscles, some of which are antagonistic to the active muscles and some of which may be spoken of as adjuvant, enter into the whole act. In flexion for instance of the forearm on the arm it is not the flexor muscles only but the extensors also which are engaged. Accolding to the immediately preceding position and use of the arm, and accolding to the kind and amount of flexion which is to be carried out, the extensor's will be cither relaxed, that is to say inhibited, or thrown into a certain amount of contraction. And in some of the more complicated voluntary movenuents the part played by adjuvant muscles is considerable. Hence in a voluntaly movement the will has to gain access not only to the active muscles, but also to the antagonistic and adjuvant muscles; and every voluntary morement, even one of the simplest kind, is a more or less complex act.

The impulses which lead to the contraction of the active muscles reach the muscles along the fibres of the anterior roots, (we may for the sake of simplicity take spinal nerves alone, neglecting the peculiar (ranial nerves,) and such evidence as we possess goes to shew that the impulses governing the antagonistic and adjuvant muscles travel by the anterior roots also; the question whether the inhibition of the antagonistic muscles when it takes place, is carried out by inhibitory impulses passing as such along the fibres, or simply by central inhilition of previously existing motor impulses need not be considered now. These anterior roots are connected as we have seen with the grey matter of the cord, and in each hypothetical segment of the cord we may recognize the existence of an area of grey matter which, though we cannot define its limits, we may, led by the analogy of the cranial nerves, call the nucleus of the nerve belonging to the segment; and we may further recognize in such a nucleus what we may call its efferent and its afferent side.

Every voluntary movement, even the simplest, is as we have repeatedly insisted a coordinated movement, and in its coordination afferent impulses play an important part. The study of reflex actions, $\$ 462$, has led us to suppose that each spinal segment presents a nervous mechanism in which a certain anount of coordination is already present, in which efferent impulses are aljusted to afferent impulses. But the results obtained by stimulating separate anterior nerve roots shew that, in the case of most muscles at all events, the especially active muscles of the limbs for instance, each muscle is supplied by fibres coming from more than one nerve root, that is to say the spinal nucleus, 
or at least the spinal motor mechanism for any one muscle, extends over two or three segments. Hence a fortiori in a voluntary movement, involving as this does in most cases more than one muscle, the spinal mechanism engaged in the act spreads over at least two or three segments, thus allowing of increased coordination. In that coordination the impulses serving as the foundation of muscular sense play an important part, but other afferent impulses, such as those from the adjoining skin, also have their share in the matter; and it is worthy of notice that not only is the skin overlying a muscle served, broadly speaking, by nerve roots of the same segment as the muscle itself, afferent in one case, efferent in the other, but in the parts of the body where coordination is especially complex, in the fingers for instance, not only is each muscle supplied from more than one segment, but also each piece of skin is supplied in the same way by the posterior roots of more than one nerve.

In the case of the frog it is clear that in reflex movements a large amount of coordination is carried out by these various spinal mechanisms: and as we have urged, we may safely infer that in the voluntary movements of the frog, the will makes use of this already existing coordination, whatever be the exact path by which in this animal the will gains access to the spinal mechanisms. In the dog we may conclude that in voluntary movements the spinal mechanisms, with coordinating functions, are also set in action, in this case by impulses passing straight from the cortex to the mechanisms by the pyramidal tract, though, apparently in the absence of the pyramidal tract, the will can work upon the mechanisms by changes travelling through other parts of the cerebrospinal axis. And in the monkey and man, subject to the doubts already expressed as to the potentialities of the human spinal cord, we may probably also infer that in each voluntary movement some, perhaps we may say much, of the coordination is carried out by the spinal mechanism set into action through impulses along the pyramidal tract. We may probably further infer that a careful adjustment obtains between the beginnings of the pyramidal tract in the cortex and its endings in the cord, so that the topography of 'areas' or 'foci' in the cortex above is an image or' projection of the spinal mechanisms below.

The complex character, on which we insisted just now, of almost every voluntary movement necessitates that in every such movement a large area of spinal mechanism is involved. But this is not all. The movements of any part, of the legs for instance, are not determined, nor is the coordination of the movements effected, simply by what is going on in the legs and the part of the spinal cold belonging to them. 'The discussion in a previous section has shewn that much of the coordination 
of the body is carried out by the middle portions of the brain, and on these the motor area must have its hold as well as on the spinal mechanisms.

The details of the nature of that hold are at present unknown to us; but it must be remembered that not all the fibres passing down from the motor region, not all those even proceeding from the densest and most clearly defined motor areas, are pyramidal fibres. With the pyramidal fibres are mingled fibres having other destinations, and some of these probably pass to the thalamus and so join the great tegmental region. Moreover the motor region must have close ties with other regions of the cortex whence fibres pass to the pons to make connections with the cerebellum. On the other hand, the cerebellum is especially connected with what we may fairly consider the afferent side of the spinal cord and bulb. These facts must merely be taken as indicating the possibilities by which the motor region is kept in touch with the great coordinating mechanism; it would be venturesome at present to say much more.

In an ordinary voluntary movement an intelligent consciousness is an essential element. But many skilled movements initiated and repeated by help of an intelligent conscious volition may, when the nervous machinery for carrying them out has acquired a certain facility, (and in all the higher processes of the brain we must recognize that, in nervous material at all events, action determines structure, meaning by structure molecular arrangement and disposition) be carried out under appropriate circumstances with so little intervention of distinct consciousness that the movements are then often spoken of as involuntary. All the arguments which go to shew that the distinctly conscious voluntary skilled movement is carried out by help of the appropriate motor area, go to shew that the motor area must play its part in the involuntary skilled movements also. So that distinct consciousness is not a necessary adjunct to the activity of a motor area. And it is worthy of notice that some of these, in their origin, purely voluntary skilled movements, which by long-continued training have become almost as purely involuntary, are hampered rather than assisted by being 'thought about.'

Lastly, withont attempting to enter into psychological questions we may at least say that the birth-place of what we call the 'will,' is not conterminous with the motor area; the will arises from a complex series of events, some of which take place in other regions of the cortex, and probably in other parts of the brain as well. With these parts the motor area has ties concerned not in the carrying out of volition, but in the generation of the will. So that, looking round on all sides, it is obvious, as we have said, that the motor area is a mere link in 
a complex chain. It is moreover a link of such a kind, that while the changes which the breaking of it makes in the daily life of a lowly animal, such as the dog, in whom the experience of the individual adds relatively little to the nervous and psychical storehouse transmitted from his ancestors, can hardly be appreciated by a bystander, those which the breaking of it makes in the daily life of a man, whose brain at any moment is not only a machine fitted for present and future work but a closely packed record of his past life, are obvious not only to the individual himself, but to his fellows. 
SEC. 4. ON THE DEVELOPMENT WITHIN THE CENTRAL NERVOUS SYSTEM OF VISUAL AND OF SOME OTHER SENSATIONS.

\section{Visual Sensations.}

$\S 494$. In the chain of events through which some influence brought to bear on the periphery of a sensory nerve gives rise to a sensation, we are able, with more or less success, to distinguish between those events which are determined by the changes at the periphery and those which are the expression of changes induced in the central nervous system. Thus when certain rays of light proceeding from an object and falling upon the eye give rise to visual perception of the object, two sets of events happen; the rays of light, by help of the mechanisms of the eye, partly dioptric, partly nervous, give rise to certain changes in the fibres of the optic nerve, which we may call visual impulses; and these visual impulses reaching the brain along the optic nerve give rise to visual sensations and so to visual perception of the object. We shall later on, under the hearding of " the senses," deal chiefly with the peripheral events, and have now to consider some points connected with the central events, to learn what we know concerning how the various sensory impulses travelling along the several kinds of sensory nerves behave within the central nervous system. In doing so we shall have from time to time to refer to peripheral events, but only occasionally, and never in any great detail. It will be convenient to begin with the special sense of sight, and we must first briefly call attention to a few points which we shall have to study in fuller detail hereafter.

The eye is so constructed that images of external objects are brought to a focus on the retina, the stimulation of which by light starts the visual impulses along the fibres of the optic nerve; and the distinctness with which, by means of the visual sensations arising out of these visual impulses, we perceive external objects is dependent on the sharpness of the retinal images. The eye is further so constructed that, in any posi- 
tion of the eye, the rays of light proceeding from a portion only of the external world fall upon the retina; or in other words in any one position of the eye only a portion of the external world is visible at the same time. 'The portion so seen is spoken of as the visual field for that position.

The image thrown on the retina is an inverted one, so that the top of an actual object is represented by the lower, and the bottom by the upper part of the retinal image; similarly the actual left-hand side of the retinal image corresponds to the righthand side of the actual object, and the right-hand side to the left-hand side. Hence the right-hand half of the visual field corresponds to the left-hand side of the retina, and the left-hand half to the right-hand side.

The eye can be moved in various directions, and since in the visual field the portion of external nature which can be seen at the same time differs with each different position, a large range of vision is thus secured; and this can be further increased by movements of the head. Moreover we normally make use of two eyes, our normal vision is binocular; and the visual field of the right eye differs from that of the left eye. There is one striking difference which must always be borne in mind. A section carried through the eye in a vertical and front-to-back plane, through what we shall learn to call the optic axis (Fig. 138, ox ) (the exact details of the plane may be left for the present), will divide the retina into two lateral halves, and in each retina one half will be on the nasal side next to the nose, and the other half will be on the malar or temporal side, next to the cheek or temple. It must be remembered that the nasal halves and temporal halves of the two retinas do not occupy corresponding positions in space. The temporal half of the left retina is on the left side of its own eye, whereas the temporal half on the right retina is not on the left but on the right side of its eye; and so with the nasal halves. Now in the right eye, the right-hand side of the visual field corresponds to the nasal half of the retina, and the left-hand side of the visual field to the temporal half of the retina, whereas in the left eye the right-hand side of the visual field corresponds to the temporal half of the retina, and the left-hand side to the nasal half. 'This is shewn in Fig. 138, where the left-hand visual field and the retinal area concerned are shewn shaded in each eye.

When we look at an object with the two eyes, though two retinal images are produced, one in one eye and one in the other, we perceive one object only, not two. This is the essential fact of binocular vision; when certain parts of each retina are stimulated at the same time we are conscious of one sensation only, not two; and the parts of the two retinas which, stimulated at the same time, give rise to one sensation are spoken of as "corresponding parts." From the structure and relations of the 


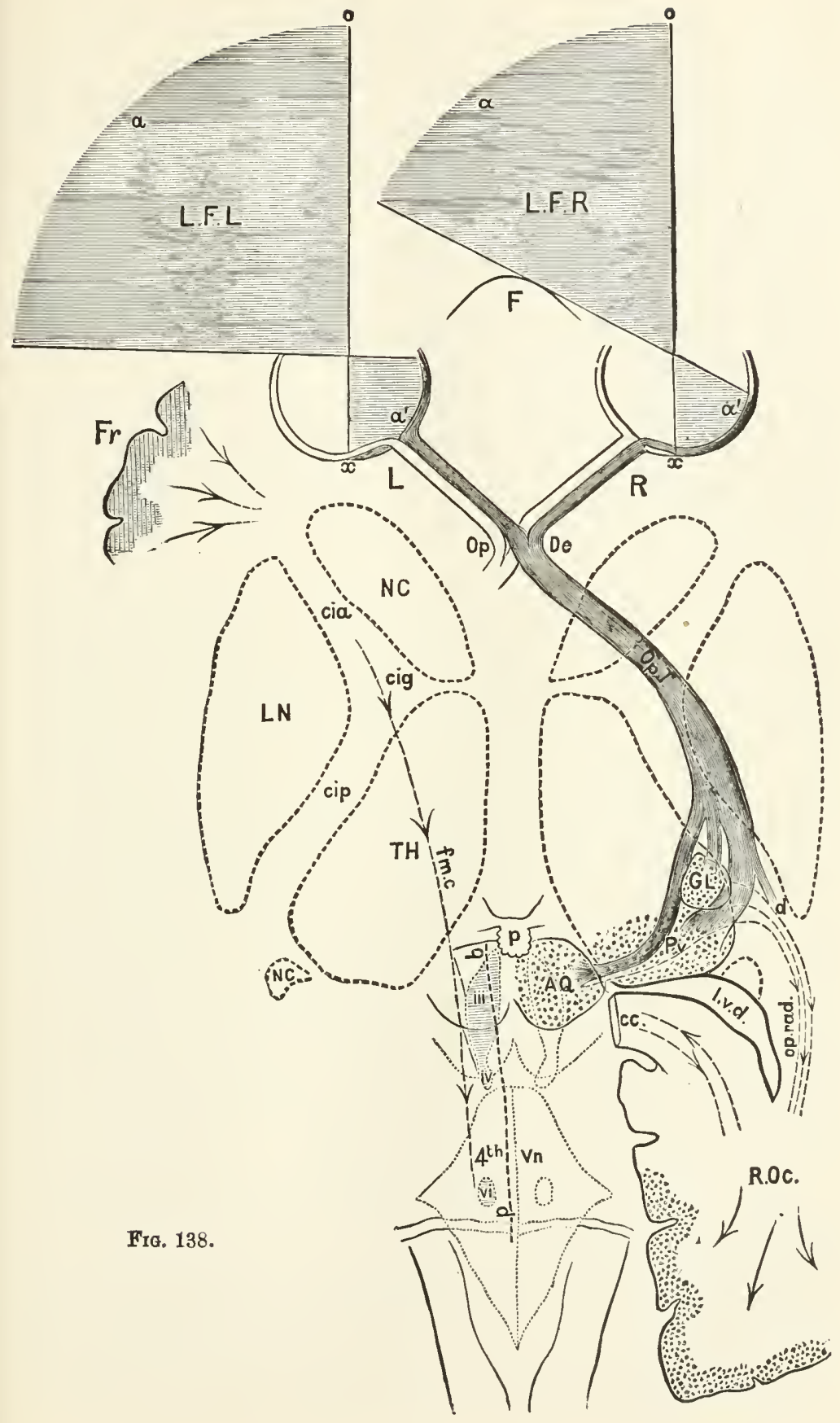


Fig. 198. Dhagram to mleustrate tule Nervols Apparatus of Vislon ix Max. (Nherrington.)

$L$. the left eye, $R$. the right eye, o.x. the optic axis. $F$. the outline of the face between the eyes, $O_{p} . T$. the right optic tract (shaded) supplying, through $O p$. De. the optic decussation, the temporal side of the retina of the right eye and the nasal side of the retina of the left eye. $L . F . L$. and $L . F$. R. the left visual fields of the left and right eye respectively; the two fields and the parts of the two retinas whose excitation produces vision over the fields are shaded, the olject $a$ in the field of the right side giving rise to an image at $a^{\prime}$, and $a$ on the left side an image at $a^{\prime}$.

The right optic tract is represented as ending in $C L$. the lateral corpus geniculatum. in $P v$. the pulvinar, and in $A Q$. the anterior corpus quadrigem inum, all three stippled; op. rad. the optic radiation from these bodies to $R$. Oc. the right occipital lobe, whose stippled cortex inclicates the 'visual area.' $d$. the 'direct ' tract to the cortex. c. c. corpus callosum, cut across at the splenium, $l . v . d$. descending horn of the lateral ventricle.

The left side has been utilized to indicate at $F$. shaded with lines, the cortical motor area for the eyes; fim. $c$. indicates the path from it to III. IV. VI. the nuclei of the third, fourth and sixth nerves. $p . b$. the posterior longitudinal bundle, shewn as a broken line. $N C$. the nucleus caudatus, L.T. the nucleus lenticularis and TH. optic thalanus shewn in ontline, Cia. the front limb, Cig. the knee, and Cip. the hind limb of the internal capsule. 'The outlines of the fourth ventricle this. Vn. and of the posterior corpora quadrigemina are shewn by dotted lines, that of the bulb is shewn by a fine line. $p$. the pineal gland.

two eyes it follows that the temporal side of the right and the nasal side of the left eye are such corresponding parts, while the nasal side of the right eye corresponds to the temporal side of the left eye. But the whole of each retina is not employed in binocular vision. Owing to the position of the two eyes in relation to the nose, it comes about that an object held very much on one side, to the left-hand side for instance, while it is capable of producing an image on the extreme nasal side of the left eye, and can be secn therefore by that eye, cannot produce an image on the temporal side of the right eye; the nose blocks the way. It is therefore not seen by the right eye, and the vision of it is monocular, by the left eye only. In Fig. 138 it may be scen that the left visual field of the left eye (L.F.L.) extends more to the left, and is larger than the left vismal field of the right eve (L.F.R.) and that the right retinal area, corresponding to the left visual field, extends farther along the nasal side of the left side $\left(a^{\prime}\right)$, than it does along the temporal side of the right eye $\left(a^{\prime}\right)$, the difference being due to the presence of the nose $\left(F^{\prime}\right)$. And similar conditions obtain with regard to the extreme right-hand side of the visual field.

$\$ 495$ After these preliminary statements, we may now turn to consider some anatomical facts concerning the ending of the optic nerve in the brain.

The optic nerve of each eye consists of nerve fibres coming from all parts of the retina of that eye; but the two optic nerves meet, ventral to the floor of the third ventricle, cross each other at the optic chiasma (Fig. 138, op. De), and are thence contimued on under the name not of optic nerves but of optic tracts (Op.'T.). 
The decussation of fibres which takes place in the chiasma has peculiar characters. At their decussation (we are speaking now of man) the fibres in the optic nerve belonging to the temporal half of the eye in which the nerve ends pass into one optic tract, namely, the optic tract of the same side, while the fibres belonging to the nasal half pass into another optic tract, namely, the optic tract of the opposite side. Thus the fibres of the temporal late of the right eye and of the nasal half of the left eye pass into the right optic tract, and the fibres of the nasal half of the right eye and of the temporal half of the left eye pass into the left optic tract. Compare Fig. 138, in which the fibres forming the right optic tract are shaded while those forming the left optic tract are left unshaded. Now, the nasal half of one retina and the temporal half of the other retina are 'corresponding' parts. Hence, while each optic tract contains fibres belonging to half of each eye, the two halves thus represented in each tract are corresponding halves.

The amount and character of the decussation taking place in the optic chiasma differs in different animal types, the differences having relation to the amount of binocular vision, which in turn depends on the position of the eyes in the head, that is, on the prominence of the face between the eyes. In the fish for instance, with laterally placed eyes, no binocular vision at all is possible, and the decussation is complete; the whole optic nerve of each eye crosses over to the other optic tract. Between this and the arrangement in man just described, various stages obtain in various animals.

The chiasma also contains at its hinder part fibres which have no connection with the optic nerves or the eyes, but are simply commissural tracts passing from one side of the brain, namely, from the median corpus geniculatum (this has nc connection with the optic nerves and is not concerned in vision) along one optic tract, through the chiasma to the other optic tract, and so to the median corpus geniculatum of the other side of the brain. 'These fibres are spoken of as the inferior or posterior (optic) commissure or areuate commissure, or Gudden's commissure. It was once thought that in a similar way fibres passed from one retina along one optic nerve, through the front part of the chiasma to the other optic nerve, and so to the other retina forming an anterior (optic) commissure; but this seems to be an errol.

The optic tract also contains fibres taking origin from the grey matter in the floor of the third ventricle and forming what is sometimes spoken of as Meynert's commissure; these filnes which belong neither to the optic nerves nor to the inferior commissure, join the optic tracts for a while, but eventually leave them to pass to the pes.

$\S 496$. That part of the optic tract which consists exclu- 
sively of fibres coming from the retinas of the two eyes, and it is this part, and this part only, which is concerned in vision. ends in three main ways, as shewn diagrammatically in Fig. 138. In the first place part of the tract ends in the lateral corpus geniculatum $(G L)$. In the second place, a very large number of fibres passing the corpus geniculatum on its ventral and lateral surfaces spread out into the pulvinar $\left(P V^{\top}\right)$. In the third place others, in considerable number, taking a more median direetion, reach the anterior corpus quadrigeminum ( $A Q)$ These thrce sets end apparently in connection with the nerve cells of the respective bodies. 'Thus the really optic fibres of the optic tract end in one of three collections of grey matter, the lateral corpus geniculatum, the pulvinar, and the anterior corpus quadrigeminum. Further, we have reasons for thinking that a considerable part at all events of the grey matter of these three bodies, especially of the first two, is associated with and, in a certain sense, dependent on the fibres of the optic nerves; the reasons are as follows. We know that when a nerve fibre is cut away from its trophic centre it degenerates; but the division, and the loss of the peripheral degenerating portion, has no obvious effect on the trophic centre; when a spinal nerve, for instance, is divided below the spinal ganglion, though the nerve below the section degenerates, the ganglion and the piece of nerve in connection with it remain very much as before. We have it, however, in our power to bring about changes of a deeper and wider character, a cessation of growth amounting to atrophy, by operative interference with nervous structures before they are fully developed. Thus in an adult animal, a section of an optic nerve or removal of the eye leads to degeneration in the optic nerve and optic tract; the optic fibres have their trophic centre in certain cells of the retina, of which we shall speak in treating of vision, and cut away from that centre they degenerate: by this means the nature of the optic decussation in animals, and indeed in man, has been ascertained. But if the eyes be removed (removal of both eyes being desirable on account of the characters of the optic decussation), in a new-born animal, not only do both the optic nerves and the greater part of both optic tracts cease to be further developed and degenerate, but the bodies mentioned above, the two lateral corpora geniculata, the pulvinar on each side, and the two anterior corpora quadrigemina do not fully develope; certain parts of them undergo atrophy. The development of these nervous structures seems therefore to be largely dependent on their functional connection with the eyes by means of the optic tracts and nerves.

The same method confirms the view expressed above that the median corpus geniculatum has $n$ o connection with vision. When the eyes of new-born animals are extirpated neither the median corpora geniculata nor the posterior corpora quadrigemina 
shew any sign of atrophy: and the part of the optic tract which does not degenerate is the inferior commissure connecting the two median corpora geniculata. Obvionsly these parts are associated with functions of the brain other than those of sight. The lateral corpora geniculata, the pulvinar and the anterior corpora quadrigemina, are, we may repeat, alone to be regarded as the chief central parts in which the optic nerves end. IVe may also repeat that owing to the peculiarity of the optic decussation each optic nerve thus finds its endings in both sides of the brain.

$\$ 497$. Though the above three bodies are undoubtedly the chief endings of the optic nerve, three primary visual centres, if we may so call them, it is also believed that some fibres of the optic tract, making connections with neither of these three bodies, pass by the crus cerebri straight to certain parts of the cerebral hemisphere (Fig. 138, d) ; but this fourth ending is by no means so clearly established as are the other three. And undoubtedly the main connection of the cerebral hemisphere with the optic tract is not a direct one, hut an indirect one, through the three bodies in question. A number of fibres proceeding from the occipital cortex and reaching the thalamus throngh the hind limb of the internal capsule form what is called the 'optic radiation.' These fibres begiming (or ending) in the cortex of the occipital region. end (or begin). (Fig. 138, op. $r a(l)$ to a large extent, in the pulvinar and in the lateral corpus geniculatum, but also in the anterior corpus quadrigeminum, reaching it by the anterior brachium. When in a newborn animal the occipital cortex, or even a certain part of the occipital cortex is removed, these three bodies atrophy, and the atrophy extends to the optic tract and nerve; conversely removal of both eyes in a new-born animal leads not only to atrophy of the three bodies in question, but also to imperfect growth of the occipital lobes. Not only so, but even in the fullgrown animal removal of the occipital cortex entails a degeneration and atrophy of these three bodies, the details differing in different animals according to their kind, the degeneration in some animals reaching into the optic tract and nerve. And even in man degeneration of the external corpus geniculatum, of the pulvinar, and to sone extent of the anterior corpus quadrigeminum and of the optic tract has been observed to result from disease of the occipital lobe. This is a remarkable circumstance; for we may assume that the fibres carrying impulses from say the corpus geniculatum to the occipital lobe, are axis cylinder processes starting from cells in the former structure to end in the latter, and therefore having their trophic centres in the former. The degeneration occurring in the corpus geniculatum as a result of the removal of or disease in the occipital cortex is a different thing from the degeneration which takes 
place in the optic tract, after removal of the retina; it seems rather a degeneration from pure want of functional activity, as if the cells in the corpus geniculatum are unable to act as they ought to do on the arrival of visual impulses along the optic fibres, if their ties with the occipital cortex are broken. We may therefore conclude that in the complex act of vision two orders of central apparatus are involved; we may speak of two kinds of centres for vision, the primary or lower visual centres supplied by the three bodies of whieh we are speaking, and a secondary or higher visual centre supplied by the cortex in the occipital region of the cerebrum. And experimental results accord with this view.

Before we proceed to discuss those results, one or two preliminary observations may prove of use.

In the first place, as we have previously urged, the interpretation of the results of an experiment in which we have to judge of sensory effects, are far more uncertain than when we have to judge of motor effects, that is of course when the experiment is conducted on an animal. IVe can estimate the motor effect quantitatively, we can measure and record the contraction of the muscles; but in estimating a sensory effect we have to depend on signs, our interpretation of which is based on analogies which may or may not be misleading. We are on safer ground when we can appeal to man himself in the experiments instituted by disease; but the many advantages thus secured are often more than counterbalanced by the diffuse characters, or the complex concomitants of the lesion. In dealing with sensory effects we must expect and be content for the present with conclusions less definite and more uncertain even than those gained by the study of motor effects.

In the second place, in dealing with vision, it will be desirable to know the meaning which we are attaching to the words which we employ. By blindness, that is 'complete' or 'total' blindness, we mean that the movements and other actions of the body are in no way at all influenced by the amount of light falling on the retina. Of partial or incomplete or imperfect vision, using the word vision in its widest sense, there are many varieties; and we may illustrate some of the defects of the visual machinery, regarded as a whole, with its central as well as its peripheral parts, by referring to certain defects of vision due to changes in the eye itself. The eye may fall into such a condition, that the mind can only appreciate, and that to a varying degree, the difference between light and darkness; the mind is aware that the retina (or it may be part of the retina) is being stimulated to a less or greater degree, but camnot perceive that one part of the retina is being stimulated in a different way from another part; a sensation of light is exeited, but not a set of visual sensations corresponding to the sets of pen- 
cils of luminous rays, which, reflected, or emanating from external objects in a definite order, arc falling upon the eye. 'The eye again may fall into another condition, in which such sets of visual sensations are excited, but on account of dioptric imperfections or for other reasons, the sereral sensations are not adequately distinct; the mind is aware through the eye of the existence of 'things,' but camnot adequately recognize the character's of those things; the visual inages are blured and indistinct. And a large number of gradations are possible between the extreme conchition in which only those objects which present the strongest contrast with their surroundings are visible, to a condition which only just falls short of normal vision. Imperfections of this kind, of varying degree, may result from failure not in the peripheral apparatus, not in the retina, or optic nelve or other parts of the eye, but in the central apparatus; the retinal image may be sharp, the retina and the optic fibres may be duly responsive, but from something wrong in some part or other of the brain, the visual sensations excited by the visual impulses mar fail in distinctness, and that in varying degree: imperfections of vision whether of central or peripheral origin, in which risual sensations fail in distinctuess are generally spoken of under the not wholly unexceptionable nane of amblyopia.

If one optic nerve be divided, total blindness of one eye will result; but if one optic tract he divided, it follows from what has been said above, that half-blindness in the corresponding halves of both eyes will result. If, for instance, the right optic tract (Fig. 138, Op. T.) be divided, the left visual fields of both eyes will be blotted out. The same condition will be brought about by failure in the optic tract at its central ending, provided of course the mischief be confined to the ending of the one tract. Such a half-blindness or half-vision is spoken of as hemianopsia, or hemianopia or hemiopia; the wolds left and right are generally used in reference to the visual field; thus left hemianopsia is the blotting out of both left visual fields, through failure of the right optic tract. If instead of the whole optic nerve being divided, certain bundles only were cut across, partial blindness in that eye would be the result, a portion of the visual field of that eye would be blotted out. If not the optic nerve but the optic tract were so treated, mischief limited to a few bundles of one tract would lead to corresponding blots in the corresponding halves of the visual fields of both eyes. Further, an affection of half the retina or of a linited area in the retina might occur of sucl a character as to lead not to complete, but to partial blindness, to a hemi-amblyopia or to a partial amblyopia. The part of the retina so affected might be central, or peripheral, and miglit be a quadrant, or any patch of any size, form and relative position. And we may further im- 
agine it at least possible that mischief in the brain might be so limited as to produce any of the above partial effects, though the retina, optic nerve, and optic tracts all remained intact.

The above visual imperfections we have illustrated by changes in the peripheral apparatus, but there is a kind of imperfection which we may still call a risual imperfection, though it is of purely central origin. In a normal state of things a visnal sensation, excited in the brain, is or may be linked on to a chain of psychieal events; we often then speak of it as a visual iclea. When we see a dog, the visual sensation, or rather the group of sensations making up the visual perception of the dog, does not exist by itself, apart from all the other events of the brain; it joins and affects them, and among the events which it so affects may be and often are psychical events; the visual perception 'enters into our thoughts' and modifies them. Between the visual impulse as it travels along the optic nerve or tract and its ultimate psychical effect a whole series of events intervene; and we may take it for granted that the ehain may be broken or spoilt at any of its links, at the later as well as at the earlier ones. We may therefore consider it possible that the break or damage may occur at the links by which the fully developed visual sensation joins on to psychical operations. We may suppose that an object is seen and yet does not affect the mind at all or affects it in an abnormal way.

These foregoing considerations emphasize the difficulty and uncertainty of interpreting the visual condition of an animal which has been experimented upon. When for instance, after an operation, an animal ceases to be influenced in its previous normal manner by the visual effects of external objects, a most careful psychical analysis is often necessary to enable us to judge whether the newly introduced disregard of this or that objeet is due to the mere visual selisations being blurred or blunted, or to some failure in the psychical appreciation of the sensations; and in most cases such an analysis is beyond our reach. The greatest caution is needful in drawing conclusions from experiments of this kind, especially from such as appear to have been hastily carricd out or hastily observed; and we must be content here to dwell on some of the broader features only of the subject.

$\$ 498$. Since we have in this matter to trust so mucli to analogics with our own experienee, we may turn at once to the monkey, as being more instructive than any of the lower animals. We have already said that electrical excitation of the occipital cortex behind the motor region may produce movements, but that these movements are in character different from those caused by stimulation of the motor region itself. In the monkey stimulation of parts of the occipital region, the occipital lobe and the angular gyrus for instance, may give rise to movements of the eyes, of the eyelicls, and of the head, that is of the neck, all the 
movements so produced being such as are ordinarily connected with vision. It will not be profitable to enter here into the details conceming the exact topography of the excitable parts or of the special characters of the movements so called forth. But it is important to note that these movements are unlike the movements excited by stimulation of the appropriate motor area in as much as their occurrence is far less certain, they need a stronger stimulus to bring them out, when evoked they are feeble, being easily antagonized by appropriate stimulation of the motor area, and they have a much longer latent period. They are not due to any indirect stimulation of the motor area, through ' association' fibres connecting the spot stimulated with the motor area or otherwise, since they persist after removal of the motor area. Movements of this kind may also be ritnessed in the dog. They are obviously the result of impulses transmitted in some direct manner from the cortex to some parts below, and may be taken as an indication that the parts of the cortex in question are in some way connected with vision. 'The exact manner however in which they are brought about is at present not clear.

$\$ 499$. The results of removal of the cortex in the monkey also shew clearly that the hind region of the cortex is specially connected with vision, though there has been and indeed still is much discordance among the various observers both as to the particular results and especially as to their interpretation.

Many observers have found that removal of the occipital lobe on one side, the region marked 'vision' in Figs. 12.3, 124, caused hemiopia, the effect on the visual fields being a crossed one; when the right lobe for instance was removed there was blindness in the left visual fields, that is in the right halves of the retinas of both eyes; in other words the visual impulses passing along the right optic tract failed to produce their usual effect, so that the animal disregarded objects on its left-hand side. IVe may remark that the decussation of the optic nerves in the monkey is very similar to that in man. When both occipital lobes were removed, total blindness resulted. Such a result seems perhaps at first sight, not only in accordance with the anatomical connections spoken of above, but also simple and satisfactory. Visual impulses originating in the corresponding halves of the two retinas travel along the appropriate optic tract to the primary visual centres of one side, and thence pass by the optic radiation to the occiprital lobe of the same side (for there is no decussation along the whole line save at the optic chiasma), where they give rise to visual perceptions. But even a little consideration will raise difficulties. The eyes are preeminently organs of bilateral use; and if, when we are looking with the two eyes at an object whose image occupies parts of the two halves of each retina, the impulses generated in the corresponding halves of the two retinas, affect exclusively the one side of the brain only, there 
must exist remarkably delicate and exact arrangements between the two halres of the brain by which in each case as it occurs, the two sets of visual sensations should be accurately pieced into a whole perception in which we can subjectively recognize no halves. It is at least strange that while the notor nervous mechanism for the two eyes is finely bilateral, the sensory mechanism should be baldly unilateral. WV need not then be surprised to meet with other experinental results discordant with the above mentioned apparently simple result. In the first place not only is the hemiopia, caused by the removal of one occipital lobe, often transient, but also, according to some observers, the lost rision may retur'n after the total removal of both lobes, though some impairment may be noticed long afterwards, so long in fact as the animal is kept alive. In the second place in the hands of other observer's destruction not of the occipital lobe but of the angular gyrus of one side (Fig. 122) has led to hemiopia, failure in the left (or right) visual fields, indicating failure in the central endings of the right (or left) optic tract, being caused by removal of the right (or left) gyrus, and destruction of both angular gyri has led to total blindness, not only the hemiopia but the total blindness being, however, apparently transitory. And cases have been observed in which the blindness due to removal of the occipital lobe which would be by itself transient has been made permanent by the subsequent removal of the angular gyrus. In other cases again destruction of one angular gyrus has produced, not hemiopia, but crossed blindnes, or crossed amblyopia, that is to say has affected the whole of the retina of one eye, and that the crossed eye, the eye of the same side not being, or being supposed not to be, at all affected; and indeed similar results have also been stated to follow upon removal of one occipital lobe.

In man clinical histories so far conform to the results of experiments on the monkey as to associate the occipital cortex, and more particularly the cuneus (see Figs. 13t, 135) with vision. A very large and increasing number of cases have been recorded in which hemiopia, a blotting out of the corresponding halves of the visual fields of the two eyes (homonymous hemiopia as it is called) has been associated with disease of the occipital lobe namely of the cuneus, or of adjoining parts of the lingual lobe, in the neighbourhood of the calearine fissure, or of adjoining portions of the occipital convolutions; and there have been similar cases where not the half, but a part only, a quadrant it may be or less, of each visual field has been blotted out. 'The teaching of such cases is in full accord with the anatomical leading, but leaves untouched the difficulty mentioned above as attaching to such unilateral cerebral action. It is worthy of note that in many such cases of hemiopia, the macula lutea, the region of distinet vision is left intact in both eyes; and this has led some to the 
view, that the macula of each eye unlike the rest of the retina is represented in both ceirebral hemispheres. But this is not very satisfactory. Certain cases on the other hand have been met with, which like some of the experiments on monkeys point to at least some share being taken by the angular gyrus.

Many experiments have been made on the dog, and the results obtained have been interpreted by some observer's as shewing that in this animal not only is an area in the occipital region of the cortex specially connected with vision, but even that particular parts of the area correspond to particular parts of the field of vision, the several parts of the retina being as it were projected on to the cortex. We need not enter liere upon the details of this view which needs some special exposition since in the dog vision is much less binocular than in man; it will become desirable to do so should evidence be fortheoming that a similar projection obtains in man: but, though some elinical histories have been held to indicate this, others are opposed to it. In short though it is clear that the occipital cortex is concerned in vision, our present knowledge does not afford a consistent view of the exact way in which it is concerned.

$\$ 500$. We may perhaps say a few words on the question what is it which actually takes place in the cortex during vision? Are we to conceive of it as if a visual impulse set going along the fibres of the optic tract underwent no essential change until it reached the cortex, as if it there suddenly developed into a "visual sensation'? W' e can hardly suppose this. Between the cortex and the optic tract, the lower vismal centres, the tegmental masses, intervene; and we can hardly suppose that interference with these bodies produces the same effect on vision as simple section of the optic tract. TVe have seen in a previons section that the frog and the bird certainly, and accorcling to some observers also the rabbit, are in the abscnce of the cerebral hemispheres not totally blind, since their movements appear to be guided by retinal impressions; and cases are recorded of the dog being obviously still guided in some measure by retinal impressions after the occipital lobes and indeed the greater part of the brain had been removed. This is a matter of no little difficulty; it is perhaps possible for simple afferent impulses to determine even complex movements without the intervention of 'consciousness,' and we may be justified in speaking of the effects of light on a brainless animal as being mere instances of 'mechanical' reflex action; still we are probably justified in assuming that the simple visual impulses, travelling along the fibres of the optic tract, undergo important transformations in the tegmental masses, and that the changes which are propagated along the fibres of the optic radiation, constitute something quite different from the impulses along the optic tract or nerve. We may perhaps assume that in 
vision the cortieal events are psyehical in nature, and that the funetion of the optic radiation is to furnish what we may call erude visual sensations for further psychical elaboration. Or perhaps we do wrong in attempting to dissociate in any such distinct way the eortical and the tegmental events. The remarkable dependence as regards nutrition between the occipital eortex and the tegmental masses, of which we spoke a little while back, shews that the two work together in the closest way. Possibly we are wrong in thinking that in the generation of a visual sensation, a something passing upwards from the one is transformed into something else in the other, and ought rather to suppose that the sensation is a produet of the two working together in a manner to the understanding of which our ordinary eoneeptions of nervous impulses gathered from the study of ordinary efferent and afferent nerves afford us little help. Possibly also the ties between the eortical and tegmental proecsses are drawn tighter in the higher animals as vision becomes more complex, so that after total removal of the eerebral cortex while the bird and possibly even the dog may be said still to 'see,' man and probably the higher' monkey's appear, as experiments and elinical histories shew, to be not only psyehically but in every way blind, and that long before degenerative changes in the tegmental masses ean have become marked.

As to the several parts played by the individual tegmental masses we at present know little or nothing. It is worthy of note that when we compare various animals, we find that the comnections of the cortex with the eorpus quadrigeminum are the more prominent in the lower animals, and in the higher animals more and more give plaee to those with the pulvinar and especially with the eorpus genieulatum. Some facts perhaps may be taken as indieating that the corpus quadrigeminum is espeeially concerned, when visual sensations influence the coordination of movements; but this is not eertain.

\section{Sensations of Smell.}

$\S 501$. In many animals in whom the sense of smell is acute, a portion of the eortex, known as the 'pyriform lobe' or 'hippoeampal lobule, and whieh is anatomically continuous with the front end of the hippoeampal gyrus (the part to which the name uneinate gyrus is often restricted), acquires relatively large dimensions. This and the anatomical relations just mentioned would lead us to suppose that a part of the cortex which is eontinuous with the front end of the hippocampal gyrus is in some way eonneeted with smell. The argument from eomparative anatomy, however, is one which must be used with eaution; since, besides the great difficulty of determining the homologies 
of parts of the brain in different animals, relative increase in the part in question might be correlated to other things than the power of smell, and might be determined by circumstances having no relation to smell.

The experimental evidence, though on the whole it gives support to the view, is conflicting; and when the difficulty of determining whether a 'dumb animal' can or cannot smell is borne in mind, this will not be wondered at. The observation that electrical stimulation of the region in question gives rise to movements of the nostrils, which have been interpreted as snifling in response to subjective olfactory sensations, cannot have much weight; and while some observers have found that the removal of this part of the brain destroys the sense of smell, others have obtained negative results.

The few clinical histories which bear upon the matter are perhaps more trustworthy. These seem to shew that a lesion involving the cortex of this region, but leaving the olfactory bulb and tract, as well as other parts of the brain, intact, may destroy or greatly impair smell. And we may perhaps give particular weight to the cases in which epileptiform attacks, preceded by an 'aura' in the form of a peculiar' smell, have been associated with disease limited to this region; for the phenomena of 'aura' seem to be connected with cortical processes.

Though the evidence on the whole goes to shew that the cortex at the front end of the hippocampal gyrus is especially connected with smell, and we have so marked it in Fig. 137, yet the whole matter stands on a somewhat different footing from the sense of sight. In man the relations of smell to the other operations of the brain (though, as we shall see in dealing with the senses, somewhat peculiar) are far more limited than are those of vision, and the psychical development of simple olfactory sensations is extremely scanty.

\section{Sensations of Taste.}

$\S 502$. This special sense though so closely associated with smell stands, together with the special sense of hearing, on a different footing from the two preceding special senses, since the nerves concerned belong to the category of ordinary cranial nerves, and we lack, in reference to them, the anatomical leading which is offered to us in the case of the optic and olfactory nerves.

We shall see in dealing with the senses that the fifth nerve and the glossopharyngeal nerve have been considered as nerves of taste, but that the matter is one subject to controversy; the gustatory function of the fifth is attributed to the peculiar chorda tympani nerve, and other questions have been raised. Whatever view we take, however, the nerves of taste are ordi- 
nary eranial nerves, and we have no anatomical guidanee as to the fibres of either of the above two nerves making special eonnections with any part of the cortex. Though sensations of taste enter largely into the life of animals, and indeed of man himself, we have no satisfactory indications which will enable us to eonnect this special sense with any part of the cortex; the view indeed has been put forward that some part of the cortex in the lower portion of the temporal lobe, not far from the centre for smell, serves as a eentre for taste; but the arguments in favour of this view are not, as yet at least, eonvineing.

\section{Sensations of Mearing.}

$\S 503$. The cochlear division of the eighth or auditory nerve may be assumed to be a nerve of the special sense of hearing, and of that alone; the vestibular division serves, as we have seen, for other functions than those of hearing, $\$ 478$, but as we shall urge in dealing with the senses is not to be regarded as wholly useless for the purposes of that sense. The cochlear division may be traeed into the bulb, and the vestibular division into the lateral auditory nucleus (which perhaps may be regarded as a continuation or segmental repetition forwards of the euneate nucleus or of part of that nucleus), and into the cerebellum, the cerebellar continuation being probably the part of the nerve which serves for coordinating functions. The connections of the auditory nerve with the cerebral hemisphere belong to the same category as those of other afferent cranial, and we may add spinal nerves; we have no very elear anatomieal guide towards any particular part of the cortex.

When we turn to the empirical results furnished by experiment and clinical observations, we find that these, though cven less definite and less accordant than in the case of the senses of sight and smell, point to part of the first or superior temporal (temporo-sphenoidal) convolution (Figs. 123, 134, 136) lying in the temporal lobe just ventral to the Sylvian fissure, as being specially eoncerned in hearing in some sueh way as the oecipital lobe is concerned in vision.

Electrical stimulation of this region of the cortex gives rise to 'pricking of the ear's,' and other movements such as are frequently connected with auditory sensations; but such phenomena are in this instance perhaps to be depended upon even less than in other similar instances. While some observers maintain that this convolution, the operation including other portions of the temporal lobe as well, may be removed from a monkey without producing any certain signs of deafness, other observers have fonnd that removal of it on one side affected the hearing of the ear on the opposite side, and removal on both 
sides brought the animal into a condition in which, without being perhaps absolutely deaf, it reacted towards sound in a very imperfect manner indeed, very different from its normal beliaviour. The scanty clinical histories bearing on this matter are not very decisive; for though deafness has been observed in connection with disease affecting the superior temporal convolution, the lesion has usually invaded other parts as well, and the deafness has been associated with other symptoms, notably aphasia. An auditory 'aura' has however at times been observed in connection with disease of this region, as also a peculiar psychical failure, known as 'word deafness,' in which, though sounds are heard, that is to say auditory sensations are felt, it may be even as usual, the perception or psychical appreciation of the sounds is lacking, and a spoken word is not recognized.

Lastly, we may add that, though as we said the anatomical learling is not definite, observers have found that, in new-born animals, on the one hand destruction of the part of the cortex probably corresponding to the region mentioned above, leads to atrophy of the median corpus geniculatum, and, to some extent, of the posterior corpus quadrigeminum ; and on the other hand destruction of the internal ear leads to an atrophy of part of the lateral fillet of the opposite crossed side which may be traced to the posterior corpus quadrigeminum, and thence to the median corpus geniculatum; and section of the lateral fillet on one side leads, among other results, to atrophy of the striae acusticae and tuberculum acusticum of the crossed side. This suggests that the path of auditory impulses is along the cochlear nerve to the lateral fillet of the crossed side, and so by the posterior corpus quadrigeminum and median corpus geniculatum to the cortex of the temporal lobe of that crossed side, the two latter bodies bearing towards hearing a relation somewhat like that borne towards sight by the anterior corpus quadrigeminum and lateral corpus geniculatum. But the matter needs farther investigation.

There remains the spccial sense of tonch, but this we had better consider in connection with sensations in general. 
SEC. 5. ON THE DEVELOPMENT OF CUTANEOUS AND SOME OTHER SENSATIONS.

$\$ 504$. The sensations with which we have just dealt arise through impulses passing along special nerves or parts of special nerves, the optic nerve, the olfactory nerve, \&c.; we have now to deal with sensations arising through impulses along the nerves of the body generally. These are of several kinds. In the first place there are sensations which we may speak of as 'cutaneous sensations,' the impulses giving rise to which are started in the skin covering the body, or in the so-called mucous membrane lining certain passages. These sensations, which as we shall see in dealing with the senses are dependent on the existence of special terminal organs in or near the skin, are sensations of 'touch,' in the narrower meaning of that word, by which we appreciate contact with and pressure on the skin, and the sensations of 'temperature' which again we may, as we shall see, divide into sensations of 'heat' and sensations of 'cold.' These sensations may be excited in varying degree by impulses passing along any nerve branches of which are supplied to the skin. Then there are the sensations constituting the 'muscular sense,' to which we have already referred, and these again may be excited in any nerve having connections with the skeletal muscles.

As we shall see in dealing with the senses, when a nerve is laid bare and its fibres are stimulated directly either by pressure, such as pinching, or by heat, or by cold, or in other ways, the sensations which are caused do not enable us to appreciate whether the stimulation is one of contact or pressure, or of temperature, or of some other kind ; we only experience a 'feeling,' which at all events when it reaches a certain intensity we speak of as 'pain.' And we have reason to think that at least from time to time impulses along various nerves give rise to sensations which have been spoken of as those of 'general scnsibility,' by which in addition to other sensations, such as those of touch and of the muscular sense, we become aware of changes in the condition and circumstances of our body. When the stimula- 
tion of the skin exceeds a certain limit of intensity, the sense of 'such or temperature is lost in, that is to say, is not appreciated as separate from the sense of pain; and under abnormal circlinstances acute sensations of pain are started by changes in parts, for example tendons, the condition of which under normal circumstances we are not conscious of appreciating through any distinct sensations, though it may be that these parts do normally give rise to feeble impulses contributing to 'general sensibility.' It may be debated whether 'pain' is a phase of all sensations, or of general sensibility alone, or a sensation sui generis. We shall have something further to say on this matter when we treat of the senses; meanwhile it will be convenient for present purposes if we consider that the sensations we have to deal with just now are the sensations of touch and of temperature, those of the muscular sense, and those of general sensibility including those of pain.

\$ 505. 'The fairly convincing evidence that the occipital cortex has special relations with vision, and the less clear evidence that other regions have special relations with smell and hearing, suggest that special parts of the cortex have special relations with the sensations now under consideration. But in the cases of the senses of sight and smell we had a distinct anatomical leading; and we have seen how nncertain is the evidence where such an anatomical leading fails or is deficient, as in hearing and taste. In the case of sensations of the body at large, the anatomical leading similarly fails us. Moreover, if our judgment concerning the visual sensations of animals operated on is difficult, how much more difficult must be our judgment concerning their sensations of touch and temperature, and even of pain?

As we liave already urged (\$\$ 488, 489) observations made on man himself whether in the cases where it has been possible to stimulate the cortex by an electric current, or in respect to the phenomena of disease, such as the amra of epileptic attacks and the like, shew most distinctly that the so-called motor region of the cortex is closely associated with sensations, and that particular areas of this region are especially associated with sensations originating in particular parts of the body. We have also seen that experiments on monkeys confirm this view; the removal of a motor area, that for instance of the hand, entails not only loss of movement in the hand, but also loss or impairment of sensation in the hand, lasting as long at least as the loss or impairment of the movements; noreover, so far as can be learnt, all sensations are affected by the removal of the cortex, those of pressure and temperature as well as those of the muscular sense and of pain. Similar results have also been obtained in the dog. So that the evidence seems convincing that the parietal region of the cortex, while it has special connections with volun- 
tary movements, has at the same time special connections with cutaneous and other sensations, and in both relations may be mapped out into areas corresponding to parts of the body.

But this matter of the sensations is one of even greater complexity than that of voluntary movements. This is shewn among other things by the fact that the sensations with which we are now dealing may be profoundly affected by operations on parts of the cortex other than the above region. In the dog, for instance, according to many observers, removal of almost any part of the cortex impairs cutaneous sensations, the amount and duration of the effect being broadly proportionate to the extent of cortex removed, though operations on the frontal region have the least effect. Other observers again have found that in the monkey removal or destruction of the gyrus fornicatus (Figs. 122, $12 t)$ on the mesial surface of the brain, ventral to the callosomarginal sulcus which forms on the mesial surface the ventral limit of the motor region (an operation of very great difficulty), has brought the whole of the opposite side of the body to a condition which has been described as an anesthesia, that is a loss of all cutaneous tactile sensations, and an analgesia, that is a loss of sensations of pain, the condition being accompanied by little or no impairment of voluntary movements and, though apparently diminishing as time went on, lasting until the death of the animal some weeks afterwards. Again, removal of the continuation of the gyrus fornicatus into the gyrus hippocampi has in other instances led to a more transient anesthesia also of the whole or greater part of one side of the body. And it is asserted that removal of no other region of the cortex interferes with cutaneous and painful sensations in so striking and lasting a manner as does the removal of parts, or of the whole of this mesial region. These contradictory results shew how complex and difficult the subject is.

$\$ 506$. We may now attack the problem in a different way, and instead of beginning with the cortex begin with afferent impulses started along afferent nerves from their peripheral endings, and attempt to trace them centralwards. And first we may call to mind what anatomical guidance we possess.

We have seen ( $\$ 452)$ that the fibres of posterior roots, the channels of afferent impulses, end in the spinal cord in at least two main ways. One set are continued on, not broken by any relays, in the median posterior tract, and by this tract representatives of all the spinal nerves are connected with the gracile nucleus in which the median posterior column cnds. The other fibres of a posterior root end in the grey matter of the cord not far from their cntrance; we have reason to think that they are brought into contact by arborescent endings collateral or terminal, with the bodics or processes of certain cells in the grey matter. Putting aside as foreign to our present subject the 
probable endings in contact with motor cells which seem to afford the mechanism for many reflex actions, we may distinguish at least two other kinds of ending. We have reason to think that some of the fibres make connections by contact with the cells of the vesicular cylinder, Clarke's column. We have further reason to think that axis-cylinder processes of these cells of the vesicular cylinder, go to form the cerebellar tract, which we may assume to be an afferent tract, and which may be traced through the restiform body to the cerebellum in connection with certain cells of which, especially those of the nucleus dentatus, the fibres of the tract end. Now though the cerebellum is connected, in an indirect way it is true, with the cerebral cortex we have no grounds for thinking that the cerebellum is concerned with the development of the sersations with which we are now concerned: when the whole cerebellum is removed there is no apparent affection of cutaneous sensations. We may therefore dismiss the cerebellar tract; the afferent impulses passing along it have probably to do with the coordination of movements affecting equilibrium, but they are not the impulses which becone developed into conscious sensations of touch or of pain.

The other fibres of the posterior root of which we are speaking end in connection with other cells of the grey matter. From these cells we have reason to think fibres originate and pass upward in the white matter. Some of these we may distinguish as the 'ascending antero-lateral' tract; but our knowledge here becomes less definite. Some of the processes of the cells, with which the posterior root fibres make connection, end as they begin within the grey matter of the cord itself, in connection with other cells of the grey matter; and even those which travel beyond the cord appear to go no farther than the bulb, ending in connection with cells in that structure, more particularly with the cells of the gracile and cuneate nuclei. So far as our present knowledge goes there is no definite tract from various parts of the cord to the brain comparable to the pyramidal tract from the brain to various parts of the cord. The passage of afferent impulses on their way to become conscious sensations is from the first a system of relays. 'There is a first relay where the ending of the root fibre makes connections with a nerve cell in the adjoining grey matter, or in the gracile nucleus. There is in the former case a further relay in the bulb at least, and possibly in some instances other relays in the grey matter of the cord on the way. The path to the bulb thus supplied by the fibres and their relays, keeps to a large extent on the same side of the cord, though some cross. ing is observed.

From the bulb onwards one definite path only, and that a narrow one, is marked out anatomically by the median fillet 
(we may as urged above neglect the ties between the bulb and the cerebellum). This, starting from the gracile and cuneate nuclei and crossing at the interolivary layer, passes on through the mid-brain to the optic thalamus with the cells of which its fibres seem to make connections, though according to some observers part of the fillet is continued, without relays, right to the cerebral cortex. But if, as is stated, the destruction of the two nuclei followed by complete degeneration of the fillet entails no obvious loss of sensation, the afferent impulses passing along it must be of a peculiar kind. Other fibres of the anterior lateral columns, some at least of which, probably most, if not all, we may regard as afferent in nature, pass into the reticular formation of the bulb, and thence onward, probably more or less by relays into the tegmentum.

$\$ 507$. How do experimental results and clinical histories accord with such an anatomical programme?

We may first call attention to a somewhat old experiment. We have seen, $\$ 153$, that afferent impulses started in afferent fibres, in those for instance of the sciatic nerve, so affect the vaso-motor centre in the bulb as to cause a rise of blood-pressure, at least in an animal under urari. Those afferent impulses must pass by some path or other from the roots which supply the sciatic nerves with afferent fibres along the thoracic and cervical cord to the bulb. If the path be blocked, the stimulation of the sciatic nerve will fail to produce the usual rise of blood-pressure. Now in a rabbit, the amount of rise of bloodpressure following upon the stimulation of one sciatic nerve with a certain strength of current having been ascertained, it is found that a much less rise of blood-pressure or none at all follows the same stimulation after division of certain parts of the cord in the mid or upper thoracic region; that is to say, the section of the cord has partially or completely blocked the path of the afferent impulses. Further, the block is conspicuous when the lateral colnmn is divided, and is not increased by other parts of the cord being divided at the same time: when both lateral columns are divided the block is almost complete. And further, supposing one sciatic, say the right, is the one which is stimulated, a block occurs both when the lateral column of the same, right, side and when that of the crossed, left, side is divided, but is greater when the division is on the crossed than when it is on the same side. And similar results were obtained when, the cxperment being conducted in a similar way, signs of pain insteal of variations in blood-pressure were taken as the tokens of the blocking of impulses. "We may infer that the impulses, which reach the lumbar eord by the roots of the sciatic nerve, travel up, the cord, or rather give rise within the lumbar cord to nervous impulses, which travel up the cord in such a manner that in the lower thoracic region they pass almost exclusively 
along the fibres of the lateral column, some having kept to the same side of the cord, but more having crossed over to the opposite side, before reaching the thoracic region. Though these vaso-motor experiments have a certain value, inasmuch as the results gained by them are more or less distinctly quantitative and measurable, many objections may be urged against their validity as affording a general proof of the course taken in the cord by impulses giving rise to sensations. 'They were conducted on rabbits, animals low in scale and especially so perhaps in respect to the spinal cord, they were limited to one region of the cord, the observations were made immediately after the division of the cord, before the immediate effects of the operation had passed off; and further, it may be urged that impulses affecting the vaso-motor centre may not be identical with those giving rise to sensations.

Many experiments have been made on dogs; and if we content ourselves with making no distinction between the different kinds of afferent impulses, and in the case of these animals it would hardly be profitable to attempt to make a distinction, we may say that the several experiments so far agree that they point to the lateral columns as being the chief paths of afferent, sensory, impulses, or to speak more exactly, to the passage of these impulses being especially blocked by section of the lateral columns. Some observers find that in the dog a section of the lateral column on one side, or' at least a hemisection of the cord, produces 'loss of sensation' on the opposite side greater than on the same side, or confined to the opposite side, and even accompanied by an exaltation of sensation, a hyperesthesia, on the same side. Other observers again, and these certainly competent observers, find that, in the dng, section of one side affects sensation on both sides, and indeed chiefly on the same side. IVe may perhaps once more repeat the warning how difficult is the quantitative and qualitative determination of sensations in such an animal as the dog; and may remark that in all these cases of unilateral section the increased blond supply due to failure of the normal vaso-constrictor tone must influence the peripheral development of sensory impulses.

In these experiments, as in those on voluntary movements, it is most important to distinguish between immediate or temporary and more lasting effects; and observers have found that the loss of sensation following a hemisection of the cord, like the loss of voluntary movement, is temporary only, and eventually disappears, though the recovery is slower and less complete than is the case with movements. As with voluntary movement (\$491) so with sensation, recovery, though less complete than that of movement, is possible when a hemisection on one side has been at a later date followed by a hemisection on the other side.

The experiments on monkeys are in like manner neither 
accordant nor decisive; and even in these animals with their more raried signs of sensations, the interpretation of these signs is besetwith fallacies. Some observer's have found that a hemisection (in the thoracic region) produced loss of sensation on the crossed side, accompanied by little or no loss on the same side; other observers again have failed to obtain after a hemisection satisfactory proof of any such marked loss on the crossed side; they find on the contrary that impulses giving rise to sensations of touch and of temperature, as well as those concerned in the muscular sense pass up the same side, while those giving rise to pain seem to pass up both sides, that is to say probably travel along the grey matter. Further, large portions of the lateral column, the more internal parts adjacent to the grey matter being left, have been removed without any very obvious and certainly without any lasting defects of sensation on the one side or on the other.

$\$ 508$. Turning now to man we find that clinical experience shews that in him the integrity of the cerebral hemispheres, and of the connection of the hemispheres with the rest of the central nervous system, is essential to the full development of sensations; and that in this respect each hemisphere is related to the crossed side of the body. A very common form of paralysis or 'stroke' is that due to a lesion of some part of one hemisphere (the exact position of the lesion need not concern us now), frequently caused by rupture of a blood vessel, in which the patient loses all power of voluntary movement and all sensations on the crossed side of his body (including the face); he is said to be suffering from hemiplegia, 'one sided stroke.' Not only do voluntary impulses fail to reach the muscles of the affected side, but sensory impulses, such as those which, started for instance in the skin, would under normal conditions lead to sensations of touch, of heat or cold, or of pain, fail to effect consciousness, when they originate on the affected side; the patient cannot on that side feel a rough surface, or a hot body, or the prick of a pin. The same is true when the loss of sensation is not complete, but partial.

Further, though perhaps anatomical considerations would lead us to expect that a great deal of the crossing took place in the spinal bulb, clinical histories moreover agree, at least to large extent, in shewing that much takes place in the cord, so that when the lesion is confined to one half of the cord, the sensations affected in the parts below the level of the lesion are chiefly or even exclusively those of the crossed side. But there is not entire accordance among observers, especially as to the crossing being complete. And with regard to the muscular sense there is a distinct conflict of opinion; the majority of cases seem to shew that in unilateral disease or in jury to the cord, the muscular sense in company with the voluntary movements, fails on the 
same side; but cases have been recorded in which the muscular sense in company with other sensations, seemed to be affected on the crossed side; it must be remembered however that it is very difficult to appreciate a deficiency of muscular sense mingled with deficiencies in other sensations, and we should i priori expect the muscular sense to run parallel with motor impulses.

The clinical histories of diseases of the spinal cord in man bring to light in a fairly clear manner a fact of some importance, namely, that the several impulses which form the bases of the several kinds of sensations, of touch, heat, cold, and pain, and of the muscular sense, are transmitted along the cord in different ways and presumably by different structures. For disease may impair one of these sensations and leave the others intact. 'Thus cases of spinal disease are recorded, in which on one side of the body or in one limb ordinary tactile sensations seemed to be little impaired, and yet sensations of pain were absent; when a needle was thrust into the skin no pain was felt, though the patient was aware that the needle had been pressed upon the skin at a particular spot; and conversely in other cases pain has been felt upon the insertion of a needle, though mere contact with or pressure on the skin could not be appreciated. Again, cases are recorded in which the skin was sensitive to touch or pain, but not to variations of temperature; it is further stated that cases have been met with in which cold could be appreciated but not heat, and vice versa; and there are some facts which point to sensations of pain being more closely associated with those of heat, and tactile sensations with those of cold, than those of pain with those of touch or those of heat with those of cold. Cases of spinal disease are also recorded in which the muscular sense appeared to be affected apart from other sensations. IVe shall return to these matters later on in dealing with the senses; we refer to them now simply as shewing that disease, limited as far as can be ascertained to the spinal cord, may affect the several sensations separately, and therefore as suggesting that the several kinds of impulses, forming the bases of the several kinds of sensation, are transmitted in different ways and follow different 'paths' along the spinal cord.

When however we appeal to clinical histories or indications as to the several paths within the spinal cord taken by these several impulses, the answer is a most uncertain one, as indeed might be expected from the too often diffuse character of the lesions of disease; and it is perhaps not too much to say that no satisfactory deductions at all can be made.

$\S 509$. Whether then we turn to experiments on animals or to the study of disease, the teachings with regard to sellsation, in contrast to those with regard to voluntary movement, are in the highest degree uncertain and obscure. A few general reflec- 
tions will perhaps help us to appreciate the value of such facts as we possess.

We have seen reason to think that in every movement whether voluntary and of cortical origin, or involuntary and started either as a simple spinal reflex or through the working of some part or other of the brain, the motor impulses, which swcep down the motor fibres to the muscles, issue marshalled and coordinated from the grey matter of the cord (for the sake of clearness we may omit the cranial nerves), from what we have called the motor mechanisms of the cord. Analogy would lead us to suppose that the afferent impulses, forming the bases of the several kinds of sensations, similarly left the afferent fibres to join the grey matter of the cord in what we may call the sensory mechanism. And such anatomical leading as we possess seems to support this view; with the exception of the median posterior tract, to which we will return immediately, all the fibres of a posterior root seem to end in the grey matter not very far from the entrance of the root. We have seen that a coordinate reflex movement may be carried out by at least a few segments of the cord; that a reflex movement may be started by stimuli of various kinds and therefore presumably by afferent impulses of various kinds; and that impulses forming the basis of the muscular sense are essential to the coordination of the movement. All our knowledge goes to shew that in a reflex movement carried out by a few segments of the cord, the whole chain of events between the arrival of the afferent impulses along the posterior root and the issue of efferent impulses along the anterior root may be carried out by grey matter, and grey matter alone. WV may further infer that, while on the one hand the same procedure might obtain not through a few segments only but along the whole length of the cord, there would be an advantage, especially in respect to the rapidity of transmission, in employing internuncial tracts of fibres between the several segments, the advantage being greater the more distant the segments which have to work together.

We might further suppose that it would be of advantage to possess some direct path between the cerebral cortex and the spinal sensory mechanism immediately connected with the posterior root, such as is afforded by the pyramidal tract between the cortex and the spinal motor mechanism immediately connected with the anterior root. But no anatomical evidence of such a tract is forthcoming; and, as we have before remarked, along all the tracts which seem to be sensory in nature, in contrast to what takes place in the motor tracts, relays of grey matter are continually being interpolated.

The median posterior tract, since it gathers up representatives of successive nerves, presents itself as the nearest approach to such a sensory homologue of the pyramidal tract, though it 
ends in the bulb, and is not continued on directly to the cortex. And possibly it does play a somewhat analogous part, in so far as it serves as a special comnection between the brain and the whole series of spinal nerves. But we are wholly ignorant as to what it really does; and whatever be the exact nature of the part which it plays, there is no adequate evidence either from clinical histories or from experiment that it has relations to one kind of sensation only. It has indeed been supposed by some to be especially a tract for the impulses of the muscular sense; but neither experiment nor clinical study affords adequate proof of this view. The condition known as locomotor ataxy, the salient feature of which is loss or impairment of muscular sense, is associated with disease of the posterior root and of its entrance into the cord, not with disease confined exclusively to the median posterior column. Moreover the tract cannot carry all the impulses of muscular sense, since some of them must pass at once into the grey matter, to take part in the coordination of reflex movements, and must therefore travel by fibres which do not form this tract. Similarly is there no adcquate proof of the tract being an exclusive channel for tactile or for painful sensations. Possibly it lias some special relations to all the different kinds of sensation.

We may also perhaps urge similar considerations with regard to the cerebellar tract, which though starting from a relay of grey matter is thence onward to the cerebellum a continuous tract. This tract also has been supposed to carry impulses of a special kind, and more particularly those of muscular sense. But even admitting that the tract does convey impulses derived from the muscles and their appendages, which impulses the cerebellum makes use of in its work of coordinating novements, especially those concerned in equilibrium, it cannot be the channel for the ordinary impulses of muscular sense, since these remain after total removal of the cerebellum. Nor does either experiment or clinical study afford in other ways any clear proof that this tract is solely or even especially concerned with the muscular sense.

With regard to the antero-lateral or other ascending tracts our knowledge is too imperfect to justify us in supposing that any one is the special or exclusive channel for any one kind of sensation, or indeed in drawing any conclusions at all concerning it.

But when we subtract from the white matter of the cord these continuous tracts of ascending degeneration of presumably sensory or afferent function, and the continuous tracts of descending degeneration, which we may confidently speak of as motor or at least efferent, there are left only the fibres which we may suppose to be longitudinal commissural or internuncial fibres between successive segments. We are thus driven to the pro- 
visional conclusion, that sensory impulses pass either by the grey matter alone, or by a series of steps as it were, by relays of grey matter connected by internuncial tracts of fibres, whose length we do not know, but which may be short. That such internuncial tracts intervene is rendered probable, on the one hand by the fact that section of the white matter, leaving the grey untouched, does affect sensations, and on the other hand by the fact that the several kinds of sensation appear to travel along the cord by separate paths, or at least may be separately blocked. But, if we accept this view, we must at the same time admit that, in animals at least, the lines provided by the internuncial tracts and their relays are not rigid, that within limits and under circumstances alternative routes are possible.

$\$ 510$. We may here perhaps raise once more, and this time more pointedly than before, the doubt whether we are justified in assuming, as we generally lo assume, that the events which take place in the fibres connecting relays of grey matter within the central nervous system, are exactly the same as those which take place in the fibres of nerves ontside the central system, during the passage of what we call a nervous impulse. Mlost of our knowledge of a nervous impulse has been gained by the study of the motor nerve of a muscle-nerve preparation. 'Our knowledge of the processes in afferent nerves is much more imperfect. And, with regard to the proeesses taking place in fibres within the central nervous system we have hardly any exact experimental knowledge at all. It has been maintained by many observers that not only the grey matter but also the tracts of white matter in the spinal cord, while they are capable of conveying impulses in one direction or the other, arc incapable of being so excited by artificial stimuli as to generate new impulses. These observers maintain that, when movements or signs of sensation follow the direct stimulation of various parts of the cord, the effects are due to issning motor fibres or entering sensory fibres having been stimulated, and not to a stimulation of the intrinsic substance of the parts themselves; they propose accordingly to call these parts 'kinesodic' and 'asthesodic' respectively, that is to say, scrving as paths for motor or sensory impulses without being themselves either motor or sensory. The evidence on the whole goes to shew that this view is a mistaken one, that the varions tracts of the spinal cord, like the pyramidal tract and indeed other parts of the brain, are excitable towards artificial stimuli. The question cannot, however, be considered as definitely closed; and the very fact that it has been raised illustrates the point on which we are now dwelling. We may further quote, in similar illustration of the same point, the following remarkable fact which was observed in the series of expcriments referred to in $\$ 491$ on the effects of repeated hemisection of the spinal cord in dogs. The animal had partially recovered voluntary movements in his hind limbs after 
a third hemisection of the thoracic cord, and yet when, at his death, a strong tetanizing current was directed through the bulb and cervical cord, no movements of the hind limbs followed: the impulses started by artificial stimulation could not pass the bridge which sufficed for volitional impulses of natural origin. It is not too much to say that our experimental knowledge as to the events which accompany the activity of the structures within the central nervous system is almost entirely limited to the rccognition of the 'currents of action' refer'red to in $\$ 486$. We are already going beyond our tether when we assume on the strength of this that the processes started in the fibres of the pyramidal tract by artificial stimulation are in all respects identical with those started in the fibres of a motor nerve. We are going still more beyond our tether when we assume that the processes started in the same pyramidal fibres as the outcome of natural events in the motor cortex are of the same kind. But these assumptions are trifles compared with the assmmption that the events taking place in the fibres of the optic radiation, passing from the pulvinar to the occipital cortex are identical with the events taking place in the fibres of the optic tract on the way to the pulvinar, or that the events travelling along the spinal cord to the brain as the result of a prick of the little finger are ilentical with those which the prick has started in the fibres of the ulnar nerve. Of the latter events we know a little; of the former events we know next to nothing. And we may here ask the question what is the meaning of these continual relays of grey matter along the sensory tract unless it be that at each relay, some transformation, some further elaboration of the impulses takes place, until what were the relatively, but only relatively, simple impulses along the fibres of the peripheral nerve are by successive steps changed in the complex events which we call a conscious sensation? We have no reason to think that the afferent impulses started at the periphery of a cutaneous nerve-fibre change essentially in character as they travel over the fibre along the stretch of nerve of which it is a part. Nor have we any satisfactory evidence that any change in the character of the impulses is effected by the nerve cell in the ront-ganglion with which the fibre is connected by means of a T piece, though this is possible. Within the cord things are different. The arborescent cnding of the fibre of the posterior root within the spinal cord (or bulb) is in contact with, not in continuity with, the cell-substance of the cell, or the processes of the cell on which it impinges. Of course it is possible that the ending shonld set up in that cell-substance molecular changes identical with those which constitute the impulse passing along the fibre; but it is much more probable that the changes which it sets up are of a different order, the transition being in a rough way comparable to the transition from a nervous impulse reach- 
ing a motor end plate to the contraction in the muscular fibre which that impulse brings about. The new changes started in the first relay cell may be vely different from those coursing along the posterior root-fible; and these again in a similar way may start still other changes in the next relay cell; and so on. IV e may therefore well hesitate to speak of or consider all the events in the central nervous system as either motor or sensory in nature. It is perhaps not an exaggeration to represent the views of some observers as if they supposed that afferent impulses, say tactile impulses, that is impulses eventually giving rise to tactile sensations, travelled unchanged from the skin to the cortex and there suddenly blossomed into sensations. If such a view were true, undoubtedly the chief task of physiology, almost the only one, would be to ascertain the tract along which these impulses passed. But if on the other hand the views just now urged have any real foundation, the question of tracts or paths sinks into insignificance compared with the almost untouched problems as to what are the several successive changes by which simple impulses are developed into full sensations, and when and low the changes are effected.

$\$$ 511. Seeing how unsatisfactory is our present knowledge with regard to the tracts or paths of sensations in the relativcly simple spinal cord, it would be useless to attempt any discussion as to their paths in the much more complex brain. If it be probable that the passage is effected by relays of grey matter in the former, the same method is much more plobable in the latter; and if neither experiment nor clinical study throws much light on the path up to the bulb, these camnot be expected to give much help in the maze of grey matter and fibres by which the bulb is joined to the cortex. The several defined areas or collections of grey matter, and the several strands and tracts of fibres must have of course a meaning; but it may be doubted whether we have everl so much as a correct glimpse of that meaning in any case, if we except those which are in immediate connection with the cranial nerves and their nuclei. Secing that the thalamus appears on the one hand to be connected with all or ncarly all parts of the cortex, and on the other hand to serve as the front of the tegmental system, it is tempting to suppose that it plays an important part in sensations pertaining to the body generally, as part of it, the pulvinar, certainly does with reference to the special sense of sight; but we lave no decisive indications as to wliat part it plays. And the part which it plays, whatever that may be, is not an exclusiveiy sensory one, since both experimental and morbid lesions of the thalamus are apt to produce disorder's of movement as well as other cfferent effects. We ought perhaps to say the parts which it plays; for it is a complex body, having many ties and probably performing many dutics. 
We have already spoken of the fillet, as seeming to be a special internuncial tract connecting what appear to be more particularly afferent or sensory parts of the bulb, such as the gracile and cuneate nuclei, with such parts of the middle brain as the optic thalamus and thus indirectly with the cortex, and of its value as a probable path of sensations of one kind or mother from the body at large.

A conspicuous part of the brain, namely the cerebellum, naturally arrests our attention on account of its large connections with what appear to be afferent structures; what may be said concerning this will be said in the next section. 


\section{SEC. 6. SOME O'THER ASPECTS OF THE FUNC- TIUNS OF THE BRAIN.}

\$512. It is difficult to say anything definite concerning the transmission of sensory impulses and the development of sensations; it is still more difficult to say anything definite, beyond what has been already incidentally said, concerning the parts played in the work of the brain by the various aggregations of grey matter and tracts of fibres forming the middle part of the brain. Neither experiment nor clinical study has as yet afforded any clear or sure leading.

Let us first speak of the cerebellum.

The commections of this body with the rest of the nervous system are strikingly manifold. By the inferior peduncle, the fibres of which end largely in the nucleus dentatus, it has an uncrossed grip on afferent structures of the spinal cord and bulb; by the cerebellar tract it is connected through the vesicular cylinder with the posterior roots of spinal nerves of the same side; it has a like connection with the cuneate and gracile nuclei of the same side, and fibres from the eighth (vestibular) nerve, as well probably as from other afferent cranial nerves of the same side, pass into the pedunele. The same inferior peduncle has a crossed connection with the lower olive of the other sile, but this is probably efferent in nature, and there are probably other efferent conncctions. By its middle peduncle, the fibres of which are especially connected with the superficial grey matter, the cerebellum has large connections with the opposite side of the pons and, through the relay of the cells forming the grey matter of the pons, with fibres passing from the frontal and temporo-oceipital regions (possibly from scattered elements of the parietal region) of the cerebral cortex to the pons. Thus each lateral half of the cerebellum has wide crossed connections with the opposite half of the cerebrum. Whether these connections are afferent, that is leading from the cerebellum to the cerebral cortex, or vice versa, or have both characters, it is difficult to say. Besides this the supcrior peduncle, 
starting largely from the nucleus dentatus, affords another connection with the cerebrum, largely crossed, though possibly partly uncrossed; but this is an indirect connection so far as the cerebral cortex is concerned, since the peduncle ends in the red nucleus and other tegmental structures. 'This connection is presumably the channel of impulses passing from the cerebellum to the cerebrum; but we can only say 'presumably.'

'The above connections are too complex to enable one to draw from them any precise physiological deductions; and when we turn to the results of experiment and clinical observation, we find even these by no means clear.

Electrical stimulation of the surface of the cerebellum, in the monkey and in other animals, has led to movements of the eyes, and of other parts of the head; but we cannot from such results draw any satisfactory inferences.

The results of removing either portion of or the whole of the cerebellum have been partly accordant, partly discordant. They agree in shewing that the organ has no special connections with the sexual functions, a view once largely held. 'They also appear to agree in so far that the effects produced by removing a lateral half are largely if not wholly confined to the same side of the body; the influence exerted by one half of the cerebellum tells on one half of the body, and that on the same side. 'They further agree in that removal of even the whole of the cerebellum has no obvious psychical effects, and does not appear to interfere with the full development of cutaneous and other sensations. Lastly, while they agree in indicating that the cerebellum plays a special part in the coordination of movements affecting equilibrium, and this is also shewn by clinical histories, they are not agreed as to how that part is exactly played.

The effects produced by removal of the cerebellum are in part immediate and temporary, in part of a more lasting character. Characteristic among the former are some of the 'forced movements' treated of in $\$ 480$, such, for instance, as rotation round the longitudinal axis of the body; this seems especially to occur after division of the middle peduncle. And the movements of the body in general are for a while in a remarkable degree incoordinate and irregular, so that locomotion and even the maintenance of a natural attitude are for the time inpossible. Tonic spasms of various muscles are also observed, those of the trunk leading to curvature of the body. Later on this condition subsides, but there remains as a lasting effect an imperfection of movement, an unsteadiness of gait, an irregularity and shortcoming in muscular actions, accompanied frequently by muscular tremors. This unsteadiness in movement is also seen in many cases of cerebellar disease, the gait of the patient in many respects resembling that of a person who is drunk. Obviously the cerebellum has some important influence over the contrac- 
tions of the skcletal muscles (the visceral muscles do not seem to be affected); but observers are not agreed as to what that influcnee exactly is or exactly low it is brought about. The view has been jut forward that influences from the cerebellum reinforce so to speak the voluntary impulses proceeding to the muscles, regulate the fusion of the several constituent simple contractions into the sustained muscular effort, which as we have secn is of the nature of a tetanus, and further, so affect the untrition of the muscle, as to keep up that proper tone of the muscle ( $\$ 470)$ which is a necessary condition of successful muscular action. But according to some observers an animal which from removal of the cerebellum has become highly irregular in its general movements, can carry out a particular muscular act with complete success, can for instance grasp powerfully and firmly with the hand: in such an act the voluntary impulses must reach the muscle in full force, the muscular sense belonging to the muscles used must be in full play, and it may even be argued that no marked loss of tone in the particular muscles can be present. If this be so, obviously the unsteadiness of the movements in general must have some other explanation than the one given above.

'Though some have maintained that injury to the cerebellum must be unsymmetrical, must be on one side only or more on one side than the other, in order to produce its effects, this does not seem to be the case. Again while some have maintained that injury to the median region is especially effective, others deny this. Indeed so far as we can judge at present, there is no localization of function in the various regions of the cerebellar cortex; all the cortex may be said, as used to be said of the cerebral cortex, to 'act as a whole.' Iastly, in respect to the relations of the cerebellum to the cerebrum, it should be noted that removal of one half of the cerebellum has appearer to produce an effect on the crossed cerebral hemisphere, of such a kind that that hemisphere is more readily excitable towards electric and other stimuli, the effect lasting long after the operation of removal. In this connection it is worthy of notice, that congenital deficiency, or atrophy of the cerebral hemisphere of one side, is frequently accompanied by a corresponding deficiency of the crossed cerebellar hemisphere.

$\S 513$. Both the anterior and posterior corpora quadrigemina are complex in structure; not only do they differ from each other, but also in each the grey matter differs in different parts, both as to its nature and appearance and as to its connections with tracts of fibres. If we have little right to speak of the 'functions of the cerebellum,' we have even less right to speak of the 'functions of the corpora quadrigemina' or of either pair of them. 'The anterior pair, as we have seen, has to do in some way with vision; but we have reason to think that a part only of 
the whole body is thus concerned; and there is some foundation for the view that of this part, one portion belongs, so to speak, to the optic tract and another portion to the cortical fibres of the optic radiation. Possibly still another part is concerned in bringing, as we have ( $\$ 500)$ suggested, visual impulses to bear on the coordination of movements.

Stimulation of the surface of the posterior pair, besides giving rise to movements of various parts of the body, has in monkers and some other animals, the singular effect of producing a vocal utterance in the form of a cry or bark. But we cannot make much use of these results for the purpose of drawing conclusions as to what share these bodies take in the whole work of the brain. In the frog, the optic lobes correspond to the two pair of corpora quadrigemina together; and the cry just mentioned may perhaps be put side by side with the fact that in the frog the optic lobes seem to furnish a mechanism for croaking: when the optic lobes are destroyed, the characteristic reflex croaking is done away with. The probable connection of the posterior corpora quadrigemina with hearing is also interesting in this connection; but we have no satisfactory evidence of any special ties between the bodies in question and either the cortical area for phonation or the vocal mechanism in general; the occurrence of the cry remains so far an isolated fact.

In frogs, in which the cerebellum is very small, the optic lobes seem to be particularly concerned in the coordination of movements. When the brain is removed by means of a section behind the optic lobes the animal loses the power of balancing itself ( $\$ 474)$, which it possesses when the section passes in tront of the optic lobes; and injury to the optic lobes produces incoordination of movement and often 'forced movements.' It has been maintained that the loss of coordination is in these cases due to removal of or injury to the central grey matter in the walls of the third ventricle, and not to mere removal of or injury to the optic lobes: but the whole evidence goes to shew that in the frog and in the bird the optic lobes do play a part in the coordination of movement, though lesions of the central grey matter around the third ventricle, or indeed of the thalamus or other parts of the tegmentum, may give rise to loss of coordination or to 'forced movements.'

In the mammal removal of or injury to the posterior corpora quadrigemina does not cause blindness, but may, like a lesion of the anterior pair, give rise to loss of coordination or to forced movements; the effect, however, is in most instances very temporary. The comnection of the anterior pair with vision suggests a clue as to how this pair takes part in coordination; but as to how the posterior pair could intervene in the matter we have hardly so much as a hint: for, even if we admit a connection between them and the sense of hearing, and, 
remembering that a loud sound will often cause a person to reel, further admit that purely aurlitory inpulses, as distinct from what we have called ampullar impulses, may take part in the general coordination of bodily movements and in the maintenance of equilibrium, as they certainly do in the special coordination of laryngeal movements, still we are not much nearer an muderstanding of the matter. We may add that section of the lateral fillet, which appears as a conspicuous tie between the posterior corpora quadrigemina and the parts of the nerrous system behind them, does not appear to have any marked effect in producing incoordination.

In fine, beyond the broad facts on which we dwelt in a previous section. namely, that we maintain our equilibrium and carry out complex movements involving often several parts of our body, through what we call coordination, that afferent impulses supply important factors of this coordination, and that the cerebellum, through the vestibular nerves in part at all events, together with other portions of the middle brain, are in some way its chief instruments, we as yet know very little. We have certainly no adequate knowledge as to how either pair of corpora quadrigemina exactly intervene in the matter, or, indeed, as to what other parts they play in the general work of the brain.

With regard to other tracts of fibres or areas of grey matter we have nothing to say, except as regards those which are more or less immediately connected with certain of the cranial nerves, such for instance as the nerves for movements of the eyes, and these it will be best to consider when we have to deal with the nerves themiselves.

$\$ 514$. Besides the somatic functions which in previous discussions we have chiefly had in view, the brain as a whole undoubtedly carries out splanchnic functions; concerning these, however, we must be very brief.

Of the respiratory and vaso-motor functions of the bulb we have already treated in their appropriate places, and we have referred ( $\$ 429)$ to the experimental evidence that a lesion of the corpus striatum, or of the front part of the optic thalamus has a remarkable influence on the development of heat in the body. We have further seen that the higher parts of the brain, acting through the bulb, exercise powerful influences on respiration, on the vaso-motor system, and on the beat of the heart. Daily cxperience affords abundant instances of actions such as thesc, as well as of the influence of the brain on other organic functions. We can bring our will to bear on the mechanism of micturition ( $\$ 348$ ) which is almost wholly, and on the mechanism of defacation ( $\$ 225)$ which is largely, splanchnic in nature. These movements, however, are not skilled movements; and as we explained in dealing with them, 
the action of the brain as regards them seems limited to augmenting or inhibiting the activity of spinal centres. We should therefore hardly expect them to be specially represented in the cortical motor region. But emotions have a much wider and more powerful intluence over the splanchnie functions than has the will, and have the power of affecting the work of ccitain organs, for instance the heart and secreting glands, which the will is unable to touch. And since we have every reason to believe that the cortex is closely associated with the emotions, we may naturally infer that the elcments of the cortex supply a link in the chain through which an emotion influences this or that splanchnic activity; we may, accordingly, expect to find that stimulation of some part or other of the cortex produces splanchnic effects. The results of cxperimental investigation, however, are both scanty and discordant; but the greater weight should perhaps be attached to the positive results. Thus, some observers find that stimulation of the cortex, the locality being in the dog some part of the sigmoir gyrus. produces movements of the bladder; and they trace the path of this influence through the front part of the thalamus and the tegmentum to the bulb and so to the cord, excluding the cerebellum, which other observers believed to be concerned in the matter. Some observers again find that stimulation of the cortex produces a flow of 'chorda saliva,' while other's maintain that the secretion, when it does occur, is an indirect and not a direct effect of the cortical stimulation; and it may be remarked that the cortical area, which is claimed to be a "salivation area,' lying in the dog on the convolutions dorsal to and in front of the Sylvian fissure, is not either the area connected with the facial nerve, or that allotted to taste or smell.

Similarly, stimulation of parts of the cortex has in the hands of various observer's led to movenents or to arrest of movements of the intestines, to changes in the beat of the heart, and to various vaso-motor and other effects; but it will not be profitable to enter into any further details. We may, however, add the remark that when the cortical motor area for a limb is removed, or suffers a lesion, the temporary paralysis which is thereby caused is accompanied by a rise of temperature in the limb; this may be at times very great indeed; in the monkey for instance, the hand or foot on the paralysed side may be as much as $5^{\circ} \mathrm{C}$. higher than that of the other side. The effect is partly due to vaso-motor paralysis, but, especially considering that the muscles of the limb are relatively quiescent and so producing less heat than usual, cannot be due to that alone. 'The remarkable result may be taken as still further illustrating the complexity of the processes connected with the cortical motor area; the area is in some way associated with the vaseular arrangements and nutrition of the muscles with whose move- 
S18 SPLANCHNIC FUNCTIONS OF THE BRAIN. [Book II.

ments it is concerned. We may add that in the cases of the dogs kept alive for some time after nearly complete removal of both cerebral hemispheres, a vastly increased bodily metabolism was a marked symptom; the animals had to be fed with enormous quantities of food. 


\section{SEC. 7. ON THE TIME TAKEN UP BY CEREBRAL OPERATIONS.}

$\S 515$. We have already seen ( $\$ 467$ ) that a considerable time is taken up in a purely reflex act, such as that of winking, though this is perhaps the most rapid form of reflex movement. When the movement which is executed in response to a stimulus involves cerebral operations a still longer time is needed; and the interval between the application of the stimulus and the commencement of the muscular contraction varies according to the nature of the mental labour involved.

The simplest case is that in which a person makes a signal immediately that he perceives a stimulus, ex. gr. closes or opens a galvanic circuit the moment that he feels an induction shock applied to the skin, or sees a flash of light, or hears a sound. By arrangements similar to those employed in measuring the velocity of nervous impulses, the moment of the application of the stimulus and the noment of the making of the signal are both recorded on the same travelling surface, and the interval between them is carefully measured. This interval, which has been called 'the reaction period' or 'reaction time,' may be divided into three stages: (1) The time during which afferent impulses are generated in the peripheral sense organs and transmitted along the afferent nerves to the central nervous system; this may be called the "afferent stage." (2) The time during which, through the operations of the central nervous system, the afferent impulses are transformed into efferent impulses; this may be called the "central stage." (3) The time taken up by the passage of the efferent impulses along the efferent nerves and the transformation of the nervous impulses into muscular contractions ; this may be called the "efferent stage." In the efferent stage the events are comparatively simple, and though not absolutely constant, do not vary largely; we are able to form a fairly satisfactory estimate of its duration and so of the share in the whole reaction period which may be allotted to it. The events of the afferent stage are much more complex and the estimates of its duration, being arrived at in an indirect manner, 
and chiefly based upon calculations of the whole reaction time, are very uncertain. Hence all attempts to estimate the length of the "central" stage, the "reduced reaction period" as it is sometimes called, by subtracting the efferent and afferent stages, must be subject to much error. But a good deal may be learnt by studying the variations under differcnt circumstances of the reaction period as a whole.

Taking first of all the cases in which the events of the central stage are simple, such as those where the subject has merely to make a signal upon feeling a sensation, we find that the length of the reaction period is dependent on the intensity of the stimulus, being shorter with the stronger stimulus. But variations in the strength of the stimulus, especially in the ease of minimal stimuli, have a much more striking effect in determining the certainty of the reaction than in affecting the length of the period. Thus when the signal is made in response to some visual sensation, upon seeing an electric spark for instance, if the spark be a very weak one the subject of the experiment often fails to make the signal at all, though he may rarely fail if the spark be a strong one.

Some of the most marked variations in the length of the reaction period are determined by the individuality of the subject. Thus with the same stimulus applied under the same circumstances the reaction period of one person will be found very different from that of another.

The length of the reaction period varies also according to the nature and disposition of the peripheral organs stimulated. In general it may be said that eutaneous sensations produced by the stimulus of an electric shock applicd to the skin (the signal for instance being made by the right hand when the shock is felt by the left hand) are followed by a shorter reaction period than are auditory sensations, while the period of these is in turn shorter than that of visual sensations produced by luminous objects; on the other hand, the shortest period of all is said to be that of visual sensations produced by direct electrical stimulation of the retina. Roughly speaking we may say that the reaction period is for cutaneous sensations $\frac{1}{7}$ th, for hearing $\frac{1}{6}$ th, and for sight $\frac{1}{5}$ th of a second.

Practice materially shortens the reaction period; indeed, after long practice, making the signal, at first a distinct effort of the will, takes on the characters of a reflex act, with a correspondingly shortened interval. Lastly, we may add that in the same individual and with the same stimulus, the length of the period will vary according to eircumstances, such as the time of year, the weather, and the like, as well as according to the condition of the individual, whether fresh or fatigued, fasting or replete, having taken more or less alcohol, and the like.

The reaction period of vision has long been known to astrono- 
mers. It was early found that when two observers were watching the appearance of the same star, a considerable discrepancy existed between their respective reaction periods, and that the difference, forming the basis of the so-called 'personal equation,' varied from time to time according to the personal conditions of the observers.

$\$ 516$. The events taking place in the central stage are of course complex, and this stage may be subdivided into several stages. Without attempting to enter into psychological questions, we may at least recognize certain elementary distinctions. The afferent impulses started by the stimulus, whatever be their nature, when they reach the central nervous system undergo changes, and as we have seen, probably complex changes before they become sensations; and further changes, now of a more distinctly psychical character, are necessary before the mind can duly appreciate the characters of these sensations and act accordingly. Then come the psychical processes througli which these appreciated sensations, or perceptions, or apperceptions as they are sometimes called, determine an act of volition. Lastly, there are the executive processes of volition, the processes which, psychical to begin with, end in the issue of coorlinate motor impulses, or, in other words, start the distinctly physiological processes of the efferent stage. We may thus speak of the time required for the perception of the stimulation, of the time required for the action of the will, and of the time required for the complex psychical processes which link these two together. Accepting this elementary analysis, it is obvious that the total length of the central stage may be varied by differences in the length of each of these parts; and a more complete analysis would of course open the way for further distinctions. Hence, by studying the variations of the whole reaction time under varying forms of psychical activity, we may form an estimate of time taken up by various psychical processes.

We may take as an instance the case in which the subject of the experiment has to exercise discrimination. The mode of making the signal being the same, and the stimulus being of the same order in each trial, that is to say, visual, or cutaneous, or anditory, \&c., and general circumstances remaining the same, two different stimuli are employed, and the subject is required to make a signal in response to the one stimulus, but not to the other; the subject has to discriminate between the psychical effects of the two stimuli. Suppose, for example, the stimulus is the sound of a spoken or sung vowel, and the subject is required to make a signal when $a$ is spoken or sung, but not when $o$ is spoken or sung. If the subject's whole reaction period be determined (i) in the usual way, with either $a$ or o spoken (and the result will be found not to differ naterially whether $a$ or $o$ be used), the subject knowing that only $a$ or 
only o will be spoken, and then be determined again (ii) when he has to discriminate in order that he may make the signal when $a$ is spoken but not when $o$ is spoken, he not knowing which is about to be spoken, the whole reaction period will be found to be distinctly longer in the second case. The experiment may be varied by making use of all the vowel sounds taken irregularly as the stimulus, the subject responding by a signal to one only, as arranged beforehand. And of course other orders of stimulus may be used, either visual, the signal being made when a red light is shewn but not when other colours are shewn, or tactile, the signal being made when one part of the body is touched but not when other parts are touched, and the like.

In such experiments where the subject has to distinguish, to diseriminate between two or more events, the prolongation of the reaction period is obviously due to the longer time required for the psychical processes taking place during what we have called the central stage. In the two cases, one without and the other with discrimination, not only are the afferent and efferent stages the same in both, but we have no reason to suppose that in the central stage is there any difference between the two cases as to the time taken up by the transformation of simple sensory impulses into perceptions, or as to that taken up by the will in gaining access to the motor apparatus and so starting the processes of the efferent stage; the delay takes place in the psychical processes intervening between these two parts, and the amount of delay is the measure of the time needed for the processes involved in the discrimination. This "discrimination period" has been found to differ in the same individual aceording to the sensation employed, visual, auditory, \&c., and according to the kind of difference in the sensation which has to be discriminated, for instance in visual sensations between colours or between objects in different parts of the field of vision. In a series of observations made in this way, the discrimination period, i.e. the prolongation of the simple reaction period due to having to discriminate, was found to range from $0.011 \mathrm{sec}$. to 0.062 sec.

Another series of observations may be made in the following way. The signal being one made with the hand, the simple reaction period for a stimulus is determined with the signal given by the right hand. Two kinds of stimuli are then employed, both of the same order, two vowel sounds for instance, and the subject is directed to respond to one rowel with the right hand and to the other with the left liand. It is found, the subject being right-handed, that the reaction period is greater when the signal is made with the left hand. In this case the delay takes place not in the recognition of the effects of the stimulns, nor in the processes through which the 
will is formed upon that recognition; these are the same in the two cases; it takes place in the processes by which the will is brought to bear on the nervous motor apparatus for making the signal, on the cortical origin, for example of the pyramidal tract; these processes take a longer time in the case of the unaccustomed left hand than in the case of the usual right hand. In this way we obtain a measure of so to speak the volitional side of psychical processes.

In a somewhat similar way we may obtain a measure of the time required for perception. A strong sensation following too closely upon a weak one will prevent the psychical recognition of the weaker one. If, for instance, two or three letters in white on a black background be presented to the eye, and a large white surface be presented afterwards at an interval which is male successively shorter and shorter, it is found that when the interval is made very brief indeed the letters cannot be perceived at all. In proportion as the interval is prolonged, the recognition of the letters increases, until at an interval of about 05 sec. they are fully and clearly recognized. That is to say, the time required for perception is in such a case of about that length.

The duration of all these psychical processes, as of the simple reaction period itself, varies of course under different circumstances, and the discrimination period may be conveniently used for measurements of the rarying effects of circumstances. Practice shortens the discrimination period as it does the simple reaction period. One of the most powerful.influences is that of attention. And it is stated that the shortening of the period is greater when the attention is concentrated on the making of the signal than when it is more especially directed to recognition of the stimulus; in other words, the volitional processes are more amenable than are the perceptive processes to the psychical action which we call attention. On the other hand, the period is distinctly prolonged if the observer be distracted by conconitant sensations. For example, the period for discriminating between two visual sensations is prolonged if powerful auditory sensations be excited at the same time.

The same method of measurement may be used in other ways and under other circumstances with reference to psychical processes. It must be remembered, however, that all such observations are open to many fallacies and need particular caution. It not unfrequently happens that false results are obtained; for instance, the subject, expecting the stimulus to be brought to bear upon him and straining his attention, makes the signal before the stimulus actually comes off. And the interpretation of the results obtained are in many cases very difficult; but it would be out of place to dwell upon these matters any further here. 


\section{SEC. 8. THE LYMPHATIC ARRANGEMENT OF THE BRAIN AND SPINAL CORD.}

\$517. The Cerebro-spinal Fluid. The speeimens of cerebrospinal fluid whieh have been examined as to their eomposition are not quite comparable with eaeh other, sinee while some (such as those obtained from eases where a fraeture of the base of the skull has plaeed the subarachnoid space at the base of the brain, where it is largely developed, in eommunication with the external meatus, and the fluid eseapes by the ear) may be regarded as normal, others (such as those obtained from eases of hydroeephalus where the ventricles eontain an unusual quantity of fluid, or from cases of spinal malformations) must be considered as abnormal. In most of the more eomplete analyses, the fluid examined has belonged to the latter elass; and the following statements apply, strietly speaking, to them alone.

With this eaution we may say that the eerebro-spinal fluid is a transparent, eolourless or very slightly yellowish fluid, of faint alkaline reaction, free from histological elements. 'The speeific gravity is about 1010 or less, the amount of solids being on an average 1 p.e. Of these by far the greater part, $\cdot 8$ or 9 p.c., is supplied by salts, the total quantity of which as well as the relative amount of the several eonstituents being about the same as obtain in blood and lymph. The eomparative defieiency of solids is due to the seantiness of the proteids, which rarely exceed 1 p.c. These are chiefly globulin and a form of albumose, or even peptone; albumin is said to be generally absent. The fluid, save apparently in exeeptional eases, does not elot, and eontains neither fibrogenous factor's, nor fibrin ferment. It very frequently contains a substance whieh like dextrose reduces Feliling's solution but which is not a sugar; it appears to be pyrocatechin or a elosely allied body.

Seeing that a fluid of such a eomposition is of a different nature from ordinary lymph, furnished entirely in the ordinary way, we might be inelined to infer that probably a very large pirt of the whole mass of the fluid is furnished by the secreting epithelium of the choroid plexus. But it must be borne in 
mind, that the foregoing analyses refer chiefly to fluid appearing under abnormal circumstanees, and it would be hazardous to draw any wide inference from them. We have little or no cxact experimental evidence as to how much fluid is actually secreted by the choroid plexuses; and if the fluids which have been analyzed do represent a mixture of ordinary lymph supplied through the pia mater with the peculiar secretion of the choroid plexus and cerebro-spinal canal, some further change beyond the mere mingling of the two fluids is needed to explain the remarkable abscnce of albumin which has been so strongly insisted upon by various authors.

$\$ 518$. We may fairly suppose that during life the fluid is continually being supplied, from the one source or the other; but we have no very exact knowledge as to the rate at whieh it is furnished. In the dog, the fluid has been observed to escape at a rate varying very largely under different circumstances, and ranging from 1 e.c. in 40 minutes to as much as 1 c.c., in 6 minutes, the total quantity discharged in 24 hours varying from 36 c.c. to 240 c.c. In the cases of fracture of the base of the skull mentioned above, a very considerable flow has been. frequently observed; but it may be doubted whether the abnormal circumstances of such cases have not raised the secretion above the normal. The rate of flow was found in the dog to be much increased by the injection of substances (normal saline solution) into the blood, but to be relatively little influenced by artificial heightening of arterial pressure. This has been put forward as indicating that the fluid is chiefly furnished as a secretion and not as an ordinary transudation of lymph; but it cannot be regarded as affording a valid argument. The pressure under which the fluid exists is also very variable; it is closcly dependent on the vascular arrangements of which we shall have to speak presently. In the $\operatorname{dog}$ the average pressure has been estimated at about $10 \mathrm{~mm}$. of mercury.

If the fluid is thus continually formed it must always find a means of escape. This is probably supplied by the tubular prolongations of the subarachnoid space along the nerve roots; these are continuous with the lymphatic vessels of the nerves, and so with the lymphaties of the body generally; and in the skull, the passages of this kind along the cranial nerves, especially along the two optic nerves into the orbits, afford a ready means of escape. It is also urged that some of the fluid escapes through the Pacchionian glands directly into the blood of the venous sinuses. In a dead body fluid introduced in to the subarachnoid space through an opening over the bulb, disappears at even a very low pressure with great rapidity. The circumstances then are, however, not the same as in life; and the few experiments which have been made seem to shew that, during life, a somewhat high pressure is required to secure the escape of fluid 
introduced in addition to that naturally secreted. Thus it is stated that when in a dog normal saline solution is introduced into the subarachnoid cavity at the lower end of the spinal cord very little resorption takes place so long as the pressure remains as low as about 10 c.c. of mercury; as the pressure is increased beyond this resorption quickly increases. But it may be doubted whether the resorption of added fluid is a fair test of the escape of fluid naturally present; and the experiment is of value rather as shewing simply that there are means of escape than as affording a measure of the rate of escape. Besides, the immediate effects of applying pressure at the caudal cnd of the spinal cord are not the same as those of applying pressure within the skull.

The rate of possible escape is not without importance as regards the mechanical importance of the cerebro-spinal fluid. Thus it has been urged that when an extra quantity of blood is driven into the skull, any injurious intercranial compression is prevented, not only by the transference of a corresponding quantity of cerebro-spinal fluid through the foramen of Majendie from the cranium into the spinal canal, the walls of which are less rigidly complete, but also by the direct escape of the fluid from the cavity of the skull along the cranial nerves in the manner described. It has also been urged that the fluid at the base of the skull, in the large subarachnoid spaces of which it gathers in larger quantity than elsewlere, acts as a sort of protective water cushion to the delicate cerebral substance, and that, in general, the presence of the fluid is mechanically useful to the welfare of the brain, removal of the fluid by aspiration being said to lead to hæmorrhage from the pia mater and to various nervous disorders. But our knowledge as to the part which the fluid plays is at present very imperfect; and its very peculiar chemical characters suggest that it has some chemical as well at least as mechanical functions. 
SEC. 9. THE VASCULAR ARRANGEMENTS OF THE BRAIN AND SPINAL CORD.

$\$ 519$. In the brain two important features of the distribution of the arteries deserve special attention. In the first place, the quadruple supply by the right and left vertebral and internal carotid arteries is made one by remarkable anastomoses forming the circle of Willis. Blood can pass along this circle in various ways; from the basilar artery along the right posterior communicating artery to the right internal carotid, and so by the right anterior cerebral artery and anterior communicating artery to the left side of the circle, and similarly from the basilar artery along the left side to the right, or from the right or from the left carotid through the circle, to the right hand or to the left hand in each case. Since the channel of the circle is a fairly wide one, the passage in various directions is an easy one; all the vessels radiating from the circle, including the basilar artery and its branches, can be supplied by the carotids alone, or by the vertebrals alone, or even by one carotid or one vertebral alone. In this way an ample supply of blood to the brain is secured in the face of any hindrance to the flow of blood aloug any one of the four channels. In what may perhaps be considered the usual arrangement, the calibre of the posterior communicating arteries is rather smaller than the other parts of the circle, so that, other things being equal, most of the vertebral blood will pass by the posterior cerebral arteries, while the carotid blood passes to the middle and anterior cerebral arteries; but many variations are met with. We may also here perhaps call to mind the fact that the left carotid coming off from the top of the aorta, offer's a more straight path for the blood than does the right carotid which comes off from the innominate artery.

Another special feature of the arterial supply to the brain is that the three large cerebral arteries, posterior, middle and anterior, are distributed almost exclusively to the cortex and to the subjacent white matter, while the deeper parts of the hemisphere, the nucleus caudatus, thalamus and the like, with the capsule and other adjoining white matter are supplied by smaller arteries 
coming direct from the circle of Willis, or from the very beginnings of the three cercbral arteries. It is stated that these two systems make no anastomoses with each other' but different individuals in respect to this appear to vary much. WVe may add that the anterior cerebral artery supplies the cortex of the dorsal aspect of the frontal lobe as well as the front and middle portions of the whole mesial surface of the hemisphere; while the middle cerebral, always large, is distributed to the side of the brain, that is, the parietal lobe, with the ventral part of the frontal lobe and the dorsal part of the temporal lobe; the posterior cerebral supplying the rest of the cortex, that is to say, the occipital lobe inclnding the hind part of the mesial surface of hemisphere, together with the ventral part of the temporal lobe. The distribution of these arteries therefore does not correspond to functional divisions, for while the middle cerebral supplies a large part of the motor region, it does not supply the whole of it, and does supply parts outside it. Though the small arterics as they run in the pia mater on the surface of the cortex anastomose freely, there is very little anastomosis between the small arteries which leaving the pia mater dip down into the substance of the brain; hence when these latter arterics are blocked, the nutrition of the part of the cortex supplied by them is apt to be impaired.

$\S 520$. The venous arrangements of the brain have very special characters.

The channels for the venous blood of the brain are not veins but sinuses, not so much tubes for maintaining a uniform current as longitudinal reservoirs, which while affording an easy onward path can also be easily filled and easily emptied, and in which the blood can move to and fro withont the restrietions of valves. This arrangement is correlated to the peculiar surroundings of the brain, which is not like other organs protected merely by skin or other extensible and elastic tissue, but is encased by a fairly complete inextensible en velope, the skull. As a consequence of this, when at any time an extra yuantity of blood is sent from the heart to the brain, room must be male for it by the increased exit of the fluids already present; for auy pressure on the brain-substance beyond a certain limit is injurious to its welfare and activity. Some room may, as we have seen ( $\$ 518$ ), be provided by the escape of cerebro-spinal fluid from the skull. But, within the limits of the normal cerebral eirculation, the charaeteristic vcuous sinnses especially serve to regulate the internal pressure; they form temporary reservoirs from which a comparatively large quantity of blood can be rapidly diseharged from the cranium, the flow from the sinuses being greatly assisted by the low or negative pressure obtaining in the veins of the neck at each inspiratory movement of the chest. The injurious effects of too 
great a pressure on the brain-substance are seen in certain maladies, where blood passing by rupture of the blood vessels out of its normal channels remains effused on the surface of the brain or elsewhere, and thus taking up the room of the proper brain-substance leads, by 'compression' as it is called, to paralysis, loss of consciousness, or deatl. They are also shewn by experiments on animals. When by driving an excess of fluid into the subdural cavity through a hole in the cranium or by other means a certain amount of pressure is established in the cranial cavity both the respiration and the circulation are affected. The breathing is slowed and eventually arrested, but may in certain cases be quickened. The heart is slowed by vagus inhibition, and a rise of blood pressure due to vaso-constriction is observed unless the slowing of the heart be sulficient to neutralize this. These phenomena point of course especially to an influence exerted on the spinal bulb; but besides these changes in the pupil and other effects are met with.

$\$ 521$. The supply of blood to the brain seems at first sight not to correspond to the importance of this the chief organ of the body. In the rabbit it would appear that hardly more than one per cent. of the total quantity of the blood of the body is present at ally one time in the brain, a quantity but little more than half that which is found in the kidneys; and while the weight of blood in the brain at any one time amounts to abont five per cent. of the total weight of the organ, being about the same as in the muscles, in the kidney it amounts to nearly twelve per cent., and in the liver to as much as nearly thirty per cent. Making every allowance for the relative small size and functional importance of the rabbit's brain, the bloorl-supply of even the human brain must still be small; and making every allowance for rapidity of current, the interchange between the blood and the nervous elements must also be small. In other words, the metabolism of the brain-substance is of importance not so much on account of its quantity as of its special qualities.

The circulation in the brain may be studied by help of various methods. A manometer may be connected with the peripheral end of the divided internal carotid artery, a second manometer being attached in the usual way to the central portion. Since the peripheral manometer records the blood-pressure in the circle of IVillis transmitted along the peripheral portion of the carotid artery, variations of pressure in the circle of Willis may thus be studied; and a comparison of the peripheral with the central manometer will indicate what general changes are taking place in the circulation through the brain. Thus a fall of pressure in the peripheral manometer unaccompanied by any corresponding fall in the central manometer would shew that the "peripheral resistance" in the brain was being lowered, in other wol'ds, that the vessels were being dilated. 
In another method, in the dog, the outflow of venous blood from the lateral sinus through the posterior facial vein has been measured. The freedom with which blood passes along the sinuses justifies the assumption that the outflow through the open vein gives an approximate measure of the rate of How under natural conditions; still the results are only approximate, and besides, the continued loss of blood introduces error.

A third method is a plethysmographic one. The skull is made to serve as the box of the plethysmograph or oncometer $(\$ 330)$; a small piece of the roof having been removed by the trephine, a membrane is fitted to the hole, and the movemeuts of the membrane are recorded by help of a piston and lever or directly by a lever. In young subjects, the fontanelle, or portion of the cranium not yet ossified, may be utilized as a natural membrane, and its movements recorded in a similar mamner. When the instrument is fitted to the hole in a watertight manner, this method records variations in internal pressure; and we may take it for granted, unless otherwise indicated, that greater or less pressure is due to more or less blood passing to the brain. But the amount of pressure brought to bear on the recording instrument will also depend on the readiness with which the cerebro-spinal fluid escapes from the cavity of the skull; if there be a hindrance to the escape, or on the other hand an increased facility of escape, the same increase of supply of blood will produce in one case a less, in the other a greater movement of the lever. If the membrane be attached loosely to the hole so as to allow free escape of the cerebrospinal fluid, the lever practically resting on the surface of the cerebral hemisphere, the method records variations in the dorsoventral diameter of the hemisphere, and these may be taken as measuring variations in the volume of the brain and so in the blood supply. In neither form, however, does the method by itself give us all the information which we want. An increase of blood in the brain, and therefore an expansion of the brain, and so a movement of the recording instrument, may result either from a fuller arterial supply or from hindrance to the venous outflow; the former condition is, at least in most cases favourable to, the latter always and distinctly injurious to, the activity of the nervous structures. Hence the teachings of the lever must be interpreted by help of a simultaneous observation of the general arterial pressure and of the bloodpressure in the veins of the neck; or the pressure in the sinuses themselves may be measured by introducing a cannula directly into the torcular Herophili. Moreover, the argument which we used ( $\$ 337$ ) in reference to the kidney may be applied here and probably with equal force, namely, that the value of the blood stream for the nutrition of the tissue is dependent not alone on the amount of blood-pressure, but also and espe- 
cially on the rapidity of the flow; indeed this second factor is of particular importance in view of the need of supplying the nervous elements with an adequate interchange of gases. Now of the rapidity of flow the plethysmographic method can give us indirect information only.

522. By one or other or all of these methods, certain important facts have been made out. The volume of the brain, as determined by the amount of blood present in it, is continually undergoing changes brought about by various causes. Each heart-beat makes itself visible on the cerebral as on the renal plethysmographic tracing, and as we have seen in speaking of respiration, the diminution of pressure in the great veins of the neck during inspiration leads to a shrinking, and the reverse change during expiration to a swelling of the brain. The plethysmograph also shews variations, larger and slower than the respiratory undulations, and brought about by various causes, such as the position of the head in relation to the trunk, movements of the limbs, modifications of the respiratory movements, and apparently phases of activity of the brain itself, as in waking and sleeping; undulations corresponding to the Traube-Hering variations (\$315) of blood-pressure niay not unfrequently be observed.

All the various methods show that the flow through the brain is largely determined by a vaso-motor action of some kind or another. And this we might indeed infer from ordinary experience. When the head is suddenly shifted from the erect to a hanging position, there must be a tendency for the blood to accumulate in the cranial cavity, and conversely when the head is suddenly shifted from a hanging to an erect position, there must be a tendency for the supply of blood within the cranium to be for a while less than normal. Either change of position, and especially perhaps the latter, would lead to cerebral disturbances, which in turn would in ourselves be revealed by affections of our consciousness. That a perfectly healthy, and especially young organism whose vaso-motor mechanisms are at once effective and delicately responsive, can pass swiftly from one position of the head to the other without inconvenience, whereas those in whom the vaso-motor mechanisms have by age or otherwise become imperfect are giddy when they attempt such rapid changes, is in itself adequate evidence of the importance of the vaso-motor arrangements affecting the circulation through the brain. The several methods agree in shewing that increased general arterial pressure, such as that for instance induced by stimulation of a sensory nerve, leads to a greater flow of blood to the brain; the volume of the brain is increased and the venous outflow by the lateral sinus is quickened. Conversely, a lowering of arterial pressure leads to a lessened flow of blood to the brain. 
Seeing that the cerebral arteries have well-developed muscular coats, the basilar artery in fact being conspicuous in this respect, one would be led to suppose that the brain possessed special vaso-motor nerves of its own; and recognising the importance of blood supply to rapid functional activity one would perhaps anticipate that by special vaso-motor action, the supply of bloor to this or that particular part of the brain might be regulated apart from changes in the general supply. The various observations, however, which have hitherto been made have failed to demonstrate with certainty any such special vaso-motor nerves or fibres directly governing cerebral vessels. It would be hazardous to insist too much on this negative result, especially since the observations have been chiefly directed to the nerves of the neck, the experimental difficulties of investigating the presence of vaso-motor fibres in the cranial nerves being very great. Still it may be urged and indeed has been urged that the flow of blood through the brain is so delicately responsive to the working of the general vaso-motor mechanism just because it has no vaso-motor nerves of its own. In such an organ as the kidney, an increase of general blood-pressure, as we have more than once insisted, may or may not lead to a greater flow through the kidney according as the vessels of the kidney itself, through the action of the renal vaso-motor' nerves, are dilated or constricted; and, as we have seen, a constriction of the renal vessels may be one of the contributors to the increased general pressure. In the brain, on the other hand, an increase of general arterial pressure seems always to lead to increase of flow. Thus in the 'Traube-Hering undulations just mentioned, the expansions of the brain are coincident with the rises of the general pressure, whereas in the normal kidney and in other organs the local Traube-Hering undulation reverses the general one, the shrinkings are synchronous with the rises of pressure, the local constriction being one of the factor's of the gencral rise. It is argued, that in the absence of vaso-motor nerves of their own, the cerebral ressels are wholly, so to speak, in the hands of the general vaso-motor system, so that when the blood-pressure is high owing to a large vaso-constriction in the abdominal viscera, more blood must necessarily pass to the brain, and when again the blood-pressure falls through the opcning of the splanchnic flood-gates (\$151) less blood necessarily flows along the cerebral vessels. And indeed one may recognize here a sort of self-regulating action; for diminishing the supply of blood to the vaso-motor centre in the bulb acts, as we know, as a powerful stimulus in producing vasoconstriction, and so leads to a rise of blood-pressure; but this very rise of blood-pressure drives more blood to the brain, including the bulb, and thus the injurious effects to the brain threatened by an anæmic condition are warded off by the very 
beginning of the anæmia itself. All these advantages are, however, quite compatible with the coexistence of special vasomotor mechanisms.

$\$ 523$. Moreover the flow of blood to, and consequent change in the bulk of, the brain, and indeed the flow of blood through the brain, as measured by the venous outflow, may be modified independently of changes in the general blood-pressure. For instance, stimulation of the motor region of the cortex quickens the venous outflow, without producing any marked change in the general blood-pressure; this feature becomes very striking at the onset of epileptiform convulsions when these make their appearance. It is difficult not to connect such a result of functional activity with some special vaso-motor nervous arrangement comparable to that so obvious in the case of a secreting gland. Again, it has been observed that certain drugs have an effect on the volume of the brain, quite incommensurate with their effect on the vaso-motor system; thus in particular the injection into the general blood stream of a weak acid produces a large and immediate expansion of the brain, while the introduction of a weak alkali similarly gives rise to similar considerable shrinking. It is suggested that these effects are produced by the acid or alkali acting directly on the muscular coats of the minute arteries and so leading to relaxation or contraction respectively. Observations go to show that the grey matter of the cortex is faintly alkaline during life and under normal conditions, but becomes acid after death or when its bloodsupply is interfered with; and it has been urged that nervous grey matter like muscular substance developes acidity during activity, as well as upon death, the acidity being probably due in each case to some form of lactic acid. And just as it has been suggested that the dilation of the minute arteries of a skeletal muscle, accompanying or following the contraction of the muscle, is brought about by the acid generated during the contraction causing a relaxation of the muscular coats of the minute arteries, so it has been suggested that a similar acidity, the product of nervous activity, similarly leads in nervous tissue to a dilation of the vessels of the part. The existence of special vaso-motor mechanisms would, however, afford a more satisfactory explanation of these and other phenomena; in spite of the negative results so far obtained, the matter is obviously one needing further investigation. Meanwhile we have abundant evidence that, however brought about, the flow of blood through the brain, and probably through particular parts of the brain, is varied in accordance with the needs of the brain itself and the events taking place elsewhere in the body. 
CHAPTER III.

SIGHT.

SEC. 1. ON THE GENERAL STRUCTURE OF THE EYE, AND ON THE FORMATION OF' THE RETINAL IMAGE.

$\$ 524$. Is dealing with the brain we have been incidentally obliged to deal with some of the facts connected with the senses; but we must now study the details of the subject. And, for the very reason that it is the most highly developed and differentiated sense, it will be convenicnt to begin with the sense of sight; we shall find that the study of it throws more light on the simpler and more obscure senses than the study of them throws on it.

A ray of light entering the eye and falling on the retina gives rise to what we call a sensation of light; but in order that distinct vision of any object emitting or reflecting rays of light may be gained, an image of the object must be formed on the retina, and the better defined the image the more distinct will be the vision. Hence in studying the physiology of vision, our first duty is to examine into the arrangements by which the formation of a satisfactory image on the retina is effected; these we may call briefly the dioptric mechanisms. We shall then have to inquire into the laws according to which rays of light impinging on the retina give rise to nervous impulses, and into the laws according to which the sensory impulses thus generater, which we will call visual impulses, give rise in turn to visual sensations. Here we shall come upon the difficulty of distinguishing between the events which are of physical origin, due to changes in the retina and optic tibres, and those which are of psychical origin, due to features of our own consciousness; for many of our conclusions are baser on an appeal to consciousness. W' shall find our difficulties further increased by the fact, that in appealing to our own consciousness we are apt to fall into error by failing to distinguish between those affections of 
consciousness which are the primary and direct results of the stimulation of the retina and those secondary, more reeondite, affections of consciousness to which the former, through the intricate working of the central nervous system, give rise, or, in familiar language, by confounding what we see with what we think we see. These two things we will briefly distinguish as visual sensations and visual judgments: and we shall find that even in vision with one eye, though more especially in binocular vision, visual judgments form a very large part of what we frequently speak of as our 'sight.'

$\$ 525$. In the structure of the eye we may distinguish two parts: the one is the retina, in which visual impulses are generated; the other comprises all the rest of the eyeball, for all the other structures serve either as a dioptric mechanism or as a means of nourishing the retina. This distinction is readily seen when we trace out the early history of the eye.

The first of the three primary cerebral vesicles, that which is the forerumner of the third ventricle, buds out on each side the stalked and hollow optic vesicle. The wall of this optic vesicle, like that of the rest of the medullary tube, consists of epithelium, and the eavity of the vesicle is at first continuous, through the canal of the hollow stalk, with that of the medullary tube. The whole is covered over by the layer of epiblast which, with scanty underlying mesoblast, is the rudiment of the future skin.

Very soon the vesicle is doubled back upon or folded in upon itself so that the originally more or less spherical hollow single-walled vesicle is converted into a more or less hemispherical cup with a double wall, one the hind or outer wall corresponding to the hind half, and the other the front or inner wall to the front half of the vesicle, the two walls of the cup coming eventually into contact so that the cavity of the vesicle is obliterated. 'The folding is somewhat peculiar, inasmuch as it takes place not only at the front but also and indeed chiefly at the side, forming at the side a cleft, the choroidal fissure, the edges of which ultimately unite. We camnot enter into the details of the matter here, and indeed only refer to the character of the folding in order to point out that it involves the stalk as well as the cup. The stalk is first flattened and then doubled up lengthwise, a quantity of mesoblastic tissue being thrust into the hollow of the fold: and eventually the originally hollow stalk becomes a solid stalk having within it a core of mesoblastic tissue, carrying blood vessels. This core of vascular mesoblast, the origin of the future central artery of the retina, is continuous with a quantity of mesoblast which enters into the hollow of the cup at the time of folding, and, as we shall see, the central artery of the stalk is up to a certain stage of development earried forward through the centre of the cup. The 
cup bccomes what we may speak of broadly as the retina, and we may call it the optic or retinal cup; the solid stalk becomes the optic nerve; and the other parts of the eyeball are formed round this retinal cup, which remains as the essential part of the eye.

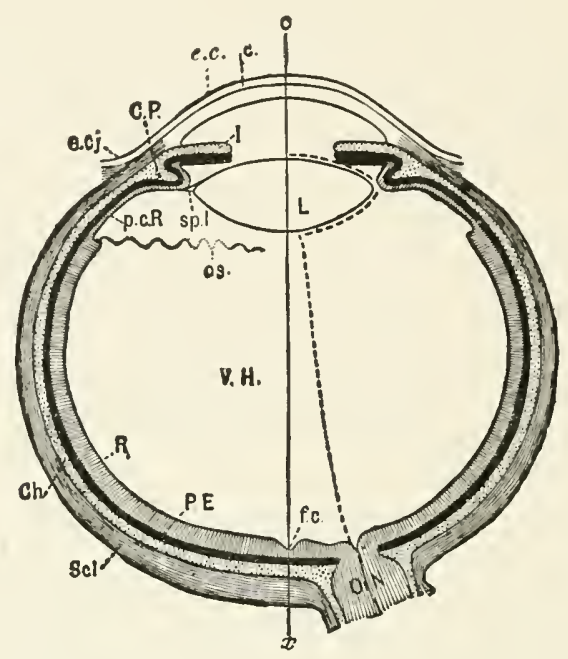

Fig. 139. Diagrammatic outline of a horizoxtal section of the eye, to lllustrate the relatioNs of the varrous parts.

The figure is to be regarded as very diagrammatic, more or less distortion of the relative sizes of the various parts and of the relative thickness of the coats being unavoidable in the effort to secure simplicity.

$\mathrm{Scl}$. the sclerotic coat, shaded longitudinally, continuous with the (unshaded) body of the cornea. e.c. the epithelium of the cornea continuous with e.cj. the epithelium of the conjunctiva.

$C h$. the choroid coat with $C . P$. the ciliary process and $I$. the body of the iris, all stippled to indicate that they are all parts of the same vascular investment.

$R$. the retina or inner wall, and $P$. $E$. the pigment epithelium or outer wall of the retinal cup. In front of the wavy line $O S$., marking the position of the ora serrata, the retina proper changes into the pars ciliaris retinæ, $p . c . R$. Both the pigment epithelium and the pars ciliaris retine are represented as continued over the back of the iris as well as over the ciliary process.

$L$. the lens. sp. $l$. the suspensory ligament. The broken line round the lens, shewn on one side only, represents the membrana capsulo-pupillaris; and the straight continuation of it through $V . H$. the vitreous humour to $O$. N. the optic nerve indicates the embryonic continuation of the central artery of the retina.

$o . x$. the optic axis, in this case made to pass through the fovea centralis $f c$.

The front or inner wall of the retinal cup is from the first distinctly thicker than the hind or outer wall (Fig. 139); it soon consists of more than one layer of epithelium, and it alone, or, more strictly speaking, part of it alone, bccomes the retina proper. The hind or outer wall remains thin, and continues to consist of a single layer of epithelium, the cells of which are never developed 
into nervous elements but soon become loaded with pigment, and the greater part of it is known in the arlult eye as the pigment epithelium of the retina, which, as we shall see, is in close functional connection with the nervous elements of the retina proper. The fibres of the optic nerve, as they are developed in the stalk of the retinal cup, become connected with the elements of the inner or retinal wall only of the cup; they pierce the outer wall of pigment epithelium, making no conncetions with the cells of that outer wall.

The retina then, in which by the action of light visual impulses arc genelated, is in reality a part of the brain, removed to some distance from the rest of the brain but remaining connected with it by means of the tract of white matter which we call the optic nerve; and, as we shall see, the retina is in structure similar to parts of the grey matter of the brain. The optic nerve is not like other nerves an outgrowth from the central nervous system, but like the olfactory tract a commissure of white matter between two parts of the brain, namely, between the outlying retina and the internally placed corpus geniculatum, pulvinar, and corpus quadrigeminum. IVe shall find accordingly that in structure it differs from ordinary cranial or spinal nerves.

Into the mouth of the retinal cup there is thrust a rounded mass of epithelium, an involution from the superficial epiblast; this becomes the lens. The hollow of the retinal cup is occupied, as we have said, by mesoblast; this ultimately becomes modified into the vitreous humour. 'The mesoblastic tissue snrrounding the cup is developed into an investment of two coats ; an inner, somewhat loose and tender, vascular and in part muscular coat, which on the one hand serves to nourish the retina, and on the other hand carries out certain movements of the dioptric apparatus, and an outer, firmer and denser coat, which affords protection to the wholc of the structures within. The inner vascnlar coat, which may be compared to the pia mater, is called the cho. roid (Fig. $139 \mathrm{Ch}$.), and in the front part of the eye, at about the level of the lens, is thrown into a number of radiating folds or plaits, the cilialy processes $C . P$. 'The outer coat, which may be compared to the dura mater, is called the sclerotic (Fig. 139 Scl.). Over the greater part of the eyeball the two coats are in apposition, or separated only by narrow lymphatic spaces, which may be compared with the subarachnoid spaces, but towards the front they diverge ; the choroid is bent inwards towards the central axis of the eye to form the diaphragm called the $i r$ is (Fig. 139 I.), while the sclerotic is continued forwards to form, beneath the epidermis into which the superficial epiblast is developed, the basis of the cornea (Fig. $139 \mathrm{C}$.). At the angle of divergence of the two coats is developed a small mass of muscular fibres, the ciliary muscle of which we shall speak in detail presently.

The inner or front wall of the retinal cup becomes as we have 
said thick, and is developed into the retina; but this takes place only over about the hind three-fourths of the cup. Along a meridian round the eye, at a wavy boundary line called the ora serrata (Fig. 139 O.S.), the retina proper eeases and the inner wall of the retinal cup in front of the ora serrata is eontinued on as a nuch thinner structure (Fig. 139 p.c.R.) eonsisting of a single layer only of cells; this is spoken of as the pars ciliaris retince. The outer or hind wall of the retinal eup consists throughout. of a single layer of epithelium cells loaded with pigment. Behind the ora serrata, that is, in the region of the retina proper, these cells have, as we shall see, peculiar features, but in front of the ora serrata they lose these features and become ordinary cubical cells, though still loaded with pigment.

Hence the ehoroid may be described as having a double lining. Over the hind part of the eye, behind the ora serrata, it is lined by the single layer of pigment epithelium and the retina. In front of the ora serrata it, including the ciliary processes, is lined by the same layer of pigment epithelium representing the outer wall, and by the single layer of cells, free from pigment, representing the inner wall of the retinal cup, the latter being called, as we have said, the pars ciliaris retine. And as the ciliary part of the choroid passes on to form the iris, these two layer's are also continued on to line the back of the iris, eoming to an end at the margin of the pupil or central opening of the iris, which may accordingly be taken as marking the extreme lip of the retinal eup. Fig. 139. Here however, as we shall see, the two layers are not so easily and distinctly recognized as in the ciliary region; and the nature of the structures forming the back of the iris has been a matter of much controversy.

At an early stage the mesoblastic tissue, which fills up the hollow of the retinal cup and surrounds the lens, is continuous at the mouth of the retinal cup with the outer investment of the cup; it here forms around the lens the membrana capsulo-pupillaris, and at the margin of the iris the membrana mupillaris blocking up the future opening of the pupil. The arteria centralis retince, which during the folding of the cup and stalk is carried into the core of the optic nerve, does not at this early stage stop at the retina, but is continued forward through the middle of the vitreous humour to the membrana capsulo-pupillaris, and furnishes the developing lens with an abundant supply of blood. But neither layer of the retinal cup stretehes over the pupillary membrane; they both stop, as we have said, at the margin of the ilis. Before birth takes place, the membrana pupillaris is, in man, absorbed and the pupil is thus established; at the same time the central artery in the vitrcous humour is obliterated beyond the retina, and the vaseular membrana capsulo-pupillaris gives place to the non-vascular capsule of the lens and the suspensory ligament of whieh we shall speak hereafter. 
Between the iris, which is the extreme front of the choroid. investment, and the cornea, which is the extreme front of the sclerotic investment, the lymphatic spaces which over the rest of the eye are narrow and linear are developed into a large conspicuous chamber, the anterior chamber of the eye, which upon the establishment of the pupil by the absorption of the pupillary membrane becomes continuous with the smaller "posterior chamber" of the eye or space between the back surface of the iris and ciliary processes on the outside and the suspensory ligament with the lens on the inside. The cavity of the conjoined anterior and posterior chamber's, being a continuation and enlargement of the flatter spaces between the choroid or pia mater of the eye, and sclerotic or dura mater of the eye, may be likened to the subarachnoid space, and like that space contains a peculiar fluid ; this, which is called the aqueous humour, like the cerebro-spinal fluid, differs from ordinary lymph, and is probably, to a large extent, furnished by the ciliary processes in some such way as the cerebrospinal fluid is furnished by the choroid plexuses (\$ 517).

\section{The Formation of the Retinal Image.}

\$526. The iris and choroid coat contain, as we have said, muscular elements, and by means of these muscular elements changes in the form and relations of some of the parts of the eye are brought about; hence we have to distinguish between the eye at rest, and the eye which is undergoing one or other of these thanges.

The eye is a camera, consisting of a series of surfaces and media arranged in a dark chamber, the iris serving as a diaphragm ; and the object of the apparatus is to form on the retina a distinct image of external objects. That a distinct image is formed on the retina, may be ascertained by removing the sclerotic from the back of an eye, and looking at the hinder surface of the transparent retina while rays of light proceeding from an external object are allowed to fall on the cornea. To understand how such an image is formed, we must call to mind a few optical principles.

A dioptric apparatus in its simplest form consists of two media of different refractive power separated by a (spherical) surface; and the optical properties of such an apparatus depend upon (1) the degree of curvature of the surface, $(2)$ the relative refractive power's of the media.

Such a simple optical system is represented in Fig. 140, where $a p b$ represents, in section, a curved (spherical) surface separating a less refractive medium, on the left hand towards $O$, from a more refractive medium on the right hand towards $A$. The surface in question is symmetrically placed as regards the line $O A$, which falling normal (perpendicular) to the surface at $p$ 
passes through the centre $n$ of the sphere with whose surface we are dealing. 'This line is called the optic axis.

All rays of light which, in passing from the first less refractive to the second more refractive medimm, cut the surface normally, such as the one, $O_{p}$, in the line of the optic axis, and other's, such as $m d, m^{\prime} e$, undergo no refraction; all such rays are continued on as straight lines, and all pass through $n$ the centre of the sphere or nodul point. All other rays passing from the first to the second medium are refracted. Of these all those which lie in the first medium parallel to the optic axis, such as $c d$, are so refracted as to meet in the second medium at a point, $F_{2}$, on the optic axis; this is called the principal posterior (or second) focus. On the optic axis in the first medium there is another important point, $F_{1}^{\prime}$, the rays of light passing from which,

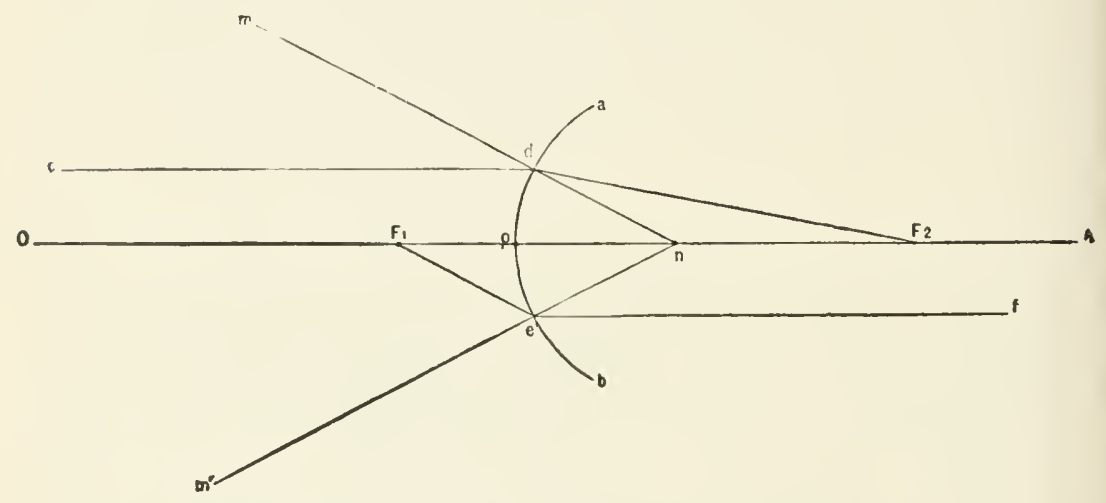

Fig. 140. Diagram of Simple Optical Srstem.

such as $F_{1} e$, are so refracted in passing into the second medium as to become parallel, ef, to the optic axis; this point is called the principal anterior (or first) focus. The point at which the optic axis cuts the surface is, for reasons which we shall see presently, called the principal point. The above points, viz. the posterior and the anterior principal foci, the nodal point, and the principal point are the cardinal points of such an optical system.

Surh a simple system, however, does not represent the optical conditions of the eye, for this consists of several media bounded by several surfaces, the latter differing from each other in curvature, though being approximately spherical. Rays of light in passing from an external object to the retina traverse in succession the following surfaces and media : - the anterior surface of the cornea, the substance of the cornea, the posterior surface of the cornea, the aqueous humour, the anterior surface of the lens, the substance of the leas, the posterior surface of the lens, 
and the vitreous humour; so that we have to deal with four surfaces, and, including the external air, four media. Indeed the matter is in reality still more complicated, for the structure of the lens, as we shall see, is such that the substance of the lens differs somewhat in refractive power in different parts, the central parts being more refractive than the peripheral parts; moreover the lens is covered in front by a capsule different in structure from the lens itself. We may, however, neglect, without fear of serious error, these smaller differences, and consider the lens as one medium of uniform refractive power bounded by an anterior and a posterior surface. The cornea again, as we shall see, is not absolutely uniform in structure, but this we may also neglect and consider the cornea as a medium, also of uniform refractive power, bounded by an anterior and a posterior. surface. Moreover, the posterior surface of the cornea is parallel to (concentric with) the anterior surface or nearly so. Now when the two surfaces which bound a medium are parallel to each other we may, in dealing with refraction, neglect the thickness of the medium entirely, we may suppose it to be absent and treat the two surfaces as if they were one. We may therefore, without serious error, neglect the substance of the cornea, and consider the cornea as affording one surface, its anterior surface, bounding the air in front from the aqueous lumour behind. Lastly, the aqueous humour differs in refractive power so little from the vitreous humour that we may consider the two as forming one medium.

We have therefore to deal with three surfaces separating three media, viz.: - first, the anterior surface of the cornea, at which considerable refraction takes place as the rays of light pass from the less refractive air into the more refractive aqueous humour; secondly, the anterior surface of the lens, at which again considerable refraction takes place as the rays pass from the less refractive aqueous humour into the more refractive substance of the lens; and lastly, the posterior surface of the lens, at which refraction takes place as the rays pass from the more refractive substance of the lens into the less refractive vitreous humour. The three surfaces, differing in curvature, are all approximately centred, symmetrically disposed around, the optic axis of the system. This optic axis meets the retina, according to some authorities, not quite at the part of the retina which, under the name of forea centratis, we shall hereafter speak of as the centre of the retina, but a little above and to the nasal side of that part; other authorities, however, maintain that it does cut the retina at the fovea centralis.

$\$ 527$. The eye, therefore, even with the simplifications which we have introduced, presents a much more complex optical system than the one described above. It has, however, been shewn mathematically that a complex optical system consisting 
of several surfaces and media centred on one optical axis may be treated as if it were a more simple system consisting of two surfaces only. In such a simplified system each of the two (ideal) surfaces has its own nodal point and its own principal fóci, anterior and posterior; moreover, the two points where the two snrfaces cut the optic axis are called principal points (and vertical planes drawn through those points principal planes), first, or anterior, and second or posterior. Hence the cardinal points of such a simplified complex system are six in number, namely, the anterior and posterior principal foci, the anterior and posterior principal points, and the anterior and posterior nodal points. (When such a system is, by removal of surfaces and media, converted into the still more simple system of one surface separating two media, the two nodal points become coincident in one point, namely, the centrc of the sphere, and the two principal points become coincident in one point, namely, the point at which the optic axis cuts the surface.)

In order to effect such a simplification of a complex optical system, it is requisite to know:-(1) The refractive index of each medium. (2) The radius of curvature of each surface. (3) The distance along the optic axis between the first surface on which the rays fall and the succeeding surfaces. These can be and have been determined for the human eye, and the following table gives the several values usually adopted with some recent corrections, the latter being placed in brackets.

\section{Refractive index of aqueous or vitreous humour 1.3376 (1.3365)}

Mean refractive index of lens ............................. 1.4545 (1.4371)

Radius of curvature of cornea ......................... 8 (7.829) mm.

$$
\begin{array}{lllll}
" 6 & \text { of anterior surface of lens } & 10 \\
6 & \text { of posterior " } & \text { " } & \ldots & 6
\end{array}
$$

Distance from anterior surface of cornea to anterior surface of lens

Thickness of lens $4(3 \cdot 6)$

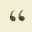

$$
66
$$$$
66
$$$$
66
$$$$
66
$$

By means of these measurements the optical system of the eye niay be simplified into an optical system of two surfaces. In this 'schematic, or diagrammatic, eyc of Listing,' as it is generally called, the two (ideal) surfaces, and the principal points where these cut the optic axis (Fig. 141, $p^{1}, p^{2}$, the two surfaces being indicated by dotted lines), lie close together in the front part of the aqueous humour, and the nodal points, $n^{1}, n^{2}$, lie, also close together, in the back part of the lens.

Further, the two principal surfaces lie so close together that, for practical purposes, no serious error is introduced, if instead of two snch surfaces wc assume the existence of one surface lying midway between the two. In this way we arrive at the 'reduced diagrammatic eyc,' or 'the reduced eye ' as it is called, in which 
the several surfaces and merlia of the actual eye are replaced by one (ideal) spherical surface (Fig. 141, P), having one nodal point, $N$; the two media which the surface separates arc supposed to be air on the one side and water on the other.

The sevcral positions of the cardinal points of this 'reduced eyc 'are as follows:

The principal point, where the one surface of the system cuts the optic axis, lies in the aqueous humour, $2.3448 \mathrm{~mm}$. behind the anterior surface of the cornea.

The nodal point lies in the back part of the lens, $.4764 \mathrm{~mm}$. in front of the posterior surface of the lens.

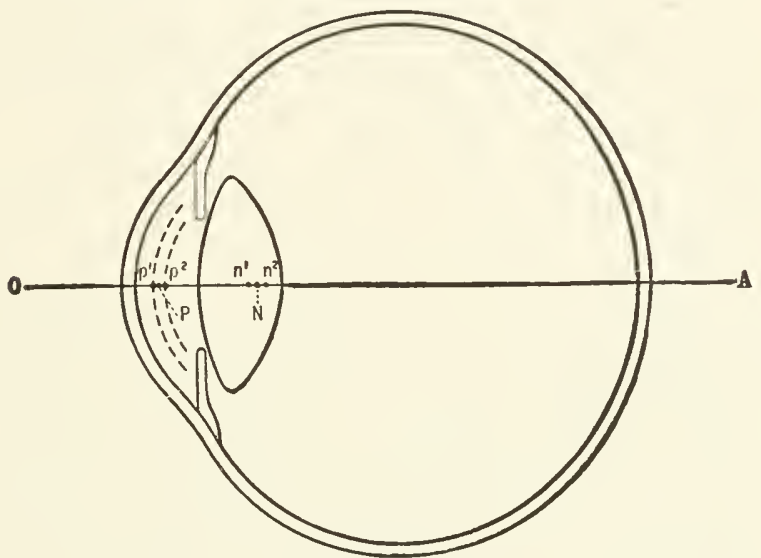

Fig. 141. Diagram of the Schematic or Diagramatic Ere.

The posterior principal focus lies $22.647(22.819) \mathrm{mm}$. behind the anterior surface of the cornea, that is to say, practically lies on the retina.

The anterior principal focus lies $12.8326 \mathrm{~mm}$. in front of the anterior surface of the cornea.

The radius of curvature of the (ideal) surface is $5.1248 \mathrm{~mm}$.; (that of the cornea is $8 \mathrm{~mm}$. and of the anterior surface of the lens $10 \mathrm{~mm}$.).

\$528. By help of this 'reduced eye' we are enabled to trace out the paths of rays of light through the actual eyc, and to study the formation of images on the retina. When an image of an external object, such as an arrow (Fig. 142), is formed in such an eye, each point of the object is considered as sending out a pencil of diverging rays, which by the system are made to converge again into the point in the image which corresponds to the point in the object. One such pencil of rays proceeds from the point at the extreme tip of the arrow, another from the extreme point at the other end, and other pencils from all the points between these 
two. Each such pencil has for its core a ray called the principal ray, a, a', around which are arranged, with increasing divergency, the other rays of the pencil, such as $b, b^{\prime}, c, c^{\prime}$. When such a pencil of rays falls on the refracting surface, such as the 'principal surface' of the reduced eye, the principal ray of the pencil, $a$, being normal to that surface, is not refracted at all, but passes struight on through the nodal point $n$, while the other rays of the pencil, $b, c$, undergoing refraction according to their respective

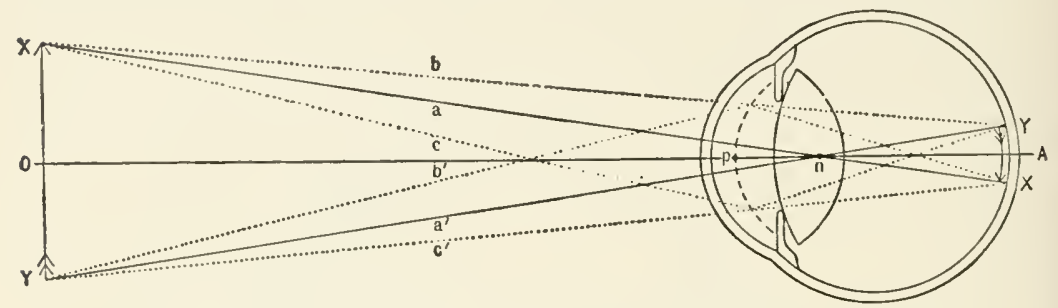

Fig. 142. Diagray of the Formation of a Retinal Image.

divergencics, are made to converge together at some point on the path of the principal ray, and thus form at that spot the image of the point from which the pencil proceeded. The exact position on the line of the principal ray, at which convergenee takes place and at which the image is formed, will depend on the refractive power of the optical system in relation to the amount of divergence of the pencil; the refractive power of the system remaining the same, it will be nearer to, or farther from, the nodal point according as the rays are less or more divergent; and the divergence of the rays remaining the same, it will be nearer to, or farther from, the nodal point according as the refractive power of the system is greater or less.

Hence supposing the eye to be in that condition in which a distinct image of the arrow is formed on the retina, we can find the position on the retina of the image of the extreme point of the tip of the arrow, by simply draiving a straight line from that cxtreme point of the arrow $X$ through the nodal point $n$ of the 'reduced' eye. Such a straight line represents the path of the 'principal ray' of the pencil proceeding from the extreme tip of the arrow, and when an image is formed on the retina the other diverging rays of that peneil will be so refracted as to converge at the point $x$, where that line meets the retina; all the rays will form together therc the image of the extreme point of the arrow. In a similar way a straight line drawn through the norlal point from the extreme point of the other end of the arrow, and continued until it meets the retina at $y$, will give us the position of the image of the other end of the arrow ; and in like manncr lines drawn from other points of the arrow through 
the same nodal point will give us the position on the retina of the images of those other points. In this way the construction of the reduced eye enables us to ascertain the position, magnitude and features of the retinal image of an object.

$\$ 529$. A ray of light, that is to say a series of waves of ether, falling upon a point of the retina stimulates certain structures in the retina and gives rise, as we have said, to visual impulses and so to a sensation of light; this we may consider as a visual sensation in its simplest form. When a number of different points of the retina are thus stimulated at the same time, as when an image of an external object falls in proper focus on the retina, the total result is a complex group of visual impulses and thus a complex sensation, by which we perceive, as we say, the object; and we frequently speak of this complex sensation as a visual image corresponding to the retinal image. The term is perhaps not a very desirable one, since it seems to imply an identity between the former which is a psychical matter, and the latter which is a physical matter; whereas, the one thing we may be sure about is that the psychical thing, though it is a sign and token of, is wholly different from the physical thing.

It will be as well perhaps thus early to call attention to the fact that, as indeed is shewn in Fig. 142, the image on the retina is an inverted one. What is the upper part of the object in the external world is represented in the lower part of the retinal image, what is on the right-hand side of the object is represented on the left-hand side of the iniage. In the visual judgment which is based upon the visual sensation, the retinal image is, as it were, reinverted; we take the left-hand side, or the bottom of the retinal image, as a token or sign of the right-hand side or the top of the object seen. We shall return to this matter later on; but in studying the dioptrics of the eye this inversion of the retinal image must always be borne in mind. 


\section{SEC. 2. THE FACTS OF ACCOMIIODATION.}

$\S 530$. When an object emitting or reflecting light, a lens, and a screen to receive the image of the object, are so arranged in reference to each other, that the image upon the screen is sharp and distinct, the rays of light proceeding from each luminous point of the object are brought into focus on the screen in a point of the image corresponding to the point of the object. If the object be then removed farther away from the lens, the rays proceeding in a pencil from each luminous point will be brought to a focus at a point in front of the screen, and, subsequently diverging, will fall upon the screen as a circular patch composed of a series of circles, the so-called diffusion circles, arranged concentrically round the principal ray of the pencil. If the object be removed, not farther from, but nearer to the lens, the pencil of rays will meet the screen before they have been brought to focus in a point, and consequently will in this case also give rise to diffusion circles. When an object is placed before the eye, so that the image falls into exact focus on the retina, and the pencils of rays proceeding from each luminous point of the object are brought into focus in points on the retina, the sensation called forth is that of a distinct image. When on the contrary the object is too far away, so that the focus lies in front of the retina, or too near, so that the focus lies behind the retina, and the pencils fall on the retina not as points, but as systems of diffusion circles, the sensation produced is that of an indistinet and blurred image. In order that objects both near and distant may be seen with equal distinctness by the same dioptric apparatus, the focal arrangements of the apparatus must be accommodated or adjusted to the distance of the object, either by changing the refractive power of the lens, or by altering the distance between the lens and the screen.

That the eye does possess such a power of accommodation or adjustment is shewn by every-day experience. If two needles be fixed upright, some tivo feet or so apart, into a long piece of wood, and the wood be held before the eye, so that the needles are nearly in a line, it will be found that if attention be directed 
to the far needle, the near one appears blurred and indistinct, and that, conversely, when the near one is distinct, the far one appears blurred. By an effort of the will we can at pleasure make either the far one or the near one distinct; but not both at the same time. When the eye is arranged so that the far needle appears distinct, the image of that needle falls exactly on the retina, and each pencil of rays reflected from each point of the needle unites in a point upon the retina: but when the far needle is seen distinctly, the focus of the near needle lies behind the retina, and each pencil from each point of this needle falls upon the retina in a series of diffusion circles; hence the image of the near needle is blurred. Similarly, when the eye is arranged so that the near needle is distinct, the image of that needle falls upon the retina in such a way, that each pencil of rays from each point of the needle unites in a point on the retina, while each pencil from each point of the far needle mites at a point in front of the retina, and then diverging again falls on the retina in a series of diffusion eircles, and the far needle is now seen indistinctly. If the near needle be gradually brought nearer and nearer to the eye, it will be found that greater and sreater effort is required to see it distinctly, and at last a point is reached at which no effort can make the image of the needle appear anything but blurred. 'The distance of this point from the eye marks the near limit of accommodation for near objects. Similarly, if the person be short-sighted, the far needle may be moved away from the eye, until a point is reached at which it ceases to be seen distinctly, and appears blurred; the far limit of accommodation is reached. In the one case, the eye, with all its power, is unable to bring the image of the needle sufficiently forward to fall on the retina: the focus lies permanently behind the retina. In the other, the eye camnot bring the image sufficiently backward to fall on the retina: the focus lies permanently in front of the retina. In both cases the pencils of rays from the needle strike the retina in diffusion circles.

\$53. The same phenomena may be shewn with greater nicety by what is called Scheiner's Experiment. If two smooth holes be pricked in a card, at a distance from each other less than the diameter of the pupil, and the card be held up, with the holes horizontal before one eye, the other being closed, and a needle placed vertically be looked at through the holes, the following facts may be observed. When attention is directed to the needle itself, the image of the needle appears single. Whenever the gaze is directed to a more distant object, so that the eye is no longer accommodated for the needle, the image of the needle appears double and at the same time blurred. It also appears louble and blurred when the eye is accommodated for a distance nearer than that of the needle. When only one needle is seen, and the eye therefore is properly accommodated for the 
distance of the needle, the only effect produced by blocking up one hole of the card is that the needle and indeed the whole field of vision seems dimmer. When, however, the image is double on account of the eye being accommodated for a distance greater than that of the needle, blocking the left-hand hole eauses a disappearance of the right-hand or opposite image, and blocking the right-hand hole causes the left-hand image to disappear. When the eye is accommodated for a distance nearer than that of the needle, blocking either hole causes the image on the same side to vanish. The following diagram will explain how these results are brought about.

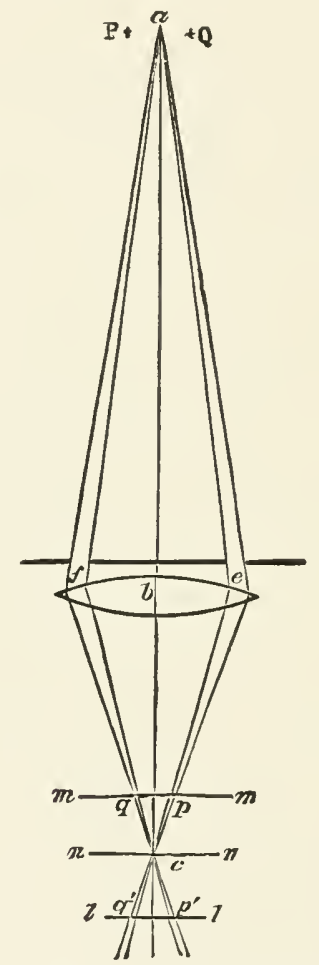

Fig. 143. Diagram of Scheiner's Experiment.

Let $a$ (Fig. 143) be a point in the needle, and ae, af the extreme right-hand and left-hand rays of the pencil of rays proceeding from that point, and passing respectively throngh the right-hand $e$, and left-hand $f$, holes in the card. (The figure is supposed to be a horizontal section of the eye, and a forms part of a transverse section of the vertically placed needle.) When the eye is accommodated for $a$, the rays $e$ and $f$ meet 
together in the point $c$, the retina occupying the position of the plane $n n$; the point in the needle appear's as one point, and the needle will appear as one needle. When the eye is accommodated for a distance beyond $a$, the retina may be considered to lie ${ }^{1}$ no longer at $n$, but nearer the lens, at $m m$ for example; the rays ae will cut this plane at $p$, and the rays of at $q$; hence the point in the needle will no longer appear single, but will be seen as two points, or rather as two systems of diffusion circles, and the single needle will appear as two blurred needles. The rays passing through the right-hand hole $e$, will cut the retina at $p$, i.e. on the right-hand side of the optic axis; but, as we have already (\$529) said, the image on the right-hand side of the retina is referred by the mind to an object on the left-hand side of the person; hence the affection of the retina at $p$, produced by the rays ae falling on it there, gives rise to an image of the spot $a$ at $P$, and similarly the left-hand spot $q$ corresponds to the right-hand $Q$. Blocking the left-hand hole, therefore, causes a disappearance of the right-hand image, aud vice versa. Similarly when the eye is accommodated for a distance nearer than the needle, the retina may be supposed to be removed to $l l$, and the right-hand $a e$ and left-hand af rays, after uniting at $c$, will diverge again and strike the retina in diffusion circles at $p^{\prime}$ and $q$. The blocking of the hole $e$ will now cause the disappearance of the image $q^{\prime}$ on the left-hand side of the retina, and this will be referred by the mind to the right-hand side, so that $Q$ will seem to vanish.

If the needle be brought gradually nearer and nearer to the eye, a point will be reached within which the image is always double. This point marks with considerable exactitude the near limit of accommodation. With short-sighted persons, if the needle be removed farther and farther away, a point is reached beyond which the image is always double; this marks the far limit of accommodation.

The experiment may also be performed with the needle placed horizontally, in which case the holes in the card should be vertical.

The determination of the accommodation of the eye for near or far distances may be assisted by using two needles, one near and one far. In this case one needle should be vertical, and the other horizontal, and the card turned round so that the holes lie horizontally or vertically according to whether the vertical or horizontal needle is being made to appear double.

$\$ 532$. In what may be regarded as the normal eye, the socalled emmetropic eye, the near limit of accommodation is about 10 or $12 \mathrm{~cm}$., and the far limit may be put for practical purposes

1 of course, in the actual eye, as we shall see, accommodation is effected by a change in the lens, and not by an alteration in the position of the retina; but for convenience sake, we may here suppose the retina to be moved. 
at an infinite distance. The 'range of distinct vision' therefore for the emmetropic eye is very great. In the myopic, or shortsighted eye, the near limit is brought much closer ( 5 or $6 \mathrm{~cm}$.) to the cornea; and the far limit is at a variable but not very great distance, so that the rays of light proceeding from an object not many feet away are brought to a focus in the vitreous humour instead of on the retina. The range of distinct vision is therefore in the myopic eye very limited. In the hypermetropic, or loug-sighted eye, the rays of light coming from even an intinite distance arc, in the passive state of the eye, brought to a focus beyond the retina. The near limit of accommodation is at some distance off, and a far limit of accommodation does not exist. The presbyopic eye, or eye of advanced years, resembles the hypermetropic eye in the near point of accommodation being at some distance, but differs from it inasmuch as the former is an essentially defective power of accommodation, whereas in the latter the power of accommodation may be good and yet, from the internal arrangements of the eye, be muable to bring the image of a near object on to the retina. When an eye becomes presbyopic, the far limit may remain the same, but since the power of accommodating for near cbjects is weakened or lost, the change is distinctly a reduction of the range of distinct vision. When no effort of accommodation is made, the principal posterior focus of the eye lies in the normal, emmetropic eye on the retina, in the myopic eye in front of it, and in the hypermetropic eye behind it.

$\S 533$. By what changes in the eye are we thus able, within the above mentioned limitations, to see distinctly objects at different distances? In directing our attention from a far to a very near object we are conscious of a distinct effort, and feel that some change has taken place in the eye; when we turn from a very near to a far object, if we are conscious of any change in the eye, it is one of a different kind. The former is the sense of an active exertion; the latter, when it is felt, is the sense of relaxation after exertion.

Since the far limit of an emmetropic eye is at an infinite distance. no such thing as active accommorlation for far distances need exist. The only change which need take place in the eye in turning from near to far objects will be a mere passive undoing of the accommodation previously made for the near object. And that $n$ such active accommodation for far distances takes place is shewn by the following facts: the eye, when opened after being closed for some time, is found adjusted not for moderately distant but for far distant objects; we are conscious of no effort in turning from moderately distant to far distant objects; and when the power of the eye to accommodate is impaired or abolished, as we shall see it may be, by atropin or nervous disease, the vision of distant objects may be maffected. The sense 
of effort often spoken of by myopic persons as being felt when they attempt to see things at or beyond the far limit of their range seems to arise from a movement of the eyelids, and not from any internal changes taking place in the eye.

What then are the changes which take place in the eye, when we accommodate for near objects? It might be thought, and indeed once was thought, that the curvature of the cornea was changed, becoming more convex, with a shorter radius of curvature, for near objects. This is disproved by the fact that accommodation takes place as usual when the eye (and head) is immersed in water. Since the refractive powers of aqueous humour and water are very nearly alike, the cornea, with its parallel surfaces, placed between these two fluids, can have little or no effect on the direction of the rays passing through it when the eye is immersed in water. Moreover we have it in our power to detect any change in the curvature of the cornea which may take place. If a luminous body such as a candle be held in front of a convex surface like the cornea an image of the body is seen reflected from the surface; and, with the body at a certain distance, the image will be of a certain size. If now the curvature of the surface be increased, if the surface be made more convex, the image will diminish in size; if the curvature of the surface be diminished the image will increase in size. Indeed by measuring carefully the changes in the size of the image we may determine the amount of change in the curvature of the surface. And accurate measurements of the dimensions of an image on the cornea have shewn that these undergo no change during accommodation, and that therefore the curvature of the cornea is not altered. Nor is there any ehange in the form of the bulb; for any variation in this would necessarily produce an alteration in the curvature of the cornea, and pressure on the bulb would act injuriously by rendering the retina anzemic and so less sensitive. In fact, there are only two changes of importance which can be ascertained to take place in the eye during accommodation for near objects.

One is that the pupil contracts. When we look at near objects, the pupil becomes small; when we turn to distant objects, it dilates. This however cannot have more than an indirect influence on the formation of the image; the chief use of the contraction of the pupil in accommodation for near objects is to cut off the more divergent circumferential rays of light.

'The other and really efficient change is that the anterior surface of the lens becomes more convex. If a light be held before the eye, three reflected images may, with care and under proper precautions, be seen by a bystander: one (Fig. $144 \mathrm{~A}, a$ ) a very bright one caused by the anterior surface of the cornea, a second less bright, $b$, by the anterior surface of the lens, and a 
third very dim, c, by the posterior surface of the lens; when the images are those of an object, such as the flame of a candle, in which a top and bottom ean be recognized, the two former images are secn to be erect, but the third inverted. When the eye is aceommodated for near objects, no change is olserved in the first, and none, or a very insignificant one, in the third of these images; but the second, that from the anterior surface of the lens, is seen to beeome distinctly smaller, shewing that the surface has become more convex. When, on the contrary, vision is direeted from near to far objeets, the image from the anterior
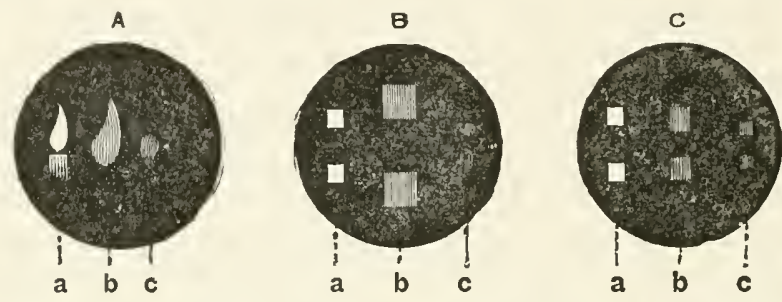

Fig. 144. Diagram of Images reflected from the Eye.

In $A$ are seen the three images of a candle reflected from $a$, the anterior surface of the cornea, $b$, the anterior surface of the lens, and $c$ the posterior surface of the lens. $a$ is bright and erect, $b$ also erect, is larger but less bright, $c$ inverted is small and dim.

B shews the images, two squares, as seen in the phakoscope when the eye is directed to a far object. $C$ the same when the eye is accommodated for a near object. 'The pair $b$ are in $\mathbf{C}$, smaller and closer together than in $\mathbf{B}$, shewing an increase of curvature.

surface of the lens grows larger, indieating that the eonvexity of the surface has diminished, while no change takes plaee in the image from the cornea, and none, or hardly any, in that from the posterior surface of the lens. And aeeurate measurements of the size of the image from the anterior surface of the lens have shewn that the ehanges in curvature whieh do take place are considerable; the radius of curvature of the lens aceommodated for near objects is $6 \mathrm{~mm}$., for far objects $10 \mathrm{~mm}$.; and this difference is sufficient to account for the power of aceommodation which the eye possesses.

The observation of these reflected images is facilitated by the simple instrument introduced by Helmholtz and called a Phakoscope. It consists of a small dark chamber, with apertures for the observed and observing eyes; a needle is fixed at a short distance in front of the former, to serve as a near object, for which accommodation lias to be made; and a lamp or candle is so disposed as to throw an image on each of the three surfaces of the observed eve. Since a change in the distance between two images is more readily appreciated than is a simple change of size of a single image, two prisms are employed so as to throw a double image in the form of bright squares on each 
of the three surfaces, Fig. 144 B, C. When the anterior surface of the lens becomes more convex the two images reflected from that surface approach each other, C, when it becomes less convex they retire from each other, B.

These observations leave no doubt that the essential change by which accommodation is effected, is an alteration of the convexity of the anterior surface of the lens. And that the lens is the agent of accommodation, is further shewn by the fact that after removal of the lens, as in the operation for cataract, the power of accommodation is lost. In the cases which have been recorded, where eyes from which the lens had been removed seemed still to possess some accommodation, we must suppose that no real accommodation took place, but that the pupil contracted when a near object was looked at, and so assisted in making vision more distinct. 
SEC. 3. THE MECHANISM OF ACCOMIIODATION AND THE NOVEMENTS OF THE PUPIL.

$\S 534$. How is this increase in the curvature of the anterior surface of the lens during accommodation for near objects brought about?

It has been supposed to be due to a compression of the circumference of the lens by a contraction of the sphineter muscle of the iris, which, as we shall see, is the cause of the narrowing of the pupil attendant upon accommodation for near objects; but this is disproved by the fact that normal accommodation may take place in eyes from which the iris is congenitally absent or has been wholly removed by operation. It has also been attributed to vaso-motor changes, to increased fulness of the vessels of the iris or ciliary processes, surrounding and pressing upon the lens; but this also is disproved, not only by the fact just mentioned but as well by the fact that aecommodation may be effected, after death, in an eye which is practically bloodless, - by stimulating the ciliary ganglion or short ciliary nerves with an interrupted eurrent or by other means; as we shall sec, these nerves govern the accommolation mechanism. The real nature of the mechanism seems to be as follows:

The lens is a body of considerable elasticity. When the curvature of the anterior surface of the lens is determined, as may be done by appropriate means (by measurements of images seen by reflection from it), in its natural position in the eye at rest, and then again determined, after the lens has been removed from the eye, the anterior surface is found to be more convex in the latter than in the former case. There seems to be in the eye in its natural eondition at rest some agency at work, keeping the anterior surface of the lens somewhat flattened. All that is neerled is some means of counteracting this agency, and thereby allowing the lens through its elasticity to assume its natural form. And the arrangements of the suspensory ligament deseribed in a previous section afford an explanation of what is the agency in question, and how it is counteracted.

The cavity of the eyeball behind the suspensory ligament is filled with the vitreous humour. If this is sufficiently abundant 
it will distend the cavity and render the suspensory ligament tense. But since the suspensory ligament passes obliquely forwards, all round, from the ciliary processes to the front of the lens, tension of the ligament will tend to flatten the lens, altering its shape but not its bulk.

'The choroid, of which the ciliary processes form the forward continuation, is loosely attached to the sclerotic along the line of the lamina fusca and suprachoroideal membrane; the one can to a certain extent be slipped backwards and forwards beneath the other.

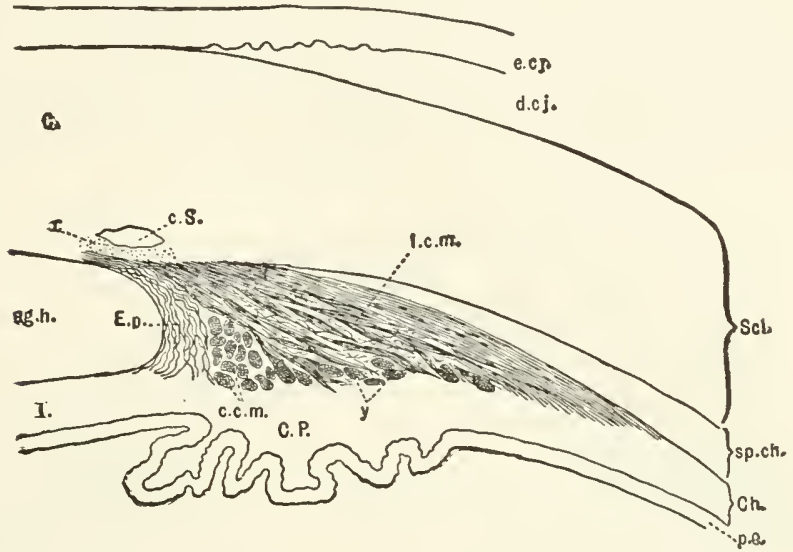

Fig. 145. Diagram of the Ciliary muscle as seen in a vertical radial SECTION OF TIIE CILIART REGION.

$E$.cj. epithelium of the conjunctiva. d.cj. dermis of the conjunctiva. Scl. Sclerotic. sp.ch. suprachoroidal layer. Ch. Choroid. p.e. pars ciliaris retinae and pigment epithelium represented as one layer. C.P. Ciliary processes. I. Iris. ag.h. anterior chamber. E.p. ligamentum pectinatum. c.S. canal of Schlemm, and $x$ tissue to inside of it.

l.c.m. longitudinal, and c.c.m. circular ciliary muscle. $y$ bundles of the longitudinal muscle cut across as they are taking a circular direction.

The (longitudinal) ciliary muscle (Fig. 145) is attached on the one hand to the junction of the sclerotic and cornea, and on the other hand to the front part of the choroid. If we suppose the former to be a fixed point, the contraction of the muscle would pull the moveable choroid and ciliary processes somewhat forward. But the pulling forward of these structures would slacken the suspensory ligament by bringing its ciliary attachment more forward. And a slackening of the suspensory ligament by relieving the pressure on the elastic lens would allow the front surface to become more convex. This is shewn diagrammatically in Fig. 146, one-half of which, the left half, is intended to represent the eye directed towards distant objects, while the other 
half represents the change taking place during accommodation for a nearer object.

$\$ 535$. It seems possible then that accommodation for near objects may be brought about by a contraction of the (longitudinal) ciliary muscle dragging forwards the choroid and ciliary processes, thus slackening the suspensory ligament, and so permitting the compressed elastic lens to bulge forward. And experimental evidence shews that this is what does take place. The ciliary muscle is governed, as we shall see presently, by the eiliary nerves. If in a living animal (dog) or in an eye immediately after removal from the body, an opening be made in the sclerotic in order to watch the choroid, it may be seen that when the ciliary nerves are stimulated the choroid does move forward at the same time that the frout surface of the leus becomes more convex; a needle, the point of which is carefully lodged in the

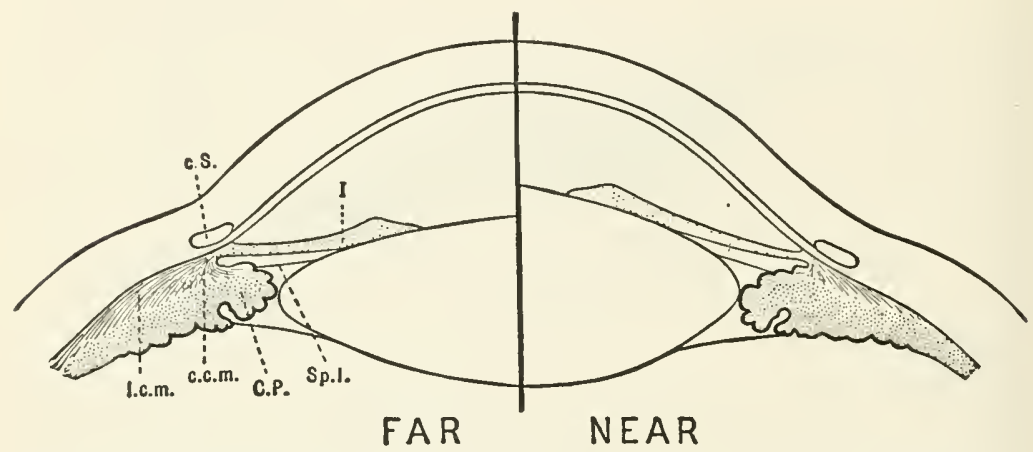

Fig. 146. Diagram to illustrate Accommodation. (After Helmholtz.)

C.P. Ciliary process. I. Iris. Sp.l. suspensory ligament. I.c.m. longitudinal ciliary muscle. c.c.m. circular ciliary muscle. C.S. canal of Schlemm.

The left half represents the arrangement for viewing far objects and the right half that for viewing near objects.

choroid, moves in such a way as to shew that the choroid moves forward, though no appreciable movement can be seen in a needle thrust into the front part of the ciliary muscle itself. If the cornea be cut away so as to leave only at one place a small fragment still connceted to the junction of the sclerotic and cornea, this piece moves backward when the ciliary nerves are stimulated, shewing that the ciliary musele does pull on the point of junction of the sclerotic with the cornea. When, however, the cornea is intact, or even when a sufficiently large part of it is left, the junction becomes a fixed point, at least relatively to the moveable choroid. Moreover not only the contraction of the ciliary muscle and movement of the choroid, but the actual slackening of the suspensory ligament and change in the curva- 
turc of the lens may be observed to follow upon stimulation of the ciliary nerves. We may conclude, therefore, that the possible explanation given above is the actual one.

One or two additional points are worth mentioning. During accommodation for near objects the pupil is narrowed; we shall speak of this presently. A narrowing of the pupil means that the edge and inner part of the iris moves over the front surface of the lens toward the centre of the pupil. In becoming more convex, the front surface of the lens, especially the central portion, projects further forward into the anterior chamber, and in so doing carries with it the pupillary edge and inner part of the iris; for the iris lies close upon and indeed in contact with the anterior surface of the lens. And when the eye is carefully watched sideways this projection forwards of the pupillary margin of the iris may be observed. While the edge of the pupil thus moves forward, and the body of the iris increases in a radial direction, becoming correspondingly thinner (cf. Fig. 146), the circumferential edge of the iris is carried slightly backwards, owing to the giving way to a certain extent of the elastic ligamentum pectinatum on which the ciliary muscle pulls; and thus additional space is afforded in the anterior chamber for the aqueous humour driven aside by the projection of the anterior surfice of the lens.

'The action of the circular', equatorial fibres of the ciliary muscle (Fig. 145) and of the fibres intermediate between these and the longitudinal meridional fibres, is not quite so clear. We may, however, suppose that the cireular fibres acting in concert with the longitudinal fibres would bring the ciliary processes nearer to the leus, and so assist in slackening the suspensory ligament. But no very decisive explanation has been given why the circular fibres are often largely developed in some eyes, it is said hypermetropic or long-sighted eyes, and scantily present in others, myopic or short-sighted eyes. And indeed there are several points in the whole action of accommodation which still require to be cleared up.

Accommodation is in a certain sense a voluntary act; we can by looking at near or far objects bring about the change whenever we please. Since, however, the change in the lens is always accompanied by movements in the ilis, it will be convenient to consider the latter before we speak of the nervous mechanism of the former.

\section{The Changes in the Pupil.}

§ 536. Although by looking at near or far objects, and so voluntarily bringing about changes in the accommodation mechanism, we can call forth the accompanying changes in the iris, and can thus at pleasure produce a constriction, narrowing, or a dilation, widening, of the pupil; it is not in our power to 
bring the will to act directly on the iris by itself. This faet alone indicates that the nervous mechanism of the pupil is of a special character, and such indeed we find it to be.

The pupil is constricted, eontracted, narrowed, (1) when the retina (or optic nerve) is stimulated, as when light falls on the retina, the brighter the light the greater being the contraction; (2) when we accommodate for near objects. The pupil is also constricted when the eyeball is turned inwards. when the aqueous humour is deficient, in the early stages of poisoning by chloroform, alcohol, and similar substances, in nearly all stages of poisoning by morphia, physostigmin, and some other drugs, in the early part of the day, in deep slumber, in the epileptic seizure, and in eertain nervous diseases. The pupil is dilated, widened, (1) when stimulation of the retina (or optie nerve) is diminished or arrested, as in passing from a bright into a dim light or into darkness, (2) when the eye is adjusted for far objects. Dilation also occurs when there is an excess of aqueous humour distending the anterior chamber, during dyspncea, during violent muscular efforts, as the result of stimulation of sensory nerves, as an effect of emotions, as an effect of fatigue, in the later stages of poisoning by chloroform, alcohol and similar substanees, in all stages of poisoning by atropin and some other drugs, and in certain nervous diseases.

$\$ 537$. We may say at once that we are able to recognize two separate nervous mechanisms regulating these ehanges in the pupil. One of these regulates the size of the pupil according to the amount of light falling upon the retina, and is by far the more important of the two; through the other, the size of the pupil is modified by other influences. We will consider the former mechanism first. During the action of this, constriction of the pupil is undoubtedly eauserl by contraction of the circularly disposed muscular fibres which form within the iris the sphincter muscle. The more or less spongy body of the iris being extensible, the shortening of the fibres and bundles of fibres of the sphincter must necessarily narrow the ring of the pupil of which the sphincter forms the almost immediate margin. Conversely, the body of the iris being elastic as well as extensible, a relaxation of the muscular fibres of the sphincter, assisted by the return to their natural position of structures displaced by the contraction, will lead to a widening of the pupil. We may in respect to this mechanism at all events consider the constricted pupil as the result of a contraction of the sphineter muscle, and the dilated pupil as the result of a diminution of that contraction. Whether a dilated pupil is always a mere negative result, dne to a lessening of the activity of the sphincter, or whether in certain cases an active dilator muscle is bronght into play we will discuss later on in connection with the other meehanism. 
\$538. Before proceeding it will be desirable to recall to mind the nervous supply of the eyeball, omitting for the present the nerves governing the six ocular muscles which move the eyeball as a whole.

The eyeball is supplied, in the first place, by the short ciliary nerves (Fig. 147 s.c.) coming from the ophthalmic or lenticular, or ciliary ganglion (l.c.) which is connected by means of its three roots, (1) through the so-called 'short root' with the third nerve (r.b.), (2) with the cavernous sympathetic plexus

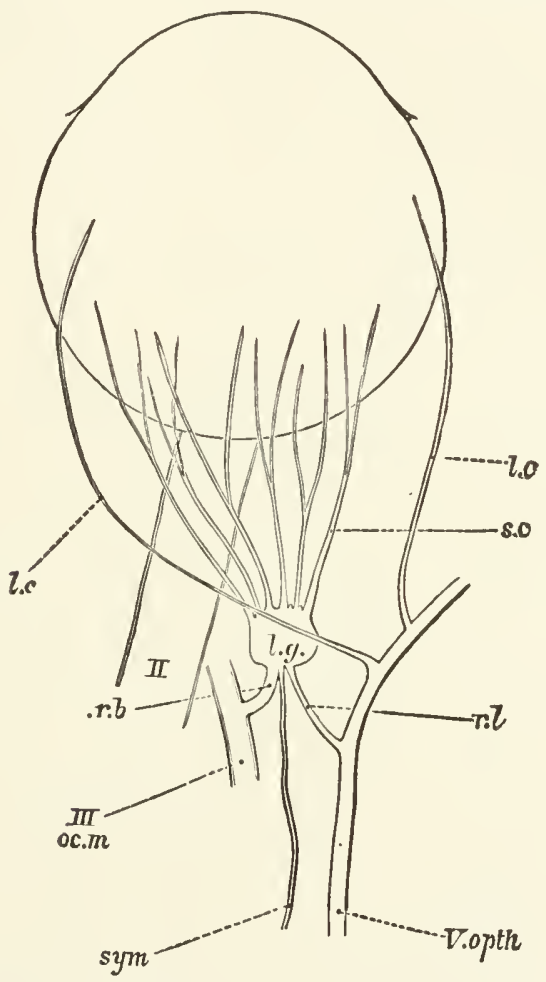

Fig. 147. Diagramiatic representation of the nerves governing the pupil.

II. Optic nerve. l.g. ciliary ganglion. r.b. its short root from III. oc.m., third or oculo-motor nerve. sym. its sympathetic root. $\quad r . l$. its long root from $V$. ophthm. the nasal branch of the ophthalmic division of the fifth nerve. s.c. the short ciliary nerves from the lenticular ganglion. l.c. the long ciliary nerves from the nasal branch of the ophthalmic division of the fifth nerve.

and so, along the internal carotid artery, with the cervical sympathetic nerve (sym.), and (3) through the so-called 'long root' with the nasal branch of the ophthalmic division of the fifth nerve $(r . l$.$) . Besides the short ciliary nerves, the eyeball is$ supplied by the long eiliary nerves (l.c.) coning direct from 
the nasal branch of the ophthalmic division of the fifth nerve. The short ciliary nerves, which are the most numerous, pierce the selerotic at the lind part of the eyeball and are distributed on the one hand to the blood vessels of the choroid, ciliary processes and iris, and on the other hand to the ciliary muscle and to the sphincter of the pupil. The less numerous long eiliary nerves, piercing the sclerotic somewhat nearer the front of the eye, are clistributed to the muscles of the iris, and probably to the ciliary muscle.

The third or oculo-motor nerve we may trace back to its nucleus in the floor of the aqueduct; the sympathetic root we may trace back along the cervical sympathetic to the spinal connections of that nerve, on which we have so often dwelt; the renarkable ophthalmic division of the fifth nerve we may trace back to the nucleus of the fifth nerve; this nerve is exceedingly complex, and indeed we have reason to consider its ophthalmic division as an independent nerve, which in the course of evolution has become annexed to other nerves to form what we call 'the fifth' nerve.

$\$ 539$. We may now make the broad statement, qualifications of which we will consider later on, that constriction of the pupil, brought about by light falling on the retina, is a reflex act, of which the optic is the afferent nerve, the third or oculomotor the efferent nerve, and the centre some portion of the brain lying in the front part of the floor of the aqueduct at the level of the anterior corpora quadrigemina. This is shewn by the following facts. When the optic nerve is divided, light falling on the retina of that eye no longer causes a constriction of the pupil: we are supposing that the observations are confined to one eye. When the third nerve is divided, stimulation of the retina or of the optic nerve no longer causes constriction; but direct stimulation of the peripheral portion of the divided third nerve causes constriction of the pupil which may be extreme. If the region of the brain spoken of above as the centre be carefully stimulated, constriction of the pupil will take place even when no light falls on the retina or after the optic nerve has been divided. After destruction of the same region stimulation of the retina is ineffectual in narrowing the pupil. But if the centre and its comnections with the optic nerve and third nerve be left intact and in thorouglily sound condition, constriction of the pupil will occur as a result of light falling on the retina, though all other parts of the brain be removed.

It might be imagined that this cerebral centre acted as a tonic centre, whose action was simply increased, not originated, by the stimulation of the retina; but this is disproved by the fact that if (still dealing with one eye) the optic nerve be divided subsequent section of the third nerve produces no further dilation. 
When the rootlets of the third nerve are separately divided as they leave the brain, it is found that section of those placed more anteriorly interferes with accommodation and constriction of the pupil, while section of the hinder ones affects the ocular muscles. Moreover if the hind part of the floor of the third ventricle and front part of the floor of the aqueduct be carefully explored (in the dog) by means of the interrupted current, the following movements may be observed in succession as the electrodes are shifted from the front backwards; first movements of accommodation, next constriction of the pupil, and then contractions of the ocular muscles. Now in this region lies the elongated nucleus of the third nerve; and it would appear that while the fibres of the third nerve concerned in accommodation arise from the extreme front of the nucleus, those which act upon the pupil start from a succeeding part, the remaining hinder part giving rise to the fibres which govern the ocular muscles. It seems therefore natural to regard the part of the nucleus from which the pupil-constricting fibres spring, as the centre of the reflex pupil-constricting mechanism, as the pupilconstrictor centre.

There is no difficulty as to the connection of the centre with the efferent limb of the reflex chain. The pupil-constrictor' fibres pass from the nucleus to the trunk of the third nerve of the same side, and so by the short root to the ciliary ganglion (Fig. $147 r . b$. ), whence they reach the pupil by the short ciliary nerves; section of the short ciliary nerves breaks the reflex chain of which we are speaking, and stimulation of them or of their peripheral ends causes narrowing of the pupil.

But considerable diffieulties are met with in determining the connection of the optic fibres, the afferent limb of the chain, with the centre. We should perhaps naturally suppose that the afferent nervous impulses which affected the pupil were the same as, or at least took the same course as those which gave rise to visual sensations; but visual sensations may be interfered with or even abolished, leaving the pupil-constrictor mechanism still active, and on the other hand the afferent limb of the latter may fail, without any impairment of visual sensations. The afferent impulses by means of which light constricts the pupil, seem therefore to take a path of their own; but the matter is not as yet fully worked out.

It is desirable to remember one important difference as to the behaviour of the pupil which obtains between man and some of the higher mammals on the one hand, and the lower mammals as well as other vertebrates on the other. In the former, the pupil-constricting nervous mechanisms of the two eyes are not completely independent; there is a functional communion between the two sides, so that when one retina is stimulated both pupils contract, and indeed, in man, as a rule, contract equally. 
Hence, when a change in the pupil of one eye is brought about by some means other than the one we are now considering, the pupil of the other eye is affected; when for instance one pupil is dilated with atropin, the larger amount of light thus admitted into that cyc causes a narrowing of the pupil of the other eye, and thus increases the difference between the pupils of the two eyes. In the lower mammals and other vertebrates, the mechanisms in question are independent, stimulation of one retina produces no effect on the pupil of the other eye.

It is by means of this reflex mechanism of which we have just given a sketch that the changes of the pupil which take place in actual life are to a large extent carried out; a constricted pupil indicates in the majority of instances an activity of the reflex mechanism, and a dilated pupil the absence of or diminution of that activity. In the normal, healthy organism the activity of the mechanism is in the first instance dependent on the amount of light falling on the retina; but even in the normal condition, and still more in an abnormal condition of the organism, other influences may become dominant. The activity of the centre may be exalted or depressed by nervous or other actions; the retina or optic nerves may be affected by the same amount of light to a degrce less than or greater than the normal, and the efferent limb of the chain may be less or more effective.

$\S 540$. Besides, however, all the various changes which may thus be induced by playing upon the optic-oculo-motor reflex mechanism, other agencies are able to act on the pupil quite apart from this reflex mechanism; some of these act through the second mechanism of which we spoke, and to which we can now turn.

If the cervical sympathetic in the neek be divided, all other influences which could possibly affect the pupil being avoided, a constriction of the pupil will be seen to take place; this however is at times (in animals) not very well marked; but, whether it be so or no, if the peripheral portion of the nerve (i.e. the upper portion still connected with the head) be stimulated, a well-dcveloped dilation is the result. The cervical sympathetic has, it will be observed, an effect on the pupil, the opposite of that which it exercises on the blood vessels of the head and neck; when it is divided, the pupil becomes constricted but the blood vessels dilate, and when it is stimulated the pupil is dilated while the blood vessels are constricted. This pupil-dilating influence of the cervical sympathetic may, as in the casc of the vaso-constrictor action of the same nerve, be traced backwards down the neck to the upper thoracic ganglion, and thence to the spinal cord along the rami communicantes and anterior roots of certain thoracic nerves. In all the higher animals the chief channel is the second thoracic nerve; in the eat, dog, monkey, and probably in man some of the impulses pass by the first thoracic nerve and a few by the third; in the rabbit a very few pass by the first 
but a good many by the third nerve. In the frog the channel is the fourth spinal nerve. Along the spinal cord the dilating influence may be further traced up through the bulb to a centre, which appears to be placed in the floor of the front part of the aqueduct not far from and apparently lateral to the centre for constriction of the pupil. Some authors have supposed that a part of the spinal cord in the lower cervical or upper thoracic region above the origin of the second thoracic nerve has a special share in carrying out the dilating action and hence have called this region the centrum ciliospinale inferius: but this seems very doubtful. Since, as a rule, a very decided amount of narrowing of the pupils follows upon mere section of the cervical sympathetic, we may infer that, unlike the case of the pupil-constrictor mechanism, tonic impulses habitually proceed from the pupildilator centre.

We may trace the path of dilating impulses in the other direction upwards along the cervical sympathetic, not to the sympathetic root of the ciliary ganglion and so to the short ciliary nerves, but to fibres which, passing over the Gasserian ganglion apply themselves to the ophthalmic division of the fifth nerve, and from thence along the nasal branch to the long ciliary nerves, and so to the iris; while the short ciliary nerves are the channels for pupil-constrictor impulses, the long ciliary nerves are the channels of pupil-dilator impulses.

$\$ 541$. But while the mode of action of the pupil-constrictor impulses seems clear, since these have simply to throw into contraction, or increase the contraction of, the fibres of the sphincter, the mode of action of the pupil-dilator impulses is a matter which has been and still is disputed. In the first place, considering how vaseular the iris is, it does not seem unreasonable to interpret some of the variations in the condition of the pupil as the results of simple vascular turgescence or depletion brought about by vaso-motor action or otherwise. When the blood vessels are dilated and filled they will cause the iris to encroach on the pupil, making the latter snall and narrow, and conversely a constricted and emptied condition of the blood vessels would lead to the pupil being large and wide. And indeed slight oscillations of the pupil, due to greater or less fulness of the blood vessels, may be observed synchronous with the heart-beat, and others synehronous with the respiratory movements. Hence, remembering how conspicuous a channel for vaso-constrictor impulses is the cervical sympathetic, it seems very natural to suppose that the widening of the pupil which follows upon stimulation of the cervical sympathetic is simply the result of the constriction of the blood vessels of the iris, and conversely that the narrowing of the pupil observed after section of the cervical sympathetic is simply the effect of a greater fulness of the iridic blood vessels resulting from the falling away of the 
usual vaso-constrictor impulses. A further support to this view is afforded by the observations that the cervical sympathetic does contain vaso-constrictor fibres for the blood vessels of the iris, and that these leave the spinal cord by the same paths as the pupil-clilator impulses, that is to say somewhat ligher up than the vaso-constrictor fibres for the ear. Nevertheless it seems clear that the pupil-dilating influence exerted by the sympathetic is something quite different from its vaso-constrictor influence; for the dilating effects of stimulating the sympathetic may be witnessed in a bloodless eye, in which vaso-motor changes could not produce their effect. Further, the changes in the pupil and in the calibre of the iridic blood vessels are not coincident; when the sympathetic is stimulated the widening of the pupil begins some time before the constriction of the blood vessels and indeed may, with a brief stimulation, be over and past, before the latter has reached its maximum. Again, in the extreme widening of the pupil which as we shall see is brought about by atropin, and which seems to be of the same nature as the widening caused by stimulation of the sympathetic, the blood vessels of the iris need not be in the least constricted. Moreover, it is stated that the long ciliary nerves which act as pupil-dilators carry no vaso-constrictor impulses; it is said that stimulation of the long ciliary nerves while it widens the pupil produces no vaso-motor effects, and after the division of the long ciliary nerves stimulation of the cervical sympathetic, while it produces vaso-constriction in the eye as in other parts of the head and face, gives rise to no widening of the pupil. The impulses along the fibres of the cervical sympathetic, which cause widening of the pupil, must act in some manner other than by giving rise to vascular changes.

Did there exist in the eyes of animals an arrangement of muscular fibres disposed radially from the circumference of the iris to the pupil as conspicuous as the circular muscle, since such a muscular arrangement would act as a dilator, there would probably be a general agreement that the widening which results from stimulation of the sympathetic is brought about by contraction of these dilator muscular fibres. But it is only in the case of nne or two kinds of animals that any such distinct radial muscles are present in the iris, and even in these cases the muscles are not conspicuous. In all other aninals including man, the only structure in the iris which can be appealed to as a dilator muscle, is a peculiar nucleated layer at the hinder surface, just in front of the pigment epithelium. The absence of any clear indubitable dilator muscle has led some to explain the pupil-dilating influence of the sympathetic as due to the impulses along that nerve inhibiting the previously existing contraction of the sphincter. These argue that the sphincter may be compared to the heart, inasmuch as it possesses an automatic power 
of contraction, manifested however not in a rhythmic but in a tonic manner, and that like the heart its action may be either augmented or inhibited by nervous impulses; and we have seen $(\$ 3+7)$ that a similar view may be taken of the actions of the plain muscular fibres of the alimentary canal and of the bladder. According to this view the sphincter of the iris, when removed from all influences, is in a state of tonic contraction, pulling against the radial strain of the elastic tissue of the iris and so maintaining a pupil of a certain size. Under the influence of light falling on the retina, impulses reaching the sphincter by the short ciliary nerves augment its contraction, and narrow the pupil in proportion to their intensity. On the other hand, inspulses reaching the sphincter from the sympathetic by the long ciliary nerves inhibit the activity of the sphincter, diminish the force with which it is pulling against the elastic tissue of the iris, and so lead to a widening of the pupil, thus either diminishing the constriction which is being caused by the action of light on the retina or otherwise, or, in the absence of all external constricting influences, causing the pupil to become wider than it naturally would when removed from all extrinsic influences whatever. In support of such a view it is pointed out that the muscular tissue forming the sphincter is peculiar, since a slip of it when directly stimulated by a weak interupted current elongates; in this respect it shews a further analogy with the heart whose activity may similarly be inhibited by the interrupted current. Again, in the extirpated eye, or even in the isolated iris, cold dilates and warmth constricts the pupil, the one relaxing, and the other increasing the contraction of the sphincter. On the other hand the following facts seem to shew that the dilation which we are discussing cammot be simply a matter of inhibition but must be due to the radial pull of some or other contractile elements. By stimulating one ciliary nerve at a time, or by partial direct stimulation of the iris with an electric current, the two electrodes being placed close together on the sclerotic near the outer margin of the iris, an unequal cliange, in the iris, an uneven pupil may be produced; and this, since it may be brought about even while the sphincter is contracting, must be due to a radial pull. Further, a mere radial slip of the iris, a slip cut out by two radial incisions carried from the margin of the pupil towards the sclerotic, the slip remaining comnected with the sclerotic, may be made by stimulation to shorten. Lastly, since this shortening does not appear, when the hind surface of the iris has been previously sharply brushed, so as to injure it, we may conclude that the layer spoken of above, peculiar though its characters be, is really a radially disposed contractile tissue, and by its contraction dilates the pupil.

Whatever be the view adopted as to the exact mode of action of the sympathetic there remains the broad fact that the pupil is 
under the dominion of two antagonistic mechanisms : one a constricting mechanism, reflex in nature, the third nerve serving as the efferent, and the optic as the afferent tract; the other a dilating mechanism, apparently tonic in nature, but subject to augmentation from various causes, and of this the cervical sympathetic is the efferent chamnel. Hence, when the third or optic nerve is divided, not only do constricting impulses cease to be manifest. but the effect of their absence is increased. on account of the tonic dilating influence of the sympathetic being left free to work. When, on the other hand, the sympathetic is divided. this tonic dilating influence falls away, and constriction results. When the optic or third ncrve is stimulated, the dilating effect of the sympathetic is overcome, and constriction results ; and when the srmpathetic is stimulated, any constricting influence of the third nerve which may be present is orercome, and dilation ensues.

'The former, optic oculo-motor mechanism is the instrument by means of which the pupil is adapted to the amount of light, the latter, sympathetic mechanism appears to be employed when other influences are brought to bear on the pupil. 'Thus the characteristic pupil-dilating effects of cmotions such as fear. of the painful stimulation of sensory nerves, of dyspncea. and in part of some drugs, appear to be carried out through the sympathetic mechanism.

We may add that both these mechanisms may he thrown into action by stimulation of certain parts of the ocular area in the cerebral cortex; constriction or dilation may be obtained by stimulation of the appropriate spot. 'That the dilation which is observed is brought about by means of the sympathetic mechanism, is shewn by the fact that it fails if the cervical sympathetic be previously divided.

$\$ 542$. In the case of many drugs, however, the effect produced is either in part or wholly independent of both these nervous mechanisms. A small quantity of atropin introduced into the system. or even directly into the eje. causes a dilation of the pupil which may be so great that the iris is reduced to a mere rim, while physostigmin (eserin) similarly introduced into the system or eye produces a constriction of the pupil which may be so great that the pupil is narrowed to a mere pin's point. Since both these drugs may produce their full effects after division of the optic oculo-motor and the sympathetic nerves, and indeed may produce their effects in an extirpated eveball, it is obvious that those effeets are not due to the drugs acting on the central parts of the above mechanisms. 'Their action is a local one. 'They do not act by means of the ciliary ganglion. for both drugs continue to produce their effects to a most marked degree after the ganglion has been excised. Nor have we any evidence that their action is dependent on any other local nervous mech- 
anism. such as might be afforded by the nerve cells lying in the choroid or even in the iris. 'They appear to act directly on the sphineter, atropin paralyzing it or producing relaxation, and physostigmin increasing or producing contraction, both often of an extreme character. Whether the drugs act on the actnal muscular tissue itself or on the endings of the nerve fibres in the muscular tissue, or on both together, and how far their effect is due to changes in the special dilator muscle, are questions which we need not discuss here. The important point is that the action of both these drugs is a local one ; hence, when they have produced their full effects, the normal nervous mechanisms on which we have been dwelling are of little or no nse ; even an abundance of light leads to no constriction in the full at ropinized eye, and removal of light produces little or no dilation in an eye fully under the influence of physostigmin.

We may here mention the fact that in certain animals at all events, for instance the eel, light falling into the cye, even into an extirpated eye, will cause constriction of the pupil ; and this seems to be brouglit about by means of some nervous connection between the retina and the iris, for the effect ceases when the retina is destroyed. But this peculiar action has not yet been satisfactorily explained.

The share of the fifth nerve in the work of the iris seems to be chicfly at least a sensory one: the iris is sensitive, and the sensory impulses which are generated in it pass from it along the fibres of the fifth nerve.

We may sum up the nervous mechanism of the pupil then somewhat as follows. The salient and most frequently repeated event, the constriction of the pupil upon exposure to light, is a reflex act, the centre of which is placed in the brain ; and the correlative widening of the pupil upon diminution of light is due to the tonic action of the sympathetic making itself felt upon the waning of its antagonist. The dilating effects of emotions, of sensory impressions, especially painful ones, and of dyspmua appear to be brought abont by an increased activity of the dilating centre, assisted possibly in the latter instance by a depression of the constricting centre. The constriction of the pmpil in the earlier stages of the action of alcohol and chloroform and in slumber is probably due to an increased action of the constricting centre, but the narrow pupil caused by such a drug as physostigmin is due, chiefly if not exclusively, to a local action. The constricted pupil of morphia appears to be due partly to central and partly to local action. The dilating effects of such a drug as atropin are chiefly if not exclusively due to a local action, but in the widened pupil of the later stages of alcohol poisoning and of some other drugs we can probably trace the effects of an exhanstion of the constricting centre, assisted possibly by an increased activity of the dilating centre. 
\$543. The nervous mechanism of accommodation. The ciliary muscle which brings about accommodation is governed in this action by fibres which may be traced, through the short ciliary nerves and ciliary ganghion, along the third nerve, to a centre which lies (in rogs ) in the extreme front of the Hoor of the aqueduct, or rather perhaps in the extreme hind part of the floor of the third rentricle, and which is especially connected with the extreme front of the moleus of, and so with the most anterior bundles of the root of, the third nerve. As we have already said stimulation of this centre, or of the third nerve, or of the short ciliary nerves, leads to a contraction of the ciliary muscle and to accommodation for near objects.

'This nervous mechanism, mlike that for the pupil, is under the command of the will, thongh the will needs to be assisted by visual sensations; it is moreover only hrought into play by the direct action of the will; we are not led to accommodate by any other influence than the desire to sce distinctly near or far objects. 'The mechanism may, however', be aftected by the local action of drugs. Such drugs as atropin and physostigmin which have a special action on the pupil, also affect the mechanism of accommodation. Atropin paralyzes it, so that the eye remains aljusted for far objects; and physostigmin thows the eye into a condition of forced accommodation for near objects. 'This double action has been explained by the supposition that, by acting on the muscular fibres, or on the nerve endings, or on both, atropin inhibits the contraction of or paralyzes, while physostigmin throws into contraction or angments the contraction of the ciliary muscle. But the phenomena, on further inquiry, are fornd to be more complieated than they appear to be at first sight. 'There are also other facts which indicate that our knowledge of the mechanism of accommodation is far from being complete. For instance, so far as we know at present, when we pass from accommodation for a near object to that for a far object, we simply 'let go' the previous effort; we cease to contract the ciliary muscle, and the return of the suspensory ligament and other parts is simply the passive result of the cessation of the contraction of the eiliary muscle. If, now the change from near to far be such a mere passive relaxation of a previous contraction we should, judging trom onr experience of ordinary muscular contractions, expect the time taken up by it to be greater, or at least not less than the time taken up by the change from far to near ; but as a matter of fact it is very much shorter, indeed the act is an exceedingly rapid one.

$\$ 544$. 'There remains a word to he said concerning the constriction of the pupil which takes place when the eye is accommodated for near objects, and when the pupil is tmined inwarls (the two being closely allied, since the two cyes converge to see near objects), and the return to the more dilated condition when 
the eye returns to rest and regains the condition adapted for viewing far objects. These are instances of what are called "associated movements." A similar instance is afforder by certain cases of blindness of one eye due to atrophy of the optic nerve; in such cases the pupil of the blind eye may be wholly insensible to light, and yet becomes narrowed when the subject looks at a near object with the sound eye. In so doing he throws into action the accommodation mechanism, and with that the pupil-constricting mechanism of both eyes. Two movements are thus spoken of as "associated" when the special central nervous mechanism employed in carrying out the one act is so connected by nervous ties of some kind or other with that employed in carrying out the other, that when we set the one mechanism in action we unintentionally set the other in action also. In this eonstriction of the pupil associated with accommodation the nervous ties between the parts of the central nervous system concerned in the generation of the will, the centre for accommodation, and the centre for the constriction of the pupil, are such that whenever the will stirs up the impulses necessary to carry out accommodation, it at the same time stirs up corresponding impulses in the pupil-constrictor mechanism. More than this we camnot at present say.

We can, as we have said, accommodate at will; few persons only can effect the necessary change in the eye unless they direct their attention to some near or far object, as the case may be, and thus assist their will by visual sensations. By practice, however, the aid of external objects may be dispensed with ; and it is when this is achieved that the pupil may seem to be made to dilate or contract at pleasure, accommodation being effected without the eye being directed to any particular object. 


\section{SEC. 4. IMPERFECTIONS IN THE DIOPTRIC APPARATUS.}

$\S 545$. Imperfections of accommodation. The emmetropic eye, in which the principal posterior focus lies on the retina, may, as we have said, be taken as the normal eye. The myopic, in which the principal posterior focus lies in front, and the hypermetropic eye, in which it lies beyond the retina, may be considered as im:perfect eyes, though the former possesses an advantage over the normal eye in so far that it can see minute objects more distinctly than can the normal eye, since these can be brought so near the eye as to give a relatively large retinal image and yet remain within the limits of accommodation. An eye may be myopic from too great a convexity of the cornea, or of the anterior surface of the lens, or from permanent spasm of the accommodationmechanism, or from too great a length of the long axis of the eyeball. The last appears to be the usual cause. Similarly, the cause of hypermetropism is in most cases the possession of ton short a bulb. In presbyopia the failure or loss of accommodation may be due either to a loss of elasticity of the lens, or to increasing weakness of the ciliary muscle, or to the parts becoming rigid: the first appears to be the more common cause; the change, which may affect not only normal but also other eyes, generally begins in the fifth decade of life.

These several defects may be remedied by the use of appropriate lenses, by wearing proper spectacles. The myopic eye needs for distant objects the rays of which fall parallel on the cornea (or at least so little divergent that they still are brought to a focus in front of the retina) a concave glass, of such a refractive power, of such a focal length, as to give to parallel rays, before they fall on the cornea, snifficient divergence to enable the dioptric mechanism of the eye to bring then to a focus on, and no longer in front of, the retina.

The hypermctropic eye needs a convex glass of such a focal length as will give to parallel rays, before they fall on the cornea, sufficient convergence to enable the eye to bring them to a focus on the retina. 
The presbyopic eye similarly needs a convex glass the focal length of which must depend on the amount of accommodation still possessed by the eye; it must give the rays just so much convergence that the weakened mechanism is able to bring them to a focus on the retina, the convexity or refractive power of the glass being increased, that is to say its focal length diminished, as the loss of accommodation increases.

$\$ 546$. Spherical aberration. In a spherical lens the rays which are refracted by the circumferential parts are brought to a focus sooner than those which pass through the more central parts ; in consequence the rays proceeding from a luminous point are no longer brought to a single focus at one point, but form a number of foci at different distances. Hence, when rays are allowed to fall on the whole of the lens, the image formed on a screen placed in the focus of the more central rays is blurred by the diffusion-circles caused by the circumferential rays which have been brought to a premature focus. In an ordinary optical instrument spherical aberration is obviated by a diaphragm which shuts off the more circumferential rays. In the eye the iris is an adjustable diaphragm; and when the pupil contracts in near vision the more divergent rays proceeding from a near object, which tend to fall on the circumferential parts of the lens, are cut off. The lens however, as we have seen, is not uniform in structure, and the refraction which it exercises does not, as in the case of the ordinary lens, increase regularly and progressively from the circumference to the centre, but varies most irregularly; hence the purpose of the narrowing of the pupil cannot be simply to obviate splierical aberration; and indeed the other optical imperfections of the eye are so great, that such spherical aberrations as are actually caused by the lens produce no obvious effect on vision.

$\$ 54 \%$. Astigmatism. We have hitherto treated the eye as if its dioptric surfaces were all parts of perfect spherical surfaces. In reality this is rarely the case, either with the lens or with the cornea. Slight deviations from the spherical shape do not produce any marked effect, but there is one deviation, known as regular astigmatism, which, present to a certain extent in most eyes and very largely developed in some, frequently leads to very imperfect vision. This defect is due to one or other of the dioptric surfaces being not spherical but more convex along one meridian than another, more convex, for instance, along the vertical than along the horizontal meridian. When this is the ease with the dioptric surface of an optical system the rays proceeding from a luminous point are not brought to a single focus at a point, but possess two linear foci, one nearer than the normal focus and corresponding to the more convex surface, the other farther than the normal focus and corresponding to the less convex surface. If the vertical meridians of the surface be more convex than the 
horizontal, then the nearer linear focus will be horizontal and the farther linear focus will be vertical and viceversa. ('This can be shewn much more effectually on a model than in a diagram in which we are limited to two dimensions.) Now, in order to see distinctly a vertical line, it is much more important that the rays which diverge from the line in a series of horizontal planes should be brought to a focus properly than those which diverge in the vertical plane of the line itsclf ; for the former contribute to a far greater extent than do the latter to the sum of rays which go to form the retinal image of and so to excite the sensation of the line. Similarly, in order to see a horizontal line distinctly it is much more important that the rays which diverge from the line in a serics of vertical planes should be brought to a focus properly than those which diverge in the horizontal plane of the line itself. When a horizontal line is held before an astigmatic dioptric surface, more convex in the vertical meridian, it will give rise to a strong image of a horizontal line at the nearer focus where the many vertical rays diverging from the line are brought to a linear horizontal focus, and to a weak image of a vertical line at the farther focus where the fewer horizontal rays are brought to a linear vertical focus. Similarly, a vertical line held before the same surface will give rise to a strong image of a vertical line at the farther focus where the horizontal rays diverging from the vertical line are brought to a lincar vertical focus, and to a weak image of a horizontal line at the nearer focus. But in the case of an astigmatic eye trying to see a horizontal or vertical line or rod such as a horizontal or vertical needle, the mind will neglect the weaker image, and take the stronger image as the only image of the object. Hence if a horizontal and a vertical needle be placed at the same distance before an astigmatic eye. which is more convex in the vertical mcridian, that eye will see a horizontal needle distinctly when the nearer, and a vertical necdle distinctly when the farther of the two foci falls on the retina; it will requirc a different accommodation to see the one and the other distinctly. If the astigmatism is such that the horizontal meridian be the more convex, the rertical needle will be seen most distinctly at the nearer, and the horizontal at the farther focus. In both forms of astigmatism the horizontal and the vertical lines which go to make up the features of the surface of an object will fail of being seen distinctly at the same timc; and the vision of the object will be imperfect.

Rays of light procceding from a line, which is ncither vertical nor horizontal but oblique, give rise in an astigmatic system to a number of foci arranged in so complex a mamncr that no distinct image can be formed on the retina; the presence of these lines accordingly adds to the imperfection of the vision of any ohject.

Most eyes are thus more or less ' regularly' astigmatic, and 
generally with a greater convexity along the vertical meridian. If a set of horizontal or vertical lines be looked at, or if the near point of accommodation be determined by Scheiner's experiment, for the needle placed first horizontally and then rertieally, the distance from the eye at which the horizontal lines or needle are seen distinetly will be found, in most cases, to be appreciably and in many cases considerably shorter than that at which the vertical lines or needle are seen with equal distinctness. In other words, in the case of most eyes, a vertical line must be farther from the eye than a horizontal one. if both are to be seen distinctly at the same time. The cause of astignatism is, in the great majority of eases, the unequal curvature of the cornea; but sometimes the fault lies in the lens, as was the case with the philosopher Young.

Regular astigmatism may be remerlied hy the use of cylindrical glasses, that is to say, glasses which are convex along one meridian but plane along the other. Thus the ordinary astigmatic eye with the greater curvature along the vertical meridian will be benefited by a cylindrical glass, plane in the vertical plane but possessing such convexity in the horizontal plane as will make up for the relatively deficient horizontal curvature of the cornea.

When the curvature of the cornea or of the lens differs not in two meridians only but in several, irregular astigmatism is the result. A certain amount of irregular astigmatism, due to the cornea or lens, exists in most eyes, thus eamsing the image of a bright point, such as a star, to be not a round dot but a radiate figure; in some cases the irregularity is so great that several imperfect images are formed of every object.

$\$ 548$. Chromatic aberration. The different rays of the spectrum are of different refrangibility, those towards the violet end of the spectrum being brought to a focus sooner than those near the red end. 'Ihis in optical instruments is

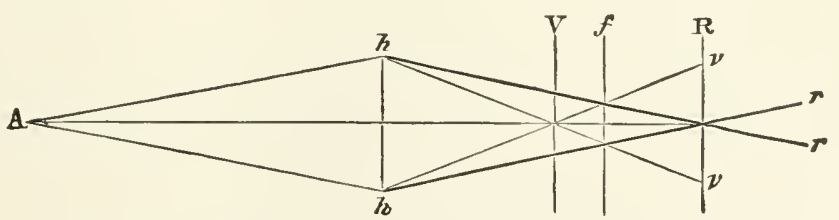

Fig. 148. Diagram illustrating Cimomatic Aberration.

$h h$ is the dioptric surface, $h v$ represents the blue, and $h r$ the red rays ; $V$ is the focal plane of the blue, $R$ of the red ray.

obviated by using compound lenses made up of various kinds of glass. In the eye we have no evidence that the lens is so constitutcd as to correct this fault; still the total clispersive 
power of the instrument is so small, that such amount of chromatic aberration as does exist attracts little notice. Nerer-theless some slight aberration may be detected by careful observation. When the spectrum is observed at some distance off the violet end will not be seen in focus at the same time as the red end. Again, if a luminous point be looked at througl a narrow orifice covered by a piece of violet glass, which while shutting out the yellow and green allows the red and bluc rays to pass through, there will be seen alternately an image having a blue centre with a red fringe, or a red centre with a blue fringe, according as the image of the point looked at is thrown on one side or other of the true focus. Thus suplosing $f$ (Fig. 148) to be the plane of the mean focus of $A$, the violet rays will be brought to a focus in the plane $T$, and the red rays in the plane $R$. If the rays be supposed to fall on the retina between $V$ and $f$, the diverging or blue rays will form a centre surrounded by the still converging red rays; whereas if the rays fall on the retina between $f$ and $R$, the converging red rays will form a centre with the still diverging blue rays forming a fringe round them. If the rays fall on the retina at $f$, the two kinds of rays will be mixed together; as will be seen from the figure, the circumferential still converging red ray $h r$ as it cuts the plane of the retina is, in ordinary vision, accompanied by the diverging riolet ray $h v$, and thus by a sort of compensation, we see together, though not in absolutely proper focus, even the rays which differ most in refraction. 'The experiment may be varied by blocking up one half of the pupil with a piece of card and using the mncovered half of the pupil to look through a picee of violet glass at a white surface or a candle flame. 'The red strip will be seen to have a blue edge.

$\$ 549$. Entoptic phenomena. The various media of the eye are not uniformly transparent; the rays of light in passing through them undergo local absorption and refraction, and thus various shadows are thrown on the retina, of which we become conscions as imperfections in the field of vision, especially when the eye is directed to a miformly illmminaterl surface. These are spoken of as entoptic phenomena, and are very varied, many forms having been described.

Tears on the cornea, or temporary uncremness of the anterior surface of the cornea after the eyelid has been pressed on it, may give rise to retinal images and so to visual sensations; but in these cascs the canse lies outside the eye and the result can hardly be spoken of as entoptic.

Changes in the margin of the pupil appear in the shadow of the iris which bounds the field of vision. If we look at a bright object or luminous surface through a pin-hole in a card placed close in front of the eye (in order to get the best image 
on the retina, the pin-hole should occupy the position of the principal anterior focus), the dark circle which bounds the field of vision is the image caused by the shadow of the margin not as might at first be supposed of the pin-hole but of the iris. This is at once shewn by the changes which it can be marle to undergo, while the pin-hole remains motionless, by alternately closing and opening the other ere; the field of vision of the eve which is looking through the pin-lole may be observed to contract when light enters, and to expand when the light is shut off from the other cye; for as we have scen (\$539) light falling on one retina learls to consensual narrowing of the pupil of the other eve. Other changes or irregularities in the iris may be observed by this method.

Imperfections in the lens or in its capsule may also give rise to entoptic images. Not unfrequently a radiate figure corresponding to the arrangement of the fibres of the lens makes its appearance.

The most common entoptic phenomena are those caused by the presence of floating bodics in the vitreous humour, the socalled musce volitantes. These are rearlily seen when the eye is turned towards a uniform surface, and are frequently very troublesome in looking through a microscope. They assume the form of rows and groups of beads, of single bearls, of streaks, patches and granules, and may be recognized by their ahnost continmal movement, especially when the head or eye is moved up and (lown. When an attempt is made to fix the vision upon them they immediately float away.

Since the images on the retina are in these cases shadows and since the strongest shadows are cast by parallel rays, the images are best seen when the rays of light giving rise to the shadows on the retina traverse the vitreous humour in parallel lines; hence the best illumination for examining the phenomena is one placed in the principal anterior focus, the rays diverging from which fall parallel on the retina $(\$ 526$, Fig. 140). The sharpuess of the images is also increased by using a small but bright source of light, as by looking at a bright light through a small hole in a screen.

The sensations which these objects in the vitreous humour excite by means of the retinal images to which they give rise do not tell us that the objects are in the vitreous humour. As we shall see we refer all affections of the retina, all visual sensations to some changes in the external world ; and if we trusted to our sensations alone in the cases of these entoptic phenomena we should suppose that the causes existed outside ourselves. It is only by means of inferences drawn from the features and behaviour of the sensations that we arrive at the conclusion that the causes lie in the vitreous humomr.

The accompanying diagram (Fig. 149) illustrates how the 
position of objects in the eye may be determined by the movements of their shadows on the retina. It represents the reduced diagrammatic eye seen in vertical section, with $n$ the nodal point, $p$ the principal plane, and $F$ the plane of the prineipal anterior focus. 1 represents an object in the anterior chamber, 2 another in the substance of the lens, and 3 a third in the vitreous humour. If a bright light be looked at through a pin-hole in a card placed

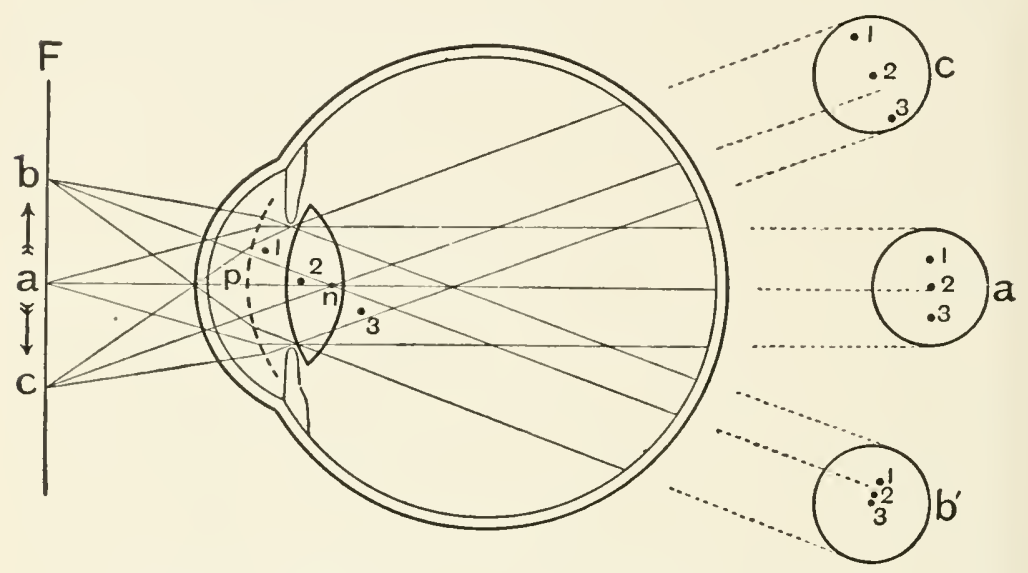

Fig. 149. Diagram to illustrate Entoptical Images.

in the plane of the principal anterior focus $F$ so that the hole is at the principal anterior focus $a$, the rays of light may be considered as diverging from $a$, and we may draw them as refracted at the principal plane $p$, and then passing parallel through the vitreous humour. 'The image on the retina in this case may be represented by $a^{\prime}$. The ficld of vision, limiterl by the shadow of the iris, will be circular ; the shadow of 2 will lie close to the optic axis, that of 1 a little above it, and that of 3 some little way below it. It will of course be remembered that in the apparent image all the features will be inverted (\$ $\$ 07$ ). If now the card be moved upward so that the light emanates from the pin-hole at $b$, and the paths of the rays of light be drawn as before, the image resulting will be that shewn at $b^{\prime}$. 'The shadow of 2 las changed very little in position; but that of 1 has moved downwards, while that of 3 has moved upwards so that all three lie closer together. If, on the contrary, the card be moved downward to $c$ the result will be that shewn in $c^{\prime}$; the shadow of 2 , as before, has moved but little, while that of 1 has moved upward, and that of 3 downwards, so that the three sharlows are farther apart.

Thus while the shadows of objects in the anterior chamber move in a direction the opposite to that of the movement of the 
source of an illumination placed in the plane of the principal anterior focus, the shadows of objects in the vitreous humour move in the same direction as the source of illumination. Hence. by observing the direction of the novement of an entoptic image resulting from the movement of the illumination, the position in the eye of the object giving rise to the image may be determined, regard of course always being had to the so-called mental inversion of the retinal image. Stated more strictly the rule would run thus. The shadows of objects in front of the nodal point $(\$ 527)$ in the lens move in a direction contrary to and those of objects behind the nodal point in the same direction as the movement of the illumination ; moreover the more distant the object from the nodal point the greater the movement of the shadow caused by the same movement of the illumination.

In this connection we may refer to one or two matters which however cannot be called dioptric imperfections.

If a white sheet or white cloud be looked at in daylight through a Nieol's prism, a somewhat bright double cone or double tuft, with the apices touching, of a faint blue colour, is seen in the centre of the field of vision, crossed by a similar double cone of a somewhat yellow darker colour. These are spoken of as Haidinger's brushes ; they rotate as the prism is rotated, and are supposed to be due to the unequal absorption of the polarized light in that part of the retina which we shall study presently as "the yellow spot." The prism must be frequently rotated, since when the prism remains at rest the phenomena fade. We may here remark that the media of the eye are fluorescent : a condition which favours the perception of the ultraviolet rays. There are other entoptic phenomena due to features of the retina, of which we shall speak in treating of the development of visual impulses.

Lastly, returning to dioptric imperfections, we may add that the optical arrangements are also to a certain extent imperfeet inasmuch as the dioptric surfaces are, according to most observers, not truly centred on the optic axis. 


\section{SEC. 5. ON SOME GENERAL FEATURES OF VISUAL SENSATIONS.}

$\$ 550$. When light falls upon the retina it produces, under favourable circumstances, a change in our consciousness which we call a sensation of light, a visual sensation. The immediate effect of the light is to stir up certain changes in the retina; these retinal changes give rise in turn to nervous changes in the optic fibres; these latter, which we have called 'visual impulscs, start in the brain a further series of events, one effect of which is a change in our consciousness; and it is this change in our consciousness which we call a sensation. We may, and often do, speak of light as a 'stimulus' to the retina, the result of the stimulation being visual impulses; but we may also speak of light as a stimulus to the whole visual apparatus, central as well as retinal, regarding the sensation as if it were the direct and immediate, instead of being the indirect and nltimate effect of the stimulus. We may, by observing certain general features of visual sensations, such as can be ascertained by means of a dircet and simple appeal to our own consciousness, study the relations which obtain between the characters of the stimulus on the one hand and those of the sensation on the other. There are certain advantages indeed in doing this before we proceed to discuss the nature of the changes in the retina through which rays of light give rise to visual impulses in the optic fibres. But in taking this course we must bear in mind how complex is the whole process through which the stimulus gives rise to the sensation. We must remember that, as we lave already said, though some of the characters of a visual sensation are impressed upon it while it is as yet immature, as yet in the stage of visual impulses, others are introduced later on in the course of the cerebral changes. Since we are now dealing for the first time with sensory impulses studied in this way, we may venture to enter into some details, for the deductions which may be drawn concerning visual sensations will apply to a large extent to other sensations.

To simplify matter's we will in the first instance suppose that the luminous object, the object enitting or reflecting light, is so 
small that the image of it on the retina may be considered as a mere point; we may speak of it as a luminous point. If for the sake of illustration or otherwise we have to consider a larger luminous objetet. we shall do so without regard to the size of the image on the retina unless this is specially mentioned.

We may hegin with the preliminary remark that in dealing with light as a stimulus of visual sensations, we have to consider not only the intensity of the stimulus but also its duration. A luminous point may appear dim and feeble, that is to say, the waves of light from it have a small amplitude and so bring little energy to bear on the retina, or it may appear bright and strong, that is to say, the waves of light have a large amplitude and so bring much energy to bear on the retina. Whether dim or bright, the luminous point may act on the retina for a longer or a shorter time; and, moreorer, during its action may remain steady, not varying in intensity, or may vary in intensity and become unsteady or flickering. In estimating the total visual effect of a luminous point, we have to consider both these features, its intensity or brightness and its duration.

Neglecting for the present the feature of duration, we find that a luminous point must possess a certain amount of brightness in order to prodnce any conscions sensation at all, in order to be visible. If the waves of light fall on the retina with less than a certain amplitude, if their energy sinks below a certain minimmm, they fail to give rise to risual impulses, or at least to such as can affect consciousness ; for we may suppose that visual impulses might be generated and yet be so feeble as not to produce in the cerebral centre changes sufficiently great to affect consciousness. It will be understood, of course, that the exact degree of brightness at which the luminous point becomes visible depends on the greater or less irritability. on the sensitiveness. of the retina. The same amount of luminous energy which, falling on one retina or on one part of a retina. produces a distinct sensation, may, falling on a less sensitive retina or on a less sensitive part of the same retina, produce no sensation whatever.

From the minimum onvards the intensity of the sensation increases with the luminous intensity of the object; a wax canclle appears brighter than a rushlight, and the sun brighter than any eandle; we are dealing now with the intensity of the light quite apart from the size of the luminous objeet. The ratio, however, of the sensation to the stimulns is not a simple one. If the luminosity of an ohject be gradually increased from a very feeble stage to a very bright one, it will be found that, thongh the corresponding sensations likewise gradually inerease, the increments of the sensations lue to the increments of the luminosity gradually diminish, and at last an increase of the luminosity produces no appreciable increase of sensation ; a light, when it reaches a certain brightness, appears so bright that if it 
becomes hrighter we lo not recognize that it is brighter. Hence it is much easier to distinguish a slight difference of brightness between two feeble lights than the same difference between two bright lights; we can easily tell the difference between a rushlight and a wax candle; lut two suns, or even two bright lamps, one of which compared with the other gave out just that additional amount of light. just that additional quantity of luminous energy, which a wax candle gives out in addition to that given out by a rushlight, would appear to us to have exactly the same hrightness. In a darkened room an object placed before a candle will throw what we consider a decp shadow on a sheet of paper or any white surface. If, however, stulight be allowed to fall on the paper at the same time from the opposite side, the shadow is no longer visible. 'The difference between the total light reflected from that part of the paper' where the shadow was, and which is illuminated by the sun alone, and that reflected from the rest of the paper which is illuminated by the candle as well as by the sum. remains the same; yet we can no longer appreciate that difference because the whole surface has become so bright.

On the other hand, when we carefully compare the visual sensations excited by measurable differences of luminosity, we come candles so as to throw two shadows of some object on a white surface, the shadow caused by each light will be illuminated by the other light, and the rest of the surface will be illuminated by both lights. If now we move one candle away we shall reach a point at which the shadow caused by it ceases to be visible, that is to say, we fail at this point to appreciate the difference between the surface illuminated by the near light alone and that illuminated by the near light and the far light together. If now, having noted the distance to which the candle had to be moved, we repeat the same experiment with two bright lamps, moving one lamp away until the shadow it casts ceases to be visible, we shall find that the lamp has to be moved just as far as the candle; that is to say, the least difference between the illumination of the bright lamps which we can appreciate is the same as in the ease of the dimmer candles. Many similar examples might be given shewing a similar result; in fact, it is found by careful observation that, within tolerably wide limits, the smallest difference of light which we can appreciate by visual sensations is a constant fraction (about $\frac{1}{100}$ th) of the total luminosity employed. As we shall see, the same relation holds good with regard to the otlier senses as well. It may be put in a general form, as a law of sensation, often called Weber's law, somewhat as follows: 'The smallest change in the magnitude of a stimulus which we can appreciate through a change in our sensation always bears the same proportion to the whole magni- 
turle of the stimulus. It should however be stated that this law holds good within certain limits only ; it fails when the stimulus is either above or below a certain range of intensity.

Hence, if we take the smallest difference which we can appreciate in a stimulus as a measure of our sensibility to differences in the stimulus, we may say that on the one hand in respect to absolute differences, such as that between two lamps and that between two rushlights, our sensibility varies inversely as the magnitude of the stimulus; we are more sensible of the same absolute difference when that is a difference between two rushlights than when it is a difference between two lamps. On the other hand, in regard to relative differences, our sensibility is independent of the magnitude of the stimulus; the difference of which we are sensible in the case of the lamp bears the same proportion to the whole luminosity of the lamp as the difference of which we are sensible in the case of the rushlight bears to the whole luminosity of the rushlight.

$\$ 551$. Returning now to consider the duration of the stimulus, as distinguished from its intensity, we find that a stimulus of extremely brief duration may give rise to a distinct sensation ; the flash of an electric spark, for instance, is readily visible. There is probably a limit in respect to duration within which the stimulus fails to produce a sensation; it is probable, for instance, that a certain number of undulations in suecession must fall on the retina in order to give rise to a visual sensation, and that a single undulation of the ether falling on the retina, if such a thing were possible, would produce no visual effect; but the exact limit will depend on the intensity and nature of the light, and we need not enter upon these details here.

It is of more importance to note that the visual sensation caused by a very brief stimulus lasts a considerable time; the sensation has a duration much greater than that of the stimulus. 'The sensation of a flash of light, for instance, lasts for a much longer time than that during which luminous vibrations are falling on the retina. In this respect, we may roughly compare a visual sensation to a simple muscular contraction caused by such a stimulus as a single induction shock. We might indeed construct a "visual sensation curve" very much after the fashion of a "muscle curve." We should find that after a very obvious latent period the sensation began, rose to a maximum and then declined. This latent period forms an important part of the "reaction period." on which we dwelt in a former part of this work (\$515). As we have said, in all the sensations with which we are now dealing, we have to distinguish at least two parts, the peripheral part, the events taking place in the retina, and the central part, the events taking place in the brain, the two being united by means of the visual impulses passing along the optic nerve. And within the latent period are comprised the 
changes in the retina which start the visual impulses, the passage of these impulses along the optic fibres, and the changes in the brain antecedent to consciousness beginning to be affected ; of these the retinal changes probably take up the most time, but into this point we cannot enter now.

The length of the sensation, as compared with that' of the stimulus, is illustrated by viewing objects in motion under a very brief illumination, such as that of a single electric spark. In such a case the light reflected from the object is sufficient to generate a distinct sensation, to give rise to a distinct image of the object, but it ceases before the object can make any appreeiable change in its position, and the image accordingly is that of a motionless object. When a moving body is illuminated by several rapid flashes in succession, several distinct images corresponding to the positions of the borly during the sereral flashes are generated; this, as we shall see presently, is because the images of the body corresponding to the several flashes fall on different parts of the retina.

The duration of the stimulus remaining the same, the characters of the sensation and the form of the sensation curve will, in accorlance with what was stated above, vary with the intensity of the stimulus; a bright flash will produce a sensation greater and of longer duration than that produced by a feeble flash, the curve will be higher and more extended. We have reason to think, too, that the form of the curve is dependent on the intensity of the stimulus in such a way that the decline from the maximum begins earlier and at all events in the first part of its course, is more rapid with the stronger than with the feebler stimulus.

When the stimulus is not a mere flash, but is of some duration leading to a prolonged sensition, we can readily distinguish between that part of the sensation which is going on while the light is still falling into the eye, and that part which goes on after the light has ceased to fall on the retina; this latter part is often spoken of as the after-image. When the light is very bright this "after-image" frequently becomes very prominent even after a very brief exposure. 'Thns, if we look, even for a moment only, at the sun, and then immediately shut the eye, an intense visual sensation, a bright visual image of the sun, remains for some considerable time. After-images, especially as they are vanishing, are marked by certain features, which we shall stndy later on, and which, as we shall see, are related to the fatigue or exhaustion of the retina; for the retina, or rather the whole vismal apparatus, is, we need hardly say, subject to fatigue.

Careful observation moreover has shewn that the visual sensation eurve is not a smooth one but broken in a remarkable mamner. When the retina is momentarily stimulated with a 
bright light, the sensation almost immediately that it has begun suddenly diminishes or even disappears and then is immediately again re-establisherl.

$\$ 552$. From the prolonged duration of visual sensations it results that when two or more stimuli, such as two or more flashes of light, follow each other at a sufficiently short interval, the two sensations or the several successive sensations are fused into one more or less uniform sensation. Thus a luminous point moving rapidly round in a circle gives rise to the sensation of a continuous circle of light. We might, in a very general manner, compare this with the way in which a series of simple muscular contractions resulting from rapidly repeated induction shocks are fused into a fairly uniform tetanus. When the stimuli succeed each other so rapidly that each sensation begins before its predecessor has had time to appreciably decline, the total sensation is as completely uniform as if the stimulus were constant. If the interval between each two stimuli be just so long that each sensation in turn has had time to distinctly diminish before the next sensation begins, the result is a "flickering "; and there are of course many degrees of flickering between a perfectly steady and an obviously intermittent light. 'The interval at which fusion takes place, that is, the interval between successive stimuli which must be exceeded in order that successive distinct sensations may be produced, varies according to the intensity of the light, being shorter with the stronger light; with a faint light it is about $\frac{1}{10}$ sec., with a strong light $\frac{1}{30}$ or $\frac{1}{50}$ sec. This may be shewn by rotating rapidly before the eye a disc arranged with alternate black and white sectors of equal width. With a faint illumination, the flickering indicative of the successive sensations from the white sectors not being completely fused, ceases when the rotation becomes so rapid that each pair of black and white sectors takes only $\frac{1}{10}$ sec. in passing before the eye. When a brighter illumination is used the rapidity must be increased before the flickering disappears; this is owing to the decline of the stronger sensation, as stated above, beginning earlier and being more rapid than that of the weaker sensation.

$\$ 553$. When a luminous point excites the retina, we recognize in the sensation not only the features of intensity, duration and constancy or steadiness, but also a character which is dependent on th position in the retina of the image of the lumi. nous point. We recognize the sensation caused by a luminous point whose image falls on the temporal side of the retina, as different and distinct from the sensation caused by a luminous point whose image falls on the nasal side of the retina, and so with other positions of the images; indeed, as we shall see presently, we are able to distinguish, to recognize as different and distinct, two sensations excited by two luminous points, the 
inages of which lie very close indeed to each other on the retina. We distinguish the sensations, however, not by reference to the parts of the retina affected, but by reference to the relations in space of the luminous points giving rise to the sensations. Since this is a matter of some importance we may treat of it in some detail.

In the vast majority of cases the changes in the retina which give rise to visual impulses, and so to visual sensations, are brought about by light falling on the retina. But the retina may be stimulated by other agencies than that of light. When this is the case the changes in the retina, however produced, if they are able to affect consciousness at all, give rise to visual sensations, and to visual sensations only. A mechanical stimulation of the retina, as when a blow is struck on the eye, produces a visual sensation, a sensation of light; pressure exerted on the eyeball so as to produce pressure on the retina gives rise to visual sensations in the form of rings of light, of coloured light. known as "phosphenes'; and when the retina is subjected in various ways to stress or strain, as by rapid accommodation, or by rapid movement of the eyeball from side to sidc, there often result visual sensations in the form of light of some kind or other, best appreciated when objective light is cut off from the retina and when the retina has by long repose been rendered unusually sensitive. Electrical stimulation also gives rise to visual sensations: not only is the induced current, or the break and make of a constant current, thus able to excite the retina, but during the whole time of the passage of a constant eurrent of adequate strength, even though it remain of uniform intensity, visual impulses, and thus visual sensations, are being generated; in this respect the retina resembles sensory and differs from motor nerves. It is stated that when the current is directed from the layer of optic fibres to the layer of rods and cones, the sensation is a positive one, a sensation of light or of increased light, but that a current in the reverse direction gives rise to a negative sensation, a sensation of diminished light, a sensation of blackness.

That the stimulation of retinal structures by other agents than light may thus give rise to visual sensations, and apparently to visual sensations alone, may be verified by experinent at any time. The occasions on the other hand are rare in which evidence can be gained as to whether stimnlation of the optic nerve apart from the retina, whether stimulation of the optic fibres themselves, and not of their special endings in the retina, also gives rise to visual sensations and to visual sensations alone. In certain cases of removal of the eye it has been stated that when the optic nerve was divided in the abscnce of anesthetics, the patient "saw a great light" accompanied by no more pain than could be accounted for by the filaments of the fifth nerve 
which are distributerl to the optic nerve as nervi nervorum. Such experiences are urged in support of the view that all impulses passing along the optic nerve however generated, whether by retinal changes or by other means, are visual impulses and visual impulses only ; they give rise to visual sensations and to visual sensations alone. On the other hand, in other cases of removal of the eye in the absence of anesthetics. neither section of the optic nerve nor subsequent stimulation of the stump has given rise to visual sensations. We shall return to this question later on when we have to speak of what is known as the "specific energy of nerves," and have only referred to it incirlentally now.

5554 . Visual sensations then may be produced in many other ways than by the falling of light on the retina ; and the point to which we wish to call attention now is that we are unable to distinguish a sensation thus produced from the risual sensation produced by light itself. WTe camot by the help of the mere sensation alone recognize the nature of the agency which has produced the changes in the retina giving rise to the sensation. 'The identity of sensations due to mechanical stimulation with those due to luminous stimulation may be illustrated by the story of the witness in a case of assault, who swore that he, in the lark. recognized his assailant by help of the flash of light produced by a blow on his eye. Since light emitted or reflected from external objects is the normal stimulus for visual sensations, all our visual sensations seem to us to be produced by rays of light proceeding from external objects; we look for their cause not in the retina itself, but in the external world; and when we wish to know why we have felt the sensation of a flash of light, we ignore the retina and seek at once in the external world for some source of the rays of light corresponding to the sensation. Hence, also, when in a particular part of the retina, in a spot for instance on the nasal side of the right eye, changes take place such as would be produced by the image of a luminous point falling on that spot, though we recognize the sensation which results as having a certain feature, owing to its being started in that particular spot, we do not through the sensation learn anything about the retina itself, we do not through it recognize that the nasal side of the retina or any particular spot in the nasal side has been affected ; what we do recognize, or infer, is the existence in the external world of such a luminous point as woukl give rise to the sensation in question. The dioptric arrangements of the eye are, as we have seen ( $\$ 529)$, such that a luminous point in order to give rise to an image in the spot in question, and so to the sensation in question. must occupy a particular position on what we call the right-hand side of the external world. We accordingly recognize the sensation as having been caused by, or refer the sensation to, a luminous point having that position on our right hand. And so with the sensa- 
tions similarly generated in all other spots in the retina; we recognize theim as caused by luminous points occupying such positions in the external world that their images fall on those spots. In each case we ignore the retina itself, and the changes taking place in it are to us simple tokens of luminous events in the external world. When with the right eye we see a luminous point on our right-hand side, if we know that changes are taking place on the nasal side of the retina of that eye, it is not because we are directly aware that the nasal part of the retina is being affected. but because our knowledge of the dioptrics of the eye teaches us that the image of the luminous point is falling on the nasal side of the retina. If we are suffering from right-sided hemiopia $(\$ 497$ ) all that our sensations can of themselves tell us is that we cannot see things on the right-hand side; they do not tell us anything about the retima itself; they camnot even tell us whether the deficiency of vision is due to changes failing to be set up in the retina or to the cerebral centres failing to be affected by the retinal changes; such questions we have to decide by some other means than a simple examination of our sensations, and by a similar roundabout way only are we able to conclude that in such a hemiopia it is the nasal sicle of the right retina, and the temporal side of the left retina, which fail to give rise to visual sensations. Our sensations, in fact, tell us of themselves nothing about the optical image on the retina; they do not tell us whether the retinal image is inverted or no ; the fact that the retinal image is an inverted one does not in itself influence our visual sensations, and hence the inversion needs no correction on our part.

\$555. As we have just said, if the images of two luminous objects, two luminous points, fall on the retina at a certain listance apart, the consequent sensations are distinct. If, however, the two objects are male to approach each other, a point will be reached at which the two sensations are fused into one. Two stars at a certain distance apart may be seen distinctly as two stars, while two stars nearer each other appear to be one star ; we cannot analyze the latter sensation into its constituent parts.

Similarly, when the images of a number of luminous points, of equal luminosity, fall on the retina sufficiently near each other, the effect is not a number of sensations of luminous points, but one sensation, that of a luminous surface. This introduces a new feature of visual sensations, namely, that of size. If the luminous points be few, so as to involve a small area of the retima, the sensation is that of a small surface; if the luminous points, equally near to each other as before, be numerous, so as to involve a large area of the retina, the sensation is that of a large surface. Noreover, such a sensation of a surface will be referred to some position in space corresponding, 
as we have just seen, to the region of the retina affeeted, and will possess features determined by the relative positions of, that is, by the fignre formed by the luminous points; it will be the sensation of a surfaee of a certain form, round, square or the like; thus the retinal area stimulated supplies data, which are used, in a manner which we shall study later on, for judging the size and form as well as the position of visible objeets.

When the images of two luminous points are at a eertain distance apart on the retina, the two sensations may have no appreeiable effeet whatever on eaeh other; but when they are within a certain distanee from each other, the sensations do affect eaeh other, in a manner which we shall study later on. Meanwhile we will merely say that when two images approaeh so elosely that the two sensations become fused into one, sueh a mutual influenee is exerted that the intensity of the total sensation produeed is greater than that of either of the sensations caused by a single image, though less than the sum of the two. A number of luminous points seattered over a wide surface would appear each to have a eertain brightness; eaeh would give rise to a sensation of a eertain intensity. If they were all gathered into one spot, that spot would appear brighter than any of the previous points; the intensity of the sensation would be greater.

$\$$ 556. The region of distinct vision. The distanee at whieh two images must be apart from eaeh other in orler that the two sensations may be separate is not the same for the whole area of the retina. If two luminous points lie near the optie axis, so that their images fall on the fovea centralis or on the yellow spot, they will be seen as two distinet points, even when their images lie very elose indeed to each other. If the lmminous points be moved aside, so that the images fall on the retina outside the yellow spot, the two luminous points, though at the same distanee apart from each other, will give rise to one sensation only, and be seen as one point; they may be moved even farther apart from each other and still give rise to one sensation; and if the two points be plaeed so much on one side that their respeetive images fall on the extreme peripheral parts of the retina near the ora serrata, the two images may be separated from eaeh other a very considerable distanee and yet give rise to one sensation only. We may vary the experiment by making use of a negative sensation, and take two blaek dots on a white surface only just so far apart that they ean be seen distinetly as two when plaeed near the axis of vision so that their images fall on or near the fovea, and then, keeping the axis fixed, move the two points outwards, so that their images travel outwards from the fovea ; it will be found that the two soon appear as one. The two sensations beeome fused, as they would do if brought nearer to each other in the centre of the field. The farther 
away from the centre of the field, the farther apart must two points be in order they may be seen as two.

It is obvious that the more sharply we can distinguish the several sensations produced by the images of the several points of which any external object may be supposed to be made up, the more distinct will be our vision of the object. In the fovea centralis our power of thus distinguishing sensations is at its maximum; in the outer parts of the yellow spot around the forca it is less; just outside the yellow spot it is much less; and thence diminishes more gradually towards the periphery of the retina. Hence we speak of the forca centralis, including more or less of the whole yellow spot, as the "region of distinct vision;" and when we wish to examine closely the featurcs of an external object, we so direct the eye, we so 'look' at the object, that its image falls as far as possible on the fovea centralis. The diminution of distinctness does not take place equally from the centre to the circumference along all meridians. The outline described by a line uniting the points where two spots at a certain distance apart cease to be seen as two when moved along different radii from the centre, is a very irregular figure; it differs very much in different individuals; is often not the same in the two eyes of the same person, and does not necessarily correspond to the figure of " $"$ the field of vision" to which we shall later on refer. "We may add that the power of distinguishing two points in the peripheral parts of the retina is much increased by practice.

As we have just said, when we look intently at an object such as a star in the heavens we so direct the eye that the image of the object falls on the fovea centralis. In the case of most people, two stars so looked at appear to become one when the angle subtended by the distance between them becomes less than 60 seconds or one minute; when they are nearer than this the two sensations become one. And similar measurements are obtained when other images are made to fall on the fovea, such as those of parallel white streaks on a black ground or black streaks on a white ground. In the case of an acute and trained observer this minimum distance may be diminished to 50 seconds; in many cases, on the other hand, it is not less than 73 seconds and may be more. Now the distance between two points subtended by an angle of 50 seconds, corresponds in the diagrammatic eye ( $\$ 527$ ) to a distance of $3.65 \mu$ in the retinal image, and of 7.3 seconds to $5.36 \mu$. Hence in the fovea centralis the elements of the retina excited by light, must lic $3 \cdot 65 \mu$ or $5 \cdot 36 \mu$ apart, or in round numbers about $4 \mu$ apart, in order that the two sensations, excited at the same time, may remain distinet.

In the periphery of the retina the distance must be much greater; thus at the extreme periphery, two black dots distant apart about $15 \mathrm{~mm}$. viewed at a distance of $20 \mathrm{~cm}$. and there- 
fore giving a distance of more than a millimeter in the retinal image, are still seen as one point.

557. In accordance with the above, we may suppose the retina to be divided into areas, stimulation of the retina within which gives rise to a single sensation; we might speak of these as visual areas, and of the stimulation of a visual area as a sensational unit. 'The areas are very small, and the sensational units very numerous in the fovea centralis and yellow spot; the areas are larger, and the sensational units fewer, over the rest of the retina, increasingly so towards the periphery. 'The smaller or larger the areas, the more numerous or fewer the sensational units in any retina or in any part of a retina, the more or less distinct will be the vision.

Now in the human eye 50 cones may be counted along a line of $200 \mu$ in length drawn through the centre of the yellow spot; this would give $4 \mu$ for the distance between the centres of two adjoining concs in the yellow spot, the average diameter of a cone at its widest part being here about : $\mu$ and there being slight intervals between neighbouring cones. Hence if we take the centre of a cone as the centre of an anatomical retinal area, these anatomical areas correspond very fairly in the region of distinct vision to the physiological visual areas just spoken of. If two points of the retinal image are less than $4 \mu$ apart, they may both lie within the area of a single cone; and it is just when they are less than about $4 \mu$ apart that they cease to give rise to two distinct sensations. It must be remembered, however, that the fusion or distinction of the sensations is ultimately determined by the brain. The retinal area must be carefully distinguished from the sensational unit, for the sensation is a process whose arena stretehes from the retina to certain parts of the brain, and the circumscription of the sensational unit, though it must begin as a retinal area, must also be continued as a cerebral area, the latter corresponding to, and being as it were the projection of, the former. Two points of the retinal image less than $4 \mu$ apart might lie both within the area of a single cone; but the reason why, under such circumstances, they give rise to one sensation only is not because one cone-fibre only is stimulated. For, two points of a retinal image might lie, one on the area of one cone and another on the area of an adjoining cone, and still be less than $4 \mu$ apart; in such a case two conefibres would be stimulated; and yet only one sensation would be produced.

In the ease where the two points lie entirely within the area of a single cone, it is exceedingly probable that, even if the adjacent cones or cone-fibres in the retina are not at the same time stimulated, impulses radiate from the cerebral ending of the excited cone into the neighbouring cerebral endings of the neighbouring cones; in other words, the sensation-area in the 
brain does not exactly correspond to and is not sharply defined like the retinal area, but gradually fades away into neighbouring sensation-areas. We may imagine two points of the retinal image so far apart that cren the extreme margins of their respective cerebral sensation-areas do not touch each other in the least; in such a case there can be no doubt about the two points giving rise to two sensations. We might, however. imagine a second case where two points were just so far apart that their respective sensation-areas should coalesce at their margins, and yet that, in passing from the centre of one sensation-area to the centre of the other, we should find on examination a considerable fall of sensation at the junction of the two areas ; and in a third case we might imagine the two centres to be so close to each other that in passing from one to the other no appreciable diminution of sensation could be discovered. In the last case there would be but one sensation, in the second there might still be two sensations if the marginal fall were great enough, even though the areas partially coalcsced.

That the ultimate differentiation of the sensations rests with the brain is still more clear in the case of sensations started in the periphery of the retina ; two points of a retinal image might stimulate two cones a considerable distance apart, or screral cones, to say nothing of the intervening rods, might be stimulated, and yet one sensation only result.

Thus, the distinction or fusion of visual sensations is ultimately determined by the disposition and condition of the cerebral centres. Hence the possibility of increasing by exercise the faculty of distinguishing two sensations, since by use the cerebral sensation-areas become more and more differentiated, though the mosaic of rods and cones fixes for the power of discrimination of each individual a limit beyond which exereise cannot carry improvement. This effect of exercise is however shewn in touch even more strikingly than in sight. 


\section{SEC. 6. ON COLOUR SENSATIONS.}

$\$ 558$. The sensation excited by a luminous point possesses still another character besides those of intensity, duration, constancy, and localization, namely the one which we speak of as colonr.

When we allow sunlight reflected from a white cloud or from a sheet of white paper to fall into the eye, we have a sensation which we call that of white light. When we look at the same light through a prism and allow different parts of the spectrum to fall in succession into the eye, we have a series of sensations, differing in character from the sensation of white light and from each other; these we call 'colour sensations,' sensations of red, yellow, and the like. In the latter case the luminous undulations are dispersed in a linear series according to their wavelengths, from the short waves of the extreme violet to the long waves of the extreme red; and we learn from the spectrum, on the one hand, that undulations having different wave-lengths produce different sensations, and on the other hand that undulations having wave-lengths longer than that of the extreme red, about $\lambda 760,{ }^{1}$ or shorter than that of the extreme violet, about $\lambda$ 390, are unable to excite the retina and are therefore invisible. When we look directly at a white object all this dispersion is absent, and the retina is excited at the same time by undulations of all the above wave-lengths. A sensation of 'colour' then is a sensation evoked by undulations of particular wavelengths, a sensation of 'white' is the sensation which results when the retina, or a part of it, is simultaneously excited by undulations of all wave-lengths which are able to affect it, that is by the whole visible spectrum. When we direct our eyes to an object in such a way that the rays of light proceeding from it might fall on the retina when we bring the object within our field of vision, and yet experience from it neither any sensation of white nor any of the various colour sensations, we call the resulting affection of consciousness a sensation of 'black,' we say that we see 'black.' Sometimes the word 'colour' is confined to the sensations other than those of white and black, sometimes it is used to comprise these two sensations as well.

$1 \lambda$ signifies a millionth of a millimeter or $.001 \mu$. 
When we examine the speetrum we are able to pereeive a very large number of different colours, we experience a multitude of sensations, no two of which are exactly alike. There are certain broad differenees which we express by common names, such as red, orange, yellow and the like. But we ean go much further than this. If we take any part of the spectrum, the green for instance, we find that a very slight change in the wave-length produees a ehange in the character of the sensation. For eonvenience' sake we call a whole group of sensations green; but we are obliged to admit that there are several kinds of green, several distinct kinds of scnsations, though we do not possess names for all of them; a trained eye will recognize that within the green of the spectrum, the sensation produeed by one part is a different sensation from that produced by an adjoining part differing in wave-length from the former by an exeedingly small amount. The same is the case with other parts of the spectrum. And in general we may say that any change in the wave-length will produce a change in the sensation, so that we might speak of almost each wave-length as producing a separate sensation.

On the other hand we also easily reeognizc that the sensations produced by the spectrum are not all wholly unlike, that some are allied to others, and that in some eases one sensation is intermediate between two other sensations and partakes of the nature of both. We reeognize the sensation produced by the part of the speetrum lying between the green and the yellow as partaking on the one liand of the nature of the sensation of green and on the other hand of yellow, and call it yellowish green or greenish yellow; we similarly recognize a greenish blue or a bluish green, and so on. This suggests that our colour sensations are in reality mixed sensations, that the multitude of different sensations to which the spectrum gives rise are brought about not by each wave-length giving rise to a separate and independent sensation, but by means of a certain smaller number of primary sensations exeited in different degrees by different wave-lengths and mixed in various proportions.

$\$ 559$. This view is confirmed when we study in a systematic nianner the results of mixing or fusing together colour sensations.

The best method of fusing colour sensations is that of allowing two different parts of the spcetrum to fall on the same part of the retina at the same time. We may also make use of surfaecs coloured with pigments, but in doing so we must bear in mind the nature of the colour of pigments. A pigment possesses colour because when white light falls upon it some of the rays are absorbed while others are reflected. Thus gamboge absorbs the blue rays very largely as well as to 
a slight extent the red rays, but reflects the yellow rays and with these many of the green rays; indigo on the other hand absorbs the red and yellow but reflects the blue and a good deal of the green. Hence when we look at a yellow gamboge patch our retina is excited not by those rays alone which form the yellow of the spectrum, but by many other rays as well: the colour is not a "pure' colour, does not correspond to one of the colours of the speetrum, but is a mixture of more than one. And this is the case with most pignents; hence when they are employed in experiments on the mixture of sensations, difficulties and even errors arise which are avoided by the use of the colours of the prism. We may here incidentally remark that mixing the sensations excited by looking at pigments gives very different results from mixing the pigments themselves. Thus when gamboge and indigo are mixed the mixture is green because the gamboge absorbs the blue and the indigo absorbs the red and yellow, while both reflect the green. We shall see presently that when the sensation excited by gamboge is mixed with the sensation excited by indigo the result is a sensation not of green but of white; and we shall see why this is. What we have just said with regard to surfaces coloured with pigments applies also to glasses stained with pigment, it being understood that the colour of stained glass, seen as a transparent object, corresponds to the rays which it does not absorb. When pure pigments, i.e. pigments corresponding as closely as possible to the prismatic colours, are user, satisfactory results may be gained, either by using the reflected image of one pigment, and arranging so that it falls on the retina at the same spot as the direct image of the other pigment, or by allowing the image of onc pigment to fals on the retina before the sensation produced by the other has passed away. The first result is easily reached by the simple method of placing two pieces of coloured paper a little distance apart on a table, one on each side of a glass plate inclined at an angle. By looking with one eye down on the glass plate the reflected inage of the one paper may be made to coincide with the direct image of the other, the angle which the glass plate makes with the table being adjusted to the distance bctween the pieces of paper. In the second method, the - colour top' is used ; sectors of the colours to be investigated are placed on a dise made to rotate very rapidly, and the image of one colour is thus brought to bear on the retina so soon after the image of another that the two sensations are fused into one.

$\$ 560$. When by any of the above methods sensations corresponding to the red and yellow of the spectrum are mixed together in certain proportions the result is a sensation of orange, quite indistinguishable from the orange of the spec- 
trum itself. Now the latter is produeed by rays of eertain wave-lengths, whereas the rays of red and of yellow are respectively of quite different wave-lengths. The orange of the spectrum cannot be made up by any mixture of the red and the yellow of the speetrum in the sense that the red and yellow rays can unite together to formin rays of the same wave-lengths as the orange rays; the three things are absolutely different. It is simply the mixed sensation of the red and yellow which is indistinguishable from the sensation of orange; the mixture is entirely and absolutely a subjective one. In the same way we may by appropriate mixtures produce the sensations corresponding to other parts of the spectrum. Now we must suppose that rays of different wave-lengths affect the retina in different ways and so give rise to different visual impulses, that, for instance, the visual impulses generated by orange rays are different from those generated by red rays or by yellow rays. Hence we are led by the fact of mixed sensations being identical with other apparently simple sensations to infer that the visual impulses and hence the visual sensations which any ray originates are of a complex character. We conclude, for instance, that the impulses which a ray in the middle of the orange gives rise to are not simple impulses answering exelusively to the colour of that ray, but eomplex impulses, parts of which may be excited by rays other than the particular orange ray in question. In saying this we must bear in mind that we possess no direct information of the nature of visual impulses, our knowledge of these being limited to what we learn through the sensations to which they give rise ; the eomplexity of the sensation may be, and indeed probably is, of a different order from that of the visual impulse; to this point we shall return.

The view that our ordinary colour sensations are mixtures of simpler sensations is further eonfirmed by an examination of the eolours of external nature. For, though we see around us very many eolours besides those present in the speetrum, yet we find that the sensations of all these eolours may be reprodueed by mixtures of sensations excited by various parts of the spectrum. 'Thus the colour purple, which is so abundant in the external world and yet so conspicuous by its absence from the spectrum, may be at onee reproduced by fusing in proper proportions the sensations of red and of blue. And very many other colours present in the external world but not seen in the spectrum itself may be produeed by mixing various speetral colours in various proportions.

Other colours in nature may be reprodueed by mixing speetral colours with white or with black. When by means of a slit we allow a certain limited part of the spectrum. say in the green, to fall on a certain area of the retina, the rays exciting that area have certain wave-lengths, lying within eertain limits. 
say from $\lambda 525$ to $\lambda 535$; no rays but these are affecting the letina at the time, and the result is the sensation which we call spectral green. But we might easily so arrange matters that a certain amount of white light, that is of light of all wave-lengths of the visible spectrum, should fall on the area in question at the same time that the gieen is falling upon it; the result would be a mixed sensation, a sensation of spectral green mixed with the sensation of white, and we should recognize this sensation as different from the sensation of spectral green. Further by varying the proportion of white to green falling on the area in question at the same time we should have a whole series of different sensations from a green in which therc was hardly any white to a white in which there was hardly any green. In such a serics of colour sensations we recognize a hue supplied by the spectral colour, and we use the plinase more or less." saturated" to cxpress the proportion of white light; when very little white is present, we speak of the colour as being highly saturated. It need hardly be said that not only individual spectral colours, but all mixtures of these also, may be thus "mixed with white."

Again, taking a given area of the retina we may, on the one hand, throw on to the area a small amount of a spectral colour in such a way that all the elements of the retina in the area are excited, to a slight degree, giving rise to a feeble sensation of that colour; but we may, on the other hand, so scatter a few rays over the area that while some elements are excited others remain at rest and yet in such way that the excitation of the whole area still gives rise to one sensation only. We may speak of each of these sensations as one in which the sensation of the spectral colour is mixed or fused with the sensation which we call black; or we may distinguish the former as merely a feeble sensation and the latter as more strictly mixed with black. Many of the colours of the external world are of this nature; thus the colours which we call "browns" are mixtures of yellow or of red or of both (and possibly of other spectral colours also) with more or less black. In a similar way we may mix, not a spectral colour, but white with black, various mixtures forming various "greys."

\$ 561. Putting aside these more or less peculiar cases of mixture with black, we may say that the character of a colour depends (1) on the wave-lengths of the particular rays which, either alone or in excess of other rays, are falling on a given area of the retina; (2) on the amount of this coloured light falling on that area in a given time; and (3) on the amount of white light falling on that area at the same time. The first determines what we call the hue, the second the intensity, and the third the amount of saturation. Our common phrases do not distinguish with sufficient accuracy these three conditions, 
which obviously may exist under various combinations. On the one liand we frequently use wholly unlike names for colours which differ only in degree of saturation, such as carmine and pink; on the other hand we often use the same adjectives for quite different conditions. It is desirable to employ the word 'pale,' to mean little saturated, largely mixed with white, and the word 'deep' or 'rich' to mean lighly saturated, slightly mixed with white. The word 'tint' might be used to express various degrees of saturation, the word 'hue' being reserved to denote the dominant wave-length. 'Tone' is frequently employed to cxpress variations of wave-length within a named colour, as for instance different tones of red. 'The word 'bright' is often used somewhat loosely, but it is desirable to employ it exclusively as identical with 'lmminous, that is to say, as indicating the intensity of the sensation; a colour is more or less bright according to the amount of luminous energy which is being expended on the retina. We may remark, in passing, that while we can easily compare the brightness or luminosity of two white lights or of the same part of the spectrum under a feeble and under a strong illumination, we may feel some difficulty in comparing the amount of brightness of one colour with that of another, the brightness for instance of a given yellow with that of a given red. Conversely the word 'dark' is used to denote feeble intensity, or admixture with black. Lastly, our appreciation of the colours of external objects is modified by the nature of the surface which is coloured, and features so arising reccive various names; but these are in reality outside actual colour sensations.

\$562. Admitting that our colour sensations may be considered to be much fewer in number than those which we appear to have when we look on the colours of the spectrum or of nature, admitting that rays of light arake in us certain "primary" colour sensations, which mixed in various proportions reproduce all our colour sensations, we have now to ask the question, What is the nature or what are the characters of these primary colour sensations?

In view of the answer to this question we must call attention to certain results which may be obtained by a further study of the mixing of colours, meaning by that the mixing of colour sensations.

We have seen that all the colours of the spectrum mixed together make white. We have now to add that white may also be produced by mixing two colours only, provided that these are properly chosen. If we take a part of the red of the spectrum, and by any of the methods given in $\$ 559$. mix it with successive parts of the spectrum, we shall find that the mixture with a particular part of the green or blue green gives white. These two coloms are said to be complementary to cach 
other. In order to get a complete white, that is a white frce from all colour, a certain proportion between the relative amounts of red and green light, that is to say between the intensities of the two sensations, must be observed. And it will be understood that the white thus produced by two small parts of the spectrum is not equal in intensity to the white which would be produced by the combined effect of the whole of the same spectrum. The following may be taken as characteristic complementary colours, the respective wave-lengths being given :

$\begin{array}{ll}\text { Rell, } \lambda 656, & \text { Blue Green, } \lambda 492, \\ \text { Orange, } \lambda 608, & \text { Blue, } \lambda 490, \\ \text { Gold Yellow, } \lambda 574, & \text { Blue } \lambda 482, \\ \text { Yellow, } \lambda \text { 564, } & \text { Indigo-blue, } \lambda 462, \\ \text { Greenish Yellow, } \lambda 564, & \text { Violet, } \lambda 433 .\end{array}$

It will be understood that the above are not the only complementary colours; as we pass from the red end of the spectrum towards the green, each snccessive part of the spectrum has its complementary part on the other, blue side of the spectrum. each wave-length on the red side has its complementary wavelength on the blue side. When we reach the greenish yellow at $\lambda 564$, the complementary colour is on the very margin of the violet end of the visible spectrum. But we may go, so to speak, outside the spectrum, for the green of the spectrmm has for its complementary colour, purple. Or, to put it in another way, while each end of the spectrum has its complementary colour at the other end, the complementary colour of the middle of the spectrum is a combination of the two ends.

The bearing of these facts on the theory of primary colour sensations is ohvious. Two complementary colours excite between them all the primary sensations which are excited by white light, though not to the same intensity. Rays of the wave-length $\lambda 656$ falling on the retina give rise to the sensation which we denote as a particular kind of red; they do this however, not by the simple and exclusive stimulation of a particular red sensation, but by exciting all the primary sensations which are not excited by the wave-length $\lambda+92$. Conversely rays of the wave-length $\lambda 492$, produce the sensation of blue green by exciting all the primary sensations which are not excited by $\lambda$ 6.56. Similarly complex is the effect of other wavelengths. We may roughly describe each of two complementary wave-lengths as stirring up about half the whole of the primary sensations which can be excited by rays of all wave-lengths.

$\$ 563$. To produce white out of two colours, out of two parts of the spectrum, we are limited to certain pairs; if we take one colour, we are limited to one other colour, to its pair ; we 
have no choice. If however we are allowed three colours instead of two, we have a much greater range. If we take any three colours, provided only that they lie a certain distance apart along the spectrum, we can produce white by mixing them in certain proportions. If we take any red, green and blue, we can by adjusting the amount of each, that is the intensity of each, produce white.

We may go further than this. By adjusting the amounts of each of the three colours we can reproduce all the colours of the spectrum. If we take, for instance, a red of a certain wave-length, a green of a certain wave-length, and a blue of a certain wave-length, we can, without calling to our aid any other wave-lengths, by varying the relative intensities of the three, produce not only white light, but also orange, yellow, and violet, with all the intermediate tints, that is to say, produce all the colours of the spectrum; and we nay in the same way proluce the non-spectral purple. Our choice however is to a certain extent limited ; the three colours which we choose must be spread over the spectrum, for we cannot obtain these results with three colours taken from the red and yellow alone, or from the green and blue alone. Moreover, the result is not a complete one ; the colour which we thus produce by combining three spectral colours differs from a true spectral colour in not being saturated ; it is "mixed with white," more so in some cases than in others; in relation to this deficiency of saturation, the green region of the spectrum behaves differently from the red end and the blue end.

$\$ 564$. These results shew that the primary colour sensations out of which our recognized colour sensations originate, may be reduced to three in number. If we suppose that we possess three primary sensations so disposed in reference to the spectrum, so arranged so to speak along the spectrum, that a ray of light affects each of the three differently according to its wave-length, we can understand how all our multitudinous colour sensations may arise from the varied excitation of these primary sensations. There may be more than three of these primary sensations, but if so they must behave as if they were three; they cannot be less, since as we have seen the results of mixing two sensations only are extremely limited. We may therefore speak of our vision as trichromic, as based on three, or the equivalent of three, primary sensations.

When we attempt to inquire further into the nature of these primary sensations, we find ourselves in the face of two rival theories.

The one, propounded by Young but more fully elaborated by Helmholtz and Maxwell, and known as the Young-Ifelmholtz theory, teaches that there are three and only three such primary sensations. As we have just seen, any three parts of the spec- 
trum, with certain restrictions, might be chosen as corresponding to these three primary sensations so far as concerns the reproduction, by means of them, of all other colour sensations; hence in determining the nature of the primary sensations we must have recourse to other considerations. We may for instance very naturally suppose that two of the three correspond to the two ends of the spectrum, and may therefore he spoken of as more or less closely corresponding to our recognized sensations of red, and of violet. If red and violet be thus two of the sensations the third one must correspond to green, for only a sensation corresponding to green would give white when mixed with the other two sensations. Or again, choosing green in the first instance as one of the primary sensations for the reason that it stands apart from the others in its complement, purple, not being a spectral colour, we may decide that the two other primary sensations ought to differ as much as possible from each other, and therefore choose red and blue rather than red and violet since violet is obviously more allied to red than is blue; indeed we may perhaps regard violet, on account of its relations to red, as the beginning of a second spectrum the greater part of which is invisible. The decision between these two forms of the same theory rests on a number of consiclerations, into the discussion of which we eamnot enter here. Unless we specially call attention to the difference between them, which acquires importance on certain occasions only, we shall treat them as identical, and use the words blue and violet in this connection indifferently.

Such a view of three primary colour sensations is represented in the diagram (Fig. 150). 'Thus the red primary sensation,

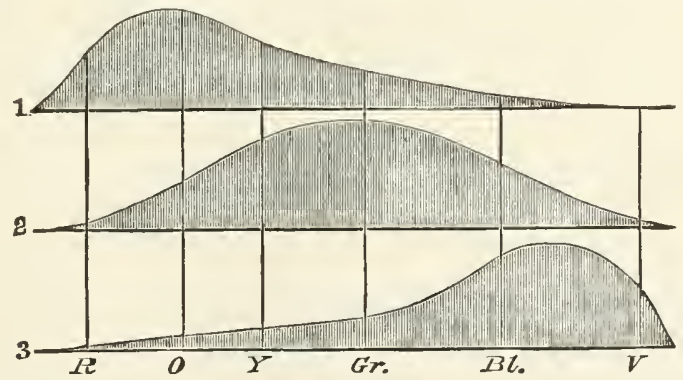

Fig. 150. Diagram of Turee Primary Colour Sensations.

1 is the so-called 'red,' 2 'green,' and 3 'violet' primary colour sensation $R, O, Y$, \&c. represent the red, orange, yellow, \&c., colour of the spectrum. 'The diagram illustrates, by the height of the curve in each case, how the several primary colour sensations are respectively excited to different extents by vibrations of different wave-lengths. But, in this, and also in Fig. 151, the curves are to be understood not as careful curves of actual variations in the intensity of the several changes, but as simply serving to illustrate roughly the nature of the theory. 
cxcited to a certain cxtent by the rays at the extrome red end, is most powerfully affected by the rays at a little distance from that end, the rays from this point onvards towaids the blue end producing less and less effect. 'The curve of the green primary sensation begins later and reaches its maximum in the green of the spectrum, while the violet or blue primary sensation is still later and only reaches its maximm towarls the blue end of the spectrum. Each ray calls forth each primary sensation though to a different degree, and the total result of each ray, or of each croup of rays, is determined by the proportionate amount of the three sensations. Thus the sensation of orange ( $O$ in the figure) is brought about by a mixture of a great deal of the primary red with much less of the primary green, and hardly any of the primary violet; the orange sensation is converted into a vellow sensation by diminishing the primary red and largely increasing the primary green, the primary violet undergoing also some slight increase. And similarly with all the other sensations. When all the three primary sensations are together excited, each to its whole extent, as when ordinary light falls on the retina, the result is a sensation of white. According to this theory, black is simply the absence of sensation from the visual apjaratus.

In the view, as originally put forward by Young, the three primary sensations were supposed to be represented by three sets of fibres, each set of fibres being differently affected by different rays of light, and the impulses passing to the brain along each set awakening a distinct sensation. No such distinetion of fibres can be found in the retina; but an anatomical basis of this kind is not necessary for the theory; we can easily conccive of the same fibre transmitting three distinct kinds of impulses; and indeed, as we shall sce later on, there are more ways than one by which we can imagine the sensations to be differentiated.

$\$ 565$. Another theory, that of Hering, starts from the observation that when we examine our own sensations of light we find that certain of these seem to be quite listinct in nature from each other, so that each is something sui generis, whereas we easily recognize all other colour sensations as various mixtures of thesc. 'Thus red and yellow are to us quite distinct: we do not recognize any thing common to the two; but orange is obviously a mixture of red and yellow. Green and blue are equally distinct from each other and from red and yellow, but in violet and purple we recognize a mixtmre of red and blue. White again is quite distinct from all the colours in the narrower scnse of that word, and black, which we must accept as a sensation, as an affection of consciousncss, even if we regard it as the absence of sensation from the field of vision, is again distinct from everything else. Hence the sensations, caused by different kinds of light or by the absence of light, which 
thus appear to us distinct, and which we may speak of as 'native, or 'fundamental' sensations, are white, black, red, yellow, green, blne. Each of these seems to us to have nothing in common with any of the others, whereas in all other colours we can recognize a mixture of two or more of these.

This result of common experience suggests the idea that these fundamental sensations are the primary sensations, concerning which we are inquiring. And Hering's theory attempts to reconcile, in some such way as follows, the various facts of colour vision with the supposition that we possess these six fundamental sensations. The six sensations readily fall into three pairs, the members of each pair having analogous relations to each other. In each pair the one colour is complementary to the other; white to black, red to green, and yellow to bline.

The little we know about the actual nature of sensations leads us to believe that the nervous processes which are at the bottom of sensations are, like other nervous processes, the outcome of metabolic changes in nervous substance. We shall presently call attention to the view that vision originates in the metabolic changes of a certain substance (or substances) in the retina, that the metabolism of this substance, which has been called visual substance, is especially affected by the incidence of light, and that the metabolic changes so induced determine the beginnings of visual impulses and thus of visual sensations. In the metabolism of living substance, we recognize $(\$ 30)$ two phases, the upward constructive analoolic phase, and the downward destructive katabolic phase; we nuy accordingly, in the absence of any distinct leading to the contrary, on the one hand suppose that different rays of light, ravs differing in their wave-length, may affect the metabolisin of the visual substance in different ways, some promoting anabolic, others promoting katabolic changes, and on the othier hand that different changes in the metabolism of the visual substance may give rise to different sensations.

We may therefore regard ourselves as at liberty to suppose that there may exist in the retina a visual substance of such a kind that when rays of light of certain wave-lengths, the longer ones for instance of the red side of the spectrum, fall upon it. katabolic changes are induced or encouraged, while anabolic clanges are similarly promoted by the incidence of rays of other wave-lengths, the shorter ones of the blue side. But, as we have already said, it is difficult in these matters of sensation, to distinguish between peripheral, retinal, and central, cerebral events; we may accordingly extend the above view to the whole visual apparatus, central as well as peripheral, and suppose that when rays of a certain wave-length fall upon the retina, they in some way or other, in some part or other of the 
visual apparatus, induee or promote katabolie changes and so give rise to a sensation of a eertain kind, while rays of another wave-length similarly induce or promote anabolic ehanges and so give rise to a sensation of a different kind.

'The theory of Hering, of which we are now speaking, applies this view to the six fundamental sensations, and supposes that

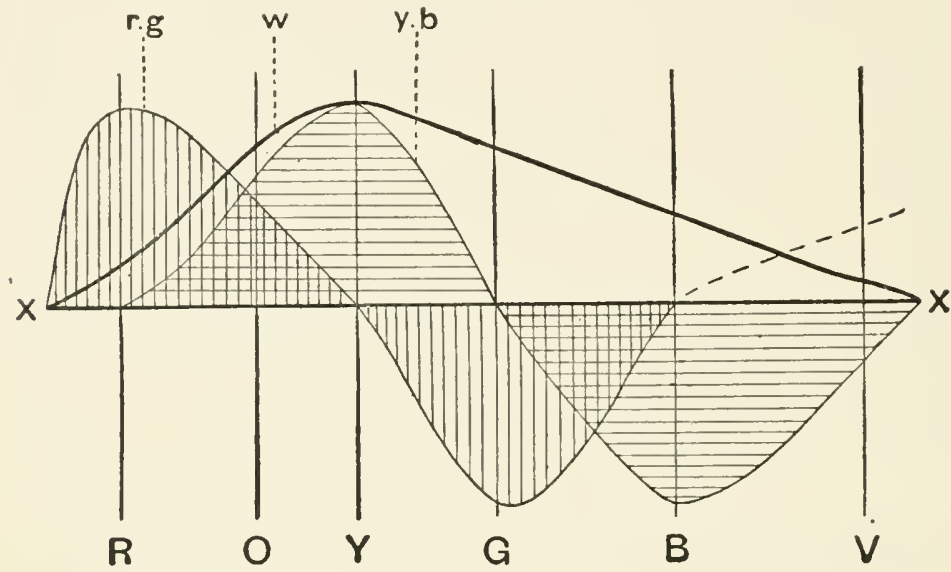

Fig. 151. Diagram to illustrate Hering's Tileory of Colour Vision.

The lines R.O.Y.G.B.V.indicate, as in Fig. 150, the position on the spectrum of red, orange, yellow, green, blue and violet.

The line $r . g .$, which indicates a space, shaded vertically, is intended to represent the effect of rays of different wave-lengths on the red-green visual substance. In the red, orange and yellow up to the line $Y$., the effect is katabolic, one of dissimilation (red sensation). $Y$. marks the position of equilibrium; beyond this the effect is anabolic, one of assimilation (green sensation). Beyond the blue, $B$. the effect (indicated by a broken line) is represented as once more katabolic.

The line $y . b$. similarly represents the behaviour of the yellow-blue substance, shaded horizontally, katabolic (yellow) up to $G$., anabolic (blue) beyond.

The line $w$. similarly indicates the white-black substance, unshaded, katabolic (sensation of white) along the whole length of the spectrum.

eaeh of the three pairs is the outcome of a particular set of katabolic and anabolic changes; these we may provisionally speak of as changes in a distinet visual substance, without attempting to decicle whether the changes are retinal or eerebral or both. The theory supposes the existence of what we may eall a redgreen visual substanee, of such a nature that so long as its metabolism is normal, katabolic and anabolic ehanges being in equilibrim, we experienee no sensation, but that when katabolic ehanges (ehanges of clissimilation is Hering s own term) are inereased, we experience a sensation of (fundamental) red, and when anabolic changes (changes of assinilation) are increased 
we experience a sensation of (fundamental) green. A similar yellow-blue visual substance is supposed to furnish through katabolic changes, a yellow, through anabolic changes a blue sensation; and a white-black visual substance similarly provides for a katabolic sensation of white and an anabolic sensation of black. The two members of each pair are therefore not only complementary but also antagonistic. Furtler these substances are of such a kind that while the white-black substance is influenced in the same way though to different degrees by rays along the whole range of the spectrum, the two other substances are differently influenced by rays of different wave-length (see Fig. 151). Thus in the part of the spectrum which we call red, the rays promote a large katabolism of the red-green substance with comparatively slight effect on the yellow-blue substance; hence our sensation of red. In that part of the spectrum which we call yellow the rays effect a large katabolism of the yellow-blue substance but their ation on the red-green substance does not lead to an excess of either hatabolism or anabolism, this substance being neutral to them; hence our sensation of yellow. The green rays, again, promote anabolism of the red-green substance, leaving the anabolism of the yellow-blue substance equal to its katabolism; and similarly blue rays cause anabolism of the yellow-blue substance, and leave the red-green substance neutral. Finally at the extreme blue end of the spectrum, the rays once more provoke katabolisun of the red-green substance, and by adding red to blue give violet. W'hen orange rays fall on the retina, there is an excess of katabolism of both the red-green and the yellow-blue substance; when greenishblue rays are perceived there is an excess of anabolism of both these substances; and other intermediate hues correspond to varying degrees of katabolism or anabolism of the several visual substances.

When all the rays together fall on the retina, the red-green and yellow-blue substance remain in equilibrium, but the whiteblack substance undergoes great katabolic changes; and we say the light is white.

Such are the two main theories of colour vision; and much may be said in favour of both of them; at the same time both of them present difficulties. We may perhaps regard as the distinetive feature of Hering's theory the view that white is an independent sensation, and not, as aceording to the YoungHelmholtz theory, the secondary result of the mixture of primary sensations. In Hering's theory rays of all wave-lengths (within the range of the visible spectrum) give rise to the seusation of white, whatever may be the colour sensation produced at the same time; a fully saturated colour, one wholly ummixed with white, according to this view does not exist. This assumption enables us to explain much more readily thas does the 
Young-Helmholtz theory the occurrence under certain dircnmstances of white sensations replacing or accompanying, that is to say diminishing the saturation of, colour sensations. On the other hand it introduces what appears to many minds a grave difficulty in reference to black. The theory supposes that the sensation of black is the result of the predominance of anabolic changes in the white-black substance. But what name are we to give to the sensation when the white-black substance is in a condition of equilibrium? We camnot investigate the corresponding conditions of equilibrium in the red-green, or in the rellow-blue substance, because we can never study these by themselves. When either of them oceurs, as when rays limited to certain wave-lengths are falling on the retina, we are by liypothesis at the same time subject to changes in the whiteblack substance; we may therefore leave these two conditions of equilibrium on one side. But we are constantly experiencing the condition of equilibrim of the white-black substance, maccompanied by any stimulation of either the red-green or yellow-bluc substance; we do so when the influence of light has for some time been wholly removed from the eye, or again taking the view, which is the more probable one, that the changes of which we are speaking are ccrebral changes, when the retina by disease or injury has become insensible to light. Under such circumstances we must suppose that the previous katabolic excitement of the white-black substance has died away, and that the substance is in equilibriun. Now when we examine our sensation under these circumstances, we find that though it is one of darkness it is one which differ's from a sensation of intense blackness. So distinct is the difference that the sensation in question has been spoken of under the phrasc "the intrinsic light of the retina." And that we may experience sensations of black different from this sensation due to the retina being at rest may be shewn in scveral ways. When we close and shade the eyes after they have been exposed to a very lright sunlight, we first experience a sensation of blackness, but this soon gives way to the sensation of mere darkness corresponding to the "intrinsic light of the retina." Again if we stare for some time at a white disc on a black field and then close the eyes, what we shall speak of presently as a negative after image is developed ; the part of the field of vision cor'esponding to the white dise appears as a black dise, which by its blackness stands out in fairly strong contrast to the rest of the field of vision, which corresponding to the area of the retina previously free from the stimulus of light, now yields the sensation of the "intrinsic light of the retina." And other examples of a similar kind might be given. Admitting then that the "intrinsic light of the retina" corresponds to a condition of equilibrium of the white black substance, we may speak 
of this as the neutral condition on one side of which we have sensations of white and on the other side sensations of black. Such a neutral condition has been spoken of as a "neutral grey," but the word grey is so often associated with a mixture of white and black sensations coexisting at the same time rather than with a neutral condition, that the term seems unsuitable. Many minds find it difficult to realize that the condition of which we are speaking is a true neutral condition, the various degrees of blackness being insignificant compared with the various degrees of intensity of white, and accordingly find it difficult to accept Hering's theory.

Both theories conform to the conclusion ( $\$ 564$ ) that normal vision is trichromic in the sense of being made up of three factors; for the three pairs of fundamental sensations of the one theory (the two members of each pair being reciprocally antagonistic, the positive and negative phase of the samc thing), play the same part in the equations of mixtures as the three primary sensations of the other theory. Indeerl it will be foumd on examination that all the results of the mixtures of colours are equally explicable on both theories. In comparing the two theories, however, especially in reference to the results of mixtures, we must bear in mind that "brightness" or " lmminosity" does not possess the same meaning in the two theories. In the Young-Helunholtz theory brightness is dependent on the extent to which the primary sensation is excitcd, on the anount of energy expended in the physical substratnm. whatever that may be, of the primary sensation. The red of the extreme red end of the spectrum has a minimum of brightness since the extreme red rays excite the red sensation to a minimum and the other two sensations hardly or not at all. Is we pass bluewards the brightness increases, partly becausc the red sensation is more powerfully excited. but also because to the brightness of the red sensation there is now added the brightness of the green sensation. And the brightness of a saturated yellow, such as that of the spectrum, is the sum of the brightnesses of the red and green sensations and nothing else; we neglect for the sake of simplicity the minute adjunct of the bine sensation. In Hering's theory the case is different. 'The lack of brightness at the red end of the spectrum is due not merely to the feeble development of the red sensation, to the feeble (katabolic) excitation of the red-green substance. but also to the fecble development of the white sensation, to the feeble (katfoolic) excitation of the white-black substance; and the brightness of the yellow of the spectrum is due not mercly to the latige development of the yellow sensation but also to the large increase of the white sensation. When, moreover, we come to examine this feature of "brightness" or "luminosity" more closely, we find that many questions of great complexity are raised; and 
many statements regarding the results of mixing sensations, such as those respecting complementary colours (\$.562) lave to be qualified by considerations touching the luminosity of the constituents; but into these questions we cannot enter here.

We may here remark when the extreme red end of the spectrum is examined it is found that along a certain length, between $\lambda 760$ and $\lambda 655$, there is 110 elange in the sensation as regards luue but only as regards luminosity; the red remains exactly the same kind of red, it only becomes brighter and more readily scen. Similarly at the other end from $\lambda$ t.30 to $\lambda 390$ the sensation of violet remains of the same hue though differing in luminosity. And these facts have becn brought forward on the Young-Helmholtz theory in support of violet being a primary sensation; it is urged that the red and violet which thus do not change in lnue but only in luminosity correspond to the actual primary sensations. The behaviour at the red end is quite intelligible on Hering's theory, since, as the waves shorten in length both the red and the white sensations are supposed to increase, though probably in this part of the spectrum the white sensation is very feeble, rapidly increasing a little farther on. The behaviour at the violet end presents diffieulties, sinee if the violet be due to admixture with a second octave so to speak of red, the violet should ehange in hue, become more red, as the rays shorten. I3ut the same diflieulty presents itself to the Young-Helmholtz theory if hlue be accepted as a primary sensation. Moreover observations on this part of the spectrum are exceedingly difficult. We cannot, however, attempt to diseuss the eontending theories properly; this would carry us beyond the limits of this book. IV e must content ourselves with ineidental referenee to some conclusions, which are suggested by the study of some other features of colour sensations as well as of abnomal colour vision, and to these we may now turn.

566. Variations in Colour Vision. Colour-Blindness. Persons differ very much in their power of appreciating and discriminatiug colours, and that quite independently of their ability to give expression to their colour sensations, that is to say, of their skill in naming colours. One person will regard as identical two colours which another person recognizes as different. In many cases such differences in the power of discriminating colours are slight, but in some eascs they are great. Cortain persols are met with who regard as quite alike, or nearly alike, colour's which to most people are glaringly distinct; such persons arc said to be "colour-blind."

The most common token of "colour-blinclness" is the inability to distinguish, or the difficulty in distinguishing, red and green. 'The great chenist Dalton, who was colour-blind, found great difficulty in recognizing at a distance his real 
(Giasgow) college gown when it was lying on the college grassplot; the colour-blind can tell a cherry among the leaves on a tree much more by its form than by its colour; and when such persons are asked to make matches' between coloured objects, such as skeins of coloured wools, they will put together a red skein and a green skein as being of the same colour. Most colour-blind people more or less confound red and green; but when a number of such colour-blind persons are tested in making matches either between skeins of wool or otherwise, it is found that they do not all make the same matches; they do not agree as to the particular red and green which they regard as identical, and they disagree in various other matches. But they all agree in this that when they are tested by the method of mixing colours it is found that all the colour sensations which they experience, including white, may be reproduced by mixtures of two colours only, whereas as we have seen (\$.56t) normal vision requires three. For instance all the colours which they see may be reproduced by varying mixtures of yellow and blue. The vision of these colour-blind people is therefore dichromic not trichromic. All their colour sensations are compounded of two not three (or two, not three pairs of) primary sensations.

On further examination it is found that these ordinary colour-blind persons may be more or less successfully divided into two classes. The nembers of one class have the following characters. The spectrum seems to them shortened at the red end; that is to say they fail to receive any visual semsation from the rays of extremely long wave-length which still give to the normal eye a distinct sensation of red. The blue-green of the spectrum seems to them less deeply coloured than the rest of the spectrum cither on the red or on the blue sidc; this part gives rise in them to a sensation like that caused by feeble white light; they have a difficulty in recognizing any hue in it and they often speak of it as grey, while in the remainder of the spectrum both to the blue and to the red side they have distinet sensations of colour. We may eall this region of the spectrum the 'neutral band': and it is one of the characters of this class that they see such a neutral band in the blue-green. They confound, as we have said, reds and greens, but when asked to make an exact match between a red and a green they choose a bright red and a dark green; they are more or less uncertain about all colours containing red or green, and when asked to match a purple they generally select a blue or a violet.

To the members of the other class, the spectrum is not shortened: they receive sensations as far to the red side as does the normal cye. 'They also see a 'neutral' band, but this is placed in the green, that is to say, nearer the red end than is 
the case with the first class. When asked to make an exact match between red and green they choose a dark red and a bright green; when asked to match a purple they generally select a green or a grey.

Persons whose vision belongs to one or other of these classes are sometimes spoken of as 'totally' colour-blind; for' there are grades of difference between such a kind of vision and normal vision, and some eyes may be ealled 'partially' colour-blind. Moreover, even among these 'totally' colourblind persons individual differences oceur in each elass; indeed not a few eases are met with which do not seem to fit into either elass, since they unite in themselves some of the characters of each elass. But even if we make allowance for these exceptions, the existence of the two classes with their respective features seems to offer a strong support to the Young-Helunholtz theory. In both elasses vision is dichromic not trichromic, that is to say according to that theory in both classes one of the three primary sensations is missing. Since the characteristic mistake which they both make is to confound red and green, we may infer that the missing primary sensation is not blue but either red or green. If we further suppose that in the first elass red is missing, in the second green, all the features of the two.classes seem intelligible.

On this view all the visual sensations which the first elass experience are made up of green and blue; and their vision might be represented by Fig. 150 with the upper curve (1) omitted. Owing to the absence of the red sensation, the extreme red rays hardly affect them at all. Since all their visual sensations are made up of various mixtures of the primary green and primary blue sensations, and since the sensation which they call white light (whatever it may be when compared subjectively with that of the normal eye) is the sensation produced when rays of all the wave-lengths of the visible spectrum are falling on the retina at the same time, that is to say when both of the two primary sensations are being equally excited at the same time, it follows that any particular wave-lengths which equally excite both the two sensations should also produce a sensation which to them is identical with that of white light. Now the blue-green rays do excite equally both the green and the blue sensation (ef. lig. 150), and it is just at this part of the spectrum that these persons see the 'neutral band' spoken of above. Further, the matches which eyes of this class make are suth as we might imagine would be made if the sensation of red were absent, and the two remaining sensations when mixed together made white. Henee members of this class are spoken of as being "red-blind."

In eyes of the second class, since red is present though green is wanting, the speetrum extends redwards as far is in the 
normal eye ; the least coloured part of the spectrum, the 'neutral band,' occupies about the same position as the green seen by the normal eye, for here the red sensation and blue sensation are excited to about the same extent; and the matches made by eyes of this class are such as might be expected in the absence of the green sensation. Members of this class are accordingly spoken of as "green-blind."

It might appear at first sight that the lack of a primary sensation, that is to say, the want of a third of all visual sensations, would lead to a general deficiency of vision ; for the lack of one-third of visual sensations would be equivalent to a diminution of the total illumination of external objects to the extent of one-third, and this, unless we suppose that the normal eye lives in a superfluity of light must, especially in feeble light, lead to dim vision; moreover a vision which has to trust to two-fold differences must be less sure than one based on threefold differences, But this does not necessarily follow; the two remaining sensations might become more highly developed, might so to speak expand in the absence of the third. Or we may suppose, as indeed has been supposed, that in these cases neither the red sensation nor the green sensation is absent, but that the two sensations coincide. We may imagine that the visual substance, or whatever it be, changes in which give rise to the red sensation, is affected by light of different wave-lengths in the same way as is the visual substance changes in which give rise to the green sensation. This might be illustrated by making the two curves for red and green in Fig. 150 coincide. If the red curve were moved bluewarls so as to coincide with the green curve, the figure would illustrate a red-blind case ; if the green curve were moved redwards to coincide with the red curve, the figure would illustrate a green-blind case. And as a matter of fact the general vision of colour-blind people seems to be as good as that of normal eyes; moreover, within the range of the colours which they can see, colour-blind people are if anything more acute than most people; though they regard as more or less alike two colours which seem to the normal eye wholly unlike, they can more easily detect minute differences such as those of shade or tone, within each of the two colours.

\$ 567. The phenomena, however, of these two classes of colour-blind eyes can also be interpreted on Hering's theory. In both of them the red-green substance may be supposed to be missing, and their dichromic vision to be made up exclusively of changes in the yellow-blue and white-black substances. Since they are thus supposed to have neither red nor green sensations, they must necessarily confound red and green; and the smaller differences, which, as we have seen, divide into two classes all those which confound red with green may be explained as follows. 
Even in eyes which may be considered normal as regards colour vision, eyes which certainly cannot be called colourblind, considerable differences will, on closer examination, be found in regard to sensations of yellow. If by means of a special arrangement we bring a certain amount of the red part of the spectrum and a certain amount of the green part of the spectrum on to the eye at the same time, the result is a sensation of yellow; according to the Young-Helmholtz theory yellow is a mixture of red and green. By the same arrangement we can bring on to the eye at the same time a certain amount of the actual vellow of the spectrum. In this way we can niake a match between a mixture of spectral red and green on the one liand, and spectral yellow on the other, comparing the mixed sensation derived from two parts of the spectrum with the sensation derived from a single (yellow) part. IVe have to adjust the quantities of red light and green light until the mixture seems of the same hue and the same brightness as the yellow, not shewing either a reddish or a greenish tone. When this is clone it is found that different people differ very materially as to the veroportion of red and green, the proportion of the intensities of the two sensations, necessary to make the mateh with yellow; with the sane quantity of red some need more green, other's less green, to make the match. 'This, on the Young-Helmholtz theory, is interpreted as meaning that the development of the red and green primary sensations differs even in people whose colour vision is considered normal. But on Hering's theory, in which yellow is a fundamental sensation, it may be interpreted as meaning that in passing along the spectrum towards the red end, the point at which the yellow-blue substance ceases to be affected by rays of light, is placed much nearer the red end in some people than in others. By Hering's hypothesis the green of the spectrum affects not only the reclgreen substance, ef. Fig. 151 (in way of anabolism), but also to some extent the yellow-blue substance (in way of katabolism); the red rays on the other hand affect the yellow-blue substance very slightly, while the (pure) yellow rays are neutral to the red-green substance producing neither katabolic red nor anabolic green, but simply yellow by katabolic action on the yellowblue substance. 'This at least represents the condition of the majority of eyes. If, however, we suppose that in other eyes the yellow-blue substance is considerably affected by red rays, if in Fig. 151, we suppose the curve representing the yellow sensation to be considerably extended towards the red end, in these eyes the red rays would give risc to a sensation of yellow at the same time that they exeited a sensation of red, the red would be mixed with yellow; hence in such eyes a certain amount of red being already mixed with yellow would need less green (with its necessarily accompanying yellow) to pro- 
duce a certain amount of yellow as the result of the mutual neutralization of the red and green. In such cases we may suppose not that the whole relation of the yellow-blue substance to wave-lengths is altered, but merely that the sensitiveness to long wave-length is increaserl; the curve of the yellow-blue is not shifted bodily along the spectrum, but the form of the curve is altered so that, the maximum of yellow remaining the same, the yellow end of the curve extends further into the red. Not only this match of red and green with yellow, but other matches of a similar nature, shew that in different eyes the yellow sensation (in Hering's sense) is more prominent in some people than in others, that some people so to speak are more yellow sighted than others.

The application of this fact to the colour-blind cases is obvious. In the one class, the red-blind of the Young-Helmholtz theory, the relations of the primary sensations, the distribution along the spectrum of the visual substances are the same as in the normal eye save that the red-green substance and the cor'responding sensations are missing; and since the visibility of the red end of the spectrum is chiefly affected by the red sensation, the whiteblack substance being as compared with the red-green substance but slightly sensitive to the extreme rays, the spectrum is shortened. The feeble white visual impulses excited are insufficient to affect consciousness unless supported by red visual impulses. In the second class, the yellow-blne substance has undergone an expansion similar to but probably greater than that which obtains in the yellow-sighted but otherwise normal eyes mentioned above, it is sensitive to even the rays at the red end of the spectrum; hence the spectrum to eyes of this class seems of the ordinary visible length.

$\S 568$. So far then both theories may be made to explain the ordinary phenomena of colour-blindness; but it is obvious that the subjective condition of the colour-blind must be different according to one theory from what it is according to the other. According to the Young-Helmholtz theory the red-blind person does not experience in any degree the sensation of either red or yellow; from the green of the spectrum to the red end he only sees some sort of green. Indeed along the whole spectrum, the sensations which he experiences are only various kinds of green and blue, with various amounts of the sensation whatever it be, whether white or simply green-blue, or some other sensation unknown to the normal eye, which results from the mixtmre of the green and blue sensations. The green-blind person, according to the same theory, has only the sensations of red and blue, with the sensation whatever it may be derived from the mixture of these two, he never has the sensation of either green or yellow. Obviously the sensations of the two classes ought to differ very widely. According to Hering's theory, both classes agree in 
seeing neither red nor green, all their sensations are made up of yellow and blue, with white and black, and the only differcnce betwecn the two classes is that the one, the green-blind of the other theory, see more yellow than do the other.

$\$ 569$. We have treated of the colour-blind as if they were confined to those who confounded red with green. According to the Young-Hclmholtz theory, another class of colonr-blind is possible, the violet- or blue-blind, those who while possessing red and green sensations lack the third, violet or blue sensation. such a kind of rision is impossible on Hering's theory; according to that theory, if a person fails to see blue, he must also fail to see yellow.

Lastly we may remark that absolute colour-blindness, a condition in which shades of black and white alone indicate the features of external objects, while possible on Hering's theory, is impossible on the Young-Helmholtz theory. According to the latter a person reduced to one primary sensation must see either red, grecn, or blue; this one sensation is excited in him both by objects which we called coloured and by objects which we call white. He would probably call it white; but it would be either red, green, or blue. According to Hering's theory he might still see white and black in the total absence of both the red-green and the yellow-blue substance. Now, cases do undoubtedly occur, though they are relatively rare, of marked colour-blindness, which arc neither red-blind nor green-blind. Cases further occur which may be described as cases of total colourless blindness; the subject recognizes only differences of luminosity. Cases, moreover, occur in which one eye only is affected, the other being normal, so that the subject can describe the sensations of the affected eye by help of those of the normal eye; and a not small number of cases occur in which the deficieney of colour sensation is not congenital, affecting the whole of the eye, but the result of disease, and limited to a part of the retina, the rest of which may be normal. And it might be thought that by an examination of these several cases it would be easy to decide definitely between the two theories. But when this cxamination is carried out, many difficnlties arise in the way of reaching such a dccision; and indeed some of the facts observed seem compatible with neither theory. A full discussion of these difficulties - and only a full discussion would be satisfactory - is impossible here. We may simply remark that when we said that absolute colour-blindness, the limitation of visual sensations to those of black and white, was impossible on the Young-Helmholtz theory, we should have added, unless it be supposed that in such cases all the three primary sensations coincide, none of them being absent, much in the same way that two may be supposed to coincide in red or green blindness. 
\$50. What we have said concerning colour vision refers to the central parts of the retina only. If a coloured object be moved so that its image travels from the central to the peripheral parts of the retina, the colour sensations change and the peripheral parts may be spoken of as colour-blind. Thus the sensation of red is lost towards the periphery, which may be spoken of as red-blind, while in the same region other sensations, at all events that of blue, are still felt. At the extreme periphery even blue is wanting, that is, all the primary sensations are wanting, and yet we receive by it uncoloured sensations, sensations of black and white. But these phenomena of peripheral colour vision also need a fuller discussion than we can afford to give them here.

$\$ 571$. Influence of the pigment of the yellow spot. In the macula lutea, or yellow spot, the yellow pigment which is diffused through the retinal structures in this region absorbs some of the greenish-blue rays of the light which falls upon it. We may use this feature of the yellow spot for the purpose of making the spot, so to speak, visible to ourselves, by the following experiment. A solution of chrome alum, which only transmits red and greenish-blue rays, is held up between the eye and a white cloud. The greenish-blue rays are absorbed by the yellow spot, and here the light gives rise to a sensation of red; whereas in the rest of the field of vision, the sensation is that ordinarily produced by the purplish solution. The yellow spot is cousequently marked out as a rosy patch. 'This very soon however dies away.

Though, when we wish our vision to be most acute, we use the fovea centralis in which the pigment is extremely scanty or absent owing to the thimness or absence of all retinal layers except the cones and cone fibres, still in ordinary vision we make large use of the whole yellow spot, and our sensations of the colour of external objects must be to a certain extent influenced by the pigment of the spot. The light which reaches the rods and cones of this region from objects which we call white, is in reality more or less tinged with yellow; in other words what we call white is more or less yellow. Indeed variations in the amount of pigment present in the yellow spot have been offered in explanation of some of the differences in colour vision discussed above.

$\$ 572$. In speaking of the relation between a visual sensation and the intensity of the stimulus $(\$ 550)$ we were confining our remarks to white light: when we inquire into the behaviour of our colour sensations under variations in the intensity of the stimulus, we come upon results which are in many ways complicated. IVe must be content with pointing out one or two only of these.

Each of our colour sensations, when the light giving rise to 
it reaches a certain intensity, ceases to be a colour sensation and becomes a sensation of white. The theory of three primary colour sensations may be used to explain this. Thus, taking violet as a primary sensation, a violet light of moderate intensity appear's violet because it excites the primary sensation of violet much more than those of green and red. If the stimulus be increased the maximum of violet stimulation will be reacherl, while the stimulation of green will continue to be increased and even that of red to a slight degree. The result will be that the light appear's violet mixed with green, that is to say, appear's blue. If the stimulus be still further increased while the green and violet are both still largely excited the red stimulation may be increased until the result is violet, green, and red in the proportions which make white light. And so with light of other colours. But the same facts may also be explained on Hering's theory, for this supposes that the stock, so to speak, of whiteblack substance is far greater than that of either of the other two vismal substances; hence under violent stimulation the white sensation wholly overpowers any accompanying colour sensation.

Conversely when the intensity of the stimulus is diminished, colour sensations may disappear before all sensation of light is lost. When the light is very dim we cease to recognize the colour of coloured objects though we continue to see the objects. And this is not merely because the white light reflected from the object (and it is through this that we chiefly become aware of the form of an object) is more powerful than the particular rays which give the object colour; since even a satmrated colour behaves in the same way. If with a feeble illumination we allow a very small part of the spectrum to fall on the retina, we are much more distinctly conscious of a sensation of light than of any particular colour sensation; indeed the minimum sensation thus felt has been called a 'grey' for all parts of the spectrum. Moreover the colour which is first recognized upon gradually increasing the illumination, appear's less saturated, that is to say apparently more mixed with white than when a large amount of light of the same refrangibility falls on the retina; and such distinct colour sensation as may be felt at the first moment of looking at such a light soon diminishes, giving way to a mere sensation of light.

When we attempt to compare one colour sensation with another in reference to their bchaviour towards variations in the intensity of the stimulus we find the results to a certain extent conflicting. When we diminish the intensity of the stimulus by diminishing general illumination, when we look for instance at objects in nature under light of varing intensity, we find that the colours change unequally as the light diminishes; as is well known the colours of flowers look very different when night is 
falling from what they do under bright daylight. In particular we find that as the light diminishes red sensations and also yellow sensations disappear earlier than blue sensations. Hence in dim lights, as those of evening and moonlight, blues preponderate, reds and yellows being less obvious, whereas in bright lights yellows and reds become prominent.

On the other hand, if we test our sensitiveness to different colours in a different way we get results which are opposed to the above. If for instance we determine the distance at which we cease to recognize the colour of a piece of coloured paper, say $1 \mathrm{~cm}$. square, we find that the blue goes first, then green and next yellow, red being recognizable at the longest distance, though the difference between red and yellow is not very great. It will be understood of course that in this experiment we are dealing not only with diminished energy, with diminished amplitude of the luminous waves, but also with a diminished area of retinal stimulation.

Or again, if we take the heating effects of rays of different wave-lengths as a measure of their energy, we may determine the amount of energy needed, in the case of the several colours, to produce a given visual effect. When this is done it is found that the rays in the green, about wave-length $\lambda 530$, are the most effective; from this part of the spectrum the efficiency declines both towards the violet and the red.

The three several methods lead to three different results, the one teaches that blue, the other that red or yellow, and the third that green is the colour to which the eye is most sensitive. It would be hazardous to found important conclusions on any of them.

There are several other facts of considerable importance bearing on the theory of colour vision, but it will be best to consider these in connection with certain modifications of visual sensations with which we shall have presently to deal. Meanwhile having acquired some general notions of visual sensations, we may turn from the study of the little we know concerning the way in which these sensations originate through retinal changes, to the study of the way in which light falling on the retina gives rise to visual impulses. 


\section{SEC. 7. ON THE DEVELOPMENT OF VISUAL IMPULSES.}

$\S 573$. We have already called attention to the important fact that the changes which give rise to visual impulses begin on the outer side of the retina, that the rays of light pass through the inner layers of the retina without, as far as we know, producing any effect, and do not begin their work until they reach the region of the rods and cones. It is in this region that the energy of light is transformed into energy of another kind; and the processes here started travel back to the layer of fibres in the inner surface of the retina and thence pass as visual impulses along the optic nerve. That on the one hand the optic fibres themselves are insensible to light and that on the other hand visual impulses do begin in the region of the rods and cones is shewn by the phenomena of the blind spot and of Purkinjé's figures respectively.

The Blind Spot. There is one part of the retina on which rays of light falling give rise to no sensations; this is the entrance of the optic nerve, and the corresponding area in the field of vision is called the blind spot. If the visual axis of one eye, the right for instance, the other being closed, be fixed on a black spot in a white sheet of paper, and a small black object, such as the point of a quill pen dipped in ink, be moved gradually from the black spot sideways over the paper away towards the outside of the field of vision, at a certain distance the black point of the quill will disappear from view. On continuing the movement still farther outward the point will again cone into view and continue in sight until it is lost in the periphery of the field of vision. If the pen be used to make a mark on the paper at the moment when it is lost to view and at the moment when it cones into sight again, and if similar nurks be made along the other meridians as well as the horizontal, an irregular outline will be drawn circumscribing an area of the field of vision within which rays of light produce no visual sensations. This is the blind spot. The dimensions of the figure drawn vary of course with the distance of the paper from the eye. If this distance be known, the size as well as the position of the area of the retina corresponding to the blind spot 
may be calculated from the diagrammatic eye ( $\$ 527)$. The position thus determined coincides exactly with the entrance of the optic nerve, and the dimensions (about $1.5 \mathrm{~mm}$. diameter) also correspond; the exact size and shape of the blind spot differs lowever in different individuals. While drawing the outline as above directed the indications of the large bianches of the retinal vessels as they diverge from the entrance of the nerve can frequently be recognized. The existence of the blind spot is also shewn by the fact that an image of light, sufficiently small, thrown upon the optic nerve by means of the ophthalmoscope, gives rise to no sensations.

The existence of the blind spot proves that the optic fibres themselves are insensible to light, that light can stimulate them only through the agency of the retinal structures in which they end.

§ 574. Purkinjés Figures. If one enters a dark room with a candle and while looking at a plain (not parti-coloured) wall, moves the candle up and down, holding it on a level with the eyes by the side of the head, there will appear in the field of vision of the eye of the same side, projected on the wall, an image of the retinal vessels, similar to that seen on looking into an eye with the ophthalmoscope. The field of vision is illuminated with a glare, and on this the branched retinal vessels appear as shadows. In this mode of experimenting the light enters the eye through the cornea, and an image of the candle is formed on the nasal side of the retina; it is the light emanating from this image which throws shadows of the retinal vessels on to the rest of the retina. In Fig. 153 the light a forms an image on the retina at $b$; the light reflected from this spot casts a shadow of the retinal vessel $\nu$ on to another part of the retina at $c$, and the image of this shadow appears in the field of vision at $d$. A far better method is for a second person to concentrate the rays of light, with a lens of low power, on to the outside of the selerotic where this is thin just behind the cornea; the light in this case emanates from the illuminated spot on the sclerotic and passing straight through the vitreous humour throws a direct shadow of the vessels on to the retina. Thus the rays passing through the sclerotic at $b$, Fig. 152, in the direction $b_{\nu}$, will throw a shadow of the vessel $\nu$ on to the retina at $\beta$; this will appear as a dark line at $B$ in the glare of the field of vision. This proves that the structures in which visual impulses originate must lie behind the retinal vessels, otherwise the shadows of these could not be perceived.

If the light be moved from $b$ to $a$, the shadow on the retina will move from $\beta$ to $a$, and the dark line in the field of vision will move from $B$ to $A$. If the distance $B A$ be measured when the whole image is projected at a known distance, $k \mathrm{~B}$ from the eye, $k$ being the nodal point ( $\$ 527$ ) of the reduced diagram- 
matic eye, then, knowing the distance $k \beta$ in the diagrammatic eye, the distance $\beta a$ can be calculated. But if the distance $\beta a$ be thus estimated, and the distance $b a$ be directly measured, the distances $\beta \nu, a \nu, b \nu, a \nu$ can be calculated; and if the appearance in the field of vision is really caused by the shadow of $\nu$ falling on $\beta$, these distances ought to correspond to the distances of the retinal vessels $\nu$ from the sclerotic $b$ on the one hand and from that part of the retina $\beta$ where visual impressions begin, on the

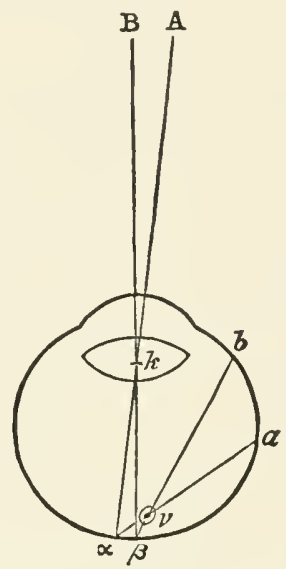

Fig. 152. Diagram illustrating the Formation of Purkinjés Figures wien the Illumination is Directed throvgh the Sclerotic.

other. When this is done it is found that the distance $\beta \nu$ thus calculated corresponds fairly well to the distance of the retinal vessels from the layer of rods and cones. Thus Purkinje's fig. ures prove in the first place that the sensory impulses which form the commencement of visual sensations originate in some part of the retina behind the retinal vessels, i.e. somewhere between them and the choroid coat; and calculations based on the movements of the shadows following movements of the illumination, even if they do not give absolutely exact results, at least go far to shew that these impulses originate at the outermost part of the retina, viz. the layer of rods and cones.

In the second method of experimenting, where the light passes through the sclerotic, the image always moves in the same direction as the light, as it obviously must do, when the spot of light on the selerotic is moved from $a$ to $b$ (Fig. 152) the shadow on the retina moves from $a$ to $\beta$, and the (inverted) image moves from A to B. In the first method, where the light enters through the cornea, the image moves in the same direction as the light when the light is moved from side to side, provided the movement does not extend beyond the middle of the comea, but in 
the opposite direction to the light when the latter is moved up and down. In Fig. 153, which represents a horizontal section of an eye, if $a$ be moved to $a, b$ (the illuminated spot on the retina, the light reflected from which easts a shadow of $\nu$ on to $c$ ) will move to $\beta$, the shadow on the retina $c$ to $\gamma$, and the image $d$ to $\delta$. If on the other hand $a$ be supposed to move above the plane of the paper, $b$ will move below, in consequence $e$ will move above, and $d$ will appear to move below, i.e. $d$ will sink as $a$ rises.

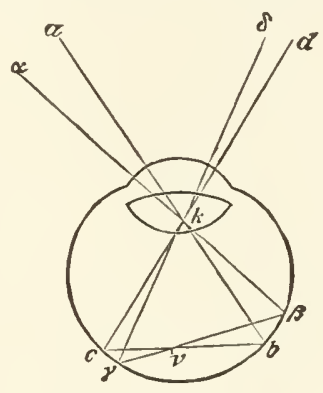

Fig. 153. Diagram illustrating the Formation of Purkinjé's Figures when the Illumination is directed tirrodgh the Cornea.

It is desirable in these cases to keep moving the light to and fro, especially in the first method, since the retina soon becomes tired, and the image fades away. To give rise to a conscious sensation of the slight difference between shadow and absence of shadow the retina must be extremely sensitive; if the shadow remains motionless, the sensitiveness rapidly decreases in the parts which are not in shadow, until the visual sensations from these parts are no stronger than those from the parts in shadow; when the light is moved the parts which were in shadow, not having been so much stimulated, are sufficiently sensitive to the light which now falls on them, while those parts which had been previously fatigued recover their sensitiveness by resting in the shadow. The experiment, like the experiment by which the yellow spot (\$571) is made visible, is incidentally useful as shewing how extremely sensitive and how soon fatigued are the retinal structures.

Some observers can recognize in the axis of vision a faint shadow corresponding to the edge of the depression of the fovea centralis.

The retinal vessels may also be rendered visible by looking through a small orifice such as a pin-hole in a card placed close to the eye, in the position of the principal anterior focus, at a bright surface such as a white cloud, and moving the orifice very rapidly from side to side or up and down. If the movement be from side to side, the vessels which run vertical will be seen; if up and down, the horizontal vessels. In this case, as in 
the similar instance of shadows cast by objects in the vitreous humour ( $\$ 549)$, the shadow is cast by the rays passing parallel through the vitreous humour; hence the change from shadow to absence of shadow is more marked with the vertical vessels when the movement is sideways and with the horizontal vessels when it is up and down. The fine capillary vessels are seen more easily in this way than by Purkinjés method. The same appearances may also be produced by looking through a microseope from which the objective has been removed and the eye-piece only left (or in which at least there is no object distinctly in focus in the field), and moving the head rapidly from side to side or backwards and forwards. Or the microscope itself may be moved; a circular movement of the field will then bring both the vertically and horizontally directed vesscls into view at the same time.

$\$ 575$. It bcing admitted that the processes which give rise to visual impulses begin somewhere in the region of the rods and cones, we have to ask the question, How do they begin and what is their nature? We are accustomed to consider light as the undulations of an ether; a nervous impulse is, so far as we can understand, a molecular change propagated along the substance of the axis cylinder of a nerve fibre; and, though as we have seen our knowledge of the subject is very limited, still the analogy of a muscular contraction, and of other responses of living substance to a stimulus, lead us to conclude that ehemical changes play a part in this molecular change. By what steps does the undulation of the ether give rise to the material molecular change? In attempting to answer this question we may adopt one or other of two views.

On the one hand we may suppose that the vibrations of the ether are able, through the means of the retinal apparatus of the rods and cones for example, to give rise in some more or less direct manner to the molecular vibrations which are the beginnings of the nervous impulses in the optic nerve. And the rapidity with which events must come and go in the retina in order that the eye may be, what it is, an instrument for appreciating rapidly repeated minute changes, lends support to this vicw. But the present state of our knowledge of physical phenomena does not afford us an adequate explanation of how such a direct transformation can be effected. 'The recent progress of science tends, it is true, more and more to lay bare the close relations which obtain between optical and electric phenomena, and the latter, as we have so often seen, play an important part in the generation of nervous impulses. 'Then again many of the phenomena of fluorescence seem to supply a bridge between the vibrations of ether, and the vibrations of molecules. But in neither of these directions is it possible, at present at all events, to frame a hypothesis which can be satisfactorily applied to retinal processes. 
On the other hand we may perhaps more naturally turn to a chemical explanation. We are familiar with the fact that rays of light are able to bring about the decomposition of very many chemical substances; and we accordingly speak of these substances as being sensitive to light. All the facts dwelt on in this book illustrate the great complexity and corresponding instability of the composition of living matter. And we might reasonably suppose that living matter itself would be sensitive to light; that is to say that rays of light falling on even undifferentiated protoplasmic substance might set up a decomposition of that substance and so bring about a molecular disturbance; in other words, that light might act as a direct stimulus to living matter. As a matter of fact, however, we meet with very little evidence of this, especially when we make a distinction between thermic rays, rays which though they produce physical results are to us invisible, and luminous rays which alone when they fall on our retina give rise in us to the sensation of light. Nor can we be surprised at this apparent indifference of living matter towards light when we reflect that living matter in what we may call its purest form is remarkable for its transparency, that is to say the rays of light pass through it with exceedingly little absorption. But in order that light may produce chemical effects, it must be alssorbed; its energy must be spent in doing the chemical work. Accordingly the first step towards the formation of an organ of vision, that is to say an organ through which the body of a living being reacts towards light, is the differentiation of a portion of the substance of the body into a pigment at once capable of absorbing light, and sensitive to light, i.e. undergoing decomposition upon exposure to light. An organism, a portion of whose body had thus become differentiated into such a pigment, would be able to react towards light. The light falling on the organism would be in part absorbed by the pigment, and the rays thus absorbed would produce a chemical action and set free chemical substances which before were not present. We have only to suppose that the chemical substances thus produced are of such a nature as to induce other chemical changes, or in some way or other to act as a stimulus to other parts of the organism, (and we have manifold evidence of the exquisite sensitiveness of living matter in general to chemical stimuli,) in order to see how rays of light falling on the organism might excite movements in it, or modify movements which were being carried on, or might otherwise affect the organism in whole or in part. A comparatively simple illustration of this is afforded by some of the lowly organisms called bacteria, especially by the one which has been called bacterium photometricum. This organism is remarkably sensitive to light, and especially reacts towards certain rays of light. It is coloured with a purple pigment, apparently allied to chlorophyll; and the rays of light, to which 
it is especially sensitive, are just those which are absorbed by the pigment.

\$576. Photochemistry of the Retina. Such considerations as the foregoing may be applied to even the complex organ of vision of the higher animals. If we suppose that the actual terminations of the optic nerve are surrounded by substances sensitive to light, then it becomes easy to imagine how light falling on these sensitive substances should set free chemical bodies possessed of the property of acting as stimuli to the actual nerve-endings and thus give rise to visual impulses in the optic fibres. We say "easy to imagine," but we are, at present, far from being able to give definite proofs that such an explanation of the origin of visual impulses is the true one, probable and enticing as it may appear. And it must be remembered that in such chemical changes electrical events may intervene and that in a special way.

One of the most striking features in the structure of the retina is the abundance of black pigment, fuscin, in the retinal epithelium. It is difficult to suppose that the sole function of this pigment is to absorb the superfluous rays of light, and that the rays thus absorbed are put to no use and simply wasted. And indeed it has been shewn that the pigment is sensitive to light; but the changes in it induced by light are excessively slow. Moreover its presence cannot be of fundamental importance, since vision is not only possible but fairly distinct with albinos in which this pigment is absent.

Then again, in the vast majority of vertebrate animals, the outer limbs of the rods are suffused with a purplish-red pigment, the so-called visual purple, which is so eminently sensitive to light that images of external objects may by appropriate means be photographed in it on the retina. When the eye of a frog or of a rabbit is examined in an ordinary way, with full exposure to light, the retina appears colourless. But if the eye be kept in the dark for some time before it is examined, the retina, if removed rapidly, will be found to be of a beautiful purplish-red or pink colour. Upon exposure to light the colonr changes to yellow and then fades arvay, leaving however the retina, not only white but more opaque than it was before. Upon examination with the microscope it is found that the purple colour is confined exclusively to the rods and to the outer limbs of the rods, the inner limbs being wholly devoid of it.

The colour of the rods is due to the presence of a distinct pigment, the "visual purple," diffused through the substance of the outer limbs; and this may be extracted from the rods by dissolving these in an aqueous solution of bile salts. A clear purple solution is thus obtaincd, which is capable of being blcached by the action of light, and in its general features and behaviour is similar to the pigment as it naturally exists in the retina. 
Visual purple is found as we have said exclusively in the outer limbs of the rods; it has never yet been found in the cones, and it is accordingly absent from (or exceedingly scanty iin) the retinas (such as those of snakes) which are composed of cones only (or contain very few rods), and from the greater part of the macula lutea and the whole of the forea centralis of the retinas of man and the ape. The intensity of the coloration varies in different animals, and the retinas even of some animals possessing rods (bat, dove, hen) seem to be wholly deroid of the visual purple; it is generally well marked in retinas in which the outer limbs of the rods are well developed. Its absence or presence is not dependent on nocturnal habits, since the intense colour of the retina of the owl is in strong contrast to the absence of colour in the bat. It has been found in the retina of the embryo.

The visual purple is bleached not only by white but also by monochromatic light. Of the various prismatic rays the most active are the greenish-yellow rays, those to the blue side of these coming next, the least active being the red. Now it is precisely the greenish-yellow rays which are most readily absorbed by the colour itself. A natural colour retina or a solution of visual purple gives a diffuse spectrum without any defined absorption bands, and according to the amount of colouring material through which the light passes, absorption is seen either to be limited to the greenish-yellow part of the spectrum or to spread thence towards the blue and, to a much less extent, towards the red. Thus the various prismatic rays produce a photochemical effect on the visual purple in proportion as they are absorbed by it. Under the action of light the visual purple, whether in solution, or in its natural condition in the rods, passes through a purplish orange to a yellow, and finally becomes colourless; and we appear to be justified in speaking of a "visual yellow" and "visual white" as products of the photochemical changes undergone by the visual purple.

For the restoration of the visual purple, after it has been destroyed by light, the maintenance of the circulation of the blood through the tissues of the eye is not essential. The retinal epithelium has by itself, provided that it still retains its tissue life, the power of regenerating the purple. If a portion of the retina of an excised eye be raised from its epithelial bed, bleached, and then carefully restored to its natural position, the purple will return if the eye be kept in the dark.

If the image of some bright object such as a lamp or a window be thrown on to the retina, either of an eye in its natural position or of one recently excised, care having been taken to keep the retina for some time previous away from all rays of light, the portion of the retina on which the rays have fallen will be found to be bleached, the rest of the retina remaining 
purple. In fact an "optogram" of external objects may thus be obtained; and if the retina be removed and treated with a 4 p.c. solution of potash alum before the retinal epithelium has had time to obliterate the bleaching effects, the retina may remain permanently in that condition: the photochemical effect may, as the photographer's say, be "fixed."

It seemed very tempting, especially upon the first discovery of it, to suppose that this visual purple is directly concerned in vision. If we suppose that visual purple itself is inert towards, produces no effect on, the endings of the optic nerve, but that either visual yellow or visual white, i.e. some product of the action of light on visual purple, may act as a stimulus to those endings, the way seems opencd to understanding how rays of light can give rise to sensory impulses in the optic nerve. And such a view receives incidental support from the fact that the visual efficiency of rays of different wave-lengths corresponds very closely to their photochemical efficiency towards visual purple; the greenish-yellow rays which are most active towards visual purple are precisely those which seem to us the brightest, most luminous, which produce the greatest effect on our consciousness. But visual purple is absent from the cones, it is in ourselves absent from the fovea centralis, the region of most distinct vision; it is further entirely wanting in some animals which undonbtedly see very well; and lastly animals such as frogs, naturally possessing the pigment, continue to see very well and even apparently to see colours when their visual purple has been absolutely bleached, as it may be by prolonged exposure of the cyes to strong light. We cannot therefore, at present at least, explain the origin of visual impulses by the help of visual purple. It is rifficult to suppose that it plays 13 o part in the origination of visual impulses; but even in a photochemical theory of vision we cannot allot to it more than a subsidiary function, possibly something analogous to the "sensitizer" of the photographer. At the same time its history suggests that some substances, sensitive like it to light, but nulike it, colourless and therefore escaping observation, may exist, and by photochemical changes be the means of exciting the optic nerves; but if so we must suppose that these substances, though colourless, are capable of absorbing light, since otherwise they would not be acted upon by it. Apart from thcir providing visual purple the cells of the retinal epithelium, with their remarkable amoboid pigment-carrying filamentous processes, lave probably in other ways to do with vision, though we cannot at present state what their exact function in this respect is. Their importance in vision is indicated by their behaviour towards light.

If an eye be fully exposed to light before removal and examination, the processcs carrying pigment are found to stretch a long way inwards between the outer limbs of the rods and 
cones, investing these outer limbs with a sheath of pigment, and even reaching between the inner limbs. If on the contrary the eye be kept in the dark before removal and examination, the processes are found to be short and to stretch a little way only inwards, not reaching much farther than the tops of the outer limbs of the rods and cones. The substance of the cell has in fact the power of amœboid movement, at one time throwing out long filamentous processes inwards between the rods and cones, and at another time retracting the processes into the body of the cell. As they move to and fro these processes carry with them the crystals of pigment with which they are studded; hence in the extended condition much of the pigment is carried away from the body of the cell inwards between the rods and cones, leaving the nucleus less covered with pigment, while in the retracted condition the pigment is carried back to the body of the cell and the nucleus becomes obscured. Further, while various circumstances may determine whether the processes are extended or retracted, the falling of light on the retina has the most marked and potent effect. When light falls on the retina the processes hurry inwards and envelope the outer limbs of the rods and cones with pigment; when the light is shut off from the retina the processes carry back the pigment to the body of the cell.

Hence in an eye exposed to light the processes and pigment being largely jammed in between the outer limbs of the rods, and these outer limbs at the same time swelling, the pigment epithelium adheres closely to the retina, and when the retina is removed is carried away with it. In an eye kept in the dark, the processes being withdrawn, and the outer limbs of the rods shrinking again, the attachment of the retina to the epithelium is much less, and the retina can be more readily removed so as to leave the pigment epithelium adherent to the choroid.

Urari has an effect on these cells of the pigment epithelium of such a kind that they cease to throw out their processes; they seem to be paralyzed. Hence in the eye of a urarized animal the pigment epithelium readily separates from the retina.

We may add that in frogs at least, this shifting of the pigment may be seen to be accompanied by a change of form in the inner limbs of the cones. Under the influence of light the inner limb becomes shorter and broader, in fact contracts, and when the influence of the light is removed elongates to its original length. Moreover these changes in the cones may be induced, not only by light falling on the retina but also, through a mechanism not at present fully understood, as the result of stimulation of the skin, by light or otherwise; in these latter cases the change of form of the cone is not necessarily accompanied by migration of the pigment. 
\$577. Whatever view we adopt, whether photochemical or other, as to the changes which lead to stimulation of the real endings of the retinal nervous mechanism, we camnot at present state anything definite concerning those nerve-endings or the manner of their stimulation.

Each outer limb of a rod is a cylinder of highly refractive material, closely packed round with the black pigment of the retinal epithelium. When an image of an external object, such as a candle-flame, is formed on the retina, at or near the layer of rods and cones, the rays of light diverge again beyond the focal plane in the form of pencils of rays from each point of the image. Of these some passing between the rods are absorbed by the pigment, while others pass into the outer limbs of the rods; of these latter some traversing the whole length of the limb, are absorbed by the pigment beyond, while others undergo "total reflection" at the sides, or are absorbed by the pigment after reflection. Hence of all the rays which fall on the layer of rods and cones, a small number only are reflected back into the vitreous humour and so through the pupil; hence the eye when looked into usually looks black. In the case of the conical outer limbs of the cones the amount of light thus thrown back into the vitreous humour must be still less. We may fairly assume that the light which thus disappears, partly in the actual outer limbs of the rods and cones, partly in their immediate surrounding, sets up changes which, whatever be their exact nature, either are or in some way assist the very beginnings of visual impulses. It also seems probable that these changes, so long as they are confined to the region of the outer limbs, ought not to be considered as nervous in nature, it seems probable that they do not take on a nature analogous to that of a nervous impulse, until they have passed the conspicuous break which divides the outer from the inner limbs. But on these matters we have no certain knowledge.

We may here turn aside for a moment to remark that when an image of a candle-flame is formed on the retina the rays reflected back, as stated above, from the retina through the pupil form a second image in the position of the candle-flame; hence to see an image of an illuminated retina the observing eye must be placed in the position of the source of illumination. This is the principle of the ophthalmoscope.

There are many forms of this instrument, but the accompanying diagram (Fig. 154) will illustrate its essential features. The rays from the lamp $L$ (or other source of illumination) are reflected by the concave mirror $\boldsymbol{M}, \boldsymbol{M}$, and brought to a focus at $a$. The rays diverging from $a$ are, by means of the lens h rendered parallel, and thus, through natural dioptric arrangements of the observed eye $B$, are brought to a focus on the retina at $a^{\prime}$. The rays reflected back from the part $a^{\prime}$ of 
the retina thus illuminated, will, as stated above, follow the same path as on entering, and so return to the focus $a$. Hence the rays reflected from a number of points on the retina, such as those forming the arrow at $\alpha^{\prime}$, will be brought to a focus in a

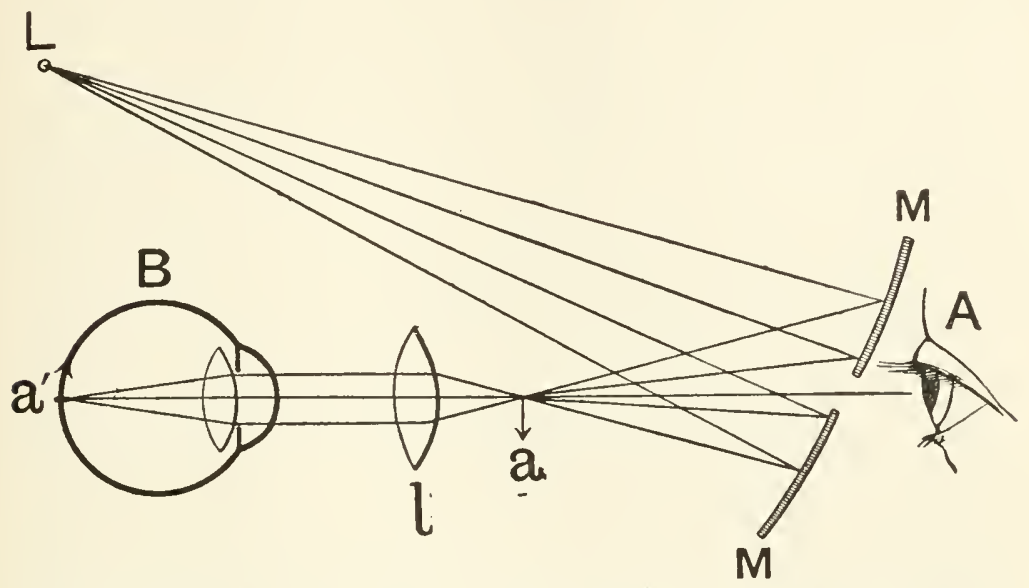

Fig. 154. Diagram to yllustrate the Privciples of a simple Fori of UPHTHA LMOSCOPE.

corresponding number of points at $a$, i.e. will form an (inverted) image of the arrow at $a$. And the observing eye placed at $A$ behind the hole in the mirror will see at $a$ an inverted image of the illuminated retina.

$\S 578$. As to the meaning of the difference between rods and cones no satisfactory statement can be made. It has, it is true, been suggested that the cones subserve the vision of colour and the rods that of form only. This, however, is in flagrant contradiction to both the theories of colour vision discussed above. For colourless vision of form is the appreciation of differences in black and white; and according to the YoungHelmholtz theory, white is simply a combination of colour sensations. Sensations of white, apart from colours ordinarily so called, are only admitted by Hering's theory, and an extension of this theory in the direction that the rods are connected exclusively with the white and black substance, and the cones exclusively with the red-green and yellow-blue substances, lands us at once in absurdity. Moreover since it is in the fovea centralis that we have the most acute vision of both form and colour, the cones alone must be able to serve as the instruments of all visual sensations. The argunent that in nocturnal animals the rods are developed almost to the exclusion of cones, because such animals do not need colour sensations, is one which can be turned against itself, since it may be urged that 
the dim light in which these creatures move calls for increased and not diminished appreciation of small differences of colour. The eoloured globules intercalated between the outer and inner limbs of cones in some of the lower animals, such as birds and reptiles, have probably no closer relation to colour vision than has the yellow pigment of our own macula lutea.

The close resemblance in their general features, apart from form, between the rods and cones, suggests that their functions differ in degree rather than in kind, and this view is supported by the rod-like character assumed by the cones in the macula lutea and especially in the fovea centralis. But we can hardly expect to be able to differentiate the functions of the two, so long as we know so little about either.

With regard to what goes on in the other layers of the retina our ignorance is complete. We may fairly suppose that the events which take place in the inner limbs of the rods and cones are different from those which take place in the optic fibres. We may conclude that the latter are of the nature of nervous impulses, though we may here repeat what we have already urged, namely, that it is hazardous to infer that the little we know of motor nervous impulses may be applied with little or no modification to sensory nervous impulses; but as to the nature of the events in the inner limbs of rod and cones, or as to what happens in the intervening layers of the retina, we know nothing.

$\$ 579$. The little objective knowledge which we possess concerning retiual processes is almost limited to the detection of electric currents. The retina and optic nerve like other nervous structures develope electric currents which may be spoken of as currents of rest and currents of action. They may be shewn by placing one electrode on the retina of a bisected eye, or on the cornea of a whole one, and the other on the optic nerve, or hind part of the eye-ball or on the cortical visual centre or even on some distant part of the body. They are also manifested by the isolated retina itself. 'The phenonena appear somewhat complicated by the appearanee now of positive, now of negative variations; but this fact comes out clearly that the incidence of light on the irritable retina developes an clectric change, the magnitude of which is to a certain extent proportionate to the intensity of the light acting as a stimulus. 'The changes gradually diminish and disappear as the retina gradually loses its irritability. IVe may add that these electric phenomena appear to be quitc independent of the condition of the visual purple. 
SEC. 8. ON SOME FEATURES OF VISUAL SENSATIONS ESPECIALLY IN RELATION TO VISUAL PERCEP'TIONS.

$\$ 580$. In our previous study of visual sensations we dealt chiefly with the more simple and fundamental characters of sensations; we considered each sensation by itself and discussed its features irrespective of the influence of other sensations excited at the same time, except so far as it became necessary, in treating of the localization of sensations, to speak of the circumstances which determined the fusing of two neighbouring sensations into one. It very rarely occurs however that any object or event in the external workd gives rise to a simple sensation such as those on which we have dwelt; each part of the external world, each external object such as a tree, is the source of many distinct sensations differing from each other in intensity and other characters. In looking at a tree we are conscions of many sensations of different colours and intensities, each having a definite localization; but these are coordinated in our consciousness into a whole and we say we "see a tree." The effect which the whole visible world has upon us is not that of a multitude of single sensations each separate from and independent of the other, but of a smaller though still large number of groups of sensations corresponding to what we call the objects of nature. And we have now to turn our attention to certain facis concerning vision which become especially prominent when we are the subject not of an isolated single visual sensation but of complex groups of simultaneous visual sensations. The sum of visual sensations and groups of sensations which are excited by images falling on the retina at any one time, we call, as we have already said ( $\$ 494)$, the 'field of vision,' or ' visual field.'

$\$ 581$. Before we proceed any further however it will be well to call to mind that in studying vision as we are now doing by means of an appeal to our own consciousness, we are deserting the ordinary methods of physiology for the methods which are more strictly speaking those of psychology. Or rather in our study of vision we are using both methods, sud- 
denly turning from one to the other. We are using ordinary physiological methods when we are studying how the rarious rays of light proceeding from a tree form an image of the tree on the retina, and how these rays thus falling on the retina give rise to visual impulses. But when we study the change in our consciousness which is brought about by the visual impulses thus cxeited through the image of the tree falling on the retina, we are dealing with psychological problems. The objeet, the tree itself, and our vision of it, the one being commonly spoken of as the cause of the other, are connected by a cliain of events; one end of the chain we study by physiological, the other end by psychological methods; and the difficulty of our task arises from the fact that we have to use these two different methods for a common purpose, namely that of explaining how the tree gives rise to the vision of it.

When we turn to the physiological side of the problem we cannot at present say much more than that the rays of light proceeding from the tree give rise to the changes in the optic fibres which we have called visual impulses. We have seen in dealing witl the brain reason to think ( $\$ 78)$ that visual impulses, like other sensory impulses, may influcnce the working of the central nervous system without producing any such change of consciousness as can be studied by psychological methods; and we further suggested ( $\$ 500)$ that in the structures of the midbrain which we called the primary visual ecntres a visual impulse underwent a development by which it bceame no longer a mere impulse but something more, and that the changes in these primary visual centres transmitted to the occipital cortex gare lise there to the changes with which the distinct affection of consciousness is associated. It is undesirable to speak of the events in the primary visual centres as "sensations," since it is convenient to reserve this term for the psychical events, the changes of consciousness of which we can bccome aware by examining onr own minds; nor is there at present any need to give them any name at all; but it is important when we arc using the psychological method to remember that between the physiological visual impulses and the psychological sensation there are events which must not be ignored.

Turning now to the psychological side of the problem we find that the psychical events are also complex, and that the psychical effects due to the same visual impulses are not all of the same kind. This is seen even in the case of simple and isolated visual sensations. Taking the effect of a luminous point, shining for a moment only, as a simple form of visual sensation, we must distinguish what we may eall the mere change of conseiousness, the mere sensation of light, from the firther psychical effect of which we have already spoken and through which we associate the sensation with a luminous point 
occupying a particular position in external nature. Though the latter always accompanies the former, though whencver we experience a visual sensation we refer it to its cause in the external world, we can dissociate the two in our minds, and can speak of the nere sensation independently of the further psychical action. When we have vision not of such a simple object as a luminous point, which we may consider as giving rise to a single sensation, but of a tree which gives rise to a complex group of sensations, the psychical actions which accompany the mere sensations are manifold and become prominent in the total visual effect produced by the tree. 'That total visual effect is determined not only by the sensations to which the retinal image of the tree is at the time giving rise, but also by various psychical events dependent on the previous knowledge of the nature of trees which we have gained by touch as well as by sight, and on other circumstances. In common language we distinguish between the mere sensation and the further psychical visual effect by saying that we 'feel' a sensation and 'perceive' an object; and, though the tern 'perception' has been employed in different meanings by different writers, we may here make use of it, in what is perhaps its most usually accepted meaning, to denote the further visual effect to which we have just called attention as distinguished from the immediate sensation. IVe feel a sensation of light, and we may feel at one and the same time a number of such sensations of different intensity and quality; wo perceive an object, it may be a simple object such as a mere transient flash of light or a complex object such as a tree or a scene.

From what we have said above it follows that, although it is perfectly true as we have insisted ( $\$ 524$ ), that our perception of external objects is based on the optical sharpness of the retinal image, and on the distinctness of the several sensations which the retinal" image excites, we should be wrong in supposing that when an image of an object is formed on the retina the visual impulses correspond exactly to the retinal inage, the sensations correspond exactly to the impulses, and the perception corresponds exactly to the sensations, so that the perception is as it were a "mental image" corresponding exactly to the retinal image and hence to the object itself. The truth lies in the contrary direction; things are not what they look, or, since the same applies to other senses besides vision, what they seem; and one object of philosophy is to ascertain the exact relations between things as they are and things as we think them to be. We must of course confine ourselves here to pointing out, in regard to vision, some of the more salient differences which obtain between the actual features of an object and our perception of the object.

Of these differences some are clearly of psychical origin. 
Our perception of a tree is in part determined by events other than the actual sensations, by psychical processes arising out of our previous experiences of trees, and in other ways. Some of these psychical processes we shall consider a little later on.

Other differences are either clearly or possibly of physiological origin; the view may at least be argued that they arise either during the retinal changes through which visual impulses are developed or during the subsequent cerebral changes, spoken of above, through which the visual impulses give rise to visual sensations; and it is to some of these that we wish first to call attention.

\$582. Irradiation. A white patch on a dark ground appear's larger, and a dark patch on a white ground smaller, than it really is. In Fig. 155, the white square on the right hand

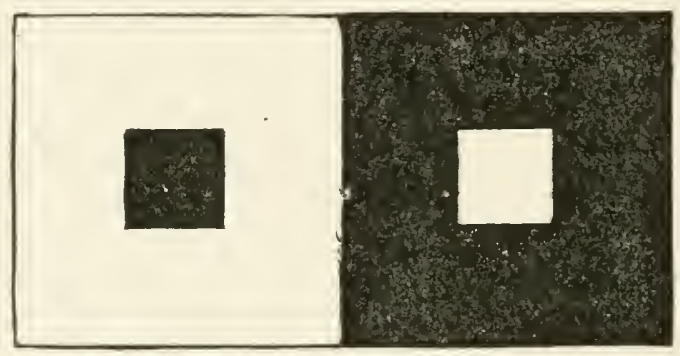

FIG. 155.

side looks larger than the black square on the left hand side though both are exactly of the same size. So also neighbouring white surfaces tend to melt together. The effect is increased when the object is somewhat out of focus, and may be then partly explained by the diffusion circles which, in each case, encroach from the white upon the dark. But over and beyond this, any sensation coming from a given retinal area occupies a larger share of the field of vision, when the rest of the retina and central visual apparatus are at rest, than when they are simultaneously excited. It is as if the neighbouring, either retinal or cerebral, structures were sympathetically thrown into action at the same time. In this way a certain difference is established between the retinal image and the perception.

$\$ 583$. Simultaneous contrast. If a white strip be placed between two black strips, the edges of the white strip, near to the black, will appear whiter than its medium portion; and if a white cross be placed on a black background, the parts close to the black will appear sometimes so white, compared with the centre of the cross, that the latter will seem dim or even shaded. This effect which occurs even when the object is well in focus, is spoken of as one of 'simultaneous contrast'; the increased 
sensation of light which causes the apparent greater whiteness of the borders of the cross is regarded as the result of the 'contrast' with the black placed immediately close to it. Still more striking results are seen with coloured objects. If a book, or pencil, be placed vertically on a sheet of white paper, and illuminated on one side by the sun, and on the other by a candle, two shadows will be produced, one from the sun which will be illuminated by the yellowish light of the candle, and the other from the candle which will in turn be illuminated by the white light of the sun. The former naturally appears yellow; the latter, however, appears not white but blue; it assumes, by contrast, a colour complementary to that of the candle-light which surrounds it. If the candle be removed, or its light shut off by a screen, the blue tint disappears, but returns when the candle is again allowed to produce its shadow. If, before the candle is brought back, vision be directed through a narrow blackened tube at some part falling entirely within the area of what will be the candle's shadow, the area, which in the absence of the candle appears white, will continue to appear white when the candle is made to cast its shadow, and it is not until the direction of the tube is changed so as to cover part of the ground outside the shadow, as well as part of the shadow, that the latter assumes its blue tint. If a small piece of grey paper be placed on a sheet of pale green paper, and both covered with a sheet of thin tissue paper, the grey paper will appear of a pink colour, the complementary of the green. 'This effect of contrast is far less striking, or even wholly absent, when the small piece of paper is white instead of grey, and generally disappears when the thin covering of tissue paper is removed. It also vanishes if a bold broad black line be drawn round the small piece of paper, so as to isolate it from the ground colour. And many other instances of this kind of contrast might be given. It is obvious that whenever in vision this effect intervenes, a discrepancy is introduced between the features of an object and our perception of them.

\$584. After-images. Successive Contrast. As we have already $(\$ 5.51)$ seen the visual sensation lasts much longer than the stimulus, and under certain circumstances the sensation is so prolonged that it is spoken of as an after-image. Such after-images are best developed when an eye, which has for some time been removed from the influence of light, is momentarily exposed to a somewhat strong stimulus. Thus if immediately on waking from sleep in the morning the eye be directed to a window for an instant and then closed, an image of the window with its bright panes and darker sashes, the various parts being of the same colour as the object, will remain for an appreciable time.

When, however, the eye has been for some time subjected 
to a stimulus, the sensation which follows the withdrawal of the stimulus is of a different kind; the result is what is called a negative after-image, or negative image, to distinguish it from a positive after-image, like the one mentioned above, which is simply a continuation of the sensation primarily excited with all its characters unchanged except that of intensity. If, after looking steadfastly at a white patch on a black ground, the eye be turned to a white ground, a grey patch is seen for some little timc. A black patch on a white ground similarly gives rise when the eye is subsequently turned towards a grey ground to a negative image in the form of a white patch. This may be explained as the result of exhaustion. When the white patch has been looked at steadily for some time, that part of the retina on which the image of the patch fell has become tired; hence the white light, coming from the white ground subsequently looked at, which falls on this part of the retina, does not produce so much sensation as in other parts of the retina; and the image, consequently, appears grey. And so in the otler instance; in this case, the whole of the retina is tired, except at the patch; here the retina is for a while most sensitive, and hence the white negative image. In speaking of the retina being tired we are using these words for simplicity's sake. We liave no right to suppose that the exhaustion takes place in the retinal structures only; it may occur in the central cerebral structures during the development of visual impulses into sensations; indeed the chief part of it is probably of such a cerebral origin.

When a red patch is looked at, and the eye subsequently turned to a white or to a grey ground, the negative image is a greenish blue; that is to say, the colour of the negative image is complementary to that of the object. Thus also orange produces a blue, green a pink, yellow an indigo-blue, negative image, and so on ; the negative image is in each case complementary to the primary one.

Similarly, when the eye, after looking at a coloured pateh, is turned not to a white or grey but to a coloured ground, the colour of the negative image is a mixture of the colour complementary to the primary image with the colour of the ground; if a yellow ground be chosen after looking at a green object, the negative image will appear as a mixture of red and yellow, a reddish yellow; and so on.

Again, when a pateh of coloured light is made to travel through the visual field with sufficient rapidity, as when a patch of light or of colour placed near the margin of a rotating dise is looked at, the image of the patch as the disc revolves is followed by a negative image in the shape of a sort of ghost having a colour more or less but not exactly complementary to that of the patch.

Though these negative images only become striking after a 
prolonged or intense excitation of the retina, such as rarely occurs in ordinary vision, still the effect must intervene, even if to a slight extent only, in our daily sight, and proportionately contribute to the discrepancy between the perception and the object.

$\$ 585$. The phenomena of 'simultaneous' and 'successive contrast' are further of interest in relation to the theory of colour vision. The mere occurrence of the negative images can be explained as a result of exhaustion on either hypothesis of colour vision. According to the Young-Helmholtz theory when the coloured patch is looked at, one of the three primary colour sensations is much exhausted, and the other two less so in varying proportions, according to the exact nature of the colour of the patch; and the less exhausted sensations become prominent in the after-image. Thus, the red patch exhausts the red primary sensation, and the negative image is made up chiefly of green and blue sensations, that is, appears to be greenish-blue, or bluish-green, according to the particular hue or tone of the red. So also the yellow patch exhausts both the red and green sensations leaving the blue only to make itself felt. On Hering's hypothesis, we may suppose that, owing to the continued effect of looking' at the red patch, the katabolic changes of the redgreen substance become less and less, leading to a prominence and indeed to an actual increase of anabolic changes in the same substance; hence, the sensation of green dominating in the negative image; and we may suppose that like events occur in the yellow-blue substance.

The Young-Helmholtz theory does not explain so readily as does Hering's theory why negative images often follow upon positive images without any stimulation of the retina subsequent to the primary one. As we have already said, if a white patch on a black ground be looked at for some time, and the eyes be then shut, a negative image of the spot will be seen on the ground of the 'intrinsic light' of the retina much blacker than the ground, and having in its immediate neighbourhood a sort of bright corona. Conversely a black patch on a white ground will give rise to a patch of exaggerated 'intrinsic light' in contrast to the blackness of the rest of the field. So also, if a window be looked at and the eyes then closed, the positive after-image with bright panes and dark sashes gives way to a negative after-image with bright sashes and dark panes. Looking at a bright red spot gives rise to a green after-image, and so with other colours. Moreover, the eyes being still shut, there may be a series of after-images; the negative after-image with its black, green, \&c., corresponding to the white, red, \&c., of the positive image, may give way to a return of the positive imag'e with all its original features, to be succeeded by a second negative image like the first, and thus often by a whole series of alternate pos- 
itive and negative images, each gradually becoming fainter and more obscure. 'These and similar' phenomena are more or less satisfactorily explained on Hering's theory as the results of rhythmic oscillations between katabolic and anabolic changes; on the other theory we have to have recoursc to psychological explanations. This is especially the case with the phenomena of simultaneous contrast. In the case for instance of the grey patch seen as pink in the midst of a green field, it is argued that the patch does not actually excite a sensation of pink but that we think it is pink because we attribute the greenness of the whole field to the covering tissue paper, and seeing the patch shine through this judge the patch to be reflecting just those rays, namely pink, which mixing with the green would give rise to white, that is to a colourless grey. Hering's theory on the other hand offers a direct physiological explanation of the effect; it supposes that when one part of the retina is stimulated, the neighbouring portions of the field of vision are affected at the same time in a manner which may be roughly but only roughly compared to electric induction, so that they undergo changes antagonistic or complementary to those going on in the part of the field of vision corresponding to the portion of the retina actually stimulated. Thus in the case of the grey patch on the green field, the anabolism of the red-green substance in the green field surrounding the grey patch leads to a certain amount of katabolic action of the red-green substance within the grey patch, and so gives rise to a red sensation.

$\S 586$. W W have seen ( $\$ 553$ ) that visual sensations may be produced in other ways than by light falling on the retina. In such eases the effect which is prodnced upon our consciousness is wholly misleading. A mechanical or electrical stimulation of the retina may give rise to a visual sensation identical with that which would be produced by the rays from a flash of light falling upon a part of the retina. In both cases we should have a perception of a flash of light occurring in a certain part of the field of vision; and so far as the perception itself is concerned we could not distinguish between the latter which is a real and the former which is a false perception.

Not only single and simple sensations, but also complex groups of sensations may be excited by other means than that of light falling on the retina, and we may thus experience varied and intricate perceptions which have no objective reality at all. Many people when they close their eyes at night, or indeed at other times, see images of faces or other objects: and though under such circumstances it is easy to recognize the subjective origin of the perception, that conclusion is reached by reasoning upon the circumstances, and not because the perecption itself differs in character from a like perception caused by looking at an external object. In such cases it is probable that some 
causes or other of a physiological nature give rise either in the lower visual centres or in the cerebral cortex to just such changes as would be induced by corresponding visual impulses, though those impulses are wholly wanting; in other words the causes in question give rise to visual sensations, in the physiological meaning of that word, which produce a psychological effect identical with that of visual sensations produced in the ordinary way through the aetion of light on the retina. In some cases perhaps the process may begin even in the retina itself; abnormal changes in one or other of the retinal structures may lead to the development of complex coordinate visual impulses.

Sometimes the sensations and perceptions thus occurring, especially those which are met with on closing the eyes at night, may be recognized as revivals, more or less altered, of sensations experienced during the day; something sets going again the series of cerebral events which were set going by actual rays of light. These are generally spoken of as "recurrent sensations."

At other times, there is no history of any like sensation having been felt in the immediate past; the psychical effect appears to have no objective cause at all. Moreover such false sensations and perceptions having a distinctness which gives them an apparent objective reality quite as striking as that of ordinary visual perceptions, may occasionally be experienced not only when the eyes are elosed, but even when the eyes are open, and when therefore ordinary visual perceptions are being generated, with which they mingle and with which they are often confused. They are then spoken of as ocular pluantoms or hallucinations. They sometimes become so frequent and obtrusive as to be distressing, and form an important element in some kinds of delirium, such as delirium tremens.

It is probable, as we have just suggested, that these false perceptions may be started by events, which in ordinary language may be called physiological; but the whole chain of events between the visual impulse or even the immediate effect of the impulse which we may consider as the physiological sensation, and the terminal psychological perception is long and complex; the discordance between the perception and its apparent cause, in other words, the falsity of the perception, may be introduced in the later, psychological, links of the chain. And an hallucination may have such an origin that it may fitly be spoken of as purely psychological.

This naturally leads to the remark that a perception may be revived in the mind, without the usual physiological antecedents, as the result of purely psychological processes; it is then generally spoken of as an 'idea.' And we find, upon examination, that each new perception which we experience is more 
or less modified by memories and ideas resulting from bygone perceptions of a like kind. But we have already determined to defer the consideration of these and other more or less distinctly psychical modifications of perceptions until we have studied certain results arising from the use of two eyes. 


\section{SEC. 9. BINOCULAR VISION.}

$\S 58 \%$. So far we have treated of vision as if it were carried out by means of one eye and have only incidentally referred to our possessing two eyes. Our ordinary vision is, however, carried out by means of two eyes, our vision is binocular not monocular; and to the characters of this binocular vision we must now turn. In dealing with monocular vision we rarely have occasion to refer specially to the movements of the eyeball; but in binocular vision these play an important part; and even before we go into details, it will be desirable to point out not only certain general facts, but also the meaning of certain terms which we shall have to use.

The eye is virtually a ball placed in a socket, the bulb or eyeball and the orbit forming a ball-and-socket joint. In its socket joint the eyeball is capable of various movements, but these are limited to those of rotation within the socket; the eyeball cannot by any voluntary effort be moved out of its socket. It is stated that by a very forcible opening of the eyelids the eyeball may be slightly protruded; but this trifling locomotion may be neglected. By disease, however, the position of the eyeball in the socket may be materially changed.

The movements of rotation to which the eyeball is thus limited are carried out round a centre in the eye which is termed the centre of rotation, and which has been determined to lie in the vitreous humour about $13.5 \mathrm{~mm}$. behind the anterior surface of the cornea, not quite $2 \mathrm{~mm}$. behind what, though the eyeball is not a sphere, may be considered as the geometric centre of the eyeball; it is of course quite different from the optical centre or nodal point of the diagrammatic eye $(\$ 527)$.

When we, in looking, direct our vision to a point, a line drawn from such a point, which we may call the fixed point of vision, to the centre of rotation, is called the visual axis; prolonged past the centre of rotation it meets the retina in the centre of the fovea centralis; hence in the view of those who hold that the optic axis, the line on which the dioptric surfaces of the eye are centred, meets the retina on one side of the fovea, the visual axis does not coincide with, and is different 
from the optic axis. When with both eyes we look straightforwards to the far distance, the visual axes of the two eyes are parallel; when we direct the two eyes to the same fixed point, the two visual axes converge to the fixed point, the amount of convergence being the greater the nearer the fixed point to the observer.

The horizontal plane in which the two visual axes lie is called the visual plane; and a vertical plane at right angles to this, midway between the two eyes, or more exactly bisecting a line, sometimes called the "base line" or "fundamental line" joining the nodal points of the two eyes, is called the median plane.

$\S 588$. As we have seen, the sum of the sensations which we can receive from the retina at the same time is spoken of as the "visual field" or "field of vision." The term therefore has properly a subjective meaning, but it is sometimes used in an objective sense to denote the space or area of the external world, rays of light from which are capable of exciting the retina at any one time; where we wish to distinguish between the two, we may call the latter the "field of sight." The dimensions of the field of sight for one eye will even in the same individual vary with the width of the pupil and other dioptric arrangements of the eye; individual variations are also considerable; but the ordinary dimensions may be stated as subtending an angle of about $145^{\circ}$ in the horizontal and about $100^{\circ}$ in the vertical meridian, the former being distinctly greater than the latter. When an extemal object lies outside the area subtending these angles we say that it is outside the field of sight for that position of the eye ; it may of course be brought into the field of sight by moving it or by moving the eye. The outline of the field is an irregular onc, and stretehes farther towards the temporal side of the fixed point, that is, towards the nasal side of the retina, than on the other side; it is somewhat larger and of a different form when the eye is turned towards the temporal side than when the eye is directed straight forwards, ef. Fig. 156. It will be understood that the two visual fields of the two eyes are unlike, ef. Fig. 157.

When we use both eyes a large part of the visual field of each eye overlaps that of the other; that is to say, the rays of light procecding from a large part of the field of sight of each eye fall upon and affect both retinas. But at the same time a certain part of each visual field does not so overlap any part of the other. If the right hand be held up above the right shoulder and bronght a little forward it soon becomes distinctly visible to the right eye, it enters into the field of sight of the right eye. But if the right eye be closed, we find that the right hand kept in the former position is not visible to the left eye; it is outside the field of sight of that eye ; it has to be brought 
much further forward until it comes into the field of sight of the left eye ; the profile of the face and especially of the nose prevent the rays reflected from the hand gaining access to the left retina until the hand is brought a certain distance forward. The right-hand side of the objective field of sight of the right

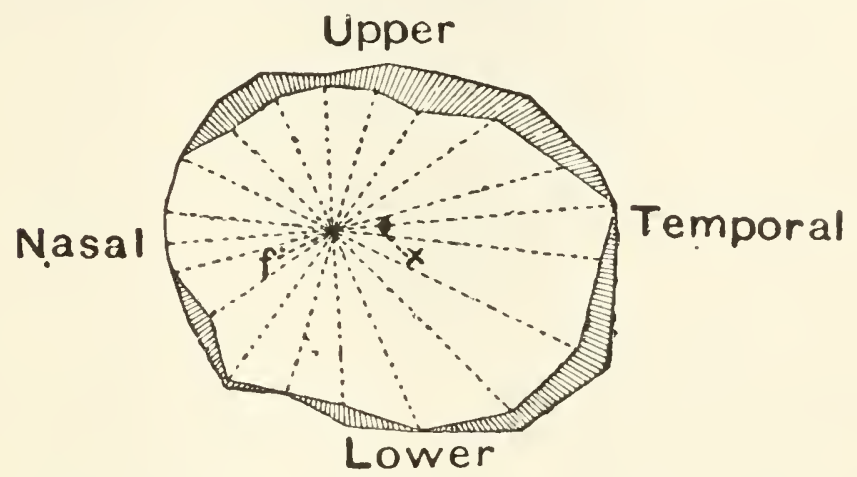

Fig。156. The visual field of the right eite. (Aubert.)

The figure represents the visual field projected into space and therefore corresponds to the objective field of sight; the temporal side of the figure corresponds to the nasal side of the retina. The shaded part indicates the increase gained by looking outwards towards the temporal side. $f$, fovea ; $x$, blind spot.

eye, corresponding to the nasal side of the retina of that eye, extends much farther to the right than does the right-hand side of the field of sight of the left eye, which corresponds to the temporal side of the retina of that eye. Cf. Fig. 157. Similarly, the left-hand side of the field of sight of the left eye extends farther to the left than does that of the right eye. Hence on the one hand the total field of sight of the two eyes together is increased in the horizontal diameter, subtending on an average an angle of $180^{\circ}$ instead of $145^{\circ}$; and on the other hand while a certain right-hand and left-hand part of the united fields of sight belong respectively to the right and left eye only, the remainder of the field is common to the two eyes. The area common to the two eyes when the visual axes converge to the sane fixed point, is shewn as the shaded part in Fig. 157. Rays of light from objects in the common part affect the retinas of both eyes at the same time, vision is here binocular; rays of light from objects at the extreme right and left affect only the right and left retina respectively, vision in these parts of each eye is never binocular, always monocular. The amount of each retina which is thus cut off from binocular vision is determined by the prominence of the nose and profile between the eyes; in some of the lower animals the position of the eyes is so completely lateral that no rays of light proceeding from the same 
object can fall on any part of the two retinas at the same time, and in these creatures vision is wholly monocular.

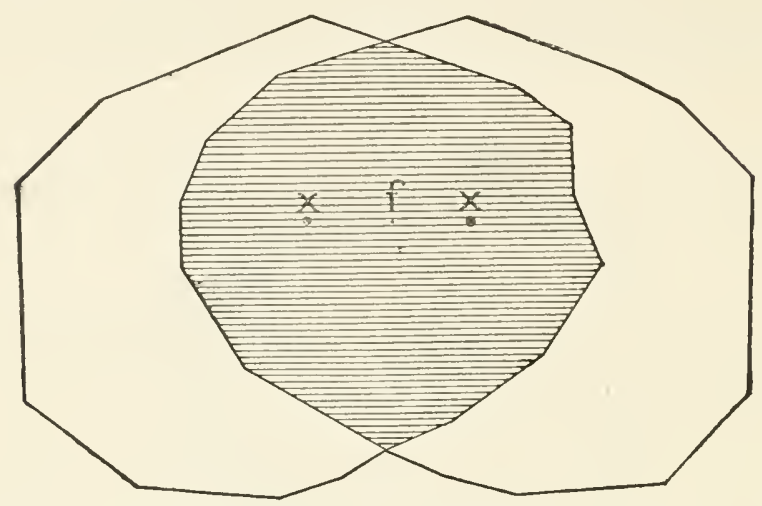

Hig. 157. The visual Fields (Fields of Sight) of tile two Eyes wiey the eYes converge to the same fixed point. (Aubert.)

The shaded part is that common to the two eyes. $f$, the fixed point, corresponding to the fovea of each eye; $x$, the blind spots of the two eyes.

§589. Corresponding or Identical Points. Though when we use two eyes, we must receive from every object in the field of sight common to the two eyes two sets of visual impulses, indeed we may say two sets of sensations, our perception of the object is under ordinary circumstances a single one; we see one object, not two. By putting either eye into an unusual position, as by squinting, we can render the perception double; we see two objects where one only exists. This singleness of the sensation under ordinary circumstances shews that certain parts of each retina are so related to each other that when an image of an object falls on these parts at the same time, the two sets of sensations excited in the two parts are blended into one; such parts are spoken of as corresponding parts; they have also been called identical parts. Since in the ordinary movements of the eyes we see objects single, and do not receive donble impressions unless we move the eyes in an unusual manner, it is obvious that the movements of the eyeballs and these corresponding parts of the two retinas are so related, the one to the other, that the former bring the images of objects to fall on the latter.

IVe can easily determine which are the corresponding parts of the two retinas by tracing out the paths of the rays of light falling on the two retinas, S5 528. As we have said, when we look at an object with one eyc the visual axis of that eye is directed to the object, and when we use two eycs the visual axes of the two eyes converge at the object, the eyeballs moving accordingly. The corresponding points of the two retinas are 
those on which the two images of the object fall when the visual axes converge at the object. Thus in Fig. 158 if $v l$ from $X$ to $x$ and $X$ to $x^{\prime}$ be the two visual axes, $x, x^{\prime}$ being the centres of the fover centrales of the two eyes, then, the object $X Y Z$ being seen single, the point $y$ on the one retina will 'correspond' to or be 'identical' with the point $y$ ' on the other', and the point $z$ in the one to the point $z^{\prime}$ in the other.
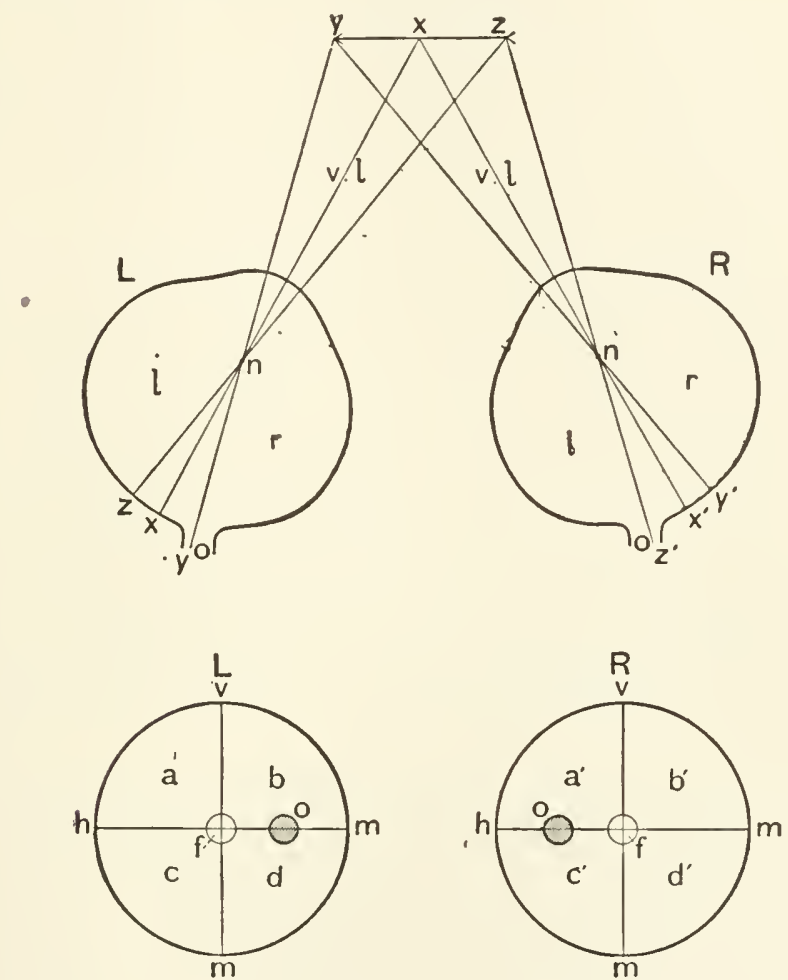

Fig. 158. Diagram illustrating Corresponding Points.

$L$ the left, $R$ the right eye, $n$. nodal point, $o$. optic nerve, $x$. fovea. $x^{\prime} y^{\prime} z^{\prime}$ are points in the right eye corresponding to the points $x y z$ in the left eye. $v . l$. visual axis. The two figures below are projections of $L$ the left and $R$ the right retina. $f$. fovea, $o$. blind spot. It will be seen that $a$ and $c$ on the temporal side of $L$ corresponds to $a^{\prime}$ and $c^{\prime}$ on the nasal side of $R . \quad v . m . h . m$. lines of separation.

When the whole area of the retina in each eye which we use for binocular vision is explored in this way we find, as follows geometrically from the paths of the rays of light, that the upper half of one retina corresponds to the upper half of the other, the lower half to the lower half, the right side to the 
right side, and the left side to the left side. But when we turn to the structure of the retina we find that the left or nasal side of the right eye, since it contains the entrance of the optic nerve, is comparable with, not the left, but the right or nasal side of the left eye, and in like manner the right or temporal side of the right eye is comparable with the left or temporal side of the left eye. Hence, considered in relation to the structure of the retina, the corresponding points appear to be reversed from side to side, though not from top to bottom. While the upper half of the retina of the left eye corresponds to the upper lialf of the retina of the right eye, and the lower to the lower, the nasal side of the left eye corresponds with the temporal side of the right, and the temporal of the left with the nasal side of the right.

It will be observed that in each eye a vertical plane through the visual axis ( $v . l$. in Fig. 158) cuts the retina in a vertical line $v$. m., which divides the retina into two lateral, temporal and nasal, halves, each temporal and each nasal half corresponding with the nasal and temporal half respectively of the other eye. When the visual axes of the two eyes are parallel, the two vertical planes in question are parallel to the median plane and to each other. Further, a horizontal plane drawn through the visual axis at right angles to the above vertical plane cuts the retina in a horizontal line $h . m$.; and this also divides the retina into two halves, an upper and lower half, the upper and the lower halves of both retinas being corresponding. 'These two lines. cach of which may be considered as a series of corresponding points, are sometimes spoken of as lines of separation.

The blending of the two sensations into one occurs, we repeat, only when the two images of an object fall on corresponding points of the two retinas. Hence it is obvious that in single vision with two eyes the ordinary movements of the eyeballs must he such as to bring the visual axes to converge at the object looked at so that the two images may fall on corresponding points. When the visual axes do not so converge, and when therefore the images do not fall on corresponding points, the two sensations are not blended into one perception, and vision becomes double. It is therefore important to study in some detail the movements of the eyeballs, by means of which, in ordinary vision, the relative positions of the two retinas are so carefully adjusted that we habitually see objects single not double.

$\$ 590$. The movements of the Eyeball. As we have said, the movements of the eyeball are movements of rotation round an immobile centre, the centre of rotation; but these movements are limited in a particular way. and it is necessary to pay attention to their characters and limitation. 
One position of the eyeball, for reasons which we shall see presently, is called the primary position, and it will be desirable to start from this position. Thougl its exact determination requires special precantions it may be described as that which is assumed when, with the head erect and vertical, we look straight forwards to the distant horizon ; the visual axes of the two eyes are then parallel to each other and to the median plane.

Let us now suppose three axes drawn througl the centre of rotation, in the three planes of space:- one, the visual axis itself, which we may call the longitudinal axis; another at right angles to this and horizontal, the horizontal axis; and a third also at right angles, but vertical, the vertical axis. Corresponding to these three axes we have three main possible movements of rotation. 'The eyeball might be rotated round the vertical axis so that the visual axis mover from side to sicle. It might be rotated round the horizontal axis so that the visual axis mover up and down. And lastly, it might be rotated round the longitudinal axis, the visual axis itself remaining motionless and the pupil turning round like a wheel.

Now we can easily carry out by an exercise of the will the first and second of these movements. We can easily move the eyes up and down, rotating them on the horizontal axis, as when we look up to the heavens or down to the ground. We can also move the eyes from side to side, rotating them round the vertical axis, as when we look to the right or to the left. We can move the two eyes sideways together in the same direction keeping the visual axes parallel, or we may move them laterally in opposite directions, as when the visual axes being parallel we make them converge, or when convergent bring them back to or towards parallelism. And we can combine rotation round the horizontal axis with rotation round the vertical axis, and so give oblique movements to the eyeball. We can do all this by an exercise of the will, but we cannot by any voluntary effort carry out the third kind of movement, we cannot rotate the eyeball round the visual axis, we cannot twist the eye in a swivel movement round its longitudinal axis. There are certain movements of the eye in which such a swivel rotation, if we may so call it, does to a certain extent take place, and when we induce these movements we do bring about such a swivel rotation: but we cannot bring about swivel rotation by itself, we can only effect it as part of the particular movements in question.

And there is a reason why we are thus limited as to our power of moving the eyeball. In both rotation round the horizontal axis, and rotation round the vertical axis and in all the various combinations of these two movements which are possible, the two "lines of separation" ( $\$ 589)$ on both the retinas keep their places; there is no dislocation of the corresponding regions of the two retinas. Obviously the two retinal circles in the lower 
part of Fig. 158 could be rotated round the vertical or round the horizontal axis or round any intermediate oblique axis without the two images of an external object ceasing to fall on corresponding parts. But if the retinal cireles were twirled round their respective visual axes, the lines of separation, $v, m$. and $h$. m., would rotate in a clock-hand fashion, and if the movements of the two eyes were unequal or in opposite directions, a dislocation of corresponding parts would ensue, and vision would become double. The limitation to the movements of the eyeball so as to avoid a swivel rotation is in the interests of binocular vision.

$\$ 591$. Not only do we find ourselves thus limited in our power when we attempt by a direct effort of our will to execute particular movements of the eyeball, but a similar limitation obtains in the natural movements of the eye in vision. The various movements of the eyeballs which we carry out when we are looking at things conform to a general law, which is known as "Listing's Law," and which may be described as follows.

We stated a little while back that the "primary position" of the eyeball is one in which the visual axis lies parallel to the median plane and is directed to the distant horizon. When the eyeball is changed from this primary position into any other position, all of which may be called secondary positions, the change is effected without any swivel rotation round the visual axis itself; the visual axis may be directed up and down, or from side to side or in any intermediate oblique manner without any such swivel rotation taking place. In other words the movements by which the eyeball is brought from the primary position into any of the secondary positions are, in all cases, movements of rotation round the horizontal axis, or round the vertical axis, or round an axis, which thongh oblique, being neither horizontal nor vertical, lies in the same plane that they do; that is to say every movement from the primary to a secondary position is a movement of rotation round an axis lying in a plane which passing through the centre of rotation is vertical to the visual axis.

The experimental proof of "Listing's law" may be obtained by the help of negative images ( $\$ 584$ ) in the following manner. Let the eye be directed to a grey wall or board which, otherwise of uniform appenrance, is marked by parallel vertical and horizontal lines, placed at some little distance from each other so as to give a pattern of squares. At one of the intersections, which is to be used as the fixed point of vision, place two narrow strips of red paper in the form of a cross, one vertical coinciding with the vertical line and the other horizontal coinciding with the horizontal line. Having brought the eye carefully into the primary position stare at the red cross until on turning the eye 
away a green negative image is produced. If now the vision be directed from the fixed point either up or down along the vertical line of the pattern on the wall, or from side to side along the horizontal line, it will be found that the cross of the negative image coincides in turn with each of the crosses of the pattern on the wall, the horizontal limb coinciding with a horizontal line and the vertical limb with a vertical line. This shews that duning the up and down and rluring the side to side movement, during the rotation of the eyeball round its horizontal or round its vertical axis, no swivel rotation has taken place, for otherwise the negative image would have been turned round, and its cross would make an angle with the image of the cross on the wall. If the pattern on the wall be changed so that the lines while still at right angles to each other are oblique, not vertical and horizontal (this is most conveniently done by using not a wall but a large board and turning the board round), and the observation be repeated except that the eye is turned not vertically or horizontally but obliquely so as to follow the lines of the pattern, it will still be found that the cross of the negative image coincides with the cross of the pattern, and that whatever be the angle round which the board has been turned. 'This shews that Listing's law holds good not only for up and down and side to side movements but also for oblique movements, for movements of rotation round an axis which whatever its obliquity lies in a plane at right angles to the visual axis.

In the ordinary movements of the eye then, a swivel rotation round the visual axis does not take place; and this limitation, since it holds good for the two eyes used together, as well as for one eye used by itself, serves to secure single vision with two eyes inasmuch as it avoids changes which might cause the images of external objects to fall on the parts of the two retinas which were not "corresponding parts." In certain movements of the eyes, however, a certain amount of swivel rotation does take place. This is especially seen in somewhat unusual movements. For instance when the head is turned down to the shoulder, or again when in directing vision to any object, the head is moved from side to side, the eyes do not move with the head; they appear to remain stationary, very much as the needle of a ship's compass remains stationary when the head of the ship is turned. The change in the position of the visual axes to which the movement of the head would naturally give rise is met by compensating movements of the eyeballs; were it not so, steadiness of vision would be impossible; and these compensating movements are found, on careful examination, to include a certain amount of swivel rotation round the visual axes. In certain other more usual movements some amount of such a swivel rotation is also present; and indeed, though so long as the visual axes remain parallel, movement in any direction may 
take place without any such rotation, a slight amount does intervene during convergence of the visual axes, as when we turn our eyes from a distant to a near object. On careful examination, however, it appears that such an amount of swivel rotation as does take place is after all for the purpose of securing the end that corresponding parts of the two retinas should be affected by the same external object; and, though we cannot here enter more fully into the subject, we may say that not only the more general movements of the eye which obey Listing's law, but also those which form an exception to it, appear to be carried out in the interests of binocular vision. We may now turn to the study of the ocular muscles, by the carefully coordinated eontractions of which the various movements, on which we have dwelt, are brought about.

\$592. The muscles of the eyeball or ocular muscles. The eyeball is moved by six muscles, four of which are straight, musculi recti, inferior, superior, internus or medialis and extermus or lateralis, and two oblique, musculi obliqui, inferior and superior. The four straight muscles, taking origin from the back of the orbit around the sphenoidal fissure and the entrance of the optic nerve, are directed, as their name indicates, straight forward, (the superior rectus, however, having a peculiar bend.) and are inserted in positions corresponding to their several names into the sclerotic, behind the cornea, the bundles of fibres of the tendons being interwoven with those of the sclerotic. The tendon of the internal rectus on the inedian or nasal side of the eyeball is the broadest of the four; that of the superior rectus on the upper surface being somewhat narrower, and those of the inferior rectus on the under surface and of the external rectus on the lateral or temporal side, still narrower (Fig. 159). The insertion of the superior rectus lies nearer to that of the external rectus than to that of the internal rectus; its position therefore is not exactly median, indeed for twothirds of its width it lies in the upper lateral quadrant of the selerotic ring. The insertions of the external and of the internal rectus are both median. The insertion of the internal rectus is the one closest to, and that of the superior rectus the one farthest away from the cornea, and the latter slants so as to be nearer the cornea at its median than at its lateral end.

The superior oblique muscle, or trochlear or pathetic muscle, taking origin from the back of the orbit near the origin of the straight muscles and rumning forward internal to the superior rectus, cnds in a tendon, which changing its direction by means of a pulley (trochlea), and passing beneath the superior rectus is inserted into the sclerotic in the upper region of the bulb towards its hind part. The line of insertion of the tendon (Fig. 159) runs obliquely from the temporal towards the nasal 
side, its mid-point lying not far from the vertical mericlian of the eyeball.

'The inferior oblique muscle arises from the front of the floor of the orbit on the nasal side; it is directed at first backwards to the temporal side, underneath the inferior rectus, between that and the floor of the orbit, and then passing upwards and backwards is inserted into the sclerotic underneath the external

\section{LEFT EYE}

FROM TEMPORAL SIDE

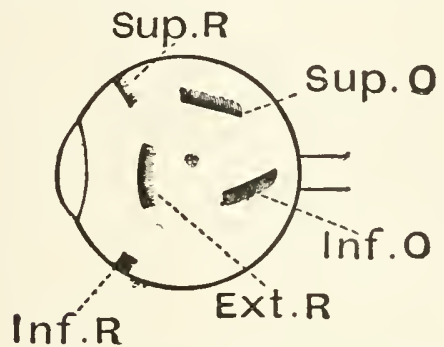

FROM ABOVE

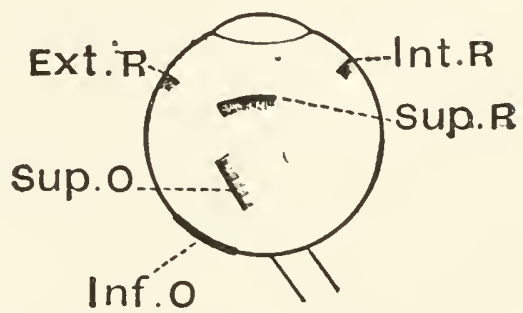

Fig. 159. The Left eye seen from A, the temporal sine. B, from above, shewisg the insertions of the Ocular Muscles. (Jessop.)

rectus in the hind temporal part of the ball. The line of insertion (Fig. 159) is also an oblique one like that of the superior oblique but it is placed somewhat farther past it; its hind end lies not far from the entrance of the optic nerve and it runs thence forwards and downwards.

$\$ 593$. The manner in which these muscles are thus severally attached to the eyeball suggests that in contracting they would move the eyeball in the following ways. Taking changes in the direction of the visual axis as indicating the nature of each movement we should expect that the superior rectus would turn the visual axis upwards, the inferior reetus downwards, the external rectus outwards towards the temporal side, and the internal rectus inwards towards the nasal side. The inferior oblique, its insertion being on the hind and lateral part of the eyeball, and the direction of the muscle being downwards, would in contracting turn the visual axis upwards, while the superior oblique having a somewhat similar insertion but acting in an opposite direction would turn the visual axis downwards. Both muscles however in thus raising or lowering the visual axis would, owing to the oblique direction of their insertions at the same time, turn it to the temporal side; the movement as the names of the muscles suggest, would be an oblique one. 
The six muscles therefore would seem to act as three pairs, the superior and inferior rectus, the internal and external reetus, and the inferior and superior oblique, each pair rotating the eyeball round a particular axis. Calculations based on a careful study of the attachments and directions of the several muscles, and the results of actual observations, shew that this is so, and that the movements carried out by the several pairs may be more aceurately described as follows.

The superior rectus and the inferior rectus (see Fig. 160) rotate the eye round a horizontal axis, which may be described

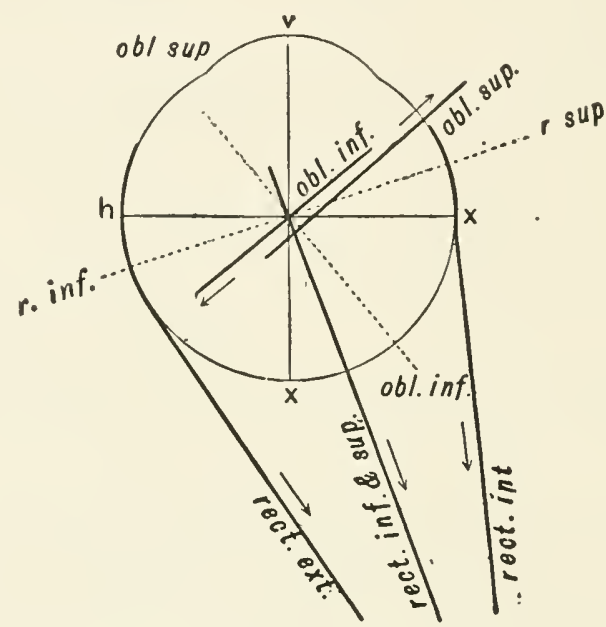

Fig. 160. Diagram to illestrate the actions of the Muscles of the Eye.

The eye represented is the left eye seen from above. The thick lines shew, by means of the arrows, the direction in which the several muscles pull, the beginning of each line also indicating the attachment of the muscle. The dotted lines indicate the axis of rotation of the superior and inferior rectus and of the oblique muscles. The axis of rotation of the internal and external rectus being perpendicular to the plane of the paper cannot be shewn. $v x$ represents the visual axis and $h x$ a line at right angles to it. ( $\Lambda$ fter Fick.)

as one directed from the root of the nose to the temple; it is therefore not a line at right angles with the visual axis but one making an acute angle $\left(20^{\circ}\right)$ with such a line. The superior and inferior oblique rotate the eye round a horizontal axis which may be deseribed as one directed from the centre of the eycball to the occiput; it again is not a line at right angles to the visual axis, but makes an angle, with such a line, larger $\left(60^{\circ}\right)$ than the similar angle made by the inferior and superior rectus, and turned in a different direction. 'The internal rectus and external rectus rotate the eyeball round a vertical axis pass- 
ing through the centre of rotation of the eyeball parallel to the median plane of the head when the head is vertical; this therefore is at right angles to the visual axis, and so differs from the other two.

When we compare the movements thus effected by these several pairs of muscles with the movements which we described above (\$590) as the ordinary movements of the eye, namely movements of rotation round a vertical and round a horizontal axis both at right angles to the visual axis, we see that it is only the movements round the vertical axis which can be carried out by one pair of muscles acting alone, the particular pair being the internal and external rectus. Neither the horizontal axis of rotation of the inferior and the superior rectus, nor that of the oblique muscles, is placed exactly at right angles to the visual axis; each of them makes an oblique angle with that axis. Hence when in carrying out the ordinary movements of the eye we rotate the eyeball round the horizontal axis, we do not employ either of these pairs of muscles alone, but combine them, making use of one muscle of one pair with one of the other. The superior and inferior rectus in moving the visual axis up and down also turn it somewhat inwards, to the nasal side; but this is corrected if the oblique muscles act at the same time; and it is found that the rectus superior acting with the inferior oblique moves the visual axis directly upwards, and the rectus inferior acting with the superior oblique directly downwards in a vertical direction; that is to say the two combinations rotate the eyeball round a horizontal axis at right angles to the visual axis.

Hence there are only two movements of the eyeball which we can carry out by the help of one muscle alone, namely that in which we simply turn the visual axis to the nasal side, employing the internal rectus, and that in which we turn it to the temporal side, employing the external rectus, the visual axis in both cases remaining in the same plane, the visual plane. In order to raise or lower the visual axis in the same vertical plane, without lateral movement, we must use two museles; and if we wish to execute an oblique movement combining an up and down with a side to side movement of the visual axis we must employ three of the ocular muscles. These several movements, with the muscles concerned, may be stated as follows, the movement in each case being described with reference to changes in the direction of the visual axis.

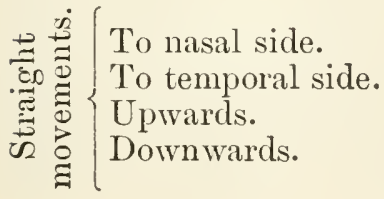

\section{Internal rectus.}

External rectus.

Superior rectus and inferior oblique. Inferior rectus and superior oblique. 


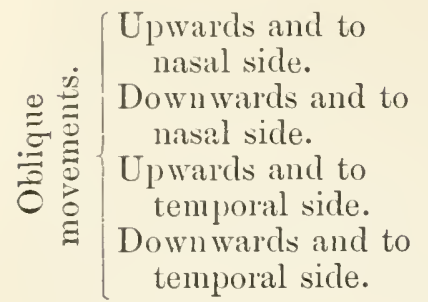

Superior rectus, internal rectus and inferior oblique.

Inferior rectus, internal rectus and superior oblique.

Superior rectus, external rectus and inferior oblique.

Inferior rectus, external rectus and superior oblique.

The fact that in our ordinary movements of the eye we do thus combine the actions of muscles, and the advantages of such a eombination are further shewn in connection with that swivel rotation of the eye round the visual axis itself, which, as we have seen, is wholly avoided in many of our movements and which we cannot carry out by a direct effort of the will. The superior rectus acting by itself, owing to the position of its insertion in reference to the direction of the fibres, not only turns the visual axis inwards while directing it upwards, but also to a slight extent rotates the eye round the visual axis, and the inferior rectus as well as both the oblique muscles in like manner tend in contracting to give the eyeball such a swivel rotation. 'This tendency of the superior rectus like its tendency to turn the visual axis inwards is counteracted by the inferior oblique, the swivel rotation of the latter being contrary in direction to that of the former; and the like tendency of the inferior rectus is in like manner counteracted by the superior oblique. Thus the movements, in carrying out which these muscles are combined, are rendered free from the swivel rotation element. On the other hand this tendency of the muscles in question is utilized in the particular movements in which the swivel rotation does take place.

$\$ 594$. The coordination of the movements of the eyes. The external rectus is governed by the sixth nerve, nervus abducens, the nucleus of which lies in the floor of the fourth ventricle in a position indicated by the eminentia teres. The superior oblique musele is governed by the fourth nerve, nervus trochlearis, the nucleus of which lies in the floor of the aqueduct, in the region of the posterior corpus quadrigeminum. All the other ocular muscles are governed by the third nerve, the nucleus of which lies in the floor of the aqueduct in the region of the anterior corpus quadrigeminum ; as we have said (\$539), the fibres of the third nerve going to these ocular muscles seem to be more especially connected with the hind part of the nucleus.

From what has been said above it is obvious that, even in the movements of one eye, a coordination of the motor nervous impulses must in most cases take place. When we turn the visual axis outwards the motor impulses are, it is true, confined 
to the sixth nerve, reaching the external rectus, and when we turn it inwards are confined to the third nerve, reaching the internal rectus; but in all other movements motor impulses must descend to at least two muscles along different nervebranches, and in many cases must start from two or even all three of the cranial nuclei just mentioned. Even in movements of one eye there must be, in most cases, more or less coordination of actual motor impulses, in order to secure due efficiency of the movement; by actual motor impulses we nean inpulses leading to the contraction of muscular fibres, irrespective of any influences which may at the same time be brought to bear on antagonistic muscles, in order to facilitate or qualify the movement.

But if this is true in the case of one eye, much more is it true when we use both eyes in binocular vision.

'Two facts about binocular vision strike our attention. 'The one is that, as may be seen by watching the movements of any person's eyes, the two eyes move together. If the right eye moves to the right, so does also the left, and, if the object looked at be a distant one, exactly to the same extent; if the right eye looks up, the left eye looks up also; and so with regard to other movements. Very few persons are able by a direct effort of the will to move one eye independently of the other; though by some the power has been acquired. IVe shall refer immediately to particular movements in which one eye only is moved, while the other remains motionless. The other salient fact is that the movements of the two eyes are limited in certain ways. As we have seen one of the simplest ocular movenents is the side to side movement of the visual axis, and one of the commonest binocular novenents is the convergence of the visual axes, as when we turn our eyes from something far off to something near, or conversely the change from considerable convergence to less convergence as when we turn our eyes from something near to something farther off. In a large number of instances this change to convergence from parallelism, or this increase or decrease of convergence takes place without any change in the visual plane, without any raising or lowering of the visual axes; in such instances the movement is carried out in convergence by the two internal rectus museles, or in decrease of convergence by the two external rectus muscles; and the only coordination necessary is one which secures that the muscle of one eye should work in harmony with the muscle of the other eye. But even this relatively simple movement is limited in a very marked way. WV can bring the visual axes of the two eyes from a condition of parallelism to one of almost any degree of convergence, but we cannot, without artificial assistance, bring them from a condition of parallelism to one of divergence. The stereoscope will enable us to ereate such a 
divergence. If in a stereoscope the distance between the pictures be increased very gradually so as earefully to maintain the impression of a single object, the visual axes may be brought to diverge, and the subject of the experiment may himself be made aware of the divergence, by the sudden removal of the instrument from his eyes; his vision of external objects is for a moment double, but for a moment only. This experiment shews the reason of the limitation of which we are speaking. so long as the visual axes are parallel or appropriately convergent the images of external objects fall on corresponding parts of the two retinas, and single vision results; when the visual axes are carried beyond parallelism, the images on the two retinas are not on corresponding parts and vision is double. Thus, as regards convergence or divergence of the visual axes, the movements of the two eyes are governed by the principle that the will can of itself only carry out those movements which are consistent with images of external objects falling on corresponding parts of the two retinas. There is an exception to this in the case of extreme convergence; we can as in squinting make the visual axes converge too much, and in consequence by a simple effort of the will can obtain double vision ; but this is probably in order to leave a margin which shall secure our being able to use to the utmost our accommodation mechanism for near objects; otherwise the rule holds good. Not only so, but as the above experiment also shews, when by artificial assistance, which is in itself directed towards securing single vision with the two eyes, we obtain divergence of the visual axes, immediately that the assistance is done away with the axes return, by an involuntary movement, to parallelism; the double vision occurring at the moment of removal of the instrument rapidly gives way to normal single vision. Other illustrations of the same principle may be met with. For instance, if a distant object be looked at with both eyes, but with a prism held horizontally before one eye, and if the image of the objeet be kept carefully single while the prism is turned very slowly from the horizontal to the vertical position, then on suddenly removing the prism a double image is for a moment seen; this shews that the eye before which the prism was placed had moved in disaccordance with the other. The double image, however, immediately after the removal of the prism, becomes single on account of the eyes coming into accordance.

When we examine all the various movements of the eyes which we are capable of making by a direct effort of the will, we find that they are all of such a kind that through them the two images of an external object are brought upon corresponding parts of the two retinas; conversely the movements which could be effected by the contractions of this or that ocular muscle, but the effect of which would be to bring the two images 
on to parts of the retina which do not correspond, are the movements which our unassisted will cannot carry out.

In an earlier part of the work (\$478) we insisted at some length on the important share taken by sensations, or at least by afferent impulses, in the coordination of motor impulses; and the movements of the eye illustrate this in a very marked degree. All the various movements of the eye are dependent on visual sensations. The issue of each efferent motor volitional impulse is dependent on afferent visual impulses. In order to move our eyes, we must either look at or for an object, when we wish to converge our axes, we look at some near object real or imaginary, and the convergence of the axes is usually accompanied by all the conditions of near vision, such as increased accommodation and constriction of the pupil. And so with other ocular movements. Above all, the careful selection of this or that ocular muscle, the extent to which it is to be thrown into contraction, its accompaniment by the contraction of other ocular muscles and the due coordination of all the several contractions - all these things are so determined by visual sensations that the two images of each object looked at fall on corresponding parts of the two retinas.

A little reflection will slew how large an amount of coordination must thus take place in daily life, how in the various movements of the eye there must be, so to speak, the most delicate picking and choosing of the muscular instruments. When we look at an object to the right, since we thereby turn the right eye to the temporal side, and the left eye to the nasal side, we throw into action the external rectus of the right eye and the internal rectus of the left; and similarly when we look to the left we use the external reetus of the left and the internal rectus of the right eye. On the other hand when we look at a near object, and therefore converge the visual axes, we use the internal rectus of both eyes; and when we look at a distant object, and bring the axes from convergence towards parallelism, we use the external rectus of both eyes. Or to take another instance. Suppose the eyes, to start with, directed for the far distance, and that it is desired to direct attention to a nearer point lying in the visual line of the right eye. In this case no movement of the right eye is required; all that is necessary is for the left eye to be turned to the right, that is, for the internal rectus of the left eye to be thrown into action. But in ordinary movements the eontraction of this muscle is always associated with either the external rectus of the right eye, as when both eyes are turned to the right, or the internal rectus of that eye, as in convergence; the muscle is quite unaccustomed to act alone. This would lead us to suppose that in the case in question the contraction of the internal rectus of the left eye is accompanied by a contraction of both the external and the internal rectus of the right eye, 
keeping that eye in lateral equilibrium. And the peculiar oscillating movements seen in the right eye, as well as the sense of effort in the right eye which is felt by the person, support this idea. We need not multiply these instances; it must be sufficiently obvious that a very large amount of coordination takes plitce in the daily use of our eyes.

$\$ 595$. Such a coordination involves the existence of what, to continue the use of a term which we have previously used, we may call a coordinating nervous mechanism. The coordinated efferent impulses issue from one or mole of the nuclei of the three cranial nerves concerned, namely the sixth, the fourth, and the third. The afferent visual impulses taking part in the coordination, we have in an earlier part of this book ( $\$ 496$ ) traced to the primary visual centres, and thence to the occipital cortex. The volitional impulses themselves are we have seen ( $\$ 484)$ connected in some way or other with an area of the cortex lying in the monkey in the frontal lobe, in the neighbourhood and in front of the precentral fissure (Figs. 122, 12:3) and probably in man occupying a corresponding position. How are these three factors of the whole nervous action brought to bear the one on the other? When it is remembered how complex and delicately balanced are the movements in question, probably the most intricate and the most delicately balanced of all the movements of the body, it will readily be understood how difficult is the answer to such a question. Stimulation of the cortical area for movements of the eyes leads as might be expected to bilateral movements, to movements of both eyes. The most common effect of stimulating the cortical area is a lateral movement of both eyes in the same direction towards the opposite side, a conjugate lateral deviation of both visual axes towards the opposite side. For instance when the cortical area of the left hemisphere is stimulated, the visual axes of both eyes are turned to the right, the external rectus of the right eye and the internal rectus of the left eye being thrown into contraction by impulses passing down the right sixth nerve and left third nerve; the efferent impulses therefore cross in the case of one nerve but not in the casc of the other. Similarly, when the right hemisphere is stimulated, impulses pass down the right third nerve and left sixth nerve.

Though these lateral movements are those most easily produced, other movements, conjugate raising or lowering of both eyeballs, oblique movements, and even movements of convergence lhave been obtainer. And, were our means of stimulation adcquately discriminating, it would probably be found that each of the several ocular movements might be ealled forth by stimulating the appropriate cortical focus. Like movements of the eyeballs may also be obtained by stimulating not the frontal motor region, but the oceipital region ( $\$ 498$ ). These latter 
movements are not due to the frontal motor area being indirectly thrown into aetion, sinee they appear even after this has been removed; they are obviously brought about by a separate mechanism. The action of the cortex, moreover, appears not to be limited to producing eontractions in these ocular muscles; it may take on the charaeter of inhibition. If the third and fourth nerves be divided on one side, so as to leave the rectus externus of that eye alone available, not only is the opposite eye moved outwards, upon stimulation of the cortex on that side, but the eye of the sime side follows it to a certain extent; that is to say the stimulation of the cortex, while it leads to contraction in the opposite rectus externus, inhibits a tonic contraction of the rectus externus of the eye of the same side and so permits that eye to move in the same direetion as its fellow. 'This is a further indication of the eomplexity of the coordination of these oenlar muscles. This eoordination is not effected or not wholly effected in the cortex, since coordinate movements may be produced by stimulating the fibres leading from the eortex. Possibly some at least of the cooldination is effeeted by help of the anterior eorpora quadrigemina, since eoordinate oeular movements may be obtained by directly stimulating these bodies. The tract of fibres known as the posterior longitudinal bundles, which seems to serve as a tie uniting the several oeular nuelei, probably also plays a part in the matter.

\section{The Horopter.}

$\S 596$. When we look at any objeet we direct to it the visual axes, so that when the retinal image of the object is small, the

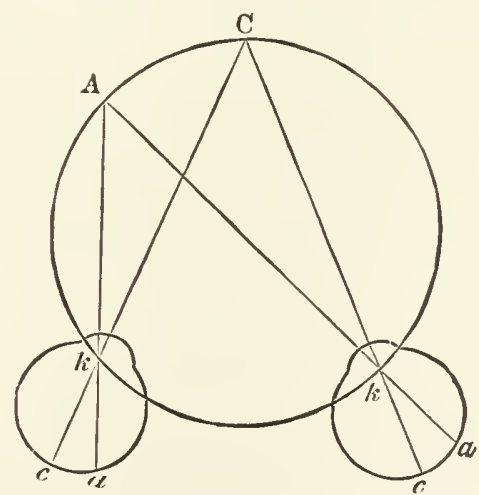

Fig. 161. Diagram illustrating a simple Horopter.

When the visual axes converge at $C$, the images $a$ a of any point $A$ on the circle drawn through $C$ and the nodal points $k k$, will fall on corresponding points.

'eorresponding' parts of the two retinas, on which the two images of the objeet fall, lie in their respective fover eentrales. 
But while we are looking at the particular object the images of other objects surrounding it fall on the retina surrounding the fovea, and thus go to form what is called indirect vision. And it is obviously of advantage that other images, besides that of the object to which we are specially directing our attention, should fall on 'corresponding' parts in the two eyes. IVere it not so, while our vision of the particular object would be single, our vision of all its surroundings would be double; and this, at least in certain cases, would be confusing. For, even when we are concentrating our attention on a particular object, we are still conscious of its surroundings, and besides, our appreciation of any image falling on the fovea is influenced by impressions which we are at the same time receiving from other parts of the retina.

Now for any given position of the eyes there exists in the field of sight a certain line or surface of such a kind that the images of the points in it all fall on corresponding points of the retina. A line or surface having this property is called a Horopter. The horopter is in fact the aggregate of all those points in space which, in any given position of the eyes, are projected on to corresponding points of the retina; hence its detcrmination in any particular case is simply a matter of geometrical calculation. In some instances it becomes a very complicated figure. The case whose features are most easily grasped is that of a circle drawn in the plane of the two visual axes throngh the point of the convergence of the axes and the nodal points of the two eyes such as is shewn in Fig. 161. It is obvious from geometrical relations that the two images of any point in such eirele, the rays from which can enter the two pupils and fall on the two retinas, will fall on corresponding points of the two retinas. When we study the various horopters of the several positions which the two eyes can take up, we find that the characters of the horopter are adapted to the needs of our daily life. 'Thus in the position assumed by the two eyes when we stand upright and look at the distant horizon the horopter is (approximately, for normal emmetropic eyes) a plane drawn through our feet, that is to say, is the ground on which we stand; the advantage of this is obvious.

Nevertheless, in most positions of the eyes a large number of the images which make up the binocular visual field, do not lie on any horopter, do not fall on corresponding points, and give rise not to one sensation only but to two sensations differing to a ccrtain extent from each other. A great deal of what we see is secn double by us, we receive fiom many objects two unequal impressions; but the inequality chiefly serves to give an appearance of "solidity" to the objects, to assist in our judgment of solidity. 'To the consideration of these and other visual judgments as well as of some other psychological features of vision we must n w turn. 
SEC. 10. ON SOME FEATURES OF VISUAL PERCEPTIONS AND ON VISUAL JUDGMENTS.

\$597. We may now turn our attention to some of those differences between the features of external objects and our perception of them which are more distinctly of psychological origin; but since the purpose of this work is physiological and not psychological we must be content to treat them very briefly.

'Taking first of all the general features of the field of vision, we find psychical processes entering largely even into these. As we have incidentally seen, the sensations which an object excites are very different according as the object is in the central or in the peripheral region of the field of sight. Two parts of the object sufficiently far apart to give rise to two sensations in the former case may give rise to one sensation only in the latter case; and the colour sensations excited by the same object may be widely different in the two cases. If we picture to ourselves the group of sensations excited by the image of an object, such as a flower, when the image falls on the fovea, and compare that group with the group of sensations excited by the same flower when the image of it falls on the periphery of the retina, supposing the comparison to be made before the sensations are moulded into psychical perceptions, the two groups would appear to belong to very unlike objects. Mloreover, when we use both eyes, the images of some of the objects in the field of sight are falling on both retinas, while others are falling on one retina only, and of those which fall on both retinas, some lie on corresponding points, so that the sensations of the two eyes are blended, while others, not lying in the horopter, give rise to sensations in one eye different from those in the other. Could we become aware of the crude sensations which go to make up, our field of vision, they would appear as a heterogeneous medley. But in the field of vision of which we are actually aware, that in which the crude sensations have by psychical operations been moulded into perceptions, we do not recognize the various discrepancies of which we are speaking; the field of vision is homogeneous. When we look at a landscape we are not aware that objects on the far left or far right hand are producing 
sensations in a way very different from that in which objects directly in the line of vision are producing sensations; it is only by special analysis that we become acquainted with the properties of the peripheral retina. In actual vision the activities of the central retina by virtue of psychical processes dominate those of the periphery. Conversely though, as we have said, when we wish to see anything very distinctly we habitually make use of the central retina; yet nevertheless in ordinary vision, at the same time that we are thus making use of the central retina we are also receiving impressions from the whole of the rest of the retina within the field of vision, and these more or less peripheral impressions influence to a certain extent the psychical effect of the central sensations. Our perception of an object, such as a flower, is not the same when we look at it as part of a landscape, making use of the whole field of rision, as when we look at it through a tube or otherwise in such a way as to exclude peripheral vision; the flower in the latter case seems much more brilliant, and more highly coloured. Some of the effect in this case may be physiological and due to retinal events, but the greater part is psychical. The influence of psychical processes is probably also illustrated by the experience that, if on turning our back on a landscape, we bend the body so as to get a view of the landscape backwards between the legs, all the objects seem to have an unusually brilliant colouring.

A striking difference between the objective field of sight and the subjective field of vision is illustrated by the fact that, though, as we have seen, that part of the retina which corresponds to the entrance of the optic nerve is quite insensible to light, we are conscious of no corresponding blank in the field of vision. When in looking at a page of print we so direct the visual axis that some of the print must fall on the blind spot, no gap in the print is perceived; we have to take special measures ( 573$)$ to discover the existence of the spot. We could not expect to see a black patch, because what we call black is the absence of the sensation of light from structures which are seusitive to light; we must have visual organs to see black. But there are no visual organs in the blind spot, and consequently we are in no way at all affected by the rays of light which fall on it. By psychical operations we "fill up," as it is said, the vacancy caused by the blind spot, so that there is in our subjective field of vision no gap corresponding to the gap in the retinal image; we treat the sensations coming from two points of the retina lying on opposite margins of the blind spot as if they were sensations excited in two points lying close together, thus preserving the contimuity of the field of vision between them. Concerning the particular psychical actions by which this is carried out, and concerning the special effects which are produced when an object in the field of sight passes 
into the region of the blind spot there has been much discussion; but into this we camnot enter here.

In ordinary vision, the existence of the blind spot is of little moment. Since it lies outside the region of distinct vision, and since moreover in each movement of the eye the image of a fresh part of the external world falls upon it, the errors to which it may lead are not serious even when we use one eye only. The deficiency is further remedied by the use of two eyes, since, the two blind spots being each on the nasal side, the image of an object will not fall on both blind spots at the same time. Other smaller or accidental imperfections in one or both eyes are similarly remedied by the use of two eyes.

\$ 598. Turning now to the psychical processes connected with the perception of particular objects, we find these to be very complex. Some of them relate to the very formation of the perception out of the sensations which the object excites, and are often of such a kind that the perceptions which they influence so distinctly fail to correspond with the actual objects that the lack of correspondence can in many cases be demonstrated: such erroneous perceptions are often spoken of as "illusions." In other cases the psychical processes relate to a further mental action by which we form judgments as to the features of external objects. It is not easy however always to draw a line between a 'visual judgment,' such as that involved in forming a conclusion as to the size of an external object, and what nay be called a mere "modified perception," as when a line appears to us shorter or longer than it really is. We may be content here to treat them all together.

The complexity of the psychical processes in question comes about in various ways. On the one hand the characters of a perception are determined not alone by the sensations which actually give rise to it but also by the psychical conditions remaining as the effect of former like sensations. In the formation of perceptions and judgments, suggestions and associations play their part; so that each perception, while it adds to, is also in part the result of our 'experience.' A simple illustration of this is seen in some of the effects of colour. Blue colours as we have seen predominate in a dim light such as that of evening, of moonlight or of winter, whereas reds and yellows are marked in a bright light such as that of full sunshine, or of a summer's day. Hence, when a landscape is viewed through a yellow glass, the yellow hue suggests to the mind bright sunlight and summer weather, although the actual illumination which reaches the eye is diminished by the glass. Conversely when the same landscape is viewed through a blue glass the idea of moonlight or winter is suggested. And many other instances might be given in which the appreciation of the present is moulded by the experience of the past. 
On the other hand the visual perception or visual judgment is not formed exclusively out of the visual sensations which are excited by the image of an object falling on one or on both cyes in a given position. In looking at an object, a movement of one or both eyes often takes place, and the perception of the object or a judgment concerning the object is formed out of the two (or more) sensations excited by the same object in different positions of the eyes. And here other factor's enter into the process, namely sensations other than visual sensations, sensations connected with the contractions of the muscles of the eye, affections of what is known as "the muscular sense." These come into play even when we use one eye only, but are especially potent when we use both eyes in binocular vision; a large number of our visual judgments are determined by the muscular sensations derived from the movements of the eyes through which we look at the object whose features we are judging.

Other influences also, such for instance as sensations of touch, take part in the psychical processes in question. The mere visual sensations which external objects excite, the immediate and direct effects of the visual impulses, form after all but a small part of what we call our vision. Such sensations and other like sensations derived through other senses are to us but symbols of things, upon which the mind puts its own interpretation. But into these matters we camot enter here. We must confine ourselves to certain common facts concerning perceptions, illusions and visual judgments, and more especially to those which relate to the size and distance of external objects and to the characters of form which are indicated by the word "solidity."

$\$$ 599. Appreciation of Apparent Size. The foundation of our judgment of the size of any object is the size of the retinal image of the object. We can distinguish a sensation involving a large retinal area from one involving a small area, and in the region of distinct vision can appreciate even small differences; this is of course only an exercise of the power of localization. We have seen however that, even in the case of a simple and single sensation such as that of a white patch on a black ground, the sensation does not correspond exactly to the objective stimulation of the retina; the white patch through irradiation $\$ 582$ appear's larger than it really is. When we come to deal with more complex groups of sensations we find that over and above any such physiological modifications of the sensations, the psychical processes mentioned above affect our perceptions and judgments of size, often giving rise to illusions. If a line such as $A C$, Fig.

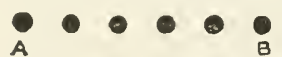

FIG. 162 .
- 162 , be divided into two equal parts $A B, B C$, and $A B$ be divided by distinct marks

into several parts, as is shewn in the figure, while $B C$ be left 
entire, the distance $A B$ will always appear greater than $C B$. The retinal images of the spaces from $A$ to $B$ and from $B$ to $C$ are equal and the corresponding primary visual sensations are also equal, but the mental appreciation of $A B$ is interfered with by the concurrent sensations of the several intervening dots and intervals, and this leads to a mental exaggeration of the interval between $A$ and $B$. So also, if two equal squares (Fig. 163) be

A

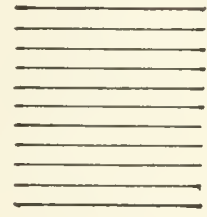

$\mathbf{B}$

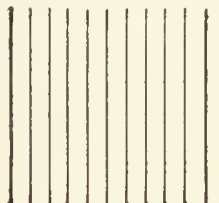

FIG. 163.

marked, one with horizontal and the other with vertical alternate dark and light bands, the former will appear higher, and the latter broader, than it really is. Hence short persons often affect dresses horizontally striped in order to increase their apparent height, and very stout persons avoid longitudinal stripes. Again, when a short person is placed side by side with a tall person, the former appears shorter and the latter taller than each.

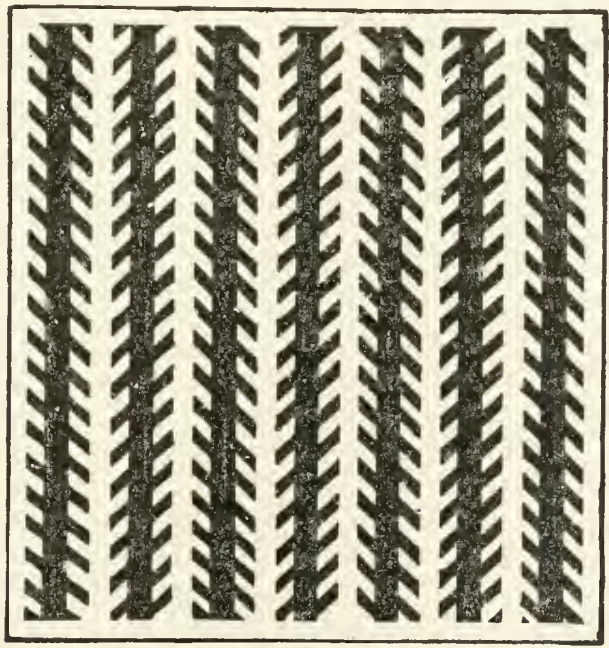

FIG. 164 .

really is. By reason of somewhat similar psychical processes two perfectly parallel lines or bands, each of which is crossed by slanting parallel short lines (Fig. 164), will appear not 
parallel, but diverging or converging according to the direction of the cross-lines; the direction of the cross-lines affects our pereeption of the distance between the parallel lines.

$\$ 600$. Judgment of Distance and Actud Size. 'The size of the retinal image gives us by itself a measure not of the real size but only of the apparent size of the object. The size of the retinal image will depend on the distance of the object and on the dioptric arrangements of the eye; with the same dioptric arrangements it will depend on the angle subtended by the diameter of the object, and this may be the same for a small object near as for a large object far off. In order to form a judgment as to the actual size of an object, we must adjust our perception of the apparent size by means of a judgment of the distance at which the object is placed; and here the great use of two eyes comes in.

Even with one eye we ean, to a certain extent, form a judgment not only as to the position of the object in a plane at right angles to our visual axis, but also as to its distance from us along the visual axis. If the object is near to us, we have to accommodate for near vision; if far from us, to relax our accommodation mechanism so that the eye becomes adjusted for distance. The muscular sense of this effort enables us to form a judgment whether the object is far or near. Seeing the narrow range of our accommodation, and the slight muscular effort which it entails, all monocular judgments of distance must be subject to much error. Every one who has tried to thread a needle or to pour out a glass of wine without using both eyes, knows such errors.

When, on the other hand, we use two eyes, we have still the variations in accommodation, and in addition have all the assistance which arises from the muscular effort of so directing the two eyes on the object that single vision shall result. When the object is near, we converge our visual axes; when distant, we bring them back towards parallelism. This necessary contraction of the ocular muscles affords a muscular sense, by the help of which we form a judgment as to the distance of the object. We can judge of the distance of a vertical line more easily than of a horizontal line, because we can converge our vision more easily upon the former'; this is seen in attempting a 'high jump' over a horizontal cord, the judgment of the distance of the cord is facilitated by hanging a vertical cord or tape to it. Conversely, when by any means the convergence which is necessary to bring the object into single vision is lessened, the object seems to become more distant; when the convergence is increased, the object seems to move towards us; this may be seen in the stereoscope.

The judgment of size is, as we said above, closely connected with that of distance. The real size of the object can be inferred 
from the apparent size, that is to say from the size of the retinal image, only when the distance of the object from the eye is known. Thus when an object gives rise to a retinal image of a certain size, that is to say has a certain apparent size, we estimate the distance from us of the object giving rise to the image, and upon that come to a conclusion as to its real size. Conversely, when we see an object, of whose real size we are otherwise aware, or are led to think we are aware, our judgment of its distance is influenced by its apparent size. Thus when part of our field of vision is occupied by the image of a man, knowing otherwise the ordinary size of a man, we infer, if the image be very small, that the man is far off. The reason of the image being small may be because the man is far off, in which case our judgnent is correct; it may be, however, because the image has been lessened by artificial dioptric means, as when the man is looked at through an inverted telescope, in which case our judgment becomes an illision. So also a picture on a magic lantern screen when gradually enlarged seems to come forward, when gradually diminished seems to recede. In these cases the influence which the absence of any muscular sense of binocular adjustment or monocular accommodation ought to bring to bear on our judgment, is thwarted by the more direct influence of the association between size and distance. An instructive illusion of a similar kind is produced by developing in the eye a strong negative image ( $\$ 584)$ and projecting the image on to a screen which is made to move backwards and forwards, or is alternately inclined at various angles; the negative image appears to change in size and shape, although it is absolutely subjective in nature and wholly independent of the movements of the screen.

The complex reaction on each other of judgments as to distance and size is illustrated by the experience that an object such as a person looks unnaturally large when seen in a fog; being seen indistinctly, he is judged to be farther off than he really is, and so appears larger than he naturally would do at the distance at which he is supposed to be; and we are similarly influenced by the greater or less brightness or saturation of colours. Conversely, distant mountains when seen distinctly in a clear atmosphere appear small, because on account of their distinetness they are judged to be nearer than they really are. The indistinctness of the image of the moon or sun when seen on the horizon, similarly contributes to its appearing larger than when seen in the zenith; our judgment however is probably in this case also due to onr being better able to compare the moon or sum with terrestrial objects. We seem moreover in this matter to be especially inflnenced by our conception (which is itself an illustration of the subject we have in hand) that the vault of the heavens is flatter than it really is ; the zenith appears to be less 
distant than the horizon; a geometric construction will shew that a body of the same size placed at different parts of the real (spherical) vault will appear greater near the horizon than near the zenith of the flatter, apparent vault. An amusing illustration of visual judgments may be obtained by asking a number of persons in succession what they regard as the size of the moon in mid heavens. Even making allowance for dioptric differences in individual eyes the size of the retinal image of the moon must be about the same in all eyes. And yet while some persons will be found ready to compare the moon in mid heavens with a three-penny piece, other's will liken it to a cart-wheel; and others will make intermediate comparisons.

$\$ 601$. Judgment of Solidity. When we look at a small circle all parts of the circle are at the same distance from us, all parts are equally distinct at the same time, whether we look at it with one eye or with two eyes. When, on the other hand, we look at a sphere, the various parts of which are at different distances from us, a sense of the accommodation, but much more a sense of the binoeular adjustment, of the greater or less convergence of the two eyes, required to make the various parts successively distinct, makes us aware that the various parts of the sphere are unequally distant; and from that we form a judgment of its solidity. As with distance of objects, so with solidity, which is at bottom a matter of distance of the parts of an object, we can form a judgment with one eye alone; but our ideas become much more exact and trustworthy when two eyes are used. We are further much assisted by the effects produced by the reflection of light from the various surfaces of a solid object, and the shadows cast by its raised parts; so much so, that raised surfaces may be made to appear depressed, or vice versa, and flat surfaces either raised or depressed, by appropriate arrangements of shadings and shadow.

Binocular vision, moreover, affords us a means of judging of the solidity of objects, inasmueh as the image of any solid object which falls on to the right eye cannot be exactly like that which falls on the left, though both are combined in the single perception of the two eycs. Thus, when we look at a truncated pyramid placed in the middle line before us, the image which falls on the right eye is of the kind represented in Fig. 165 R, while that which falls on the left eye has the form of Fig. 165 L; yet the perception gained from the two images together corresponds to the form of which Fig. $165 \mathrm{~B}$ is the projection. Whenever we thus combine in one perception two dissimilar images, one of the one, and the other of the other cye, we judge that the object giving rise to the images is solid.

This is the simple principle of the stereoscope, in which two slightly dissimilar pictures, such as would correspond to the vision of each eye separately, are, by means of reflecting mir. 
rors, as in Wheatstone's original instrument, or by prisms, as in the form introduced by Brewster, made to cast images on corresponding parts of the two retinas so as to produce a single perception. Though each picture is a surface of two dimensions only, the resulting perception is the same as if a single object, or group of objects, of three dimensions had been looked at.

It might be supposed that the judgment of solidity which arises when two dissimilar images are thus combined in one perception, was due to the fact that all parts of the two inages

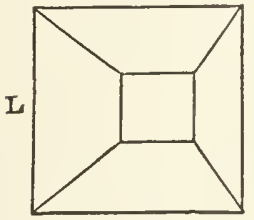

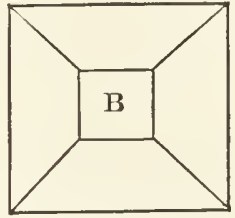

FIG. 165.

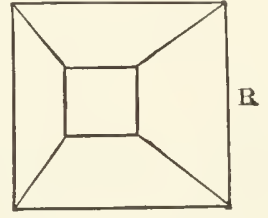

B.

camnot fall on corresponding parts of the two retinas at the same time, and that therefore the combination of the two needs some movement of the eyes. 'Thus, if we sipperimpose R on L. (Fig. 165), it is evident that when the bases coincide the truncated apices will not, and vice versa; hence, when the bases fall on corresponding parts, the apices will not be combined into one image, and vice versa; in order that both may be combined, there must be a slight rapid movement of the eyes from the one to the other. 'That, however, no such movement is necessary for each particular case is shewn by the fact that solic objects appear as such when illuminated by an electric spark, the duration of which is too short to permit of any movements of the eyes. If the flash occurred at the moment that the eyes were binocularly adjusted for the bases of the pyramids, the two summits not falling on exactly corresponding parts would give rise to the perceptions of two summits, and the whole object ought to appear confused. That it does not, but, on the contrary, appears a single solid, must be the result of psychical operations, resulting in what we have called a juidgment.

As we have seen, in any one position of the two eyes, only a small portion of the field of sight lies in the horopter and falls on corresponding points of the two retinas. Most of the objects in a scene on which we look give rise to dissimilar images in the two eyes; and we attribute solidity to them by reason on the one hand of the movements of the eyes, and on the other hand of the psychical processes just mentioned. Conversely the same processes which thus give rise to apparent solidity assist us in forming judgments of distance.

602. If the images of two surfaces, one black and the other white, are made to fall on comesponding parts of the eye, 
so as to be united into a single perception, the result is not always a mixture of the two impressions, that is a grey, but, in many eases, a sensation similar to that produced when a polished surface, such as plumbago, is looked at: the surface appears brilliant, is said to have a "lustre." The reason probably is because when we look at a polished surface the amount of reflected light which falls upon the retina is generally different in the two eyes; and hence we associate an unequal stimulation of the two retinas with the idea of a polished lustrous surface.

We may in this connection refer to what is known as "the struggle of the two fields of vision," though the matter is one of sensations and not of judgments or intricate psychical processes. When the impressions of two colours are united in binocular vision, the result is in most cases not a mixture of the two colours, as when the same two impressions are brought to bear together at the same time on a single retina, but a struggle between the two colours, now one, and now the other, becoming prominent, intermediate tints however being frequently passed through. This may arise from the diffieulty of accommodating at the same time for the two different colours ( $\$ 5+8$ ); both eyes will be accommodated at the same time and to the same degree, but if two eyes, one of which is looking at red, and the other at blue, be at one moment both accommodated for red rays, the red sensation will overpower the blue, while if at another moment they are both accommodated for blue, the blue will prevail. It may be however that the tendency to rhythmic action, so manifest in activity of other simpler forms of living matter makes its appearance also in the cerebral changes involved in binocular vision. 


\section{SEC. 11. THE NUTRITION OF THE EYE.}

$\S 603$. The main blood-vessels of the eye are, the arteria centralis supplying the retina, and the (posterior) ciliary arteries supplying the choroid, ciliary processes and iris, the vessels going to the choroid being called the short ciliary arteries, and those reaching forward to the ciliary processes and iris, the long ciliary arteries. From the arteria centralis retince the blood is returned by the vena centralis, while the venie vorticosa of the (posterior) ciliary veins gather up the blood of both the long and the short (posterior) ciliary arteries. These two systems communicate to some extent with each other by anastomoses at the entrance of the optic nerve, but on the whole are independent.

In addition to the above, the anterior ciliary arteries pass to the eyeball with each of the four straight ocular muscles, supplying the front part of the sclerotic as well as the edge of the cornea, and sending through the sclerotic "perforating "arteries to end in the iris, ciliary processes, and front part of the choroid, and so join the system of the posterior ciliary arteries. Corresponding to these anterior ciliary arteries are veins which make their way back to the ocular muscles, and the roots of which are especially connected with the circular canal of Schlemm. Further, the edge of the cornea is in addition supplied by conjunctival blood-vessels.

'The blood-supply of the various parts of the eye is therefore somewhat as follows. The inner layers of the retina are supplied in a direct manner by the arteria centralis retins, but the outer layers together with the pigment epithelium in an indirect manuer by the close set choroidal network "choriocapillaris" of the posterior ciliary arteries. The choroid proper, that part which serves as an investment to the retina and specialized pigment epithelium, is supplied by the short (posterior) ciliary arteries; but the front ciliary part of the choroid, together with the ciliary processes and iris, receives blood from the long (posterior) ciliary arteries, and also from the anterior ciliary arteries. The cornea is supplied by the conjunctival as well as by the 
anterior ciliary arteries, the blood-vessels as we have said extending a short distance only within the circle of the corneal eircumference, while the scanty supply of the sclerotic is furnished in the front part of the eyeball by the anterior and in the hind part by the posterior ciliary arteries.

The nutritive supply of the lens, with its capsule, and of the vitreous humour is an indirect one, by means of lymph; the anterior surface of the former is bathed by the aqueous humour; the lymph streams in the vitreous humour, of which we shall speak immediately, furnish that substance with the scanty nourishment it needs, and sweep by the posterior surface of the lens.

$\$ 604$. In speaking of the movements of the pupil we referred to vaso-motor changes in the eye. So far as our present information goes, we have evidence chiefly of vaso-constrictor fibres which passing from the sympathetic to the ciliary ganglion ( $\$ 536$ ) reach the posterior ciliary arteries by the short ciliary nerves; but there are facts which seem to shew that the fifth nerve supplies vaso-dilator fibres through the ophthalmic branch.

The separate distribution of the short ciliary arteries to the hinder part of the choroid investment which is busy with the nourishment of the retina and which takes little or no share in the movements of accommodation, and of the long ciliary arteries to the front part of the investment which, as iris, ciliary processes and muscle, and front part of the choroid itself, is concerned in the movements of the pupil and of accommodation, suggests that a corresponding separate distribution of raso-motor nerves also exists; but we have no exact experimental evidence of this.

We saw in speaking of the brain ( $\$ 522)$ that clear evidence of the cerebral vessels being subject to vaso-motor influences was wanting; and in this respect once more the retina behaves like a part of the brain. Though by help of the ophthalmoscope changes of calibre in the retinal vessels can easily be observed, we have as yet no decisive proof that such changes can be brought about by vaso-motor nerves acting directly on the arteria centralis retine. The changes which are observed seem to be determined not by the greater or less contraction of the muscular coat of the retinal vessels themselves, but by the pressure to which the blood in the vessels is subjected, and that may be varied by many extraneous causes.

\section{The Lymphatics of the Eye.}

$\$ 605$. Though the lymph in the large serous cavities may be considered to play a mechanical part inasmuch as it facilitates the movements of the viscera, and though in such a tissue as the skin, the lymph in the cavities and vessels of the dermis may 
similarly perform a mechanical task in assisting to give at once firmmess and suppleness to the skin, yet over the body at large the function of the lymph is preeminently a nutritive one, and its mechanical duties are insignificant. As regards the eye the case is different. 'The eyeball is broadly speaking a shell filled with fluid, the aqueous and vitreous humours; and for the various functions of the eye it is necessary that this shell should be filled to a certain extent, should be tense to a certain degree, not more and not less; and this fulness, this tension, "intraocular" tension," which is considerable, probably much higher than the ordinary pressure in the lympli-spaces of the body at large, is provided by the lymphatic arrangements. If the retina were not adequately supported by the vitreous humour, if it could flap about or in any way alter its curvature, the dioptric arrangements of the eye would be upset; if the vitreous humour at one time slirank, at another expanded, the movements of accommodation could not be carried on; if the aqueous humour were now abundant, now scanty, the movements of the pupil would become irregular and uncertain; and if the whole globe were so flabby as to give way under the pull of each ocular muscle, the delicate movements of the eyeball on which we lately dwelt would become impossible. Hence the lymphatics of the eye have a double importance, inasmuch they not only, as elsewhere, assist in maintaining the due nutrition of the several tissues, but also in a mechanical way help to make the eyc an adequate dioptric instrument. In accordance with this double duty we find a special lymph apparatus added to the more general lymphatic arrangements such as exist elsewhere.

As belonging to the more general arrangements we may note the following. The lymph-spaces of the cornea pass at the margin of the cornea into the lymphatic vessels of the conjunctiva. The scanty lymph-spaces of the sclerotic pass at the extreme front into the conjunetival lymphatics, but elsewhere are continuous either on the inner surface with the perichoroidal lympli-spaces, or on the outer surface and that more freely, with the large lymphatic Tenonian cavity. 'Tenon's capsule is a loose thin investment of connective tissue lying between the sclerotic and the ocular muscles and forming sheaths round the tendons of the latter. Between the looser capsule and the denser sclerotic is a large irregular lymphatie cavity bearing the above name. The perivascular and other lymph-spaces of the choroid join the perichoroidal spaces, which in turn communicate with the Tenonian cavity by lymph-spaces or lymplatics aceompanying the eiliary veins and to some extent the ciliary arteries as these pierce the sclerotic. The Tenonian eavity itself joins a large lymphatic cavity surrounding the optic nerve, 'the supravaginal' cavity, whence the lymph is carried away by the ordinary lymphatics of the orbit. 
The perivascular and other lymph-spaces of the retina are in connection with the lymph-spaces of the optic nerve, which in turn join the subarachnoid space of that nerve, and this is continuous with the corresponding space in the brain. There appear to be also paths uniting these lymphatics of the retina and optic nerves with the perichoroidal spaces and Tenonian cavity, and so witl the external lymphatic system.

$\$ 606$. In the special lymph apparatus the ciliary processes, the iris, the aqueous humour and the vitreous humour are concerned.

The aqueous humour. We have more than once spoken of the anterior chamber as a lymphatie cavity; nevertheless the aqueous humour contained in it differs greatly from ordinary lymph. Not only does it contain much more water, the total solids being not much more than 1 p.c. ( $1 \cdot 3$ p.c.) but also the relative proportion of the solids between thenselves is different from that of lymph, and special substances are present in it. The proteids are particularly scanty, not more than about 1 p.c.; these are serum-albumin, globulin, and apparently fibrinogen. Inorganic salts are present in about the same proportion as in blood and lymph, viz. 8 p.c.; and these, chiefly sodium chloride, with an unusual proportion ( $\cdot t$ p.c.) of so-ealled extractives, furnish nearly all the solid matter. Among these extractives is a substance which reduces cupric solutions but which is not a sugar, though its exact nature is as yet muknown; urea and sarcolactic acid (in some combination) arc also said to be, at least often, present. The reaction is neutral or faintly alkaline.

Like the 'serous fluid' in the large serous cavities and the cerebro-spinal fluid in the cavities of the central nervous system, the aqueous humour comes and goes; the particular fluid which at any given moment is present in the eye has not always becn there; some of the fluid is continually passing away and fresh fluid continually arriving. If fluid be withdrawn from the anterior chamber by puncture of the cornea, the chamber is soon refilled; incleed, under certain circumstances, a considerable quantity of fluid may be drained away from the ehamber, fresh fluid taking the place of that which escapes. And, though under normal conditions the quantity of aqueous humour is fairly constant, the fluid may be in excess or may be deficient, and the one phase may pass into the other: The question therefore arises, Whence comes the fluid and whither does it go?

The characters of aqueous humour just given shew that in many respects it resembles cerebro-spinal fluid though differing in several features. That fluid, we have seen reason to believe, is in part at all cvents furnished by the choroid plexuses, by a process which presents some analogies with the aet 
of secretion. And the resemblance between the ciliary processes and the choroid plexuses, for both are vascular folds of pia mater covered with epithelium derived from the lining of the primitive medullary canal, suggests that the former furnish the aqueous humour in some such way as the latter furnish the cerebro-spinal fluid. There is a certain amount of experimental evidence in favour of this view, for when such a substance as fluorescin, which can be detected by the greenish tinge which it gives to the fluids and tissues, is injected into the body, into the subcutaneous connective tissue or peritoneal cavity for instance, not only does it speedily appear in the aqueous humoul, but the ciliary processes are said to be the parts of the eye in which its presence may be first detected. It may be urged that, unlike the epithelium covering the choroid plexuses, the pars ciliaris retinæ bear's no distinctive histological indications of secretory activity : but, as we shall presently have occasion to point out, a wholly analogous layer of epithelium, that lining the eavities of the internal ear, though possessing no marked secretory features, certainly furnishes, by an act very similar to secretion, a more or less lymph-like fluid, the so-called endo-lymph. The phrase 'secretion' however must not be strained. The somewliat specialized loose strona of botli the ciliary processes and iris undoubtedly contains in its meshes a large quantity of what we may suppose to be ordinary lymph; and what is intended by the above view is that while some of this lymph may pass by the perichoroidal spaces and so away as ordinary lymph, a much larger proportion passes on to the free surfaces abutting on the posterior and anterior chambers, and in so passing becomes modified in nature.

The fluid thus furnished by the ciliary processes makes its way, in the first place, into the posterior chamber; but though the iris, as we have seen $(\$ 535)$ lies close on the lens, there is undoubtedly a communication between the two chambers sufficiently free to allow fluid to pass readily from one to the other and so to fill the anterior chamber from the posterior. It is difficult to suppose that some of the lymph with which the sponge-like stroma of the iris is laden, does not find its way direct through the anterior surface of the iris into the anterior chamber; and such a transit would probably be assisted by the continual changes of the pupil. On the other hand the extent of surface furnished by the ciliary processes, which moreover also have the advantages of movement in each act of accommodation, is very large compared with that of the iris; hence we may probably with confidence conclude that the greater part of the aqueous humour is furnished by the ciliary processes, though the iris may contribute. We may add that probably the iridic contribution differs in nature from the rest, since the epithelium which the fluid has to traverse is a thin layer of flat epithelioid plates. 
The answer to the question. How does the aqueous humour leave the anterior chamber? presents perhaps less difficulties. 'The anterior' chamber at the 'iridic angle' communicates freely with the spaces of Fontana, and these with the eanal of Schlemm, which in turn is in direct eonnection with the radicles of the anterior ciliary veins. Sinee the ciliary muscle pulls on the tissue surrounding the canal of Sclulemm it is possible, or even probable that the movements of aecommodation help alternately to close and open the canal, and thus to pump, its contents into the veins; by this means the exit of fluid from the anterior chamber is rendered less depenclent on the relative pressures of the blood in the vein and of the fluid in the anterior chamber. By this ehamnel the aqueous humour gains a ready, relatively direct, and short access to the bloodstream. And clinical experience shews that if this way be blocked an aceumulation of aqueous hnmour results.

$W^{T}$ e may conclude then that the aqueous humour is a reservoir intercalaterl in a strean of a peculiar fluid whieh is passing from the ciliary processes through the small posterior and larger anterior chamber, the spaees of Fontana and the eanal of Schlemm into the venous system. 'This reservoir on the one hand serves a mechanical jurpose in preserving the natural form of the eye and in affording an adequate fluid bed for the movements of the iris, and on the other hand, by bringing new food material and earrying away waste products, enables the lens to earry out the slow and seanty metabolism neeessary for its life.

$\$ 607$. For mechanical purposes the due eondition of the vitreous humour is perhaps even more important than that of the aqueous humour. We have already ealled attention to the faet that the vitreous humour in spite of its being originally a plug of mesoblastic tissue, in adult life closely resembles the aqneous humour in its chemical features; and indeed it is practieally an attenuated mesoblastic sponge through which is eontinually streaming, though at a low rate, a fluid identical with or exeeeding like to the aqucous humour. 'Through the optic dise the fluid of the vitreous humonr has aecess to the lymph-spaees of the optie nerve; material injected into the pial sheath of the optie nerve finds its way through the optic disc into the vitreous humour passing aloug a 'central canal,' 'lyaloid canal,' whieh remains after the disappearance of the prolongation of the arteria centralis retina ( $\$ 525)$. And probably some of the fluid of the vitreous humour finds its way by this path into the subarachoid spaee.

But the greater part of the fluid of the vitreous humour seems to belong to the same system as the aqueous humour. Flnirls pass readily in some way or other through the suspensory ligament; fluid injected into the vitreous humour finds its way 
1nto the anterior chamber, and a block at the iridic angle leads to undue distension, not of the anterior and posterior chambers only, but of the whole globe of the eye; the pressures of the aqueous and vitreous humour are the same and vary similarly and concurrently. We have no satisfactory evidence that any large amount of fluid passes direct from the choroid through the retina, past the internal limiting and hyaloid membranes into the vitreous humour; as far as we know the whole of the lymph of the retina is carried away by the optic nerve in the manner mentioned above; and we must therefore conclude that the region of the zonule of Zimn serves as the door both for the entrance and exit of fluid, the circulation through the vitreous humour between its indistinct concentric lamellie being secured by diffusion assisted by the movements of the eyeball.

This important flow of what we may call modified lymph like that of the more ordinary lymph in other parts of the body, is determined in the first instance by the blood flow, and we may apply to the eye the remarks which were made when ( $\$ 24 t)$ we treated generally of the relations of lymph to blood-supply. Broadly speaking the intraocular pressure rises and falls with the general blood-pressure; the dim cornea and sunk eye that betoken the approaching end are duc to the fall of blood-pressure which accompanies death. A local fall, preceded by a transient rise, may be brought about by stimulation of the cervical sympathetic, and a local rise by stimulation of the ophthalmic branch of the fifth nerve, stimulation of the third nerve having apparently little effect in either direction. We may add that, tempting as the view may seem that the lymph arrangements of the eye are under the direct control of the nervous system, we have no evidence that such is the ease.

Concerning the influence of the nervous system on the general nutrition of the eye, and the disorders which follow upon section or injury to the fifth nerve we have already, in an earlier part of the work ( $\$ 439)$, said all that at present we have to say. 
$\$ 608$. The eye is protected by the two eyelids, each of which is strengthened and rendered firm by a curved plate of dense connective tissue called the tarsus (or incorrectly the tarsal cartilage), which is larger in the upper than in the lower eyelid. Elevation of the upper eyelid assisted by some depression of the lower eyelid is spoken of as "opening the eye"; depression of the upper eyelid assisted by elevation of the lower eyelid is spoken of as "shutting the eye." The latter movement is brought about by the contraction of the orbicularis oculi, a muscle of eircularly disposed striated fibres placed beneath the skin of each eyelid and stretching also over the adjoining bony orbit. The muscle is governed by a branch of the seventh, facial nerve, and may be thrown into action as part of a reflex act or of a voluntary effort. When the facial nerve becomes incapable, through injury or disease, of carrying motor impulses, the eye cannot be shut and remains widely open. There are some reasons however for thinking that the motor fibres for the orbicularis, though forming part of the facial nerve outside the brain, take origin within the brain, not from the facial nucleus but from the hind end of the third, oculo-motor nucleus. In the reflex contraction of the orbicularis, known as ' winking' or 'blinking," which is so familiar as an almost typical reflex movement, but which in the waking loours is repeated so regularly, twice a minute or so, as to take on almost the character's of a rhythmic automatic act, the exciting afferent impulses are earried along the fibres of the fifth nerve distributed to the cornea and conjunctiva, and probably, but not certainly, pass some way down the ascending root of that nerve.

The eye is opened mainly by the raising of the upper eyelid through the contraction of the levator palpebres superioris. This muscle, taking origin from the back of the orbit in company with the oeular muscles, is inserted into the upper surface of the tarsus of the upper eyelid, beneath the orbicularis. It is governed by a branch of the third nerve; hence injury or disease of this nerve is frequently the cause of a drooping of the upper eyelid and an inability to open the eye fully. 
A portion of the tendon of the levator palpebræ closely united with an extension of the tendon of the superior rectus is inserted into the hinder part of the upper eyelid, where the conjunctiva lining it is about to be reflected over the eyeball, and a similar extension of the inferior rectus is similarly inserted into the lower eyelid. Hence a contraction of the superior rectus, while elevating the visual axis, at the same time raises somewhat the upper eyelid; and in like manner the inferior rectus, while depressing the visual axis, lowers the lower eyelid.

Between the main tendon of the levator palpebræ and the tendinous slip just mentioned lies a small bundle of plain, unstriated muscular fibres, which starting from the levator, ends in the hind border of the tarsus; it is sometimes spoken of as the middle insertion of the levator. A similar bundle of plain muscular fibres connects the insertion of the inferior rectus with the tarsus of the lower eyelid. These two small plain unstriated muscles appear to be governed by nervous filaments proceeding from the cervical sympathetic, stimulation of the cervical sympathetic leading to contraction of these muscles and so to a partial opening of the eye, and section of the same nerve preventing their being thrown into contraction and so contributing to closure of the eyelids. In some of the lower animals this closure of the eye upon section and opening upon stimulation of the cervical sympathetic is very distinct. In those animals which possess a third eyelid this is retracted by stimulation and comes forward upon section of the cervical sympatletic.

Stimulation of the cervical sympathetic also causes some protrusion and section causes recession of the whole eyeball; this is seen at times in man in disease.

$\$ 609$. The conjunctiva which lines the ocular surface of the eyelids and is reflected from them over the eyeball, the line along which reflection takes place being spoken of as the formix conjunctivce, consists like the skin of the body of which it is a continuation, of an epithelium or epidermis resting on a dermis of connective tissue. It differs from the skin in the dermis being delicate and in the epidermis being thin with a tendency for the constituent cells to become columnar; hence it is sometimes spoken of as a "mucous membrane." On the ocular surface of the eyelids the conjunctiva is thrown into irregular ridges or imperfect and fused papille, giving rise to a satiny appearance; here the epithelium consists of several layers of cells, the uppermost of which are flattened. Over the fornix, the epithelium consists of two or three layers only, the cells in the uppermost layer being cubical or columnar; over the bulb the epithelium consists also of a few layers only, the upper cells being somewhat flattened and the dermis being thrown up into scattered papillæ.

Imbedded in the tarsus, stretehing from the hind border t"r 
the free edge of the lid lies, in each eyelid, a row of thirty or fewer largely developed sebaceous glands the Meibomian glands. Sebaceous glands are also attached to the follicles of the eyelashes, and into the dnets of some of these open the glands of Moll, which have the strueture of a sweat gland. Small mucous glands are morcover found in the conjunctiva especially in the neighbourhood of the fornix.

These several glands contribute to kecp the surface of the eye and eyelids moist; but this is ehiefly effected by the secretion of the lachrymal gland which is placed above the upper eyelid in the lateral region of the orbit, and which, imperfectly divided by an extension of the tendon of the levator palpebrarum into two masses, discharges its secretion by several ducts opening along the fornix conjunctivæ. Under ordinary circumstances the fluid thus secreted is carried away through the punctum lachrymale of the upper and of the lower eyelid, at the inner angle of the eye, into the lachrymal canalieuli, and so into the lachrymal sac, and finally into the cavity of the nose. When the secretion becomes too abundant to escape in this way it overflows on to the cheeks in the form of tears.

The structure of the lachrymal gland is in its main features identical with that of an albuminous salivary gland, or with that of the parotid, save that the epithelium of the ducts is never striated. In some animals a somewhat peculiar gland, the Harderian gland, lies in the inner (median) region of the orbit; this varies in structure in different animals, being in some a sebaceous gland united with a gland similar in structure to the lachrymal gland.

If a quantity of tears be collected, they are found to form a clear faintly alkaline fluid, in many respects like saliva, containing about 1 p.c. of solids, of which a small part is proteid in nature. Among the salts present sodium chloride is conspicuous.

The nervous mechanism of the secretion of tears, in many respects, resembles that of the secretion of saliva. A flow is usually brought about either in a reflex manner by stimnli applied to the conjunctiva, the nasal mucous membrane, the tongue, and the interior of the mouth, or more directly by cmotions. Powerful stimulation of the retina by light will also cause a flow, as will electrical or other stimulation of any of the cranial or upper spinal afferent nerves. Venous congestion of the lead is also said to cause a flow. The efferent nerves are the lachrymal and orbital branches of the fifth nerve, especially the former, stimulation of these causing a copious flow. It is said that stimulation of the cervical sympathetic will also cause a somewhat scanty flow of turbid tears, but on this point all observers are not agrecd.

The chief use of the act of blinking is to keep the surface of 
the cornea moist, and so transparent; if the cornea be kept uncovered for a few minutes its dried surface soon becomes dim. But besides this, blinking undoubtedly favours the passage of tears through the lachrymal canaliculi into the lachrymal sac, and hence when the orbicularis is paralyzed tears do not pass so readily as usual into the nose; but the exact nechanism by which this is effected has been much disputed. According to some authors, the contraction of the orbicularis presses the fluid onwards out of the canals, which, upon the relaxation of the orbicularis, dilate and receive a fresh quantity. Others maintain that a special arrangement of muscular fibres keeps the canals open even during the closing of the lids, so that the pressure of the contraction of the orbicularis is able to have full effect in driving the tears through the canals. 


\title{
CHAPTER IV.
}

\author{
HEARING.
}

SEC. 1. ON THE GENERAL STRUCTURE OF THE EAR, AND ON THE STRUCTURE AND FUNCTIONS OF THE SUBSIDIARY AUDITORY APPARATUS.

$\S 610$. WE have seen that the eye consists on the one hand of the special modified epithelium, the retina, so constituted that light falling upon it gives rise to visual impulses in the optic nerve and thus to visual sensations in the brain, and on the other hand of a special dioptric mechanism, into the construetion of which several tissues enter and which is so arranged as to cause the rays of light to fall in a proper manner on the retina. In the ear we meet with a somewhat similar arrangement; we may recognize on the one hand a specially moditied epithelium, which we may call the auditory epithelium, so constituted that the vibrations of matter, the rapidly alternating variations of pressure, which we call "waves of sound," generate in the auditory nerve connected with it, auditory impulses, developed in the brain into auditory sensations, and on the other hand an acoustic apparatus so arranged that waves of sound are conducted in a proper manner to the auditory epithelium. But while, as we have seen, the optic nerve conveys, so far as we know, visual impulses only, we have reason to think (\$478) that some fibres at least of the anditory nerve convey impulses which do not give rise to auditory sensations, but enter in a peculiar manner into the mechanism of coordinated movements.

The retina as we have secn is developed out of the optie vesicle, and the subsidiary dioptric mechanism is built up around the optic vesicle; and in a somewhat similar way the anditory epithelium is devcloped into an otic vesicle, and the subsidiary acoustic apparatus is built up around the otic vesicle. The otic vesicle, like the optic vesicle, is lined by an epithelium of epiblastic origin, but is not like that vessel budded off from the medullary canal. It takes origin in an involution of the 
skin covering the head; for a time the epithelium of the vesicle is continuous with the epidermis of the skin, and wholly unconnected with the developing brain; later on the epithelial involution separates from the skin, becomes a closed independent vesicle, and makes connections with the brain through the auditor'y nerve growing out to meet it. The otic vesicle therefore is not like the optic vesicle a part of the brain, and we find accordingly the structure of the auditory epithelium much more simple than that of the retina: it corresponds to a part only of the retina, to the more external layers of the retina, not to all of them.

We have seen that the optic fibres are comnected with a part only of the optic vesicle, with the anterior wall only of the retinal cup and not with the whole of this ; the part of the anterior wall which forms the pars ciliaris retinse and the whole of the posterior wall make no connections with the optic fibres and remain in the form of a relatively simple epithelium. The connection of the auditory nerve with the walls of the otic vesicle is still more partial; the nerve fibres become connected with the epithelium in a few limited areas. It is only in these areas that the epithelium lining the otic vesicle becomes differentiated into the special auditory epithelium; elsewhere it possesses relatively simple characters.

The cavity of the optic vesicle is, as we have seen, soon obliterated by the coming together of the anterior and posterior walls. The cavity of the otic vesicle is permanent, growing with the growth of the organ and becoming filled with a peculiar' fluid secreted by the walls, called endolymph. The vesicle as it grows soon loses its early simple, more or less spherical form and assumes a most complicated shape, becoming divided into the parts known as the utricle with the semicircular canals, the saccule, and the canalis cochlearis; of these we shall speak in detail later on.

$\S 611$. While the vesicle is assuming this complicated shape, the mesoblastic tissue investing it undergoes a differentiation. The tissue immediately in contact with the epithelium becomes connective tissue serving as a dermis to support the epithelium, so that the vesicle becomes a (complicated) sac with membranous walls lined with epithelium specially modified into auditory epithelium at particular places, at which places and at which places alone, the auditory nerve makes connections with the walls.

The outer portion of the mesoblastic tissue is converted into bone of a somewhat dense character, and thus furnishes a bony shell or envelope enclosing and to a large extent following the contour of the complicated membranous sac. Between the outer bony envelope and the inner membranous sac is developed a large irregular lymphatic space which (Fig. 166) follows to 
a great extent the contour of the sac, but is broken up by broad adhesions of the membranous sac to the periosteum lining the bony envelope or by narrower bridles of connective tissue crossing the space; some of these form beds for the branches of the auditory nerve on their way to the auditory epithelium. 'The

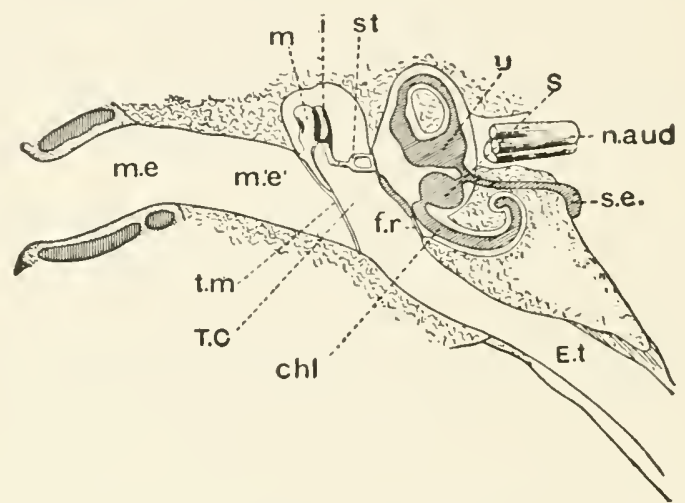

Fig. 166. Diagra to illustrate the general Structure of the Ear. (After Schwalbe.)

The figure is purely diagrammatic, intended only to shew in one view all the several important parts in relation to cach other; such a view is in the actual ear impossible.

$m . e$. the external meatus or auditory passage, in the outer part where the walls are cartilaginous, $m^{\prime} \cdot e^{\prime}$. the same in the inner part where the walls are osseous.

T.C. the tympanic cavity. $t . m$. the tympanic membrane. $m$. malleus, $i$. incus, st. stapes, attached to the fenestra ovalis. f.r. fenestra rotunda. E.t. Eustachian tube.

$U$. the utricle, with the perilymph space around. One semicircular canal with its ampulla is shewn, with the bony core of the hoop. S. Saccule. s.e. sacculus endolymphaticus lying within the cranial cavity, and connected by the ductus endolymphaticus with both saccule and utricle. chl. the canalis cochlearis, connected with the saccule by the canalis reuniens, and surrounded by its perilymph space, scala vestibuli, and scala tympani, the latter ending at the fenestra rotunda, the former continuous with the perilymph space of the vestibule around the utricle and saccule; the cochlea is shewn diagrammatically as a simple curve, the scala vestibuli and scala tympani being continuous at the top.

$N$. aud. the auditory nerve shewing the three main divisions of the trunk.

fluid in this space, which is lymph and which has access to the lymphatics of neighbouring parts, receives the special name of perilymph. A portion of the sac, with its surrounding perilymph space and bony envelope, undergocs a development differing materially from that of the rest of the sac, and is known as the cochlea. The bony envelope surrounding the parts of the membranous sac known as the utricle and saccule does not follow closely the contour of those parts but remains an undivided part called the vestibule (Fig. 167); the parts of 
the membranous sac called the semicircular canals are however (v) lowed somewhat closely by the bony envelope. The whole bcrsy envelope may be dissected out from the spongy bone surrounding it, and may be obtained as a separate mass (Fig. 167),
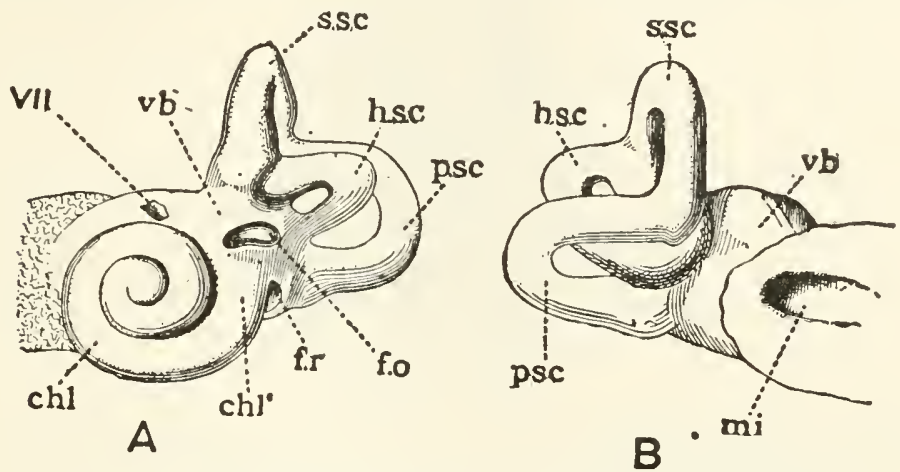

Frg. 167. The Bony Labyrinth. Left Ear. (Schwalbe.)

A. seen from the outside. B. seen from the median side. Both magnified twice.

$\boldsymbol{V} b$. vestibule. $C h l$. cochlea. $C h l^{\prime}$. the beginning of the first turn of the cochlea. F.o. fenestra ovalis, f.r. fenestra rotunda, s.s.c. superior, p.s.c. posterior, h.s.c. horizontal semicircular canals. m.i. meatus auditorius internus, canal for the auditory nerve. VII. opening of the canal containing the seventh nerve.

known by the name of the labyrinth, or bony labyrinth to distinguish it from the membranous labyrinth which lies within it, separated from it by the perilymph space. The bony labyrinth consists of cochlea, vestibule and semicircular canals, but the part of the membranous labyrinth corresponding to the vestibuie is divided into utricle and saccule. The auditory nerve pierces the bony labyrinth at the so-called meatus auditorius interms (Fig. $167 \mathrm{m.i}$.) on its way to be distributed to the walls of the membranous sac.

All these structures, lying at first not far beneath the skin and forming together the 'internal ear,' as they grow come into close connection with a passage on the side of the head leading from the exterior into the pharynx and known as the "first" or" " hyomandibular visceral cleft." By a series of changes, which we need not describe here, and indeed about which there is some divergence of opinion, this simple primitive passage is replaced in the adult by two passages separated from each other by a partition known as the membrana tympani (Figs. 166 t.m., 168), or tympanic membrane. On the outer side of the membrane lies a tubular channel, the external auditory meatus (Figs. 166 m.e., $m^{\prime} . e^{\prime} ., 169$ m.e.), lined by skin, and opening on to the exterior by an orifice guarded with the "pinna" or "auricle." On the inner side of the membrane lies the drum-shaped 
tympanic cavity (Fig. 166 T.C.), often called the "middle ear," which through the tubular Eustachian tube (Figs. 166 E.t., 169 E.t.), opens into the pharynx, and which is lined throughout by mucous membrane continuous with that of the pharyux.

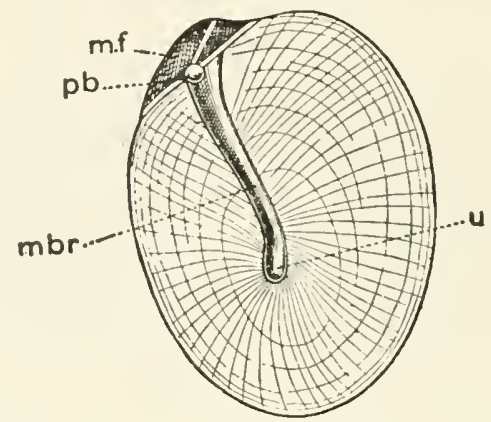

Fig. 168. The Membrana Trmpani. (After Schwalbe.) (Magnified four times.)

The membrane is seen from the external meatus and the handle of the malleus, $m b r$, is represented as shining through. m.f. the membrana flaccida, the folds of which are represented radiating from p.b., the projection outwards caused by the end of the short process of the malleus. $u$. the umbo of the membrane, to which is attached the end of the handle of the malleus. The figure shews diagrammatically, the radial and circular fibres of the membrane.

The 'internal ear' forms the mesial side of the more or less flattened and drum-shaped tympanic cavity opposite to the outer side which is to a large extent formed by the tympanic membrane; and at two places the osseous tissue of the bony envelope of the internal ear is wanting, the gaps giving rise

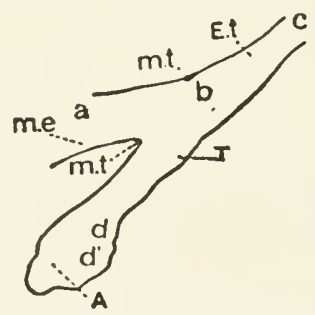

Fig. 169. Diagram to illestrate the relations of Auditory Passage, Trmpanum and Eustachian Tube. (After Schwalbe.)

The figure represents a section not quite horizontal, being inclined downwards in front; the right-hand edge of the page may be taken to represent the median plane of the head.

m.e. external meatus, T. the tympanic cavity. E.t. the Eustachian tube. $A$. is placed in the antrum mastoideum. m.t. indicates the attachment of the tympanic membrane.

a.h. the axis of the external meatus, c. b. $d$. that of the Eustachian tube. $d d^{\prime}$. shews the curved axis of the antrum. 
to what in the dried skull appear as two foramina, but in the fresh state are two membranous fenestræ. One of these, oval in shape, called the fenestra ovalis (Figs. 166, 170, 171 f.o.), lies between the tympanic cavity on the outside and that part of the perilymph space which surrounds the division of the mem-

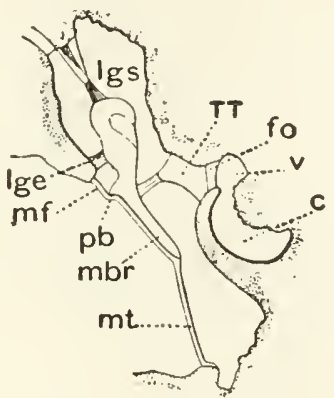

Fig. 170. Frontal (transverse vertical) section through the TYMPANum. (Left Ear.) (Schwalbe.)

The figure, partly diagrammatic, is magnified twice, and shews the front part of the tympanum as seen from behind; the incus has been removed, the articular surface on the head of the malleus being indicated.

$m t$. The membrana tympani. mf. membrana flaccida. mbr. handle of the malleus. p.b. short process of the malleus.

lg.e. external ligament, lg.s. the superior ligament of the malleus.

$T T$. The bony projection from which the tendon of the tensor tympani passes to the malleus. f.o. the fenestra ovalis. $v$. the front part of the vestibule. c. the beginning of the first (basal) turn of the cochlea.

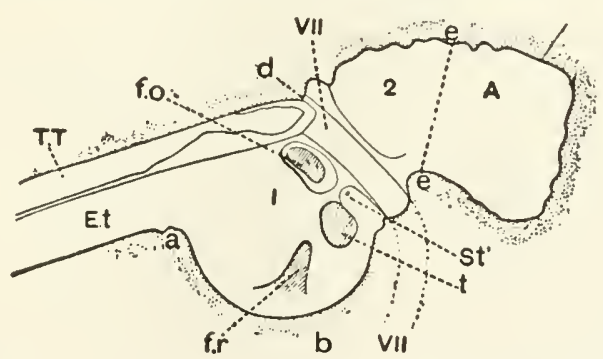

Fig. 171. Diagram of the median Wall of the Trmpanum of the Left EAr. Magnified twice. (After Schwalbe.)

1. The tympanic, 2. the epitympanic region; below the reference figure is seen the gentle prominence due to the ampullæ. $A$. the antrum mastoicleum, the line ee marking its limits. E.t. the Eustachian tube. T.T. the groove for the tensor tympani. f.o. the depression of the fenestra ovalis, the fenestra itself being shaded. f. $r$. the depression leading to the fenestra rotunda; above, and obliquely to the left of this, lies the projection caused by the base of the cochlea. $S t$. the prominence for the stapedius, with the orifice for the exit of the tendon. VII. the course of the facial nerve. The tympanum proper lies within the letters $a . b$. . d.e. 
branous labyrinth known as the utricle on the inside; in the dried bony labyrinth (Fig. 167 F.o.) it appear's as a hole in the vestibule. The other, round in shape, called the fenestra rotunda (Figs. 166, $171 \mathrm{f.r}$.), lies between the tympanic cavity and a part of the perilymph space which enter's into the construction of the cochlea; as we shall see, the perilymph space of the cochlea may be regarded as a peculiar tubular prolongation of that of the vestibule, and the membrane of the fenestra rotunda closes as it were the end of this prolongation.

Certain bones of the skull, converted by striking developmental changes into a jointed chain of minute bones, the auclitory ossicles (Figs. $166 \mathrm{~m}$. i. st., 172), are by processes of growth thrust into the tympanic cavity in such a way that they eventually seem to lie wholly in the cavity, and to form

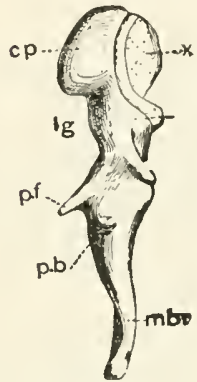

A

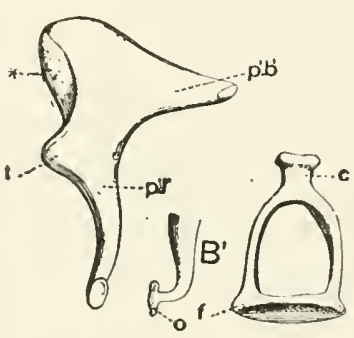

B

$\mathrm{C}$

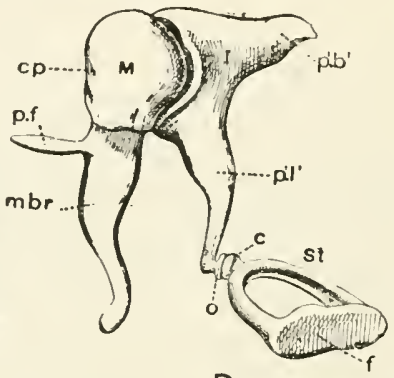

D

Fis. 172. The Audrory Ossicles. (After Schwalbe and Helmholtz.) Magnified four times.

A. The malleus. cp. the head (caput). * the articulating surface for the incus. $t$. tooth locking with tooth of incus. $l g$. is placed opposite the attachment of the ligaments. $p_{i} f$. processus gracilis or Folianus, represented as short. $p . b$. processus brevis. m.6r. handle (manubrium).

B. The incus. * surface articulating with malleus. $t$. tooth locking with tooth of malleus. $p^{\prime} \cdot b^{\prime}$. processus brevis, $p^{\prime} \cdot l^{\prime}$. processus longus.

$B^{\prime}$. The lower end of the processus longus seen sideway; 0 . its expanded termination or os orbiculare.

C. The stapes. $c$. the head. $f$. the foot-plate.

D. The three ossicles in connection. $\boldsymbol{H}$. malleus, $I$. incus, st. stapes; the other letters as above.

a bridge across the cavity between the tympanic membrane on the outer side, and the fenestra ovalis on the mesial side (Fig. 173). The ossicles are three in number; to the tympanic menbrane is attached the malleus; this is joined to the incus, which in turn is joined to the stapes, the end of which is attached to the fenestra ovalis.

$\$ 612$. The affections of consciousness, which we call sensations of sound, are the result of auditory impulses reaching 
certain parts of the brain along the auditory nerve; and these auditory impulses are generated through vibrations, or rhythmically repeated variations of pressure which we call, "waves of sound,' in some way or other acting upon the terminations of the auditory fibres in the auditory epithelium. The waves of sound gain access to the epithelium by means of the peri-

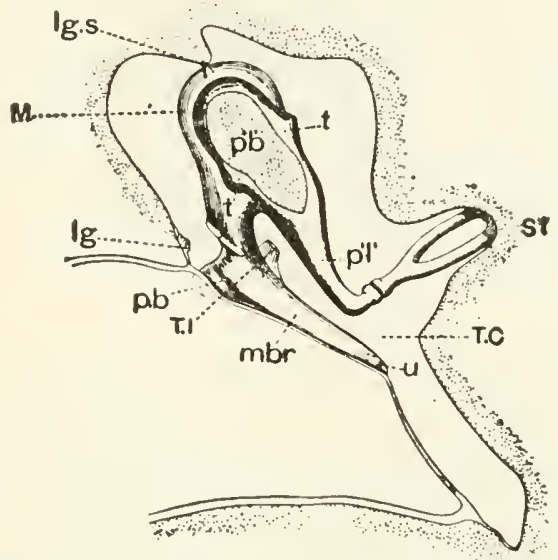

Fig. 173. Tine Ossicles in Position. Magnified four times. (After Hensen.)

The figure represents a section through tympanum in the line of the long axis of the malleus and incus; the short process of the incus $p^{\prime} b^{\prime}$ has been cut through.

T.C. The tympanic cavity. mbr. handle of malleus. $u$. umbo. p.b. short process of the malleus shewn in dotted outline as pushing outwards the membrana flaccida. T.T. the attachinent of the tendon of the tensor tympani. $l g$. the attachment of the external ligament of the malleus. $l g . s$. the superior ligament of the malleus. t.t. the teeth of the incus. $p^{\prime} l^{\prime}$. the long process, shaft, of the incus. St. the stapes.

lymph, passing probably in some parts directly through the dermis of the membranous sac to the overlying epithelium, and being in other parts transmitted to the endolymph from the perilymph across the membranous walls, and acting on the epithelium through the endolymph.

Waves of sound may be and to a certain extent are conducted in a direct manner to the perilymph, through the tissues, especially the harder bony tissues, of the head, reaching the perilymph across its bony envelope. The vast majority however of the waves of sound which fall upon the head travel through the medium of the air, and in order to reach the perilymph have to pass from a gaseous medium, the air, into the solid and liquid media of the head. Now the vibrations of particles constituting waves of sound are not readily communicated from a gaseous to a liquid or solid medium; special conditions are required to effect this. The transference of sound from the air to the 
perilymyh is attended with considerable difficulty; and the parts of the ear which we have spoken of above as constituting the middle and outer ear, serve as an acoustic apparatus for facilitating this transference and thus bringing the aerial waves to act on the auditory epithelium, the action of the apparatus being somewhat as follows.

Waves of sound falling on the side of the head reach the tympanic nembrane by the external meatus, and throw that membrane into vibrations. These vibrations are transmitted through the chain of ossicles to the membrane of the fenestra ovalis and so to the perilymph lying on its far side; sweeping over the perilymph in its continuous cavity the waves eventually break upon the fenestra rotunda, having on their way affected the auditory epithelium. We have first to inquire low this subsidiary acoustic apparatus perfor'ms its work.

\section{The conduction of sound through the Tympanum.}

$\S 613$. The chain of ossicles, jointed together, attached to the tympanic membrane at one end, and to the fenestra ovalis at the other, and secured by ligaments, may be regarded as a lever. Observations and experiments shew that the end of the short process of the incus serves as the fulcrum, the power being applied at the umbo in which the handle of the mallens ends, and the effect being brought to bear on the end of the long process of the incus attached to the stapes. In thus acting

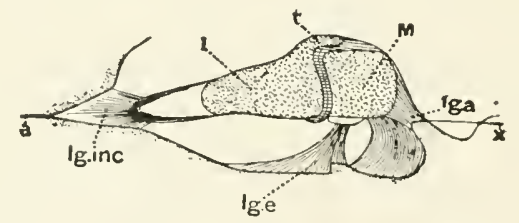

Fig. 174. The Ligaments of the Ossicles. (After Hensen.)

The figure represents a nearly horizontal section of the tympanum, carried through the heads of the malleus and incus.

$M$. malleus. $I$. incus. $t$. articular tooth of incus. lg.a. anterior and lg.e. external ligament of the malleus. lg.inc. ligament of the incus.

The line $a x$ represents the axis of rotation of the two ossicles.

as a lever the heads of the malleus and incus rotate round a horizontal line drawn through them in the direction of the line $a x$ in Fig. 174. Such a lever may be represented by the line $x x^{\prime}$ in Fig. 175. Careful measurements shew that the whole length of the line from $F$ the fulcrum to $P$ where the power is applied, is about $9.5 \mathrm{~mm}$., while tise length from $F$ to $W$, where the effect is brought to bear, is about $6.3 \mathrm{~mm}$. Hence when 
the tympanic membrane is driven inwards, the corresponding inward movement of the stapes in the fenestra is as far as extent is concerned only about two-thirds of that of the tympanic membrane. By the principle of the lever, however, the amount of pressure exerted by the movement of the stapes, the

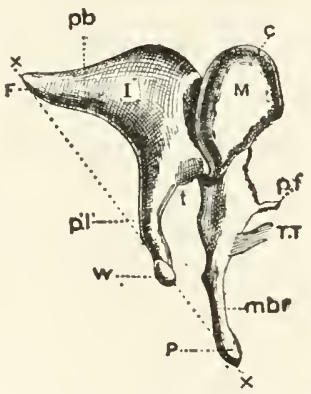

Fig. 175. The Mallees and Incus, in position. (Helmholtz.)

$\boldsymbol{M}$. The malleus. $c$, the head, $m b r$, the handle, $p \cdot f$, processes Folianus. $T . T$, the tendon of the tensor tympani.

I. The incus. $p^{\prime} b^{\prime}$, the short process, $p^{\prime} l^{\prime}$. the long process, $t$. tooth locking with the malleus.

The line $X X$ represents the lever formed by the two ossicles, with, $F$, the fulcrum at the attachment of the short process of the incus, $P$, the point where the power is applied at the end of the handle of the malleus, $I V$, the point where the effect is produced at the os orbiculare of the incus.

force of the movement, is one and a half times greater than the force expended in producing the movement of the tympanic membrane. 'The arrangement of the lever' of ossicles therefore is such as to convert a relatively large movement in to a smaller movement of greater intensity; the benefit of such a conversion is obvious.

$\S 614$. The conduction of sound from the external air to the labyrinth takes place by means of the tympanic membrane and the chain of ossicles acting as a lever in the manner just described.

Stretched membranes have the property of being readily thrown into vibrations by aerial waves of sound, and of transmitting the vibrations to bodies in contact with themselves. The tympanic membrane is a stretched membrane which, by its size, nature and conformation is specially adapted to take up and transmit a great variety of vibrations. Sound is a vibration of the particles of matter, a series of movements of the particles from and to a fixed point. In air and other gases the movements of the particles lead to alternating condensation and rarefaction of the medium, the sound is propagated as waves of alternating condensation and rarefaetion, which since the toand-fro movement of the particles is in the same direction as 
that in which the undulations are travelling, are spoken of as 'longitudinal' waves. In liquids the transmission of sound also takes place by longitudinal waves of alternating condensation and rarefaction, and sound may travel through solids in the same way. But solids in the form of nembranes or plates, strings, and rods may also give rise to sounds by being thrown into bodily vibrations, a rod for instance bending alternately to-and-fro in rapid succession. In such a case the particles of the rod move sensibly in a direction transverse to the long axis of the rod; and the vibrations of this kind, thus giving rise to sounds, are spoken of as "transversal" vibrations. It will be understood that a rod, membrane, plate or string, may also be the subject of longitudinal vibrations; but the sound given out by such longitudinal vibrations differs from that given out by transversal vibrations of the same body. A rod, string, or membrane thrown into sufficiently rapid and strong transversal vibrations, will communicate its vibrations to the surrounding air, and so give forth a sound, which will travel through the air in the form of waves of longitudinal vibrations. Conversely, sound travelling through the air in waves of longitudinal vibrations, and striking upon a rod, string or membrane, may throw it into transversal vibrations. And this is what takes place in the ear. Aerial waves of sound, in the form of longitudinal vibrations, alternating condensations and rarefactions, of the air, travelling along the meatus, fall upon the tympanic membrane, and throw it into transversal vibrations; the membrane bends bodily inwards and outwards in time with the condensations and rarefactions of the air in the meatus on its outer surface.

The vibrations of a rod, a tuning-fork for example, are comparatively simple in character; and we find, correspondingly, that a tuning-fork is very limited in its power of 'taking up' sounds from the air, of being thrown into vibrations by sounds falling upon it; it will only take up from the air the particular sounds, the particular tones as we shall presently call them, which it itself gives forth when thrown into vibrations by being struck. The vibrations of a membrane are much more complex; and for this reason a membrane takes up much more readily a variety of different sounds reaching it through the air. Still every membrane has its fundamental tone or tones, as they are called, those which it naturally gives forth when thrown into vibrations; and it takes up these from the air much more readily than any other sounds. It is a feature of the tympanic membrane that it takes up, without any marked distinction, a very great variety of sounds within a very large range. It probably has a fundamental tone of its own, but this is kept in the background; it is not prominent, and does not materially influence our hearing. Were it otherwise, were the tympanic membrane 
thrown into vibration much more readily by a particular sound than by any others, that sound would be dominant in all our hearing; and unless, as in vision, psychical experience intervened to correct the mere sensation, we should be misled in our judgments as to what was taking place around us.

This general usefulness of the tympanic membrane is secured partly by features proper to itself, partly by the fact that it is 'damped' by the attachment to it of the chain of ossicles. Without attempting to enter into a discussion of a matter which is in many ways complex, we may say that the following features contribute to make the tympanic membrane sensitive to a large variety of sounds. In the first place its dimensions are relatively small. In the second place the material of which it is composed is peculiar, so that it is in a special way unyielding and rigid; it retains its form when cut away from its bony attachments by a circular incision, and the malleus, including its handle, may be removed from it without distorting it. In the third place, its remarkable form, that of a shallow fumnel with sides gently convex towards the meatus, has a marked effect upon its capabilities of vibration. The chain of ossicles, attached at its far end, to the membrane of the fenestra ovalis has a 'damping' effect, similar to that, familiar to every one, of lessening or stopping the sound of a vibrating empty wine-glass or tumbler by pressing the finger on it, and this 'damping' while it diminishes to a certain extent all the vibrations of the membrane is especially effective in the case of excessive vibrations, such as those which might be produced by the sound which is the fundamental note of the membrane.

$\$ 615$. The vibrations thus set going in the tympanic membrane are transmitted from it to the chain of ossicles. The transmission might take place in two ways. In the first place the vibrations, the alternate bendings inwards and outwards of the membrane, might, by carrying with it the attached handle of the malleus, work the chain of ossicles as a lever, in the manner described in $\$ 613$, so that each inward flexion of the tympanic membrane led to the membrane of the fenestra ovalis pushing the perilymph of the labyrinth inwards, while the retur'n outwards again of the one led to a like return of the other. In the second place the transversal vibrations of the tympanic membrane might set up longitudinal vibrations in the substance of the malleus, which would travel as longitudinal vibrations through the chain, and so reach the perilymph. In the one case the whole chain of ossicles swings to and fro, in the other case the sound is propagated by molecular movement. That the ossicles do move en masse has been proved by recording their movements in the usual graphic method. A very light style attached to the end of the incus or to the stapes is made to write on a travelling surface; when the tympanic membrane is 
thrown into vibrations by a sound, the curves described by the style indicate that the chain of bones moves with every vibration of the membrane. On the other hand, the comparatively loose attachments of the several ossieles is an obstacle to the molecular transmission of sonorous vibrations through them. Moreover, sonorous vibrations can only be transmitted to or pass along such bodies as either are very long compared to the length of the sound-waves, or, as in the case of membranes and strings, have one dimension very much smaller than the others. Now the bones in question are not only not especially thin in any one dimension, but are in all their dimensions exceedingly small compared with the wave-lengths of the vibrations of even the shrillest sounds we are capable of hearing; hence they must be useless for the molecular propagation of vibrations. IVe may conclude then that when waves of sound throw the tympanic membrane into vibrations, each inward excursion of the membrane is followed by a corresponding impulse given by the foot of the stapes to the perilymph. As we have seen the space through which the end of the incus moves is less than that through which the handle of the malleus moves, and the movements of the stapes are in addition restricted by the manner of its attachment to the rim of the fenestra ovalis; but the energy with which the end of the incus and hence the stapes moves is proportionately increased, so that we might almost speak of the gentle swingings of the tympanic membrane being converted into smart taps on the perilymph of the labyrinth.

The impulses thus given to the perilymph at the fenestra ovalis travel along the intricate passages of the perilymph spaces, and finally break upon the fenestra rotunda; if the membrane which closes this orifice be watched it may be observed to pulsate in sequence with the pulsations of the fenestra ovalis. During their passige these impulses are communicated to the endolymph and in some way or other affect the endings of the auditory nerve. How they do this we shall presently study; but we may here call attention to the fact that the waves of sound which fall on the tympanic membrane are for the most part not simple in character but complex, and in many cases exceedingly so. This complexity is carried on into the vibrations of the tympanic membrane and so into the impulses given to the perilymph; the waves which sweep past the endings of the auditory nerve are, so to speak, reproductions of the complex aerial waves passing down the meatus.

$\$ 616$. By far the greater number of sounds which we hear reach the tympanic membrane by passing through the air down the meatus. One great use of the long external passage is probably to protect the delicate tympanic membrane from the accidents to which it would be subject were it freely exposed on the surface of the body; but it has also a use in transmitting to the 
tympanic membrane sounds travelling to the ear in certain directions more readily than those coming in other directions. The constriction of the meatus at the junction of the outer and middle third serves as a sort of diaphragm by which waves of sound travelling too much out of the line of the meatus are turned back. 'The external ear, auricle, or pimna has also probably a similar effect, reflecting into the meatus waves which fall upon it in a particular direction or waves of a particular kind. But of these uses, which are of more importance in some animals than in man, we shall speak again in considering the manner in which we recognize the directions of sounds.

Sounds however may reach the ear by paths other than the meatus. If a tuning-fork be struck and then held near the ear it will after a while cease to be heard, the sound dies away; but the sound is heard again if the handle of the fork be placed between the teeth; and when the sound again dies away, it may be revived by gently closing the external meatus, care being taken not to cause conpression of the air within. When the tuning-fork is held between the teeth its vibrations are transmitted, through the bones of the head to the tympanic membrane, which thus set in motion acts in the same way as when it is set in motion through the air of the meatus. 'That the vibrations which thus reach the internal ear are, for the most part at least, conducted through the tympanum, and not brought to bear on the perilymph directly through the bony walls of the labyrinth is not only indicated by the effect just mentioned of closing the meatus, for this could have no influence on the labyrinth itself, but may be also proved by experiment. If a style be attached to the stapes laid bare in the skull, the vibrations of a tuning-fork brought into contact with the skull, will lead to corresponding movements of the style.

Not only may vibrations be transmitted from the skull to the tympanic membrane, but also conversely the vibrations of the membrane, brought about in the usual way through the meatus, may be transmitted to the bones of the skull. If a long tube introduced into one meatus be spoken or sung into, the sounds may be heard by help of a stethoseope placed over various parts of the head. They are heard best perhaps at the opposite meatus; the vibrations of the bones of the skull set going by one tympanic membrane throw the other tympanic membrane also into vibrations.

$\$ 617$. Two muscles act upon the auditory apparatus of the tympanum; one, the tensor tympani, acts upon the malleus and hence upon the tympanic membrane, the ether, the stapedius, acts upon the stapes.

The tensor tympani (Fig. 176) is a slender muscle, lying in a groove above the bony canal of the Eustachian tube, and having very much the direction of that tube. The tendon in which it 
ends, turns round, almost at right angles to the line of the muscle, over a bony prominence at the end of the groove, and passing athwart the cavity of the tympanum from the median side outwards (Fig. 170 T.T.) is attached to the upper part of the handle of the malleus.

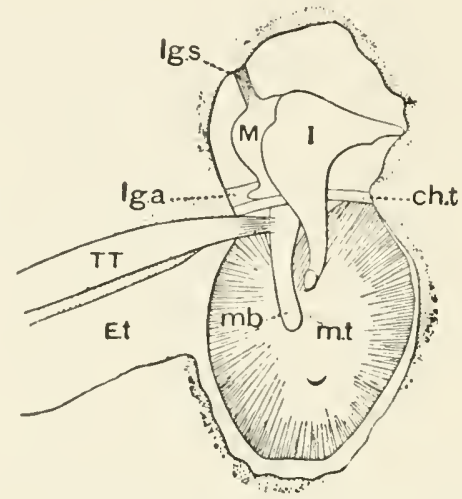

Fig. 176. Diagram of the outer wall of the Trupanum as seen from the mesial sibe. Magnified twice. (After Schwalbe.)

m.t. membrana tympani. $m b$. handle of $M$ the mallens. $T$. the incus. E.t. Eustachian tube. T.T. tensor tympani, the tendon of which is seen attached to the upper part of the handle of the malleus. $7 g . a$. the anterior and $l g . s$. the superior ligament of the malleus. ch.t. the chorda tympani nerve traversing the tympanic cavity.

The effect of the contraction of the muscle is to pull the handle of the malleus and so the tympanic membrane inwards towards the median side. Even in a quiescent state it may be of use in keeping up a certain amount of tension and in preventing the tympanic membrane being pushed out too far. When it contracts it certainly renders the tympanic membrane more tense; hence it has been supposed on the one hand to act as a damper lessening the amount of vibration of the membrane in the case of too powerful sounds, and on the other hand to accommodate the apparatus to the sounds falling upon it since the more tense membrane is more readily thrown in to vibrations by higher notes and is less sensitive to lower notes. It has been urged that it is readily thrown into contraction at the commencement of a sound, especially of a moise, and returns to rest during the continuance of a prolonged musical note, the contraction being a simple contraction or twitch, rather than a continued tetanic contraction; it is suggested that this may serve to tune the membrane as it were for the sound which follows. Efferent impulses reach it through fibres of the fifth nerve from the otic ganglion, and its activity is regulated by reflex action, vibrations 
of the tympanic membrane starting the afferent impulses. In some persons the muscle seems to be partly under the dominion of the will, since a peculiar crackling noise which these persons can produce at pleasure appears to be caused by contraction of the tensor tympani.

The stapedius is a small muscle imberded in the bone of the median wall of the tympanum, the tendon issuing by a hole close to the fenestra ovalis (Fig. $171 \mathrm{St}$.) and being inserted into the head of the stapes (Fig. $177 S T$ ). It is supposed to regulate the movements of the stapes, and especially to prevent the foot-plate being driven too far into the fenestra ovalis during

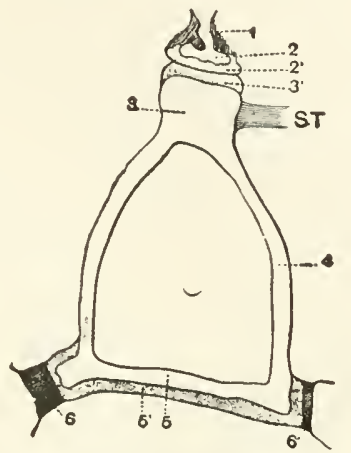

Fig. 177. The Stapes in Position. Much magnified. (Schwalbe.)

1. The end of the shaft of the incus. 2. Its expansion or os orbiculare. $2^{\prime}$. The articular cartilage of the same. 3. The capitulum of the stapes. $33^{\prime}$. Its articular cartilage. 4. The hoops of the stapes. 5. The foot-plate of the stapes. $5^{\prime}$. Its articular cartilage. 6 . The membrane of the fenestra ovalis. stapes.

$S T$. The tendon of the stapedius muscle attached to the capitulum of the

large or sudden movements of the tympanic membrane. Contractions of the muscle pull the front part of the stapedial footplate towards the tympanum, the hind part being thereby pressed somewhat into the labyrinth and the whole membranous ring round the foot being rendered more tense; but the total effect is to diminish the pressure in the labyrinth. It perhaps may be regarded as the antagonist of the tensor tympani. It is governed by fibres from the facial nerve.

$\$ 618$. The cavity of the tympanum is, as we have seen, continuous with the Eustachian tube. The walls of the tube in the first third of its length arjoining the tympanum are osseous, but in the remaining two-thirds are cartilaginous and $\cdot m e m-$ branous. The tube, whose lumen is of varying diameter and special shape, passes obliquely forwards, downwards, and towards the median line (Fig. 171, E.t.) to open at the side of the upper part of the pharynx. The mucous membrane 
lining the tube consists of a ciliated epithelium resting on a dermis rieh in retieular and adenoid tissue, and bearing glands. The action of the cilia is such that the movement which they effect is directed from the tympanum to the pharynx. The mucous membrane lining the tympanum is a continuation of that lining the tube and, like that, ciliated except over the tympanic membrane, the chain of ossicles, and probably some other parts; in these situations the epithelium consists of a single layer of flat non-ciliated cells, and a similar epithelium lines the antrum and mastoid cells which continue the cavity of the tympanum backwards and upwards.

One use of the Eustachian tube is to carry down to the pharynx the fluid, normally very small in amount, which is secreted by the mucous lining of the tympanum, but a far more important use is that of placing the air in the tympanum in communication with that in the pharynx and so with the external air, by which means the pressure on the two sides of the tympanic membrane is equalized. If as happens sometimes the tube is definitely closed, the absorption of the gases in the air at first present in the tympanum diminishes the pressure on the inner side of the tympanic membrane, and so interferes with the vibrations of the membrane. Moreover it is desirable that general changes of pressure in the cxternal atmosphere should be rapidly followed by corresponding ehanges in the pressure within the tympanum, since the tympanic membrane would not vibrate normally if any marked difference of pressure on the two sides were brought about; and this would result if the way from the tympanum to the external air through the tube were blockerl.

The lumen of the tube has in its lower part the form of an obliquely vertical slit, the sides tonching or nearly so; and much dispute has taken place as to whether the tube is normally closed or open. It is undoubtedly opened during the act of swallowing, and during the act, by the action of certain muscles of the palate, air is forced up into the tympanum. It may be opened also by a forced inspiration or a forced expiration when the nose and mouth are kept closed; in the former case the pressure of the air in the tympanum is diminished, in the latter case increased. Although under normal circumstances the lumen is so far patent as to allow the escape of the fluid driven by the cilia, the evidence goes to shew that it is praccally closed; sounds for instance generated in the pharynx do not throw the tympanic membrane into vibrations in such a way as they would do if the tube were thoroughly open. Apparently the occasional opening, such as that effected by swallowing, is sufficient to kcep the pressure within the tympanum at its proper level. When the general pressure of the external atmosphere is rapidly increased or diminished, tempo- 
rary deafness, especially to low notes, frequently ensues, in consequence of the pressure within the tympanum not following the changes of the pressure without. This however is soon remedied by the act of swallowing, which opens the tube and thus equalizes the pressure.

An abnormal permanency in the closure of the tube is recognized as a cause of deafness, and may be remedied by catheterism of the tube, that is to say, opening up the tube by passing an instrument into it from the pharynx. 


\section{SEC. 2. ON AUDITORY SENSATIONS.}

$\S 619$. The vibrations which we call sound are transmitted as we have seen to the perilymph through the fenestra ovalis, by means of the tympanic membrane and chain of ossicles. The vibrations of the perilymph in some way or other, by help of the auditory epithelium, give rise in the fibres of the auditory nerve to auditory impulses, and these reaching the brain are developed into auditory sensations. Before we attempt to consider how the vibrations of the perilymph thus give rise to auditory impulses it will be convenient to adopt the plan which we pursued in the case of vision, and to deal first with some of the leading characters of auditory sensations such as can be ascertained by psychological methods.

We readily recognize two classes of sensations; the objective causes of the one class we speak of as noises, those of the other class as musical sounds. When we inquire into the physical features of the two classes we find that the vibrations which constitute a musical sound are repeated at regular intervals, and thus possess a marked periodicity or rhythm. When no marked periodicity is present in the vibrations, when the repetition of the several vibrations is irregular, the sensation produced is that of a noise. There is however no abrupt line between the two. Between a pure and simple musical sound produced by a series of vibrations each of which has exactly the same period, and a harsh noise in which no consecutive vibrations are alike, there are numerous intermediate stages. Much irregularity may present itself in a series of sounds called music, and in some of the roughest noises the regular repetition of one or more vibrations may be easily recognized. Still it will be desirable to consider the two classes as distinct, and it will be convenient to deal first with musical sounds.

$\$ 620$. The sensations which are produced by musical sounds possess three marked characters. In the first place our auditory sensations, like our other sensations, may be more or less intense; and the character in a musical sound which corresponds to the intensity of the sensation we call loudness. 'This is detcrmined by the amplitude of the vibrations, by the amount of 
energy which is expended in producing the vibratory movements; the greater the disturbance of the air (or other medium) the louder the sound. Using the term 'wave' to denote the characters of the vibrations, the loudness of a sound is indicated by the height of the wave.

In the second place we recognize a character which we call pitch. This is determined by the frequency of repetition of the vibrations, by the time taken up by each vibration; the greater the number of consecutive vibrations which fall upon the ear in a second, the shorter the time of each vibration, the higher the pitch. Hence the pitch of a sound is indicated by the length of the wave, a low note having a long, a high note a short wavelength. We are able to distinguish a whole series of musical sounds of different pitch, from the lowest to the highest audible note.

In the third place, we distinguish musical sounds by what is usually called their quality (timbre); the same note sounded on a piano and on a violin produces very different sensations, even though the two instruments give rise to vibrations having the same period of repetition. This arises from the fact that the musical sounds generated by most musical instruments are not simple but compound vibration; the instrument sets going in the surrounding air not one series only of vibrations of one wave-length, but several series of different wave-lengths; as we shall see however, the several vibrations travel through the air, not as a group of waves but as one compound wave. IVhen the note $\mathrm{C}$ in the bass clef is struck on the piano, and we analyze the total sound, we find that it can be resolved partly into a series of vibrations with a period characteristic of the pure tone of $\mathrm{C}$ of the bass clef, and partly into other series of vibrations with periods characteristic of the $\mathrm{C}$ in the octave above (midlle $\mathrm{C}$ ), of the $\mathrm{G}$ above that, of the $\mathrm{C}$ of the next octave, and of the E above that. And the sensation which we associate with the sound of the $\mathrm{C}$ in the bass clef on the piano is determined by the characters of the complex viluration arising out of these several constitutent simple vibrations. Almost all musical sounds are thus composed of what is called a fundamental tone accompanied by a number of partial tones. When a violin string gives out a musical note, the fundamental tone is produced by the string vibrating along its whole length, the partial tones by the string vibrating at the same time in segments or definite parts of the whole length; and so with other instruments; hence the name 'partial.' Since these partial tones have a higher pitch than the fundamental tone they are frequently spoken of as "partial uppertones or overtones' or simply as 'overtones.' The partial tones vary in number and relative prominence in different instruments and thus give rise to at difference in the sensation caused by the whole sound. Hence 
while a 'tone' is a single series of simple vibrations, a "note' may be and generally is a number of series of different vibrations oceuring together. While the fundamental tone determines the pitch of a note, the quality of the note is determined by the number and relative prominence of the partial tones.

$\$ 621$. In much the same way that rays of light of more than or of less than a certain wave-length are incapable of exciting the retina, our vision being limited to the range of the visible spectrum, waves of sound of mole than or of less than a certain wave-length are unable to affect the ear so as to produee a sensation of sound. Vibrations having a recurrence below about 30 a second are unable to produce a sensation of sound; the note of the 16 -feet organ pipe, 33 vibrations a second, gives us the sensation of a droning sound; a tone of 40 vibrations is quite distinct. Some authors however place the limit at 24 or even 15 a second. If waves of long wave-length are powerful enough we may feel them by the sense of touch, though not by that of heariug. What we have just said applies to vibrations which are simple, such as give rise to a pure tone; if the fundamental tone is accompanied by partial tones we may hear one or other of these, and are thus apt to say we hear the former when in reality we only hear the latter. As regards the limit of ligh notes, it is possible to hear a note caused by vibrations as rapid as 40,000 a second; at least some persons have this power, though the limit for most persons is far lower, about 16,000 . Some persons hear low sounds more easily than high ones, and vice versa. This may be so pronounced as to justify the subjects being spoken of as deaf to low or high tones respectively, a condition which may be compared in a general way to color blindness. The range in different animals differ's very widely, the high notes of the instrument known as Galton's whistle, though inaudible to man, are distinctly heard by some other animals, for instance, cats.

'The limitations which are thus imposed on our hearing do not wholly correspond to the limitations of our vision. In the latter case the limits are fixed wholly by the capacities of the retina and cerebral centres; radiant rays of longer wave-length than the extreme visible red are able to get access to the retina through the dioptric apparatus though they are unable to excite visual impulses, or at least such visual impulses as can affect consciousness. In the case of hearing, though the auditory cpithelium is probable, like the retinal structures, limited in its powers, narrower limits are fixed by the subsidiary acoustie apparatus; the tympanic membrane, extensive as is its range compared with that of most artificial membranes, cannot respond to all vibrations; and hence its powers fix the limits of hearing. The reason why we appreciate high notes more readily than low ones is probably to be referred to the tympanum rather 
than to the auditory epithelium. And the condition of the tympanal apparatus as affected by disease will determine the relative appreciation of low or high tones; in certain states of the tympanum the ear becomes unusually sensitive to high notes; an instance of this is seen in the paralysis of the stapedius muscle due to injury or disease of the seventh nerve.

$\$ 622$. We dwelt, in speaking of vision, on our power of appreciating differences of brightness or luminosity; we have a similar power of appreciating differences in loudness; and that relation between differences in the intensity of the stimulus and differences in the intensity of the sensation, which we spoke of as WVeber's law ( $\$ 550)$, holds good for hearing as well as for vision.

The power of distinguishing difference of pitch, the power of recognizing the difference between two notes of different pitch, and the appreciation of the qualities of various musical sounds which is built up on this, may in a general way be compared to acuteness of colour vision. It is, however, very different from that in many respects, and varies much more widely than does that. As is well known the difference in this power between different individuals, according as they have or have not a 'musical ear,' is very great. Some persons, even though fairly sensitive to differences of loudness, are unable to distinguish two notes differing considerably in wave-length. On the other hand a well-trained ear can distinguish the difference of a single or even of a half vibration a second, and that through a long range of notes. As might be expected the power of appreciating difference of pitch is not the same for all audible notes. The range of an ordinary appreciation of tones lies between 40 and 4000 vibrations a second, i.e. between the lowest bass $\mathrm{C}\left(\mathrm{C}_{1} 33\right.$ vibrations $)$ and the highest treble $\mathrm{C}\left(\mathrm{C}^{5}\right.$ 4224 vibrations) of the piano; tones above and below these, even though audible, are distinguished from each other with great difficulty. The power of recognizing, and being able to name, a note when heard, is an extension of and based upon this power of recognizing differences of pitch, though not by itself exactly the same thing.

$\S 623$. We said, in speaking of vision ( $\$ 551$ ), that, probably, several undulations falling in succession upon the retina were necessary for the development of a visual sensation. In like manner, in order that a distinct sensation of a musical sound may be developed, several, or at least more than one wave of sound must fall on the ear. The various observer's are not agreed as to the lower limit of the number of vibrations necessary in order that the affection of consciousness may take the form of a definite musical sound; some place it at five, others higher, while it has been asserted that two vibrations are sufficient. When the vibrations are thus lim- 
ited in number, the sound, even though it is recognized as a musical sound, is not elearly appreciated; its pitch is not distinctly recognized. In such a case the recognition may be made more full and certain by increasing the number of vibrations; in order that we may appreciatc the pitch of a sound the ear must receive a larger number of vibrations than are necessary merely to enable us to recognize that the sound is a definite one. Conversely even when the vibrations are too few to give rise to a scnsation of a definite tone, consciousness is not wholly unaffected, an auditory sensation is produced, though it cannot be called one of tone. These facts indicate the complex nature of the nervous processes which form the basis of auditory sensations; we might say this of sensations in general, for similar results are observed in the case of all sensations.

$\$ 624$. As we said above (\$619) noises are not sharply defined from musical sounds, they differ only in being more complex and less regular; and what has just been said in respect to musical sounds, holds good to a large extent for noises. W'e readily distinguish, in noises, difference of loudness; we may also in many cases recognize a dominance of pitch, dne to the fact that among the multifurious vibrations certain groups of vibrations are repeated periodically; we distinguish a rumbling noise in which vibrations of slow recurrence are promincnt from a harsh shrill noise in which rapid vibrations are similarly prominent; we also recognize qualities in noises, we distinguish one noise from the other by the characters of the predominant constituent vibiations. Owing to the fact to which we just now referred, that in a musical sound the effect on consciousness is a summation of the individual effects of the several vibrations, we are more sensitive to a musical sound of not too short duration, than to a noise involving an equal expenditure of energy. On the other hand the limit of the number of movements necessary to give rise to a sensation of noise is less than that required for a musical sound; a few vibrations insufticient in number to give rise to the sensation of a tone are able to give rise to an auditory sensation which we may call a noise, and probably onc movement of the tympanic membrane might if ample enough give rise to such an auditory scnsation. Moreover owing to the very irregularity of a noise, to the varied character of the constituent molecular movements, we have a very great range in distinguishing various noises; persons who have great difficulty in detecting different notes can often readily recognize differences in noises.

5625 . In treating of vision we dwelt at some length on the phenomena of cxhaustion which make their appearance when the stimulus is continued. These occur in hearing also, and indeed are indicated by such common phrases as "a deafening noise;" but they are not so prominent as in vision, and do not so distinctly serve as the basis for theoretical discussions. They are 
best studied by means of musical somnds, since with these owing to their very nature the stimulation is more uniform than with noises. With almost any note, the sensation diminishes and finally disappears if the sound be maintained long enough; but the exhaustion comes on more rapidly with high than with low notes, especially with very high ones. If a sounding tuningfork be held up to one ear, and then, just as the sound becomes inaudible be transferred to the other ear, the sound may be distinctly heard; the fresh untired sensory apparatus of the one side is sensitive to the vibrations which the tired apparatus of the other side can no longer feel. Or, if the tuning-fork which the tired ear can no longer hear, be replaced by one vibrating at the moment so far as can be arranged with the same intensity as it, but of distinctly different pitch, this will be heard; the first tuning-fork only tired certain parts of the sensory apparatus, those affected by vibrations of a certain period characteristic of the pitch of that tuning-fork, but left mntired the parts of the sensory apparatus responding to the vibration of other periods, such as those of the second tuning-fork. Again, the quality of a note struck on a musical instrument depends as we have seen on the presence of partial tones, having certain relations to the fundamental tone. Now, if immediately before striking a note on an instrument, choosing especially an instrument whose notes are 'rich' by virtue of the number or prominence of the partial tones, we cause one of the partial tones of the note to be sounded powerfully in the ear, the note when subsequently struck does not possess its full quality; it appears 'thin' or 'poor.' 'This is because the previous sounding of the partial tone has tired the particular part of the auditory apparatus with which we hear the partial tone, and in the whole sensation of the subsequent full note the constituent sensation corresponding to that particular partial tone is absent or at least is below its normal intensity. Thus we have in auditory sensations something analogous to the "negative image" of visual sensations.

$\mathrm{W}$ e do not in hearing experience a sensation analogous to the visual sensation of white light, a simultaneous stimulation of the apparatus by vibrations of all kinds, and cannot therefore experience an auditory sensation corresponding to the visual sensation of black; the nearest approach perhaps to such a psychological condition is that in which we are placed upon the sudden cessation of powerful and varied music; at such times we seem to be the subject of a "silence which can be heard."

$\$ 626$. As in the case of visual sensations, so likewise in the case of auditory sensations the duration of the sensation is longer than that of the action of the stimulus, the auditory sensation lasts after the waves of sound have ceased to fall upon the ear. Hence when two sensations follow each otler within a sufficiently short interval, they are fused into one. Since a 
membrane, thrown into vibrations by a passing sound may continue to vibrate after the sound has eeased, we might perhaps expect that this would be the case with the tympanic membrane, and that hence the interval of fusion would be longer in the case of hearing than in that of vision, for in the latter case we have no corresponding behaviour of any part of the dioptric apparatus. But we have seen (\$614) that the acoustic arrangements of the tympanum very rapidly damp the tympanic membrane; and, as a matter of fact, the interval in question is decidedly shorter in hearing than in vision. Visual sensations separated by less than $\frac{1}{10}$ see. become fused ( $\left.\$ 552\right)$; but auditory sensations separated by not more than $\frac{1}{100}$ sec. may remain distinet; if two seconds pendulums be set swinging not quite in accord with each other and made to tick, the tick of the one can be distinguished from that of the other even when they differ in time by not more than $\frac{1}{100}$ sec.

$\$ 627$. When two notes are sounded at the same time the two sound waves (we may suppose the notes to be pure ones, consisting of a fundamental tone only without partial tones) do not travel as two separate waves, but are compounded as we have already said, into a single wave, the characters of which will depend on the relative characters of the two constituents. If the two notes have the same period, that is to say are identical, the effect will be simply an increase in amplitude; the compound wave will have its crests higher, and its troughs decper than those of either of the single waves, but will otherwise be like both of them. If two tuning-forks of exactly the same pitch be struck, the sensation which we experience is the same is that which we experience from either of them alone, only more intense; the sound is louder.

If, however, the two tuning-forks are not of the same pitch, but so related that the period of vibration of the one is not an exact multiple of that of the other, the sensation which we experience when the two sound together has certain marked features. We hear a sound which is the effect on our ear of the compound wave formed out of the two waves; but the sound is not uniform in intensity. As we listen the sound is heard now to grow louder and then to grow fainter or even to die away, but soon to revive again, and once more to fall away, thus rising and falling at regular intervals, the rhythmic change being either from sound to actual silence or from a louder sound to a fainter one. Such variations of intensity are due to the fact. that, owing to the difference of pitch, the vibratory impulses of the two sounds do not exactly correspond in time. Since the vibration periorl, the time during which a particle is making an cxcursion, moving a certain distance in one direction and then returning, is shorter in one sound than in the other, it is obvious that the vibrations belonging to one sound will so to speak get 
ahead of those belonging to the other; hence a time will come when, while the impulse of one sound is tending to drive a particle in one direction, say forwards, the impulse of the other sound is tending to drive the same particle in the other direction, backwards. The result is that the particle will not move, or will not move so much as if it were subject to one impulse only, still less to both impulses acting in the same direction; the vibrations of the particle will be stopped or lessened, and the sensation of sound to which its vibrations are giving rise will be wanting or diminished; the one sound has more or less completely neutralized or "interfered" with the other, the crest of the wave of one sound has more or less coincided with the trough of the wave of the other sound. Conversely at another time, the two impulses will be acting in the same direction on the same particle, the movements of the particle will be intensified, and the sound will be augmented. And the one condition will pass gradually into the other. 'The repetitions of increased intensity thus brought abont are spoken of as beats.

The length of the interval at which the beats recur will depend on the difference of period of the two sounds in relation to the actual period or pitch of each. It may be stated generally that the number of beats in a second is equal to the difference between the number of vibrations per second of the two sounds; thus two very low pitched tuning-forks, vibrating respectively at 64 and 72 a second, will give 8 beats a second, and two very high pitched tuning-forks, vibrating respectively at 4224 and 4752 a second, will give 528 beats a second; but in this respect there are complications which we cannot consider here.

Beats are produced when the periods of the coincident sounds are not exact multiples of each other. When the periods are exact multiples no beats occur; two tuning-forks, for instance, the period of one of which is exactly double that of the other, give rise to no beats when sounding together; and so in other instances.

By beats then a continuous musical sound may be broken up into a series of discontinuous sounds. When the beats are repeated a few times only in a second the discontinuous sounds give rise to discontinuous sensations; we hear the separate beats. But if the beats are repeated sufficiently rapidly the successive sensations are fused in one, we cease to hear the beats as such, though we have other evidence that the beats continue to be produced. Just as a series of simple vibrations when repeated sufficiently rapidly, say 40 times a second, gives rise, by summation, to a single musical sound, to a tone, so a series of groups of vibrations, each group corresponding to the interval between two beats, gives rise when the groups follow each other rapidly, by a similar summation, to a continuous sensation. And, though 
the matter is one which has been much disputed, the evidence secms to shew that the continuous sensation thus produced is a musical sound, a tone, which has been called a "beat-tone," whose pitch is determined by the number of beats repeated in a second.

The rapidity however with which beats must be repeated in order to give rise to a continuous sensation, is different from that with which single vibrations must be repeated in order to give rise to a musical sound. Beats repeated 30 or 40 times a second are readily distinguished as such; it is not until they reach a rapidity of repetition of about 132 a second that they cease to be distinctly recognized. Before they disappear or as they disappear, at the time when they can no longer be recognized as separate beats, but have not as yet become fused into a completely continuous sensation, they give to the sound which they accompany a peculiar quality, a particular roughness and harshness. This quality if excessive is disagreeable to the ear ; we speak of it as dissonance.

From what has been said it is obvious that when a picce of music is played on an instrument and still more when it is played, as in a concert, on several instruments of different kinds, the disturbance in the air, and the consequent vibrations of the tympanic membrane and of the perilymph, are in the highest degree complex. If the disturbance has certain characters, the sound gives us pleasure, if other characters, we regard the sound as disagreeable; and it is found that the disagreeable features of music are associated with the presence of beats, and still more with the presence of that ill-defined roughness which, as we said just now, is the characteristic of beats when, through rapidity of repetition, they are about to disappear. At the same time there are reasons for thinking that it is the prominence rather than the mere presence of this element which offends the ear, that the element is a necessary ingredient of effective music, and that even the very quality of a musical sound is dependent in part on a certain minute ardmixture of vibrations disagreeing in period with the funclamental tone and with the regular partial tones. But this is a matter into which we cannot enter herc; we have referred to it because it illustrates the extreme complexity of the processes which underlie our sensations of sound. 


\section{SEC. 3. ON THE DEVELOPMENT OF AUDITORY IMPULSES.}

$\S$ 628. We may now turn for a little while to the obscure question, How the vibrations of the perilymph give rise to auditory impulses and so to auditory sensations.

In speaking of the ossicles (\$ 615) we gave reasons for thinking that the vibrations of the tympanic membrane are carried onward by the chain of ossicles swinging as a whole, and not conveyed through the chain from molecule to molecule. A similar argument may be applied to the perilymph. The dimensions of the whole labyrinth compared with the length of the waves of sound are so minute that molecular vibrations may be neglected. Moreover the walls of the labyrinth may, as a whole, be regarded as absolutely rigid so that, the perilymph being incompressible, each blow given at the fenestra ovalis is transmitted instantaneously through the whole mass of perilymph; the fluid driven in by the inward thrust of the stapes has to find room for itself elsewhere, and that room is furnished by the outward bulge of the membrane of the fenestra rotunda, for we may neglect other means of escape such as the lymph spaces around the endolymphatic duct, the nerves and the blood vessels. Hence at each movement of the stapes the whole mass of the perilymph swings bodily, the membrane of ferestra rotunda moving outwards and inwards at the same instant that the stapes moves inwards and outwards; and each such mass-vibration of the perilymph repeats the characters of the vibration of the ossicles and tympanic membrane, of which it is the continuation.

As they sweep over the vestibule, these vibrations are communicated through the walls of the enclosed membranous labyrinth to the endolymph. The vibrations of the endolymph, or of the walls themselves, affect in some way or other the auditory epithelium of the three cristre and the two maculie.

The vibrations also travel from the vestibule into the scala vestibuli of the cochlea, ascending the spiral from below upwards. As they ascend they are transmitted across the mem- 
brane of Reissner, the endolymph of the canalis cochlcaris, and the basilar membrane to the scala tympani, and so reach the fenestra rotunda. The bulk of the vibrations ascending the scala vestibuli thus reach the scala tympani by crossing the canalis cochlearis, and in so crossing affect in some way or other the auditory epithelium of the organ of Corti; it is probably only a remmant which at the summit of the spiral passes directly from the one scala to the other. The features of the basilar membrane point to its being readily thrown into vibrations, and we may conclude that the vibrations started at the fenestra ovalis and transmitted from the scala vestibuli to the scala tympani throw the basilar membrane into corresponding vibrations. By the vibrations of the basilar membrane, or by a more direct action of the vibrations of the endolymph, the auditory epithelium is so affected as to give rise to auditory impulses.

We now come upon matters of no little difficulty. We have seen reason to think that the eighth nerve serves as the chan-

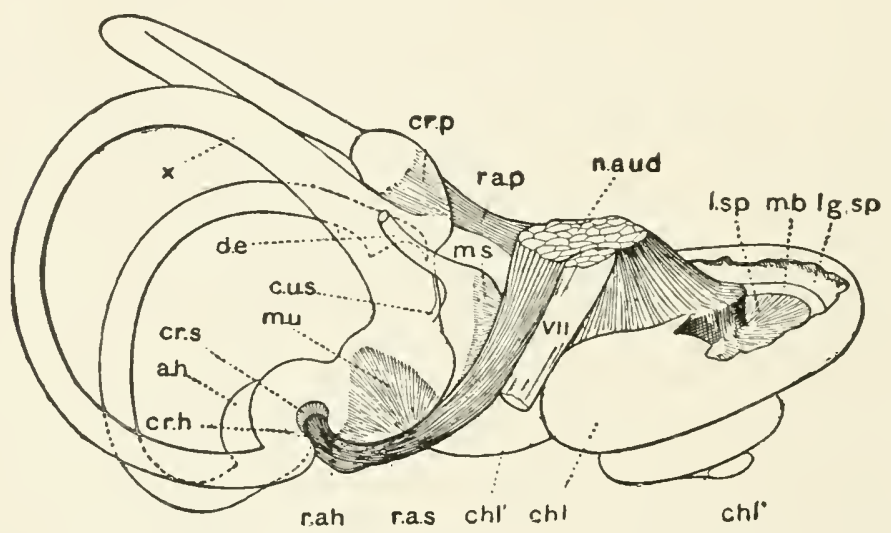

Fig. 178. The Membrayons Labrinth (of the Right Ear) as seen FROM ABOVE, MAGNIFIED SIX TIMES. (After Retzius.)

The bony envelope has been wholly removed from the vestibular division, but only in part broken through in the cochlear division.

$c h l$ the cochlea, $c h l^{\prime}$ the first part of the basal whorl, $c h l^{\prime \prime}$ the summit. To the right, where the bony wall has been broken through, are seen: $l . s p$ the spiral lamina, m.b the basilar membrane, lg.sp the spiral ligament.

$n$ aud the auditory nerve, lying alongside of which is seen VII, the seventh, facial nerve.

$m . s$ macula of the saccule. m.u macula of the utricle. $c r . p$ the crista of the posterior semicircular canal, with $r_{0} \cdot x_{0} p$ the branch of the auditory nerve distributer to it, $c r . s$ crista of the superior canal with r.a.s its nerve. a.h ampulla and $c r . h$ crista of the horizontal canal, with $r$.ah its nerve.

$x$ the conjoined posterior and superior canals. d.e ductus endolymphaticus, with $c . u . s$ its junction with the utricle. 
nel for impulses other than auditory impulses, for impulses which take part in the development of, and in the maintenance of, the sense of equilibrium. We have further seen reason to think that the whole of the vestibular division of the nerve, the part which is connected with the maculæ of the utricle and saccule (Figs. 178,179) as well as the part which is connected with the cristie of the semicircular canals, acts in this way. But there is no reason to think that, in the ligher animals possessing a

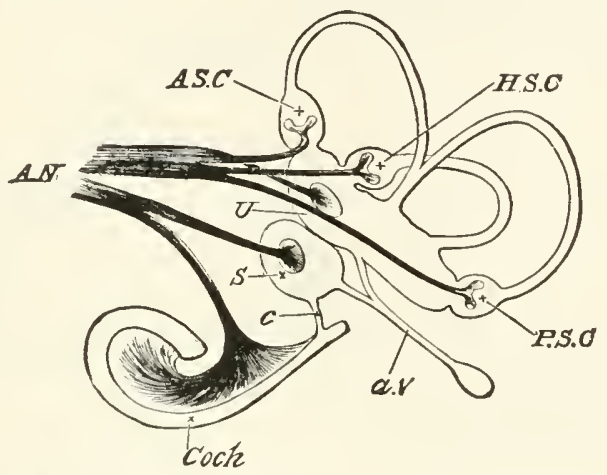

Fig. 179. Tile Membranots Labyrinth and the Endings of the Auditory Nerve.

The figure is wholly diagrammatic, and is introduced as giving a simpler view of the essential parts of $\mathrm{Fig} .178$; it should be used only in conjunction with that.

U. utricle. S. saccule. A.S.C. Superior (or anterior), P.S.C. posterior, H.S.C. horizontal, semicircular canals.

Coch. The canalis cochlearis represented as a tube partially unrolled. $c$. canalis reuniens, joining the saccule with the canalis cochlearis. a.v. ductus endolymphaticus, shewing its origin from both saccule and utricle, and its dilated blind end, the saceus endolymphaticus.

$A . N$. The auditory nerve ending in the cristæ of the ampullæ, in the maculæ of the utricle and saccule, and along the whole length of the canalis cochlearis. The branch of the vestibular division of the nerve ending in the saccule remains in close contact with the cochlear division, longer than does the rest of the vestibular division ending in the utricle and ampulla (the branch to the posterior canal should have been represented as lying in contact with that to the saccule).

well-developed cochlea, the cochlear division of the nerve, distributed solely to the cochlea, has any such function; this division of the nerve seems to carry auditory impulses only. We may therefore in the first instance confine ourselves to the cochlea exclusively. Now in the cochlea the connection of the fibres of the auditory nerve seems to be exclusively limited to the hair-cells, inner and outer; and we may conclude that these hair-cells are in some way or other concerned in the development of auditory impulses. This view is supported by the analogy of vision; for we have seen reason to think that visual impulses begin in the rods and cones, which like the hair-cells are 
modified epithelium cells, and we shall presently find that modified epithelium cells also play an essential or important part in the development of sensations other than those of vision and hearing. We may conclude that the vibrations of the perilymph in some way or other bring about such changes in the hair-cells as to give rise to auditory impulses. What is the exact nature of these changes and what is the exact way in

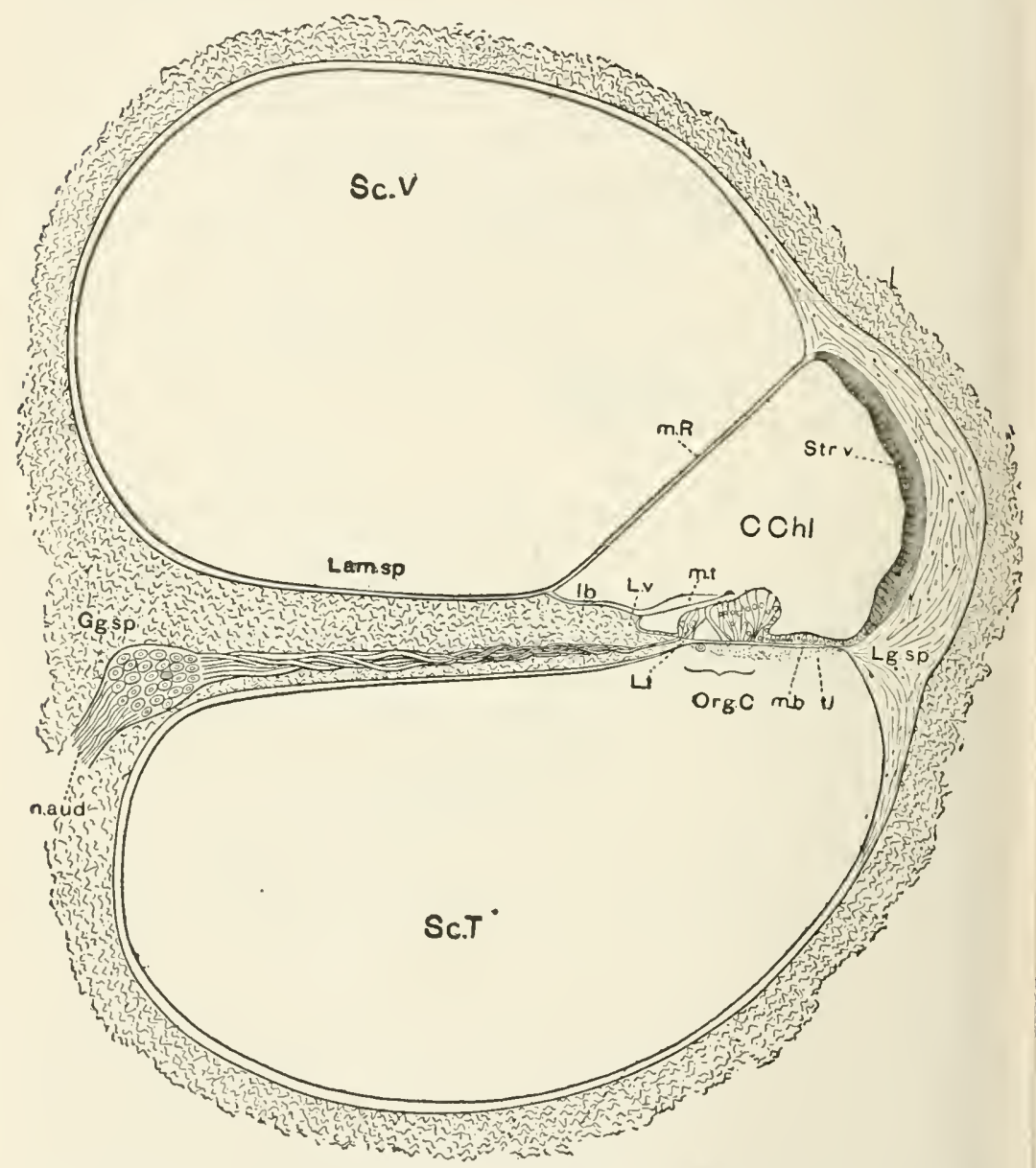

Fig. 180. Diagram of a transverse section of a Whorl of the Cochlea.

Sc.v. Scala Vestibuli. Sc.T. Scala Tympani. C.chl. Canalis cochlearis. n.aud. auditory nerve. Gg.sp. Spiral ganglion. Lam.sp. Lamina spiralis. lb. limbus. L.v. labium vestibulare. Lt. labium tympani. m.R. membrane of Reissner. I.a.sp. spiral ligament. Str.v. stria vascularis. Org.C. organ of Corti. m.b. basilar membrane. t.l. lymphatic epithelioid lining of the basilar membrane on the tympanic side. m.t. tectorial membrane. 
which they are brought about? To these questions we can at present give no satisfactory answer at all. Any attempt to answer them leads us at once into speculations. The rod-like appendages of the hair-cells, the so-called laairs (Figs. 181, 182) are too short and uniform, to permit us to suppose that they are vibrating organs responding by their vibrations to the vibrations of the perilymph and so bringing those vibrations to bear on the substance of the hair-cells. The vibrations find their way, so to speak, to the hair-cells in some other way. The membrana tectoria (Figs. 180, 181, m.t.) has the aspect of an organ serving to

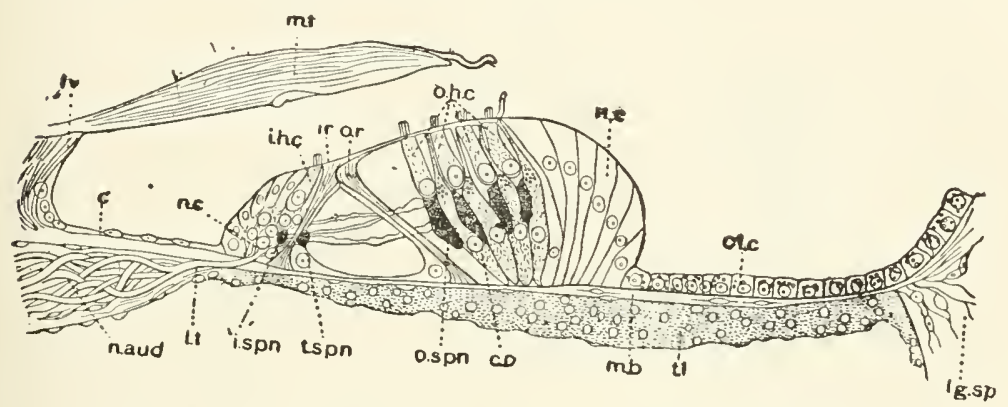

Fig. 181. Diagram of the Organ of Corti. (After Retzius.)

i.r. inner rod of Corti, o.r. outer rod of Corti.

i.h.c. inner hair-cells, n.c. the group of nuclei beneath it. o.h.c. outer haircell, or cell of Corti, of the first row, c.D. its twin cell of Deiters; four rows of these twin cells are shewn.

n.aud. the auditory nerve perforating the tympanic lip l.t, and lost to view among the nuclei beneath the inner hair-cell. $i . s p . n$. the inner spiral strand of nerve-fibrills. t.sp.n. the spiral strand of the tumnel. o.sp.n. the outer spiral strand belonging to the first row of outer hair-cells ; the three succeeding spiral strands belonging to the three other rows are also shewn. Nerve-fibrilla are shewn stretching radially across the tumnel.

H.c. Hensen's cells, Cl.c. Claudius cells, m.b. basilar membrane, $t l$. lynphatic epithelioid lining of the basilar membrane, on the side towards the scala tympani. lg.sp. spiral ligament. $c^{\prime}$. cells lining the spiral groove, overhung by l.v. the vestibular lip. m.t. the tectorial membrane; a fragment of it is seen torn from the rest and adherent to the organ of Corti just outside the outermost row of outer hair-cells.

'damp' the vibrations of the basilar membrane, and the hairs of the hair-cells may perhaps rather serve the purpose of bringing their damping action to bear directly on the substance of the hair-cells; for the membrane in question comes down into direct contact with them. We may further suppose that in the development of auditory impulses, the peculiar rods of Corti (Fig. 182) play some special part. But concerning all these matters we can at present do hardly more than make guesses, and those unprofitable ones. 
One point deserves mention. We saw reason to think ( $\$ 573$ ) that visual impulses cannot be generated in the optic fibres otherwise than through the intervention of the retinal structures; in the absence of the retina an animal is wholly blind. In pigcons, however, from which the labyrinths of both ears have been entirely removed, a certain apparent power of response to sounds has been observed; the animals still seemed to hear. And it has been contended that such cases are instances of the mere fibres of the auditory nerve apart from their special terminations being sensitive to the vibrations of sound; it is suggested

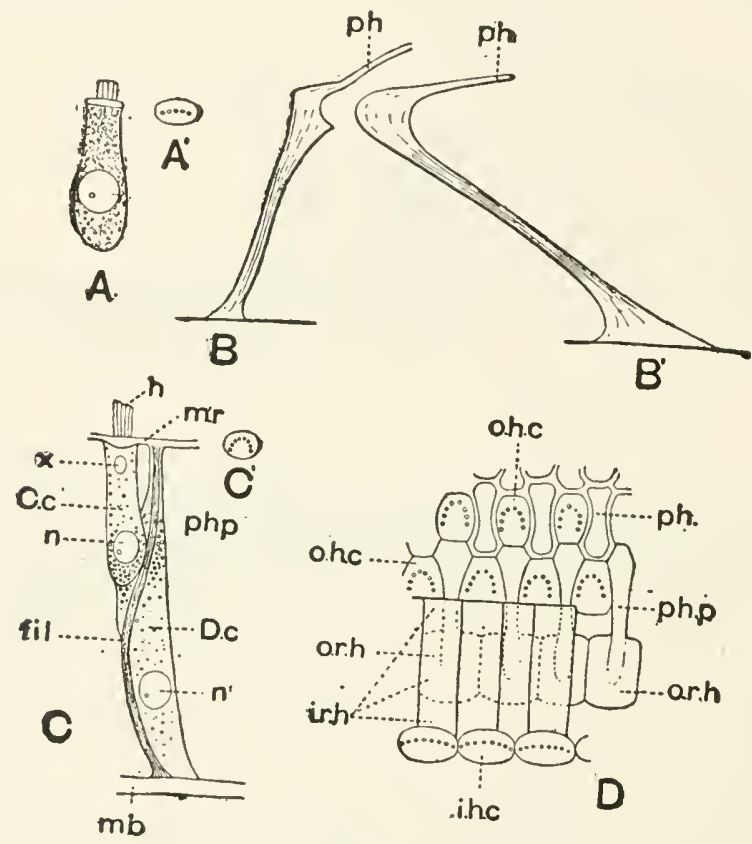

Fig. 182. Diagram of the constituents of the Organ of Corti. (After Retzins.)

A. Inner hair-cells, $A^{\prime}$, the head seen from above.

B. Inner, $\mathrm{B}^{\prime}$. outer rod of Corti, $p h$. (in each) plalangar process.

C. The twin outer hair-cell. C.c. cell of Corti, $h$. its auditory hairs, $n$. its nucleus, $x$, Hensen's body. D.c. cell of Deiters, $n^{\prime}$. its nucleus, ph.p. its phalangar process, fil. the cuticular filament, m.b. basilar membrane, m.r. reticulate nembrane.

$\mathrm{C}^{\prime}$. The head of the cell of Corti as seen from above.

D. The organ of Corti seen from above. i.h.c. the heads of the inner haircells. ir.h. the head and phalangar process of the inner rod. o.r.h. the head of the outer rod, with ph.p. its phalangar process, covered to the left hand by the inner rods, but uncovered to the right. o.h.c. the heads of the cells of Corti supported by the rings of the reticulate membrane. $p h$. one of the phalangæ of the reticulate membrane. 
that the fibres are directly stimnlated by the vibrations passing through the bone, in canals of which the fibres lie. Sucl a conclusion presents great difficulties; we shall have to refer to it again later on.

$\$ 629$. Leaving this view for the present on one side, and assuming that the waves of sound are converted into auditory impulses by means of the hair-cells, we may now turn to another question, also one of great difficulty. How do the different vibrations which determine the natire of different sounds so differently affect the hair-cells as to give rise to sensations of corresponding difference? A complex sound, consisting of vibrations of more than one period, travels as we have said, not as a group of discrete waves, each corresponding to a vibration of a particular period, but as a complex wave in which the simple waves are compounded into one; and the vibrations of the tympanic membrane, followed by the vibrations of the perilymph, have the same composite eharacter. When for instance a note is sung, or sounded on a musical instrument, the air in the external auditory passage is not the subject of one set of waves corresponding to the fundamental tone, and of other sets corresponding to the several partial tones, but vibrates in the pattern of one composite wave; the tympanic membrane executes one complex vibration, and a corresponding single comnlex vibration excites the auditory epithelium. And this holds good not for a single sound only but for a mixture of sounds. We can in a clumsy way take a graphic record of the vibrations of a dead tympanic membrane, by attaching a marker to the stapes; coull we take an adequate record of the movements of the living tympanum of one of the audience at a concert, we should obtain a curve, a phonogram, which though a single curve only would be on the one land a record of the multitudinous vibrations of the concert, and on the other hand a picture of the actual blows with which the perilymph had struck the auditory epithelium.

Now, whatever be the exact nature of the process by which the vibrations of the perilymph give rise to auditory impulses, we may consider it as probable that, in giving rise to those impulses, the complex vibration is analyzed again into its constituent simple vibrations, that the vibrations start afresh so to speak in the auditory epithelium, marshalled in the same array as that in which they started from the sounding instruments, as if the auditory epithelium itself constituted the band playing the music. And indeed that something of this kind does take place is indicated by the fact that an adequately sensitive ear can in a musical sound detect one or more of the partial tones as distinct from the fundamental tone, or still more easily can in a mixed concert detect the several notes of the several instruments, though as we have just said in the movements of the 
tympanic membrane, all the constituent factors are merged into one complex sweep. We may conclude then that we possess some means of analyzing the composite wares of sound which sweep through the perilymph, and of sorting out their constituent vibrations.

There is at hand a simple and easy physical method of analyzing composite sounds. If a person standing before an open pianoforte, the loud pedal being held down, sings out any note, it will be observed that a number of the strings of the pianoforte will be thrown into vibration, and on examination it will be found that those strings which are thus set going correspond in pitch to the fundamental tone and to the several partial tones of the note sung. The note sung reaches the strings as a complex wave, but the strings are able to analyze the wave into its constituent vibrations, each string taking up those vibrations and those vibrations only which belong to the tone given forth by itself when struck. If we suppose that each terminal fibril or each group of fibrils of the auditory nerve is connected with a terminal organ so far like a pianoforte-string that it will readily vibrate in response to a series of vibrating impulses of a given period and to none other, and that we possess a number of such terminal organs sufficient for the analysis of all the sounds which we can analyze, and that each terminal organ so affeeted by particular vibrations gives rise to a sensory impulse and thus supplies the basis for a sensation of a distinct character-if we suppose these organs to exist, our appreciation of sounds is in part explained.

When the rods of Corti were first discovered, it was thought that they were specially connected with the nerve fibres, and served mechanically to stimulate the fibrils passing along their limbs, by striking them after the fashion of minute hammers. Since these rods, to whose striking resemblance to the keys of a pianoforte we have already called attention, are arranged in a long series the members of which vary regularly in the length and in the span of their arch, from the bottom to the top of the spiral, it was supposed that each pair would vibrate in response to a particular tone, and hence that the whole series served for the analysis of sound.

But this view proved untenable. Whatever purpose they serve, the rods of Corti produce their effect, not by acting directly on nerve fibrils, but by contributing in some way or other to the play of the hair-cells; and, whatever be the way in which they intervene, they do not vary in length and arrangement along the spiral to such an extent as the above view demands. Moreover, they are wholly absent from the rudimentary cochlea of birds, though these creatures very clearly can appreciate musical sounds. This last fact proves indubitably that the rods in question are not absolutely essential for the recognition of tones, 
since it is in the highest degree improbable that birds are able to recognize tones in some manner absolutely different from that employed by mammals.

In the face of these difficulties it has been suggested that the basilar membrane, which is present in birds as well as in mammals, and which, being tense radially but loose longitudinally, i.e. along the spiral of the cochlea, may be considered as consisting of a number of parallel radial strings, each capable of independent vibrations, is the sought-for organ of analysis; for it may be shewn mathematically that a membiane so stretched in one direction only is capable of vibrating in such a manner. And the radial dimensions of the basilar membrane increasing as they do upwards from the bottom of the spiral to near the top give a much greater range of difference than do the rods of Corti. According to this view, when a composite vibration sweeps along the cochlea it throws into sympathetic vibrations those small portions and those portions only of the basilar membrane, the vibrations of which correspond to the single vibrations of which the composite vibration is made up; and the vibrations in turn so affect the overlying structures, that auditory impulses are generated in particular groups of fiblils of the auditory nerve. These auditory impulses reaching the brain give rise to a corresponding sensation of a particular sound.

But the dimensions of even the basilar membiane do not seem wholly adequate for the purpose; since the latest measurements shew that in man its range is very limited. If we take the whole width of the membrane, the range is from $.21 \mathrm{~mm}$. at the base to $36 \mathrm{~mm}$. at the top, though if we take the specially modified part reaching from the outer feet of the rods to the spiral ligament, we get a wider range, namely, from $.075 \mathrm{~mm}$. at the base to $.126 \mathrm{~mm}$. at the top. On the other hand the estimated number of radial fibres of the membrane is very large, 24,000 ; and even if we suppose that several fibres always vibrate together, this would still leave some thousands of groups of strings, each group acting as an analyzer.

In the present state of our knowledge the whole matter must be left as uncertain. Even if the basilar membrane acts in some such way as suggested, the other structures in the auditory epithelium present problems as yet insoluble. The true function of the rols of Corti and of the reticulate membrane of which these form a part, of the cells of Deiters, of the inner hair-cells as distinguished from the outer hair-cells, as well as the reason there are four rows of the latter (whereby probably the effect of the vibritions of a group of the basilar fibres is increased) and only one of the former, all these are as yet merely questions which cannot be answered.

$\S 630$. Even admitting that, in some way or another, sets of vibrations or, to use a more general term, sets of molecular 
movements are started in the auditory epithelium, in more or less complete correspondence with the sets of vibrations which originate from the musical instrument or other sounding body, and admitting further that each set of such molecular movements in the auditory epithelium starts a particular nervous impulse in a fibril or in a set of fibrils of the auditory nerve, we are very far from laving solved the problem of hearing.

It must be borne in mind that making the fullest allowance for the assistance afforded us by the organ of Corti, the appreciation of any sound is ultimately a psychical act. 'The malysis of the vibrations by help of the basilar membrane or otherwise is simply preliminary to a syuthesis of the auditory impulses so generated into a complex sensation. We do not receive a distinct series of specific auditory inpulses resulting in a specific sensation for every possible variation in the wave-length of sonorous vibrations any more than we receive a distinct series of specific visual impulses for every possible wave-length of luminous vibrations. In each case we have probably a number of primary sensations, from the various mingling of which, in different proportions, our varied complex sensations arise; but there is this difference between the eye and the ear that whereas in the former the number of primary sensations appears to be limited to three or at least to six, in the latter the number is probably very large; what the exact number is has not at present been even suggested. Our appreciation of a sound is at bottom an appreciation of the combined effect produced by the relative intensities to which the primary auditory sensations are, with the help of the organ of Corti, excited by the sound. The appreciation and the subjective analysis of sounds is ultimately a psychical process; and though there are individual differences in the structural finish and physical capabilities of the auditory epithelium as of other parts of the ear, the differences in the psychical or at least cerebral powers of individuals are far greater' and when we speak of a musical ear we really mean a musical mind or a musical brain.

$\S 631$. If the organ of Corti, as appears from the above, affords the means by which we appreciate tones, it is evident that by it also we must be able to estimate loudness, for the quality of a musical sound is dependent on the intensity, as well as on the pitch, of the partial tones in relation to the fundamental tone and to each other. Further, as we said above the distinction between noise and music is a quantitative and fluctuating one; indeed the tendency of inquiry seems to shew that the quality or timbre of a sound, and it is this which so largely contributes to the value of a sound as an element of music, is in part dependent on vibrations, which being irregular, that is, having no exact arithmetical relation to the fundamental 
tone, may be spoken of as noise. But, if noise is only confused music, and nusic more or less orderly noise, the cochlea must be a means of appreciating noises as well as musical sounds.

We may therefore reject the view which has been put forward by some that while by the cochlea we appreciate musical sounds, our knowledge of noises is gained by auditory impulses leaching us through the vestibule.

Are we to conclude then that the vestibule has nothing to do with hearing, is concerned only with equilibrium? A certain support is given to this view by cases in man where deafness seems to have been due to disease confined to the cochlea; and in animals deafness is said to have been produced by division of the cochlear nerve, the vestibular nerve being left intact. Moreover animals possessing a cochlea certainly continue to hear and to hear well after division of both vestibular nerves; but this is not a valid argument against no auditory impulses at all passing along this nerve, since the cochlea is obviously adequate by itself for ordinary hearing, and the loss of the vestibule might simply entail in the character of the sense changes too fine to be readily recognized in a dumb animal.

On the other hand vertebrates, lower in the scale than birds and reptiles, namely, fishes, though they have a well-developed vestibular labyrinth, possess either no cochlea at all or the merest trace of one, and yet undoubtedly are the subject of auditory sensations, in some cases of acute sensations. The evidence that fishes hear seems irresistible, they are said to respond to musical sounds; and yet those who hold the views just explained are driven to maintain either that fishes do not hear in the true sense of the word but only feel vibrations, or that they hear by means of an insignificant fragment of their relatively large vestibule. The structure of the piscine and amphibian vestibular auditory epithelium is in the main putting aside smaller matter's, such as the length of the auditory hairs, the size and abundance of otoliths and otoconia and the like, so identical with that of birds and reptiles and of mammals, that it is impossible to resist the conclusion that it serves the same purpose in all the several classes. In birds and reptiles the short rudimentary nearly straight tubular cochlea possesses a short basilar membrane, an auditory epithelium in which a distinction of outer and inner hair-cells is foreshadowed, and a tectorial membrane. But if we are to suppose that these creatures receive auditory impulses exclusively from the cochlea, and none at all from the vestibule, it is a matter of wonder that the cochlea of the, for the most part, dumb crocodile should appear almost as highly developed as that of the vocal bird. Or again, if the bird and reptile already possessing a cochlea still derive auditory sensations by means of the vestibule, we may conclude that mammals also do the same. 
The changes in the endolynph which give rise to the impulses affecting equilibrium, namely a simple change in the amount of pressure or a simple shifting of position, a simple flowing, are so different from the changes, the rapid repeated vibrations produced by sound, that it seems permissible to conceive of the cristre and naculæe reacting differently towards the two agencies, and so giving rise to impulses of different natures, one auditory and the other not. The value of the hairs of the cristie and ampullie as vibrating organs have probably been exaggerated; anong other things the medium in which they move, the somewhat riscid endolymph, is unfavourable to vibrations; and the otoliths and otoconia, if they have any relations to vibrations, probably serve as 'dampers.' Still the hairs probably do vibrate as the endolymph vibrates; and we may inagine that the changes in the hair-cells and hence in the nerve fibres and hence in the brain are different when the hairs are thrown into series of vibrations from what they are when the hairs are gently pressed or gently moved. Further we may reflect that in ourselves the sensations gained by the cochlea are so dominant, that we may be at the same time receiving sensations through the restibule without being aware that we are doing so; these latter may, further, be of a different nature from the former, and the vestibular hearing of a fish may be something very different from our, mainly, cochlear hearing. At any rate we may hesitate to accept the view that no auditory impulses travel along the vestibular nerve. But if we do thus hear by means so to speak of a double organ, then the origin and nature and effects of auditory impulses must be still more complex and difficult than appears from the study of the cochlea alone, perplexing as they even then seem.

The difficulties attending an adequate conception of the nature and origin of auditory impulses are further increased by the following observation. Two tuning-forks, not quite in unison, produce 'beats' when they are sounding together'; the beats are due to the influence exerted by one set of waves on the other set ( $\$ 627)$. But it is stated that, if the two forks be listened to, one with onc ear and the other with the other, precautions being taken so that the vibrations reaching the one auditory nerve by the onc ear, cannot, by conduction through the bones of the head or otherwise, also gain access to the other auditory nerve, the beats are still heard. 'This observation, unless there be some hidden fallacy in it, seens to shew that the beats arise in the brain itself, that the impulses travelling along the auditory nerve so far resemble in their nature and character, the waves of sound giving rise to them, that the two sets of impulses along the two nerves meeting in the brain give rise to beats, just as do the two sets of waves meeting in the air. If we further couple with this conclusion the view referred to above 
(\$ 628), that waves of sound falling on the auditory fibres themselves may give rise to auditory sensations, we arrive at the conception that the auditory impulses are mere copies so to speak of the physical sound waves, a conception which, if substantiated, would necessitate a revisal of all our views concerning nervous impulses. 


\section{SEC. 4. ON AUDITORY PERCEPTIONS AND JUDGMENTS.}

$\S 632$. In spite of the many and striking differences between the two senses, it is possible to draw several parallels between auditory and visual sensations. When we are the subject of a visual sensation we refer the cause not to changes taking place in the retina, but to some luminous object in the external world. So also, when we are the subject of an auditory sensation we refer the canse not to changes taking place in the internal ear, but to some sounding body outside the ear and in the vast majority of cases to some sounding body outside ourselves. We do not simply feel auditory sensations, we perceive sounds, ef. §. 581.

IVe have seen that in the case of the eye, visual sensations, excited by events taking place in the visual apparatus itself, may be confounded with sensations excited by objects in the external world, and much the same happens with the ear also. The tympanic membrane for instance may be thrown into vibrations not by waves of sound, but by objects coming mechanically into contact with it; particles of the dried secretion of the external auditory passage, the 'wax of the ear', playing on the tympanic membrane, may give rise to auditory sensations, a 'buzzing' or" 'singing in the ears,' which we cannot by the mere psychological examination of the sensations themselves distinguish from auditory sensations excited in the ordinary way by sonorous viblations reaching us from some sounding body at a distance. And in a general way, we may speak of entotic phenomena, corresponding to the entoptic phenomena on which we dwelt ( $\$ 549)$ in speaking of vision.

Auditory sensations moreover may arise, in the complete quiescence of the tympanic apparatis and perilymph, as the result of changes either in the auditory epithelium or in the central auditory nervous apjaratus. We may be subject to auditory phantoms or hallucinations, corresponding to oeular phantoms or hallucinations, and like them often misleading or distressing. Few persons, moreover, can listen to exciting music or can hear impressive cries without experiencing "recurrent" auditory sensations. 
§ 633. In one important respect the parallel between hearing and sight fails. When we see an object, the rays of light coming from the object excite a particular part of the retinal expansion; and our appreciation of the position which that object holds in space is based on our power of "localizing" retinal changes. The terminal expansion of the auditory nerve however has no such definite relations to the positions in space of objects from whence sounds are proceeding; we have no evidence that any particular part either of the organ of Corti or of the maculæe is alone or specially affected by sounds coming from a particular quarter, and the evidence that sounds affect the three cristre differently according to the direction of the sound is at least doubtful. Hence we possess no "auditory field" which can be directly compared with the "visual field;" and our conclusions as to the direction in which the sounds which reach our ears have travelled, our judgments as to the position in space of bodies exciting auditory sensations are formed in an indirect manner.

The vast majority of the sounds which we hear reach the auditory epithelium by way of the tympanic membrane and chain of ossicles; even the sounds which are conducted to the ear through the bones and hard parts of the head pass to a large extent by this way ( $\$ 616$ ); in normal hearing the auditory sensations which are generated by vibrations transmitted directly through the bony walls of the labyrinth to the perilymph are probably insignificant. Now it is only in relation to these latter that the disposition in space of the three semicircular canals can possibly have any meaning; the vibrations reaching the perilymph by way of the tympanic membrane, whatever their original direction, have all the same direction when they enter at the fenestra ovalis, and fall in the same way upon the three semicircular canals. We may therefore conclude that the position in space of the three canals in question has nothing to do with our ordinary judgments as to the direction of sounds. In forming those judgments we are assisted mainly by two things.

In the first place a peculiar character of the outwardness which we attribute to our usual auditory sensations, that by which we judge the sound to arise not only outside the internal ear but outside our whole body, seems, in some way, largely dependent on the vibrations which cause the sensation having travelled along the external auditory passage. If the two passages be filled with fluid the hearer refers the sounds which he hears, in spite of their starting at some distance off, not to the external world outside himself, but to the inside of his own head; the sounds appear to him to come, not it may be remarked from the internal ear or any part of it, but from the roof of the mouth, or the top of the skull or the back of the head. So also if the ear-pieces of a binaural stethoscope be 
pushed well up into the auditory passages, the sounds heard through the instrument seem to come from the roof of the observer's own mouth.

The difference between such an abnormal mode of hearing and ordinary hearing does not lie in the fact that in the former case the tympanic membrane is not used at all; for even when the external passage is filled with fluid, a layer of air which always adheres to the tympanic membrane permits at least a certain amount of vibration of that membrane; and on the other hand when the sound is actually generated in the roof of the mouth, and rightly judged to be generated there, the tympanic membrane by its vibrations conducts the greater part of the sound to the internal ear. How it is that the passage of the vibrations through the external passage imparts to the sensation this attribute of outwardness is not clear. Indeed certain sounds may be made to lose this particular outwardness, though the external passage be still employed. If two musical sounds of the same pitch be listened to with the two ears separately by means of two telephones, the sound will, under certain conditions, appear to originate somewhere in the head of the observer.

$\S 634$. In the second place our appreciation of the particular quarter from which a somd, recognized by help of the external passage to be of outward origin, has travelled is dependent on our using two ears. As onu ordinary vision is largely binocular, so our ordinary hearing is, to a still larger extent, binaural. In the case of the ear there are no sharp limitations to the range of the organ of either side; through the merlium of the air and external auditory passage or of the hard parts of the head a sound which affects one ear affects to a certain extent the other ear also ; hence all our hearing is, under ordinary circumstances, binaural. And in some such way as two visual sensations excited in "corresponding parts" of the two retinas are fused into one, so every sound which reaches us is heard not as two sounds, one by one ear and the other by the other, but as one sound by the two ears together.

When the sounding body is on one side of the head, say the right side, the sensations excited through the right internal ear are more powerful than those excited through the left internal ear; we are not distinctly conscious of the difference between the two sensations, the combined effect is a single sensation; but the difference does affect our consciousness in a certain way, and that affection of conscionsness serves as a basis for the judgment that the sounding body is somewhere on our right hand. Hence we are able to judge the lateral much more readily than the fore and aft position of a somding body. If a tuning-fork be held in the median vertical plane over the head, the eyes being shut, though it is easy to recognize it as being in 
the median plane, it is very difficult to say what is its position in that plane, i.e. whether it is more towards the front or back of the head. Hence also a man who is absolutely deaf of one ear lias great difficulty in recognizing the direction of sounds.

Further, when we desire to judge particularly as to the direction of a sound, we listen to it, and in the act move the head into the position in which we hear the sound most ristinctly. In this way the movements of the head in hearing play a part somewhat analogous to the movements of the eyes in vision.

Even in the case of ourselves, and still more in the case of some animals, the form of the external ear favours the entrance into the meatus, and hence the access to the tympanic mem. brane, of sounds travelling in a particular direction; this also assists our judgment of the direction of sounds. Hence, by tying back the ears and affixing artificial ears, differing in shape or position from the natural ones, we may make false judgments in this matter.

Moreover, in forming a judgment as to the direction of sounds we appear to be guided by something more than the mere relative intensity of the sounds falling on the two ears. When a complex sound emanates from a boly on one side of us, the constituent vibrations do not travel equally and uniformly over and around the head; some are refracted more than others, so that they do not reach the two ears equally; and besides when they reach them are not equally reflected by the two pinnx. In this way partial tones of different pitch, and this applies especially to high tones, reach the two tympanic membranes in unequal intensities, and the sound of which they form part appears as heard by the one ear of a quality slightly different from that heard by the other ear ; this difference of quality, like the difference in mere intensity of the sound as a whole, serves as a basis for recognizing the direction of the sound. Such a difference will be morc marked in the complex sounds which we call noises than in purer and more simple musieal sounds; and, as a nratter of fact, our appreciation of direction is more accurate in the case of noises than of musical sounds. An exception to this rule is met with in the case of the human voice, the direction of which, though it is as a whole a musical sound, can be judged better than even that of a noise; but noises enter largely into the human voice, and besides we are much more practised in relation to it than in relation to any other kind of sound. All our judgments of the direction of sounds are however at the best imperfect.

$\$ 635$. Our judgment of the distance of sounds is even still more limited. $\ddot{A}$ sound whose characters we know appears to us near when it is loud, and far off when it is faint. A blindfold person will be unable to distinguish between the difference 
of intensity produced on the one hand by a tuning-fork being held before him, first with the broad edge of the fork toward him and then with the narrow edge, and the difference on the other hand caused by the removal of the tuning-fork to a distance. And our judgments in this respect may be false, as is seen in the effects produced by the ventriloquist. We can on the whole better appreciate the distance of noises than of musical sounds, differences of quality as well as of intensity playing the same part in the judgment of distance as of direction; when a sound becomes distant the intensity of the fundamental tone diminishes more rapidly than do those of the higher partial tones, and hence the quality of the sound is affected. 


\section{CHAPTER V.}

\section{TASTE AND SMELL.}

\section{SECTION 1. OLFACTORY SENSATIONS.}

\$ 636. Particles of odoriferous matters present in the inspired air, passing through the lower nasal chambers, diffuse into the upper nasal chambers, and falling on the olfactory epithelium produce sensory impulses which, ascending to the brain, give rise to sensations of smell. If we assume that the rod cells, and not the cylinder cells, are the special functional elements, we may suppose that the sensory impulses are originated by the contact of the odoriferous particles with the free endings of the rod cells; but they may be duc to contact with the cylinder cells: in either casc we are wholly in the dark as to the manner in which the contact of the particles with the cells brings about the molecular changes constituting a nervous impulse. We camnot even say whether we ought to speak of the first step by which the contact of the particle begins the series of changes as a chemical or as a physical process.

In nearly all cases the odoriferous particles are conveyed to the membrane in a gaseous medium, namely, the atmosphere; but before they can gain access to the cells they must become dissolved or at least suspended in fluid; for the whole olfactory membrane is kept moist by a layer of fluid, secreted by the glands, and the odoriferous particles must pass into this layer of fluid before they can gain access to the cells. Indeed, the proper condition of this layer of fluid is one of the essential conditions of the exercise of the sense. If on the one hand the membrane be too dry, or if on the other hand the secretion be too abundant or altered in quality, the power of smelling is diminished or even wholly suspended. It is a matter of common experience that a nasal catarrh interferes with smell. When the nostril is filled with rose-water the odour of roses is not perceived; and simply filling the nostrils with distilled water suspends for a time all smell, the sense gradually return- 
ing after the water has been removed; the water apparently acts injuriously on the delicate olfactory cells. If insteal of using rose water, the rose scent be dissolved in "normal saline solution" which (\$ 14) more elosely resembles the natural secretion, the cells ean perform their function, and the scent is perceived. The glands of the olfactory membrane form an important subsidiary apparatus for the development of olfactory sensations.

The other subsidiary apparatus of smell is exceedingly meagre. By the forced nasal inspiration, called sniffing, we draw air so foreibly through the nostrils that currents pass $1 p$ into the upper as well as the lower nasal chamber's; and thus a more complete contact of the odoriferous particles with the olfactory membrane than that supplied by mere diffusion is provided for.

$\$ 637$. We have every reason to think that any stimulus applied to the olfactory cells will produce the sensation of smell; but the proof of this is not absolutely clear ; and we have $n 0$ definite evidence as to what is the result of directly stimulating the fibres of the olfactory nerve. The olfactory membrane however is certainly the only part of the body in which odour's as such can give rise to any sensations: and the sensations to which they give rise are always those of smell. The mucous membrane of the nose is however also an instrument for the development of afferent impulses other than the specific olfactory ones. Chemical stimulation of the nasal mucous membrane by pungent substances such as ammonia gives rise to a sensation distinct from that of smell, a sensation which does not afford us the same information concerning the chemical nature of the stimulus, as does a real olfactory sensation, and which is much more allied to the sensations produced by chemical stimulation of other surfaces sensitive to chemical action. This scnsation moreover seems to be developed both in the nonolfactory, and in the olfactory regions of the nasal mucous membrane; and it is probable that these two kinds of sensations, the one produced by odours, the other by pungent substances, thus arising in the olfactory nembrane are conveyed by different nerves, the former by the olfactory, the latter by the fifth nerve.

Each substance that we smell eauses a specific sensation, and we are not only able to recognize a multitude of distinct odours, but also in certain eases to distinguish individnal odlonrs in a mixed smell. And though we may reengnize certain odonss as more like to each other than to other odours, or can even make a rough classification of odours, we cannot, as we can in the case of visual colour sensations, reduce our multifarious olfactory sensations to a smaller 11 mmber of primary sensations mixed in varions proportions. Nor have we at present any satisfactory 
guicle to connect the characters of an olfactory sensation with the chemical constitution of the body giving rise to it.

The sensation takes some time to develope after the contact of the stimulus with the olfactory membrane, and may last very long. When the stimmlus is repeated the sensation very soon dies out: the sensory terminal organs speedily become exhausted. 'The larger, apparently, the surface of olfactory membrane employed, the mole intense the sensation; animal. with acute scent have a proportionately large area of olfactory membrane. The greater the quantity of odoriferous material brought to the membrane, the more intense the sensation up to a certain limit; and an olfactometer for measuring olfactory sensations has been constructed, the measurements being given by the size of the superficial area, impregnated with an odoriferous substance, over which the air must pass in order to give rise to a distinct sensation. The limit of increase of sensation however is soon reached, a minute quantity producing the maximum of semsation and further increase giving rise to exhaustion. The minimum quantity of material required to produce an olfactory sensation may be in some cases, as in that of musk, almost immeasurably small.

In ordinary circumstances odoriferous particles reach both mostrils, and we receive two sets of olfactory nervous impulses, one along each olfactory bulb. These however are fused into one sensation; our olfactory sensations are almost exclusively binasal. When two different odours are presented separately to the two nostrils, by means of two tubes for instance, the effect is not always the same. Sometimes an oscillation of sensation similar to that spoken of in binocular vision ( $\$ 602)$ takes place. At other times, the particular result depending on the nature of the odours, one sensation only is felt, the one sensation wholly destroys the other. And we may infer from this that when, as frequently happens, in a mixture of odours we can only recognize one dominant odour, the suppression of the missing sensations is not due to the chemical action of one odnur upon another, or to the one odour preventing the other from acting on the olfactory cells; but from a central cerebral obliteration of all the sensations but one.

$\$ 638$. As in the cases of the previous senses, we project our olfactory sensations. into the external world; the smell appears to be not in our nose, but somewhere outside us. We can judge of the position of the odour however even less definitely than we can of that of a sound. Our chief guide seems to be that we by turning the head ascertain in which direction we experience the strongest sensations.

The sense of smell seems to play a far more important part in the lives of the lower animals than it does in our own life : and what we now possess is probably the mere remnant of a once 
powerful mechanism. We may perhaps connect with this on the one hand the fact that, even in ourselves, the olfactory fibres have allotted to them what is virtually a whole segment of the brain, namely the olfactory lobe, and on the other hand the fact that olfactory sensations seem to have an unusually direct path to the inner working of the central nervous system. Mental associations cluster more strongly round sensations of smell tham round almost any other impressions we receive from without. And powerful reflex effects are very frequent, many people fainting in consequence of the contact of a few odorous particles with their olfactory cells.

The assertion that the olfactory nerve is the nerve of smell has been disputed. Cases have been recorded of persons who appeared to have possessed the sense of smell, and yet in whom the olfactory lobes were found after death to be absent. Direct experiments on animals however shew that loss of the olfactory lobes entails loss of smell. On the other hand, it is stated that section or injury of the fifth nerve causes a loss of smell though the olfactory nerve remains intact; but in these cases it has not been shewn that the olfactory membrane remains intact, and it is quite possible that, as in the casc of the eye, changes may take place in the nasal membrane as the result of the injury to the fifth nerve, sufficient to prevent its performing its usual functions. 


\section{SEC. 2. GUSTATORY SENSATIONS.}

\$ 639. The word taste is frequently used when the word smell ought to be employed. We speak of 'tasting' odoriferous substances, such as an onion, a wine, a savoury dish, and the like, when in reality we only smell them as we hold them in our month; this is proved by the fact that the so-called taste of these things is lost when the nose is held, or the nasal membrane rendered inert by a catarrh. If the nose be held and the eyes shut, it is very difficult to distinguish in eating between an apple, an onion and a potato; the three may be recognized by their texture, but not by their "taste." Most of what we call 'flavours' appeal in reality to the sense of smell not to that of taste.

We also experience by means of the surfaces with which we taste sensations other than those of taste. We feel by means of the mucous menbrane of the mouth sensations of the same kind as those which we feel by means of the skin, and which we shall study presently as tactile sensations or sensations of pressure, sensations of heat and of cold; indeed the tactile sensations of the tip of the tongue are remarkably acute. We also experience by means of the mouth sensations of pain and other more or less indefinite sensations which we shall presently speak of as phases of "general "or "common sensibility;" and in this respect the mucous membrane of the mouth is much more sensitive than the skin towards chemical substances; an acid for instance or other corrosive liquid, in such a concentration as when applied to the skin produces a sensation not essentially different from that of mere contact with an innocuous liquid, may when applied to the mouth produce a very painful sensation. Again, when the interrupted current is applied to the tongue we not only feel the contact of the electrodes but experience a peculiar sensation which is probably due to the contractions excited by the current in the muscular fibres of the tongue; we say we "feel the current."

$\$ 640$. There are however certain sensations quite distinct from those just mentioned and quite independent of smell which 
we experience when various substances are placed in the mouth; and these, which are the ginstatory sensations proper, may be broally classified into 'bitter,' 'sweet,' 'acid' or 'sour,' and 'salt,' to which perhaps should be arlded 'metallic' and 'alkaline.' 'The sensation of bitterness, such as that produced by quinine, and the sensation of sweetness, such as that produced by sugar, are very definite and specific sensations; they appear to be of an order different from those of acidity or sourness and of saltness; indeed an acid 'taste' is apt to merge into an affeetion of general sensibility mentioned above. The character's 'metallic' and 'alkaline' should perhaps be regarded as qualifying one or other of the other sensations rather than as being inclependent sensations.

In the ordinary course of things these sensations are excited by the contact of specific sapid substances with the mucous membrane of the mouth, the substances acting in some way or other, by virtue of their chemical constitution, on the endings of the gustatory fibres. When we taste quinine, the particles of the quinine, we must suppose, set up chemical changes in the cells of the taste-buds or in other parts of the epithelium, and by means of those changes gustatory impulses are started. But mechanical or electrical stimuli, in the absence of sapid substances, will give rise to gnstatory sensations. When the tongue is smartly tapped, in addition to the sensation of touch or the more or less painful sensation which may be produced, a sensation, which we must call a sensation of taste, is developed and often lasts for some considerable time. If a constant current be applied to the tongue, sensations of taste are developed at the two electrodes, that at the anode differing from that at the kathode, and the exact nature of each being dependent upon the region of the mouth stimulated. It is probable that in this ease electrolysis either of the fluids covering the epithelium or of the substance of the epithelial cells themselves generates bodies which act as chemical stimuli; and it is possible that the mechanical disturbance of the cells, when the tongue is tapped, also sets free chemical stimuli. But sensations of taste may be provoked by an interrupted induced current, so feeble as not to be felt as an electric current, and so arranged that the make and break shocks are equalized; in this case there can be little or no electrolysis, and we may infer that the eurrent acts in some way or another on the specifie nerve endings. It is somewhat singular that heat when applied to the tongue appear's not to produce any sensations of taste.

As we shall presently see, the nerve fibres concerned in taste belong either to the fifth nerve or to the glossopharyngeal nerve or to both nerves. We saw in dealing with vision that the evidence as to whether direct stimulation of the optic fibres without the intervention of the retinal structures could produce visual 
sensations was uncertain. We have no satisfactory evidence whatever that direct stimulation of the gnstatory fibres along their coursc in either the above two nerves will produce sensations of taste. As far as the sense of taste is concerned we have no adequate evidence that specific gustatory impulses can be developed in the gustatory fibres apart from changes in the nerve endings. But the evidence is negative only; and the case is one not suited for experiment, since both nerves along their whole course are mixed nerves containing other afferent fibres than those of taste.

$\$ 641$. It is essential for the development of taste, that the substance to be tasted should be dissolved; hence, the value of the glands, which are especially abundant in the neighbourhood of the taste-buds. The effect is also increased by friction; and the tongue and lips may be regarded as a subsidiary apparatus which by their movements assist in bringing the sapid substances into contact with the mucous membranc of the mouth. A substance may give rise to hardly any sensation of taste when simply placed on the extended tongue, and yet excite very distinct sensations when rubbed between the tongue and the soft palate; indeed we generally make use of this movement known as "smacking the lips," when we desire to obtain strong taste sensations. In this act howevel we not only make use of the most sensitive surfaces and call in the aid of friction, but we also increase the sensation by employing a large area of sensitive surface; for the larger the surface the more intense is the sensation.

'The sensation takes some time to develope, and endures for a long time, though this may be in part due to the stimulus remaining in contact with the terminal organs.

A temperature of about $40^{\circ}$ is the one most favourable for the production of the seusation. At temperatures much above or below this, taste is much impaired. A weak solution of quinine readily tasted at the normal temperature of the mouth is not tasted if, immediately before, very cold or very hot water be held in the mouth for a little while.

WTe may cxperience at the same time coincident taste sensations of different kinds, such for instance as one of bitterness with one of saltness; but in some cases one sensation interferes with the other, as for instance bitterness and sweetness. A taste sensation following upon a previous sensation of a different kind, is frequently influenced by its predecessor, being sometimes augmented, sometimes inhibited.

Though we can hardly be said to project our sensations of taste into the external world, as we do those of sight, hearing and smell, we assign to them no subjective localization. When we place quinine in our month, the resulting sensation of taste gives us no information as to where the quinine is, though we 
may learn that by concomitant general sensations arising in the buccal mucous nembrane. And it must be remembered that all our gustatory sensations are always accompanied by tactile or other sensations; we do not, as in the case of smell, experience the specitic sensation alone and apart by itself. And not infrequently, as when substances at once sapid and pungent are placed in the mouth, the general sensation of pungency overconres and hides the specific gustatory sensation. In the case of acids, it is often difficult to distinguish between the acid taste and the more general effect of the acid on the common sensibility of the buccal membrane of which we spoke above $\S 639$.

Though we possess a gustatory apparatus with separate nerves on each side of the mouth all our sensations are single. Nor can we distinguish a pure gustatory sensation developed on one side only from one developed on both sides, if the two are equally intense.

As in the case of the senses previously dealt with we may experience subjective gustatory sensations, sensations of central origin due to changes in the central sensory organs ( $\$ 502)$ : and these, though originated not by gustatory impulses but by other events, may seem to us identical with those set up in an ordinary way by gustatory impulses reaching the centre along the gustatory fibres.

$\$ 642$. Sensations of taste are not originated, either by sapid substances or otherwise, cqually in all parts of the lining membrane of the mouth. The part in which they are best developed, and always developed if developed at all, is the back of the tongue, in the neighbourhood of the circumvallate papillix. They are also developed at the tip and along the sides of the tongue, but to a variable extent in different individuals; some persons have very acute and distinct taste sensations in these parts, others little or none at all. On the dorsal surface of the middle of the tongue very feeble taste sensations, if any at all, are developed; they are always wholly absent from the undersurface of the tongue. Some taste sensations are also developed in the soft palate and front surface of the palatine arches; bnt these again vary much in distinctness in different individuals. In the cases recorded in which taste remained after the entire extirpation of the tongue including the circumvallate papilla, the sensations seem to have been chiefly developed in the soft palate. There is also some evidence that taste sensations may be developed on the hinder surface of the epiglottis.

In individuals who receive sensations from all or several of the various parts above mentioned, it commonly happens that bitter things are most readily appreciated at the back of the tongue and sweet things at the tip: and this distribution may perhaps be considered as the normal one; but individual varia- 
tions in this respect are met with; many persons taste both bitter and sweet things best at the back of the tongue: and some persons taste bitter things quite distinctly at the tip. The salt taste is said to prevail at the tip and the acid taste at the sides of the tongue; but many persons experience acid and salt tastes in those regions and those regions only in which they experience bitter and sweet tastes.

We have already said that bitter and sweet tastes seem to be on a different footing from acid and salt tastes; and we have a certain amount of evidence that the two former sensations are brought about by means of terminal organs different from those by means of which the two latter are brought about. If some of the leaves of a plant which grows in India and is called Gymnema sylvestre, be chewed, or if the mouth be washed with a decoction of the leaves, for some little time afterwards bitter. and sweet tastes are lost, neither quinine nor sugar exciting the usual sensations, though acid and salt tastes remain maffected. We may interpret this result as indicating that the drug in some way or other 'paralyzes,' that is to say, suspends the action of. the terminal organs, whatever they may be, by means of which bitter and sweet tastes are developed, but leaves untouched those by which other gustatory sensations are developed. 'The action of the same drug supports the further conclusion that the terminal organs of bitter tastes are different from those of sweet tastes; since by using an adequately weak dose of the drug the sweet taste may be abolished while the bitter taste remains distinct.

Inceed it is probable that the distribution of the several kinds of tastes over different regions of the mouth, which we mentioned above, is dependent on the distribution of different kinds of terminal organs; it is probable that we experience bitter tastes by means of the back of the tongue because the terminal organs of the bitter taste are limited to, or at least most abundant in, the back of the tongue, those of the sweet taste by the front of the tongue because the terminal organs of the sweet taste are more abundant there; and so on. If a small quantity of a particular bromine derivative of the substance which from its remarkably sweet taste has been called 'saccharine,' be placed carefully on the tip of the tongue, a sweet taste is developed; but if the same substance be carefully placed on the back of the tongue the result is not a sweet but a bitter taste. At least this is the result in the case of those individuals who taste bitter at the back of, and sweet at the tip of, the tongue. From this we may infer that, in such tongues, the specific terminal organs of the sweet taste are more or less completely limited to the front, and those of the bitter taste to the back of the tongue, both sets of terminal organs being of such a nature that while quinine affects the one 
only and sugar the other only, the substance of which we are speaking is able to affect both of them. In a somewhat similar way certain salts, magnesium sulphate for instance, when applied to the back of the tongue cxcite a bitterish taste, but when applied to the tip of the tongue excitc an acid or a sweetish acid taste.

We said a little while back that a weak intermupted current, so applied as to produce little or no electrolytic effect, was able to develope sensations of taste, varying in kind according to the region of the tongue stimulated. When the electrodes are applied to the tip of the tongue, the more usual result is that though an acid taste is the most prominent, a mixed gustatory sensation is dereloped, in which a sweet taste may be often recognized as a constituent. In like manner a bitter constituent may be recognized in the sensation developed when the electrodes are placed at the back of the tongue. If the tongue be previously subjected to the influence of Gymnema, the taste at the tip is free from all sweetness and that at the back free from all bitterness, the sensations which are then experienced being variously described as simply "metallic," or "salt," or" "acid." From this result we may draw the important inference that the interrupted current developes a bitter and a sweet taste by acting in some way or other directly on the specific terminal organs of the two respective tastes, very much in the same manner as do bitter and sweet things.

We have already said that when an acid, especially a somewhat strong acid, is placed on the tongue or in the mouth, the pure gustatory acid sensation is apt to be confused with the sensation of pungeney, the affection of general sensibility which the acid also brings about and which speedily merges into pain. These two sensations may be differentiated by means of cocaine. If the tongue be painted with a weak solution of eocaine, the general sensibility, the groundwork so to speak of pain, is abolished, while the pure gustatory sensations are at first hardly affected at all; a relatively strong acid which previously made the tongue "smart" so that real gustatory sensations were obseured, now developes a pure 'rich' acid taste alone. It is morenver said that cocaine applied to the tongue in increasing strength of solution abolishes the several classes of sensations in the following order: general sensibility and pain, bitter taste, sweet taste, salt taste, acid taste, tactile sensations.

Taking all these facts, and others which we might bring forward, into consideration, we are led to the conclusion that the development of the several kinds of gustatory sensations depends on the presence of specific terminal organs in the surfaces by means of which we taste. There appear to be distinct terminal organs for bitter tastes, for sweet tastes, for acid tastes, for salt tastes, and possibly for other tastes, all differing from 
the terminal organs for tactile sensations, and from the structures whatever they may be which are concerned in general sensibility. Further, we have a certain anount of evidence that these terminal organs are at least chiefly present in the fungiform and circumvallate papillae. By careful manipulation it is possible, uncler a lens, by means of a finely pointed brush to limit the application of a minute drop of a sapid liquid, such as syrup, solution of quinine and the like to a single papilla, and to appreciate the sensation thus caused before the material has had time to spreal by diffusion. When this is done, it is found that taste sensations are readily produced if the sapid substance be applied to a papilla, but not at all or less readily if it be applied between the papilla. Further, some papilla are found especially sensitive to sweet, or to bitter or to acid substances, or to two of these to the exclusion of the other. And somewhat similar results are obtained by a limited application of the electric current. Since the taste-buds are especially developed on these circumvallate and fungiform papiilse, we may infer that the name taste-bud has been wisely chosen. But the development of taste sensations, including bitter sensations, at the tip of the tongue, from which taste-buds are said to be absent, presents a difficulty. Unless we suppose that taste-buds, though often absent from the tip of the tongue, are present in those cases in which sensations are developed, we must conclude that gustatory sensations may originate by the help of some kind of nerve ending other than that of tastc-buds. It might be suggested that bitter and sweet tastes are devcloped by means of taste-buds and acid and salt tastes by means of other endings; but there is no satisfactory evidence of this.

$\$ 643$. The question which nerve or nerves subscrve taste and what is the course of the gustatory fibres is one which presents great difficulties. The front surface of the tongue is supplied by the lingual or gustatory branch of the fifth nerve, the hind surface by the glossopharyngeal nerve, which nerve also supplies the soft palate, though a branch (palatine) of the fifth nerve goes there also. 'The nerves traced to the taste-buds in the papilla foliatie and circumvallatie belong to the glossopharyngeal nerve, and it can hardly be doubted that gustatory fibres run in the branches of that nerve which go to the back of the tongue. On the other hand in the cases in which sensations are distinctly developed in the tip of the tongue we must infer that gustatory fibres run in the lingual branch of the fifth, since no glossopharyngeal fibres are distributed to this part of the tongue.

But it by no means follows from this that gustatory fibres pass straight both up the trunk of the glossopharyngeal nerve, and up the trunk of the fifth nerve to their respective nuclei in the bulb. 
On the one hand there is a good deal of evidence to shew a connection between sensations of taste and the chorda tympani nerve. Cases have occurred in which disease of the ear, involving destruction of the chorda tympani within the tympanum, has been followed by loss of taste in the tongue on the same side; and stimulation of the chorda tympani within the tympanum has been known to give rise to sensations of taste. Neither of these results is conclusive. The chorda tympani contains afferent fibres which have a remarkable effect on the nutritive processes of the tongue, and the loss of taste due to destruction of the chorda might be due to disordered nutrition of the tongue, and so be analogous to the loss of smell which may follow injury of the fifth nerve. Again, where stimulation of the chorda within the tympanum produces sensations of taste, these may be due to efferent impulses producing changes in the tongue, which in turn give rise to sensations of taste rcaching the brain by other channels than the chorda itself; we have no satisfactory evidence that direct stimulation of the central stump of a divided chorda will give rise to sensations of taste. The connection between the chorda and taste, however, may be of a more real kind.

On the other hand we must bear in mind how varied and complex are the junctions in the skull between the fifth nerve, the seventh nerve, and the glossopharyngeal nerve, by way of the Vidian nerve, the petrosal nerves, the tympanic plexus, Jacobson's nerve, and the otic and sphenopalatine ganglia. And it seems possible to suppose that fibres leaving the brain by the fifth nerve might find their way not directly to the lingual branch but by a roundabout way through the chorda tympani, and that at the same time other fibres from the same fifth nerve might ultimately join the glossopharyngeal nerve and reach the back of the tongue by that nerve. 'There are no cases on record in which disease of the glossopharyngeal nerve within the cranial cavity has led to distinct loss of taste; but cases have been recorded in which disease of the fifth nerve within the cranial eavity, and so far as could be ascertained limited to the fifth nerve, has led to an entire loss of taste over the whole of one side of the tongue, both back and tip. Such cases lead to the at least provisional conclusion that the gustatory fibres are fibres belonging to the fifth, though they may reach the tongue partly by way of the glossopharyngeal, partly by way of the chorda tympani. 


\section{CHAPTER VI.}

\section{ON CUTANEOUS AND SOME OTHER SENSATIONS.}

\section{SEC. 1. THE GENERAL FEATURES OF CUTANEOUS SENSATIONS.}

$\S 644$. THE sensations which we experience by means of the skin and cutaneous nerves appear, in the first instance, to be of at least three kinds. In the first place, all bodies, whatever their chemical or physical nature, be they gaseous, liquid or solid, when brought into contact with the skin, when made to exert mechanical pressure on the skin, produce sensations of a certain kind; these sensations, whose characters depend mainly on the amount of pressure exerted and on the region and area of the skin pressed upon, may be conveniently spoken of as tactile sensations or sensations of touch proper. In the second place, when either by actual contact with, or by the mere proximity of hot or cold bodies, of whatever nature, the temperature of an area of the skin is changed with sufficient rapidity, we experience sensations of a kind different from the tactile sensations just mentioned; these we may speak of as sensations of temperature, sensations of heat and cold. In the third place, when too violent a pressure is exerted on the skin, or when the changes of temperature are excessive, or when certain changes giving rise neither to tactile nor to temperate sensations are produced, or take place in the skin, we experience sensations which we call sensations of pain. This third kind of sensation stands, in many respects, apart from the other two, and it will be convenient to study sensations of pain by themselves. Sensations of touch proper and of heat and cold are much more akin and may be treated of together.

\section{Tactile Sensations or Sensations of Pressure.}

$\S 645$. Many of the characters of tactile sensations are of the same order as those of visual sensations, which we studied somewhat fully, and indeed similar characters may be more or 
less distinetly recognized in all sensations. The amount, that is to say the intensity of the sensation, valies with the amount of the stimulus, with the amount of pressure brought to bear on a given area of the skin. Taking the same spot of skin, the tip of the forefinger for instance, we can experimentally ascertain the minimum of pressure of which we can become conscious, such for example as that exerted by a minute fragment of some light body, pith or wool, falling through a certain small height. Starting from this minimum and increasing the pressure, we find the sensation also to increase up to a certain limit; and Weber's ław (\$5.50) holds good for tactile sensations, indeed may be more easily verified in their case than perhajs in the case of other sensations.

When two sensations follow each other in the same spot of skin at a sufficiently short interval they are fused into one; thus, if the finger be brought to benr lightly on the edge of a rotating card cut into a series of teeth, the teeth cease to be felt as such when they follow each other at a rapidity of about $1500 \mathrm{in}$ a second. The vibrations of a cord cease to be appreciable by touch wlien they reach the same rapidity.

When two sensations are generated at the same time at two points of the skin too close together they becone fused into one; but to this feature, which is of a different nature from the preceding, we shall return presently.

The sensation caused by pressure is at its maximum soon after its beginning, and thenceforward diminishes. The more suddenly the pressure is increased, the greater the sensation; and if the increase be sufficiently gradual, even very great pressure may be applied without giving rise to any sensation. A sensation in any spot is increased when the surrounding areas of skin are not subject to pressure at the same time. Thus if the finger be dipped into mercury the pressure of the mercury will be felt more at the surface of the fluid adjoining the skin which is not in contact with the mereury, than in the parts of the skin wholly covered with the mercury; and if the finger be drawn up and down, the sensation caused will be that of a ring moving along the finger. This effect may be compared with those of 'contrast' in visual sensations ( $\$ 583$ ).

All parts of the skin are not equally sensitive to pressure; the minimum of pressure which can be felt or the smallest difference of pressure which can be appreciated differs very much at different parts of the skin. Measured in this way, tactile sensations are much more acute on the palmar surface of the finger, or on the forehead, than on the arm or on the sole of the foot or on the back. In making these determinations all muscular movements should be avoided in order to eliminate the museular senses of which we shall speak later on; and the area stimulated should be as small and the contact as uniform as possible. 
In a similar manner small eonsecutive variations of pressure, as in counting a pulse, are more readily appreeiated by certain parts of the skin, such as the tip of the finger, than by other's. In all cases variations of pressure are more easily distinguished when they are suceessive than when they are simnltaneous.

$\$ 646$. The localization of tactile sensations. When anything touches a spot of our skin, we not only experience a pressure sensation of greater or less intensity according to the amount of pressure exerted and the particular region of skin pressed upon, we are also at the same time aware that the sensation has been started in that spot, that the spot in question and not another has been touched. When we are touched on the finger or on the back we refer the sensations to the finger or to the back respectively, and when we are touched at two places on the same finger at the same time we refer the sensations to two parts of the finger. We localize our touch sensations with reference to the surface of our body after the same fashion that we localize our visual sensations with reference to the external world. Our whole skin serves us as a 'field of touch' analogous to the 'visual field' of the eye: and as when experiencing a visual sensation, we refer it to its presumed canse and say we perceive a light in some part or other of the field of sight, so when we experience a tactile sensation we say we perceive that something has touched this or that part of our skin; the tactile sensation has become a tactile perception. As the accuracy of our visual perceptions is largely dependent on the smallness of the retinal interval which must separate two simultaneous retinal stimulations in order that these shall give rise to two separate sensations, vision being most distinct in the fovea centralis where this interval is smallest, so also the areuracy of our tactile pereeptions is dependent on the smallness of a like cutaneous interval. Where, as in the tip of the finger, the interval is small, contact with even a small area of surface may give rise to several simultaneous but distinct sensations, each of whieh we localize; and we thus obtain by means of one contact several perceptions affording a considerable amount of information concerning the nature of the surface. Where, as in the skin of the back, the interval is great, contact with even a large area of surface may give rise to one sensation, which we do not resolve into its components, all the several sensory impulses from the skin fusing into one common sensation: we only localize this one sensation, we have only one perception of something touching that part of our back, and the information which we thus acquire enncerning the nature of the surface in contact with the skin is limited.

As the above remark indicates, the interval in question varies very widely in different parts of the surface of the body ; our power of localization is mnch finer in certain parts than in 
others. Morcover the distribution of the fineness of localization is not identical with that of the mere appreciation of pressure; some parts may be very sensitive and yet possess imperfect localization. The magnitude of the interval of space which must separate two simultaneous stimulations of the skin in order that the two consequent sets of impulses may give rise to two distinct sensations may be conveniently determined for different regions of the skin by measuring the distance at which two points (preferably blunted) of a pair of compasses must be held apart, so that when the two points are in contact with the skin, the two consequent sensations can be localized with sufficient accuracy to be referred to two points of the body, and not confounded together as one.

The following tabular statement of results thus obtained may be taken as shewing in a general way the relative power of locali zation in the more important regions of the surface of the skin.

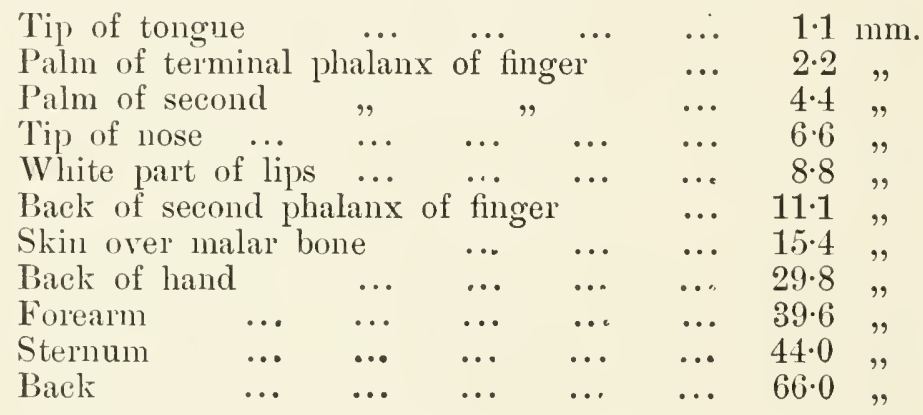

As a general rule it may be said that the more mobile parts, or those which execute the widest movements, or execute movements most easily and frequently, such as the hands and lips, are those by which we can thus discriminate sensations most readily. The lighter the pressure used to give rise to the sensations, provided that the impulses generated are adequate to excite distinetly appreciable sensations, the more easily are two sensations distinguished; thus two compass points which, when touching the skin lightly, appear as two, may, when firmly pressed, give rise to one sensation only. The distinction between the sensations is obscured by neighbouring sensations arising at the same time. Thus two points readily distinguished as two when the skin is under ordinary conditions, are confused into one when brought to bear inside a ring of heavy metal pressing on the skin.

It need hardly be said that these tactile perceptions, like all other perceptions, are increased by exercise. We may speak of our 'field of touch,' as being eomposed of tactile areas or units, in the same way that we spoke $(\$ 557)$ of our field of vision as 
being composed of visnal areas or units; but all that was there said concerning the subjective nature of the limits of visual areas, applies equally well, mutatis mutandis, to tactile areas. When two points of the compasses are felt as two distinct sensations, it is not necessary that two, and only two, nerve-fibres should be stimulated, or, putting the matter nore generally, that two or only two discrete sets of sensory impulses, should travel along separate paths to separate cerebral centres. All that is necessary is that the two cerebral sensation-areas should not be too completely fused together. The improvement by exercise of the sense of touch must be explained not by an increased development of the terminal orgains, not by a growth of new nervefibres in the skin, but by a more exact limitation of the sensational areas in the brain, as for example by the development of a resistance which limits the radiation taking place from the centres of the several areas.

\section{Sensations of Heat and Cold.}

$\S 647$. When we bring into contact with, or even into the immediate neighbourhood of a spot of skin, a body distinctly hotter than is the skin at the spot for the time being, we experience a special sensation; we feei something in the skin that was not there before, but that something is wholly unlike the effect of pressure, and we call the sensation a sensation of heat. The sensation is obviously due to the rise in the temperature of skin which is the direct effect of the contact with or the nearness of the hot body. Our skin has a certain temperature which varies from time to time, according to circumstances, and is not the same in all regions of the skin at the same time. A given spot of skin at a given time will have a certain temperature; that temperature does not give rise to a distinct sensation though its effects may enter into what we may call general sensibility; we may not be directly conscious, for instance, that the forehead has one temperature and the hand another, though the two temperatures may differ widely. It appears then that we are only conscions of a cutaneous sensation of heat when the temperature of a region of the skin which has previously been faily constant is raised; we may add suddenly raised, for in sensitions of heat as of pressure the stimnlus must act with a certain rapirlity in order to produce a distinct effect on consciousness.

If the body brought into contact with or near to the skin, instead of being distinctly hotter is distinctly colder than the skin we also experience a special sensation, a seusation of cold; and this sensation differs in kind not only from that of pressure, but also from that of heat. We might expect perhaps that since cold only differs from heat in degree, both being degrees of temperature, that the sensations of heat and cold would also 
be alike, differing only in degree; but when we appeal to our consciousncss we recognize that they differ in kind. So long as sensations of heat and cold remain sensations of heat and cold, they appear to us not as merely different phases of the same thing but as quite unlike : when the exciting heat or cold is excessive we perhaps may fail to distinguish between the two, but that is because both are lost in the sensation of pain. It appears then that we are conscious of a specific sensation of cold when the temperature of a region of the skin which has previously been fairly constant is with suffieient rapiclity lowered. 'To how large an extent we are, under ordinary circumstances, unconscious of the actual temperatiure of the skin and how sensitive we are to even slight changes of temperature may be illustrated by using one region of the skin as a stimulus of heat or cold for another. At a time, for instance, when we are not directly conscious of the hand being either colder or hotter than the forehead, by putting the one up to the other we may experience a distinct sensation telling us that the hand decidedly differs in temperature from the forehead; we feel at once that one is warmer or colder than the other, though it may take some little time to recognize which is the warmer or the colder.

$\$ 648$. These sensations of heat and eold behave very much in the same way as sensations of pressure. We have alrcady said that the change of temperature like the change of pressure must be effected with a certain rapidity in order to produce a distinct sensation, and in general the more gradual the change the less intense is the sensation.

As might be expected from the fact that it takes a longer time to produce a change of temperature than to cxert pressure, the sensation of either heat or cold is somewhat slowly developed and lasts some considerable time; hence consecutive sensations readily fuse into one.

Since it is the changed temperature and not the particular temperature arrived at which is the basis of the sensation, a hot body or a cold body gives rise to a sensation only at the first contact or approach and for some little time afterwards, the effect diminishing from the very moment that the change has been established. Hence a hot body or a cold body applied to the skin, even when kept itself at a constant temperature and not cooled or heated by contact with the cooler or warmer skin, ceases after a while to be felt as hot or cold. For this reason the repeated dipping of the hand into hot or cold water produces a greater sensation than when the hand is allowed to remain all the time in the water, though in the latter case the temperature of the skin is most affected.

The effects of eontrast are obvious in scnsations of heat and cold as in those of pressure; when the hand is dipped in hot water the sensation is most intense at the ring where the hand emerges from the surface of the water. 
We can with some accuracy distinguish small differences of temperature, especially those lying near the normal temperature of the skin. In this respect these sensations follow Weber's law, though apparently slight differences of cold are more easily recognized than those of slight heat. The range of the greatest sensitiveness seems to lie between $27^{\circ}$ and $33^{\circ}$.

The regions of the skin most sensitive to variations in temperature are not identical with those most sensitive to variations in pressure. Thus the cheeks, eyelids, temples and lips, are more sensitive than the hands. The least sensitive parts are the legs, and front and back of the trunk; to this matter however we shall return.

As with pressure sensations so also with sensations of heat and cold, two sensations excited at a certain distance apart may or may not be fused into one, the distance necessary for the separation of the sensations varying in different regions of the body, and being, as might be expected from the ease with which heat and cold are conducted, much greater than in the case of pressure sensations. We also 'localize' the sensations of heat and cold; we can recognize which region of the skin is being heated or cooled ; and thus these sensations also enter into our perceptions of the external world.

$\$ 649$. We have treated of the sensations of touch and of heat and cold as cutaneous sensations; but they are not confined to the skin commonly so called. We experience the same sensations in varying degree by help of the lining of the mouth and pharynx, which is called a mucous membrane; and they may also be traced for a short distance up the rectum beyond the margin of the skin proper. But in both these situations, the lining membrane is by origin and in structure epiblastic. that is to say cutaneous, and in possessing cutaneous functions shews its real nature. These functions are most marked at the beginning of the passages, the tip of the tongue being very sensitive to touch and heat and cold, with a well-developed power of localization; they are very rapidly lost in the rectum. and more gradually disappear at the lower part of the pharynx and in the esophagus; a fluid which in the mouth is felt distinctly as hot gives rise to a sensation of pain not of heat when it is swallowed, and a cold or warm drink is only felt as cold or warm when swallowed in quantity sufficient to affect by conduction the abdominal skin. The maintenance of these eutaneous functions in the initial parts of the alimentary canal, which are under the dominion of the will, is, like the sense of taste, a safeguard against the introduction into the canal of noxious substances; in the subsequent parts, no longer subject to the will, any warning which such sensations might give would be too late and useless. 


\section{SEC. 2. ON PAINFUL AND SOME OTHER KINDS OF SENSATION.}

$\S 650$. When excessive pressure is exerted on the skin, or when the change of temperature passes certain limits, the sensation which is excited ceases to be recognized as one either of touch or of temperature and takes on characters of its own; we then call it a sensation of pain. In this respect the skin as a sensory organ aplears at first sight to differ from the other organs of sense which we have studied. We have no evidence that simple stimulation of the retina, however excessive, will give rise to pain, meaning by pain the kind of sensation we feel when the skin is ent or burnt. We often speak it is true, especially in cases of disease of the eye, of exposure to light causing pain, but the pain in such cases is felt through the eyeball, not through the retina and optic nerve; the pain is not an excessive development of visual sensations, it is a phase of that sensibility which the subsidiary structures of the eye share, in common as we shall see presently, with not only the skin but nearly all other structures of the body. In like mamner we have no evidence that an auditory or an olfactory or a gustatory sensation can, through mere intensity, become converted into a sensation of pain, though we may, in the act of hearing, smelling or tasting, receive sensations of pain from the ear, nose or mouth. We often of course apply the word 'painful' to a sound, or a gromp of visual sensations, or a smell or a taste; but that is in the sense of being exceedingly disagreeable, and has reference to our classification of the complex psychical effects of all our sensations into those which are pleasurable and those which are painful. Without entering into any psychological analysis, we may assume that the pain which we feel when the finger is cut is a wholly different thing from the pain which is given to a most delicately musical ear by even the most horrible discord; and in what follows we shall use the word pain in the first of these two meanings.

$\$ 651$. The above considerations suggest that in the case of the skin as in the cases of the other organs of special sense, a 
sensation of pain is not simply an exaggeration of a sensation of pressure or of a sensation of temperature, but is a separate sensation, developed in a different way in the skin, a sensation which may overide and so seen to replace the semsation of pressure or temperature developed at the same time, but which must not be confounded with it. And this view derives support from the fact that events taking place in many other parts of the body, from which we experience sensations neither of touch nor of temperature, may under favomrable circumstances give rise to pain in varying degree. When, for instance. a tendon is laid bare contact with a body will not produce tactile sensations, heating or cooling will not produce temperatne sensations; one cannot by means of the tendon as one can by means of the skin perceive that a rough or smooth body, that a hot or cold body, has been brought to act upon it. Indeed in respect to all structures other than the skin and nerves, to such structures namely as muscles, tendons, ligaments, bones, and the viscera generally, there is a large amount of experinental and clinical evidence shewing that, so long as these are in a normal condition, experimental stimulation of them does not give lise to any distinct change of consciousness; a muscle or a tendon, the intestine, the liver or the heart may be handled. pinched, cut or cauterized without any pain or indeed any sensation at all being felt or any signs given of consciousness being affected. Nevertheless when the parts are in an abnormal condition even slight stimulation may produce a very marked effect on consciousness. If, for instance, a tendon becomes inflamed, any movement causing a change in the tendon, especially one putting the tendon on the stretch, will affect consciousness and give rise to a sensation. But the sensation is one of pain and not of any other kind. Moreover we simply 'feel' the pain, we do not 'perceive' the cause of it; because we feel the pain we infer that something has caused it, but we cannot from the nature of the pain itself decide whether that something is a stretching of the tendon, the contact of a hard or soft body, the approach of some hot or cold body, the application of some chemical substance, the passage of an electric current, or intrinsic events taking place in the tendon itself as the result of physiological changes. And so in other instances; there is hardly a part of the body changes in which may not, under certain circumstances, give rise to sensations of pain. We can to a variable extent, in a more or less ill-defined manner, localize the sensation; we can distinguish a pain in the foot from one in the leg, a pain in the thrumb from one in a finger; we may occasionally fix the pain in a very small limited area, though especially if the sensation be intense, the pain radiates and its localization becomes obscure. And we may here remark that when we thus localize a pain arising in the structures of which we are speaking, we refer the pain not 
to the structures themselves but to neighbouring parts and especially to the skin; the intense pain, for instance, of "renal colic," cansed hy the impact of a calculus in the ureter is referred by us not to the ureter itself but to adjoining parts, to the corresponding somatic segment; and so in other instances. We can also recognize certain characters in different pains, beyond that of the mere degree of intensity; we speak of pains as being burning, aching, gnawing, cutting, throbbing and the like. But in all cases the pain remains a mere sensation; when it comes, all we can say is that we feel in a particular region of the body a pain of a certain intensity and having a certain character. We infer that something is wrong, but the pain in no way tells us what the wrong is; we may call the pain a burning one because it is more or less like the pain which we feel when the skin is burnt; but in the vast majority of cases heat has nothing whatever to do with pains of a burning character; and so with other kinds of pain, the character of the pain does not in itself tell us anything about its cause.

Are we then to regard pain as a sensation of a kind by itself, the very threshold of which, the very least amount of which that can in any way affect our consciousness, must be regarded as already pain? In attempting to answer this question the following considerations deserve attention.

We are in a certain obscure way aware of what we may call the general condition of our body. To put an extreme case, if the whole of our abdominal viscera were removed we should be aware of the loss. We should be aware of this through more ways than one. The tactile sensations from the abdominal skin would be in such a case different from the normal, and moreover the muscular sense of the abdominal walls and of all the muscles whose actions bear on the abdomen, would make us aware of the void. But beyond all these indirect ways, it is probable that we should in a more or less obscure manner be directly conscious of the loss. It is probable that sensory impulses, not of the character of pain, are continually, or from time to time, passing upwards from the abdominal viscera to the central nervous system. 'These do not affect our consciousness in such a distinet manner as to enable us to examine them psychologically in the same way that we are able to examine special sensations such as those of sight, or even sensations of pain; they are even less well defined than those of the muscular sense; nevertheless they do enter, though obscurely, into our consciousness, so that we become aware of any great change in them, and they have been spoken of under the title of "common" or "general sensibility." In discussing the manner in which the manifold coordinate movements of the body were carried out we saw reasons for thinking that the central processes of the nervous system were largely determined by varied afferent impulses which 
produced their effects without giving rise to any sharp and decided change of consciousness; many of these are probably afferent impulses of the common sensibility of which we are now speaking.

If we suppose that the skin in common with the other tissues of the body possesses this common sensibility, and if we further suppose that in the skin as elsewhere, these afferent impulses when developed, as is the case under normal circumstances, to a slight extent only are not distinctly recognized by consciousness, and that when they do assume such a magnitude or intensity as to break in upon consciousness the change of consciousness which they produce is of the kind which we call pain, we reach a conclusion which is also supported by other considerations. On the one hand such a view is in accord with the conclusion that cutaneous sensations of pain are wholly distinet from and developed in a wholly different way from sensations of touch and temperature; and, as we shall see, to this conclusion we are led by several different arguments. On the other hand it relicves us from the following difficulty. It may happen to a man to suffer pain in a particular region or tissue of the body, once only in the course of his lifetime or possibly not even once; nay, we may suppose that in this or that region or tissue pain is felt once only in one individual among a large number of persons. If we suppose that pain is not as suggested above an excessive phase of something which is continually going on in a lower phase, but a something by itself quite distinct from all other sensations, we are driven to conchude, since such a sensation must have a special mechanism, including special afferent nerve fibres to carry it out, that in the case in question such a mechanism of pain has been preserved intact but unused through whole generations in order that it may once in a while come into use; which is in the highest degree improbable. This difficulty disappears if we suppose that the constantly smouldering embers of common sensibility may be at any moment fanned into the flame of pain.

We may conclude then that the skin in common with other tissues possesses common sensibility, and that when this is excited in excess, so as to distinctly affect consciousness, we call it pain. We thus experience through the skin three kinds of sensations, those of touch, of temperature, and of common sensibility, but the two former only are developed by further psychical processes into perceptions; it is by them alone that we obtain through the skin knowledge of external objects.

$\$$ 652. There is another consideration to be taken into view. The agents which applied to the skin produce pain, act violently on the skin, in many eases injuring the epidermis and affecting the dermis. Moreover if the epidermis be removed, and the stimulus, mechanical, thermal or chemical, be applied to the 
dermis or to the nerves ruming in it, we still cxperience sensations of pain, though no longer those of touch and temperaturc; when a sharp or hot body is made to touch, not the intact skin but a wound, we suffer pain, but do not recognize the sharpness or the heat which is causing the pain. This suggests that the special sensations of touch and temperature are brought about by special, epithelial structures serving as the differentiated ends of nerve fibres, but that common sensibility and pain need no such special endings; this however opens up questions which we must consider separately by themselves.

\$ 653. Hunger and thirst. We may introduce here the few words that we have to say concerning two affections of consciousness, which may perhaps be considered as kinds of sensation, namely, hunger and thirst.

We refer our feelings of thirst to, or at least we associate them with, a particular condition of the mucous membrane of the mouth, especially of the soft palate. When the mucous membrane of this region becomes drier than normal, as for instance by being exposed to too great an evaporation, we feel 'thirsty,' and the feeling is at once removed by adequatcly moistening the membrane. Under ordinary circumstances however the condition of thirst is brought about, not by anything bearing specially or exclusively on the mucous membrane of the soft palate or even of the whole mouth, but by the diminution of the water present in the body either through restriction of the intake, or through excess of the output in the secretions, such as that of sweat, or through both together. This is often spoken of as diminution of the water of the blood; but most probably the specific gravity of the blood is kept constant by the withdrawal of water from the lymph, so that the loss falls on the latter fluid. Such a diminution of the water of the bolly may be brought about by circumstances such as excessive sweating which in themselves do not cause special dryness of the mucous membrane of the soft palate; this part then undergoes a loss of water in common with the other tissues, but not in a special degree. Nevertheless thirst thus brought about may be temporarily assuaged by simple moistening of the soft palate. From this we may infer that the sensation of thirst is brought about by afferent sensory impulses started in the mucous membrane of the soft palate by a deficiency of water in that membrane, perhaps by a drain on the lymph spaces of that membrane.

We are in the habit of assuaging thirst by drinking water, or watery fluids, and in doing so produce both a direct local effect on the palate and a general indirect effect on the body. In the absence of the local effect, the indirect effect is slow in coming and needs a large quantity of thuid; when in cases of gastric 
fistula water is introduced into the stomach through the fistulous opening, large quantities may be given before thirst is assuaged.

The sensation of lunger is in a somewhat similar mamer referred to, or associated with, the condition of the gastric mucous membrane. We feel hungry when the stomach is empty. But even more distinctly than in the ease of thirst the main cause of the sensation seems to be a general condition of the body, namely, that produced by the products of digestion ceasing to be thrown into the blood. The sensation is not due to the mere emptiness of the stomach, though the emptiness of the stomach is one of the results of the abstinence from food, for the feeling of humger may disappear though the stomach may remain empty, if adequate nourishment be conveyed in other ways, as by injection into the bowels; conversely even we ourselves may under abnormal conditions feel hungry on a full stomach, and in some animals, herbivora, the stomach is always more or less full. The sensation however does seem to be in some way specially commected with the condition of the gastric walls, much in the same way that thirst is specially comnected with the palate; the products of digestion have a much greater power in appeasing hunger when they act locally and directly on the gastric membrane than when they are simply brought to bear on the body at large, and a small quantity of food will immediately satisfy hunger when introduced into the stomach, though it will have no effect when introduced otherwise. Moreover our own consciousness clearly connects the sensation in some way or other with the stomach.

As to what is the particular change in the gastric membrane which thus gives rise or assists in giving rise to the sensation we know little or nothing; indigestible substances such as cannot be properly called food when taken into the stomach at least temporarily remove the sensation. And we have little or no knowledge as to the particular nerves which serve as the patlis for the afferent impulses which we may suppose to be generated in the gastric membrane. Division of the vagus nerve on both sides is said to have no effect on hunger, from this we may conclude that the impulses do not pass up this nerve, though it appears to be the sensory nerve of the stomach. But we have no evidence that the impulses pass along the sympathetic nerves.

Allied somewhat to hunger is the peculiar feeling which we may perhaps also speak of as a sensation, known as nausca, the precursor of vomiting and brought about like vomiting by a variety of events. We have little or no knowledge of it viewed as a sensation.

The affection of consciousness which is produced by the form of cutaneous stimulation known as "tickling" is of a peculiar character, differing from tactile scnsations. Indeed it is probably undesirable to speak of it or of other like psychical effects 
of cutaneous stimulation as a sensation, since it seems to be not the direct effect of the sensory cutaneous impulses, which are probably ordinary tactile impulses, but rather the effect on consciousness of changes in the central nervous system brought about by those sensory impulses. 


\section{SEC. 3. ON THE MODE OF DEVELOPMENT OF CUTANEOUS SENSATIONS.}

$\S 654$. Our studies so far point to the conclusion that sensations of touch and temperature stand on the same footing as visual, auditory and other special sensations, and it will be profitable now to compare in some detail the former with the latter. In doing so we may, in order to make the matter more simple, confine ourselves in the first instance to sensations of touch proper, that is to sensations of mere contact and pressure, discussing later on the relations of these to sensations of heat and cold.

In studying vision we came to the conclusion that the undulations of the ether so affect the rods and cones and other retinal structures as to give rise to visual impulses, and that these visual impulses, travelling up the fibres of the optic nerve to the visual centres, gave rise by means of those centres to the affections of consciousness which we call visual sensations; we may leave aside in the present instance all reference to the complexity of the visual centres.

We obtained absolute proof that the only way in which light can give rise to visual impulses in the optic fibres is by acting on the retinal structures. Since the optic fibres are the only nerve fibres in direct connection with the retinal structures visual impulses can be carried by them alone. As we pointed out we know absolutely nothing about the nature of visual impulses themselves; our conclusions concerning the various characters and kinds of visual impulses are simply deductions from the psychological examination of our sensations; our objective knowledge of them is limited to the fact that when light falls on a functionally active retina an electric change is developed in the optic fibres. As we mentioned in $\$ 553$ the statement that stimulation of the optic fibres themselves, as when the optic nerve is cut with a knife, gives rise to visual sensations, has led to the adoption of the view that any impulse passing along the optic fibres, however started, whether by the action of light on the retina, or by direct stimulation of the fibres themselves, gives rise 
to a visual sensation and must therefore be regarded as a visual impulse. This view, under the title of the doctrine of " the specific energy of nerves," has been extended to the nerves of the other special senses and indeed to nerves in general. This doetrine teaches that, owing either to the constitution of the central ending of a sensory fibre or to that combined with the nature of the fibre itself (the view may also be adapted to motor fibres), whatever impulses are generated in the fibre ean give rise to those events only which are specifie to that eentral ending, impulses of all kinds along an optic fibre giving rise to visual sensations, impulses of all kinds along an auditory fibre giving rise to auditory sensations, and so on. Hence under this view the purpose of the specific terminal organ is simply to allow the specific stimulus of the sense. light in the case of the retina, to develope impulses in the speeific nerve, a result which, in the absence of the terminal organ, it is powerless to athieve. We saw however (\$553) that according to some observers direct stimulation of the optic fibres, as when the nerve is cut, does not produce visual sensations, and therefore does not give rise to visual impulses; so far as ean be ascertained such a stimulation of the fibres appears to produce no effeet at all on the central nervous system. In arguing from this result we must remember that the optic nerve is not a true nerve and that its fibres are not comparable with the fibres of a true nerve; the optic nerve is a part of the brain, and its fibres are analogous to the internuncial fibres of the white matter of the central nervous system, concerning which as we saw ( $\$ 510$ ) the view has been urged that they are not capable of being stimulated directly. Neglecting however this view which is at best very doubtful, we are led, if we accept the insensitiveness of the optic nerve to direct stimulation as true, to modify the doctrine of the specific energy of nerves in the following way. We must suppose that the visual centres are so constituted that they are stirled up to the development of visual sensations by the advent only of those kind of impulses which are started by means of the terminal organ. Since electric changes are developed in the optic fibres as in other nerve fibres when the optic fibres are directly stimulated, we may infer that direct stimulation does lead to nervous impulses; and we may further infer that these reach the visual centres but are unable to develope visual sensations because they are not true visual impulses such as are generated by help of the terminal organs.

The facts which we mentioned in speaking of hearing ( $\$ 628$ ) as seeming to shew that the fibres of the auditory nerve in the absence of the labyrinth ma be directly stimulated by sound, if we accept them as valid, afford a strong support to the simpler conception of the specific energy of nerve fibres. On the other hand the modified view is supported, though the support is of a negaive kind only, by the bchaviour of the other organs of 
special sense. We have no satisfactory experimental or other evidence that stimulation of the olfactory fibres otherwise than through the terminal organs will give rise to olfactory sensations. We have evidence that stimulation of the centres by various means will give rise to the specifie sensations, but not that stimulation of the fibres of the nerves themselves will. The branches of the glossopharyngeal and fifth nerves distributed to the organs of taste are, unlike the above, mixed nerves, and when they are stimulated sensations other than specific taste sensations are also developed, and the former might obscure the latter; still the evidence so far as it goes supports the view that stimulation of gustatory fibres otherwise than through their terminal organs does not lead to the development of gustatory sensations. In the case of touch the evidence is perhaps still stronger. TVe must in any case suppose that each cutaneous nerve distributed to a given area of skin contains fibres which subserve the sense of touch exercised by that area, and which pass from the terminal organs in that area, whatever their nature, to the parts of the central nervous system, whatever they may be ( $\$ 505)$, which act as centres of touch sensations. If these fibres when directly stimulated, apart from their terminal organs, necessarily give rise to touch sensations, stinulation of the nerve itself while running in the subeutaneous tissue should give rise to touch sensations. But experience shews, as we said a little while ago, that this is not the case. Whenever the nerve fibres themselves are directly stimulated, as for instance when the epidermis is removed from the skin or when a nerve is laid bare, then however they be stimulated, be the stimulus weak or strong, if consciousness be affected at all, the affection takes on the form of pain; psyehological examination of the subjective result discloses nothing that can be called a sensation of touch. A familiar instance of the difference between the effects of stimulating a nerve trunk, and those of stimulating the cutaneous terminal organs of special sense, is seen in the effect of dipping the elbow into a freezing mixture. The cold affects the skin of the elbow and gives rise to sensations of cold in that part; but the cold, if intense enough, also affects the underlying trunk of the ulnar nerve, and by direct stimulation of the fibres in the trunk developes sensory impulses ; these impulses however are those not of sensations of cold, but of pain; and the pain, in accordance with a principle to which we shall presently call attention, is referred to the terminal distribution of the ulnar nerve on the ulnar side of the hand and arm. In speaking above (\$ 651) of pain we said that excessive pressure or excessive heat or excessive cold applied to the skin, overrides or annuls pressure and temperature sensations and gives rise to mere sensations of pain; and it might be urged that when a nerve is directly stinulated the specific sensations of touch and temperature are similarly annullerl. But in the 
case of the skin an excessive or violent stimulation is necessary to produce this effect, whereas a nerve may be directly stimulated by so slight a stimulus as to give rise to hardly more than discomfort without distinct pressure or temperature sensations being felt; and we can hardly suppose that in such a case these are present but are annulled by an amount of pain so slight as that which is produced. 'Thus making every allowance for the suggestion that sensations of pain may override and obscure concomitant sensations of touch and temperature, we seem driven to the conclusion that the latter sensations ean only be developed by lielp of special terminal organs, and that a stimulation of the nerve fibres themselves if it produces any effect at all on conscionsness gives rise to pain, and to pain alone.

We are in this way led to conceive of the skin as provided on the one hand with specific fibres ending in specific terminal organs and serving for sensations of touch and temperature, and on the other hand with fibres of common sensibility having no such specific terminal organs, the two kinds of fibres being mixed together in the common cutaneous nerve. These fibres moreover have not only different peripheral but also different central endings, and during at least some part of their course run in different tracts or in a different manner in the central nervous system; for as we saw in treating of the central nervous system ( $\$ 508)$ cases of disease of the central nervous system have been recorded in which over certain cutaneous areas sensations of touch had been lost, while common sensibility and sensations of pain remained, or vice versa. IVe may add that the difference between the central paths or endings of the nerves of touch and those of pain is further shewn by the fact that in certain nervous diseases (tabes) when the skin is pricked with a pin, the contact of the pin may be felt as mere touch for so long a time as one or two seconds before pain is felt; the diseased condition enormously delays the transmission of the impulses of pain but has not so much effect on those of touch.

$\S 655$. We may go a step further; there is a certain amount of cvidence that the terminal organs and fibres concerned in touch proper, in sensations of pressure, are different and separate from those concerned in sensations of heat and cold. In the first place the general topographical distribution over the surface of the body of sensitiveness to pressure is different from that of sensitiveness to temperature. A familiar instance of this is seen in bringing the palm of the hand to touch the forehead. In the former the sense of tonch is highly developed, in the latter the sense of temperature; hence with the forehear we feel that the hand is warm or cold, with the hand we feel that the forehcad is rough or smooth; at least these two feelings respectively preponderate, the one in the one part, the other in the other. In the second place, if the stimulation of the 
skin be confined to extremely minute areas, if the pressure, or the change of temperature be brought to bear as much as possible on a mere point of the skin, it is found that some points of the skin are sensitive to pressure but not to ehange of temperature, while others again are sensitive to change of temperature but not to pressure. If a blunt pointed but otherwise fine needle be used to exert pressure, a little exploration will ascertain that at some points the amount of pressure tan readily be recognized, the sense of touch is acute, while at other points, and these may be quite near the others, the amount of pressure cannot be recognized, and indeed no sensation is experienced until the pressure is excessive and then the sensation felt is not one of touch proper but of pain. Similarly if heat or cold be applied by means of a metal tube or rod narrowed to a fine point, it will be found that some points of the skin are very sensitive to changes of temperature, while other points are insensitive to temperature, the applieation of heat or cold giving rise to pain only and not to specific sensations of heat or cold. Further, the points of the skin which are sensitive to pressure are those which are not sensitive to heat or cold, and vice versa. Such results as these are only intelligible on the supposition that the terminal organs for pressure are different from those for heat and cold and differently distributed over the surface of the skin.

$\$ 656$. The punctiform method of exploring the sensitiveness of the skin has further led to a result which is unexpected and indeed presents difficulties. Heat and cold in themselves differ only in degree; they are positive and negative phases of the same thing. We should therefore naturally expect that the same terminal organs would be employed for sensations both of heat and of cold, and that the same points of the skin would be alike sensitive both to heat and to cold. But the results of experimentation by the method in question contradict this expectation. It is found that some points are sensitive to heat, that is to say a sensation is developed when the temperature of the point of the skin is raised above what it happens to be at the time of experimenting, but are not sensitive to cold, that is to say no sensations are developed when the temperature of the point of the skin is lowered below what it happens to be at the time of experimenting; and other points may similarly be found to be sensitive to cold but not to heat. Moreover this result is in accord with results gained otherwise. If the arm or leg be "sent to sleep" by pressure on the brachial or sciatic nerves the skin will be found at a certain stage to be little sensitive to warmth though distinctly sensitive to cold. So also the whole surface of the glans penis, in eontrast to the prepuce, is very slightly sensitive to cold, but distinctly sensitive to warmth. Moreover cases of disease of the central nervous system have been recorded 
in which the skin of a limb was sensitive to warmth, that is to degrees of temperature above that of the limb, but insensitive to cold. It may be remarked that in these cases, as in that of the limb "gone to sleep," the scnsations of touch proper and of cold seem to run together and sensations of pain and of heat also to run together.

It seems probable then from these considerations that we possess three sets of terminal organs and three sets of fibres, one for pressure, a second for heat and a third for cold. It must be borne in mind however that the three sensations are not wholly independent, since sensations of pressure are modifier if changes in temperature be taking place at the same time in the same spot of skin. Thus a penny cooled down nearly to zero and placed on the forehead will be judged by most people to be as heavy or even heavier than two pennies of the temperature of the forehead itself, that is to say the sensation of pressure is increased by a concomitant sensation of cold ; and a similar modification of the sensation of pressure is also often observed when the object pressing is not colder but warmer than the skin pressed on. A similar effect seems to be shewn in certain cases of disease of the central nervous system in which it has been recorded that a hot body such as a heated spoon was felt when brought in contact with the skin, though the same spoon applied at the temperature of the skin itself produced no sensation at all, and the heated spoon was recognized not as a hot body, but simply as something touching the skin. The exact explanation of these facts is not very clear, but it may perhaps be argned that the effect is brought about amid the central processes through which the sensations are developed and does not shew that the sensations have common terminal organs.

$\$ 65 \%$. In attempting to understand the nature of the peripheral events through which the sensory impulses giving rise to sensations of pressure of heat and of cold are developed two or or three matters must be borne in mind. In the first place, as we have alrearly said, though the skin has a temperature of its own, we are not directly conscious of that, or at all events are not distinctly conscious of it in the same way that we become conscious of any sudden change in that temperature; nor indeed are we, except in extreme cases, distinctly conscious that the temperature of one region differs from that of another, or that the temperature of the same region gradually varies from time to time. It would seem as if the development of a clear and distinct sensation was largely dependent on the contrast as to temperature between an area of the skin and surrounding areas; and indeed we have already pointed out the marked effects of tontrast. The same applies to pressure; we are not, at least distinctly and directly, eonseious of the uniform pressure of the atmosphere over the whole surface of the body, when we stand 
naked in still air. We are not however justified in assuming that under the above circumstances nothing whatever is taking place in the sensory nerves of the skin, that when we feel a sensation the change in the sensory apparatus (using that phrase in its widest sense to include both peripheral and central parts) is one from absolute quiescence to activity; it is not impossible, and some facts indeed seem to suggest, that even when we feel no distinct cutaneous sensations, afferent impulses still continue to stream onwards from the periphery to the central nervous system, supplying as it were a groundwork of nervous events which enter largely in various ways into the conduct of the whole body, but which do not distinetly affect consciousness. If this be so, we may infer that the affection of consciousness which we call a sensation is the immediate effect of an adequately large change in this groundwork, rather than of a set of quite new isolated impulses passing straight up from the peripheral organ to the "seat of conscionsness."

In the second place when we do experience sensations of temperature the sensation is caused not by the mere change of temperature but by the altered condition of the skin which results from that change. When an area of the skin having a normal temperature is brought in contact with a cold body, the skin undergoes a change from a normal to a lower temperature, and we experience a sensation of cold. Now, if it were only the change from a normal to a lower temperature which gave rise to the sensation, though the sensation might and probably would last much longer than the change itself, it could not be prolonged by the mere maintenance of the lower temperature when once the change had been established. But experience shews that it is: we still feel a sensation of cold, at a time when the contact of the cold body is not producing any further lowering of temperature and at most is only maintaining the lower temperature already brought about. Nay, more, the sensation of cold continues after the cooling body has been removed, at the time when the skin is returning to its normal temperature, that is to say is undergoing the very opposite change of temperature, namely, one from cold to heat. And the same considerations apply to sensations of heat.

$\$ 658$. We may conclude then that when the application of cold or of heat to the skin causes a sensation of cold, the cold or heat produces a condition in the material of the skin, which condition starts nervous impulses in the afferent nerves of cold and heat sensations. Since the application of cold or of heat to the nerve fibres underlying the skin does not produce a sensation of cold or heat, but only a sensation of pain, we may further conclude that the material whose condition starts the sensation is placed in the skin itself, in the epidermis or in the immediately underlying dermis. Since we experience sensations of 
cold and heat in regions of the skin, not only free from touch corpuscles but also free from any dermic terminal organs as yet known, the "points" of the skin determined experimentally to be points of cold and heat sensations, having been repeatedly found when extirpated to be free from all such dermic organs, we may, though with less certainty, still further infer that the material exists somewhere in the epidermis. We may add that sensations of temperature may be felt in the cornea, from which all dermic terminal organs seem certainly to be absent. And our knowledge that the nerve fibres end as fine fibrillæ between and among the cells of the Malpighian layer brings us to the final conclusion that the material of which we are speaking is to be sought for either in the fine nerve fibrillæ themselves, or, as seems more likely, in some or other of the cells of the Malpighian layer specially connected with those fibrillæ.

Beyond this we camnot go ; and even admitting thus much, it is difficult to understand how, if the change be one from a higher to a lower temperature, the lower temperature, whatever mily have been the exact degree of the higher temperature, should in giving rise to sensations of cold affect one set of fibres only, or how the higher temperature should similarly affect another set of fibres only; but we must leave the matter here.

'The considerations which have just been brought forward in relation to sensations of heat and cold, may also be applied to sensations of pressure; with regard to them also we are driven to the conclusion that they take origin in the lower layer of the epidermis through sone condition brought about by the pressure. We can appreciate pressure by the cornea, from which as we have said dermic organs are absent. If the "points of skin' in various parts of the body, determined experimentally to be points of pressure sensation, be extirpated and examined it is found that dermic organs are not necessarily present; indeed such points of pressure sensations do not differ essentially in structure from points of heat or cold sensations, though some slight difference in the manner of distribution of the dermic nerve filaments has been described.

We are thus brought to the conclusion that the so-called touch corpuscles are in no way essential to touch. At the same time their remarkable prominenee in those parts of the skin in which touch is most sensitive would seem to shew that, even if not neceswary, they are in some way adjuvant to pressure sensations. But what that aid may be is at present a mere matter. of speculation; and we are perhaps still more in the dark as to functions of the end-bulbs and of the Pacinian bodies. 


\section{SEC. 4. THE MUSCULAR SENSE.}

$\S 659$. Before we go on to deal with some of the psychical aspects of cutaneous sensations it will be desirable to speak of certain sensations accompanying and belonging to the movements of the body which are carried out by means of the skeletal muscles; for these sensations, often spoken of as constituting a "muscular sense," are in many ways related to or mixed up witl cutaneous sensations.

When we examine our own consciousness we find that we are aware of the position not only of the whole body (this matter we discnssed some time back), but also of the several parts of our body. In this we are under ordinary circumstances assisted by sight; but sight is not necessary. If for instance, with the eyes shut, we place the arm in any attitude, we are aware of the attitucle and can describe, or by movements of the other arm initate with considerable accuracy the details of the attitude, the relative positions of the upper arm, forearm, hand, finger's and the like. If we change the attitude by moving the arm or part of the arm we can, though the eyes be still shut, tell the amount and characters of the change.

Again, when we examine our own consciousness we find that we possess a measure of the amount of resistance to our movements which we from time to time meet with. When we come into contact with an external object we are conscious not only of the pressure exerted by the object on our skin, but also of the pressure which we exert on the object; we can appreciate the amount of effort which we make to produce by pressure an effect upon the object. A similar appreciation of our own efforts assists us largely in forming a judgment as to the weight of an object. If we place the hand and arm flat on a table, we can estimate the pressure exerted by a body resting on the paln of the hand, and so come to a conclusion as to its weight; in this case we are conscious only of the pressure exerted by the body on our skin. If however we hold the body in the hand, we not only feel the pressure of the body, but we are also aware of the exertion required to support and lift it. And we find by experience that when we trust to this appreciation of the amount 
of effort needed to lift an object as well as to sensations of pressure. we call form much more accurate judgments concerning the weight of the object than when we rely on sensations of pressure alone. When we want to tell how heavy a thing is, we are not in the habit of allowing it simply to press on the hand laid flat on a table or otherwise at rest; we hold it in our hand and lift it np and down.

The above instances deal with three things which it might be desirablc to keep separate, namely, " position," "movement" and 'effort;" it might seem desirable to speak of " a sense of position," "a sense of movement," and "a sense of effort." But, if we leave out of consideration the problems connected with our appreciation of the position of the head, which as we have seen seems especially dependent on afferent impulses passing up the auditory (vestibular) nerve, we may say that the position of the varions parts of our body is so closely dependent on movement, that is on the contraction of skeletal muscles, some muscle or other playing its part in almost every position and every change of position, that in the discussion on which we are now entering it will be hardly profitable to distinguish between the two; and we may use the term " muscular sense" to denote our appreciation both of movement and of position resulting from movement.

$\$ 660$. There are more valid reasons for distinguishing between our appreciation of an effort and our appreciation of the movement which is the result of that effort. For the view has bcen put forward and supported by argument that when we make a muscular effort, we are directly conscious of the nervous processes of the central nervous system underlying the effort, that the changes in the central nervous system involved in initiating and executing a movement of the body so affect our consciousness that we have a sense of the nervous effort itself, of the innervation as it has been called; and it is urged that the condition of the central nervous system through which we appreciate the nature and magnitude of the effort is thus the direet effect of central changes, and not the outcome of afferent impulses proceeding from the part moved.

Whether it be the case or not that consciousness is thus directly affected by changes in the central nervous system, such for instance as those taking place in the motor cortical area or in the pyramidal tract, the evidence goes to shew that any such affection has, at most, very little share in that appreciation of our movements which is generally called "the museular sense." Not only is our appreciation of passive movements very similar to our appreciation of active movements (we are as well aware of an attitude in which our arm has becn placed by others as of one in which we have placed it ourselves), but also if a muscular contraction be brought about not by any action at all 
of the central nervous system, but by the direct electric or other stimulation of the muscles or motor nerves, the muscular sense of the movement which results differs little from that of a like voluntary movement. If for instance, while our eyes are shut, the wrist be bent by direct stimulation of the flexor muscles, we ire aware of the movement and can appreciate its character and amount; we can even use such an artificial movement to judge of weight and resistance. It is indeed urged that our jurgment under such conditions is less secure than when the movement is a voluntary one; and from this it is argued that our judgment is at least assisted by our appreciation of the central changes by a "sense of the effort" as distinguished from a muscular sense of peripheral origin; but even this is disputed. We may conclude that our appreciation of our movements and muscular efforts is largely, if not wholly, dependent on what may be called a muscular sense which is the outcome of afferent inpulses proceeding from the periphery and started in the parts concemed in the movement.

$\$ 661$. Coming next to the questions, What is the exact nature of these afferent impulses? In what tissues are they started, and along what paths do they travel? we find the answers beset with considerable difficulties. Every movement of the body, even a simple one, is in reality a conplex affair, and the carrying it out involves changes in several tissues. In the first place there are changes in one or more muscles, changes, of contraction in active movements, of extension and relaxation in passive movements. In the second place there are changes in the skin which during a movement is in one spot stretched, in another relaxed or folded; and in movements of locomotion the pressure of the foot on the ground is continually changing. In the third place, by far the majority of movements affect a joint, and hence involve changes in the relations of the articular surface, in the capsule and ligaments and in the tendons. All these are possible sources of afferent impulses.

Now we know that the skin is a source of afferent impulses and so of sensations, namely, the sensations of pressure, of temperature and of pain; and we may fairly suppose that stretching or slackening the skin gives rise to impulses either analogous to those caused by the pressure of an external object or, it may be, of a nature more akin to those which belong to general sensibility. Hence it is possible that these do at least contribute, under normal circumstances, to what as a whole we call the muscular sense.

Indeed it is maintained by some that these cutaneous impulses furnish the whole basis of what is called the muscular sense, the name on this view being of course erroneous. In attempting to judge of such a view we may appeal on the one hand to our own consciousness, and on the otlier hand to the 
phenomena of ineoordinate movements. In a previous part of this work, we dwelt upon the importance of afferent impulses as factors in the eoordination of movements. We have had occasion repeatedly to insist that all the movements of the body, a large number of those whieh are involuntary as well as all those whieh are voluntary, are guided by afferent impulses, and that in the absence of these afferent impulses the movements are apt to beeome uncertain and imperfeet, or even to fail altogether. We need not here repeat what we have previously urged; it is sufficient for our present purpose to say that eonspieuous among these afferent impulses are those which form the groundwork of the museular sense; at times they may do their work without directly affecting eonsciousness but at other times they bring about a distinct affeetion of conseiousness, and it is this affection of consciousness which is more properly ealled the museular sense.

Now, on the one hand, we find upon examination that eoordination of movements is not distinetly affected by the diminution of cutaneous sensations, but may be maintained in the absence of cutaneous sensations and indeed in the absence of the skin. Thus frogs are said to be able to execute their ordinary movements without signs of ineoordination after the whole skin has been removed. Cases of nervous diseases have been reeorded in whieh, if not eomplete absence of, at least great failure in, eutaneous sensations has not been aecompanied by any decided loss of eoordination. And if we appeal to our own eonsciousness we do not find the muscular sense notably diminished by temporary anrsthesia of the skin; if, for instance, the skin of the arm be rendered for a while anxsthetic, we do not find any marked ehange in our power of judging weights or resistance, or in appreeiating, with the eyes shut, the position of the limb.

On the other hand we find recorded eases of nervous diseases in which loss of eoordination, and loss of the muscular sense, as indicated by the difficulty or inability to judge weights and resistance and to reeognize with the eyes shut the position of the limbs or other parts of the body, have oceurred without notable loss of cutaneous sensations. This is often strikingly shewn in eases of the disease or group of diseases known as "tabes dorsalis," often spoken of from one of its prominent symptoms as, "loeomotor ataxy," the conspieuous pathological condition of whieh is a structural ehange in the posterior columns of the lower part of the cord. In eertain stages of this disease the patient may retain good eutaneous sensations, he may experienee taetile, temperature and painful sensations in the skin of his legs, for instanee, and possess adequate museular strength in his legs, and yet, from want of eoordination, be unable to move them properly unless he be assisted by sight. 
So long as his eyes are open he may be able to stand and walk, but if his eycs are shut he often falls, and when he moves, moves with a staggering uncertain gait; he fears, in the dark, to go up or down stairs even though he knows them well. When a direct appeal is made to his consciousness he appears to possess little ol no muscular sense; he is unaware, so long as his eves are shut, of the position of the limbs affected by the disease, and if the arms are affected is unable properly to judge weights. These cases of "tabes" are very varied in their symptoms, which indeed alter as the disease advances. Concerning them and similar phenomena presented by other allied nervous diseases there has been much discussion; but the evidence afforded by them, supported as it is to a certain extent by experimental results, is strongly in favour of the view that the afferent impulses which determine coordination and which go to make up what we are now calling the muscular sense are other than those started in the skin.

We may therefore dismiss cutaneous sensations as not being essential factors of the sense.

$\$ 662$. There remain on the one hand the muscles, with which we may in the first instance include the belonging tendons, and on the other hand the joints with their belonging ligaments; the afferent impulses under discussion must come from one or other or both of these. We cannot by an appeal to our own consciousness localize the muscular sense so as to lodge it exclusively either in the one or the other of these parts and must trust to indirect indications. On the one hand there seems to be a close connection between the muscular sense and the 'sense of fatigue;' and the latter appears to be determined by the condition of the muscles. Again, in many of our movements we employ a part only of a muscle, and it is difficult to suppose that the afferent impulses which guide us in using that part only, depend alone on the effect which the partial use of the muscle produces on the joints or other parts. On the other hand, when we have a muscular sense of the movements of the fingers, we can hardly suppose that the sense is afforded by impulses coming exclusively from the muscles moving the fingers, distant as these often are from the joints which they move. And, again, the movements of which we are most distinctly sensible, are especially the movements affecting joints: indeed we have some difficulty in appreciating the amount and character of a movement not necessarily involving a joint such as one caused by contractions of the orbicular muscle of the mouth or of the eye, even though in these cases we are assisted by cutaneous sensations.

We have evidence both that the joints and that the muscles can supply the necessary afferent impulses. The joints are well supplied with afferent nerve fibres, and undoubtedly give 
rise to afferent impulses. On the other hand afferent impulses may proceed from muscles. When, for instance, a nerve twig going to a musele is stimulated, centripetally, after division, reflex movements result; if the stimulus is wcak the movement is confined to the muscle itself (we are supposing that other nerve twigs going to the muscle are left intaet); if the stimulus is strong, the movement spreads to neighbouring muscles. Again, the phonomena often spoken of as 'muscle reflexes' such as the 'knee-jerk' and the like $(\$ 515)$ are all so many proofs of afferent impulses passing up from the muscles. In speaking of the knee-jerk, we called attention to the influence exerted upon the movement of the muscle employed, by afferent impulses proceeding from the antagonistic muscles, and instances might be multiplied of the action and 'tone' of one muscle being modified by afferent impulses passing up from its antagonist to the nervous centre. And undoubtedly muscles are well supplied with afferent fibres. When the anterior roots, fibres from which supply a given muscle, are cut, a very large number of the nerve fibres present in the muscle remain undegenerated; these, which end partly in the tendon by a plexiform arrangement of fibrils terminating in minute end-bulbs known as the organ of Golgi, but partly and indeed largely in a somewhat similar manner, in comnection with the muscular fibres themselves, seem to be undoubtedly afferent fibres. Hence we have anatomical support for the view that the afferent impulses of the muscular sense may come from the muscles and their tendons no less than from the joints. In attempting further to distinguish between the actual museular fibres themselves and the tendons as the source of these impulses while admitting that the tendon form part of the source, we may conclude that the above-mentioned terminations of afferent fibres amoug the muscular fibres themselves indicate that these also form another part; and this view is supported by the connection, also mentioned above, of fatigue with the nuscular sense.

Against the view that the afferent impulses of the muscular scnse cone from the muscular fibres themselves has been urged the fact that these, tested cxperimentally, possess in a normal condition a very feeble general sensibility; when a muscle is cut or pinched comparatively little or, according to some observers, no pain is felt; it is only under abnormal circumstances, as when a muscle is inflamed, that direct stimulation of this kind causes pain: and the pain which we feel in cramp is similarly the product of an abuormal condition, for evcu an extremely violent muscular effort docs not cause us actual pain. 'This argument however is not valid, for not only may it equally well be applied to the other set of tissues, tendons, ligaments and the like, which in a normal con- 
dition possess a similarly feeble general sensibility, but it supposes that the muscular sense is merely a development of general sensibility not a special sense, like that of touch. IVe have no positive reasons for this supposition, and arguments based on the analogy of the skin oppose it. We have seen reason to regard the cutaneous sensations of pressure and temperature as wholly distinct from those of general sensibility, that is to say of pain; and we may conclude that the muscular sense is similarly a special sense, similarly distinct from affections of common sensibility in either muscular fibres or their connective tissue appendages.

We ought therefore probably to conclude that the muscular sense though based in part on impulses derived from the museles, and further from the muscular fibres themselves as well as from the tendons, is also, and possibly to a large extent, based on impulses derived from the joints, though we cannot as yet assign accurately the relative share. If this be so the "muscular" sense is not a wholly appropriate term; but it would be undesirable, at present at least, to attempt to replace it by a new one.

This muscular sense, using the term in its broad meaning, enters largely into our life. By it we are not only enabled to coordinate and execute adequately the various movements which we make, but through it we derive much of our knowledge of the external world. Through it we are also conscious of the varying condition of the several parts of our body even when the muscles are at rest; the tired and especially the paralyzed limb is said to 'feel heavy.' In this way the state of our muscles and other tissues largely determines our general feeling of health and vigour, of weariness, ill health and feebleness.

The fact that the Pacinian bodies are found around joints has led to the suggestion that these serve as the terminal organs of the inuscular sense; but especially bearing in mind what has just been said, the argument which we used against considering the touch corpuseles as the terminal organs of touch may, with perhaps still greater force be applied against regarding the Pacinian bodies as the terminal organs of the muscular sense. 


\section{SEC. 5. ON TACTILE PERCEPTIONS AND JUDGMENTS.}

$\$ 663$. As a means of gaining knowledge of external things the sense of touch ranks next in importance to that of sight. Auditory sensations enter largely and in several ways into our life: they serve as an important neans of communication; together with smell and taste they afford pleasure and guide our acts; but, as regards our direct knowlerlge of the external world, we learn by means of them very little compared with what we learn by sight and touch. To a certain extent we make use of touch by itself ; we bring the surface of a body into contact with some region of the skin such as the finger, and by the several sensations which we receive either from several points of that region at the same time, or from one or more points in succession, we learn certain characters of the surface, whether for instance it is rough or smooth. We thus also ascertain whether the body be lot or cold; and we may, within certain limits, form a judgment of the size of the surface by simply estimating the size of the area of our skin with which the body can be in contact at the same time.

But though we may and do thus base conclusions on tactile perceptions alone, we most frequently employ touch in association with sight on the one hand and with the muscular sense on the other.

'The ties indeed between touch and the muscnlar sense are many and close. When we explore the nature of a body by touch we press the skin, of the finger for instance, on the body; and we do that not merely in order to determine to what extcnt the tactile sensation is increased by the increase of pressure, but also and indeed chiefly to ascertain the amount of resistance to pressure which is offered by the boly. But that resistance, through which chiefly we judge whether the body be soft or hard, is appreciated not by the tactile but by the muscular sense.

Or again, placing the finger on the surface of a body, and moving the finger over the surface in such a way that the contact, as judged by the pure tactile sensation, remains the same, we find that in one case the movement has been continued in the same plane, whereupon we judge the surface to be flat, that 
in another case the finger has been gradually carried out of the plane, whereupon we judge the surface to be curved, and that in the third case the movement of the finger has been irregular, whereupon we judge that the surface is irregular: and so on. In each case we estimate the movement by the muscular sense, and thus by a combination of muscular sense and of touch we form a judgment of the conformation of external bodies. In the same way, and indeed as part of the same process, by a combination of the muscular sense and of touch we estimate the size of external objects. By a like double act we estimate the position in space in relation to our body of such objects as are within our reach, such as can be touched either directly by one of our limbs or indirectly by help of a stick or otherwise. So closely bound together are the muscular sense and the scnse of touch proper, that in common language we speak of learning this or that by touch, when we really employ both senses.

$\$ 664$. No less close are the ties between sight and touch; indeed a very large part of our psychical life is built up on the association of visual and tactile sensations. There is no part of the external world, including our own bodies, which we can explore by touch, which we cannot, either directly or by optical aids such as mirrors, also explore by vision; and our conceptions of the nature of all such things is the outcome of a combination of the two senses, or rather bearing in mind what has just been said, of the three senses, sight, touch, and the muscular sense. It is relatively easy to recognize blindfold, by touch alone, the characters of objects with which we are already previously familiar by help of vision; but it is very difficult to form by touch alone an accurate judgment of the form and size of objects which we have never seen. Were we limited to sight alone, we should form one set of conceptions of the world, -were we limited to touch we should form another; and the two sets would be different.

In the conceptions which we form in actual life the two are combined. The congenitally blind are limited to one set only; and, when, as has happened in cases of congenital cataract, those who have been blind from birth are restored to vision after they have grown up and have accumulated a store of tactile conceptions, they fail at first to connect their new visual sensations with their old tactile experience. The stories of the first experiences in vision of such persons, as that for instance of the man who had to feel a cat in order to connect the visual image with his previous tactile image, and having carefully felt it all over said "Now, Puss! I shall know you again," illustrate the close dependence on each other of visual and tactile normal perceptions. This is also indicated by the zeal with which in former days the question was discussed whether a man 
who had been born blind and restored to sight in adult life, could recognize at first sight and by sight alone a cube, a square, and a sphere. It is perhaps especially in relation to size and space, that the two senses work together.

There are no converse eases of persons who, born without touch, and trusting to sight alone have, in later life, had touch restored to them; but there are many things within our vision, which are beyond our touch at the moment and some which we can never touch at any time: our conceptions of these latter are more or less uncertain, and the direct visual sensations have to be strengthened or corrected not by mere sensations but by intellectual efforts and reasoning. A group of visual sensations, constituting a visual image, may have an ordinary objective cause, but may be an ocular illusion; and the test which we at once apply to determine this is that of touch; the ordinary idea of a "ghost' is that of a something which we can see but camnot touch, which excites visual sensations but affords no tactile sensations. Conversely a touch by something invisible, a touch as of a body which we ought to be able to see but cannot, we also recognize as unreal. The concordance of touch and vision affords in fact to a large extent the standard by which we judge of the reality of things.

$\$ 665$. The last remark naturally leads to the statement that as in the case of the other sensations, so in the case of the several cutaneous sensations, we may have sensations which are not due to their ordinary objective causes.

We have seen that visual sensations may arise from changes in the retina started not by light but by other agents, mechani cal and others; and the question presents itself, Can touch proper, the sensation of pressure, be excited otherwise than by pressure and sensations of temperature by changes in the skin other than those of temperature? No very definite answer can be given to this question, though the case quoted above $(\$ 656)$. in which a leated spoon applied to the skin produced a sensation not of heat but of contact, points perhaps to the affirmative, as does also the fact that electric currents applied to the skin may produce sensations, pricking sensations, which if not identical with, maty at least be confused with those of pressure.

Cutaneous sensations of all kinds may however be of centril origin, may be due to changes in the central nervous system quite independent of all events in the skin, and may yet be referred to this or that region of the skin and to the objective cause which ordinarily gives rise to the sensation. Painful sensations indeed may rise from changes not only in the central organs but at any part of the whole length of the nerve, all being referred to the cutaneous terminations of the nerves on which the cause of pain is usually brought to bear. Tactile and temperature sensations as we have sail camnot originate in 
changes in the nerves themselves, but they may arise through changes in the central organs; we may be subject to tactile phantoms comparable to ocular phantoms. Compared with visual sensations however our tactile sensations are so to speak fragmentary. A momentary exposure of the retina may fill the mind with a complex visual image, full of the most varied incident, but the tactile impressions which we can receive at any one moment are few and simple. Hence our tactile phantoms are also simple; we may fancy that some invisible garment has swept past us, or that a scorching flame has passed near us, we may feel that the hand or that the head is swollen and large, and we may experience an imaginary pain in every region of the skin in turn: but the most that we can thus feel is simple compared with the possible complexity of an ocular or even an auditory pliantom.

$\$ 666$. Like other sensations our tactile sensations while they sometimes give us trustworthy information of the external world at other times may give rise to illusions. 'This is well illustrated by the so-called experiment of Aristotle. It is impossible in an ordinary position of the fingers to bring the radial side of the midcle finger and the ulnar side of the ring finger to bear at the same time on a small object such as a marble. Hence when with the eyes shut we cross one finger over the other, and place a marble between them so that it touches the radial side of the one and the ulnar side of the other, we recognize that the object is such as could not under ordinary conditions be touched at the same time by these two portions of our skin, and therefore judge that we are touching not one but two marbles. Upon repetition however we are able to correct our judgment and the illusion disappears. 


\title{
CHAPTER VII.
}

\section{ON SOME SPECIAL MUSCULAR MECHANISMS.}

\author{
SEC. 1. THE VOICE.
}

$\$ 667$. If a small mirror, warmed in order to avoid the condensation of moisture upon it, be placed in an appropriate slanting position, namely, at about an angle of $45^{\circ}$ with the horizon, in the back of the pharynx with its upper margin resting against the base of the uvula and be adequately illuminated, a view of the interior of the larynx may be obtained. Sueh a mirror with its various appurtenances is called a laryngoscope. The details of the view thus gained will of course vary with the exact position and inclination of the mirror, but the following may be taken as the average appearance (Fig. 183).

In front (reversed of course in the mirror image) will be seen the edge of the back of the tongue $(L)$, and immediately in front of this the top of the epiglottis (e). 'These parts will of necessity appear much foreshortened, and peeping out from underneath the top edge of the epiglottis may be seen the swelling at its base known as the "tubercle" or "cushion of the epiglottis" $\left(e^{\prime}\right)$. The curved sides of the epiglottis will be seen sweeping away to the right and to the left, and emerging from near the end of each will be visible the ary-epiglottic fold (ar.ep.t.) on which are obvious first the round swelling due to the cartilage of IVrisberg (u') and next that due to the cartilage of Santorini (s). If at the time when the view is being taken, the voice is being uttered and especially if a high note is being given (Fig. $187 A$ ) the two cartilages of Santorini are in close apposition, and the mucous membrane between is folded 11). If no voice is being uttered and cspecially if a deep inspiration be taken (Fig. $187 \mathrm{~B}$ and $\mathrm{C}$ ), the cartilages of Santorini are far apart and the mucous membrane between them appears as a ridge completing at the hind part the rim of the aperture to the larynx; there may also be seen on each side lying immediately to the median side of the prominence of the cartilage 


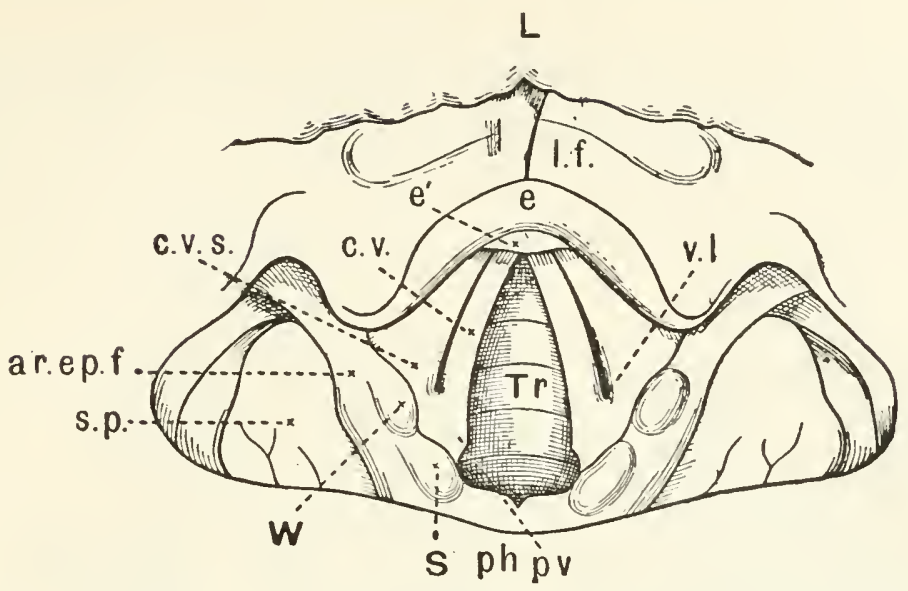

Fig. 183. Diagram of a Laryxgoscopic view of the Laryxx (magnified twice).

$L$. the base of the tongue. $e$. the epiglottis, seen foreshortened with $e^{\prime}$ its cushion, ar.ep.f. the ary-epiglottic folds. $W$. the Capitulum Wrisbergi, $S$. Capitulum Santorini; the mucous membrane between the arytenoids is stretched straight, the notch being merely indicated. c.x. vocal cords, c.v.s. ventricular bands, v.l. the opening into the ventricle of the larynx seen between them. 'The former, bounding the widely open glottis of more or less triangular form, through which a view of the trachea $(T r$.) is obtained are seen to end in the processus vocales $(p \cdot v$.).

On each side of the larynx is seen $s . p$. the pyriform recess. $p h$. the hind wall of the phlarynx. l.f. the median glosso-epiglottic fold.

of Santorini a shallower prominence due to the top of the arytenoid itself, shewn at $\alpha$ in Fig. $187 \mathrm{~B}$. Between the two phases of complete apposition and of the widest separation of the tubercles of Santorini, intermediate phases may from time to time be seen, such as those shewn in Fig. 18:3, Fig. $187 \mathrm{~B}$.

These several structures define the superior aperture of the larynx which in the laryngoscopic view, owing to the foreshortening, is not seen as it is in a dissection (Figs. 18t, 185, 186) namely as a slanting orifice with a long fore and aft diameter but appears as a rhomboidal space with the transverse diameter generally the longer one. If no voice is being uttered, and the breathing be gentle and quiet, the glottis may be seen within this aperture as a slit, more or less in the form of an elongated isosceles triangle with the apex ripping beneath the cushion of the epiglottis, the sides formed by the vocal cords, and the base by the arytenoids with the membrane between them. In a farourable view (Fig. 183) the voeal cords (v.c.) may be seen to be attached to the processus vocales and the distinction between the membranous and cartilaginous glottis observed. On the outside of each vocal cord, separated from it by the mouth of 
the corresponding ventricle of the larynx and reaching to the side of the laryngeal aperture, may be seen the ventricular band (c.v.s.). By their white colour the vocal cords present a strong contrast to the rest of the larynx.

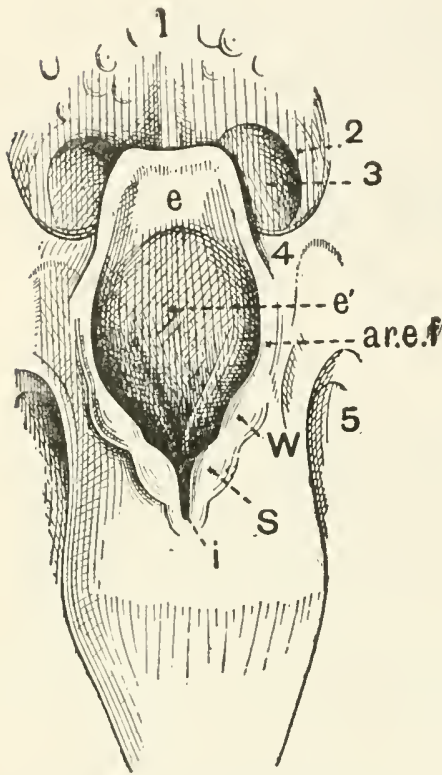

FIG, 184 .

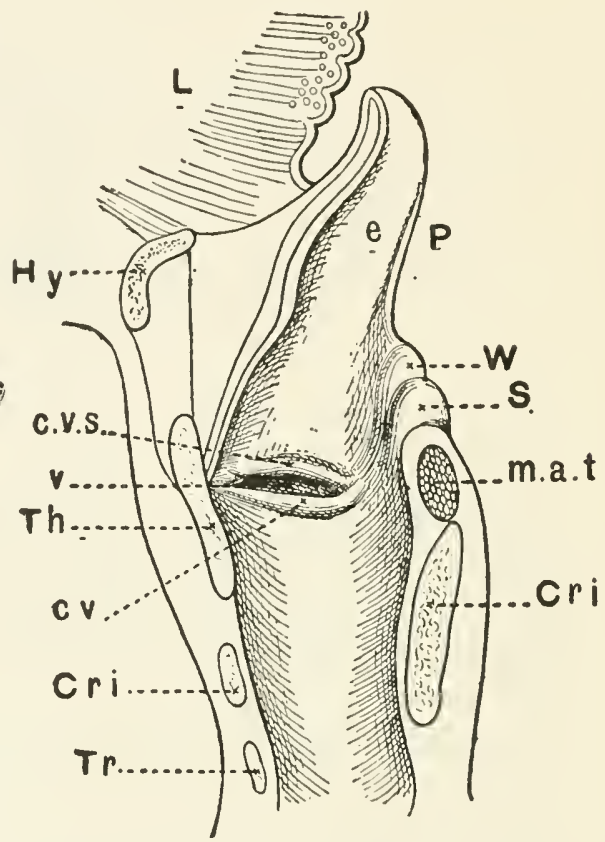

FIG. 185.

Fig. 184. DMagram of the Superior Aperture of tile Larynx.

The cesophagus and pharynx are supposed to be lair open from behind.

e. the epiglottis with $e^{\prime}$ its cushion. ar.e.f. the ary-epiglottic fold, on which are seen the swellings or "capitula" caused ( $W$ ) by the cartilage of Wrisberg and $(S)$ by the cartilage of Santorini. $i$. the notch or incisura in the mucous fold uniting transversely the two arytenoid cartilages.

1. (placed in the middle line of the base of the tongue) the median and (2) the lateral glosso-epiglottic folds, the latter forming the boundary of the depression (3) called the vallecula. 4. the pharyngo-epiglottic fold. 5. the pharyngolaryngeal or pyriform recess.

\section{Fig. 185. Diagram of tile Larynx in vertical section.}

e. the epiglottis. $l$. the base of the tongue. Hy. hyoid bone. Th. thyroid cartilage ; Cri. cricoid cartilage ; $T r$. tracheal cartilage; all cut across.

$W$. the swelling due to the cartilage of Wrisberg and $S$. that due to the cartilage of Santorini ; from these eminences folds descend towards the processus vocalis of the arytenoid. c.v. the true, and $c \cdot v . s$. the false vocal cord or "ventricular band," with the mouth of the ventricle of the larynx $v$. between them. m.a.t. the transverse arytenoid muscle cut across. $P$ is placed in the cavity of the pharyux. 
If the voice, and especially if a high note, be uttered the view changes (Fig. $187 \mathrm{~A}$ ), besides an alteration in the form of the laryngeal aperture, the vocal cords are seen to be brought close together and nearly parallel so that the glottis becomes a mere slit. If no voice is being uttered and a deep inspiration be taken changes of another kind may be observed (Fig. $187 \mathrm{C}^{\gamma}$ ); the glottis becomes a wide aperture with the form of a truncate?

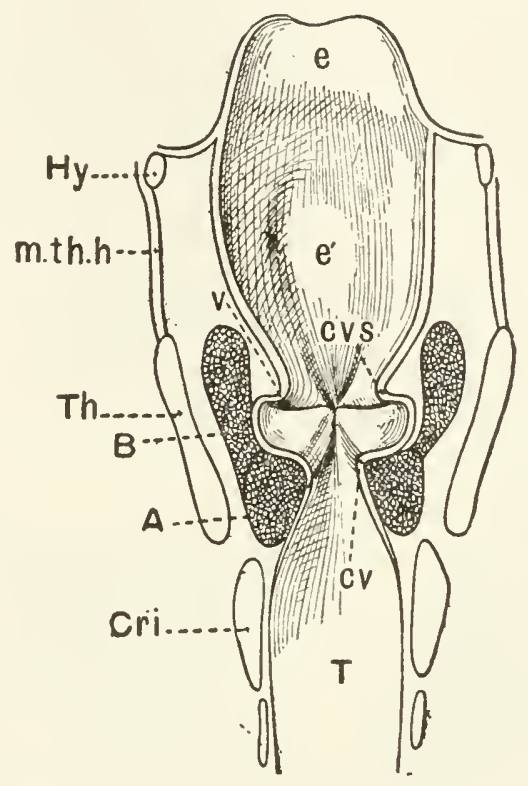

Frg, 186. Diagram ov the mouyx in vertical traysterse section.

Hy. hyoid bone. Th. thyrozd cartilage; Crt. cricoid cartilage; m.th.h. thyro-liyoid membrane, all cut across.

$e$ epiglottis, $e^{\prime}$ its cushion. c.v.s. ventricular bands, c.v. vocal cords, with $v$. the ventricles of the larynx between them, $T$. the trachea.

$A$. the internal thyro-arytenoid muscle, cut across; it is seen to form the bulk of the wedge-shaped projection of which the vocal cord is the extreme edge. $B$. the external thyro-arytenoid, cut across.

rhomboid, the obtuse angle on each side marking the attachment of the vocal cord to the processus vocalis; through this wide opening the tracheal rings are clearly visible, and indeed with an appropriate position of the mirror the bifurcation of the trachea into the bronchi may under favourable circumstances be observed. When changes in the voice or in the breathing are being made, the white glistening vocal cords may be seen to come together or to go apart like the blades of a pair of scissors. 
$\$ 668$. Laryngoscopic observation then teaches that the larynx is nsed not only for the utterance of roice, for phonation, but also for breathing; and indeed in speaking of respiration we called attention to this; but the former is its nore important use and we may chiefly dwell on this, referring incidentally to the respiratory functions.

In order that the membranous edges of an aperture may be readily thrown into sonorous vibrations by a blast of air, the edges should be brought near together and the aperture reduced to a mere slit. Ilence the fundamental condition for the formation of the voice, and indeed speaking generally of voices of all kincls, is the approximation and consequent more or less parallelism of the vocal cords.

In the voice, as in other sounds (cf. $\$ 620$ ), we distinguish three fundamental features: (1) Loudness. 'This depends on the strength of the expiratory blast. (2) Pitch. This depends on the rapidity of the vibrations, and this we may in a broad way consider as determincd on the one hand by the length and on the other hand by the tension of the vocal cords. IVhat we may call the natural length of the vocal cords is constant, or varies only with age; and the influence of this factor bears on the general range of the voice, not on the particular note given out at any one time. The tension of the vocal cords on the contrary is very variable, and the pitch of any particular note uttered depends in the main on this; hence great importance attaches to the mechanisms by which changes in the tension of the vocal cords are brought about. But, as we shall see, the problems connected with the compass of a voice and with changes of pitch are very complex; in considering these things we have to do with much more than mere variations in the tension of the vocal cords along the whole of what we have called their natural length. These matters however we shall deal with later on. and may for the present considel tension as the main factor of changes in pitch. (3) Quality. This, as we have seen $(\$ 620)$, depends on the number and claracter of the partial tones accompanying any fundamental note sounded, and is determined by a varicty of circumstances, ehief among which are, on the one hand the form, thickness and other physical qualities of the cords, and on the other hand, the disposition of the resonance chamber, or parts of the respiratory passage other than the glottis itself.

We may confine ourselves in the first instance to the conditions which determine the merc ntterance of the voice and to the mechanisms which affect the tension of the vocal cords, and henee the pitch of the voice. 'The problems thercfore which we have to attack are, first, By what means are the cords brought near to each other or drawn asunder as occasion demands? and secondly, By what means is the tension of the cords made to vary? IVe 
may speak of these two actions as narrowing or widening of the glottis, adduction or abduction of the edges of the glottis, and tightening or relaxation of the vocal cords. We may first dwell on the muscular aspects of the mechanisms by which these results are brought about, taking the nervous factors into consideration afterwards. The change of form of the glottis is best understood when it is borne in mind that each arytenoid cartilage is, when seen in horizontal section (Fig. 187), somewhat of the form of a triangle, with a median, an external, and a posterior side, the processus vocalis being placed in the anterior angle at the junction of the median and external sides. When the cartilages are so placed that the processus vocales are approximated to each other and the internal surfaces of the cartilages nearly parallel,

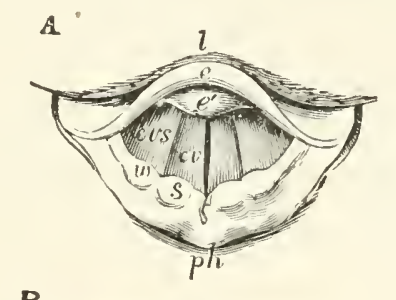

B
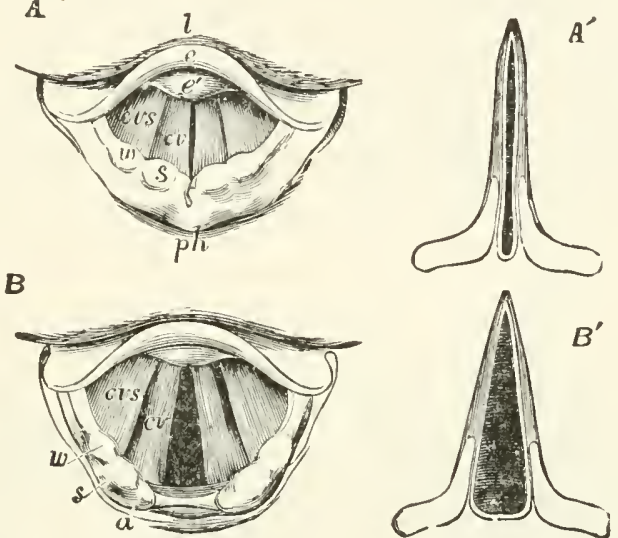

c
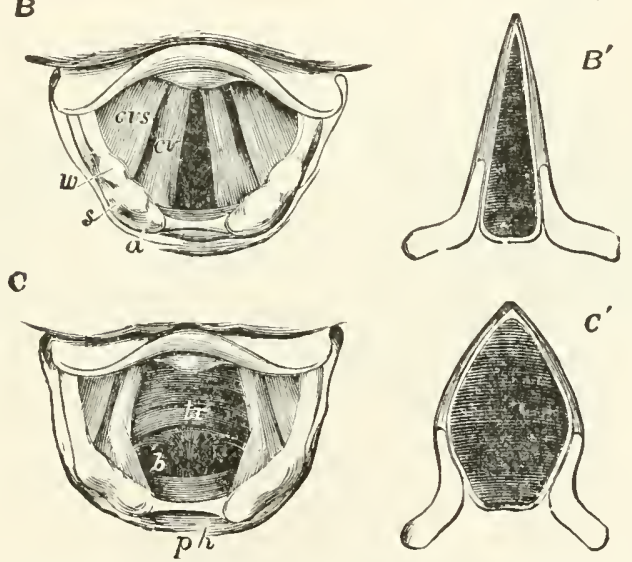

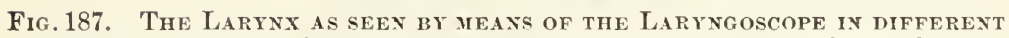
conditions of the Glottis. (From Quain's Anatomy, after Czermak.)

$A$ while singing a high note; $B$ in quiet breathing; $C$ during a deep inspiration. 'The corresponding diagrammatic figures $A^{\prime}, B^{\prime}, C^{\prime}$, illustrate the changrs in position of the arytenoid cartilages, and the form of the rima vocalis anci rima respiratoria in the above three conditions.

$l$ the base of the tongue; $e$ the upper free part of the epiglottis; $e^{\prime}$ the tubercle or cushion of the epiglottis ; $p h$. part of the anterior wall of the pharynx behind the larynx; $w$ swelling in the aryteno-epiglottidean fold causer by the cartilage of Wrisberg; $s$ swelling caused by the cartilage of Santorini; $a$ the summit of the arytenoid cartilage; $c v$ the vocal cords ; $c v s$ the ventricular bands; $t r$ the trachea with its rings ; $b$ the two bronchi at their commencement. 
the glottis is narrowed (Fig. $187 \mathrm{~A}^{\prime}$ ). When on the contrary the cartilages are wheeled round on the pirots of their articulations, so that the processus vocales diverge, and the internal surfaces of the cartilages form an angle with each othen, the glottis is widened (Fig. 18T $B^{\prime}, C^{\prime}$ ). Moreover the two cartilages may to a certain extent be bodily drawn together, or dragged apart, the two hind angles, between the median and posterior sides, being now close together, now apart.

$\$ 669$. The museles of the larynx though small, are numerous and complicated, and are so disposed in respect to their origins and insertions and to the sweep of their fibres, that the effect of the contraction of one muscle will depend upon whether or no and how far other muscles are thrown into contraction at the same time; moreover in the case of some of the muscles at least the effect is different according as the whole muscle or a part only contracts.

The first muscle to which we may call attention is the transverse arytenoid (M. arytenoideus posticus s. transversus) (Fig. 188). This is a relatively thick muscle covering the hind sur-
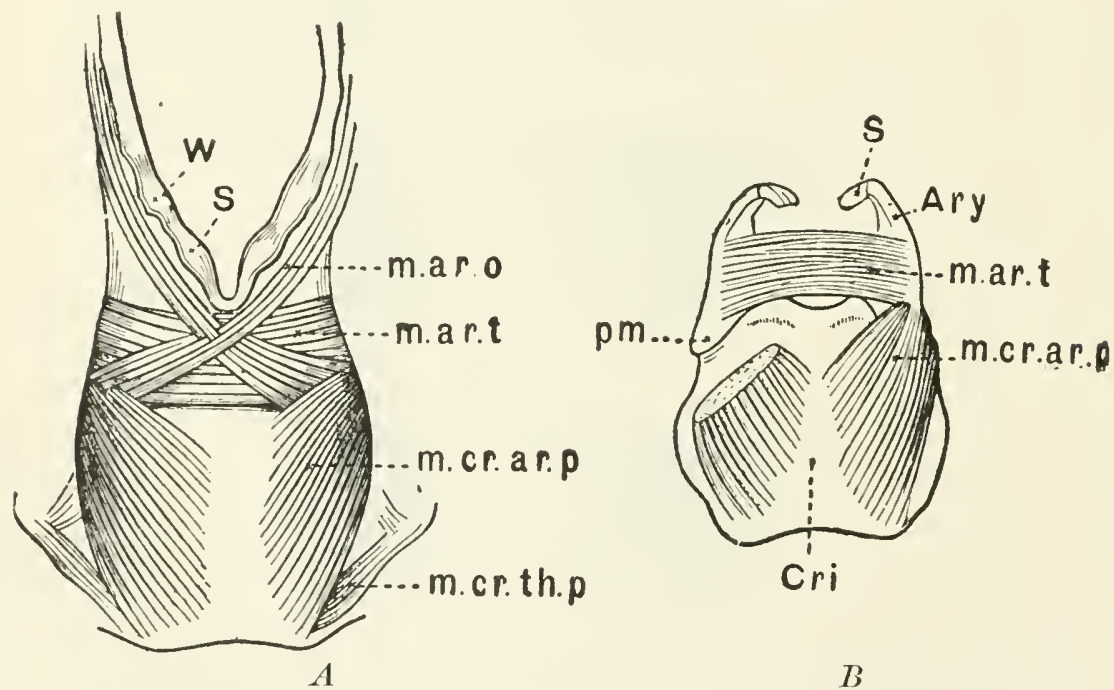

Fig. 188. Diagram of the Transverse and Oblique Artenoid and of the l'osterior Crico-arytenold Muscles.

A. shews the three inuscles in position in reference to the aperture of the larynx ; $B$. shews the attachments of the transverse arytenoid and posterior cricoarytenoid.

m.ar.t. transverse arytenoid muscle. m.criar.p. posterior crico-arytenoid muscle. m.ar.o. oblique arytenoid muscle. Cri. cricoid cartilage. Ary. arytenoid cartilage. p.m. processus muscularis of arytenoid cartilage. W. prominence of cartilage of Wrisberg. S. prominence of cartilage of Santorini (in $B$, it marks the cartilage itself). m.cr.th.p. is the small posterior crico-thyroid muscle. 
faces of both arytenoid cartilages; the fibres starting from the outer edge of onc eartilage r'un transversely across to the outer edge of the other cartilage, and the belly of the muscle occupies the concave hind surfaces of the two cartilages together with the intervening space. The effect of the contraction of this muscle is to bring the two cartilages closer together and so to narrow the glottis; indeed if in an animal it be divided, the glottis remains widely open behind. It is an important closer of the glottis, adductor of the vocal cords. When it is not contracting the cartilages come apart through the elastic reaction of their connections.

Most inportant is a mass of muscular fibres, which starting from the lower part of the reentering angle of the thyroid pass horizontally but inclined somewhat upwards to the arytenoids at about the level of the vocal cords. The whole mass is described as forming two museles. The outer or lateral part ending in the outer edge of the arytenoid and upper part of its processus muscularis is called the external thyro-arytenoid (M. thyro-arytenoideus externus) (Figs. 189, m.th.ar.e.186 B.) The direetion of the muscle as a whole is lorizontally backwards, though inclined outwards and upwards, but the constituent individual bundles run in various ways and some even pass vertically

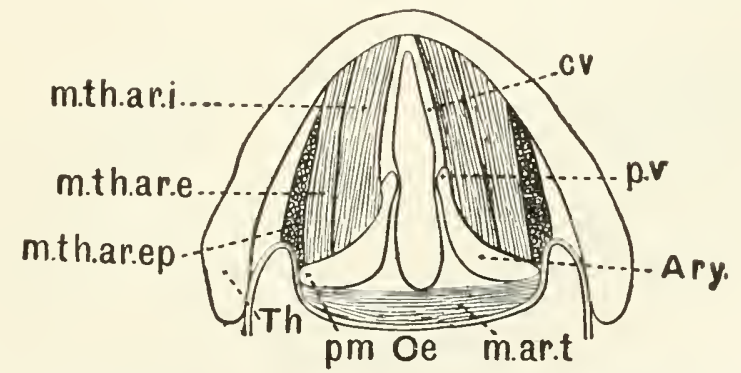

Fig. 189. Diagram to illustrate the Thyro-arytenoid Muscles.

The figure represents a transverse section of the Larynx through the bases of the arytenoid cartilages.

Ary. arytenoid cartilage. p.m. processus muscularis. p.v. processus vocalis. Th. thyroid cartilage. c.v. vocal cords. Oe is placed in the cesophagus.

m.th.ar.i. internal thyro-arytenoid muscle. m.th.ar.e. external thyro-arytenoid muscle. m.th.ar.ep. part of the thyro-ary-epiglottic muscle cut more or less transversely.

into the ventricular bands. To the inner or median sir?. of this external muscle, between it and the correspondings vocal chord, lies the inner muscle which running from the reentering angle of the thyroid to the processus vocalis and outer surface of the arytenoid forms a wedge-shaped mass, the thin edge of which is covered by the actual vocal 
cord. It is called the internal thyro-arytenoid (II. thyro-arytenoideus internus s. M. vocalis) (Figs. 189, 190, in.th.ar.i. 186 A) and has by some authors been subdivided into a median and lateral division. The general direction of the muscle is

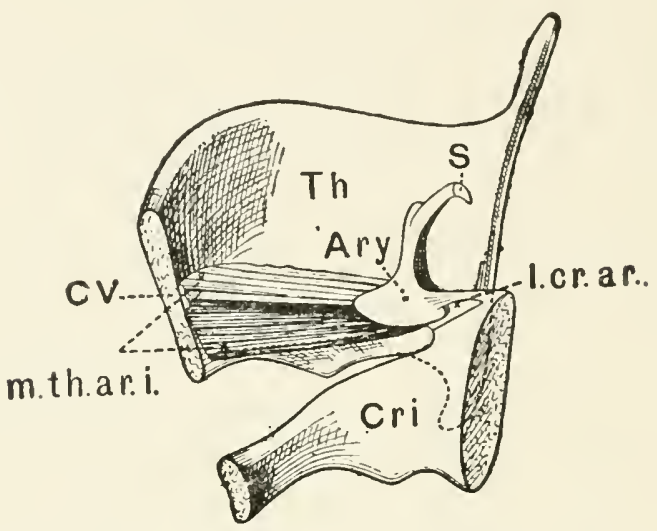

Fig. 190. The Interyal Thyro-Arytenoid Muscle.

The left halves of the thyroid and cricoid have been removed so as to shew the right arytenoid in position.

Th. thyroid. Cri. cricoid. Ary. arytenoid. S. cartilage of Santorini. l.cr.ar. the crico-arytenoid ligament. m.th.ar.i. the internal thyro-arytenoid muscle, with c.v. the vocal cord.

horizontally backwards, but, as in the external muscle, the constituent bundles run in various directions and some are said to end or begin in the vocal cord itself. One most important action of these two muscles is undoubtedly to bring the arytenoids nearer to the thyroid and so to slacken the vocal cords; but they produce other effects, and their contractions, especially those of the external muscle, help under circumstances to bring the vocal cords together and so to narrow the glottis. They also, as we shall see, produce changes in the form and thickness of the cords.

Of less importance than any of the above is a small muscle which starting from the processus muscularis of one arytenoid passes (Fig. 188 A, m.ar.o.) obliquely upwards towards the summit of the other arytenoid, crossing its fellow obliquely at the back of the transverse arytenoid muscle, which it thus partially covers; some of the fibres seem to end in the cartilage of Santorini but most of them are continued to the thyroid, the ary-epiglottic fold, and the base of the epiglottis. It is called the oblique arytenoid (M. arytenoideus obliquus) or it may be regarded as part of a flat, irregular muscle, the thyro-ary-epiglottic muscle (Fig. 186 m.th.ar.ep.). Its action is to approx- 
imate the two arytenoids and so to help in closing the glottis. It, with the transverse arytenoid and the external thyro-arytenoid muscles, may be looked upon as forming together a sort of sphineter of the larynx; their combined contractions certainly tend to close the glottis.

A relatively large and very important muscle is the posterior crico-arytenoid (MI. crico-arytenoideus posticus) (Fig. 188 m.criar.p.). This, starting from the lower part of the hind surface of the cricoid near to the median line, passes obliquely upwards to be inserted into the outer edge of the arytenoid just below the insertion of the transverse arytenoid muscle, at the upper part of the processus muscularis. Its chief action is by wheeling the outer corners of the arytenoids backwards to throw the processus vocalis outwards and so to widen the glottis; it is in this way a special, we may perhaps say the only, dilator of the glottis, or abductor of the cords; but it is maintained that it has other actions.

The above muscle acting as a dilator meets its antagonist in the lateral crico-arytenoid (M. crico-arytenoideus lateralis s. anterior) (Fig. 191 m.cr.ar.l.), which taking origin from a large

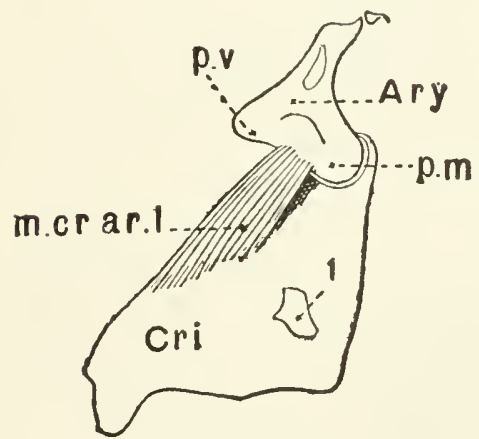

FIG. 191.

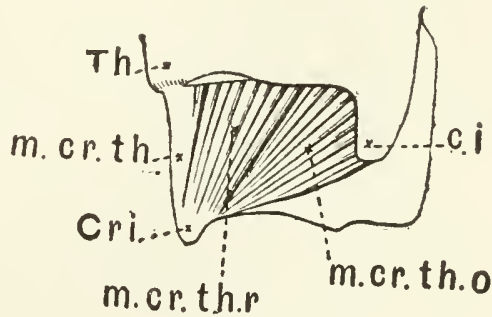

Fig. 192.

Fig. 191. The Lateral Crico-arytenoid Muscle.

Ary. arytenoid. p.v. processus vocalis, p.m. processus muscularis. Cri. cricoid. 1. surface for articulation of lower cornu of thyroid. m.cr.ar. $l$. the lateral crico-arytenoid muscle.

\section{Fig. 192. The Crico-thyroid Muscle.}

Th. thyroid; c.i. its inferior cornu. Cri. cricoid; m.cr.th.r. the straight part, m.er.th.o. the oblique part of the crico-thyroid muscle. m.cr.th. crico-thyroid membrane.

portion of the upper border of the cricoid cartilage in its lateral parts in front of the thyro-cricoid articulation, passes upwards and backwards to be inserted into the processus muscularis and outer side of the arytenoid in front of and below the insertion 
of the posterior crico-arytenoid. Its main action is to wheel the outer corner of the arytenoid forwards and inwards and thus, by converging the processus vocales, to adduct the cords and to narrow the glottis; and it has been urged that it may in this action be assisted not antagonized by a part of the preceding muscle.

The last muscle to which we need call attention, and which in some respects stands apart from the rest, is the crico-thyroid (M. crico-tliyroideus anticus). This (Fig. $192 \mathrm{cr}$.th.) starts from the front lateral surface of the cricoid, near its lower border, and passing obliquely backwards and upwards is inserted into the lower edge and inner lateral surface of the thyroid. It is sometimes subdivided into a front part (cr.th.r.) the fibres of which run more directly upwards (M. cr. thy. reetus) and a lateral part (cr.th.o.) the fibres of which run in a more oblique direction (M. er. thy. obliquus). The action of the muscle is a somewhat complicated one, but the effect of its contractions as a whole is, if the thyroid be regarded as the more moveable of the two cartilages, to pull the thyroid downwards and forwards over the front part of the cricoid, or, if the thyroid be supposed to be the more fixed, to rotate the cricoid on its tranverse axis, pulling upwards the front part and tilting downwards the hind part on which the arytenoids sit; the latter is probably its real action. Upon either view, its contractions increase the distance between the rcentering angle of the thyroid and the processus vocalis and so stretch the vocal cord; it is in fact the main tightener of the vocal cords.

There are other small muscles in the larynx as well as muscles connecting the larynx with surrounding parts; but it is not necessary for us to dwell on them here. Meanwhile it is obvious from what we have said that narrowing or widening the glottis, and slackening or tightening the vocal cords, are brought about by the above muscles acting somewhat as follows.

$\$ 670$. Narrowing of the glottis; adduction of the vocal cords. The glottis is narrowed by the combined contraction of the three muscles which we spoke of above as forming a sort of sphincter for the larynx, namely, the transverse arytenoid, the oblique arytenoid and the (external) thyro-arytenoid. These produce their effect chiefly by bringing the two cartilages near to each other in the middle line, and in this action the transverse arytenoid muscle is the most potent. Hence this muscle may be regarded as the most effective of the constrictors of the glottis.

The glottis is also narrowed by the lateral crico-arytenoid, but this produces its effect by rotation of the arytenoid cartilages; it pulls the processus muscularis forwards and so throws the processus vocalis inwards.

Widening of the ylottis; abduction of the vocal cords. The 
chief if not the only agent for the widening of the glottis is the posterior crico-arytenoid. This, pulling the outer edge of the arytenoid backwards, throws the processus vocales outwards, and so abducts the vocal cords. It has been argued that the transverse arytenoid acting alone or in concert with the above, or at least in the absence of any contraction of the other members of the sphincter group, would also wheel the outer edge of the arytenoid in the same way and so also abduct the vocal cords; but the evidence seems to be against this view.

Tightening of the vocal cords. This is especially effected by one muscle on each side, namely by the crico-thyroid which, by bringing the thyroid and the front part of the cricoid nearer to each other, increases the distance between the thyroid and the arytenoids when the latter are fixed. Supposing the transverse arytenoid and posterior crico-arytenoid to fix the arytenoids, the direct effect of the contraction of the crico-thyroid is to tighten the vocal cords. There is besides a special action of the internal thyro-arytenoid by which this muscle becomes, in contrast to the external thyro-arytenoid, a tightener of the cord; of this action we shall speak later on.

Stackening of the vocal cords. This is effected by the whole sphincter group just mentioned, but more especially by the external thyro-arytenoid and to a certain extent by the internal thyro-arytenoid; these acting alone, produce an effect the reverse of that of the crico-thyroid, bringing the arytenoid cartilages nearer to the thyroid cartilage, and so shortening the distance between the processus vocales and that body.

These several acts, however, the widening or narrowing of the glottis, the tightening or slackening of the vocal cords, are only the gross acts, so to speak, of the movements of the larynx. When a voice of any kind has to be uttered the cords must be approximated and to a certain extent tightened; and for the earrying out of even these gross acts not one muscle only but more than one, and often several are brought into play; the movements which give rise to any kind of voice are combined and coordinated movements. But, as we shall see presently, when this or that particular kind of voice is being uttered or when changes in the voice are being effected, the above words. widening, tightening and the like, very imperfectly describe what is taking place in the larynx; changes of a very complex nature are brought about, and for these the greatest nicety of combination is necessary.

$\$ 671$. We may now turn to the nervous mechanisms of the larynx. Fibres of the superior laryngeal branch of the vagus nerve are distributed to the mucous membrane of the larynx, and serve as the afferent channels by which impulses from the exquisitely sensitive surface pass upwards to the central nervous system. 
The same superior laryngeal nerve also contains motor fibres for the crico-thyroid muscle; and in this respect this muscle, the chief tensor of the vocal cords, stands apart from all the rest of the muscles of the larynx, for these are all supplied by the recurrent laryngeal branch. These motor fibres, both of the superior and of the recurrent laryngeal nerves, though rumning in the trunk of the vagus are generally believed to belong not to the vagus proper but to the spinal accessory nerve, and to the division of that nerve which we have called the bulbar accessory nerve; but on this point opinions are not agreed.

There are some reasons for thinking that the superior laryngeal contains afferent fibres not only for the crico-thyroid but also for at least some of the muscles whose motor fibres come from the recurrent laryngeal; and it has been suggested that these afferent fibres of the superior laryngeal convey the afferent impulses of the 'muscular sense;' but this needs further investigation.

In dealing with the nervous mechanism we must now distinguish between the larynx as a part of the mechanism of breathing and as an organ of voice. During breathing the glottis is open, and at least during at all deep or laboured breathing undergoes as we have previously said an increased widening during inspiration followed by narrowing during the succeding expiration. In many animals this rhythmic respiratory movement is very marked; but careful laryngoscopic observations shew that in man during quite quiet breathing there is no appreciable change in the width of the glottis.

Much difference of opinion has been expressed as to whether the width of the glottis thus permanently maintained during quiet breathing is identical with that assumed after death. But careful laryngoscopic measurements shew that during life the glottis is distinctly wider than after death; the average width during quiet breathing, is in man about $14 \mathrm{~mm}$., in woman about $12 \mathrm{~mm}$.; after death in man $5 \mathrm{~mm}$., in woman $4 \mathrm{~mm}$. This points to a continued 'tonic' contraction of some or other of the dilators of the glottis; and the muscle especially concerned in this action appears to be the posterior crico-arytenoid. Whether this tonic dilator action, whose centre lies in the bulb, close to or forming part of the general respiratory centre, is automatic in nature or maintained in a reflex manner by afferent impulses, we need not stay now to discuss; nor need we dwell on the question whether the widened glottis is the result merely of the action of the dilator muscle, or whether it is the balance of a struggle between antagonistic muscles; though analogy would perhaps lead us to expect the latter to be the case, the cvidence appears to be in favour of the former view.

'The rhythmic alternation of widening and of narrowing observed in laboured breathing is, through the activity of the bul- 
bar nervous mechanism, brought about by the various muscles spoken of above, the sphincter group being especially ised for narrowing. When occasion requires, a powerful action of this group leads, as in the first step of a 'cough, to complete closure of the glottis; and further security in the act is obtained by the narrowing of the vestibule or space above the vocal cords, the ventricular bands being brought together by the thyro-ary-epiglottic assisted by other muscles.

Both the continued patency and the rhythmic clanges are carried out by means of the recurrent laryngeal nerves. When in a living animal both these nerves are divided, the glottis becomes narrowed, assuming what may be considered its natural dimensions, namely, those proper to it after death, when all muscular contractions have ceased. Owing to the narrowing the entrance and exit of air into and out of the lungs is less easy than before, and a certain amount of dyspnoea, especially obvious if the breathing be hurried, may result; but the extent to which this occur's differ's much in different kinds of animals and indeed in different individuals. It need hardly be said that when both the recurrent nerves are divided the rhythmic widening and narrowing wholly cease, the glottis remaining immobile; the voice also is lost. When the nerve is divided on one side only, the glottis becomes deformed; when an attempt to utter voice is made, the vocal cord on that side remains farther away from the middle line than its fellow, owing to the failure of the adductor muscles on that side, and no voice is produced, since the approximation and parallelism of the vocal cords can no longer be effected. On the other hand during a deep inspiration the glottis is deformed by the vocal cord on that side being nearer the middle line than its fellow, owing to the failure of the posterior crico-arytenoid on that side. When the peripheral portion of one recurrent nerve is stimulated, the vocal cord of the same side is approximated to the middle line; when both nerves are stimulated, the vocal cords are brought together and the glottis is narrowed; though the nerve is distributed to both dilating and constricting, to abductor and adductor muscles, the latter overcome the former when the nerve is artificially stimulated. But this is true only when the stimulus is adequately strong; if the stimulus be weak, the abductors alone are thrown into contraction and the glottis is widened. We may, in this connection, add the remark that ether paralyzes the adductors before the abductors, and has this effect, even after division of both recurrent nerves; the more general respiratory function of the larynx, the maintenance of a wide passage by means of the abductors, is preserved, while the more speeial function of phonation, the narrowing of the glottis by the adductors, is lost. A like differentiation of the two functions is shewn, in a reverse way, by the clinical experience that while functional ner- 
vous disorders, such as hysteria, are marked by failure of the adductor's alone, the characteristic loss of voice being due to this, the first effect of structural changes in the bulb or other parts of the nervous mechanism is to bring about failure of the abductors; indeed the condition of the larynx as shewn by the laryngoscope may be used as an aid to the diagnosis of commencing organic disease.

$\$ 672$. When the larynx is used for voice the recurrent laryngeals are brought into play in order to produce the essential condition of voice, the approximation of the vocal cords. The vocal cords having been adequately approximated, low notes may be uttered without any further change in the larynx; in their natural position of rest the vocal cords are sufficiently tense to permit their being thrown into vibrations when brought near enough together and subjected to a sufficient blast. In order however to utter notes at all high, the tension of the corls must be increased; and this as we have said is brought about chiefly by means of the superior laryngeal nerves and the crico-thyroid muscles. Hence when these nerves are divided or fail through disease, high notes can no longer be uttered; and the division or failure of the nerve even on one side only will bring about this result.

When in using the voice a change has to be made from a higher to a lower note, while the action of the crico-thyroid ceases or is lowered, that of the antagonistic thyro-arytenoid comes into play, and the recurrent laryngeal nerve is again used.

$\S 673$. Utterance of the voice is a conspicuously voluntary act and in the vast majority of cases an eminently skilled act. Hence we find, as we have already ( $\$ 484-488)$ seen, an area in the motor region of the cerebral cortex devoted to phonation. This in the monkey (Fig. 123) lies at the lowest part of the ascending frontal convolution wedged in between the sylvian fissure and the lower end of the precentral fissure; in man as we have seen (Fig. 136) the more highly developed area for 'speech' is situate at the posterior end of the third frontal convolution, having as we have also seen an importance on the left side of the brain which it does not possess on the right.

Stimulation of the area in question in the monkey or of the corresponding area in the dog leads to adduction of the cords and closure of the glottis, the resulting movement being bilatcral. As in the case of other areas, the effect is more pure, the laryngeal movement is less mixed with other movements, when the stimulation is strictly limited to a certain part of the whole area, in this case to the lower part. But stimulation of the cortex near the pure centre for phonation leads to an acceleration in the rhythm of and exaggeration of 
the laryngeal respiratory movements, as indeed of the respiratory movements as a whole; though the respiratory laryngeal movements are in the main worked by a bulbar mechanism, they can be influenced by cortical changes.

As in the case of the other cortical motor areas, the path from the cortical area for phonation to the muscles whose actions it governs runs in the pyramidal tract through the internal capsule. Moreover in the bulb there appears to be a subordinate nervous mechanism, with which the impulses or influences descending the pyranidal tract make connection before they issue as coordinate motor impulses along the laryngeal nerves; and indeed by local electrical stimulation of the bulb, in the floor of the fourth ventricle, adduction or abduction of the cords may be brought about. The bulbar mechanism for abduction is placed higher up than that for adduction, and stimulation of either side produces in both cases bilateral movements.

$\S 674$. So far we have mainly spoken of the voice as the result of two gross acts, the narrowing of the glottis and the tightening of the cords. We must now say a few words on some other changes in the larynx, especially in reference to the various qualities and kinds of voice. Many of the features of the voice are conferred upon it by means of the parts of the respiratory passage above or below the vocal cords, by what we have spoken of as the resonance chamber or tube; these we shall deal with in treating of 'speech,' and may here confine ourselves, in the main, to changes in the larynx itself. It should be noted however that whencver voice is uttered the larynx is more or less firmly fixed by the extrinsic laryngeal muscles, such as the thyro-hyoid, sterno-thyroid, pharyngeal muscles and others. The exact position in which it is fixed will depend on the pitch of the notes which are uttered; it is raised for high notes and lowered for low ones, and may be fixed either above or below or at the natural position of rest.

We are accustomed to classify voices according to the range of pitch within which the voice can sing truly and with ease, and we thus distinguish, in ascending scale, such voices as bass, barytone and tenor in the male, alto, mezzo-soprano and soprano in the female. Could we consider the vocal cord as a membranous edge, possessing a form and nature which was constant or varied only with age, so that the rapidity of its vibrations, and hence the pitch of the voice, depended solely upon its length, fixed by the growth of the individual, and upon its varying tension, deternined by muscular contraction, the result being influenced by the varying width of the glottis, the structural basis of the distinction between the several kinds of voice would be simple enough; the bass and the contralto voices would have long vocal cords, and the other voices in each sex would be 
in asconding scale successively shorter. The vocal cord, however, is not of such a permanent nature; it undergoes under the influence of muscular contraction changes other than those of tension affecting its whole length; its form may be altered and the positions or attitudes which it may assume cannot he described as simply those of greater or less distance from its fellow along its whole length. It is in section as we have said wedge-shaped; and the projecting angle of the wedge may be an open broad one, or a narrow acute one; the vibrating cord may be thick or thin; and its vibrations will vary accordingly.

The change from thick to thin is apparently brought about by muscular contraction; it has been suggested that a partial contraction of some of the fibres of the thyro-arytenoid muscle, external and also intermal, more particularly of the bundles which take a more or less vertical direction, produce the result; but the exact mechanism is by no means clear, though special examination of the larynx shews that such a change of thickness may take place.

Again, there are reasons for thinking that contraction of the internal thyro-arytenoid muscle as a whole affects the form and the physical condition of the vocal cord, of which it furnishes so to speak the body; the strand of elastic fibres which forms the surface of the cord lies upon the muscle somewhat after the fashion of a fascia, and when the muscle, which in a state of rest is somewhat curved with the concavity towards the glottis, thickens and shortens in its contraction, carrying with it the overlying layer of elastic fibres, it brings the whole cord into a different form and different physical condition; and this must affect the character of the vibrations. Again, it is maintained that some of the fibres of the internal thyro-arytenoid running forward from the processus vocalis and outer surface of the arytenoid are inserted into the layer of elastic fibres at varying distances from the thyroid. If some of these bundles were to contract by themselves they might render the front part of the cord tense and the hind part relatively lax, or might modify in particular parts that general tension of the whole cord which was being effected by the crico-thyroid.

Further, the closure of the glottis, the adduction of the cords may take place in different ways, according as this or that muscle or part of a muscle is being especially used. While the vocal cords are being sufficiently approximated to allow the expiratory blast to throw them into vibrations the cartilaginous glottis may remain fairly open, or may be nearly or quite closed; and each of these conditions must affect the voice in a different way. Again, we have seen that the two vocal cords are close together at their insertion into the thyroid, and diverge from the middle line on each side so that the membranous glottis, when the larynx is at rest, is an isosceles triangle. We might infer 
from this that when the cords are adducted, the glottis must always remain an isosceles triangle with the angle at the apex, next to the thyroid, becoming more and more acute as adduction proceeds, and that the parts of the cords in front, nearer the thyroid, must come into actual contact before the parts behind, nearer the processus vocales, do. But the laryngoseope shews that the form of the membranous glottis is very varied; it may be open behind and closed in front, or closed both behind and in front and open, even widely so, in the middle, or may be along almost its whole length a slit with parallel sides, and in that case either very narrow, a mere linear cleft, or of appreciable width. And though the exact mechanisms are obscure, we cannot doubt but that these several phases result from special muscular contractions.

$\$ 675$. We might dwell on other changes which may by help of the laryngoscope be observed in the larynx during the production of the voice, all shewing that muscular contractions may produce complex and varied changes in the larynx besides simple alduction or abduction and general tension or slackening of the vocal cords; but we have said enough for our present purpose, which is to insist that in the production of voice the mere dimensions of the larynx, and we might add other natural inborn features, serve but as the playground for muscular skill; it is the latter much more than the former which determines the characters and the powers of the voice. A laryngoscopist, even the most experienced, would probably hesitate from a mere inspection of the larynx to predicate the nature of the singing voice. He could not even predicate the possession of a singing voice of any kind. Of two larynges, provided they were both of normal structure, he would be unable to say which belonged to the man who could and which to the man who could not sing; for the power to sing is determined not by the build of the larynx but by the possession of an adequate nervous mechanism through which finely appreciated auditory impulses are enabled so to guide the impulses of the will that these find their way with sureness and precision to the appropriate muscular bundles. And what is true of the difference between singing and not singing at all is in a similar way true of the difference between singing low and singing high, as well as of the difference between singing superbly and singing indifferently well. The physiological difference between a bass voice and a tenor voice, between a contralto and a soprano probably lies not so much in the mere natural length of the vocal cords as in the constitution of the nervous and nuscular mechanism; experience shews that cords of the same natural length may in one individual be the instrument of a bass, in another of a tenor voice, or in one individual of a contralto, in another of a soprano voice. Again, though the "magnificent organ" of a distinguished artist may 
have certain inborn qualities which lighten the labours of the nervous mechanism, it is the latter which is the real basis of the artist's fame; the former may be so slight or so abstruse as to escape observation, and a larynx, the notes of which have charmed the world, may yield through the laryngeal mirror a picture of the most commonplace kind.

That the build of the larynx is thus wholly subordinate to the nervous mechanism is further illustrated by the fact that the same larynx may and indeed does produce different kinds of voice. The difference in the kind of voice which may be brought about by the nervous system working the same larynx in different ways is strikingly shewn by comparing what is called the chest voice and the head voice. In the former, which deals with relatively low notes, the sounds are full and strong, and the lower resonance chamber which is supplied by the trachea, bronchi and indeed by the whole chest, is thrown into powerful and palpable vibrations; hence the name 'chest voice.' 'The latter, which deals with relatively high notes, is thin and poor, being deficient in partial tones, is not accompanied by the same conspicuous vibrations of the chest but is accompanied by vibrations of the head; hence the name 'head voice.'

It is obvious that the dispositions of the larynx must be very different in the two voices; but what the differences exactly are has been and still is a matter of controversy, and indeed extended laryngoscopic observation leads to the conclusion that the change from the one voice to the other is not effected in precisely the same way by all larynges. The evidence however scems to shew that in the chest voice the vocal cords are relatively broad and thick, and that the membranous glottis is open along its whole length. The cords will of course vary as to their tension through the lange of the voice, being more tense with the higher notes, and the width of the glottis is not always the same; but it is probable that throughout the voice the cords, in producing the fundamental tone of any note sung, vibrate along their whole length, and also through their whole breadth, the partial tones being due of course, as in other musical instruments, to vibrations of segments. In order to throw into vibration along their whole length such relatively broad and thick cords a powerful blast of air is needed, and hence the transmission of the vibrations downwards to the chest.

When the same larynx shifts to the head voice the vocal cords appear to become narrow, thin and always distinctly tense. In some cases the membranous glottis is closed before and behind, so that the cords are free to vibrate in their middle portion only, and here the slit is sometimes a relatively wide elliptical space; in other cases the glottis seems to be open 
along its whole extent, though reduced to a mere linear slit; but it is probable that in all cases the cords vibrate along a part only and not along the whole of their length. In order to throw into adequate vibrations the thin edges now presented, a less powerful blast is required, and the vibrations are no longer felt in the chest, though they are transmitted through the pharyngeal passages to the head.

As subsidiary conditions we may mention that in the chest voice the superior aperture of the larynx is widely open, the transverse diameter being perhaps especially long, while in the head voice the aperture is constricted, at times remarkably so. In the chest voice the epiglottis is usually depressed so as to hide from sight, in the laryngoscopic view, the front part of the cords, while in the head voice it is usually raised, but many variations in the attitude of the epiglottis may be observed. In the head voice the cartilaginous glottis seems always to be completely closed, whereas in the chest voice it is found in some cases to be closed, in other cases to be more or less open.

Making all allowance for diseordance of opinion as to what are the exact conditions of each kind of voice, and admitting the imperfection of our knowledge as to both the purpose and the mode of production of many of the differences observed, we may at least draw the conclusion that in the case of each kind of voice a certain general disposition of the mechanism is made, that a certain 'setting' of the machine takes place, by which the quality of the voice is determined, and that the machine thus set is played upon so as to produce a series of notes differing in pitch but all retaining the same particular quality. The setting of the machine in the chest voice is such that the notes produced by it are lower notes reaching up to a certain pitch only, the setting not being adapted for higher notes, and conversely the setting of the head voice allows of the production of high notes only, being incompatible with the production of low notes.

It may be urged that the setting for the chest voice is really the natural disposition of the larynx and that of the head voice a strange and artificial condition into which the larynx is forced (and indeed the latter is in certain cases called a "falsetto" voice, which term however has a technical meaning not always coincident with head voice); but a closer examination of voices shews that there is in reality no one natural condition of the larynx, and that there are other dispositions or settings of the larynx besides those of the chest voice and the head voice, these being so to speak extreme cases.

When a singer sings a series of notes in an ascending scale it will be observed that beginning with the lowest notes the voice during a certain range remains through all the notes of the same quality, differing in pitch only, but that at or about a 
certain note, the voice in passing from one note to the next above is not merely raised in pitch but absolutely altered in quality, and further maintains the new features in the succeeding higher notes. 'This sudden change is spoken of as the 'bleak' in the voice, and a lange of notes of differing pitch but of the same quality which is thus separated by a 'break' from another range of notes of a different quality is called a 'register.' laryngoscopic observations, especially recent ones in which photographic aid has been called in, shew that during a register there is a particular' 'setting' of the larynx, which is maintained throughout the whole register, the chief change observable being an increasing tension of the cords as the notes rise in pitch, and that at the break there is a sudden shifting of the setting, the new setting bcing maintained during the ensuing register with changes which as before are chiefly directed to a tension of the cords increasing with the rising notes.

In most voices the ear may recognize two such breaks, separating three registers, lower, middle, and upper, the lower and upper being usually the chest and head voice described above. But some voices are marked by three breaks separating four registers, the differences being distinctly recognizable by the ear, and there are some reasons for thinking that a break, that is a change in the setting of the larnyx, may take place without being evident to the ear, though visible by the help of the laryngoscope. We may add one part of the training of a singing voice consists in rendering the break, the transition from one register to another, as little obvious to the ear as possible.

It would be beyond the scope of this work to enter upon the details of the several registers of the different kinds of voices, beyond the little we have said touching the chest voice and head voice; these are matter's of great difficulty subject to much controversy, and indeed, as we have already said, observations tend to shew that exactly the same disposition does not obtain for the same register in all larynges; it seems probable that two larynges may gain the same end by two different manouvres, may produce the same kind of voice by different dispositions of the larynx. In any case the subject is one of extreme complexity, and we have ventured to dwell on it, even so long as we have, because it affords a striking illustration of what we have more than once insisted upon, the complicated character so often belonging to the muscular contractions by which the animal body gains its ends, and the delicately adjusted coordination of efferent nervous impulses needed to secure for the effort a complete success. We have so repeatedly. in plevious parts of this work, insisted on the importance of afferent impulses to the coordination of complex movements that it is hardly neccssary here to do mole than to point out that the comnection of the use of the voice with auditory sensations affords striking instances of the truth of 
what we have insisted upon. Auditory sensations are at least as important for the proper management of the voice as are visual sensations for the movements of the eyes, and more important than are visual sensations for the movements of the body and limbs. Indeed they are in a way essential to the very utterance of the voice; the dumbness which is so conspicuous a concomitant of congenital deafness is in most cases due not to deficieney in the muscular apparatus or even in the nervous mechanism on what we may call its motor side, but to the lack of afferent impulses from the auditory nerve. And in popular language we recognize this dependence of the management of the laryngeal muscles on auditory sensations when we talk of such or such one, who is deficient in this respect, as "lhaving no ear."

$\$ 676$. The ventricles of the larynx appear to be useful in allowing the vocal cords sufficient room for their vibrations; they also supply a secretion by which the vocal cords are kept adequately moist. The purpose of the ventricular bands is not exactly known; it has been suggested that they may at times exert a damping action by being brought down to touch the vocal cords; but this is very doubtful. The epiglottis, the position of which as we have seen varies in different kinds of voice, has also an influence on the character of the voice; and further influences which we shall consider under 'speech' are exerted by the form of the pharynx and the mouth.

$\S 677$. At the age of puberty a rapid development of the larynx takes place, leading to a change in the range of the voice. The peculiar harshness of the voice when it is thus 'breaking' seems to be due to a temporary congested and swollen condition of the mucous membrane of the vocal cords accompanying the active growth of the whole larynx. The change in the mucous membrane may come on quite suddenly, the voice 'breaking' for instance in the course of a night. 


\section{SEC. 2. SPEECH.}

$\S 678$. All sounds as we have seen ( $\$ 619$ ) may be divided into musical sounds, in which the vibrations are regular, and noises, in which the vibrations are irregular'; but we have also seen that the distinction between the two is not a sharp one. The vibrations into which the air in the larynx is thrown by the vibrations of the vocal cords in ordinary voice are on the whole regular' the sound so produced is a musical sound. The vibrations of the glottis may however vary as to the degree of their regularity; and under certain circumstances they may be so irregular that the sound becomes an indeniable noisc; as for instance in the sound which we call a 'cry' or a 'shriek.'

The sounds produced in the larynx like other musical sounds consist of partial tones added to a fundanental tone, and are in many cases very rich in partial tones. By modifying the shape of the passage leading through the pharyngeal, the buccal, and to a certain extent the nasal cavities, to the opening of the mouth, which we have spoken of as a resonance tube or chamber, and which, for reasons which we shall see, we may now call the vowel chamber, we are able to render loud and prominent one or other of the partial tones of a sound which is produced by the larynx and thus to affect its quality as it leaves the mouth.

We are also able, quite independently of the larynx (and indeed independently of breathing), to create sounds by means of parts of the mouth or other portions of the vowel chamber. These are for the most part noises but, as for instance in whistling, may be musical sounds.

In speech we make use on the onc hand of laryngeal sounds, more or less modifierl in quality by the vowel chamber, and on the other hand of sounds generated in various parts of that chamber; our speech in fact consists of a basis of musical sounds with an addition of noises.

$\S 679$. One great feature of speech is that it is "articulate;" it consists of syllables jointed together, the parts of specch which we call words being formed of two or more syllables, or at times of one only. In the great majority of syllables we recognize two kinds of sounds which we call voreels and consonants. 'Though it 
is easy to say which is a vowel and which is a consonant, it is difficult to frame a definition which shall be free from all objections. It has been said that vowels are formed by the voice, that is by the vibrations of the vocal cords (hence the name vowel, vocalis), and consonants by the mouth, lips or other parts of the chamber above the larynx; but as we shall see, on the one hand the vowels, as indeed the name which we have adopted for the chamber indicates, are formed by help of that chamber, and on the other hand many consonants are formed by help of the voice. 'The word 'consonant' expresses the view that what we call consonants are always sounded with some vowel or other and cannot be sounded alone by themselves; but several consonants can be so sounded; hence the name is inappropriate. We may make the distinction that whereas in a vowel the form assumed by the resonance tube merely modifies the sound produced by the larynx, in a consonant a change of form in the same tube creates a noise which may exist by itself or may mingle with the sound produced by the larynx: but this is not exact, since as we shall see such a consonant as $M$ may be used (as for instance in 'bottom,' in which though we write we do not sound the second $o$ ) in such a way that the form of the mouth only modifies a laryngeal sound, and the utterance may be continued indefinitely, like that of a vowel. Indeed we employ $M$ and certain other consonants in two ways; we use $M$ as a true consonant in company with a vowel as in 'my' or, as in the above instance, we may use it by itself, it alone forming a syllable. In this latter function $M$ may conveniently be called a sonant, the sounds of speech being divided into 'sonants' and true 'consonants.' IWe may however leave these definitions and turn at once to the mode of formation of the several vowels and consonants, or rather to the more common of these, since each language has its own vowels and sounds, and while some are common to all, some are special to a few, or even to one. We may merely remark that in speech the vowels bear the brunt of the work, they carry the 'accent,' and the consonants are, so to speak, built upon them as on a foundation. Some consonants (sonants) however may be used like vowels to carry accent.

$\S 680$. Vowels. With the utterance, either in singing or speaking, of each vowel the vowel chamber is moulded into a particular shape. Taking the most common vowels $\mathrm{U}, \mathrm{O}, \mathrm{A}, \mathrm{E}, \mathrm{I}$ pronounced in the way in which most nations pronounce them and so corresponding to our oo, o, broad $a(a h), e$ as in bet, and $e e$, we find the following.

In $U$ the vowel chamber is large with a narrow opening at the mouth. The larynx is depressed, or at least not raised above the position of rest, the tongue is flattened, especially in front, and the lips are protruded so as to reduce the mouth to a narrow round opening. The form of the vowel chamber, with a wide 
pharyngeal and a narrow buccal orifice, may be compared to that of a round flask without a neck. In A, the mouth is opened wide, the larynx is somewhat raised, the tongue flattened and at the same time driven somewhat backwards towards the hind wall of the pharynx, so that the entrance fiom the pharynx to the larynx is narrowed. The vowel chamber thus assumes a form which may be compared to that of a funnel, the wide end being at the mouth and the narrow end at the larynx. In $O$ the shape is intermediate between that of $U$ and that of $A$, the exact shape depending on the kind of $\mathrm{O}$ which is being uttered.

In I the shape is very different. The larynx is raised and the tongue is carried forwards and upwards in such a way that it touches the teeth and the hard palate at the sides and nearly so in the middle leaving only a narrow canal in the middle line. At the same time the lips instead of being protruded are drawn back and the soft palate is raised high up. In this way there is developed above the larynx a relatively large pharyngeal space which communicates with the exterior by a narrow canal; the form of the vowel chamber may now be compared to that of a round flask with a long narrow neck. In E, and the other vowels between $\mathrm{A}$ and $\mathrm{I}$, the shape of the resonance is correspondingly intermediate; in passing from $\mathrm{A}$ to $\mathrm{I}$, the tongue is brought forwards and upwards, the buccal orifice narrowed and the larynx raised.

In each of the above cases what we have called the vowel chamber acts as a resonance chamber; that is to say in cach case, owing to the shape of the cavity (in relation to the nature of its walls), the air in the chamber is more readily thrown into vibrations by certain tones than by others, and when a sound containing those particular tones is sounded into the chamber, those particular tones are reinforced and rendered loud and prominent. The shape of the vowel chamber in uttering $U$ is such that the cavity acts as a resonator towards a particular tone, namely, the bass $f$, or more probably the bass $b$, and while the laryngeal sound with its fundamental and partial tones is passing through it, reinforces and renders loud the tone $b$, occurring as a constituent of the whole sound. And similarly with the other vowels. In fact vowel sounds are musical sounds in which a particular constituent tone is reinforced and rendered loud out of proportion to the other tones; in the case of some vowels two toncs are so reinforced. The tone thus reinforced is generally a partial tone, but may be the fundamental tone. When the vowel is sung or spoken in notes of different pitch the particular partial tone which is reinforced will occupy different positions in the series of partial tones; it may be the first, second, third or other partial tone according to the pitch of the fundamental tone.

That the vowel chamber does act in this way as a resonator 
for a particular tone is shewn by moulding the cavity into the proper form for uttering a particular vowel, and bringing before the mouth a series of sounding tuning-forks of different pitch; it will be found that it is the sound of one tuning-fork and one in particular which is reinforced and made louder, namely, the one whose pitch corresponds to the fundamental tone of the particular vowel cavity; in the case of the vowel $U$ for instance it will be the tuning-fork having the pitch $b$. On the other hand that what we recognize as vowel sounds do result from the reinforcement in a musical sound of a particular tone or of particular tolles may be shewn by setting into vibration a series of tuningforks of different pitch, in imitation of a musical sound with its constituent tones, and then in turn reinforcing the sound of particular tuning-forks by the help of artificial resonators. When this is done the reinforcement of the appropriate tone gives rise to a vowel sound, the reinforcement of $b$ giving rise to $U$ and so on. 'The curves moreover described by the vowel sounds in the phonograph, in which the vibrations of the air transmitted to a thin plate or membrane are made to write on a recording surface, are in form such as would be described by sounds in which particular constituent tones were reinforced in the manner described. Again, as we said in dealing with hearing (\$ 629), when a note is sung into the open piano, the particular strings of the piano corresponding to the constituent tones of the note sung are thrown into sympathetic vibration; in the sound thus returned by the piano the nature of the vowel on which the note was sung may be recognized; the string corresponding to the characteristic tone of the vowel is thrown into appropriately strong vibrations.

The nature of the vowel sounds is especially well illustrated by the kind of speech which we eall whispering. In this, in contrast to audible speech, no musical sounds are generated by the vocal cords. A laryngeal sound is generated but it is a noise, not a musical sound, and is caused by the friction of the air as it passes through the glottis, which assumes a peculiar form, the processus vocales projecting inwards towards each other, leaving the cartilaginous glottis as well as the greater part of the membranous glottis more or less open. This noise, like the musical sound of audible speech, is modified by the parts of the mouth and pharynx, and in it we may recognize vowels and consonants. The noise of the whisper, though weak, contains multifarious vibrations, contains among other and irregular vibrations, the regular vibrations corresponding to the several vowels. When we whisper a vowel, we 'set' the vowel chamber so that it may reinforce the set of vibrations of the particular pitch characteristic of the vowel; and a well-trained ear may recognize in the whispered vowel the dominant tome.

A vowel then is essentially a musical sound of a special 
quality, due to the dominance of a particular tone, originating in the glottis and reinforeed by the conformation of the vowel chamber. 'This vicw at least, as expounded above, is the one gencrally held; but recent observations based on graphic records have, it is right to say, thrown some doubt on the matter; these we cannot discuss here. There are noreover many subsidiary questions connected with the formation of vowels into which we cannot enter here. IVe will merely add that in uttering a true diphthong, the conformation of the vowel chamber proper to the initial vowel is changed rapidly but gradually and without any obvious break into that proper to the final vowel.

$\S 681$. Consonants. These as we have already said in some cases so far resemble vowels in that they are modifications of the voice, that is to say of laryngeal sounds, caused by the disposition of the parts of the vowel chamber, the disposition however being in all such cases different from that giving rise to a vowel, and moreover the very assumption of the disposition taking part in the generation of the whole sound. Such consonants however are relatively few, the great majority of consonants are caused by changes which set up vibrations in some part or other of the vowel chamber or in the larynx itself; they are noises which may or may not be accompanicd by voice, the vibrations producing a different consonant according as they are or are not accompanied by voice. Such vibrations may be set up in several ways. In the case of many consonants vibrations are set up by the passage being closed and then suddenly opened at a particular part; such consonants are spoken of as explosives. In the case of these consonants the noise which is their essential part cannot be prolonged, it is momentary in duration, though when it is accompanied by voice the latter may continue for some time. In the case of other consonants the noise is caused by the rush of air through a narrow space and the consonants may be described as 'frictional'; or the noise may be produced by the vibratory movements of particular parts. Both these kinds of consonants can naturally be prolonged for an indefinite time, and have been called continuous consonants.

On the other hand the characters of a consonant are also dependent on the particular part of the passage at which they are generated; and this also may be used as a means of classification. Some consonants are produced at the lips by the movement or position of the lips in reference to each other or to the teeth; these are called labial and labio-rental consonants. Others again are produced by the movement or position of the tongue in reference to the tecth or to the tecth and hard palate; these are called dental. Yet other's are produced by the movement of the back of the tongue in relation to the soft palate and pharynx or fauces; these are called yuttural consonants, the name being also applied to certain consonants which are essen- 
tially noises generated in the larynx itself. These names are useful for a general broad classification; the term dental however is used to include consonants which are formed by the tongue in relation to, not the teeth, but the front part of the hard palate; hence it is to that extent open to objection. 'There are also other classifications into which we cannot enter here.

$\$ 682$. When the various languages of the world are examined the number of consonants, that is to say of sounds used in speech and having the characters on which we are dwelling, is found to be very large; and concerning the nature and mode of formation of many of them much discussion has taken place. We must content ourselves here with very briefly indicating the chief facts concerning the mode of formation of the most important and common.

The group of consonants represented by $\mathrm{M}, \mathrm{N}, \mathrm{NG}$, are very closely allied to vowels. In each of these as in a vowel the larynx is thrown into vibrations; but instead of the vibrations passing out by the mouth through a passage which has assumed a form belonging to this or that vowel, the passage to the mouth is closed, and the vibrations find their way out through the nasal cavity which acts as a resonance chamber. When a vowel is sounded the soft palate either completely shuts off the nasal cavity from the vowel chamber, or at least offers such resistance that an insignificant proportion of the expiratory blast passes into the nasal cavities. A vowel may be sulg powerfully, and yet a flame exposed to the nostrils only will shew 110 movements; in the case at least of some vowels however, a piece of cold polished steel will become dim, shewing that some air is passing through the nostrils. When the communications between the nasal and pharyngeal cavities are sufficiently free, and the other conditions are favourable for the nasal cavities to act as a resonance chamber, the vowel sounds are apt to take on a nasal character; and this occurs more readily when the vowel is said than when it is sung. In the group of consonants in question the nasal cavities become all important, the passige through the mouth being blocked. In M the passage is closed by shutting the lips, in $\mathrm{N}$ by the application of the tongue to the front of the hard palate and upper teeth, in NG by the application of the tongue to the soft palate.

While in the above group no new vibrations are added to the laryngeal vibrations, in the ordinary L, which like them is based on laryngeal sounds, new vibrations, constituting a noise, are added. 'The passage is not completely, only partially closed; the front of the tongue is pressed against the hard palate in such a way that the passage is blocked in the middle but the air escapes through narrow channels on each side. It is the noise caused by the rush of air through these narrow spaces which added to the voice produces the sound we distinguish as L. In certain forms 
of $\mathrm{L}$, for instance in the Welsh $l l$, the noise is not accompanied by laryngeal vibrations.

$\mathrm{R}$ is also allied to the above in so far as it too needs voice, and is bascd on laryngeal vibrations. But these vibrations are modified in a special way; they are rendered intermittent by vibratory movements started in some part of the passage, there being different kinds of $R$ according as the interruption takes place at the tongue, or at the fauces. The common $R$ is produced by the vibrations of the point of the tongue raised against the front of the hard palate, and the guttural $R$ by the vibrations of the uvula against the root of the tongue. In the feeble English $R$ there appear to be no vibrations; the vowel chamber is simply narrowed in front by the tip of the tongue.

It will be observed that $\mathrm{L}$ and the common $\mathrm{R}$ resemble each other to a considerable extent in the position of the tongue; the chief difference is that in $\mathrm{L}$ the tongue is not itself the subject of muscular movement, and the vibrations are produced by the friction of the expiratory blast through the narrow channel, whereas in $\mathrm{R}$ the vibratory interruptions are produced by the movements of the tongue. If in pronouncing $\mathrm{L}$ the tongue is suddenly set in movement, or in pronouncing $R$ the tongue is suddenly arrested in its movements while in approximation to the palate, the one consonant is changed into the other; and, as is well known, certain persons, for instance the Chinese, are apt to use the one instead of the other.

The explosives differ according to the part where the interruption takes place; and in each kind of interruption the sound is different and receives a different name according as voice is used or no. $\quad \mathrm{P}$ is uttered when the lips, being first closed and an expiratory blast driven against them, are suddenly opened. During this act $n$ voice is uttered. If voice is uttered the $P$ becomes $B$. These are labial explosives. In $\mathrm{T}$, the interruption which is suddenly removed is caused by the application of the tongue to the front part of the hard palate in the case of the English, to the teeth in the case of most other languages; it is called a dental explosive, dental being used in the wide meaning stated above. With $\mathrm{T}$, there is no voice; if voice be added the sound becomes $D$. Since P differs from B, and T from D, only in the absence or presence of voice, the removal or atdition of voice will at once convert in each case the one consonant into the other; and by certain nations $\mathrm{P}$ and $\mathrm{B}$ are used the one for the other, as are also $\mathrm{T}$ and $\mathrm{D}$.

It will be observed that $B$ and $D$, both with voice, have certain relations to $\mathrm{M}$ and $\mathrm{N}$ respectively. In $\mathrm{B}$ and $\mathrm{M}$ the action is labial, in $\mathrm{D}$ and $\mathrm{N}$ dental, voice being present in all; the difference is that in $\mathrm{M}$ and $\mathrm{N}$, the action consists in the establishment of an obstruction in the buceal passage, in B and D in the sudden removal of an obstruction. If there be, as in nasal 
catarih, an adequate obstruction to the exit through the nasal passages of the expiratory blast which ereates the sound, it becomes difficult if not impossible to establish the obstruction in the mouth, since in that case there is no exit at all for the expiratory blast. Hence in nasal catarih there is a tendency for the effort to pronounce $M$ to result in $B$, and that to pronounce $\mathrm{I}$ in D; 'name' becomes 'dabe.'

If the tongue be brought to the back instead of to the front of the hard palate the consonant $K$ (hard $C$ ) is uttered ; if roice be added the sound is G (hard). These are guttural explosives. Allied to them is the brief sound which in certain cases inaugurates a vowel and which, due to the sudden openimg of the closed glottis, is immediately followed by the vibrations of the cords and so by the true vowel sound. This is the spiritus lenis as distinguished from $\mathrm{H}$ or the spiritus asper, of which, formed as it is in a different manner, we shall speak directly.

Certain other consonants are continuous, and like Is are formed by the rush of air through a constriction formed somewhere in the passage; they are frictional in origin. They differ however from the ordinary $\mathrm{L}$ in that they are not always accompanied by voice; like the explosives they may be uttered without any vibrations of the vocal cords, and when these do accompany the frictional sound the consonant is altered in its characters and receives anotlier liame. As in the case of the explosives, in forming the different members of the group, the vibrations giving rise to the sound are started in different parts of the passage, at the lips, at the teeth or hard palate, or at the fauces.

When the constriction is caused by the lip being brought into contact with the teeth (and generally the lower lip and upper teeth are used), so as to reduce the outlet to a narrow space, the vibrations started at the constriction give rise to $\mathrm{F}$, when no voice is uttered at the same time. If voice be also uttered the $\mathrm{F}$ becomes $\mathrm{V}$. If the teeth take no part in the constriction, and this be made exclusively by the two lips, the vowel chamber at the same time assuming the shape proper to the vowel $U$, the sound if voice be uttered is $W$, the English $W$ and the allied French ou (in oui) may be regarded as the vowel $U$ (in two different forms) turned into consonants. The sound which is formed by the two lips alone, in the absence of voice is the English (North Country) Wh.

When the constriction is formed between the tongue and the teeth in such a way that the tip of the tongue protruckes between the partially open rows of teeth the sound is called Th: a 'hard' 'Th as in 'thin' if without voice, a 'soft' $T$ ' as in 'this' if with voice. The effect of this manouvre does not differ greatly from that of forming $\mathrm{F}$, and certain persons in attempting to pronounce a hard Th give utterance to $F$, as in the cockney 'nuffin.'

When the constriction takes place between the tongue and 
the teeth in sucn a way that a narrow channel is formed between the upper incisors and the tip of the tongue curved into a groove the sound is called $\mathrm{S}$ (soft $C$ ) if without voice, and $\%$ if with voice. If the constriction be formed a little farther back behind the front teeth by the approximation of the tongue to the front of the hard palate, the sound uttered without voice is $S h$; if voice be added the sound becomes the French $j$, which we represent by $z$ as in 'azure' or by $g$ as in 'badger.'

If instead of being formed by the teeth the constriction be carried farther back from the region of the teeth and hard palate to that of the soft palate and fauces, a guttural aspirate is formed. Without voice this is the hard $c^{t} h$ as in the Scotch 'loch,' with roice the soft $C h$.

$Y$ appears to be the vowel $I$ (ee) used as a consonant, much in the same way that, as stated above, $W$ is $U$ used as a consonant.

Lastly, a consonantal sound may be formed by the glottis itself supplying a constriction but in such a way that the vocal cords are not thrown into musical vibrations. When in uttering a vowel we begin with the glottis not closed as in the spiritus lenis but open, and send through the glottis an expiratory blast which creates irregular vibrations by friction before the cords are brought into a proper position for their regular vibrations, the result is the aspirate $\mathrm{H}$, the spiritus asper. 'The particularly powerful $\mathrm{H}$ of Arabic is produced by bringing the processus vocales into contact with or near to each other but so as to leave the cartilaginous glottis widely open and the membranous glottis more or less open; at the same time the ventricular bands are approximated, and the superior aperture of the larynx is forcibly constricted. The expiratory blast driven through the series of irregular passages gives rise to the irregular vibrations which constitute the sound, the vocal cords being motionless or at least not giving rise to the regular vibrations of voice.

We have seen that in whispering no true voice is uttered, no regular vibrations are generated in the vocal cords, though the passage of the air through the glottis produces vibrations which serve as the basis of the whisper, being modified by the vowel cavity so as to form vowels. 'To these vibrations may be added the vibrations of the consonants, so that a whisper becomes complete though feeble speech. Since the irregular glottic vibrations of a whisper are very weak compared with the relatively powerful true vocal vilbrations, the distinction between consonants with voice and without voice is in a whisper largely obscured; it is difficult for instance in a whisper to distinguish between $P$ and $B$. 


\section{SEC. 3. ON SOME LOCOMOTOR MECHANISMS.}

$\$ 683$. The skeletal muscles are for the most part arranged to act on the bones and cartilages as on levers, examples of the first kind of lever being rare, and those of the third kind, where the power is applied nearer to the fulcrum than is the weight, being more common than the second. This arises from the fact that the movements of the body are chiefly directed to moving comparatively light weights through a great distance, or through a certain distance with great precision, rather than to moving heavy weights through a short distance. The fulcrum is generally supplied by a (perfect or imperfect) joint, and one end of the acting muscle is made fast by being attached either to a fixed point, or to some point rendered fixed for the time being by the contraction of other muscles.

There are indeed few movements of the body in which one muscle only is concerned; in the majority of eases several muscles act together in concert; the movements of the larynx which we have just studied afford a striking illustration of this. The relations of the muscles which thus act together are many and varied. When one muscle is contracting, the contractions of another muscle or of other museles may, as just stated, serve to secure a fixed point, or may enforce the effect of the first muscle, or, and this is perhaps the most common case, may give a special direction to the action, the movement effected being the resultant of the forces employed in combination. Many muscles are, either partially or wholly, antagonistic in action to each other, such for instance as the flexors and extensors, and such museles as those of the face, which act bilaterally in opposite directions on parts placed in the middle line; and the relations of these antagonistic muscles seem to be specially complex. When a muscle contracts it is, as we saw in treating of nerve and muscle, of advantage that the muscle should at the moment of contraction be already "on the stretch;" this is provided by the anatomical disposition of the parts assisted probably, as we saw ( $\$ 470)$, by skeletal tone, but is also further secured by the action of its antagonists, which moreover, after 
the muscle has contracted, assist its return to its proper position of rest. When a point has to be fixed the two sets of museles which act as antagonists on the point are both thrown into contraction, in proportion to their relative effect. If the action of one muscle (or set of muscles) is to be dominant its antagonist may take no part in the action, being neither contracted nor relapsed; but there are reasons for thinking that, in many cases at all events, the action of one muscle though remaining dominant is tempered and guarded so to speak by a concomitant feebler action of its antagonist; on the other hand we have evidence as in the case of the ocular muscles ( $\$ 595$ ) that it may be assisted by an inhibition, a relaxation of the antagonist. These several phases are governed by the nervous system, and the behaviour of antagonistic muscles and groups of muscles affords many instances of what we have so often insisted upon, namely, that nearly all the various movements of our body are coordinate movements, and that in many cases the coordination is extremely complex.

$\$ 684$. The erect posture, in which the weight of the body is borne by the plantar arches, is the result of a series of contractions of the muscles of the trunk and legs, having for their object the keeping the body in such a position that the line of gravity falls within the area of the feet. That this does require muscular exertion is shewn by the facts that a person when standing perfectly at rest in a completely balanced position falls when he becomes unconscious, and that a dead body cannot be set on its feet. The line of gravity of the head falls in front of the occipital articulation, as is shewn by the nodding of the head in sleep. The centre of gravity of the combined head and trunk lies at about the level of the ensiform cartilage, in front of the tenth thoracic vertebra, and the line of gravity drawn from it passes behind a line joining the centres of the two hip-joints, so that the erect body would fall backward were it not for the action of the muscles passing from the thighs to the pelvis assisted by the anterior ligaments of the hip-joints. 'The line of gravity of the combined head, trunk and thighs falls moreover a little behind the knee-joints, so that some, though little, muscular exertion is required to prevent the knees from being bent. Lastly, the line of gravity of the whole body passes in front of the line drawn between the two ankle-joints, the centre of gravity of the whole body being placed at the end of the sacrum; hence some exertion of the muscles of the calves is required to prevent the body falling forwards.

$\S 685$. In walking advantage is taken of this forward position of the centre of gravity, and the tendency to fall forwards is utilized to swing each leg in turn forwards after the fashion of a pendulum. In each step there is a moment at which the 
body is resting vertically on one leg, say the right, while the other is inclined obliquely behind. The two legs and the plane of the ground form a right-angled triangle, of which the left leg: is the hypothenuse, the right angle being between the right leg and the ground. At a certain moment the foot of the right leg will be flat on the ground and the line of gravity will pass through its heel. But the centre of gravity is moving forwards: even if there had been no previous steps, and so no momentum, the body and with it the centre of gravity, unless prevented by muscular effort, would have fallen forward; we may therefore speak of the line of gravity as travclling forwards; it passes from the heel to the toe (of the right foot). If the body were simply falling forwards the centre of the hipjoint would move downwards as well as forwards, describing a circle with the leg as a radius. But at the moment of which we are speaking the (right) leg is somewhat flexed, both at the ankle and still more at the knee. And, as the line of gravity is travelling forward from the heel to the toe, the active part of the performance intervenes. The foot is raised from the ground from the heel forwards, until it is only the ball of the great toe which is resting on the ground, and the whole leg is, by muscular effort, straightened. In this act the right leg acts as a lever, the ball of the great toe serving as a fulcrum; and the effect of the act is to prevent the centre of gravity, or the hip-joint, from moving downwards, and to carry it forwards only in more nearly a straight line. In thus carrying the hips (and body) forward the leg has changed its position; from being vertical and flexed with the whole sole resting on the ground, it has become inclined forwards obliquely, extended straight, with the toes only resting on the ground. It has assumed the same posture as that of the left leg at the moment at which we started.

Even at that moment the left leg was behind the line of gravity, and unless it moved would become more and more so as the changes in the right leg went on; hence if left to itself it would swing forward much as a pendulum which had been raised up would swing forward when let go. And during the elianges in the right leg which we have just described the left does swing forward, its movement being chiefly determined like that of a pendulum by gravity, though it may be assisted by direct muscular effort, and is certainly so guided, being for instance slightly flexed during the transit. It swings forward in front of the line of gravity and is thus brought to the ground, the toes in proper walking making contact first and the heel later, though many people who wear shoes bring the heel down at least as soon as the toes. It swings we say in front of the line of gravity; but that line of gravity is travelling forwards, so that in a very short time the body is resting 
vertically on the left leg, with the line of gravity falling at the left heel. That is to say, the left leg has now assumed the position which the right leg had when we began; meanwhile, as we have seen, the right leg has assumed the former position of the left leg; the step is completed, and the movements of the next step merely repeat those of the one which we have described.

It is obvious from the above that in walking there are in each step periods when both feet are touching the ground, and periods when one or the other foot is raised from the ground, but there is no period when both feet are off the ground. This is shewn in the diagram, Fig. 193, which represents two steps.

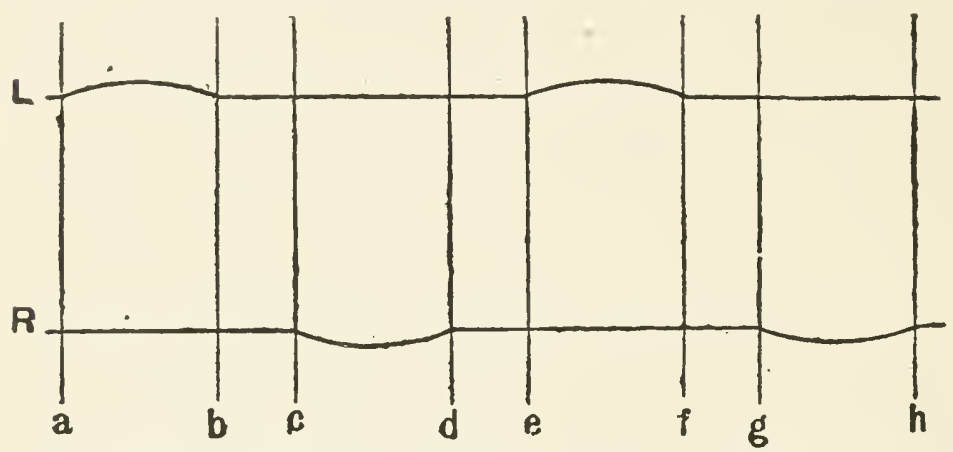

Fig. 193. Diagram to illustrate the contact of the Feet with the Grouxd In WaLking.

$R$, the right foot. $L$, the left foot. In each case the curved line represents the time when the foot is not in contact with the ground, and the straight line when it is in contact.

During $a-b$, the left leg (L) leaves the ground as indicated by the curving of the line. During $b-c$ both feet are on the ground. During $c-d$ the right leg (R) is above but the left (L) is still on the ground. During $d-e$ both are on the ground and the double step is completed, the next step beginning again at $e$ with the left leg leaving the ground.

IVe have said that the centre of gravity is in walking prevented from moving downwards as well as forwards, as it would do in the act of falling forwards. It does not however describe a straight line forwards, it, and with it the top of the head, rises and falls at each step of each leg, and hence describes a serics of consecutive curves not unlike the line of flight of many birds.

Since in standing on both feet the line of gravity falls between the two feet, a lateral displacement of the centre of gravity is necessary in order to balance the body on one foot. Hence in walking the centre of gravity describes not only a series of vertical, but also a series of horizontal curves, inas- 
much as at each step the line of gravity is made to fall alternately on each standing foot. While the left leg is swinging, the line of gravity falls within the area of the right foot, and the centre of gravity is on the right side of the pelvis. As the left foot becomes the standing foot, the centre of gravity is shifted to the left side of the pelvis. The actual curve described by the centre of gravity is therefore a somewhat complicated one, being composed of vertical and horizontal factors.

The natural step is the one which is determined by the length of the swinging leg, since this acts as a pendulum; and hence the step of a long-legged person is naturally longer than that of a person with short legs. The length of the step however may be diminished or increased by a direct muscular effort, as when a line of soldiers keep step in spite of their having legs of different lengths. Such a mode of marching must obviously be fatiguing, inasmuch as it involves an umecessary expenditure of energy.

In slow walking, which Fig. 193 may be taken to illustrate, there is an appreciable time during which, while one foot is already in position to serve as a fulcrum, the other, swinging, foot has not yet left the ground. In fast walking this period is so much reduced, that one foot leaves the ground the moment the other touches it; hence there is practically no period during which both feet are on the ground together; this might be shewn by omitting $b-c$ and $d-e$ in Fig. 193.

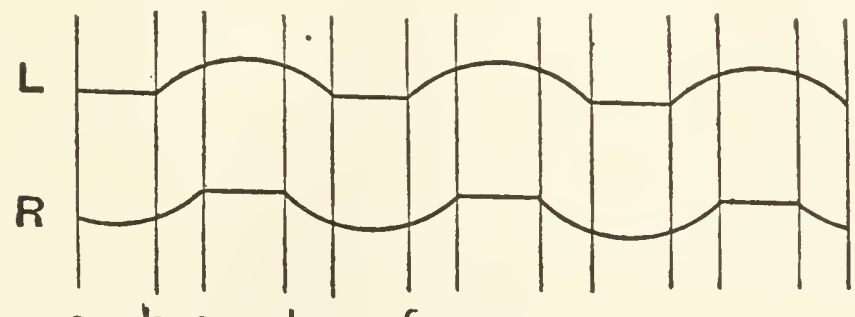

a b c d e $f g$

Fig. 194. Diagram to illustrate Running.

$L$, the line of contact with the ground of the left, $R$ of the right foot; in each case the curved portion of the line represents the time during which the foot leaves the ground.

When the body is swung forward on the one foot acting as a fulcrum with such energy that this foot leaves the ground before the other, swinging, foot has reached the ground, as shewn in Fig. 194, there being an interval, $b-c, d-e$ in the figure, during which neither foot is on the ground, the person is said to be running, not walking.

In jumping this propulsion of the body takes place on both feet at the same time; in hopping it is effected on one foot only. 



\section{BOOK IV.}

THE TISSUES AND MECHANISMS OF REPRODUC'TION. 



\section{REPRODUCTION.}

\$686. MANy of the individual constituent parts of an animal body are capable of reproduction, i.e. they can give rise to parts like themselves; or they are capable of regeneration, i.e. their places can be taken by new parts more or less closely resembling themselves. The elementary tissues undergo during life a very large amount of regeneration. Thus the old epithelium scales which fall away from the surface of the body are succeeded by new scales from the underlying layers of the epidermis; old blool-corpuscles give place to new ones: wornout muscles, or those which have failed from disease, are renewed by the accession of fresh fibres; divided nerves grow again; broken bones are united ; connectire tissue seems to disappear and appear almost without limit; new secreting cells take the place of the old ones which are cast off; in fact, with the exception of some cases, such as cartilage, and these doubtful exceptions, all those fundamental tissues of the body which do not form part of highly differentiated organs are, within limits fixed more by bulk than by anything else, capable of regeneration. To that regeneration by substitution of molecules, which is the basis of all life, is added a regeneration by substitution of mass.

In the higher animals regeneration of whole organs and member's, even of those whose continued functional activity is not essential to the well-being of the body, is never witnessed, though it nay be seen in the lower animals; the digits of a newt may be restored by growth, but not those of a man. And the repair which follows even partial destruction of highly differentiated organs, such as the retina, is in the higher animals very imperfect.

In the higher animals the reproduction of the whole individual can be effected in no other way than by the process of sexual generation, through which the female representative element or ovum is, under the influence of the male representative or spermatozoon, developed into an adult individual.

We do not purpose to enter here into any of the morpholog- 
ical problems connected with the series of changes through which the ovum becomes the adult being; or into the obscure biological inquiry as to how the simple, all but structureless orum contains within itself, in potentiality, all its future developments, and as to what is the essential nature of the male action. These problems and questions, which are fully discussed in other works, do not properly enter into a work on physiology, except under the view that all biological problems are, when pushed far enough, physiological problems. We shall limit ourselves to a brief survey of the more important physiological phenomena attendant on the impregnation of the ovum, and on the nutrition and birth of the embryo, incidentally calling attention to some of the leading structural features of the parts concerned. 


\section{CHAPTER I.}

\section{IMPREGNATION.}

\section{SEC. 1. MENSTRUATION.}

$\S 687$. Frow puberty, which may be said to occur at from 13 to 17 years of age, to the climacteric, which may be said to arrive at from 45 to 50 years of age, the exact time in each case varying considerably and being apparently determined by various conditions, the human female is subjected monthly to a discharge from the vagina known as the 'menses,' 'catamenia,' and by many other terms. The discharge is the result of changes in the lining membrane of the uterus. A similar change in the uterus occurs in the lower animals, being repeated at intervals differing in length in different animals, and is usually accompanied by sexual excitement and changes in the external genital organs; the phenomena are then spoken of by such names as 'heat,' 'rut,' \&c.

The female human, and other, is also subject to another recurring process, the escape of an ovum from its Graafian follicle in the ovary, a process spoken of as "ovulation." The changes of the uterus during menstruation have the appearance of being a preparation for the reception of a discharged ovum so that the latter if fertilized may be developed into an embryo; and there are many reasons for thinking that the acts of ovulation and menstruation are coincident with a causal connection between the two. But this cannot be regarded as definitely proved, and many observers maintain that menstruation may and does occur without any discharge of an ovum, and conversely that an ovum may be discharged, as in copulation, quite apart from menstruation.

The discharge of an ovum, whether at menstruation, or at another time appears to take place as follows. The whole ovary at this time becomes congested, the blood vessels being so dilated and filled with blood that we may almost speak of the 
condition as one of erection; and the ripe follicle, whose ovum is about to escape, bulges from its surface. "The most projecting portion of the wall of the follicle, which has previously become excessively thin, is now ruptured, apparently by the mere distension of the cavity, and the orum, now lying close under the projecting surface of the follicle, escapes, invested by some of the cells of the discus proligerus, into the Fallopian tube. Much discussion has taken place as to how the entrance of the orum into the Fallopian tube is secured. It is probable that under ordinary circumstances the ovary is embraced by the trumpet-shaped fimbriated mouth of the Fallopian tube, and the contact is probably rendered more complete by the turgid and congested condition of both organs; it is possible that the plain muscular fibres present in the mouth of the tube mav assist, and indeed gliding movements of the mouth of the tube over the ovary have been observed in animals. It has, however, been asserted that the turgescence of the tube does not occur until after the orum has become safely lodged in the tube, and it is argued that the ovum is carried in the proper direction by currents set up by the action of the ciliated epithelium lining the tube, currents whose direction and strength seem, as shewn by experiment, to be adequate to carry into the uterus particles present in the peritoneal fluid; and the groove in the ciliated surface of the ovarian fimbria especially connected with the ovary, suggests itself as the natural path for the ovum. Arrived in the tube, the ovum travels downwards very slowly, by the action probably of the cilia lining the tube, though possibly its progress may occasionally be assisted by the peristaltic contractions of the muscular walls. The stay of the ovum in the Fallopian tube may extend to several days; the channel, as we have seen, is a narrow one, especially at the entrance into the uterus. The escape of the ovum is followed by changes in the follicle and rest of the ovary, leading to the formation of a corpus luteum.

Concerning the exact nature of the changes in the uterus which lead to the menstrual flow there has been and is much discussion. The opportunities for an exact histological investigation of a human menstruating uterus are rare; but from what has been observed under the most favourable circunstances, aided by the results of the study of the monkey which is subject to a periodic change cxceedingly like human menstruation, we may conclude that the changes which occur are somewhat as follows. In the first stage of the period not only does the whole uterus, the cervix excepted, become congested, the blood vessels being distended, and the mucous membrane especially red, thick and swollen, but a new growth takes place in the mucous membrane at least in its more superficial layer's. This growth affects not only the epithelial but also the connective tissue, stroma, 
elements, and the blood vessels become enlarged, more or less irregular spaces filled with blood being developed close under the surface. In this way a modified superficial portion of the mucous membrane becomes differentiated from the underlying portion; and from this modified portion, which may be compared to the decidua of pregnancy of which we shall presently speak, a certain amount of hamolrhage into the cavity of the uterus early takes place. But the blood thus escaping does not form the main menstrual flow. The growth of the modified mucous membrane is immediately followed by its rapid degeneration, and the part so degenerated is cast off, laying bare the deeper, less-changed layers of the mucous membrane, from the torn and open irregular blood spaces of which a more copious flow of blood takes place. It is this freer escape of blood which, mixed with the detritus, or even with conspicuous pieces of the shed membrane and containing many cells resembling leucocytes, constitute the menstrual flow; as the hæmorrhage diminishes, these constituents other than actual blood become more prominent, and the discharge becomes less and less coloured. The degeneration and shedding is in turn followed by new growth from the deeper parts of the mucous membrane left behind, whereby the normal mucous membrane is restored in its entirety. The amount of change which takes place probably differs in different individuals; in some cases possibly the amount of proliferation and subsequent degeneration is relatively slight, the hæmorrhage being more comparable to that from any congested mucous membrane, such as nasal hrmorrhage; in other cases again, according to some observers, the whole thickness of the mucous membrane may be removed, the muscular coat beneath being laid bare. The blood as it passes through the vagina becomes somewhat altered, probably by the influence of the other constituents of the discharge, and when scanty coagulates but slightly; when the flow however is considerable, distinct elots may make their appearance. 


\section{SEC. 2. THE MALE ORGANS.}

$\S 688$. The tail of a spermatozoon may be regarded as a single cilium, the movements of which are of an undulatory character, the waves travelling from the middle piece to the end of the tail; and the statements previously made ( $\$ 86)$ concerning ciliary action may be applied generally to the movement of a spermatozoon. The motion is apparently not a very rapid one, for it has been calculated that a half vibration takes at least a quarter of a second. It has also been calculated that a spermatozoon progresses at the rate of about 2 or $3 \mathrm{~mm}$. a minute.

When discharged semen is left to itself the movements continue for some ( 24 or 48 ) hours, but they appear to last much longer in the female passages. Spermatozoa have been observed in movement when removed from the neck of the living human uterus 5 or even 7 days after coitus; and in some of the lower animals the duration of vitality may be enormously long. Making all allowance for any possible direct nutrition of the living substance of the spermatozoon by means of the fluid of the semen, we must conclude that the encrgy of the movement is derived from the expenditure of what we niay venture to call the contractile material stored up in the middle piece and tail of the organism at its formation; the material of the head we may suppose to be devoted entirely to the work of impregnation. So small a store must be soon exhausted; hence it is difficult to suppose that vigorous movements can be continued for very long periods; and probably the activity of the spermatozoa is largely dependent on the circumstances by which it is surrounded; it may remain motionless in one medium, and become active when the medium is changed. The spermatozoon is probably quiescent so long as it remains in the seminal tubes, but we have no exact information as to whether or no movements begin in the epididymis and vas deferens without exposure to air; and it is possible that after coitus the beginning and maintenance of its vigorous movements may largely depend on the condition of the secretions in the vagina and uterus. In this connection it may be noted that 
the movements of a spermatozoon, like ciliary movements, are favoured by fluids having a weak alkaline reaction, whereas almost any degree of acidity (unless used to neutralize excessive alkalinity) arrests them; and the mucous secretion of the uterus while it is alkaline at the neck of the uterus becomes acid as it passes down the vagina. Hence it might be inferred that those spermatozoa only which rapidly find their way into the os uteri manifest vigorous movements; but it would be dangerous to lay too great stress on this.

$\$ 689$. The semen contains a relatively large quantity of solid matter, and this in turn is to a great extent furnished by the spermatozoa; indeed the spermatozoa form so large a portion of the semen that the chemical substances present in the former are dominant in the latter. The head of a spermatozoon appears to be largely composed of the body or group of bodies known as nuclein or nucleo-albumin, a result which supplies chemical evidence of the nuclear nature of the spermatozoan head; and nuclein forms a considerable portion of the solid matter of the whole semen. Lecithin is also present in the semen in considerable quantity ; otherwise the chemical features of the secretion, which are as yet imperfectly known, present no special interest. The crystals found in dry semen are not as was once thought of a proteid nature but are compound phosphates containing an organic base. As discharged in coitus the semen proper from the testicle is mixed with the prostatic and other secretions.

From the testicle itself various forms of proteid of the globulin class have been extracted; and glycogen is not unfrequently present.

The cavity of the vesicula seminalis serves as a temporary receptacle for the semen, though some secretion, and in some animals a decided quantity, takes place from its interior. In certain animals the secretion clots, and then appears to contain a substance identical with or allied to fibrinogen; in these animals the clot which is thus formed by the mixture of the male secretion with the bloody secretion of the rutting female helps to secure the retention of the former within the female passages. The secretion of the prostate presents no special features, except that it is apt to contain peculiar concentric corpuscles; but the fact that the prostate remains undeveloped in castrated animals suggests that the secretion plays some part in coitus. The glands of Cowper afford a thick mucous secretion.

$\S$ 690. Erectile Tissue. The erectile tissue of the corpora cavernosa and corpus spongiosum consists of an irregular labyrinth formed by trabeculæ composed of connective tissue with abundant elastic elements mixed up with a large but variable amount of plain muscular tissue. The spaces of the trabeculie are lined by spindle-shaped epitheloid plates, resting in some 
cascs on a layer of plain muscular fibres, and are venous sinuses, into which blood finds its way chiefly through the terminal capillaries of the numerous arteries lying in the trabecule but also in some cases by minute arteries opening directly into the spaces; from the sinus the blood finds its way out into smaller regular veins. In the corpora cavernosa, and to a less extent in the corpus spongiosum, the small arteries in the trabeculæ are extremely twisted up and looped, bulging into the venous sinuses as arterial coils, the so-ealled 'helicine arteries.' When the arteries supplying these masses of erectile tissue, namely, the branches of the pudic arteries and dorsal artery of the penis, are constricted, and when the plain muscular fibres of the trabecula are in a state of contraction, whereby the venous spaces are largely elosed, the greater part of the blood flowing through the arteries finds its way by ordinary capillaries into the efferent veins, little blood passes into the venous sinuses, and the whole tissue is relatively small in bulk. When on the other hand the arteries are dilated and in addition the muscular bundles of the trabeculice are relaxed, a large quantity of blood passes into the venous sinuses, these become greatly distended with blood; the whole mass of erectile tissue becomes turgid, and in proportion to the resisting nature of the outer envelope, as is especially seen in the corpora cavernosa, hard and rigid.

$\S 691$. In the $\operatorname{dog}$ and cat, fibres from the anterior roots of the second and first, or sometimes from the third, sacral nerves form the nervi erigentes, which passing to the pelvic plexus are distributed to the penis and to other organs; in the monkey the fibres are supplied by the seventh lumbar and first sacral, sometimes also by the second sacral nerves. They receive this name because stimulation of them leads to erection of the penis; and this results from a vaso-dilator action on the arteries supplying the erectile tissue. Erection of the penis is hence to a large extent a vaso-dilator effect. But not wholly so; the entrance of the blood from the dilated arteries into the venous sinuses is facilitated by the relaxation of the muscular bundles in the trabeculæ, whose contraction would offer an obstacle to the spaces becoming filled. Further the filling of the venous sinuses tends of itself to compress the large longitudinal veins running in the centre of the corpora cavernosa and thus to increase the distension already begun; moreover contractions of the striated muscles, the transversus perinaei, and the bulbocavernosus, between the bundles of which the veins pass, also tend to check the outflow and so to increase the erection. In the dog even powerful stimulation of the nervi erigentes will not produce complete erection; the factors just mentioned are absent, and the blood, though it more or less fills the venous sinuses, flows freely away by the veins.

The dilating action of the nervi erigentes and the nervous 
impulses leading to the subsidiary acts in erection may be set going as part of a reflex action, by stimulation of the glans penis. Of such a reflex act the centre lies in the lumbar spinal cord and erection, with emission of semen, has been witnessed in a $\operatorname{dog}$ after division of the spinal cord in the thoracic region. But erection also takes place as the result of emotions, in which case we may suppose that impulses descending from the brain affect the lumbar centre in a direct manner; and indeed erection has been experimentally brought about by stimulation of certain parts of the brain.

The antagonistic act, namely, constriction of the blood vessels and retraction of the penis may, in the cat, be brought about by stimulation of fibres coming from the upper lumbar (and possibly the lower thoracic) region, and reaching their destination by way of the sympathetic.

$\S 692$. The emission of semen, for. which act erection is preparatory, is carried out by a succession of agencies. The epididymis with its coni vasculosi may be regarded as a reservoir filled by the secretory activity of the seminal tubes; hence its relatively enormous length. It is possible that the act may begin with an increase of secretory activity on the part of the seminal tubes, bearing perhaps especially on the fluid parts of the semen, by which the epididymis becomes overfilled; we have no positive evidence of this. Nor have we evidence of any pressure, either intrinsic by means of the plain muscular fibres which are said to occur scantily in the septa of the testis, or extrinsic through the cremaster or other muscles, being brought to bear on the contents of the seminal tubes. Hence we may conclude provisionally that the act begins with a propulsion of the contents of the distended epididymis by means of peristaltic contractions of the muscular walls of that tube. In any case the flow of fluid having reached the vas deferens, is carried along that tube by the peristaltic contractions of its much stouter and much more muscular walls. In the monkey stimulation of the anterior roots of the second and third lumbar nerves leads to a powerful contraction of the vas deferens, sweeping down it in a single wave.

One effect, possibly a chief effect, of the flow along the vas deferens is to fill and distend the vesiculæ seminales; or we may suppose that preparatory feeble contractions of the epididymis fill and distend both the vas deferens and the vesiculæ seminales, and that the act really begins with a more powerful contraction of both these distended organs by which their contents are rapidly ejected into the prostatic urethra; at the same time contractions of the muscular fibres of the prostate discharge the secretion of that gland into the urethral canal. So far plain muscular fibres only are brought into play; but the act is completer hy the aid of striated muscles, namely, by forcible 
contractions of the levator ani, of the constrictor urethræ including the external sphincter of Henle, of the ischio-cavernous muscle, which starting from the ischium on each side embraces the root of the penis, and of the bulbo-cavernosus muscle (or ejaculator urinæ) which starting from the perinæum embraces the beginning of the urethri and corpus spongiosum. A contraction begins in the external sphincter ani, extends to the levator ani and then passes to the other muscles, progressing in a wave-like manner from behind forwards, and is repeated in a more or less distinctly rhythmic manner until all the semen is ejected from the urethra.

These expulsive contractions, especially the last named, appear like erection to be carried out by the help of a centre in the lumbar region of the cord, and for them afferent impulses generated in the sensitive surface of the glans penis are more essential than for simple erection. In the $\log$ stimulation of the internal pudic nerve throws the whole group of striated muscles just named into successive contractions as described, but each muscle may be made to contract separately by stimulation of its own individual branch.

The semen being received into the vagina, the walls of which, and especially the external appendages of which, are at the time in a state of turgescence resembling the erection of the penis, but less marked, lies, probably, at the far end of the vagina in a pool into which the os uteri dips; and it is possible that contractions of the round ligaments (which contain striated muscular fibres) by tilting the cervix backwards assist in bringing the os uteri into the semen. In this manner the spermatozoa find their way into the uterus and so into the Fallopian tube, where (probably in its upper part) they come in contact with the ovum. In the rabbit spermatozoa may reach the ovary within two hours after coitus. In the case of some animals impregnation may take place at the ovary itself. The passage of the spermatozoa is most probably effected mainly by their own vibratile activity; but in some animals a retrograde peristaltic movement travelling from the uterus along the Fallopian tubes has been observed; this might assist in bringing the semen to the ovum, but inasmuch as these movements are probably parts of the act of coitus and impregnation may be deferred till some time after that event, no great stress can be laid upon them. 


\section{CHAPTER II.}

\section{PREGNANCY AND BIRTH.}

\section{SEC. 1. THE PLACENTA.}

$\S$ 693. THe spermatozoa travelling up the female passages come in contact with the ovum. Making their way through the cells of the discus, which by this time are undergoing degenerative changes, and piercing the zona pellucida, they enter the vitellus; it is stated that as a rule one spermatozoon only actually reaches the vitellus. Here the tail, which by its vibratile activity has thus brought the spermatozoon to its destination, ceases to move and soon disappear's; but the head (which is a prepared and, so to speak, purified nucleus, a male pronucleus) unites with the pronucleus of the ovum to form the nucleus of the now inpregnated ovum.

As the result of this action of the spermatozoon on the ovum, the latter, instead of dying as when impregnation fails, awakes to new nutritive activity. It undergoes segmentation, the one cell becomes by cell-division a mass of cells, which, passing through a series of remarkable morphological changes, into the details of which we cannot enter here, developes into an embryo.

\$ 694. No sooner, however, have these changes begun in the ovum than correlative changes, brought about probably by reflex action, but at present most obscure in their causation, take place in the uterus. The mucous membrane of this organ, whether the coitus, which was the cause of the impregnation, took place at a menstrual period or at some time in the interval, undergoes changes which though more intense are at first not unlike those of menstruation; it becomes congested, and a rapid growth takes place, characterized by a proliferation of the epithelial and other tissues. Unlike what takes place in menstruation, however, this new growth does not give way to hæmorrhage and immediate decay; it remains, and may be dis- 
tinguished as a new temporay lining to the uterus, the socalled decidua. Into this decidua the ovum, on its descent from the Fallopian tube, in which it has already undergone some developmental changes, is received; and in this it becomes embedded, the new growth closing in over it. Meanwhile the rest of the uterine structures, especially the muscular tissue, become also much enlarged; as pregnancy advances a large number of new muscular fibres are formed.

As the ovum, now developing into the embryo and its appendages, continues to increase in size, it bulges into the cavity of the uterus, carrying with it the portion of the decidua which has closed over it. Henceforward, accordingly, a distinction is made in the now rapidly developing decidua between the decidua reflexa, or that part of the membrane which covers the projecting ovum, and the decidua vera, or the rest of the membrane lining the cavity of the uterus, the two being continuous round the base of the projecting ovum. That part of the decilua which intervenes between the ovum and the nearest uterine wall is spoken of as the decidua serotina. As the embryo with its appendages continues to enlarge, carrying with it the decidua reflexa, the latter becomes pushed against the decidua vera, gradually obliterating the cavity of the uterus, except at the cervix; about the end of the third month, in the human subject, the two come into complete contact all over, and ultimately the distinction between them is lost.

The decidua reflexa, and the decidua vera in general, undergo various changes, but these are of subordinate interest compared with those which take place in that part of the decidua vera which is called the decidua serotina, and which lead to a special union between maternal tissues and tissues belonging to the growing embryo, a union which gives rise to the structure known as the placenta or' 'after-birth.'

$\S 695$. During the development of the ovum while some of the cells, arising by cell-division from the primordial cell, become the embryo proper, others form the appendages of the embryo; to the latter belongs the double bag which encloses the embryo, and which consists of an inner bag, the true amnion and an onter bag, the false amnion. The latter over the whole of its surface is in contact with the decidua, and developes a number of branched villi, consisting, like the rest of the membrane, of an epithelium (epiblast) resting on a dermic (mesoblastic) basis; these villi are embedded in or applied to the decidual surface. The false amnion, bearing villi, often called the chorion, is at first devoid of blood vessels; but a diverticulum of the hinder part of the developing alimentary canal of the embryo, called the allantois, grows out rapidly into the space (containing fluid) between the false and the true ammion, and soon applies itself to the former. As it grows, two 
arteries, continuations of the primitive aorta, the allantoic arteries, subsequently called umbilical arteries, make their appearance. These carry the blood of the embryo to the villi of the chorion; from thence it is returned at first to two veins, but ultimately to a single vein running in company with the umbilical arteries, and called the umbilical vein.

At first all the villi over the whole surface of the chorion except at two opposite poles are thus supplied with blood, but ultimately the supply is restricted to that part of the chorion which is applied to the decidua serotina. Here the villi become developed into large and conspicuous vascular tufts, whereas over the rest of the chorion they soon atrophy; and here the placenta is formed through a number of complex changes, the details of which have been and still are the subject of much discussion, changes by which the whole region, stretching from the basal portion of the uterine glands, or even from the uterine muscular coat, to the connective tissue which carries the capillary loops in which the umbilical arteries end, is so altered that it becomes difficult to say which are maternal, which are embryonic elements, which structures are of glandular and true epithelial origin, which of connective tissue or epithelioid origin.

There is evidence that in the formation of the placenta, the hypertrophied glandular mucous membrane, having done its work in nourishing by secretory activity the embryo at an early stage, is, at least in its more superficial portions, absorbed, eaten as it were, by the advancing chorionic vascular tufts. 'This is introductory to the special vascular arrangements of the placenta, the uterine glands making way for the system of blood sinuses; but even in the full-grown placenta we may recognize that the interchange between mother and fotus is effected not in a wholly mechanical manner by the mere bringing into close juxtaposition the maternal and fotal blood, but also by an activity which we may venture to call secretory on the one hand of the epithelium covering the villi, and on the other hand of the decidual cells, whatever may be the exact origin and nature of each of these kinds of cell.

As the nutrition of the embryo becomes more and more concentrated in the altered decidua serotina or placenta, the decidua vera and reflexa, having played their part, are done away with. They are not, however, shed abruptly as in menstruation; they are returned piecemeal by absorption into the maternal system; they atrophy until the whole reflexa and the superficial part of the vera is reduced to a mere membrane adherent to the expanded chorion, while the basal portion of the vera remains to grow up after the birth of the fœetus into a normal mucous membiane.

The serotina having become the maternal portion of the 
placenta continues its functions during the whole of the intrauterine life of the embryo. When the term of the maternal nutrition of the embryo is ended and birth takes place, there is a sudden disruption of tissue along the line of the decidual layer, either where this joins the muscular coat, the whole mucous coat being subsequently renewed, or at some little distance from it, the 'basal remnants' of the glands being left to grow up into the new mucous lining; and the transformed serotina, like the changed mucous membrane of menstruation but even more suddenly and abruptly, is shed as the "after-birth." With the placenta there are also shed the so-called 'membranes,' that is to say the amniotic membranes together with the membranous remnants of the vera and reflexa, which have become adherent to and fused with these. Hence ultimately the whole decidua, the whole transformed mucous niembrane of the pregnant uterus, like the changed mucous membrane of menstruating uterus is, though in a different manner, cast off.

We may add that the form and structure of the placenta and the mode of connection between the mother and the embryo differ in different placental animals; in all cases, however, the blood of the chorionic villi of the embryo are bathed in sinus-like blood-spaces of the mother. In all cases too there is a development around the villi of epithelial structures of a secretory character' in ruminant animals collections of such cells form what is called 'uterine milk.' It is in these cells belonging to the border line between mother and infant, whether they are of maternal or of embryonic origin, that the glycogen, which is so often present in the placenta, is placed, and the presence of this substance may be taken as a token of the metabolic activity of these cells. 


\section{SEC. 2. THE NUTRITION OF THE EMBRYO.}

$\S 696$. In a hen's egg a very small part only of the whole egg, namely, a minute collection of cells called the blastoderm, is actually developed into the chick and its appendages; by far the greater part of the mass included within the egg-shell, namely the 'yolk' and the 'white,' is mere nutritive material. Through the porous egg-shell the oxygen of the air has adequate access to the contents within, and through the same egg-shell carbonic acid can escape. The yolk and the white supply all the food needed by the developing chick until it is hatched, and either directly or indirectly by means of the allantoic vessels the tissues of the embryo and its appendages breathe through the shell.

In the mammal the supply of yolk is insignificant; almost from the first the developing ovum receives nutritive material from the mother. Within the ovary the ovum is fed by the cells of the Graafian follicle; and a similar mode of feeding is continued for some little time in the uterus. The repeated cell division of the ovum produces a compact mass of cells, the 'mulberry mass,' and this in turn is converted into the 'blastodermic vesicle,' which consists of a cellular membrane investing fluid contents; during this conversion a considerable increase in the total bulk of the ovum takes place, water and nutritive material passing into the ovum from the mother, probably from the cells lining the Fallopian tube. Received within the uterus and covered up by the decidua, the developing embryo is supplied with food and oxygen by the cells of the uterine mucous membrane with which it lies in contact, very much in the same way that the growing ovum was supplied by the cells of the Graafian follicle; and the same uterine cells carry away the scanty waste matters of the embryo's nutritive activity.

The amount of food which the embryo needs and receives is at first small but continually and rapidly increases; the amount of oxygen which the embryo needs is at first insignificant, but the need of oxygen also increases continually and rapidly, though especially during the early stages it is limited by the fact that the processes going on in the embryonic tissues are largely 
synthetic, directed to the building up of the tissues, and snch processes consume very little oxygen compared with the processes leading to expenditure of energy in movement and heat. Hence the simple method of nutrition and respiration by means of the direct contact of the cells of the uterine mucous memlrane is exchinged for the special vascular mechanism of the placenta, by which the embryo lives upon and breathes through the uterine blood of the nother. From an early period up to birth the placental circulation is the chief, we may almost say the only means by which the embryo breathes and is fed; but the details of the placental events are changing during the whole of this time. 'The embryo, all the while increasing in bulk, passes through phase after phase; the structural features of one day give way to those of the next, its morphological history being as it were a series of dissolving views; and each new structural phase entails new functional events both in the embryo itself and in the placenta. 'This is perhaps especially seen in the earlier stages at a time when the placental circulation has becn established in its main outlines, but in the embryo most of the future organs are still in a shadowy inchoate condition. At this epoch, of the total bulk of blood coursing from the embryo towards the tissues of the mother and back again, the greater part is at any one moment to be found in the placenta and only a small part in the tissues of the embryo itself ; later on the blood is equally divided between the placenta and the embryo; and still later the embryo has the larger share, and it is the smaller part which is at any one moment flowing through the chorionic villi of the placenta. There can be no doubt that in the earlier phase the influences which the placental structures exert on the foetal blood are in many ways different from those which are exerted later on. IVe find that during the earlier phases the cellular placental elements are correspondingly prominent, indicating that much labour of the kind for which cells are necessary is being then carried on, whereas in the later stages the placental mechanism approaches though it never quite reaches the more mechanical conditions of a simple membrane separating the foetal and maternal blood. We camnot enter at all fully here into the successive phases; we must confine ourselves chiefly to the main features of what is going on during the latter months of gestation when the placental eirculation is in full swing.

$\$ 697$. At this time the somewhat rapid strokes of the fœtal heart drive the foetal blood through the umbilical arteries to the capillaries of the chorionic villi, from whence it is returned by the umbilical vein. From experiments on lambs and other animals it would appear that the blood pressure in the umbilical artery is moderately high ( 40 to $80 \mathrm{~mm}$. Fg.) and that in the umbilical vein very considerable (15 to $30 \mathrm{~mm}$. IIg.), higher than the venous pressure in the mother in a vein of correspond- 
ing size; the difference between the arterial and venous pressure is therefore relatively less than in the mother. Corresponding to this the velocity of the blood flow is relatively low. 'The number of red corpuscles in a given bulk of foetal blood, which was of course at first very scanty, has by this time much increased, but as a rule remains up to the end less than that of the mother, though this has become diminished by the pregnancy. In many cases no marked distinction of colour can be observed between the blood in the umbilical arteries and that in the umbilical vein, but such difference as can be noted is in the direction of the blood in the vein being brighter than that in the arteries, and at times this is conspicuously the case. If, for instance, the foetus at the time of observation happens to make prolonged movements, the contrast between the clark blood of the umbilical arteries and the bright blood of the umbilical vein may become striking. An examination of the gases of the blood shews that the blood in the vein contains more oxygen and less carbonic acid than that of the arteries; the former for instance has been found to contain from 7 to 20 p.c. of oxygen and 40 p.c. of carbonic acid, the latter 2 to 6 p.c. of oxygen and 46 p.c. of carbonic acid. Hence the blood in the umbilical vein is essentially arterial blood, and that in the umbilical arteries essentially venous blood. It may be observed that while as regards the amount of carbonic acid the blood of the foetus runs parallel to that of the mother, the arterial blood of the foetus (in the umbilical vein) contains less oxygen than that of the mother. This is not due alone to the relatively smaller annount of hæmoglobin, for as shewn by experiment the hamoglobin of the foetal arterial blood is far from being saturated with oxygen, whereas as we have seen ( $\$ 286$ ) that of the adult is, or very nearly so. WVe may add that the foetal blood left to itself uses up its free oxygen rapidly, very much more rapidly than does adult blood.

The maternal blood is conveyed to the placental sinuses by arteries which open directly into the sinuses. Hence, though independently of any influence exerted by the foetal blood the blood returned from the sinuses by the uterine veins is venous blool, rendered venous by the maternal tissues themselves, yet the blood in the sinus to which the capillaries of the villi are exposed may be regarded as rather arterial than venous, and in any case contains more oxygen and less carbonic acid than does the foetal blood arriving by the umbilical arteries. Seeing that the relatively narrow uterine arteries open out suddenly in the wide placental sinuses the flow in the latter must be slow; the flow in the foetal vessels is also as we have seen not rapid; hence ample time is given for the interchange of gases. The change which is thus effected is probably carried out by diffusion, the amount of change being determined by the relative percentages 
of the gases in the maternal and fotal blood. At least we have no more evidence in the case of this placental respiration than we had in the case of the pulmonary respiration that the interchange is in any way assisted by cellular activity of a secretory kind. The placental respiration of the mammal seems in fact exactly to repeat the branchial respiration of the fish; in the former the foetus breathes by means of the maternal blood in the same way that in the latter the fish breathes by means of the water in which it lives.

It follows from the above that the fœtus may be asphyxiated in two ways: on the one hand by interference with the access of foetal blood to the placenta, as when the cord is tied, and on the other hand by the maternal circulation being arrested, or by the maternal blood being wanting in oxygen. When the mother is asphyxiated the fotus is asphyxiated too, the oxygen passing from the foetal blood to that of the mother. In such a case, owing to the more imperious demands of the maternal blood, the store of oxygen in the foetal blood is sooner exhausted and asphyxia is more rapidly developed than in the case when the cause lies in the fœtus, not in the mother, and the oxygen simply disappears from the fotal blood as it is slowly used up by the fotal tissues; for the rate of fotal oxidation though it increases continually during the intra-uterine life, especially in the later stages, is slow compared to what it becomes some time after birth.

$\S 698$. The foetus not only breathes but also feeds and probably excretes by means of the placenta; the blood returning by the umbilical vein is not only richer in oxygen and poorer in carbonic acid but also richer in nutritive material and poorer in waste products than the blood of the umbilical arteries. In dealing however with the nutrition of the embryo we must bear in mind a special condition under which the embryo lives. As we have sair the embryo proper becomes at an early date invested with the double membranous bag of the amnion, consisting of the immer ammion and outer (false) amnion. Between the two there is at first a space, into which as we have seen the allantois grows in order to become the placenta; but, as the fluid, which from the first is present within the inner bag, increases in amount, without any corresponding increase in the fluid between the inner and outer bag, the (true) amnion in its expansion after the formation of the placenta reaches and unites with the false amnion which by this time is known as the chorion. The whole interior of the uterus is lined, next to the decidua, by a menbrane apparently simple but composed of united amnion and chorion, and within this, surrounding and supporting the embryo, lies the ammiotic fluid, which at first scanty rapidly increases in amount until in the later stages of pregriancy it may amount to 800 c.c. or even much more. 
In the roof of the uterus, in the region of the placenta, the amniotic fluid is in close proximity not only to the branching umbilical arteries and veins of the fœetus, but also to many of the maternal blood vessels, being separated from the maternal blood by nothing more than the thin wall of the blood vessel and the membrane just spoken of. The fluid is also over the rest of the internal surface of the uterus, in close proximity to the blood vessels of the maternal decidua, and indeed in the later stages, when the decidua apart from the placenta has largely retrograded, to the blood vessels of the uterine mucous membrane. The conditions therefore are favourable for the transudation of material from the blood of the mother into the amniotic cavity; and we have experimental evidence that not only water but various substances may pass in this way from the one to the other. If indigo-carmine ( $\$ 336)$ be injected into the veins of the mother, none passes by the umbilical vein into the tissues of the fotus; these remain wholly uncoloured. Yet the amniotic fluid becomes deeply tinged with the pigment, which obviously must have passed directly from the mother into the amniotic cavity. Hence we may conclude that though the amniotic fluid is at first derived exclusively from the foetus, and during the whole time is partly derived from the same source, it is also, and especially in the later stages, largely derived by direct transudation from the mother.

Into this amniotic space the passages of the fœtus, the mouth, anus, \&c. open, and it serves as we shall see as a repository for the excretions of the foetus. Into it is discharged such urine as the foetus secretes, into it are shed. the fotal epidermic scales, and appendages such as hair's, and into it may be discharged the contents of the alimentary canal, known as the meconium. Now, hairs, epidermic scales, in the case of hoofed mammals portions of shed hoofs, and at times meconium have been found in the fotal stomach; they arrived there by the foetus swallowing the amniotic fluid; we have other evidence that the foetus in the uterus may execute swallowing movements, and if these are executed they must lead to swallowing of the amniotic fluid, since this will pass into the mouth and pharynx whenever the mouth is opened. If these swallowing movements occur frequently, and there is some evidence that they do, nutritive material contained in the fluid and derived directly from the mother, might thus be conveyed to the foetus; the latter might be nourished by means of the amniotic fluid. But, even making all allowance for any possible nourishment in this way, we may probably regard it as insignificant compared with that which is carried on by the placental and umbilical vessels; we may assume that the food of the futus reaches it mainly by passing from the maternal sinuses into the capillaries of the chorionic villi. 
$\$$ 699. Judging from analogy we may conclude that the food of the foutus consists, like that of the adult, of proteids, fats, carbohydrates and salts conveyed in water. In attempting to understand how these materials pass from the blood of the matermal sinus to the blood of the foutal villus, we have to face problems of the same kind as those which we met with in considering absorption from the alimentary canal (\$253).

Here as there diffusion and filtration play their parts; but here also as there the passage of material does not follow the laws of diffusion and filtration which regulate the passage of material through non-living nembranes. We have evidence that diffusible substances pass readily from mother to foetus and from foetus to mother. When sugar is injected in considerable quantity into the vessels of the mother, it is found in excess in the tissues of the futus. When such a drug or poison as atropin is injected into the mother it passes to the futus, and manifests its presence there by dilation of the pupils. Not only may the foetus be killed by injection of strychnine into the mother, but the mother may be killed by the injection of strychnine carefully restricted to the foetus. Again, if curare, which is inert towards the foetus at least up to a certain dose, be injected into the fotus, the mother is affected by the drug, the fact that the drug does not poison the foetus assisting in its transmission to the mother ; this result is especially worthy of notice since curare has a very low diffusible power. The influence of diffusion seems to be further illustrated by the fact that if large quantities of sugar or other diffusible substance be injected into the blood vessels of the mother, while the thickened plasma of the maternal blood is diluted by the entrance of water, as shewn by the diminished proportion of red corpuscles, that of the foutus as shewn by the same method undergoes concentration; water passes from the fotal blood to meet the needs of the maternal blood.

Nevertheless that in the passage of nutritive material from the mother to the foetus, and of waste products from the foetus to the mother, we liave to deal with something more than ordinary diffusion, is shewn by the fact that the specific gravity of the fotal blood differs from, being definitely above, that of the matermal bloor; if diffusion had its full power the specific gravities of the two bloods would soon become equalized. Although exact information concerning the matter is at present very linited or almost wholly wanting, it is probable that the epithelium cells of the placenta, either those of the villi or the 'decidual' cells or both, play a part not unlike that played by the epithelium of the alimentary canal or even play a more important part. Whether the proteids of the maternal blood undergo a change analogous to peptonification in passing to the fotus, whether the mother furnishes fat to the foutal blood, 
and if so how, - to these and other questions which suggest themselves no very satisfactory answer can at present be given. With regard to fat, leaning on the analogy of the conclusion at which ( $\$ 486)$ we arrived, that in the adult the fat of the food is probably not taken up by the tissues as fat during the nutrition of the tissues by the blood, we may perhaps suppose that the mother does not supply the foetus with fat as such. We have already referred to the significant presence of glycogen in the placenta; and it would almost seem as if the placenta exerted at one and the same time on the material passing from the mother to the fotus influences comparable not only with those exerted by the walls of the alimentary canal but also with those subsequently exerted by the hepatic cells on the material which passes by way of the portal vein from the intestines to the right side of the heart. Again the very phrase "uterine milk" suggests that the placenta epithelial cells exercise a secretory and metabolic influence comparable to that of the mammary gland. But how far these analogies are false or true future research must letermine; and putting aside for a while the special problems thus suggested we may, in a broad way, say that the foetus lives on the blood of its mother, very much in the same way that all the tissues of any animal live on the blood of the body of which they are the parts.

$\S 700$. For a long time all the embryonic tissues are 'protoplasmic' in character; that is to say, the gradually differentiating elements of the several tissues remain still embedded in undifferentiated material; and during this period there must be a general similarity in the metabolism going on in various parts of the body. As differentiation becomes more and more marked, it obviously would be an economical advantage for partially elaborated material to be stored up in various foetal tissues, so as to be ready for immediate use when a demand arose for it, rather than for a special call to be made at each occasion upon the mother for comparatively raw material needing subsequent preparatory changes. Accordingly, we find the tissues of the futus at a very early period loaded with glycogen. The muscles are especially rich in this substance, but it occurs in other tissues as well. The abundance of it in the former may be explained partly by the fact that they form a very large proportion of the total mass of the fortal body, and partly by the fact that, while during the presence of the glycogen they contain much undifferentiated substance, they are exactly the organs which will ultimately undergo a large amount of differentiation, and therefore need a large amount of material for the metabolism which the differentiation entails. It is not until the later stages of intra-uterine life, at about the fifth month, when it is largely disappearing from the muscles, that the glycogen begins to be deposited in the liver. By this 
time histological differentiation has advanced largely, and the use of the ghrogen to the economy has become that to which it is put in the ordinary life of the animal; lience we find it deposited in the usual place. We do not know how much carbohydrate material finds its way into the umbilical vein; and we cannot therefore state what is the source of the foetal glyeogen; but it is at least possible, not to say probable, that it arises, in part at all events, from a splitting up of proteid material in the foetal body.

$\$ 701$. Concerning the rise and development of the func tional activities of the embryo, our knowledge is almost a blank. We know scarcely anything about the various stejs by which the primary fundamental qualities of the living matter of the ovum are differentiated into the complex phenomena which we have attempted in this book to expound. We can hardly state more than that while muscular contractility becomes early developed, and the heart probably, as in the chick, beats even before the blood-corpuscles are formed, movements of the foetus are in the human subject first felt about the sixteenth week; they probably oceur before but are not easily recognized, while from that time onward they increase and subsequently become very marked. They are often spoken of as reflex in character, and some of them are undoubtedly of this nature. When the uterus of a pregnant animal is prematurely opened, various reflex movements of the foetus may be excited by appropriate stimulation, different kinds of animals differing in this respect as they do with regard to the powers of the new-born animals. Such reflex movements may be witnessed before the placental circulation has been interrupted, but they are increased if the fuetus be made to breathe. We have already referred to swallowing movements; and may add that an immature fortal animal may be made to bite by introdueing the finger into its mouth. Some of these normal intra-uterine movements appear however to be not reflex but automatic if not voluntary in nature. Movements of the limbs, apparently automatic, have been observed in foetuses in which the brain has not been developed. We may ard that in the human subject the occurrence of intra-uterine convulsions is fully acknowledged.

$\$ 702$. The digestive functions are naturally, in the absence of all food from the alimentary eanal, in abeyance. Though pepsin may be found in the gastric membrane at about the fourth month, it is doubtful whether a truly peptic gastric juice is secreted during intra-uterine life; trypsim appears in the pancreas somewhat later, but an amylolytic ferment cannot be obtained from that organ till after birth. The date however at which these several ferments make their appearance in the embryo appears to differ in different animals. 'The excretory functions of the liver are developed early, and about the third 
month bile-pigment and bile-salts find their way into the intestine. The quantity of bile secreted during intra-uterine life accumulates in the intestine and especially in the rectum, forming, together with material secreted by the walls of the alimentary canal and sone desquamated epithelium, the so-called meconium. Human meconium is found to contain about 20 p.c. of solids. These consist of a considerable quantity of cholesterin ( $\cdot 7$ p.c.), some fatty acids, bile salts with bile pigments, both largely unaltered, and calcium and sodium salts; the ash is rather more than 1 p.c. Though bile contributes normally to form the meconium, it is not essential, for a considerable quantity has been found in the futus in cases where the liver has been absent.

The distinet formation of bile is an indication that the products of fuetal metabolism are no longer wholly carried off by the maternal circulation; and to the excretory function of the liver there are now added those of the skin and kidney. Since in man, and in many other animals, such substances as are secreted by the kidney find their way at an early date into the cavity of the amnion, the determination of the history of the renal secretion is a matter of difficulty, for as we have seen the amniotic fluid is derived in part at least directly from the mother, and substances present in it may or may not have been discharged into it by the fotus. The amniotic fluid varies not only in quantity but also in specific gravity $(1.002$ to 1.086$)$ and in composition, and there does not seem to be any definite relation between its specific gravity and the quantity in which it occurs, or between its specific gravity and the size or age of the foetus. It may be said to contain on the average about 1.6 p.c. of solid matter, of which about $\cdot 2$ are proteids, $\cdot 8$ extractives and $\cdot 6$ salts. The proteids are serum albumin and probably paraglobulin, mucin or a mucin-like body being also present. Sugar appears to be sometimes present, sometimes absent. The most important constituent is perhaps urea, which seems to be always present. Since this is found at quite an early stage, before any secretion from the fotal kidney could take place, it may be thus considered as derived from the mother and comparable in origin to the urea found in serous fluids; but since urine containing urea is found in the fortal bladder at lcast as early as the seventh month, we may conclude that during the later stages of pregnancy, and possibly much earlier, part of the urea of the amniotic fluid comes from the fotal kidney. In some animals, ex. gr. ruminants, the cavity of the allantois remains for a long time permanent and filled with fluid, instead of as in man becoming at an early date obliterated in its distal portion. In these animals the kidneys discharge their secretion into this allantoic sac, and in the contents of the sac is found the body allied to urea, allantoin, so called from its having 
bcen first discovered in this situation. 'Traces of allantoin have also been found in human amniotic fluid, which result suggests that this substance is at any early stage formed by the kidney but subsequently gives place to the permanent urea.

There is no evidence that any sweat is secreted by the foetus in the uterus; and indeed if any such secretion does take place this can only be for the discharge of solid matter, and not as in the adnlt for the discharge of water; but the epidermic seales are undoubtedly shed, and may be detected in the amniotic fluid.

$\$$ 703. About the middle of intra-uterine life, when the fortal circulation is in full development, the blood flowing along the umbilical vein (see Fig. 195) is chietly carried by the ductus venosus into the inferior vena cava and so into the right auricle. 'Thence it appears to be directed by the valve of Eustachius through the foramen ovale into the left auricle, passing from which into the left ventricle it is driven into the arorta. Part of the nmbilical blood, however, instead of passing directly to the inferior cava, enters with the blood carried by the portal vein into the hepatic circulation, from which it returns to the inferior cava by the hepatic veins. 'The inferior cava also contains blood coming from the lower limbs and lower trunk. Hence the blood which passing from the right auricle into the left auricle through the foramen ovale is distributed by the left ventricle through the aortic arch, though chiefly blood coming direct from the placenta, is also blood which on its way from the placenta has passed through the liver and blood derived from the tissues of the lower part of the body of the foutus. The blood descending as fwetal renous blood from the head and limbs by the superior vena cava appear's not to mingle largely with that of the inferior vena cava, but to fall into the right ventricle, from which it is discharged through the ductus arteriosus (Botalli) into the aorta below the arch, whence it flows partly to the lower trunk and limbs, but chiefly by the umbilical arteries to the placenta. A small quantity only of the contents of the right ventricle finds its way into the lungs. Now the blood which comes from the placenta by the umbilical vein direct into the right aur;ele is, as far as the respiration of the foetus is concerned, arterial blood; and the portion of umbilical blood which traverses the liver probably loses at this epoch very little oxygen during its transit through that gland, the liver being at this period much more a simple excretory than an actively metabolic organ. Ilence the blool of the inferior vena cava, though mixed, is on the whole arterial blood; and it is this blood which appears to be sent by the left ventricle through the arch of the aorta into the carotid and subclavian arteries. Thus the head of the fortus is provided with blood comparatively rich in oxygen. 'The blood descending from the head 
and upper limbs by the superior vena cava is distinctly venous; and this passing from the right ventricle by the ductus arteriosus is driven along the descending aorta, and together with some of the blood passing from the left ventricle round the

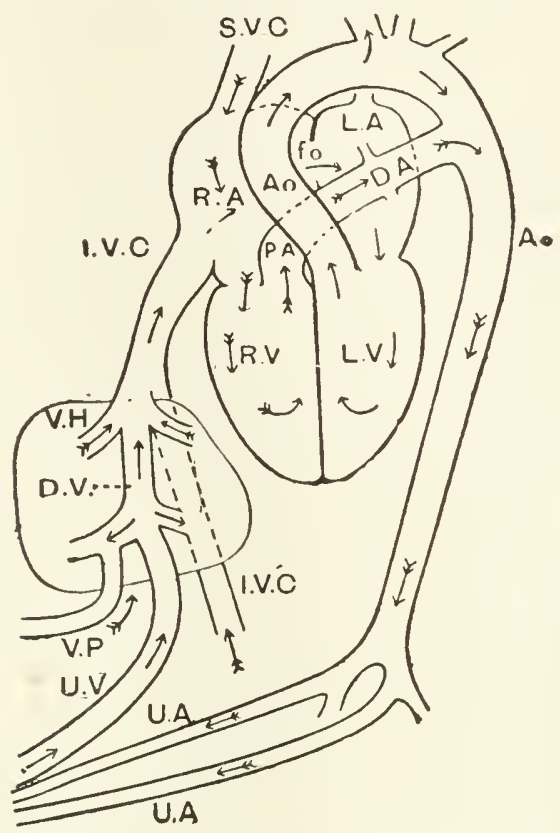

Fig. 195. Diagran to illestrate the Fetal Circulation.

It will be understood that the figure is purely diagrammatic and constructed simply to shew in a convenient manner the general course taken by the blood.

The winged arrow indicates venous, the plain arrow arterial, or, in parts, mixed blood.

$U V$. The umbilical vein, passing in part to the liver (indicated in outline), joined by blood from the alimentary canal along the mesenteric, becoming the portal vein $V . P$., but chiefly flowing on by the ductus venosus $D . V$. (into which fall the hepatic veins $V . H$.) into the inferior vena cava, $I . V . C$.

This chiefly arterial but still mixed blood passes through the right auricle $R$. A., the foramen ovale f.o. to the left auricle $L . A$., thence to the left ventricle $L . V$. and so by the arch of the aorta $A o$. to the arteries of the head and upper limbs.

The venous blood of the head and upper limbs passes from the superior vena cava $S . V . C$. through the right auricle to the right ventricle $R \cdot V^{\top}$. and thence by the pulmonary artery P.A. and ductus arteriosus $D . A$. to the descending aorta, and so to the umbilical arteries $U$.A.

aortic arch falls into the umbilical arteries and so reaches the placenta. The fotal circulation then appears to be so arranged that, while the most distinctly venous blood is driven by the right ventricle back to the placenta to be arterialized, the most 
distinctly arterial (but still mixed) blood is driven by the left ventricle to the cerebral structures, which, we may conclude, have more need of oxygen than have the other tissues. Contrary to what takes place afterwards, the work of the right ventricle is in the foetus greater than that of the left; and, accordingly, that greater thickness of the left ventricular walls, so characteristic of the adult, does not become marked until close upon birth.

$\$ 704$. In the later stages of pregnancy the mixture of the various kinds of blood in the right auricle increases preparatory to the changes taking place at birth. But during the whole time of intra-nterine life the amount of oxygen in the blood passing from the aortic arch to the brain is sufficient to prevent any inspiratory impulses being originated in the bulbar respiratory centre. This, during the whole period elapsing between the date of its structural establishment, or rather the consequent full development of its irritability and the epoch of birth, remains dormant; the oxygen-supply to its substance is never brought so low as to set going the respiratory molecular explosions. As soon however as the intercourse between the maternal and umbilical blood is interrupted by separation of the placenta or by ligature of the umbilical cord, or when, as by the deatl of the mother, the umbilical blood ceases to be replenished with oxygen by the maternal blood, or when in any other way blood of sufficiently arterial quality ceases to find its way by the left ventricle to the bulb, the supply of oxygen in the respiratory centre sinks, and when the fall has reached a certain point an impulse of inspiration is generated and the foetus for the first time breathes. This action of the respiratory centre may be assisted by adjuvant impulses reaching the centre along various afferent nerves, such as those started by exposure of the body to the air, or to cold ; but these are subordinate, not essential. A retarded first breath may be hurried on by dashing water on the face of the new-born infant; but so long as the placental circulation is intact, stimulation, even varied and strong, of the fotal skin, though it may give rise to reflex movements of the limbs and other parts, will not call forth a breath; whereas, on the other hand, upon the cessation of the placental circulation, the fotus may make its first respiratory movements while it is still invested with the intact membranes and thus sheltered from the air and indeed from all external stimuli.

$\S 705$. When the first breath is taken, as under normal circumstances it is, with free access to the atmosphere, and the lungs become inflated with air (we dwelt in dealing with respiration, $\$ 257$, on some features of this first breathing), the scanty supply of blood which at the moment was passing from the right ventricle along the pulmonary artery returns to the 
left auricle brighter and richer in oxygen than ever was the foetal blood before. With the diminution of resistance in the pulmonary circulation caused by the expansion of the thorax, a larger supply of blood passes into the pulmonary artery instead of into the ductus arteriosus, and this derivation of the contents of the right ventricle increasing with the continued respiratory movements, the current through the latter canal at last ceases altogether, and its channel shortly after birth becomes obliterated. The obliteration is ultimately secured by proliferation of the internal coat, in which even before birth the sub-epithelial layer is unusually developed, a thrombus ( $\$ 33$ ) at times helping, but before this takes place the closure seems to be assisted by the mechanical arrangements of the parts. Corresponding to the greater flow into the pulmonary artery, a larger and larger quantity of blood returns from the pulmonary veins into the left auricle. At the same time the current through the ductus venosus from the umbilical vein having ceased, the flow from the inferior cava has diminished; and the blood of the right auricle finding little resistance in the direction of the ventricle, which now readily discharges its contents into the pulmonary artery, but finding in the left auricle, which is continually being filled from the lungs, an obstacle to its passage through the foramen ovale, ceases to take that course. Any return of blood from the now vigorous and active left auricle into the right auricle is prevented by the valve which, during the latter stages of intra-uterine life, has been growing up in the left auricle over the foramen ovale. At birth the edge of this valve is to a certain extent free so that, in case of an emergency, as when the pulmonary circulation is obstructed, a clirect escape of blood into the left auricle from the overburdened right auricle can take place. Eventually, in the course of the first year, adhesion takes place, and the separation of the two auricles becomes complete. With its larger supply of blood and greater work the left ventricle acquires the greater thickness characteristic of it during life. Thus the foetal circulation, in consequence of the respiratory movements to which its interruption gives rise, changes its course into that characteristic of the adult. 
SEC. 3. PARTURITION.

$\$$ 706. Owing to the growth of the fotus, and also to the accumulation of the amniotic fluid, the uterus towards the end of pregnancy has become much distended and has risen into the eavity of the abdomen, displaeing the abdominal viseera. 'The expansion of the uterus during pregnancy is a complex proeess in whiel the meehanical effects of the inereasing internal pressure are mingled with those of growth. Though the uterine walls are, as we have said, much thickened by the addition of new museular fibres as well as by the inerease in length, breadth, and thiekness of the individual fibres, and also enlarged by the vaseular development, they become somewhat thinned again towards the end of pregnaney by reason of the great distension of the cavity. The Fallopian tubes and the round ligaments share in the uterine enlargement, in so far as their muscular tissue is inereased; but the mucous membrane of the former does not alter, and the only changes taking place in the ovary are those concerned with the corpus luteum left by the shed ovum. The walls of the vagina are congested, soft and hypertrophied. Previous to labour the foxtus oecupies in the womb a position whieh it assumes at a quite early date, namely, one in which the head is direeted downwards towards the pelyis; this is at least the normal position, though deviations from it not infrequently oeeur.

From an early date waves of contraetion, at times rhythmieal, sweep over the enlarged uterus and towards the end of pregnancy beeome more marked. As a rule these are "insensible" contractions, that is to say the mother is not eonseious of them, though at times they may be distinetly felt; and in all cases they are temporary, produeing no permanent effeet on the uterus or its eontents. Though, as shewn by the cases of premature labour and abortion, whether occurring from natural causes or induced artificially, the uterine museles are capable at even an early date of earrying out the systematie contraetions whieh lead to the expulsion of the futus, they do not in normal parturition enter upon this phase of activity until a certain time has 
been run. In the human subject the period of gestation generally lasts from 275 to 280 days, i.e. about 40 weeks, the general custom being to expect parturition at about 280 days from the last day of the last menstruation. Seeing however that, in many cases, it is uncertain whether the ovum which developes into the embryo left the ovary in connection with the last menstruation or with the first one missed or during the intervening weeks, an exact determination of the duration of pregnancy is difficult if not impossible.

In some animals the period of gestation is longer, in others shorter than in man, being in the mare about 350 days, in the cow about 280 days, sheep about 150 days, dog about 60 days, rabbit about 30 days.

Immediately preceding labour a secretion of mucus, coming from the os uteri and at times mixed with blood, is often a sign or 'show' that the efficient uterine contractions, are about to begin.

$\$ 707$. The onset of labour is marked by rhythmically repeated contractions of the uterus which most distinctly affect consciousness and are recognized as "labour pains." The first effect of these is the opening up or widening of the os uteri constituting the "first stage of labour." The contractions may perhaps be spoken of as "peristaltic" in character, but the arrangement of the bundles of muscular fibres in the hody of the uterus is a complex one, and the gross effect of the contractions is to exert pressure, probably of a fairly uniform kind, on the uterine contents, that is on the amniotic fluid or "waters" enclosed in the "membranes" and surrounding the foetus. "These membranes are the amnion, the chorion and the decidua, the fir'st being easily separated from the other two along the loose connective tissue joining it to the chorion, and thus forming an inner and outer sheet or membrane. Over the os uteri the decidua consists of decidua reflexa only; and here the membranes with the contained fluid act as a hydraulic plug directing the force of the uterine contractions towards expanding the mouth. As labour goes on a special character of the uterine contractions becones prominent. In the contractions of which we spoke above as oceurring during pregnancy before labour really conmences, the relaxation of each muscular fibre following upon a contraction is a complete one. But in labour the muscular fibres while with each pain they contract and relax, are all the while becoming permanently and progressively thicker and shorter. This change by which the uterine wall becomes progressively thicker and more compact has been spoken of under the not very desirable term "retraction," as distinguished from "contraction," but appears to be a sort of tonic contraction or perhaps rather a residue of contraction like that seen in skeletal muscles under certain conditions; 
at each recurring "pain" the shortening of the muscular fibres starts so to speak from this more permanent shortening instead of from complete relaxation, and the return is to it not to complete relaxation.

This more permanent tonic contraction or "retraction" does not however affect the whole uterus; it is, broadly speaking, confined to the body and absent from the cervix. Indeed in the latter region all contractions are wanting, the muscular fibres appear to be inhibited, and the walls yielding to the pressure exerted upon them become thinner instead of thicker: as the pressure increases the fibres possibly become lamed or paralyzed. In this way a distinction is established between an "upper segment" of the uterus corresponding to the body, the walls of which become thicker and shorter through the continued and progressive "retraction," and a "lower segment," corresponding to the cervix but possibly including the lower part of the body, the walls of which become stretched and thinner, the line of demarcation between the two segments being often called "the retraction ring." As the pressure in the body of the uterus continues and waxes greater, the month becomes wider and wider, until the head of the foetus begins to pass through it into the vagina, the walls of which like those of the "lower segment" have meanwhile become stretched and thinner; and as the foetus is thus leaving the uterus the progressive tonic contraction adapts the uterine walls to the lessening cavity. Sometimes the membranes are ruptured, with escape of the "waters," before the head has left the uterus, at other times they form a bulging cushion preceding and making way for the fotus.

When the os uteri has become fully expanded and is ready to allow the head of the foetus to pass through it into the vagina, the intrinsic contractions of the uterus begin to be assisted by an extrinsic force, by contractions of the abdominal walls which thus exert on the uterus and its contents a pressure very similar to that exerted on the rectum in defrecation (\$225). These contractions, which mark the onset of the "second stage" of labour, are rhythmical in nature like those of the uterus itself, and synchronous with them. The expulsive power of the uterus is thus greatly increased, and the head of the foetus followed by the rest of its body is driven through the vagina and then through the vulva, these playing apparently a wholly passive part in the matter, and the child is thus literally "thrust upon the world.'

At the very beginning of labour there takes place at the internal os a cleavage of the decidua vera between a deeper less altered and a superficial more altered layer, so that the latter. attached to the chorion and thus forming part of the "membranes," separates from the utcrine surface. This separation, 
the beginning of which is the cause of the "show" spoken of above, and which is considered to be a mechanical effect of the uterine contractions but which must be prepared for by histological changes, during the early stages of labour extends upwards for two or three inches only; but, at the last, it is carried right through the "decidual layer" of the placenta. Hence, after the expulsion of the foetus, the uterus contains within its cavity, separated from and now foreign to itself, the placenta and membranes, the latter consisting of amnion, chorion, the whole of the remains of the decidua reflexa and a variable part of the decidua vera; and, under normal conditions, these are by the last expulsive efforts ejected with or immediately after or soon after the child. As a rule the membranes are ruptured and the amniotic fluid escapes before the head extrudes, but at times the child is born still surrounded by the intact membranes with their contained fluid; it comes into the world in its "caul."

When the placenta and membranes have left the uterus (they not unfrequently are lockged for a while in the vagina), the tonic contraction or "retraction" spoken of above, which during the whole of labour has been following up the advance of the fotus, and progressively lessening the uterine cavity, continues is work and serves an important purpose. When the last pain of labour, by which the emptied uterus is gathered up into a sniall hard ball, passes away, the walls under normal conditions do not wholly relax, a permanent tonic contraction keeps the walls thick and in contact, thus closing the uterine cavity; and over this compact and closed uterus waves of rhythmical contraction, the "after-pains," still for a while pass without altering its permanent condition. By this continued contraction or retraction, not only the open, torn ends of the vessels of the decidua but all the vessels throughout the thickness of the uterine walls are so compressed that all extensive bleeding is prevented. Should this continued contraction give away to relaxation, hrmorrhage or "flooding" follows. 'This retraction or tonic contraction, whatever be its exact nature, which is so conspicuous in the uterus but which perhaps may be recognized in a lesser degree as mere ordinary tonic contraction in other rhythmically contracting organs, in the bladder, in the intestine, and even in the heart, appears to serve more than one purpose in the work of the uterus; by continually lessening the uterine cavity it renders more efficient during labour the rhythmic uterine "pains," by compressing the blood vessels during labour it gradually shuts off the extravagant blood supply now no longer needed, and by continuing that compression after labour and by closing the uterine cavity it prevents hrmorrhage and wards off the evil effects which the free entrance into the uterine cavity of foreign organisms might bring about. And 
probably it is on account of its great usefuhness that this peculiar form of muscular activity is so prominent in the uterus.

Even before labour proliferation of the epithelioid cells may be observed in the lining membrane of the uterine vessels; these arc rapirly increased after labour is completed, and form part of the healing processes which follow. 'The tonic contraction of which we have been speaking is maintained mntil the blood vessels are permanently closed by these nutritive healing processes. After birth the muscular elements of the uterus dwindle, many of the fibres undergoing fatty degeneration, and thus the mucons and muscular walls are gradually brought back to their natural condition. During the early days of this process of involution a discharge, the lochia, takes place from the internal surface of the uterus.

\$ 708. The whole process of parturition may be broadly considered as a reflex act, the nervous centre of which is placed in the lumbar cord. In a dog, whose thoracic cord had been completely severed, parturition took place as usual; and the fact that, in the human subject, labour will progress in a natural nnanner while the patient is unconscious from the administration of chloroform, though it is of ten retarded and sometimes arrested, shews that in woman also the contractions both of the uterus and of the abdominal muscles are involuntary, however much the latter may be assisted by direct volitional efforts.

Observations on animals shew that even in a virgin uterus and in one which is not enlarged by pregnancy movements can be excited in a reflex manner through the central nervous system and may oceur rhythmically in an apparently spontaneous manner ; but the latter are often absent or are so slight as readily to escape observation, and the former are often feeble and excited with difticulty. In a pregnant animal on the other land, especially if pregnancy be advanced, powerful rhytlmic expulsive movenents repeatedly oceur in the apparent absence of all cxtrinsic stimuli and are very readily provoked by the stimulation of varions afferent nerves. They may also be induced by direct stimnlation of the spinal cord at any part of its whole length as well as of various regions of the brain; the analogy with the movements of the urinary bladiler lcads us to suppose that the impulses thus started in the brain and upper part of the spinal cord do not pass directly to the nterus but throw into activity the reflex centre in the spinal cord. Movements of the uterus are readily cxeited when the blood ceases to be duly arterialized, extrusion of the fretus being a common result when a pregnant animal is asphyxiated; and though the venous blood may aet in part as a direct stinulus to the uterine muscles the contractions are mainly due to the blood exciting the nervons centre. Drugs such as ergot which increase uterine eontractions probably in like manner 
produce their effect chiefly at least through their action on the nervous centre. The ready way in which the uterus enlarged by pregnancy responds by reflex contraction to the stimulation of various afferent nerves is illustrated in the human subject by the means usually adopted to secure after the birth of the child that continued contraction by which hemorrhage is avoided. Should for any reason such a contraction fail to take place, it may be secured by applying cold or pressure to the abdominal walls or by introilucing a liand or some foreign body into the vagina, or, what perhaps best illustrates the reflex nature of the matter, by applying the child to the nipple; in the latter case the relatively feeble afferent inpulses generated in the mammary nerves by the sucking of the child are especially potent in producing by reflex action contraction of the uterine muscles.

$\$$ 709. The nerves of the uterus reach that organ chicfly along the broad ligament in company with the blood vessels, are partly medullated, partly non-medullated, and are derived from the pelvic plexus lying between the rectum and the vagina. 'The pelvic plexus, on which as also on the nerves passing to the uterus, numerous small ganglia are scattered, is a continuation on each side of the body of the medially placed hypogastric plexus, but it is joined by branches coming directly from the sacral nerves. In the lower animals (dog) the roots which supply fibres to the uterus are on the one hand the upper lumbar, which traverse the sympathetic strand known as the hypogastric nerve, and on the other liand probably the first and second sacral. In the human subject the corresponding roots are probably the upper lumbar and third, fourth and second sacral.

Stimulation, in the dog, either of the hypogastric nerve or of the sacral nerves produces contractions in the pregnant uterus; it is stated that the mode of contraction is different in the two cases, in the latter the longitudinally disposed fibres, in the former the circularly disposed fibres being especially thrown into action. Moreover, it is said that while the fibres passing by the hypogastric nerve are vaso-constrictor towards the uterine arteries, those passing by the sacral nerves are vasodilator. Other observers have failed to obtain any such difference between circular and longitudinal contractions, and find that in some animals at least, while contractions of the uterus may be readily brought about by stimulation of the sympathetic nerves from the lumbar region, passing through the hypogastric nerves, contractions camnot with certainty be obtained by stimulating the sacral nerves. On the other hand, stimulation of the sacral nerves, of the second, third, and sometimes of the first, readily produces movements of the vagina. It may be added that stimulation of certain areas of the cerebral cortex will give rise to movements of the uterus and of the vagina. 
§ 710. Though under normal circunstances efficient uterine contractions do not set in until the full period of gestation is completed, yet by reason of changes in the uterus or its contents, occurring from natural causes or induced artificially, the full swing of movements may, at almost any time, though at some times more readily than at others, be brought about. On the other hand it may be delayed for a considerable time beyond the proper term. We may be said to be in the dark as to why the uteris, after remaining for months subject only to futile contractions, is suddenly thrown into powerful and efficient action, and within it may be a few hours or even less gets rid of the burden which it has borne with such tolerance for so long a time. None of the various hypotheses which have been put forward can be considered as satisfactory. There is no evidence for the view, based on the occur'ence of contractions in consequence of an asphyxiated condition of the blood, that the onset of labour is caused by a gradual diminution of oxygen or accumulation of carbonic acid in the blood, reaching at last to a climax. Nor are there sufficient facts to connect parturition with any condition of the ovary resembling that aecompanying menstruation. Nor can much stress be laid on the supposition that the real exciting cause is the separation of the decidua from the permanent uterine wall, the separation being the outcome of the precerling processes of growth, since the aetual separation itself seems to be caused by the initial contractions of labour, and the histological changes which precede it are only one set of changes among many others all having their goal in labour. We ean only say that labour is the culminating point of a series of events, and must come sooner or later, though its immediate advent may at times be decided by accident; but it would not be profitable to discuss this question here.

The action of the abdominal muscles in parturition, at least so much as takes place independently of the will, is, in contrast to that of the uterine muscles, obviously a reflex act of a more ordinary kind carried out by means of the spinal cord; and we may suppose that, though the mere contractions of the uterus may serve as a possible source, the necessary stimulus is supplied by the pressure of the futus in the vagina; in support of this it may be noted that the action becomes much intensified towards the end of labour as the stress and strain caused by the advancing liead tell more and more on the external skin.

$\$ 711$. Hence as we have said the whole act of parturition may with reason be considered as a reflex one. Whether it be wholly a reflex or in a certain sense an antomatic one, the act can readily be inhibited by other contemporary actions of the central nervous system. Thus cmotions very frequently become a lindrance to the progress of parturition; as is well known, 
the entrance into the bedroom of a stranger often causes for a time the sudden and absolute cessation of 'labour' pains, which previously may have been even violent. Judging from the analogy of micturition, we may suppose that this inhibition of uterime contractions is brought about by an inhibition of the centre in the lumbar cord leading to a sudden cessation of the augmentor action of which we spoke above as far as the uterus itself is concerned, and in a more direct way to a cessation of the contractions of the abdominal muscles. Some observations tend to shew that a region of the bulb exerts such an inhibitory influence; but the matter needs fuller investigation. 


\section{CHAPTER III.}

\section{THE PHASES OF LIFE.}

$\$$ 712. THE child has at birth, on an average, rather less than onc-third the maximum length, and about one-twentieth the maximum weight, to which in future years it will attain.

'I : composition of the body of the new-born babe, as compared "th that of the arlult, will be seen from the following table, in which the details are more full than those given in $\S 413$; the figures in brackets are more recent observations.

\begin{tabular}{|c|c|c|c|}
\hline \multirow[t]{2}{*}{ 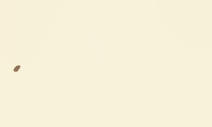 } & \multicolumn{2}{|c|}{$\begin{array}{l}\text { Weight of organ in percentage } \\
\text { of Body-weight. }\end{array}$} & \multirow{2}{*}{$\begin{array}{l}\text { Weight of organ in } \\
\text { adult, as compared } \\
\text { with that of new-born } \\
\text { babe taken as } 1 \text {. }\end{array}$} \\
\hline & New-born babe. & Adult. & \\
\hline Eye & $\cdot 28$ & .023 & $1 \cdot 7$ \\
\hline Brain & $14 \cdot 34(12 \cdot 28)$ & $2 \cdot 37(2 \cdot 25)$ & $(3 \cdot 34)$ \\
\hline Kidneys & .88 & $\cdot 48$ & 12 \\
\hline Skin & $11 \cdot 3$ & $6 \cdot 3$ & 12 \\
\hline Liver & $4 \cdot 39 \quad(5 \cdot 03)$ & $2 \cdot 77(3.05)$ & $13 \cdot 6(11 \cdot 05)$ \\
\hline Heart & $.89 \quad(\cdot 73)$ & $.52(.49)$ & $15(12 \cdot 1)$ \\
\hline $\begin{array}{l}\text { Stomach and } \\
\text { Intestine }\end{array}$ & $2 \cdot 53$ & $2 \cdot 34$ & 20 \\
\hline Lungs & $2 \cdot 16$ & $2 \cdot 01$ & 20 \\
\hline Skeleton & $16 \cdot 7$ & $15 \cdot 35$ & 26 \\
\hline Muscles, \&c. & $2 \cdot 34$ & $4 \cdot 31$ & 48 \\
\hline Testicle & $\cdot 037$ & $\cdot 08$ & 60 \\
\hline
\end{tabular}

It will be observed that the brain and eyes are, relatively to the whole body-weight, very much larger in the babe than in the adult. This disproportion is a very marked embryonic feature, and has a morphological or phylogenic, as well as a physiological or teleological, significance. Inasmuch as the smaller body has relativcly the larger surface, the skin is naturally proportionately greater in the babc; but the same disproportion is observed in the kidncys, these like the skin increasing 
in weight twelve times only between birth and full growth, whereas the whole boly increases twenty times. The heart and the liver according to the newer observations behave very similarly, and even according to the older observations lag considerably behind the whole frame, whereas the lungs and the alimentary canal almost exactly keep pace with it, and the skeletal framework, in spite of its being specifically lighter in its earlier cartilaginous condition, maintains throughout life very nearly the same relative weight. The muscles on the contrary grow more than twice as fast as the whole body; the great increase in these covers the relative decrease of the other parts, and it is largely by the laying on of flesli and fat that the babe gains the bulk of the man.

$\$ 713$. We usually measure growth by taking account of two sets of changes, changes of stature and changes of weight; and we may study both these changes in more than one way.

If we measure the height at intervals we may plot out the curve of growth of stature, and when we do this we find that the curve rises rapidly at first but afterwards more slowly, shewing that the increment is decreasing, and at about the twenty-fifth year ceases to rise at all. From thence to about fifty years of age the height remains stationary, after which there may be a decrease, especially in extreme old age. 'The curve moreover is not regular, but indicates by its changes that the increment of height in a given time is now smaller, now greater.

The curve of weight is, on the whole, at first very similar to that of height, rising in a somewhat similar way and shewing similar irregularities; but instead of ceasing to rise at about the twenty-fifth year it continues to rise, though marked with many irregularities, and may continue to do so until about the fortieth year. After the sixtieth year a decline of variable extent is generally witnessed. It should be noted that in the first few days of life, so far from there being an increase, there is an actual decrease of weight, so that, even on the seventh day the weight still continues to be less than at birth; and a similar post-natal loss of weight is observed in animals. If we take the curve of growth from the impregnation of the ovum onwards this post-natal loss of weight will appear as an abrupt change in the curve due to the so to speak violent act of birth. It should be added that the curves both of height and weight exhibit differences dependent on sex, circumstances, race, climate and the like.

We may also study the progress of growth by measuring the increment of growth in a given time, in a year for instance, and plotting out the curve of the yearly increment. When we do this we obtain very instructive results. We find that the yearly increment decreases very rapidly during the first two 
or threc years, then remains nearly stationary or even rises, and at about the seventh or eighth year undergoes a marked fall. This fall, howcrer, is temporary only; the curve soon rises again and with some irregularities attains a maximum between the twelfth and fifteenth year, from which point onwards it falls rapidly with some minor' irregularities. These marked variations in the increment of growth which are obviously connected with and preparatory to the important change which we call puberty, are seen in the curves both of stature and of weight, the changes in weight occurring however rather later than those of stature, and both being somewhat different in boys from what they are in girls. Both are also influenced by the conditions of life; but a stuly of the curves of growth of young pcople living under various surroundings, while it teaches the great importance of properly administering to the wants of youth, at the same time illustrates the recuperative clasticity of the bodily frame; it may often be observed that the ill effects of adverse circumstances, provided they be not too great, are soon recovered from under the influence of a happy change; food and comfort will turn the abnormal fall in the curve of growth of a starved waif into a sharp rise.

Lastly, we may study growth by observing the actual ratc of growth, by measuring the magnitude of the fraction of the total weight which is added to the weight in a given time; we take weight because this is the most significant element of growth. When this method is adopted, an examination of such statistics as are available with regard to man, confirmed by the results of careful observations on young animals, tends to shew that the rate diminishes continually from birth onwards, the diminution being rapid at first but slower afterwards, and being broken by various irregularities. In other words, the power of growth diminishes continually though somewhat irregularly throughout life, and a like diminution apparently obtains in intra-uterine existence. It scems as if the impetus of growth given at impregnation gradually dies out.

$\$ 714$. The saliva of the babe, very scanty at first and not abundant until teething begins, is active on starch though less so than in the adult, and its gastric juice, unlike that of many new-born animals, has good peptic powers, and its pancreas good tryptic powers, though apparently the pancreatic action on starch is feeble. From this we may infer that its digestive processes are in general identical with that of the adult though ill suited for any large amount of starch in the food; and they are feeble, since the fieces of the infant contain a considerable quantity of undigested food (fat, casein, \&c.), as well as unaltered bile-pigment, and undecomposed bile-salts.

The heart of the babe, as shewn in the preceding Table, is, relatively to its body-weight, larger than the adult, and the 
frequency of the heart-beat much greater, viz. about 130 or 140 per minute, falling to about 110 in the second year, and about 90 in the tenth year. Corresponding to the smaller bulk of the body, the whole circuit of the blood system is traversed in a shorter time than in the adult (12 seconds as against 22 ) ; and consequently the renewal of the blood in the tissues is exceedingly rapid. Relatively to the body-weight there is also considerably more blood in the babe than in the adult. The respiration of the babe is quicker than that of the adult, being at first about 35 per minute, falling to 28 in the second year, to 26 in the fifth year, and so onwards. The respiratory work, while it increases absolutely as the body grows, is, relatively to the body-weight, greatest in the earlier years. It is worthy of notice, that the absorption of oxygen is said to be during these earlier years relatively more active than the production of carbonic acid ; that is to say, there is a continued accumulation of capital in the form of a store of oxygen-holding explosive compounds (cf. \$289). 'This, indeed, is the strikimg feature of infant metabolism. It is a metabolism directed largely to constructive ends. The food taken represents, undoubtedly, so much potential energy; but before that energy can assume a vital mode, the food must be converted into tissue; and, in such a conversion, morphological and molecular, a large amount of energy must be expended. The metabolic activities of the infant are more pronounced than those of the adult, for the sake, not so much of energies which are spent on the world without, as of energies which are for a while buried in the rapidly increasing mass of flesh. Thus the infant requires over and above the wants of the man, not only an income of energy corresponding to the energy of the flesh actually laid on, but also an income corresponding to the energy used up in making that living sculptured flesh out of the dead amorphous proteids, fats, carbohydrates and salts, which serve as food. Over and above this, the infant needs a more rapid metabolism to keep up the normal bodily temperature. This, which is no less, indeed slightly ( .30$)$ higher, than that of the adult, requires a greater expenditure, inasmuch as the infant with its relatively far larger surface, and its extremely vascular skin, loses heat to a proportionately much greater degree than does the grown-up man. It is a matter of common experience that children are more affected by cold than are adults. The bodily temperature is moreover less stable in the infant than in the adult, and departures from the normal temperature have not the grave significance they have in the adult.

This rapid metabolism is however not manifest immediately upon birth. During the first few days, corresponding to the loss of weight mentioned above, the respiratory activities of the tissues are feeble; the embryonic habits seem as yet not to have 
been completely thrown off, and, as was stated in $\$ 306$, newborn animals bear with impunity a deprivation of oxygen, which would be fatal to them later on in life.

Assoeiated probably with these constructive labours of the growing frame is the prominence of the lymphatic system. Not only are the lymphatic glands largely developed and more active (as is probably shewn by their tendency to disease in youth), but the quantity of lymph circulation is greater than in later years. Characteristic of youth is the size of the thymus body, which increases up to the second year, and may then remain for a while stationary, but generally before pubcrty has suffered a retrogressive metamorphosis, so that very often liardly a vestige of it remains behind. The thyroid body is also relatively greater in the babe than in the adult; the splcen, on the other hand, relatively to the body-weight does not change greatly, though rather smaller in the adult. As we have already said the recuperative power of infancy and early youth is very marked.

The quantity of urine passed, though scanty in the first two days, rises rapidly at the end of the first week, and in youth the quantity of urine passed is, relatively to the bodyweight, larger than in adult life. 'This may be, at least in quite early life, partly due to the more liquid nature of the food, but is also in part the result of the more active metabolism. For not only is the quantity of urine passed, but also the amount of urea and of some other urinary constituents excreted, relatively to the body-weight, greater in the child than in the adult. The presence of uric acid, of oxalic acid, and according to some, of hippuric acid in unusual quantities is a frequent characteristic of the mine of children. It is stated that caleic phosphates, and indeed the phosphates generally, are deficient, being retained in the body for the building up of the osseous skeleton.

$\S 715$. It would be beyond the scope of this work to enter into the psychical condition of the babe or the child, and our knowledge of the details of the working of the nervous system in infancy is too meagre to permit of any profitable discussion. It is hardly of use to say that in the young the whole nervous system is more irritable or more excitable than it is in later year's; by which we probably to a great extent mean that it is less rigid, less marked out into what, in preceding portions of this work, we have spoken of as nervous mechanisms. In new-born puppies and some other animals stimulation of the various cerebral areas does not give rise to the usual localized movements; these do not appear until some time after birth ; but in this respect differences are observed in different kinds of animals corresponding to the well-known differences between different kiuds of animals in the powers possessed at birth : 
the human babe as regards the latter is intermediate between the puppy and the young guinea-pig. As we have seen, the fibres of the various tracts in the central nervous system acquire their medulla at different epochs; there is experimental evidence in support of the view, otherwise probable, that the assumption of functional activity follows in the same order; and the pyramidal tract is as we have seen the one in which the fibres are very late in acquiring their medulla. It has been asserted that in a new-born animal stimulation of the vagus produces no cardiac inhibition and that this does not appear for several days; other observers however have obtained positive results and that even in the uterus; probably in this respect also animals differ. In the human infant the sense of touch, both as regards pressure and temperature, appears well developed, as does also the sense of taste, and possibly, though this is disputed, that of smell. The pupil (larger in the infant than in the man) acts fully, and normal binocular movements of the eyes have been observed in an infant less than an hour old. The eye is from the outset fully sensitive to light, though of course visual perceptions are imperfect. Auditory sensations on the other hand, seem to be dull, though not wholly absent, during the first few days of life; this may be partly at least due to absence of air from the tympanum and to a tumid condition of the tympanic mucous membrane. As the child grows up his senses sharpen with constant exercise, and in his early years he possesses a general acuteness of sight, hearing, and touch, which frequently becomes blunted as his psychical life becomes fuller. Children however are said to be less apt at distinguishing colours than in sighting objects; but it does not appear whether this arises from a want of perceptive discrimination or from their being actually less sensitive to variations in hue. A characteristic of the nervous system in chillhood, the result probably of the more active metabolism of the borly, is the necessity for long or frequent and deep slumber.

$\$$ 716. Dentition marks the first epoch of the new life. At about seven months the two central incisors of the lower jaw make their way through the gum, followed immediately by the corresponding teeth in the upper jaw. The lateral incisors, first of the lower and then of the upper jaw, appear at about the ninth month, the first molars at about the twelfth month, the canines at about a year and a half, and the temporary dentition is completed by the appearance of the second molars usually before the end of the second year.

About the sixth year the permanent dentition commences by the appearance of the first permanent molar beyond the second temporary molar ; in the seventh year the central permanent incisors replace their temporary representatives, fol- 
lowed in the next year by the lateral ineisors. In the ninth year the temporary first molars are replaeed by the first bicuspicls. and in the tenth year the second temporary molars are similarly replaced by the second bicuspids. The canines are exchanged about the elerenth or twelfth year, and the second permanent molars are eut about the twelfth or thirteenth rear. 'There is then a long pause, the third or wisdom tooth not making its appearanee till the seventeenth, or even twentyfifth rear, or in some cases not appearing at all.

$\S 71 \%$. Shortly after the conclusion of the permanent dentition (the wisdom teeth excepted) the oeeurrence of puberty marks the beginning of a new phase of life; and the difference between the sexes, hitherto merely potential, now becomes funetional. In both sexes the maturation of the generative organs is acempanied by the well-known ehanges in the body at large; but the events are much more obvious in the typical female than in the aberrant male. Though in the boy, the breaking of the voice and the rapid growth of the beard which aeeompany the appearance of active spermatozoa, are striking features, yet they are after all superficial; and though, as we have seen ( $\$ 713)$, the curves of his inereasing weight and height undergo before and at this period, characteristic variations, the general events of his economy pursue for a while longer an unchanged course; the boy does not become a man till some years after puberty; and the decline of his functional manhood is so gradual that frequently it ceases only when disease puts an end to a ripe old age. With the occurrence of menstruation, on the other hand, at from thirteen to seventeen year's of age, subsequent to the acceleration of growth noted above $\$ 713$, which indeed appears preparatory to it, the girl almost at once becones a woman, and her functional womanhood eeases suddenly at the climacteric in the fifth decennium. During the whole of the child-bearing period her organism is in a comparatively stationary condition. 'The variations in the yearly inerement of the girl before puberty' though not so marked are more complex than those of the boy, and she reaches the maximmm of yearly increment sooner than does he; during this whole period indeed she precedes him in growth and she has nearly reached her maximum, while he is still continuing to grow. Her eurve of weight from the nineteenth ycar onward to the climacteric, remains stationary, being followed subsequently by a late increase, so that while the man reaches his maximum of weight at about forty, the woman is at her greatest weight about fifty.

Of the statical differences of sex, some, such as the formation of the polvis, and the costal mechanism of respiration, are directly connected with the act of child-bearing, while others have only an indirect relation to that duty; and indications at least of nearly all the characteristic differenees are seen at birth. 
The baby boy is heavier and taller than the baby girl, and the maiden of five breathes with her ribs in the same way as does the matron of forty. 'The woman is lighter and shorter than the man, the limits in the case of the former being from 1.444 to $1 \cdot 740$ metres of height and from $39 \cdot 8$ to $93 \cdot 8$ kilos of weight, in the latter from $1 \cdot 467$ to 1.890 of height, and from $49 \cdot 1$ to 98.5 kilos of weight. The muscular system and skeleton are both absolutely and relatively less in woman, and her brain is lighter and smaller than that of man, being about 1272 grammes to 1424. Her metabolism, as measured by the respiratory and urinary excreta, is also not only absolutely but relatively to the body-weight less, and her blood is not only less in quantity but also of lighter specific gravity and contains a smaller proportion of red corpuseles. Her strength is to that of man as about 5 to 9, and the relative length of her step as 1000 to 1157.

$\$ 718$. From birth onward (and indeed from early intrauterine life) the increment of growth as we have seen, though undergoing certain variations, continues to diminish. At last a point is reached at which the curve cuts the abscissa line, and the increment becomes a decrement. After the culmination of manhood at forty and of womanhood at the climacteric, the prime of life declines into old age. The metabolic activity of the body, which at first was sufficient not only to cover the daily waste but to add new material, later on is able only to meet the daily wants, and at last is too imperfect even to sustain in its entirety the existing frame. Neither as regards vigour and functional capacity, nor as regards weight and bulk, do the turning-points of the several tissues and organs coincide either with each other or with that of the body at large. We have already seen that the life of such an organ as the thymus is far shorter than that of its possessor. The eye is in its dioptric prime in childhood, when its media are clearest and its muscular mechanisms most mobile, and then it for the most part serves as a toy; in later years, when it could be of the greatest service to a still active brain, it has already fallen into a clouded and rigid old age. The skeleton reaches its limit very nearly at the same time as the whole frame reaches its maximum of height, the coalescence of the various epiphyses being pretty well completed by about the twenty-fifth year. Similarly the muscular system in its increase tallies with the weight of the whole body. 'The brain, in spite of the increasing complexity of structure and function to which it continues to attain even in middle life, early reaches its limit of bulk and weight. At about seven year's of age it attains what may be considered as its first limit, for though it may increase somewhat up to twenty, thirty, or even later year's, its progress is much more slow after than before seven. The vascular and 
digestive organs as a whole may continue to increase even to a very late period. From these facts it is obvious that though the phenomena of old age are, at bottom, the result of the individual decline of the several tissues, they owe many of their features to the disarrangement of the whole organism produced by the premature decay or disappearance of one or other of the constituent bodily factors. 'Thus, for instance, it is clear that were there no natural intrinsic limit to the life of the muscular and nervous systems, they would nevertheless come to an end in consequence of the nutritive disturbances caused by the loss of the teeth. And what is true of the teeth is probably true of many other organs, with the addition that these cannot, like the teeth, be replaced by mechanical contrivances. 'Thus the term of life which is allotted to a muscle by virtue of its molecular constitution, and which it could not exceed werc it always placed under the most favourable nutritive conditions, is, in the organism, further shortened by the similar life-terms of other tissues; the future decline of the brain is probably involved in the early decay of the thymus.

Two changes characteristic of old age are the so-called calcareous and fatty degenerations. These are seen in a completely typical form in cartilage, as, for instance, in the ribs; here the cell-substance of the cartilage corpuscle becomes hardly more than an envelope of fat globules, and the supple matrix is rendered rigid with amorphous deposits of calcic phosphates and carbonates, which are at the same time the signs of past and the cause of future nutritive decline. And what is obvious in the case of cartilage is more or less evident in other tissucs. Everywhere we see a disposition on the part of the living substance of the tissue to fall back upon the easier task of forming fat rather than to carry on the more arduous duty of manufacturing new material like itself; everywhere almost we see a tendency to the replacement of a structured matrix by a deposit of amorphous material. In no part of the system is this more evident than in the arteries; one common feature of old age is the conversion by such a change of the supple elastic tubes into rigid channels, whereby the supply to the various tissues of nutritive material is rendered increasingly more difficult, and their intrinsic decay proportionately hurried.

Of the various tissues of the boty the muscular and nervous are however those in which functional decline, if not structural decay, becomes soonest apparent. The dynamic coefficient of the skeletal muscles diminishes rapidly after thirty or forty years of life, and a similar want of power comes over the plain muscular fibres also; the heart, though it may not diminish, or even may still increase in weight, possesses less and less force, and the novements of the intestine, bladder. and other organs, diminish in vigour. In the nervous system, 
the lines of resistance, which, as we have seen, help to map out the central organs into mechanisms, and so to produce its multifarious actions, become at last hindrances to the passage of nervous impulses in any direction, while at the same time the molecular cnergy of the impulses themselves becomes less. The eye becomes feeble, not only from cloudiness of the media and presbyopic nuscular inability, but also from the very bluntness of the retina; the sensory and motor impulses pass with increasing slowness to and from the central nervous system, and the brain becomes a more and more rigid mass of nervous substance, the molecular lines of which rather mark the history of past actions than serve as indications of present potency. The epithelial glandular elements seem to be those whose powers are the longest preserved; and hence the man who in the prime of his manhood was a 'martyr to dyspepsia' by reason of the sensitiveness of gastric nerves and the reflex inhibitory and other results of their irritation, in his later years, when his nerves are blunted, and when therefore his peptic cells are able to pursue their chemical work undisturbed by extrinsic nervous worries, eats and drinks with the courage and success of a boy.

$\$$ 7i9. Within the range of a lifetime are comprised many periods of a more or less frequent recurrence. In spite of the aids of a complex civilization, all tending to render the conditions of his life more and more equable, man still shews in his economy the effects of the seasons. The birth-rate for instance shews an increase in winter, and most people gain weight in winter and lose weight in summer. Careful observations of school children shew that these increase in length rapidly in the spring but hardly at all in the autumn, and very slowly in the winter, while their increase in weight is most marked in the autumn, being very slight or even negative in the spring, and not great in winter. Some of these apparent effects of the season are the direct results of varying temperature, but some probably are habits acquired by descent, and in some again the comnection is a very indirect or possibly not a real one. Within the year, an approximately monthly period is manifested in the female by menstruation, though there is no exact evidence of even a latent similar cycle in the male. The phenomena of recurrent diseases, and the marked critical days of many other maladies, may be regarded as pointing to cycles of smaller duration than that of the moon's revolution, save in the cases in which the recurrence is to be attributed rather to periodical phases in the disease-producing germ itself, than to variations in the medium of the disease.

$\S$ 720. Prominent among all other cyclical events is the rhythmic rise and fall in the activities of the central nervous system; most animals possessing a well-developed nervous sys- 
tem, must, night after night, or day after day, or at least time after time, lay them down to sleep. 'The salient feature of sleep is the cessation or extreme lowering of the psychical activity of the brain and of the nervous processes which serve as the basis of that activity. When sleep is at its height, the afferent nervous impulses which external agents set going in the afferent somatic nerves such as those of the special senses, are no longer the starting points of complex cerehral processes; not only do they fail to excite consciousness and to leave their mark on memory, bitt they may be unable to call forth even a simple reflex movement. And yet they are not wholly without effect; for though a set of feeble afferent impulses may proluce no visible reaction and leave no impression on the mind of the sleeper, yet impulses of the same kind, if made stronger in proportion to the depth of the sleep, may be followed by their wonted cerebral consequences, and may thus awake, as we say, the sleeper. It would seem as if the afferent impulses met in their course with an unwonted resistance to their progress, as if the wheels of the cerebral machinery worked stiffly so that the lesser shocks of molecular change which otherwise would have moved them, were broken and wasted upon them. Corresponding to this block or lessened inroad of afferent impulses, the outflow of efferent impulses is stopped or largely diminished; the body gives no sign of the working of a conscious will, the eyelids drop and the head nods, and the various actions by which the erect posture is maintained are let go for lack of the governing motor impulses. And psychological self-inquiry tells us that in complete sleep this absence of outward signs of cerebral activity has its fellow. in the absence of inward marks ; the interval between falling asleep and awakening is a blank and gap in the history of the mind.

We say 'complete slecp' since there are many degrees of sleep, the state which we call that of dreaming being one of them; and between the most perfect wide-awakefulness and that deepest slumber which refuses for a long time to give way before even the strongest stimuli. no clear line of demarcation can be drawn. When we fall asleep the tie between 'ourselves' and the external world is not suddenly snapped, we do not by onc step pass from consciousness to unconsciousness; and the same when conversely we awake; as the world vanishes from us or comes back to us, the afferent impulses of sight, of sound and of other kinds. for a period which may be brief but always exists, produce, before they cease or begin appreciably to affect us at all, effects in ascending or descending seale which we call unreal. And the outward signs of sleep may vary from one in which volition is present and even dominant, to one in which even the simplest reflex movenients of the skeletal muscles are with difficulty evoked, and the maintenance of some skeletal tone 
$(\$ 470)$ and of breathing afford, so far as the skeletal muscles are concerned, almost the only token that the central nervous system is alive. But we cannot enter here into the psychology of sleep and dreams.

Though the phenomena of sleep are largely confined to the central nervous system and especially to the cerebral hemipheres, the whole body shares in the condition. The pulse and breathing are slower, the intestine, the bladder, and other internal muscular mechanisms are more or less at rest, and the secreting organs are less active, some apparently being wholly quiescent; the secretion of mucus attending a nasal catarrh is largely diminished during slumber, and the sleeper on waking rubs his eyes to bring back to his conjunctiva its needed moisture. The output of carbonic acid, and the intake of oxygen, especially the former, is lessened; the mine is less abundant, and the urea falls. Indeed the whole metabolism and the dependent temperature of the body are lowered; but we cannot say at present how far these are the indirect results of the condition of the nervous system, or how far they indicate a partial slumbering of the several tissues.

Thoracic respiration is said to become more prominent than diaphragmatic respiration during sleep, and a rise and fall of the respiratory movements, resembling if not identical with the Cheyne-Stokes rhythm of respiration ( $\$ 305)$, is frequently observed. During sleep the pupil is constricted, during deep sleep exceedingly so; and dilation, often unaccompanied by any visible movements of the limbs or body, takes place when any sensitive surface is stimulated; on awaking also the pupils dilate. The eyeballs have been generally described as being during sleep directed upwards and converging, or according to some anthors, diverging; but other's maintain that in true sleep the visual axes are parallel and directed to the far distance. The eyes of children have been deseribed as continually executing during sleep movements, often irregular and unsymmetrical and unaccompanied by changes in the pupils. The contraction of the pupils is worthy of notice, since it shews that the condition of sleep is not merely the simple and direct result of the falling away of afferent impulses; when the eyes are closed in slumber the pupils ought, since the retina is then quiescent, to dilate; that they are constricted, the more so the deeper the sleep, shews that important actions in the brain, probably in the middle portions of the brain, are taking place.

We are not at present in a position to trace out the events which culminate in this inactivity of the cerebral structures. The analogies between ordinary sleep and winter sleep or hibernation are probably real; the chief difference appears to. be that in the latter the diminished activity is due to an extrinsic cause, cold, and in the former to intrinsic causes, to 
clanges in the organism itself: but we saw in treating of hibernation. that intrinsic changes prepared the way for the action of external cold. It has been urged that during sleep the brain is anemic, and though observations have yielded conflicting results, the evillence seems to be in favour of this riew; but even if this anæmia is a constant accompaniment of sleep, it must, like the vascular condition of a gland or any other active organ. be regarded as an effect. or at least as a subsicliary event, rather than as a printary cause. Nor can the view which regards sleep as the result of a change in the mechanical arrangements of the cranial eirculation, such as either a retardation or acceleration of the venous outflow, be considered as satisfactory. 'The essence of the condition is rather to be sought in purely molecular changes, though we cannot, lowever, at present make any definite statements concerning the nature of these molecular changes.

The phenomena of sleep shew very clearly to how large an extent an apparent automatism is the ultimate outcome of the effects of antecedent stimulation. When we wish to go to sleep we withdraw our automatie brain as much as possible from the influence of all extrinsic stimuli. We lie down in order to relieve the skeletal muscles and indeed the heart too from the labour entailed by the erect posture; we put off the tight garments which eontinually spur the skin; we empty the bladder to a void the stimulus of its distension; and we choose for sleep the night and a quiet place, drawing the curtains, in order that our eyes may be withdrawn fron light and our ears from sounds. In this connection may be quoted the interesting case which is recorded of a lad whose sensory tie with the external world was, from a complicated ancesthesia, limited to that afforded by a single eye and a single ear; the lad could be sent to sleep at will, by elosing the eye and stopping the ear.

$\S 721$. The cycle of the day is however manifested in many other ways than by the alternation of slceping and waking, with all the indirect effects of these two conditions. There is a diurnal curve of temperature, apparently independent of all immerliate circumstances, the hereditary impress of a long and ancient sequence of days and nights. Even the pulse, so sensitive to all bodily changes, shews, running through all the immediate effects of the changes of the minute and the hour, the working of a diurnal influence which camnot be accounted for by waking and slceping, by working and resting, by meals and abstinence between meals. And the same may be said concerning the rhythm of respiration, and the products of pulmonary, eutaneous and urinary excretion. There seems to be a daily curve of bodily metabolism, which is not the product of the day's events. Within the day we have the narrower rhythm of the respiratory centre with the accompanying rise 
and fall of activity in the vaso-motor centres. And lastly, there stands out the fundamental fact of all bodily periodicity, that alternation of the heart's systole and diastole which ceases only at death. Though, as we have seen, the internittent flow in the arteries is toned down in the capillaries to an apparently continuous flow, still the constantly repeated cycle of the cardiac shuttle must leave its mark throughout the whole web of the body's life. Our means of investigation are, however, still too gross to permit us to track out its influence. 


\title{
CHAPTER IV.
}

\author{
DEATH.
}

$\S$ 722. WHEN the animal kingdom is surveyed from a broad standpoint, it becomes obvious that the ovum, or its correlative the spermatozoon, is the goal of an individual existence; that life is a cycle beginning in an ovum and coming round to an ovum again. The greater part of the actions which, looking from a near point of view at the higher animals alone, we are apt to consider as eminently the purposes for which animals come into existence, when viewed from the distant outlook whence the whole living world is surveyed, fade away into the likeness of the mere byplay of ovum-bearing organisms. The animal body is in reality a vehicle for ova; and after the life of the parent has become potentially renewed in the offspring, the body remains as a cast-off envelope whose future is but to die.

Were the animal frame not the complicated machine we have seen it to be, death might come as a simple and gradual dissolution, the 'sans everything' being the last stage of the successive loss of fundamental powers. As it is, however, death is always more or less violent; the machine comes to an end by reason of the disorder caused by the breaking down of one of its parts. Life ceases not because the molecular powers of the whole body slacken and are lost, but because a weakness in one or other part of the machinery throws its whole working out of gear.

We have seen that the central factor of life is the circulation of the blood, but we have also scen that blood is not only useless, but injurious, unless it be duly oxygenated; and we have further seen that in the higher animals the oxygenation of the blood can only be duly affected by means of the respiratory muscular mechanism, presided over by the respiratory centre in the bulb. Thus the life of a complex animal is, when reduced to a simple form, composed of three factors: the maintenance of the circulation, the access of air to the hemoglobin of the blood, and the functional activity of the respiratory centre; and death may come from the arrest of any one of these three. As it has been put, death may begin at the heart or at the lungs or at the 
brain. In reality, however, when we push the analysis further, the central fact of death is the stoppage of the heart, and the consequent arrest of the circulation; the tissues then all die, because they lose their internal medium. The failure of the heart may arise in itself, on account of some failure in its nervous or muscular elements, or by reason of some mischief affecting its mechanical working. Or its stoppage may be due to some fault in its internal medium, such for instance as a want of oxygenation of the blood, which in turn may be caused by either a change in the blood itself, as in carbonic oxide poisoning, or by a failure in the mechanical conditions of respiration, or by a cessation of the action of the respiratory centre. The failure of this centre, and indeed that of the heart itself, may be caused by nervous influences proceeding from the brain, or at least brought into operation by means of the central nervous system ; it may, on the other hand, be due to an imperfect state of blood, and this in turn may arise from the imperfect or perverse action of various secretory or other tissues. The modes of death are in reality as numerous as are the possible modifications of the various factors of life; but they all end in a stoppage of the circulation, and the withdrawal from the tissues of their internal medium. Hence we come to consider the death of the body as marked by the cessation of the heart's beat whenever that cessation is one from which no recovery is possible; and by this we are enabled to fix an exact time at which we say the body is dead. We can, however, fix no such exact time to the death of the individual tissues. They are not mechanisms, and their death is a gradual loss of power. In the case of the contractile tissues, we have apparently in rigor mortis a fixed term, by which we can mark the exact time of their death. If we admit that after the onset of rigor mortis recovery of irritability is impossible, then a rigid muscle is one permanently dead. In the case of the other tissues, we have no such objective sign, since the rigor mortis of other tissues manifests itself chiefly by obscure chemical signs. And in all cases it is obvious that the possibility of recovery, depending as it does on the skill and knowledge of the experimenter, is a wholly artificial sign of death. I tet we can draw no other sharp line between the seemingly dead tissue whose life has flickered down into a smouldering ember which can still be fanned back again into flame, and the handful of dust, the aggregate of chemical substances into which the decomposing tissue finally crumbles.

Moreover, the failure of the heart itself is at bottom loss of irritability, and the possibility of recovery here also rests, as far as is known at present, on the skill and knowledge of those who attempt to recover. So that after all the signs of the death of the whole body are as artificial as those of the death of the constituent tissues. 



\section{APPENDIX.}

\section{THE CHEMICAL BASIS OF THE}

ANIMAL BODY.

BY

A. SHERIDAN LEA, M.A., Sc.D., F.R.S., Fellow and Lecturer of Gonville and Caius College, Cambridge. 


\section{PREFACE.}

The following Appendix is an abridgement of the larger work published in 1892 as Part V. of Professor Foster's Text-book. The reduction in bulk has been effected by the omission of the discussional parts in the original, as also of many less essential details as to methods, etc., and of the references to the literature of the subject.

\section{A. SHERIDAN LEA.}

July, 1896. 


\section{APPENDIX.}

\section{THE CHEMICAL BASIS OF THE ANIMAL BODY.}

THE animal body, from a chemical point of view, may be regarded as a mixture of various representatives of three large classes of chemical substances, viz. proteids, carbohydrates, and fats, in association with smaller quantities of varions saline and other crystalline bodies. By proteids are meant bodies containing carbon, oxygen, hydrogen, and nitrogen in a certain proportion, varying within narrow limits, and having certain general features; they are frequently spoken of as albumiuoids. By carbohydrates are meant starches and sugars and their allies. IV e have also seen that the animal body may be considered as made up on the other hand of actual "living substance," sometimes spoken of as protoplasm (see $\$ 5$ ) in its various modifications, and on the other liand of numerous lifeless products of metabolic activity. We do not at present know anything definite about the molecular composition of the active living substance; but when we submit living substance to chemical analysis, in which act it is killed, we always obtain from it a considerable quantity of the material spoken of as proteid. And many authors go so far as to speak of living substance or protoplasm as being purely proteid in nature: they regard the living protoplasm as proteid material, which in passing from death to life, has assumed certain characters and presumably has been changed in construction, but still is proteid matter; they sometimes speak of protoplasm as "living proteid" or "living albumin." It is worthy of notice however that even simple forms of living matter, like that constituting the body of a white corpuscle, forms which we may fairly consider as the nearest approach to native protoplasm, when they cau be obtained in sufficient quantity for cliemical analysis, are found to contain some representatives of carbohydrates and fats as well as of proteids. We might perhaps even go as far as to say, that in all forns of living substance, the proteid basis is found upon analysis to have some carbohydrate and some kind of fat associated with it. Further, not only does the normal food 
which is eventually built up into living substance consist of all three classes, but, as we have seen in the sections on nutrition, gives rise by metabolism to members of the same three classes; and as far as we know at present, carbohydrates and fats, when formed in the body out of proteid food, are so formed by the agency of living snbstance, by the action of some living tissue. Hence there is at least some reason for thinking it probable that the molecnle of living substance, if we may use sueh a phrase, is far more eomplex thin a molecule of proteid matter, that it contains in itself residues so to speak not only of proteid but also of carbohydrate and fatty material.

Whether this be so or not, for at present no dogmatic statement can be made, there is no doubt that when we examine the various tissues and fluids of the animal body from a chemical point of view we find present in different places, or at different times in the same tissue or fluid, several varieties and derivatives of the three chief classes ; we find many forms of proteids, and bodies elosely allied to proteids, in the forms of mucin, gelatin, etc.; many varieties of fats; and several kinds of carbohydrates.

We find moreover many other substances which we may regard as stages in the constructive or destructive metabolism of the varions forms and phases of living matter, and which are important not so much from the quantity in which they oceur in the animal body at any one time as from their throwing light on the nature of animal metabolism; these are such substances as urea, uric acid, other organic crystalline bodies, and the extractives in general.

In the following pages the chemical features of the more important of these various substances which are known to occur in the animal body will be briefly considered, such characters only being deseribed as possess or promise to possess physiological interest. The physiological function of any substance must depend nltimately on its molecular (including its chemical) nature; and though at present our chemical knowledge of the constituents of an animal body gives us but little insight into their physiological properties, it cannot be doubted that sirch chemieal information as is attainable is a necessary preliminary to all physiological study.

\section{PROTEIDS.}

These form the principal solids of the nuscular, nervous, and glandular tissnes, of the sermm of blood, of serous fluids, and of lymph. In a healthy condition, sweat, tears, bile, and urine contain mere traces, if any, of proteids. Their general 
percentage composition may be taken as lying within the following limits :

$$
\begin{aligned}
& \text { C } 50 \cdot 0 \text { to } 55 \cdot 0 \ldots .50 \cdot 0 \text { to } 55 \cdot 0 \\
& \mathrm{H} \quad 6 \cdot 9, \quad 7 \cdot 3 \ldots . .66 .8,7 \cdot 3 \\
& \text { N } 15 \cdot 0,18 \cdot 0 \ldots 15 \cdot 4,18 \cdot 2 \\
& \text { O } 20 \cdot 0,23 \cdot 5 \ldots .22 \cdot 8,24 \cdot 1 \\
& \text { S } 0 \cdot 3,, 2 \cdot 0 \ldots 0 \cdot 4,5 \cdot 0 \\
& \text { (Hoppe-Seyler.) (Drechsel.) }
\end{aligned}
$$

The composition of the true proteids lies so constantly within the above limits that conclusions as to the proteid nature of any substance whose purity is assured may be drawn with safety from the results of its ultimate analysis.

In addition to the above constituents, proteids ordinarily leave on ignition a variable quantity of ash. In the case of egg-albumin the principal constituents of the ash are chlorides of sodium and potassium, the latter exceeding the former in amount. The remainder consists of sodium and potassium, in combination with phosphoric, sulphurie, and earbonic acids, and very small quantities of calcium, magnesium, and iron, in union with the same acids. There may be also a trace of silica. The ash of serum-albumin contains an excess of sodium chloride, but the ash of the proteids of muscle contains an excess of potash salts and phosphates. The sulphur in proteids is present partly in a stably combined condition, partly loosely combined. 'The latter is removed by boiling with alkalis, the former' is not. The proportions of the two differ in the several proteids.

Proteids met with in the animal body are all amorphous, the only apparent exception being hemoglobin: this substance is however not a pure proteid but a compound of a proteid globin with the less complex hæmatin. It is to the latter that the power of crystallizing is due.

Some are soluble, some insoluble in water, some are characteristically soluble in moderately concentrated solutions of neutral salts, and all are for the most part insoluble in alcohol and ether; they are all soluble in strong acids and alkalis, but in becoming dissolved mostly undergo decomposition. 'Their solutions exert a left-lianded rotatory action on the plane of polarization, the amount depending on various cireumstances, and differing for the several proteils.

Crystals into whose composition certain proteid (globulin) elements largely entered were long since observed in the aleurone-grains of many seeds. Similar crystalloid compounds are also described as occurring occasionally in the egg-yolk of some animals (Amphibia and Fishes). By appropriate methods they may be separated and reerystallized from their solution in distilled water. 


\section{General reactions of the proteids.}

1. Heated with strong nitric acid, they or their solutions turn yellow, and this colour is, on the addition of ammonia, or caustic soda or potash, changed to a deep orange luue. (Xanthoproteic reaction.)

2. With Millon's reagent ${ }^{1}$ they give, when present in sufficient quantity, a presipitate, which turns red on boiling. If they are only present in traces, no precipitate is obtained, but nnerely a red colouration of the solution when boiled.

3. If mixed with an excess of concentrated solution of sodinm hydrate, and one or two drops of a dilute solution of sulphate of copper, a violet colour is obtained, which deepens in tint on boiling. (Piotrowski's reaction.)

The above serve to detect the smallest traces of all proteids.

4. Render the fluid strongly acid with acetic acid, and add a few drops of a solution of ferrocyanide of potassium; a precipitate shews the presence of proteids, except true peptones and some forms of albumose.

No general method can be given for the quantitative estimation of the various proteids. For this some special manuals should be consulted and use made of the reactions which are specifically characteristic of each proteid as given below.

\section{Classification of the Proteids.}

The following classification is both convenient and concise.

\section{Class I. Native albumins.}

Soluble in distilled water. Solutions coagulated on heating, especially in presence of a dilute (acetic) acid. Not precipitated by carbonates of the alkalis or by sodium chloride, or generally by solutions of neutral salts.

\section{Egg-albumin. 2. Serum-albumins.}

\section{Class II. Derived albumins ${ }^{2}$ (Albuminates).}

Insoluble in distilled water and in dilute neutral saline solutions; soluble in acids and alkalis. Solutions not coagulated by boiling.

1. Acid-albumin. 2. Syntonin. 3. Alkali-albumin.

1 A solution of mixed mercurous and mercuric nitrates prepared by dissolving mercury in nitric acid.

2 Casein was at one time placed in this group with reference chiefly to its solubilities and precipitability. It is now known to be a nucleo-albumin, and is classed as such. (See p. 1207.) 


\section{Class III. Globulins.}

Insoluble in distilled water, soluble in dilute saline solutions. Soluble in very dilute acids and alkalis: if the acids and alkalis are strong they are rapidly changed into members of Class II. Readily precipitated by saturating their dilute saline solutions with neutral salts such as sodium chloride or magnesium sulphate.

1. Crystallin, the globulin of the crystalline lens. 2. Vi. tellin. 3. Paraglobulin or Serum-globulin. 4. Fibrinogen. 5. Myosin. 6. Globin.

\section{Class IV. Fibrins.}

Insoluble in water. Soluble with difficulty in strong acids and alkalis, and undergoing a simultaneous change into members of Class II. Soluble by the prolonged action of moderately strong (10 p.c.) solutions of neutral salts, with simultaneous change into members of Class III.

\section{Class V. Coagulated proteids.}

Products of the action of heat on members of Classes I., III., and IV., or of Class II. when precipitated by neutralization and heated in suspension in water. They are also obtained by the prolonged action of alcohol in excess upon members of Classes I., III., and IV. 'Their' solubilities, except in solutions of neutral salts, in which they are insoluble, are in genera] similar to, but less than, those of Class IV.

\section{Class VI. Albumoses and peptones.}

The true peptones are extremely soluble in water. They are not precipitated by acids, alkalis, neutral salts or many of the reagents which precipitate other proteids. They are precipitated but not coagulated by even the prolonged action of alcohol. Peptones are readily diffusible, albumoses less so. Some of the albumoses are readily soluble in water, some are less soluble. They are distinguished from peptones by being precipitated when their solutions are saturated with neutral ammonium sulphate. They yield precipitates with many of the reagents which precipitate other proteids, and it is specially characteristic that the precipitates they yield with nitric acid and with ferrocyanide of potassium in presence of acetic acid disappear when warmed and reappear on cooling. 


\section{Class VII. Lardacein or amyloid substance.}

Insoluble in water, dilute acids and alkalis and saline solutions. Converted into members of Class II. by strong acids and alkalis.

\section{The Chemistry of the several Proteids.}

\section{Class I. Native Albumins.}

\section{Egg-albumin.}

As obtained in the solid form by evaporating its solutions to dryness at $40^{\circ}$, preferably in vacuo, it forms a seni-transparent, brittle mass, of a pale yellow colour, tasteless and inodorous. Dissolved in water it yields a clear neutral colourless solution. This solution coagulates on heating, but the temperature at which the coagulation takes place varies considerably with the concentration and is largely dependent upon the presence or absence of salts. The more commonly observed temperature is 70 - $73^{\circ}$, but it is stated that coagula may also be obtained at $54^{\circ}$ and $63^{\circ}$. The more dilute the solution is the higher is the temperature at which it coagnlates, thus finally resembling a solution of albumin from which the salts have been removed by dialysis. When precipitated from solution by excess of alcohol it is readily coagulated by the precipitant, so that it is now usually insoluble in water. In this respect it differs somewhat characteristically from serum-albumin which is not so immediately though it is ultimately coagulated by the action of aleohol.

Strong acids, especially nitric acid, cause a coagulation similar to that produced by heat or by the prolonged action of alcohol; the albumin becomes profoundly changed by the action of the acid and does not dissolve upon removal of the acid. Mercuric chloride, nitrate of silver and lead acetate, precipitate the albumin, forming with it insoluble compounds of variable composition.

Strong acetic acid in excess gives no precipitate, but when the solution is concentrated the albumin is transformed into a transparent jelly. A similar jelly is produced when strong canstic potash is added to a concentrated solution of eggalbumin. In both these cases the substance is profoundly altered, becoming in the one case acid- in the other alkalialbumin.

According to recent researches egg-albumin may be obtained in a crystalline form by slow evaporation of its solutions in presence of neutral ammonium sulphate. The separation takes place at first in the form of minute spheroidal globules of 
various sizes and finally minute needles, either aggregated of separate, make their appearance. It has not as yet been found possible to obtain these so-called crystals from solutions which have been freed by dialysis from the ammonium salt.

\section{Serum-albumin.}

This is the sole proteid, apart from the globulins, which occurs in serum. Pure solutions of this proteid closely resemble those of egg-albumin in their general reactions, but the difference of the two is clearly shewn by the following statements.

1. When free from salts and in $1-1.5$ p.c. solution it coagulates on heating to $50^{\circ}$. 'The addition of sodium chloride raises the coagulating point to $75-80^{\circ}$. Under the conditions in which it occurs in serum it is not found to shew any opalescence on heating at any temperature below $60^{\circ}$, and it may be regarded as coagulating completely at $75^{\circ}$.

By fractional heat-coagulation of serum freed from globulin, evidence has been obtained of the existence in the serum of many animals of three albumins coagulating at $70-73^{\circ}, 77-78^{\circ}$, and $82-85^{\circ}$. In some serums only two of these albumins occur.

2. It is not readily coagulated by alcohol or precipitated by ether: egg-albumin is, and most readily by alcohol.

3. It is more strongly lævo-rotatory than egg-albumin.

4. It is not very readily precipitated by strong hydrochloric acid and the precipitate is readily soluble on the further addition of acid: the reverse is the case for egg-albumin.

5. Precipitated or coagulated serum-albumin is more readily soluble in nitric acid than is egg-albumin.

6. Egg-albumin if injected subcutaneously or into a vein, reappear's unaltered in the urine; serum-albumin similarly injected does not thus normally pass ont by the kidney.

Serum-albumin is found not only in blood-serum, but also in lymph, both that contained in the proper lymphatic channels and that diffused in the tissues; in chyle, milk, transudations, and many pathological fluids. urine.

It is this form in which albumin generally appears in the

\section{Class II. Derived Albumins (Albuminates).}

\section{Acid-albumin.}

When a native albumin in solution, such as egg-or serumalbumin, is treated for some little time with a dilute acid, such as hydrochloric, its properties become entirely changed. 'The most marked changes are (1) that the solution is no longer 
coagulated by heat; (2) that when the solution is carefully neutralized the whole of the proteid is thrown down as a precipitate; in other words, the serum-albumin, which was soluble in water, or at least in a neutral fluid containing only a small quantity of neutral salts, has become converted into a substance insoluble in water or in similar neutral fluids. The body into which serum-albumin thus becomes converted by the action of an acid is spoken of as acid-albumin. Its characteristic features are that it is insoluble in distilled water, and in neutral saline solutions, such as those of sodic chloride, that it is readily soluble in dilute acids or dilute alkalis, and that its solutions in acids or alkalis are not coagulated by boiling. When suspended, in the undissolved state, in water, and heated to $75^{\circ} \mathrm{C}$., it becomes coagulated, and is then undistinguishable from coagulated serum-albumin, or indeed from any other form of coagulated proteid.

Globulins are more readily converted into acid-albumin than are the native albumins. Coagulated proteids or fibrin require for their conversion the application of the acids, preferably hydrochloric, in a concentrated form, the products thus obtained being practically undistinguishable from the products of the action of dilute acids on the more readily convertible proteids.

\section{Syntonin.}

Although this substance is merely the acid-albumin which results from the action of acids on the globulin (myosin) contained in muscles, and in its nore obvious properties is at first sight identical with other acid-albumins, it merits a short and separate description, not only on account of its historical interest in the chemistry of muscles but also because recent work has shewn it to be distinctly different from the similar products of the action of acids on other proteids, and its properties and reactions have been more fully studied than those of any other form of acid-albumin.

The reactions specially characteristic of this substance and its distinctions from other forms of acid-albumin and from alkali-albumin are indicated in the following statements.

1. It is soluble in lime-water, and this solution is coagulated, though incompletely, by boiling.

2. It is insoluble in acid phosphate of soda $\left(\mathrm{NaH}_{2} \mathrm{PO}_{4}\right)$, other acid-albumins are soluble. In presence of this salt it does not pass into solution on the addition of alkali until the whole of the acid phosphate has been converted into the neutral $\left(\mathrm{Na}_{2} \mathrm{HPO}_{4}\right)$. In this respect it differs from alkali-albumin, which is soluble under the same conditions long before the conversion of the acid into the neutral phosphate is complete.

3. It is soluble in dilute sodium carbonate. 
4. When precipitated from its acid solution by neutralization the precipitate is more gelatinous than that of the other acid-albumins, and less readily soluble in alkalis.

\section{Alkali-albumin.}

If serum- or egg-albumin or washed muscle be treated with dilute alkali instead of with dilute acid, the proteid undergoes a change in many ways similar to that which was brought about by the acid. The alkaline solution, when the change has become complete, is no longer coagulated by heat, the proteid is wholly precipitated on neutralization, and the precipitate, insoluble in water and in neutral solutions of sodium chloride, is readily soluble in dilute acids or alkalis.

Alkali-albumin may be prepared by the action not only of dilute alkalis but also of strong caustic alkalis on native albumins as well as on coagulated albumin and other proteids. The jelly produced by the action of caustic potash on white of egg (p. 1168) is alkali-albumin; the similar jelly produced by strong acetic acid is acid-albumin. In short the general statement may be made that under otherwise similar conditions, if an alkali is employed instead of an acid to act on proteids, alkalialbumin is formed instead of acid-albumin.

Notwithstanding their very similar general reactions acidand alkali-albumin are distinct, though very closely allied substances, and we might go even so far as to say that probably every proteid yields its own kind of either the one or the other proteid on treatment with acids and alkalis. But as yet we do not possess any means of distinguishing between the several forms of each substance by any ordinary reactions.

The chief evidence which is advanced as to the difference of the two products is the following.

1. Alkali-albumin is in general more soluble than acidalbumin.

2. When precipitated by neutralization the former (alkali) is flocculent, the latter (acid) is more viscid, transpareut, and gelatinous.

3. When dissolved in a minimum of alkali and heated to $100^{\circ}$ in sealed tubes, alkali-albumin coagulates, acid-albumin does not.

4. Alkali-albumin possesses, strongly marked, the properties of an acid.

5. Acid-albumin can be converted into alkali-albumin by the action of strong alkalis, but the reverse conversion of the product thus obtained or of an ordinary prepared alkali-albumin into acid-albumin is stated to be impossible. 


\section{Class III. Globulins.}

Besides the derived albumins there are a number of native proteids which differ from the albumins in not being soluble in distilled water; they need for their solution the presence of an appreciable, though it may be a small, quantity of a neutral saline substance such as sodium chloride. Thus they resemble the albuminates in not being soluble in distilled water, but differ from them in being soluble in dilute sodium chloride or other neutral saline solutions. 'Their general characters may be stated as follows.

They are insoluble in water, soluble in dilute (1 p.c.) solutions of sodium chloride; they are also soluble in dilute acids and alkalis, being changed on solution into acid- and alkalialbumin respectively unless the acids and alkalis are exceedingly dilute and their action is not prolonged. The saturation with solid sodium chloride or other neutral salts of their saline solutions, precipitates most members of this class.

\section{Crystallin. (Globulin of the crystalline lens.)}

This form of globulin is usually regarded as identical with vitellin. It is however convenient to treat it separately inasmuch as it can be prepared in a pure form, whereas vitellin has not as yet been olstained free from lecithin.

Crystalline lenses are rubbed up in a mortar with a little fine sand and a few crystals of rock salt; the mass is then extracterl with water and filtered. The filtrate contains the crystallin and some serum-albumin. The former is separated from the latter by copious dilution with distilled water and passing a current of carbon dioxide through the diluted mixture, whereupon the crystallin is precipitated.

According to the latest researches the lens contains two globulins which differ slightly in their precipitability and in the temperatures at which their solutions coagulate.

\section{Vitellin.}

This constitutes the characteristic proteid constituent of egg-yolk. Some at least of the globulins present in regetable protoplasm and more particularly in the crystals of the aleurone grains, appear to be identical in their general properties and reactions with vitellin. As obtained in conjunetion with some lecithin by exhaustion of egg-yolk with ether, it consists of a white, pasty, granular nass, insoluble in water, readily soluble in solutions of sodium chloride which may be easily filtered. Unlike other true globulins it cannot be precipitated from this solution by saturation with sodium chloride. Its saline solu- 
tions ( 10 1). NaCl) are coagulated by heating to $75^{\circ}$. It is readily soluble in 1 p.c. sodium earbonate, is incompletely precipitated from this solution by dilution with water, but fairly completely by the additional passing of a stream of carbon dioxide through the diluted solution.

Vitellin has not as yet been obtained free from lecithin and is also possibly in egg-yolk loosely combined with a nuclein. Further investigation is needed to determine its real nature.

\section{Paraglobulin. (Serum-globulin.)}

This proteid occurs most characteristically in blood-serum (also in lymph), in amounts now known to be much larger than was at one tine supposed, and thus constituting about one-half of the total proteids of the serum.

'The most satisfactory method of preparing it pure and in considerable quantity is as follows: serum is saturated at $30^{\circ}$ with magnesium sulphate, by means of which paraglobulin is quantitatively precipitated. The precipitate collected by filtration is distributed through a small volume of a saturated solution of the magnesium salt, collected on a filter and washed with saturated solution of $\mathrm{IgSO}_{4}$. By this means it is separated from the larger part of the serum-albumin.

'To effect its final and complete separation from this latter proteid, two methods mat be alopted. (a) The precipitate is dissolved in water, then largely diluted and the paraglobulin further separated out by passing a stream of $\mathrm{CO}_{2}$. ( $\beta$ ) 'The precipitate is dissolved as before in water, the paraglobulin again salted out by $\mathrm{MgSO}_{4}$, this process repeated several times, and the final product separated from the magnesium salt by dialysis.

Pure paraglobulin is insoluble in water. If dissolved in a minimal amount of alkali it is precipitated by .03 to .5 p.c. of $\mathrm{NaCl}$. On the addition of more than $.5 \mathrm{p} . \mathrm{c}$. of the salt it goes again into solution and does not begin to be reprecipitated on the addition of more salt until at least 20 p.c. NaCl has been added. It is not completely precipitated by saturation of its solutions with $\mathrm{NaCl}$. Its dilute saline solutions coagulate on heating to $75^{\circ}$.

Paraglobulin occurs in smaller amounts $\left(\frac{1}{3}-\frac{1}{2}\right)$ in chyle, lymph, and serous fluids.

Cell-globulins. A name given to some forms of globulin which occur in lymph-corpuscles and may be extracted from them by solutions of sodium-chloride. Of these, one, cell-globulin- $\alpha$, occurs in minute quantities only and is characterized by coagulating at $48-50^{\circ}$. The other, cell-globulin- $\beta$, is more copiously present in the corpuscles and coagulates in dilute saline solutions at $65^{\circ}$. The latter resembles paraglobulin very closely in properties other than the identity of 
their temperatures of heat coagulation in dilute saline solntions, e.g. precipitability, ete. Cell-globulin- $\beta$ is stated to differ from true paraglobulin, by possessing the power of lastening the clotting of diluted salt-plasma, and the so-called 'fibrin-ferment' is accordingly regarded by some as identical with cell-globulin- $\beta$ and arising from the disintegration of leucocytes.

The proteid constituent of the stroma of red blood-corpuseles consists chiefly of a globulin usually regarded as identical with paraglobulin, since its saline solutions coagulate at $75^{\circ}$ and it is precipitated from the same by saturation with sodium chloride and a current of carbon dioxide.

\section{Fibrinogen.}

This globulin occurs in blood-plasma together with paraglobulin and serum-albumin. During blood-clotting it is converted largely, if not entirely into fibrin. It is also found in chyle, serous fluids and transudations, more particularly in hydrocele fluids.

In its general reactions it resembles paraglobulin but is markedly distinguished from the latter by the following characteristies. (1) As it oecurs in plasma or in dilute solutions of sodium chloride ( $1-5$ p.c.), it coagulates at $55-56^{\circ}$. (2) It is very readily precipitated by the addition of sodium chloride to its saline solutions until the whole contains 16 p.e. $\mathrm{NaCl}$, whereas paraglobulin is not appreciably precipitated until at least 20 p.c. of the sodium salt has been added.

Salted plasma, obtained by centrifugalizing blood whose clotting is prevented by the addition of a certain proportion of magnesium sulphate, is mixed with an equal volume of a saturated $\left(35.87\right.$ p.c. at $14^{\circ} \mathrm{C}$.) solution of sodium chloride; the fibrinogen is thus precipitated while the paraglobulin remains in solution. The adhering plasma may be removed by washing with a solution of sodium chloride, and the fibrinogen finally purified by being several times dissolved in and reprecipitated by sodium chloride.

When a fluid containing purified fibrinogen is made to yield fibrin by the action of fibrin-ferment, the amount of fibrin formed is always less than that of the fibrinogen which disappears at the same time. The deficit thms observed is at least partly accounted for by the simultaneous appearance of a globulin which coagulates, when leated in saline solution, at $64^{\circ}$.

\section{Myosin.}

When an irritable contractile musele passes into rigor, the substance of which the muscle-fibres are chiefly composed undergoes a change, analogous to the clotting of blood-plasma, which results in the formation of a clot of myosin. By appro- 
priate methods (see $\$ 56$ ) the muscle-fibres may be broken up and their contents obtained as a viseid slightly opalescent fluid (muscle-plasma), which filters with difficulty and clots at temperatures above $0^{\circ}$. The muscle-plasma may be diluted with solutions of varying strengths of several neutral salts, whereby its clotting may be delayed, and the nature and phenomena of the processes involved in the clotting investigated along the lines previously employed in the clucidation of the phenomena of the clotting of blood-plasma. The more important facts which have thus been made out may be briefly summarized as follows. Muscle-plasma contains a globulinforerumer of myosin ('myosinogen') which resembles fibrinogen in coagulating at $56^{\circ}$. This proteid is converted into myosin on the occurrence of clotting by the action of a specific ferment, which is regarded as being closely related to, if not irlentical with an albumose. The sermm, which is left in small quantities only after the formation of the clot, contains proteids which coagulate at $47^{\circ}$ (paramyosinogen), $63^{\circ}$ (myoglobulin), and $73^{\circ}$ an albumin closely resembling serumalbumin.

Apart from the general reactions which characterize myosin as a globulin, it is distinguished by the low temperature $\left(55-56^{\circ}\right)$ at which its saline solutions constantly coagulate. It leaves a large ash residue on incineration, consisting chiefly of salts of lime. As already stated, it is converted into an insoluble proteid by the prolonged action of water, and into syntonin by the action of acids. It is also stated that if myosin is dissolved in $\mathrm{NaCl}$ or $\mathrm{MgSO}_{4}$ (10 and 5 p.c. respectively) it yields a renewed clot on mere dilution with water.

Globulins to which the name of myosin is applied are described as occurring in vegetable protoplasm and in the cells of the liver.

\section{Globin.}

When hæmoglobin is allowed to undergo decomposition spontaneously by exposure to the air an insoluble proteid is obtained of which very little is known, but to which the name of globin has been given. It appears to be perhaps an outlying nember of the globulin class of proteids, but unlike a true globulin is scarcely soluble in dilute acids and imperfectly soluble in alkalis and solutions of sodium cliloride. It is converted into acid and alkali-albumin by the action of strong acids and alkalis respectively, and is stated to yield no trace of ash on incineration.

\section{Class IV. Fibrin.}

This proteid is ordinarily obtained by 'whipping' blood with a bundle of twigs until clotting is complete; the fibrin which 
adheres to the twigs is then washed in a current of water until all the hemoglobin of the entangled corpuscles is removed and it is now quite white. The washing is greatly faeilitated if the fibrin is very finely chopped before it is washed, and if it is frequently kneaded and squeezed with the hand during the washing. In this way it may be obtained quite white in a few hours. The washing is also much facilitated if the blood is mixed with an equal bulk of water before it is whipped. It is obvious that fibrin prepared by the above method must be in an extremely impure condition, for it contains a not inconsiderable admixture of the remains of the white corpuscles and the stronata of the red. It can only be prepared pure during the clottiug of either filtered or centrifugalized iced-plasma or saltplasma, or by the action of purified fibrin-ferment on pure fibrinogen. In accordance with this, fibrin as ordinarily obtained leaves a variable amount of granular residue which contains plosphorus during its digestion by pepsin. No such residue is observed when fibrin from fittered plasma is digested with pepsin, but in no other essential respect does the one fibrin differ from the other.

Fibrin, as ordinarily obtained, exhibits a filamentous structure, the component threads possessing an elasticity much greater than that of any other known solid proteid.

If allowed to form gradually in large masses, the filamentous structure is not so noticeable, and it resembles in this form pure india-rubber. Such lumps of fibrin are capable of being split in any direction, and no definite arrangement of parallel bundles of fibres can be made out.

Fibrin is insoluble in water and dilute saline solutions. It is also ordinarily insoluble in dilute acids ( $\mathrm{HCl}$ ) if their action takes place at ordinary temperatures and is not prolonged, merely becoming swollen and transparent in the acid and returning to its original state if the acid is removed by an excess of water or eareful addition of an alkali. By prolonged action at ordinary temperatures, or a shorter action at $40^{\circ}$, the fibrin is profoundly changed and certain forerumners of the peptones which may be finally formed (at $40^{\circ}$ ) are produced. It is similarly insoluble in dilute alkalis and ammonia, but passes more readily into solution in these reagents, if their action is prolonged or the temperature is raised, than is the case with dilute acids. 'The behaviour of fibrin towards solutions of neutral salts is peculiar and important. As already stated, fibrin prepared by simply whipping blood is insoluble in dilute saline solutions. When fibrin is subjected to the prolonged action of more eoncentrated ( 10 p.c.) solutions of nentral salts, and the salt solution is fiequently renewed, the fibrin may be finally completely diss.nferd, leing converted into members of the globnlin class. Most observers agree that the globulin thus chiefly formed 
coagulates at $55-56^{\circ}$, but in addition one may be obtained coagulating at $59-60^{\circ}$, the two differing further in their solubilities in 1 and 10 p.c. solutions of NaCl. These changes are brought about by the salts in the entire absence of any putrefactive phenomena, and the resulting globulins cannot be made to yield fibrin again by any treatment with fibrin-ferment.

When fresh unboiled fibrin is simply washed till it is white and digested with pure active trypsin, it is largely converted into coagulable proteids during the initial stages of the ferment action. These proteids are eharacteristically globulins, and one is closely related to paraglobulin, as judged of by its coagulating in saline solutions at $75^{\circ}$. The second coagulates at $55-56^{\circ}$, and in this respect more closely resembles fibrinogen.

The purest fibrin always leaves a small but fairly constant ash-residue on incineration. Of the inorganic constituents of which this residne is composed it is probable that sulphur is the only element which enters essentially into the composition of the fibrin.

When boiled in water or treated for some time with alcohol it loses its elasticity, becomes much more opaque, is much less soluble in the various reagents which dissolve the original fibrin with comparative ease, is attacked with much greater difficulty by pepsin and trypsin, and is in fact indistinguishable from all other coagulated proteids.

A peculiar property of this body remains yet to be mentioned, viz. its power of decomposing hydrogen dioxide. Pieces of fresh fibrin placed in this fluid, though themselves undergoing no change, soon become covered with bubbles of oxygen ; and guaiacum is turned blue by fibrin in presence of hydrogen dioxide or ozonized turpentine.

\section{Class V. Coagulated Proteids.}

These are insoluble in water, dilute acids and alkalis, and neutral saline solutions of all strengths. In fact they are really soluble only in strong acids and strong alkalis, though prolonged action of even dilute acids and alkalis will effect some solution, especially at high temperatures. During solution in strong acids and alkalis a destructive decomposition takes place, but some amount of acid- or alkali-albumin is always produced, together with some peptone and allierl substances.

Very little is known of the chemical characteristics of this class. 'They are produced by heating to $100^{\circ} \mathrm{C}$., solutions of egg- or serum-albumin, globulins suspended in water or dissolved in saline solutions; by boiling for a sliort time fibrin suspended in water, or precipitated acid-and alkali-albumin suspended in water. They are readily converted at the tem- 
perature of the body into peptones, by the action of gastric juice in an acid, or of pancreatic juice in an alkaline medium.

All proteids in solution are precipitated by an excess of strong alcohol. If the precipitant be rapidly removed they are again solnble in water, but if the precipitated proteids are subjected for some time to the action of the alcohol they are, with the exception of albumoses and peptones, coagulated and lose their solubility.

\section{Class VI. Albumoses and Peptones.}

When any of the proteids already described are submitted to the digestive action of pepsin or trypsin, certain substances are formed, in the earlier stages of the action, which are intermediate between the proteid undergoing digestion and the proteid product (peptone) which finally results from the action of the enzymes. When the digestive fluid employed is pepsin in presence of dilute (.2 p.c.) hydrochloric acid, a small portion of the proteid nuay be at first converted into a form of ordinary acid-albumin. ${ }^{1}$ It is obtained by neutralizing a peptic digestive mixture at an early stage of the digestion, and has been frequently and almost usually confomided with the 'parapeptone' of Meissner. As will be explained later on, the two substances are quite distinct forms of protcid. At a later stage of the digestion the first-formed acid-albumin disappears, a considerable amount of parapeptone is formed and other products make their appearance, which are known collectively under the name of albunoses. By a more prolonged action of the pepsin a considerable portion of these albumoses is further changed into the final product peptones; beyond this stage no further change can be brought about by the action of pepsin. If trypsin be employed in an alkaline solution $\left(.25\right.$ p.c. $\left.\mathrm{Na}_{2} \mathrm{CO}_{3}\right)$ the decomposition of the proteid is much more complicated and profound. Instead of acid-albumin a small amount of alkali-albunin makes its appearance, together with more or less (see above, p. 1177) of coagulable globulins in the earliest stages of the digestion. Albumoses speedily make their appearance, to be somewhat rapidly and it may be largely converted into peptones, of which some are in their turn partially, though never completely, converted into leucine, tyrosine, and other less well-defined crystalline products. Similar products of the decomposition of proteids may be ohtained by the action of acids alone, in the absence of all enzyme, preponderance of any one or more of the products being dependent upon the concentration of the acids, the temperature at which they are employed, and the

${ }^{1}$ To this substance the name 'syntonin' was formerly applied; this term is however most appropriately used to denote that form of acid-albumin which results from the action of acids on myosin. (See above, p. 1170.) 
duration of their action. Proteids may also be peptonized by means of water acting at high temperatures under considerable pressure.

Meissner's Researches (1859-1862). When an alkali was added to the tiltered fluid resulting from the acid peptic digestion of any proteid, to an amount just short of that required for exact neutralization, a precipitate was obtained which he named parapeptone. In its general reactions it resembled acidalbumin or syntonin, but was distinctively claracterized by its incapability of undergoing conversion into a peptone by the further action of pepsin. He pointed out at the same time that it might be digested by an infusion of the pancreas. After the removal of the parapeptone he occasionally obtained a further precipitate by the addition of acid, to not more than $\cdot 05$ to $\cdot 1$ p.c., to the filtrate; this substance he named metapeptone. He further described a residue insoluble in dilute acids, but soluble in dilute alkalies, which made its appearance during the digestion of casein and to which lie gave the name of dyspeptone. After the removal of the above products there still remained in solution three substances called respectively $a$-, $b$-, and $c$-peptone and characterized as follows:

$a$-peptone; preeipitated by strong nitric acid and by potassium ferrocyanide in presence of weak acetic acid.

$b$-peptone; not precipitated by strong nitric acid nor by potassium ferrocyanide unless in presence of an excess of strong acetic acid.

$c$-peptone; not precipitated by nitric acid nor by the potassium salt, whatever be the amount of acetic acid simultaneously added.

These statements of Meissner led to considerable subsequent controversy, and the occurrence of the several products he described was, with the exception of parapeptone and $c$-peptone, denied by those who repeated his experiments.

The Researches of Kïhne. From what has been already said it is at once evident that Meissner"s views implied a decomposition or splitting-up of the primary proteid molecule, inasmuch as he held that his parapeptone wits incapable of conversion into peptone by the further action of pepsin. Kühne, impressed with the profound and obvious decomposition which trypsin brings about when it acts on proteids, reverted once more to the possibilities implied in Meissner's views. In so doing he found further confirmation of the idea that even in gastric peptonization the proteid is not merely changed but split up, in the fact that only a portion of the gastric peptones can be made to yield lencine and tyrosine by the action of trypsin; from which it follows that during a complete gastric peptonization at least two distinct peptones are formed. In accordance with this he assumed that the original p"oteid molecule must itself consist 
of two parts, of which each yielded its corresponding peptone during the hydration which leads to the formation of peptones. He found also further confirmation of this probability in the work of Schützenberger (1875). This observer, decomposing proteids witl acids at $100^{\circ} \mathrm{C}$., cane to the conclusion that half the proteid molecule is readily decomposable by the acids, while the other half is peculiarly resistent and is obtained in the final products as an extraordinarily indigestible but true proteid. to which he gave the characteristic name of 'hemiprotein.' Convinced thus of the double nature of the proteid molecule, and seeing but little hope of separating from each other in a mixture the two peptones which must presumably result from the gastric peptonization of a proteid, Kühne endeavoured to establish their existence by trying to discover the primary products intermediate between the proteid and the peptones, - antipeptone on the one hand and hemipeptone on the other. ${ }^{1}$ In this his endeavour's were at once assisted by his being in possession of a large amount of a proteid identical with that first described and carefully examined by Bence-Jones (1St8), and hence called by his name. A renewed examination of this substance revealed that it was capable of conversion by pepsin into a peptone which was readily further decomposed by trypsin. It was in fact the product intermediate between the original proteid and the hemipeptone, and to it Kühne gave the name of hemialbumose. It now was only necessary to obtain the corresponding albumose precursol of the antipeptone, to peptonize this and shew that the peptone thus obtained would yield no lencine or tyrosine by even prolonged treatment with trypsin. This Kühne succeeded in doing by a fractionated peptic digestion and thus established his own views, and in cloing so shewed how accurate as a whole Meissner's statements were. 'This will be evident from the detailed description of the several products of the decomposition of proteids by pepsin, trypsin, and acids, which is given below. The fundamental notion then of Kühne's view is that an ordinary native albumin or fibrin contains within itself two residues, which he calls respectively an anti-resiche and a hemi-residne. The result of either peptic or tryptic digestion is to split up the albumin or fibrin, and to produce on the part of the anti-residue antipeptone, and on the part of the heni-residue hemipeptone, the latter being distinguished from the former by its being susceptible of further change by tryptic digestion into leucine, tyrosine, \&e., each peptone being preceded by a corresponding anti- or hemi-albumose. Antipeptone remains as antipeptone

'The name 'hemipeptone' was given in order to convey the idea that it is the peptone formed from one half of the original proteid molecule, 'antipeptone' on the other hand that it is that form of peptone which withstands or is opposed to $(\dot{a} \nu \tau i)$ any further decomposing action of the agents which led to its appearance. 
even when placed under the action of the most powerful trypsin, provided putrefactive changes do not intervene. Kühne's views may be conveniently exhibited in the accompanying tabular forms.

\section{Deconposition of Proteids by Acids.}

1.

By $\cdot 25$ p.c. $\mathrm{HCl}$ at $40^{\circ} \mathrm{C}$.

Albumin.

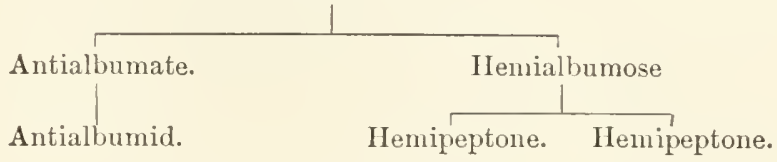

2 .

By $3-5$ p.c. $\mathrm{H}_{2} \mathrm{SO}_{4}$ at $100^{\circ} \mathrm{C}$.

Albumin.

Antialbumid.

Hemialbumose.

19

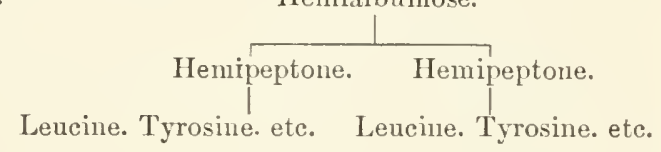

Decomposition of Proteids by Digestive Ferments (Enzymes).

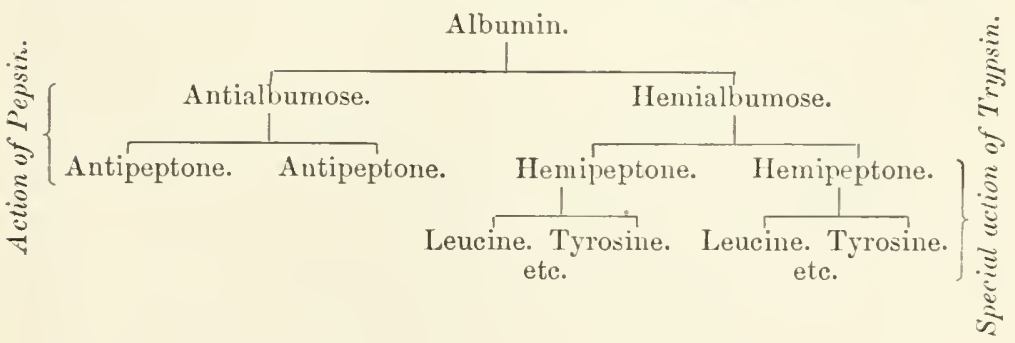

Having thus briefly stated the steps by which our present knowledge has been reached of the possible products of a digestive conversion of proteids, it now remains to deal with these products seriatim. In so doing it will be best to describe first such products as arise most largely and characteristically during the action of acids, and to treat of the albumoses and peptones subsequently.

Antialbumate. This substance is according to Kïhne identical with Meissner's parapeptone. It is most readily formed by the fairly prolonged action of dilute acids at $40^{\circ}$, but it may also make its appearance, but to much smaller extent, during a peptic digestion in which but little pepsin is present. It is 
obtained, mixed in some cases with variable quantities of an ordinary acid-albumin, by neutralizing the digesting mixture, from which it is thus precipitated. As alrcady stated, it is characterized by the property that it cannot be converted into a peptone by the most prolonged action of cren the most active pepsin, while on the other hand it is readily peptonized by trypsin and yields then antipeptone, but no lencine or tyrosine. Apart from its behaviour witl pepsin and trypsin, it resembles ordinary acid-albumin and syntonin in its general ehemical reactions. But the latter are chemically quite distinct from antialbumate or parapeptone, for either of them may be peptonized by pepsin, and the peptones thus formed maty be partly made to yield leucine and tyrosine by the subsequent action of trypsin.

Antialbumid. By the further prolonged or active treatment of antialbumate with acids it is converted into the substance to which Kühne gave the name of antialbumid. It is in all respects identical with the 'hemiprotein' of Schiitzcuberger, and also probably with the dyspeptone of Meissner, so far as the latter was not perhaps largely composed of nucleins. It also makes its appearance, but in very small amount, during a peptic digestion, and in considerable quantity during a pancreatic. It is characterized by its relatively great insolubility in dilute acids and alkalis, so that it separates out as a granular residue during a pancreatic digestion. This residue is readily soluble in 1 p.c. caustic soda; if reprecipitated by neutralization, it is now soluble in 1 p.c. sodium carbonate. From either of these solutions it is very completely precipitated by the addition of a little sodium chloride. In dilute alkaline solution (1 p.e. $\mathrm{Na}_{2} \mathrm{Co}_{3}$ ) it may be partly converted into a peptone by the action of trypsin, during which process the larger part separates out into a gelatinous coagulum or clot, which is quite unacted upon by pepsin and can only be peptonized by the prolonged action of very active trypsin in presence of a considerable amount (5 p.c.) of sodium carbonate. The peptone thus produced is antipeptone, for it yields no leucine or tyrosine by the action of trypsin.

The Albumoses. These are the primary products of the action of the proteolytic enzymes on proteids, and give rise by the further action of the ferments to the corresponding peptones. In accordance with Kühne's views already stated there must of necessity be at least two albumoses, antialbumose the forerumer of antipeptone, and hemialbumose of hemipeptone.

Antialbumose. 'This substance is obtained as a neutralization precipitate at a certain early stage of a fractionated peptic digestion of proteids. In its ordinary chenical reactions it is indistinguishable from acid-albumin or syntonin. It may be converted into a peptone by the further action of pejsin, and 
still more readily by the action of trypsin, so that it does not make its appearance in the final products of either a prolonged peptic or a short tryptic digestion. The peptone, into which it may be converted by either pepsin or trypsin, is antipeptone, for it cannot be made to yiekl any trace of leucine or tyrosine by even the most prolonged and energetic treatment with trypsin, and in this fact lies the distinction between antialbumose and either acid-albumin or syntonin. During its peptonization by trypsin some antialbumid is simultaneously formed. Antialbumose differs from parapeptone by the fact that the latter can only be peptonized by trypsin, the former by either pepsin or trypsin.

Hemialbumose. This is the best known, most characteristic, and most frequently obtained by-product of proteid zymolysis. ${ }^{1}$ It was first noticed and isolated by Meissner under the name of $a$-peptone, is identical with Bence--Jones' proteid in the urine of osteomalacia, and has also been known under the name of 'propeptone.' Of late years it has been recognized as occurring not infrequently in urine, and it is more than probable that many of the older statements as to the occurrence of peptones in urine and other fluids referred really to the oceurrence of hemialbumose. It is also stated to occur normally in the marrow of bones, and in ecrebrospinal fluid. Since it is readily peptonized by trypsin with the simultaneous formation from the peptone of much leucine and tyrosine, hemialbumose scarcely makes its appearance in any appreciable quantity in the final products of a pancreatic digestion. It is best prepared by the action of a small amount of very active pepsin on a considerable mass of fibrin, previously swelled up into a gelatinous mass by the action of 2 p.e. $\mathrm{HCl}$ at $40^{\circ}$. Under the action of the pepsin the fibrin liquefies : as soon as this is complete, dilute sodium carbonate is added until the reaction is just faintly alkaline, by which means a bulky precipitate is obtained. 'This is removed by filtration and the filtrate now contains a large amount of hemialbumose and but little peptone, and may be utilized directly for the tests characteristic of the albumose.

Reactions of Hemialbumose. The pure dry substance is not readily soluble in distilled water, but readily soluble in traces of acids, alkalis, and neutral salts (sodium chloride). These solutions give the following characteristic reactions :

1. Acidulate fairly strongly with acetic acid and add a few drops of saturated solution of sodium chloride; a precipitate is formed which disappears on warming and comes down again on cooling. If excess of the salt is added the precipitate does not dissolve on warming.

1 This expression may be conveniently used to denote generally the changes produced by the unorganized ferments. 
2. Add carefully a few drops of pure nitric acid; a precipitate is formed if the acid is not in excess, which disappears on warming and comes again on cooling.

3. Add acetic acirl, avoiding all excess, and then a trace of potassium ferrocyanide; a precipitate is formed which disappears on warming and reappears on cooling.

4. On the addition of caustic sola in excess and a trace of sulphate of enpper the ordinary biuret reaction is obtained. This reaction distinguishes hemialbumose from other soluble proteids, with the exception of peptones.

Hemialbumose has so far been spoken of as if it were one uniform substance only. More recent researeh has shown that four closely allied but distinct forms of proteid have to be dealt with under the name hemialbumose, so that this word has now acquired a historic rather than an actual designative significance. These substances are distinguished by the following names aud reactions. 1. Protoulbumose. Soluble in water and precipitable by saturation with sodium chloride. 2. Heteroalbumose. Insoluble in water but soluble in dilute (5-10 p.e.) solutions of sodium chloride, from which, like protoalbumose, it is precipitable by saturation with the salt. 3. Dysalbumose. Insoluble in either water or solutions of sodium chloride. It appears to be merely a modified form of heteroalbumose resulting from the prolonged action of water and neutral salts or of being kept dry. It may be readily reconverted into heteroalbumose by solution in dilute hydrochloric acid (.2 p.c.) or caustic soda (1 p.c.) and reprecipitation by neutralizing either of these solutions. 4. Deuteroalbumose. Soluble in water and not precipitable from this solution by saturation with sodium chloride unless an acid be added at the same time.

Solutions of protoalbumose and heteroalbumose yield precipitates directly on the addition of nitric acid; deuteroalbumose does so only in presence of sodium chloride. The latter is not precipitated by sulphate of copper; the two former are. Heteroalbumose is not preeipitable by mercuric chloride, whereas proto- and deutero-albumose are so.

The albumoses are slightly diffusible, but less so than the peptones.

The peptones. Since the albumoses and peptones must be regarded as a series of progressive products of the hydrolytic decomposition of proteids, it is difficult to decide the exact point at which the former pass into the latter, or in other words to obtain a distinctive criterion for a true peptone. Hence in all probability the rarious substances which have at earlier dates been described as peptones have consisted to some extent, if not largely, of a mixture of true peptones with variable quantities of albumoses. Of late years it has become customary to discriminate between the two classes of substances by reference 
to their behaviour towards neutral ammonium sulphate. The albumoses are precipitated when their solutions are saturated with this salt, and the true peptones are characterized as those final products of the digestion which are not preeipitable by the ammonium salt. The peptones are thus obtained in the filtrate from the saturated solntion and may be separated from the excess. of admixed salt by processes which are tedious and admit of no, suitably brief clescription.

The general properties and reactions of peptones as thus obtained may be stated as follows. Precipitated by alcohol they consist of a white or yellowish powder, which is hygroscopic and extraordinarily soluble in water, and hence often deliquescent. Unless thoroughly dehychated the powder may melt on gentle warming. They have such an affinity for water that a small portion of the dry substance when moistened hisses as does phosphoric anhydride under similar conditions. Fron their nentral aqueous solutions they are precipitated with clificulty by a large excess of alcohol, being unchanged in the process and not becoming coagulated or insoluble by prolonged exposure to the action of the precipitant. The precipitation occurs with difficulty if at all in presence of hydrochloric acid. Peptones are strikingly non-precipitable by many of the reagents by which other proteids may be precipitated, more especially by ferrocyanide of potassium in presence of acetic acid, a reagent by which practically all other proteids in solution are precipitated, but are precipitated by tannic acid, nercurie chloride, nitrates of mereury, and by phosphotungstie and phosphomolybdic acids in presence of hydrochloric or other mineral acids; also by the double iodides of potassium and mercury or potassium and bismuth, in presence of strong mineral acids. A very characteristic reaction is the "biuret or pink coloration which is obtained on the addition of an excess of caustic soda and a mere trace of sulphate of copper. The slightest excess of the copper salt gives a violet colour, as is the case with all other proteids, which decpens in tint on boiling. This biuret reaction is however yielded also by the albumoses but to a less striking degree (see above). Peptones are all lavorotatory and more diffusible than the albumoses.

Amphopeptone. This is the mixture of anti- and hemi-peptone resulting from the action of pepsin on proteids.

Antipeptone may be obtained by the action of either pepsin or trypsin on antialbumose, or by the action of trypsin on antialbumate or antialbumid. When purified no leucine or ty rosine can be obtained by the most prolonger action of trypsin on this peptone.

Hemipeptone. Although occurring in amphopeptone, it cannot as yet be separated with any precision from the antipeptone with which it is mixed.

Notwithstanding the probable formation of peptones in large 
quantities in the stomach and intestine, to judge from the results of artificial digestion, a very small quantity only can be found in the contents of these organs. They are probably absorbed as soon as formed.

It is now generally considered that the peptones are products of the hydrolytic decomposition of the proteids from which they are formed. This view is based partly upon general considerations as to the probable nature of the change from observations of the conditions under which they are formed, and which are known to be hydrolytic in other cascs, e.g. the conversion of starch into sugar by the action of enzymes and acids. The one important fact in comnection with the relationship of the peptones to the mother proteids is that they are, as already stated, products of the decomposition of the latter and of smaller molecular weight, an assumption which is warranted not only by the whole tendency of recent investigation and of actual cryoscopic determinations but more especially by the fact that whereas ordinary proteids are non-diffusible, peptones, and to a less degree the albumoses, are diffusible.

It was at one time stated that when peptones are injected into the blood-vessels, the blood speedily loses its power of clotting after removal from the body. This action is now known to be due to the albumoses with which the peptones were mixed. The clotting may similarly be prevented by the injection of a 1 p.c. $\mathrm{NaCl}$ extract of the pharynx and gullet of the leech: the cause of this has not as yet been fully worked out.

During the pancreatic digestion of proteids some by-product makes its appearance which gives a characteristic violet or pink coloration on the addition of bromine, or of chlorine in the presence of acetic acid. The substance to which the colour is due has been called tryptophan.

\section{Class VII. Lardacein, or the so-called amyloid substance.}

The substance, to which the above name is applied, is found as a pathological deposit in the spleen and liver, also in numerous other organs, such as the blood-vessels, kidneys, lungs, cte.

It is insoluble in water, dilute acids and alkalis, and nentral saline solutions.

In percentage composition it is almost identical with other proteids.

The sulphur in this body exists in the oxidized state, for boiling with caustic potash gives no sulphicle of the alkali. The above results of analysis would lead at once to the ranking of lardacein as a protcid, and this is strongly supported by other facts. Strong hydrochloric acirl converts it into acid-albumin, and caustic alkalis into alkali-albunin. When boiled with dilute sulphuric acid it yields lencine and tyrosine; by pro- 
longed putrefaction indole, phenol, etc. On the other hand, it exhibits the following marked differences from other proteids: It wholly resists the action of ordinary digestive fluids; it is coloured red, not yellow, by iodine, and violet or pure blue by the joint action of iodine and sulphuric acid. From these last reactions it has derived one of its names, 'amyloid, ' though this is evidently badly chosen; for not only does it differ from the starch group in composition, but by no means can it be made to yield sugar: this latter is one of the crucial tests for a true member of the carbohydrate group.

Purified lardacein is readily soluble in moderately dilute ammonia, and can, by evaporation, be obtained from this solution in the form of tough, gelatinous flakes and lumps; in this form it gives feeble reactions only with iodine. If the excess of ammonia is expelled, the solution becomes neutral, and is precipitated by dilute acids.

In opposition to the older statements it has recently been stated that lardacein may be digested by pepsin in presence of hydrochloric acid.

The known products of decomposition of proteids are very numerous, varying in nature and relative amount with the conditions and reagents by means of which they are produced, and it may be similarly, though to a much less extent, with the kind of proteid employed. They belong for the most part to wellknown elasses of chemical substances and in many cases representatives of several consecutive members of any given homologous series are obtained during the decompositions. A study of these products has not up to the present time thrown any extended light upon the more minute molecular structure of the proteids and it cannot as yet be said that we possess any real knowledge of their constitution. When proteids are decomposed by mineral acids ( $\mathrm{HCl}$, in presence of stannous chloride) at $100^{\circ}$, the products formed are chiefly amido-acids such as leucine, tyrosine, glutamic and aspartic acids with some ammonia. (Most recently two new nitrogenous crystalline bases, lysine and lysatine, have been additionally obtained.) The former is probably diamido-caproic acid, the latter a homologue of creatine. These bases, as also ammonia, are also formed during a pancreatic digestion of proteids. When decomposed by barium hydroxide in sealed tubes at $200^{\circ}-250^{\circ}$, the products already named (except lysine and lysatine) are obtained, together with amido-acids homologous with leucine, leuceïnes $\left(\mathrm{C}_{\mathrm{n}} \mathrm{H}_{2 \mathrm{n}-1} \mathrm{NO}_{2}\right)$, ete., and additionally carbonic, oxalic and acetic acids. 


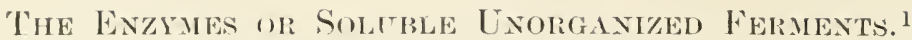

In the chemistry of animal and regetable cells it is found that in many cases substances may be extracted from them which possess to a most striking degree the property of inducing change in an indelinitely large nilss of certain other substances withont themselves undergoing any observable alteration. These agents are known as the enzymes or soluble ferments, and the essential conception of an enzyme is smmmed up in the above statement of the most remalkable characteristic of their activity. Further investigation of these enzynes shows that their activity is dependent upon many subsidiary factors which are mole or less common to them all. 'Thus their activity is largely depenclent upon temperature, being absent at sufficiently low temperatures, increasing as the temperature is raised to a certain optimal point which varies slightly for different enzymes, then again diminishing as the temperature is further raised and finally disappearing. By the action of a sufficiently high temperature they permanently lose their characteristic powers and are now spoken of as being ' killed.' Again the enzymes are extremely sensitive to the reaction, whether acid, alkaline or neutral, of the solutions in which they are working, also to the presence or absence of rarions salts, some of which merely inhibit their action while otlers permanently destroy it; and their activity is in all cases lessened and finally stopjed by the presence of an excess of the poducts to whose formation they have given rise. It has been already said that an enzyme may be killed by exposure to a high temperature, but this only holds good when they are in solution, or if in the solid form they are heated in a moist condition. When perfectly diy they may be heated to $100^{\circ}-160^{2}$ without any permanent loss of their powers. It will be seen that so far the enzynes have been characterized solelv with reference to the peculiarity of their morle of action and to the influence of smounding conditions mpon that activity, and the question of their probable chemical composition has been left untouched. Notwithstanding the frequent endeavours which have been made to prepare the enzymes in a pure condition, it is unwise to lay any great stress upon the results of the analysis of these so-called "pure ferments," bearing' in mind that, as in the ease of the proteids, no criterion of their purity exists. This much however may be said. In the majority of cases, an-

1 It appears advisable to use the term 'enzyme' to denote the soluble unorganized ferments generally, reserving the older name of 'ferment' for the organized agents such as yeast to which it was first applied. If this be done it will be convenient to use the expression 'zymolysis' to denote the changes proluced by the enzymes in their action on other substances, and to apply the term 'fermentation' to the action of the organized ferments. 
alysis shews that their composition approximates more nearly to that of a proteid than of any other class of substances, and this is apparentlytrue even when they do not yield to any marked degree the reactions (xanthoproteic, etc.) which are character. istic of a true proteid. Ordinarily it is almost impossible to obtain an enzyme solution of any considerable activity which is free from proteid reactions, and hence many authors are inclined to regard these bodies as being really of proteid nature. But this is a point which is as yet by no means settled; the balance of recent opinion appears to be in favour of the view that the enzymes are proteid in nature, but this is still an open question.

The enzymes are possessed of certain properties more or less common to them all, by means of which they may be separated from the tissues in which they primarily occmr, and isolated from the solutions thus obtained. Soluble in water, they may he precipitated unchanged from this solution by the addition of an excess of absolute alcohol. They mat also in many cases be precipitated from their aqueous or other solution by saturation with neutral ammonimm sulphate. They are conveniently soluble in glycerin, from which they may as before be precipitated by an excess of alcoliol. None of the enzymes are diffusible and hence they may readily be freed from auy admixed diffusible substances by means of dialysis. They possess further the remarlsable property of adhering with great tenacity to any finely divided precipitate which is formed in the solutions in which they are present, more particularly if the precipitate is of a viscid or gelatinous nature. In some cases the enzymes do not exist in the free and active conditions in the cells of the respective tissues but in the form of an inactive antecerlent to which the name of 'zymogen' is usually applied. Hence to obtain an active extract it is frequently necessary to treat the tissue with some such reagent as shall ensure the conversion of the zymogen into the active enzyme.

During prolonged digestions it is essential to ensure the absence of any changes due to the development of bacteria or other organisms. The most suitable antiseptics for this purpose ale salicylic acid (.1 p.c.) aud thymol ( 5 p.c.). These reagents are dissolved in a small quantity of alcohol and added in the above proportions to the digestive mixture.

It is frequently a matter of the utmost importance to determine whether the hydrolytic power of any given preparation is due to the action of a soluble enzyme or of a ferment (organized). The discrimination is most readily effected by carrying on the digestion in presence of chloroform, which is inert towards the enzymes but inhibits the activity of ferment orgranisms. 


\section{Special Description of the More Important Enzymes.}

\section{Ptyalin.}

Whilc occurring chiefly and characteristically in saliva, a similar enzyme may be obtained in minute amount, but fairly constantly from almost any tissue or fluid of the body, more particularly in the case of the pig. Most recently this enzyme has been prepared as follows. Saliva is diluted with an equal volume of water and saturated with neutral ammonium sulphate. The precipitate thus formed is treated on the filter for five minutes with strong alcohol, removed from the filter and further treated with absolute alcohol for one or two days. It is now dried at $30^{\circ}$ and yields, on extraction with a volume of water equal to that of the original saliva, a solution which is actively zymolytic and is stated to be free from all proteid reactions. The hydrolytic activity of ptyalin is most marked in neutral or nearly neutral solutions.

An amylolytic enzyme is found in urine.

Evidence of the existence of a zymogen of ptyalin (ptyalinogen) has been obtained in the case of the saliva of the horse.

\section{The amylolytic enzymes of the pancreas and intestine.}

The secretion of the pancreas is even more active than saliva in effecting the hydrolysis of starch. This property is dependent upon the presence in this secretion of an enzyme which in many ways closely resembles ptyalin, but differs from it markedly in its greater power of effecting a more complete decomposition of the starch than can ptyalin. Under ordinary conditions the only sugar formed by the action of ptyalin on starch is maltose. The pancreatic enzyme on the other hand not only rapidly converts starch into maltose, but further converts this maltose in to dextrose in considerable quantity during a digestion of relatively short duration. The secretion of the pancreas is of extremely complicated composition and contains in addition to the amylolytic at lcast two other well-characterized enzymes; from these the former has as yet been only very imperfectly separated, so that scarcely anything is known of its chemical nature as distinct from its converting powers. An atcive amylolytic extract is best prepared by extracting finely minced pancreas for five or six days with four times its weight of 25 p.c. alcohol, the mixture being frequently stirred. The pancreas of the pig yields the most certainly active cxtracts and more particularly if the gland is liept for $2+$ hours after removal from the body, and is then treated for a few hours with dilute $(.5$ p.c.) acetic acid before its tinal extraction with alcohol. Extracts made with strong solutions of sodium cliloride are also frequently very active. 
The secretion and extracts of the small intestine possess to a slight extent the power of slowly hydrolyzing stareh into maltose, the conversion being more rapid if portions of the mucous membrane of the intestine be finely divided and immersed in the starch solution. The tissue and its extracts on the other hand possess to a very marked extent the power of rapidly effecting a conversion of maltose into dextrose; this is of great physiological significance inasmuch as it points to the probability that the carbohydrates are absorbed from the intestine as dextrose and not as maltose, a view which is supported by the fact that maltose does not appear to be capable of direct assimilation, but is excreted largely unchanged if injected into the blood. The intestinal mucous membrane also yields an inverting enzyme which, similarly to the well-known 'invertin' of yeast, decomposes cane sugar into a mixture of dextrose and lævulose (see p. 1221).

\section{Pepsin.} juice.

This is the characteristic proteolytic enzyme of gastric

Preparation of peptic digestive fluids. If a few drops of a glycerin extract of gastric mucous membrane be added to dilute ( 2 p.c.) hydrochloric acid or if the tissue be simply extracted for a short time with the dilute acid and the extract be filtered a solution is obtained which suffices for demonstration and ordinary purposes. When however a peptic extract is required for research purposes it is essential to arlopt some more elaborate method which yields a product as free as possible from admixed substances; one of the best is as follows. The mucous membrane is digested with phosphoric acid and the fluid precipitated with lime-water. The precipitate of calcium phosphate is then filtered off, washed, and dissolved in dilute hydrochloric acid, and this solution is then dialyzed until it is free from chlorine and phosphates, and on acidulating with hydrochloric acid is ready for use.

Pepsin does not exist preformed in the cells of the gastric glands but as a zymogen to which the name of pepsinogen is given; this is readily converted into pepsin by the action of hydrochloric acid.

The hydrolytic activity of pepsin is manifested only in presence of an acid. The most efficient acid in this respect for artificial digestions is hydrochloric of a strength of 2 p.c. The average percentage of this acid may be stated as $\cdot 2$ p.c. in normal gastric juice, but it varies slightly in the case of different animals. Other acids may be substituted for the hydrochloric, the optimal percentage varying for the several acids. 
Traces of pepsin and other enzymes are frequently found in unine.

\section{Trypsin.}

The proteolytic enzyme of pancreatic juice. The composition of the enzyme as prepared by Kühne was found to be remarkably complex, as shewn by the fact that when dissolved in water and boiled it is split up with the formation of 20 p.c. coagnlated proteid and 80 p.c. albumose.

Preparation of solutions of trypsin for digestion experiments. 'The following method due to Kühne yields an extraordinarily pure and active tryptic solution; unfortunately it is a somewhat lengthy process.

One part by weight of pancreas which has been extracted with alcohol and ether is digested at $40^{\circ}$ for 4 hours with 5 parts of 11 r.c. salicylic acid. The residue after being squeezed out is further digested for 12 hours with 5 parts of 25 p.c. $\mathrm{Na}_{2} \mathrm{CO}_{3}$. and the residue is again squeezed out. The acid and alkaline extracts are now mixed together, the whole made up to $25-5$ p.c. $\mathrm{Na}_{2} \mathrm{CO}_{3}$, and digested for at least a week in presence of 5 p.c. thymol. By this means all the first formed albumoses are fully converted into peptones: this is essential. At the end of the week the fluid is allowed to stand in the cold for 24 hours, filtered, faintly acidulated with acetic acid and saturated with neutral ammonium sulphate. By this means all the trypsin is separated out and may be collected on a filter, where it is washed with the ammonium salt (sat. sol.) till free from peptones. It is now finally dissolved off the filter in a little $\because 5$ p.e. solution of $\mathrm{Na}_{2} \mathrm{CO}_{3}$, to which thymol is added and thus an extremely active and very pure digestive solution is obtained. Ten grams of the original pancreas yield $80-100$ c.e. of extract.

Although trypsin exhibits its hydrolytic powers to the greatest advantage in presence of an alkali, its activity is searcely so directly relater to the alkali as is that of pepsin to dilute hydrochloric acid. Thus it will digest proteids, although much more slowly, in a neutral solution and even in presence of dilute (.012 p.c.) hydrochloric acid, but the slightest excess $(\cdot 1$ p.c.) of the acid destroys it.

This comparative independence of tryptic activity in its relations to the reaction of the digestive mixture is doubtless of considerable physiological significance. 'The reaction of the contents of the small intestine is very variable. The chyme as discharged from the stomach is of course acid, and this acidity is largely diminished by the advent of the strongly alkaline bile and pancreatic juice, so that the reaction may become alkaline within a short distance of the pylorus. On the other hand the alkaline reaetion may not be at all appreciable until the lower end of the intestine is reached and frequently, at 
least in dogs, the reaction is faintly acid throughout, whether they are fed on proteids or on a mixture of carbohydrates and fat. The acidity in the latter case is not surprising bearing in mind the readiness with which the carbohydrates undergo a lactic fermentation, especially inside the intestine, and it might therefore have been abnormal in the dog whose food rloes not normally contain carbohydrates. On the other hand in man. living on a mixed diet, the possibility of a lactic fermentation is always present. It is impossible to make any general statement as to the reaction of the contents of the small intestine: it varies at different times, and depends upon the kind and relative amount of the several food-stuffs, the changes these undergo and the amount of alkaline secretions with which they are mixed. All the evidence we do possess leads to the belief that intestinal digestion to be of use must be capable of being carried on in a mixture which may be alkaline, or neutral or even frequently acid. Although the acidity of the intestinal contents may be due to hydrochloric acid in the upper end of the duodenum, the acidity is elsewhere much more probably due to lactic or butyric acids, and it is interesting in this connection to notice that the former of these two acids exerts a distinctly favouring influence on tryptic digestion, especially in presence of bile and sorlium chloride. Thus in presence of .02 p.c. lactic acid and $1-2$ p.c. bile and sodium chloride fibrin may be digested more rapidly than in a neutral solution and fully as quickly as in a solution of morlerate alkalinity. But the presence of $\cdot 05$ p.c. of lactic acid stops the digestion.

Traces of trypsin have been stated to be found in urine; this is somewhat doubtful.

The pancreas contains, in its absolntely fresh and normal condition, no ready-made enzyme but an antecedent (zymogen) of the same. This substance is readily converted into the active enzyme by the action of dilute acids ( 1 c.e. of 1 p.e. acetic acid to each $1 \mathrm{grm}$. of gland-substance) and a conversion also takes place if the gland is liept for some time, especially in the warm, this resulting most probably from the spontanenus acidification which it thus undergoes. 'The zymogen is soluble in strong glycerin withont conversion into the enzyme, it is also soluble in water in which it is gradually changed in to the enzyme, most rapidly when warmed, probably under the influence of the acid reaction which the solution acquires.

\section{Lipolyn. ${ }^{1}$}

In addition to the two pancreatic enzymes which have already been described both the secretion and the gland-sub-

${ }^{1}$ From $\lambda i \pi$ os $=$ fat and $\lambda \dot{\epsilon} \epsilon \nu=$ to split up or decompose. 
stance contain a third substance which has not as yet been isolated, of which therefore but little is known from a chemical point of view, but which must be regarded as an enzyme in virtue of the typical conditions under which it is able to effect a hydrolytic decomposition of neutral fats into glycerin and free fatty acid. It is most actively present in the substance of the fresh gland or in its secretion, and may be cxtracted from the former by means of glycerin or water. In every case it is essential to ensure that the gland had not acquired an acid reaction before extraction and that all acidification in the extract is absent, since the enzyme is peculiarly sensitive to acids other than fatty and is readily destroyed by them. Hence a dilute alkaline solution should be employed, and sodium bicarbonate mixed with the normal carbonate is the most efficient solvent.

The enzymic nature of the active agent is shewn by the fact that its lipolytic activity is greatest at about $40^{\circ}$, is destroyed by boiling, and is dependent upon the reaction of the digestive mixture, being greatest in presence of a dilute alkili although it will show itself in a neutral solution. It will also be observed that the decomposition which lipolyn effects is typically liydrolytic.

\section{Rennin.}

Extracts of the mucous membrane of the stomach of young animals and more especially of the calf have been known, from time immemorial, to possess a most remarkable power of causing milk to elot, and rennet was commonly employed by the Romans for the manufacture of cheese. The substance to which the clotting is due is an enzyme to which the name of remin may be conveniently given. ${ }^{1}$ The cnzymic nature of the active agent in rennet is clearly shown by the typical relationship which it exhibits in its activity to the reaction of the solution in which it is present, to the temperature at which its activity is greatest, to the fact that the briefest exposure to $100^{\circ}$ or the more prolonged exposure to lower temperatures ( $70^{\circ}$ or above) suffices to destroy its active properties and to the fact that a minute trace suffices to clot a relatively enormous amount of casein.

Aqueous and glycerin extracts of the gastric mucous membrane are usually found to be active in clotting milk, but the activity of a faintly acid extract is in all cases greater. This is due to the existence of a remnin zymogen (remninogen) which is readily converted into the enzyme by the action of acids. The preparation of highly active and permanent solutions of rennin is of considerable commercial importance in connection with the cheese-making industry. 'The most efficient extrac-

1 This name seems more convenient than the more commonly used expressions 'the rennet ferment' or 'the milk-curdling ferment.' 
tive is sodium chloride 5-15 p.c., and permanency is attained by the addition of alcohol.

Although rennin is most copiously present in the gastric mucous membrane of the calf, it may be obtained from the tissue of almost any stomach, if not as ready-made enzyme at least in the form of a zymogen. It occurs also in the stomach of children and of man, and has been described as present in the pancreas of the pig, ox and sheep. Rennin is stated to occur in traces in urine.

\section{Fibrin-ferment.}

For ordinary purposes an extremely active ferment solution may be most readily obtained by extracting the so-called 'washed blood clot' with 8 p.c. solution of sodium chloride. 'The solution in this case contains a large amount of globulins in solution, as also does the similar extract which may be equally efficiently prepared from ordinary washed fibrin.

In no case as yet has the fibrin-ferment been obtained in a condition of such purity as to justify any definite statement as to its chemical composition.

In addition to the undoubted relationship of lencocytes to fibrin-formation it appears that the protoplasm of many other cells, both animal and vegetable, may exert an influence similar to that of the white corpuscles of blood. The present state of knowledge and the conflicting views of various observers render it impossible to make any dogmatic statement as to the origin of this fibrin-ferment.

The information which we possess as to the nature of the fibrin-ferment is much less complete and satisfactory than in the case of other enzymes. But that it is properly placed in the class of these substances is shewn by the typical facts that its activity is closely dependent upon temperature, being destroyed by heating to $70^{\circ}$; that it does not affect the amount but only the rate of change of fibrinogen into fibrin; that it is carried down by gelatinous precipitates formed in its solutions, produces a change which is out of all proportion to the mass of enzyme employed, and is not, so far as we know, used up in the change which it induces since it is present in serum.

\section{Muscle-enzyme.}

The phenomena of the clotting of muscle-plasma compared with those of blood-plasma and the relationship of the process to the presence of neutral salts and to temperature suggest at once that the change is probably one in which some enzyme plays a part. Immediately after the discovery of the fibrinferment the question of the existence of a myosin-ferment was investigated and resulted in the discovery of the existence in 
muscles of an enzyme which appeared to be identical with fibrin-ferment rather than specifically myosinic. More recently it has been shewn that from museles which have been treated for some time with alcohol, a solution may be obtained which hastens the clotting of diluted muscle-plasma, does not facilitate the formation of fibrin in bloor-plasma and, unlike fibrin-ferment, requires to be heated to $100^{\circ}$ before it loses its activity. 'The ative agent in the solution is therefore not identical with fibrin-ferment and may be spoken of as a myosin-ferment.

\section{Urea-ferment.}

When urine is exposed to the air its acidity at first increases. but in most eases this speedily gives way to a marked alkalinity which is accompanied by the evolution of ammonia. 'This is due to a hydrolytic fermentative change resulting from the appearance and development in the mine of certain microorganisms of which the best known is the 'lorula nere. Normally mine is free from these organisms and may be kept in the excised bladder for an indefinite period without exlibiting any tendency to become alkaline; in certain abnormal conditions it may modergo an active alkaline fermentation while still in the bladder. When urine which by exposure to the air has entered into active alkaline fermentation and, as shewn by microscopic examination, is full of 'Torula, is efficiently filtered no enzyme capable of hydrolizing urea can be precipitated by alcoliol from the clear filtrate. If on the other hand the unfiltered nime be precipitated with an excess of alcolol and the precipitate washed with alcohol and died in the air, a powder is obtained which is itself extraordinarily active and yields to an aqueous extract a soluble enzyme which rapidly converts urea into ammonia and carbon dioxide. 'The rapidity of the conversion precludes the intervention of any devcloping organism, and that the change is truly due to an enzyme is shewn by the fact that it takes place with equal readiness in presence of chloroform.

The most prolific source of the urea enzyme is in all cases the mucous urine passed in inflammatory conditions of the bladder. In this case the enzyme appears to be closely associated with the mucin and is presumably a secretory product of the mucous membrane, for it is frequently obtained when there has been no operative use of surgical instruments which conld account for the introduction of micro-organisms from the exterior.

Nitrogenous Noy-Crystaldine Bodes allied to ProTEIDS.

These resemble the proteids in many general points, but exhibit among themselves much greater differences than do 
the proteids. Their percentage composition approaches that of the proteids, and like these they yield, under hydrolytic treatment, large quantities of leucine and in some cases tyrosine and other characteristic products.

\section{Mucins.}

These are the substances which give to many animal secretions, such as saliva, bile, synovial fluid, etc., their characteristic ropy consistency. They may also be obtained by the use of appropriate solvents from the tissues themselves, such as sub-maxillary gland, tendons and umbilical cord. The general phenomena of the formation of mucin by mucous cells, and more particularly the characteristic behaviour of the mucous granules in relation to the secretory activity of the sub-maxillary gland, leave but little doubt that mucin is to be regarded as lerived from the true proteids; in conformity with this it yields many of the reactions characteristic of the proteids (Millon s and xanthoproteic), and by the action of mineral acids some form of acid-albumin is usually obtained. During this treatment (or with alkalis) moreover a second product generally. makes its appearance, which belongs to the group of carbohydrates, is known by the name of animal gum and by heating with acids may be made to yield a reducing sugar. Notwithstanding the views which have frequently been advanced that mucin is in reality a mixture of proteid and carbohydrate material, it is now known with considerable certainty that it is a unitary substance which, from what has been already said, may be regarded as an animal glucoside to which the general name of glycoproteid may be given. It further appears that the substance at first secreted by the mucous cells may not be typical mucin but a sort of mucinogen which readily gives rise to mucin on treatment with dilute (.01 p.c.) caustic potash. If it be assumed for the moment that there is only one kind of mucin, then the following general statements as to this substance may be additionally made. It is precipitated from its solutions by acetic or hydrochloric acids, the precipitate being soluble in excess of the latter but not of the former acid. In its precipitated form it swells up strongly in water but does not go into true solution; the addition of dilute alkalis ( 1 - .2 p.c.) or of lime-water leads to its ready solution, from which it can again be precipitated by the addition of an acid. It may be extracted from any mucigenous tissue by the use of dilute alkalis or lime-water, and in solution is somewhat characteristically precipitated by basic lead acetate. Our knowledge of mucin is however in an extrenely transitional condition, and recent investigations have shewn that probably the mucins derived from different sources are really distinct substances, just as we 
are familiar with different forms of proteids. From this it follows that no general statement of the properties of the mucins ean be as yet made whieh would be other than mislealing, and it will conduce to elearness to give a brief aecount of this substanee as obtained from eaeh of the ehief sources from which it has been derived.

The mucin of bile. Normal bile is not viseid when freshly seereted, but becomes so during its stay in the gill-bladder. The substanee to whieh the viscidity is due is seereted by the internal epithelium of the gall-bladder and was until reeently regarded as being a muein. But that it is an entirely different bolly is shewn by the fact that it yields no reducing (earbohy(lrate) snbstanee when boiled with mineral acids. Furthermore the preeipitate formed on the careful addition of acetic acid is soluble in exeess of the aeid and moreover it contains phosphorus, whieh the true mueins do not. The so-called mucin of bile is henee not a muein, but belongs to that class of substances now known as nueleo-allumins (see p. 1206).

A eertain very small amount of true mucin has however been stated to exist in human bile.

The mucin of the sub-maxillary gland. The gland is finely minced, washed and extracted with watcr: the extriet is filtered and hydrochloric aeid is added up to 1.5 p.c. The mucin is thus preeipitated at first, but at once passes into solution, from whieh it is preeipitated by the addition of a volume of water equal to three to five times that of the original solution. This preeipitate is then again dissolved in dilute hydrochloric acid and repreeipitated by water, the process being repeated several times. As thus prepared and thoroughly washed it possesses a distinctly aeid reaetion; it may be dissolved to a neutral solution, by the cautious addition of very dilute alkalis, and now exhibits the following properties. It is readily precipitated by aeetic aeid, much less readily in presence of sodium chloride; this salt on the other hand greatly faeilitates the precipitation of muein by aleohol, whieh again does not take plaee in presence of a trace of free alkali. Any exeess of alkali, especially on warning, at onee ehanges the substanee so that its eharaeteristie ropiness is permanently lost, and boiling with dilute mineral aeids yields a redueing substance. It gives the usual reactions for proteids and is strongly preeipitated by the aeetates of lead and by $\mathrm{CuSO}_{4}$ and by excess of $\mathrm{NaCl}$ and $\mathrm{MgSO}_{4}$.

The mucin of tendons. 'The tendo Achillis of the ox is eut into thin sliees, washed with distilled water and extracted with half-saturated lime-ivater; the mucin is thus dissolved and is purified by preeipitation with either acetic or hydroehloric acids, re-solution in dilute alkali and repreeipitation with acids. In its general reactions it resembles the mucin previously deseribed, but differs from it in being insoluble in 1-2 p.e. 
hydrochloric acid and in its distinctly greater resistance to the action of acids and alkalis.

Mucin of the umbilical cord. May be prepared by the method employed for the mucin of the sub-maxillary gland. It appears to differ from the other mucins in containing more nitrogen and a considerable amount of sulphur: it lies in fact somewhat midway between the proteids and true mucins.

By prolonged boiling with sulphuric acid mucins yield lencine and tyrosine, but the products of their decomposition have not been as yet fully studied.

Analyses of the several mucins exhibit differences in percentage composition which lie within somewhat similar linits to those ahearly assigned (p. 1165) to the proteids. But a comparison of these shews that the mucins contain slightly less carbon, markedly less nitrogen and correspondingly more oxygen than do the proteids.

Mucin-like substances, to which the general name of mucoids or mucinoids has been given, are found in ascitic fluids, the vitreous humour, the cornea and in the white of egg. They all yield a reducing substance when boiled with mineral acids and otherwise resemble the true inucins.

\section{Gelatin or Glutin. ${ }^{1}$}

The ultimate fibrils of connective tissue and the organic matter of which bones are largely composed consist of a substance named in the first case 'collagen,' in the second 'ossein.' 'They are obtained either by digesting carefully cleansed tendons with trypsin, which dissolves up all the tissue-elements except the true collagenous (gelatiniferous) fibrils, or by extracting bones with dilute acids in the cold, by means of which the inorganic salts are dissolved and the ossein remains as a swollen elastic mass which retains the shape of the original bone. As thus prepared they are insoluble in water, saline solutions and either cold dilute acids or alkatis; in the former however (acids) they swell up to a transparcnt gelatinous mass. When subjected to prolonged boiling with water, more especially under pressure as in a Papin's digester, they are gradually dissolved, and the solution now contains true gelatin into which they have been converted by hydrolysis, and has acquired the characteristic property of solidifying into a jelly on cooling. The conversion of collagen into gelatin may be still more easily effected by a shorter boiling in presence of dilute acids, but in this case, unless the process be carefully regulated, the firstformed gelatin is further hydrolyzed into what are often spoken of as gelatin-peptones. Although insoluble in dilute acids

1 Glutin must not be confounded with the vegetable proteid 'gluten.' 
collagen is readily dissolved by digestion with pepsin in presence of an acid passing rapidly though the condition of gelatin into that of gelatin-peptone, and although collagen is not acted upon by trypsin in alkaline solution, it is readily hydrolyzed by this enzyme after a short preliminary treatment with dilute acid or boiling water, the products as before being known as gelatin-peptones. When gelatin is exposed for some time in the dry condition to a temperature of $130^{\circ}$ it is reconverted into a substance closely resembling collagen, which may be again converted into gelatin by treatment with water under pressure at $120^{\circ}$.

Gelatin obtained by the above means from connective tissue or bones is, when dry, a transparent, more or less coloured and brittle substance. It is insoluble in cold water, but swells up into an elastic flexible mass which now dissolves readily in water when warmed. When the solution is again cooled it solidifies characteristically into a jelly even when it contains as little as 1 p.c. of gelatin; it is also readily soluble in the cold in dilute acids and alkalis. The proteid reactions of gelatin are so feeble that they must be regarded as due entirely to unavoidably admixed traces of proteid inpurities; more particularly is it to be noticed that the usual reaction of proteids with Millon's reagent is entirely wanting, a fact which indicates the probable absence of aromatic (benzene) residues in its molecule and corresponds to the absence of tyrosine among the products of its decomposition. Gelatin is precipitated by but few salts, viz.: mereuric chloride and the double iodide of mercury and potassium in acid solution. Several acids on the other hand precipitate it readily, such as phosphotungstic and metaphosphoric, also taurocholic and tannic. Of the two lastnamed acids the former yields an opalescence in presence of 1 part of gelatin in 300,000 of solution, and the latter in still more dilute solutions.

When decomposed in sealed tubes with caustic-baryta gelatin yields on the whole the same products as do the proteids, with the exception of tyrosine; neither this nor any other substance of the typically aromatic series, is ever obtained during any decomposition of gelatin whether by chemical or putrefactive processes. By prolonged hoiling with hydrochloric acid it yields glycine (glycocoll), lencine, glutamic acid and ammonia, and with sulphuric acid aspartic acid as well.

Gelatin-peptones. By prolonged boiling with water (1 p.e. solution boiled for 30 hours), or shorter treatment in a Papin's digester, also by heating with hydrochloric acid $\left(4\right.$ p.c. at $\left.40^{\circ}\right)$, or still more readily by pepsin in presence of acid or by trypsin, gelatin loses its power of solidifying on cooling, and is converted into more highly soluble and now diffusible substances, to which the name of gelatin-peptomes has been given. A similar change 
occurs when gelatin is taken into the stomach. From the conditions under which the change is effected and from certain evidence deducible from analysis there can be but little doubt that the conversion takes place as the result of hydrolysis, as in the case of the formation of true peptones from proteids.

Recent rescarches have shewn that, the hydrolytic decomposition of gelatin by digestive enzymes gives rise to products analogous to those obtainable by the same method from the proteids. 'Thus during both its peptic and tryptic digestion certain prinary products are formed to which the name gelatoses or glutoses may be applied and which have so far been distinguished as proto- and deutero-gelatose. Accompanying these, in variable amount, are other products known as gelatin-peptones. The latter are to be regarded as a product of the further action of the enzymes on the first formed gelatoses and, like the true peptones in their relationship to the albumoses, may be separated from them by their non-precipitability on saturation with ammonium sulphate, a reagent which completely precipitates the gelatoses. Protogelatose is partially precipitated by saturation of its solution with common salt, and completely so on the simultaneous addition of acetic acid. Denterogelatose is not precipitated by either of the above reagents. The so-called true gelatin-peptones have not yet been obtained in sufficient quantity to admit of their complete examination. 'The products of the digestion of gelatin appear to give a distinct biuret reaction with caustic soda and sulphate of copper, and like the peptones (and albumoses) are not precipitated by taurocholic acid, which precipitates gelatin from its solutions.

Reticmlin. This is the name given to the substance of which the connertive-tissue fibrils of reticular or retiform tissue, as met with in lymphatic glands or the mucous membrane of the intestine, are composed. It resembles gelatin in several respects but requires further investigation.

\section{Chondrin.}

The matrix of hyaline cartilage is composed of an elastic, semitransparent substance which is insoluble in cold or hot water and does not swell up appreciably by treatment with either water or dilute acetic acid. By prolonged treatment with water under pressure in a Papin's digester it is gradually dissolved and yields a solution which gelatinizes on cooling and now contains the substance usually spoken of as chondrin. The hyaline matrix of cartilage appears thus to bear the same relationship to chondrin that the ground-substance of connectivetissue (collagen) does to gelatin, and is therefore frequently spoken of as 'chondrigen.'

The substance known as chondrin, which is obtained in 
solution by the action of superheated water on hyaline cartilage, exhibits the following characteristic reactions. It is precipitated by acetic acid which does not, even if in considerable excess, redissolve the precipitate; minute quantities of mineral acicls similarly cause a precipitate to appear which is in this case readily soluble in the slightest excess of the acids. Thesc reactions suffice to distinguish between chondrin and gelatin, and a further distinction may be made on the basis of the fant that solutions of chondrin are precipitated by several reagents such as alum, normal lead acetate, and other metallic salts (of $\mathrm{Ag}$ and $\mathrm{Cu}$ ), which yield no precipitate with gelatin, while on the other hand mereuric chloride and tamnin do not precipitate chondrin but are characteristic precipitants of gelatin (see above). The above reactions also indicate a possible relationship to muein, which is confirmed by the fact that chondrin when boiled with dilute mineral acids yields a reducing substance with marked carbohydrate affinities. Hence the view was some years ago expressed that chondrin is not a lefinite unitary substance, but a mixture of gelatin and mucin, and recent research has confirmed the general accuracy of this statement. The following is a somewhat synoptic account of the views now held as to the chemistry of cartilage. By appropriate methods of extraction, of which the details are too complicated to admit of any adequately concise description, the matrix of cartilage yields a substance with marked affinities to mucin, and hence called chondromucoid. This resembles mucin in several of its reactions and like mucin yields, when boiled with mineral acids, a form of acid-albumin and a reducing body. 'The latter is not an immediate product of the decomposition of the chondromucoid, but makes its appearance in the final stage of the cleavage of the products first formed. When boiled with acids chondromucoid yields at first an acid called chondroitic acid, of which a certain amount is also present in the free state in the cartilage and may be extracted from it by means of dilute caustic soda. Chondroitic acid is regarded as being an ethereal sulphate of a substance called chondroitin, and the latter when boiled with mineral acids splits up into acetic acid and the above-mentioned reducing substance now named chondrosin, to which the formula $\mathrm{C}_{12} \mathrm{H}_{21} \mathrm{NO}_{11}$ is assigned. Chondrosin is soluble in water, lievorotatory, reduces cupric oxide in an alkaline solution somewhat more strongly than does glucose, has thus marked carbohydrate affinities, and from the products obtained when it is decomposed by barium hydrate may perhaps be regarded constitutionally as similar to a compound of glyeuronic acid (see p. 1220) and glucosamine (see p. 1205). The residue of cartilage which remains after the extraction of chondromucoid consists chiefly of a collagenous substance which by the action of superheated water, as in a 
Papin's digester, is converted into true gelatin. During the conversion an extremely insoluble residue is formed which has affinities on the one hand with keratin and on the other with elastin.

\section{Elastin.}

'This is the characteristic component of the elastic fibres which remain after the removal of gelatin, mucin, fats, etc., from tissues such as "ligamentum nucha." Some of the more important ways in which it differs from the substances which have been previously described are sufficiently stated by describing the method of its preparation in a pure form. Ligamentum nuche of an ox is cut into fine slices, treated for three or four days with boiling water, then for some hours with 1. p.c. caustic potash at $100^{\circ} \mathrm{C}$. and subsequently with water. This process is then repeated with 10 p.c. acetic acid. Finally it is treated for 24 hours in the cold with 5 p.c. hydrochloric acid, washed with water, boiled with 95 p.c. alcohol and extracted for at least two weeks with ether to remove every trace of adherent fat. By the above method it may be obtained as a pale yellowish powder in which the shape of fragments of the original elastic fibres may be still distinguished under the microscope. When moist it is yellow and elastic, but on drying it becomes brittle and may with difficulty be pulverized in a mortar. Sulphur probably does not enter into its composition (?). It may be dissolved by strong alkalis at $100^{\circ} \mathrm{C}$., and it also goes into solution when treated with mineral acids at the same temperature ; but in the latter case the solution involves decomposition with the formation of much leucine $(30-40$ p.c.) and traces ( .25 p.c.) of tyrosine when the acid employed is sulphuric. If strong hydrochloric acid be employed with chloride of zine the same crystalline products are obtained together with ammonia, glycine, and an amidovalerianic acid, but no glutamic or aspartic acids. In this respect it differ's from both ordinary proteids and gelatin, since the former when similarly treated yield the glutamic and aspartic acids but no glycine, and the latter never yiclds the least trace of tyrosine. During the putrefactive decomposition of elastin products similar to the above are obtained together with some peptone-like substance. When treated with superheated water, or with dilute hydrochloric acid at $100^{\circ} \mathrm{C}$. or with pepsin or trypsin in acid and alkaline medium respectively, elastin is more or less rapidly dissolved and undergoes a true digestive change, during which products are formed, many of whose general reactions are analogous to those of the digestive products of proteids. It is however as yet uncertain whether a true elastinpeptone can be obtained; it is more probable that during the digestion only 
some of the primary substances (elastuses) make their appearance, since they are completely precipitated by saturation with neutral ammonium sulphate. Elastin is also rapidly corroded and dissolved by the action of papainı.

\section{Keratin.}

Hair, nails, feathers, horn and the epidermal structures in general are composed chiefly of keratin, admixed however with small quantities of proteids and other substances, from which it may be freed by thorough extraction with water, alcohol, ether and dilute acids in suceession, followed by digestion with pepsin and trypsin and a renewed washing with the above reagents. A convenient sonree which readily yields a pure prodnct, owing to the comparatively simple composition of the mother substance, is found in the shell-membrane of ordinary egrs. The percentage composition of keratin is in general allied to that of the true proteids, but varies within somewhat wide limits according to the source from which it has been prepared and particularly with regard to the sulphnr which it contains. This latter element varies in amount from $\cdot 5$ to $5 \cdot 0$ p.c. and leads to the formation of sulphides of the metal when keratin is dissolved in alkalis. Unlike the proteids, gelatin and elastin, keratin is quite mnaffected by the most prolonged and active digestion with either pepsin or trypsin. On the other hand, when deeomposed at high temperatures by either caustic baryta or strong hydrochloric acid, it yields large quantities of leueine (15 p.e.), tyrosine (3-4 p.c.) and other produets which are in general identical with those obtained by the similar treatment of proteids. It is soluble in strong alkalis when heated, and is further stated to be dissolved by prolonged treatment with superheated water; in the latter case a product is obtained to which, since it somewhat resembles an albumose, the name keratinose has been given, and which may now be digested by means of pepsin. Further investigation in this direetion is however neerled before any positive statements can be made respecting any truly digestive products derivable from keratin, or indeed as to the characteristic differenees of the keratins from different sources.

\section{Neurokeratin.}

When the substance of the brain or any mass of medullated nerves is thoroughly extracted with water, alcohol and ether, and then digested with pepsin and trypsin in snecession, a residue is obtained which closely resembles the ordinary keriltins, and constitutes about $15-20$ p.e. of the whole brain after it has been freed from its medullary constituents by alcohol an!. (ofler. 'This residue is nemokeratin, so named from the 
source from which it is obtained. It is characterized by its somewhat greater resistance to those decomposing agents whose action on keratin has been already described.

\section{Chitin.}

Although it is not found as a constitnent of any mammalian tissue, this substance composes the chief part of the exoskeleton of many invertebrates. It is by many regarded as the animal analogue of cellulose of plants, and from this point of view it possesses considerable morpholugical interest. The most convenient source from which it may be prepared is the cleansed exoskeleton of crabs or lobsters. This is first thoroughly extracted with dilute hydrochloric acid and caustic potash, after which it is treated with boiling alcohol and ether, and nay be finally completely decolorized by the action of permanganate of potash. It is a white amorphous substance which often retains the shape of the integument from which it has been prepared. It is insolnble in any reagents other than concentrated mineral acids, such as sulphuric or hydrochloric. The immediate addition of water to these solutions probably reprecipitates the chitin in an unaltered form. When heated with concentrated hydrochloric acid it is decomposed into glucosamine and acetic acid, of which the former is the characteristic product. A similar decomposition is observed when sulphuric acid is employed.

Glucosamine $\left(\mathrm{C}_{6} \mathrm{H}_{11} \mathrm{O}_{5} \cdot \mathrm{NH}_{2}\right)$. Crystallizes from alcohol in fine needles, is dextrorotatory, and reduces Fehling's fluid to the same extent as does dextrose, but is not fermentable. By treatment with nitrous acid a carbohydrate $\left(\mathrm{C}_{6} \mathrm{H}_{12} \mathrm{O}_{6}\right)$ is obtained which also reduces cupric oxide, but is similarly unfermentable. This is doubtless the substance which led to certain erroneous statements as to the production of a true dextrose from chitin.

\section{Nuclein.}

The nuclei of cells, both animal and vegetable, differ distinctly in chemical composition from the remaining substance of the cells. As a result of this difference it is possible to separate the nuclei approximately by various means from the adjacent cell-substance, and the name nuclein was given originally to the material of which the nuclei or parts of nuclei thus isolated seemed chiefly to eonsist. As research proceeded the fact soon becane apparent that the various investigators were dealing with distinctly different substances under the one name nuclein, and it is only recently that our knowledge of the nucleins has begun to take a more definite and systematic shape. It is however still in a too transitional condition to 
admit of more than a somewhat synoptic statement of the views at present held.

The nucleins are all obtained as an undissolved residue, left when the cells, tissue, or other parent-substance is subjected to artificial digestion with pepsin and hydrochloric acid. This residue is then purified by solution in very dilute alkali, precipitation with hydrochloric acid and washing with water, alcohol and ether. As thus prepared the nucleins arc white amorphous powders, characteristically rich in phosphorus, which may be split off as phosphoric acid by boiling with acids or alkalis. During this decomposition some proteid is simultaneously obtained so that the nucleins are regarded as compounds of proteid with a substance to which the name of nucleic acid has been given. In accordanes with these facts the nucleins exhibit distinctly acid properties, give the reactions characteristic of proteids and have a marked affinity for basic staining reagents.

Further study of the products obtained when nucleins are boiled with mineral acids has led to their being divided into two classes; of these the true nucleins, or as it may best be called simply 'nuclein,' yields not merely proteid and phosphoric acid, but characteristically members of the xanthine group (see p. 1263), the so-called xanthine bases or nuclein bases. These nucleins oceur preponderatingly in nuclei, are often ferruginous and some of them yield a reducing substance when decomposed by boiling acids, while others do not. The other form of nuclein has received the name of pseudo- or paranuclein; it gives the same general reactions as does true nuclein, but is sharply characterized by not yielding any of the xanthine bases when decomposed by boiling with dilute mineral acids. This form of nuclein is known chiefly as forming the undissolved residue left during a peptic digestion of casein or' other typical nucleo-albumin.

As already stated the true nucleins are regarded as compounds of a proteid with nucleic acid. This acid is the source of the xanthine bases obtained by the cleavage of nuclein, and since the nucleins from various sources yield preponderatingly and hence characteristically some one of the xanthine bases, it appears probable that there are several forms of nucleic acid. The nuelein of spermatozoa is regarded by many observers as being a native nucleic aeid, since it yiclds only xanthine bases and phosphoric acid but 110 proteid when decomposed by acids.

\section{Nucleo-albumins.}

While nuclein is now regarded as a compound of nucleic acid with a proteid, it is found that the nucleins may themselves unite with a further and often large amount of proteid, to form 
well-characterized classes of substances, to one of which the name of nucleo-albumin is given. Whereas nuclein occurs chiefly in the nuclei, nucleo-albumin is present chiefly in the cell-protoplasm but is also found in large quantity in certain secretions, such as milk and bile.

'The more characteristic reactions of the nucleo-albumins may be stated as follows. Soluble in very dilute alkalis they are readily reprecipitated by acetic acid and the constancy in properties of the product obtained by repeated solution and precipitation shews that they are not mere mixtures of nuclein and proteid. Their behaviour towards alkalis and acetic acid is such as to lead to an easy confusion with the mucins, from which however they differ by being highly phosphorized and not yiekding a carbohydrate as a product of hydrolytic decomposition. When digested with pepsin they yield peptones and albumoses and a phosphorized residue which is in most respects identical with nuclein, but since it does not yield products of the xanthine series when decomposed by acids, is known as pseudo- or paranuclein. They are, like the globulins, precipitated from solution by neutral salts, the precipitate becoming swollen and slimy when the precipitant is sodium chloride or magnesium sulphate, but not so when sodium sulphate is employed.

\section{Casein.}

This is the well-known proteid existing claracteristically in milk and in no other fluid or secretion of the body.

It may be readily obtained by diluting milk with four volumes of water and adding acetic acid to faint acidity. The casein is thus precipitated and after several washings by decantation is freed from fats by extraction with alcohol and ether.

Pure casein is a fine, snow-white powder. It is practically insoluble in water, but is soluble in alkalis, carbonates and phosphates of the alkalis, lime- and baryta-water. From these solutions it may be precipitated by excess of neutral salts such as sodium chloride, and by dilute acids, in which it is again soluble if any excess of acid is present. Its reactions thus correspond closely to those of acid-and alkali-albumin, but it is a perfectly distinct substance. Solutions of pure easein are not coagulated by boiling, but if heated to $130-150^{\circ}$ in sealed tubes a coagulation is obtained.

When casein is digested with pepsin in presence of hydrochloric acid it leaves a phosphorized residue of nuclein, which since it cannot be made to yield any menbers of the xanthine series by boiling with mineral acids is characterized by the name of paranuclein or pseudonuclein. Casein is therefore now regarded as a compound of a true proteid with this nuclein and is the typical and best-known instance of a nucleo-albumin. 
Action of remnin on casein. Contrary to the older views that the formation of the clot is rather of the nature of a precipitation than a true ferment action, we now know that by the action of rennin the clotting of casein is due to a specific action of the enzyme which results in the formation of a substance (which may be conveniently called tyrein) differing essentially from casein. The specific action of the enzyme is further shewn by the fact that simultaneously with the formation of the clot, a by-product is formed having the properties of a soluble albumin. Further the clot is entirely different from casein: it is much less soluble in acids and alkalis than the latter, always leaves as ordinarily prepared a large and constant residne of ash (calcium phosphate) on ignition, and even if it be freed from the calcium salt by special methods and dissolved in dilute alkalis, is not capable of being made to yield a clot by the renewed action of rennin.

The calcium salt plays an all-important part in the clotting of casein. Casein freed from this salt and dissolved in dilute alkali will not yield a clot; dialyzed milk similarly yields no clot, but if the dialysate be concentrated and added to the milk it now elots on the addition of remnin. When pure casein is dissolved in lime-water and neutralized with phosphoric acid it now clots with rennin. The action of the salt in the whole process appears to be that it determines not so much the action of the enzyme on the casein, but rather the subscquent separation from solution of the altered product. Neither is the calcium salt alone essential, for it may be replaced, but with less efficient results, by the similar salts of magnesium, barium and strontium.

After the removal of casein from milk by precipitation, the filtrate contains a small amount of coagulable proteid sometimes spoken of as 'lactalbumin,' closely resembling serum-albumin in its general properties, but differing slightly as to its specific rotatory power and the temperature at which it coagulates when heated. Also a minute quantity of a globulin closely similar to that of blood serum.

When milk is kept for some time at a temperature above $50^{\circ}$ and below its boiling point, a firm skin is formed over its surface composed largely of casein. Its formation is not to be regarded as being spccially claracteristic of milk, for purc casein dissolved in dilute alkalis cxhibits the same phenomenon, as also do alkali-albumin, chondrin, gelatin and the filtrate from 1 p.c. starch when it is concentrated on a water-bath. Its formation is probably duc to the rate of evaporation from the surface of the milk being more rapid than the fluid diffusion into the upper layer; and in accordance with this it is found that its appearance is considerably facilitated by blowing a r:thid stream of air or any indiffercnt gas over the surface of the warmed milk. 
Our knowledge of the chemical properties of casein as already described is based cntirely upon researches carried out upon the milk of cows. 'There is no reason to suppose that all that has been said does not apply equally to the milk of other animals. Nevertheless human milk shews, apart from the difference of composition (see $\$ 407$ ), certain differences from cow's milk, which are due to a distinct but characteristic difference in the reactions of the casein contained in cach. This is shewn by the following facts. (1) Human milk elots less firmly than cow's milk and sometimes not at all with rennin. (2) The casein in human milk, on the arldition of acetic acid, yields a very imperfect precipitate which is finely flocculent, almost granular as compared with the compact and coarsely flocculent precipitatc yielded by cow's milk. (3) The casein in human milk is, as already stated, very incompletely precipitated by the addition of acids and can only be completely precipitated by saturation with magnesium sulphate. (4) Casein from human milk is less soluble in water than is that of the coiv.

Some very rceent researches seem to shew that casein from human milk may not be a nucleo-albumin.

\section{The mucleo-albumin of bile.}

Attention has already been drawn to the fact that the 'mucin' of bile is in reality a nucleo-albumin (see p. 1198). It is best prepared as follows. Bile is mixed with five volumes of absolute alcohol and centrifugalized. 'The precipitate thus obtained is then dissolved in water and the above process repeated two or three times, leaving the substance for as short a period as possible in contact with the alcohol.

Recent research has shown that the 'nucin' of normal urine is in reality chiefly a nucleo-albumin, although of course true mucin is present in large amount in catarrhal affections of the bladder. According to somc observers the vitellin of eggyolk is a nucleo-albumin, but this is a matter of some doubt.

\section{Nucleo-proteids.}

The nucleo-albumins have been regarded as compounds of pseudoor para-nuclein with proteids. Very recently substances have been prepared which in their general reactions as to precipitability, etc., closely resemble the nucleo-albumins and leave a residue of nuclein on digestion with pepsin, but when they are decomposed by boiling with acids, members of the xanthine series are obtained. From this it aplears that they are compounds of proteid with nuclein, not with pseudonuclein, and it is proposed to mark this difference by applying to them the name nucleo-proteid. They have so far been obtained chiefly from nuclei and cell protoplasm, but one is said to be present in serum. Some substances with the general reactions of a 
nucleo-proteid yield additionally some form of carbohydrate by hydrolytic cleavage. To these the name of nucleo-glycoproteid may per. haps be conveniently applied. They have been so far described as obtained from the panereas and mammary gland.

\section{CARBOHYDRATES.}

Certain members only of this extensive class have been found in the human body; of these, the most important and wide-spread are glycogen, grape-sugar or dextrose (glucose), with which diabetic sugar seems to be identical, maltose and milk-sugar.

Although the above-mentioned carbohydrates may be detected in various tissues and secretions of the animal body, their presence in the several cases is not so much due to their introduction into the body in the form in which they there occur as to their production from other members of the carbohydrate group existing in food. The chief of these is starch, and it will perhaps conduce to completeness to deal first very briefly with this parent-substance and some of the products of its decomposition.

\section{The Starch Group.}

\section{Starch $\left(\mathrm{C}_{6} \mathrm{H}_{10} \mathrm{O}_{5}\right)_{n}$.}

Starch occurs characteristically in plants and is formed in their green parts under the determinant influence of the chlorophyll-corpuscles. The exact mode of its formation is however as yet undecided. It exists in plant-tissues in the form of grains which vary in size and shape according to the plant, but which possess the common characteristic of exhibiting a stratified structure, which is much more marked in some cases (potato-starch) than in others, and the phenomena of doublerefraction when examined in polarized light. Considered as a whole the grains appear to be composed of two substanees, of which the chief both in quantity and importance is called granulose and the other cellulose. The former, which yields the blue colour characteristic of starch on the addition of iodine, may be dissolved out by the action of saliva or malt-extract, leaving a cellulosic skeleton of the original grain. This so-called cellulose is not identical with ordinary cellulose, as shewn by its ready solubility in several reagents which do not dissolve the latter. When treated with boiling water the grains swell up and finally burst, yielding a uniform viseous mass of starch-paste of which the chief component is the granulose. The mass thus obtained eannot be regarded as a trile solution of stareh, and it filters with extraordinary difficulty, leaving 
a gelatinous residue on the filter, however dilute the starchpaste may be which is used for the filtration. When subjected to hydrolytic agencies such as superheated water, dilute acids and enzymes the starch passes rapidly into true solution, yielding at the same time a series of successive products to be described below.

\section{Soluble starch (Amylodextrin) $\left(\mathrm{C}_{6} \mathrm{H}_{10} \mathrm{O}_{\tilde{5}}\right)_{n}$.}

When starch-paste, heated to $40^{\circ} \mathrm{C}$. on a water-bath, is digested with a small amount of saliva and the whole stirred so as to effect a thorough mixture of the two, the paste rapidly loses its opalescent appearance, becoming limpid and clear like water: the moment this change has taken place the digesting mixture should be boiled to cut short the further action of the ptyalin. The fluid thus obtained contains the first product of the hydrolysis of starch to which the name of 'soluble starch' has been given. Its solution filters readily, and the filtrate yields with iodine the pure blue characteristic of the original unaltered starch. On the addition of an excess of alcohol the soluble starch is precipitated, the precipitate after drying being but little soluble in cold water, although it readily dissolves in water at $60-70^{\circ} \mathrm{C}$. It also yields a characteristic precipitate with tannic acid, and differs in this respect from the dextrins. It is dextrorotatory and does not reduce Fehling's fluid. The same substance may be similarly obtained by the limited action of malt-extract or pancreatic juice.

\section{The dextrins $\left(\mathrm{C}_{6} \mathrm{H}_{10} \mathrm{O}_{5}\right)_{n}$.}

When the hydrolytic action of saliva, malt-extract, or pancreatic juice on starch-paste is prolonged, the first-formed soluble starch is rapidly changed into a number of successive substances to which the general name of dextrin is given. These products are intermediate between soluble starch and the sugars which result from the complete hydrolysis of starch, and are probably very numerous, the similarity in the properties of the successively formed dextrins rendering their separation and characterization extremely difficult. 'They are all precipitable by alcohol, and differ from soluble starch in yielding no precipitate with tannic acid.

(i) Erythrodextrin. If during the earlier stages of the hydrolysis of stareh-paste, successive portions of the solution be tested by the addition of iodine, it may be observed that the pure blue which it yields at first passes gradually through violet, and reddish-violet to reddish-brown, the latter colour being supposedly due to the presence in the solution of erythrodextrin, whence the name. But little is definitcly known of this dextrin as a chemical individual, its chief characteristic 
being the colour it yiclds with iodine. 'The violet observed during the earlier stages of hydrolysis is due to an admixture of the blue due to soluble starch with the red of the erythrodextrin.

Commercial dextrin, which is very impure, containing dextrose and frequently unaltered starch, usually yields a very strong red coloration on the addition of iodine.

(ii) Achroodextrin. When, during the prolonged enzymic hydrolysis of starch under ordinary conditions, the addition of iodine ceases to give any coloration, the fluid now contains much sugar together with a considerable but variable proportion of this dextrin, which has received its name from its behaviour towards iodine, yielding no colour with this reagent. It is the characteristic dextrin obtained during the prolonged artificial digestion of starch with saliva (or pancreatic juice) and may be separated from its solution by concentration and the addition of an excess of alcohol. As thus prepared it is mixed with much allherent maltose, from which it cannot be entirely freed by washing with alcohol or by successive solution in water and reprecipitation with alcohol. A partial separation may be obtained by fermenting off the sugar with yeast or by dialysis, since dextrin is non-diffusible. If however the mixture be warmed with a slight excess of mercuric cyanide and caustic soda, the whole of the sugar is destroyed in reducing the mercuric salt, leaving in solution a non-reducing dextrin.

Maltodextrin. This substance is described as appearing during the earlier stages of a limited hydrolysis of starch-paste with diastase, and it may perhaps similarly occur when saliva or pancreatic juice is employed. It differs from the dextrins previously described as follows. It is more soluble in alcohol and distinctly diffusible; it reduces Fehling's fluid, has a lower specific rotatory power and is completely convertible into maltose by the further action of diastase. It will therefore not be found among the products of a prolonged liydrolytic degradation of stareh.

When starch-paste is hydrolyzed outside the body with diastase or with animal enzymes some dextrin is always obtained together with the sugars which make their characteristic appearance during the process. There is however no evidence that in the body any carbohydrate is absorbed as dextrin from the alimentary canal. We shall therefore not be far wrong in concluding that in the animal body starch is completely converted into sugar previous to absorption, and if this be the case the interest of the plyysiologist in the primary products of starch hydrolysis becomes very small, cxcept so far as a study of these products is essential to the elucidation of the probable molecular magnitude and structure of the parent-substance. 
When starch is treated with dilute boiling acids, the products which have been so far described are formed in rapid succession, the whole being finally converted into dextrose.

\section{Animal-gum $\left(\mathrm{C}_{12} \mathrm{H}_{20} \mathrm{O}_{10}+2 \mathrm{H}_{2} \mathrm{O}\right)$ (?).}

This is described as a form of carbohydrate which may be extracted by the prolonged action of superheated water from salivary and mncous glands, and is found also in milk and urine. It yields no coloration with iodine, is very feebly dextrorotatory and appears to form a compound with cupric oxide; the latter is obtained when caustic soda and sulphate of copper are added to its solution, and may be used for the separation of animal-gum from urine. It is non-reducing, but yields a reducing sugar when boiled with mineral acids.

\section{Glycogen $\left(\mathrm{C}_{6} \mathrm{H}_{10} \mathrm{O}_{5}\right)_{n}$.}

This substance is from a purely chemical point of view extremely like starch, the similarity being most marked when the hydrolytic products of the two are compared. A study of its oceurrence, behaviour and fate in the animal body leaves but little doubt that it may be regarded from the physiological side as truly the animal analogne of the vegetable starch, and as such it is frequently spoken of as 'animal starch.' It was first described as a constituent of the liver, and in more recent times it has been found to occur in greatcr or less quantities in many tissues of the adult borly, as for instance the museles, also in white blood-and pus-corpuscles and other contractile protoplasm, in which its presence is significantly connected with their specialized activity, not as an esscntial, as some have supposed, but as a convenient accessory. It is also conspicuously found in the tissues of the embryo before the liver is functionally active, and is present in large quantities in many molluses, as for instance the common oyster (9.5 p.c.).

Preparation of glycogen. The liver of an animal (rabbit or dog), previonsly fed with copious meals of carbohydrate, is excised as rapidly as possible, ent into small pieces and thrown into an excess of boiling water, at least 400 e.c. to each $100 \mathrm{gr}$. of liver. After being boiled for a short time, the pieces are removed, ground up as finely as possible in a mortar with sand or powdered glass, returned to the original water and boiled again for some time. On faintly acidulating the boiling mass with acetic acid a large amount of the proteid matter in solution is coagulated and may be removed by filtration. The filtrate is now rapilly cooled, and the proteids finally and connpletely precipitated by the alternating addition of hydrochloric acid and of a solution of the double iodide of mercury and 
potassium (Brïcke's reagent), ${ }^{1}$ as long as any precipitate is formed. The precipitated proteids are again removed by filtration, the glycogen precipitated by the addition of two volumes of 95 p.c. alcohol, ${ }^{2}$ collected on a filter, washed thoroughly with 60 p.c. spirit, and finally with absolute alcohol and ether.

The above method suffices in cases where there is much glycogen present and no quantitative result is desired; as a matter of fact there is a not inconsiderable loss during its application. The accurate determination of glycogen in tissues is a matter of some difficulty.

Glycogen is, when pure, an amorphous white powder, readily soluble in water, with which it yields a solution which is usually, but not always, opalescent. This solution contains no particles which are visible under the microscope and filters readily without diminution of the opalescence; the latter may be largely removed by the addition of free alkalis or acetic acid. Under ordinary conditions it is readily precipitated by alcohol when the mixture contains 60 p.c. of the precipitant, but if pure, and in $0.5-1.0$ p.c. solution, even an excess of absolute alcohol is stated not to cause its precipitation. The precipitation takes place at once on the addition of a trace of sodium chloride.

It gives a characteristic port wine coloration with iodine, which does not however distinguish it from erythrodextrin, since in both cases the colour, contrary to the older and current statements, disappears on warming and returns on cooling. On the other hand, dextrins are not precipitated by 60 p.c. alcohol, even the most insoluble of these substances requiring at least 85 p.c. of alcohol for their precipitation, and usually more. It appears that the reaction with iodine is most delicate in presence of sodium chloride.

The hydrolytic products obtained by the action of enzymes and dilute boiling acids on glycogen have not been as fully studied as they have in the case of starch, but the general course of the decomposition is the same in both cases. Thus when treated with dilute mineral acids at $100^{\circ} \mathrm{C}$., the opalescence disappears, some dextrin is formed en passunt, and finally the solution contains only dextrose. On the addition of saliva or pancreatic juice to a solution of glycogen at $40^{\circ}$, the first change observed is an immediate disappearance of the opalescence, followed by a rapid conversion into some form of dextrin and a considerable proportion of a sugar which is apparently identical with maltose. Some trace of dextrose may perhaps at the same time be formed.

1 Prepared by saturating a boiling 10 p.c. solution of potassinm iodide with freshly precipitated iodide of mercury; on cooling this is filtered and the filtrate employed as directed.

2 So that the mixture contains 60 p.c. of alcohol. 
The change which glycogen in the liver undergoes postmortem and presumably also during life is strikingly different from that which has been described above. Whereas by ordinary enzymic hydrolysis, maltose is the chief final product obtained, there is now no doubt that in the liver little if any maltose is formed, the so-called liver-sugar being apparently identical with true dextrose.

\section{Cellulose $\left(\mathrm{C}_{6} \mathrm{H}_{10} \mathrm{O}_{5}\right)_{n}$.}

Although true cellulose is never found as a constituent of the animal tissues, it possesses no inconsiderable interest for the physiologist in view of the fact that in the herbivora a large amount of cellulose is digested and absorbed so that it does not reappear cxternally in the excreta. In man also there is no doubt that some digestion and absorption of cellulose may occur, the process being facilitated by the fact that in those more succulent vegetables and fruits in which it is taken by man, the cell-walls are comparatively non-lignified and hence more easily acted upon by the digestive agents. Further, although at present but little is known as to how the digestion of cellulose is brought about in the alimentary canal, there is increasing evidence of the possible existence of a specific enzyme to whose solvent action the change is dne. But as yet this evidence rests almost entirely upon experiments with and observations on vegetable organisms.

Cellulose is related to starch and in some cases plays the part of a store of reserve material, being dissolved, presumably by some enzyme, and utilized during germination.

By treatment with strong sulphuric acid cellnlose may be dissolved with the formation of a dextrin-like product: on diluting with water and boiling it is finally converted into a sugar which is apparently identical with clextrose.

As already stated, cellulose is undoubtedly digested in the alimentary canal more especially of herbivora, but also to a less extent of man. We know however but little of the real nature of the digestive processes which are involved in this. It may take place under the influence of putrefactive organisms, and this accords with the marked occurrence of marsh-gas in the gases of the intestine of herbivora and its increased presence in the intestine of man when largely fed with a vegetable diet. On the other hand it is possible that the digestion may turn out to be due to some definite enzyme, but as yet no such enzyme has been obtained with certainty from the secretions or tissues of the alimentary canal.

\section{Tunicin $\left(\mathrm{C}_{6} \mathrm{H}_{10} \mathrm{O}_{5}\right)_{n}$.}

This substance constitutes the chief part of the mantle of 
Tunicata (Ascidians) and is closely similar to if not actually identical with vegetable cellulose. but more resistant to the action of acids than is true cellulose. It is coloured blue by the addition of iodine after preliminary treatment with sulphuric acid. It is soluble in concentrated sulphnric acid, and if water be added to this solution, and it be boiled for some time, a sugar which is apparently identical with ordinary dex. trose is obtained.

It is prepared in the pure form by treating the mantles for some days with water in a Papin's digester, then in succession with boiling dilute hydrochloric acid, strong caustic potash and water. As thus obtained it retains the form of the parent tissue.

\section{THE SUGARS.}

The researches of Emil Fischer have thrown a flood of light on the chemistry of the sugars. In phenyl-hydrazine $\left(\mathrm{C}_{6} \mathrm{H}_{5} \cdot \mathrm{NH}\right.$. $\mathrm{NH}_{2}$ ) he discovered a reagent which forms with the sugars compounds known as hydıazones and osazones. These provided for the first time by their various solubilities, melting-points and rotatory powers an adequate means of detecting, separating and characterizing the several members of this class of carbohydrates. Hence it became possible to investigate the occurrence of sugars among the complicated products of the reactions employed in the effort to effect their transformations and synthetic production.

The osazomes. The compounds of the sugars to which this generic name is applied are formed when solutions of the sugar's are warmed for some time on a water-bath with phenyl-hydrazine and dilute acetic acid, and separate out either in an anorphous or erystalline state. As alleady stated the osazones of the variois sugars differ characteristically in their solubilities, melting-points and rotatory powers. They hence afford an invaluable means not only for detecting and isolating the sugars but also for discriminating between sugars whose optical and reducing powers may afford an insufficient distinction.

\section{MoNosACCHARIDES.}

This division of the sugars comprises those which contain from two to nine atoms of carbon, and they may hence conveniently be called dioses, trioses, tetroses, cte., up to nonoses. The well-known and typical sugar glucose (dextrose) belongs to the hexose group, as also do lievulose and galactose. Riecently the pentoses have acquired a physiological interest, whereas so far the others, with the exception of the hexoses, are of purely chemical importance. 'The monosaccharides are 
either aldehydes (aldoses) or ketones (ketoses) of polyatomic alcohols.

\section{The Pentose Group $\left(\mathrm{C}_{5} \mathrm{H}_{10} \mathrm{O}_{5}\right)$.}

Typical members of this group are arabinose and xylose, obtained by the hydrolytic action of mineral acids on vegetable gums. They are erystalline, dextrorotatory, and reduce Fehling's fluid (sce note 2, p. 1222), but are not fermentable. Their osazones melt at characteristically low temperatures, $155-160^{\circ}$. When heated with hydrochloric acid they yield furfuraldehyde, the aldelyde $\left(\mathrm{C}_{5} \mathrm{H}_{4} \mathrm{O}_{2}\right)$ of pyromucie acid, which serves for both the detection and estimation of these sugars. When warmed with phloroglucin (triliydroxy benzene, $\left.\mathrm{C}_{6} \mathrm{H}_{3}(\mathrm{OH})_{3}\right)$ in presence of strong hydrochloric acid, they give rise to a brilliant red colour, which is characteristic and shows an absorption band between $\mathrm{D}$ and $\mathrm{E}$.

Pentoses have recently been found in the urine of an opium eater and in several cases of diabetes. It also appears probable that the carbohydrate obtained during the decomposition of a nucleo-proteid prepared from the pancreas is a pentose. When pentoses are administered to animals (rabbits and fowls), they are absorbed and assimilated and lead to an accumulation of glycogen, which is howerer of the ordinary kind. 'There is some difference of opinion as to their fate when introduced into the human body, but the balance of evidence seems to shew that they largely reappear unaltered in the urine.

\section{The Hexose or Glucose Group.}

1. Dextrose (Glucose, Grape-sugar).

$$
\mathrm{C}_{6} \mathrm{H}_{12} \mathrm{O}_{6} . \quad\left[\mathrm{COH}-(\mathrm{CH} \cdot \mathrm{OH})_{4}-\mathrm{CH}_{2} \cdot \mathrm{OH}\right] \text {. }
$$

Is found in minute but fairly constant quantities as a normal constituent of blood, lymph and chyle. Its occurrence in the liver has been already referred to $(\$ 369)$ in commection with diabetes, a disease which is characterized by the large amount of dextrose which is present in the blood and the still larger amount in the urine. The question whether dextrose is a normal constituent of urine has led to much dispute, but it now appears probalble that it is present in minute amounts. The experimental difficulties of deteeting traces of sugar in this excretion are considerable. There is no dextrose normally in bile.

The probability that it is as dextrose that the carbohydrates are finally absorbed from the alimentary canal has alrealy been referred to (p. 1191). 'This corresponds with the fact that dextrose is the most readily assimilable sugar, as is known from comparative injections of the various sugars into the blood-res- 
sels and observations on their subsequent appearance in the urine.

When pure, dextrose is colourless and crystallizes from its aqueous solution in six-sided tables or prisms, often agglomerated into warty lumps. 'The erystals will dissolve in their own weight of eold water, requiring however some time for the process; they are very readily soluble in hot water. Dextrose is somewhat sparingly soluble in cold ethyl-alcohol, more soluble in warm; slowly soluble, but in considerable quantity, in methylalcohol and insoluble in ether.

A freshly prepared cold aqueous solution of dextrose possesses a specific rotatory power for monochromatic yellow light of $(a)_{\mathrm{D}}=+100^{\circ}$. This rapidly falls, especially on warming, until it may be taken as $(a)_{\mathrm{D}}=+52 \cdot 5^{\circ}$ for solutions which do not contain more than 10 p.c. of the sugar. The rotation is however dependent on the concentration of the solution being least with very dilute solutions.

Dextrose readily forms compounds with alkalis and salts, which are in many cases characteristic, as for instance those with caustic alkalis and sorlium chloride. When heated, many of these compounds, more particularly those of bismuth, copper and mercury, are decomposed, the decomposition being accompanied by the precipitation either of the metal $(\mathrm{Hg})$ or of an oxide $\left(\mathrm{Cn}_{2} \mathrm{O}\right)$. This fact provides the basis for the more important methods of detecting the presence of dextrose and other singars with similar reducing powers, and of estimating them quantitatively in solution, since it is found that the anount of reduction effected by any given sugar is, under given conditions, a constant quantity.

\section{Phenyl-glucosazone. $\mathrm{C}_{18} \mathrm{H}_{22} \mathrm{~N}_{4} \mathrm{O}_{4} \cdot \quad\left[\mathrm{C}_{6} \mathrm{H}_{10} \mathrm{O}_{4}\left(\mathrm{C}_{6} \mathrm{H}_{5} \cdot \mathrm{N}_{2} \mathrm{H}\right)_{2}\right]$.}

This compound of dextrose with phenyl-hydrazine crystallizes in yellow needles. It is almost insoluble in water, very slightly soluble in hot alcohol, melts at about $205^{\circ}$ and is levorotatory when dissolved in glacial acetic acid.

An important property of dextrose is its power of undergoing fermentations. Of these the principal are: (1) Alcoholic. This is produced in aqueous solutions of dextrose, under the influence of yeast. 'The decomposition is the following: $\mathrm{C}_{6} \mathrm{H}_{12}()_{6}=2 \mathrm{C}_{2} \mathrm{H}_{6} \mathrm{O}+2 \mathrm{CO}_{2}$, yielding (ethyl) alcohol and carbon dioxide. Higher alcohols of the fatty series are found in traces, as also are glycerin, succinic acid and probably many other bodics. The fermentation is most active at about $25^{\circ} \mathrm{C}$. Below $5^{\circ} \mathrm{C}$. or above $45^{\circ} \mathrm{C}$. it almost entirely ceases. If the saccharine solution contains more than 15 per cent. of sugar it will not all be decomposed, as excess of alcohol stops the reac- 
tion. (2) Lactic. This is best known as occurring in miik when it turns sour owing to the conversion of lactose into lactic acid. But dextrose and other sugars may also be converted into lactic acid $\left(\mathrm{C}_{6} \mathrm{H}_{12} \mathrm{O}_{6}=2 \mathrm{C}_{3} \mathrm{H}_{6} \mathrm{O}_{3}\right)$, the conversion being ordinarily due to the presence of some specific micro-organism which is specially active in presence of decomposing nitrogenous material such as decaying cheese. A similar change is rapidly produced when dextrose is mixed with finely divided gastric mucous membrane. There is also some evidence of the existence of an unorganized ferment (enzyme) in the mucous membrane of the stomach which can convert lactose and dextrose (?) into lactic acid. On prolongerl standing the lactic fermentation is apt to pass into (3) Butyric. This results from the appearance and action of another specific organized ferment on the first formed lactic acid, the change being accompanied by the evolution of hydrogen and carbon dioxide -

$$
2 \mathrm{C}_{3} \mathrm{H}_{6} \mathrm{O}_{3}=\mathrm{C}_{3} \mathrm{H}_{7} \cdot \mathrm{COOH} .+2 \mathrm{CO}_{2}+2 \mathrm{H}_{2} \text {. }
$$

Lactic and butyric fermentations are most active at $35^{\circ}$ and $40^{\circ}$ respectively; they probably occur constantly in the alimentary canal with a carbohydrate diet and may in some cases be remarkably predominant. The hydrogen evolved during butyric fermentation possibly plays some important part in the production of the fuecal and urinary pigments from those of bile.

\section{Lævulose.}

$$
\mathrm{C}_{6} \mathrm{H}_{12} \mathrm{O}_{6} \cdot \quad\left[\mathrm{CH}_{2} \cdot \mathrm{OH}-\mathrm{CO}-(\mathrm{CH} \cdot \mathrm{OH})_{3}-\mathrm{CH}_{2} \cdot \mathrm{OH}\right] \text {. }
$$

This is the ketone corresponding to the aldehyde dextrose. It is best known as occurring mixed with dextrose in many fruits, also in honey, and is stated to occur occasionally in urine. It is a characteristic product of the action of dilute mineral acids on cane-sugar, which is hereby decomposed into equal parts of dextrose and lievulose, and since, when the change is complete, the original dextrorotatory power of the solution has become lievorotatory, the cane-sugar is said to have been 'inverted.' A similar inversion takes place in the stomach and small intestine. In its general reactions levulose behaves like dextrose, but may be at once distinguished from the latter by its powerful lavorotatory action on polarized light: this varies considerably with the temperature and concentration of the solution. It yields with phenyl-hydrazine an osazone identical with that derived from dextrose. It for'ms a compound with calcium hydrate, which unlike that rielded by dextrose is extremely insoluble and may thus be 1) loyed for the separation of the two sugars. 


\section{Galactose (Cerebrose). $\quad \mathrm{C}_{6} \mathrm{H}_{12} \mathrm{O}_{6}$.}

When milk sugar (lactose), see p. 1223, is boiled with dilute mineral acids it is decomposed into a molecule of dextrose and one of galactose -

$$
\mathrm{C}_{12} \mathrm{H}_{22} \mathrm{O}_{11}+\mathrm{H}_{2} \mathrm{O}=\mathrm{C}_{6} \mathrm{H}_{12} \mathrm{O}_{6}+\mathrm{C}_{6} \mathrm{H}_{12} \mathrm{O}_{6} .
$$

The two sugars may be separated by crystallization and by taking advantage of the greater solubility of galactose in absolute alcohol. In its general reactions and behaviour galactose resembles dextrose but is possessed of a considerably greater specific rotatory power $\left[(a)_{D}=+83^{\circ}\right]$ which increases with the concentration and rise of temperature. It yields with phenylhydrazine an osazone (phenyl-galactosazone) which has the same composition as phenyl-glucosazone and very similar solubilities. It differs however from the latter in melting at $190-193^{\circ}$ and in being optically inactive when dissolved in glacial acetic acid. It has recently been shewn that the sugar which results from the action of boiling dilute sulphuric acid on certain constituents of the brain substance and has been named cerebrose, is really irlentical with galactose.

Galactose is fermentable with yeast, but less readily so than is dextrose.

\section{Glycuronic acid. $\mathrm{C}_{6} \mathrm{H}_{10} \mathrm{O}_{7} . \quad\left[\mathrm{COH}-(\mathrm{CH} \cdot \mathrm{OH})_{4}-\right.$ $\mathrm{COOH}]$.}

This acid was first obtained as a compound, campho-glycuronic acid, in the urine of dogs after the administration of eamphor, and subsequently as mrochloralic acid after the administration of clloral. Since then it lias been found in urine as ethereal or glucoside-like compounds with an extensive series of members of the fatty or aromatic series after the introduction of the appropriate substances into the animal body. It is probable that traces of compounds of this acid occur normally in urine, since this excretion is usually slightly levorotatory and it is known that indole and skatole which are formed in the alimentary canal readily reappear in the urine as compounds of glycuronic acid, viz. indoxyl-and skatoxyl-glycuronic acid, when introduced into the body. The compounds of glycuronic acid are all lievorotatory and some of them reduce metallie salts on boiling and may hence lead to errors in the determination of sugar in urine.

Glycuronic acid does not oceur in the free state in the animal body. Chemically it is closely related to dextrose. and like dextrose is dextrorotatory but to a less extent, reduces Fehling's fluid to the same extent as does dextrose and forms with phenyl-hydrazine a yellow erystalline compound which 
melts at $114-115^{\circ}$. The acid is known only as a syrup soluble in alcohol and water. It gives the reaction with phloroglucin and yiclds furfuraldehyde as do the pentoses (see p. 1217).

'The formation of the compounds of glyeuronic acid to which attention has been drawn is of great and increasing interest.

\section{DISACCHARIDES.}

\section{The Cane-Sugar Group.}

\section{Saccharose (Cane-sugar). $\mathrm{C}_{12} \mathrm{H}_{22} \mathrm{O}_{11}$.}

Although it is not found as a constituent of any animal tissue this sugar possesses no inconsiderable interest in view of the fact that it is a food-stuff which is largely consumed by man and may constitute in many cases no small part of the total carbohydrates with which the body is supplied.

Cane-sugar is chiefly distinguished from the other's by the fact that it does not reduce metallie salts, and does not form a compound with phenyl-hydrazine, but the property which is of greatest interest to the physiologist is the ease with which it may be 'inverted' or converted into equal parts of dextrose and lievulose -

$$
\mathrm{C}_{12} \mathrm{H}_{22} \mathrm{O}_{11}+\mathrm{H}_{2} \mathrm{O}=\mathrm{C}_{6} \mathrm{H}_{12} \mathrm{O}_{6} \text { (dextrose) }+\mathrm{C}_{6} \mathrm{H}_{12} \mathrm{O}_{6} \text { (lievulose). }
$$

This inversion is readily brought about by treatment with dilute mineral acids at $100^{\circ}$ or even at $40^{\circ}$ or below if the action is more prolonged; it is also the result of the action of enzymes, more especially of invertin from yeast, and is characterized experimentally by the change in the rotatory power of the solution, which from being originally dextrorotatory becomes lavorotatory; hence the name 'inversion.' For cane-sugar $(\alpha)_{\mathrm{D}}=+66^{\circ}$; for lævulose $(\alpha)_{\mathrm{D}}=-100^{\circ}$. The rotatory power of the latter is largely dependent upon temperature and concentration.

When cane-sugar is injected into the blood-vessels or tissues of an animal it is eliminated in an unaltered condition and is thus shewn to be non-assimilable. On the other hand, it may be introduced in large amounts into the alimentary canal without reappearing externally in the urine. From this it nuay be concluded that it undergoes some change before or during absorption and this change is most probably that of inversion. 'This change may take place in the stomach, partly under the influence of the acid of the gastric juice but also as the result of the action of a soluble enzyme; it is even more marked in the small intestine, where the active agent is without doubt an enzyme. From this it appear's that cane-sugar conforms to the apparently general rule that the carbohydrates leave the alimentary canal as dextrose. 
Cane-sugar readily undergoes a lactic-acid fermentation in presence of sour milk to which zinc oxide is added for the fixation of the acid as it is formed.

2. Maltose. $\mathrm{C}_{12} \mathrm{H}_{22} \mathrm{O}_{11}+\mathrm{H}_{2} \mathrm{O}$.

This is the sugar which is characteristically formed, together with dextrins, by the action of malt-extract (diastase) on starchpaste. It is similarly the chief sugar which is formed by the action of saliva and pancreatic juice upon starch-paste or upon glycogen, being accompanied in the case of pancreatic juice by a variable but distinct amount of dextrose if the action of this secretion be prolonged. Maltose is also formed by the action of dilute acids upon starch-paste, but in this case it is difficult to prevent the simultaneous formation of dextrose, into which it is readily converted by acids, yielding $98-99$ p.c. of the latter sugar. It is therefore usually prepared from the products of the action of malt-extract on starch-paste.

Maltose is very soluble in water, also in alcohol, but less so in the latter solvent than is dextrose. It crystallizes in fine needles which are however not very easily obtained. Solutions of maltose are dextrorotatory and reduce metallic salts; it is therefore not easily distinguished from dextrose by merely qualitative tests. As the necessity of discriminating between the two sugars is one of frequent occurrence, the following characteristic differences between their optical and reducing powers are of great importance. For maltose in 10 p.c. solution at $20^{\circ} \mathrm{C}$. $(\alpha)_{\mathrm{D}}=+140^{\circ} ;^{1}$ for dextrose $(\alpha)_{\mathrm{D}}=+52 \cdot 5^{\circ}$. When maltose is boiled with Fehling's fluid ${ }^{2}$ the amount of cuprous oxide which separates out is only about two-thirds of that which would be reduced by an equal weight of dextrose, or in other words 66 parts of dextrose reduce as much as 100 parts of maltose. Bearing in mind that maltose may be readily converted into dextrose by boiling with dilute acids with a corresponding change of its optical and reducing powers, while dextrose is of course unaltered by this operation, it is easy to base upon the above facts a method of identifying the two sugars. As a further difference between the two it may be stated that Barfoed's reagent ${ }^{3}$ is not reduced by maltose, whereas it is by dextrose. In this respect maltose resembles lactose (milk-sugar) which also does not reduce this reagent.

\section{Phenyl-maltosazone. $\mathrm{C}_{24} \mathrm{H}_{32} \mathrm{~N}_{4} \mathrm{O}_{9}$.}

This compound of maltose is obtained by the action of

${ }^{1}$ Some authors make it less $=+195 \cdot 4$.

2 Solution of hydrated cupric oxide in caustic soda in presence of the double tartrate of sodium and potassium (Rochelle salt).

${ }^{3}$ Dissolve 1 part of cupric acetate in 15 parts of water: to $200 \mathrm{c}$.c. of this solution add 5 c.e. of acetic acid containing 38 p.c. of glacial acid. 
phenyl-liydrazine upon it in presence of acetic acid. It crystallizes readily in minute yellow needles and is characterized by being (unlike phenyl-glucosazone) soluble in about 75 parts of boiling water, and still more soluble in hot alcohol. Its melting-point, $206^{\circ}$, is practically the same as that of phenyl-glucosazolle.

The researches referred to above (p. 1190) shewed that whereas pancreatic juice rapidly converts starch-paste into maltose and a little dextrose, an extract of the mucous membrane of the small intestine or the tissue itself, while acting but feebly on starch-paste, rapidly converts maltose into dextrose. It was hence surmised that maltose would be found to be a non-assinilable sugar, requiring like cane-sugar to be converted into the simpler dextrose before absorption. More recent experiments have confirmed this view, for it has been found that if maltose be injected into the blood-vessels it is largely excreted in an unaltered form in the urine. The converting action of extracts of the intestinal mucous membrane is strikingly less than that of the tissue itself; from this it may perlaps be inferred that the change into dextrose takes place rather during than previous to absorption. This fact corresponds elosely to the well-known views as to the changes which peptones similarly undergo during their passage through the walls of the intestine into the ncighbouring blood-vessels (see $\$ 250$ ).

\section{Lactose (Milk-sugar). $\quad \mathrm{C}_{12} \mathrm{H}_{22} \mathrm{O}_{11}+\mathrm{H}_{2} \mathrm{O}$.}

It is found characteristically and solely in milk, in quantities varying with the class of animal and at different times with the same animal. The percentage is relatively high in human milk. It is also said to occur in the urine of lying-in women and sucklings.

Preparation. The casein is precipitated from diluted milk by the addition of acetic acid. The filtrate from this is boiled to coagulate the remaining proteids which are then removed by filtration. This final filtrate is then concentrated and on prolonged standing yields crusts of millk-sugar which are purified by recrystallization from hot water.

It yields, when pure, hard colourless crystals, belonging to the rhombic system (four-sided prisms). It is less soluble in water than dextrose, requiring for solution six times its weight of cold, but only two parts of boiling, water; it is entirely insoluble in alcohol and in ether. It is fully precipitated from its solutions by the addition of basic lead acetate and ammonia.

Solutions of many metallie salts are readily reduced by boiling with lactose, but the reducing power is less than that of dextrose. Thus 1 c.c. of Fehling's fluid, which is reduced by 
$5 \mathrm{mgr}$. of dextrose, requires $6 \cdot 7 \mathrm{mgr}$. of lactose, provided that certain conditions as to the dilution of the solution, duration of boiling, etc., are attended to. These are important for the accurate volumetric estimation of lactose. The specific rotatory power of lactose is $(a)_{1}=+52 \cdot 3^{\circ}$, and is independent of the concentration in solutions which contain up to 35 p.c. at ordinary temperatures. Its rotatory power is thus identical with that of dextrose. It is, however, readily distinguishable from dextrose by its smaller solubility in water, insolubility in alcohol, and ineapability of undergoing direct alcoholic fermentation with yeast. It also does not rednce Barfoed's reagent, and in this resembles maltose. When boiled with dilute mineral acirls it yields equal molecules of dextrose and galactose (see p. 1220), and since the specific rotatory power of the latter of these is high $\left[(a)_{\mathrm{v}}=+83^{\circ}\right]$, this increase of rotatory (and reducing) power on treatment with acids affords a further convenient means of discrimination between lactose and dextrose.

\section{Phenyl-lactosazone. $\quad \mathrm{C}_{24} \mathrm{H}_{32} \mathrm{~N}_{4} \mathrm{O}_{9}$.}

This compound of lactose with phenyl-hydrazine is formed under conditions similar to those employed for the preparation of the analogous compound of dextrose. It is soluble in $80-90$ parts of boiling water and melts at about $200^{\circ}$. It crystallizes readily in the form of yellow needles which, unlike the crystals of phenyl-maltosazone, are usually aggregated into clusters.

Lactose is readily capable of undergoing a direct lactic fermentation and this occurs characteristically in souring milk. The exciting cause is doubtless ordinarily an organized ferment, but there is also some evidence of the existence in the alimentary canal of an enzyme which can effect the same conversion. The circumstances and products of the conversion are the same as for dextrose and sacharose.

Although isolated lactose is unaffected by yeast, milk itself is capable of undergoing, under the influence of certain ferments, an alcoholic fermentation, and this has been employed from very early times by the inhabitants of certain districts of Russia in the preparation of Kumys from mare's milk, and Kephir from cow's milk. Of late years these fluids have attracted much attention in virtue of their supposed therapeutic action in certain wasting diseases. Very little is as yet known as to the real nature of the changes which occur during the fermentation, but they are probably extremely complex and due to the presence of several organized ferments. Kephir ferment is a commercial article in Russia, obtainable at the apothecaries.

The non-assimilability of saccharose and maltose lias already been referred to, and experiment has shewn that lactose is similarly incapable of assimilation, for when injected into the 
blood-vessels it appears unaltered in the urine. It is therefore presumably changed in the alimentary canal into some form of sugar which is assimilable, it may be into dextrose and galactose. It does not appear that any such conversion can be markedly observed, if at all, under the action of any of the secretions of the alimentary canal, hence the change may more probably take place, as in the ease of maltose, rather during than before the passage of the sugar through the intestinal walls.

\section{FATTY ACIDS AND FATS, THEIR DERITATIVES} ANI) ALLIES.

\section{Acids of the Acetic Series.}

General formula $\mathrm{C}_{n} \mathrm{H}_{2^{n+1}}$. COOH (monobasic).

The free acids are found only in small and very variable quantities in various parts of the body; their derivatives on the other hand form most important constituents of the human frame, and will be considered further on.

Some of the lower acids of the series have been obtained by treating proteids with molten caustic potash. They also oceur among the products of the putrefaction of proteids, as for instance in old cheese.

Of the primary alcohols from which this series of acids is derived only two have as yet been obtained from animal tissues or secretions, viz. ethyl-alcohol, $\mathrm{C}_{2} \mathrm{H}_{5}$. $\mathrm{OH}$, and cetyl-alcohol, $\mathrm{C}_{16} \mathrm{H}_{33}$. OH. 'The former from muscle, brain and liver, the latter in union with palmitic acid in spermaceti and the secretion of the caudal glands of birds.

\section{Formic acid. H. COOH.}

When pure is a strongly corrosive, fuming fluid, with powerful irritating odour, soliclifying at $0^{\circ} \mathrm{C}$., boiling at $100^{\circ} \mathrm{C}$., and capable of being mixed in all proportions with eitler water or alcohol. It has been obtained from various parts of the body, such as the spleen, thymus, pancreas, muscles, brain, and blood; in the latter its presence may be due to the action of acids on the hemoglobin. It also occurs in minute traces in urine. It is exereted by some ants (Formica rufa) in a fairly concentrated form and may be present to the surprisingly large extent of 40 p.c. in the secretion of certain caterpillars. 'The separation of so acid a fluid from the alkaline cell-substance is remarkable and of considerable interest. When heated with strong sulphuric acid it is decomposed into carbon monoxide and water. It is further characterized by readily effecting the reduction of metallic salts, as of mercury or silver, when heated with their solutions. 


\section{Acetic acid. $\mathrm{CH}_{3}, \mathrm{COOH}$.}

Is distinguished by its characteristic odour; its boilingpoint is $118^{\circ} \mathrm{C}$.; the anhychrous acid solidifies at about $17^{\circ}$. It is soluble in all proportions in alcohol and in water.

It may be formed in the stomach as the result of fermentative changes in the food, and is frequently present in diabetic urine, as also in traces in normal urine. In other organs and fluids it exists only in minute traces.

With ferric chloride it yields a blood-red solntion, decolourized by hydrochloric acid. (It differs in this last reaction from sulphocyanide of iron.) Heated with alcohol and sulphuric acid, the characteristic odour of acetic ether (ethyl-acetate) is obtained.

\section{Acetone. $\mathrm{CH}_{3} \cdot \mathrm{CO} \cdot \mathrm{CH}_{3}$.}

This substance is the typical member of the general class known as ketones and may be prepared by the dry distillation of calcium or barium acetate.

Acetone is a volatile liquid, soluble in water, boiling at $56^{\circ}$, and possessed of an agreeable ethereal odour. It may be obtained in considerable quantity by distillation from the urine and blood of diabetic patients and accounts for the peculiar ethereal odour which these frequently evolve. This symptom is of serious prognostic importance, and it has been supposed by many authors that the fatal diabetic coma which rapidly supervenes is caused by the presence of acetone. 'The urine of diabetic patients frequently exhibits a reddish-violet coloration with ferric chloride, supposedly due to the presence of aceto-acetic acid $\left(\mathrm{CH}_{3} \cdot \mathrm{CO} \cdot \mathrm{CH}_{2} \cdot \mathrm{COOH}\right)$ which readily yields acetone by its decomposition.

Acetone is also not infrequently found in the urine and breath (?) of children in apparently normal health.

Acetone gives a characteristic reaction with iodine in presence of an alkali (formation of iodoform) and colour-reactions with sodium nitro-prusside and fuchsine.

\section{Propionic acid. $\mathrm{C}_{2} \mathrm{H}_{5} \cdot \mathrm{COOH}$.}

This acid closely resembles the preceding one. It possesses a very sour taste and pungent odour'; is soluble in water, boils at $141^{\circ} \mathrm{C}$., and may be separated from formic and acetic acid by taking advantage of the superior solubility of its lead salt in cold water.

It occurs in small quantities in sweat, in the contents of the stomach, and in diabetic urine when undergoing fermentation. It is similarly produced, mixed however with other products, during alcoholic fermentation.

It is stated to have been found occasionally in normal urine. 


\section{Butyric acid. $\mathrm{C}_{3} \mathrm{H}_{7} \cdot \mathrm{COOH}$.}

There are two possible isomeric acids of the general formula $\mathrm{C}_{3} \mathrm{H}_{7} \cdot \mathrm{COOH}$, the normal or primary, $\mathrm{CH}_{3} \cdot \mathrm{CH}_{2} \cdot \mathrm{CH}_{2} \cdot \mathrm{COOH}$ and iso- or secondary, $\mathrm{CH}\left(\mathrm{CH}_{3}\right)_{2}$. COOH.

Normal butyric acid. An oily colourless liquid, with an odour of rancid butter, soluble in water, alcohol, and ether, boiling at $162^{\circ} \mathrm{C}$.

Found in sweat, the contents of the large intestine, fæces, and in urine. It occurs in traces in many other fluids, and is plentifully obtained when diabetic urine is mixed with powdered chalk and kept at a temperature of $35^{\circ} \mathrm{C}$. It exists, in union with glycerin as a neutral fat, in small quantities in milk, and gives the characteristic odour to butter which has become rancicl.

It is the principal product of the second stage of lactic fermentation (see p. 1219), and is ordinarily prepared from this source.

Isobutyric acid. Occurs in freces and among the putrefactive products from proteids, also in certain fruits such as the banana.

\section{Valeric or Valerianic acid. $\mathrm{C}_{4} \mathrm{H}_{9} \cdot \mathrm{COOH}$.}

Four isomeric forms of this acid exist. Of these the one here described is the isoprimary $\mathrm{CH}\left(\mathrm{CH}_{3}\right)_{2} \mathrm{CH}_{2} \cdot \mathrm{COOH}$. (Isopropyl-acetic acid.)

An oily liquid, of burning taste and penetrating odour as of decaying cheese; soluble in 30 parts of water at $12^{\circ} \mathrm{C}$., readily soluble in alcohol and in ether. Boils at $175^{\circ} \mathrm{C}$.

It is found in the solid excrements, and is formed readily by the decomposition, through putrefaction, of impure leucine, ammonia being at the same time evolved; hence its occurrence in urine when that fluid contains leucine, as in cases of acute atrophy of the liver.

Caproic acid. $\mathrm{C}_{5} \mathrm{H}_{11} \cdot \mathrm{COOH}$.

Caprylic acid. $\mathrm{C}_{7} \mathrm{H}_{15}$. COOH.

Capric (Rutic) acid. $\mathrm{C}_{9} \mathrm{H}_{19}$. COOH.

These three occur together (as fats) in butter, and are contained in varying proportions in the freces from a meat diet and the first two in sweat. The first is an oily fluid, slightly soluble in water, the others are solids and scarcely soluble in water; they are soluble in all proportions in alcohol and in ether. They may be prepared from butter, and separated by the varying solubilities of their barium salts. 


\section{Lauric or Laurostearic acid. $\mathrm{C}_{11} \mathrm{H}_{23}$. COOH.}

\section{Myristic acid. $\mathrm{C}_{13} \mathrm{H}_{27}$. COOH.}

These occur as neutral fats in spermaceti, in butter and other fats. They present no points of interest.

\section{Palmitic acid. $\mathrm{C}_{15} \mathrm{H}_{31}$. COOH.}

\section{Stearic acid. $\quad \mathrm{C}_{17} \mathrm{H}_{35} \cdot \mathrm{COOH}$.}

These are solid, colourless when pure, tasteless, odourless, crystalline bodies, the former melting at $62^{\circ} \mathrm{C}$, the latter at $69 \cdot 2^{\circ} \mathrm{C}$. In water they are quite insoluble; palmitic acid is more readily soluble in cold alcohol than stearic: both are readily dissolved by hot alcohol, ether, or chloroform. Glacial acetic acid dissolves them in large quantity, the solution being assisted by warming. 'They readily form soaps with the alkalis, also with many other metals. 'The varying solubilities of their barium salts afford the means of separating them when mixed: this method may also be applied to many others of the higher members of this series.

These acids in combination with glycerin (see below), together with the analogous compound of oleic acid, form the principal constituents of human fat. As salts of calcium they occur in the freces and in 'adipocire,' and probably in chyle, blood and serous fluids, as salts of sodium. 'They are found in the free state in decomposing pus, and in the caseous deposits of tuberculosis.

Adipocire. When animal (proteid) tissues are buried for some time in damp ground or otherwise exposed to moisture in the absence of any free supply of oxygen they are frequently found to have undergone a peculiar change by which they are converted into a waxy or fatty substance. This is known as adipocire. It consists not of true neutral fats but of the ammonium, and in some cases calcium, salts of the highest fatty acids palmitic and stearic, or of the frce acids themselves. Practically nothing is definitely known as to the agencies and mode of this conversion. It may be the result of a purcly chemical ehange or perhaps it is more probably due to the action of some micro-organism. On either view of its formation the occurrence of adipocire is of extreme interest as shewing a possible direct formation of the higher fatty acids and hence of fats from proteids. It is however smpposed by some author's that the adipocire is formed entirely by change and aggregation from the fats present in the tissues at death. 'This view is probably incorrect. 


\section{Acids of the Oleic (Acrylic) Series. $\mathrm{C}_{n} \mathrm{H}_{2 n-1}$. COOH (monobasic).}

The acids of this series bear the same relationship to the olefines $\left(\mathrm{C}_{2} \mathrm{H}_{4}\right)$ that those of the acetic do to the paraffins $\left(\mathrm{CH}_{4}\right)$. Some of the higher members of the series are found as glycerin compounds in various fats.

They bear an interesting relation to the acids of the acetic series, breaking up when heated with caustic potash into acetic acid and some other member of the same series : - thus,

$$
\begin{aligned}
& \text { Oleic acid. } \\
& \text { Potassium acetate. Potassium palmitate. } \\
& \mathrm{C}_{17} \mathrm{H}_{33} \cdot \mathrm{COOH}+2 \mathrm{KHO}=\mathrm{KC}_{2} \mathrm{H}_{3} \mathrm{O}_{2}+\mathrm{KC}_{16} \mathrm{H}_{31} \mathrm{O}_{2}+\mathrm{H}_{2} \text {. }
\end{aligned}
$$

\section{Oleic acid. $\mathrm{C}_{17} \mathrm{H}_{33} \cdot \mathrm{COOH}$.}

This is the only acid of the series which is pliysiologically important. It is found united with glycerin in all the fats of the human body.

When pure it is, at ordinary temperatures, a colourless, odourless, tasteless, oily liquid, solidifying at $4^{\circ} \mathrm{C}$. to a crystalline mass. Insoluble in water, it is soluble in alcohol and in ether. It cannot be distilled without decomposition. It readily forms, with potassium and sodium hydroxide, soajs which are soluble in water: its compounds with most other bases are insoluble. It may be distinguished from the acids of the acetic series by its reaction with nitrous acid which converts it into a solid (elaïdic acid) and by the changes it undergoes when exposed to the air. It may be converted into stearic acid

$$
\mathrm{C}_{17} \mathrm{H}_{33} \cdot \mathrm{COOH}+\mathrm{H}_{2}=\mathrm{C}_{17} \mathrm{H}_{35} \cdot \mathrm{COOH} \text {. }
$$

\section{The Neutral Fats.}

These may be considered as ethereal salts formed by replacing the exchangeable atoms of hydrogen in the triatomic alcohol glycerin (see below), by the acid radicles of the acetic and oleic series. Since there are three such exchangeable atoms of hydrogen in glycerin, it is possible to form three classes of these ethereal salts; only those, however, which belong to the third class occur as natural constituents of the human body: those of the first and second are of theoretical importance only.

The following reaction, which represents the formation of tri-palmitin from glycerin and palmitic acid, is typical for all the others.

$$
\begin{gathered}
\text { Glycerin. } \\
\mathrm{C}_{3} \mathrm{H}_{5}(\mathrm{OH})_{3}
\end{gathered}+3\left(\mathrm{C}_{15} \mathrm{H}_{31_{6}} \mathrm{COOH} \text { (O) }\right)=\mathrm{C}_{3} \mathrm{H}_{5}\left(\mathrm{C}_{15} \mathrm{H}_{31} \cdot \mathrm{CO} \cdot \mathrm{O}\right)_{3}+3 \mathrm{H}_{2} \mathrm{O} .
$$

They possess certain general characteristics. Insoluble in water and but slightly in alcohol, they are readily soluble in 
ether, chloroform, benzene, ete. ; they also dissolve one another. They are neutral bodies, colourless and tasteless when pure; they are not capable of being distilled without undergoing decomposition, and yield as a result of this decomposition, solid and liquid hydrocarbons, water, fatty acids, and a peculiar substance, acrolein, resulting from the decomposition of the glycerin. (See below.)

They possess no action on polarized light.

They may readily be decomposed into glycerin and their respective fatty acids by the action of caustic alkalis or of superheated steam.

\section{Palmitin (Tri-palmitin). $\quad \mathrm{C}_{3} \mathrm{H}_{5}\left(\mathrm{C}_{15} \mathrm{H}_{31} \cdot \mathrm{CO} \cdot \mathrm{O}\right)_{3}$.}

Palmitin is but slightly soluble in alcohol either cold or hot, readily so in ether, from which, when pure. it crystallizes in fine needles; if mixed with stearin, it generally forms shapeless lumps, althongh the mixture may at times assume a crystalline form, and was then regarded as a distinct hody, namely margarin. When pure it melts at $62^{\circ}$ and solidlities again at $45^{\circ}$.

It is most conveniently obtained from palm-oil by removing the free palmitic and oleic acids by alcohol and repeatedly crystallizing the residue from ether.

\section{Stearin (Tri-stearin). $\quad \mathrm{C}_{3} \mathrm{H}_{5}\left(\mathrm{C}_{17} \mathrm{H}_{35} \cdot \mathrm{CO} . \mathrm{O}\right)_{3}$.}

This is the hardest and least fusible of the ordinary fats of the body; is also the least soluble, and hence is the first to crystallize out from solutions of the mixed fats. Readily soluble in ether and in boiling alcohol. It crystallizes usually in square tables or glittering plates. It presents peculiarities in its fusing points, melting first at $55^{\circ}$ then soliclifying as the temperature is further raised and melting finally and permanently at $71^{\circ}$.

Preparation. From mutton suet, its separation from palmitin and olein being effected by repeated crystallization from ether, stearin being the least soluble. It is however very difficult to obtain it pure by this process.

\section{Olein (Tri-olein). $\quad \mathrm{C}_{3} \mathrm{H}_{5}\left(\mathrm{C}_{17} \mathrm{H}_{33} \cdot \mathrm{CO} . \mathrm{O}\right)_{3}$.}

Is obtained with difficulty in the pure state, and is then fluid at ordinary temperatures. It is somewhat soluble in alcohol, very soluble in ether. It readily undergoes oxidation when exposed to the air, and is converted by mere traces of nitrous acid into a solid isomeric fat tri-elaïdin. Olein is saponified with much greater difficulty than are palmitin and stearin. 
Preparation. From olive oil, either by cooling to $0^{\circ} \mathrm{C}$. and pressing out the olein that remains fluid, or by dissolving in hot alcohol and cooling, when the olein remains in solution while the other fats crystallize out.

The fats which oceur in the animal body are mixtures of the above three substances in varying proportions. 'The normal fat of each animal or class of animals is however characterized by the constant preponderance of one of the three; thus in the fat of man and carnivora palmitin is in excess over the other two. In the fat of herbivora stearin predominates and in that of fishes olein. Butter contains, in addition to the above, several fats formed by the union of glycerin with the radicles of the lower acids of the acetic series.

\section{Glycerin (Glycerol). $\mathrm{C}_{3} \mathrm{H}_{5}(\mathrm{OH})_{3}$.}

As already stated, glycerin is a triatomic alcohol, the neutral fats being ethereal salts formed from it with the radicles of the higher fatty acids and oleic acid.

When pure, gilycerin is a viscid, colourless liquid, of a wellknown sweet taste. It is soluble in water and in alcohol in all proportions, insoluble in ether. Exposed to very low temperatures it becomes almost solid; it boils at $290^{\circ}$ and may be distilled without decomposition in the absence of air.

It dissolves the alkalis and alkaline earths, also many oxides, such as those of learl and copper; many of the fatty acids are also soluble in glycerin.

It possesses no rotatory power on polarized light.

It is easily recognized by its ready solubility in both water and alcohol, its insolubility in ether, its sweet taste, and its reaction with bases. When sufficiently heated, especially in pesence of a dehydrating agent, glycerin is decomposed, loses two molecules of water and yields acrolein. $\mathrm{C}_{3} \mathrm{H}_{5}(\mathrm{OH})_{3}$ $=\mathrm{C}_{3} \mathrm{H}_{4} \mathrm{O}+2 \mathrm{H}_{2} \mathrm{O}$. This substance possesses an intensely penetrating, irritating and pungent odour, so that its formation enables glycerin to be readily identified. It is the cause of the peculiar smell arising from overheated fats. Chemically it is the aldehyde of allyl alcohol (derived from the olefines) and is intermediate between this substance and acrylic acid, which is a homologue of oleic acid. (See above.)

Glycerin is formed in traces during the alcoholic fermentation of sugar. It is prepared in bulk by distilling in a current of superheated steam the fluid residue left after the saponification of fats with lime.

\section{Soaps.}

When neutral fats are heated with lime or canstic alkalis under pressure they are decomposed, the metal combining with 
the free fatty or oleic acid to form a salt, leaving the glycerin in solution. These salts are called soaps, being soluble in water if the metal is an alkali, insoluble if it is calcium, lead, or other similar metal. 'The reaction which takes place during the above saponification is as follows.

Tri-stearin.

Potassium stearate. Glycerin.

$\mathrm{C}_{5} \mathrm{H}_{5}\left(\mathrm{C}_{17} \mathrm{H}_{35} \cdot \mathrm{CO} \cdot \mathrm{O}\right)_{3}+3 \mathrm{KHO}=3\left(\mathrm{C}_{17} \mathrm{H}_{35} \cdot \mathrm{COOK}\right)+\mathrm{C}_{3} \mathrm{H}_{5}(\mathrm{OH})_{3}$.

A sinilar decomposition into glycerin and free fatty acid ean be effected by pancreatic juice (see p. 1193), the acid uniting with the alkali of the juice or of the bile to form a soap. This decomposition is however quantitatively inconsiderable but qualitatively of great importance for the absorption of fats, owing to the extraordinarily great emulsifying power of a mixture of bile, free fatty acids and soluble soaps. The same decomposition takes place when fats, more especially butter, turn rancid.

\section{Acids of the Glycolic and Oxalic Series.}

Glycolic Acid Series.

\section{Lactic (a-hydroxy-propionic) acid. $\mathrm{C}_{3} \mathrm{H}_{6} \mathrm{O}_{3}$.}

This, after carbonic acid, is to the physiologist the most important acid of the selies.

If lactic acid is regarded as derived from propionic acid, $\mathrm{CH}_{3} \cdot \mathrm{CH}_{2}$. COOH, it may be noticed at once that two isomeric lactic acids must be capable of being formed from it. These acids will have the following formulæ respectively; $\mathrm{CH}_{3} \cdot \mathrm{CH}(\mathrm{OH}) \cdot \mathrm{COOH}$ and $\mathrm{CH}_{2}(\mathrm{OH}) \cdot \mathrm{CH}_{2} \cdot \mathrm{COOH}$. Of these the first is known as ethylidene-lactic acid, the second as hydracrylic acid.

In addition to the above a third acid, isomeric with ethylidene-lactic acid, is known, namely sarcolactic or paralactic acid. Of these three acids only two occur in the body, hydracrylic being absent. A fourth acid, to which the name of ethylene-lactic acid has been given, has also been describerl as isomeric with hydracrylic acid. It is however probable that this acid is really acetyl-lactic acid, hydracrylic acid being the true ethylene-lactic acid. (See below.)

The several forms of lactic acid are all sirupy colourless Huids, soluble in all proportions in water and in alcohol, and to a slight extent in ether. They possess an intensely sour taste, and a strong acid reaction. When heated in solution they may partially distil over in the escaping vapour but are usually decomposed during the process. They form salts with metals, of which those with the alkalis are very soluble and crystallize with diffieulty. The calcium and zine salts are of the greatest 
importance, as will be seen later on, inasmuch as by their varying solubilities they afford a means of separating the several acids each from the other.

\section{Ethylidene-1actic acid. $\mathrm{CH}_{3} \cdot \mathrm{CH}(\mathrm{OH}) \cdot \mathrm{COOH}$.}

This is the ordinary form of the acid, obtained characteristically as the chief product of the lactic fermentation of sugars.

Lactic acid occurs in the contents of the stomach and intestine, more particularly during a diet rich in carbohydrates, and may be rearlily formed by the digestion of gastric mucous membrane with solutions of dextrose or saecharose. It has been found also in muscles, and according to some observers in the ganglionic cells of the grey substance of the brain.

The most important salts of this acid are those of zinc and calcium.

Zinc lactate. $\mathrm{Zn}\left(\mathrm{C}_{3} \mathrm{H}_{5} \mathrm{O}_{3}\right)_{2}+3 \mathrm{H}_{2} \mathrm{O}$. Soluble in 53 parts of water at $15^{\circ}$; in 6 parts at $100^{\circ}$. Almost insoluble in alcohol.

Culcium lactate. $\quad \mathrm{Ca}\left(\mathrm{C}_{3} \mathrm{H}_{5} \mathrm{O}_{3}\right)_{2}+5 \mathrm{H}_{2} \mathrm{O}$. Soluble in $9 \cdot 5$ parts of cold water; soluble in all proportions in boiling water. Insoluble in cold alcohol.

\section{Sarcolactic acid.}

This form of the acid is isomeric with the preceding one. In its general chemical behaviour as tested by the various decompositions it can undergo it is found to be identical with ethylidene-lactic acid, the sole observable difference being in the different solubility of its calcium and zine salts. But both sareolactic acid and its salts differ strikingly from the preceding acid and its salts as regards their physical properties, for the former exert a distinct rotatory action on polarized light while the latter do not.

This acid has not yet been prepared synthetically and is only known as occurring characteristically in muscles to which it gives their acid reaction, and in blood. In the latter it is found more particularly, as might be expected, after the muscles have been in a state of contracting activity. It is also found in urine, very markedly in case of phosphorus poisoning and in the same excretion after violent muscular exertion, or artificial stimulation of groups of muscles, and very strikingly after extirpation of the liver in birds, and frogs. It is also stated to be formed in variable and slight amount during the lactic fermentation of dextrose. Lactic acid has also been frequently described as a constituent of various pathological fluids; in these cases it is probable that the acid is often the sarcolactic acid. 
As occurring characteristically in muscles it is hence found in large quantities in Liebig's ' extract of meat,' which is the most convenient source for its preparation.

The zinc and calcium salts of sarcolactic acid are much more soluble both in water and alcohol than are those of ethylidenelactic acid.

Zinc sarcolactate. $\mathrm{Zn}\left(\mathrm{C}_{3} \mathrm{H}_{5} \mathrm{O}_{3}\right)_{2}+2 \mathrm{H}_{2} \mathrm{O}$. Soluble in $17 \cdot 5$ parts of water at $15^{\circ}$ or 964 parts of boiling 98 p.c. alcohol.

Calcium sarcolactate. $\quad \mathrm{Ca}\left(\mathrm{C}_{3} \mathrm{H}_{5} \mathrm{O}_{3}\right)_{2}+4 \mathrm{H}_{2} \mathrm{O}\left[? 4_{2}^{1} \mathrm{H}_{2} \mathrm{O}\right]$. Soluble in 12.4 parts of cold water, soluble in all proportions in boiling water or alcohol.

The free acid is dextrorotatory, but the true value of $(a)_{\mathrm{D}}$ is unknown owing to uncertainty as to the purity of the acid. The salts on the other hand are all lavorotatory. For the zine salt, when one part is dissolved in 18 of water $(a)_{\mathrm{D}}=-7 \cdot 6^{\circ}$.

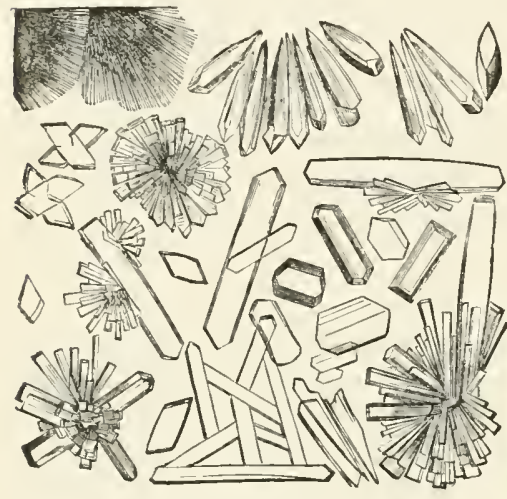

Fig, 190. ZINC SARcolactate. (After Kühne.)

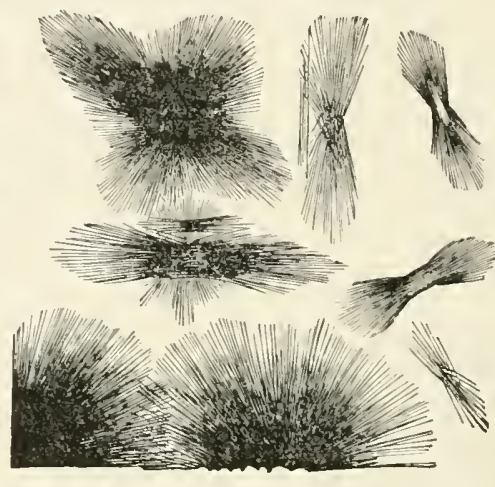

Fig. 197. Calchum siacolactate. (After Kühne.)

Both this acid and the preceding one yield an intense yellow coloration when added to an extremely dilute (almost colourless) solution of ferric chloride. This reaction is sometimes useful.

The lævorotatory form of this acid which should exist according to chemical theory has quite recently been obtained by a bacterial fermentation of cane-sugar. Its salts are dextrolotatory and if equivalent amounts of its zine salt are mixed with the same lievorotatory salts of sarcolactic acid and warmed for some time, on subsequent crystallization the optically inactive salt is obtained of ordinary lactic acid as it arises during the fermentation of sugars.

\section{Ethylene-lactic acid. $\mathrm{CH}_{2}(\mathrm{OH}) \cdot \mathrm{CH}_{2}$. COOH.}

This acid has been usually described as accompanying sarcolactic acid in extracts of muscles and as being isolable 
from this by taking advantage of the varying solubilities of the zine salts of the two acids.

More recent researches have however made it probable that what has usually been described as ethylene-lactic acid, obtainable from muscle-extract, is really acetyl-lactic acid, $\mathrm{CH}_{3}$. CH $\left(\mathrm{C}_{2} \mathrm{H}_{3} \mathrm{O}_{2}\right) \mathrm{COOH}$, the true ethylene-lactic acid being hydracrylic acid, which does not oceur in the animal body.

\section{Hydroxy-butyric acid. $\mathrm{CH}_{3}$. $\mathrm{CH}(\mathrm{OH}) \cdot \mathrm{CH}_{2}$. COOH.}

This acid is the next homologne to the lactic acids in the glyeolic series. It is frequently found in the urine of acute diabetes, usually accompanied by aceto-acetic acid $\left[\mathrm{CH}_{3} . \mathrm{CO}\right.$. $\mathrm{CH}_{2} \cdot \mathrm{COOH}$. The pure acid is sirupy and lævorotatory, $(a)_{\mathrm{D}}=-23 \cdot 4$.

\section{Oxalic Acid Series.}

Oxalic acid. $(\mathrm{CO} . \mathrm{OH})_{2}$.

This acid does not occur in the free state in the human body. Calcium oxalate, however, is a not unfrequent constitu-

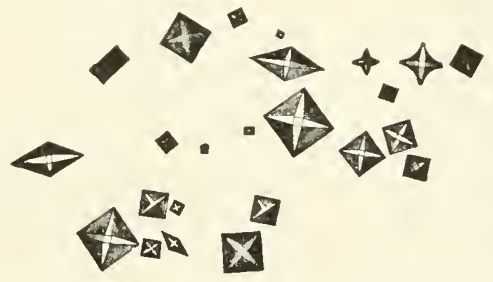

Fig. 198. Calcium Oxalate. (After Funke.)

ent of urine, and enters into the composition of many urinary calculi, the so-called mulberry calculus consisting almost entirely of it, and it is very commonly found in urinary deposits. As ordinarily precipitated from solutions of calcium salts by the addition of a salt of oxalic acid, the caleium oxalate is usually amorphous. To obtain it in the crystalline form dilute solutions of the two reagents must be allowed to mix very slowly, as by diffusion. In urine the case is different; the oxalate is at first in dilute solution, probably dissolved by the sodium dihydrie phosphate $\left(\mathrm{NaH}_{2} \mathrm{PO}_{4}\right)$ to which the acidity is normally due. On standing the urine cools and the oxalate separates ont in a erystalline form, viz. rectangular octohedra, which is eharacteristic and striking, and usmally unlike that of any other constituent of urinary deposits.

In some cases it presents the anomalous forms of rounded lumps, dumb-bells, or square columns with pyramidal ends, but these forms are uncommon. 
The crystals are insoluble in anmonia and acetic acid, bnt readily soluble in hydrochloric or other mineral acid, also slightly so in solutions of acid phosphates and urates of sodium. The above characteristies serve to identify this salt, but in practice the microscopical appearance is usually of most use.

\section{Succinic acid. $\mathrm{COOH} . \mathrm{CH}_{2} \cdot \mathrm{CH}_{2} \cdot \mathrm{COOH}$.}

This is the third acid of the oxalic series, being separated from oxalic acid by the intermediate malonic acid, $\mathrm{CH}_{2}(\mathrm{COOH})_{2}$. It may occur in the spleen, the thymus, and thyroid bodies, hydrocephalic and hydrocele fluids. It has also been stated to occur normally in urine, but this is very doubtful, as also is the statement that it is found in this excretion after taking food rich in asparagin, e.g. asparagus. It is obtained as a product of the putrefaction of proteids.

Succinic acid crystallizes most usually in the form of large four-sided prisms, occasionally as rhombic tables. It is soluble in about 20 parts of cold water, much more so in hot; it is also soluble in alcohol, more especially if hot, and is but very slightly so in ether.

The crystals melt at $180^{\circ} \mathrm{C}$, and boil at $235^{\circ} \mathrm{C}$, , being at the same time decomposed into the anhydride and water. The alkali salts of this acid are soluble in water, insoluble in alcohol and in ether.

Some of the amido-derivatives of succinic acid, viz. asparagin and aspartic acid, are of considerable interest; they will be described later on.

Cholesterin. $\mathrm{C}_{26} \mathrm{H}_{44} \mathrm{O}$ or $\mathrm{C}_{27} \mathrm{H}_{46} \mathrm{O}$.

This substance is described here rather for the sake of convenience than from its possessing any relationship to those which have preceded it.

Cholesterin is the only alcohol which occurs in the human body in the free state. (The triatomic alcohol glycerin is always found combined as in the fats; and cetyl-alcohol is obtained only from spermaceti.) It is a glittering white crystalline substance, soapy to the touch, crystallizing in fine needles from its solution in ether, chloroform or benzene; from its hot alcoholic solutions it is deposited on cooling in rhombic tables; this is the characteristic form and of great importance for the identification of cholesterin.

When dried it melts at $145^{\circ}$, and distils in closed vessels at $360^{\circ}$. It is quite insoluble in water and in cold alcohol, but soluble in solutions of bile salts.

Solutions of cholesterin possess a left-handed rotatory action 
on polarized light $(a)_{1}=-3 \cdot 5$ in ethereal solution, $=-37$ in chloroformic.

Cholesterin occurs in small quantities in the blood and many tissues, and is present in abundance in the white matter of the cerebro-spinal axis and in nerves. It is a constant constituent of bile, and forms frequently nearly the whole mass of some gall-stones. It is found in many jathologieal fluids, hydrocele, the fluid of ovarial cysts, etc.. also in faces and milk. It also occurs in the substance of the crystalline lens, more especially in 'cataract.'

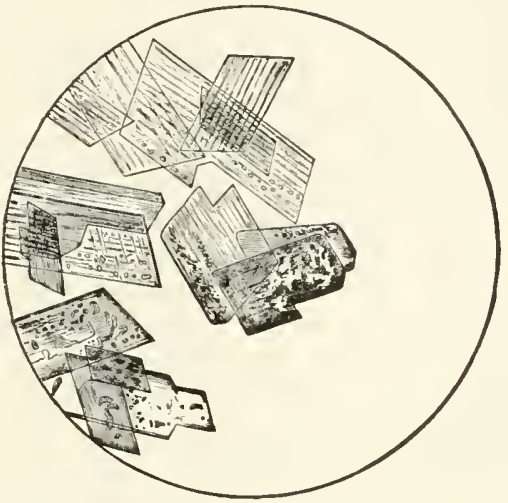

Fig. 199. Cholesterin Crystals. (After Funke.)

Preparation. Gall-stones supply the most convenient source of cholesterin. These are pounded, extracted with boiling water and dissolved in boiling alcohol. The solution is filtered through a heated filter, and the cholesterin separates out in a fairly pure condition as the filtrate cools. It is purified by resolution in boiling alcohol to which some caustic soda has been added, from this it again separates on cooling, and is finally washed with cold alcohol and water.

Cholesterin is characterized, apart from its crystalline form, by some striking reactions which may be obtained even with microscopic quantities.

(i) When the crystals are treated with concentrated sulpluric acid they usually turn violet or red. On the addition of a little indine the play of colours is very marked, the crystals being variously coloured, _ blue, red, green, violet.

(ii) When dissolved in chloroform, the solution turns blood-red on the addition of an equal volume of concentrated sulphuric acid: this turns to blue, green and finally yellow, the change of colour being very rapid if the solution is freely exposed to the air in an open dish. The sulphuric acid under the chloroform exhibits a green fluorescence. 
(iii) When evaporated to dryness on porcelain with a few drops of concentrated nitric acid, a yellow residue is obtained, which turns red if treated, while still hot, with ammonia.

\section{Conplex Nitrogenous Fats and their Derivatives.}

\section{Lecithin. $\mathrm{C}_{44} \mathrm{H}_{90} \mathrm{NPO}_{9}$.}

Occurs widely spread throughout the body. Blood (redcorpuscles), bile, and serous fluids contain it in small quantities, while it is a conspicuous component of the brain, nerves, yolk of eggs, semen, pus, white blood-corpuseles, and the electrical organs of the ray. It occurs also in yeast and other vegetable cells and in small amount in milk.

When pure, it is a colourless, slightly crystalline substance, which can be kneaded, but often crumbles during the process. It is rcadily soluble in cold, exceedingly so in hot alcohol; ether dissolves it freely though in less quantities, as also do chloroform, fats, benzene, carbon disulphide, etc. It is often obtained from its alcoholic solution, by evaporation, in the form of oily drops. It swells up in water, and during the action, as observed under the microscope, extremely curious curling filamentous processes can be seen to protrude from the edge of the solid. 'These are the so-called 'myelin forms.'

Preparation. Usually from the yolk of egg, where it occurs in union with vitellin. Its isolation is too complicated to admit of any brief description.

Lecithin is easily decomposed; not only does this decomposition set in at $70^{\circ} \mathrm{C}$., but the solutions, if merely allowed to stand at the ordinary temperature, acquire an acid reaction, the substance being decomposed. Acids and alkalis, of course, effect this much more rapidly. If heated with baryta-water it is completely decomposed, the products being choline, glycerinphosphoric acid, and barium stearate. This may be thus represented: -

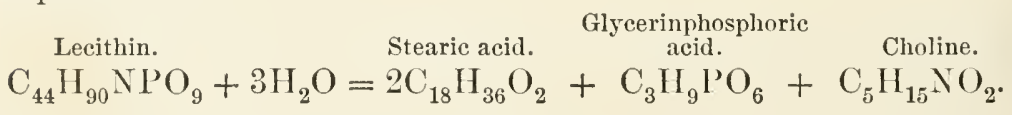

When heated in an ethereal solution with dilute sulphuric acid, it is merely split up into choline and distearyl-glycerinphosphoric acid. Hence it has frequently been regarded as a sort of salt of choline with distearyl-glycerinphosphoric acid. It appears however more probable from the most recent researches that it is really an ethereal compound of this acid with the choline. It appears also that there probably exist other analogons compounds in which the radicles of oleic and palmitic acids take part. 
In accordance with these views the constitution of lecithin may be most adequately represented by the following formula

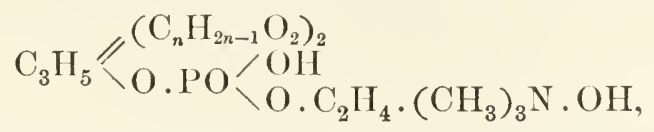

where $\mathrm{C}_{n} \mathrm{H}_{2 n} \mathrm{O}_{2}$ represents the radicle of a fatty acid which in ordinary lecithin appear's to be that of stearie, viz. $\mathrm{C}_{18} \mathrm{H}_{35} \mathrm{O}_{2}$.

\section{Glycerinphosphoric acid.}

$$
\mathrm{C}_{3} \mathrm{H}_{9} \mathrm{PO}_{6} \cdot \quad\left[\mathrm{C}_{3} \mathrm{H}_{5} \cdot(\mathrm{OH})_{2} \cdot \mathrm{O} \cdot \mathrm{PO}(\mathrm{OH})_{2}\right] \text {. }
$$

Occurs as a product of the decomposition of lecithin, and hence is frequently found in those tissues and fluids in which the latter is present. It may occur occasionally in urine.

The acid is dibasic and forms salts which are usually, so far as they are known, soluble in cold water, bnt the lead salt is an exception to this rule and may hence be used as a precipitant. 'The salts are insoluble in alcohol.

Jecorin. This is a substance at first prepared from the liver (whence its name), but subsequently from spleen, blood, muscles, and brain. In its solubilities, etc., it resembles lecithin, and similarly contains phosphorus, but unlike the latter it reduces Fehling's fluid. Very little is yet known as to its composition and nature.

$$
\text { Choline. } \mathrm{C}_{5} \mathrm{H}_{15} \mathrm{TO}_{2} \cdot\left[\left(\mathrm{CH}_{3}\right)_{3} \equiv \mathrm{N} / \mathrm{CH}_{2} \cdot \mathrm{CH}_{2}(\mathrm{OH})\right] \text {, }
$$

trimethyloxyethyl-ammonium hydroxide.

Discovered originally among the products of the decomposition of pigs'-bile and subsequently of ox-bile, whence the name choline. It does not occur in the free state except as a product of the decomposition of lecithin, but has been recently obtained in extracts of the suprarenals.

Choline when pure is an oily liquid with a strong alkaline reaction, soluble in alcohol or ether. It yields crystalline compounds with acids and with some salts, of which the double salts formed with hydrochloric acid and the chlorides of either gold or platinum erystallize readily and are employed for the detection and separation of the base. The platinum salt is readily soluble in water, insoluble in alcohol. The gold salt is but slightly soluble in cold water, but soluble in hot alcohol.

When boiled in concentrated solution choline is decomposed into glycol and trimethylamine

$$
\left(\mathrm{CH}_{3}\right)_{3} \equiv \mathrm{N} / \mathrm{CH}_{2} \cdot \mathrm{CH}_{2}(\mathrm{OH})=\mathrm{C}_{2} \mathrm{H}_{4}(\mathrm{OH})_{2}+\mathrm{N}\left(\mathrm{CH}_{3}\right)_{3} .
$$

By oxidation with concentrated nitric acid it yields the 
extremely poisonous alkaloid muscarine $\mathrm{C}_{5} \mathrm{H}_{15} \mathrm{NO}_{3}$. Choline is itself possessed of poisonous properties, and arising as it does from the decomposition of lecithin and protagon is now recognized as one of the alkaloidal products or ptomaines (see below) which occur in putrefying animal tissues.

vinyl-ammonium hydroxide.

Neurine. $\mathrm{C}_{5} \mathrm{H}_{13} \mathrm{NO} \cdot\left[\left(\mathrm{CH}_{3}\right)_{3} \equiv \mathrm{N} / \mathrm{CH}=\mathrm{CH}_{2}\right]$, trimethyl-

This substance is closely related to choline both in composition and origin, but is much more powerfully toxic than that body. It was first described as a product of the decomposition of protagon by caustic baryta, and until recently the names choline and neurine were applied interchangeably to the basic product of the action of baryta on lecithin or protagon first described under the name choline. Later researches have however shewn that neurine differs distinctly both in composition and properties from the older choline, and have further identified it as one of the most commonly occurring and actively toxic of the alkaloidal basic products of the putrefactive decomposition of animal tissues known under the name of the ptomaines. Like choline it is in the pure state a sirupy fluid, with strongly alkaline reaction and is extremely soluble in water. It forms with hydrochloric acid and platinum chloride characteristic double salts which crystallize readily. The double salt which neurine forms with gold chloride crystallizes in yellow needles; it is but slightly soluble in cold water, though soluble in hot water.

\section{Protagon. $\mathrm{C}_{160} \mathrm{H}_{308} \mathrm{~N}_{5} \mathrm{PO}_{35}$ (?).}

A crystalline substance, containing nitrogen and phosphorus, obtained originally and chiefly from the brain. Protagon separates out from warm alcohol on gradual cooling in the form of very sullall needles, often arranged in groups: it is slightly soluble in cold, more soluble in hot alcohol, and in ether. It is insoluble in water, but swells up and forms a gelatinous mass. It melts at $200^{\circ}$ and forms a brown sirupy Hluid.

Preparation. Finely divided brain substance, freed from bloodvessels and connective tissue, is digested at $45^{\circ} \mathrm{C}$. with alcohol (S.) p.e.) as long as the alcohol extracts anything from it. The united extracts are filtered while hot and the protagon separates out from the filtrate on cooling to $0^{\circ}$. It is next thoroughly extracted with ether to get rid of all cholesterin and other bodies soluble in ether, and finally purified by repeated crystallization from warm alcohol.

By treatment with boiling solution of caustic baryta prota- 
gon is decomposed, yielding the several products which result from the decomposition of lecithin under the same conditions, together with an additional product known as cerebrin.

\section{Cerebrin.}

Is obtained as a product of the decomposition of protagon when brain-substance is boiler with baryta-water and then extracted with hot alcohol. It separates out from this latter solvent when cooled at $0^{\circ}$, and may be purified by recrystallization.

It is a light, colourless, slightly hygrosenpic powder, which swells up in water. When heated to $80^{\circ}$ it turns brown, and -at a higher tempcrature $\left(160^{\circ}\right)$ melts, bubbles up and finally burns away. It is insoluble in cold alcohol or ether ; warm alcohol dissolves it readily. It appears probable that at least three closely allied substances may be present in the product as above described, a fact which points to the possible existence of several forms of protagon. The cerebrins, when boiled with dilute sulphuric acid, yield a sugar which has recently been shewn to be identical with galactose. (See above, p. 1220.)

\section{Charcot's Crystals.}

These remarkable crystals, whose chemical nature and significance have been the subject of much surmise, were first

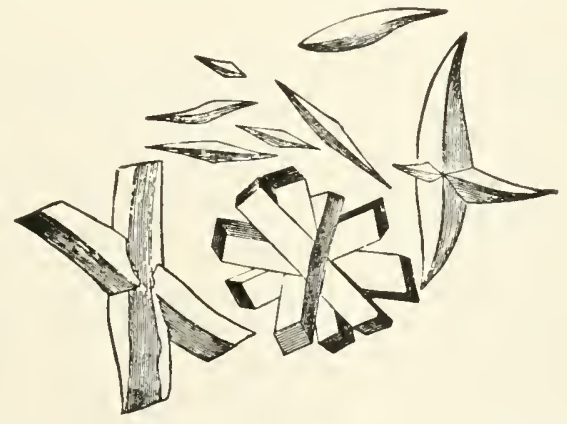

Fig. 200. Charcot's Crystals. (Krukenberg.)

described by Charcot in the spleen and blood of leukhrmic patients. Later researches have confirmed their characteristic appearance in this disease and have further shewn that they occur in health, more particularly in semen, but also in various tissues; they are also found in asthmatic expeetorations. 'They may be readily obtained fiom semen by extracting, with warm water to which a little ammonia has been added, the resilue which remains after semen has been treated with boiling 
alcohol. The crystals separate out from this solution on concentration, and may be purified by recrystallization.

The crystals are insoluble in alcohol, ether and chloroform, slightly soluble in cold and readily so in hot water. Dilute acids and alkalis also dissolve them readily.

It has been stated that the crystals are in reality a compound of phosphorie acid with a nitrogenous base to which the name spermine has been given, and the formula $\mathrm{C}_{2} \mathrm{H}_{5} \mathrm{~N}$ (?) has been assigned. This base is obtained by the ardition to the crrstals of baryta-water, which forms a phosphate of barium and liberates the base. It is soluble in water and alcohol, yielding strongly alkaline solutions; it may be reconverted into Charcot's crystals by the action of phosphoric acid. 'This base was at one time regarded as closely related to, if not identical with ethylinimine, $\mathrm{C}_{2} \mathrm{H}_{4}$. NH. It has however been recently shewn that the two substances are not identical, and it has further been stated that the composition of spermine is most probably represented by the formula $\mathrm{C}_{10} \mathrm{H}_{26} \mathrm{~N}_{4}$.

\section{AMIDES AND AMIDO-ACIDS. THEIR DERIVA- TIVES AND ALLIES.}

Amido-Acids of the Acetic Series.

\section{Amido-formic acid. $\mathrm{NH}_{2}$. $\mathrm{COOH}$.}

This substance is identical with carbamic acid, one of the amido-derivatives of carbonic acid, the first acid of the oxalic acid series. It will be described under the oxalic group.

2. Glycine. $\mathrm{C}_{2} \mathrm{H}_{5} \mathrm{NO}_{2}$. $\left[\mathrm{CH}_{2}\left(\mathrm{NH}_{2}\right)\right.$. COOH]. (Amidoacetic acid.) (Also called Glycocoll and Glycocine.)

Does not occur in the free state in the animal body, but enters into the composition of several important substances, more especially hippuric and glycocholic acids. It is also a product of the action of hydriodic acid on uric acid, and of boiling acids and caustic alkalis on gelatin: hence the name glycocoll or gelatin-sugar, since it possesses a sweet taste. It crystallizes in large, colourless, hard rhombohedra, or foursided prisms, which are easily soluble in water (1 in $4 \cdot 3$ ), insoluble in cold, slightly soluble in hot alcohol, insoluble in ether.

Its solutions possess an acid reaction but a sweet taste. Glycine has also the characteristic property of uniting with both acids and bases to form erystallizable compounds, as also with salts. In this it exhibits its amidic nature, which is fur- 
ther clearly evidenced by the method of its synthetic production by the action of monochloracetic acid on ammonia:-

$$
\mathrm{CH}_{2}(\mathrm{Cl}) \cdot \mathrm{COOH}+2 \mathrm{NH}_{3}=\mathrm{CH}_{2}\left(\mathrm{NH}_{2}\right) \cdot \mathrm{COOH}=\mathrm{NH}_{4} \mathrm{Cl} \text {. }
$$

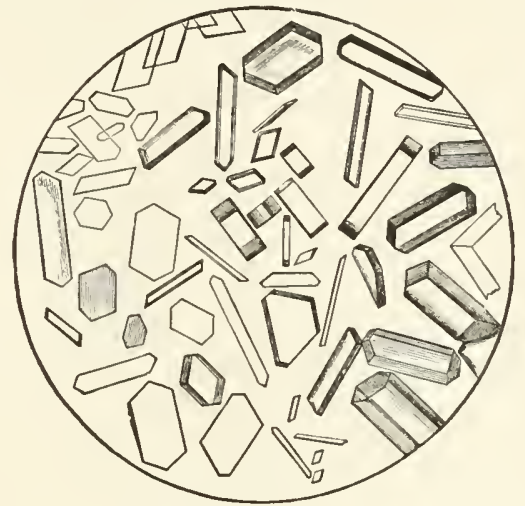

Fig. 201. Glycine Crystals. (After Funke.)

Preparation. Either synthetically as above, or more usually by the decomposition of hippuric acid by prolonged boiling with hydrochloric acid, whereby it is split up into glycine and benzoic acid, the latter being separated by chrystallization and shaking up with ether in which glycine is insoluble.

\section{Sarcosine.}

$\mathrm{C}_{3} \mathrm{H}_{7} \mathrm{NO}_{2} \cdot\left[\mathrm{CH}_{2} \cdot \mathrm{NH}\left(\mathrm{CH}_{3}\right) \cdot \mathrm{COOH}\right]$ (Methylglycine.)

Like glycine in its general chemical properties it further resembles it in that it is never found in the free state as a constituent of the animal body. It is however a substance of considerable interest and importance, not merely on account of its chemical relationship to creatine (see below), but as having been employed in a well-known series of experiments intended to elucidate the probable mode of formation of urea in the body.

\section{Diamido-acetic acid. [CH. $\left.\left(\mathrm{NH}_{2}\right)_{2} \cdot \mathrm{COOH}\right]$.}

This acid has recently been found among the products of the decomposition of easein by boiling with hydrochloric acid and stannous chloride.

\section{Amido-valerianic acid. $\left[\mathrm{C}_{4} \mathrm{H}_{8}\left(\mathrm{NH}_{2}\right)\right.$. $\left.\mathrm{COOH}\right]$.}

Has been described once as obtained from pancreas tissue or the products of its putrefactive decomposition. Is crystalline, soluble in water and somewhat insoluble in alcohol and ether. 


\section{Diamido-valerianic acid. $\left[\mathrm{C}_{4} \mathrm{H}_{7}\left(\mathrm{NH}_{2}\right)_{2} \cdot \mathrm{COOII}\right]$.}

When benzoic acid is administered to fowls it is not converted into hippuric acid as it is in mammals (see p. 1273), but gives rise to an acid called ornithuric. When this is boiled with hydrochloric acid it splits up into benzoic acid and a base called ornithin, which has the composition of diamidovalerianic acid.

7. Leucine. $\mathrm{C}_{6} \mathrm{H}_{13} \mathrm{NO}_{2}$ ( $(\alpha$-Amido-caproic acid). Recent research has shewn that of the various possible isomeric amidocaproic acids the leucine dealt with by physiologists is a-amidoisobutylacetic acid, $\left(\mathrm{CH}_{3}\right)_{2} \mathrm{CH}, \mathrm{CH}_{2}$. $\mathrm{CH}\left(\mathrm{NH}_{2}\right)$. $\mathrm{COOH}$.

It is a characteristic product of the decomposition of proteids and gelatin whether by the action of boiling acids, caustic alkalis or putrefactive influences. It occurs normally in variable amounts in the pancreas, spleen, thymus, thyroid, salivary glands, liver, etc., and also in plants, more especially in those parts in which reserve materials are accumulated, such as bulbs, tubers and seeds. It is also typically formed during the tryptic (pancreatic) digestion of proteids to an extent which amounts on

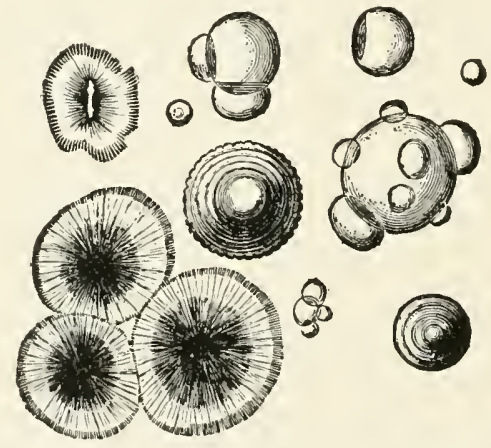

Fig. 202. Leucine Crystals. (Krukenberg.)

the average to some $8-10$ p.c. on the proteid digested, and is in this case always accompanied by tyrosine. It may occur in the urine, more particularly in cases of acute yellow atrophy of the liver; but its presence in this excretion in other and more general diseased conditions of the liver is by no means so constant or certain as it presumably would be on the conmon assumption that a large part of the urea leaving the body is due to its formation from leucine under the converting action of the liver.

As usually obtained in a more or less impure form it crystallizes in rounded fatty-looking lumps which are often collected 
together and sometimes exhibit radiating striation. When pure, it forms very thin, white, glittering flat crystals. It is extremely soluble in hot water, less so but still very soluble in coll water, soluble in alcohol, insoluble in ether. 'The crystals feel oily to the touch, and are without smell and taste. Leucine is particularly solnble in presence of acids and alkalis. The aqueous solutions are lievorotatory, acid and alkaline solntions on the other hand dextrorotatory.

Preparation. (i) From horn-shavings by prolonged boiling with sulphuric acid, 5 of acid to 13 of water. (ii) From the products of the molonged tryptic (pancreatic) digestion of proteids.

For ordinary practical purposes the microscopic appearance of the crystals affords the most convenient means for recognizing leucine, and in this way very minute traces may be determined with certainty. The confirmation of the clue thus afforded by the application of chemical tests is however not easy unless a fair anount of material is at hand, and that in a pure condition. When carefully heated to $170^{\circ}$ leucine sublimes and yields a characteristic odour of anylamine.

8. Lysine. $\mathrm{C}_{6} \mathrm{H}_{14} \mathrm{~N}_{2} \mathrm{O}_{2} \cdot \quad\left[\mathrm{C}_{5} \mathrm{H}_{9}\left(\mathrm{NH}_{2}\right)_{2} \cdot \mathrm{COOH}\right]$ (Diamido-caproie acid.)

This base has been recently discovered among the crystalline products formed when casein is decomposed by being boiled with hydrochloric acid and stannous chloride under exclusion of oxygen. It may be separated from the mother liquor as a donble salt with platinum chloride. It may be separated from this salt as a hydrochloride by the action of sulphuretted hydrogen, and from the hydrochloride it is obtained in the free state by boiling with hydroxide of lead. As thus obtained it is optically active, being dextrorotatory, but by heating to $150^{\circ}$ in presence of baryta it becomes inactive.

Further investigation has shewn that lysine may be similarly formed by the decomposition of egg-albumin, gelatin and keratin. It has also been found among the products of a pancreatic digestion of proteids (fibrin).

\section{Taurine. $\mathrm{C}_{2} \mathrm{H}_{7} \mathrm{NSO}_{3} \cdot \quad\left[\mathrm{CH}_{2}\left(\mathrm{NH}_{2}\right) \cdot \mathrm{CH}_{2}\left(\mathrm{SO}_{2} \cdot \mathrm{OH}\right)\right]$.} (Amido-ethylsulphonic acid.)

Isethionic acid, $\mathrm{CH}_{2}(\mathrm{OH}) \cdot \mathrm{CH}_{2} \cdot \mathrm{SO}_{2}(\mathrm{OH})$, like glycolic acid, $\mathrm{CH}_{2}(\mathrm{OH})$. COOII, contains two hydroxyls replaceable by amidlogen $\mathrm{NH}_{2}$, so that two isomeric amido-derivatives can be formed from it. Of these one is amido-isethionic acid, $\mathrm{CHI}{ }_{2}(\mathrm{OH}) \cdot \mathrm{CH}_{2} \cdot \mathrm{SO}_{2}\left(\mathrm{NH}_{2}\right)$, the other amido-ethylsulphonic acid or taurine. 
Taurine is stated to occur in traces in the juices of muscles and of the lungs, but it is known chiefly as a constituent of taurocholic acid, which is one of the characteristic acids of bile, more especially of the carnivora and above all of the dog.

It crystallizes in colourless, regular, four- or more usually six-sided prisms; these are readily soluble in water, less so in alcohol. The solutions are neutral. It is a very stable compound, resisting temperatures of less than $240^{\circ} \mathrm{C}$; it is not acted on by dilute alkalis and acids, even when boiled with them. It is not precipitated by metallic salts.

Preparation. Ox-bile is boiled for several hours with dilute hydrochloric acid. The fluid residue is separated from the resinous scum, and freed from any remaining traces of bile acids by means of lead acetate, the excess of precipitant being removed by sulphuretted hydrogen, The final filtrate is then concentrated to crystallization, and the taurine finally purified by recrystallization from water. The use of the lead salt may be omitted in many cases and the taurine purified by several crystallizations from water.

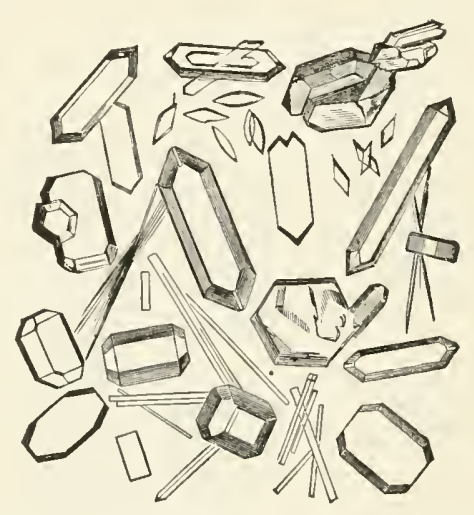

Fig. 203. 'Taurine Crystals. (After Kühne.)

The behaviour of taurine when introduced into the alimentary canal is remarkable. In the case of man the larger part reappears in the urine in combination with carbamic acid as tauro-carbamic acid. In dogs a large part is excreted unaltered together with some tauro-carbamic acid. In herbivora (rabbit), on the other hand, a portion of it is excreted in the urine, but the larger part is oxydized, leading to a large increase of sulphates in the urine together with some hyposulphites. Injected subeutaneously it is largely excreted in an unaltered form.

Tauro-carbamic acid. $\mathrm{NH}_{2} \mathrm{CO} \cdot \mathrm{NH}\left(\mathrm{CH}_{2}\right) \cdot \mathrm{CH}_{2} \cdot\left(\mathrm{SO}_{2} \mathrm{OH}\right)$. It is most easily obtained as a potassium salt by the action of potassium cyanate on tamine. 


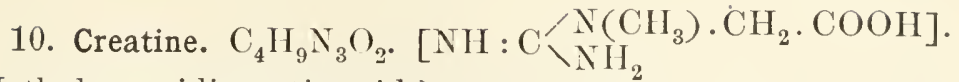
(Methyl-guanidinacetic acid.)

By the union of ammonia with cyanamide a strongly alkaline base, guanidine, is obtained: $\mathrm{CN} . \mathrm{NH}_{2}+\mathrm{NH}_{3}=\mathrm{NH}$. C $\left(\mathrm{NH}_{2}\right)_{2}$ (see below). When sarcosine is employed instead of ammonia a similar reaction takes place, resulting in the formation of creatine:

$$
\mathrm{CN} \cdot \mathrm{NH}_{2}+\mathrm{CH}_{2} \cdot \mathrm{NH}\left(\mathrm{CI}_{3}\right) \cdot \mathrm{COOH}=\mathrm{NII}: \mathrm{C}\left(\mathrm{NH}_{2}\right) \cdot \mathrm{N}\left(\mathrm{CH}_{3}\right) \cdot \mathrm{CII}_{2} \cdot \mathrm{COOH} \text {. }
$$

Since sarcosine is methyl-amidoacetic acid it is at once obvious that creatine may be regarded as being methyl-guanidinacetic acid. When cyanamide is treated with boiling baryta-water it takes up a molecule of water and yields urea, $\mathrm{CN} .\left(\mathrm{NH}_{2}\right)+$ $\mathrm{H}_{2} \mathrm{O}=\mathrm{CO}\left(\mathrm{NH}_{2}\right)_{2}$, hence, as might be expected, creatine yields by similar treatment sarcosine and urea. This is to the physiologist the most important chemical property of ereatine, bearing as it does so closely upon one possible source and mode of formation of urea in the body.

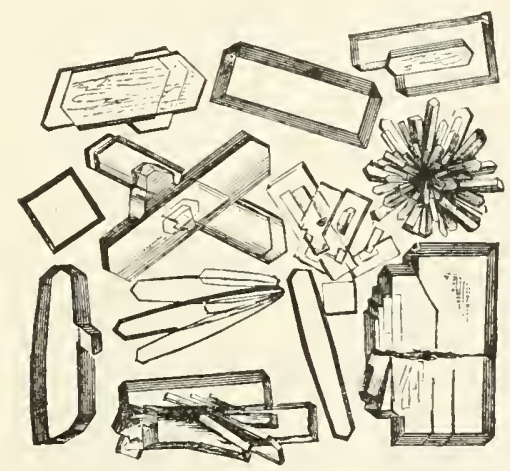

Fig. 204. Creatine Crystals. (Krukenberg after Kühne.)

Creatine occurs as a constant and characteristic c mstituent of muscles and their extracts to an amount which is variable but may be taken as from $0.2-0.3$ p.c. on the weight of the muscle. It is also found in nervous tissue and is said to occur in traces in several fluids of the borly. It mist however be carefully borne in mind that creatine very rearlily loses a molecule of water and thus becomes creatinine, an 1 that the latter with equal readiness takes up a molecule of water to form creatine. Hence the creatine obtained during any analysis need not at all necessarily imply its presence as such in the original tissue or fluid unless due allowanc ${ }^{2}$ has been made for the possible effect of the methods employed upon the reciprocal 
conversions of creatine and creatinine. This is the cause of the conflicting statements as to the occurrence of creatine in urine; as a matter of fact this excretion always contains creatinine. It is on the whole most probable that any creatine which may be found in urine is due to the conversion of creatinine into creatine during its extraction, since it has been shewn that the more rapidly the separation is effected, the less is the quantity of creatine obtained, and the greater the amount of creatinine.

In the anhydrous form creatine is white and opaque, but crystallizes with one molecule of water in colourless transparent rhombic prisms.

'The crystals are soluble in 75 parts of cold water, extremely soluble in hot; slightly soluble in absolute alcohol, they are more soluble in dilute spirit and are insoluble in ether. The aqueous solutions are neutral in reaction.

Creatine is a very weak base, scarcely neutralizing the weakest acids, with which it forms soluble crystalline compounds.

Preparation. Most conveniently from 'Liebig's Extract.' This is dissolved in 20 parts of water and precipitated by a slight excess of basic acetate of lead. The filtrate is then freed from the lead salt by means of sulphuretted liydrogen and concentrated at moderate temperature (avoid boiling) to a thin syrup. On standing in a cool place for two or three days the creatine crystallizes out. The crystals are removed by filtration, washed with $S S$ p.c. alcohol and purified by recrystallization from water.

Creatine yields no very striking reactions by means of which it can realily be identified. It reduces Fehling's fluid by prolonged boiling without any separation of cuprous oxide. On boiling in presence of alkaline mercuric oxide, a transient red colour is obtained and finally a separation of metallic mereury. Tile reactions of ereatinine on the other hand are striking (see bel(w), and lience creatine may be identified with most certainty by conversion into creatinine, and the determination of the presence of the latter substance. The conversion is readily effected by boiling with dilute mineral aeids, during which process creatine loses one molecule of water : $\mathrm{C}_{4} \mathrm{H}_{9} \mathrm{~N}_{3} \mathrm{O}_{2}=\mathrm{C}_{4} \mathrm{H}_{7} \mathrm{~N}_{3} \mathrm{O}$ $+\mathrm{H}_{2}(\mathrm{O}$.

Mention has already been made of the possible and very probable genetic relationship of urea to muscle-creatine (see \$382).

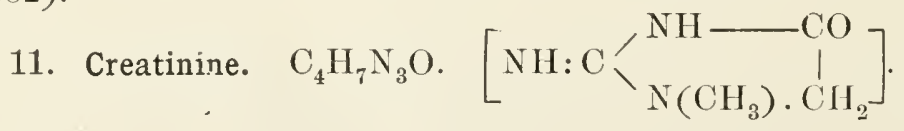

Creatinine as already stated is simply a dehydrated form of creatine. It occurs normally as a constant constituent of urine, varying however in amount from $0 \cdot 5$ to $4 \cdot 9$ grin. per diem according to the amount of proteid food (meat) eaten. It is 
not a normal constituent of mammalian muscle but is found in the muscles of some fishes, and has been obtained from sweat. It crystallizes in colourless prisms or tables according to the conditions under which the separation takes place and the mode of preparation, and frequently, owing to imperfect development, the crystals assume a very cliaracteristic 'whetstone' form.

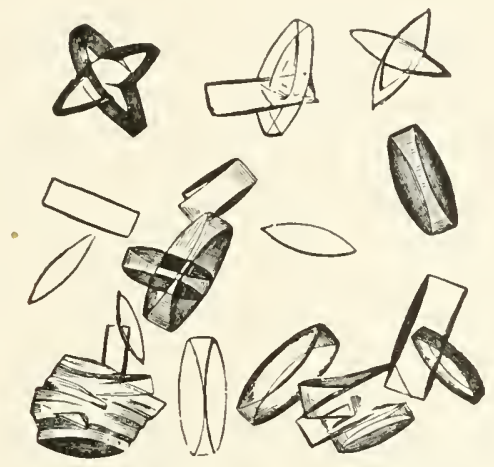

Fig. 205. Creatinine Crystals. (Kurukenberg after Kühne.)

Creatinine is readily soluble in cold water ( 1 in 11.5$)$ also in alcohol, but is scarcely soluble in ether. 'The aqueous solutions are usually alkaline, but some observers regard the alkalinity as due to impurities. It acts as a powerful base, forming

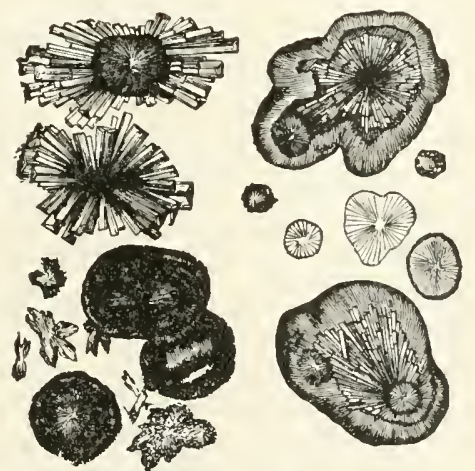

Fig. 206. Creatinine-Zinc-Cillonide Cristals. (Krukenberg after Kühne.)

compounds with acids and salts which erystallize well. Of these the most important is the salt with chloride of zine $\left(\mathrm{C}_{4} \mathrm{H}_{7} \mathrm{~N}_{3} \mathrm{O}\right)_{2}{ }^{\prime} / \mathrm{nCl}_{2}$, botll on account of its characteristic erystalline form and of its general insolubility in comparison with the other compounds of this substance. Hence its formation is employed not merely for the determination of ereatinine but for 
its separation from solutions. It crystallizes in warty lumps composed of aggregated masses of prisms, or fine needles.

This compound is formed when a concentrated neutral solution of the zine salt is added to a not too dilute solution of creatinine, and since it is almost insoluble in alcohol it is frequently convenient to employ alcoholic rather than aqueous solutions of the two substances.

Preparation. This does not admit of any useful brief description.

Apart from the characteristic formation of the compomd with zine chloride, creatinine yields several well-marked reactions, of which the following are the more striking.

1. Weyl's reaction. To the suspected solution a few drops of very dilute sodium nitro-prusside $\left[\mathrm{Na}_{2}(\mathrm{NO}) \mathrm{FeCy}_{5}\right]$ are added, and then, drop by drop, some dilute caustic soda. If creatinine is present a fine but transient ruby-red colour is obtained which speedily passes into yellow. If the solution is now acidulated with acetic acid and boiled it turns at first greenish and finally blue. This last colour is due to the formation of Prussian-blue. Weyl's reaction is extremely delicate and suffices to detect .0287 p.c. of creatinine in pure solution or .066 p.c. in urine.

When applied to urine the absence of acetone should be ascertained, since it also gives a similar ruby-red colour, but no subsequent blue can be obtained from it, and the solution when yellow turns red again on the addition of strong acetic acid. Hydantoin or methyl-hydantoin also yields the red coloration.

2. Jaffe's reaction. On the addition of an aqueous solution of picric acid and a few drops of dilute caustic soda, an intense red coloration is produced. This suffices to detect $\cdot 1$ part of creatinine in 5000 of water. Acetone alone gives a similar coloration but to a comparatively very feeble extent.

By prolonged boiling of creatinine with Fehling's fluid, reduction takes place, but there is no simultaneous separation of cuprous oxide, and it appears that creatinine may prevent the separation of the oxide when the reduction is due not to itself but to such a substance as dextrose.

\section{Lysatine. $\mathrm{C}_{6} \mathrm{H}_{13} \mathrm{~N}_{3} \mathrm{O}_{2}$.}

This most interesting substance has been recently obtained during the decomposition of casein by boiling with hydrochloric acid and stannous chloride and was first separated from the mother liquor left after the preparation of lysine (see p. 1245). The methods of obtaining lysatine admit of no suitably concise description. It is a white crystalline solid, soluble in water and crystallizable from its aqueous solution by the addition of some alcohol and ether. It forms a well-characterized double salt with 
nitrate of silver, $\mathrm{C}_{6} \mathrm{H}_{13} \mathrm{~N}_{3} \mathrm{O}_{2}$. $\left.\mathrm{HNO}_{3}+\lg _{\mathrm{NO}}\right)_{3}$. From this its empirical formula is seen to be that of a homologue of creatine, but there is some doubt as to whether one molecule of water should not be subtracted from the above, in which case it would be homologous with creatinine $\left(\mathrm{C}_{4} \mathrm{H}_{7} \mathrm{~N}_{3} \mathrm{O}_{2}\right)$ and receive the name lysatinine $\left(\mathrm{C}_{6} \mathrm{H}_{11} \mathrm{~N}_{3} \mathrm{O}_{2}\right)$.

Lysatine may also be found among the products, similarly obtained, of the decomposition of gelatin, keratin and elastin, in the latter case unaccompanied by lysine, and in considerable amount from the products of a pancreatic digestion of fibrin. Its production in the latter case is important as a preliminary to the most interesting fact regarding lysatine which must now be stated. It has already been said (p. 12+7) that when creatine is boiled in baryta-water it is split 1 p into sarcosine and urea. Now it is found that when lysatine is similarly treated it also yields urea as a product of its decomposition, so that in this way it has become possible for the first time to obtain urea from proteids by a decomposition not involving oxidation, and thus an endeavour often made in the past has at last been realized. Apart from the more purely theoretical interest of this result it is at once seen that we have here a possible source of some of the urea which leaves the body.

A crystalline product of the decomposition of at first keratin and subsequently gelatin, egg-albumin and casein has recently been described under the name of arginine $\left(\mathrm{C}_{6} \mathrm{H}_{14} \mathrm{~N}_{4} \mathrm{O}_{2}\right)$.

\section{Amido-acios of the Lactic Series.}

\section{Cystine. $\left(\mathrm{C}_{3} \mathrm{H}_{6} \mathrm{NSO}_{2}\right)_{2}$. \\ $\left[\mathrm{S} \cdot \mathrm{C}\left(\mathrm{CH}_{3}\right)\left(\mathrm{NH}_{2}\right) \cdot \mathrm{COOH}\right]_{2}$.} (Amido-sulpholactic acid.)

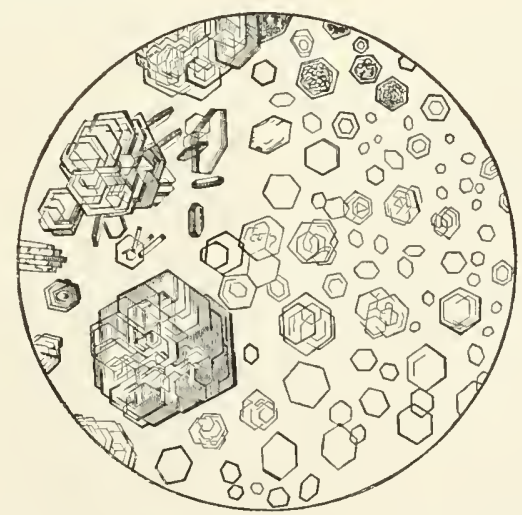

Fig. 207. Crstine Crystals. (After Funke.)

Is the chief constituent of a rarely occurring urinary calculus in men and dogs. It may also occur in renal concretions, 
and in gravel, and is occasionally found in urine, from which it separates out as a greyish serliment on standing. It is prepared from this sediment, or better still from cystic calculi, by solution in ammonia. This solution is then allowed to evaporate spontaneously and yields the cystine in regular, colourless, six-sider tables of very characteristic appearance. Cystine may be separated from urine by taking advantage of the formation of a sodium salt of henzoyl-cystine when it is shaken up with a few drops of benzoyl chloride.

Cystine is insoluble in either water, alcohol or ether, readily soluble in ammonia, differing in this respect from uric acid, also in many alkaline carbonates and in mineral acids. Its solutions are strongly lievorotatory.

Cystine is one of the few crystalline substances, occurring physiologically, which contain sulphur, hence its detection is easy.

\section{Amino-acids of the Oxalic Series.}

\section{Carbamic acid. $\mathrm{CO} / \mathrm{NH}_{2}$.}

Carbanic acid is a substance of peculiar interest to the physiologist on account of the important part it is frequently supposed to play in the formation of urea in the animal body.

Carbamic acid is unknown in the free state; its best known salt is that with ammonium, but many others have been prepared. It further appears that some of its salts occur in serum and its caleium salt in the urine of the horse, and it is also stated to be formed during the oxidation of glycine, leucine, and tyrosine by means of potassium permanganate in alkaline solution. Ammonium carbamate is extremely soluble in water, in which solution it is gradually converted into the carbonate. At ordinary pressures when heated to $60^{\circ}$ it is decomposed into ammonia and carbon clioxide, but under pressure at $130^{\circ}-140^{\circ}$ it yields urea. When electrolyzed in cold aqueous solution by a rapidly and continuously commutated current the salt similarly loses water and yields urea.

\section{Aspartic (or asparaginic) acid. $\mathrm{C}_{4} \mathrm{H}_{7} \mathrm{NO}_{4}$. [COOHI. $\mathrm{CH}_{2}$. CH $\left.\left(\mathrm{NH}_{2}\right) \cdot \mathrm{COOH}\right]$. (Amido-succinic acid.)}

This acid is chiefly obtained from plant extracts, and occurs notably in beet-sugar molasses. It may be synthetized, but is most conveniently prepared by boiling asparagine with caustic alkalis or mineral acids. It is also a typical product of the action of boiling mineral acids and caustic baryta on both vegetable and animal proteids and of acirls on gelatin, being usually aceompanied by its homologue, glutanic acid. It is also now recognized as a product in minute quantities of the 
pancreatic digestion of fibrin and regetable gluten, although it does not oceur as a constituent of any animal tissue or secretion. It crystallizes in rhombie prisms which are but sparingly soluble in eold water or alcohol, but readily soluble in boiling water. Its solutions, if strongly acid, are dextrorotatory, but if alkaline, lævorotatory. It forms a characteristic readily crystallizable compoumd with oxide of copper, which is practically insoluble in cold, but soluble in boiling water, and may be used for the separation of aspartic acid from solutions in which it is mixed with other substances.

3. Glutamic (or glutaminic) acid. $\mathrm{C}_{5} \mathrm{H}_{9} \mathrm{NO}_{4}$. (Amidopyrotartaric acid.)

This acid is homologous with aspartic acid. The eireumstances and conditions under which it occurs are in general the same as for aspartic acicl, but it has not as yet been ohtained by the action of pancreatic enzymes on proteids and is never found in any animal tissues or"secretions. But as a product, often to a large amount, of the artificial decomposition of proteids it acquires some considerable importance. It is always prepared by treating proteids with boiling mineral acids.

It crystallizes in rhombic tetrahedra or octahedra; is not very soluble in cold, but readily soluble in hot water ; insoluble in alcohol and in ether. Its aqueous and acid solutions possess a strong dextrorotatory power.

4. Asparagine. $\mathrm{C}_{4} \mathrm{H}_{8} \mathrm{~N}_{2} \mathrm{O}_{3}+\mathrm{H}_{2} \mathrm{O} . \quad\left[\mathrm{COOH} \cdot \mathrm{CH}_{2} \cdot \mathrm{CH}\right.$ $\left.\left(\mathrm{NH}_{2}\right) \cdot \mathrm{CONH}_{2}\right]$. (Amido-succinamie acid.)

Although asparagine is not found as a constituent of the animal body it is a substance of considerable interest to the physiologist. Not only is it closely related to aspartic acid, into which it may be converted by the action of boiling acids and alkalis, yielding at the same time ammonia, but it undoubtedly plays a most important part in the constructive proteid metabolism of plants. Further, it exists in not inconsiderable amount in many plant-tissues used as food by man, and is known, like so many of the members of the numerous class of amido-acids to which it belongs (leucine, glycine, ete.), to give rise to urea when taken into the body of carnivora, and to uric acid in that of birds.

Asparagine erystallizes readily in large rhombic prisms which are not very soluble in cold, but readily soluble in hot water, and are insoluble in absolute alcohol and in ether. Its solutions are dextrorotatory. It may be prepared synthetically, but is usually obtained by crystallization from the expressed juice or extracts of the seedlings of peas, beans or lupins. 
Mercuric nitrate yields a precipitate with asparagine which may be used for its separation from vegetable extracts. Ureaferment converts it into succinic acid.

\section{THE UREA AND URIC ACID GROUP.}

\section{Urea. $\left(\mathrm{NH}_{2}\right)_{2} \mathrm{CO}$. (Carbamile.)}

This is the chief nitrogenous constituent of normal urine in mammalia and some other animals. 'The urine of birds also contains a small amount, more particularly on a meat diet. Average normal human urine contains from $2 \cdot 5-3 \cdot 2$ p.c., the average total daily excretion varying from 22-35 grams or as a mean 30 grams. It is also found in minute quantities in normal blood ( 025 p.c.), serous fluids, lymph and aqueous humour : it is not usually met with in the tissues excent that of the liver. It is never present in normal mammaiian muscles, but may make its appearance there under certain pathological

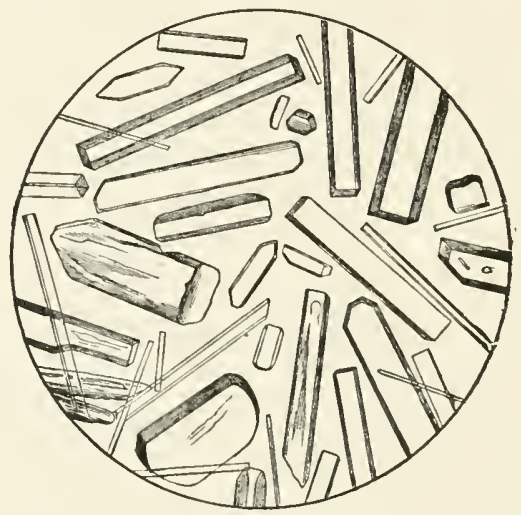

Fig. 208. Urea Crystals separatev iy slow eyaporation from aqueous solution. (After Funke.)

conditions. Under ordinary conditions the amount of urea in sweat is almost inappreciable, but the older statements of its occurrence in this excretion have recently received confirmation, and it appears that this source of nitrogenous loss to the body may liave to be taken into account.

When pure it crystallizes from a concentrated solution in the form of long, thin glittering needles. If deposited slowly from dilute solutions, the form is that of four-sided prisms with pyramidal ends; these are always anhydrous. When the separation occurs rapidly, as for instance from a strong alcoholic solution on a glass-slide, the typical crystalline form is 
not readily observed, but rather that of irregular dendritic crystals.

Urea is very soluble in cold water, distinctly less soluble in cold alcohol, readily so in hot; it is insolnble in anhydrous ether and in petroleum-ether. It possesses a somewhat bitter, cooling taste, resembling saltpetre.

Urea readily forms compounds with acids and bases; of these the following are important as a means of detection and identification.

\section{Nitrate of urea. $\left(\mathrm{NII}_{2}\right)_{2} \mathrm{CO} \cdot \mathrm{HNO}_{3}$.}

Obtained by the addition of a slight excess of pure colourless nitric acid to a moderately concentrated solution of urea. 'The nitrate should separate out rapidly in the form of six-sided or rhombic tables, frequently aggregated in piles, but the successful obtaining of typical crystals requires some attention to the concentration of the solution.

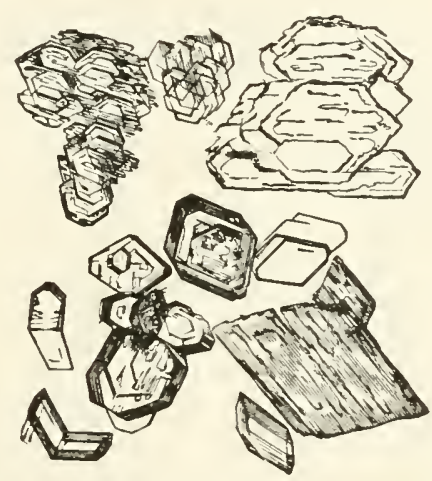

Fig. 209. Cristals of Nitrate of Urea. (Krukenberg after Küüne.)

The crystals are but slightly soluble in nitric acid, or alcohol, more soluble in cold water and much more so in hot water. They are insoluble in ether.

\section{0xalate of urea. $\left[\left(\mathrm{NH}_{2}\right)_{2} \mathrm{CO}\right]_{2} \cdot \mathrm{H}_{2} \mathrm{C}_{2} \mathrm{O}_{4}+\mathrm{H}_{2} \mathrm{O}$.}

Obtained by the addition of concentrated aqueous solution of oxalic acid to a concentrated aqueous solution of urea. This salt crystallizes out in rhombic tables closely resembling those of the nitrate, but they are frequently aggregated into a characteristic prismatic form. As in the case of the nitratc some care is required with respect to the concentration of the respective solutions during its preparation.

The crystals are less soluble in oxalic acid than in water, 
but may in other respects be taken as resembling those of the nit rate in respect of their solubilities.

Of the many salts which urea forms with other bases and salts, those which it yields with mereuric oxide and nitric acid are of most importance. W'hen a solution of mereuric nitrate is added to one of urea a precipitate is formed which, dependently upon the conecntration and relative amounts of the two solutions, may contain some one of three possible salts, consisting of $\left[\left(\mathrm{NH}_{2}\right)_{2} \mathrm{CO}\right]_{2} \cdot \mathrm{Hg}\left(\mathrm{NO}_{3}\right)_{2}$ united with 1,2 or 3 molecules of mercuric oxide $(\mathrm{HgO})$. When the solutions are fairly neutral and dilute, the salt with 3 molecules of $\mathrm{HgO}$ is formed $\left[\left(\mathrm{NH}_{2}\right)_{2} \mathrm{CO}\right]_{2} \cdot \mathrm{Hg}\left(\mathrm{NO}_{3}\right)_{2} \cdot 3 \mathrm{HgO}$. This is the salt formed in the reactions on which Liebig's volumetric method for the determination of urea is based.

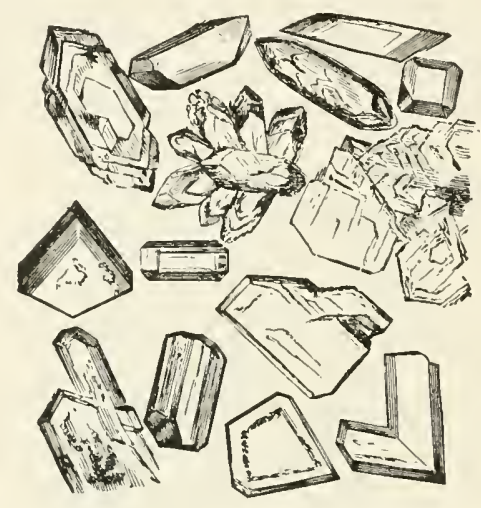

Fig. 210. Crystals of Oxalate of Urea. (Krukenberg after Kühne.)

Urea may be heated dry in a tube to $120^{\circ}$ without being decomposed, on further raising the temperature it melts at $13 \cdot 26^{\circ}$ and afterwards gives off ammonia, and if heated to $150^{\circ}$ for some time is converted largely into biuret: $2\left(\mathrm{NH}_{2}\right)_{2} \mathrm{CO}$ $=\mathrm{NI}_{2} \cdot \mathrm{CO} \cdot \mathrm{NH} \cdot \mathrm{CO}\left(\mathrm{NH}_{2}\right)+\mathrm{NH}_{3}$. On further heating to a higher temperature $\left(200^{\circ}\right)$ it is largely converted into cyanuric acid. When biuret is dissolved in water it yields on the addition of caustic soda and dilute sulphate of copper the wellknown pink colour employed for the detection of peptones, and hence called the 'biuret reaction.'

When treated with nitrous acid, e.g. impure yellow nitric acid, it is decomposed finally into carbon dioxide, nitrogen and water: $\left(\mathrm{NH}_{2}\right)_{2} \mathrm{CO}+2 \mathrm{HNO}_{2}=\mathrm{CO}_{2}+2 \mathrm{~N}_{2}+3 \mathrm{H}_{2} \mathrm{O}$. A similar decomposition is obtained by the action of sodium hypochlorite or hypobromite: $\left(\mathrm{NH}_{2}\right)_{2} \mathrm{CO}+3 \mathrm{NaBrO}=3 \mathrm{NaBr}^{2}+\mathrm{CO}_{2}+$ $\mathrm{N}_{2}+2 \mathrm{H}_{2} \mathrm{O}$. Since the volnme of nitrogen evolved is constant 
for a given weight of urea, this latter reaction forms the basis of a method for the quantitative determination of urea.

When a crystal of urea is treated with a drop of concentrated freshly prepared aqueous solution of furfuraldehyde $-\mathrm{C}_{5} \mathrm{H}_{4} \mathrm{O}_{2}$ (aldehyde of pyromucic acid) and then immediately with a drop of hydrochloric acid (sp. gr. =1.10) a play of colours is observed which passes rapidly from yellow through green, blue and violet to a final brilliant purple. The test may be also applied by the addition of three drops of the acid to a mixture of one drop of 1 p.e. aqueous urea solution and 5 c.c. of aqueous furfuraldehyde solution.

Detection in solutions. In addition to the microscopic appearance of the crystals obtained on evaporation, the nitrate and oxalate should be formed and examined. Another part should give a precipitate with mercuric nitrate, in the absence of sodium chloride, but not in the presence of this last salt if in excess; in presence of sodium chloride the mercuric nitrate reacts first with the sodium salt in preference to the urea. A third portion is treated with nitric acid containing nitrous fumes; if urea is present, nitrogen and carbon dioxide will be obtained. To a fourth part pure nitric acid in excess and a little mercury are added, and the mixture is warmed. In presence of urea a colourless mixture of gases $\left(\mathrm{N}\right.$ and $\left.\mathrm{CO}_{2}\right)$ is given off. A fifth portion is treated, after evaporation to dryness, in the way above described for the application of the biuret reaction.

Quantitative determination. For this some special manual should be consulted.

The determination of the total nitrogen in urine is also of great importance and is now usually carried out by converting all the nitrogen of a measured portion of urine into ammonia by boiling with fuming sulphuric acid and the subsequent addition of potassium permanganate. The ammonia is then expelled from the acid solution by distillation with an excess of caustic soda or potash, the ammonia being received into a measured volume of standardized acid, whose diminution of acidity due to the absorption of ammonia is finally determined by titration with standard alkali.

Substituted ureas. The hydrogen atoms of urea can be replaced by alcohol- and acid-radicles. The results are substituted ureas in the first case, or ureides as they are called in the second, when the hydrogen is replaced by the radicle of an acid. Many of them are called acids, since the hydrogen from the amido group, if not all replaced as above, can be replaced by a metal. Thus the substitu- 
tion of oxalyl (oxalic acid) gives parabanic acid, $\mathrm{CO} / \mathrm{NH} . \mathrm{CO}$; of tartronyl (tartronic acid), dialuric acid, $\mathrm{CO} / \mathrm{NH} . \mathrm{CO} \backslash \mathrm{CHOH}$; of mesoxalyl (mesoxalic acid), alloxan $\mathrm{CO} /{ }_{\mathrm{NH} . \mathrm{CO}}^{\mathrm{NH} . \mathrm{CO}} \backslash \mathrm{CO}$. These substances are interesting as being also obtained by the artificial oxidation of uric acid.

2. Uric acid. $\mathrm{C}_{5} \mathrm{H}_{4} \mathrm{~N}_{4} \mathrm{O}_{3}$.<smiles>O=C1NOC2NC(=O)NC2N1</smiles>

The chief constituent of the urine in birds and reptiles; it occurs only sparingly in this excretion in man $(\cdot 2-1$ grm. in 24 hours) and most mammalia. It is normally present in the spleen, and traces of it have been found in the lungs, muscles of the heart, pancreas, brain and liver. Urinary and renal calculi often consist largely of this substance, or its salts. In gout, accumulations of uric acid salts may occur in various parts of the body, more especially at the joints, forming the so-called gouty concretions.

It is when pure a colourless, crystalline powder, tasteless, and without odlour. The crystalline form is very variable,

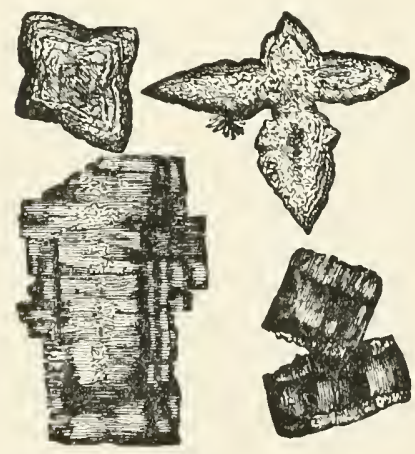

liapidly separated.

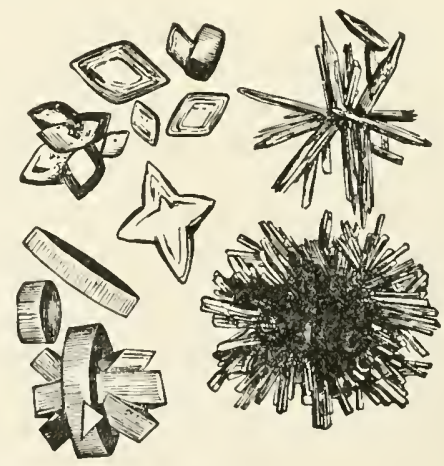

Slowly separated.

Fig. 211. Cristals of Uiric Acm. (Krukenberg after Külne.)

differing according to the concentration of the solution from which the crystals are obtained, the rate at which they are formed, and whether they are separated out spontaneously or 
by the addition of acids to either solutions of the acid or to urine. Hence it is extremely difficult to illustrate them within reasonable limits, and for figures of the various possible forms some special work must be consulted. The impure acid crystallizes much more readily than does the purified. The following figure shews additionally some very characteristic forms in which uric acid separates out from urine either spontaneously or after the addition of hydrochloric acid.

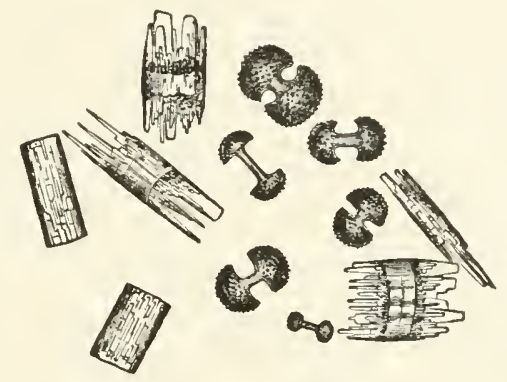

Fig. 212. Cristals of Uric Acid. (After Funke.)

Uric acid is remarkably insoluble in water ( 1 in 14,000 or 15,000 of cold water, 1600 of boiling). Ether and alcohol do not dissolve it appreciably. On the other hand, sulphuric acid

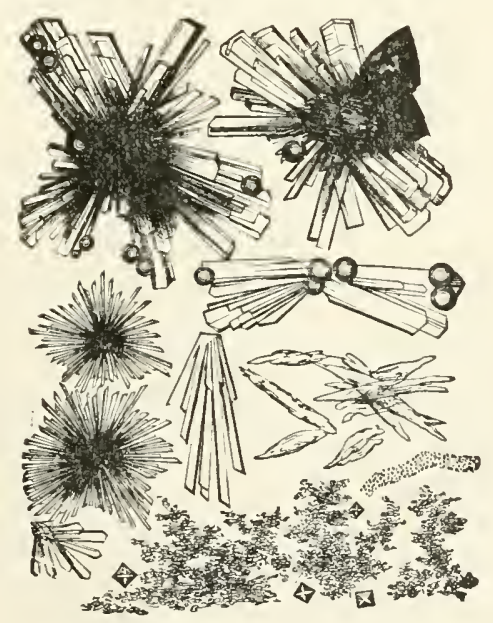

FIG. 213. (Krukenberg after Kühne.)

Urinary sediment, showing chiefly the most usual form of crystals of acid sodium urate, $\mathrm{C}_{5} \mathrm{H}_{3} \mathrm{NaN}_{4} \mathrm{O}_{3}$.

takes it up in the cold without decomposition, and it is also readily soluble in many salts of the alkalis, as in the caustic 
alkalis themselves; ammonia however scarcely dissolves it, and in this respect it differs conveniently from cystine. It is fairly soluble in glycerin, and soluble to some extent in solutions of lithium earbonate.

Salts of uric acid. Of these the most important are the acid urates of sodium, potassium and ammonium; these salts are frequently still called 'lithates,' the term 'lithic' acid being used for urie acid. 'The sodium salt which is the most common constituent of many urinary sediments crystallizes in many different forms, these not being characteristic, since they are almost the same for the corresponding compounds of the other two bases. It is very sparingly soluble in cold water ( 1 in 1100 or 1200), more soluble in lot (1 in 125). It is the prineipal constituent of several forms of urinary sediment, and composes a large part of many calculi; the excrement of snakes contains it largely. The potassium resembles the sodium salt very closely, as also does the compound with ammonium; the latter occurs generally in the sediment from alkaline urine.

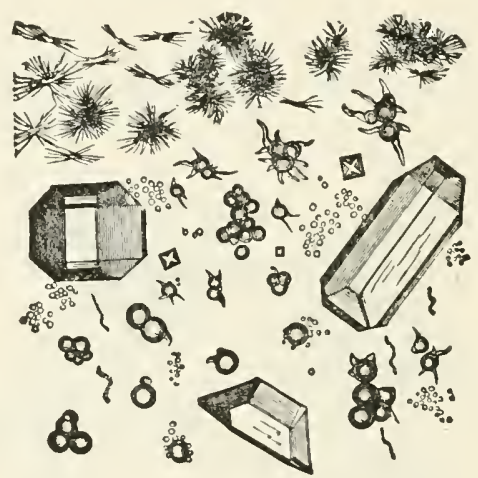

Fig. 214. (Krukenberğ after Küühne.)

Urinary sediment from alkaline urine. The large crystals consist of ammonio-magnesium phosphate (triple phosphate, $\mathrm{NH}_{4} \mathrm{Mgl} \mathrm{O}_{4}+6 \mathrm{H}_{2} \mathrm{O}$ ). A few crystals (octahedra) of calcium oxalate are also slıwn. The remaining crystals represent the form of acid ammonium urate, $\mathrm{C}_{5} \mathrm{H}_{3}\left(\mathrm{NH}_{4}\right) \mathrm{N}_{4} \mathrm{O}_{3}$. The rounded objects are urinary fungi.

The amount of uric acid in mammalian urine is too small to make it a source of the acid. Crystals may however be rearlily obtained from human mine by adding to it $2-3$ p.c. of strong hydrochloric acid and letting it stand for one or two days in a cool place. The crystals form on the sides of the containing vessel.

On the large seale it is usually prepared from guano, or from snake's or fowl's excrement. 
Identification of uric acid. The crystalline forms afford some clue, but are so numerous that some forms which may at any time present themselves are scarcely characteristic. 'The rhombie tables, 'dumb-bell' and 'whetstone' crystals, are on the whole most characteristic.

i. Murexide test. 'The suspected substance is treated in a porcelain dish with a few drops of strong nitric acid and evaporated carefully to dryness, by preference on a water-bath. The residue thus obtained will, if uric acid is present, be of a yellow or more frequently red colour, which turms to a brilliant reddish purple on exposure to the vapours of ammonia. On the subsequent addition of a drop of caustic soda the colour is changed to a reddish blue. 'This disappear's on warming, whereas the similar colour obtained by the above process from guanine does not. This is an important means of distinguishing betwen the two substances.

The test depends on the formation of murexide, which is the acid ammonium salt of purpuric acid, the acid itself being unknown in the free state. Uric acid is decomposed when heated with nitric acid, yielding alloxan and then alloxantin; by the action of ammonia the latter is converted into murexide, $\left(\mathrm{NH}_{4}\right) \mathrm{C}_{8} \mathrm{H}_{4} \mathrm{~N}_{5} \mathrm{O}_{6}+\mathrm{H}_{2} \mathrm{O}$.

The murexide test is so striking and characteristic that it suffices completely for the identification of uric acid. The following tests may be applied in confirmation if required, but not for the purposes of initial detection.

ii. Schiff's reaction. The substance is dissolved in sodium carbonate, and a drop is then placed on filter paper previously moistened with nitrate oi silver. A yellow or almost black coloration, due to the formation of metallic silver by reduction of its nitrate, is at once obtained.

iii. When a solution of mic acid in caustic soda is boiled with a small amount of Fehling's fluid, reduction oceurs with production of a greyish precipitate of urate of cuprous oxide. If the copper salt is in excess red cuprous oxide is obtained.

Estimation of uric acid in solutions (urine). The accurate quantitative determination of uric acid is a matter of some difficulty; for details some standard work should be consulted.

An inspection of the constitutional formula of uric acid suggests at once that it contains the residues of two molecules of urea. This corresponds to the fact that nearly all the possible decompositions of uric acid yield either a molecule of urea along with the more specific product of the decompositions, frequently itself derivative of urea, or else some substance which can by further change be decomposed into urea and some other product which is as before frequently a derivative of urea. The close cliemical relationship of urea and uric acid is thus clearly shewn. 
3. Oxaluric acid. $\mathrm{C}_{3} \mathrm{H}_{4} \mathrm{~N}_{2} \mathrm{O}_{4}$. (Hydrated parabanic acid.)

Occurs in minute traces in normal urine, from which it is extracted by filtering a large quantity of urine very slowly through a relatively small amount of animal charcoal. 'The charcoal after being washed with distilled water is extracted with boiling alcohol, to which it yields the oxaluric aeid as an ammonium salt. The free acid is a white crystalline powder, not very soluble in water: its alkaline salts are readily soluble.

\section{Allantoin. $\mathrm{C}_{4} \mathrm{H}_{6} \mathrm{~N}_{4} \mathrm{O}_{3}$. (Diureide of glyoxylic acid.)}

The characteristic constituent of the allantoic fluid, more especially of the calf, as also of foetal urine and amniotic fluid; it occurs also in the urine of nany animals for a short period after their birth. Traces of it are sometimes detected in this excretion at a later date. It is obtained in urine after the internal administration of uric acid. It has also been found in

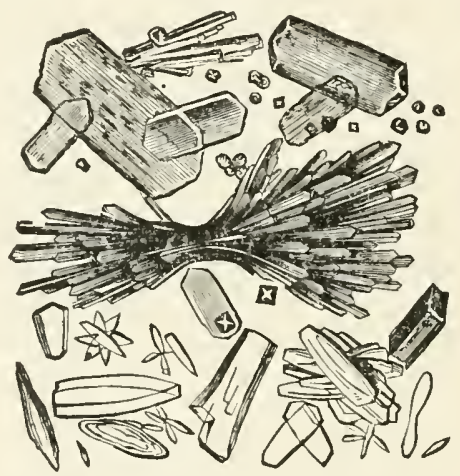

Fig. 215. Crystals from concentrated Urine of Calf. (After Kühne.)

The large central crystal composed of an aggregation of small prisms is allantoin: those below it are crystals of creatine, creatinine, and oxalate of lime. The large prisms in the upper part of the figure consist of magnesium phosphate.

vegetable tissues. It crystallizes in small, shining, colourless, hexagonal prisms. They are soluble in 160 parts of cold water, more soluble in hot, insoluble in cold alcohol and ether, soluble in hot alcohol. Carbonates of the alkalis dissolve them, and compounds may be formed of allantoin with metals but not with acids. The salts with silver and mercury are important as providing a means of separating allantoin from its solutions.

Allantoin gives no reactions which are sufficiently striking to admit of its detection in urine or other fluids: it must therefore in all cases first be separated out and then examined. The separation may be effected in several ways, of which those more 
usually employed consist in its precipitation with nitrate of nercury or silver. From the urine of calves or from their allantoic fluid, allantoin may usually be obtained in crystals by mere concentration and subsequent standing till crystallization occurs.

Allantoin may be easily obtained by the careful oxidation of uric acid with potassium permanganate.

As prepared artificially it crystallizes readily in large prismatic hexagonal crystals.

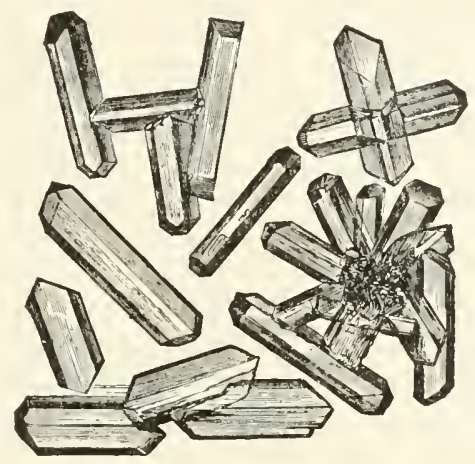

Fig. 216. Crystals of Allantoin prepared by tie oxidation of Uric Acro. (After Kïhne.)

In addition to the crystalline form and precipitability with nitrates of mercury and silver, allantoin is further characterized by yielding Schiff's reaction with furfuraldehyde (see above, p. $1257, s u b$ urea), but less readily and with less intense coloration than does urea. It also reduces Fehling's fluid on prolonged boiling.

\section{THE XANTHINE GROUP.}

This group comprises a number of substances closely related to uric acid and to each other. Some of them occur in small amounts in the tissues (muscles) and excretions (urine) of the body and are to be regarded as being, like urea and uric acid, typical products of the downward destructive metabolism of proteids. They are also obtained as products of the decomposition of the true nucleins when boiled with acids. Some of them are closely related to certain alkaloids which occur in plants (theobromine and caffëine), and which probably play some not unimportant part in the nutritional change of the animal body, since they are constantly consumed, in some form or other, by the larger part of the human race. This relationship of the xanthine-bodies to certain vegetable alkaloids is further interesting when it is remembered that the latter are 


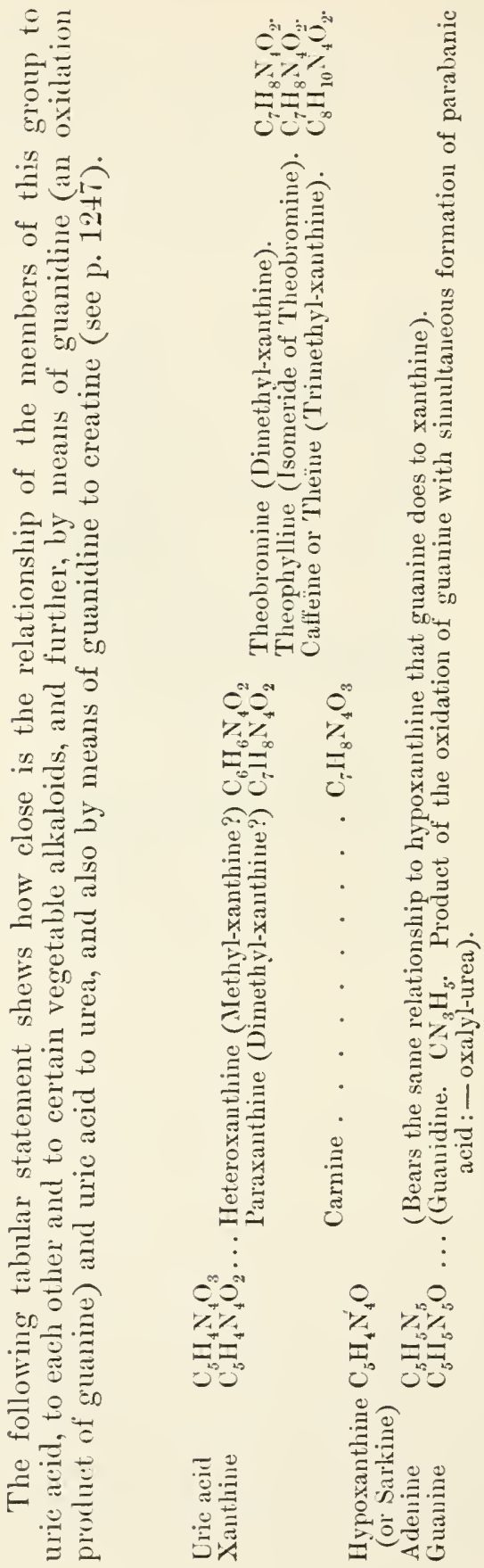


regarded by plant-physiologists as waste-products of the vegetable organism, and are thus found chiefly in those parts of the plant which are on their way to removal, viz. the bark, leaves and seeds.

1. Xanthine. $\mathrm{C}_{5} \mathrm{H}_{4} \mathrm{~N}_{4} \mathrm{O}_{2} \cdot \quad \mathrm{NH}-\mathrm{CH}$<smiles>C=C(NC(C)=O)NC(=O)O</smiles>

First discovered in a urinary calculus, and called xanthic oxide. More recently it has been found as a normal, though very scanty, constituent of urine, muscles, and several other tissues, such as the liver, spleen, thymus, brain-substance, etc. It occurs in larger quantities, together with hypoxanthine, in 'extract of meat,' and is also found in traces in vegetable tissues, - lupins, malt seedlings and tea. In nearly all cases it is accompanied by hypoxanthine. 'The amount which is present in any of the above tissues and fluids is so small that none of then, except perhaps the extract of meat, affords a convenient source for its preparation. To obtain it in quantity

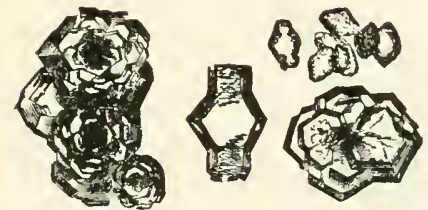

Fig. 217. XiNTHiNe hyDRoChloRide, $\mathrm{C}_{5} \mathrm{H}_{4} \mathrm{~N}_{4} \mathrm{O}_{2} \cdot \mathrm{HCl}$. (liühne.)

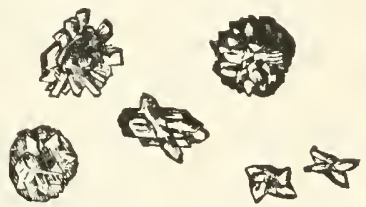

Fig. 218. Xanthine vithate, $\mathrm{C}_{5} \mathrm{H}_{4} \mathrm{~N}_{4} \mathrm{O}_{2} \cdot \mathrm{HNO}_{3}$. (Külnne.)

guanine is treated with nitrous acid, and the nitro-product thus obtained is reduced in ammoniacal solution with ferrous sulphate. It may also be prepared artificially from hydrocyanic acid and water in presence of acetic acid. When pure it is a colourless powder, requiring about 14,000 parts of water for its solution at ordinary temperatures and 1400 at $100^{\circ}$. Insoluble in alcohol and in ether, it dissolves readily in dilute aeids and alkalis (characteristically in ammonia) forming crystallizable conpounds.

Reactions. The discrimination of members of the xanthine group is not easy, since, from their close relationship, they yield many reactions in common. The following are characteristic of ximthine.

i. Weidel's reaction. The substance is warmed with freshly prepared chlorine-water and a trace of nitric acid as long as any gas is evolved: it is then carefully evaporated to dryness and, if xanthine is present, the residue turns pink or purplish-red 
on the access of ammonia fumes. Carnine gives a similar coloration if but little chlorine-water is used, while guanine and adenine do not.

ii. Hoppe-Seyler's reaction. When xanthine is introduced into some caustic sola with which some chloride of lime has been mixed, each particle of the substance surrounds itself with a dark green ring which speedily turns brown and then disappears.

iii. Strecker's test. When evaporated to dryness on porcelain with nitric acid a yellow residue is obtained which turns reddish-yellow on the addition of caustic soda or potash (not of anmonia) and reddish-violet on subsequent warming. Distinctive from uric acid.

iv. Xanthine is more readily soluble in ammonia than is uric acid.

v. Xanthine vields in solution in dilute nitric acid a characteristic crystalline compound with nitrate of silver, which differs from the similar compound of hypoxanthine both in the forms which it presents and in its greater solubility in nitric acid of sp. gr. $1 \cdot 1$ at $100^{\circ}$. It is therefore used as a means of separating xanthine and hypoxanthine.

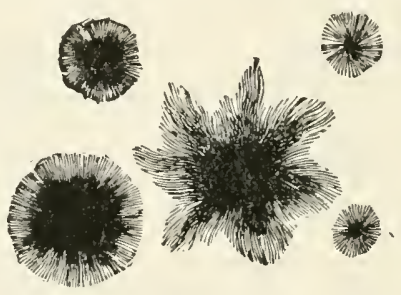

Fig. 219. Crystals of Xanthine Silver-mitrate, $\mathrm{C}_{5} \mathrm{H}_{4} \mathrm{~N}_{4} \mathrm{O}_{2} \cdot \mathrm{AgNO}_{3}$.

(Krukenberg after Kühne.)

vi. The compound of xanthine with hydrochloric acid is far less soluble in water than are the similar compounds of hypoxanthine and guanine, and hence affords a further means of separating these bases.

\section{Heteroxanthine. $\mathrm{C}_{6} \mathrm{H}_{6} \mathrm{~N}_{4} \mathrm{O}_{2}$. (Methyl-xanthine.)}

This substance occurs in minute quantities in the normal urine of man and the dog, along with xanthine and hypoxanthine and another closely allied xanthine base, paraxanthine. It oceurs in larger amount in the urine of leukhemic patients. It is crystalline, but not very characteristically so, is soluble with difficulty in cold water, much more soluble in hot water, is insoluble in alcohol and in ether. It may, as also may paraxanthine, be separated from other xanthine bases by taking advantage of the relatively slight solubility of its sodium salt 
in caustic soda. It also yiclds with hydrochloric acid a relatively insoluble salt which crystallizes readily, whereas the corresponding salt of paraxanthine is readily soluble. They may by this means be separated the one from the other.

Heteroxanthine does not give the ordinary reaction for xanthine with nitric acid and caustic soda, but yields a brilliant coloration on the application of IVeidel's test (see sub xanthine). Like the other xanthine bases it gives an insoluble salt with an ammoniacal solution of nitrate of silver.

\section{Paraxanthine. $\mathrm{C}_{7} \mathrm{H}_{8} \mathrm{~N}_{4} \mathrm{O}_{2}$. (Dimethylxanthine.) Isom-} eride of Theobromine.

Like heteroxanthine it occurs in very small amounts in urine. It is soluble with difficulty in cold water, but is more soluble than xanthine; is much more soluble in hot water, insoluble in alcohol and in ether. It crystallizes readily in characteristic flat, somewhat irregular, six-sided tables when its solutions are slowly evaporated, or in needles if rapidly. It forms, as do the preceding subtsances, a crystalline salt with nitrate of silver; this like the corresponding compound of xanthine is soluble in strong nitric acid (sp. gr. 1.1) at $100^{\circ}$, and may thus be separated from hypoxanthine. It may be separated from xanthine by means of its greater solubility in cold water, and from heteroxanthine by the difference in the solubility of its salts with sodium and hydrochloric acid.

Paraxanthine gives Weidel's reaction but not the ordinary xanthine test with nitric acid and caustic soda.

\section{Carnine. $\mathrm{C}_{7} \mathrm{H}_{8} \mathrm{~N}_{4} \mathrm{O}_{3}$.}

Closely allied in composition to the precerling base, but as yet of unknown constitution, carnine occurs only as a constituent of "extract of meat, of which it forms about one per cent., although it has been stated to oceur also in urine (?).

It crystallizes in white masses composed of very small irregular crystals; it is soluble with great difficulty in cold, readily soluble in hot water, insoluble in alcohol and in ether. It unites with acids and salts to form crystallizable compounds. Of these the more important are the salts with basic lead acetate, soluble in boiling water, and with nitrate of silver, insoluble in strong nitric acid and ammonia. Carnine gives Weidel s reaction when only a small amount of chlorine water' is employed, but the test fails if any excess is used.

Carnine bears an interesting relationship to hypoxanthine, into which it may be converted by treatment with chlorine or nitric acid, or still more readily by bromine.

$$
\mathrm{C}_{7} \mathrm{H}_{8} \mathrm{~N}_{4} \mathrm{O}_{3}+\mathrm{Br}_{2}=\mathrm{C}_{5} \mathrm{H}_{4} \mathrm{~N}_{4} \mathrm{O} \cdot \mathrm{HBr}+\mathrm{CH}_{3} \mathrm{Br}+\mathrm{CO}_{2} \text {. }
$$


5. Hypoxanthine. $\mathrm{C}_{5} \mathrm{II}_{4} \mathrm{~N}_{4} \mathrm{O}$.<smiles>N#Cc1cncnc1NC=O</smiles>

Closely related to xanthine and usually occurring with it in the tissnes and fluids of the body. Hypoxanthine may be obtained from normal muscles, and hence is found in larger amounts in 'extracts of meat.' It occurs also in the spleen, liver, and medulla of bones, and in considerable quantity in the blood and urine of lenkhæmic patients; also in normal urine and in vegetable tissues - lupins, malt-seedlings and tea.

It may be separated from xanthine by taking advantage of the slighter solubility of its salt with nitrate of silver in boiling nitric acid (sp. gr. 1·1). The crystalline form of this salt is characteristic.

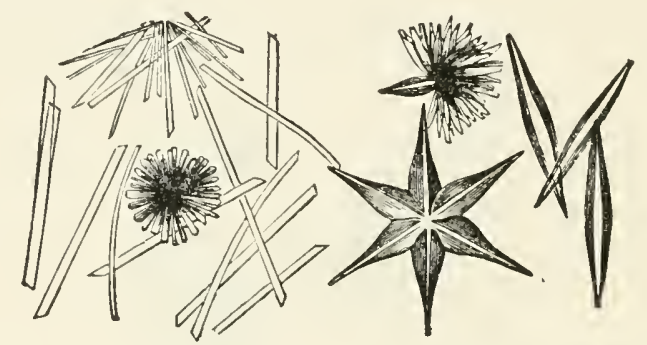

Fig, 220. Hypoxantinine Silver-Nitrate, $\mathrm{C}_{5} \mathrm{H}_{4} \mathrm{~N}_{4} \mathrm{O} \cdot \mathrm{AgNO} \mathrm{g}_{3}$. (Krukenberg after Kühne.) acids.

It also yields crystalline salts with nitric and hydrochloric

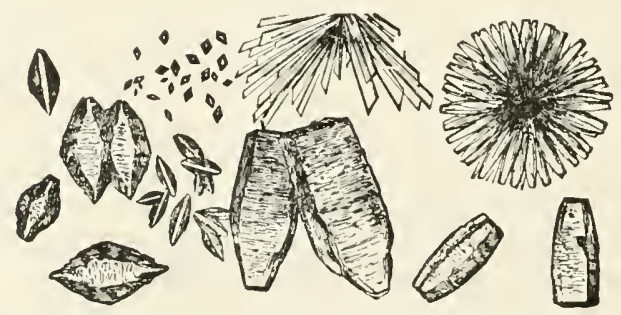

Fig. 221. Hypoxanthine-nitrate, $\mathrm{C}_{5} \mathrm{H}_{4} \mathrm{~N}_{4} \mathrm{O}$. $\mathrm{HNO}_{3}$. (Kühne.)

Hypoxanthine is soluble in 300 parts of cold and 78 of boiling water, insoluble in cold alcoliol and in ether, soluble in 900 parts of boiling alcohol. It does not yield either 
Weidel's reaction or the reaction with nitrie acid and caustic soda so characteristic of the other xanthine bases. It gives no green coloration with caustic soda and chloride of lime such

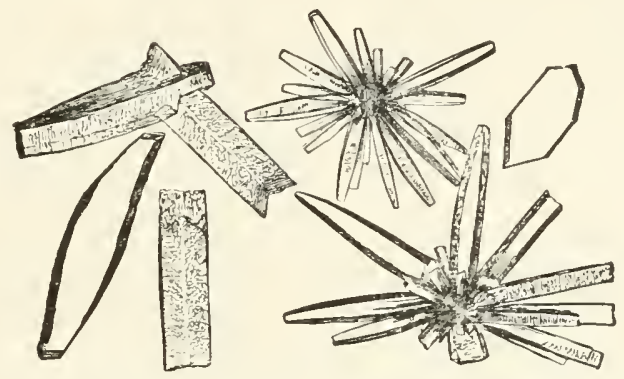

Fig. 222. Hypoxanthixe-hydrochloride, $\mathrm{C}_{5} \mathrm{H}_{4} \mathrm{~N}_{4} \mathrm{O}$. HCl. (Kühne.)

as xanthine does, but after treatment with hydrochloric acid and zinc, it yields a ruby-red coloration on the adtition of an excess of caustic soda. In this reaction it resembles adenine.

6. Adenine. $\mathrm{C}_{5} \mathrm{H}_{5} \mathrm{~N}_{5}$. (Imido-hypoxanthine.)<smiles></smiles>

This base was first obtained during the treatment of pancreatic tissue for the preparation of hypoxanthine. It is a product of the decomposition of the nucleins and may therefore be prepared from any tissue rich in nuclei such as the spleen, thymus and lymphritic glands. It is present in considerable amount in the leaves of tea and is found in the urine of leulihimia. It bears the same relationship to hypoxanthine that guanine does to xanthine, and can similarly be converted into hypoxanthine by the action of nitrous acid.

When pure it crystallizes in needles from aqueous solutions. Is soluble in 1086 parts of cold water, readily in hot water, insoluble in ether, slightly soluble in hot alcohol. Tichds crystalline compounds with acids, also with some salts. 'The compound with nitrate of silver is soluble in hot nitric acid (sp. gr. 1.1), and is thus separable, together with hypoxanthine. from xantline. It also yields a readily crystalline compound with picric acid, which is very insoluble in cold water ( 1 in 3500) and may be used for its quantitative separation from solutions. It does not give the ordinary reactions char- 
acteristic of the xanthine bodies, but like hypoxanthine shews a red coloration on the addition of an alkali after treatment with hydrochloric acid and zinc.

7. Guanine. $\mathrm{C}_{5} \mathrm{H}_{5} \mathrm{~N}_{5} \mathrm{O}$. $\mathrm{NH}-\mathrm{CH}$<smiles>C=C(N)C(=N)NC=O</smiles>

It was first obtained from Peruvian guano, which still provides the most convenient source for its preparation.

Guanine is also found in small quantities in the pancreas, liver and muscle extract, and among the products of the action of acids on some nucleins. It may also oceur in urine, more especially of pigs, in which case it is also found in many of their tissues; additionally in leukhæmic blood in the retinal tapetum of fishes and in their scales, as also in the integument of amphibia and reptiles and in vegetable tissues.

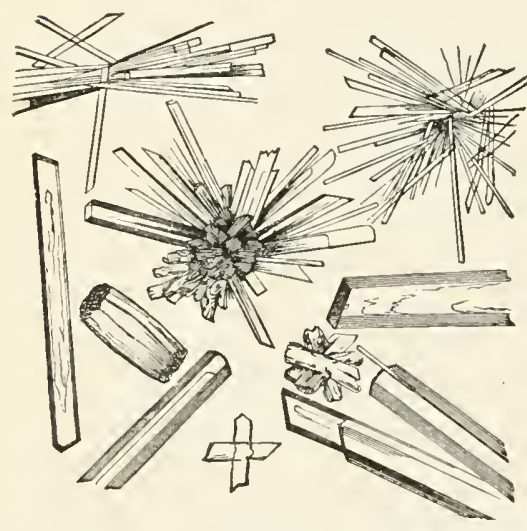

Fiti. 22:3. Guaxine in yorochloride, $\left.\mathrm{C}_{5} \mathrm{I}_{5} \mathrm{~N}_{5}{ }^{\prime}\right) \cdot \mathrm{HCl}+\mathrm{H}_{2} \mathrm{O}$. (After Kiilnne.)

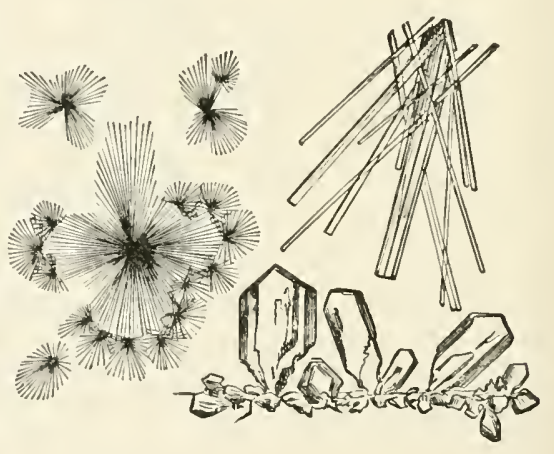

Fig. 224. Guanine nitrate, $\mathrm{C}_{5} \mathrm{II}_{5} \mathrm{~N}_{5} \mathrm{O} \cdot \mathrm{HNO}_{3}+1 \frac{1}{2} \mathrm{H}_{2} \mathrm{O}$. (After Kühne.)

It is a white amorphous powder, insoluble in water. alcohol, ether and ammonia. Its insolubility in the latter distinguishes it from xanthine and hypoxanthine. It unites with acids, alkalis and salts to form crystallizable compounds. Of its compounds with acids the most characteristic are those with hydrochloric and nitric acids.

The compound with nitrate of silver is extremely insolubie in strong boiling nitric acid.

Reactions. Bytreatment with nitric acid and caustic soda (Strecker's test) it yields a coloration closely resembling that 
given by xanthine, but does not respond to Weidel's test. (See above, p. 1265.)

Capranica's reactions. (i) A yellow crystalline precipitate on the addition of a saturated aqueous solution of picric acid to a solution of guanine-hydrochloride; insoluble in cold water. (ii) An orange-coloured crystalline precipitate, very insoluble in water, on the addition of a concentrated solution of potassium chromate. (iii) Prismatic yellowish-brown crystals on the addition of a concentrated solution of ferricyanide of potassium. Xanthine and hypoxanthine when similarly treated do not yield the last two precipitates.

Several new crystalline substances of the xanthine series have recently been isolated from urine, in which, however, they occur in most minute quantities. Of these episcurkine, $\mathrm{C}_{4} \mathrm{H}_{6} \mathrm{~N}_{3} \mathrm{O}$ (?), is very slightly soluble in water. It forms a double salt with nitrate of silver, which is very insoluble in nitric acid. It does not give the ordinary reactions of xanthine, but yields a colour as of murexide (see p. 1261) with hydrochloric acid and potassium chlorate and subsequent exposure to ammonia vapours. Epiguanine, to which the formula $\mathrm{C}_{10} \mathrm{H}_{13} \mathrm{~N}_{9} \mathrm{O}_{2}$ is assigned, is also but slightly soluble in water. It yields erystalline compounds with acids and salts, of which the double salt with platinum is peculiarly characteristic. It also gives the ordinary reaction for xanthine with nitric acid and caustic soda.

8. Guanidine.

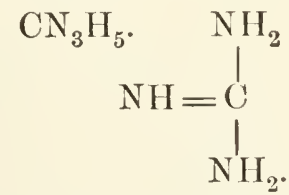

Although this substance does not occur in the free state in any tissue or fluid of the animal body, it is of considerable interest, for it has been obtained by the direct oxidation of proteids and may be made to yield urea by treatment with boiling dilute sulphuric acid or baryta-water. NH:C $\left(\mathrm{NH}_{2}\right)_{2}+$ $\mathrm{H}_{2} \mathrm{O}=\left(\mathrm{NH}_{2}\right)_{2} \mathrm{CO}+\mathrm{NH}_{3}$. Further, it affords a connecting link between the xanthine series and creatine (p. 1247), the latter substance being, as already stated, methylguanidinacetic acid, while guanidine is itself the chief product of the oxidation of guanine.

\section{Tanthine derivatives.}

There can be but little doubt that the xanthine bodies (and uric acid) are typically products of the downward, excretionary nitrogenous metabolism of animals. The alkaloidal principles of plants, in this case theobromine and caffeïne, may be similarly regarded as excretionary products, and are hence found collected in those parts of the plant which are more immediately or ultimately cast off, viz. the leaves, seeds and bark. The facts already stated render the 
consumption of theobromine and caffeine in some form or other by practically the whole human race less surprising than it might at first sight appear. Their universal use also indicates that they supply some distinct want of the economy which cannot as yet be explained purely with reference to their relationship to the nitrogenous extractives of animal tissues, but rather to the physiological effect their ingestion produces. In moderate doses they exert an agreeable stimulating action whereby the sensations of fatigue and drowsiness are removed, the body being thus enabled to exert itself with less sense of effort and less initial stimulus, and the mind is more active, clear-sighted and resistent to the depressing effects of unpleasant influences. There is no evidence, as was at one time assumed, that they act in any way by reducing the activity of nitrogenous metabolism. In the case of cocoa and chocolate we have to deal not merely with the stimulating effects of the theobromine they contain, but also with the fact that they are of extreme nutrient value owing to the large amount of fats ( 50 p.c.), proteids ( 12 p.c.), and carbohydrates which enter into their composition.

\section{THE AROMATIC SERIES.}

\section{Benzoic acid. $\mathrm{C}_{6} \mathrm{H}_{5}$. COOH.}

This is not found as a normal constituent of the body. When it occurs in (chiefly herbivorous) urine its presence is usually due to a fermentative decomposition of hippuric acid whereby benzoic acid and glycine (glycocoll) are formed.

$$
\begin{aligned}
\mathrm{C}_{6} \mathrm{H}_{5} \cdot \mathrm{CO} . \mathrm{NH} . \mathrm{CH}_{2} \cdot \mathrm{COOH} .+\mathrm{H}_{2} \mathrm{O} \\
=\mathrm{C}_{6} \mathrm{H}_{5} \cdot \mathrm{COOH} \cdot+\mathrm{CH}_{2}\left(\mathrm{NH}_{2}\right) \cdot \mathrm{COOH} .
\end{aligned}
$$

The acid is usually prepared by the above decomposition of hippuric acid, which is readily effected by a short boiling with mineral acids or, less readily, with caustic alkalis. It is also obtained by the dry distillation of gum-benzoin, from which the acid separates by sublimation. The sublimed acid generally crystallizes in fine needles which are light and glistening. It is soluble in about 200 parts of cold or 25 of boiling water and very soluble in alcohol, ether and petroleum-ether, ${ }^{1}$ in which latter hippuric acid is insoluble. When precipitated from solutions, either by cooling or the addition of acids to its salts in the cold, the crystalline form is usually much less distinct.

A part from the crystalline form benzoic acid is characterized by its property of readily subliming, even at $100^{\circ}$, thus resembling leucine and differing markedly from lippuric acid. As a result of this it passes off freely in the vapours arising from its

1 Petroleum-ether consists ordinarily of a mixture of the more volatile hydrocarbons obtained by distillation during the fractionating of crude petroleum and boils up to about $120^{\circ}$. The most volatile petroleum-ether boils up to about $80^{\circ}$. 
boiling aqueous solutions, so that in eoneentrating fluids, such as urme, in which its presence is conjectured, they should be first rendered alkaline with sodium carbonate, thus forming a non-volatile salt. Benzoic acid may be additionally recognized by the following tesı: when treated with a little boiling nitric acid and evaporated to dryness, the residue thus obtained yields, on further leating, an unmistakable odour of nitrobenzene.

When introduced into the body benzoic acid is readily and largely converted into hippuric acid, while at the same time small quantities of succinic acid may make their appearance. The chief interest in the acid centres in the above relationship to hippuric acid, a fact discovered by Wöhler in 1824 and specially interesting as being the first known instance of a well-defined synthesis effected by the animal body, and the starting-point for the disproval of Liebig's views as to the fundamental difference in the metabolic processes of animal and plant tissues.

2. Hippuric acid. $\mathrm{C}_{7} \mathrm{H}_{6} \mathrm{O}_{2}, \quad\left[\mathrm{C}_{6} \mathrm{H}_{5}, \mathrm{CO} \cdot \mathrm{NH} \cdot \mathrm{CH}_{2}\right.$. COOH.] (Benzoyl-glycine.)

This acid is found in considerable quantities $(1.5-2.5$ p.c.) in the urine of herbivora, and also, though to a much smaller

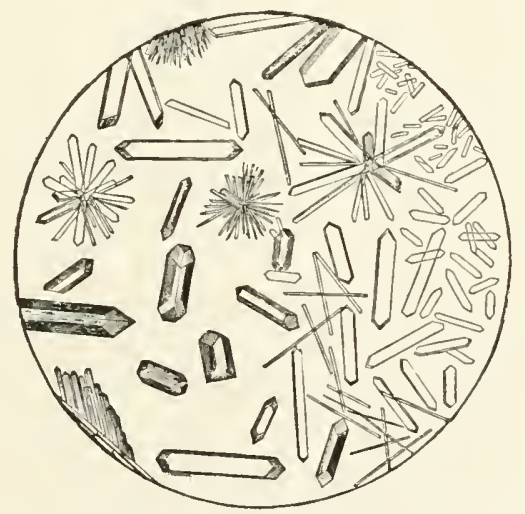

Fig. 225. Hippuric Acid Crystals. (After Funkc.)

amount $(0 \cdot 1-1 \cdot 0 \mathrm{grm}$. per diem $)$ in the urine of man. It is undoubtedly formed in the body by the union, with dehydration, of benzoic acid and glyeine (see $\$ 339$ ).

It may be readily obtained from the urine of horses or cows, more particularly when they are out to grass. The perfectly fresh ${ }^{1}$ urine is boiled with milk of lime in slight excess, by

1 To avoid fermentative decomposition into benzoic acid and glycine. 
which means the acid is fixed as a hippurate of calcium. It is then filtered, the filtrate concentrated to a small bulk and treated when cold with hydrochloric acid in slight excess; this decomposes the calcium salt, liberating hippuric acid, which separates out at once owing to its comparatively slight solubility. It is then purified by several recrystallizations from boiling water, but it is extremely difficult to obtain it colourless.

When lapidly separated out from its aqueous solutions, as in the above method of its preparation, it assumes the form of fine needles. By slower crystallization it yields long four-sided prisms or columus with pyramidal ends; these are frequently arranged in groups and present a semitransparent, milky appearance.

When pure they are odourless and of a somewhat bitter taste. They require 600 parts of water for their solution at $0^{\circ}$, are very readily soluble in hot water, also in alcohol and to a less cxtent in ether. They are conveniently insoluble in petroleum-ether, in virtue of which hippuric acid can be readily separated from benzoic acid, which is soluble in this reagent. Its solutions redden litmus-paper.

Apart from the characteristics already stated the acid may be recognized by the following reactions. When gently heated in a small tube the acid does not at once sublime as does benzoic acid, but melts and solidifies again on cooling. If more strongly heated it molts as before but is now decomposed, yielding a sublimate of benzoic acid accompanied by an odour like that of new hay, while oily red drops are observed in the tube. When treated with boiling nitric acid (see above sub benzoic acid) and evaporated to dryness, the residue, on bcing heated, yields the marked and characteristic odour of nitrobenzene.

\section{Tyrosine. $\mathrm{C}_{9} \mathrm{H}_{11} \mathrm{NO}_{3} \cdot \quad\left[\mathrm{OH} \cdot \mathrm{C}_{6} \mathrm{H}_{4} \cdot \mathrm{CH}_{2} \cdot \mathrm{CH}\left(\mathrm{NH}_{2}\right)\right.$.} COOH.] (Para-oxyphenyl-a-amidopropionic acid.)

This substance always accompanies lencine, though less in amount, as a product of the pancreatic digestion of proteids, but not of gelatin, also as a product of their putrefactive decomposition as well as of the action of boiling mineral acids and alkalis. It is also perhaps found normally in small quantities in the pancreas and its secretion and in the spleen, and traces have been described as obtained from various tissues of the boly. It is normally absent in urine but makes its appearance together with leucine in this excretion in several discased conditions of the liver, notably acute ycllow atrophy, also in phosphorus poisoning; there is however some conflict of opinion as to its constancy in such cases. It is also present in not inconsiderable quantities, along with leucine, in many plant tissues. 
Tyrosine crystallizes in exceedingly fine needles which are usually collected into feathery masses. The crystals are snowwhite, tasteless and odourless. If crystallized from an alkaline solution tyrosine often assumes the form of rosettes composed of fine needles arranged radiately.

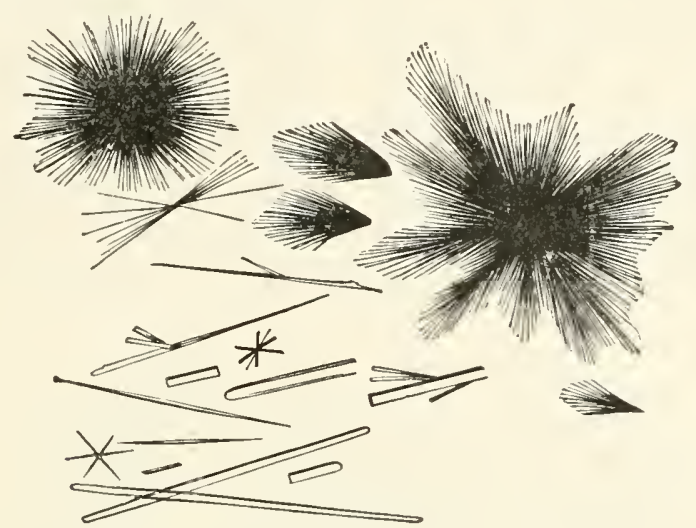

Fig. 226. Trrosine Crystals. (Krukenberg.)

The crystals are very sparingly soluble in cold water ( 1 in 2000 at $20^{\circ}$ ), much more soluble in boiling water ( 1 in 150); they are almost insoluble in strong alcohol $(1$ in 13,500$)$ and quite insoluble in ether. They are readily soluble in acids and particularly so in ammonia and other alkalis and in solutions of alkaline salts.

Preparation. (i) The products of a prolonged panereatic digestion of proteids are neutralized and filtered; the filtrate when concentrated usually yields crusts of tyrosine erystals, which may be readily purified by solution in a little boiling water from which they separate out on cooling after concentration if necessary. (ii) Horn shavings are boiled for 24 hours with sulphurie acid ( 5 of acid to 13 of water). The sulphuric acid is then separated by the addition of lime, and the filtrate from the calcium sulphate yields as before crusts of tyrosine crystals on concentration and cooling. These are then purified by recrystallization from boiling water. Any leucine at first present in the crystalline crusts remains in the mother-liquors from which the tyrosine has been separated.

Apart from its crystalline form and characteristic solubilities tyrosine may be readily recognized by several well-marked reactions.

Hoffmann's reaction. When heater with Millon's reagent, solutions of tyrosine yield a brilliant crimson or pink coloration which, if much tyrosine is present, is accompanied finally by a similarly coloured precipitate. 
Piria's reaction. If tyrosinc is moistened on a watch-glass with concentrated sulphuric acid and warmed for five or ten ninutes on a water bath, it turns pink owing to the formation of tyrosine-sulphonic acid $-\mathrm{C}_{9} \mathrm{H}_{10}(\mathrm{SO} 2 \mathrm{OH}) \mathrm{NO}_{3}+2 \mathrm{H}_{2} \mathrm{O}$. 'This is then diluted with water, warmed, nentralized with barium sarbonate, and filtered while hot. The filtrate yields a violet colour on the careful ardition of very dilute perchloride of iron. The colour is readily destroyed by any excess of the iron salt.

4. Kynurenic acid. $\mathrm{C}_{10} \mathrm{I}_{7} \mathrm{NO}_{3} . \quad\left[\mathrm{C}_{9} \mathrm{H}_{5} \mathrm{~N}\right.$. OH . COOH. $]$ (IIydroxyquinoline-carboxylic acid.)

This acid occurs characteristically but in variable amounts in the urine of $\log$, but does not appear to have been found

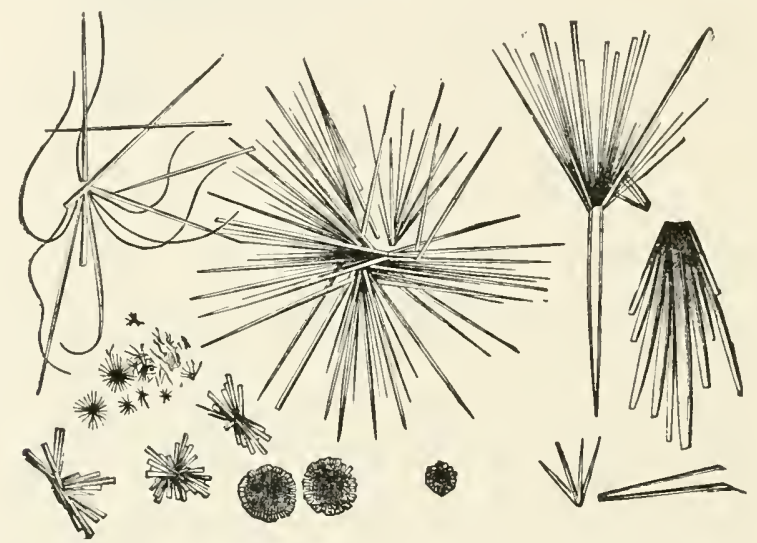

Fig. 227. Cristals of Kixurexic Acid. (After Kiuhne.)

normally in that of man. It is practically insoluble in cold water, slightly so in boiling water and realily soluble in hot alcohol and in dilute ammonia. It crystallizes in long brilliant

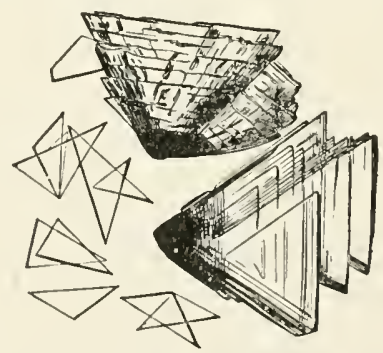

Fig. 228. Cristals of Bariug Kinglexate. (After Kühne.)

white neerlles which when kept under acidulated water are often changed into long glittering four-sided prisms. 
This acid forms salts of which that with barium crystallizes readily and in a very characteristic triangular form.

Apart from its crystalline form and that of its barium salt this acid may be readily recognized by the following reaction. When heated on a water bath with hydrochloric acid and chlorate of potash and evaporated to dryness a reddish residue is obtained which turns at first to a brownish green on the addition of ammonia and finally to an emerald green.

The amount of kynurenic acid in the urine is increased on the ingestion of isatin, a product of the oxidation of indigo. Under ordinary conditions its amount in this excretion is dependent upon the nature of the food supplied to the animal, being greatest under a proteid diet, and is not related to the occurrence or absence of putrefactive processes in the alimentary canal.

\section{Inosite. $\mathrm{C}_{6} \mathrm{H}_{12} \mathrm{O}_{6}+2 \mathrm{H}_{2} \mathrm{O}$. [CH.OH $]_{6}$.}

This substance has the same percentage composition as a sugar and possesses a distinctly sweet taste, in virtue of which properties it appears to have been usually classed with the

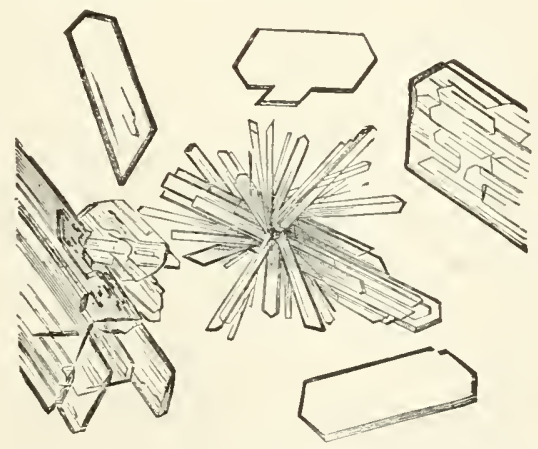

Fig. 229. Ixosite Cristals. (After Kïihne.)

carbohydrates. It does not however vield any of the reactions most typical of this class of substances ; for instance it exerts no rotatory power on polarized light, does not reduce metallic salts, does not undergo alcoholic fermentation and does not react with phenyl-hydrazine. On aceount of these peculiarities the view was long ago expressed that it is not a carboliydrate at all, and it has more recently been shewn to belong to the benzene series. Structurally it may be represented by a closed ring of six CH . OH groups.

Inosite occurs but sparingly in the human body; it was found originally in the muscles. It is found in the lungs, kidneys, spleen, liver, and brain, and occurs also in diabetic 
urine, and in that of 'Bright's disease, and is found in abundance in the vegetable kingdom, more especially in unripe beans, from which it may be conveniently prepared. It is also found in the urine after the ingestion of an excess of water into the body.

Pure inosite for'ms large efflorescent crystals (rhombic tables); in microscopic preparations it is usually obtained in tufted lumps of fine crystals.

Readily soluble in water, it is only slightly so in dilute alcohol, and is insoluble in absolute alcohol and ether.

Although inosite admits of no direct alcoholic fermentation it has been stated to be capable of undergoing a lactic fermentation in presence of decomposing proteid (cheese) and chalk, yielding ordinary (ethylidene-) lactic acid and some butyric acid. It had been previously stated that the acid thus obtained is sarcolactic (ethylene- or para-lactic acid).

\section{Reactions of inosite.}

(i) Scherer's test. 'The suspected substance is treated with strong nitric acid and evaporated nearly to dryness on porcelain. On the addition of a little ammonia and a few drops of freshly prepared and not too dilute solution of calcium chloride, a bright pink or rose-coloured residue is obtained on renewed evaporation if inosite is present.

(ii) Gallois' test. When inosite in concentrated solution is treated with a few drops of 2 p.c. mercuric nitrate solution, or Liebig's solution for the estimation of urea, and the mixture is evaporated to dryness it yields a yellow residue which on being more strongly heated turns rosy red; this disappears on cooling and returns again on renewed heating.

(iii) Seidel's reaction. A small amount ( $03 \mathrm{gr}$.) of the suspected substance is evaporated to dryness in a platinum crucible with a little nitric acid (sp. gr. 1.1-1.2) and the residue is treated with ammonia and a few drops of a solution of strontium acetate. If inosite is present a greenish coloration is observed together with a violet precipitate.

6. Phenol. $\mathrm{C}_{6} \mathrm{H}_{5} \cdot \mathrm{OH}$. Hydroxybenzene. (Carbolic or phenylic acid.)

This substance is formed, together with indole and skatole, during the putrefactive decomposition of proteids, more especially in prolonged putrefactive pancreatic digestions. From these it may be obtained by simple distillation. In accordance with this it is formed in not inconsiderable quantity in the alimentary canal, more especially when putrefactive processes in its contents are increased either pathologically or as the result of experimental interference. Of the phenol thus formed 
a small proportion is passed out in the faces, the larger part however is excreted in the urine as an ethereal salt of sulphuric acid, viz. phenylsulphate of potassium. 'The latter' is typical of an extensive series of similar ethereal sulphates which make their appearance in urine after the ingestion of aromatic substances.

Phenyl-sulphuric acid. ${ }^{1} \mathrm{C}_{6} \mathrm{H}_{5}$. O . $\mathrm{SO}_{2} \mathrm{OH}$. Apart from its abundant presence in urine as an alkaline salt after the administration of phenol this compound occurs normally in small quantities in most urines, more particularly in those of herbivora, since in these animals the conditions for its formation are especially provided by the preponderance of aromatic compounds in their food and the more marked activity of putrefactive changes in their alimentary canal. The total sulphates in urine consist therefore partly of this ethereal sulphate (together with the similar compounds of cresol, indole and skatole, see below) and of ordinary sulphates.

\section{Cresol. $\mathrm{C}_{6} \mathrm{H}_{4} \cdot \mathrm{OH}, \mathrm{CH}_{3}$. Methylphenol.}

This homologue of phenol exists in three isomeric forms, ortho-, para- and metacresol. It is now known that the phenols which may be obtained by the distillation of urine with acids consist preponderatingly of paracresol, accompanied in some cases by orthocresol and possibly (?) by metacresol in minute amounts. Like phenol it is not found free in urine but as cresylsulphuric acid, $\mathrm{C}_{7} \mathrm{H}_{7} \mathrm{O} . \mathrm{SO}_{2} \mathrm{OH}$. The general conditions of its presence in urine are practically identical with those for the occurrence of phenylsulphuric acid. When introduced into the animal body the three isomeric cresols undergo distinctly different oxidational changes.

\section{Pyrocatechin. $\mathrm{C}_{6} \mathrm{H}_{4}(\mathrm{OH})_{2}$. Ortho-dihydroxybenzene.}

This substance occurs in small amounts in human urine, united with sulphuric acid as a mono-ethereal compound, $\mathrm{OH} . \mathrm{C}_{6} \mathrm{H}_{4} \cdot \mathrm{O} . \mathrm{SO}_{2} \mathrm{OH}$. It is more plentifully present in the urine of the herbivora, especially of the horse, and is largely increased in amount by the administration of benzene or phenol. It is also stated to occur in cerebrospinal fluid. When present in urine it (together with hydroquinone) confers on this exeretion, especially if alkaline, the property of turning successively greenish, brown and finally dark-brown or almost black on exposure to the air, and of readily redueing solutions of metallic salts, a fact to be taken into account when dealing with the presence or absence of sugar in the urine. Solutions of pyrocatcchin turn emerald green on the addition of a few drops

${ }_{1}^{1}$ Not to be confounded with phenolsulphonic acid, $\mathrm{C}_{6} \mathrm{H}_{4}(\mathrm{OH}) \cdot \mathrm{SO}_{2} \mathrm{OH}$. 
of very dilute solution of ferric chloride, avoiding all excess of the reagent. If the green solution is now acidulated with tartaric acid, it turus violet on the subsequent addition of a little ammonia and purplish-red on the addition of excess. 'The green colour may be restored by excess of acetic acid. It may be distinguished from hydroquinone by yielding a precipitate with normal acetate of lead which is soluble in acetic acid, whereas the latter substance does not.

But little is knowu as to the source of this substance in urine apart from its probable formation from the phenol produced by putrefactive changes in the alimentary canal. In herbivora there is some evidence that it is derived from certain aromatic constituents of their food.

\section{Hydroquinone. $\mathrm{C}_{6} \mathrm{H}_{4}(\mathrm{OH})_{2}$. Para-dihydroxybenzene.}

Has not yet been described as occurring normally in urine, but only as the result of the ingestion of phenol. It exists in urine as an ethereal compound with sulphuric acid and is largely the cause of the dark colour which this excretion assumes after the absorption of phenol on exposure to the air. It resembles pyrocatechin in effecting the reduction of metallic salts, but differs from it in being nearly insoluble in cold benzene and in not yielding any precipitate with normal lead acetate. This latter property suffices for its separation from pyrocatechin. It is readily converted by oxidation into quinone, $\mathrm{C}_{6} \mathrm{H}_{4} \mathrm{O}_{2}$, whose characteristic odour affords a further means of identification, and when heated in an open test-tube it yields a blue sublimate.

The third known isomeric dioxybenzene viz. meta-dihydroxybenzene or resorcin has not yet been found in the animal body or in urine.

\section{THE INDIGO SERIES.}

\section{Indole. $\mathrm{C}_{8} \mathrm{H}_{7} \mathrm{~N} . \quad\left[\mathrm{C}_{6} \mathrm{H}_{4} \backslash \mathrm{CH} / \mathrm{CH}\right.$. $]$}

Indole occurs characteristically in the fieces, to which with skatole it inparts their peculiarly unpleasant odour. Its presence here is due to its formation during the putrefactive decomposition of proteids which usually occurs to a greater or less extent in the alimentary canal, part of the indole leaving the body in the urine as a potassium salt of indoxylsulphuric acid (see below), the remainder being excreted with the faces. It may readily be obtained, contaminated by varying quantities of phenol and skatole (see below), by acidulating and distilling the produets of a not too prolonged alkaline putrefactive pan- 
creatic digestion of proteids, preferably of liver or fibrin. Indole passes over into the distillate, from which it is extracted by shaking up with ether, and is left behind as an impure oily liquid when the ether is driven off by heat. It may also be prepared by heating moist proteids slowly to a red-heat with excess of caustic potash, the indole as before passing over into the distillate. Indole is a crystalline body which when pure nelts at $5: 3^{\circ}$. It is soluble in boiling water, alcohol and ether.

Reactions. A strip of pine-wood moistened with hydrochloric acid is coloured bright crimson when dipped into an alcololic solution of indole. ${ }^{1}$ Its alcoholic solution turns red when treated with nitrous (fuming nitric) acid and its aqueous solution gives a copious red precipitate with the same reagent. This reaction is more delicate if carried on by the addition of strong nitric acid first and of a 2 p.c. solution of potassium nitrite subsequently. When indole in dilute solution is mixed with a little sodium nitroprusside and then with a few drops of caustic soda it turns at once violet-blue, and pure blue on subsequent acidulation with acetic acid. Skatole yiclds neither of the above reactions. Indole also forms a well-marked crystalline compound picric acid (trinitro-phenol) when added to a solution of the acid in benzene, so also does skatole.

\section{Indoxylsulphuric acid. $\mathrm{C}_{8} \mathrm{H}_{6} \mathrm{~N} \cdot \mathrm{O} . \mathrm{SO}_{2} \mathrm{OH}$. The indi-} can of urine.

Indole as previously stated is a characteristic product of the putrefaction of proteids. When administered to amimals, it leads to a correspondingly increased output of urinary indican, an increase which is similarly observed as the result of either a nornally. pathologically or experimentally increased activity of putrefactive processes in the alimentary canal. Hence indican is uncler normal conditions more plentiful in the urine of herbivora than of carnivora. It is also increased in carnivorons urine under a meat diet, is not increased by the administration of gelatin and is least during starvation, although in the latter case it may not entirely disappear. 'These facts correspond again to the experimental observations that gelatin does not yield indole during its putrefactive decomposition whereas muein does, and the latter substance constitutes a part at least of the contents of the alimentary canal during starvation. These statements show clearly the origin and morle of formation of urinary indican, the first-formed indole undergoing oxidation into indoxyl, which is subsequently united to the elements of sulphuric acid and excreted as an ethereal compound.

1 This reaction depends on the presence of coniferin in the pine-wood. Phenol under similar conditions yields a blue coloration. 
Indoxylsulphuric acid is not known in the free state; its most important salt is that with potassium, the form in which it occurs in urine. When warmed in aqueous solution with hydrochloric acid it decomposes into indoxyl and potassium sulphate: -

$$
\mathrm{C}_{8} \mathrm{H}_{6} \mathrm{~N} \cdot \mathrm{O} \cdot \mathrm{SO}_{2} \cdot \mathrm{OK}+\mathrm{H}_{2} \mathrm{O}=\mathrm{C}_{8} \mathrm{H}_{6} \mathrm{~N}(\mathrm{OH})+\mathrm{KHSO}_{4} .
$$

Indoxyl by oxidation is converted into indigo-blue:-

$$
2 \mathrm{C}_{8} \mathrm{H}_{6} \mathrm{~N}(\mathrm{OH})+\mathrm{O}_{2}=\mathrm{C}_{16} \mathrm{H}_{10} \mathrm{~N}_{2} \mathrm{O}_{2}+2 \mathrm{H}_{2} \mathrm{O} \text {. }
$$

The blue coloration which results from the above reaction affords the one test for the presence of indican in urine. The lest is applied as follows (Jaffé). A small volume of urine (10 c.c.) is mixed with an equal volume of strong hydrochloric acid and 2-3 e.c. of ehloroform. A strong solution of chloride of lime is then added drop by drop, shaking after the addition of each drop. If indican is present the layer of ehloroform which settles on standing will be coloured more or less brilliantly blue according to the amount of indican in the urine. 'The formation of indigo-blue is also the basis for the quantitative estimation of indican. The latter is converted into indigoblue by oxidation and the indigo-blue is estimated either by weighing or colorimetrically or spectrophotometrically.

\section{Indigo-blue. $\mathrm{C}_{16} \mathrm{H}_{10} \mathrm{~N}_{2} \mathrm{O}_{2}$.}

It is formed, as stated above, from indican, and gives rise to the bluish colour sometimes observed in sweat and urine.

It may, by slow formation from indican, be obtained in fine crystals; these are insoluble in water, slightly soluble, with a faint violet colour, in alcohol and in ether. Chloroform dissolves them to a slight extent, as also does benzol. Indigo is soluble in strong sulphuric acid, forming at the same time two compounds with the acid, indigo mono- and di-sulphonic acids. The sodium salts of these acids are solnble in water and, mixed with sodium sulphate, constitute the crude 'indigocarmine' of commerce, and in a purer form the sulphindigotate of soda used in certain experiments on the nature of the excretory activity of the kidney and other glands (see $\$ 336$ ). These soluble sulphonates give an absorption band in the spectrum which lies to the red side of and close to the D line. This may be used to detect indigo.

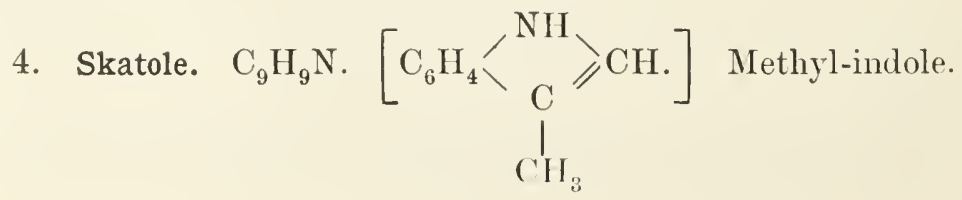


The conditions of its production are in general the same as those for the formation of indole, so that the two substances occur mixed in variable proportions anong the products of the putrefactive decomposition of proteids or brain-substance and of the action of caustic potash at high temperatures on proteids. In the former case it appears to be produced at a later stage than is indole, so that on the whole it is most preponderant the longer the putrefactive change is allowed to proceed. Its presence in the frees is thus due to causes similar to those which account for the presence of indole, and the resemblance is further shewn by the fact that a portion of the first-formed skatole is absorbed, oxidized, and appears extemally in the urine as skatoxyl-sulphuric acid '(see below).

Skatole is a crystalline substance which melts when heated to $93^{\circ}$, and has a powerfully unpleasant odour, somewhat like that of indole.

Reactions. Many of the reactions of skatole resemble so closely those of indole that they provide no means for distinguishing between the two substances. Skatole is, however, characterized by yielding only a milky opacity instead of a red coloration on the addition of fuming nitric acid to its aqueous solutions (see sub indole), in not giving the reaction with a strip of pine-wood dipped in hydrochloric acid which indole does, ${ }^{1}$ by its lesser solubility in water and greater resistance to the action of caustic soda.

\section{Skatoxyl-sulphuric acid. $\mathrm{C}_{9} \mathrm{H}_{8} \mathrm{~N} . \mathrm{O} \cdot \mathrm{SO}_{2} \mathrm{OH}$.}

The close relationship between indole and skatole is further shewn by the fact that the latter, like the former, after absorption from the alimentary canal, is oxidized, the product being skatoxyl, $\mathrm{C}_{9} \mathrm{H}_{8} \mathrm{~N} . \mathrm{OH}$, which unites, as does indoxyl, with the elements of sulphuric acid and leaves the body in the urine as a potassium salt of the above acid.

Our knowledge of the quantitative formation of skatole in the alimentary canal and of its relationship to the simultaneous prorluction of indole is far less complete than is that respecting the latter substance. Notwithstanding the close chemical relationship of the two it appears that their physiological behaviour is markedly different. In the first place it seens that the absorption of skatole is less complete than that of indole, since it preponderates in the normal frees: in accordance with this, but little of its ethereal sulphate is found normally in urine. Further, whereas by the ingestion of indole nearly the whole of the sulphates of the urine may be

I When however a strip of pine-wood is dipped into an alcoholic solution of skatole and subsequently into strong hydrochloric acid, it is coloured first crimson, which turns to bluish-violet. 
converted into the ethereal compound with indoxyl, when skatole (synthetically prepared) is similarly employed a large part reappen's in the freces; and although at first the ethereal sulphates are increased they subsequently diminish even with continued injection of skatole and are stated to finally disappear. Indoxyl-sulphuric acid may be regarded as a urinary chromogen, since it yields a pigment, indigo, by oxidational decomposition; so also may skatoxyl-sulphuric acid, but it is found that the amount of pigment-forming material specifically present in the urine of a dog fed with skatole is not so directly proportional to the amount of skatoxyl-sulphuric acid as it is to the similar compound of indoxyl when indole is administered. It has been suggrested that a large part of the skatolic chromogen exists as a compound of skatoxyl and glycuronic acid. When Jaffés test (see p. 1282) for urinary indiean is applied to urine which contains skatoxyl compounds the results obtained are as follows. The urine turns dark red or violet on the addition of hydrochloric acid, bright crimson on the addition of nitric acid, and a similar colour is obtained if it is warmed with hydrochloric acid and ferric chloride. The colouring matter thus obtained is probably formed from the skatoxyl (not known in the free state), and by reduction may be made to yield skatole.

Skatole has recently been described as occurring in a vegetable tissue, namely, the wood of an East Indian tree, Celtis reticulosa.

\section{THE PTOMAINES.}

Although the substances to which the above name has been given (from $\pi \tau \hat{\omega} \mu \alpha$, a corpse) are now known to belong chiefly to the class of compounds called amines, so that they provide no chemical sequence to the bodies previously described, their characteristic production during the putrefactive decomposition of animal tissues seems to make this a suitable place for treating of them.

The ptomaines as a group may be said to closely resemble the class of substances long known under the name of alkaloids and obtained from plant tissues. The resemblance is shewn not merely in their chemical constitution, but more obviously in their similar solubilities in various fluids, in their general behaviour towards reagents, and in some cases even in their specific reactions, and more especially in their frequently powerful (poisonous) action on the animal organism, the actions of certain ptomaines being almost identical with those of certain vegetable alkaloids. The ptomaines may therefore be regarded as alkaloids of animal origin. The close similarity of the two classes of substances has hence endowed the ptomaines 
with very considerable interest from a medico-legal point of view in virtue of the not infrequent use of the vegetable alkaloids for eriminal purposes and the now obvious possibility that the detection of alkaloids in the corpse may afford no reliable information as to the administration of the same during life. They are further of considerable and increasing pathological interest and that from two points of view. In the first place, as products of the general putrefactive changes which animal tissues undergo, they may account for the severe symptoms and not infrequent death which results from the consumption as food of fish, sausages and timned-meats. In the second, there appears to be increasing evidence of the formation of special ptomaines by the organisms characteristic of specific diseases, so that the pathological conditions may be due rather to the products formed by the organisms than to the organisms themselves directly, a possibility of no small importance in the light of recent prophylactic research.

While the general reactions of the ptomaines place them as already stated side by side with the vegetable alkaloids, their specific reactions and properties exhibit considerable differences both in comparison with each other and with those of the alkaloids. Some are liquid and lighly volatile so that they pass off readily during distillation of their aqueous solutions. others ane liquid and non-volatile, others again solid and crystalline. They exhibit equally marked differences in their. solubilities. 'Thus neither' benzene nor petroleum-ether will extract them from their acid aqueous solution. Ether on the other hand dissolves out a few of the ptomaines from an acid solution and a large majority from an alkaline solution. Some are more particularly soluble in chloroform, a few are insoluble in any of these reagents. Amyl-alcohol is the one reagent in which as a class they appear to be almost generally soluble. 'Their behaviour with the usual alkaloidal precipitants (mercuric and platinic chlorides, tannic acid, the double iodides of potassium with mercury and other metals, etc.) is equally varied. They are all precipitated by phospho-molybdic acid and most of them yield crystalline compounds with a solution of iodine in hydriodic acid. Possessed of an alkaline reaction they further act as powerful reducing agents, many of them converting ferri- into ferrocyanides, the reduction being evidenced by the formation of Prussian blue on the simnltaneous addition of ferric chloride. This property is however possessed by many vegetable alkaloids and not by every ptomaine; it cannot therefore be regarded as a specific class-reaction for these substances. Some of the ptomaines (toxines) are extraordinarily poisonous, producing effects which are frequently specific but in many cases similar to those of certain vegetable alkaloids. Others again are quite inert. 
Two of the most clearly defined ptomaines are cadaverine and putrescine. These are found in corpses which have undersone putrefactive decomposition, cadaverine appearing in the earlier stages of putrefaction and putrescine preponderating in the later. The latter is largely present in putrid herrings. The former is identical with pentamethylene-diamine $\mathrm{NH}_{2}\left(\mathrm{CH}_{2}\right)_{5} \mathrm{NII}_{2}$. 'The latter has been shewn to have the constitution of tetramethylene-diamine, $\mathrm{NH}_{2}\left(\mathrm{CH}_{2}\right)_{4} \mathrm{NH}_{2}$. They have both recently been obtained as conspicuous eonstituents of urine from a case of eystinuria and appear to owe their origin to putrefactive processes occurring in the intestine. They are both somewhat viscid fluids which erystallize at low temperatures, and yield readily crystallizable compounds with acicls and salts of gold, platinum and mereury. Their benzoyl compounds are insoluble in water and hence afford a convenient means for their separation. Choline and the highly toxic nenrine, which really belong to this class, have already been (lescribed. (See above, pp. 1239, 1240.)

Leukomaines. ${ }^{1}$ This name has been applied to those basic (alkaloidal) substances which occur in living tissnes and are to be regarded as products of their normal metabolism and thus distinct from ptomaines. They are obtained by extracting finely minced ox-flesh with an cxtremely dilute aqueons solntion of oxalic acid. This extract may contain the following six bases: Xanthocreatinine, $\mathrm{C}_{5} \mathrm{H}_{10} \mathrm{~N}_{4} \mathrm{O}$; Chrysocreatinine, $\mathrm{C}_{5} \mathrm{H}_{8} \mathrm{~N}_{4} \mathrm{O}$; Amphicreatinine, $\mathrm{C}_{8} \mathrm{H}_{19} \mathrm{~N}_{7} \mathrm{O}_{4}$; Pseudoxanthine, $\mathrm{C}_{4} \mathrm{H}_{5} \mathrm{~N}_{5} \mathrm{O}$, and two, as yet unnamed, with the composition $\mathrm{C}_{11} \mathrm{H}_{24} \mathrm{~N}_{10} \mathrm{O}_{5}$ and $\mathrm{C}_{12} \mathrm{H}_{25} \mathrm{~N}_{11} \mathrm{O}_{5}$ respectively. They probably stand in elose relationship to paraxanthine, $\mathrm{C}_{7} \mathrm{H}_{8} \mathrm{~N}_{4} \mathrm{O}_{2}$, heteroxanthine, $\mathrm{C}_{6} \mathrm{H}_{6} \mathrm{~N}_{4} \mathrm{O}_{2}$, and adenine, $\mathrm{C}_{5} \mathrm{H}_{5} \mathrm{~N}_{5}$ (see above, p. 1264), and it is interesting to note that comparing the formula of the leukomaines with each other and with those of creatinine, $\mathrm{C}_{4} \mathrm{H}_{7} \mathrm{~N}_{2} \mathrm{O}$, and creatine, $\mathrm{C}_{4} \mathrm{H}_{9} \mathrm{~N}_{3} \mathrm{O}_{2}$, they are found to differ in several cases by the gromp CNH.

The leukomaines are regarded by some authors as feebly toxic alkaloidal produets of metabolism from which the organism is normally freed either by their excretion, since they are found in urine, or by destructive oxidation, and it has further been suggested that their abnormal retention in the economy may be the cause of certain obseure pathological conditions.

\section{THE BILE-ACIDS.}

\section{Cholalic (or cholic) acid. $\mathrm{C}_{24} \mathrm{H}_{40} \mathrm{O}_{5}$.}

This acid occurs in traces as a product of the decomposition

1 The name is derived from $\lambda \epsilon u ́ \kappa \omega \mu a$, occasionally used to denote white of egg and hence to indicate their origin from proteids. 
of the bile-acids in the small intestine, in larger quantities in the contents of the large intestine, and in the freces of man and many animals. In icterus the urine is also stated to frequently contain traces of this acid. Its principal interest lies in its being the starting-point, by its union with glycine or taurine, for the acids which, as sodium salts, exist characteristically in bile.

Owing to the readiness with which ox-bile can be obtained in large quantities, this has been most frequently used for the preparation of cholalic acid, whose properties as usually given hence refer to the acid as obtained from this source. IIore recent researches have however demonstrated comparatively unimportant but still distinct differences in the composition and properties of the acid as it occurs in the bile-acids of different animals. The description of the acid which here follows refers to that form which is obtained from ox-bile.

Preparation. This depends upon the decomposition of the bile-acids (glycocholic and taurocholic) by means of alkalis at boiling temperature.

The acid possesses the following properties. The crystals obtained from hot alcoholic solutions are usually in the for'm of large rhombic tetrahedra or octahedra, containing 21 molecules of water of crystallization which may be driven off by heating to $100^{\circ} \mathrm{C}$. 'The crystals are but slightly soluble ( 1 in 750 ) either in water, even when boiling, or in ether, but readily soluble in alcohol. This acid may also be obtained in an amorphous form by concentrating its solutions to dryness and is now less insoluble than when crystallized. The alkali and barium salts of cholalic acid are soluble in water and in alcohol especially when warm, and yield, like the free acid, dextrorotatory solutions. For solutions of the anhydrous acid $(a)_{\mathrm{D}}=+50^{\circ}$. When crystallized with $21 \mathrm{H}_{2} \mathrm{O},(a)_{\mathrm{D}}=+35^{\circ}$. In alcoholic solutions of the sodium salt $(a)_{\nu}=+31 \cdot 4^{\circ}$.

The constitution of cholalic acid is scarcely as yet definitely known but may be represented by $\mathrm{C}_{20} \mathrm{H}_{31}\left\{\begin{array}{l}\mathrm{COOH} \\ \left(\mathrm{CH}_{2} \mathrm{OH}\right)_{2} \\ \mathrm{CHOH}^{2}\end{array}\right.$ yields with iodine a compound which like that resulting from the interaction of iodine and starch possesses a brilliantly blue colour and is specifically distinctive, since it cannot be obtained either from the bile-acids or choleic acid (see below) or the products of the decomposition of cholalic acid.

When cholalic acid is prepared from luman bile it exhibits certain differences, more especially as regards the lesser solubilities of its alkali and barium salts, which led to its being regarded as distinct from that obtained from ox-bile and hence it was called anthropocholalic acid. It appears however that the bulk of the acid is identical with that from ox-bile, the 
slight difference being due to an admixture with another acid either cholalic, as was first supposed, or fellic.

Choleic acid, $\mathrm{C}_{25} \mathrm{H}_{42} \mathrm{O}_{4}$ or $\mathrm{C}_{24} \mathrm{H}_{40} \mathrm{O}_{4}$. Is obtained in small amounts mixed with cholalic arid during the preparation of the latter from ox-bile. It differ's from cholaliv: acid in the solubility of its salts and the products of its oxidational decomposition.

Fellic acid, $\mathrm{C}_{23} \mathrm{H}_{40} \mathrm{O}_{4}$ or $\mathrm{C}_{23} \mathrm{H}_{38} \mathrm{O}_{4}$. Obtained in small amounts from human bile during the preparation of ordinary cholalic acid. It is characterized by the extreme insolubility of its barium and magnesium salts. It also yields a less brilliant Pettenkofer reaction (see below) than does cholalic acid.

The bile-acids of the pig and goose when decomposed yield forms of cholalic acid called respectively hyo-cholalic acid, $\mathrm{C}_{25} \mathrm{H}_{40} \mathrm{O}_{4}$, and cheno-cholalie, $\mathrm{C}_{27} \mathrm{H}_{41} \mathrm{O}_{4}$.

\section{Dyslysin. $\mathrm{C}_{24} \mathrm{H}_{36} \mathrm{O}_{3}$.}

When cholalic acid is heated to $200^{\circ} \mathrm{C}$. or boiled for some time in solution with hydrochloric or sulphuric acid it loses two molecules of water and yields a resinous anhydride called dyslysin, from its insolubility in water, alcohol and alkalis. As resulting from the delyydration of cholalie aeid it is fonnd sometimes in small anount in the freces. It is a non-crystalline substance which is soluble in an excess of ether, also in solntions of cholalic acid or of its salts. By treatment witl boiling alkalis it may be reconverted by hydration into cholalie acid.

The various forms of cholalic acid which may be prepared from the bile of different animals each yield a corresponding form of dyslysin.

\section{Glycocholic acid. $\mathrm{C}_{26} \mathrm{H}_{43} \mathrm{NO}_{6}$.}

It is found as a sodium salt chiefly in ox-bile but also in that of man, mixed in both cases with a much smaller and variable amount of taurocholic acid, also present as a sodium salt. In carnivora it occurs, if at all, in such minute traces only, that it may be said to be absent from the bile of these animals; hence their bile-acid consists entirely of taurocholic acid. In icterus the urine may contain small quantities of glyeocholic acid.

Preparation. This may be effected as already described in $\$ 207$.

The acid crystallizes in fine glistening needles which require about 300 parts of cold but only 120 of hot water for their solution. They are also very soluble in alcohol but practically insoluble in ether. The salts of this acid, more especially those with the alkalis, are extremely soluble even in eold water, also in alcohol, but very slightly so if at all in ether. Both the 
free acid and its salts are dextrorotatory: for the former, in alcoholic solutions, $(a)_{\mathrm{D}}=+29 \cdot 0$, for the latter $(a)_{\mathrm{D}}=+25 \cdot 7^{\circ}$.

\section{Taurocholic acid. $\mathrm{C}_{26} \mathrm{H}_{45} \mathrm{NSO}_{7}$.}

This acid is found to some extent in ox-bile, and is more plentifully present in that of man. The bile of the dog contains taurocholic acid alone, unmixed with glycocholic.

Preparation. As already described in $\$ 207$, using dog-bile as material.

'This acid is extremely soluble in water and in alcohol, but not in ether, so also are its salts with the exception of the one formed on the addition of basic lead acetate in presence of ammonia, which is insoluble in water and in alcohol. The acid and its salts are dextrorotatory; for the sodium salt in alcoholic solution $(a)_{\mathrm{D}}=+24.5^{\circ}$. If dissolved in water the rotatory power is less, and in this respect it resembles glycocholic acid.

Pettenkinfer's reaction for bile acids.

The following is the more usual method of obtaining the reaction. Bile, which may be very considerably diluterl, or a dilute solution of bile-salts or acids is mixed in a porcelain dish with a few drops of a 10 p.e. solution of cane-sugar. Concentrated sulphuric acid is now added to the mixture with constant stirring to an extent not exceeding $\frac{2}{3}$ of its volume, the addition of the acid being so regulated that the temperature of the mixture is not allowed to rise above $70^{\circ} \mathrm{C}$. Hereupon a brilliant cherry-red colour makes its appearance and rapidly assumes a magnificent purple tint. On standing for somt time the colour becomes darker and of a more distinctly blue tint. The reaction may also be obtained by the addition of first the acid and then the sugar solution. The success of the test depends on the careful avoidance of any excessive rise of temperature during the addition of the sulphuric acid and more especially of any excess of sugar which by being charred by the acid gives a lirown roloration and masks the typical purple. The purple solution if diluted with alcohol (not with water which destroys the colour), shews with a spectroscope a characteristic absorption spectrum consisting of two absorption bands, one between $L$ and $E$ abutting on $E$ and a second adjoining the $F$ line. In the earlier stages of the reaction a third narrow band near $D$ makes its appearance but disappears later on.

It is important to remember that an extended series of substances other than cholalic acid and the bile-acids (pigments and other substances which are charred by sulphuric acid) either interfere with the brilliancy of the reaction or else themselves yield a purple colour which closely resembles that due to the bile-acids. Among the latter those of chief importance are proteids, amyl-alcohol, oleic acid, the higher fatty acids and cholesterin. 


\section{THE COLOURING MATTERS AND PIGMENTS OF THE ANIMAL BODY.}

\section{Henoglobin and its Derivatives. ${ }^{1}$}

1. Hæmoglobin. ${ }^{2}$ This is the well-known constituent of the red corpuscles to which the dark colour of the blood from an asphyxiated animal is due. It is also present to a less and somewhat variable amount in ordinary venous blood, in presence of correspondingly variable amounts of the compound which it forms with oxygen, namely oxy-hæmoglobin. In normal arterial blood it is probably present in mere traces, if at all, since here its affinities for oxygen are completely satisfied to form oxy-hæmoglobin.

Owing to the ease and avidity with which hæmoglobin unites with oxygen to form the distinct and stable compound known as oxy-hæmoglobin, its investigation is attended with considerable experimental difficulties, hence our knowledge of it as a chemical substance is on the whole less complete than is that of oxy-hemoglobin. Hremoglobin may be obtained in a crystalline form, but with some considerable difficulty owing to its extreme solubility in water. The crystals may be prepared by sealing up a concentrated aqueous solution of oxy-hamoglobin in glass tubes from which, if necessary, all remaining air is displaced by hydrogen: on prolonged standing all the oxygen disappears during the putrefactive reduction which ensues, and finally, more readily on exposure to a low temperature, crystals of hæmoglobin make their appearance in the fluid. A similar production and formation of crystals is frequently observed when crystals of oxy-hæmoglobin are sealed up with Canada balsam under a cover-slip and kept for some time. The form of the crystals obtained from the blood of different animals has not yet been fully investigated.

As ordinarily seen the erystals of liæmoglobin have a dark red appearance, unlike the bright scarlet of oxy-hæmoglobin, with a strong purple or bluish tint. They are extremely soluble in water, much more so than the crystals of oxy-hæmoglobin.

In addition to the absorption band ordinarily described for hæmoglobin, it alsa shows one in the extreme violet end of the spectrum between $G$ and $H$, its centre corresponding to W. L. 426.

1 The more important characteristics and properties of these substances have been previously deseribed in some detail ( $\$ 275$ to $\$ 282$ ) and may therefore be here dealt with very brietly.

2 'The single name hæmoglobin is used here to denote what is more frequently and usually called 'reduced' hemoglobin, as distinct from oxy-hæmoglobin. The adoption of the name as here used is both simpler and more logical. 
When decomposed in the absence of oxygen (air), as for instance by the action of organic acids, more dilute mineral acids or best of all by caustic alkalis, it yields a proteid, of which but little is known (see p. 1175), and a coloured substance called hemochromogen. 'The latter' on exposure to air absorbs oxygen and becomes ordinary hrematin; it is in fact the substance usually spoken of as reduced hæmatin. (See below.)

2. 0xy-hæmoglobin. ${ }^{1}$ When hremoglobin is exposed to the air it rapidly unites, molecule for molecule, with oxygen, thus becoming oxy-hremoglobin, the characteristic constituent of the red-corpuscles to which the scarlet colour of arterial blood is due. It may be readily set free from the corpuscles by the addition to detibrinated blood of such fluids as alcohol, ether, chloroform, water and solutions of bile-salts or by repeatedly freezing and thawing the blood; when thus set free it passes into solution in the adjacent serum. From this solution it may be obtained as crystals with more or less readiness, dependently upon the kind of animal whose blood is used for its preparation (see $\$ 275$ ), the difference being due partly at least to the varying solubility of the several hæmoglobins.

To obtain rapidly a microseopic preparation of oxy-hæmoglobin crystals it suffices to take a drop of the blood of some animal whose hæmoglobin crystallizes readily (rat, guinea-pig or $\operatorname{dog}$ ), to mix a drop of it on a slide with a minute drop of water and allow the mixture to evaporate until a ring of dried substance is formed at the periphery. If it be now covered with a cover-slip, crystals usually form in a short time, espccially if it be kept cooled. Crystals may be also readily obtained even from human blood by admixture with serum in a state of slight putrefactive decomposition. For laboratory purposes large quantities of crystallized oxy-hæmoglobin may be very readily obtained from dog's blood as follows. The blood is defibrinated and strained through fine muslin: it is then placed in a flask and ether is added with frequent shaking until the blood is just 'laky,' i.e. transparent. The flask is now surrounded by a freezing mixture of ice and salt and in a short time its contents usually become almost pasty from the mass of crystals which form in it. 'These are then centrifugalized off, dissolved in a minimal amount of water, filtered, cooled to $0^{\circ}$, and after the addition of one quarter of its bulk of cooled alcohol again immersed in a freezing mixture. The second crop of crystals thus obtained may be again recrystallized as already described. The crystals are finally washed with

1 IIæmoglobin is united to corpuscles in the blood of all vertebrates with two exceptions, Amphioxus and Leptocephalus. In invertebrate blood it is usually found in solution in the plasma, but there are a few (eight) exceptions to this rule. 
water at $0^{\circ}$ containing 25 p.c. of alcohol, and may be dried in vacuo over sulphuric acid at $0^{\circ}$, and are now fairly stable.

The crystals obtained from the hromoglobin of various animals differ in their crystalline form. The following figurc shews some of the most typical forms.

Numerous analyses of oxy-hemoglobin have been made, but these while they tell us at most that it consists of oxygen, hydrogen, nitrogen, and carbon together with iron as a characteristic constituent and some sulphur, and seem to indicate that it differs in composition as obtained from different animals, do not as yet enable us to assign with any certainty a definite formula to its composition. It is however certain that its molecular weight is enormously great $(13,000-14,000)$.
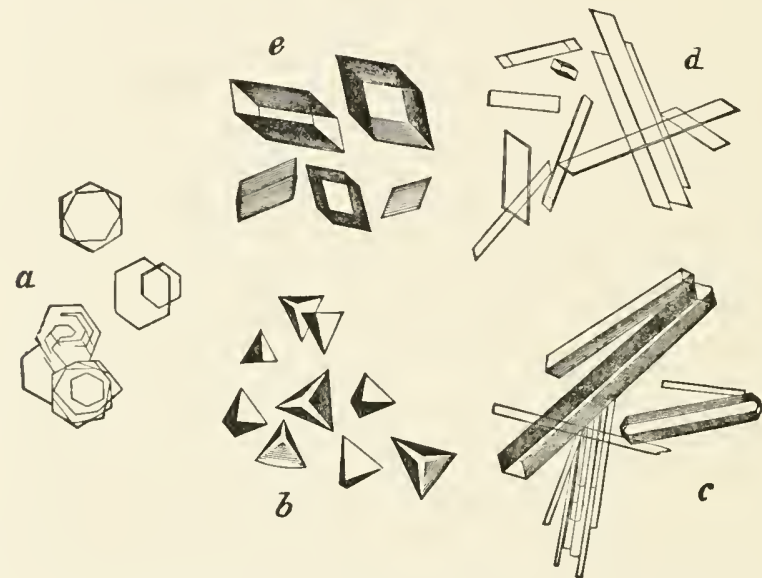

Fig. 230. Crystals of Oxy-hemoglobin. (After Funke.)

a. Squirrel, b. Guinea-pig, c. Cat, or Dog, d. Man, e. Hamster.

The spectroseopic appearances of solutions of oxy-hæmoglobin have been already sufficiently described and figured (\$276), but in addition to the bands there figured, it shews also a band in the extreme violet between $G$ and $I$, whose centre corresponds to W. L. 414.

There appear's to be a consensus of opinion that hæmoglobin, and more particularly oxy-hamoghobin, possesses to a slight degree the properties of an acid. This view appears to be biserl on the following facts. Oxy-hæmoglobin is extraordinarily soluble in alkalis and in this solution appears to be more stable than ordinarily. It is further stated that it has a feeble power of facilitating the evolution of carbon-dioxide from dilute solutions of sodium carbonate. It is hence often supposed that in the red blood-corpuscles the hrmoghobin is united to the alkalis of which their stroma partially consists. If the above views 
are correct they may assist in explaining to some slight extent the difficulties in mnderstanding the causes of the exit of carbondioxide from venous blood during its passage through the lungs. (See $\$ 288$.$) But the possibility here indicated must$ be received with the greatest caution.

3. Carbon-monoxide hæmoglobin. When a current of carbon-monoxide is passed through a solution of oxy-hæmoglobin the oxygen is driven off and its place taken by the first-named gas. The compound thus formed results, like oxy-haemoglobin, from the union of one molecule of the gas with one of hæmoglobin. It further resembles oxy-hæmoglobin in being readily crystallizable in forms isomorphous with those of the former, but the erystals are on the whole less soluble, brighter coloured and more stable than are those of oxy-hrmoglobin. The compound of carbon-monoxide with hrmoglobin is much more stable than is oxy-hæmoglobin, so that the gas is not again expelled by the action of oxygen, a fact which fully explains the fatal result of breathing carbon-monoxide. Finally the spectrum of carbon-monoxide hamoglobin, while very similar at first sight to that of oxy-hæmoglobin, differs distinetly from it in the position of its two absorption bands (see Fig. 89, No. 6). The absorption band in the extreme violet between $G$ and $H$ has its centre at W. L. 420. Since the determination of this compound in blood is frequently of considerable importance in medical jurisprudence, many tests for its presence have been devised additionally to the evidence afforded by the spectroscope. One of the oldest and best consists in adding to the suspected blood twice its volume of caustic soda of sp. gr. 1.3. If carbon-monoxide hamoglobin is present it yields a brilliant red precipitate, differing entirely in appearance from the brownish-green mass observed if oxy-hamoglobin is present.

4. Nitric oxide hæmoglobin. If a current of nitric oxide be passed through a solution of carbon-monoxide hemoglobin, the carbon-monoxide is displaced by the former gas. The compound thus obtained is still more stable than is carbon-monoxide hæmoglobin. It may be crystallized and in solution exhibits two absorption bands very similar to those of oxy-hrmoglobin but slightly nearer the red end of the spectrum; these bands are not affected by reducing agents. The position of its absorption band in the extreme violet is the same as that of the carbon monoxide compound. If prepared by passing the gas through ordinary blood, the latter should first be freed from oxygen by a current of hydrogen and care must be taken to neutralize the nitrous acid formed during the process.

5. Carbon-dioxide hæmoglobin. There appear's to be no 
doubt that a solution of hæmoglobin takes up a larger volume of carbon-dioxide than can be accounted for as the result of a merely physical absorption. 'Tluus in one set of experinents it was found that $1 \mathrm{gr}$. of hamoglobin could unite with $2 \cdot 366$ c.c. of the gas at a temperature of $18.4^{\circ}$ and partial pressure of $31.98 \mathrm{~mm}$. of $\mathrm{Hg}$, the latter being a mean average partial pressure of carbon-dioxide in venous blood according to the older established data, while that in arterial blood is $21.28 \mathrm{~mm}$. It is further stated that the stronger solutions of hæmoglobin absorb relatively less carbon-dioxide than the weaker, and that, as in the case of oxy-hemoglobin various modifications of hemoglobin exist possessing different powers of uniting with this gas. On comparing the amounts of carbon-dioxide and of oxygen or $\mathrm{CO}$ or NO which may unite with a given weight of hæmoglobin it is at once evident that the mode of union of the former gas must be different from that of the latter three, with which, as alrearly stated, hæmoglobin unites molecule for molecule. This difference in behaviour is very probably due to the decomposition which hæmoglobin undergoes when a current of carbondioxide is passed through it, and indeed it is hence probable that the so-called carbon-dioxide hæmoglobin is rather a compound of the gas with a coloured product of the decomposition of hæmoglobin, viz. hæmochromogen, which has been shewn to unite with carbon-monoxide (see below). The compound, whatever be its true nature, is stated to exhibit a one-banded absorption spectrum closely similar to that of hemoglobin, but the centre of the band lies slightly more towards the violet end of the spectrum.

6. Methæmoglobin. When blood or solutions of hæmoglobin which have been exposed to the air for some time are examined with the spectroscope they are frequently found to exhibit, in addition to the more or less persistent absorption bands of oxyhiemoglobin, a marked band of absorption between $C$ and $D$, closely resembling but differing slightly in position from the band which hamatin shews in acid solution (see Fig. 90, No. 4). This band may also frequently be observed in many pathological fluids, such as those from ovarial cysts, etc., which are coloured by blood, and in urine when similarly coloured. The substance to which the band is due is known as methenoglobin. It may be readily prepared in the laboratory by the action of many reagents such as acids or alkalis, or more conveniently of certain salts, on solutions of oxy-hremoglobin. Of these salts those which may perhaps on the whole be most advantageously employed to obtain the spectrum of methemoglobin are nitrites, potassium ferricyanide, or potassium permanganate. With the two latter salts the spectrum of methemoglobin maty be obtained as follows. To 10 c.c. of 
a moderately strong solution of oxy-hiemoglobin add a few drops of a dilute $(\cdot 5-1 \cdot 0$ p.c.) solution of either of the salts and warm very gently. If on examination with a spectroscope the two bands of oxy-hamoglobin are still strongly visible, let the mixture stand for a short time, and if the band eharaeteristic of methrmoglobin has not made its appearance add one or two drops more of the solution of the salt and proceed as before. As soon as the bands of oxy-hemoglolin have markedly disappeared, acidulate very faintly and examine again. 'The band which should now be visible as characteristic of methæmoglobin lies in the red part of the spectrum, between $C$ and $D$, nearer to the former line. As already renarked its position is closely similar to that of hrmatin in acid solution, but comparison will shew that it lies nearer $D$ than does the hrmatin band, the centre of the latter being situated at W. L. 640, while that of the former is at W. L. 630 (see Fig. 90, Nos. $t$ and 5 ).

In the preparation of large quantities of crystallized oxyhaemoglobin from pig's blood it was observed that during the recrystallizing essential to its purification a copious crop of reddish-brown crystalline needles was obtained. These were found on examination to be crystals of methrmoglobin. They are most casily obtained if the oxy-hremoglobin is first converted into methemoglobin by the action of potassium ferricyanide (one or two minute crystals of the salt to half a litre of warm concentrated solution of oxy-hemoglobin); the mixture is then shaken until it has a dark brown colour, and is cooled to $0^{\circ}$ after the addition of one quarter of its bulk of alcohol also cooled to $0^{\circ}$. They have also been obtained from the blood of the $\mathrm{dog}$, horse and other animals, and resemble in crystalline form the crystals of oxy-hamoglobin from the same sources. These crystals are doubly refracting, readily soluble in water though less so than oxy-hamoglobin, and the solution, unlike that of the latter substance, yields a precipitate with basic lead acetate in presence of ammonia; they are identical in percentage composition with those of oxy-hemoglobin.

The behaviour of methemoglobin towards reducing agents is interesting and also important as affording a means of diserimination between this substance and hematin. If some ammonium sulphide be added to an alkaline solution of methamoglobin the mixture may be observed to yield the spectrum of (reduced) liemoglobin, and on now shaking up with oxygen (air) it shews the spectrum of oxy-hanoglobin. When a solution of hematin is similarly treated it yields the spectrum of hremochromogen (reduced hamatin) in alkaline solution (see below).

7. Hæmocyanin. As previously stated (p. 1291) the bloodplasma of nuany invertebrates contains hæmoglobin in solution; 
in a few cases this is united to special corpuscles in the blood. But in the case of other invertebrates this respiratory pigment is replaced by another to which, since it turns blue on exposnre to air (oxygen), the name hemocyanin has been given. Hence the arterial blood of those animals in which it occurs is blue, while the venous is colourless.

Hemocyanin is a proteid of the globulin class; it is therefore partially precipitated by a current of carbon-dioxide, by saturation of its solutions with sodium chloride and completely by saturation with magnesium sulphate. Unlike hemoglobin it has not yet been erystallized, and contains copper, presumably as a constituent of its molecule, in place of the iron characteristic of hamoglobin. It exhibits no absorption bands when examined spectroscopically.

Another animal pigment is known, into whose composition copper ( 5 -s p.c.) enters; this is the substance called turacin. It gives the chararteristic colour to the plumage of certain African birds known as 'Touracos or Plantain-eaters, whence the name turacin. It differs entirely from hrmocyanin in its general properties, and is only mentioned here because it contains copper, as does the former pigment. It is slightly soluble in water, readily soluble in dilute alkalis, the solutions in either of these solvents shewing two absorption bands between $D$ and $E$ very similar to, though not identical with, the bands of oxy-hæmoglobin and a third faint broad band at $F$. It is not however a respiratory pigment.

\section{Hæmochromogen. $\mathrm{C}_{34} \mathrm{H}_{36} \mathrm{~N}_{4} \mathrm{FeO}_{5}(?)$.}

When (reduced) hæmoglobin is treated with acids, or, better still, with alkalis in the entire absence of oxygen it is decomposed into a proteid and a coloured substance to which the name hamochromogen is given. When alkalis are used in its preparation the solution obtained is of a brilliant purplish-red colour and is characterized by two marked absorption bands, the stronger lying halfway between $D$ and $E$, the other and fainter between ' $E$ ' and $b$. These are identical with the bands of Stokes reduced hematin in alkaline solution (see Fig. 90, No. 3). When exposed to the air (oxygen) the solution rapidly loses its brilliant colour, becomes dichroic, viz. red in thick and greenish in thin layers and now yields an absorption spectrmm which exhibits one not very strongly marked band in the yellow, to the red side of $D$ and touching the latter line. This is the spectrum of hamatin in an alkaline solution (see Fig. 90, Nos. 1 and 2). When the decomposition of the hamoglobin is brought about by acids instead of alkalis, the coloured product is similarly hamochromogen, but in this case mnless special precautions are taken some of the hemochromogen is itself further decomposed and yields hematoporphyrin or iron-free 
hæmatin (see below). When a solution of hæmatin in alkali is reduced with Stokes' fluid ${ }^{1}$ or ammonium sulphide the solution obtained shews two absorption bands identical with those alrearly described as characteristic of hamochromogen. From these facts it would at first sight appear that reduced hæmatin in alkaline solution and hemochromogen in a sinilar solution are identical substances, and this is the view which has been most generally adopted. Solutions of hæmochromogen show a very strong absorption band in the extreme violet between $h$ and $G$, its centre corresponding to w. L. 420. By appropriate methods hamochromogen may be obtained in a crystalline form.

\section{Hæmatin. $\mathrm{C}_{32} \mathrm{H}_{32} \mathrm{~N}_{4} \mathrm{FeO}_{4}(?)$.}

When oxy-hrmoglobin is decomposed by either acids or alkalis it yields a proteid and a coloured substance known as hematin. This decomposition may take place in old bloodclots or extravasations and is readily produced by the action of either gastric or pancreatic juice on oxy-hremaglobin, so that hematin is frequently found in the contents of the alimentary canal and in the faces, more especially with a flesh diet. It has also been found in urine as the result of poisoning with sulphuric acid or arseniuretted hydrogen.

For ordinary purposes hematin is characterized chiefly by the spectroseopic appearances of its solutions. When dissolved in an alkali (ammonia) it shews one absorption band in the yellow adjoining $D$ to the red side of this line, while at the same time there is great absorption at the blue end of the spectrum (Fig. 90, Nos. 1 and 2). On treatment with a reducing agent, Stokes' fluid or ammonium sulphide, this band is replaced by two others in the green of which the one nearest $D$ is remarkably dense, the other less sharply defined. Very little absorption of the red end is observed while that of the blue is as before very marked (Fig. 90, No. 3). This is the spectrum of reduced hrematin and is identical with that of hæmochromogen. Alkaline solutions of hematin are strongly dichroic, being ruby-red in thick layers and greenish in thin layers viewed by reflected light.

The acid alcoholic solution of hæmatin is characterized by one absorption band between $C$ and $D$, adjoining $C$, whose centre is situated at W. L. 640 . 'This band is somewhat similar to that of methemoglobin, but it is less dense, and careful observation shews that the centres of the respective bands do not coincide (Fig. 90, Nos. 5 and 4). Acid solutions of hrematin are monochromatic and of a dull reddish-brown colour. If

1 Prepared by adding tartaric acid to a solution of ferrous sulphate and then ammonia until it is strongly alkaline. 
blood or a strong solution of oxy-hæmoglobin be made strongly acid by the addition of acetic acid the hremoglobin is decomposed. hrematin is set free, and if the solution be shaken up with ether and allowed to stand, the ether rises to the surface and is more or less coloured owing to the presence of hiematin held in solution in the acid ether. This acid ethereal solution shews, in addition to the one band already described as characteristic of hematin in an acid solution, three other bands whose positions and relative intensities are sufficiently shewn in Fig. 90, No. 6. Solutions of hæmatin in an alkali shew no definite absorption band in the extreme violet. In acids, or with its acid compounds, a strongly marked band may be seen between $h$ and $L$.

Pure hæmatin is a scaly but not crystalline mass of bluishblack colour and metallic lustre, strongly resembling iodine. When finely powdered it appears dark or light brown according to the fineness of the powder. It is a remarkably stable substance; may be heated to $180^{\circ}$ without decomposition, but by stronger heating, if finally decomposed, liberates an odour of hydrocyanic acid and leaves a residue (12.5 p.c.) of pure oxide of iron. It is quite insoluble in either water, alcohol, ether, chloroform or benzene. It is somewhat soluble in strong acetic acid, especially if warm, also in alcohol (not water) to which some acid has been added, and readily soluble in alkaline solutions or in alcohol containing alkalis. It is not affected either by strong caustic alkalis even when heated, or by hydrochloric or nitric acids. It may be dissolved in strong sulphuric acid, but is now found to have undergone a change during solution which results in the removal of iron and the production of hæmatoporphyrin or iron-free hæmatin.

If the decomposition of hæmatin by sulphuric acid be brought about in the absence of oxygen an iron-free insoluble substance is obtained known as hæmatolin, to which the formula $\mathrm{C}_{68} \mathrm{H}_{88} \mathrm{~N}_{8} \mathrm{O}_{7}$ is assigned.

If potassium cyanide be added to an alkaline solution of hæmatin, this now shews one broad absorption band extending from $D$ to $E$. By the action of reducing agents, this band is replaced by two other bands. The substance to which these appearances are due is known as cyan-hæmatin, but all further information is still wanting.

10. Histohæmatins. This is the name assigned to a elass of pigments which are stated to be of wide-spread occurrence in the tissues of both vertebrates and invertebrates, and to be related to though quite distinct from hemoglobin and hæmatin. They are regarded as respiratory pigments, playing towards the muscles or other tissues in which they more particularly Dccur the same part that hæmoglobin does to the tissues generally. Our knowledge of these pigments is however as yet 
limited to the spectroscopic appearances which they present either in situ in the mother-tissue or in solutions obtained by the action of ether, while their respiratory function is assumed from the changes which they exhibit under the influence of reducing agents and subsequent exposure to oxygen. Of these histohiematins the one most fully described is known as myohematin from its characteristic presence in muscles.

Myohamatin. 'To observe the spectrum of this substance a slice of tissue, such as that of the heart, is squcezed in a compressorium until sufficiently thin to transmit light. It is then examined with a microspectroscope under strong illumination. Or, on the other hand, the tissue may be treated with excess of ether, under whose influence an aqueous juice is extruded in which the myohæmatin is in solution. Speaking generally, for the appearances vary slightly according to the source of the pigment, myohrematin yields a four-banded absorption spectrum. The first band lies close to $D$, but towards the red end of the spectrum. The next two bands are situated close together abont midway between $D$ and $E$. The remaining band lies in the region between $E$ and $b$. Solutions of myolicmatin are when weak of a reddish-yellow colour, but if strong they are pure red. By the action of warm alcohol containing a little sulphuric acid a spectrum is obtained closely similar to that of hæmatin in acid solution, and by the use of concentrated sulphuric acid a substance is produced which in both acid and alkaline solutions shews bands similar to those of hæmatoporphyrin in the same solvents. Under certain conditions myohematin becomes 'modified' and now yields two bands similar to those of hemochromogen, but situated nearer the violet end of the spectrum.

The conclusions drawn from the above spectroscopic facts have been the subject of some controversy and adverse criticism, the appearances being regarded as due not to a specific pigment, but rather to hæmochromogen or mixtures of other products of the decomposition of hæmoglobin.

11. Hæmin. ${ }^{1} \quad \mathrm{C}_{34} \mathrm{H}_{35} \mathrm{~N}_{4} \mathrm{FeO}_{5}$. HCl. (Hæmatin-hydrochloride or 'Teichmann's crystals.)

These crystals may be readily obtained for microscopic examination by heating a drop of fresh blood on a glass slide under a cover-slip with a little glacial acetic acid. In the case of blood which has been dried, as in an old blood clot or stain, the residue should be powdered as finely as possible with a minute quantity (trace) of sodium chloride. A little of the powder is then placed on a slide and covered with a slip

1 According to more recent work the formula of true hæmin is $\mathrm{C}_{32} \mathrm{H}_{30} \mathrm{~N}_{4} \mathrm{FeO}_{3}$, the crystals being a hydrochloride of this substance. 
under which some glacial acetic acid is now run in. It is then warmed carefully to a temperature just short of that which would cause the acid to boil. If the operation has been successful, on cooling crystals of hemin will be seen under a microscope mixed in either case as in Fig. 231 with a granular débris.

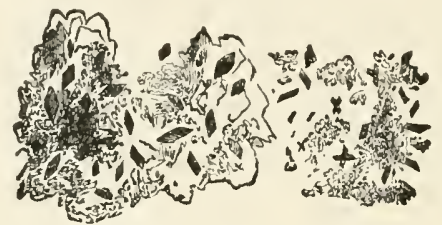

Fig. 231. Hжmin Cristals from a drop of blood. (Külne.)

If they are absent, warm again, adding more acid if necessary. The crystals are dark brown, frequently almost black, elongated rhombic plates and prisms belonging to the triclinic system. In a purified specimen they are arranged singly or in groups as shewn in Fig. 232, and apart from their form are characterized by being strongly doubly-refracting: when examined under the microscope between crossed Nicol prisms those crystals whose axes are suitably inclined to the incident light stand out bright yellow or orange on the dark field. They are quite insoluble

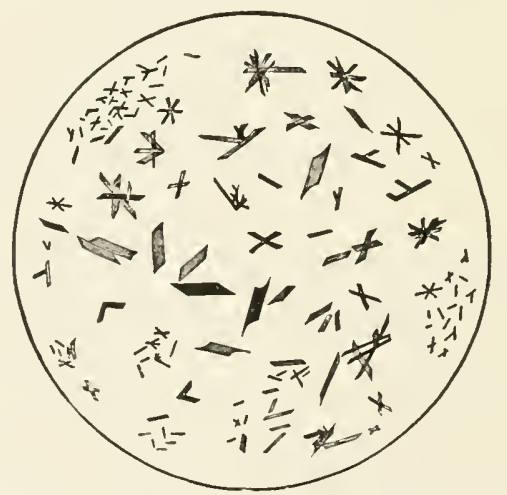

Fig. 232. Hemin Crystals. (After Preyer.)

in either water, alcohol, ether, chloroform or dilute acids : they may however be dissolved to some extent in glacial acetic or hydrochloric acids, especially if warmed, and are readily soluble in alkaline carbonates or dilute caustic alkalis, being at the same time decomposed by the latter solvent into hematin and a chloride of the alkali. This fact provides the best means for obtaining pure hæmatin.

A solution of hrematin hydrochloride shews an intense ab- 
sorption band in the extreme violet, the greatest absorption taking place between $h$ and $L$.

Although it is quite eas y to obtain typical crystals moler the microscope from minute amounts of hemoglobin or hæmatin, their preparation on a large scale is somewhat tedious.

The successful preparation of hrenin crystals from minute quantities of hæmoglobin or methrmoglobin is of the greatest importance for medico-legal purposes, since they snffice, even in the absence of all other confirmatory evidence, to establish the nature of the material used in their preparation. In the detection of blood-stains it is usual first to examine with a spectroscope an aqueous solution of the colouring matter if it can be obtained, for the characteristic absorption bands of oxyhremoglobin or methæunglobin. In old stains the hrmoglolin is frequently decomposed, in which case it is insoluble in water, and alkaline extracts must be marde and examined for the spectra characteristic of hrematin. The residnes from the spectroscopic examination are lastly used to prepare liamin crystals, in final confirmation of the evidence previously obtained.

Using amyl-alcohol in the preparation of hæmin erystals it is stated that the crystals have the following composition

$$
\left(\mathrm{C}_{32} \mathrm{H}_{30} \mathrm{~N}_{4} \mathrm{FeO}_{3} . \mathrm{HCl}\right)_{4} \mathrm{C}_{5} \mathrm{H}_{9} \text {. OH. }
$$

The group $\mathrm{C}_{32} \mathrm{H}_{30} \mathrm{~N}_{4} \mathrm{FeO}_{3}$ is regarded as the true hæmin, Teichmann's crystals consisting of $\mathrm{C}_{32} \mathrm{H}_{30} \mathrm{~N}_{4} \mathrm{FeO}_{3}$. $\mathrm{HCl}$. When the crystals thus prepared are decomposed by caustic alkalis as in the ordinary method for preparing hæmatin from them, the hæmin is supposed to take up one molecule of water and yield hæmatin, $\mathrm{C}_{32} \mathrm{H}_{32} \mathrm{~N}_{4} \mathrm{FeO}$. 'By treating this hæmatin with strong sulphuric acid, it loses its iron and nniting with oxygen yields hæmatoporphyrin or iron-free hæmatin, $\mathrm{C}_{32} \mathrm{H}_{32} \mathrm{~N}_{4} \mathrm{O}_{5}$, which is however further regarded as derived by dehydration from a true hæmatoporphyrin whose composition is $\mathrm{C}_{16} \mathrm{H}_{18} \mathrm{~N}_{2} \mathrm{O}_{3}$. The latter is thus identical in composition with bilirubin, whose formula is undoubtedly $\mathrm{C}_{16} \mathrm{H}_{18} \mathrm{~N}_{2} \mathrm{O}_{3}$. This is regarded as affording the desired chemical proof of the genetic relationship of the bile- and blood-pigments, the derivation of the former from hæmatin being represented as follows, $\mathrm{C}_{32} \mathrm{H}_{32} \mathrm{~N}_{4} \mathrm{FeO}_{4}+2 \mathrm{H}_{2} \mathrm{O}-\mathrm{Fe}=2\left(\mathrm{C}_{16} \mathrm{H}_{18} \mathrm{~N}_{2} \mathrm{O}_{3}\right)$.

\section{tin.) \\ 12. Hæmatoporphyrin. $\mathrm{C}_{32} \mathrm{H}_{36} \mathrm{~N}_{4} \mathrm{O}_{6}$ (?). (Iron-free hæma-}

If hematin is dissolved in concentrated sulphuric acid it yields a solution which, after filtration through asbestos, is of a brilliant purple-red colour. By the action of the acid, the iron is removed from the hrematin and hæmatoporphyrin is formed. If this solution is diluted with snlphuric acid it shews with spectroseope two absorption bands of which one adjoins $D$ to the red side of this line, while the other is very strongly marked 
and lies midway between $D$ and $E$. By the addition of water to the solution in sulphuric acid the colouring matter is largely precipitated, especially if some alkali be carefully added to neutralize the acid. 'The precipitate thus obtained is readily soluble in dilute alkalis, and this solution is characterized by four absorption bands, one half-way between $C$ and $D$, two between $D$ and $E$, and one conspicuons band adjoining $b$ and extending nearly to $F$. Acid and alkaline solutions of liæmatoporphyrin show an absorption band in the extreme violet between $h$ and $h$. Hrematin also yields hrmatoporphyrin by the action of strong hydrochloric acid at $130^{\circ}$ in sealed tubes.

Some interest attaches to this substance owing to its not infrequent occurrence in minute quantity in normal urine and in larger amounts in the urine of many diseases in forms which may shew slightly different absorption spectra but are probably closely related if not identical. It is stated to be markedly present in urine after the administration of sulphonal and has then been obtained in a crystalline form. It is also found in the integument of some invertebrates and in the egg-shells of certain birds. It is further interesting to notice that in hrmatoporphyrin we have a strongly coloured pigment derived from hrematin with removal of the iron which the latter contains, a fact which facilitates our conception of a possible derivation of the iron-free bile-pigments from the iron-containing hæmoglobin or hæmatin.

\section{Hæmatoidin. $\mathrm{C}_{16} \mathrm{H}_{18} \mathrm{~N}_{2} \mathrm{O}_{3}$.}

This substance is found as reddish or orange rhombohedral crystals in old blood-clots as of cerebral hæmorrhages, in corpora lutea, in the urine in cases of transfusion of blood and in

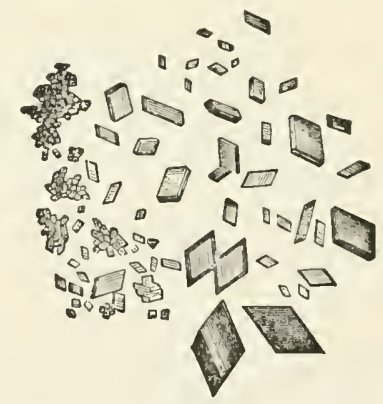

Fig. 233. Hamatoidn Cirstils. (Frey after Funke.)

cases of hæmaturia. There is no doubt that as occurring in the above cases it is directly derived from some metamorphosis of hæmoglobin. Apart from the similarity of crystalline form 
and colour it was further found that hæmatoidin erystals readily give the characteristic (Gmelin's) reaction for bilirubin by treatment with nitrie acid, and thus its identity with bilirubin was at once asserted and supported by very convincing evidence. The identity was however at one time disputed. There is however no doubt that hæmatoidin is really identical with bilirubin, so that now the name is of interest rather from a historical point of view, and physiologically as indicating the undoubted genetic relationship of the pigments of bile to those of blood.

\section{Bile-Pigments And their Derivatives.}

The bile is in all animals a characteristically lighly coloured secretion. 'The colour of the fresh bile is as a general rule golden-red in man and carnivora, and more or less bright green in herbivora. These colours are due to the presence of a pigment known as bilirubin in the first case and biliverdin in the second; but since the latter pigment may be readily formed by simple oxidation from the former, bile may frequently contain both these colouring matters and hence possess a colour intermediate to the above, though usually with a preponderance of either the golden-red or green shade. In addition to these two pigments others are occasionally present in bile, as evidenced by the fact that while neither bilirubin nor biliverdin exhibits any absorption bands when examined spectroscopically, fresh bile of herbivora ${ }^{1}$ frequently does shew bands, three or four in number, due to substances of which but little is known beyond these spectroseopic appearances.

\section{Bilirubin. ${ }^{2} \mathrm{C}_{16} \mathrm{H}_{18} \mathrm{~N}_{2} \mathrm{O}_{3}$.}

It occurs chiefly and characteristically in the fresh bile of man and carnivora, to which it imparts the well-known goldenred colour. It frequently constitutes the larger part of some kinds of gall-stones, more especially of the ox and pig, not as free bilirubin but as a compound with chalk, and amounting to some 40 p.e. of the concretions. It is also found in the urine in icterus, also constantly in the serum from horses' blood, though not from that of man or the ox, and frequently as crystals under the name 'haematoidin' (see p. 1302) in old bloodclots (extravasations) and fluids from ovarial and other cysts.

1 Bile of carnivora does not usually shew bands until it has been treated with an acid.

2 'This is the generally accepted formula. It is possible that the formula is really twice the above, viz. $\mathrm{C}_{32} \mathrm{H}_{36} \mathrm{~N}_{4} \mathrm{O}_{6}$ as required to represent the formula of a well-defined tribromo-substitution product, $\mathrm{C}_{32} \mathrm{H}_{33} \mathrm{Br}_{3} \mathrm{~N}_{4} \mathrm{O}_{6}$. This doubling of the formula is also necessary to express the derivation of hydrobilirubin $\left(\mathrm{C}_{32} \mathrm{H}_{40} \mathrm{~N}_{4} \mathrm{O}_{7}\right)$ from bilirubin. 
Bile-pigments are also stated to occur normally in the urine of logs, more particularly in the summer.

Bilirubin is insoluble in water and almost insoluble in either ether or alcohol, though distinctly more soluble in alcohol than in ether. It is on the other hand readily soluble in alkalinc solutions, hence its solution in bile, also in glycerin carbon-disulphide, and benzene, and above all in chloroform. From its solution in the latter it may be separated out by extrenely slow evaporation of the solvent in a crystalline form as rhombic plates or prisms. The general shape of these is shewn above in Fig. 233; but as obtained from solution in either carbon-rlisulphide or chloroform the crystals usually exhibit somewhat blunt ends and slightly convex surfaces. As

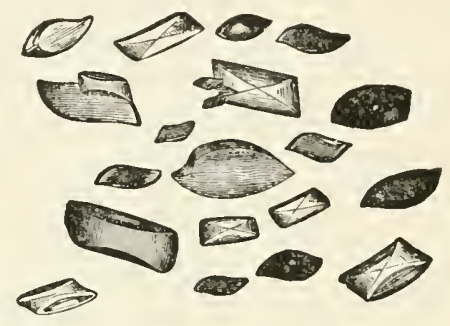

Fif. 234. Bilirebin crystallized from Carbon-disulphide. (Krukenberg.)

ordinarily prepared it is an amorphous powder of the colour of sulphicle of antimony. It rearlily forms compounds with bases, e.\%. sodium, barium and caleium, the latter providing a convenient means for the separation of bilirubin from bile, urine or other dilute solution.

When carnivornus bile is exposed to the air it turns more or less rapidly green; this is due to its oxidational conversion into biliverdin, the normal pigment of herbivorous bile. A similar change is at once produced by an oxidizing agent such as nitric acid containing nitrous acid, but in this case the chinge of colour does not stop short with green but passes successively throngh blue, violet and red to a final yellow. These later colours are due to products of the progressive oxidation of the first formed biliverdin, but with the exception of the final substance (choletelin), they are as yet but imperfectly characterized. The play of colours observed when either bilirubin or biliverdin is oxidized, constitutes the well-known Gmelin's reaction. This is extremely delicate and maty be applied in either of the two following ways. A few dops of the suspected solution are placed on a porcelain slab and a drop of yellow fuming nitric acid is bronght into contact with it. A filaty of colours is observed at the junction of the fluids if bile-pigments are present. Or on the other hand some of the 
acid may be poured into the bottom of a test-tube and the suspected fluid carefully adkled so as not to mix with the acid but float on its surface. If bile-pigments are present coloured rings (layers) appear at the junction of the two liquids, being yellow nearest the acid and progressively red, violet, blue and green passing upwards. It is stated that this test will detect as little as 1 part of bilirubin in 70,000-80,000 parts of solvent.

\section{Biliverdin. ${ }^{1} \mathrm{C}_{16} \mathrm{H}_{18} \mathrm{~N}_{2} \mathrm{O}_{4}$.}

This is, as already stated, the first product of the oxidation of bilirubin. It gives the characteristic colour to the bile of herbivora, probably accounts for the colnur of biliary vomit in carnivora (man), is possibly found in the urine in icterus, has been stated to occur in the edges of the placenti in pregnant animals (bitches), while on the other hand it occurs in mere traces in gall-stones whether of man or other animals. It has also been described as occurring in egg-shells and the integuments of certain invertebrates.

Apart from its colour biliverdin differs most characteristically from bilirubin in its solubilities. It is (like bilirubin) soluble in alkalis but insoluble in water and ether, whereas (unlike bilirubin) it is insoluble in either chloroform, carbonbisulphide or benzenc, but readily soluble in alcohol and in glacial acetic acid. It has further never been obtained in a crystalline form, and like bilirubin it shews no absorptinn bands but a somewhat strong absorption of the violet end of the spectrum. Treated with fuming (yellow) nitric acid it gives Gmelin's reaction, starting now with a blue colour as a product of the first stage of its oxidation.

\section{Bilicyanin. (Cholecyanin, Choleverdin.)}

This is the substance which results from the oxidation of biliverdin and is the cause of the blue colour observed when bile is treated with fuming (yellow) nitric acid as in Gmelin's reaction.

Bilicyanin is for practical purposes characterized solely by its marked absorption spectrum. This consists of three bands, - nne on each side of $D$, that to the red side of $D$ being the darkest, and one between $b$ and $F$. The latter is probably iclentical with the baud seen in acid solutions of choletelin and due to the production of this substance in small quantity during the nxidation of bilirubin. The position of the bands varies somewhat according to the solvent employed and as to whether the solution is acid or alkaline.

During the application of Gmelin's test for bile-pigments the

1 The formula here given should probably be doubled. 
blue due to bilicranin is bordered by a violet colour and this by a red, the final and permanent colour being yellow. Of these three the first is not as yet known to be definitely due to one specific substance; it is most jrobably the result of a mixture of the blue of bilicyanin with the red of the next product. The red colour is on the other hand supposedly due to a definite pigment sometimes called bilipurpurin, of which however nothing definite is as yet known. The yellow marks the final formation of choletelin.

\section{Choletelin. ${ }^{1} \quad \mathrm{C}_{16} \mathrm{H}_{18} \mathrm{~N}_{2} \mathrm{O}_{6}$. (?)}

This is the fimal product of the oxidation of bile-pigments. It is readily soluble in alkalis, as also in either alcohol, chloroform or etlier, but least so in the two last solvents. None of the solutions exhibit any fluorescence even after the addition of zine chloride. In this it differs markedly from urobilin, a well-known yellow urinary pigment. In alkaline solutions choletelin shews no absorption band; in acid solutions there is a distinct absorption of light, resulting in an ill-defined band, between $b$ and $F$.

\section{Hydrobilirubin. $\mathrm{C}_{32} \mathrm{H}_{40} \mathrm{~N}_{4} \mathrm{O}_{7}$.}

When bilirubin is dissolved in dilute caustic potash or soda or suspended in water and treated with sodium-amalgam in successive portions, air being at the same time carefully excluder, it is observed that at first no hydrogen is evolved; the dark-coloured solution becomes gradually lighter in colour and more transparent, until at the end of two or three days it is bright yellow or brownish-yellow, and now hydrogen begins to come off from the mixture. At this stage the supernatant fluid should be poured off from the metallic mercury which has accumulated, and if it is now acidulated strongly with either hydrochloric or acetic acid, it yields a more or less copious flocculent precipitate of a dark reddish-hrown colour. This precipitate is impure hydrobilirubin. When dried it takes the form of a dark reddish-brown amorphous powder, which is reatily soluble in alcohol and chloroform, and but sparingly soluble in pure ether. It is also very soluble in alkalinc solutions, to which it imparts a yellow colour as of normal urine: when acidulated the solutions tum red.

The acirl solutions of hydrobilirubin shew a marked absorption band between $b$ and $F$ which becomes fainter if ammonia is arded until the reaction is alkaline. But on the subsequent addition of a few drops of a solution of zinc chloride, the band reappears with usually increased intensity, thougl shifted slightly towards the violet end of the spectrum.

1 The formula here given should probably be doubled. 
This alkaline solution to which the zine salt has been added also shews, in marked distinction to the acid solntions, a brilliant fluorescence which is most characteristic of the substance, being of a bright rosy-red colour by transmitted and bright green by reflected light.

Previously to the discovery of hydrobilirubin, a well-characterized urinary pigment had been isolated and described under the name of urobilin (see below), while about the same time a pigment had been obtained from freces and described, under the name of stercobilin, as identical with urobilin. Careful comparison of hydrobilirubin with urobilin has led to the view of the elose relationship if not identity of the two substances. This view has been most generally adopted and is correct as a broad statement of facts. When hrematin in acid solution is reduced by means of tin and hydrochloric acid it assumes a pale yellow colour and a substance is obtained which is characterized by an absorption band between $b$ and $F$. A similar result follows when hrmatoporphyrin is treated in the same way. The product to which the absorption band is due may be regarded as practically identical with urobilin or hydrobilirubin, and if this be so we have here the best and most direct chemical evidence of the relationship between the colouring matters of the blood and bile. For if one and the same substance, viz. urobilin, can be prepared by the same means, namely reduction (hydrogenation) from both hematin (hemoglobin) and bilirubin, these two substances must be themselves closely related. It has not however as yet been found possible to prodnce a bile-pigment directly from hemoglobin or hematin by any artificial process outside the animal body. Solutions of hremoglobin when injected into the subcutaneous tissue of the horse become after a few days partially converted in situ into granules and flakes which are of a yellow or orange colour and yield an intense Gmelin's reaction. Finally by the action of phenylhydrazin on hæmatin and on bilirubin products are obtained which in each case exhibit a similar and marked play of colours under the action of fuming (yellow) nitric acid.

\section{The Pigments of Urine.}

\section{Urobilin. $\mathrm{C}_{32} \mathrm{H}_{40} \mathrm{~N}_{4} \mathrm{O}_{7}$. (?)}

This is the best known and most definitely characterized of the urinary pigments. In fresh normal urine the amount is frequently extremely small, but increases on standing exposed to the air (oxygen), a result due to the probable presence in the urine of some chromogen or mother-substance of the urobilin. 
A solution of urobilin may be readily obtained as follows. Urine is made alkaline with ammonia and then saturated with nentral anmonium sulphate. The precipitate thus formed is collected on a filter freed by pressure from adhering fluid, dried in the air till free from ammonia and extracted with absolute alcohol. This solution is yellowish-brown or yellow if more dilute, and shews a strong green fluorescence. If made acid with liydrochloric acid the colour deepens and the fluoreseence disappears. Alkaline solutions are yellow or yellowish-green, according to the concentration. The fluorescence is usually increased by the addition of zine chloride, the solutions appearing rose-coloured by trinsmitted light and bright green by reflected.

Spectra of urobilin. Neutral or alkaline alcoholic solutions shew one absorption band between $b$ and $F$. In alkaline solution the band is frequently very faint, but may be more strongly marked after the addition of zinc chloride. In acid solutions a similar band is seen, situated however in this case slightly more towards the violet end of the spectrum.

In fevers the urine contains additionally a colouring substance to which the name of febrile nrobilin has been given. It is closely related to normal urobilin, but differs in the red colour of its acid alcoholic solution as compared with the yellow of ordinary urobilin, and this solution shews an absorption band in the yellowish-green of the spectrum on the red side of the line $E$ :

The colouring power of urobilin is inconsiderable, hence the normal colonr of urine is chiefly due to some other pigment of which as yet but little is definitely known. It has been called urochrome.

\section{Uroerythrin.}

This is a pigment of which but little is known. It is regarded as the colouring substance of certain bright red (pink) nrinary deposits and as possibly occurring in the highly coloured urines of rheumatism, etc. It appears to be an anorphous reddish substance, with an acid reaction, slowly soluble in either water, alcohol or ether, readily soluble in amyl-alcohol. Treated with eanstic alkalis it turns green, more particularly when in the solid form. In alcoholic solution obtained by boiling pink urates with alcohol it shews two ill-defined absorption bands between $D$ and $F$.

\section{Urohæmatoporphyrin.}

This pigment is described as occasionally occurring in certain pathological urines as of acute rheumatisin, Addison's disease, etc., and receives its name from certain resemblances of 
its spectra to those of hematoporphyrin. It is obtained from urine by the method employed for the separation of urobilin, or artificially by the action of reducing agents on hematin, this being the supposed source of its origin in the body. It is soluble in either alcohol, ether, benzene or chloroform. In acid alcololic solution it shews three absorption bands, one narrow arljoining $D$ on the red side of this line, one half-way between $D$ and $E$ and one between $b$ and $F$ closely resembling the band of urobilin. There is also occasionally a fourth very faint band between the first two bands described above. In alcoholic solution made alkaline by ammonia it yields a spectrum elosely resembling that of hematoporphyrin. But unlike the latter substance its solutions shew a very faint green fluorescence on the addition of zinc chloride and ammonia. 'The occurrence of hæmatoporphyrin in urine has been frequently recorded, and from the spectroscopic appearances described above, some observers are inclined to the view that mohrmatoporphrin is not a single substance but a mixture of hæmatoporphyrm with some pigment closely resembling urobilin.

\section{Humus pigments.}

When carbohydrates are treated with acids or alkalis, among the numerous products which arise are certain pigmentary bodies of a more or less dark brown colour. A similar coloration is well known as occurring in fruits when bruised or exposed to the air, and generally in decaying vegetable tissues. These substances are known under the name of 'humus.' When urine is treated with acids in presence of oxygen it acquires a markedly darker colour, and since carbohydrates in small amount are probably present in all urines, there is at once a possibility that some at least of the observed coloration is due to the production of humus-pigmented substances by the action of the acids on the carbohydrates. In accordance with this view certain so-called humus pigments have been prepared from urine, but our knowledge of them is as yet very incomplete. They are stated to be practically insoluble in any solvents other than amyl-alcohol, strong ammonia and caustic alkalis: the solutions shew no absorption bands when examined spectroscopically. They are further said to account for the usually dark colour of normal herbivorous urine and of urine after the cutaneous absorption of carbolic acid and several other aromatic compounds.

\section{Urinary melanin.}

Certain tumours are not infrequently observed which from their extremely dark pigmentation are spoken of as 'melanotic,' 
the colouring substance being known as melanin. ${ }^{1}$ The urine of patients suffering from these tumonrs is either dark brown or black when voided or speedily assmmes this colour after brief exposure to the air or by the action of nitric acid or other oxidizing argents, the pigment to which the colour is due being apparently identical with that present in the tumour. This aetion of oxidizing agents indicates that here also, as in the case of other urinary pigments, there is primarily some chromogenic forermmer (melanogen) of the actual pigment. This chromogen as also the fully formed pigment may be partially precipitated by baryta-water, the remainder being precipitable by the addition of normal lead acetate. The purified pigment is partly insoluble, partly soluble in acetic acid of $50-75$ p.c. Of these portions the former when dried is a brownish-black amorphous powder, insoluble in either water, alcohol, ether, chloroform or dilute (mineral) acids, but readily soluble in alkalis. The latter has been obtained in too small amounts to admit of complete investigation. Some melanins may contain iron ( $\cdot 2$ p.c.) and some a considerable amount of sulphur (9 p.c.) and none shew any absorption bands when their solutions are examined spectroscopically.

When melanotic urines are treated with oxidizing agents or solutions of ferric chloride, they yield, according to the concentration of the reagent, either a dark brown cloudiness or else a black precipitate soluble in excess of the precipitant: this test is both delicate and characteristic.

\section{Indoxyl-pigments.}

Of the total indole formed in the alimentary canal, a portion is excreted with the freces, while the remainder is absorbed and reappears in the urine united with potassium as ethereal comlounds of indoxyl with either glycuronic acid (p. 1220) or sulphuric acid (p. 1281), the latter being known as urinary indican. When warmed with hydrochloric acid these componnds are dccomposed, yielding indoxyl and the potassium salt of the corresponding acid. If the decomposition is effected in the absence of oxygen, the indoxyl may be in part gradually ehanged into an amorphous reddish sulsstance, indigo-red, which is insoluble in water, but yields a red solntion when dissolved in alcohol, ether or chloroform. These solutions shew no eertainly eharacteristic absorption bands. In presence of oxygen and with most ecrtainty by the action of an oxidizing agent, the indoxyl is readily converted into indigo-blue, whose propertics and solubilities have been already sufficiently described (p.1282).

1 The name melanin is more usually applied as a generic title for the dark brown or black pigments such as occur in the hair, epidermis, retinal epithelium, choroid, etc. 


\section{Skatoxyl-pigments.}

The skatole formed in the alimentary canal gives rise, like indole, to compounds of skatoxyl with either sulphuric acid or glyeuronic acid (see p. 1220). These compounds when decomposed by hydrochloric acid or oxidizing agents give rise to a colouring matter which is more or less red and may exhibit a distinct and strong purple tint. The pigment is insoluble in water, but soluble in either alcohol or chloroform, also when freshly prepared in ether but less so if it has been kept some time. Alcoholic solutions are of a reddish-violet colour; ethereal solutions may shew a green fluorescence, which on exposure to the air takes on a reddish tinge. It is also soluble in hydrochloric and sulphuric acids, giving bright red or pink solutions, and in alkalis yielding yellow solutions. No absorption bands for this substance have as yet been described and the whole subject requires further investigation.

The urinary pigments so far dealt with may be regarded as either normal or pathological, or as resulting from the spontaneous or artificial decomposition of urinary eonstituents which are at the outset colourless. In addition to these, other colouring substances are not infrequently observed, or colour-reactions obtained, in urines passed after the administration of certain drugs or the consumption of certain vegetable tissues. They are in nany cases not unimportant as leading at first sight to possibly erroneous conclusions as to the presence in urine of pathologically important pigments, e.g. of bile or blood. After the administration of rhubarb or senna the urine may be yellow or greenish-yellow, due to the presence of chrysophanic acid $\left[\mathrm{C}_{14} \mathrm{H}_{5}\left(\mathrm{CH}_{3}\right)(\mathrm{OH})_{2} \mathrm{O}_{2}\right]$, and similarly after the use of santonin $\left(\mathrm{C}_{15} \mathrm{H}_{18} \mathrm{O}_{3}\right)$. In such cases if the urine is strongly alkaline it may be of a red colour; this is changed to yellow on the addition of hydrochloric acid, and if it is initially acid, it turns red on the addition of an excess of alkali. After the internal administration of copaiba, the urine turns pink or rose-coloured on the addition of hydrochloric acid and shews three absorption bands, one (narrow) in the orange to the red side of $D$, one broad band in the green between $D$ and $E$, similar to that of fuchsine, and one in the blue. Tammin leads to the appearance in urine of gallic acid $\left[\mathrm{C}_{6} \mathrm{H}_{2},(\mathrm{OH})_{3} . \mathrm{COOH}\right]$, which is hence sometimes found normally in the urine of herbivora (horse). In such cases the urine, if made alkaline with caustic potash, turns brown, and bluish-black on the addition of ferric chloride. It also vields a pink coloration with Millon's reagent, similar to that given by proteids or tyrosine. After doses of antipyrin $\left[\mathrm{C}_{9} \mathrm{H}_{6} \mathrm{~N}_{2} \mathrm{O}\left(\mathrm{CH}_{3}\right)_{2}\right]$ the urine may be dark-coloured and gives a brownish-red colour on the addition of ferric ehloride. Fuchsine (hydrochloride of rosaniline, $\mathrm{C}_{20} \mathrm{H}_{19} \mathrm{~N}_{3} . \mathrm{HCl}$ ) reappears partly unchanged in the urine, to which it imparts a reddish tinge. It is detected by making the urine alkaline with ammonia and shaking with an equal volume of ether: the latter extracts the colouring matter and into the solution thus obtained a thread of white wool is dipped and allowed to dry spontaneously. 
If fuchsine is present the wool is stained red. Salicylic acid (ortholiydroxybenzoic acid, OH. $\mathrm{C}_{6} \mathrm{H}_{4}$. COOH) is exereted partly in an unaltered form, partly as salicyluric acid, OH. $\mathrm{C}_{6} \mathrm{H}_{4}$. $\mathrm{CONH}$. $\mathrm{CH}_{2}$. $\mathrm{COOH}$. 'These may be detected by the intense violet colour they yield on the addition of ferric chloride. Finally, after the absorption of carbolic acit (phenol) and many other aromatic compounds such as pyrocatechin, hydroquinone, etc., the urine turns greenish-brown and finally dark brown on exposure to air.

\section{Retinal Pigments.}

\section{Fuscin (Retinal melanin).}

This pigment is found as minute granules imbedded in the cell-substance and processes of the retinal epithelium (see § 576). These granules may be either irregular, as they always are in the choroid, or may, especially as in birds, possess an elongated form with sharply pointed ends distinctly suggestive of a crystalline structure. It is obtained by extracting the tissues with boiling alcohol, ether and water, and then digesting for some time with trypsin. The residue is freed from nucleins by dissolving the latter in caustic alkalis and from nenrokeratine (p. 1204) by decantation and straining though fine gauze. The pigment when freshly prepared is practically insoluble in all ordinary reagents, but is partially dissolved if boiled for some time with strong caustic alkalis or sulphuric acid. By prolonged treatment with dilute nitric acid it becomes soluble in alkalis, yielding yellow solutions. It becomes similarly soluble by prolonged exposure to light with free access of air (oxygen), and may be again precipitated from these solutions by the addition of an acid. It is remarkable that notwithstanding its extreme insolubility and resistance to the action of most reagents fuscin is gradually bleached by exposure to light, a result due to some oxidational change, since it only occurs in presence of oxygen. The product to which the above description refers contains much nitrogen, and leaves on incineration a slight ash-residue containing traces of iron.

Later investigations of the pigment (from the choroid and iris) confirm the above statements of its insolubility in most reagents, and further shew that it contains neither sulphur nor iron. The black pigment from hairs is stated to contain less nitrogen and a not inconsiderahle amount of sulphur but no iron, and to be readily soluble in alkalis.

\section{Lipochrin.}

The fat globules in the retinal epithelium from which this

1 The pigments of the retinal epithelium and choroid are apparently identical. 
pigment is obtained are more especially abundant in the frog. It is soluble in chloroform, ether, benzene, carbon-sulphide, etc. When dissolved in ether it gives two absorption bands between $F$ and $G$; in carbon-bisulphicle two bands, one each side of $F$. The pigment of the body-fat of frogs gives similar absorption spectra when dissolved in the same solvents. Solutions of lipochrin are slowly bleached by exposure to a strong light. The pigment is probably closely allied to the yellow colouring matter of many other animal fats.

\section{Chromophanes.}

These are the colouring substances of the red, green and yellow fat-globules which occur in the outer end of the immer limbs of the retinal cones. They are prepared, as yet, chiefly from the eyes of hirds, as follows. The retinas are deliydrated with alcohol and extracted with ether. 'The ethereal solntim of the fats is then evaporated to dryness, the residne dissolved in hot alcohol and saponified with canstic soda. The hard coloured soaps thus obtained are then extracted in succession with petrolenm ether (see note p. 1272). ether and benzene: of these solvents the first dissolves out the yellowish-green chlorophane, the second the yellow xanthophane and the third the red-coloured rhodophane.

(i) Chlorophane. Soluble in petroleum ether, ether, carbon-bisulphide and in alcohol. When dissolved in the first two of these solvents it shews two absorption bands between $F$ and $G$; in solution in the latter, the two bancls lie one each side of $F$.

(ii) Xanthophane. Soluble in ether, carbon-bisulphide and in alcohol. In ethereal solution it shews only one absorption band near $F$ towards the blue end of the spectrum. In earbon-bisulphide it shews similarly one band near, and to the blue side of $b$. It is thus distinguished from the yellow pigment (lipochrin) of the retinal epithelium previously describer.

(iii) Rhodophane. Soluble in turpentine, benzene and in alcohol. In benzolie solution it shews one bancl close to, but on the red sirle of $F$; in solution in turpentine the band is similarly near, but now on the blue side of $F$.

Solutions of the chromophanes are slowly bleached by the action of light, chlorophane losing its colour fairly rapidly. xanthophane more slowly and rhodophane only after prolonger exposure.

\section{Visual-purple (Rhodopsin).}

Its distribution in the retina has been sufficiently deseribed in $\$ 576$.

Preparation in solution. The most suitable material is 
afforded by the retins of frogs which have been kept in the dark for two or three hours, since in these animals not only is the visual purple very marked and somewhat persistent inder the action of light, but further the retina can be scparated from the adjacent epithelium with great ease and is free from blood. The necessary operation for the removal of the retince, as alio all subiequent manipulations, must be carried on in a feeble light from a sodium flame to avoid bleaching. The retine (20-30 suffice) are then extracted for an hour in the dark with about 1 c.c. of a freshly-prepared $2-5$ p.c. solution of bile salts from ox-bile, which is tinally filtered. If brought into daylight and examined the solution is seen to possess a brilliant pinkish-purple colour, which rapidly becomes red, vellow and finally colourless under the action of light. A similar initial colour is observed in the retina in situ, followed by the same change of colour when exposed to light, the yellow being regarded as due to a 'visual yellow' (xanthopsin), and perhaps the final colourless stage, since it admits of regeneration in the dark into visnal purple if the retina is fresh and in contact with its epithelium (see $\$ 576$ ), may be spoken of as a 'visual white' (leukopsin).

Spectroscopic properties. Neither visual purple nor visual yellow gives any distinct absorption band; there is a general absorption of the central parts of the spectrum easily seen between $E$ and $G$ in the case of visual purple, which changes into a general absorption of the violet end of the spectrm from $F$ onwards as the purple changes into yellow and finally disappears altogether.

Action of light. White light, as also that from an electric lamp or magnesium flime, bleaches visual purple with extreme rapidity, dependently upon the intensity of the illumination: direct sunlight destroys the colour almost instantaneously. When monochromatic light (of the spectrum) is used it is found that the yellowish-green, i.e. the region most strongly absorbed by the pigment, is most active, followed seriatim by green, blue, greenish-yellow, yellow, violet, orange and red: the ultra-red rays have no such bleaching power. At low temperatures the effect of light is less. increases with rise of temperature, and at $75^{\circ}$ the colon is destroyed even without exposure to light.

Action of reagents. The colour is at once destroyed by the action of caustic alkalis, most acids, alcohols, chloroform and ether: it is on the other hand persistent in presence of ammonia, solutions of ordinary alum, of sodium chloride, carbonates of the alkalis and a large number of other salts. One of the most important factors in determining the bleaching of visual purple by either light or heat is the presence or abscnce of water. If the entire retina be dried in vacuo over sulphuric 
acid, or if a solution of the pigment be similarly evaporated to dryness, the visual purple is comparatively resistent to the action of light, although it is bleached by a sufficiently prolonged exposure.

\section{Lipochromes OR Lutervs.}

After the rupture of the ovarian follicle which accompanies the discharge of an ovum, the cavity of the follicle becomes filled with a mass of cells, traversed by ingrowths of connective tissue from the neighbouring stroma and frequently contains blood resulting from hæmorrhage at the time of rupture. This is followed, most strikingly if impregnation of the discharged ovmm takes place, by a fatty degeneration of the contained cells, resulting in the formation of a bright pigmented mass of a brilliant yellow or orange colour, while at the same time some of the colouring matter of the blood may be converted into that crystalline substance already described under the name hematoidin (1. 1302) as being identical with bilirubin. The structure which results from the above changes is known as a 'corpus luteum.' The pigment at first received the name lutein, under which designation as a class-name these fatty pigments have usually been known. Since, howerer, as we have alrearly seen in the case of the chromophanes, and as will appear subsequently in the case of the pigments of egg-yolk and of the substance tetronerythrin, we have to deal with pigments which while they give the reactions characteristic of the group exhibit colours other than yellow, it is perhaps advisable now to use the term 'lipochrome ' as generic and to retain lutein as specific for certain yellow pigments only. The lipochromes are characterized by exhibiting absorption bands, which though varying somewhat in position according to the solvent employel, are usually situated towards the violet end of the spectrum. They are characterized chemically by giving (i) a transient violet colour followed by a bright blue when treated with concentrated sulphuric acid. (ii) A transient bluish-green under the action of strong (yellow) nitric acid.

\section{Lutein.}

This pigment may be obtained from corpora lutea by extraction with chloroform. It is insoluble in water but readily soluble in alcohol, ether, chloroform and benzene. These solutions exhibit two absolption bands, one inclosing $F$, the other about half-way between $F$ and $G$.

If egg-yolk be extracted with a little alcohol and much ether, the solution shews two bands similar to those already described for lipochrin or frog's fat (p. 1312), while sometimes 
a third faint band near $G$ may be seen, espeeially if the residue from the ethereal extract be clissolved in carbon-bismlphicle and examined. If the residnes from the ethereal extratts of egg-yolk and corpora lutea be saponified and extracted with carbon-bisulphide, the solutions yield identical absorption spectra.

\section{Serum lutein.}

The sermm from the blond of almost all animals is usually of a more or less yellow colonr; it is specially marked in the case of the horse and ox, is also marked in the case of sheep and man, aud is but slightly present under normal conditions in the serum of the dog, rabbit or cat. The colour has by different observers been ascribed to different pigments. In some cases it may be due, at least partly, to the presence of bile-pigments or their derivatives, these being much increased in certain rliseases such as jaundice. But in addition to these it appears that the colour of all pigmented serums is due to a specific pigment, which while it may differ (?) slightly as obtained from the blood of different animals, belongs in each case to the general class of substances known as lipochromes. By shaking serum with ethyl or amyl alcohol a coloured extract is obtained which contains a fatty pigment, evidently belonging to the class of lipochromes as judged by the fact that it is soluble in aleohol, ether, chloroform, benzene, carbon-bisulphide, etc., shews the two (in the case of birds only one) bands in the blue part of the spectrum, and gives the chemical reactions (p. 1315) with nitric acid and sulphuric acid characteristic of these substances. It is in many cases identical with the pigment which can be extracted from the fat of the animal from whose blood the serum was obtained. Serum lutein is blcacled by the action of light.

\section{Tetronerythrin.}

This name was first given to a substance extracted by chloroform from the red excrescences over the eycs of certain birds. It was described later as occurring in some sponges, fishes and feathers. More recently it has been found as a pigmentary constituent of the blood of crustacea. The pigment is readily soluble in alcohol, ether, chloroform, benzene and carbonbisulphide, is readily bleached by light, yields the chemical reactions with sulphuric acid and nitric acids which are chatracteristic of the lipochromes (see p. 1315), like these shews an absorption band near $F$ somewhat similar to that of xanthophane and rhodophane, and is slowly bleached by the aetion of light. 
In conclusion it must suffice to describe two pigments which do not naturally fall under any of the above groups into which these substances have been divided.

Pyoryamin. Pus, which ordinarily presents a more or less bright yellow colour, is frequently greenish and sometimes blue. 'The blue colour is due to a pigment (pyocyanin) which is apparently formed in the pus by the action of specific organisms. It is olstained either from pus or the bandages into which it has been absorbed by extraction with dilute alcohol or with water to which a trace of ammonia has been arlded. The aleoholic extract is then evaporated to a small bulk and the residue extracted with chloroform, or it may be extrated at once from the aqueous solution by shaking with chloroform. It may be obtained in a crystalline form by slow evaporation of the chloroformic solutions, the crystals being readily soluble in water and alcohol, but only slightly in ether. Acids change the blue colour to red and alkalis restore the original blue. None of the solutions shew any distinct absorption hands. When kept the crystals turn greenish, due to a deconposition which takes place most readily in alkaline solutions exposed to the air and light, and results in the formation of a yellow pigment, pyoxanthose. The latter' is, unlike pyocyanin, only slightly soluble in water but readily soluble in ether, by which property the two pigments admit of being separated. Pyoxanthose is crystalline, soluble in alcohol and chloroform, is coloured red by acids and violet by alkalis. Since pyoxanthose appears to be a product of the decomposition of pyocyanin. both pigments may occur simultaneously in pus, in which case the fluid is green. According to some more recent observations pyocyanin as judged of by its reactions with the chlorides of grold and platinum and with other alkaloidal precipitants, as also from the formation of crystalline compounds with acids, is closely related to the alkaloids.

Sweat is also occasionally coloured blue, in some cases by indigo-blue (p. 1282) as in urine, and it nay be (?) by a pigment similar to pyocyanin.

Pigment of the suprarenal bodies. A suprarenal body when a section is male through it is found to consist of an outer or cortical portion, of a yellow colour, which constitutes the chief part of its structure, and an inner, medullary part of a darker colour. When the latter is acted upon by ferric chloride it assumes a dark bluish- or greenish-black colour, and if an aqueous extract of its substance (or the tissue itself) be treated with an oxidizing agent it turns red. It appears therefore that the suprarenals contain some form of chromogen or pigmentforerumer which gives rise under appropriate conditions to a pigment. According to some observers extracts of the cortex shew a spectrum similar to that of the histohamatins 
(p. 1298), while the medulla gives one resembling hremochronogen. The pigment is obtaimable from the suprarenals as a brownish-red substance with an acid reaction, soluble in water and alcohol, but neither of the solutions shew any distinet absorption bands. The whole subject requires further investigation, which might be of interest in connection with the origin and causation of the increased pigmentation of the skin observed when the suprarenals are diseased. 


\section{INDEX.}

Aberration, spherical, 871; chromatic, 873 Abscissa line, mode of measuring curves on (footnote), 186

Absorption from alimentary canal, 398, 412

Achroodextrin, 315

Acid-albumeu, formation of, 19, 88, 324

Acid, benzoic, an antecedent of hippuric acid, 539 ; renal action on, 540 ; butyric, etc., in fat, 606

- carbonic, clotting retarded by its presence, 21; development of, in rigor mortis, 91, 92; set free during muscular contraction, 94,637 ; in expired and inspired air, 440 ; amount of daily excretion of, 441, 624 ; percentage of, in expired air, 442, 466; its relations in the blood, 461; causes of its escape from the blood, 466 ; its exit from the lungs, 465 ; in the blood, effect of excess of, 487 ; thrown off by the skin, 552 ; lessened 'output' of, in sleep, 1155

-, cholalic, 587; hippuric, chemical composition, 517; how formed in kiduey, 539

- - hydrochloric, in gastric juice, 352 ; lactic in the blood, 48

_- lactic, isomeric variations of (footnote), 90 ; its effect on the heart, 259 ; fermentation, 394

—, uric, chemical composition, 516 ; a result of special metabolism, 596

Acids, fatty, 361, 390; in milk, 613; in sebum, 551

- , organic, in urine, 518 ; various, in spleen-pulp, 583

Actiou, currents of, $100,102,107,108,753$

- peristaltic, in plain muscular tissue,

133 ; of the stomach, 377 ; of intestine,

383 ; increased in asphyxia, 386; of ureter, 532,544 ; of bladder, 546

—, reflex, 144-14ti, 548 ; purposive nature

of, 146; of spinal cord, 696-710

-, automatic, 146,711

Addison's disease, 602
Adenoid tissue in lymphatic glands, 42; multiplication of leucocytes iu, 42,43

Adipose tissue, structure of, 604

After-birth, formation of, 1120

After-images, 882 ; negative and positive, 934

After-pains, services affected by, 1139

Age, vascular changes due to, 299; old age, phenomeua of, 1151

Air, tidal and stationary in lungs, 425; complementary, supplemeutal, and residual, 426 ; expired air, changes of, 440,443

Albumin, acid and alkali, 19, 20, 88

Albumose, clottiug prevented by, 29 ; distinguished from peptone, 325

Alcohol, chauges in proteids produced by, 24 ; physiological action of, 304 ; its probable action on cardiac tissues, 306,307 ; use in diet, 662

Alimentary canal, vaso-coust rictor nerves of, 279,368 ; structure, 311 ; circular and longitudiual coats of, 371, 372; spoutaueous movements of, 383-385; nerve supply to coats, 384,385 ; peristaltic action, 383 ; mutually destructive juices of, 392 ; absorption from, 398,417

Alkaloids, vegetable, their kinship to urea, 654

Allantoin, 1132

Allantois, growth of the, 1120

Altitudes, high, diminished oxygen pressure in, 464

Alvergniat's gas-pump, 445

Amblyopia, 789

Ammonia, mode of conversion into urea, 595,596

Amnion, true and false, 1120, 1126

Amniotic fluid, 1126 ; function, 1127 ; composition, 1131

Amobæ, characteristies of, 3-7

Amœboid movements of white corpuscles, 36, 40, 137, 292

Amphibia, double vaseular supply to kidney, 533 
Ampulla of the semieircular eanals, 732

Amylolytic action of sitiva, 317

Anabulic changes of living substance, 39

Anarotic pulse, nsually patloologiral, 230,$236 ;$

Anamia, lessened number of red corpuscles in, 3:3; respiration impeded by, 510

Anelectruns, 113; its relation to irritability, 117

"Animal stareh," $\pi t$

Animals, cold-blouterl, temperature of, 641 ; warm-blooded, temperature of, (i)1

Amulus of Vieussens, 247, 253-256

Anole, the, 5i

Aorta, proportion of sectional area of eapillaries to the, 151; eomparative blood-pressure in, 193, 203-210, 236.

Aortic valves, 189,207

Aphasia, connection of, with cortical lesion. 766, 769; phenomena of, 770

A punoa, how produced, 459,490

Aqueous humour, $97: 2$

Area, cortical motor, in $\operatorname{dog}, 710$; in monkey, 74t; in anthropoid ape, 748 ; in man, 767; mode of actiou in, 769; for movements of the eyes, $7 ! 0$; for speech, 766; 108t; for movements of laryıx, 10st; for respiratory movements, 10st

-, cortical, for vision, 791 ; for smell, 794 ; for cutaneous sensations, 799

_, diabetic, 575 ; visual, 889 ; tactile, 1010

Aristotle, experiment of, 1069

Arterial pressure, 15.; see also Blood, pressure of ; tracings of , 158, 159, 168, 1199; heart-beat in inverse ratio to, 261 ; as affected by tonic contraction, 26t; by quantity of blood, 297; by exereise, 304; vaso-motor action on, $275,276,283$

- scheme, model of, 167 ; tone, 264

Arterialization, effect of deficient, 509

Arteries, effect of ligature on, 28, 154; elasticity and contractility of, 151, 166; 245 ; pulse in, 15i3, 161, 180; changes of calibre in, 221, 262; supply of vasomotor nerves to, 262, 275; effect on blood-pressure of their contractility, 276 ; as affected by age, 299,1152 ; of the brain, 827 ; of the eye, 969 ; renal, 5:5; renal, in amphibia, 533; radial, 273-271; umbilical, 11:24; umbilical, venous blood in the, 1125; helicine, in erectile tissne, 1116 ;

Artificial pulsc, tracings of, 168, 223

Arytenoid cartilanes, $11 \% 6$

Ash of nutuscle, silts in, 93; of nerves, salts in, 106
Asphyxia, phenomena of, 48., 4.91; increased peristaltic action in, 386

Astigmatism, 861

Ataxy, locomotor, 807

Atroply, acute, of liver, decrease of urea in, $5 ! 14$

Atropin, cardiac inhibition counteracted by, 2̌- 5 ; salivary secretion al'rested by, 338 , 351 ; its action on sweating, 556 , 557 ; its effect on the pupil, 867 ; on accommodation, 868

Auditory sensatious, 998 ; impulses, 1007 ; atulitory epithelium, structure, 101:; anditory hairs, 1009

Aura, forms of, preceding epileptiform attacks, 795

Automatic action, 146,711

Axis-cylinder process, in nerve cells of spinal cord, $1+3$

Babe, the, composition of as compared with adult, 114t; digestive processes of, 1146 ; nervous system of the, $114 \mathrm{~s}$

Bacteria, ingestion of by white corpuscles, 44; results of their presence in alimentary canal, 361,393

Bacterium photometricum, its reaction towards light, 921

Banting, his metlod of reducing fatness, (6)!)

Basis, molecular, of chyle, 403

Bat, the gastric glands of, $3 \pm 6$

Beats, in musical sounds, 1005

Bechive, temperature of, (it1

Bidder's ganglia in heart of frog, 243

Bile, characters and composition, 354; secretion of, 366,367 ; action on food, 357 ; oll fats, 3.92; antagonistic to peptic action, 358, 393; storage of in gall-bladder, 367: resorption of under pressure, 370 ; osmotic passage of fat facilitated by, 417 ; relation of to formation of urea, 589; toetal sceretion of, $i 130$

Bile-acids, the;r formation, 586

Bile pigmenta, 36 , 356, 581; salts, 356

Bilin, its eomjersition, 357

Bilirubin, its relations with hamatin, 34 , 584 ; composition of, 356 ; formation of, 586

Biliverdin, its composition, 356

Birtli, changes of the lungs at, 427 ; changes of circulation at, 1134

Bladder, muscles of,, 7.5

Blastodermic resicle, 1123

blind spot of retina, 916

Blindness, total and partial, 788

Blood, the, 12-50; an internal medium of interchange, 13, 149: clotting of, 1530; circumstances affecting clotting, 20 ; causes of 'lotting, 26 ; its relation to vascular walls, 27, 294; colpuseles 
(see Corpuscles): laky blood, how formed, 31, 450 ; results of injection of, 538 ; chemical composition of, $46-$ 48 ; specific gravity of, 46 ; quantity aud distribution of, 49, 50; in a part, morle of measuring, 270; results of changes in, 278; rate of flow in ressels, 172, 177, 178; its lependence on vaso-motor action, 275 ; amouut driveu by each heart-beat, 153,217 ; time occupier by one circnlation, 218; quality of, its effect ou heart-beat, 258 ; its effect on peripheral resistance, 294; as affected by exercise, 304; by deficient aeration, 457 ; quality of, in infant, 1147

Blood, the, odema due to changes in, 411 ; respiratory changes in, $43-461$; gases of, 447; how measmred, 445 ; entrance of oxygeu iuto by diffusiou, 42t; exit of carbonic acid from, 466 ; relations of oxygen in, 447; of carbouic acid in, 461 ; of nitrogen in, 461

- , vellous, colour of, 455 ; spectrum of, 455; an excitaut of respiratory centre, 484; its slowing effect on heart-beat, 504; in umbilical arteries, 1125

of percentage of sugar in, 572; course of, in foetus, 1132; circulation of (see Circulation) ; platelets, 44 ; in relation to inflammation, 292

- pressure, arterial and venous comparcd, 153-161, 166; in arteries, $153-$ 161; how measured, 154 et supra: in rcins, 155, 159; mode of registering, 156; in capillaries, 160-163, 166; phenomena of, 163; its relation to peripheral resistance, $163-170$; artificial scheme of, 167-170; endocardiac, 191197 ; aortic and ventricular compared, 203-210, 236: negative, 217 ; as affected by cardiac inhibition, "251; by stimulation of depressor, 281; of sciatic, 282; by action of drugs, 295; by changes in amount of blood, 296,297 ; by respiration, 498

- , in the kidneys, 525, 529, 532; in the brain, 829; in umbilical vein and artery, 1124

- , serum, constituents of, 19,20

- , supply, its influence on muscular irritability, 128

- vessels, their influence on fluidity of blood, 27, 28

Blushing, its cause, 286, 303

Body, the, characteristics of, in life and deatl, 1, 2 ; a verage composition of, 619 ; metabolic processes of, 55.7; changes during starvation, 620 ; potential and actual energy of, 632; expenditure of energy by, 634 ; sense of position of, 729
Bois-Reymond, dn, his 'key,' 58; on muscle-currents, 100,101

Brachial plexus, constrictor and dilator fibres in, 270

Brain, the, reaction of, 833 ; its action on spinal reflexes, 708

- cerebral hemispheres, relation of, to crossed side of boly, s04; results of removal of, in frog, 719 ; results of removal of, iu birds, 723 ; results of removal of, in mammals, 725,727

- cortex, experimental interference with, $7+0$; localization of function in, $7+1$; results of removal, 762

_, grey matter of, corpora quadrigemina, anterior, their connection with vision, 786,814 ; corpora genicnlata, 786 _- fibres of, smperior peduncles of cerebellum, 812

- optic tracts, 784; endings of, 786 , 787 ; splanchnic functions, 816 ; venous sinuses of, 828; circulation in, 829; supply of blood to, 831; its condition during sleep, 1155

Breaking of the voice at puberty, 1091

Breath, the first, cause of, 1134 ; effect of, 1135

Breathing, normal rate of, 433 ; male and female, differences between, 434; an involuntary act, 472 ; laboured, nervous mechanism of, 1082

Bright's disease, cedema of, 411

Broca's convolution, 766

Brownian movements in molecular basis of cliyle. 403

Buffy coat of clotted blood, 16

Burdach, column of, 682

Burdon-sanderson, his stethometer, 431

Calcium salts, their presence necessary in clotting of blood, 26

Calcic phosphate, insolubility of curd dependent on, 3:31; in milk, 613

Calories, cumbustion of food expressed in, 633

Calorimeters, 635

Canals, senicircular, result of injury to, 730

Capacity, vital, 428

Capillaries described, 13; their permeability, 13, 150-152; blood-interchanges effected in, 13, 150; structure of, 150 ; proportion of sectional area of, to aorta, $151,152,162$; measurenients of bloodpressure in, 160; disappearance of pulse in, 161; peripheral resistance in, 160-166; calibre, 289; plasmatic layer in, 290

Capillary circulation, normal phenomena of, 161,289 ; as affected by inflammation, 291

Capsule, Tenon's, 971 
Capsules, supra-renal, histology of, 601

Carbo-hydrates in white corpuseles, 40 ; in muscle substance, 92 ; in food-stuffs, 312 ; various forms of, 314 ; iu food, presence of glycogen depeudent on, 564 , 567 ; formation of fat from, 608 ; as food, potential energy of, 633

Carbon nomoxide, asphyxia from, 494

Catrbonic acid, see Acid, carbonic

Cardio-graphic traciugs, 201, 209

Cardio-inhibitory centre, 2i9, 252

Cardiometer, Roy and Adami's, 199

Casein. 323 ; precipitation of, 330 ; a constituent of milk, 612 ; its formation in mammary gland, 611

Cartilage, thyrc.d, 1077, 1078; cricoid and arytenoid, 1076,1079 ; of Santoriui, 1070 ; of IV risberg, 1070, 1072

Cells, albumiuous, changes in, 342 ; cardiac muscle, in frog, 243 ; in mammal, 244 ; ceutral, of gastric glands, 352 ; ciliary, 134; action of chloroform on, 136 ; ciliary, of Claudius, 1011 ; columnar fat, absorbed by, 417 ; of Corti, 1012; of Deiters, 1015; cylinder, of auditory epithelium, 1008; of olfactory mucous membrane, 1026; differentiation of, during development of ovum, 6 ; epithelial, 134; epithelium, 135 ; fat, 604; ganglionic, 143; of grey matter, 143; hair, inner and outer, of auditory epithelium, 1011, 1017 ; hepatic, continuous activity of, 369 ; changes in, 566 ; glycogen in, 568 ; action on hæmoglobin, 588; of mammary gland, 610 ; mucous, of salivary glands, 344 ; 'loaded,' 345; nerve, of spinal cord, 140-143; of central nervous system, 143; ovoid, or parietal, of gastric glands, 347 ; of paucreas, 341 ; of parotid, 343

_ـ of Lieberkiihn, 422

350,352 ; double function of cells of villi, 422

Cell-action in absorption, 422

Cell-substance, milk partly formed from, 615

Cellulose, digestion of, in large intestine, 396 ; a food-stuff for the herbivora, 661

Centres, nervous, cardio-inhibitory, 249; vaso-motor, 280, 284; limits of, 282, 283 ; pupil-const rictor, 860

- for deglutition, 373,376 ; for lactation, 618; for micturition, 547; for micturition, inhibition of, 707; for movements of eyeball, 956; for parturition, 1140; for phonation, 1084; for vomiting, 37 ?

- for respiration, 473 ; for respiration, automatic action of, 476, 488; as affected by blood-supply, 48t, 509; activity of, increased by exercise, 488 ; for sweating, 557

trophic, for uutrition of nerves, 679; visual, higher and lower, 793

Cerebeliar tract, 689

Cerebellum, see Brain, cerebellum

Cerebral operations, time taken by, 819

Cerebrin in nerve tissue, 105

Changes, anabolic and katabolic in living substauce, 39 , (551; nervous regulation of, 654; diurnal, in functions, 1156

Chauveau and Lortet, their hrematachometer, 174,178

- and Marey, their mode of measuring endocardiac pressure, 191, 192

Chest, expansion and contraction of, during respiration, 426

Chest-voice, how produced, 1089

Cheyne-Stokes respiration, 490, 1155

Chiasma, optic, decussation of fibres in, 784

Chloral, its effect on stimulation of depressor, 282

Chlorides, their presence in serum, 47

Chloroform, its effect on ciliary action, 136

Cholesterin, composition of, 355 ; a constituent of bile, $i b . ;$ its presence in blood, 47; in red corpuscles, 48; in nerve substance, 105 ; in gallstones, $i b$.; in milk, 613

Chondrin, action of gastric juice on, 329

Chorion, the, 1120

Choroid, development of, 837 ; bloodsupply of, 969

Chromogens, their presence in urine, 519

Chyle, characters of, 402 , molecular basis of, 403 ; passage of fat into, 413, 417; presence of sugar in, 414 ; absence of peptone in, 416 ; elaboration of, in the villus, 418

Chyme, how formed, 388

Cilia, 134

Ciliary movements, 52, 134; circumstances affecting, 13 $1-136$; plexuses, aqueous humour furnished by, 972

Circulation of the blood, main facts of, 153 ; capillary, $162,175,289$; hydraulic principles of the, 163,164 ; aids to, 171 ; rate of flow, 172-178; time occupied by circuit, 178, 225; constant and variable factors of, 299; as affected by bloodsupply, 301; changes in, 300 ; causes of irregularity in, 301; of cessation of, 302; placental, 1124; early fotal, 1124 ; late fotal, 1132; changes of, taking place at birth, 1135 ; renal, 525

Circus movements, $7: 35$

Clarke's column of spinal cord, 682

Clotting of blood, 15-30; retarded by cold, 16 ; by addition of saline solu- 
tions, 16, 22; by oil, 21; by carbonic acid iu the blood, 21; by injection of albumose, 29; causes of, 26; in the living body, 29; favoured by presence of foreign bodies, 21, 29, 45 ; clotting of Huids iu the body other than blood, 23 ; clotting of nuscle plasma iu rigor mortis, 90 ; clotting of lymph, 400

Coagulation of proteids by heat, 18

Cochlea of ear, 982

Coitus, behaviour of spermatozoa after, 1119

Cold, its influence on clotting, 16,21 ; on irritability of muscle and nerve, 127, 128; on vaso-constrictor action, 304; on skin action, 5t1; great, lowering of metabolism by, 650; terminal organs for sensation of, 1045 ; sensatious of, due to changes of skin temperature, 1041

Colostrum, composition of, 614

Colour sensations, many kiuds of, 891 ; mixing of, 892, 894, 898; characters of, 895 ; Young-Helmholtz's theory of, 898 ; due to metabolic changes, 901 ; iu relation to intensity of stimulus, 913 ; unequal change of, under diminishing light, 914

Colour vision, variations in, 906

— bliudness, 906 ; different kinds of, 907 ; dichromic iu nature, 907 ; YoungHelmholtz's theory of, 908; Hering's theory of, 909; absolute, 912

Colours, complementary, $896^{\circ}$; primary, 8.18 ; of arterial and venous blood, $443-$ 457

Combustion of various foods, rates of, compared, 633

Commissure, inferior optic, 785

Cones of retina, approximate dimensions of, 889

Coujunctiva, structure of, 977

Connective tissue, action of gastric juice on, 330 ; in relation to lymphatic vessels, 398

Constant current, its action, 110 ; as compared with induction shock, 118

Consonants and vowels, 1093; manner of formation, 1096 ; classification of, 1097

Coustriction of arteries, 265 ; of pupil a reflex act, 860

Constrictor fibres, 270

Contractile tissues, the, 51-138

Contractility, 53

Contraction of muscle, movements of body due to, 51; simple and tetanic, 55 ; graphic method of recording, 55; simple, phenomena of, 65 ; tetanic, 7580 ; of skeletal muscles, tetanic in character, 79 ; wave of, 83 ; optical changes, 85 ; chemical changes due to, 94 ; thermal changes due to, 95 ; electrical changes during, 104; 'making and breaking,' 110,111 ; influeuced by nature of stimulus, 119; isometric and isotonic, $i b$. ; prolonged, of red muscle, 122 ; as iufluenced by load, 123 ; idiomuscular, 125; exhausting effects of the products of, 130 ; of plain muscle, 131-134; spontaneous, 133 ; tonic, 134 ; relation of to amceboid movements, 137, 138; of heart, 216 ; features of heart contraction, 243,244 ; of villus, 420

Contractions, peristaltic, in plain, muscular tissue, 133; of ureter, 532, 54t; of bladder, 546 ; of the stomach, 377 ; of the intestine, 383

— the uterus duriug pregnancy, 1136; during 'labour,' 1137; after parturition, 1139

— of the abdominal walls, 383,1138

Contrast visual, simultaneous, 932 ; successive, 933

Conus medullaris, 689

Convulsions, anæmic, how produced, 492

Coordination of moveuents, machinery of, in birds, 730 ; in mammals, 731 ; in man, 733 ; parts of middle brain concerned in, 737

— of ocular movements, 952 ; nervous mechanism governing, 956

Cord, spinal, 139, 675-718; diagrammatic metamere of, 140 ; ganglia of the, 143; reflex actions manifested by the, 1t4; general features, 681; white matter, structure, 693 ; white matter, tracts of, delimitation of, 686 ; neuroglia, 690 ; grey matter, 691; nature of, 693; ascending and descending degeneration, 686 ; special features of the several regions, 689-695; variation in sectional area of white matter, 6.10 ; of grey matter, 691 ; course of pyramidal tracts in the, 686 ; cerebellar tract, 689 ; reflex actions of, $141,696-710$; complexity of, 700 ; reflex actious of, in man, 704; inhibition of, 707; time required for, 709 ; automatic actions of, 711-718; action in disease, 717 ; lyypothetical segmentation of, 777 ; motor mechanisms of , 806 ; lymphatic arrangements of, 824

Cornea, blood-supply to, 969

Cornu, anterior, of cord, 681,687 ; connection of efferent fibres in, 144

Corpora Arantii, 181

— geniculata, 786; quadrigemina, connection of with vision, 786 ; considerations touching the, 814 ; corpora cavernosa, $1115,1116^{\circ}$

Corpus spongiosum, 1115

Corpuscles of blood, not an essential part 
of clot, 16; relations of, with the plasma, 27

Corpuscles of blood, cartilage, presence of glyeogen in, 574: colostrum, 614

- red and white, relative proportions, 3t; composition, 37-48

_, red, microscopic appearance, 31; structure, 3:2; chemical composition, 32; methol of counting, 34; their life and death, 3i; their destruction in the liver, $i b . ;$ as oxygen bearers, 32, 34,36 ; formed in red marrow of bones, 3.5 ; their passage through the capillaries, 200 ; diapedesis of. 293 ; proportion of in foetal blool, 1125

_- white (see also Leucocytes), their connection with clotting, 29; appearance and structure of, 36,37 ; composition of, 37, 48; type of all living tissue, 39,42 ; amroboid movements, 40, 137 , 290,292 ; origin, 42 ; work, 44; grannlation $\mathrm{i} 1 \mathrm{l}, 45$; behaviour in inflammation, 2!12-294; their migration, 292

Cortex, see Brain, cortex

Corti, organ of, 100s, 1016; rods of, 1011, 1014 ; inner and onter, 1011 ; cells of, 1012

Coughing, 512

Cowper, glands of, 1115

Cramp, abolished by anelectrotonus, 117

Crassamentum, or clot, $\mathbf{1 5}$

Cream, 613

Cretinism, 601

Crico-arytenoid muscle, the posterior, 1079

Crico-thyroid muscle, 1079

Cricoid cartilage, 1079

Croaking of frog, connection of, with corpora quadrigemina, 815

Crying, 512

Curd, 330; curdling of milk, phenomena of, $i b$.

Currents of action, in a muscle, 102; in a nerve, 107 ; as started by excitation of cortex, 748

- of rest, in a muscle, 98 ; in a nerve, 107 ; in electrotonus, 115

- electrical, constant and induced, 56,57 ; interrupted or Faradaic, 62, 63; electrotonic, 114

Curves, mode of measuring (footnote), $186 ;$

Cntaneous sensations, see Sensations, cutaneous

Cyanogen componnds, their relation to urea, 516,598

Cycle, carliac, described, 182, 212 ; duration of phases, 214

Death, a gradual process, 1 ; slow clotting of blood after, 27 ; of blood corpuscles, 36, 44; from failure of heart's action, 301; from high temperature, phenomena of, 650; phenomena of, 1158

Decidua, formation of, 1120; reflexa, 1120 ; absorption of, 1121 ; vera, 1120 ; absorption of, 1121; serotina, 1120; its transformation into the placenta, 1120 ; expulsion of, after parturition, 1139

Decussation of the pyramids, 758; of optic fibres, 784

Defrecation, how effected, 381

Degeneration of severed nerve, 126; of muscle after severance of nerve, $i b$. ; of constrictor prior to dilator fibres in severed nerve, 270 ; of nerve fibres in mixed nerve, 179 ; calcareons and fatty, 1152

Deglutition, low effected, 372; different stages of, 374 ; a retlex act, 375 ; movenents of osophagus in, 376

Dentition, temporary, 1149 ; permanent, 1149

Depressor nerve, 281

Despretz signal, 70,71

Dextrins, characters of, 315

Dextrose, reactions of, 315 ; appearance of, in liver after death, 562 ; as food of muscle, 652

Diabetes, natural and artificial, 575

Diapedesis of red corpuscles, $2 ! 13$

Diaphragm, method of recording movements of, 431 ; its movements during respiration, 434 ; tetanus of, produced by stimulation of vagus, 478

Diastole of heart's action, 182, 185

Dicrotic wave, origin of, 232-236

Dicrotism in pulse tracings, 230 ; causes of, 232 ; less marked in rigid arteries, 234

Diet, average, 633; normal, composition of, 658 ; need of the three classes of food-stuffs in, 660 ; value of alcohol in, 602 ; vegetable, physiological value of, 664,$666 ;$; modifications of, with regard to size of body, 665 ; to climate, fi68; to labour, 669; to mental work, 671

"Differential capacity, extrene," 428

Differential manometer of Hürthle, 204, 205

Diffusible substances, absorption of, 420

Diffusion, laws of, 421 ; passage of gases in the tissues by, 424; in air of lungs, 425

Digestion, tissues and mechanisms of, 311-313; of living tissues, 353; muscular mechanisms of, 371 ; effects on, of presence of bacteria, 361, 393; main products of, 412 ; course taken by products of, 413

- , gastric, 321 ; circumstances affecting, 327 ; gross effect of, 388 ; time needed for, 38 ! 
Digestion, pancreatic, 359 ; salivary, 314 ; infantine, 1146

Digitalis, physiological action of, 542

Dioptric mechanisms, 834; apparatus, simple form of, 839 ; imperfections iu, 870

"Discrimination period," 821

Distance, judgmeut of, 964

Distension of lungs after birth, cause of, 427

Diuretics, their mode of actiou, 542

Diurual curves of fuuctions, 1156

Division of labour, pliysiological, 6

Dog, pancreas of, 341; submaxillary gland of, 338; submaxillary nerve, supply to, 334 ; succus entericus of, $36: 3$; nerves of alimentary canal, 384 ; saliva of, 387 ; composition of hremoglobin in blood of, 450 ; cortical motor area in, 740

Dropsy, character of lymph in, 401

Drowniug, 493

Ductus venosus, foetal blood carried to the heart by, 1132; arteriosus, foetal circulation through the, 1132 ; obliteration of, after birth, 1135

Dudgeon's splyggmograph, 221

Dura mater (of the eye), 839

Dyspnoea, at high altitudes, 465 ; nature of, 485,486 ; cardiac, 509 ; its effect on the kidney, 529; sweatiug caused by, 557

Ear, structure, 980-986; embryonic history of, 980 ; otic vesicle, 980 ; general relation ef parts, 981; cochlea, 982, 983 ; general use of parts, 986 ; tympanum, conduction of sound through, 989; muscles of, 993 ; auditory ossicles, 986; Eustachian tube, 995

- of rabbit, vaso-motor control of circulation in, 263

Egg-albumin, coagulation of, 323 ; its conversion into acid albumin, 324

Elasticity, diminished, in exhausted muscles, 130; of arteries, as affecting circulation, 16t; as affecting dicrotism, 234 ; of lungs, amount of pressure exerted by, $4: 27$

Electric stimuli described, 56; electric changes during muscle contraction, 97 ; in a nerve impulse, 107 ; electric spark, vision by illumination of, 881 ; electric currents, their development by retinal processes, 928

Electrotonic currents, 114

Electrotonus, features of, 112

Enıbryo of mammal, undifferentiated protoplasm in, 35 ; development of red corpuseles in, 35 ; glycogen in muscles of, 92, 573; growth of embryo of mamnal, 1120 ; respiration of, 1123 ; nutri- tion of, 1123-1135; supply of oxygen to the, 1123; development of adipose tissue in, 606

Emetics, various, action of, 380

Emission of semen, 1117: the striated muscles concerned in, 1118; the nervous centre for, 1118

Emotions, as affecting respiration, 476 ; respiratory mechanism a means of expressing, 511: their effect on secretion of urine, 543 ; of saliva, 335 ; ou splanchnic functions, 817

Emulsion of fats, action of bile and pancreatic juice on, $358,391,417$

End-plates of uerves, probable action of urari on, 55

Eudolymph of semicircular canals, in relation to coordinate movements, 732 ; secretiou of, in otic vesicle, 981

Energy, potential, of bodies, living and dead, 1 ; set free by breakiug down of living substance, $i b$.; of living body expended in work, 2, 636; of dead body shewn as heat, 3; renewed and set free by different tissues, 6 ; euergy of the body, income of, 632 ; expenditure of, 634 ; potential euergy of various diets, 632,658

Entoptic phenomeua, 874

Epididymis, action of in emission, 1117

Epiglottis, the, 1073 ; cushion of the, 1075 Epithelium, ciliated, 134

— cells, their action in absorption, 421 ; renal, secretiou by the, 533

Equilibrium, nitrogeuous, 626; sense of, 729

Erect posture, how preserved, 1102

Erectile tissue, structure and action of, 1115

Erection of penis, see Penis

Eructation, compositiou of gases of, 390

Erythrodextrin, 315

Eserin, pupil-contraction caused by, 866

Evaporation, temperature of body regulated by, 555

Eupncea, 485

Eustachian valve in adult life, 182; in fotal life, 1132

— tube, 984,996

Excretin, a freal constituent, 397

Excretion, tissues of, 8

Exercise, effect of, on the muscles, 128130 ; on vascular mechanism, 304,305 ; on respiration, 508 ; on the secretion of urea, 638; on the production of heat, 644 ; production of carbonic acid increased by, 637 ; visual discrimination increased by, 890 ; tactile perceptions increased by, 1040

Exhaustion of muscle and nerve tissue, $125,129,130$; of muscles, 129; auditory effects of, 1002 
Expiration, low effected, 437

Extractives, various, of spleen pulp, 583; of the thymus, 602 ; their value in diet, 631,662

Eye, the, nature of movements caused by stimulation of coltex, 790; general structure, 835 ; development of, 835 ; changes in during accommodation, 851 ; selerotic coat, 837; clioroid coat, 837 ; lens, clianges, of curvature of, 844 ; humour of vitreous, $537,97 t$; aqueous bumour, 839, 972; diagranmatic, 842

, acconmulation of, $846-853$; for far and near objects, 846 ; changes during, 8.5); mechanism of, 854; mechanism, nervous, 868 ; associated movements iu, 869); imperfections of, 870

- constrictor influences on, 857-862; dilator intluences on, 863-866; emınetropic, 8:9, s70; myopic, 850,870 ; hypermetropic, 850 , 870 ; presbyopic, 8. 0

- pupil, changes of, 857-869; constriction and dilation of, 857 ; nerves supplying, 859; coustrietion of, a reflex act, 860 ; changes in through action of cervical sympathetic, 862 ; nature of dilating mechauism, 863 ; aetion of drugs and ot her agencies in, 866

- , retina, development of, 835 ; a part of the brain, 837 ; rods and cones, 1111 ; function of, 925 ; possible differences between, 927 ; rods, preseuce of visual purple in the, 924 ; pigment epithelium, 926 ; stimulation of, ly other agencies than liglit, 884 ; visual areas of, 889 ; intrinsic light of, 904; colour blindness of periplery, 913 ; blind spot of, 916 , 960 ; photochemistry of, 922 ; correspouding or identical points, 942, 957; liues of separation, 944

- , retinal structures, fatigue of, 919 ; retinal processes, electric currents developed by, 928; muscles, ocular, 948 ; nutrition of, 969-975 ; arrangement of bloor vessels, 9t9; vaso-motor changes in, 970; lymphaties and lymphspaces, 970; protective mechauisms, 976 ; eyelids and their muscles, 976; conjunctiva and its glands, 977; Meibomian and lachrymal glands, 978; eye in old age, 1153

Fyeball, rotation of, 939, 945; movements of, 944; primary position of, 945 , 946 ; muscles of, 948; simultaneous movements, 952

Frces, composition of, 396

Fainting, a result of cardiac inhibition, 253,302

Fallopian tube, 1112-1123; reception of ovum by the, 1112
Falsetto voice, 1089

Faradization, 63

Fat, its preseuce in chyle, 402; amount alssorbed during digestion, 413; mode of absorption, 417; formation of, 571, 606,628 ; listory of, $604-609$; increase of, in cell-substance, 604; disappearance of, from cells, 605 ; nature of, in adipose tissue, 606; limits to construction of, tog; its potential euergy as food, 633

Fat-cells, structure of, 604

Fatigue, its effect on muscular irritability, 122, 129, 2591; sense of, its nature, 129; retiual, negative images produced by, 935: auditory, 1002

Fats in white corpuscles, 40; in blood, 47 ; in nerve tissue, 105 ; in food-stuffs, 312; iu cliyle, 403; action of gastric juice on, 321 , 388 ; of bile, 358 ; of pancreatic juice, 362 ; emulsification of, during digestion, 358, 391, 417; course taken by, in digestion, 413,417 ; change of, in the lacteal radicle, 418 ; various, melting-poiuts of, 606 ; various, of milk, 613 ; as food, metabolism lessened by, 616,627

Fattening, aids to, 668

Feet, sweating in $\operatorname{dog} s$ and eats only present in the, 556

Feliling's fluid, as test for dextrose, 315

Fenestra ovalis and fenestra rotuuda of ear, 985,986

Ferment, fibrin, efficient cause of coagulation, 24; its action on fibrinogen, 26 ; the amylolytic, in saliva, 318 ; the amylolytic, destroyed by gastric juice, 321

Ferments, organized and unorganized, (footnote), 318 ; their presence in urine 519

Fever, metabolism heightened by, 649

Fibres, muscular, see Muscle

- nerve, see Nerves of brain, see Brain

Fibrin, of the blood, 15; its development during elotting, 16 ; its proteid nature, 17 ; structure, 15 ; causes of its appearance, 20; actiou of gastric juice ou, 323,$326 ;$ in elotting lymph, 400

Fibrin-ferment, 24, 26

Fibrinogen, its precipitation from plasma, 23; its conversion iuto fibrin, $25,2(i, 2), 30$

Fick, spring-manometer of, 219, 220; his pneumatograph, 430,431

Fingers, clubbed, in phthisis, 656

Flatuleuce, 390

Flavours, seuse of smell appealed to by, 1029: localization of seat of perception of, $10: 33$

Flourens, 'nceud vital' of, 473 
Flnid, serons, 23; its identity with lymph, $40^{\circ}$; in diet, $66^{\circ} 2$; amniotic, 1127 ; its functions, 1127 ; composition, 1131

Fluidity of living blood, 26; of blood in the vessels after death, 27

Foetus, nourishment and respiration of, 1123; swallowing movemeuts executed hy, 1127, 1130; transmission of foodmaterial to the, 1127; growing differentiation of tissue in, 1129 ; movements cf, 1130; digestive functions, 1130; circulation in, 1132; expulsion of, 1139

Follicle, Graafian, 1123

Food, amoboid absorption of, 3 ; carried to the tissues by the blood, 8 ; its gradual change into living substance, 40; ingestion of, by white corpnscles, 40 , 44; its effect on vascular mechanism, 306; effect of its presence on gastric secretion, 338; on pancreatic secretion, 366 ; on bile secretion, 367 ; on stomach movements, 383 ; on intestiual movements, 386; as acted on hy saliva, 314 ; by gastric juice, 321 ; hy bile, 357 ; hy pancreatic juice, 362 ; hy succus entericus, 363; changes of, in the alimentary canal, 387 ; as income compared with output of material, 622 ; potential energy supplied by, 632

Food-stuffs, classification of, 311 ; changes of, in the body, 513; relative digestibility of, 5tit; fatty and carhohydrate, 627 ; peptones and salts, $629-630$; various, in normal diet, 658-660

Foramen ovale, course of foetal circulation through the, 1132; gradual occlusion of the, 1.135

Foruix conjunctivx, 977

Fovea centralis, the, 888 ; region of distinct vision in, 887

Freezing, its effect on innscle, 89

Frey and Krehl's manometer, 196, 197

Friction, peripheral, as affecting circulation, 163,166

Frog, rheoscopic, 102 ; capillary circulation in, 161

- - brainless, phenomena shewn by, $55,66,74,144,145$

_- lymph-hearts of, 405 ; winter storage of hepatic glycogen in, 566

Fuscin, its presence in the retina, 922

Gad, manometer of, 196

Gall-bladder, changes in hile effected by the, 254 ; storage of hile in the, 367

Gallstoues, cholesterin, present in, 105 ; composition of, 364

Galvanic hattery described, 57

Ganglia, spinal, 141; of splanchnic system, 141; spinal, of the posterior root, 678

Gangliou stellatum, 253-255
Gases, absorption of, by liquids, 447 ; their presence in blood, 447, 504; in nrine, 520; various, their effects on respiration, 494

Gaskell, his method of recording heartbeat, 240

Gastric juice, normal composition of, 320 ; the anylolytic ferment destroyed by, 321; its action on fats, ib. ; artificial, how prepared, 321,322 ; its action ou proteids, 323-331; nature of its action, 328 ; secretion of, 338 ; secretion of, influenced hy ahsorption of food, 340 ; formation of free acid in, 352; clianges iu its character as digestion proceeds, 388

Gelatin, in food-stuffs, 311; action of gastric juice on, 329 ; its effect as food, 629

Gestation, human period of, 1137

Giddiuess, a result of disarrangement of coordinating machinery, 733

Gland, salivary, venous pulse in, 236 ; submaxillary, of log, double nerve supply of, 267

Glands, alhuminous, 342; of Cowper, 1115; gastric, secretion from, intermittent, 338; of newt, 346 ; of bat, $i b$. ; changes in central cells of, $i b$.; lachrymal, 978; of Lieberkühn, 363 ; 1ymphatic, multiplication of leucocytes in, 42 ; mammary, structure of, 610 ; mammary, at hirth, 615; Meihomian, 978; of Moll, 978; storage of granular matter in, 348 ; oxyutic, of frog, 352 ; parotid, douhle nerve supply to, 338 ; parotid, changes of, during secretion, 342 ; salivary, venous pulse in, 236 ; submaxillary, of dog, double nerve supply of, 267, 333, 338; effect of stimulation of chorda, 336 ; effect of cervical sympathetic, 337 ; cell changes in, 344 ; therma! chauges in, 639

Globin, the proteid constituent of hrmoglobin, 460

Globulins, a group of proteids, 19; their changes to acid- and alkali-albumin, 88

Glomeruli, the, secretion hy, 535; special snbstances excreted hy, 534; effect of blood-pressure on the, 536 ; complexity of their action, 538

Glossopharyngeal nerve, 335

Glottis, the, 1073-1075; clanges in, during utterance of voice, 1075,1086 ; narrowing and widening of the, 1080

Glycerin, its effect on the hepatic cell, 5,8

Glycin, a product of metaholism, 539, 586

Flycocholic acid, 356

Glyeogen, its presence in white corpuscles, 38 ; in muscle snbstance, 92,573 ; 
in plain muscle, 1:31; in embryonic, 573 ; in liep:tic cells, 566 ; in the placenta, 57t, 112:, 1129; in the testis, 1115 ; in the fortus, 1129

Glycogen, characters of, 561 ; its conversion by the liver into sngar, 562 ; storage of, in the liver, $5(55,570$; manufacture of, 769,770 ; winter storage of licpatic of, in $\mathrm{frog}, 566$

Golvi, organ of, 10 it

Goll, cohmmn of, ti8t

Goltz and Gaule, their maximum manometer, 210

Gout, accumulation of uric acid in the blood in, 5!);

Graafian follicle, 1123

Granules in white corpuscles, 37, 40; of resting glandular cells, 343 ; of resting albuminous cells, $i b$.; of resting mi1cous cells, 344 ; in leucocytes, 419 ; in hepatic cells, 566

Growth, human, curve of, 1145

Gritzner's method of preparing fibrin, 327

Guanin, presence of in urine, 517

Gudden's commissure, 785

Gustatory sensations, see Sensations, gustatory

Gymnema sylvestre, gustatory sensations affected by, 1033

H:macytometer described, 34

Hremadromometer of Volkmann, 172

Hrmatachometer of Vierordt, 173

of Chauveau and Lortet, $17 t$

Hrematin, 32 ; its relations with bilirubin, 34,584 ; oxygen-holding power of, 460 ; iron-free, $460,58+$

Hrmatoblasts, development of, 43

Hrenatoidin, 585

Hrematoporphyrin, 585

Hemin, erystals of, 460

Hamoglobin, 32, 450; an oxygen-bearer, $34,3 i, 456 ;$ its proportion in red corpuscles, 47; in red muscle, 89; crystals of, 451 ; spectroscopic features of, 451 ; spectroscopic featnres of, reduced, 45.3; reduced, change of colour in, 4.54; absorption of oxygen by, 455; its combination with gases other than oxyuen, $45 \overline{7}$ : proluets of decomposition of, 458 ; its respiratory functions, 460 , 510 ; its relation to bilirubin, 34,584 ; of fastal arterial blood, 1125

Hremorrhage, its effect on blood-pressure, 296

Haidinger's l)rushes, 877

Hallucinations, ocular, 937; auditory, 1020

Head-voice, how prodnced, 1088

Hearing, sensations of, 796 ; mechanisms of, 950 ; binaural, 1022
Heart, visible movements in, 181; changes in, during cardiac cycle, 182; a uriculo-ventricular valves, action of, 183 ; auricnlar systole, 183 ; ventricular systole, 184; auriculo-ventricular valves, action of, 183 ; semilunar valves, action of, 184 ; change of form, 18j; cardiac impulse, 187; sounds of, 188 ; pressure exerted by (endocardiac pressure), 191 ; graphic record of, 193-197 ; negative pressure in, 192,212 ; pressure in ventricle, the phases, 210; duration of cardiac pliases, 213, 215; summary of events in, 215; work done by, 217; sequence of events in beat of, 241 ; power of independent rhythm in the several parts, 242; characters of the contraction of inuscular fibres of, 243 ; rhythmic beating due to impulses proceeding from nerve cells of the ganglia, 243 ; inhibition of beat of in frog, 245 ; in mammal, 239; augmentation of beat of in frog, 246-250; in mammal, 250; inhibitory and angmentor fibres in frog, 245, 247; in mammal, 250-253; inhibition, reflex of, 249; centre of inhilbition of (cardio-inhibitory centre), 250 ; inhibition and augmentation of beat, nature of, 256 ; inhibition of, sus pended by at ropiu, 257

Heart-beat, regulation of, 238 ; intrinsic regulation of, 300,712 ; development of normal, 181; influences other than nervous affecting, 260; relations of with vasomotor system, 306; normal rate of, 297; slowing effects of venous blood on, 505; during asplyyxia, 507; of the babe, 1146 ; death marked by cessation of, 1159

Heat, given out by contracting muscle, 95 ; loss of energy in the form of, 636 ; bodily, measnrement of, 635 ; sources and distribution of, 638 ; moles of loss of, 640 ; regulation of, by variations in loss, 641 ; production of, 643 ; increased by labour, (14t; regulation of, by the nervous system, 615; increased productiou of, by injury to parts of the brain, $6+7$; great, effects of, 649

Heat and cold, sensations of, 10\$1-1013; separate terminal organs for, $1056-$ 1058 ; epiclermal seat of sensations of, 10.57

Helmholtz, magnetic interruptor, 64

Hemianopsia, 78!

Henle, splincter of, 545

Hepatic cells, changes of, 566 ; glycogen in, $5 t^{7} 7$; vein, temperature of blood in, 640 Hering, his theory of colour vision, 900 , 92 ; colour blindness explained by, (109; as to simultaneous and successive contrasts, 935 
Hermann on muscle currents, 101

Hiccough, 511

Hippuric acid, its presence in urine, 517; how formed in the kidney, 539

Hopping, how effected, $\mathbf{1 1 0 5}$

Horopter, the, 957

Horse, sweat of, 551

Humour, aqueous, 972; how furnished, 972 ; vitreous, 974

Hunger and thirst, sensations of, 1048

Hürthle, membrane manometer of, 191196; tracings of ventricular and aortic pressure by apparatus of , 203, 204, 208, 209, 233; differential matometer of, 205; tambour sphygmoscope of, 220

Hydrochloric acid, free, in gastric juice, 329

Hydrogen, evolution of, in small intestine, $3 ! 5$

Hyperpncea, 485

Hypoxauthin, presence of, in urine, 517

Illusions, visual, 961 ; tactile, 1069

Images, retinal, formatiou of, $839-845$; in relation to sensatious excited by, 845 ; entoptical, 876

Impulses, nervous, 54, 106; electrical changes accompanying, 107; cardiac, 209 ; mode of recording, 213; afferent and effereut, 676 ; their paths along the cord, $800,801,810$; relays in course of, 802,810 ; crossing of, 804 ; sensory, different paths for different, 80.5 ; transmitted by grey matter and interumcial tracts, 808 ; ampullar, 732 ; motor, effect of efferent impulses on the coordination of, 733, 955; visual, 781, 834, 908; auditory, how excited, 980, 983; auditory, development of, 980,986 ; volitional, time required for transmission, 774 ; volitioual, course of, in man along the pyramidal tract, 775

Impurities in expired air, 442

Iucome and output of material in nutrition, 622

Incus, the, 986

Indol, a product of bacterial action, 361 , 393

Induction coil, construction of, 59

Infancy, characteristics of, 1148

Inflammation, phenomena of, 290 ; œdema due to, 410

Infusoria, ciliary motions in, 136

Inhibition, cardiac, phenomena of, 245 et supra: fainting a result of, 253 , 302 ; effect of at ropin on, 257

- of secretion of saliva, 335 ; of respiration, 479; of reflex actions, 707; of parturition, 1142

Inhibitory nerves, 148; fibres in vagus of frog, 248 ; in vagus of mammal, 250 ; cardiac inhibitory fibres, contin- uous action of, 253; their analogy with vaso-dilator fibres, 269

Inspiration, mechanism of, 433; movements of, 42t; laboured, phenomena of, 436

Intercostal muscles, their work in respic ration, 435

Intermediate line, in muscle fibre, 85

Intermittence, cardiac, 260,301

Interruptor, magnetic, 63

Iutestine, absorption of fats in the, 417 ; absorption of diff usible substances aud water, 420

- large, movements of, 381 ; changes of food in, 395 ; digestion of celluluse in the, 396

—_, small, movements of, 380 ; changes of food iu the, 390,391 ; fermentative changes of food in the, 394 ; fluidity of food maintained in the, 422

" Intrinsic light " of retina, 904

Iodine, coloration of starch by, 316

Iris, development of the, 8:37; muscular and vascular changes in the, 857

Iron, its presence in hrematin, 460; iu hrmoglobin, 450; in bile, 355,585 ; in the spleen, 582

Irradiation, 932

Irritability, muscular and nervous, 53-80 ; their mutual independence, 53, 54; diminution and disappearauce of, after death, 80,81 ; as affected by electrotonus, 112; circumstances deteruining, 125; centrifugal loss of, in severed nerve, 126; influence of temperature on, 127 ; influence of blood-supply on, 128 ; influence of functional activity on, $i b$. ; presence of oxygen a conditiou of, 130 ; prolonged, of heart, 242

Irritants, inflammatory action of on tissues, 291

Islets, extra-vascular, 149

Jaundice, how caused, 370,587

Judgments of distance and size, how formed, 965 ; of solidity, 960 ;

Juice, gastric, see Gastric juice

- - pancreatic, see Pancreatic juice

_ intestinal, see Succus eutericus

Jumping, how effected, 1105

Katabolic changes in living tissue, 39,41 ; heat liberated by, 638

Katelectronus defined, 113

Kathode or negative electrode, 57

Key, galvanic, various forms of, 58

Kidney, the, duplexity of its mechanism, 524; vaso-motor mechanisms of, 525; relations, various, of How of blood through, 528; vaso-constrictor nerves of, 530 ; effect of chemical changes in the blood, 531; secretion by the renal 
epithelium, 533; double vascular supply to, in amphibia, 533; work of the epithelium of the tubules, 538 ; kidney aud skin, mutual relations of secretory activity of, $5+1$; its relations to water absorbed by the alimentary caual, 541 ; influence of central nervous system ou, 543 ; futal, urea secreted by, 1131

Kilogran-meters, daily work of heart estimated in, 218; encrgy of food and body and day's work estimated in, 634 Knee-jerk, 705,717

Kreatiu, its presence in the blood, 47 ; chemical compositiou of, 93 ; in uustriated muscle, 131; the product of muscle metabolism, 590

Kreatiniu, its presence in urine, 517 ; the urinary form of kreatiu, 591; difficulties presented by its presence in urine, 591

Kymograph, Ludwig's, for recording blood-pressure, 160

Labour, physiological division of, 6 ; circumstances governing capacity for, 510 ; increased production of heat from, 644

"Labour," the events of, 1137 ; first stage of, 1137; second stage of, 1138; causes determining its ouset, 1142

Labyrinth of ear, bony and membranous, 983 ; perilympl cavity of, 987 ; connections of aulitory nerve with, 983 ; the cochlea, 982; vestibular, parts of, 1007, 1008; probable fuuctions, 1017 ; transmission of impulses through the, 1007

Lachrymal gland, structure of, 978

Lactalbumin, 612

Lactation, nervous centre for, 618

Lacteal radicle of intestinal villus, passage of fat into, 413,418

Lacteals, the, absorption by, 398; chyle contained by, in fasting animals, 402; passage of products of digestion into, 413

Lactic acid, its presence in the blood, 48 ; isomeric variations of (footnote), 90 ; its effect on the heart, 259 ; fermentation, 3.14 ; a product of muscular metabolism, 65.3

Lactoprotein, 612

Lactose, ready fermentation of, 613 ; its formation in the mammary gland, 617

"Laky" blood, how formed, 31

Laryngeal nerves, 1081

Laryngoseope, larynx as seen by the, 1070 Larynx, the, its condition in respiration, 438; cartilages of, 1073,1078 ; ventricles, uses of, 1091; miscles of, 1076, 1080; nervons mechanisms of, 1082; respiratory movements of, 1082 ; cortical area for movements of, 1085
Laughter, mechauism of, 512

Lecithin, in stroma of red corpuscles, 32 ; in white corpuscles, 38 ; iu the blood, 48 ; in muscle substauce, 92 ; in nervous tissue, 105; in milk, 613; its composition, 105

Leus, the, development of, 837 ; mechau isms for changing curvature of, 854 ; action of the suspensory ligament on, 855

Leucin, composition of, 361 ; in intestinal couteuts, 393 ; a product of nitrogenous metabolism, 594; its couversion into urea, 595

Leucocytes, in the lymphatic system, 42; their origiu, 43 ; their preseuce in the villi, 419; among epidemic cells, 554

Lencocythremia, increase of white corpuscles in, 44

Levatores costarum, their work in respiratiou, 436

Lieberkühn, glands of succus entericus probably furnished by, 363 ; cells of, 422

Life, processes of, compared with those of death, 1 ; its existeuce possible without organs, 3 ; periodic events of, 1153 ; factors of, 1158

Light, as stimulus to visual apparatus, 858, 878; "intrinsic," of retina, 904; changes in retina produced by, 878; sensitiveness of living matter to, 921; decomposition of, 891

Listing, diagrammatic eye of, 842 ; his law, 946

Liver, the, destruction of red corpuscles in, 35 ; blood-supply to, 367 ; quality of as affecting bile secretion, 368,369 ; liver of frog, 564 ; storage of glycogen in, 565; mammalian, 561-568; nervous control of glycogenic function, 575 ; " acute yellow atrophy" of, 587, 594; presence of urea in, 594; conversion of leucin into urea in, 5.95 ; heat set free in, 639 ; its action on lactic acid, 653; foetal, deposition of glycogen in, 1129

Living substance, food and waste of, 3

ILocomotor mechanisms, 1101

Ludwig, his stromuhr, 173 ; his mereurial gas-pump, 414

Lungs, the, their function chiefly mechanjeal, 4:4; eutrance into and exit of air from, 425; air, tidal and stationary in, 425; air, complementary, supplementary and residual, 426 ; results of opening into pleural chamber, 426; conditiou of, before birth, 427 ; elasticity of, pressure exerted by, 427 ; respiratory changes in, 462-466; effects of inflatioll and suction, 482 ; first inflation of, 1134 
"Luxus consumption" of food, 393,627 Lymph, the, a medium of exchange between blood aud tissues, 13, 14, 403; salts present in, 39 ; migration of white corpuseles into, 292 ; coagulable, in inflammation, ib.; microscopical characters of, 400 ; clotting of, 400 ; chemical composition of, varying, 401; total diurnal flow, 403; movements of, 403 ; its flow increased by muscular movemellts, 405 ; transudation of, nature of the process, 406; its functions in the eye, 971

_ capillaries, compared with blood capillaries, 398

_- corpuscles, 400

- spaces, passage of the white corpuscles into, 43

Lymphatic arrangements of brain and cold, $82 \pm$

Lymphatic glands, their influence on lymph, 401; lymphatic system, 398; prominence of, in infancy, 1148

Lymphatics of the eye, 970-975

Magnetic interruptor, 63

Majendie, foramen of, 826

Making and breaking currents and shocks, 57-65; contractions with the constaut current, 111

Male breathing, diaphragmatic character of, 433

Male organs of reproduction, 1114

Malleus, the, 986

Maltose, 315

Mammary gland, 610 ; changes in, during secretion, 610; dormant, characters of, 611 ; at birth, 615 ; relations of, to the nervous system, $\mathbf{6 1 8}$

Manometer, for measuring blood-pressure, 156, 157 ; maximum and minimum, 210, 211; endocardiac pressure shewn by, 191-197; Gad's manometer, 196; Krehl's, ib.; Fick's, 220; vaso-motor actions observed by, 277

Marey's pneumograph, 430; tambour, 192

Marrow, red, formation of red corpuseles in, 35 ; yellow, of bones, 605

Massage, metabolism excited by, 669

Mastication, how effected, 372

Meatus, auditory, external, 983 ; internal, 993

Meconium, 1127 ; sources of, 1131 ; chemjcal composition, 1131

Medulla, loss of, in vaso-constrictor fibres, 274 ; retention of in vaso-dilator fibres, 275

- oblongata, cardiac effect of stimulation of, 249; centre for nerves of taste in, 277 ; for vaso-motor impulses in, $277-280$; for constrictor impulses in, 279-284; for secretion of saliva, 335 ; for deglutition, 375 ; for vomiting, 380 ; for respiratiou, 473 ; effect on bloodpressure of successive sections of, 283; diabetic area of, 575; see also Bulb

Meibomian glands, 975

Melting-point of various fats, 606

Membrana pupillaris, absorption of, before birtl, 838; tympani, 983, 989

Membrane-manometer of Hürthle, 194196,219

Méuière's disease, 733

Meustruation, 1111-1113; causation of, 1112

Mercurial gas-pump, Ludwig's, 444, 445; Pflüger's, 445; Alvergniat's, 446

Metabolic processes of body, 559

Metabolism, defined, 39 ; water of, 41, 42; increased by exercise, 305 ; by proteid food, $617,626,654$; of muscle, products of, 591; of nervous tissue, 592; of glands, 593; proteid, its complexity, 599; nitrogenous, 625 ; products of, 653 ; of muscle, the chief source of heat, 639; conducted in the tissues, 651 ; course of products of, 653 ; nervous control of, 654 ; rapidity of, in infancy, 1147

Metals, retention of, in the liver, 355

Metameres, hypothetical, of spinal cord, 139,140

Methrmoglobin, spectrum of, 460

Micro-organisms, their actions in digestion, 394 ; in expired air, 442

Micro-unit of heat defined (footnote), 96

Micturition, mechanism of, 545 ; nervous mechanism of, 546 ; centre for, 547 ; voluntary and involuntary, 548

Migration of the white corpuscles, 43 ; in inflammation, 292; aided by changes in vascular walls, 294

Milk, actiou of gastric juice on, 327,329 ; of renuet on, 330 ; double mode of secretion of, 616 ; nature of, 612 ; constituents of, 613 ; uterine, 1122, 1129

Millon's reagent for detection of protein, 17

Mitral valves, their action, 185

Molecular basis of chyle, 403; where elaborated, 418

Moll, glands of, 978

Morse key, 59

Movements in living bodies, 2; amœboid, 36, 37, 137, 292; of body, how accomplished, 51 ; ciliary, 52,134 ; muscular, heat given out during, 95, 644; cardiac, visible, 181; of alimentary canal, 371-387; gastric, 383; intestinal, ib. ; amœboid, of lymph corpuseles, 400 ; Brownian, in chyle globules, 403 ; muscular, flow of lymph increased by, 404; respiratory, 428; bilateral, 760 ; coordinating machinery of, 729; of 
cortical origin, how effected, 753 ; of dog, 740,772 ; of monkey, 744,763 ; of authropoicl ape, 749 ; voluntary, 739 780 ; action of motor area in effecting, 769 ; as influenced by sensory impulses, 772 ; skilled, correlation of with pyramidal tract, 760; 'foreed, 735, 813; 'forced,' from injury to optic lobes in frog, 815; fortal, 1130 ; of locomotion, 1101 ; ocular, !4!9; sense of, 1059

Mucin, a constitnent of saliva, 313

Mulberry gallstones, 364

Muscre volitantes, 875

Muscarin, its action on cardiac tissue, 257 Muscle, irritability of, 53 et supra: phenomena of contraction of, $65-134$; tetanic contraction of, 75-81; gross structure of, 82 ; wave of contraction, 83 ; striated, 86 ; striated under polarized light, $i b$. ; chemist ry of, $i b$, ; living and dead, contrasted, 86, 87; dead, chemistry of, $87-95$; frozen, 89 ; rigid, acid reaction of, 90 ; living, reaction of, 91 ; chemical changes due to contraction, 94 ; thermal changes due to contraction, .55; electrical changes iu, 97; action of the constant current on, 110-116; work done by, as influenced by fatigue, 12:, 129; by load, 123; by size and form of muscle, 124; by temperature, 127; by blood-supply, 128; by functional activity, ib. ; oxygen consumed during contraction of, 304 ; plain, structure of, 131; chemistry of, ib.; characters of contraction of, 131,132 ; spontaneous contraction of, 133 ; tonic coutraction of, 134; nutrition of, 128; vascular changes in, 271; changes due to contractiou of, 304; vaso-rlilator fibres in nerve supply to, $2(i)$; embryouic, glycogen in, 573; respiration of, 469 ; governance of nutrition of, 714

Muscles, skeletal, result of metabolism in, 590; their proportion in borly, 619; tone of, 713 ; rigidity of, 717 ; their mode of action, 1101

Muscle-currents, 98-102 ; velocity of, 99; negative variation of, 102

Muscle-curves, 65 ; analysis of, 71 ; variations of, 74 ; tetanic, 75

Muscle-nerve preparation, 55-81, 102; as a machine, 119; muscle current shewn iII, 102, 103

Muscle-plasma, 8?

Muscle-serum and elot, 89

11uscle-sonnd, 122, 123

Musieal somnds, character of, 998

Myocaroliograms, 202

Myoglobulin, 89

Myograph, $66 ;$; pendulum, 68

Myosin in dead muscle, 87, 88; in white corpuseles, 88

\section{Myosinogen in living muscle, 90}

Myxoedema, its conuection with disease of thyroid, 601

Nasal passages, inspired air warmed in tlie, 438

Nausea, sensation of, 1049

Negative pressure in heart, 210, 211, 217

Nerves, irritability of, 53 et supra; tested by constant current, 118; endplates of, probable action of urari on, 55 ; connection of with muscular fibre, 82 ; chemistry of, 105 ; mixed, $1+1$; inlibitory, 148; vaso-motor, 262-28s; specific energy of, 1052 ; special sensations not cansed by stimulation of trunk, 1053; abdominal splanchnic, 141 ; vaso-constrictor fibres in, 262, 272, 277, 385; inhibitory fibres in, 385 of alimentary canal, 383,384

, brachial plexus, constrictor and dilator filores in, 270, 271

- cardiac, 253

- cervical sympathetic, eardiac augmeutor fibres, in frog, 247-249; fibres of, to salivary glands, 338 ; vaso-motor fibres in, 263, 269, 278; pupil-dilating action of, 862

—_, chorda tympani, vaso-motor fibres in, 267, 269; secretory fibres to submaxillary gland, $333,331,351$; its connection with sense of taste, 1035

— , depressor, vaso-motor functions of, 280,281

_- of eye-ball, 859

, glosso-pharyngeal, 335 ; its connection with sense of taste, 1035

- of larynx, 1081

- , lingual, 333, 334; its connection with sense of taste, 1035

- - optic, decussation of, in optic chiasma, 781; development of, 8.55

- phrenic, functions in respiratiou, 472

_ , sacral, regulation of bladder action by, 546

- sciatic, constrictor and dilator fibres in, 269-271

fibres in, 252-255

-, spinal, 140; anterior and posterior roots of, 141, 676; cfferent and afferent fibres of, their separate patlis, 667 _ of stomacli, 339; submaxillary, 333 ; thoracic, 27 ?

- vagus, inhibitory action of, 148; government of heart-beat by, in frog, 249,258 ; a mixed nerve, 141 ; of mammal, inhibitory fibres in, 250; supply to the stomach, 339 , 383; to the intestines, 383 ; cardiac augmentor and inhibitory fibres in, $249,268,385$; in- 
fluence on respiration of, 476; on the circulation, 451

Nerve-endings, specific teruinal organs of, for tastes, 1034; for pressure, 1037; cutaneous, 1054; for heat and cold, $105 t$

Nerve-fibres, efferent and afferent, 141, 143; revchent, 142; in spinal cord, 143; inedullated and non-medullated, $1+7$; vaso-constrictor and vaso-dilator, 269; vaso-constrictor, course of, 273 ; vasodilator, course of, 275 ; inhibitory and angmentor, 250; secretory and trophic, 351 ; nutrition of, 679

Nervi erigentes, vaso-dilator fibres of, 278 ; action of, on the rectum, 385 ; action on penis, and roots of, 1116

Nervous system, central, centres for automatic and reflex actions in, 143, 141 ; vaso-motor functions of, 277,282 ; regulation of temperature by the, 645 , 647 ; metabolism governed by the, 654

— mechauism, coordinating, 702, 729, 737, $951 ;$

Neurin in nervous tissue, 105

Neuroglia, of white and grey matter of cord, 690

Neurokeratin in nerve medulla, 106

Newt, chief cells of gastric glands in, 346

Nitrogen in proteids, 17; in expired air, 441 ; relations of, in the blood, 461 ; free, inassimilable by living beings, 623

Nitrogenous waste not increased by muscle contraction, 95,97 ; equilibrium, 626

"Noeud vital" of Flourens, 473

Noises and musical sounds, 998; characters of, 1002

Nostrils, their work in inspiration, 438

Noteh, dierotic, in pulse tracings, 231

Notes, how proluced vocally, 1075, 1084

Nuclein, in white corpuscles, 38; a modified proteid, 41 ; a coustituent of milk, 616; of semen, 1115

Nutrition, statistics of, 619 ; income and output of material in, 622 ; summary of phenomena of, 6.51 ; of musele, 652 ; of muscle increased by activity, 12 $\delta$; influences determining, 6.5t; nervous control of, 654,707 ; disordered, phenomena of, 6ivi; of nerve-fibres, 679 ; of embryo, $1123,112 \mathrm{~s}$

Odours, perception of, 1025 ; discrimination of, 1026

Clema, possible causes of, 399,409 ; inflammatory, 410; of Bright's disease, 411

Esophagus, movements of in deglutition, 376 ; force of contraction in the, $i b$.

Oil, clotting of blood prevented by presence of, 21
Old age, phenomena of, 1151; degenerations characteristic of, 1152

Olein, presence of, in blood, 47; a constituent of animal fat, 6006

Olfactory bulb and tract, sensations, 1025 ; julgments, 1027

Oncograph, renal, $52 \tau$

Oncometer, renal, 521;, 830

Ophthalmoscope, principle of the, 926

Optic chictsma. 784

- lobes, results of removal in frog, 737

- nerve, its decussation in chiasma, 785; an extension from the brain, 8:7; opt ic cup, 8.36; fibres, their insensibility to light, $417,10.22$

- thalaui, results of removal in frog, 737

Optical systems, simple and complex, 840

Optogram, how obtainable, 924

Ora serrata, $\$: 38$

Ordeal by rice, its mode of action, 325

Organs, definition of, s; terminal, for sensations of touch and temperature, 1051 ; for sensations of pressure, 105t; for sensations of heat different from those for sensations for cold, 1055; nature of, 1057

Orgaus of reproduction, female, 11111113 ; male, 1114-1118

Os uteri, expansion of during 'labour,' 1137

Ossicles, auditory, 986; attacliments of, 986 ; conduction of vibratious through, 989

'Output' of blood by ventricle, 197; increased by augmentur action, 256

Ovum, escape of the, 1111; transference to the uterus, 1111; impregnation of the, 1119; nutrition of, in the uterus, 1123

Oxidation in the tissues, seat of, $\mathbf{4 6 9}$

Oxygen, its absorption by the living body, 2; borue by the blood to the tissues, 13; in proteids, 17 ; borne by hremoglobin, 32-35, 461; its entrance to the blood by diffusion, 424; in air expired and inspired, 440 ; relative proportions of in arterial and venous blood, 443; varying amounts of in venous blood, 447; relations of in the blood, 447 ; absorption of, by blood not according to 'law of pressures,' 448; its access in the lung to the corpuscle, 463 ; its relations in laboured breathing and asphyxia, 46.5 ; mode of storage in muscle tissue, 468; effect on respiration of deficiency of, 487 ; effect of breathing, 49.3; results of high pressure of, 496; mode of measuring amount consumed, 623 ; consumption of, as af- 
fected by temperature, 646; absorptiou of, in infancy, 1147

Oxyhamoglobin defiued (footnote), 454; colour of, $450^{\circ}$

Oxyutic gland of frog, 352

Pacchionian glands, 825

l'ain, scnse of, 10+4-1050; localization of, 1045; special nerve-endings not needed for, 1047

"Pains" of labour, 1137

Pallor caused by cmotion, 286

Palmitin, preseut in blood, 47 ; a constitnent of animal fat, 606

Palpitation of heart, its causes, 302

Pancreas, histological chauges during secretion, 340: of $\log , 341$

Pancreatic juice, its action on food-stuffs, $359-36^{\circ} \%$; on fats, $36^{\circ}$; on proteids, 362 ; secretion of, 36.5 ; circumstances affecting, 366 ; trypsin a coustituent of, 349 ; its compositiou, 358

Panniculus adiposus, 604

Paraglobulin, a constituent of bloodserum, 19; precipitated from plasma, 23 ; in white corpuscles, 38

Parapeptone, 326

Paraphlegia, reflex action in, 706

Parotid gland, nerve supply to, 338 ; cellchanges in, 342

Parturition, 1136-1143; mechanisms of, 1137 ; a reflex act, 1140 ; inhibition of, 1142

Peduncles of the cerebellum, 812

Pendulum myograph, 66, 68

Penis, erection of, 1116 ; nerves concerned in mechanism of, 1116 ; striated muscles assisting, 1116; nervous centre for, 1117

Pepsin, the ferment body of gastric juice, 329: proteids converted into peptone by, ib.; secreted by the 'chief' gastric cells, 352; in the fotal gastric membrane, 1130

Pepsinogen, an antecedent of pepsin, 350

Peptone formed from proteids by gastric juice, 321, 325; by pancreatic juice, 32, ; test for, 325, 326; its absence from chyle, 416; its course during absorption, 416 ; as food, 629

Perceptions, visual, time required for, 823 ; and judgments, 959 ; psychical modifications of, 931

— and judgments, auditory, 1006, 1023; olfactory, 1025 ; tactile, 1066

Pericardial fluid, its persistent fluidity in pericardial bag, 28

Perioflic events of life, 1153

Peripheral region, blood-pressure in, 161 — resistance, defined, 163; its action iu the circulation, 170; illustrated by model, 167; lowered by action of depressor nerve, 281; affected by vaso-motor changes, 275; by conditiou of vascular walls, :214; by changes iu character of blood, $i b$.

zone, iu capillary contents, 290; white corpuscles present in, 291; blood platelets in, during jutlammation, 203

Peristaltic contractions of plain muscle, 131

- movements of alimentary canal, 371; excited by stimulatiou of vagus, 383 ; influences bearing ou, 386 ; of ureter, 544 ; of bladder, 547

Personal equation as affecting reactiontime, 819

Perspiration, nature and amount of, 550; secretion of, 555; regulation of temperature by, $64^{\circ} 2$

Pflüger's gas-pump, 445

Phagocytes, 14

Phakoscope, Helmholtz's, 852

Phantoms, ocular, 937; auditory, 1020; tactile, 1069

Phases of life, 1144

Phenol, a bacterial product in digestion, 394 ; compounds of, in urine, 517

Phloridzin, temporary diabetes produced by, 576

Phonation, nervous mechanism of, 1083 ; centre for, 1085

Phosphates in muscle ash, 93; in nerve ash, 106; in urine, 518

Phosphenes, 884

Phosphorus, a constituent of nuclein, 38, 630 ; of serum, 47 ; of lecithin, 105 ; of uerve tissue, 106; of milk, 613

—, its importance in organisms, 630

Plotochemistry of the retina, 922

Physiology, divisions of, 3 ; problems of, 9

Physiological unit defined, 6

Plyssostigmin, its effect on pupil contraction, 866 ; its effect on accommodation, 868

Pigment, yellow, of serum, 47

- epithelium of retina, 926

Pigmeuts, their possible formation from hremoglobiu, 36 ; of bile, 356 ; of urine, 519

Pilocarpin, its action on the sweat glands, 5.57

Piteh of somnds, discrimination of, 1001

Pituitary body, the, structure of, 601

Placenta, the, glycogen present in, 574; formation of, from the decidua scrotina, 1120; vasenlar events of the, 1121; shedding of the, 1121 ; cxpulsion of, after parturition, 1139

Plasmatic layer in eapillary coutents, 290 Plasmine, properties of, 23

Plateau, systolic, 197

Platelets, blood, 44, 292 
Pletlysmograph, principles of its action, $174,178,198$; amomut of blood in parts determined by, 270 ; for kidney measnrements, 525; for measnrement of blood-supply to brain, 830

Pleural cavity, result of access of air to the, 426

Plexus, brachial, constrictor and dilator fibres i11, 271

Pneumatograph of Fick, 431

Pneumograpl, Marey's, 430; tracing of respiratory movements by, 432

Polarizing current, irritability of nerve affected by, 112-114

Posture, ereet, how maintained, 1102

Potassium salts in cell tissue, 39,47 ; in mnscle tissue, 90,91 ; in urine, 516

Predicrotic wave, its causes, 235

Pregnaney and birth, 1119-1143

Pressure, arterial, as compared with vellous, 154,159 ; as affecting pulse tracings, 223; heart-beat in inverse ratio to, 261 ; blood, iu the small vessels, peripheral region, 161,168 ; flow of lymph regulated by, $403-409$; endocardiac, 191 ; graphic records of, 193199; negative dnring each cardiac cycle, 192, 193; how produced, 201; auricular and ventricular compared, 182, 193; of salivary secretion, 337 ; of bile secretion, 369 ; pulmonary, 427 ; thoracic, 431 ; thoracic, negative, 500 ; partial, of gases, 448; absorption of oxygen, dependent on, 462 ; results of, 496 ; atmosplieric, effect of diminntion of, 494 ; increase of, 496 ; of carbonic acid in pulmonary alveoli. $46 \%$; within the bladder, 547; intra-ocnlar, conditions affecting, 975 ; sensations of, 1037; modified by temperature, 1055 ; sensibility of skin to changes of, 1039,1056

Pressures, Henry-Dalton law of, 449

Primordial ntricle, 4

Processus vocalis, and muscularis, 1075

Proteids, general composition of, 17 ; changes in, produced by alcohol, 24; in food-stuffs, 311; action of gastric juice on, 321 ; of pancreatic juice on, 359 ; classification of, in order of solubility, 322; path taken by, during digestion, 415; a source of fat, 605 ; metabolism of body increased by, 617 ; disruption of, during digestion, 626 ; probable molecular composition of, 361 ; possible storage of, in the body, 627 ; "tissue," or morphotic and "floating " or circulating, 627

Proteid material, a constituent of living matter, 41 ; potential energy of, expressed in calories, 633; the pivot of metabolism, 654
Protoplasm, definition of, 4 ; "differentiated," 4; nndifferentiated in the embryo, 37

Pseudopodia of the white corpuscles, amoboid movements by means of, 137

Psychical processes, analysis of, 821 ; duration of, 523 ; visnal, complexity of, 961

Ptomaines, their bacterial origin, 394

Ptyalin, a constituent of saliva, 318

Pnberty, phenomena of, 1150

Pulse, the, 153, 219; methods of recording, 219-203: artificial, 223, 227 ; characters of, 227 ; disappearance of, 228 ; dicrotism in, 230-23t; allacrotic, $i b$. ; venons, 236; venous, respiratory, 237

Pulse-volume, 200, 217

Pnlse-wave, changes of, in the arteries, 227 ; velocity of the, 229); length of tlie, $i b$.

Pulvinar, the, ending of part of the optic tract in, 787

Punctum lachrymale, 978

Punctnre of pleura, result of, 426

Pupil, the, see Eye, pnpil

Purkinjé, figures of, 917

Purple, visual, 922 ; bleaching of by light, 923

Pus corpuscles, their formation, 43

Pylorus, ejection of chyme through the, 389

Pyramidal tract of cord, 686; efferent natnre of impulses of, 753 ; not indispensable for voluntary movements, 772

Pyramids of the bulb, decussation of, 758 Pyrexia, causes of, $6+8$

Radial artery, tracings of the pulse in, 221-223, 22t; 231

Radical, lacteal, contents of, 418

Ranke's diet table, 6.33

Reaction-period, subdivision of, 819 ; for vision, 881

Rectum, nervons control of movements of, 385

Recurrent sensations, 937

Peflex actions, general features of, 114146 ; doubtful if carried ont by ganglia, 144; not always proportioned to stimulus, 145 ; often purposive in character, 146 ; vaso-motur, 277

- actions of the cord, 698 ; features of dependent on afferent impulses, 698; nervons mechanisms of, 699 ; their relations to intelligence, 701 ; coordination of, 702; determined by intrinsic condition of cord, 703 ; other tban movements, 707 ; inlibition of, 705 ; inlibitory action of the brain on, 708 ; time required for, 709

Regeneration of organs in lower animals, 1109 
Registers of the voice, 1090

Relaxation of muscular fibre an essential part of contraction, 72,269

liennet, curlling action of, on milk, 330

Rennin, its direct action on casein, 331 ; its formittion in gastric cells, 352

Reprocluction, tissues and mechanisms of, 1109; general features of, 1129; female organs of, 1111 ; male organs of, 1114

Respiration, 424-512; pulmonary, circulation aided by, 171; its mechanism, 425-433) work of the muscles of the ribs in, 435 ; laboured, muscles of, 436 ; expiration, the expiratory muscles, 437 ; change of temperature of air in, 40 ; change of aqueous vapour in, 440; changes in blood caused by, 412 ; chemical aspects of, 4i0; an involuntary act, 472 ; sequence of muscular contractions in, 472

-, pulmonary, centre for, medullary, 473 ; antomatic action of, 474 ; influence by afferent impulses, 476 ; duplexity of its action, 479; ; effects of inflation and suction, 452; double action of vagus on, 483; nature of action, 484 ; two lateral halves of, 484 ; intluenced by character of blood-supply, 485 ; by deficiency of oxygen, 487, 1134; by excess of carbonic acid, 488 ; by other changes in the blood, 488; centre for apnooa, phenomena of, 489

- Cheyne-Stokes, 490; affected by clanges in atmospheric pressure, 491, 494 ; its effect on arterial pressure, 497; artificial, its effect on the circulation, 503 ; impeded, its effect on heart-beat, 507 ; as affected by muscular work, 510 ; regulation of temperature by, 642 ; as affected by sleep, 1155

—, facial and laryngeal, 438 ; cutaneous, 552; of muscle, 4ti9; of other tissues, 467 ; of the embryo, 1124; placental compared with branchial, 1126

Respiratory quotient in herbivora and carnivora compared, 629

Retina, see Eye, retina

Rheometer of Ludwig, 172

Rheoscopic frog, 102; current of action shewn in, 107

Rhythm, secondary respiratory, 490

Rhythmic clianges of calibre in artery, 262 ; beat of heart, spontaneous nature of, 239; ; beat of eardiac substance, 300 ; contractions of uterus during pregnancy, 1136

Rilss, movements of, in respiration, 434, 435

Rigor mortis, characteristics of, 86,87 ; development of carbonic acid during, 91-95; conversion of myosinogen into myosin during, 95; progressive order of, 128; accession of heat at onset of, (i4)

Rima glottidis, the, see Glottis

Ritter Valli law, 126

Rods, of retina, 122,926

Roots of spinal nerve, 141,677

Rosenthal's calorimeter, 635

Round ligament of uterus, contractions of, 1118

Roy, sphygmotonometer of, 220, 222; perfusion cannula of, 240

Roy and Adami, cardiometer of, 199, 202

Roy and Rolleston, method of recording endocardiac pressure, 193, 194

Sacculi of large intestine, peristaltic contractious of, 381

Saline solution, normal, defined (footnote), 16

Saliva, characters and properties of, 313 ; its properties, 314 ; its amylolytic action, 316, 387; characters of parotid, submaxillary, sublingual, and mixed, 318; amount daily secreted, 332 ; retlex secretion of, 333 ; centre for secretion of, in medulla oblongata, 335; of $\operatorname{dog}$, mechanical use of, 387; of the babe, $11+6$

Salivary glands, venous pulse observable in, 236

Salts, neutral, needed for formation of fibrin. 26 ; calcium, clotting as affected by, ib.; pulsation of 'washed-out' heart as affected by, 259 ; in foodstuffs, 312; absorption of, 414; as food, 630; importance of, for nutrition of nervous system, 630 ; essential to life of muscle, 652 ; in diet, 661

Santorini, cartilage of, 1070,1078

Sarcolemma, structure of, 86

Scaleni muscles, the, their service in respiration, 435

Scheiner's experiment, 817,873

Schlemm, cirenlar canal of, 969 ; passage of aqueous humour by means of, 974

Sclerotic coat of eye, development of, 837

Secretion of saliva, nervous mechanism of, 332; of gastric juice, 338 ; changes in gland coustituting act of, 340 ; changes in albuminous cells, 342 ; changes in mucous cells, 344 ; by central cells of stomach, 346 ; special substances elaborated during, 350; of pancreatic juice, 365 ; of bile, 3665 ; of urine, 524 ; glomerular and tubular in the kidney compared, 534; glomerular, its nature, 535; of sweat, 551 ; mechanism of, 555 ; of milk, 615

Secretions, carbonic acid in, 470; their constituents manufactured by glandular action, 538 
Segmentation of the ovum, 6

Self-ligestion, 352, 353

Self-induction, 62

Semen, chemical composition of, 1115; emission of, see Emission of semen

Semicircular canals, effects of injury to the, 730

Semilunar valves, their aetion, 184; dierotic wave as affected by closure of, 233,234

Sensations, special anditory, 998-1006; limits of, 1000; fusion of, 1003

—, cutaneous, 79S-811, 1037-1058; importance of contrast in, 1056 ; of pressure, 1037; localization of, 1039; of heat and cold, 1041; of pain, 1044; of touch and temperature, terminal orgaus necessary for, 1051 ; of pressure, terminal organs for, $10 j 4$; of heat, terminal organs for, different from those of cold, 1056; connection of with the muscular sense, 1066

- olfact ory, 1025, 1026

— of taste, $795,102 y-1036$; usually accompanied by other sensations, 1029; caused by electrical or mechanical stimuli, 1030; conditions of, 1031; localization of, 1032 ; distribution of terminal organs for, 1033; theories as to mode of origin, 1034 ; nerves for, 1035

_ velopment of, 793 ; general features of, 878 ; fusion of, 883 , 886; localization of, 885 ; of colour, 891 ; of colour, due to metabolic changes, 901; psychological features of, 129 ; their want of agreement with perceptions, 931; recurrent, 937

_- afferent, as factors in coordination of movement, 733 ; crossing of, from opposite hemispheres of brain, 804 ; development of, along the spinal cord, 805; transmission of, within the brain, 808; coordination of motor impulses regulated by, 95.5

Sense, the muscular, 1059; of movement, of position, and of effort, 1060 ; afferent impulses forming basis of, 1061

Sensibility, general, 1046; recurrent, 678 Serons cavities, fluid of, 402

_ fluids, artificial clotting of, 23 ; characters of, 402

Serum left after elotting of fibrin, 15 ; chemical composition of, 18-21, 46

Serum-albumin, its characters, 20; action of gastric juice and hydrochloric acid on, :324; importance of, in nutrition of muscle, 6.2

Sex, differences of, 1150

Shivering from cold, temperature raised by, 647

Shock, induction, 59; nature of, 697
Short-circuiting, 58

Sight, see Vision

singing, power of, dependent on nervous mechanism, 1087

Sinuses, venous, of brain, 828 ; placental, 1121; quality of blood in, 1125

Size, judgment of, 962

Skiu, as regulator of heat, 642; different kinds of seusations experienced tlirough the, 1037; as field of touch, 1039

Sleep, phenomena of, 1154; afferent impulses as affected by, 1154 ; respiration during, 1155 ; tle brain duriug, 1156

Smell, sensations of, 1025-1028; cortical area for, 794

Sobbing and sighing and sneezing, 511,512

Sodium chloride, its action on plasma, 22, 26; on muein, 313; glycholate and taurocholate, 3.56; hydrate, its effect on the heart, 259; snlphindigotate, excretion of by kidney, 5:34

Solidity, judgment of, 966

Somatic nerves, 141

Sound, musical, of contracting muscle, 123, 190; of the leart, 188-191, 213, 216 ; waves of, 987 ; complex, analysis of, 1013; psychical nature of appreciation of, 1016

Sounds, musical, characters of, 1021 ; appreciation of outwardness of, 1021; judgment of direction of, 1023; judgment of distance of, 1023

Spectrum, limitations of visibility of, 891

Speech, cortical area for, 76ti, 1085; a skilled movement, 766 ; movements of, bilateral, 766 ; causes of various imperfections of, 769; special mechanisms of, 1092-1100; sounds made use of in, 1092

Spermatozoa, movements of, 1114 ; action of, in the ovum, 1119

Sphincters of stomach, their action during digestion, 377 ; tone of dependent, on cord, 713

Sphincter ani, its nerve-supply, 381; versicæ, 545 ; iridis, 858

Sphygmograpli, Dudgeou's, 221

Sphygmoseope, 220

Spinal cord, see Cord, spinal

Spirometer, 428

Splanchnic nerves, 141, 271; inhibitor and angmentor fibres in, 385 ; ganglia, 141, 142 ; abdominal nerve, $266^{\circ}$

Spleen, the, possible formation of red corpuscles in, 36; rhythmical action of muscle fibres in, 171; its action during digestion, 368 ; movements of the, 579; chemical constituents of, 582 ; uric acid in the, 583

"Spleen-curve," 580

Spleen-pulp, destruction of red corpuscles in, 35 
Spot, blind, of retina, 916

ipring-manometer, 195

Stagnation stage of intlammation, 293

Stapes, or stirup bone, 986

Starch, action of saliva on, 314 ; chemieal composition of, 314 ; action of pancreatic juice on, 314, 315; 'animal,' 569 ; its value in diet, 661

Starvation, its effect in checking production of glycogen, 563,567 ; changes in body during, 620 ; fall of temperature attending, 649

Stearin, its presence in blood, 47 ; a constituent of animal fat, 606

Stellate gauglion, composite nature of, 255

Stereoscope, ocular movements affected by the, 953 ; principle of construction, 966

Stethometer of Burdon-Sanderson, 431

Stimuli defined, 53; various kinds of, 56 ; necessary characters of, 121

Stimulus, reflex actions varied according to nature of, 699

Stolnikoff's method of measuring the 'out-put' of the heart, 197

Stomach, nervous supply to, 339 ; its secretion of gastric juice, $i b ., 340$; movements of, 377 ; changes of food in the, 388

Storage of bile in gall-bladder, 367 ; of glycogen in the liver, 565,571

Striation of muscle tissue, 86

Stroma of red corpuscles, its composition, 32 ; embryonic formation of, from protoplasm, 35

Stromuhr of Ludwig described, 173

Strychnia, reflex action as affected by, 703

Substance, living, compared with dead, 3 , 86 ; metabolic elianges in, 39-41; chemical composition, 41

Substances, visual, hypothetical, 901

Suceus entericus, its nature and action, 363

Sugar, its presence in the blood, 48, 573; normally present in blood and chyle, 414; formed by saliva from starch, 316; course taken by, during digestion, 414 , 421: in rlabetic urine, 5zz; its conversion into glycogeu, 569; a product of netabolic changes, 570 ; its value in diet, 6661

Sulcus, erueial and sigmoid, of dog's brails, 740

Sulphur in proteids, 17, 630; in urine, 518

Suprarenal bodies, the, 601

Swallowing, nechanism of, 373 ; its action on tympanic air pressure, 996

Sweat, how secreted, 551, 555; composition of, 552
Sweat-fibres of different animals, course of, 557

Sweat-glands, 55 ; action of pilocarpin on, 557

Sweat-nerves, 558

Sweating in lower animals, 556 ; nervous mechanism of, 555 ; a retlex act, 557

Sympathetic system, fibres to plain muscles supplied by, 133 ; its connection with spinal nerves, 141

Syntonin, 88

Systole, auricular and ventricular, 181185 ; ventricular, a simple contraction, 190; and liastole, comparative duration of, 21:-214; amount of blood driven by each, 153, 217; work of papillary nuscles in, 183,184

Systolic plateau, the, 197, 202, 207, 216

Tactile sensations, 1037; localization of, 1039

Tambour, Marey's, 192

Tambour-sphygmoscope of Hürthle, 220

Tarsus of the eyelids, 976

Taurocholic aeid, 356

Tears, secretion of, 978

Tectorial membrane, 1011

'Teeth, order of their appearance, 1149

Tegmentum, the, 815

Temperature of living bodies, 2; as affecting elotting, 20 ; irritability, 125 , 12S; plain muscle, 134; ciliary action, 136 ; vaso-motor fibres, 271,288 ; action of gastric juice, 328 ; action of renuet, 330 ; point of saturation of gas, 440 ; absorption of oxygen by liquids, 462; the cntaneous vessels, 555; perspiration, 550, 555; storage of glycogen, 566 ; sense of taste, 1031

- of expired air, 440; regulation of, by evaporation from the skin, 555 ; by variations in loss of heat, 641,643 ; by the nervons system, 645, 646 ; of coldblooded animals, 641 ; of warm-blooded animals, 641; normal, range of, 647 ; higl, phenomena of deatl from, 649 ; low, effects of, 650 ; its relation to amount of food needed, 668 ; of body, maintenance of, 645 ; sensations of, 1041, 10.6; terminal organs for sensations of, 1056; sense of, in parts other than external skin, 1043

'Tendon reflexes, 'knee-jerk,' 705, 717

Tenonian cavity and 'Tenon's capsule, 971

Terminal organs, special sensations due to, 1053; for sense of touch, 1054; for sense of pressure, 1056 ; for sense of heat different from those for sense of cold, 1056 ; cutancous, their uature, 1057

Tetanic contraction, its nature, 55; due to repetition of stimuli, $i b,, 121$ 
Tetanus, phenomena of, 75-81, 111 ; carbonic acid evolved during, 94 ; exhaustiou of irritability from, 130

Thermopile, various forms of, 96

Thermotaxis, centre for, 646,647

Thirst, sensation of, 1048

Thoracic duct, characters of lymph from the, 400

Thorax, effect on blood-flow of pressure in the, 499,502

Thrombi, white, their nature, 45

Thymus body, structure of the, 602 ; nature and functious, 602 ; its size in infancy, 1148

Thyroid body, 600; diseases connected with, 601 ; in infancy, 1148

Thyroid-arytenoid muscles, 1077, 1078

Thyroid cartilage, 1073

Tigerstedt, his method of measuring cardiac output, 198

Tissue, conuective, 149,150

Tissues not indispensable for life, 3 ; classification of, 6 ; built up by the blood, 13; similarity of histological elemeuts of, 39 ; contractile, 55-138 ; nervous, 139-148; vascular, 149 et supra; digestive, $311-423$; respiratory, 421-512 ; relative proportions of, in the body, 619, 620; metabolism of, 651 ; their death gradual, 1159

Tone, arterial, 264, 284; general, 275283 ; bulbar vaso-motor centre for, 280-284; centre for, iu medulla, 279 ; maintained by automatic actiou of cord, 713

— of skeletal muscles, 713 ; due to central nervous system, 714

Tomes, musical, fundamental and partial, 999

Tongue, localization of taste sensations in, 1034

Tortoise, heart-beat in, independent of cardiac nerves, 213

Trachea, effect on respiration of its closure, 481

Tract, optic, course of, 785 ; ascending and descending antero-lateral, 686 ; cerebellar, 689 ; as to functions of, 807 ; median posterior, $6 \$ t$; as to functions of, 806 ; pyramidal, crossed, 686 ; direct, 687 ; relations to volition, $719,769,773$

Tracts, afferent, in spinal cord, 800 ; intermuncial, for afferent impulses, 807

Transudation into lymph spaces, 406 ; uot merely a filtration, 107 ; conditions determiuiug, 408; opposite currents of, through capillary walls, 407

Traube-Hering curves, their origin, 507 ; undulations in kidney, 528 ; variations in cerebral blood-pressure, 831,832

Tricuspid valves, 182

Trypsin, a constituent of pancreatic juice, 349,360 ; in the fotal pancreas, 1130

Trypsinogen, an antecedent of trypsin, 350

Tube, Fallopian, 1112

Tubules, uriniferous, epithelium of the, 533 ; work of the, 538; special substauces excreted by, 534

Tuniug-fork for the measurement of velocity, 67-71

Tympauum of ear, 983 ; conduction of sound through, 988 ; structure and relations, 986; membrane of, 981; muscles of the, 993 ; its connection with sense of outwardness of sounds, 1021

Tyrosin, a product of pancreatic digestion, 360 ; chemical composition, 361 ; a result of proteid decomposition outside the body, 598

Umbilical cord, 1134

- arteries, 1121; pressure in, 1124; venous blood in the, 1125

- vein, pressure iu, 1124

Undulations, respiratory, phenomena of, 498, 504; lumiuous, 891

Uuit, physiological, defined, 6

Urari, the nature of its action, 54, 83; diabetes in frogs produced by, 576

Urea, a coustituent of the blood, 18 ; absent from muscle-tissue, 93, 590; as nitrogenous waste, 93, 513, 589, 593, 598 ; its relations to kreatin, 590,591 ; its presence in the blood autecedent to kidney action, 538; its actiou on the tubules of kidney, 542 ; brought to the kidneys by the blood, 589, 59!; its formation in the liver, 594; synthesis of, 595 ; its relation with eyanogen compounds, 598; diminished excretion of, during starvation, 621; excretion of, not increased by exercise, 636; its kinship to vegetable alkaloids, $65 t ;$ a constituent of amniotic fluid, 1131

Ureter, peristaltic contractions of, 544

Uric acid, 596; chemical composition of, 516; relations to urea, circumstances determining its appearance, 596 ; constant pressure of in the spleen, 583

Urina hysterica, 543

Urine, composition and characters of, 515; normal organic constituents of, 516 , 520 ; inorgauic salts of, 517 ; average composition of, 520; abnormal constituents of, 520,577 ; secretion of, 524; vaso-motor mechanisms for, 525; its relations to the renal circulatiou, 533 ; albumillous, 537 ; pigments of, 540 ; discharge of, 54t; its secretion continuous, 544 ; changes of, in the bladder, 519; during starvation, 621 ; sugar present in diabetes, 575 ; of children, characteristics of, 1148 
Urobilin, 519

Use, muscle substance increased by, 128; skilled movements facilitated by, 779

"Uterine milk," 1122, 1129

Uterus, the, reception of the ovum by, 1111; changes in mucous membrane of, during menstruation, 1112 ; changes after impregnation, 1119; expansion of, during pregualcy, 113t; " retraction" of, 1137, 1139; rhythmical contractions of, during pregnaney, 1136 ; during ' labour,' 11:37; nerves of, 1141

Utricle, primoldial, 4 ; of labyrinth, 1009

Vagus, see Nerves, vagus

Valves of the veins, 171

- of the heart, their action in circulation, 182-185; sounds caused by their closure, 189, 190; tricuspid, their action, 183; semilunar, of the pulmonary artery, 185: their action, 186; semilunar, of aorta, 189, 207; Eustachian, 182

- ileo-crecal, mechanism of, 381 ; of the lymphatic vessels, 404

Vapour, aqucous, in expired air, 140

Vascular mechanism, 14!-307; main features of the apparatus, 150 ; main regulators of, a pparatus, 238, 24, 262

- walls, their action on the blood, 27 ; alteration of, in inflammation, 293,294

Vas deferens, contraction of in emission, 1117

Vaso-motor action, 262-288; arterial tone due to, 26t; effects of, 275 ; cutaneous and splanchnic, compensatory, 305; compensatory in loss and increase of blood, 296; simmmary of, 284 ; its rhythmic tendency, 507 ; regulation of temperature by, 612

- centre, 280-28t; limits of, 283; relations of, to other centres, 284

- fibres, constrictor, 266-269, 272; course of, $273,278,284$; loss of medulla in, 274,285 ; tonic action of, 275-283; chief parts of body supplied by, 278; dilator, 269; course of, 275; usually employed in reflex action, 278 ; retention of medulla in, 285

- functions of the central nervous system, 277 ; nerves of veins, 288

Vegetable cell, storage of metabolic products in, 654

- diet, results of, 664; large amount required, ific;

Veins, structure of, 152 ; minute, $i b$.; their capacity as compared with arteries, $i b$.; walls of , $i b$. ; blood-pressure in, 155,159 ; valves of, 171 ; vaso-motor nerves of, 288

Velocity of nervous impulse, 72,73 ; of muscular contraction, 83,84 ; compara- tive, of arterial, venous, and capillary circulation, 161-171; of arterial current, 172; of tlow in capillaries, 177 ; of flow in veins, $176 ;$; of blood current, 229 ; of pulse wave, $i b$.

Venous circulation, aids to, 171; pulse, 230 ; sinuses of brain, 828

Ventilation, positive and negative of lung, 482

Ventricle of heart of frog, its action in heart-beat, 2t0-2ts; of tortoise, isolated, spontaneous heart-beat of, 243

Ventricles of the heart, synchronism of their action, 152; their cbange of form in carliac cycle, 18.5 ; four stages of action of, 21:; tonic contraction of, 260 Vertigo, causes of, 733

Vesicles, cerebral, 835 ; optic, 835 ; otic, $9 \$ 0$

Vesicula seminales, their action in emission, 1117

Vestibule of ear, 980; parts of, 1008; perilymph cavity of, 1013

Vibrations of muscle sound, 122, 123; sonorous, longitudinal and transversal, 990 ; of the tympanic membrane, 989; through the auditory ossirles, 991; through the bones of the skull, 993

— of sound and light compared, 1000

- interference of, 1005

Vierordt, his hrematachometer, 173

Vieussens, a mulus of, 247, 253-256

Villi, the, columnar epithelium of, 417 ; pumping action of, 419 ; of chorion, foetal, 1121

Vision, 781; binocular, 782, 939-958; its action in judging of distance, 964 ; in judging of solidity, 966 ; mechanism of, 782; central apparatus for, 788 ; imperfections of, 790 ; dioptric mechanisms of, 834 , 8:39; astigmatism, 871 ; spherical aberration, 871; entoptic phenomena, 874; distinct limits of, 887 ; trichromic nature of, s9s, 90\%; colour, YoungHelmholtz's theory of, 898, 935; Hering's theory of, 900 , 935; field of, 782,929 , 940 ; corresponding or identical points, 942; struggle of the two fields of, 959 , 968

Visual areas in fovea centralis, 888 ; axis, 939; centres, lower, 793; impulses, development of, 916; impulses, origin of, 120 ; perceptions and judgments, 959 ; perceptions, psychical processes in, 961 ; plane. 940 ; purple, 922

_- sensations, 781-794; probable mode of development of, 793 ; fusion of, 886 , 887 ; in relation to visual perceptions, 929-938; simultaneous, 929

units, retinal, 889

Vitreons humour, the, 974

Vocal cords, 1071; voice produced by the 
vibration of the, 1074, 1085; tightening and slackeniug of the, 1081

Voice, the, 1070-1091; how produced, 1071; fundamental features of the, 1074; different qualities of, 1085 ; chest and head voices, 1058 ; registers of the, 1090; breaking of the, 1091

Volkmann, his hæmadromometer, 172

Voluutary movements, their tetanic character, 122; nervous mechauisms for, 739,777

Vomiting, mechanism of, 378

Vowel chamber, 1092

Vowels, how formed, 1093

Walking, how effected, 1102

Walls, vascular, their influence on transudatiou, 407

Warmth, its effect on skin action, 541

Waste matters, their discharge from the living body, 2 ; given out by amcebse, 4 ; not necessarily useless, 40,41 ; nitrogenous, 93 ; not increased by muscle contraction, $94,97,636$; elimination of, 513

Water, secretion of, by the glands, 351 ; varying amouut of, in living tissue, 409 ; its absorption into the portal system, 414; intestinal secretion of, 422 ; its discharge by the kidney, 541 ; by the skill, 555

Wave-pulse, dicrotic, origin of, 232-236; predicrotic, 235 ; anacrotic, 236

Waves of contraction, muscular, 83 ; of nerve and muscle impulse, 109; of sound, 987,999 ; of light, 891
Web of frog, arterial changes visible in, 262

Weber's law, \$80, 1001, 1038

Weight, human, eurve of, 1145

Whispering, how effected, 1095, 1100

White, sensations of, produced from mixing of colour sensations, 897

Willis, circle of, 827

Winking, how effected, 976; chief use, 978,979

"Word-deafuess," 797

Work, mechanical, in living body, 2; done by a muscle-nerve preparation, 119 et supra; amount of, done by the heart, 217 ; daily, estimate of, 634 ; mechanical source of energy of, 626 ; production of heat increased by, 644

Xanthin, a constituent of urine, 517, 597 ; present in the thymus, 602

Xanthoproteic test for protein, 17,18

Yawning, 511

Yellow spot, colour sensations as affected by, 913

Young-Helmholtz, theory of primary colour seusations, 898; as applied to colour blindness, 908 , 911; of simultaneous and successive contrasts, 935

Zinn zonule, passage of fluid by the, 975

Zone, peripheral of capillaries, 290 ; Lissauer's, 685, 686

Zymogens, 350, 352. 



\section{IN D EX}

To

\section{THE CHEMICAL BASIS OF THE ANIMAL BODY.}

Acetic acid, 1226

Acetone, 1226

Acliroodextrin, preparation of, 1212

Acid, $\alpha$-amido-caproic, 1244

, acetic, 1226

, amido-acetic, 1242

amilo-caproic, 1244

amido-ethylsulphonic, 1245

, amido-formic, 1242

,, ainido-pyro-tartaric, 1253

, amido-succinamic, 1253

, amido-succinic, 1252

, amido-sulpholactic (cystin), 1251

" amido-valeriauic, 1243

, aspartic or asparaginic, 1252

, benzoic, 1272

" butyric, 1227

, capric, 1227

" caproic, 1227

, caprylic, 1227

, carbamic, 1252

", carbolic or phenylic, 1278

, cholalic or cholic, 1286

," choleic, 1288

, cresylsulphuric, 1279

,, diamido-acetic, 1243

,, diamido-valerianic, 1244

", ethylene-lactic, $12: 34$

, ethylidene-lactic, 1233

,, fellic, 1288

, formic, 1225

, glutamic, 1253

,, glycerinpliosphoric, 1239

, glyeocholic, 1288

," glycolic, 1232

" glyeuronic, 1220

, hippuric, 1273

, hydrated parabanic, 1262

, hydrochloric, percentage of in gastric juice, 1191

, hydroxy-butyric, 1235

", hydroxy-propionic, 1232

", hydroxyquinoline-carboxylic, 1276

,, indoxyl-sulphuric, 1281

", isethionic, 1245

" isobutyric, 1227
Acid, kynurenic, 1276

," lactic, 1232

, lauric or laurostearic, 1228

, 'lithic,' 1260

, methyl-guanidinacetic, 1247

," methyl-glycine, 1243

, myristic, 1228

, oleic, 1229

," oxalic, 1235

" oxaluric, 1262

" palmitic, 12:8

, paralactic, 1233

", phenylic, 1278

,, phenyl-sulphuric, 1279

" propionic, 1226

," sareolactic, 1233

, skatoxyl-sulphuric, 1283

," stearic, 1228

," suceinic, 1236

," tauro-carbamic, 1246

,, taurocholic, 1289

, uric, 1258

, valeric or valerianic, 1227

Acid-albumin, 1169

Acids of the acetic series, 1225

, , , aromatic series, 1272

, " , glycolic series, 1232

, = , oleic (acrylic) series, 1229

, , oxalic series, 1235

, fatty, 1225

Acrolein, 1230

Acrylic series, acids of the, 1229

'Adenine,' 1264, 1269

Adipocire, formation of, 1228

Albumin, its decomposition by acids and enzymes, 1181

Albumins, derived and uative, 1166, 1168, 1169

Albuminates, 1169

chemistry of, 1168,1170

'Albuminose,' 1178

Albumoses and peptones, 1167

$$
\begin{array}{lll}
, \quad & \text { " } & \text { chemistry of, } 1178 \\
\text {, } & \text { preparation of, } 1182
\end{array}
$$


Alcohols of the human body, 1225

Aleurone-grains of plants, 1172

Alkali-albumin, 11tit, 1171

$\begin{array}{ll}, & \text { chemistry of, } 1171 \\ , & \text { preparation of, } 1171 \\ , & \text { its relations tocasein, } 1207\end{array}$

Alkaloids, certain vegetable, their relation to the xanthins, 1263, 12 it

vegetable, their resemblance to ptomaines, 1284,1285

Allantoin series, 1262

$$
\begin{array}{ll}
, & \text { sources of, } 1262 \\
, & \text { preparation, } 1263
\end{array}
$$

Alloxan, 1261

Amides and anico-acids, 1242

Amido-acids of the acetic series, 1242

$$
\begin{array}{lll}
, & \text {, } & \text { lactic series, } 1251 \\
, & \text {, } & \text { oxalic series, } 1252
\end{array}
$$

Amido-acetic acid, 1242

$a$-amido-caproic acid, 1244

Amido-cthylsulphonic acid, 1245

Amido-formic acid, 1242

Amido-pyro-tartaric acid, 1253

Amido-snceinamic acid, 1253

Amido-suceinic acid, 1252

Amido-sulpholactic acid (cystin), 1251

Anido-valerianic acid, 1203, 1243

Ammonia, its relations to urea, 1257

Amphicreatinin, 1286;

Amphopeptone, 1185

Amylodextrin, 1211

Animal body, chemical basis of the, 1163

'Animal gum,' Landwehr's, 1213

Antialbumate, 1181

$$
\text { , } \quad \text { characters of, } 1182
$$

Antialbumose, 1181

Antipeptone, 1181

$$
\text { characters of, } 1182
$$

Arginine, 1251

$$
\text { preparation of, } 1185
$$

Aromatic series, the, 1272

Ascirlians, tunicin prepared from mantle of, 1216

Ash of egg-albumin, 1165

, of proteids, 1165

,, of casein, 1207

,, of fibrin, 1177

Asparagine, 1253

Asparagine, its function in vegetable metabolism, 1253

Aspartic or asparaginic acid, 1252

Bananas, presence of isobutyric acid in, 1227

Barfoed's reagent, composition of, 1222 , note

Beans, preparation of inosit from, 1278

Benzoic acid, 1272

$$
\begin{gathered}
\text {, its relations to hippuric } \\
\text { acid, } 1273
\end{gathered}
$$

Benzol-glyein, 1273

Bile-acids, the, 1286

$$
\begin{aligned}
& \text { variations in, according to } \\
& \text { source, 1287 } \\
& \text { Pettenkofer's reaction for, } \\
& 12 s ?
\end{aligned}
$$

Bile, the mucin of, 1198

, and free fatty acids, emulsifying power of, 1231

Bile-pigments and their derivatives, 1303 , their relation to blood-

Bilicyanin, 1305 pigments, $130 \overline{7}$

Bilirubin, its identity with hamatoidin,

\begin{tabular}{|c|c|c|}
\hline " & ", & ," coloring matter \\
\hline ', & ", & $\begin{array}{l}\text { white, their connection } \\
\text { with fibrin for- } \\
\text { mation, } 1195\end{array}$ \\
\hline ," & ", & $\begin{array}{l}\text { glycogen present } \\
\text { in, } 1213\end{array}$ \\
\hline
\end{tabular}
$1: 03$

,, sources of, 1303

" preparation of, 1304

Biliverdin, 1305

Blood and bile, relationship between coloring natters of, $1300,1306-$ 1307

" dextrose a constituent of, 1217

," presence of sarcolactic acid in, 1233

Blood-corpuscles, red, proteid constituent of, 1174

Blood-plasma, fibrinogen a constituent of, 1174

, paraglobulin a constituent of, 1177

Blood-stains, detection of, 1301

Body, coloring matters of the, 1290

Brain-substance, neurokeratin obtained from, 1204

, ethyl-alcohol obtained from, 1225

, inosit present in, 1277

" preparation of cerebrin from, 1241

, protagon obtained from, 1240

Brucke's reagent for glycogen, 1214

Butter, fats present in, 1231

Butyric acid, 1227

,, fermentation, 1227

Cadaverin, 1286

Caffeï, its relations to xanthin, 1263,1271

," an excretionary product of plants, 1272

Calcium lactate, 1233

, oxialate, 1235

, salts, their action in elotting of casein, 1208 
Calcium sarcolactate, 1234

Calculi, cystic, 1252

" mulberry, 1235

Cane-sugar, digestive changes in, 1191, 13is)

," 'inversion' of, 12:1

,. bacterial fermentation of, 1234

Cane-sugar group, the, 1221

(a)ric (rutic) acid, 1227

Caprole acid, 1227

Caprylic acid, 1227

Carbanice acicl, 1252

Carbamide, 12.4

Carboliydrates, 1210-1:25

$$
\begin{gathered}
, \quad \text { in what form assimilated, } \\
1191,1217,1221
\end{gathered}
$$

Carbolic acid, 1278

Carbon-tloxicle hamoglobin, 1293

Carbon-monoxide hamoglobin, 1293

Carnine, 1267

Carnivora, nature of bile-acid of, 1288

Cartilage, elemistry of, 120)

Casein, a nucleo-albumin, 1166, note , chemistry and preparation of, $120 \%$

, action of remnin on, 1208

, its relations to nuclein, 1207

Caterpillars, formic acil in the secretion of certain, 1205

Cell-globulins, 117.3

Cells, chemical composition of nuclei of, 1205

Cellulose of stareh grains, 1210

, chemistry of, 1215

, digestion of, 1215

Celtis reticnlosi, presence of skatole in, $128 t$

Cerebrin, 1:241

Cerebrose, 12:20

Cetyl alcohol, 12:5

Cluacot's crystals, 1241

Cheese, curil of, produced only by rennin, $11 ! 4$

Chitin, preparation of, 1205

Chloroform, discrimination between enzymes and ferments by means of, $11 \times 9$

Chlorophane, 1313

Chloropliyll, starch formed under the influence of, 1210

Cholalic or clolic acid, 1286 ; ,, ., , , preparation, 1287

Cholecyanin or choleverdin, 1305

Choleic arid, 1288

Cholesterin, 12:3

$$
\begin{array}{ll}
., & \text { preparation, } 1237 \\
., & \text { reactions of, } 1237
\end{array}
$$

Choletelin, 1306;

Cholin, 1230

Chondrigen, 1:01
Chondrin, preparation and reactions of, $1201-120^{\circ}$

Chondrommeoid, 1202

Chromogens, 1307, 1310

Chromophanes of the retina, 1313

Chrysocreatinine, 1286

action of light on the, 131;

Chyle, presence of globulins in, 1174

, dextrose a constituent of, 1217

Clotting of casein, 1208

,, of blood, 1174, 1195

, of nmscle-plasma, 1174, 1195

Collagen, 11999

,$\quad$ its conversion into gelatin, 1199)

Copler, its presence in animal pigments, 1296

Corpus lutenu, pisment of the, 1315

Corpuscles, see Blond-corpuseles

Creatinine, 12ts

Creatine, 1247

$$
\begin{array}{ll}
\text {, } & \text { reparation, } 1250 \\
\text {, } & \text { reacfions of, } 1250
\end{array}
$$

, its relation to creatinine, 1217

, preparation of, 1248

, its relation to urea, 1248

Cresol, 127!

Cresylsulpluric aeid, 12799

Crystallin, chemistry and preparation of: $117^{2}$

Crystals, Chareot's, 1241

, cholesterin erystals, 1237

, creatine, 1247

, proteirl-containing, 1165

, Teichmann, 129!9

Cresylsulphuric acid, 1279

Cystiu, 1251

Deuteroalbmuose, 1184

Deuterogelatose, 1201

Dextrins, the, preparation of, 1211

Dextrose (glneose, gràje-sugar), 1217

, fermentations of, $121 \mathrm{~s}$

, diserinination of from maltose, 1262

Diabetes, chemical clianges in, 12:6, 12:35 , pentoses in cases of, 1217

Diamido-acetic acid, 1243

, valerianic acicl, 1244

, caproic arcid, 1245

Diastase, formation of maltose by, 1222

Digestion of proteirls, prolucts of, 1178

, intestinal, 1190, 1192, 1193

.. gastric, 11!1

,. tryptic, 1192

, of cellulose, 1215

Diseases, ptomaine-formation by organisms charavteristic of specific, 1285

Drechsel, 1165

Dysalbumose, 1184

Dysilysin, 1288

Dyspeptone, Meissner's, 1179, 1181 
Egg-albumin, chemistry of, 1168

,, preparation, 1168

, $\quad$ crystalline form of, 1168

Egg-yolk, the proteid constitueuts of, 1172

,. pigment of, 1315

Elastin, preparation of, 1203

Elastoses, 1214t

Euzymes, 11s8, 1196

,, chatacteristics of, 1188

, discrimination of from organized ferments, 1189

" of the pancreas, 1190

, of gastric juice, 1191

" their action on cane-sugar, 1221

Epidermal structures, keratin the chief ('onstituent of, 1204

Erythrodextrin, 1211

Ethyl-alcohol, presence of, in animal tissues, 1225

Ethyl-glycol, 1232

Ethylene-lactic acid, 1234

Ethylidene-lactic acid, 1233

Extract of Meat, preparation of sarcolactic acid from, 1234

, " preparation of carnine from, 1267

presence of hypoxanthine in, 1268

Fats, their derivatives and allies, 1225

," the neutral, 1229

, complex nitrogenous, 1238

Fehling's fluid, composition of, 1222, note

Fellic acid, 1288

Ferment, restriction of the term, 1188, note

Ferments, organized, discrimination of, from enzymes, 1189

Fermentations of dextrose, 1218

$$
\text { " lactic, of souring milk, }
$$
1224

bacterial, of cane-sugar, 1234

Fibrin, 1175

,, varying forms of, 1176

, ash of, 1177

" its action on hydrogen dioxide, 1177

Fibrin-ferment, 1195

Fibrinogen, 1174, 1195

Fishes, presence of keratinin in muscles of, 1249

Fond. the thrce classes of, 1164

Fornic acid, secreted by ants and certain caterpillars, $12: 5$

Formica rufa, formic acid, excreted by, 1225

Fruits, presence of lævulose in, 1219

Fuscin, 1312
Galactose, or cerebrose, reactions of, 1220

, a product of lactose, 1224

Gall-stones, cholesterin a constituent of, 1237

,, bilirubin prepared from, 130

Gallois' test for inosite, 1278

Gastric glauds, pepsinogen in the cells of, 1191

Gastric juice, the proteolytic enzyme of, 1191

percentage of hydrochloric acid in, 1191

Gelatin or glutin, 1199

Gelatin peptones, preparation of, 12001201

Gclatoses, the, 1201

Gland, submaxillary, mucin of, 1198

Globin, 1175

Globulin of the crystalline lens, 1172

, as compared with myosiu and fibrin, 1177

Globulins, the, 1167,1175

" their conversion into acillalbumin, 1170

, their relations to fibrin, 1276

Glucosamine, 1205

Glucose, 1:217

Glutamic or glutaminic acid, $125: 3$

Glutin or gelatin, 1199

Glutoses, the, 1200

Glycerin (glycerol), the chemistry of, 1231

Glycerinphosphoric acid, 1239

Glycin, glyeocoll, or glycocine, 1242

, preparation of, 1243

," a product of gelatin decomposition, 1200

Glycocholic acid, preparation of, 1288

Glycogen, hepatic, its conversion into sugar, 1214

the animal analogue of stareh, 1213

1. its presence in various tissues and in molluses, 1213

preparation of, 1213

," reactions of, 1214-1215

" diminution of, in muscles during aetivity, 1234

Glycolic acid series, 1232

Glyeuronic aciel, chenistry of, 1220

, , compounds of, 1290

Gmelin's reaction for bile-pigments, 1305

Gout, areumulation of uric acid salts in, 1258

Grape-sugar, chemistry of, 1217

Guanidine, its comnection with ereatine, 1247,1271

, $\quad$, , with irea, 1264 
Guanidine, chemistry of, 1271

synthesis of, 1271

Guanine, connexious of, with uric acid, 1264

, preparation of, 1270

, its conversion into xanthine, 1271

, Capraniea's reactions, 1271

Guano, Peruvian, preparation of uric acid from, 1260

, preparation of guanine from, 1270

Hæmatin, 1297

,, spectroscopy of, 1297

Hrematoidin, 1302

Hrematoporphyriu (iron-free hæmatin), 1301

Hamin (hrematin hydrochloride), 1299

Hamochronogen, 1291, 1296;

Hamocyanin, 1295

Hæmoglobin, 1290

, in the plasma of invertebrates, 1291, note carbon-monoxide, 1293

, nitric-oxide, 1293

" carbon-dioxide, 1293

Hemialbumose of Kühne, 1180-11s1

, characters of, 1183

, various forms of, 1183

Hemipeptone, 1180, and note, 1180

, how obtained, 46

Hemiprotein of Schützenberger, 1180

Herbivora, digestion of cellulose by the, 1215

, preclominance of stearin in fat of, 1231

, sources of hippuric acid in the, $127: 3$

, pigment of the bile of the, 1304

Heteroalbumose, 1184

Heteroxanthine, 1261-1266

Hippuric acid, 1273

, reactions, 1274

Histohrmatins, 1298

Honey, levulose present in, 1219

Hoppe-seyler's reaction for xanthine, $1260 ;$

Humus pigments, 1309

Hydrazones, 1216;

Hydrobilirubin, 1306

Hydroquinone, $12 \$ 0$

its probable identity with urobilin, 1307

Hydrogen, evolution of, in butyric fermentation, 121!)

Hydroxy-benzene, 1278

Hydroxy-butyric acid, 1235

Hydroxy-propionic acid, 1232

Hydroxyquinoline-carboxylic acid, 1276
Hypoxanthine, $126 t$

discrimination of, from
xanthin, 1266
sources of, 1268
its relation to carnine,
1267

Indican, urinary, 1281,1310

Indigo series, the, 1250-1284

Indigo-blue, formation of, 1282

Indigo-carmine, 128:

Indole, its combination with glycuronic acid, 1220

, sources of, 1280

," reactions of, 1281

, fate of, in the body, 1310

Indoxyl-pigments, 1310

Indoxyl-sulphurie aeid, 1281

Inosite, preparation of, 1277

, reactions of, 1278

Intestine, small, hydrolyzing power of secretion of, 1191

, variable reaction of its contents, 1193

its inverting action on canesugar, 1221

Inversion of lavulose, 1219

, of cane-sugar, 1221

Invertebrates, chitin in the exoskeletons of, 1205

, tunicinin the exoskeletons of, 1216

, haemoglobin in bloodplasma of, 1291 , note

, hæmocyanin in bloodplasma of, 1295

Iron-free hamatin, 1301

Isethionic acid, 1245

Isobuty ric acid, 1227

Isomerism, 1233

Jaffé's reaction of creatinine, 1250

,, test for indiean, 1282

, , skatoxyl, 1284

Kepliir, preparation of, from mare's milk, 1224

Keratin, composition of, 1204

Keratillose, 1204

Küunne's hemialbumose. 1180

Kumys, preparation of, from mare's milk, $122 t$

Kynurenie acid, 1276

Lactalbumin, 1208

Lactic acid series, the, 1232-1235

Lactic (hydroxy-propionic) acid, 1232 , its presence in the body, 1232

Lactic fermentation of dextrose, 1219

Lactose, preparation and reactions of, 1223 
Lactose, lictic fermentation of, 1224

, its ineapability of assinilation, 1224

Lævulose, svitlesis of, 1216

$$
\text { , clemistry of, 121! }
$$

Larlacein, or anyloid substance, 1168

$$
, \quad \text { chemistry of, } 1186 \text {; }
$$

Lamric or lanrostearic acid, 1228

Lecithin, 1238

,. a eonstitnent of (2)-yolk, 1172

, preparation of, 12:35

.. eonstitution of. 12:3!

Lens, erystalline, globulin of the, 1172

Leucin, 1:4t

, preparation of, 1245

, a result of deromposition of proteicls, 1181, 1157, 1199, 1200, $1203,1204,1244$

Leukomaines, 12sis

Leukopsin, 1314

Liebig's lixtract of Meat, 1234, 1267, 1268

Light, its bleaching action on chloroplianes, 1313,1314

Lipochrin in certain retinal epithelia, 131:-1313

Lipochrnmes or luteins, 1315

Lipolyn, 11!;

'Lithates, '1260

'Jithic acid,' 1260

Liver, eonversion of glycogen into sugar in the, 1215

, its work in the formation of urea, 1258

Liver-sugar, its apparent identity with dextrose, 1215

Lobster, chitin obtained from the exoskeleton of, $120 \%$

Jupins, xanthine found in, 1265

Lutein, souree of, $1: 315$

Luteins, the, 1315

Lymph, dextrose a constituent of, 1217

Lysatine, 1250

, preparation, 1250)

, its relation to area, 1250

- Lysatin,' 1187

Lysine, 124.j

Malt-seellings, xanthine present in, 1265 Maltodextrin, 1:212

Maltose, its conversion into dextrose, $1190,1191,1222$

,, formatiou of, 1222

Mantle of 'Tunisata, tunicin prepared from, 1211;

Marrow of bones, hemialloum in in, 1183

Marsh-gas fermentation of rellulose, 1215

Meissner, 'parapeptone of,' 117s

, his researohes on the products of rigestion, 1179

Mclanin, urinary, $1: 309$

Melanogen, 1310
Metapeptone, Meissuer's, 1179

Methemoglobin, preparation of, 1:94

, spectroseoply of, 1295

, its relation to oxylapemoglobin, 129)

Methyl-glycine, 1213

Methyl-granidinacetie acid, 1247

Methyl-indole, 12's'

Methylphenol, 127!

Miero-organisms, their appearanee in nrine, $1196 ;$

, $\quad$ conversion of dextrose by means of, 1219

Milk, preparation of casein from, $120 \mathrm{~s}$

, elotting of, 1:20s

"luman, and of cows compared, $120 ! 9$

" conversion of lactose into lactic acid in, 1219

, varying amounts of lactose in, $12: 3$

,, alcoholie fermentation of, 12:24

Milk-sngar, 1203

Millon's reagent for proteids, 1166, note, $119 \%$

Monosacelharides, 1216

Mucin, reactions of, $119 \%$

, chief sompes of, $119 \%$

Mnrexile test for uric acid, 1261

Mnsele, othyl-alcohol obtained from, 1225

Muscles, presence of glycogen in the, 1:213 ,,$\quad$ of inusite in the, $127 \%$

," , of laetic aeid in the, $12: 33$

, , of sarcolactic acid in the, 1233

Insele-enzyme, 1195

of hypoxanthine, 1268

Musele-plasma, elotting of, 1175, 1196

'Mrelin forms' of lecithin, 1238

Mroglobulin, 117\%

Myohituatin, 12:9)

Myosin, rhemistry of, 1174

Myosin-ferment, 1195

Myosinogen, 1175

Myristic aciu, 1228

Nerves, molnllaterl, nenrokeratin obtainer from, 1204

Neurin, 1240

Neurokeratin, 1204

Neutral fats, 12:39?

Nitric-oxide hamoglobin, 1293

Nitrogen in nrine, method of determination, 12: 6 ;

Nitrogenoms bodies allied to proteids, $119 ;$

Nnelein, preparation and properties of, 120)5-1:206;

Nueleo-albumins, reactions of, 1206

Nucleo-albunin of bile, 1209

Nucleo-proteids, 1209 
Olcfines, relation of oleic acids to the, 1229

Uleic acid, a constituent of human fat, $122 !)-1230$

(Hein (tri-olein), preparation of, 1230

Ortho-dihydroxybenzene, 1279

Osazones, the, 1216 ,. formation of the, 1216

()ssein, 1199

()xalic acid series, the, 1225

$$
\text { , , , anido-acids of the, } 1252
$$

Oxaluric acid, 1262

Oxy-hremoglobin, preparation, 12:91

$$
\begin{aligned}
& \text {, } \quad \text { difference in crystals of, from } \\
& \text { (Jifferent sources. 1291 } \\
& \text { spectra of, } 1292
\end{aligned}
$$

Oyster, presence of glycogen in the, 1213

Palm oil, palmitin obtained from, 1230

l'almitic acid, 1225, 1230

Palmitin (tri-palmitiu), 1230

Pancreas, the amylolytic enzyme of the, 1190

Pancreatic juice, its action on starch, 1202,1223

Papaïn, elastin dissolved by, 1204

Para-dilighoxybenzene, 1280

l'araglobuliu (serum-globuliu), chemistry of, 1173

Paramyosinogen, 1175

Parapeptone, 1178,1179

Paraxanthine, 1267

$$
\text { ,, isomer of theobromine, } \mathbf{1 2 6 7}
$$

Pentuse gronp) 1217

$$
\text { , in diabetes, } 1217
$$

P'epsin, preparation of, 1191

l'epsinogen, an antecedent of pepsin, 1191

Peptones, 116i, 1178

," preparation of, 1184

, their absorption and fate in the body, 1186

Petroleum-ether, 1272

Pettenkof $c r$ 's reaction for bile-acids, 1289

Phenol, 1278

Phenylic aciı, 1278

Phenyl-glueosazone, 1218

Phenyl-hydrazin, as reagent for the sugars, 1216

, in formation of osazones, 1216

, its action on miltose, 1223

Phenyl-lactosazone, 1224

Phenyl-malt osazone, preparation of, 1222

Phenyl-sulphuric acid, 1279

Phosphorus, its presence in casein, 1207

, a constituent of mucin, 1198

, percentage of in nuclein, 1206

., its presence in protagon, 1240

Pigments of the animal body, 1290

$$
\begin{array}{ll}
, & \text { humus, } 1: 309 \\
,, & \text { indoxyl-, } 1310 \\
,, & \text { retinal, } 1312
\end{array}
$$

Pigments of nrine, 1307

, , as affecterl by drugs, 1311

," of the suprarenal bodies, 1317

Piotrowski's reaction for proteils, 1166

Piria's reaction for tyrosine, 1276

Plants, oceurrence of leucin in, 1244

, proteid metabolism of, 1253

, alkaluidal principles of, 1271

Propeptome, 11s:

Propionic atid, 1226, 1232

Protagni, 12 40

Protalbumose, 1184

Proteids, 1164-1189

," composition of, 1165-1187

, (rystalline, 116.5

,. ash of, 1165

, general reactions of, 1166

, (lassification of, 116 if

,. coagulated, 1167, 1177

, ligestive changes of, 1178, 1179

, duplexity of molecule of, $\mathbf{1 1 7 9}$

", their decomposition by acids, $1178,1181,1187$

prodncts of decomposition of, 118i;

" theories of the coustitution of, 1187

Protogelatose, 1201

Psendoxanthine, 1286;

Ptomaines, the, 1284-1286

," their similarity to vegetable alkaloids, 1285

Ptyalin, preparations of, 1190

, its action on starch, 1190

Ptyalinogen, in saliva of horse, 1190

Purple, visual, 1313

Pus-cells, nnclein prepared from, 1205

Pus-corpuscles, presence of glycogen in, 1213

Putrefactive ormanisms, action on cellulose of, 1215

Putrescin, 1286

Pyocyauin, 1317

Pyoxanthose, 1317

Pyrocatechin, 1279

Rennet, milk-curdling ferment, 1194 note , use of, in cheese-making, 1194

Rennin, its clotting action on milk, 120s, 1194

,. its enzymic nature, 1195

Renninogen, 1194

Reticulin, 1201

Retina, pigments of the, 1312-1315

Rhorlophane, 1313

Rhodopsin, 1313

Rotation of light, mode of measurement of, 1218

Saccharose, 1221

Saliva, ptyalin a constituent of, 1190 
Saliva, mucin a constituent of, 1197

its action on starch-paste, 1222

Sarcolactic or paralactic acid, 1233-1234

Sarkosin, 1243

Scherer's test for inosite, 1278

Schiff's reaction for uric acil, 1261

Seidel's reaction for inosite, 1278

Serum-albumin, chemistry of, 1169

Serum-globnlin, 1173

Serum-lutein, 1316

Skatol, its combination with glycuronic acill, 1220

" preparations of, 1283

", reactions of, 1283

, ocenrence of, in a vegetable tissue, $128 t$

, compounds of, 1311

Skatoxyl-pigments, 1311

Skatoxyl-sulphuric acid, 1283

Soaps, formation of, with stearic and palmitic acids, 1228

, composition of, 1231

Soda, sulplindigotate of, 1282

Soluble starch, preparation of, 1211

spermaceti, cetyl-alcohol obtained from, 1225

Spermine, 1242

Spleen, presence of inosite in the, $\mathbf{1 2 7 7}$

Starch, hydrolysis of, by ptyalin, 1190

, , by pancreatic secre-

sources of, 1210 tion, 1190

" soluble, 1211

" its conversion into sugar in the body, 1212

Starch group of the carbohydrates, 1210 Starch-paste, action of saliva on, 1222

Stearic acid, 1228

Stearin (tri-stearin), preparation of, 1230

Stokes's fluid, 1297

Strecker's test for xanthine, 1266

Stroma of red blool-corpuseles, proteid constituent of, 1174

Sub-maxillary glaud, muein of the, 1197 Snceinic acid, 1236

Sugar, diabetic, 1215

Sugars, the, chemistry of, 1216

, discrimination of, 1216

Sulphindigotate of soda, 1282

Sulphur, a constituent of fibrin, 1177

, its presence in lardacein, 1186

Sulphur, its presence in keratin, 1204

, a constituent of cystin, $125^{\circ}$

Sulphuric acid, 1187

Suprarenal bodies, pigment of, 1317

Sweat, presence of urea in, 1254

Syntonin, chemistry of, 1170

, definition of, 1178, note

Taurine, 1245

," preparation, 1246
Tauro-carbamic acid, 1246

Taurocholic acid, preparation of, 1289

Tea, traces of xanthine present in, 1265

," hypoxanthine present in, 1268.

Teichman's crystals (hremin), 1299

Tendons, mucin of the, 1198

Tetronerythrin, sonrces of, 1316

Theine, its relations to xanthine, 12fit

Theobromine, its relations to xanthine, $1263,12 \nmid 4,1271$

, isomer of paraxanthine, 1266

, an excretionary product of plants, 1271

Theophylline, its relations to xanthine, 1264

Tinned meats, possible development of ptomaines in, 1285

Torula ureat, enzyme developed by, 1196

Tourace, presence of copper in phumage of, 1296

Toxines, 1285

'Irimethylviuyl-ammonium hydroxide, 1240

Trypsin, its action on fibrin, 1177

, its action on proteids, 1178, 1179

, preparations of, 1192

, zymogen of, 1193

Tunicin, 1215

Turacin, 1296

Tyrein, formation of, in clotting of casein, 1208

Tyrosine, a result of decomposition of proteids, 1181

,

a product of decomposition of nncin, 1199

" constitution of, 1274

, preparation of, $\mathbf{1 2 7 5}$

," Hoffman's reaction for, 1275

Umbilical cord, mucin of the, 1199

Urea, 125t-1263

, average daily excretion of, 1254

, synthesis of, 1255,1257

," nitrate of, 1255

, oxalate of, $1255^{5}$

, detection of, in solutions, 1257

" quantitative determination of, 1257

, its relations to nric acid, 1261

Urea-ferment, its enzymic nature, 1196

Ureas, substituted, 125i-1258

Uric acid, 1258-1263

, salts of, 1260

, preparation of, 1260

, tests for, 1261

Urinary melanin, 1309

Urine, fermentative changes in, 1196

, pathologieal changes in, 1196,1217 , $1235,1277,1283,1286,1309,1311$ presence of creatininc in, 1248

", urea the chief nitrogenous constituent of, 1254 
Urine, determination of nitrogen in, 1257 , phenylsulphuric acid in, 1279

" pyrocatechin in, 1279

,, pigments of, 1307,1308

Urobilin, its identity with hydrobilirubill, $1306,130 \%$

, preparation of, 1308

,, spectra of, 1308

Urochrome, 1308

Uroerythrin, 1308

Urohama toporphyrin, 1308

Valeric or valerianic acid, 1227

Vegetable alkaloids, their analogy with ptomaines, 1284,1285

Vegetable tissues, allantoin found in, 1262 , , , xanthine found in, 1265 , , , occurrence of hypoxanthine in, 1268

, , occnrrence of guanine in, 1270

, , , ocenrrence of skatolin, 1284

Visual-purple, 1313

,. preparation in solution, 1313

, action of light and reagents on, 1314
Visual-purple, spectroscopic properties, 1314

Vitellin, chemistry and preparation of, 1172

Weidel's reaction for xanthine, 1265

Weyl's reaction for creatinine, 1250

Xanthine group, the, 1263-1272

Xantline, its relationship to uric acid, 1264

", preparation of, 1265

" reactions for, 1265

, derivatives of, 1271

, physiological action of, 1272

Xanthocreatinine, 1286

Xanthophane, 1313

Xanthoproteic reaction for proteids, 1166

Xanthopsin, 1314

Zine lactate, 1233

, sarcolactate, 1234

Zymogen, all antecedent of the enzymes, 1189

,, of pepsin, 1191

,. of trypsin, 1193

Zymolysis, 1183 , note, 1188 



\section{Works on Medical Science and Cognate Subjects.}

PUBLISHED BY

\section{THE MACMILLAN COMPANY.}

ANDERSON (J. W.). - Lectures on Medical Nursing. I6mo. 75 cents.

Essentials of Physical Diagnosis of the Chest and Abdomen. 75 cents.

"Dr. Anderson has written in a style as compact as possible, with a view to the economy of time, yet has satisfactorily covered his subject." $-N$. Y. Times.

BALFOUR. - The Senile Heart. Its Symptoms, Sequelæ, and Treatment. By

George William Balfour, M.D. $12 \mathrm{mo}$. Cloth. \$1.50.

"Cleverly written in a thoroughly interesting and practical style." - American Medico-Surgical Bulletin.

"A very clearly expressed and readable treatise upon a subject of great interest and importance. We can heartily commend this work as a valuable contribution to the literature of old age." - Medical Record.

BALLANCE (C. A.) and EDMUNDS (W.).- A Treatise on the Ligation of the Great Arteries in Continuity. With Illustrations. Royal 8vo. $\$ 4.00$.

"This book will become a classic. It is on a timely and important subject, and is the fruit of deep and thorough research." - Medical and Surgical Yournal.

BARWELL (R.). - Lateral Curvature of the Spine. \$I.75.

BICKERTON (T. H.).- On Colour-blindness. Illustrated. Nature Series. (In the press.)

BLYTH (A. W.). - A Manual of Public Health. 8vo. \$5.25.

Lectures on Sanitary Law. 8vo. \$2.50.

BRODIE. - Dissections Illustrated. A Graphic Handbook for Students of Human Anatomy. By C. Gordon Brodie, F.R.C.S., Senior Demonstrator of Anatomy, Middlesex Hospital Medical School ; Assistant Surgeon, Northwest London Hospital. With plates drawn and lithographed by PERCY Highley. Complete in one volume. \$9.00. 
Part I. The Upper Limb. With seventeen Coloured Plates, two-thirds natural size, and ten Diagrams. $\$ 2.00$.

"The plates are exceedingly well drawn and placed on the stone. . . The explanatory letter. press is clear and concise." - The Lancet.

"Of such high merit are the plates of this, the first volume, that the publication of the three remaining numbers of the series will be awaited with impatience by all students of anatomy." - The Hospital.

"The scheme is admirably carried out and the plates most reliable.... We can confidently recommend the work." -Guy's Hospital Gazette.

Part II. The Lower Limb. With twenty Coloured Plates and six Diagrams. $\$ 2.50$.

Part III. Head, Neck, and Thorax. With 20 Plates. \$2.50.

Part IV. Abdomen. With I6 Plates, 13 Diagrams. \$2.50.

BRUNTON. - Works by T. LAUder Brunton, M.D., F.R.S.

On Disorders of Digestion: their Consequences and Treatment. 8vo. \$2.50.

Pharmacology and Therapeutics; or, Medicine Past and Present. \$I.50.

An Introduction to Modern Therapeutics. Svo. \$I.50.

BURDON-SANDERSON (J.).- Memoirs on the Physiology of Nerve, of Muscle, and of the Electrical Organ. Edited by J. BurdoNSANDERSON, M.D., F.R.SS.L. \& E. 8vo. \$5.25.

CARRINGTON (R. E.).-A Manual of Dissections of the Human Body. Second Edition, revised and enlarged. \$2.50.

CARTER (R. B.).-Eyesight: Good and Bad. Second Edition, enlarged. With Illustrations. 8vo. \$I.00.

CREIGHTON. - A History of Epidemics in Great Britain. By Charles Creighton, M.A., M.D., formerly Demonstrator of Anatomy in the University of Cambridge. 8vo.

Vol. I. From A.D. 664 to the Extinction of the Plague. \$4.50.

Vol. II. From the Extinction of the Plague to the Present Time. Svo. $\$ 5.00$.

CROCKER. - Atlas of the Diseases of the Skin. In a Series of Illustra. tions (coloured) from Original Drawings, with Descriptive Letterpress. By H. Radcliffe Crocker, M.D., F.R.C.P., Physician, Skin Department, University College Hospital, London; formerly Physician to the East London Hospital for Children ; Examiner in Medicine, Apothecaries' Hall, London. Superbly illustrated in colours. The plates ( 96 in number) represent fully 200 life-size figures, and are reproduced by chromo-lithography from the original watercolour drawings. Each fasciculus consists of six full-page plates, with descriptive letterpress. The letterpress not only describes the cases from which the drawings are taken, but also gives a general account of each disease, its differential diagnosis, and treatment. To be issued in 16 folio bi-monthly parts. Each part, \$6.00. Sold only on subscription to the eritire set.

DOLAN (T. M.).-Pasteur and Rabies. By Thomas M. Dolan, M.D., F.R.C.S. I6mo. 50 cents.

DOWNIE. - Clinical Manual for the Study of Diseases of the Throat. By James Walker Downie, M.B. \$2.50. 
ECCLES. - Sciatica. A Record of Clinical Observations on the Causes, Nature, and Treatment of Sixty-eight Cases. By A. Symons Eccles. \$1.00.

"The generally accepted views touching the origin, symptoms, and treatment of sciatica, are lucidly presented in this brief record of personal observations. The author does not aim to furnish an elaborate monograph on the subject. Is a clinical contribution to sciatica the little book will be read with interest." - Medical Record.

The Practice of Massage: Its Physiological Effects and Therapeutic Uses. By A. Symons Eccles, M.B. Aberd., Member Royal College Surgeons, England; Fellow Royal Medical and Chirurgical Society, London; Member Neurological Society, London; etc. 8vo. \$2.5o.

FAYRER. - On the Preservation of Health in India. By Sir J. FAYRER, K.C.S.1., M.D., F.R.S. ISmo. 35 cents.

FEARNLEY (W.). - A Course of Elementary Practical Histology. \$2.0o.

FITZGERALD (C. E.).-Lectures on Physiology, Hygiene, etc. With Illustrations and Diagrams. 75 cents.

FLÜCKIGER (F. A.) and HANBURY (D.). - Pharmacographia. Second Edition, revised. 8vo. $\$ 5.00$.

FOSTer. - A Text-Book of Physiology. By Michael Foster, M.A., M.D., LL.D., F.R.S., Professor of Physiology in the University of Cambridge, and Fellow of Trinity College, Cambridge. 8vo. With Illustrations. Sixth Edition, largely revised.

Part I. Blood; the Tissues of Movement; the Vascular Mechanism. $\$ 2.60$.

Part II. The Tissues of Chemical Action; Nutrition. $\$ 2.60$. (In the press.)

Part III. The Central Nervous System. \$1.75.

Part IV. The Central Nervous System (concluded); The Tissues and Mechanisms of Reproduction. \$2.00.

Part V. (Appendix) The Chemical Basis of the Animal Body. By A. Sheridan Lea, M.A., Sc.D., F.R.S. \$1.75.

"The present edition is more than largely revised. Much of it is re-written, and it is brought quite abreast with the latest wave of progress of physiological science. A chief merit of this work is its judicial temper, a strict sifting of fact from fiction, the discouragement of conclusions based on inadequate data, and small liking shown toward fanciful though fascinating hypotheses, and the avowal that to many questions, and some of foremost interest and moment, no satisfying answers can yet be given." - Nerv England Medical Fournal.

"It is in all respects an ideal text-book. It is only the physiologist, who has devoted time to the study of some branch of the great science, who can read between the lines of this wonderfully generalized account, and can see upon what an intimate and extensive knowledge these generalizations are founded. It is only the teacher who can appreciate the judicial balancing of evidence and the power of presenting the conclusions in such clear and lucid forms. But by every one the rare modesty of the author in keeping the element of self so entirely in the background must be appreciated. Reviewing this volume as a whole, we are justified in saying that it is the only thoroughly good text-book of physiology in the English language, and that it is probably the best text-book in any language." Edinburgh Medical fournal.

"From its first issue as a single octavo volume of moderate size, in 1876 , it has so grown that each of the five Parts is, in this sixth edition, nearly as large as the entire original work. From the beginning it was regarded as a masterpiece, and at once took a prominent place among text-books of physiology. ... If one seeks for the reason 'of the high estimate in which this work is held on both sides of the Atlantic, by the most advanced students as well as by general readers, it may be found in the beauty and simplicity of the style, in the lack of personal prejudice on the part of the author, in 
his thorough familiarity with the progress of physiological knowledge, and in the rare judgment with which purely hypothetical ideas and those founded on sufficient evidence are discriminated. The work is therefore a most admirable guide to physiological progress as well as general physiological knowledge." - The Vation.

FOSTER. - Text-Book of Physiology. In one volume. By MichaeL Foster, M.A., M.D., etc, etc. Abridged and revised from the Sixth Edition of the Author's larger W'ork published in five octavo volumes. Svo. Cloth, $\$ 5.00$; Sheep, $\$ 5.50$.

This new Edition will contain all the Illustrations included in the larger work, and will be published in one octavo volume of about rooo pages. It will contain all of the author's more important additions to the complete work, and be like the sixth edition of that copyrighted in this country.

FOミTER (M.) and BALFOUR (F. M.). - Practical Embryology. With Illustrations. \$2.60.

"A book especially adapted to the needs of medical students, who will find in it all that is most essential for them to know in the elements of vertebrate embryology." - Academy.

FOSTER (M.) and LANGLEY (J. N.). - A Course of Elementary Practical Physiology. Fifth Edition, enlarged. \$2.00.

"This work will prove of great value to the teacher of physiology, as an aid to the preparation of an eminently practical course of lectures and demonstrations of elementary experimental physiology. Its chief utility, however, will be to the intelligent student, who, armed with a dissecting case, a microscope, and the book, will be enabled to pass his summer vacation in a manner at once interesting and profitable." - Medical Record.

FOSTER (M.) and SHORE (L. E.). - Elementary Physiology. 16 mo. Illustrated. 75 cents.

"It is a veritable multum in parvo, and will be fully appreciated by those for whom it is intended." - American Medico-Surgical Bulletin.

"Nothing at once so scientific and so simple has appeared on the subject. It is unreservedly commended as a text-book for secondary schools." - The Educational Reviez.

GALTON. - Healthy Hospitals. Observations on some points connected with

Hospital Construction. With Illustrations. By Sir Douglas Galton, K.C.B.,

F.R.S. \$2.75.

"This is a valuable contribution to the literature of a most important subject. The eminence in engineering circles of Sir Douglas Galton would alone determine its merit. After a brief introduction into the origin of hospitals, he defines them as places 'not only for the reception and cure of the sick poor .. . but also as technical schools in which the medical student must learn his profession, and as experimental workshops in which the matured physician or surgeon carries on scientific research.' . . While the subject-matter is largely technical, it is presented in a clear style, and its meaning is clear to any intelligent person. We commend Sir Douglas Galton's book to all builders of hospitals," Medical Record.

GAMGEE. - A Text-book of the Physiological Chemistry of the Animal Body. Including an Account of the Chemical Changes occurring in Disease. By Arthur Gamgee, M.D., F.R.S. 8vo.

Vol. I. The Proteids. $\$ 4 \cdot 50$.

Vol. II. The Physiological Chemistry of Digestion. With two chromolithographic Charts by Spillon and Wilkinson. \$4.50.

GILLIES. - The Theory and Practice of Counter-irritation. By $\mathrm{H}$. Cameron Gillies, M.D. 8vo. \$2.50.

GOODFELLOW(J.). - The Dietetic Value of Bread. \$1.50.

GRESWELL (D. A.).-A Contribution to the Natural History of Scar. latina. 8vo. \$2.50. 
GRIFFITHS (W. H.). - Lessons on Prescriptions. 18 mo. \$1.00.

HAMILTON (D. J.). - A Systematic and Practical Text-book o Pathology.

Vol. I. Technical. General Pathological Processes. Diseases of Special Organs. \$vo. $\$ 6.25$.

Vol. II. Diseases of Special Organs (continued). Bacteriology, etc. 2 Parts. Sro. Each $\$ 5.00$.

"This is beyond question the most complete work on Pathology in the English language to-day. The author has accomplished his laborious task most successfully. We cannot better criticise it than by saying it is beyond criticism."-Canada Medical Record.

HAVILAND. - The Geographical Distribution of Disease in Great

Britain. By Alfred Havilani. Second Edition, 8vo. $\$ 4.50$.

HAWKINS (H. P.). - On Diseases of the Vermiform Appendix. With a consideration of the symptoms and treatment of the resulting forms of Peritonitis. By Herbert P. Hawkins, M.A., M.D., F.R.C.P. Svo. Cloth, pp. 139. \$2.25, net.

HILTON (J.). - Rest and Pain. Edited by W. H. A. Jacobson. \$2.00.

HOBLYN. - Dictionary of Medical Terms. Twelfth Edition. \$2.25.

HULLAH (J.).-The Cultivation of the Speaking Voice. Clarendon Press Series. Second Edition. I6mo. 60 cents.

HUXLEY (T. H.). - Lessons in Elementary Physiology. With numerous Illustrations. Revised by Dr. Foster. I6mo. \$I.Io.

"Unquestionably the clearest and most complete elementary treatise on this subject that we possess in any language. In this adınirable little work; the outlines of the physiology of the human body are set forth in the plainest English, with a simplicity and earnestness of purpose which command our highest admiration." - Westminster Review.

JENNER ( 1849-1879. Svo. \$4.00.

"This volume is a fitting exemplar of the careful and scientific work that has placed the author in the foremost rank of his profession. It cannot fail to prove interesting to physicians." - Neru York Medical fournal.

Clinical Lectures and Essays on Rickets. Tuberculosis, Abdominal Tumours, and Other Subjects. Svo. \$4.00.

JEX-BLAKE (SOPHIA). - The Care of Infants. I 8 mo. 40 cents.

KANTHACK (A.A.). - A Course of Elementary Practical Bacteriology, including Bacteriological Analysis and Chemistry. By A. A. KANTHAck, Lecturer on Pathology and Bacteriology, St. Bartholomew's Hospital, and J. H. Drysdale. I2mo. Cloth. \$I.Io.

KIMBER. - Text-book of Anatomy and Physiology for Nurses. Compiled by Diana Clifforn Kimber, graduate of Bellevue Training School; Assistant Superintendent New York City Training School, Blackwell's Island, N. Y.; formerly Assistant Superintendent Illinois Training School, Chicago, Ill. Fully Illustrated. Svo. \$2.50.

"A well written and unusually thorough work for its purpose. Although intended for nurses, it would make an admirable text-book in our schools and academies." - American Medico-Surgical Bulletin. 
KLEIN (E.).-Micro-organisms and Disease. Revised, with 120 Engravings. \$1.00.

The Bacteria in Asiatic Cholera. \$1.25.

KOCHER. - Text-Book of Operative Surgery. By Dr. THEODOR Kocher, Prufessor of Surgery and Director of the Surgical Clinic in the University of Bern. Translated with the Special Authority of the Author from the Second Revised and Enlarged German Edition by Harold J. Stiles, M.B., F.R.C.S., Edin., Senior Demonstrator of Surgery and formerly Demonstrator of Anatomy in the University of Edinburgh, etc. With 185 Illustrations. 8vo. \$3.5o.

MACALISTER. - A Text-Book of Physical Anthropology. In press.

MACDONALD (GRevtlle). - A Treatise on Diseases of the Nose and its Accessory Cavities. Second Edition. Svo. \$2.50.

MACEWEN. - Pyogenic Infective Diseases of the Brain and Spinal Cord, Meningitis, Abscess of Brain, Infective Sinus Thrombosis. By William Macewen, M.D., Glasgow. Svo. Buckram. \$6.oo.

Atlas of Head Sections. 53 Engraved Copperplates of Frozen Sections of the Head, and 53 Key Plates with Descriptive Tests. Folio. Bound in buckram. \$21.00.

"The 'Atlas' should certainly be in the hands of every surgeon who aspires to enter the field of brain surgery, as a careful inspection of these plates will teach more than many volumes written upon the subject. ... The plates themselves have been executed with the greatest care, the illustrations being very fine photogravures from photographic plates, about two-thirds of the actual size of the head. The appearance of the book is a credit both to the author and to the publisher." - Medical Record.

M'KENDRICK (J. G.). - A Text-book of Physiology. In 2 volumes.

Vol. I. General Physiology. 8vo. \$4.00.

Vol. II. Special Physiology. 8vo. \$6.0o.

Life in Motion; or, Muscle and Nerve. Illustrated. \$I.50.

MACLAGAN (T.). - The Germ Theory. 8vo. \$3.oo.

MACLEAN (W. C.). - Diseases of Tropical Climates. \$3.00.

MARSHALL. - Pain, Pleasure, and Æsthetics. By Henry Rutgers MarSHALL. Svo. \$3.00.

"The book must necessarily have a deep interest for physicians, and especially neurologists, for it is concerned largely with the explanation of a phenomenon-pain, the prevention and relief of which form such a good portion of their duty. We can heartily recommend it to their perusal and study." - Fournal of Nervous and Mental Disease.

MAUDSLEY. - The Pathology of Mind : A Study of its Distempers, Deformities, and Disorders. Second Edition. By Henry Maudsley, M.D. 8vo. Cloth, pp. 571. \$5.00, net.

MERCIER (C.). - The Nervous System and the Mind. 8vo. \$4.00.

MIERS (H. A.) and CROSSKEY (R.). - The Soil in Relation to Health.

By Henry A. Miers and Roger Crosskey. \$1.io.

MIGULA (W.). - An Introduction to Practical Bacteriology. Translated

by M. Camprell, and edited by H. J. Campbel, M.D. Illustrated. \$r.6o.

"Its practical character fits it for a guide for students desiring a knowledge of the elementary principles of this interesting and important topic." - Popular Science Monthly. 
MIVART (ST. Geokge). - Lessons in Elementary Anatomy. With 400 Illustrations. 16mo. \$1.75.

PALMBERG (A.). - A Treatise on Public Health and its Application in Different European Countries (England, France, Belgium, Germany, Austria, Sweden, and Finland). Edited by Arthur Newsholme. 8vo. $\$ 5.00$.

" The book is rich in descriptions and illustrations of sanitary appliances, modern and practical." - Popular Science Morthiy.

PIFFARD (H. G.). - An Elementary Treatise on Diseases of the Skin. With Illustrations. 8vo. \$4.00.

REYNOLDS. - A Primer of Hygiene. By Ernest S. Reynolds, M.D. (lond.), Senior Physician to the Ancoats Hospital, Manchester, etc., etc. With 50 lllustrations. 1Smo. 35 cents.

"This is a rare bit of text-book making. The pedagogical features are eminently creditable; the literary work, from the text-book standpoint, is admirable. The illustrations are simple, but ideal in their clearness and illustrative qualities." - Fournal of Education.

RICHARDSON, - Works by B. W. RICHARDSON, M.D.

On Alcohol. Paper, 30 cents.

Diseases of Modem Life. New Edition. (In the Press.)

ROLLESTON and KANTHACK. - Manual of Practical Morbid Anatomy. Being a Hand-book for the Post-mortem Room. Cambridge Natural Science Manuals: Biological Series. 1 2mo. \$1.60.

"To those interested in post-mortem work, this book can be heartily recommended as a most valuable, complete, and efficient guide." - American Medico-Surgical Bulletin.

STEVEN (J. L.). - Practical Pathology. \$1.75.

STRAHAN. - Suicide and Insanity. A Physiological and Sociological Study. By S. A. K. Strahan, M.D., Author of "Marriage and Disease," "Instinctive Criminality," etc. $12 \mathrm{mo}$, cloth. \$1.75.

“The subject treated of in Dr. Strahan's excellent little work has always possessed, and, in the nature of things, must in future possess keen interest, not only for physicians and jurists, moralists and sociologists, but for all thinking persons. That such a fundamental instinct as the desire to live should ever so far yield, either to reason or imperative craving, as to culminate in self-destruction, is a phenomenon so opposed to the principles of animal existence as to affect even the most unimaginative very much as would the sudden reversal of the law of gravitation."-Medical Record.

"The book is a most interesting contribution to the literature of a subject that is of increasing interest to the alienist and psychologist." $-N$. Y. Medical Fournal.

"The author has presented a very interesting and unbiased study of a topic that is engaging more and more attention, for it is not one of the least of the charges against modern society that its organ1. zation is such that men and women are unwilling to continue as associates thereof." - Popular Science Monthly.

THORNE. - Diphtheria. Its Natural History and Prevention. By R. Thorne $\$ 2.00$. 
VON KAHLDEN. - Methods of Pathological Histology. By C. voN Kahiden, Assistant Professor of l'athology in the University of Freiburg. Translated and Edited by H. Morley Fletcher, M.D., Casualty Physician to St. Bartholomew's Hospital, and Assistant Demonstrator of Physiology in the Medical School. With introduction by G. Sims Woodhead, M.D., Director of the Laboratories of the Royal Colleges of Physicians and Surgeons, etc. \$I.40.

"This manual of methods reconmends itself to every student of histological research. In the form of footnotes are presented additions and modifications of great practical value, which bring the book up to date. There are chapters on the microscope and apparatus requisite for histological work, examination of fresh tissues, methods of hardening, decalcification of tissues, methods of embedding, infection of tissues, preparing and mounting sections, staining processes, bacteria, moulds, fungi, animal parasites, and microscopical examinations for medico-legal purposes." - Nedical Summary.

WHiTE (W. Hale, M.D.). - A Text-book of General Therapeutics. \$2.50.

"In no other text-book of Therapeutics can the same interesting, varied, and necessary material be found so usefully, successfully, and agreeably presented." $-N$. E. Medical Monthly.

WILLIAMS.-Aero-Therapeutics. The Treatment of Lung Diseases by Climate. Being the Lumleian Lectures for 1893 delivered before the Royal College of Physicians. With an address on the high altitudes of Colorado. By Charles Theonore Willians, M.D., Senior Physician to the Hospital for Consumption and Diseases of the Chest, Brompton, and late President of the Royal Meteorological Society. 8vo, cloth. \$2.00.

"It is not an exhaustive work, it is true, but it is sufficiently full for the practitioner's needs, accurate, practical, and charmingly written. For those who wish a small work on the climatic treatment of pulmonary diseases, written by a recognized authority on the subject, and presenting practically and usefully most reliable information on a very important subject, we must heartily recommend Dr. Williams's work." N. Y. Medical Fournal.

WILLOUGHBY (E. F.).-Handbook of Public Health and Demography. $16 \mathrm{mo}$. \$I.5O.

"An admirably concise and lucid treatment of preventive medicine, alike commendable to general readers, teachers, students, physicians, and sanitary inspectors." - The Sanitarian.

ZIEGLLER. - A Text-book of Pathological Anatomy and Pathogenesis.

With Illustrations. Svo.

Part I. Special Pathological Anatomy. Sections I.-VIII. \$4.0o.

Part II. Special Pathological Anatomy. Sections IX.-XII. \$4.0o.

Part III. General Pathological Anatomy. In preparation.

ZIEHEN (THEODORE). - Introduction to Physiological Psychology.

Translated by C. C. VAN Lifw and Dr. OtTo Beyer. With 21 Illustrations. $\$ 1.50$.

"The book can be highly recommended to all who wish a short and clear outline of the science." - Eaucational Rezieru.

"The general reading of this book would, we think, do more for the schools of America than the investment of the same amount of time and money in anything else." - Fournal of Education.

\section{THE MACMILLAN COMPANY.}

NEW YORK: 66 FIFTH AVENUE.

CHICAGO: ROOM 23, AUDITORIUM. 




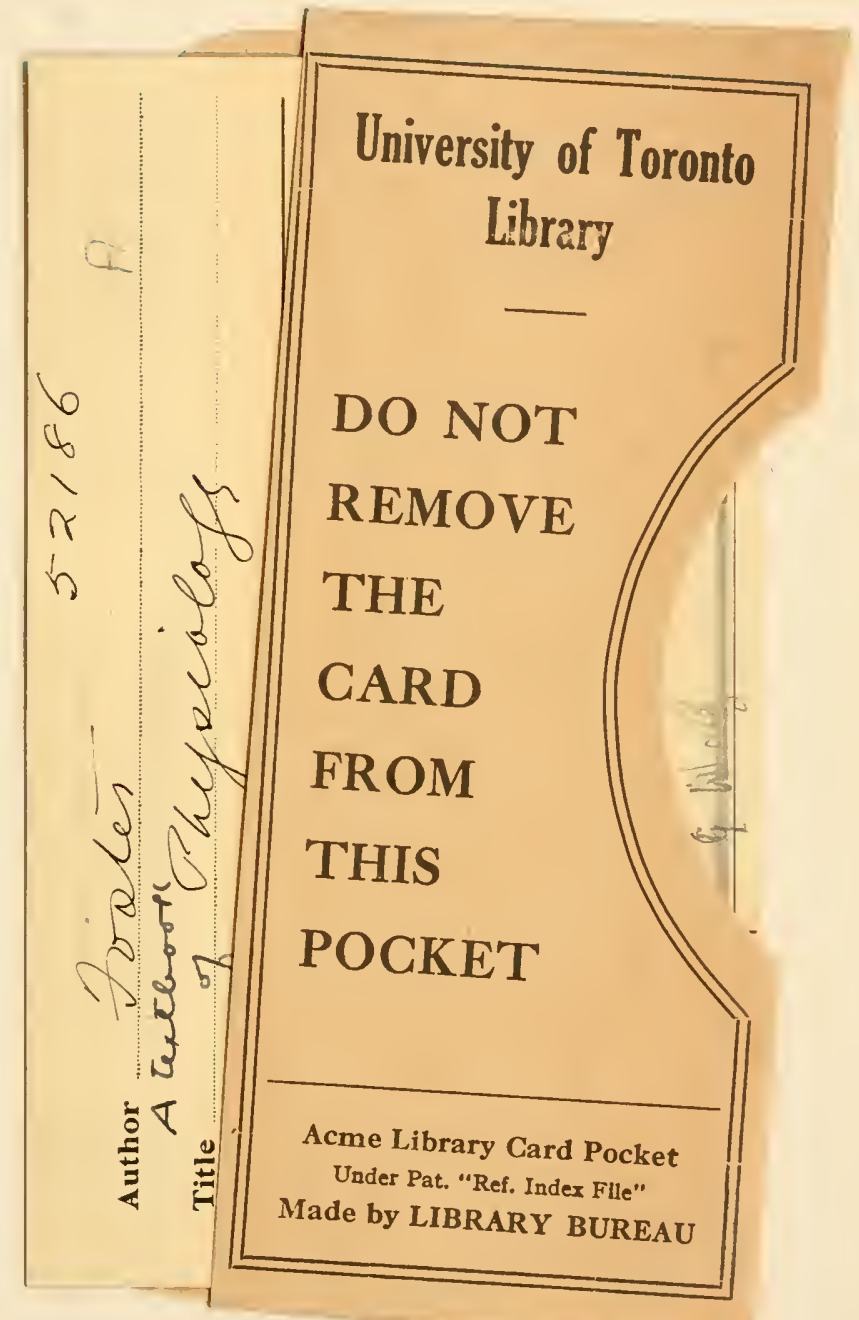


